\title{
Diet, physical activity and behavioural interventions for the treatment of overweight or obese children from the age of 6 to 11 years (Review)
}

Mead E, Brown T, Rees K, Azevedo LB, Whittaker V, Jones D, Olajide J, Mainardi GM, Corpeleijn E, O'Malley C, Beardsmore E, Al-Khudairy L, Baur L, Metzendorf MI, Demaio A, Ells LJ

Mead E, Brown T, Rees K, Azevedo LB, Whittaker V, Jones D, Olajide J, Mainardi GM, Corpeleijn E, O'Malley C, Beardsmore E, AlKhudairy L, Baur L, Metzendorf MI, Demaio A, Ells LJ.

Diet, physical activity and behavioural interventions for the treatment of overweight or obese children from the age of 6 to 11 years.

Cochrane Database of Systematic Reviews 2017, Issue 6. Art. No.: CD012651.

DOI: 10.1002/14651858.CD012651.

www.cochranelibrary.com

Diet, physical activity and behavioural interventions for the treatment of overweight or obese children from the 
TABLE OF CONTENTS

HEADER 1

ABSTRACT

PLAIN LANGUAGE SUMMARY

SUMMARY OF FINDINGS

BACKGROUND

OBJECTIVES

METHODS

RESULTS

Figure 1.

Figure 2.

Figure 3.

Figure 4.

Figure 5.

Figure 6.

DISCUSSION

AUTHORS' CONCLUSIONS

ACKNOWLEDGEMENTS

REFERENCES

CHARACTERISTICS OF STUDIES

DATA AND ANALYSES

Analysis 1.1. Comparison 1 Behaviour-changing interventions versus no treatment/usual care, Outcome 1 Change in BMI (all trials).

Analysis 1.2. Comparison 1 Behaviour-changing interventions versus no treatment/usual care, Outcome 2 Change in BMI z score (all trials).

Analysis 1.3. Comparison 1 Behaviour-changing interventions versus no treatment/usual care, Outcome 3 Change in weight (all trials).

Analysis 1.4. Comparison 1 Behaviour-changing interventions versus no treatment/usual care, Outcome 4 Serious adverse events.

Analysis 1.5. Comparison 1 Behaviour-changing interventions versus no treatment/usual care, Outcome 5 Health-related quality of life (parent-report measures).

Analysis 1.6. Comparison 1 Behaviour-changing interventions versus no treatment/usual care, Outcome 6 Health-related quality of life (child-report measures).

Analysis 1.7. Comparison 1 Behaviour-changing interventions versus no treatment/usual care, Outcome 7 Self-esteem (Harter global score).

Analysis 1.8. Comparison 1 Behaviour-changing interventions versus no treatment/usual care, Outcome 8 Waist circumference.

Analysis 1.9. Comparison 1 Behaviour-changing interventions versus no treatment/usual care, Outcome 9 Overweight. ........ Analysis 1.10. Comparison 1 Behaviour-changing interventions versus no treatment/usual care, Outcome 10 Body fat. .......... Analysis 1.11. Comparison 1 Behaviour-changing interventions versus no treatment/usual care, Outcome 11 Diet. ................ Analysis 1.12. Comparison 1 Behaviour-changing interventions versus no treatment/usual care, Outcome 12 Television viewing.

Analysis 1.13. Comparison 1 Behaviour-changing interventions versus no treatment/usual care, Outcome 13 Physical activity (accelerometer MVPA).

Analysis 1.14. Comparison 1 Behaviour-changing interventions versus no treatment/usual care, Outcome 14 Change in BMI type of control.

Analysis 1.15. Comparison 1 Behaviour-changing interventions versus no treatment/usual care, Outcome 15 Change in BMI z score - type of control.

Analysis 1.16. Comparison 1 Behaviour-changing interventions versus no treatment/usual care, Outcome 16 Change in weight - type of control.

Analysis 1.17. Comparison 1 Behaviour-changing interventions versus no treatment/usual care, Outcome 17 Change in BMI type of intervention.

Analysis 1.18. Comparison 1 Behaviour-changing interventions versus no treatment/usual care, Outcome $18 \mathrm{Change}$ in $\mathrm{BMI}$ z score - type of intervention.

Diet, physical activity and behavioural interventions for the treatment of overweight or obese children from the age of 6 to 11 years 
Analysis 1.19. Comparison 1 Behaviour-changing interventions versus no treatment/usual care, Outcome 19 Change in weight - type of intervention.

Analysis 1.20. Comparison 1 Behaviour-changing interventions versus no treatment/usual care, Outcome 20 Change in BMI attrition bias.

Analysis 1.21. Comparison 1 Behaviour-changing interventions versus no treatment/usual care, Outcome 21 Change in BMI z score - attrition bias.

Analysis 1.22. Comparison 1 Behaviour-changing interventions versus no treatment/usual care, Outcome 22 Change in weight - attrition bias.

Analysis 1.23. Comparison 1 Behaviour-changing interventions versus no treatment/usual care, Outcome 23 Change in weight - setting.

Analysis 1.24. Comparison 1 Behaviour-changing interventions versus no treatment/usual care, Outcome 24 Change in BMI z score - setting.

Analysis 1.25. Comparison 1 Behaviour-changing interventions versus no treatment/usual care, Outcome 25 Change in BMI setting.

Analysis 1.26. Comparison 1 Behaviour-changing interventions versus no treatment/usual care, Outcome 26 Change in BMI post-intervention follow-up.

Analysis 1.27. Comparison 1 Behaviour-changing interventions versus no treatment/usual care, Outcome 27 Change in BMI z score - post-intervention follow-up.

Analysis 1.28. Comparison 1 Behaviour-changing interventions versus no treatment/usual care, Outcome 28 Change in weight - post-intervention follow-up.

Analysis 1.29. Comparison 1 Behaviour-changing interventions versus no treatment/usual care, Outcome 29 Change in BMI type of parental involvement.

Analysis 1.30. Comparison 1 Behaviour-changing interventions versus no treatment/usual care, Outcome $30 \mathrm{Change} \mathrm{in} \mathrm{BMI} \mathrm{z}$ score - type of parental involvement.

Analysis 1.31. Comparison 1 Behaviour-changing interventions versus no treatment/usual care, Outcome 31 Change in weight - type of parental involvement.

Analysis 1.32. Comparison 1 Behaviour-changing interventions versus no treatment/usual care, Outcome 32 Change in BMI z score - baseline BMI z score.

Analysis 2.1. Comparison 2 Behaviour-changing interventions plus component versus behaviour-changing intervention without component, Outcome 1 Change in BMI.

Analysis 2.2. Comparison 2 Behaviour-changing interventions plus component versus behaviour-changing intervention without component, Outcome 2 Change in BMI z score.

Analysis 2.3. Comparison 2 Behaviour-changing interventions plus component versus behaviour-changing intervention without component, Outcome 3 Change in weight.

Analysis 3.1. Comparison 3 Cluster RCTs versus comparator, Outcome 1 Change in BMI.

Analysis 4.1. Comparison 4 Maintenance intervention versus no treatment/usual care, Outcome 1 Change in BMI z score. ....... ADDITIONAL TABLES

APPENDICES

WHAT'S NEW

HISTORY

CONTRIBUTIONS OF AUTHORS

DECLARATIONS OF INTEREST

SOURCES OF SUPPORT

DIFFERENCES BETWEEN PROTOCOL AND REVIEW 
[Intervention Review]

\section{Diet, physical activity and behavioural interventions for the treatment of overweight or obese children from the age of 6 to 11 years}

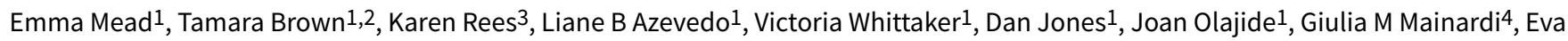
Corpeleijn ${ }^{5}$, Claire O'Malley², Elizabeth Beardsmore ${ }^{1}$, Lena Al-Khudairy³, Louise Baur6, Maria-Inti Metzendorf7 , Alessandro Demaio ${ }^{8}$, Louisa J Ells 1

${ }_{1}^{1}$ Health and Social Care Institute, Teesside University, Middlesbrough, UK. 2 School of Medicine, Pharmacy and Health, Durham University Queen's Campus, Durham, UK. 3Division of Health Sciences, Warwick Medical School, University of Warwick, Coventry, UK. 4Department of Preventive Medicine, School of Medicine, University of São Paulo, São Paulo, Brazil. ${ }^{5}$ Department of Epidemiology, University Medical Centre Groningen, Groningen, Netherlands. ${ }^{6}$ Department of Paediatrics and Child Health, The University of Sydney, Westmead, Australia. ${ }^{7}$ Cochrane Metabolic and Endocrine Disorders Group, Institute of General Practice, Medical Faculty of the HeinrichHeine-University Düsseldorf, Düsseldorf, Germany. ${ }^{8}$ The World Health Organization, Geneva, Switzerland

Contact address: Louisa J Ells, Health and Social Care Institute, Teesside University, Middlesbrough, TS1 3BA, UK. L.Ells@tees.ac.uk.

Editorial group: Cochrane Metabolic and Endocrine Disorders Group.

Publication status and date: New, published in Issue 6, 2017.

Citation: Mead E, Brown T, Rees K, Azevedo LB, Whittaker V, Jones D, Olajide J, Mainardi GM, Corpeleijn E, O'Malley C, Beardsmore E, Al-Khudairy L, Baur L, Metzendorf MI, Demaio A, Ells LJ. Diet, physical activity and behavioural interventions for the treatment of overweight or obese children from the age of 6 to 11 years. Cochrane Database of Systematic Reviews 2017, Issue 6. Art. No.: CD012651. DOI: 10.1002/14651858.CD012651.

Copyright $(2017$ The Cochrane Collaboration. Published by John Wiley \& Sons, Ltd.

\section{A B S T R A C T}

\section{Background}

Child and adolescent overweight and obesity has increased globally, and can be associated with significant short- and long-term health consequences. This is an update of a Cochrane review published first in 2003, and updated previously in 2009. However, the update has now been split into six reviews addressing different childhood obesity treatments at different ages.

\section{Objectives}

To assess the effects of diet, physical activity and behavioural interventions (behaviour-changing interventions) for the treatment of overweight or obese children aged 6 to 11 years.

\section{Search methods}

We searched CENTRAL, MEDLINE, Embase, PsycINFO, CINAHL, LILACS as well as trial registers ClinicalTrials.gov and ICTRP Search Portal. We checked references of studies and systematic reviews. We did not apply any language restrictions. The date of the last search was July 2016 for all databases.

\section{Selection criteria}

We selected randomised controlled trials (RCTs) of diet, physical activity, and behavioural interventions (behaviour-changing interventions) for treating overweight or obese children aged 6 to 11 years, with a minimum of six months' follow-up. We excluded interventions that specifically dealt with the treatment of eating disorders or type 2 diabetes, or included participants with a secondary or syndromic cause of obesity. 


\section{Data collection and analysis}

Two review authors independently screened references, extracted data, assessed risk of bias, and evaluated the quality of the evidence using the GRADE instrument. We contacted study authors for additional information. We carried out meta-analyses according to the statistical guidelines in the Cochrane Handbook for Systematic Reviews of Interventions.

\section{Main results}

We included 70 RCTs with a total of 8461 participants randomised to either the intervention or control groups. The number of participants per trial ranged from 16 to 686 . Fifty-five trials compared a behaviour-changing intervention with no treatment/usual care control and 15 evaluated the effectiveness of adding an additional component to a behaviour-changing intervention. Sixty-four trials were parallel RCTs, and four were cluster RCTs. Sixty-four trials were multicomponent, two were diet only and four were physical activity only interventions. Ten trials had more than two arms. The overall quality of the evidence was low or very low and 62 trials had a high risk of bias for at least one criterion. Total duration of trials ranged from six months to three years. The median age of participants was 10 years old and the median BMI z score was 2.2.

Primary analyses demonstrated that behaviour-changing interventions compared to no treatment/usual care control at longest followup reduced BMI, BMI z score and weight. Mean difference (MD) in BMI was $-0.53 \mathrm{~kg} / \mathrm{m}^{2}$ (95\% confidence interval $(\mathrm{Cl})-0.82$ to -0.24$) ; \mathrm{P}<$ 0.00001; 24 trials; 2785 participants; low-quality evidence. MD in BMI z score was -0.06 units $(95 \% \mathrm{Cl}-0.10$ to -0.02$) ; \mathrm{P}=0.001 ; 37$ trials; 4019 participants; low-quality evidence and MD in weight was $-1.45 \mathrm{~kg}(95 \% \mathrm{Cl}-1.88$ to -1.02$) ; \mathrm{P}<0.00001 ; 17$ trials; 1774 participants; lowquality evidence.

Thirty-one trials reported on serious adverse events, with 29 trials reporting zero occurrences RR 0.57 (95\% Cl 0.17 to 1.93 ); P = 0.37 ; $4 / 2105$ participants in the behaviour-changing intervention groups compared with $7 / 1991$ participants in the comparator groups). Few trials reported health-related quality of life or behaviour change outcomes, and none of the analyses demonstrated a substantial difference in these outcomes between intervention and control. In two trials reporting on minutes per day of TV viewing, a small reduction of 6.6 minutes per day $(95 \% \mathrm{Cl}-12.88$ to -0.31$), \mathrm{P}=0.04 ; 2$ trials; 55 participants) was found in favour of the intervention. No trials reported on allcause mortality, morbidity or socioeconomic effects, and few trials reported on participant views; none of which could be meta-analysed.

As the meta-analyses revealed substantial heterogeneity, we conducted subgroup analyses to examine the impact of type of comparator, type of intervention, risk of attrition bias, setting, duration of post-intervention follow-up period, parental involvement and baseline BMI $\mathrm{z}$ score. No subgroup effects were shown for any of the subgroups on any of the outcomes. Some data indicated that a reduction in BMI immediately post-intervention was no longer evident at follow-up at less than six months, which has to be investigated in further trials.

\section{Authors' conclusions}

Multi-component behaviour-changing interventions that incorporate diet, physical activity and behaviour change may be beneficial in achieving small, short-term reductions in BMI, BMI z score and weight in children aged 6 to 11 years. The evidence suggests a very low occurrence of adverse events. The quality of the evidence was low or very low. The heterogeneity observed across all outcomes was not explained by subgrouping. Further research is required of behaviour-changing interventions in lower income countries and in children from different ethnic groups; also on the impact of behaviour-changing interventions on health-related quality of life and comorbidities. The sustainability of reduction in BMI/BMI z score and weight is a key consideration and there is a need for longer-term follow-up and further research on the most appropriate forms of post-intervention maintenance in order to ensure intervention benefits are sustained over the longer term.

\section{PLAIN LANGUAGE SUMMARY}

Diet, physical activity and behavioural interventions for the treatment of overweight or obese children from the age of 6 to 11 years

\section{Review question}

How effective are diet, physical activity and behavioural interventions in reducing the weight of overweight or obese children aged 6 to 11 years?

\section{Background}

Across the world more children are becoming overweight and obese. These children are more likely to suffer from health problems, both while as children and in later life. More information is needed about what works best for treating this problem.

\section{Study characteristics}

We found 70 randomised controlled trials (clinical trials where people are randomly put into one of two or more treatment groups) comparing diet, physical activity, and behavioural (where habits are changed or improved) treatments to a variety of control groups delivered to 8461 overweight or obese children aged 6 to 11 years. We reported on the effects of 64 multicomponent interventions (different combinations of diet and physical activity and behaviour change), four physical activity interventions and two dietary interventions

Diet, physical activity and behavioural interventions for the treatment of overweight or obese children from the age of 6 to 11 years 
compared with no intervention, 'usual care' or some other therapy if it was also delivered in the intervention arm. The children in the included studies were followed up between six months and three years.

\section{Key results}

The average age of the children was 10 years. Most studies reported the body mass index (BMI) z score: BMI is a measure of body fat and is calculated by dividing weight (in kilograms) by the square of the body height measured in metres $\left(\mathrm{kg} / \mathrm{m}^{2}\right)$. In children, BMI is often measured in a way that takes into account sex and age, weight, and height changes as children grow older (BMI z score).

We summarised the results of 37 trials in 4019 children reporting the BMI z score, which on average was 0.06 units lower in the intervention groups compared with the control groups. We summarised the results of 24 trials in 2785 children reporting BMI, which on average was $0.53 \mathrm{~kg} / \mathrm{m}^{2}$ lower in the intervention groups compared with the control groups. We summarised the results of 17 trials in 1774 children reporting weight, which on average was $1.45 \mathrm{~kg}$ lower in the intervention groups compared with the control groups.

Other effects of the interventions, such as improvements in health-related quality of life were less clear. No study investigated death from any cause, morbidity or socioeconomic effects. Serious adverse events were rare: only two of 31 trials with data reported any serious adverse events (4/2105 participants in the behaviour-changing intervention groups compared with 7/1991 participants in the comparator groups). This evidence is up to date as of July 2016.

\section{Quality of the evidence}

The overall quality of the evidence was low or very low, mainly because of limited confidence in how studies were performed, and the results were inconsistent between the studies. Also there were just a few studies for some outcomes, with small numbers of included children. 


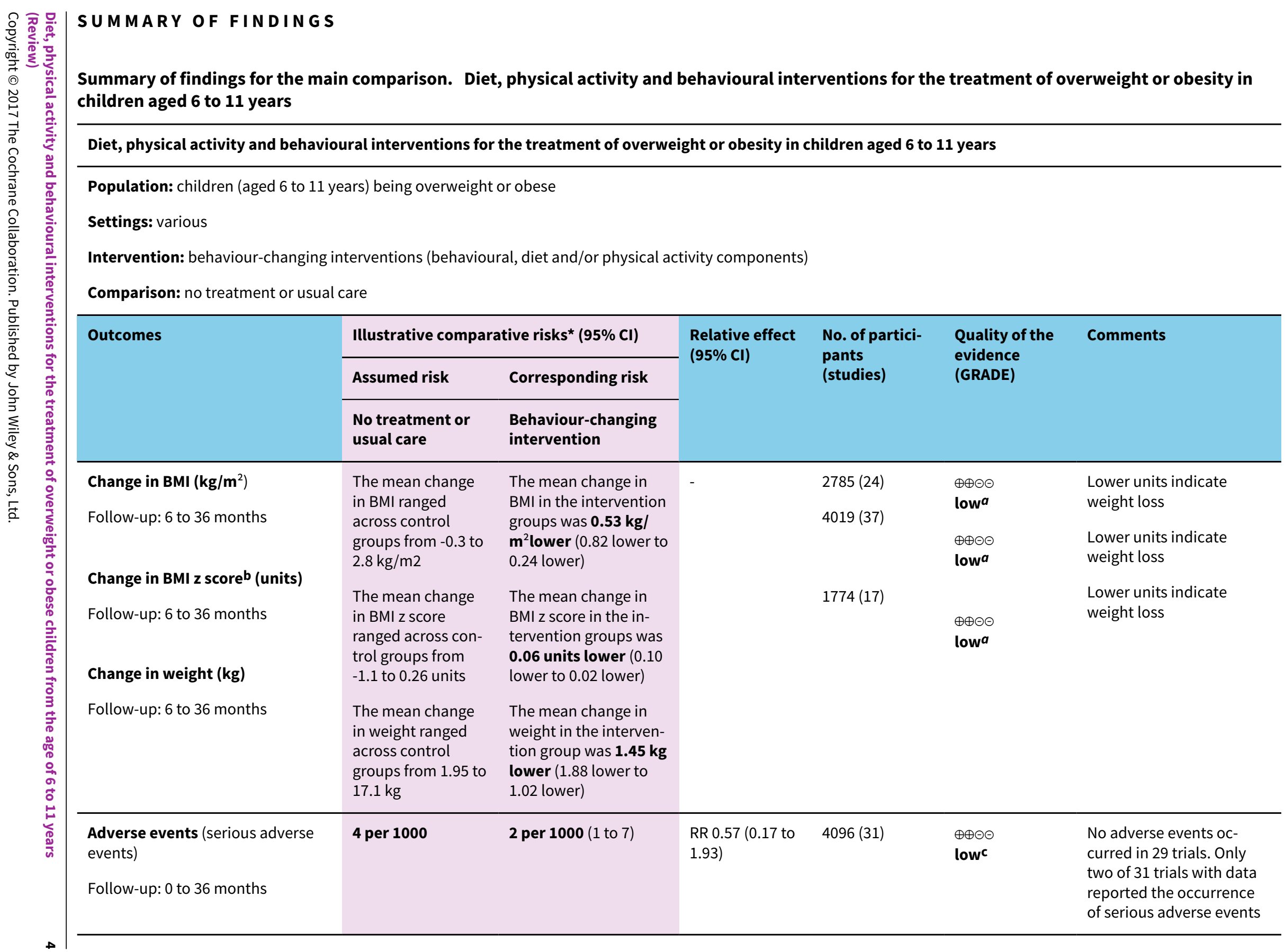


Change in health-related quality of life (SMD)

\section{Parent-reported measures}

Instruments: PedsQL parent proxy: 23 items that yield total, physical summary, and psychosocial summary scores, each with a possible range of $0-100(100=$ best possible health); Child Health Questionnaire, parent version (CHQ-PF50), physical and psychosocial concepts

Follow-up: 6 to 15 months

\section{Child-reported measures}

nstrument: PedsQLchild self-report: 23 items that yield total, physical summary, and psychosocial summary scores, each with a possible range of $0-100$ (100 = best possible health); KINDL-R questionnaire: total score includes domains of well-being, emotional well-being, self-esteem, family, friends, school. 5-point Likert scale

Follow-up: 6 months
The mean in care giver PedsQL

ranged across con-

trol groups from

-4.2 units to 3.6

units

(a)

mean change in child PedsQL in the intervention group was $\mathbf{0 . 1 5}$ units higher $(0.34$ lower to 0.64 higher)

$718(5)$

vention groups was

\subsection{3 units higher $(0.06$}

lower to 0.32 higher)

The mean in child

PedsQL ranged

across control

groups from -1.4

units to 4.01 units

Higher units indicate improvements. The minimal clinically important difference (MCID) for a PedsQL parents' proxy report is 4.50 raw units. When converting the SMD back to raw units, the MCID was not met in either metaanalysis

very lowe
164 (3) $\oplus \odot \ominus \odot$ Higher units indicate im-
provements. The minimal clinically important difference (MCID) for a PedsQL child's self-report is 4.36 raw units. When converting the SMD back to raw units, the MCID was not met in either meta-analysis

\begin{tabular}{|c|c|c|c|c|c|c|}
\hline All-cause mortality & See comment & See comment & See comment & See comment & See comment & $\begin{array}{l}\text { No deaths were reported } \\
\text { in any of the trials }\end{array}$ \\
\hline Morbidity & See comment & See comment & See comment & See comment & See comment & $\begin{array}{l}\text { No trials reported morbid- } \\
\text { ity }\end{array}$ \\
\hline Socioeconomic effects & See comment & See comment & See comment & See comment & See comment & $\begin{array}{l}\text { No trials reported socioe- } \\
\text { conomic effects }\end{array}$ \\
\hline
\end{tabular}

${ }^{*}$ The basis for the assumed risk (e.g. the median control group risk across studies) was derived from the event rates in the comparator groups. The corresponding risk (and its 95\% confidence interval) is based on the assumed risk in the comparison group and the relative effect of the intervention (and its $95 \% \mathrm{Cl}$ ). BMI: body mass index;Cl: confidence interval; PedsQL: Pediatric Quality of Life Inventory;RR: risk ratio; SMD: standardised mean difference

GRADE Working Group grades of evidence 


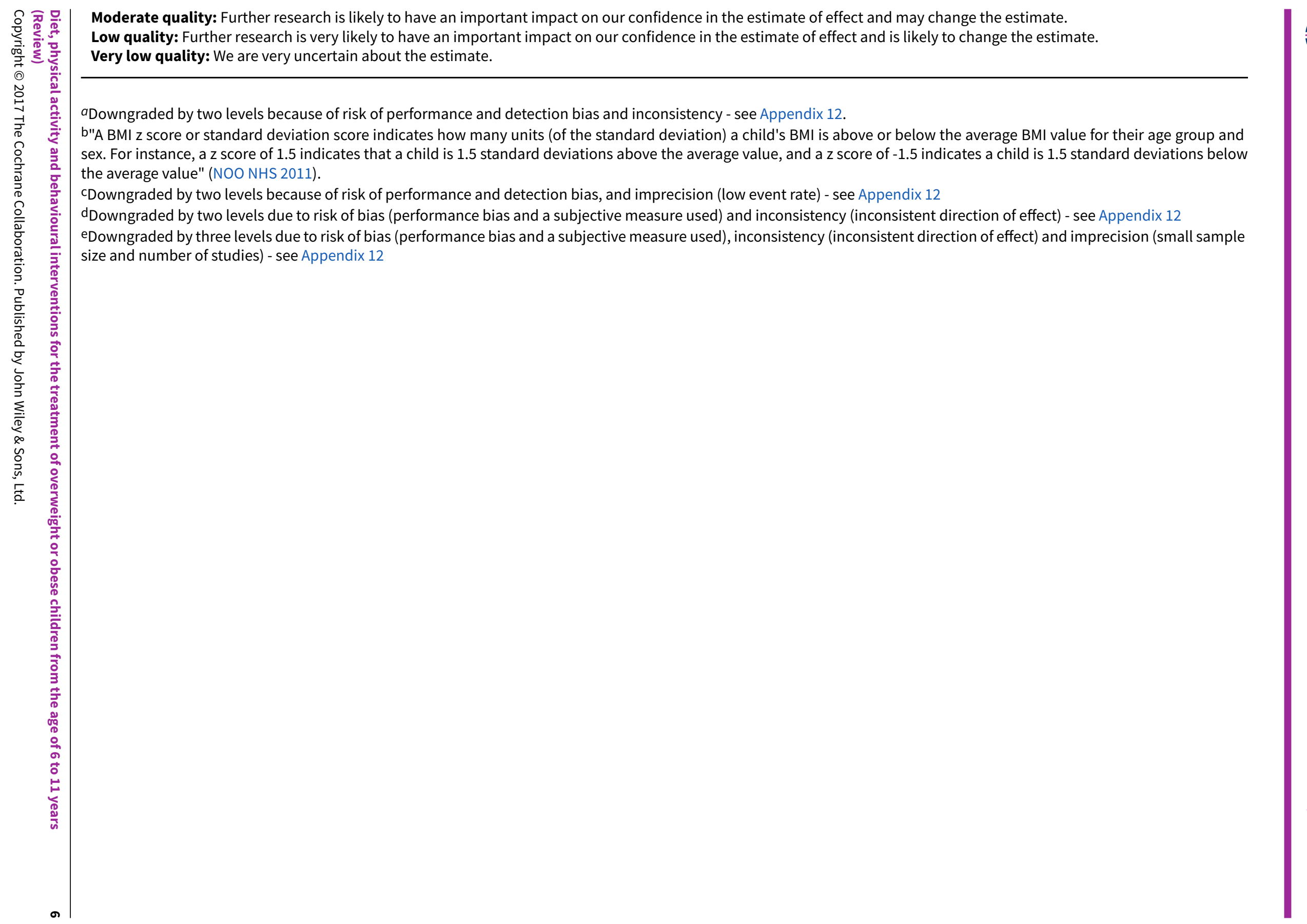




\section{B A C K G R O U N D}

The prevalence of overweight and obese children and adolescents has increased throughout the world, presenting a global public health crisis (Ng 2014; WHO 2015). Although once considered to be a condition affecting only high-income countries, rates of paediatric overweight and obesity have recently started to rise dramatically in some low- and middle-income countries (Wang 2012). Using the International Obesity Task Force (IOTF) standard definition, the age-standardised prevalence of overweight and obesity in children and adolescents has increased in low-, middle-, and high-income countries over the last 30 years (Cole 2000). In 2013, the prevalence of overweight and obese children and adolescents in high-income countries was estimated at $23.8 \%$ (95\% confidence interval (CI) 22.9 to 24.7$)$ for boys and $22.6 \%(95 \% \mathrm{Cl} 21.7$ to 23.6$)$ for girls. In low- and middle-income countries, the prevalence was estimated as $12.9 \%$ (95\% Cl 12.3 to 13.5$)$ for boys and $13.4 \%(95 \% \mathrm{Cl} 13$ to 13.9$)$ for girls (Ng 2014).

Inequalities in overweight and obesity prevalence have also been documented. Generally, socioeconomically disadvantaged children in high-income countries (Knai 2012; NCB 2015; Shrewsbury 2008), and children of higher socioeconomic status in low- and middle-income countries (Lobstein 2004; Wang 2012), are at greater risk of becoming overweight. However, this relationship may vary by population demographics (for example age, gender, ethnicity), and environment (for example country, urbanisation) (Wang 2012). The prevalence of obesity has been shown to vary by ethnicity, with large data sets showing substantial ethnic variation in English (HSCIC 2013), American (Freedman 2006; Skinner 2014), and New Zealand (Rajput 2014) child populations.

Whilst there is some evidence that the rate of increase in paediatric obesity may be slowing in some high-income countries, current levels remain too high, and continue to rise in many low- and middle-income countries (Olds 2011; Rokholm 2010). However, an additional concern in some high-income countries such as the USA, in Kelly 2013 and Skinner 2014, and the UK, in CMO 2015 and Ells 2015a, is the rise in severe paediatric obesity. Whilst the IOTF published an international definition for severe paediatric (morbid) obesity in 2012 (Cole 2012), often severe obesity prevalence is reported using country-specific cut points, making international comparisons difficult. However, data from the USA, in Skinner 2014, and England, in Ells 2015a, have shown that the prevalence of severe paediatric obesity varies by socioeconomic status and ethnicity, and may result in a greater risk of adverse cardiometabolic events and severe obesity in adulthood (Kelly 2013).

\section{Description of the condition}

Childhood overweight and obesity results from an accumulation of excess body fat, and can increase the risk of both short- and longer-term health consequences. Numerous obesityrelated comorbidities can develop during childhood, which include muscular skeletal complaints (Paulis 2014); cardiovascular risk factors such as hypertension, insulin resistance, and hyperlipidaemia (Reilly 2003), even in very young children (Bocca 2013); motor and developmental delays (Cataldo 2016); and conditions such as sleep apnoea (Narang 2012), asthma (Egan 2013), liver disease, and type 2 diabetes (Daniels 2009b; Lobstein 2004). The condition can also affect psychosocial well-being, with obese young people being susceptible to reduced self esteem and quality of life (Griffiths 2010), as well as stigmatisation (Puhl 2007; Tang-Peronard 2008). Evidence also shows that childhood obesity can track into adulthood (Parsons 1999; Singh 2008; Whitaker 1997), and is therefore associated with an increased risk of ill health later in life (Reilly 2011).

\section{Description of the intervention}

Given the serious implications associated with childhood and adolescent obesity, effective treatment is imperative. Whilst the fundamental principles of weight management in children and adolescents are the same as in adults (that is, reduced energy intake and increased energy expenditure), the primary aim of treatment (that is, weight reduction or deceleration of weight gain) and the most suitable intervention approach vary, and are dependent on the child's age and degree of excess weight, among other considerations. Family-based interventions combining dietary, physical activity, and behavioural components have been shown to be effective and are considered the current best practice in the treatment of childhood obesity in children under 12 years of age (Oude Luttikhuis 2009).

\section{Adverse effects of the intervention}

It is not anticipated that diet, physical activity, and behavioural interventions will lead to adverse outcomes. However, as with all obesity treatment interventions in children and young people, potential adverse effects should be considered, including effects on linear growth, eating disorders and psychological well-being.

\section{How the intervention might work}

Obesity is a complex multifactorial condition with numerous possible biological, behavioural and environmental determinants (Butland 2007). Many children now grow up in an obesogenic environment that promotes energy imbalance through the marketing, affordability and availability of energy dense foods, coupled with decreases in physical activity and increases in screenbased sedentary pursuits (Kremers 2006). Therefore, behaviourchanging interventions that aim to improve dietary intake, increase physical activity levels and reduce sedentary behaviours are often prescribed, and were recommended as a treatment option for childhood obesity in the preceding Cochrane Review on the treatment of child and adolescent obesity (Oude Luttikhuis 2009). Behaviour-changing interventions may target just one behavioural component (e.g. diet, physical activity or sedentary behaviour) or combine several components, and are often supported by theorybased behaviour-change techniques to help sustain positive changes and prevent relapse. As the family environment (e.g. home activities, meal times and availability of unhealthy food) plays an important role in the aetiology of obesity, parents can be defined as the 'agents for change' particularly in children under 12 years of age (Golan 2004). Given the number of interacting components, difficulty of the target behaviours and variability in possible outcomes, behaviour-changing interventions are regarded as 'complex interventions' (Craig 2008).

\section{Why it is important to do this review}

The first version of this systematic review was published in 2003 and included analysis of childhood obesity treatment trials published up until July 2001 (Summerbell 2003). The second version was published in 2009, updating the 2003 review (Oude Luttikhuis 2009). 
To reflect the rapid growth in this field, the third update to this review has been split across six reviews focusing on the following treatment approaches: surgery (Ells 2015b); drugs (Mead 2016a); parent-only interventions (Loveman 2015); diet, physical activity, and behavioural interventions for young children up to the age of six years (Colquitt 2016); schoolchildren aged 6 to 11 years; and adolescents aged 12 to 17 years.

The current review examines the effects of interventions for schoolaged children aged from 6 years to 11 years. The results of this current review and other systematic reviews in this series will provide information on which to underpin clinical guidelines and health policy on the treatment of childhood obesity.

\section{O B J E C T IVES}

To assess the effects of diet, physical activity and behavioural interventions (behaviour-changing interventions) for the treatment of overweight or obese children aged 6 to 11 years.

\section{METHODS}

\section{Criteria for considering studies for this review}

\section{Types of studies}

We included randomised controlled trials (RCTs) or cluster RCTs. Included studies observed participants for a minimum of six months (this time frame refers to the intervention itself or to a combination of the intervention with a follow-up phase).

\section{Types of participants}

Overweight or obese participants, with a mean age of six years and over, and under 12 years at the commencement of the intervention. Trials involving participants with comorbid disorders were eligible for inclusion as long as the primary focus of the intervention was to treat overweight and obese children. Parents could be involved in the intervention; however, interventions focused solely on the parents (with no child involvement) were excluded from this review as they are evaluated in another Cochrane Review: 'Parent-only interventions for childhood overweight or obesity' (Loveman 2015).

\section{Types of interventions}

Any behaviour-changing intervention (with any one or any combination of behavioural, nutritional and physical activity component) delivered as a single or multicomponent intervention, in any setting, using any delivery method, which aimed to treat paediatric obesity using any of the following intervention versus control sequences.

\section{Intervention}

- Behaviour-changing intervention (any forms of dietary, physical activity and/or behavioural therapy delivered as single- or multicomponent interventions)

\section{Comparator}

- No treatment (including wait-list control)

- Usual care

- Concomitant intervention (another behaviour-changing intervention, which was also delivered in the intervention group).

\section{Minimum duration of intervention}

No restriction on the length of intervention

\section{Minimum duration of follow-up}

Minimal duration of follow-up was six months from baseline.

\section{Specific exclusion criteria}

- Studies with pregnant participants

- Studies that included critically ill participants

- Interventions that specifically dealt with the treatment of eating disorders or type 2 diabetes

- Studies that included participants with a secondary or syndromic cause of obesity

\section{Types of outcome measures}

We did not exclude trials if one or several of the review primary or secondary outcomes were not reported.

\section{Primary outcomes}

- Changes in measured (not self-reported) body mass index (BMI), BMI z score and weight

- Adverse events

\section{Secondary outcomes}

- Health-related quality of life

- Self-esteem

- All-cause mortality

- Morbidity

- Anthropometric measures other than change in BMI, BMI z score and weight

- Behaviour change

- Participants' views of the intervention

- Socioeconomic effects

\section{Method and timing of outcome measurement}

- Changes in BMI $\left(\mathrm{kg} / \mathrm{m}^{2}\right)$ and body weight $(\mathrm{kg})$ : measured at baseline and any time-point from six months' follow-up.

- Adverse events: defined as adverse outcome that occurs during or after the intervention but is not necessarily caused by it and measured at any time-point after the start of the intervention.

- Health-related quality of life: evaluated by a validated instrument such as Paediatric Quality of Life Inventory and measured at baseline and any time point from six months.

- Self-esteem: evaluated by a validated instrument and measured at baseline and any time point from six months.

- All-cause mortality: measured at any time-point after the start of the intervention.

- Morbidity: defined as illness or harm associated with the intervention and measured at baseline and any time point from six months' follow-up.

- Anthropometric measures other than change in BMI: defined by the use of validated tools (such as waist circumference, skin fold thickness, waist-to-hip ratio, dual X-ray absorptiometry or bioelectrical impedance analysis) and measured at baseline and any time point from six months' follow-up.

Diet, physical activity and behavioural interventions for the treatment of overweight or obese children from the age of 6 to 11 years 
- Behaviour change: defined as validated measures of diet or physical activity and measured at baseline and any time point from six months' follow-up.

- Participants' views of the intervention: defined as documented or accounts from participant feedback and measured at baseline and any time point from six months' follow-up.

- Socioeconomic effects: defined as a validated measure of socioeconomic status such as parental income or educational status and measured at baseline and at least at six months.

\section{Summary of findings}

We have presented a 'Summary of findings' table to report the following outcomes, listed according to priority.

- Changes in BMI, BMI z score and weight

- Adverse events

- Health-related quality of life

- All-cause mortality

- Morbidity

- Socioeconomic effects

\section{Search methods for identification of studies}

\section{Electronic searches}

On 14 July 2016 we searched the following sources from inception of each database and placed no restrictions on the language of publication

- Cochrane Central Register of Controlled Trials (CENTRAL; 2016, issue 6,) in the Cochrane Library

- Epub Ahead of Print, In-Process \& Other Non-Indexed Citations, Ovid MEDLINE(R) Daily and Ovid MEDLINE(R) (from 1946 to Present)

- Embase Ovid (1974 to 2016 Week 28)

- PsycINFO (1806 to July Week 12016$)$

- CINAHL

- LILACS (Latin American and Caribbean Health Science Information database) (last update 08/07/2016)

- ClinicalTrials.gov (www.clinicaltrials.gov)

- World Health Organization International Clinical Trials Registry Platform (ICTRP) (www.who.int/trialsearch/)

For details on search strategies and search platforms see Appendix 1.

\section{Searching other resources}

We tried to identify other potentially eligible trials or ancillary publications by searching the reference lists of retrieved included trials, (systematic) reviews, meta-analyses and health technology assessment reports. We also contacted study authors of included trials to identify any further studies that we may have missed.

\section{Data collection and analysis}

\section{Selection of studies}

Two review authors (two of CO, EC, EM, KR, LA, LA-K, LE) independently scanned the abstract, title, or both, of every record we retrieved in the literature searches, to determine which trials we should assess further. We obtained the full texts of all potentially- relevant records. We resolved any discrepancies through consensus or by recourse to a third review author (EM, LE, TB). We have presented a PRISMA flow-chart showing the process of trial selection (Liberati 2009).

\section{Data extraction and management}

For trials that fulfilled our inclusion criteria, two review authors (two of CO, DJ, EB, EC, EM, GM, JO, KR, LA, LA-K, LB, LE, TB) independently abstracted key participant and intervention characteristics. We reported data on efficacy outcomes and adverse events using standard data extraction sheets from Cochrane Metabolic and Endocrine Disorders. We resolved any disagreements by discussion or, if required, by consultation with a third review author (EM, KR, LE, TB) for details, see Characteristics of included studies; Appendix 2; Appendix 3; Appendix 4; Appendix 5; Appendix 6; Appendix 7; Appendix 8; Appendix 9; Appendix 10; Appendix 11).

We have provided information including trial identifier about potentially relevant ongoing studies in the Characteristics of ongoing studies table. We attempted to locate the protocol of each included study and reported primary, secondary and other outcomes in comparison with data in publications in Appendix 6.

We attempted to email all authors of included trials to enquire whether they were willing to answer questions regarding their trials; Appendix 11 shows the results of this survey. Thereafter, we sought relevant missing information on the trial from the primary author(s) of the article, if required.

\section{Dealing with duplicate and companion publications}

In the event of duplicate publications, companion documents or multiple reports of a primary trial, we tried to maximise yield of information by collating all available data, and used the most complete dataset aggregated across all known publications.

We listed duplicate publications, companion documents, multiple reports of a primary trial and trial documents of included trials (such as trial registry information) as secondary references under the study ID of the included trial. Furthermore, we also listed duplicate publications, companion documents, multiple reports of a trial and trial documents of excluded trials (such as trial registry information) as secondary references under the study ID of the excluded trial.

\section{Data from clinical trial registers}

In case data from included trials were available as study results in clinical trials registers such as ClinicalTrials.gov or similar sources, we made full use of this information and extracted data. If there was also a full publication of the trial, we collated and critically appraised all available data. If an included trial was marked as a completed study in a clinical trials register but no additional information (study results, publication or both) was available, we added this trial to the table Characteristics of studies awaiting classification.

\section{Assessment of risk of bias in included studies}

Two review authors (two of EM, TB, LE, KR, DJ, JO, GM, EC, CO, $E B, L A, L A-K, L B$ ) independently assessed the risk of bias of each included trial. We resolved any disagreements by consensus or by consultation with a third review author (EM, TB, LE, KR). In case

Diet, physical activity and behavioural interventions for the treatment of overweight or obese children from the age of 6 to 11 years 
of disagreement, we consulted the rest of the group and made a judgement based on consensus. If adequate information was not available from trial authors, trial protocols, or both we contacted trial authors for missing data on 'Risk of bias' items.

We used the Cochrane 'Risk of bias' assessment tool (Higgins 2011a) and judged 'Risk of bias' criteria as having low, high, or unclear risk. We evaluated individual bias items as described in the Cochrane Handbook for Systematic Reviews of Interventions according to the criteria and associated categorisations contained therein (Higgins 2011b).

\section{Random sequence generation (selection bias due to inadequate} generation of a randomised sequence) - assessment at trial level

For each included trial we described the method used to generate the allocation sequence in sufficient detail to allow an assessment of whether it should produce comparable groups.

- Low risk of bias: the trial authors achieved sequence generation using computer-generated random numbers or a random numbers table. Drawing of lots, tossing a coin, shuffling cards or envelopes, and throwing dice are adequate if an independent person performed this who was not otherwise involved in the trial. We considered the use of the minimisation technique as equivalent to being random.

- Unclear risk of bias: insufficient information about the sequence generation process.

- High risk of bias: the sequence generation method was nonrandom or quasi-random (e.g. sequence generated by odd or even date of birth; sequence generated by some rule based on date (or day) of admission; sequence generated by some rule based on hospital or clinic record number; allocation by judgement of the clinician; allocation by preference of the participant; allocation based on the results of a laboratory test or a series of tests; or allocation by availability of the intervention).

\section{Allocation concealment (selection bias due to inadequate concealment of allocation prior to assignment) - assessment at trial level}

We described for each included trial the method used to conceal allocation to interventions prior to assignment and assessed whether intervention allocation could have been foreseen in advance of or during recruitment, or changed after assignment.

- Low risk of bias: central allocation (including telephone, interactive voice-recorder, web-based and pharmacy-controlled randomisation); sequentially-numbered drug containers of identical appearance; sequentially-numbered, opaque, sealed envelopes.

- Unclear risk of bias: insufficient information about the allocation concealment.

- High risk of bias: using an open random allocation schedule (e.g. a list of random numbers); assignment envelopes were used without appropriate safeguards; alternation or rotation; date of birth; case record number; any other explicitly unconcealed procedure.
Blinding of participants and study personnel (performance bias due to knowledge of the allocated interventions by participants and personnel during the trial) - assessment at outcome level

We evaluated the risk of detection bias separately for self-reported ('subjective outcomes') versus investigator-assessed ('objective outcomes') outcomes (Hróbjartsson 2013). We noted whether endpoints were self-reported, investigator-assessed or adjudicated outcome measures (see below).

- Low risk of bias: blinding of participants and key study personnel is ensured, and it was unlikely that the blinding could have been broken; no blinding or incomplete blinding, but we judge that the outcome is unlikely to have been influenced by lack of blinding.

- Unclear risk of bias: insufficient information about the blinding of participants and study personnel; the trial does not address this outcome.

- High risk of bias: no blinding or incomplete blinding, and the outcome is likely to have been influenced by lack of blinding; blinding of trial participants and key personnel attempted, but likely that the blinding could have been broken, and the outcome was likely to be influenced by lack of blinding.

\section{Blinding of outcome assessment (detection bias due to knowledge of the allocated interventions by outcome assessment) - assessment at outcome level}

We evaluated the risk of detection bias separately for self-reported ('subjective outcomes') versus investigator-assessed ('objective outcomes') outcomes (Hróbjartsson 2013). We noted whether endpoints were self reported, investigator-assessed or adjudicated outcome measures (see below).

- Low risk of bias: blinding of outcome assessment is ensured, and it was unlikely that the blinding could have been broken; no blinding of outcome assessment, but we judge that the outcome measurement was unlikely to have been influenced by lack of blinding.

- Unclear risk of bias: insufficient information about the blinding of outcome assessors; the trial did not address this outcome.

- High risk of bias: no blinding of outcome assessment, and the outcome measurement was likely to have been influenced by lack of blinding; blinding of outcome assessment, but likely that the blinding could have been broken, and the outcome measurement was likely to be influenced by lack of blinding.

\section{Incomplete outcome data (attrition bias due to amount, nature or handling of incomplete outcome data) - assessment at outcome level}

For each included trial and for self-reported ('subjective outcomes') versus investigator-assessed ('objective outcomes') outcomes, we described the completeness of data, including attrition and exclusions from the analyses. We stated whether the trial reported attrition and exclusions, and the number of participants included in the analysis at each stage (compared with the number of randomised participants per intervention/comparator groups). We also noted if the trial reported the reasons for attrition or exclusion and whether missing data were balanced across groups or were related to outcomes. We considered the implications of missing outcome data per outcome such as high dropout rates (e.g. above

Diet, physical activity and behavioural interventions for the treatment of overweight or obese children from the age of 6 to 11 years 
$15 \%$ ) or disparate attrition rates (e.g. difference of $10 \%$ or more between trial arms).

- Low risk of bias: no missing outcome data; reasons for missing outcome data unlikely to be related to true outcome (for survival data, censoring unlikely to introduce bias); missing outcome data balanced in numbers across intervention groups, with similar reasons for missing data across groups; for dichotomous outcome data, the proportion of missing outcomes compared with observed event risk was not enough to have a clinically-relevant impact on the intervention effect estimate; for continuous outcome data, plausible effect size (mean difference or standardised mean difference) among missing outcomes is not enough to have a clinically relevant impact on observed effect size; appropriate methods, such as multiple imputation, were used to handle missing data.

- Unclear risk of bias: insufficient information to assess whether missing data in combination with the method used to handle missing data were likely to induce bias; the trial did not address this outcome.

- High risk of bias: reason for missing outcome data was likely to be related to true outcome, with either imbalance in numbers or reasons for missing data across intervention groups; for dichotomous outcome data, the proportion of missing outcomes compared with observed event risk enough to induce clinically-relevant bias in intervention effect estimate; for continuous outcome data, plausible effect size (mean difference or standardised mean difference) among missing outcomes enough to induce clinically-relevant bias in observed effect size; 'as-treated' or similar analysis done with substantial departure of the intervention received from that assigned at randomisation; potentially inappropriate application of simple imputation.

\section{Selective reporting (reporting bias due to selective outcome reporting) - assessment at trial level}

We assessed outcome reporting bias by integrating the results of Appendix 5, 'Matrix of trial endpoints (publications and trial documents)' (Boutron 2014; Jones 2015b; Mathieu 2009), with those of Appendix 6, 'High risk of outcome reporting bias according to ORBIT classification' (Kirkham 2010). This analysis formed the basis for the judgement of selective reporting.

- Low risk of bias: the trial protocol was available and all of the trial's pre-specified (primary and secondary) outcomes that were of interest in the review had been reported in the prespecified way; the study protocol was unavailable, but it was clear that the published reports included all expected outcomes (ORBIT classification).

- Unclear risk of bias: insufficient information about selective reporting.

- High risk of bias: not all of the trial's pre-specified primary outcomes review were reported incompletely so that we could not enter them in a meta-analysis; the trial report failed to include results for a key outcome that we would expected to have been reported for such a trial (ORBIT classification).

\section{Other bias (bias due to problems not covered elsewhere) - assessment at trial level}

- Low risk of bias: the trial appears to be free of other sources of bias.
- Unclear risk of bias: there was insufficient information to assess whether an important risk of bias existed; insufficient rationale or evidence that an identified problem introduced bias.

- High risk of bias: the trial has a potential source of bias related to the specific trial design used; the trial has been claimed to have been fraudulent; or the trial had some other serious problem.

We have presented a 'Risk of bias' graph and a 'Risk of bias' summary figure.

We distinguished between self-reported, investigator-assessed and adjudicated outcome measures.

We defined the following endpoints as potentially self-reported outcomes.

- Adverse events, if reported by participants

- Health-related quality of life

- Self-esteem

- Participants views of the intervention

- Behaviour change, if reported by participants

We defined the following outcomes as potentially investigatorassessed outcomes.

- Changes in BMI and weight, if measured by trial personnel

- Adverse events, if measured by trial personnel

- All-cause mortality

- Morbidity

- Behaviour change, if measured by trial personnel

\section{Summary assessment of risk of bias}

Risk of bias for a trial across outcomes: some 'Risk of bias' domains such as selection bias (sequence generation and allocation sequence concealment), affect the risk of bias across all outcome measures in a trial. In case of high risk of selection bias, we marked all endpoints investigated in the associated trial as high risk. Otherwise, we did not perform a summary assessment of the risk of bias across all outcomes for a trial.

Risk of bias for an outcome within a trial and across domains: we assessed the risk of bias for an outcome measure by including all entries relevant to that outcome (i.e. both trial-level entries and outcome-specific entries). We considered low risk of bias to denote a low risk of bias for all key domains, unclear risk to denote an unclear risk of bias for one or more key domains and high risk to denote a high risk of bias for one or more key domains.

Risk of bias for an outcome across trials and across domains: these were our main summary assessments that we incorporated into our judgements about the quality of evidence in the 'Summary of finding' tables. We defined outcomes as at low risk of bias when most information came from trials at low risk of bias, unclear risk when most information came from trials at low or unclear risk of bias, and high risk when a sufficient proportion of information came from trials at high risk of bias.

\section{Measures of treatment effect}

When at least two included trials were available for a comparison and a given outcome, we expressed dichotomous data as a risk ratio (RR) or odds ratio (OR) with $95 \%$ confidence interval

Diet, physical activity and behavioural interventions for the treatment of overweight or obese children from the age of 6 to 11 years 
(Cl). For continuous outcomes measured on the same scale (e.g. weight loss in $\mathrm{kg}$ ) we estimated the intervention effect using the mean difference with $95 \% \mathrm{Cl}$. For continuous outcomes measuring the same underlying concept (e.g. health-related quality of life) but using different measurement scales, we calculated the standardised mean difference (SMD). We expressed time-to-event data as hazard ratio with $95 \% \mathrm{Cl}$.

\section{Unit of analysis issues}

We took into account the level at which randomisation occurred, such as cross-over trials, cluster-randomised trials and multiple observations for the same outcome. If more than one comparison from the same trial was eligible for inclusion in the same metaanalysis, we either combined groups to create a single pair-wise comparison (if the groups were suitably similar interventions) or appropriately reduced the sample size so that the same participants did not contribute multiple times (splitting the 'shared' group into two or more groups). While the latter approach offers some solution to adjusting the precision of the comparison, it does not account for correlation arising from the same set of participants being in multiple comparisons (Deeks 2011).

We analysed cluster RCTs separately from individually randomised trials.

\section{Dealing with missing data}

If possible, we obtained missing data from the authors of the included trials. We carefully evaluated important numerical data such as screened, eligible, randomly-assigned participants as well as intention-to-treat, as-treated and per-protocol populations. We investigated attrition rates (e.g. dropouts, losses to follow-up, withdrawals), and we critically appraised issues concerning missing data and use of imputation methods (e.g. last observation carried forward).

Where standard deviations for outcomes were not reported, and we did not receive information from trial authors, we calculated these following the methods presented in the Cochrane Handbook for Systematic Reviews of Interventions ( Higgins 2011c). Where papers did not report results as change from baseline, we calculated this and for the standard deviation differences followed the methods presented in the Cochrane Handbook for Systematic Reviews of Interventions for imputing these (Section 16.1.3.2 Imputing standard deviations for changes from baseline; Higgins 2011c), and assumed a correlation of 0.5 between baseline and follow-up measures as suggested by Follmann 1992.

\section{Assessment of heterogeneity}

In the event of substantial clinical, methodological or statistical heterogeneity, we did not report trial results as the pooled effect estimate in a meta-analysis.

We identified heterogeneity (inconsistency) by visually inspecting the forest plots and by using a standard $\mathrm{Chi}^{2}$ test with a significance level of $\alpha=0.1$ (Higgins 2002). In view of the low power of this test, we also considered the $I^{2}$ statistic (Higgins 2003), which quantifies inconsistency across trials to assess the impact of heterogeneity on the meta-analysis, where an $\mathrm{I}^{2}$ statistic of $75 \%$ or more indicates a considerable level of inconsistency (Deeks 2011).
When we found heterogeneity, we attempted to determine potential reasons for it by examining individual study and subgroup characteristics.

\section{Assessment of reporting biases}

If we included 10 studies or more for a given outcome, we used funnel plots to assess small study effects. Due to several explanations for funnel plot asymmetry we interpreted results carefully (Sterne 2011).

\section{Data synthesis}

We undertook a meta-analysis only if we judged participants, interventions, comparisons and outcomes to be sufficiently similar. We included all relevant trials regardless of risk of bias assessments using random-effect models; subgrouping was undertaken according to risk of bias (high, low, unclear risk). We performed statistical analyses according to the statistical guidelines presented in the Cochrane Handbook for Systematic Reviews of Interventions (Deeks 2011).

\section{Quality of evidence}

We have presented the overall quality of the evidence for each outcome specified under 'Types of outcome measures: Summary of findings' according to the GRADE approach (gradeworkinggroup.org), which takes into account issues related to internal validity (risk of bias, inconsistency, imprecision, publication bias) and also to external validity, such as directness of results. Two review authors (EM, TB) independently rated the quality of the evidence for each outcome. We have presented a summary of the evidence in a 'Summary of findings' table. This provides key information about the best estimate of the magnitude of the effect, in relative terms and as absolute differences, for each relevant comparison of alternative management strategies, numbers of participants and trials that address each important outcome and a rating of overall confidence in effect estimates for each outcome. We created the 'Summary of findings' table based on the methods described in the Cochrane Handbook for Systematic Reviews of Interventions (Schünemann 2011) using Review Manager 5 (RevMan 5) table editor (RevMan 2014). We have included an appendix titled 'Checklist to aid consistency and reproducibility of GRADE assessments' (Meader 2014), to help with standardisation of the 'Summary of findings' tables. Alternatively, we planned to use the GRADEpro Guideline Development Tool (GDT) software (GRADEproGDT 2015) and would have presented evidence profile tables as an appendix. We have presented results for the outcomes as described in the Types of outcome measures section. If metaanalysis was not possible, we presented the results narratively in the 'Summary of findings' table. We justified all decisions to downgrade the quality of trials using footnotes and we made comments to aid the reader's understanding of the Cochrane Review where necessary.

\section{Subgroup analysis and investigation of heterogeneity}

We expected the following characteristics to introduce clinical heterogeneity, and we planned to carry out the following subgroup analyses including investigation of interactions (Altman 2003).

- Type of control (no treatment, usual care or another intervention with the same components) 
- Type of intervention (diet, physical activity and/or behavioural therapy)

- Attrition bias (low, high, unclear)

- Setting

- Duration of post-intervention follow-up

- Parental involvement

- Baseline BMI z score

There is no single accepted classification for severe obesity in school children; we used the $2.67 \mathrm{BMI} z$ score which equates to the 99.6th centile for severe obesity (Ells 2015a). We put studies into subgroups based on a whether their mean baseline BMI z score was less than 2.67 units, or 2.67 units or over.

\section{Sensitivity analysis}

We investigated the impact of imputation on meta-analyses by performing sensitivity analyses, and we reported per outcome which trials were included with imputed SDs.

\section{RE S U L T S}

\section{Description of studies}

For an overview of study populations please see Table 1; for a detailed description of trials, see 'Characteristics of included studies', 'Characteristics of excluded studies, and 'Characteristics of ongoing studies' sections.

\section{Results of the search}

One overarching search was conducted for all the behaviourchanging reviews:

- Diet, physical activity, and behavioural interventions for the treatment of overweight or obesity in adolescents aged 12 to 17 years.

- Diet, physical activity, and behavioural interventions for the treatment of overweight or obesity in schoolchildren from the age of 6 to 11 years.

- Diet, physical activity, and behavioural interventions for the treatment of overweight or obesity in preschool children up to the age of 6 years.

- Parent-only interventions for childhood overweight or obesity.

Our comprehensive literature searches identified 25,483 records, after duplicates were removed this left 16,106 records. From these 15,491 records were excluded based on the title/abstract. We obtained 615 records as full-text articles and screened them for inclusion or exclusion (see Figure 1 for the PRISMA flow diagram) (Liberati 2009). We included 70 trials (164 articles) in the review and 55 trials in the meta-analyses. Twenty trials are awaiting classification (Characteristics of studies awaiting classification) and 20 trials are ongoing (Characteristics of ongoing studies). 
Figure 1. Trial flow diagram

25,483 records identified through database
searching
CENTRAL: 3208
MEDLINE: 4772
Embase: 9345
PsycINFO: 3248
CINAHL: 2302
LILACS: 115
WHO International Clinical Trials Registry
Platform: 1548
ClinicalTrials.gov: 945

0 additional records identified through non-database sources (contacts with experts, manufacturers, handsearching of literature)

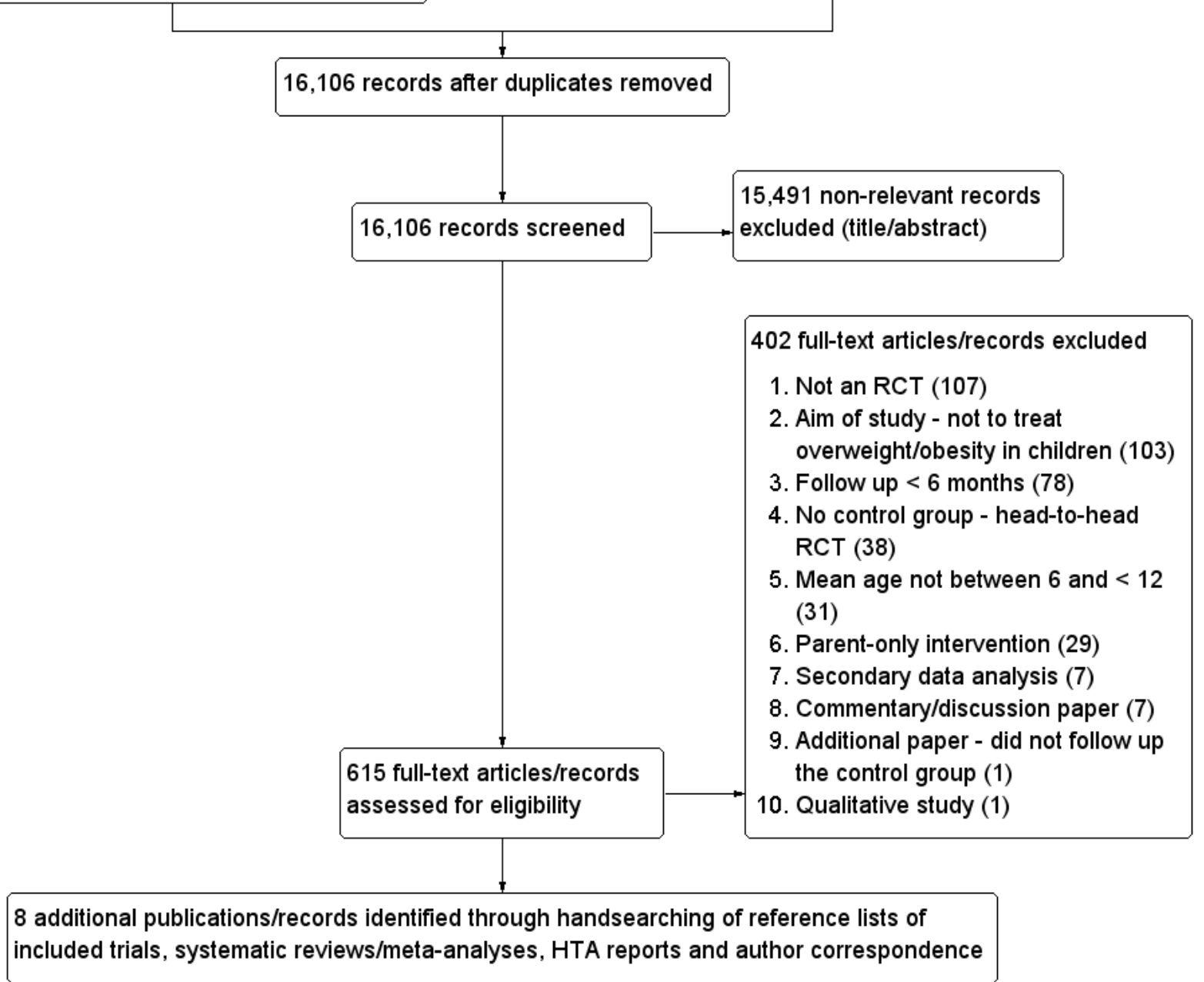

Diet, physical activity and behavioural interventions for the treatment of overweight or obese children from the age of 6 to 11 years

20 ongoing trials

(25 records/publications)

20 trials awaiting assessment (32 records/publications) 
Figure 1. (Continued)

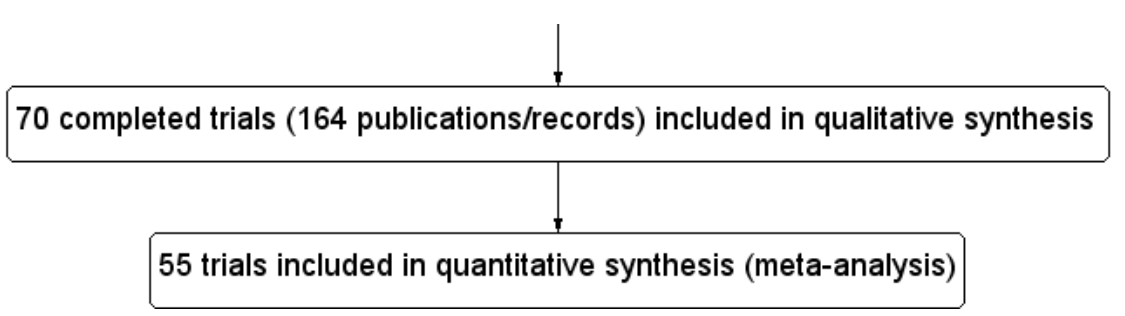

\section{Ongoing studies}

We found 20 ongoing RCTs, see Characteristics of ongoing studies. All but one are parallel RCTs. Five of the 20 ongoing studies only include participants within the age range of this review (between six years and less than 12 years). This includes: ChiCTRIOB-15005874; NCT01642836; NCT02258126; NCT02343367; RBR-8ttw64. Eleven studies have age ranges which include children 12 years or older. This includes: DRKS00007879 (8 years to 16 years old), Moore 2013 (11 years to 12 years old), NCT01221220 (8 years to 15 years old), NCT01574352 (10 years to 13 years old), NCT01736748 (6 years to 18 years old), ACTRN12613001037796 (7 years to 16 years old), NCT02082080 (9 years to 14 years old), NCT02560493 (10 years to 12 years old), NCT02711488 (9 years to 15 years old), NCT02720302 (9 years to 12 years old), and NCT02773823 (8 years to 12 years old). Three studies include children younger than six years old: ISRCTN81798055 (4 years to 11 years old), NCT02573142 (5 years to 11 years old), and NCT02684214 (4 years to 10 years old). In addition, one study has an age range that include children both younger and older than six years to less than 12 years old: NCT02124460 (2 years to 12.9 years old). Many studies include both overweight and obese participants, while eight studies only include obese participants. In one ongoing trial (NCT02720302) only overweight children are included.

The majority of interventions ( $N=14$ ) have a behavioural, diet and physical activity component. The remaining studies are diet and exercise component only (ChiCTR-IOB-15005874; NCT02082080; RBR-8ttw64), physical activity and behavioural only (NCT01736748), physical activity only (ACTRN12613001037796) and behavioural only (DRKS00007879; no mention of a diet or physical activity component). Eleven studies have a usual care/ standard treatment control group while four studies include a no-treatment control group (NCT02082080; NCT02560493; NCT02711488; NCT02773823). The remaining five trials compare a behaviour-changing intervention plus component with the same behaviour-changing intervention without the additional component (DRKS00007879; NCT01221220; NCT02560493; NCT02684214; NCT02720302). The most common primary outcome is $\mathrm{BMI} / \mathrm{BMI} \mathrm{z}$ score ( $\mathrm{N}=17$ trials). No trial reported adverse events as a primary outcome.

\section{Studies awaiting classification}

Twenty studies are awaiting classification. Seventeen trials were identified on a clinical trial register website as completed; however, no results are available. For the remaining three studies we were unable to obtain a full publication; therefore, we are unable to assess whether the trial met the inclusion of this review.

\section{Included studies}

We have presented a detailed description of the characteristics of the 70 included trials in Characteristics of included studies, and in Appendix 2; Appendix 3; Appendix 4. The following is a succinct overview.

\section{Source of data}

We obtained the majority of data presented in the review from published literature, including supplementary published data and trials registers where available. Some data were requested from study authors (see Appendix 11 for an overview). Only one study did not have data published in a journal article and all data were obtained from the clinical trial record (NCT02436330).

\section{Comparisons}

Of the 70 studies included in this review, only 21 studies included a true control; hence, the control groups received no treatment throughout the duration of the study (Arauz Boudreau 2013; Boutelle 2014; Bryant 2011; Coppins 2011; Croker 2012; de Niet 2012; Eddy Ives 2012; Epstein 1984a; Maddison 2011; Maddison 2014; Markert 2014; McCallum 2007; Nowicka 2009; Reinehr 2010; Sacher 2010; Satoh 2007; Siwik 2013; Vann 2013; Wafa 2011; Wake 2009; Wake 2013).

Control group participants in 34 studies were given usual/standard care, either as defined by the study authors or assessed by the review authors (Alves 2008; Barkin 2011; Berry 2014; Davis 2013; Davoli 2013; Diaz 2010; Epstein 2000a; Faude 2010; Gillis 2007; Gunnarsdottir 2011a; Hamilton-Shield 2014; Ho 2016; Hughes 2008; Kalarchian 2009; Kalavainen 2007; Kirk 2012; Lison 2012; Lochrie 2013; Looney 2014; Mirza 2013; Nemet 2005; Nova 2001; O'Connor 2013; Rodearmel 2007; Saelens 2013; Serra-Paya 2015; Taveras 2015; Taylor 2015; Waling 2012; Warschburger 2016; Weigel 2008; Weintraub 2008; Wilfley 2007; Wright 2012)

The remaining 15 studies (Bathrellou 2010; Berry 2007; Duffy 1993; Duggins 2010; Epstein 1985a; Epstein 1985b; Epstein 1985c; Epstein 2001; Epstein 2005; Epstein 2015; Flodmark 1993; Larsen 2015; NCT02436330; Schwingshandl 1999; Woo 2004) included a control condition in which the participants received an intervention that was also provided in the intervention group, with the intervention group also receiving an additional component (for example, diet plus physical activity plus behavioural therapy versus diet plus physical activity). Five of these trials had multiple comparator arms and could also be evaluated as intervention versus control (either usual care or no treatment) (Epstein 1984a; Epstein 2000a; Looney 2014; Taveras 2015; Vann 2013); hence, both comparator types were evaluated where appropriate. 


\section{Overview of trial populations}

Individual study sample size at randomisation ranged from 16 (eight in the intervention group, eight in the control group) (Gunnarsdottir 2011a) to 686 (336 in the intervention group, 350 in the control group) (Warschburger 2016). Twenty-one studies had a sample size less than 50 at baseline, 21 studies had between 50 and 100 participants, 17 studies had between 100 and 200 participants, four studies had between 200 and 300 participants, and finally, seven studies had more than 300 participants at baseline; these included Berry 2014; Davoli 2013; Maddison 2011; Markert 2014; Taveras 2015; Warschburger 2016 and Wright 2012. Only 39 studies clearly reported using a power calculation in their methods; only 10 of these studies actually achieved their target sample size at followup, after dropout (Croker 2012; Davis 2013; Davoli 2013; Hughes 2008; Lison 2012; McCallum 2007; Nemet 2005; Wafa 2011; Wilfley 2007; Wright 2012).

A total of 8461 participants were randomised to either the intervention or control groups. In three studies, it was unclear how many participants were measured at the endpoint (i.e. completed the whole study) (Berry 2014, Epstein 2015, Woo 2004). Therefore, in the remaining 67 studies, 5960 participants out of the 7997 randomised were measured at the study's endpoint (74.5\%). The endpoints varied across studies with the shortest follow-up time from baseline being 24 weeks and the longest being three years. The number and proportions of participants completing the study, where reported, ranged from 2899 participants $(71.9 \%)$ in the intervention groups and 2737 participants $(76.9 \%)$ in the control groups.

\section{Trial design}

All 70 studies had a superiority design. All but six studies were parallel RCTs; four studies (Berry 2007; Berry 2014; Taveras 2015; Wright 2012) were cluster RCTs. Coppins 2011 and Siwik 2013 were presented as cross-over trials; these were treated as parallel RCTs where only the first phase was analysed before crossover and the control groups were treated as waiting-list controls.

Twenty trials were multi-centre (Barkin 2011; Berry 2014; Davis 2013; Davoli 2013; de Niet 2012; Duggins 2010; Eddy Ives 2012; Gillis 2007; Hamilton-Shield 2014; Larsen 2015; McCallum 2007; O'Connor 2013; Reinehr 2010; Sacher 2010; Satoh 2007; Serra-Paya 2015; Taveras 2015; Wake 2009; Wake 2013; Wright 2012), ranging from 2 to 69 centres.

Trials were published between 1984 and 2016.

One study (Hamilton-Shield 2014) was terminated before the endpoint due to recruitment issues and technical problems with the intervention equipment.

The length of the interventions ranged from 10 days to two years. Just over half $(\mathrm{N}=37)$ trials had a period of post intervention followup (defined as the period after the active intervention and up to the final measurement) with a median duration of 10 months; followup from end of the intervention period ranged from one month to two years. We did not extract any information on whether the postintervention period was passive (i.e. just measurement) or active (i.e. a maintenance intervention period with the aim of helping children to sustain the weight status they had achieved).

\section{Settings}

Thirty of the included studies were conducted in the USA, six in the UK, five in Germany, four in Australia, three each in Sweden, New Zealand and Spain, and two each in Israel and in Italy. The remaining studies were conducted in Austria, Brazil, Canada, Denmark, Finland, Greece, Hong Kong, Iceland, Japan, Malaysia, Mexico and the Netherlands. Twenty-five studies were conducted in secondary care, eleven in primary care, seven in university research clinics, seven in the community, four in homes and four in schools. Ten studies were based in more than one setting and in two studies the setting was unclear (Duffy 1993, NCT02436330).

\section{Participants}

All participants included in this review were overweight, obese or severely obese at baseline; various diagnostic criteria were applied across the trials. Thirty-two studies included children who were overweight or obese (including morbidly obese) while 26 studies only included children who were obese (including morbidly obese). Overweight children only (not obese) were included in five studies (Davoli 2013; Duffy 1993; Faude 2010; Larsen 2015; Reinehr 2010). Six studies included both overweight and obese children but did not include morbidly/severely obese children (Eddy Ives 2012; Epstein 2001; McCallum 2007; O'Connor 2013; Saelens 2013; Wake 2009). Only one study included just severely obese children (Kalarchian 2009).

All but three studies were conducted in upper-income countries (defined using the World Bank classification). Alves 2008; Diaz 2010 and Wafa 2011 included participants from upper middle-income countries.

Of the 38 studies that clearly reported the ethnic group(s) of their participants, six studies reported that all of their participants were white (Coppins 2011; Epstein 1985a; Epstein 1985b; Epstein 1985c; Lison 2012; Warschburger 2016). In 23 studies participants were of mixed ethnic groups, but the majority ethnic group was white (Alves 2008; Boutelle 2014; Bryant 2011; Croker 2012; Davis 2013; Epstein 2000; Epstein 2001; Epstein 2005; Hamilton-Shield 2014; Kalarchian 2009; Kirk 2012; Lochrie 2013; Looney 2014; NCT02436330; Reinehr 2010; Rodearmel 2007; Sacher 2010; Saelens 2013; Siwik 2013; Taveras 2015; Wake 2009; Wake 2013; Wilfley 2007). Berry 2007 had a similar number of white and black participants, and also some Hispanic participants. Berry 2014 and Vann 2013 had a higher percentage of African American children in their studies while O'Connor 2013 had a higher percentage of Hispanic/Latino/ Mexican American participants. In Mirza 2013, Weintraub 2008 and Wright 2012, the majority of participants were Hispanic/Latino. Woo 2004 included participants who were all Hong Kong Chinese and Wafa 2011 included participants who were of Malay ethnicity.

The mean age (SD) of participants at baseline ranged from 6.2 (1.2) years (Larsen 2015) to 11.9 (2.4) years (Berry 2007), with the majority of studies including participants with a mean age over nine years but under 12 years of age; only 17 studies included participants with a mean age under nine years old (Alves 2008; Bryant 2011; Coppins 2011; Davis 2013; Davoli 2013; Epstein 1985c; Hughes 2008; Kalavainen 2007; Larsen 2015; Looney 2014; McCallum 2007; Nova 2001; O'Connor 2013; Taylor 2015; Wake 2009; Wake 2013; Wright 2012). One study (Lison 2012) had three groups with one group having a mean age of 12.3 years; however, the average age across all three groups fell under the cut-off of 12 years.

Diet, physical activity and behavioural interventions for the treatment of overweight or obese children from the age of 6 to 11 years 
Twenty-six studies had roughly an equal number of boys and girls at baseline, while in 27 studies, $55 \%$ to $69 \%$ of participants were female at baseline. In six studies, there were $70 \%$ or more girls at baseline; this included two studies that only recruited girls (Epstein 1985b; Epstein 1985c). Seven studies had more boys than girls at baseline but only two of these had more than $70 \%$ boys (Davis 2013; Maddison 2011). No study included boys only. In five studies it was unclear how many boys and girls were included at baseline (Epstein 1984a; Gunnarsdottir 2011a; Nowicka 2009; Weintraub 2008). Socioeconomic status was recorded in 32 studies at baseline (no studies reported on socioeconomic effects as an outcome); however, the variables and tools used varied greatly between the studies.

Mean BMI or BMI z score, or both, at baseline were reported in 63 studies. Mean BMI $\left(\mathrm{kg} / \mathrm{m}^{2}\right)$ value at baseline ranged from $18.3 \mathrm{~kg} / \mathrm{m}^{2}$ to $41.1 \mathrm{~kg} / \mathrm{m}^{2}$ in the intervention group and $18.2 \mathrm{~kg} / \mathrm{m}^{2}$ to $36.7 \mathrm{~kg} /$ $\mathrm{m}^{2}$ in the control group with a median values of $26.6 \mathrm{~kg} / \mathrm{m}^{2}$ and 26.5 $\mathrm{kg} / \mathrm{m}^{2}$, respectively. Mean BMI z score at baseline ranged from 1.3 units to 5.6 units in the intervention group and 1.3 units to 5.3 units in the control group with median values of 2.2 units and 2.2 units, respectively. Only one study reported the mean duration at which their participants had been overweight or obese prior to starting the trial. Davoli 2013 reported $63.6 \%$ and $64.3 \%$ of intervention and control participants, respectively, were overweight before five years old.

Comorbidites at baseline were reported in five studies (Eddy Ives 2012; Gunnarsdottir 2011a; Kalavainen 2007; Satoh 2007; Waling 2012) and included asthma, type 2 diabetes, metabolic syndrome, depression, anxiety and fatty liver diagnoses. None of the interventions had a pharmacological component; however participants in all three groups in one study were encouraged to take a vitamin/mineral supplement throughout the study (Kirk 2012).

\section{Diagnosis}

A number of different growth chart references/criteria were used to categorise overweight and obesity. The 'United States Centers for Disease Control and Prevention (CDC) 2000 growth reference' (cdc.gov/growthcharts) was used to define overweight and obesity in 31 studies while the 'International Obesity Task Force (IOTF) cut-offs' (worldobesity.org/resources/child-obesity/ newchildcutoffs) were used in 12 studies. Four studies based in the UK used the 'British 1990 growth reference (UK90)' (noo.org.uk) to define the weight status categories (Bryant 2011; HamiltonShield 2014; Hughes 2008; Sacher 2010), while only one study used the 'World Health Organization (WHO) Child Growth Standard' (who.int/childgrowth) (Eddy Ives 2012). The remaining studies used references specific to their country, raw BMI or percentage overweight cut off references - but in four studies it was unclear which growth references were used to define overweight and obesity (Duffy 1993; Ho 2016; NCT02436330; Schwingshandl 1999).

\section{Interventions}

The majority of studies in this review had a behavioural, diet and physical activity component $(\mathrm{N}=49)$. Two studies included both a behavioural and diet component but had no physical activity (Boutelle 2014; Flodmark 1993). Barkin 2011 and Maddison 2014 were the only studies to have both a behavioural and physical activity intervention without a diet component. Four studies had only a physical activity arm (Alves 2008; Faude 2010; Maddison 2011; Weintraub 2008). Eleven studies had no behavioural arm (Duggins 2010; Eddy Ives 2012; Kirk 2012; Larsen 2015; Lison 2012; Nova 2001; Nowicka 2009; Rodearmel 2007; Schwingshandl 1999; Vann 2013; Woo 2004). Ho 2016 and Satoh 2007 were the only studies to include a diet component alone.

The majority of studies ( $\mathrm{N}=65)$ included the child and parent/ caregiver (or child's family). Four of these 65 studies involved both the child and parent/caregiver; however, the main aim of the intervention was to target the parent (McCallum 2007; Taveras 2015; Wafa 2011; Warschburger 2016). Five studies only involved the child in the intervention and there was no input from the parent/caregiver (Alves 2008; Faude 2010; Maddison 2011), Schwingshandl 1999, Vann 2013). One study directly investigated whether parental involvement or parental control would add benefit to an intervention aimed at the child (Bathrellou 2010).

Participants in two studies were given treatment before randomisation, this included de Niet 2012 where a behaviouralchanging treatment (BFC) programme was given to all participants, then they were randomised to receive a short message service maintenance treatment (via text messages) plus BFC follow-up sessions or BFC follow-up sessions only for an additional nine months. Wilfley 2007 included a weight-loss treatment and then participants were randomised to three different maintenance arms. These two studies were the only two studies that specifically investigated the impact of a maintenance programme (rather than treatment programme).

Treatments provided to the intervention and comparator groups were mainly led (or co-led) by registered dietitians, therapists or psychologists. Other professionals involved in providing treatment included nutritionists, paediatricians, nurses, physical activity teachers/coaches, exercise consultants/ specialists, undergraduates/postgraduates studying nutrition or physical activity-related or medical degrees, GPs, physicians, physiotherapists, exercise psychologists, health educators/ trainers, research assistants, trained study members and community workers.

\section{Outcomes}

Fifty-one trials explicitly stated a primary/secondary endpoint in their publications (Appendix 5). The most commonly defined primary outcome was BMI or BMI z score (SDS). A median of seven outcomes was collected by the 70 studies, ranging between two and 27 outcomes. All 70 studies measured at least one outcome defined in this review - for a detailed description of how each outcome was measured in each study see Appendix 7. A total of 67 studies reported measuring BMI or BMI z scores in their publications. Only six studies reported adverse events occurring (it was unclear whether any adverse events occurred in 29 studies). Forty-seven studies measured additional body fat distribution measures such as waist circumference, body fat percentage and percent overweight. Fifty-six studies measured behaviour-change outcomes using validated tools, such as physical activity via accelerometry data, and dietary behaviours via food frequency questionnaires. Health-related quality of life or selfesteem was measured by 21 studies, while participants' views of the intervention was reported by nine studies. Only two studies reported morbidity data such as number of participants with 
metabolic syndrome. No studies reported socioeconomic effects or all-cause mortality.

We found differences between defined primary outcomes in publication and trials registers/protocols eight studies (Boutelle 2014; de Niet 2012; Epstein 2015; Kalarchian 2009; Kirk 2012; Lochrie 2013; Looney 2014; Taveras 2015) - see Appendix 5 and Appendix 6 for more details on outcome reporting bias.

\section{Excluded studies}

We excluded 402 full-text articles after evaluation, see Characteristics of excluded studies. The main reasons for exclusions was the trial not being an RCT, mean age was not six years to less than 12 years old, the aim of the study was preventing overweight/ obesity, and length of follow-up was less than six months from baseline.

\section{Risk of bias in included studies}

For details on the risk of bias of the included trials see Characteristics of included studies. For an overview of review authors' judgements about each risk of bias item for individual trials and across all trials see Figure 2 and Figure 3.

Figure 2. Risk of bias graph: review authors' judgements about each risk of bias item presented as percentages across all included studies (blank cells indicate that the particular outcome was not investigated in some studies)

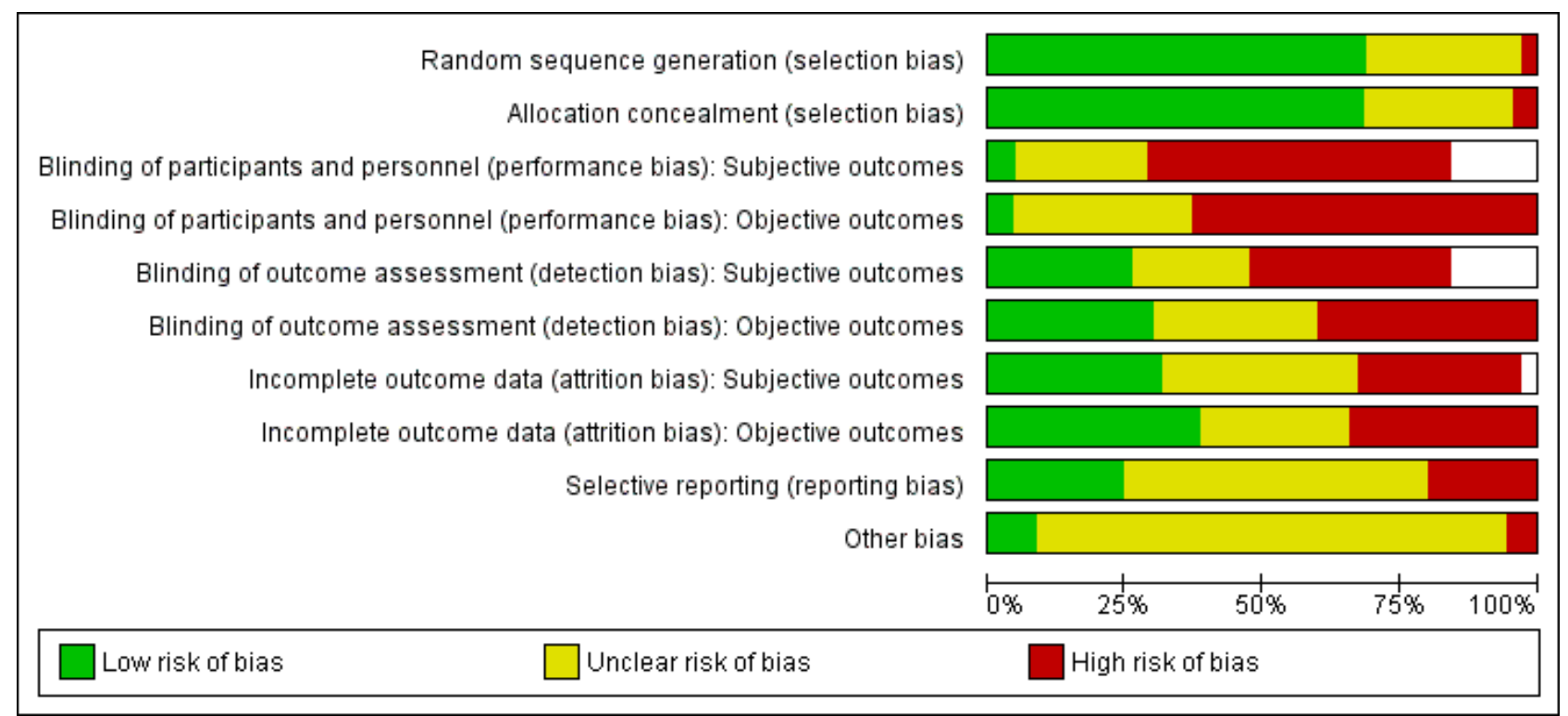

Diet, physical activity and behavioural interventions for the treatment of overweight or obese children from the age of 6 to 11 years 
Figure 3. Risk of bias summary: review authors' judgements about each risk of bias item for each included study (blank cells indicate that the study did not report that particular outcome)

\begin{tabular}{|c|c|c|c|c|c|c|c|c|c|c|}
\hline & 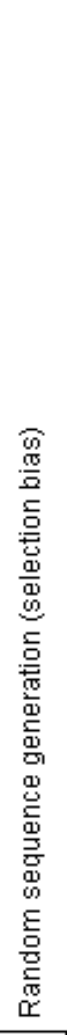 & 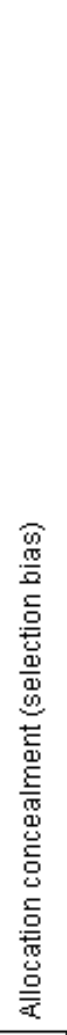 & 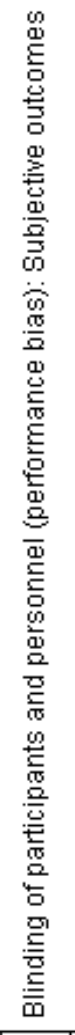 & 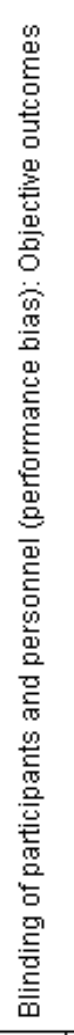 & 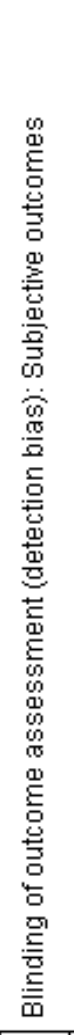 & 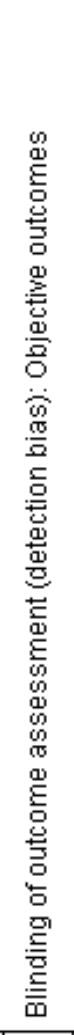 & 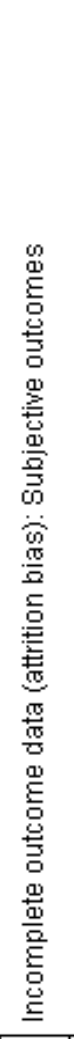 & 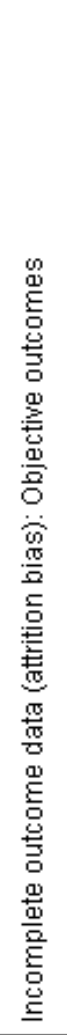 & 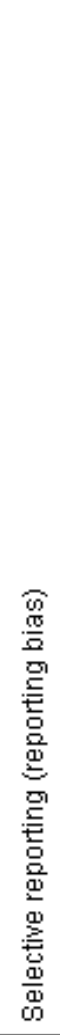 & 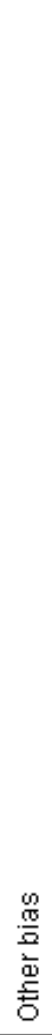 \\
\hline Alves 2008 & $\odot$ & $\odot$ & & $\odot$ & & - & $?$ & + & $?$ & $?$ \\
\hline Arauz Boudreau 2013 & $?$ & $?$ & $?$ & $\odot$ & 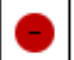 & - & $\odot$ & $\Theta$ & $?$ & $?$ \\
\hline Barkin 2011 & $?$ & $?$ & & $?$ & & $?$ & $?$ & $\Theta$ & 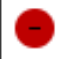 & $?$ \\
\hline Bathrellou 2010 & $?$ & $?$ & & $?$ & & $?$ & $?$ & $\Theta$ & $?$ & $?$ \\
\hline Berry 2007 & $\odot$ & $\odot$ & + & 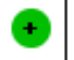 & + & + & $?$ & $?$ & $?$ & - \\
\hline Berry 2014 & $?$ & $\odot$ & $\odot$ & $\odot$ & $\odot$ & $\odot$ & $\odot$ & $\odot$ & $\odot$ & $?$ \\
\hline Boutelle 2014 & $\odot$ & + & O & $\odot$ & + & + & + & + & $?$ & $?$ \\
\hline Bryant 2011 & $\odot$ & $\odot$ & $\odot$ & $\odot$ & $\odot$ & 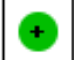 & $?$ & $?$ & $?$ & $?$ \\
\hline Coppins 2011 & $?$ & $\odot$ & $O$ & $\odot$ & 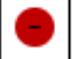 & 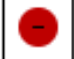 & + & + & $?$ & $?$ \\
\hline Croker 2012 & $\odot$ & $\odot$ & $\odot$ & $\odot$ & $?$ & $\odot$ & $\odot$ & $\Theta$ & $\odot$ & $?$ \\
\hline Davis 2013 & $\odot$ & $\odot$ & $?$ & $?$ & + & + & $?$ & $?$ & + & $?$ \\
\hline Davoli 2013 & $\odot$ & $\odot$ & - & $\theta$ & $\theta$ & $\theta$ & $\odot$ & + & + & $?$ \\
\hline de Niet 2012 & + & + & $O$ & $\odot$ & $O$ & O & $?$ & $?$ & $?$ & $?$ \\
\hline Diaz 2010 & + & + & & $\odot$ & & + & & $?$ & $?$ & $?$ \\
\hline Duffy 1993 & $?$ & $?$ & & $?$ & & $?$ & $?$ & $\Theta$ & $?$ & $?$ \\
\hline Duggins 2010 & $\odot$ & + & & & & & & $?$ & $?$ & $?$ \\
\hline
\end{tabular}

Diet, physical activity and behavioural interventions for the treatment of overweight or obese children from the age of 6 to 11 years 
Figure 3. (Continued)

\begin{tabular}{|c|c|c|c|c|c|c|c|c|c|c|}
\hline \multirow{2}{*}{ Duggins 2010} & \\
\hline & + & $\odot$ & $\odot$ & $\odot$ & $\odot$ & $\odot$ & $\odot$ & $?$ & $?$ & $?$ \\
\hline Eddy lwes 2012 & + & $\odot$ & $\odot$ & 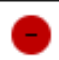 & $\odot$ & $\odot$ & $?$ & $?$ & $?$ & $?$ \\
\hline Epstein 1984a & $?$ & + & 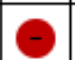 & - & $\odot$ & ○ & + & + & $?$ & $?$ \\
\hline Epstein 1985a & $?$ & $\odot$ & $O$ & $\odot$ & $\odot$ & $\odot$ & + & + & $?$ & $?$ \\
\hline Epstein 1985b & + & $\odot$ & $\odot$ & $\odot$ & $\Theta$ & $\odot$ & $?$ & $?$ & $?$ & $?$ \\
\hline Epstein $1985 \mathrm{c}$ & $?$ & $\odot$ & $O$ & $\odot$ & $\Theta$ & $\odot$ & $?$ & $?$ & $?$ & $?$ \\
\hline Epstein 2000a & + & + & 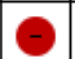 & - & $\odot$ & ○ & $\theta$ & 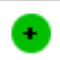 & $\odot$ & $?$ \\
\hline Epstein 2001 & + & $\odot$ & $O$ & $\odot$ & $\Theta$ & $\odot$ & + & + & $?$ & $?$ \\
\hline Epstein 2005 & + & $\odot$ & $\odot$ & $\odot$ & $?$ & $?$ & $?$ & $?$ & $?$ & $?$ \\
\hline Epstein 2015 & + & $\odot$ & $O$ & $\odot$ & $?$ & $\odot$ & $?$ & $?$ & $\oplus$ & $?$ \\
\hline Faude 2010 & $?$ & $?$ & $?$ & $?$ & $?$ & $?$ & 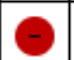 & - & $?$ & $?$ \\
\hline Flodmark 1993 & $?$ & $?$ & & $?$ & & $?$ & $?$ & $\oplus$ & $?$ & $?$ \\
\hline Gillis 2007 & $O$ & $\Theta$ & 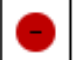 & $\odot$ & $\Theta$ & $\odot$ & $O$ & $\odot$ & $?$ & $?$ \\
\hline Gunnarsdottir 2011a & $?$ & $?$ & $?$ & $?$ & $?$ & $?$ & $?$ & $?$ & $\odot$ & $?$ \\
\hline Hamilton-Shield 2014 & + & $\odot$ & $?$ & $?$ & $?$ & $?$ & 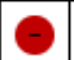 & $\odot$ & $\odot$ & - \\
\hline Ho 2016 & $\odot$ & $\odot$ & $\odot$ & $\odot$ & $?$ & $?$ & $\odot$ & $\odot$ & $\odot$ & $\odot$ \\
\hline Hughes 2008 & $\odot$ & $\odot$ & $\odot$ & $\odot$ & $\odot$ & $\odot$ & $\odot$ & $\odot$ & $\odot$ & $?$ \\
\hline Kalarchian 2009 & + & $\odot$ & $O$ & $\odot$ & $?$ & $?$ & + & + & $\odot$ & $?$ \\
\hline Kalavainen 2007 & + & 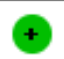 & & $\odot$ & & $\odot$ & $?$ & + & $?$ & $?$ \\
\hline Kirk 2012 & $\odot$ & $\odot$ & $\odot$ & $\odot$ & $\odot$ & $\odot$ & $?$ & $?$ & $\oplus$ & $?$ \\
\hline Larsen 2015 & + & $\odot$ & & $?$ & & $?$ & $?$ & $\odot$ & $?$ & $?$ \\
\hline Lison 2012 & $\theta$ & $\Theta$ & & $\odot$ & & $\odot$ & $?$ & $?$ & + & $?$ \\
\hline Lochrie 2013 & + & $\odot$ & $O$ & $\odot$ & $\odot$ & $\odot$ & $O$ & $\odot$ & $\odot$ & $?$ \\
\hline Looney 2014 & + & $\odot$ & $\odot$ & $\odot$ & $\Theta$ & $\odot$ & + & 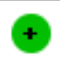 & $?$ & $?$ \\
\hline Maddison 2011 & 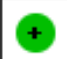 & $\odot$ & $\odot$ & $\odot$ & $\Theta$ & $\odot$ & $?$ & $?$ & $\odot$ & $?$ \\
\hline Maddison 2014 & + & + & $\odot$ & - & 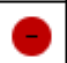 & ○ & + & + & + & $?$ \\
\hline Markert 2014 & $\odot$ & $\odot$ & $?$ & $?$ & $?$ & $?$ & $O$ & $\odot$ & $?$ & $?$ \\
\hline McCallum 2007 & $\odot$ & $\odot$ & $\odot$ & $\odot$ & $\odot$ & $\odot$ & + & $\oplus$ & $\oplus$ & $\odot$ \\
\hline Mirza 2013 & $\odot$ & $?$ & $\odot$ & $\odot$ & $\odot$ & $\odot$ & 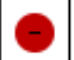 & $\odot$ & $\odot$ & $?$ \\
\hline NCT02436330 & $?$ & $?$ & $\odot$ & - & $\odot$ & $\odot$ & $\theta$ & - & $\odot$ & $?$ \\
\hline Nemet 2005 & $\odot$ & $?$ & $?$ & $?$ & $?$ & $?$ & $?$ & $?$ & $?$ & $?$ \\
\hline
\end{tabular}

Diet, physical activity and behavioural interventions for the treatment of overweight or obese children from the age of 6 to 11 years 
Figure 3. (Continued)

\begin{tabular}{|c|c|c|c|c|c|c|c|c|c|c|}
\hline Nemet 2005 & $\odot$ & $?$ & $?$ & $?$ & $?$ & $?$ & $?$ & $?$ & $?$ & $?$ \\
\hline Nova 2001 & $?$ & $?$ & $?$ & $?$ & $?$ & $?$ & $\odot$ & $\odot$ & $\odot$ & $?$ \\
\hline Nowicka 2009 & $?$ & $\odot$ & $O$ & $\odot$ & $\odot$ & $\odot$ & $?$ & $?$ & $?$ & $?$ \\
\hline O'Connor 2013 & $\odot$ & $\odot$ & 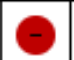 & $\odot$ & $\odot$ & $\odot$ & + & + & $?$ & $?$ \\
\hline Reinehr 2010 & $\odot$ & $\odot$ & $\theta$ & - & $\odot$ & $\odot$ & + & + & $\odot$ & $?$ \\
\hline Rodearmel 2007 & $?$ & $\odot$ & $?$ & $?$ & $\ominus$ & $\odot$ & $\odot$ & + & $?$ & $?$ \\
\hline Sacher 2010 & + & $\odot$ & - & ○ & $\odot$ & $\oplus$ & $O$ & $\odot$ & $\odot$ & $?$ \\
\hline Saelens 2013 & $\odot$ & $\odot$ & $?$ & $?$ & $\odot$ & $\odot$ & $O$ & $\odot$ & $?$ & $?$ \\
\hline Satoh 2007 & $?$ & $?$ & $?$ & $?$ & $?$ & $?$ & $\odot$ & $\odot$ & $?$ & $?$ \\
\hline Schwingshandl 1999 & $?$ & $?$ & & $?$ & & $?$ & $?$ & $\odot$ & $\odot$ & $?$ \\
\hline Serra-Paya 2015 & $\odot$ & $?$ & + & + & $\odot$ & $?$ & $?$ & $?$ & $?$ & + \\
\hline Siwik 2013 & $\odot$ & $?$ & $?$ & $?$ & $?$ & $?$ & $\odot$ & + & $\odot$ & $?$ \\
\hline Taveras 2015 & + & $\odot$ & $?$ & $?$ & $\odot$ & + & + & $\oplus$ & $?$ & + \\
\hline Taylor 2015 & + & $\odot$ & 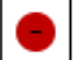 & $\odot$ & $\odot$ & $\odot$ & $\odot$ & $\odot$ & $\odot$ & $?$ \\
\hline Vann 2013 & + & $\odot$ & $?$ & $?$ & $\odot$ & $\odot$ & $O$ & $\odot$ & $?$ & $?$ \\
\hline Wafa 2011 & $\odot$ & $\odot$ & $\odot$ & $\odot$ & $\odot$ & $\odot$ & 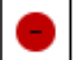 & $\odot$ & $\odot$ & $?$ \\
\hline Wake 2009 & $\odot$ & $\odot$ & $\odot$ & $\odot$ & $\odot$ & $\odot$ & $\odot$ & $\odot$ & $?$ & $\odot$ \\
\hline Wake 2013 & $\odot$ & $\odot$ & - & $\odot$ & $\odot$ & $\odot$ & $\odot$ & 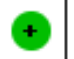 & $\odot$ & 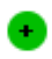 \\
\hline Waling 2012 & $\odot$ & $?$ & $\odot$ & $\odot$ & $?$ & $?$ & $\odot$ & $\odot$ & $\odot$ & $?$ \\
\hline Warschburger 2016 & + & $\odot$ & $\odot$ & $\odot$ & $\odot$ & $\odot$ & $\odot$ & $\odot$ & $?$ & $?$ \\
\hline Weigel 2008 & $?$ & $?$ & & $?$ & & $?$ & & 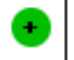 & $?$ & $?$ \\
\hline Weintraub 2008 & 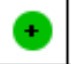 & $\odot$ & $?$ & $?$ & $\Theta$ & $\odot$ & + & $\odot$ & $\odot$ & $?$ \\
\hline Wilfley 2007 & + & $?$ & $?$ & $?$ & $\odot$ & $\odot$ & + & $\oplus$ & $\odot$ & $?$ \\
\hline Woo 2004 & + & $\odot$ & $\ominus$ & ○ & $\odot$ & $\odot$ & $?$ & $?$ & $?$ & $\odot$ \\
\hline Wright 2012 & $?$ & $?$ & $?$ & $?$ & $?$ & $?$ & $O$ & $\odot$ & $?$ & $\theta$ \\
\hline
\end{tabular}

\section{Allocation}

Forty-eight studies reported adequate sequence generation (i.e. low risk), 20 were unclear, and two were high risk due to the randomisation method they used. We rated 49 studies low risk (i.e. adequate allocation concealment), 18 were unclear and three were high risk of allocation concealment. Overall, the risk of selection bias was low for 42 studies, unclear for 26 studies and high for two studies (Gillis 2007; Lison 2012).

\section{Blinding}

Forty-four studies did not blind their participants or study personnel to study group allocation with regards to objective measures and we assessed them as high risk. We rated 23 studies as unclear and three studies as low risk for performance bias because participants and study personnel were both blinded to study group allocation. With regards to subjective measures, we judged all bias assessments to be at the same level of risk as the objective measures unless a study did not have any subjective outcomes, 
then the risk was left blank in the risk of bias table and figures (this also applied to detection and attrition bias).

Outcome assessors collecting objective outcomes were blinded to the study group in 21 studies and we assessed them as low risk, while in 21 studies it was unclear whether outcome assessment was blinded; we rated 28 studies as high risk of detection bias because outcome assessment was not blinded. If a study had subjective outcomes, then we gave the detection bias assessment the same classification as for objective measures.

\section{Incomplete outcome data}

Dropout rates were classed as low if less than 15\%; high if more than $25 \%$ in studies with follow-up from baseline of six to 12 months or more than $30 \%$ in studies with over 12 months' follow-up; unclear if more than $15 \%$ but less than $25 \%$ in studies with follow-up from six to 12 months, or less than $30 \%$ in studies with follow-up more than 12 months. We also took into consideration whether a study used intention-to-treat and also what method it used to impute missing data. For objective outcomes, we rated 27 studies as low risk; 24 studies at high risk; and 19 studies at unclear risk.

\section{Selective reporting}

To assess selective outcome reporting we checked whether publications reported outcomes described in the publication itself and in a protocol/clinical trials register entry. We rated 17 studies as low risk because they provided results for all outcomes described. Studies could only be rated as low risk if they had published a protocol or registered the trial on a clinical trials website because there was no other way to determine if the publication had reported all outcomes intended to be measured.

We classified 14 studies as having a high risk of selective outcome reporting. In Kalarchian 2009 the clinical trials register stated BMI and cardiovascular risk factors as the primary outcome; however, in the publication it was percentage overweight. In Barkin 2011 they did not report BMI outcome results for the intervention and control groups separately, only for the group combined. Epstein 2000a also combined all three groups together in the additional publication (Epstein 2001), likely due to non-significant results. Lochrie 2013 did not report raw data at baseline and follow-up (or mean change) for each group, while Gunnarsdottir 2011a failed to compare intervention and control outcomes and did not present raw results for many of its intended measured outcomes, including health-related quality of life. Reinehr 2010 did not provide qualityof-life measures separately for each group. Mirza 2013 also failed to present the results for many of its outcomes, including outcomes described on a clinical trials website. Croker 2012 also did not provide the results for all outcomes reported on the clinical trials website. Nova 2001 did not provide behavioural outcome results at follow-up, or results at 24 months' follow-up (endpoint) while Schwingshandl 1999 did not provide any BMI data at the study's endpoint (12 months). Hamilton-Shield 2014 terminated the trial before its endpoint; however, it failed to provide any data on outcomes collected before termination.

The remaining 39 studies we rated as unclear risk of selective outcome reporting bias primarily because the trial protocol was not published in advance of the study or registered on a clinical trials website. There were however, additional reasons why we classified risk of bias as unclear: Boutelle 2014 had a clinical trials entry but we rated it as unclear because the entry stated that there were three intervention groups and one control group; however, in the publication there was only one intervention and one control group. In addition, Bryant 2011; Coppins 2011; Eddy Ives 2012; Markert 2014; Wake 2009 and Warschburger 2016 had clinical trials entries but they were retrospectively entered, while O'Connor 2013 only provided one outcome measure (family attendance) on its clinical trials register entry. Potential bias may also occur in Coppins 2011 due to only reporting some outcomes as significant or non-significant (no raw results). Looney 2014 reported measuring cost-effectiveness on the clinical trials entry; however, this is not reported in the publication. In addition Sacher 2010; Serra-Paya 2015 and Taveras 2015 reported a number of outcomes in their clinical trials register entries that were not reported in the main publications.

\section{Other potential sources of bias}

We rated 60 studies as unclear, mainly because of a lack of detail in the publication or an unclear risk of bias for the other domains resulting in uncertainty of the presence of other biases. Six studies were low risk because the trials were generally well-conducted and well-reported (Ho 2016; McCallum 2007; Serra-Paya 2015; Taveras 2015; Wake 2009; Wake 2013). Four studies were high risk - Berry 2007 and Wright 2012 were cluster RCTs but did not adjust for clustering in their analyses, Woo 2004 non-randomly split their intervention group into two groups at six weeks, and HamiltonShield 2014 was terminated before the study's endpoint because of problems with recruitment and equipment.

\section{Effects of interventions}

See: Summary of findings for the main comparison Diet, physical activity and behavioural interventions for the treatment of overweight or obesity in children aged 6 to 11 years

\section{Baseline characteristics}

For details of baseline characteristics, see Appendix 3 and Appendix 4.

\section{Behaviour-changing interventions versus no treatment or usual care}

Fifty-five trials compared behaviour-changing (diet and/or physical activity and/or behavioural therapy) interventions, usual care, enhanced usual care, information control, or wait-list control. Excluding cluster RCTs and weight maintenance trials $(N=5)$ there were 20 trials in which the control groups received no treatment throughout the duration of the study and 30 trials in which the control group participants were given usual care, either defined by the trial author or assessed by the review authors. We considered outcomes here at the longest follow-up point reported for each trial.

\section{Primary outcomes}

\section{Changes in body mass index (BMI), BMI z score and body weight}

Twenty-four trials reported BMI change data that could be metaanalysed. Pooling the effects in a random-effects meta-analysis (Analysis 1.1; Figure 4) demonstrated a reduction in BMI in the intervention groups compared with controls at the final follow-up: MD - $0.53 \mathrm{~kg} / \mathrm{m}^{2}$ (95\% Cl -0.82 to -0.24$) ; \mathrm{P}=0.0004 ; 24$ trials; 2785 participants; low-quality evidence. 
Figure 4. Forest plot of comparison: 1 Lifestyle intervention versus no treatment/usual care, outcome: 1.1 Change in BMI (all trials) $\left(\mathrm{kg} / \mathrm{m}^{2}\right)$

\begin{tabular}{|c|c|c|c|c|c|c|c|c|c|c|}
\hline Study or Subgroup & $\begin{array}{r}\text { Interv } \\
\text { Mean }\left[\mathbf{k g} / \mathbf{m}^{2}\right]\end{array}$ & Intervention & Total & $\begin{array}{r}\text { Col } \\
\text { Mean }\left[k g / \mathrm{m}^{2}\right]\end{array}$ & \multicolumn{2}{|c|}{ Control } & Weight & $\begin{array}{c}\text { Mean Difference } \\
\mathrm{I}, \text { Random, } 95 \% \mathrm{Cl}\left[\mathrm{kg} / \mathrm{m}^{2}\right]\end{array}$ & \multicolumn{2}{|c|}{$\begin{array}{c}\text { Mean Difference } \\
\text { IV, Random, 95\% CI [kg/m²] }\end{array}$} \\
\hline Alves 2008 & -0.27 & 0.93 & 39 & 0.26 & 1.38 & 39 & $7.1 \%$ & $-0.53[-1.05,-0.01]$ & $\rightarrow-1$ & \\
\hline Boutelle 2014 & -0.1 & 4.7292 & 21 & 0.6 & 4.6584 & 18 & $0.9 \%$ & $-0.70[-3.65,2.25]$ & & \\
\hline Croker 2012 & -0.36 & 1.06 & 33 & -0.03 & 1.07 & 30 & $7.1 \%$ & $-0.33[-0.86,0.20]$ & - & \\
\hline Davoli 2013 & 1.52 & 1.59 & 186 & 1.56 & 1.5856 & 185 & $8.3 \%$ & $-0.04[-0.36,0.28]$ & & \\
\hline Diaz 2010 & -0.6 & 2.2562 & 33 & 0.6 & 1.9741 & 33 & $4.3 \%$ & $-1.20[-2.22,-0.18]$ & - & \\
\hline Eddy lves 2012 & -0.23 & 2.4993 & 61 & -0.3 & 3.176 & 64 & $4.4 \%$ & $0.07[-0.93,1.07]$ & - & - \\
\hline Faude 2010 & 0.5 & 3.1209 & 11 & 0.6 & 3.834 & 11 & $0.9 \%$ & $-0.10[-3.02,2.82]$ & & \\
\hline Ho 2016 & 0.02 & 1.64 & 37 & 0.14 & 1.52 & 36 & $5.9 \%$ & $-0.12[-0.85,0.61]$ & - & - \\
\hline Kalarchian 2009 & 1.5 & 2.9547 & 97 & 1.72 & 2.0468 & 95 & $5.9 \%$ & $-0.22[-0.94,0.50]$ & $\longrightarrow$ & - \\
\hline Kalavainen 2007 & 2.1 & 1.9 & 34 & 2.3 & 2.7 & 34 & $4.0 \%$ & $-0.20[-1.31,0.91]$ & & - \\
\hline Lison 2012 (1) & -0.8 & 4.0765 & 64 & 1.6 & 3.716 & 20 & $1.9 \%$ & $-2.40[-4.31,-0.49]$ & 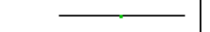 & \\
\hline Maddison 2011 & 0.09 & 1.0119 & 160 & 0.34 & 1.0182 & 162 & $8.8 \%$ & $-0.25[-0.47,-0.03]$ & - & \\
\hline Maddison 2014 & 0.12 & 4.5971 & 117 & 0.13 & 5.2407 & 113 & $3.3 \%$ & $-0.01[-1.29,1.27]$ & & \\
\hline McCallum 2007 & 1.2 & 2.761 & 70 & 1.2 & 2.162 & 76 & $5.4 \%$ & $0.00[-0.81,0.81]$ & - & - \\
\hline Nemet 2005 & -1.6 & 4.2575 & 20 & 0.6 & 5.5231 & 20 & $0.8 \%$ & $-2.20[-5.26,0.86]$ & & \\
\hline Reinehr 2010 & -0.85 & 1.02 & 34 & 0.76 & 0.99 & 32 & $7.4 \%$ & $-1.61[-2.09,-1.13]$ & $\infty$ & \\
\hline Sacher 2010 & -1.5 & 3.5158 & 37 & 0.6 & 5.058 & 45 & $1.9 \%$ & $-2.10[-3.96,-0.24]$ & & \\
\hline Siwik 2013 & 0.314 & 3.6751 & 15 & 0.716 & 6.362 & 17 & $0.6 \%$ & $-0.40[-3.95,3.15]$ & & \\
\hline Taylor 2015 & 0.8 & 2.9811 & 89 & 1.2 & 2.2924 & 92 & $5.6 \%$ & $-0.40[-1.18,0.38]$ & & \\
\hline Wake 2009 & 0.6 & 2.592 & 127 & 0.7 & 2.1984 & 115 & $6.6 \%$ & $-0.10[-0.70,0.50]$ & - & - \\
\hline Wake 2013 & 0.9 & 3.3899 & 56 & 0.8 & 4.193 & 49 & $2.8 \%$ & $0.10[-1.37,1.57]$ & & \\
\hline Waling $2012(2)$ & 0.3 & 2.868 & 36 & 0.6 & 2.6267 & 35 & $3.3 \%$ & $-0.30[-1.58,0.98]$ & & - \\
\hline Weigel 2008 & -1.5 & 3.042 & 36 & 2.8 & 3.8614 & 30 & $2.2 \%$ & $-4.30[-6.00,-2.60]$ & & \\
\hline Weintraub 2008 & 0.22 & 5.217 & 9 & 0.79 & 4.8359 & 12 & $0.4 \%$ & $-0.57[-4.94,3.80]$ & & \\
\hline Total $(95 \% \mathrm{Cl})$ & & & 1422 & & & 1363 & $100.0 \%$ & $-0.53[-0.82,-0.24]$ & $\bullet$ & \\
\hline $\begin{array}{l}\text { Heterogeneity: } \text { Tau }^{z} \\
\text { Test for overall effect }\end{array}$ & $\begin{array}{l}0.24 ; \mathrm{Chi}^{2}=66.4 \\
Z=3.56(P=0.0\end{array}$ & $\begin{array}{l}49, \mathrm{df}=23(\mathrm{P} \\
1004)\end{array}$ & $\theta<0.00$ & $0001) ; I^{2}=65 \%$ & & & & & $\begin{array}{ccc}1 & 1 & \\
-4 & -2 & 0 \\
\text { Favours intervention }\end{array}$ & $\begin{array}{ccc} & 2 & 4 \\
\text { Favours control }\end{array}$ \\
\hline
\end{tabular}

Thirty-seven trials reported BMI z score change data that could be meta-analysed. Pooling the effects in a random-effects metaanalysis (Analysis 1.2; Figure 5) demonstrated a reduction in BMI z score in the intervention groups compared with controls at the final follow-up: $\mathrm{MD}-0.06$ units $(95 \% \mathrm{Cl}-0.10$ to -0.02$) ; \mathrm{P}=0.001 ; 37$ trials; 4019 participants; low-quality evidence. 
Figure 5. Forest plot of comparison: 1 Lifestyle intervention versus no treatment/usual care, outcome: 1.2 Change in BMI z score (all trials)

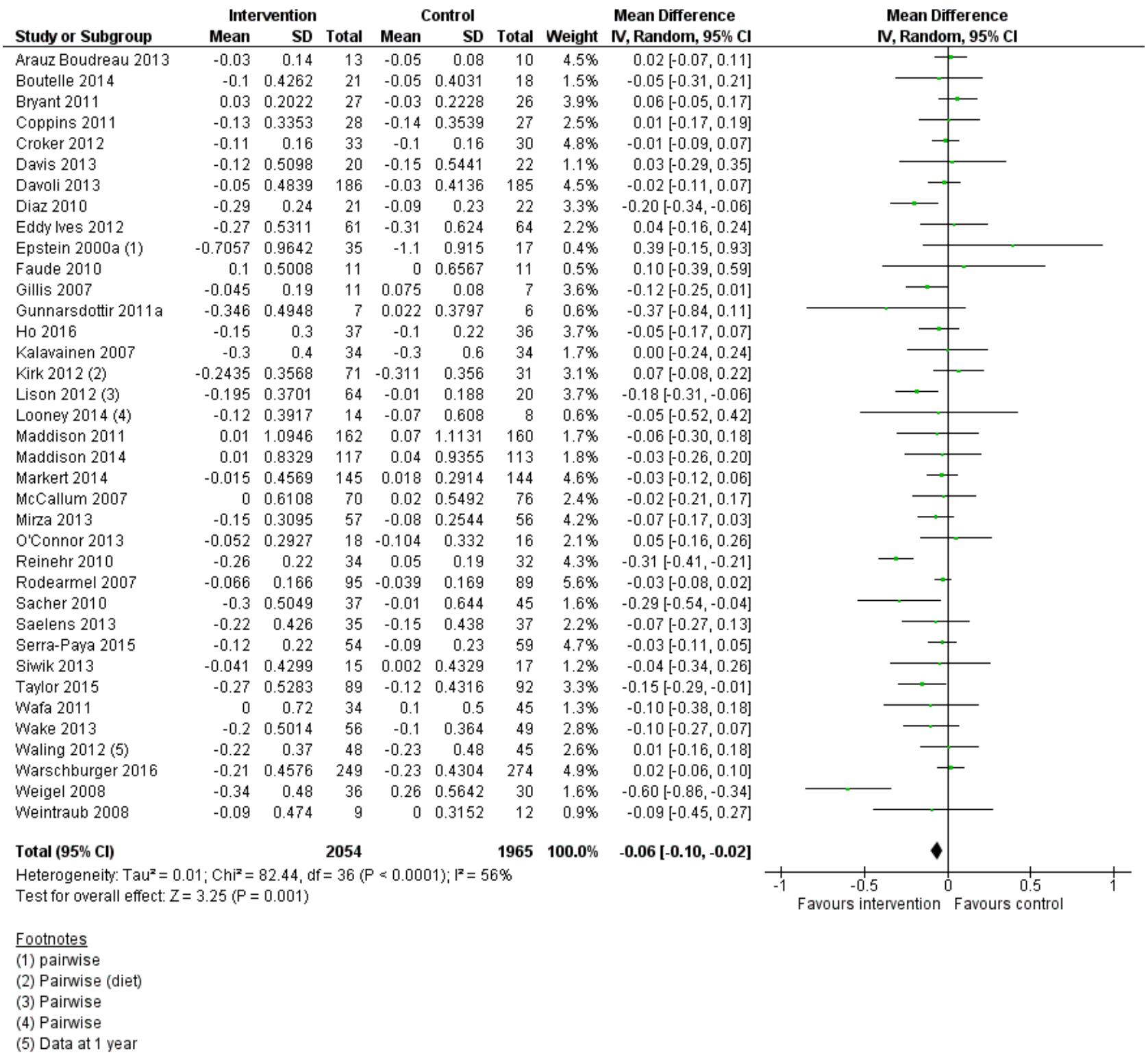

Seventeen trials reported data on change in body weight that could be meta-analysed. Pooling the effects in a random-effects metaanalysis (Analysis 1.3; Figure 6) demonstrated a reduction in body weight in the intervention groups compared with controls at the final follow-up: MD - $1.45 \mathrm{~kg}(95 \% \mathrm{Cl}-1.88$ to -1.02$)$; $\mathrm{P}<0.00001 ; 17$ trials; 1774 participants; low-quality evidence. 
Figure 6. Forest plot of comparison: 1 Lifestyle intervention versus no treatment/usual care, outcome: 1.3 Change in weight (all trials)

\begin{tabular}{|c|c|c|c|c|c|c|c|c|c|c|}
\hline Study or Subgroup & \multicolumn{3}{|c|}{ Intervention } & $\begin{array}{r}\text { Co } \\
\text { Mean }[\mathbf{k g}] \\
\end{array}$ & \multicolumn{2}{|c|}{ Control } & Weight & $\begin{array}{c}\text { Mean Difference } \\
\text { IV, Random, 95\% Cl [kg] }\end{array}$ & \multicolumn{2}{|c|}{$\begin{array}{c}\text { Mean Difference } \\
\text { IV, Random, } 95 \% \mathrm{Cl}[\mathrm{kg}]\end{array}$} \\
\hline Alves 2008 & 0.58 & 1.34 & 39 & 1.95 & 1.45 & 39 & $48.5 \%$ & $-1.37[-1.99,-0.75]$ & $\square$ & \\
\hline Coppins 2011 & 3.9 & 5.5189 & 28 & 5.1 & 5.8141 & 27 & $2.1 \%$ & $-1.20[-4.20,1.80]$ & ? & - \\
\hline Croker 2012 & 0.79 & 2.84 & 33 & 2.78 & 2.85 & 30 & $9.4 \%$ & $-1.99[-3.40,-0.58]$ & -- & \\
\hline Diaz 2010 & 2.1 & 6.0916 & 33 & 5.6 & 5.9224 & 33 & $2.2 \%$ & $-3.50[-6.40,-0.60]$ & 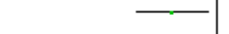 & \\
\hline Eddy lves 2012 & 3.2 & 9.4035 & 61 & 3.35 & 11.432 & 64 & $1.4 \%$ & $-0.15[-3.81,3.51]$ & & \\
\hline Epstein 2000a (1) & 8.9756 & 12.9568 & 45 & 7.2 & 17 & 32 & $0.4 \%$ & $1.78[-5.23,8.78]$ & & \\
\hline Faude 2010 & 4.1 & 13.4721 & 11 & 3.8 & 11.7475 & 11 & $0.2 \%$ & $0.30[-10.26,10.86]$ & & \\
\hline Но 2016 & 2.55 & 5.16 & 37 & 2.67 & 5 & 36 & $3.4 \%$ & $-0.12[-2.45,2.21]$ & - & - \\
\hline Kalarchian 2009 & 11.77 & 6.8942 & 97 & 13.35 & 5.3607 & 95 & $6.1 \%$ & $-1.58[-3.32,0.16]$ & & \\
\hline Kalavainen 2007 & 17.3 & 5.2 & 34 & 17.1 & 7.4 & 34 & $2.0 \%$ & $0.20[-2.84,3.24]$ & & \\
\hline Maddison 2011 & 2.03 & 13.0843 & 162 & 2.75 & 14.7236 & 160 & $2.0 \%$ & $-0.72[-3.76,2.32]$ & & - \\
\hline Maddison 2014 & 2.8 & 16.2899 & 117 & 2.59 & 18.4327 & 113 & $0.9 \%$ & $0.21[-4.29,4.71]$ & & \\
\hline Nemet 2005 & 0.6 & 16.6677 & 20 & 5.2 & 24.2211 & 20 & $0.1 \%$ & $-4.60[-17.49,8.29]$ & & \\
\hline Siwik 2013 & 3.437 & 8.842 & 15 & 3.934 & 15.9111 & 17 & $0.2 \%$ & $-0.50[-9.28,8.29]$ & & \\
\hline Taylor 2015 & 7.5 & 10.4151 & 89 & 8.1 & 8.0186 & 92 & $2.5 \%$ & $-0.60[-3.31,2.11]$ & $\longrightarrow$ & - \\
\hline Wafa 2011 & 1.5 & 2.5 & 34 & 3.5 & 2 & 45 & $17.8 \%$ & $-2.00[-3.02,-0.98]$ & $\rightarrow-$ & \\
\hline Waling 2012 (2) & 6.7 & 11.436 & 36 & 8.8 & 10.8738 & 35 & $0.7 \%$ & $-2.10[-7.29,3.09]$ & & \\
\hline Total $(95 \% \mathrm{Cl})$ & & & 891 & & & 883 & $100.0 \%$ & $-1.45[-1.88,-1.02]$ & 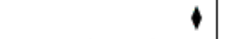 & \\
\hline $\begin{array}{l}\text { Heterogeneity: Tau } \\
\text { Test for overall effec }\end{array}$ & $\begin{array}{l}0.00 ; \mathrm{Chi}^{2}= \\
Z=6.59(\mathrm{P}=\end{array}$ & $\begin{array}{l}8.95, d f= \\
0.00001)\end{array}$ & $16(P=$ & $0.92) ;\left.\right|^{2}=09$ & & & & & $\begin{array}{ccc}1 & 1 \\
-10 & -5 & 0 \\
\text { Favours intervention }\end{array}$ & $\begin{array}{cc}5 & 10 \\
\text { Favours control }\end{array}$ \\
\hline
\end{tabular}

Some meta-analyses revealed substantial heterogeneity which we explored by subgroup analysis by type of control, type of intervention, risk of attrition bias, setting of intervention and period of post-intervention follow-up. The heterogeneity was not fully explained by any of these factors (see "Subgroup analyses" section below).

We were unable to include nine trials with no treatment or usual care control groups in the BMI and BMI z score meta-analyses and so they are narratively reported: Satoh 2007, Epstein 1984a and Nova 2001 only presented data for percent overweight. Nowicka 2009 reported no differences in follow-up outcome measures between the intervention and control groups. We could not include Vann 2013 in the meta-analyses because they did not provide SDs for $\mathrm{BMI}$ values at baseline and follow-up (or effect sizes and $\mathrm{P}$ values). A small decrease of $0.1 \mathrm{~kg} / \mathrm{m}^{2}$ was seen in the pedometer plus DVD group; however, an increase in BMI was observed in the two other intervention groups and the control group. Barkin 2011 only provided BMI data for the intervention and control groups combined - in a linear regression model they observed that parentchild dyads in the control group were more likely to decrease their BMI over the six-month study. Lochrie 2013 only provided means and standard errors at follow-up - the SD at baseline was unclear. The study found a larger reduction in BMI z score at 12 months' follow-up in the intervention group. Finally, Hughes 2008 only presented change in BMI $z$ score as median and IQR, which cannot be converted into mean and SD (or $95 \% \mathrm{Cl}$ ). At six months and 12 months the median difference between groups was not substantially different $(P=0.4$ and $P=0.5$, respectively). No BMI/ BMI z score data were available in Hamilton-Shield 2014 because the study was terminated.

In the weight meta-analyses, we were unable to include Hughes 2008 as this study only presented weight data as median and IQR a non-substantial difference between groups was found at both six months' and 12 months' follow-up ( $P=0.1$ and $P=0.9$, respectively). In addition, Lison 2012 did not provide any SD values for weight at follow-up so could not be included in the analyses. The control group increased their weight over the six-month period while a smaller increase in weight was seen in the hospital clinic group along with a small reduction in weight in the home-based group. Epstein 1984a provided weight data in an additional secondary analysis paper; however, they only presented data for the two intervention groups. Hence, we decided not to include these data in the weight meta-analyses because data were not reported for the control group. The authors found a reduction in weight in the two treatment groups combined. We were unable to include the remaining studies in the weight meta-analysis because no weight data were provided in the publications.

\section{Adverse events}

The majority of publications did not report whether or not any adverse events occurred; hence, we had to contact most study authors to obtain this information. As a result, it was confirmed that no adverse events occurred in 28 trials with a no-treatment/usualcare control group. In 16 trials it was unclear whether any adverse events had occurred. The remaining six studies reported adverse events occurring: Maddison 2011 and Maddison 2014 provided data on serious adverse events, as described below. Croker 2012 reported that one participant in the control group had a very high reduction in BMI and standardised BMI (BMI SDS). Kirk 2012 reported that some participants in both groups developed elevated triglycerides (12.2\%), elevated blood pressure (3.6\%), elevated LDL cholesterol (3.5\%) and/or elevated fasting glucose (3.5\%); however, there were no substantial differences by group. Weintraub 2008 reported that three adverse events occurred in the intervention group (skin rash, diagnosis of hypothyroidism, car collision) and six events in the control group (foot injury, eye pain and headaches, ingrown toenail, ear infection, knee pain, skin rash); however, none of these were considered to be related to the study, and it was unclear if any of these were considered serious adverse events. Mirza 2013 reported that no serious adverse events occurred; however, one child in the control group reported feeling faint

Diet, physical activity and behavioural interventions for the treatment of overweight or obese children from the age of 6 to 11 years 
during the three-month blood taking. Adverse events and the level of severity were author-assessed, often using pre-defined criteria; however, these criteria varied between the studies leading to potential inconsistency between the studies and this should be borne in mind when evaluating the adverse events outcomes.

Thirty-one trials reported serious adverse event data that could be meta-analysed. Pooling the effects in a random-effects metaanalysis (Analysis 1.4) demonstrated a RR of $0.57(95 \% \mathrm{Cl} 0.17$ to 1.93); $P=0.37 ; 31$ trials; 4096 participants; low-quality evidence), in favour of the intervention group. Only two of the 31 trials reported any serious adverse events; the other 29 reported zero serious adverse events. Serious adverse events were reported by Maddison 2011 and these included seasonal influenza that required hospitalisation $(\mathrm{N}=3)$, hip surgery due to a chronic condition $(\mathrm{N}=$ $1)$, an ankle injury $(\mathrm{N}=1)$, diagnosis of type 1 diabetes $(\mathrm{N}=1)$, a blood clot $(\mathrm{N}=1)$ and observation after a fall $(\mathrm{N}=1)$; none of these were seen as related to the study. Maddison 2014 also reported a small number of serious adverse events but none of these were considered as related to the study; they included two events in the intervention group (bowel replacement surgery and a dislocated hip) and three events in the control group (an operation to remove a cyst, a broken ankle, and two broken fingers).

These data were based on the total number of participants who suffered at least one serious adverse event (4/2105 participants in the behaviour-changing intervention groups compared with $7 / 1991$ participants in the comparator groups). We were unable to include studies where they reported adverse events but did not define if they were serious or if they did not provide the number of participants in each group who had at least one adverse event.

We aimed to provide a meta-analysis showing the number of participants in each group who discontinued due to adverse events. However, of those studies that reported adverse events occurring, only three actually reported if any participants discontinued (Croker 2012; Mirza 2013; Weintraub 2008) and they all reported that no participants dropped out due to adverse events.

\section{Secondary outcomes}

\section{Health-related quality of life and self-esteem}

Appendix 13 details the instruments that were used for analysis of health-related quality of life in the included trials. However, we were unable to meta-analyse all of the studies for the following reasons: unable to calculate mean change from data provided (Wake 2013, Warschburger 2016); no raw data were provided (Bryant 2011; Hamilton-Shield 2014; Lochrie 2013; Markert 2014; Reinehr 2010), no SDs given (Arauz Boudreau 2013), the study only presented results via domains, not overall score (Taylor 2015), and data were presented as median and interquartile ranges (IQR) (Hughes 2008). Four trials (Croker 2012, McCallum 2007; Wafa 2011; Wake 2009) reported the Pediatric Quality of Life Inventory (PedsQL) generic core scales, using the total score, either via parental or child report (Analysis 1.5; Analysis 1.6). An additional study measured health-related quality of life using the CHQ-PF50 global score (parental-report) (Kalarchian 2009) and Faude 2010 used the KINDL-R questionnaire (childreport). Using standardised mean differences (SMD), there were no substantial differences between intervention and control (higher scores indicate better quality of life) in the change in health-related quality of life at the final follow-up for parent/caregiver-reported data, demonstrating a SMD of 0.13 units $(95 \% \mathrm{Cl}-0.06$ to 0.32$)$;
$P=0.17 ; 5$ trials; 718 participants; low-quality evidence. There were no substantial differences between intervention and control (higher scores indicate better quality of life) in the change in healthrelated quality of life at the final follow-up for child-reported data, demonstrating a SMD of 0.15 units $(95 \% \mathrm{Cl}-0.34$ to 0.64$) ; \mathrm{P}=0.55$; 3 trials; 164 participants; very low-quality evidence. The minimal clinically important difference (MCID) for a PedsQL child's selfreport is 4.36 units and for PedsQL parents' (proxy) report 4.50 units (Varni 2007); when converting the SMD back to raw units, the MCID was not met in either meta-analysis.

Two trials reported a measure of self-esteem using the Harter global score that could be meta-analysed (Analysis 1.7). There were no substantial differences between intervention and control (higher scores indicate better self-esteem) in the change in self-esteem found at the final follow-up, demonstrating a MD of 0.19 units (95\% $\mathrm{Cl}-0.04$ to 0.42$) ; \mathrm{P}=0.11 ; 2$ trials; 144 participants; very low-quality evidence.

\section{All-cause mortality}

No deaths were reported in any of the trials.

\section{Morbidity}

No trials measured morbidities.

However, metabolic syndrome (which is a composite of risk indicators such as elevated blood lipids, insulin resistance, obesity and high blood pressure) was mentioned in Mirza 2013 using the National Cholesterol Education Program (Adult treatment panel III). Approximately $40 \%$ of the low glycaemic index dietary group (intervention) and $30 \%$ of the low fat dietary group (comparator) had the metabolic syndrome at baseline; at 24 months there was slight reduction in the percentage of participants with metabolic syndrome in both groups. However, there were no substantial differences between groups. Waling 2012 also measured the metabolic syndrome prevalence at baseline and follow-up using the International Diabetes Federation (IDF) definition. At baseline one participant in the intervention group and two participants in the control group had the metabolic syndrome; at one year's follow-up the number of participants with the metabolic syndrome was three in the intervention group and two in the control group.

\section{Anthropometric measures other than change in BMI}

Eleven trials reported waist circumference data that could be metaanalysed (Analysis 1.8). Meta-analysis demonstrated a reduction in waist circumference in the intervention groups compared with controls at the final follow-up: MD $-2.41 \mathrm{~cm}(95 \% \mathrm{Cl}-3.59$ to -1.23$)$; $\mathrm{P}<0.0001 ; 11$ trials; 1325 participants.

Three trials reported percentage overweight data that could be meta-analysed (Analysis 1.9). Meta-analysis demonstrated no substantial difference in percentage overweight in the intervention groups compared with controls at the final follow-up: MD $-3.27 \%$ (95\% $\mathrm{Cl}-7.47$ to 0.92$) ; \mathrm{P}=0.13 ; 3$ trials; 347 participants).

Eleven trials reported percentage body fat data that could be meta-analysed (Analysis 1.10). Meta-analysis demonstrated no substantial difference in percentage body fat in the intervention groups compared with controls at the final follow-up using (1) bioelectrical impedance analysis: MD $-1.25 \%(95 \% \mathrm{Cl}-2.62$ to 0.12$)$; $\mathrm{P}=0.07 ; 5$ trials; 1004 participants; and (2) using dual energy X-ray

Diet, physical activity and behavioural interventions for the treatment of overweight or obese children from the age of 6 to 11 years 
absorptiometry (DEXA): MD $-1.04 \%(95 \% \mathrm{Cl}-2.88$ to 0.80$) ; \mathrm{P}=0.27$; 5 trials; 443 participants.

\section{Behaviour change}

Two trials reported total kcals per day data that could be meta-analysed (Analysis 1.11). Meta-analysis demonstrated no substantial difference in total kcals per day in the intervention groups compared with controls at the final follow-up: MD -161.53 total kcals/day (95\% Cl -583.79 to 260.73$) ; \mathrm{P}=0.45 ; 2$ trials; 168 participants.

Two trials reported total minutes per day for television viewing data that could be meta-analysed (Analysis 1.12). Meta-analysis demonstrated a reduction in total minutes per day in the intervention groups compared with controls at the final follow-up: MD -6.60 minutes per day $(95 \% \mathrm{Cl}-12.88$ to -0.31$) ; \mathrm{P}=0.04 ; 2$ trials; 55 participants.

Six trials reported physical activity using accelerometers and total minutes per day data that could be meta-analysed (Analysis 1.13). Meta-analysis demonstrated no substantial difference in total minutes per day of physical activity in the intervention groups compared with controls at the final follow-up: MD -0.76 minutes per day ( $95 \% \mathrm{Cl}-5.30$ to 3.78$) ; \mathrm{P}=0.74 ; 6$ trials; 744 participants.

\section{Participants' views of the intervention}

Eight studies asked parents, the children or both for their views on the intervention (or comparator) given. Gunnarsdottir 2011a used an acceptability questionnaire to rate how satisfied families were with the intervention; the majority gave ratings of 1 to 3 (Likert scale, 1 = very satisfied, $5=$ not satisfied). The most liked components were the individual sessions and the traffic light diet food guide, and the least liked was a behavioural change technique called "token economies" which were defined as establishing goals, determining preferred rewards and providing them contingently upon achieving behavioural goals) and self-monitoring diet and physical activity.

Boutelle 2014 also asked all children and parents in the intervention group whether they liked the intervention: $50 \%$ of children liked the intervention a lot or loved it and $85 \%$ of them believed other children would like the intervention; $67 \%$ of parents in the intervention group liked it a lot or loved it, while $47 \%$ believed their child liked it a lot. Participants in Looney 2014 also undertook a process evaluation at the end of the study. There were no substantial differences in ratings between the three groups and $90 \%$ of families rated the programme as very good or excellent; $90 \%$ also said it was easy to understand. O'Connor 2013 also reported that $85 \%$ of the intervention group were positive about the treatment given.

Satoh 2007 interviewed 17 out of 21 children who completed the one-month intervention. Sixteen children said the intervention was easy to understand; however 14 children said completing the meal chart was a burden. Wake 2013 reported that the majority of parents thought the clinicians and GPs providing the intervention understood the challenges faced by the family and were confident that the intervention would make a difference.

Bryant 2011 randomly selected $10 \%$ of their sample to answer feedback about the study. The majority of parents and children reported positive experiences; however, those in the waiting list control group were disappointed that they had to wait for the intervention. Children were generally positive about the assessment but thought the worst part was the blood taking.

Hamilton-Shield 2014 collected qualitative data on treatment acceptance. The study involved an electrical device which included a weighing scale to measure food and provided feedback on satiety. Even though some parents gave some positive comments on the intervention, there were many technical problems with the device and some found it confusing to use. This may have contributed to the early termination of the study.

\section{Socioeconomic effects}

No trials measured socioeconomic effects.

\section{Behaviour-changing intervention plus additional component versus behaviour-changing intervention alone}

These interventions had the same components in the intervention and comparator groups to establish fair comparisons, and an additive component in the intervention arm. For example, diet plus physical activity plus behaviour therapy versus diet plus physical activity (with behaviour therapy being the additive component). We identified 15 trials in this category.

Of these studies, five studies also had a no-treatment or usual-carecondition group as they were at least three-arm studies (Epstein 1984a; Epstein 2000a; Looney 2014; Taveras 2015; Vann 2013). Davis 2013 compared the addition of telemedicine to standard physician visits and Duggins 2010 investigated adding a YMCA membership (physical activity) to nutrition classes led by dieticians. Epstein 2015 investigated whether adding a different nutritional component to a multi-component intervention was more beneficial. One study compared whether increasing physical activity or decreasing sedentary behaviours was more beneficial (Epstein 2001), while Epstein 1985a investigated adding a physical activity component (aerobic or behaviour-changing activity) to diet and behaviour therapy (with calisthenic exercise as a placebo).

Five studies investigated whether adding a physical activity component to a nutritional intervention improved weightrelated outcomes (Duggins 2010; Epstein 1984a; Epstein 1985b; Schwingshandl 1999; Woo 2004). NCT02436330 added an exergaming component (classed as physical activity) to a didactic health teaching intervention. Vann 2013 also included two trial arms adding a physical activity component (fitness DVD or pedometers).

Bathrellou 2010 investigated whether adding a parental involvement to a diet and physical activity intervention would be beneficial. Duffy 1993 added cognitive self-management to a behavioural intervention and Epstein 2005 added a behavioural element with regards to alternative behaviours to eating. Behavioural therapy was also an additional component in Epstein 1985c and Flodmark 1993. Larsen 2015 added an educational consultation to a diet and physical activity intervention, and Epstein 2000a assessed adding problem solving with or without parental involvement. Looney 2014 added a behavioural therapy component to a growth-monitoring intervention. Taveras 2015 (cluster RCT) also looked at adding individual family coaching to a clinical-support intervention.

Diet, physical activity and behavioural interventions for the treatment of overweight or obese children from the age of 6 to 11 years 


\section{Primary outcomes}

\section{Changes in body mass index (BMI), BMI z score and weight}

Four trials reported BMI data that could be meta-analysed (Analysis 2.1). Meta-analysis demonstrated a reduction in BMI in the intervention groups compared with controls at the final followup: $M D-0.75 \mathrm{~kg} / \mathrm{m}^{2}(95 \% \mathrm{Cl}-1.42$ to -0.09$) ; \mathrm{P}=0.03 ; 4$ trials; 195 participants.

Five trials reported BMI $\mathrm{z}$ score data that could be metaanalysed (Analysis 2.2). Meta-analysis demonstrated no substantial difference in $\mathrm{BMI} z$ score in the intervention groups compared with controls at the final follow-up: MD -0.03 units $(95 \% \mathrm{Cl}-0.10$ to 0.04 ); $\mathrm{P}=0.37 ; 5$ trials; 212 participants.

Four trials reported data for change in body weight that could be meta-analysed (Analysis 2.3). Meta-analysis demonstrated no difference in body weight in the intervention groups compared with controls at the final follow-up: MD $1.59 \mathrm{~kg}(95 \% \mathrm{Cl}-4.58$ to 7.77$) ; \mathrm{P}$ $=0.61 ; 4$ trials; 106 participants.

We were unable to include seven trials in the BMI/BMI z score meta-analyses. Bathrellou 2010 and Epstein 2015 only presented BMI values at baseline but did not present them at follow-up (only gave percent overweight). Epstein 1985a measured BMI but did not provide any data (only provided data for weight and percent overweight). Duffy 1993 and Epstein 1985b did not measure or present BMI values. Duggins 2010 presented mean change in BMI at the end of the study but did not provide any SDs. Hence, we could not use these data in the meta-analyses. At 12 months, a mean change of +10.2 units in the treatment group versus +6.5 units in the control group was reported (no $P$ value was given). Schwingshandl 1999 found a change in BMI SDS of -0.53 units in the intervention group versus -0.51 units in the control group after the 12-week intervention. The participants were followed up one year after baseline; however, the publication only provides results for fat free mass at one year, no BMI results were given.

\section{Adverse events}

In two trials, no adverse events occurred in either group (Woo 2004 - confirmed through author correspondence, and NCT02436330 - data given in clinical trials register). In 12 trials it was unclear whether adverse events occurred. This included six Epstein studies (Epstein 1985a; Epstein 1985b; Epstein 1985c; Epstein 2001; Epstein 2005; Epstein 2015) where it was unclear from the publications whether any adverse events occurred; however, after correspondence with the studies' author they highlighted that no adverse events were related to study participation but it was still unclear which studies had adverse events and what they were.

\section{Secondary outcomes}

The additive components across the studies varied greatly, therefore we analysed these comparisons in a separate metaanalysis from the usual-care and no-treatment controls for the primary analyses (see above) and have not used these comparisons in subgrouping. We have narratively described the secondary outcomes, as meta-analyses were not possible because the additive components that were investigated varied greatly between the studies.

\section{Health-related quality of life and self-esteem}

No trials measured health-related quality of life. However, NCT02436330 measured physical self-worth and global self-worth using the Children and Youth Physical Self-Perception Profile; no substantial differences between groups were found in changes from baseline to six months' follow-up.

\section{All-cause mortality}

No deaths were reported in any of the trials.

\section{Morbidity}

No trials measured morbidity.

\section{Anthropometric measures other than change in BMI}

Fourteen studies reported measuring other anthropometric measures; three of the eight studies that reported percent overweight found a significant difference in favour of the intervention group at the longest follow-up (Epstein 1985a; Epstein 1985c; Epstein 2015).

Waist circumference was measured in two studies (Larsen 2015; NCT02436330) but only Larsen 2015 found a difference in favour of the intervention group at the study's two-year endpoint (a similar finding was also seen for waist-to-height ratio). Woo 2004 measured waist-to-hip ratio, but found no substantial differences between groups.

Skinfold thickness was measured in Flodmark 1993 and found differences in reduction of all three skinfold measurements (triceps, subscapular and suprailiac) in favour of the intervention. Woo 2004 was the only study to measure body fat via DEXA - they found no substantial differences between groups.

\section{Behaviour change}

No studies used accelerometry to measure physical activity but NCT02436330 used pedometers to measure weekly steps - no substantial differences between groups were observed. Epstein 2005 used a three-day physical activity recall method to measure MVPA but found no substantial differences between groups. Three studies measured physical work capacity/physical fitness using a bicycle ergometry test and two of these studies found a treatment difference (Epstein 1985b; Flodmark 1993) while the remaining study found no substantial difference between groups (Epstein 1985a). NCT02436330 measured after school and Saturday screen time but found no substantial differences between groups at six months.

NCT02436330 measured dietary intake using "The Block Alive food frequency questionnaire (FFQ)". An increase in carbohydrates was seen in the treatment group compared to the control; however, the number of fruit servings was higher in the control group after six months. No substantial differences were found between groups in the other dietary domains (total calorie intake, percent fat, number of vegetable servings, sugar-sweetened beverage intake). Dietary intake was also measured by two studies (Duffy 1993; Epstein 2015) using a Traffic Light Diet questionnaire but only Epstein 2015 found a treatment effect for the reduction in red foods (unhealthy foods) and also fat intake, but they did not observe a substantial difference between groups in total calorie intake. Epstein 2005 measured dietary intake through a habit book and found a treatment effect

Diet, physical activity and behavioural interventions for the treatment of overweight or obese children from the age of 6 to 11 years 
at six and 12 months in alternatives to eating (activities that did not involve eating) but did not see a substantial difference between groups in eating periods. Two studies used the O'Neil 1979 questionnaire (Epstein 1985a; Epstein 1985c) to assess eating behaviours but only differences in favour of the intervention group were observed in Epstein 1985c.

\section{Participant views}

No studies measured participants' views of the intervention

\section{Socioeconomic effects}

No trials measured socioeconomic effects.

\section{Cluster RCTs}

All cluster RCTs had a usual care or no treatment control group except Berry 2007 which added a coping skills training element to a family behavioural therapy intervention.

\section{Primary outcomes}

\section{Changes in body mass index (BMI), BMI z score and weight}

We meta-analysed two cluster RCTs (Berry 2007; Taveras 2015) (Analysis 3.1) and demonstrated no substantial difference in BMI in the intervention groups compared with controls at the final followup: mean difference (MD) $-0.49 \mathrm{~kg} / \mathrm{m}^{2}(95 \% \mathrm{Cl}-1.24$ to 0.27$) ; \mathrm{P}=$ 0.20; 2 trials; 629 participants. Taveras 2015 also reported the BMI $z$ score - compared with the usual care group, children in the two intervention arms (clinical decision support and clinical decision support plus individual family coaching) showed a small mean change in BMI z score: $-0.06(95 \% \mathrm{Cl}-0.11$ to -0.02$)$ and $-0.05(95 \%$ $\mathrm{Cl}-0.09$ to 0.00 ), respectively. No substantial differences were found between the two treatment groups.

Wright 2012 presented changes in BMI and BMI z score at 12 months' follow-up; however, there were concerns over the $95 \% \mathrm{Cls}$ presented which we suspected were ranges rather than Cls. We tried to contact the study author to clarify but did not receive a response. Therefore, we did not include this study in the metaanalysis. The publication reports that there were between-group differences in BMI and BMI z score, in favour of the intervention group. We did not include Berry 2014 in the meta-analyses for BMI/ BMI z score because it was not clear from the publication how many children were included in the follow-up analysis. The publication reported that there were no substantial differences between groups for BMI percentile at both 12 and 18 months' follow-up.

There were no cluster trials that reported data on weight.

\section{Adverse events}

In the four cluster trials in this review, Berry 2007, Berry 2014 and Taveras 2015 had no adverse events in either group (confirmed through study author correspondence). It was unclear if any adverse events occurred in Wright 2012.

\section{Secondary outcomes}

\section{Health-related quality of life and self-esteem}

No trials measured health-related quality of life or self-esteem.

\section{All-cause mortality}

No deaths were reported in any of the trials.

\section{Morbidity}

No trials measured morbidity.

\section{Anthropometric measures other than change in BMI}

Berry 2007 measured body fat percentage using bioelectrical impedance analysis (BIA) but found no substantial differences between groups at the study's endpoint. Berry 2014 measured waist circumference and found a treatment effect at 12 months' follow-up but not at 18 months. Triceps and subscapular skinfolds were also measured and a treatment effect was found at 18 months' follow-up.

\section{Behaviour change}

Activity was measured using pedometers (number of steps) in Berry 2007 but no substantial differences between groups were observed at follow-up.

Berry 2014 used the Child and Adolescent (CATCH) questionnaire to measure diet and physical activity changes, but only dietary knowledge was improved in the intervention group at 18 months compared to the control. Berry 2014 also used the Child Health behaviour survey by the Department of Health and Human Services 2004 to measure dietary habits and only found a treatment effect for reduced soda consumption at 18 months. Wright 2012 used the Child and Adolescent Trial for Cardiovascular Health AfterSchool Student Questionnaire (ASSQ) to assess dietary intake and eating behaviours and found treatment effects for some outcomes (e.g. fruit and vegetable intake, food intentions); however, others showed no substantial differences (e.g. sweets intake, always reading food labels).

\section{Participants' views}

Participants' views were measured in one cluster trial (Taveras 2015) that involved two clinical-led interventions compared against a usual-care group; the most intensive intervention was highly rated by parents $(81.3 \%$ were satisfied) while only $46.9 \%$ of the parents in the less intensive intervention were satisfied.

\section{Socioeconomic effects}

No trials measured socioeconomic effects.

\section{Maintenance intervention following weight reduction}

\section{Primary outcomes}

\section{Changes in body mass index (BMI), BMI z score and weight}

Two trials reported BMI z score data (de Niet 2012; Wilfley 2007) that could be meta-analysed (Analysis 4.1) and demonstrated no difference in BMI $z$ score in the intervention groups compared with controls at the final follow-up: mean difference (MD) -0.07 units ( $95 \% \mathrm{Cl}-0.19$ to 0.04 ); $\mathrm{P}=0.22 ; 2$ trials; 263 participants). There were no maintenance trials that reported data for BMI or for body weight suitable for meta-analysis.

\section{Adverse events}

Both trials had no adverse events (de Niet 2012 confirmed through study author correspondence, and Wilfley 2007 through information in the publication).

Diet, physical activity and behavioural interventions for the treatment of overweight or obese children from the age of 6 to 11 years 


\section{Secondary outcomes}

\section{Health-related quality of life and self-esteem}

de Niet 2012 used The Child Health Questionnaire-PF50 (CHQ-PF50) to measure health-related quality of life. A treatment effect was found at three and six months' follow-up in the physical domain but this was lost at nine months' follow-up. de Niet 2012 also measured self-esteem using the Self-Perception Profile for Children (SPPC)/ Harter global score but found no substantial differences between groups at nine months.

\section{All-cause mortality}

No deaths were reported in any of the trials.

\section{Morbidity}

No trials measured morbidity.

\section{Anthropometric measures other than change in BMI}

Wilfley 2007 measured percentage overweight at two years' followup but found no substantial differences between the treatment and control groups. The BMI z score meta-analysis for maintenance trials (Analysis 4.1) showed no substantial differences between groups.

\section{Behaviour change}

The Dutch Eating Behaviour Questionnaire (DEBQ) was used to measure behaviour change in de Niet 2012. A treatment effect was seen for external eating at three months from baseline, but not at six or 15 months. No substantial differences were observed in emotional eating or restrained eating.

Wilfley 2007 used a Child Dietary Self-efficacy scale and found a treatment effect at two-year follow-up for the social facilitation maintenance intervention group when compared against the control group; the behavioural-skills maintenance intervention group showed a treatment effect compared with control but only at one-year follow-up. There were no substantial differences between the two treatment groups. Wilfley 2007 also used a Self-efficacy Scale for Children's Physical Activity but only found a difference in favour of the social facilitation maintenance for 'positive alternatives to unhealthy habits' (increasing healthy foods and decreasing sedentary behaviour) at two years; no substantial differences were found for barriers between treatment groups.

\section{Participants' views}

No studies measured participants' views of the intervention.

\section{Socioeconomic effects}

No trials measured socioeconomic effects.

\section{Subgroup analyses}

We performed a number of subgroup analyses to test the effects of different types of comparators, the type of intervention, the setting, risk of attrition bias, duration of post-intervention followup, the involvement of parents, and mean baseline BMI z score on outcomes of BMI, BMI-z score and weight.

We did not perform subgroup analyses on the different durations of follow-up from baseline, combining those studies reporting six months' follow-up, those reporting 12 months' follow-up and those reporting 18 months' follow-up or more. Neither did we perform subgroup analyses based on the length of the interventions, combining studies with a duration of intervention of six months or less and studies with duration of intervention of greater than six months. This would have resulted in some studies being included in more than one subgroup for the duration of follow-up because some studies reported follow-up at multiple time points. Also, grouping studies according to whether they were six months or less or greater than six months would not assess all studies immediately post-intervention and would not evaluate the actual length of active intervention for all studies. We were most interested in the longer-term effects of weight-management interventions and the sustainability of weight reduction. Due to the relatively large number of included studies in this review we were able to subgroup according to duration of post-intervention follow-up, that is, we could assess whether follow-up after the active intervention, and the duration of that follow-up period, impacted on BMI, BMI z score and weight outcomes.

\section{Type of control}

We did not see any subgroup differences for change in BMI, BMI z score and weight when comparing studies with controls described as 'no intervention' and studies with controls described as 'usual care' (Analysis 1.14; Analysis 1.15; Analysis 1.16).

\section{Type of intervention}

The majority of studies were multi-disciplinary interventions, however, some studies were single or dual interventions. We did not see any subgroup differences for change in BMI (Analysis 1.17), change in BMI z score (Analysis 1.18) or change in weight (Analysis 1.19).

\section{Risk of attrition bias}

We did not see any subgroup differences when combining studies according to high, low or unclear risk of attrition bias for change in BMI (Analysis 1.20), change in BMI z score (Analysis 1.21) or change in weight (Analysis 1.22).

\section{Setting of intervention}

For setting, the studies were divided into eight subgroups, school, community, home, primary care, secondary care, university research clinics, hospital inpatient and mixed settings. We did not see any subgroup differences for change in BMI (Analysis 1.25), change in BMI z score (Analysis 1.24) or change in weight (Analysis 1.23).

\section{Duration of post-intervention follow-up}

We put studies into subgroups based on whether they had a period of post-intervention follow-up (defined as the period after the active intervention and up to the final measurement) and the duration of that period: no post-intervention follow-up ( $N=15)$, less than six months $(\mathrm{N}=3)$, six months to less than 12 months $(\mathrm{N}=2)$ and post-intervention follow-up lasting 12 months or longer $(\mathrm{N}=$ 4). We calculated the duration of no post-intervention follow-up by subtracting the active intervention period from the total duration of the study (i.e. intervention and all follow-up duration).

For change in BMI (Analysis 1.26), combining studies by postintervention follow-up indicated a statistically significant subgroup difference $(P=0.03)$, however this is not reliable because all the

Diet, physical activity and behavioural interventions for the treatment of overweight or obese children from the age of 6 to 11 years 
Cls overlap (to a small degree, regarding the $\mathrm{Cl}$ for studies with post-intervention follow-up 12 months or more versus no postintervention follow-up). There were no subgroup differences for BMI z score change (Analysis 1.27) or change in weight (Analysis 1.28).

\section{Parental involvement}

We put studies into subgroups based on whether the intervention involved the parent and child, whether only the child was treated without any parental involvement and whether the parent was specifically targeted (but the child was included in the intervention). There was no subgroup difference on change in BMI (Analysis 1.29), change in BMI z score (Analysis 1.30) or change in weight (Analysis 1.31).

\section{Mean baseline BMI z score}

We put studies into subgroups based on whether the mean baseline BMI z score was less than 2.67 units or 2.67 units or greater (which equates to the 99.6th centile for severe obesity). There was no subgroup difference on change in BMI z score (Analysis 1.32).

\section{Sensitivity analyses}

We performed sensitivity analyses restricting the main BMI, BMI z score and weight meta-analyses (Analysis 1.1; Analysis 1.2; Analysis 1.3) to those studies that provided change score data (along with an SD, SE and $95 \% \mathrm{CI}$ ). Hence, we excluded studies where the mean change score SD was not provided but was imputed following the guidelines in the Cochrane Handbook for Systematic Reviews of Interventions (Section 16.1.3.2 Imputing standard deviations for changes from baseline; Higgins 2011c) and assumed a correlation of 0.5 between baseline and follow-up measures as suggested by Follmann 1992. All three sensitivity analyses were very similar to the original analyses; which showed that our original analyses were robust (see Table 2).

\section{Assessment of reporting bias}

We generated funnel plots for the primary outcomes of BMI, BMI z score and weight, as these analyses included the highest number of studies on which to assess publication bias. Inspection of the funnel plots for BMI and weight (but not BMI z score) showed an uneven distribution of studies and suggested a possibility of small study bias (data not shown).

\section{DISCUSSION}

\section{Summary of main results}

We included 70 trials in this review, with 55 comparing a behaviourchanging intervention with no treatment or usual-care control and 15 testing an additional component added to a behaviour-changing intervention. The vast majority of trials were multicomponent ( $\mathrm{N}$ $=64$ ) and individual trial sample sizes ranged from 16 to 686 participants. Total duration of trials ranged from six months to three years; duration of active intervention ranged from 10 days to two years. Just over half (37) of the trials had a period of postintervention follow-up with a median duration of 10 months.

A total of 8461 participants were randomised to either the intervention or control groups; approximately $69.5 \%$ of participants were measured at the study's endpoint. Primary analyses demonstrated that behaviour-changing interventions compared to no treatment or usual-care control reduced BMI, BMI z score and body weight. We could pool data from 24 trials reporting $\mathrm{BMI}$ for analysis, which demonstrated a reduction in BMI in favour of the intervention (measured at the last available point of followup) of $-0.53(95 \% \mathrm{Cl}-0.82$ to -0.24$) ; 24$ trials; 2785 participants; low-quality evidence). Thirty-seven trials reported BMI z score data suitable for meta-analysis, which resulted in a reduction in favour of intervention (measured at last available point of follow-up) of -0.06 units ( $95 \% \mathrm{Cl}-0.10$ to -0.02$)$; 37 trials; 4019 participants; low-quality evidence). Seventeen trials reported change in body weight that could be meta-analysed, and demonstrated a reduction in body weight in the intervention groups compared with controls at the final follow-up: MD $-1.45 \mathrm{~kg}(95 \% \mathrm{Cl}-1.88$ to -1.02$) ; \mathrm{P}<0.00001 ; 17$ trials; 1774 participants; low-quality evidence).

We excluded from the main analysis the 15 trials that evaluated an additional component to a behaviour-changing intervention, as the additive elements under investigation were extremely diverse and not comparable to the other interventions.

Thirty-five trials had no adverse events, 29 trials were unclear as to whether adverse events occurred and six trials reported a range of adverse events in a small percentage of participants. Thirty one trials documented serious adverse events, although the vast majority $(\mathrm{N}=29)$ reported zero occurrence.

Six trials (718 participants) reported paediatric quality of life inventory, two trials (144 participants) reported a measure of selfesteem, two trials (168 participants) reported change in caloric intake and six (744 participants) reported accelerometry-measured physical activity; however, none of these analyses demonstrated a significant difference between intervention and control. In the two trials reporting on minutes per day of TV viewing, a small reduction of 6.6 minutes per day $(95 \% \mathrm{Cl}-12.88$ to -0.31$), \mathrm{P}=0.04 ; 2$ trials; 55 participants) was found in favour of the intervention.

No trials reported on all-cause mortality, morbidity or socioeconomic effects, and few trials reported on participant views; none of which could be meta-analysed.

As the meta-analyses revealed significant heterogeneity, we conducted subgroup analyses to examine the impact of type of comparator, type of intervention, risk of attrition bias, setting of intervention, duration of post-intervention follow-up period, type of parental involvement and mean baseline BMI z score. No substantial subgroup effects were shown for any of the subgroups on any of the outcomes (BMI, BMI z score or weight). There was an indication of an effect for duration of post-intervention follow-up for BMI only, which demonstrated that intervention effects between groups differed only immediately post intervention (heterogeneity increased) and for post-intervention follow-up of less than six months (heterogeneity reduced to zero), however this hypothetical finding has to be further investigated in independent studies. These findings align with data from the two trials (263 participants) identified in this review that specifically examined the impact of a maintenance period following weight loss on BMI z score and found no substantial difference between intervention and control.

\section{Overall completeness and applicability of evidence}

This review contains the largest number of trials and participants, compared to the other systematic reviews in this series (surgery; drugs; parent-only interventions; diet, physical activity and 
behavioural interventions for young children aged 0 to 6 years, and adolescents aged 12 to 17 years).

The bulk of the evidence was derived from multicomponent interventions that involved the parent and child. The interventions varied in duration including longer-term interventions (up to three years) and follow-up after a period of no active intervention in half of the trials. The majority of evidence relates to trials published from 2000 onwards; however, there was no evidence included from trials conducted in lower middle-income countries. The review included evidence from a wide variety of settings. There was less evidence relating to younger children (median age was 10 across the trials) and for non-white children; however, both girls and boys were equally represented. These limitations call into question the transferability of the findings to cultural and geographic settings other than upper- and upper middle-income countries. Therefore, the results should be interpreted carefully within the context of local population needs (i.e. age, sex, socioeconomic status, ethnicity, religion, culture, disabilities/complex needs, severity of obesity) and local political and health systems.

All participants included in this review were overweight, obese or severely obese at baseline. Whilst any reduction in body mass in overweight or obese children may be of benefit, the small reduction observed in the studies included in this review may not be sufficient to improve or prevent obesity-related comorbidities. Indeed there was a lack of data reported on obesity-related comorbidities. The authors of a recent study in England (in older school aged children median age 12.4 years) reported that a reduction of $0.25 \mathrm{BMI} z$ score units was required to improve adiposity and metabolic health (Ford 2010). This is a reduction much higher than that observed in this review.

Very few studies measured any of the review's secondary outcomes other than anthropometric outcomes, the results of those that did were inconsistent and used a variety of measurement tools. Outcome results were also inconsistent depending on the timing of measurements within the studies. In summary, the data were too limited and heterogeneous to enable any meaningful synthesis of secondary outcomes for those studies that investigated adding a component to a behaviour-changing intervention, maintenance trials and cluster RCTs. Meta-analyses of secondary outcomes for usual care/no treatment comparators showed no substantial differences between groups or wide $95 \% \mathrm{Cls}$, or both.

\section{Quality of the evidence}

We rated over half $(\mathrm{N}=48)$ of the 70 included studies as having a low risk of selection bias based on the randomisation method they used. We rated 49 studies as low risk of bias for allocation concealment. However, we rated a majority of trials as high risk of bias for blinding (for both performance and detection bias). Fortyfive studies did not blind their participants or study personnel to study group allocation with regards to objective measures. Only eight trials did not have a high risk of bias on at least one criterion.

GRADE assessments of the outcomes in this review led to trials being downgraded for risk of bias, inconsistency and also imprecision. This made overall interpretation of the data difficult. Overall the quality of included trials was low for BMI, BMI z score, weight, adverse events and parent-reported health-related quality of life, and very low for child-reported health-related quality of life.

\section{Potential biases in the review process}

The review identified all relevant trials with searches from inception of databases to July 2016 and all efforts were made to include studies published up until the start of November 2016 and to obtain any additional data.

There is a potential bias in terms of the wider applicability of the findings, with the vast majority of included studies conducted in high-income countries, with a heavy reliance on data from the USA. It is also unclear as to applicability of the findings in populations of different socioeconomic status and ethnicity, due to lack of reporting of ethnicity data in the majority of trials.

The impact of the comparator group should also be considered, given that a significant proportion of studies used a 'usual care' condition which varied greatly in terms of content and intensity; there was an element of subjectivity introduced in that review authors had to sometimes assess whether the comparator was 'usual care' if not reported by the study authors as such.

We attempted to contact all study authors whenever there were missing data or clarification was needed. The majority of studies did not report if adverse events occurred; hence, we contacted study authors for this information. Some study authors did not reply and this may have introduced bias. However, we felt it was important to contact study authors about adverse events because reporting was so poor. Furthermore, we had concerns that some studies never measured or documented adverse events, so if any did occur, they would not have been captured.

\section{Agreements and disagreements with other studies or reviews}

This review is a partial update to a previous Cochrane Review: the original review 'Interventions for treating obesity in children and adolescents' (Oude Luttikhuis 2009) was split into six separate reviews, with a specific intervention and age focus.

- Diet, physical activity, and behavioural interventions for the treatment of overweight or obesity in adolescents aged 12 to 17 years

- Diet, physical activity, and behavioural interventions for the treatment of overweight or obesity in children from the age of 6 to 11 years

- Diet, physical activity, and behavioural interventions for the treatment of overweight or obesity in preschool children up to the age of 6 years (Colquitt 2016)

- Drug interventions for the treatment of obesity in children and adolescents (Mead 2016a)

- Parent-only interventions for childhood overweight or obesity (Loveman 2015)

- Surgery for the treatment of obesity in children and adolescents (Ells 2015b)

This review is now a stand-alone review of all the RCT evidence relating to the treatment of overweight or obesity in children aged 6 to 11 years. The evidence base contained in this review has increased substantially; the majority of these new trials have focused on multi-component interventions with a mix of diet, physical activity and behaviour-change elements. However, despite the substantial increase in available evidence, the overall effects in terms of BMI/BMI $z$ score and weight reductions in

Diet, physical activity and behavioural interventions for the treatment of overweight or obese children from the age of 6 to 11 years 
favour of interventions remain similar to the previous Cochrane Review of interventions to treat childhood obesity (Oude Luttikhuis 2009), with continued heterogeneity in terms of comparators, intervention content and delivery. The previous Cochrane Review (Oude Luttikhuis 2009) found very similar reductions in BMI z scores, in favour of the intervention for children under 12 years old, at 6 and 12 months' follow-up: $-0.06(95 \% \mathrm{Cl}-0.12$ to -0.01$)$ and -0.04 $(95 \% \mathrm{Cl}-0.12$ to 0.04$)$, respectively.

When comparing the findings of this review to the other behaviourchanging intervention reviews in this series (preschool, adolescent and parent-only), our findings are very similar. The preschool review (Colquitt 2016) found slightly larger reduction in BMI z score in favour of the intervention group than in this review: -0.3 units $(95 \% \mathrm{Cl}-0.4$ to -0.2$)$ for 6 to 12 months' follow-up, and -0.4 units (95\% Cl -0.6 to -0.2 ) for 12 to 18 months' follow-up. However very similar reductions in $\mathrm{BMI} z$ score were found when comparing parent-only interventions to parent-child interventions (-0.04 units, $95 \% \mathrm{Cl}-0.15$ to 0.08 ) and parent-only interventions with a waiting list control $(-0.10,95 \% \mathrm{Cl}-0.19$ to -0.01$)$ (Loveman 2015). Therefore, the other two reviews support the findings found in this review small reductions in $\mathrm{BMI}$ and $\mathrm{BMI} \mathrm{z}$ score occur when comparing behaviour-changing interventions to control groups.

The effects of behaviour-changing interventions for overweight/ obese children were assessed in another recent systematic review and meta-analysis (Ho 2012). When comparing behaviour-changing treatments to no care or waiting list controls they saw a reduction of $-1.00 \mathrm{~kg} / \mathrm{m}^{2}(95 \% \mathrm{Cl}-1.91$ to -0.08$)$ in favour of the intervention group for children 12 years old or less. A similar reduction was found when they compared the treatment group to a usual-care/minimalintervention control group. A recent review assessing the effects of educational interventions to treat obesity in 6- to 12-year-old children (Sbruzzi 2013) found very similar reductions in BMI and BMI z scores to this review: $-0.86 \mathrm{~kg} / \mathrm{m}^{2}(95 \% \mathrm{Cl}-1.59$ to -0.14$)$ and -0.06 units $(95 \% \mathrm{Cl}-0.16$ to 0.03$)$, respectively.

An overview of reviews for childhood obesity is underway that examines interventions for the treatment of obesity in children using Cochrane methodology (Ells 2016 [pers comm]). This overview will bring together all the evidence for any type of intervention to treat childhood obesity and highlight any evidence gaps that remain.

All types of treatment interventions should also be viewed within the context of prevention interventions. It is interesting that the effect size for BMI $z$ score reduction (measured at longest followup) observed in this treatment review of behaviour-changing interventions (MD -0.06 units $(95 \% \mathrm{Cl}-0.10$ to -0.02$) ; \mathrm{P}=0.001 ; 37$ trials; 4019 participants; low-quality evidence) is very similar to the BMI z score reduction (measured at first available point of followup after 12-weeks) observed in the recently updated (Brown 2016 [pers comm]) obesity prevention review (Waters 2011) of children aged up to 18 years ( -0.05 units $(95 \% \mathrm{Cl}-0.07$ to -0.03$)$; $\mathrm{P}<0.00001$; 58 studies; 53,777 participants; low-quality evidence).

\section{AUTHORS' CONCLUSIONS}

\section{Implications for practice}

Multi-component behaviour-changing interventions that incorporate diet, physical activity and behaviour-change components may be beneficial in achieving small, short-term reductions in body mass index (BMI), BMI $\mathrm{z}$ score and weight in children aged 6 to 11 years. The evidence was low quality for BMI, BMI z score and weight; and there was a limited number of trials reporting low- to very low-quality evidence for health-related quality of life including self-esteem. Although data on adverse events were not well reported and of low quality, where provided, the evidence suggests a very low occurrence of adverse events. The heterogeneity observed across all outcomes was not explained by subgrouping based on the type of intervention, type of comparator, setting, risk of bias, parental involvement or severity of obesity at baseline. The sustainability of any observed reduction in BMI/BMI z score and body weight is a key consideration and there is a need for longer-term follow-up of these children. The evidence highlights a focus in paediatric obesity on initial weight reduction interventions rather than longer term maintenance interventions. This review demonstrates that interventions show effects at the end of the intervention and up to six months post-intervention; the fact that these intervention effects might not persist is not a failure of the initial intervention, but due to a lack of maintenance interventions. Obesity is a severe chronic relapsing disease becoming manifest in an obesity-conducive environment, therefore it is unsurprising that short-term effects do not persist. Continued support through obesity maintenance interventions are required to build upon behaviour changes which increase resilience to obesity-conducive environments.

\section{Implications for research}

The systematic review identified 20 ongoing trials of behaviourchanging interventions, which will contribute data to the results of an updated review. Further research is required of interventions in lower income countries and in children from ethnic minority groups. We still do not understand what the key components of multicomponent interventions are that contribute to success, and for which children. Study designs other than randomised controlled trials may be helpful in improving our understanding.

Children aged 6 to 11 years are likely to require the support of families (hence only five of the 70 included interventions targeted the child and did not involve parents) which adds another layer of complexity, particularly given that we know parents are also likely to be suffering from excess weight; further research into the optimal ways of involving parents in paediatric obesity interventions is required. Despite this review including many more studies compared with the original review (Oude Luttikhuis 2009), the effect size on BMI z score is almost identical. Although the evidence is of low or very low quality according to GRADE, the review authors believe that it is unlikely that any subsequent update would dramatically alter the effects on BMI, BMI z score or weight. Perhaps a change of focus is required, for example, qualitative research to further our understanding of what works for who, when and why, in the context of the family, in order to tailor and target future obesity interventions. Future research could examine family-based approaches that treat both obese parents and children simultaneously, similar to two studies included within this review (Berry 2007; Berry 2014).

Further research is required on the impacts of these interventions for health-related quality of life, long term diet and activity behaviour change and obesity-related comorbidities. There is a need for standardised reporting of key outcomes and moderators (e.g. ethnicity, health-related quality of life, diet and physical activity changes and socioeconomic status). Cost data were not

Diet, physical activity and behavioural interventions for the treatment of overweight or obese children from the age of 6 to 11 years 33 (Review)

Copyright @ 2017 The Cochrane Collaboration. Published by John Wiley \& Sons, Ltd. 
considered within the remit of this review; nine of the $70(13 \%)$ included studies measured costs associated with resource use or cost effectiveness of the intervention (Bryant 2011; Coppins 2011; Hughes 2008; Kalavainen 2007; Lison 2012; McCallum 2007; Reinehr 2010; Wake 2009; Wake 2013). Nine studies reported on cost data using a variety of different reporting methods. Whilst Wake 2013 planned a full economic evaluation, this was not conducted, as the programme did not prove to be effective, and Lison 2012 simply reported that the hospital-based intervention was more expensive when compared to the home-based approach. Kalavainen 2007 reported a cost per 0.1 decrease in BMI SDS of EUR 168 for the intervention group, whilst Reinehr 2010 reported a cost per family of EUR 652. The remaining five studies provided an estimated cost of the intervention per person ranging from GBP 108 (Hughes 2008), GBP 403 (Coppins 2011), GBP 858 (Bryant 2011), AUD 873 (McCallum 2007) and AUD 1317 (Wake 2009). However, not all of these studies conducted formal cost-effectiveness analyses. As these outcomes are vitally important for practice implications and decision-makers, it is import that these outcomes are systematically reviewed.

A UK tracking study (Mead 2016b) using data from the Millenium cohort showed that overweight and obese children at $4 / 5$ years old are very likely to remain overweight and obese at $11 / 12$ years old. In addition, obese deprived boys at age $4 / 5$ were more likely to remain obese at age 11/12 compared with non-deprived obese boys (trend not seen in girls). Therefore, interventions targeted at children aged 6 to 11 years are capturing an important timeframe, however there is a complete lack of data reporting on the potential moderating effect of socioeconomic status on obesity.
Further work is required to determine the most appropriate and effective forms of post intervention maintenance, including the level of intensity and different modes of maintenance intervention, in order to ensure intervention benefits are sustained over the longer term.

\section{ACKNOWLEDGEMENTS}

We would like to thank the study authors who provided additional information from their trials. In addition, we would like to thank the Cochrane Metabolic and Endocrine Disorders (CMED) editorial base staff for their assistance with this review.

We would like to acknowledge Emma Loveman and Jill Colquitt at Effective Evidence LLP, Eastleigh, UK, who assisted with the selection of studies. We would also like to thank Congchao Lu and Jessica Verbeek from the University of Groningen, Netherlands; Megan Gow from the University of Sydney, Australia; Dora Machaira and Heather Clements from Teesside University and Sarah Smith from Durham University, UK; for their assistance with data extractions.

The World Health Organization (WHO) and Emma Mead, Tamara Brown, Karen Rees, Liane Azevedo, Victoria Whittaker, Dan Jones, Joan Olajide, Giulia Mainardi, Eva Corpeleijn, Claire O'Malley, Elizabeth Beardsmore, Lena Al-Khudairy, Louise Baur, Maria-Inti Metzendorf and Louisa Ells retain copyright and all other rights in their respective contributions to the manuscript of this updated review as submitted for publication. 


\section{REFERE N CE S}

\section{References to studies included in this review}

\section{Alves 2008 \{published data only\}}

Alves JG, Gale CR, Souza E, Batty GD. Effect of physical exercise on bodyweight in overweight children: a randomized controlled trial in a Brazilian slum [Efeito do exercício físico sobre peso corporal em crianças com excesso de peso: ensaio clínico comunitário randomizado em uma favela no Brasil]. Cadernos de Saude Publica 2008;24(Suppl 2):S353-9.

\section{Arauz Boudreau 2013 \{published data only\}}

Arauz Boudreau AD, Kurowski DS, Gonzalez WI, Dimond MA, Oreskovic NM. Latino families, primary care, and childhood obesity: a randomized controlled trial. American Journal of Preventive Medicine 2013;44(3 Suppl 3):S247-57.

\section{Barkin 2011 \{published data only\}}

Barkin SL, Gesell SB, Poe EK, Ip EH. Changing overweight Latino preadolescent body mass index: the effect of the parent-child dyad. Clinical Pediatrics 2011;50(1):29-36.

\section{Bathrellou 2010 \{published data only\}}

Bathrellou E, Yannakoulia M, Papanikolaou K, Pehlivanidis A, Pervanidou P, Kanaka-Gantenbein C, et al. Development of a multi-disciplinary intervention for the treatment of childhood obesity based on cognitive behavioral therapy. Child \& Family Behavior Therapy 2010;32(1):34-50.

* Bathrellou E, Yannakoulia M, Papanikolaou K, Pehlivanidis A, Pervanidou P, Kanaka-Gantenbein C, et al. Parental involvement does not augment the effectiveness of an intense behavioral program for the treatment of childhood obesity. Hormones 2010;9(2):171-5.

\section{Berry 2007 \{published data only\}}

Berry D, Savoye M, Melkus G, Grey M. An intervention for multiethnic obese parents and overweight children. Applied Nursing Research 2007;20(2):63-71.

\section{Berry 2014 \{published data only\}}

Berry D, McMurray R, Schwartz TA, Skelly A, Sanchez M, Neal $M$, et al. Rationale, design, methodology and sample characteristics for the family partners for health study: a cluster randomised controlled study. BMC public health 2012;12:250.

* Berry DC, Schwartz TA, McMurray RG, Skelly AH, Neal M, Hall, EG, et al. The family partners for health study: a cluster randomized controlled trial for child and parent weight management. Nutrition \& Diabetes 2014;4:e101.

NCT01378806. Family partners for health (WEIGHT). clinicaltrials.gov/ct2/show/NCT01378806 (last accessed 6 December 2016).

\section{Boutelle 2014 \{published data only\}}

* Boutelle KN, Zucker N, Peterson CB, Rydell S, Carlson J, Harnack LJ. An intervention based on Schachter's externality theory for overweight children: the regulation of cues pilot. Journal of Consulting \& Clinical Psychology 2014;39(4):405-17.
NCT01442142. A study to determine the effectiveness of a self-regulation program to treat pediatric obesity (ROC). clinicaltrials.gov/ct2/show/NCT01442142 (last accessed 6 December 2016).

\section{Bryant 2011 \{published data only\}}

* Bryant M, Farrin A, Christie D, Jebb SA, Cooper AR, Rudolf M. Results of a feasibility randomised controlled trial (RCT) for WATCH IT: a programme for obese children and adolescents. Clinical Trials 2011;8(6):755-64.

ISRCTN95431788. Empowering parents of obese children: development and controlled evaluation of an obesity-specific parenting skills training. www.isrctn.com/ISRCTN95431788 (last accessed 6 December 2016).

Rudolf M, Christie D, McElhone S, Sahota P, Dixey R, Walker J, et al. Watch It: a community based programme for obese children and adolescents. Archives of Disease in Childhood 2006;91(9):736-9.

Spoor C, Sahota P, Wellings C, Rudolf MC. Costing a pilot complex community-based childhood obesity intervention. Journal of Human Nutrition and Dietetics 2013;26(2):126-31.

Twiddy M, Wilson I, Bryant M, Rudolf M. Lessons learned from a family focused weight management intervention for obese and overweight children. Public Health Nutrition 2012;15(7):1310-7.

\section{Coppins 2011 \{published data only\}}

* Coppins DF, Margetts BM, Fa JL, Brown M, Garrett F, Huelin S. Effectiveness of a multi-disciplinary family-based programme for treating childhood obesity (the Family Project). European Journal of Clinical Nutrition 2011;65(8):903-9.

\section{ISRCTN55734850. Comparing the effectiveness of} multidisciplinary family approach with traditional dietetic treatment in managing the weight of overweight or obese children. www.isrctn.com/ISRCTN55734850 (last accessed 6 December 2016).

\section{Croker 2012 \{published data only\}}

* Croker H, Viner RM, Nicholls D, Haroun D, Chadwick P, Edwards C, et al. Family-based behavioural treatment of childhood obesity in a UK National Health Service setting: randomized controlled trial. International Journal of Obesity 2012;36(1):16-26.

ISRCTN51382628. Treating childhood obesity: a randomised, controlled trial of a family-based behavioural treatment. www.isrctn.com/ISRCTN51382628 (last accessed 6 December 2016).

\section{Davis 2013 \{published data only\}}

* Davis AM, Sampilo M, Gallagher KS, Landrum Y, Malone B. Treating rural pediatric obesity through telemedicine: outcomes from a small randomized controlled trial. Journal of Pediatric Psychology 2013;38(9):932-43.

Gallagher KS, Davis AM, Malone B, Landrum Y, Black W. Treating rural pediatric obesity through telemedicine: baseline data from

Diet, physical activity and behavioural interventions for the treatment of overweight or obese children from the age of 6 to 11 years 
a randomized controlled trial. Journal of Pediatric Psychology 2011;36(6):687-95.

\section{Davoli 2013 \{published data only\}}

Broccoli S, Davoli AM, Bonvicini L, Fabbri A, Ferrari E, Montagna $\mathrm{G}$, et al. Motivational interviewing to treat overweight children: 24-month follow-up of a randomized controlled trial. Pediatrics 2016;137(1):e2 0151979.

* Davoli AM, Broccoli S, Bonvicini L, Fabbri A, Ferrari E, D'Angelo $S$, et al. Pediatrician-led motivational interviewing to treat overweight children: an RCT. Pediatrics 2013;132(5):e1236-46.

NCT01822626. Pediatrician-lead motivational counselling to control BMI in overweight children: a RCT in primary care. clinicaltrials.gov/ct2/show/NCT01822626 (last accessed 6 December 2016).

\section{de Niet 2012 \{published data only\}}

* De Niet J, Timman R, Bauer S, Van Den Akker E, Buijks H, De Klerk C, et al. The effect of a short message service maintenance treatment on body mass index and psychological well-being in overweight and obese children: a randomized controlled trial. Pediatric Obesity 2012;7(3):205-19.

De Niet J, Timman R, Bauer S, Van Den Akker E, De Klerk C, Kordy $\mathrm{H}$, et al. Short message service reduces dropout in childhood obesity treatment: a randomized controlled trial. Health Psychology 2012;31(6):797-805.

ISRCTN33476574. A pilot study of the effectiveness of maintenance treatment by short message service (SMS) in overweight and obese children after short-term multidisciplinary cognitive behavioural group therapy. www.isrctn.com/ISRCTN33476574 (last accessed 6 December 2016).

\section{Diaz 2010 \{published data only\}}

* Diaz RG, Esparza-Romero J, Moya-Camarena SY, RoblesSardin AE, Valencia ME. Lifestyle intervention in primary care settings improves obesity parameters among Mexican youth. Journal of the American Dietetic Association 2010;110(2):285-90.

Díaz-Zavala RG, Esparza-Romero J, Moya-Camarena SY, Quihui L, Cisneros-Tapia R, Valencia ME. Lifestyle intervention in primary care settings improves obesity parameters among Mexican youth [Mejora en la sensibilidad a la insulina con un programa intensivo de cambio en el estilo de vida para control de obesidad en niños y adolescentes en el primer nivel de atención]. Archivos Latino Americanos de Nutricion 2012;62(3):258-66

\section{Duffy 1993 \{published data only\}}

Duffy $G$, Spence SH. The effectiveness of cognitive self management as an adjunct to a behavioural intervention for childhood obesity: a research note. Journal of Child Psychology and Psychiatry 1993;34(6):1043-50.

\section{Duggins 2010 \{published data only\}}

Duggins M, Cherven P, Carrithers J, Messamore J, Harvey A. Impact of family YMCA membership on childhood obesity: a randomized controlled effectiveness trial. Journal of the American Board of Family Medicine 2010;23(3):323-33.

Eddy Ives 2012 \{published data only\}

* Eddy Ives L, Moral Peláez I, Brotons Cuixar C, de Frutos Gallego E, Calvo Terrades C, Curell Aguilà, N. Lifestyle habits in overweight and obese adolescents (Obescat Study) [Hábitos de estilo de vida en adolescentes con sobrepeso y obesidad (Estudio Obescat)]. Revista Pediatría de Atención Primaria 2012;14:127-37.

Eddy LS, Moral I, Frutos E, Brotons C, Calvo M, Aloy G. Evaluation of self-awareness of adolescents with overweight and obesity (Obescat Study) [Avaluació de l'autoconcepte dels adolescents amb excésde pes (Estudi Obescat)]. Pediatria Catalana 2013;73(3):107-12.

ISRCTN35399598. OBESCAT Study 2006: randomised clinical trial to assess the efficacy of an intervention in order to reduce the body mass index in adolescents in Catalonia. www.isrctn.com/ISRCTN35399598 (last accessed 6 December 2016).

\section{Epstein 1984a $\{$ published data only\}}

Epstein LH, Kuller LH, Wing RR, Valoski A, McCurley J. The effect of weight control on lipid changes in obese children. American journal of diseases of children 1989;143(4):454-7.

* Epstein LH, Wing RR, Koeske R, Valoski A. Effects of diet plus exercise on weight change in parents and children. Journal of Consulting and Clinical Psychology 1984;52(3):429-37.

\section{Epstein 1985a \{published data only\}}

Epstein LH, Wing RR, Koeske R, Valoski A. A comparison of lifestyle exercise, aerobic exercise, and calisthenics on weight loss in obese children. Behaviour Therapy 1985;16(4):345-56.

Epstein 1985b \{published data only\}

Epstein LH, Wing RR, Penner BC, Kress MJ. Effect of diet and controlled exercise on weight loss in obese children. The Journal of Pediatrics 1985;107(3):358-61.

\section{Epstein 1985c \{published data only\}}

Epstein LH, Wing RR, Woodall K, Penner BC, Kress MJ, Koeske R. Effects of family-based behavioural treatment on obese 5-to-8year-old children. Behaviour Therapy 1985;16(2):205-12.

\section{Epstein 2000a \{published data only\}}

* Epstein LH, Paluch RA, Gordy CC, Saelens BE, Ernst MM. Problem solving in the treatment of childhood obesity. Journal of Consulting and Clinical Psychology 2000;68(4):717-21.

Epstein LH, Paluch RA, Saelens BE, Ernst MM, Wifley DE. Changes in eating disorder symptoms with pediatric obesity treatment. Journal of Pediatrics 2001;139(1):58-65.

Epstein 2001 \{published data only\}

Epstein LH, Paluch RA, Raynor HA. Sex differences in obese children and siblings in family-based obesity treatment. Obesity 2001;9(12):746-53.

Diet, physical activity and behavioural interventions for the treatment of overweight or obese children from the age of 6 to 11 years 


\section{Epstein 2005 \{published data only\}}

* Epstein LH, Roemmich JN, Stein RI, Paluch RA, Kilanowski CK. The challenge of identifying behavioral alternatives to food: clinic and field studies. Annals of Behavioral Medicine 2005;30(3):201-9.

Epstein 2015 \{published data only\}

* Epstein LH, Kilanowski C, Paluch RA, Raynor H, Daniel TO. Reducing variety enhances effectiveness of family-based treatment for pediatric obesity. Eating Behaviors 2015;17:140-3.

NCT01208870. Translating habituation research to interventions for pediatric obesity (EAT). clinicaltrials.gov/ct2/show/ NCT01208870 (last accessed 6 December 2016).

\section{Faude 2010 \{published data only\}}

Faude O, Kerper O, Multhaupt M, Winter C, Beziel K, Junge A, et al. Football to tackle overweight in children. Scandinavian Journal of Medicine \& Science in Sports 2010;20(Suppl 1):103-10.

Flodmark 1993 \{published data only\}

Flodmark CE, Ohlsson T, Ryden O, Sveger T. Prevention of progression to severe obesity in a group of obese schoolchildren treated with family therapy. Pediatrics 1993;91(5):880-4.

\section{Gillis 2007 \{published data only\}}

Gillis D, Brauner M, Granot E. A community-based behavior modification intervention for childhood obesity. Journal of Pediatric Endocrinology \& Metabolism 2007;20(2):197-203.

\section{Gunnarsdottir 2011a \{published data only\}}

Gunnarsdottir T, Sigurdardottir ZG, Njardvik U, Olafsdottir AS, Bjarnason R. A randomized-controlled pilot study of Epstein's family-based behavioural treatment for childhood obesity in a clinical setting in Iceland. Nordic Psychology 2011;63(1):6-19.

\section{Hamilton-Shield 2014 \{published data only\}}

* Hamilton-Shield J, Goodred J, Powell L, Thorn J, Banks J, Hollinghurst $\mathrm{S}$, et al. Changing eating behaviours to treat childhood obesity in the community using Mandolean: the Community Mandolean randomised controlled trial (ComMando) - a pilot study. Health Technology Assessment 2014;18(47):1-75.

ISRCTN90561114. Changing eating behaviours to treat childhood obesity in the community using Mandolean. www.isrctn.com/ISRCTN90561114 (last accessed 6 December 2016).

\section{Ho 2016 \{published data only\}}

* Ho J, Pedersen SD, Virtanen H, Nettel-Aguirre A, Huang C. Family intervention for obese/overweight children using portion control strategy (FOCUS) for weight control: a randomised controlled trial. Global Pediatric Health 2016;3:1-7.

NCT00881478. Family intervention for obese children using portion control strategy for weight control (FOCUS). clinicaltrials.gov/ct2/show/NCT00881478 (last accessed 6 December 2016).
Hughes 2008 \{published data only\}

* Hughes AR, Stewart L, Chapple J, McColl JH, Donaldson MD, Kelnar CJ, et al. Randomized, controlled trial of a best-practice individualized behavioral program for treatment of childhood overweight: Scottish Childhood Overweight Treatment Trial (SCOTT). Pediatrics 2008;121(3):e539-46.

ISRCTN41383109. Scottish childhood obesity treatment trial. www.isrctn.com/ISRCTN41383109 (last accessed 6 December 2016).

Stewart L, Chapple J, Hughes A, Poustie V, Reilly JJ. Parents' journey through treatment for their child's obesity: a qualitative study. Archives of Disease in Childhood 2008;93(1):35-9.

Stewart L, Chapple J, Hughes A, Poustie V, Reilly JJ. The use of behavioural change techniques in treatment of paediatric obesity: qualitative evaluation of parental perspectives on treatment. Journal of Human Nutrition and Dietetics 2008;21(5):464-73.

Stewart L, Houghton J, Hughes AR, Pearson D, Reilly JJ. Dietetic management of pediatric overweight: development and description of a practical and evidence-based behavioral approach. Journal of the American Dietetic Association 2005;105(11):1810-5.

\section{Kalarchian 2009 \{published data only\}}

* Kalarchian MA, Levine MD, Arslanian SA, Ewing LJ, Houck PR, Cheng Y, et al. Family-based treatment of severe pediatric obesity: randomized, controlled trial. Pediatrics 2009;124(4):1060-8.

NCT00177229. KidQuest family-based weight control. clinicaltrials.gov/ct2/show/NCT00177229 (last accessed 6 December 2016).

Wildes JE, Marcus MD, Kalarchian MA, Levine MD, Houck PR, Cheng Y. Self-reported binge eating in severe pediatric obesity: impact on weight change in a randomized controlled trial of family-based treatment. International of Journal of Obesity 2010;34(7):1143-8.

\section{Kalavainen 2007 \{published data only\}}

Heale R. A group intervention for parents and children achieved greater weight loss in obese children than routine care. Evidence Based Nursing 2008;11:43.

Kalavainen M, Karjalainen S, Martikainen J, Korppi M, Linnosmaa I, Nuutinen 0 . Cost-effectiveness of routine and group programs for treatment of obese children. Pediatrics International 2009;51(5):606-11.

Kalavainen M, Korppi M, Nuutinen O. Long-term efficacy of group-based treatment for childhood obesity compared with routinely given individual counselling. International Journal of Obesity 2011;35(4):530-3.

Kalavainen M, Utriainen P, Vanninen E, Korppi M, Nuutinen O. Impact of childhood obesity treatment on body composition and metabolic profile. World Journal of Pediatrics 2012;8:31-7. 
* Kalavainen MP, Korppi MO, Nuutinen OM. Clinical efficacy of group-based treatment for childhood obesity compared with routinely given individual counseling. International Journal of Obesity 2007;31(10):1500-8.

Kirk 2012 \{published data only\}

* Kirk S, Brehm B, Saelens BE, Woo JG, Kissel E, D'Alessio D, et al. Role of carbohydrate modification in weight management among obese children: a randomized clinical trial. Journal of Pediatrics 2012;161(2):320-327.e1.

NCT00215111. Role of carbohydrate modification in weight management among obese children. clinicaltrials.gov/ct2/ show/NCT00215111 (last accessed 6 December 2016).

\section{Larsen 2015 \{published data only\}}

Larsen LM, Hertel NT, Mølgaard C, Christensen RD, Husby S, Jarbøl DE. Early intervention for childhood overweight: a randomized trial in general practice. Scandinavian Journal of Primary Health Care 2015;33(3):184-90.

\section{Lison 2012 \{published data only\}}

* Lison JF, Real-Montes JM, Torro I, Arguisuelas MD, AlvarezPitti J, Martinez-Gramage J, et al. Exercise intervention in childhood obesity: a randomized controlled trial comparing hospital-versus home-based groups. Academic Pediatrics 2012;12(4):319-25.

NCT01503281. Effects of group vs. home-based combined exercise-diet program in childhood obesity: a randomised controlled trial. clinicaltrials.gov/ct2/show/NCT01503281 (last accessed 6 December 2016).

\section{Lochrie 2013 \{published data only\}}

* Lochrie AS, Wysocki T, Hossain J, Milkes A, Antal H, Buckloh L, et al. The effects of a family-based intervention (FBI) for overweight/obese children on health and psychological functioning. Psychology 2013;1(2):159-70.

NCT01146314. A family based intervention to reduce the risk of type 2 diabetes in children (FBI). clinicaltrials.gov/ct2/show/ NCT01146314 (last accessed 6 December 2016).

\section{Looney 2014 \{published data only\}}

* Looney SM, Raynor HA. Examining the effect of three lowintensity pediatric obesity interventions a pilot randomized controlled trial. Clinical Pediatrics 2014;53(14):1367-74.

NCT01358448. A pilot study of the efficacy and program cost-effectiveness of prevention plus for childhood obesity. clinicaltrials.gov/ct2/show/NCT01358448 (last accessed 6 December 2016).

\section{Maddison 2011 \{published data only\}}

ACTRN12607000632493. Electronic games to aid motivation to exercise (eGAME). www.anzctr.org.au/Trial/Registration/ TrialReview. aspx?id=82468 (last accessed 6 December 2016).

Foley L, Jiang Y, Ni Mhurchu C, Jull A, Prapavessis H, Rodgers A, et al. The effect of active video games by ethnicity, sex and fitness: subgroup analysis from a randomised controlled trial.
International Journal of Behavioral Nutrition \& Physical Activity 2014;11(1):46.

Maddison R, Foley L, Jiang Y, Ni Mhurchu C, Jull A, Rodgers A, et al. Electronic games to aid motivation to exercise: a randomized controlled trial. Obesity Reviews 2010;11:50.

Maddison R, Foley L, Mhurchu CN, Jull A, Jiang Y, Prapavessis H, et al. Feasibility, design and conduct of a pragmatic randomized controlled trial to reduce overweight and obesity in children: the electronic games to aid motivation to exercise (eGAME) study. BMC Public Health 2009;9:146.

* Maddison R, Foley L, Ni Mhurchu C, Jiang Y, Jull A, Prapavessis $\mathrm{H}$, et al. Effects of active video games on body composition: a randomized controlled trial. American Journal of Clinical Nutrition 2011;94(1):156-63.

Maddison R, Mhurchu CN, Jull A, Prapavessis H, Foley LS, Jiang Y. Active video games: the mediating effect of aerobic fitness on body composition. International Journal of Behavioral Nutrition \& Physical Activity 2012;9:54.

\section{Maddison 2014 \{published data only\}}

ACTRN12611000164998. Screen-time weight-loss intervention targeting children at home. www.anzctr.org.au/Trial/ Registration/TrialReview.aspx?id=336430 (last accessed 6 December 2016).

* Maddison R, Marsh S, Foley L, Epstein LH, Olds T, Dewes O, et al. Screen-Time Weight-loss Intervention Targeting Children at Home (SWITCH): a randomized controlled trial. International Journal of Behavioral Nutrition and Physical Activity 2014;11:111.

Maddison R, Mhurchu CN, Foley L, Epstein L, Jiang Y, Tsai M, et al. Screen-time weight-loss intervention targeting children at home (SWITCH): a randomized controlled trial study protocol. BMC Public Health 2011;11:524.

\section{Markert 2014 \{published data only\}}

DRKS00000803. T.A.F.F. - Telephone-based adiposity prevention for families. apps.who.int/trialsearch/Trial2.aspx? TrialID=DRKS00000803 (last accessed 6 December 2016).

Markert J, Alff F, Zschaler S, Gausche R, Kiess W, Blüher S. Prevention of childhood obesity: recruiting strategies via local paediatricians and study protocol for a telephone-based counselling programme. Obesity Research \& Clinical Practice 2013;7(6):e476-86.

* Markert J, Herget S, Petroff D, Gausche R, Grimm A, Kiess W, et al. Telephone-based adiposity prevention for families with overweight children (T.A.F.F.-Study): one year outcome of a randomized, controlled trial. International Journal of Environmental Research \& Public Health 2014;11(10):10327-44.

\section{McCallum 2007 \{published data only\}}

Gibbons K, McCallum Z, Wake M. A primary care intervention for childhood obesity: six-month results from LEAP (Live, Eat And Play), a randomised controlled trial. International Journal of Obesity 2004;28:S194. 
Gunn J, McCallum Z, Sanci L, Gerner B, Harris C, Wake M. What do GPs get out of participating in research? - experience of the LEAP trial. Australian Family Physician 2008;37(5):372-5.

ISRCTN45068927. Randomised controlled trial of a brief GP intervention to reduce overweight in Victorian primary school children (LEAP). www.isrctn.com/ISRCTN45068927 (last accessed 6 December 2016).

McCallum Z, Wake M. A primary care intervention for childhood overweight/obesity (leap): methodology of a randomised controlled trial. Journal of Paediatrics and Child Health 2003;39(6):A16.

McCallum Z, Wake M, Baur L. The Leap (live, eat and play) trial: results of a randomized controlled trial of a primary care intervention for childhood overweight/mild obesity. Obesity Research 2004;12:A15-6.

* McCallum Z, Wake M, Gerner B, Baur LA, Gibbons K, Gold L, et al. Outcome data from the LEAP (Live, Eat and Play) trial: a randomized controlled trial of a primary care intervention for childhood overweight/mild obesity. International Journal of Obesity 2007;31(4):630-6.

McCallum Z, Wake M, Gerner B, Harris C, Gibbons K, Gunn J, et al. Can Australian general practitioners tackle childhood overweight/obesity? Methods and processes from the LEAP (Live, Eat and Play) randomized controlled trial. Journal of Paediatrics and Child Health 2005;41(9-10):488-94.

McCallum Z, Wake M, Gerner B, Sheehan J, Gibbons K, Harris C, et al. Six month results from the LEAP (Live, Eat and Play) trial: a randomised controlled trial of a primary care intervention for childhood overweight/mild obesity. Pediatric Research 2004;55(4):220A-221A.

\section{Mirza 2013 \{published data only\}}

* Mirza NM, Palmer MG, Sinclair KB, McCarter R, He J, Ebbeling CB, et al. Effects of a low glycemic load or a lowfat dietary intervention on body weight in obese Hispanic American children and adolescents: a randomized controlled trial. American Journal of Clinical Nutrition 2013;97(2):276-85.

NCT01068197. Low glycemic load diets in Latino children at risk for type 2 diabetes (COOL Kids). clinicaltrials.gov/ct2/show/ NCT01068197 (last accessed 6 December 2016).

\section{NCT02436330 \{unpublished data only\}}

NCT02436330. Exergaming for health: impact of a communitybased active video gaming curriculum in pediatric weight management. clinicaltrials.gov/ct2/show/NCT02436330 (last accessed 6 December 2016).

\section{Nemet 2005 \{published data only\}}

Nemet D, Barkan S, Epstein Y, Friedland O, Kowen G, Eliakim A. Short- and long-term beneficial effects of a combined dietarybehavioral-physical activity intervention for the treatment of childhood obesity. Pediatrics 2005;115(4):e443-9.

\section{Nova 2001 \{published data only\}}

Nova A, Russo A, Sala E. Long-term management of obesity in paediatric office practice: experimental evaluation of two different types of intervention. Ambulatory Child Health 2001;7(3/4):239-48.

\section{Nowicka 2009 \{published data only\}}

Nowicka P, Lanke J, Pietrobelli A, Apitzsch E, Flodmark CE. Sports camp with six months of support from a local sports club as a treatment for childhood obesity. Scandinavian Journal of Public Health, 2009;37(8):793-800.

\section{O'Connor 2013 \{published data only\}}

Ledoux T, Hilmers A, Watson K, Baranowski T, O'Connor TM. Development and feasibility of an objective measure of patient-centered communication fidelity in a pediatric obesity intervention. Journal of Nutrition Education and Behavior 2013;45(4):349-54.

NCT01195012. Pilot RCT of an obesity intervention for primary care pediatrics: Helping HAND. clinicaltrials.gov/ct2/show/ NCT01195012 (last accessed 6 December 2016).

* O'Connor TM, Hilmers A, Watson K, Baranowski T, Giardino AP. Feasibility of an obesity intervention for paediatric primary care targeting parenting and children: Helping HAND. Child: Care, Health \& Development 2013;39(1):141-9.

\section{Reinehr 2010 \{published data only\}}

Finne E, Reinehr T, Schaefer A, Winkel K, Kolip P. Changes in self-reported and parent-reported health-related quality of life in overweight children and adolescents participating in an outpatient training: findings from a 12-month follow-up study. Health and Quality of Life Outcomes 2013;11:1.

Finne E, Reinehr T, Schaefer A, Winkel K, Kolip P. Overweight children and adolescents - is there a subjective need for treatment?. International Journal of Public Health 2009;54(2):112-6.

NCT00422916. Treatment of overweight children and adolescents. clinicaltrials.gov/ct2/show/NCT00422916 (last accessed 6 December 2016).

* Reinehr T, Schaefer A, Winkel K, Finne E, Toschke AM, Kolip P. An effective lifestyle intervention in overweight children: findings from a randomized controlled trial on "Obeldicks light". Clinical Nutrition 2010;29(3):331-6.

Schaefer A, Winkel K, Finne E, Kolip P, Reinehr, T. An effective lifestyle intervention in overweight children: one-year followup after the randomized controlled trial on "Obeldicks light". Clinical Nutrition 2011;30(5):629-33.

\section{Rodearmel 2007 \{published data only\}}

Holm KE, Wyatt HR, Murphy JR, Hill JO, Ogden LG. Parental influence on child change in physical activity during a familybased intervention for child weight gain prevention. Journal of Physical Activity \& Health 2012;9(5):661-9.

\footnotetext{
* Rodearmel SJ, Wyatt HR, Stroebele N, Smith SM, Ogden LG, Hill JO. Small changes in dietary sugar and physical activity as an approach to preventing excessive weight gain: the America on the Move family study. Pediatrics 2007;120(4):e869-79.
}

Diet, physical activity and behavioural interventions for the treatment of overweight or obese children from the age of 6 to 11 years 


\section{Sacher 2010 \{published data only\}}

ISRCTN30238779. Improving health outcomes in obese children: a randomised controlled trial of the MEND programme. www.isrctn.com/ISRCTN30238779 (last accessed 6 December 2016).

* Sacher PM, Kolotourou M, Chadwick PM, Cole TJ, Lawson MS, Lucas A, et al. Randomized controlled trial of the MEND program: a family-based community intervention for childhood obesity. Obesity 2010;18(Suppl 1):S62-8.

\section{Saelens 2013 \{published data only\}}

NCT00746629. Familial overweight: comparing use of strategies (FOCUS). clinicaltrials.gov/ct2/show/NCT00746629 (last accessed 6 December 2016).

* Saelens BE, Lozano P, Scholz K. A randomized clinical trial comparing delivery of behavioral pediatric obesity treatment using standard and enhanced motivational approaches. Journal of Pediatric Psychology 2013;38(9):954-64.

\section{Satoh 2007 \{published data only\}}

Satoh A, Menzawa K, Lee S, Hatakeyama A, Sasaki H. Dietary guidance for obese children and their families using a model nutritional balance chart. Japan Journal of Nursing Science 2007;4(2):95-102.

\section{Schwingshandl 1999 \{published data only\}}

Schwingshandl J, Sudi K, Eibl B, Wallner S, Brokenstein M. Effect of an individualised training programme during weight reduction on body composition: a randomised trial. Archives of Diseases in Childhood 1999;81(5):426-8.

\section{Serra-Paya 2015 \{published data only\}}

NCT01878994. Evaluation of a family intervention program for the management of overweight or obese children (NEREU). clinicaltrials.gov/ct2/show/NCT01878994 (last accessed 6 December 2016).

* Serra-Paya N, Ensenyat A, Castro-Vinuales I, Real J, Sinfreu-Bergues $X$, Zapata A, et al. Effectiveness of a multicomponent intervention for overweight and obese children (Nereu Program): a randomized controlled trial. PLoS One 2015;10(12):e0144502.

Serra-Paya N, Ensenyat A, Real J, Castro-Vinuales I, Zapata A, Galindo $G$, et al. Evaluation of a family intervention programme for the treatment of overweight and obese children (Nereu Programme): a randomized clinical trial study protocol. BMC Public Health 2013;13:1000.

Siwik 2013 \{published data only\}

NCT01674920. CHOICES: family physicians treat overweight children. clinicaltrials.gov/ct2/show/NCT01674920 (last accessed 6 December 2016).

* Siwik V, Kutob R, Ritenbaugh C, Cruz L, Senf J, Aickin M, et al. Intervention in overweight children improves body mass index (BMI) and physical activity. Journal of the American Board of Family Medicine 2013;26(2):126-37.

\section{Taveras 2015 \{published data only\}}

NCT01537510. Study of technology to accelerate research (STAR). clinicaltrials.gov/ct2/show/NCT01537510 (last accessed 6 December 2016).

Taveras EM, Marshall R, Horan CM, Gillman MW, Hacker K, Kleinman KP, et al. Rationale and design of the STAR randomized controlled trial to accelerate adoption of childhood obesity comparative effectiveness research. Contemporary Clinical Trials 2013;34(1):101-8.

* Taveras EM, Marshall R, Kleinman KP, Gillman MW, Hacker K, Horan CM, et al. Comparative effectiveness of childhood obesity interventions in pediatric primary care: a cluster-randomized clinical trial. JAMA pediatrics 2015;169(6):535-42.

Taylor 2015 \{published data only\}

ACTRN12609000749202. Screening, feedback and treatment in overweight 4-8 year old children: the MInT study. www.anzctr.org.au/Trial/Registration/TrialReview.aspx? id=308336 (last accessed 6 December 2016).

Dawson AM, Brown DA, Cox A, Williams SM, Treacy L, Haszard J, et al. Using motivational interviewing for weight feedback to parents of young children. Journal of Paediatrics and Child Health 2014;50(6):461-70.

Dawson AM, Taylor RW, Williams SM, Taylor BJ, Brown DA. Do parents recall and understand children's weight status information after BMI screening?. BMJ Open 2014;4(7):e004481.

Haszard JJ, Williams SM, Dawson AM, Skidmore PML, Taylor RW. Factor analysis of the comprehensive feeding practices questionnaire in a large sample of children. Appetite 2013;62:110-8.

Taylor RW, Brown D, Dawson AM, Haszard J, Cox A, Rose EA, et al. Motivational interviewing for screening and feedback and encouraging lifestyle changes to reduce relative weight in 4-8 year old children: design of the MInT study. BMC Public Health 2010;10:271.

* Taylor RW, Cox A, Knight L, Brown DA, Meredith-Jones K, Haszard JJ, et al. A tailored family-based obesity intervention: a randomized trial. Pediatrics 2015;136:281-9.

Taylor RW, Williams SM, Dawson AM, Taylor BJ, MeredithJones K, Brown D. What factors influence uptake into familybased obesity treatment after weight screening. Journal of Pediatrics 2013;163(6):1657-62.e1.

Vann 2013 \{published data only\}

Vann LH, Stanford FC, Durkin MW, Hanna A, Knight LM, Stallworth JR. "Moving and losing": a pilot study incorporating physical activity to decrease obesity in the pediatric population. Journal of the South Carolina Medical Association 2013;109(4):116-20.

Wafa 2011 \{published data only\}

Hamzaid H, Talib RA, Azizi NH, Maamor N, Reilly JJ, Wafa SW. Quality of life of obese children in Malaysia. International Journal of Pediatric Obesity 2011;6(5-6):450-4.

Diet, physical activity and behavioural interventions for the treatment of overweight or obese children from the age of 6 to 11 years 
ISRCTN14241825. Malaysian childhood obesity treatment trial (MASCOT). www.isrctn.com/ISRCTN14241825 (last accessed 6 December 2016).

Sharifah WW, Nur HH, Ruzita AT, Roslee R, Reilly JJ. The Malaysian Childhood Obesity Treatment Trial (MASCOT). Malaysian Journal of Nutrition 2011;17(2):229-36.

Wafa SW, Ruzita AT, Nur Hana H, Roslee R, Ng LO, Ayiesah R, et al. Family-focused weight management programme for childhood obesity in Malaysia: randomised controlled trial. Obesity Reviews 2010;11:85-6.

* Wafa SW, Talib RA, Hamzaid NH, McColl JH, Rajikan R, Ng LO, et al. Randomized controlled trial of a good practice approach to treatment of childhood obesity in Malaysia: Malaysian Childhood Obesity Treatment Trial (MASCOT). International Journal of Pediatric Obesity 2011;6(2-2):e62-9.

Wake 2009 \{published data only\}

ISRCTN52511065. Randomised controlled trial of a brief GP intervention to reduce overweight in Victorian primary school children - live, eat and play (LEAP 2). www.isrctn.com/ ISRCTN52511065 (last accessed 6 December 2016).

* Wake M, Baur LA, Gerner B, Gibbons K, Gold L, Gunn J, et al. Outcomes and costs of primary care surveillance and intervention for overweight or obese children: the LEAP 2 randomised controlled trial. BMJ 2009;339:b3308.

\section{Wake 2013 \{published data only\}}

ACTRN12608000055303. Better outcomes for obese children in general practice: randomized controlled trial of a new sharedcare model vs usual care. www.anzctr.org.au/Trial/Registration/ TrialReview. aspx?id=82549 (last accessed 6 December 2016).

* Wake M, Lycett K, Clifford SA, Sabin MA, Gunn J, Gibbons K, et al. Shared care obesity management in 3-10 year old children: 12 month outcomes of HopSCOTCH randomised trial. BMJ 2013;346:f3092.

Wake M, Lycett K, Sabin MA, Gunn J, Gibbons K, Hutton C, et al. A shared-care model of obesity treatment for 3-10 year old children: protocol for the HopSCOTCH randomised controlled trial. BMC Pediatrics 2012;12:39.

\section{Waling 2012 \{published data only\}}

Backlund C, Sundelin G, Larsson C. Effect of a 1-year lifestyle intervention on physical activity in overweight and obese children. Advances in Physiotherapy 2011;13(3):87-96.

Backlund C, Sundelin G, Larsson C. Effects of a 2-year lifestyle intervention on physical activity in overweight and obese children. Advances in Physiotherapy 2011;13(3):97-109.

NCT01012206. Overweighed children's health - studies of the effect of lifestyle and food habits (SELFH). clinicaltrials.gov/ct2/ show/NCT01012206 (last accessed 6 December 2016).

* Waling M, Backlund C, Lind T, Larsson C. Effects on metabolic health after a 1-year-lifestyle intervention in overweight and obese children: a randomized controlled trial. Journal of Nutrition and Metabolism 2012;2012(913965):1-10.
Waling M, Lind T, Hernell O, Larsson C. A one-year intervention has modest effects on energy and macronutrient intakes of overweight and obese Swedish children. Journal of Nutrition 2010;140(10):1793-8.

Waling MU, Larsson CL. Improved dietary intake among overweight and obese children followed from 8 to 12 years of age in a randomised controlled trial. Journal of Nutritional Science 2012;1:e16.

\section{Warschburger 2016 \{published data only\}}

ISRCTN24655766. Empowering parents of obese children: development and controlled evaluation of an obesity-specific parenting skills training. www.isrctn.com/ISRCTN24655766 (last accessed 6 December 2016).

* Warschburger P, Kroeller K, Haerting J, Unverzagt S, van Egmond-Fröhlich A. Empowering Parents of Obese Children (EPOC): a randomized controlled trial on additional long-term weight effects of parent training. Appetite 2016;103:148-56.

Warschburger $\mathrm{P}$, Kroller $\mathrm{K}$, Unverzagt $\mathrm{S}$, Haerting J. What is the parents' part in long-term weight management of their obese child? Results from the EPOC study. Obesity Facts 2013;6:230.

\section{Weigel 2008 \{published data only\}}

Weigel C, Kokocinski K, Lederer P, Dotsch J, Rascher W, Knerr I. Childhood obesity: concept, feasibility, and interim results of a local group-based, long-term treatment program. Journal of Nutrition Education \& Behavior 2008;40(6):369-73.

Weintraub 2008 \{published data only\}

NCT00186173. Sports to prevent obesity: feasibility and pilot RCT. clinicaltrials.gov/ct2/show/NCT00186173 (last accessed 6 December 2016).

* Weintraub DL, Tirumalai EC, Haydel KF, Fujimoto M, Fulton JE, Robinson TN. Team sports for overweight children: the Stanford Sports to Prevent Obesity Randomized Trial (SPORT). Archives of Pediatrics \& Adolescent Medicine 2008;162(3):232-7.

\section{Wilfley 2007 \{published data only\}}

Goldschmidt AB, Stein RI, Saelens BE, Theim KR, Epstein LH, Wilfley $D E$. Importance of early weight change in a pediatric weight management trial. Pediatrics 2011;128(1):e33-9.

NCT00301197. Childhood obesity treatment: a maintenance approach. clinicaltrials.gov/ct2/show/NCT00301197 (last accessed 6 December 2016).

Theim KR, Sinton MM, Goldschmidt AB, Van Buren DJ, Doyle AC, Saelens BE, et al. Adherence to behavioral targets and treatment attendance during a pediatric weight control trial. Obesity 2013;21(2):394-7.

* Wilfley DE, Stein RI, Saelens BE, Mockus DS, Matt GE, HaydenWade HA, et al. Efficacy of maintenance treatment approaches for childhood overweight: a randomized controlled trial. JAMA 2007;298(14):1661-73.

Diet, physical activity and behavioural interventions for the treatment of overweight or obese children from the age of 6 to 11 years 
Woo 2004 \{published data only\}

* Woo KS, Chook P, Yu CW, Sung RY, Qiao M, Leung SS, et al. Effects of diet and exercise on obesity-related vascular dysfunction in children. Circulation 2004;109(16):1981-6.

\section{Wright 2012 \{published data only\}}

Wright K, Giger JN, Norris K, Suro Z. Impact of a nurse-directed, coordinated school health program to enhance physical activity behaviors and reduce body mass index among minority children: a parallel-group, randomized control trial. International Journal of Nursing Studies 2013;50(6):727-37.

* Wright K, Norris K, Newman Giger J, Suro Z. Improving healthy dietary behaviors, nutrition knowledge, and self-efficacy among underserved school children with parent and community involvement. Childhood Obesity 2012;8(4):347-56.

Wright K, Suro Z. Using community-academic partnerships and a comprehensive school-based program to decrease health disparities in activity in school-aged children. Journal of Prevention \& Intervention in the Community 2014;42(2):125-39.

\section{References to studies excluded from this review}

Adam 2009 \{published data only\}

Adam S, Westenhofer J, Rudolphi B, Kraaibeek HK. Effects of a combined inpatient-outpatient treatment of obese children and adolescents. Obesity Facts 2009;2:286-93.

Albala 2008 \{published data only\}

Albala C, Ebbeling CB, Cifuentes M, Lera L, Bustos N, Ludwig DS. Effects of replacing the habitual consumption of sugarsweetened beverages with milk in Chilean children. American Journal of Clinical Nutrition 2008;88(3):605-11.

\section{Alberga 2013 \{published data only\}}

Alberga AS, Farnesi BC, Lafleche A, Legault L, Komorowski J. The effects of resistance exercise training on body composition and strength in obese prepubertal children. Physician \& Sports medicine 2013;41(3):103-9.

\section{Alexander 2014 \{published data only\}}

Alexander AG, Grant WL, Pedrino KJ, Lyons PE. A prospective multifactorial intervention on subpopulations of predominately Hispanic children at high risk for obesity. Obesity 2014; Vol. 22, issue 1:249-53.

\section{Amini 2016 \{published data only\}}

Amini M, Djazayery A, Majdzadeh R, Taghdisi MH, SadrzadehYeganeh $\mathrm{H}$, Abdollahi Z, et al. A school-based intervention to reduce excess weight in overweight and obese primary school students. Biological Research for Nursing 2016;29:29.

\section{Andre 2015 \{published data only\}}

Andre N, Beguier S. Using motivational interviewing as a supplement to physical activity program in obese adolescents: a RCT study. Eating and Weight Disorders 2015; Vol. 20, issue 4:519-23.

\section{Astrup 2013 \{published data only\}}

Astrup A, Damsgaard CT, Papadaki A, Jensen SM, Dalskov SM, Hlavaty P, et al. 1 The use of an ad libitum higher-protein, lowglycemic index diet in overweight children: the Diogenes Study. FASEB Journal 2013;27:249.8.

\section{Bachman 2010 \{published data only\}}

Bachman J, Raynor H. Parent and child dietary change during a family-based behavioral childhood obesity intervention. Obesity 2010;18:S117.

\section{Baker 2012 \{published data only}

Baker J, Saunders K. Fitter, healthier, happier families: a partnership to treat childhood obesity in the West Midlands. Public Health 2012;126(4):332-4.

Ball 2012 \{published data only\}

Ball GD, Ambler KA, Keaschuk RA, Rosychuk RJ, Holt NL, Spence JC, et al. Parents as agents of change (PAC) in pediatric weight management: the protocol for the PAC randomized clinical trial. BMC Pediatrics 2012;12:114.

\section{Banks 2011 \{published data only\}}

Banks J, Shield JP, Sharp D. Barriers engaging families and GPs in childhood weight management strategies. British Journal of General Practice 2011;61(589):e492-7.

Banks 2012a \{published data only\}

Banks J, Sharp DJ, Hunt LP, Shield JP. Evaluating the transferability of a hospital-based childhood obesity clinic to primary care: a randomised controlled trial. British Journal of General Practice 2012;62(594):e6-12.

Banks 2012b \{published data only\}

Banks J, Williams J, Cumberlidge T, Cimonetti T, Sharp DJ, Shield JP. Is healthy eating for obese children necessarily more costly for families?. British Journal of General Practice 2012;62(594):e1-5.

\section{Banks 2014 \{published data only\}}

Banks J, Cramer H, Sharp DJ, Shield JP, Turner KM. Identifying families' reasons for engaging or not engaging with childhood obesity services: a qualitative study. Journal of Child Health Care 2014;18(2):101-10.

\section{Banos 2009 \{published data only\}}

Banos RM, Cebolla A, Zaragoza I, Botella C, Alcaniz M. Electronic PDA dietary and physical activity registers in a weight loss treatment program for children: a description of the ETIOBE personal digital assistant program. Journal of Cybertherapy and Rehabilitation 2009;2:235-41.

\section{Baranowski 2011 \{published data only\}}

Baranowski T, Baranowski J, Thompson D, Buday R, Jago R, Griffith MJ, et al. Video game play, child diet, and physical activity behavior change a randomized clinical trial. American Journal of Preventive Medicine 2011;40(1):33-8.

\section{Barbeau 2007 \{published data only\}}

Barbeau P, Johnson MH, Howe CA, Allison J, Davis CL, Gutin B, et al. Ten months of exercise improves general and

Diet, physical activity and behavioural interventions for the treatment of overweight or obese children from the age of 6 to 11 years 
visceral adiposity, bone, and fitness in black girls. Obesity 2007;15(8):2077-85.

\section{Bau 2016 \{published data only\}}

Bau AM, Ernert A, Krude H, Wiegand S. Hormonal regulatory mechanisms in obese children and adolescents after previous weight reduction with a lifestyle intervention: maintain paediatric part - a RCT from 2009-15. BMC Obesity 2016;3:29.

\section{Bauer 2010 \{published data only\}}

Bauer S, de Niet J, Timman R, Kordy H. Enhancement of care through self-monitoring and tailored feedback via text messaging and their use in the treatment of childhood overweight. Patient Education and Counseling 2010;79(3):315-9.

\section{Bayat 2014 \{published data only\}}

Bayat E, Rahimian Boogar I, Talepasand S, Yousefi Chaigan P. The effectiveness of family-based cognitive behavioral therapy in weight reduction among children with obesity. Iranian Journal of Endocrinology and Metabolism 2014;16:254-61.

\section{Bean 2012 \{published data only\}}

Bean MK, Wilson DB, Thornton LM, Kelly N, Mazzeo SE. Dietary intake in a randomized-controlled pilot of NOURISH: a parent intervention for overweight children. Preventive Medicine 2012;55(3):224-7.

\section{Bean 2014 \{published data only\}}

Bean MK, Jeffers AJ, Tully CB, Thornton LM, Mazzeo SE. Motivational interviewing with parents of overweight children: study design and methods for the NOURISH + MI study. Contemporary Clinical Trials 2014;37(2):312-21.

\section{Benestad 2014 \{published data only\}}

Benestad B, Lekhal S, Hertel JK, Odegard R, Hjelmesaeth J. Long-term effectiveness of two family based life style intervention programs on childhood obesity. A 2-years randomized controlled pragmatic trial. Obesity Facts 2014;7:131.

\section{Benson 2008 \{published data only\}}

Benson AC, Torode ME, Fiatarone Singh MA. The effect of high-intensity progressive resistance training on adiposity in children: a randomized controlled trial. International Journal of Obesity 2008;32(6):1016-27.

\section{Bernstein 2015 \{published data only\}}

Bernstein HM. An ecological systems approach to pediatric weight management: a psychoeducational group manual for caregivers [Dissertation]. Vol. 75, Available from gradworks.umi.com/36/30/3630291.html, 2015.

\section{Best 2016 \{published data only\}}

Best JR, Goldschmidt AB, Mockus-Valenzuela DS, Stein RI, Epstein LH, Wilfley DE. Shared weight and dietary changes in parent-child dyads following family-based obesity treatment. Health Psychology 2016;35(1):92-5.

\section{Bloom 2013 \{published data only\}}

Bloom T, Sharpe L, Mullan B, Zucker N. A pilot evaluation of appetite-awareness training in the treatment of childhood overweight and obesity: a preliminary investigation International Journal of Eating Disorders 2013;46(1):47-51.

Bocca 2014 \{published data only\}

Bocca G, Corpeleijn E, Van den Heuvel ER, Stolk RP, Sauer PJ. Three-year follow-up of 3-year-old to 5-year-old children after participation in a multidisciplinary or a usual-care obesity treatment program. Clinical Nutrition 2014;33(6):1095-100.

\section{Bohnert 2013 \{published data only\}}

Bohnert AM, Ward AK. Making a difference: evaluating the Girls in the Game (GIG) after-school program. The Journal of Early Adolescence 2013;33(1):104-30.

\section{Boutelle 2011 \{published data only\}}

Boutelle KN, Zucker NL, Peterson CB, Rydell SA, Cafri G, Harnack $L$. Two novel treatments to reduce overeating in overweight children: a randomized controlled trial. Journal of Consulting \& Clinical Psychology 2011;79(6):759-71.

Boutelle 2013 \{published data only\} Boutelle KN, Norman GJ, Rock CL, Rhee KE, Crow SJ. Guided self-help for the treatment of pediatric obesity. Pediatrics 2013;131:e1435-42.

Braden 2014 \{published data only\}

Braden A, Rhee K, Peterson CB, Rydell SA, Zucker N, Boutelle K. Associations between child emotional eating and general parenting style, feeding practices, and parent psychopathology. Appetite 2014;80:35-40.

Braet 1997a \{published data only\} Braet C, Mervielde I, Vandereycken W. Psychological aspects of childhood obesity: a controlled study in a clinical and nonclinical sample. Journal of Pediatric Psychology 1997;22:59-71.

\section{Braet 1997b \{published data only\}}

Braet C, Van Winckel M, Van Leeuwen K. Follow-up results of different treatment programs for obese children. Acto Paediatrica 1997;86:397-402.

\section{Braet 2000 \{published data only\}}

Braet C, Van Winckel M. Long-term follow-up of a cognitive behavioral treatment program for obese children. Behavior Therapy 2000;31:55-74.

\section{Buhari 2014 \{published data only\}}

Buhari SSS, Talib RA, Koon PB. The HEBAT! program: an obesity prevention exploratory cluster randomized controlled trial. Obesity Reviews 2014;15:153.

\section{Burrows 2008 \{published data only\}}

Burrows T, Warren JM, Baur LA, Collins CE. Impact of a child obesity intervention on dietary intake and behaviors. International Journal of Obesity 2008;32:1481-8.

\section{Burrows 2010a \{published data only\}}

Burrows T, Berthton B, Garg M, Collins C. A novel approach to validating dietary change in children participating in the

Diet, physical activity and behavioural interventions for the treatment of overweight or obese children from the age of 6 to 11 years 
HIKCUPS (Hunter Illawarra Kids Challenge Using Parent Support) obesity intervention. Obesity Reviews 2010;11:374.

\section{Burrows 2010b \{published data only\}}

Burrows T, Warren JM, Collins CE. The impact of a child obesity treatment intervention on parent child-feeding practices. International Journal of Pediatric Obesity 2010;5:43-50.

\section{Burrows 2011 \{published data only\}}

Burrows T, Janet WM, Collins CE. Long-term changes in food consumption trends in overweight children in the HIKCUPS intervention. Journal of Pediatric Gastroenterology \& Nutrition 2011;53:543-7.

\section{Bush 2007 \{published data only\}}

Bush CL, Pittman S, McKay S, Ortiz T, Wong WW, Klish WJ. Parkbased obesity intervention program for inner-city minority children. The Journal of Pediatrics 2007;151:513-7.

\section{Bustos 1997 \{published data only\}}

Bustos LG, Moreno MF, Calderin MMA, Martinez QJJ, Diaz ME, Arana CC. Comparative study of medical advice and cognitivebehavioral group therapy in the treatment of child-adolescent obesity. Anales Espanoles de Pediatria 1997;47:135-43.

\section{Caballero 2003a \{published data only\}}

Caballero B, Clay T, Davis SM, Ethelbah B, Rock BH, Lohman T, et al. Pathways: a school-based, randomized controlled trial for the prevention of obesity in American Indian schoolchildren. American Journal of Clinical Nutrition 2003;78:1030-8.

\section{Caballero 2003b \{published data only\}}

Caballero B, Himes JH, Lohman T, Davis SM, Stevens J, Evans M, et al. Body composition and overweight prevalence in 1704 schoolchildren from 7 American Indian communities. American Journal of Clinical Nutrition 2003;78:308-12.

\section{Canas 2012a \{published data only\}}

Canas JA, Damaso L, Altomare A, Killen K, Hossain J, Balagopal PB. Insulin resistance and adiposity in relation to serum beta-carotene levels. Journal of Pediatrics 2012;161:58-64.e1-2.

\section{Canas 2012b \{published data only\}}

Canas JA, Damaso L, Mann KJ, Sweeten S, Balagopa P. Fatty acid binding proteins (FABP4 and FABP5), beta-carotene and insulin resistance (IR): a 6-month double blind placebocontrolled study on the effect of a fruit and vegetable juice concentrate (FVJC) in boys. Endocrine Reviews 2012;33 (3 Meeting Abstracts): No pagination.

\section{Canas 2014 \{published data only\}}

Canas J, Lochrie A, McGowan AG, Schettino C, Hossain J, Balagopal P. Effect of a 2-week intense life-style intervention followed by 6-month carotenoid supplementation on fat depots, aditonectin and palmitoleate: a 6 -month double blind placebo-controlled pilot study in obese children. FASEB Journal 2014;1:645.6.

\section{Carrel 2005 \{published data only\}}

Carrel AL, Clark RR, Peterson SE, Nemeth BA, Sullivan J, Allen DB. Improvement of fitness, body composition, and insulin sensitivity in overweight children in a school-based exercise program: a randomized, controlled study. Archives of Pediatrics \& Adolescent Medicine 2005;159:963-8.

\section{Carrel 2007 \{published data only\}}

Carrel AL, Clark RR, Peterson S, Eickhoff J, Allen DB. Schoolbased fitness changes are lost during the summer vacation. Archives of Pediatrics \& Adolescent Medicine 2007;161:561-4.

\section{Cash 2009 \{published data only\}}

Cash AJ. Evaluation of a multi-faceted intervention to prevent obesity in elementary school children. Dissertation Abstracts International: Section B: The Sciences and Engineering. 2009; Vol. 69:7446.

\section{Catenacci 2014 \{published data only\}}

Catenacci VA, Barrett C, Odgen L, Browning R, Schaefer CA, Hill J, et al. Changes in physical activity and sedentary behavior in a randomized trial of an internet-based versus workbookbased family intervention study. Journal of Physical Activity \& Health 2014;11:348-58.

\section{Cespedes 2014 \{published data only\}}

Cespedes EM, Horan CM, Gillman MW, Gortmaker SL, Price S, Rifas-Shiman SL, et al. Participant characteristics and intervention processes associated with reductions in television viewing in the High Five for Kids study. Preventive Medicine: An International Journal Devoted to Practice and Theory 2014;62:64-70.

\section{Chen 2013 \{published data only\}}

Chen JL, Kwan M, Mac A, Chin NC, Liu K. iStart smart: a primary-care based and community partnered childhood obesity management program for Chinese-American children: feasibility study. Journal of Immigrant \& Minority Health 2013;15:1125-8.

\section{Chen 2015 \{published data only\}}

Chen J, Kwan M, Liu K, Yu F. The feasibility and efficacy of healthy weight management program for low income Chinese American overweight and obese children in a primary care clinic. Journal of Pediatric Nursing 2015;30:821-8.

\section{Chen 2016 \{published data only\}}

Chen J, Kwan M. Short-term efficacy and correlates of change in health weight management program for Chinese American children. Clinical Pediatrics 2016;55:463-9.

\section{Chirita-Emandi 2014 \{published data only\}}

Chirita-Emandi A, Puiu M. Outcomes of neurofeedback training in childhood obesity management: a pilot study. Journal of Alternative \& Complementary Medicine 2014;20:831-7.

\section{Chongviriyaphan 2010 \{published data only\}}

Chongviriyaphan N, Sangthien N, Suthutvoravut U. The nutrition counselling with a behavior modification is effective in obese school-aged children. Obesity Reviews 2010;11:292-3.

Diet, physical activity and behavioural interventions for the treatment of overweight or obese children from the age of 6 to 11 years 
Cohen 2012 \{published data only\}

Cohen TR, Hazell T, Vanstone CA, Moore M, Plourde H, Rodd C, et al. Body composition of 6 to 8.5 year old overweight and obese children: 12-week follow-up from an eating and exercise behaviour family-centered lifestyle intervention in Quebec (Canada). FASEB Journal 2012;26:811-2.

\section{Collins 2010 \{published data only\}}

Collins CE, Morgan PJ, Okely AD, Burrows TL, Cliff DP, Jones RA, et al. HIKCUPS (Hunter Illawarra Kids Challenge Using Parent Support) reduces BMI z-score up to 2 years: results of a multisite randomized trial for overweight children. Obesity Reviews 2010;11:280

\section{Cooperberg 2014 \{published data only\}}

Cooperberg J. Food for thought: a parental internet-based intervention to treat childhood obesity in preschool-aged children. Dissertation Abstracts International: Section B: The Sciences and Engineering. 2014; Vol. 74.

\section{Coppinger 2016 \{published data only\}}

Coppinger T, Lacey S, O'Neill C, Burns C. 'Project Spraoi': a randomized control trial to improve nutrition and physical activity in school children. Contemporary Clinical Trials Communications 2016;3:94-101.

\section{Cradock 2016 \{published data only\}}

Cradock AL, Barrett JL, Giles CM, Lee RM, Kenney EL, deBlois ME, et al. Promoting physical activity with the Out of School Nutrition and Physical Activity (OSNAP) initiative a cluster-randomized controlled trial. JAMA Pediatrics 2016;170:155-62.

\section{Crova 2014 \{published data only\}}

Crova C, Struzzolino I, Marchetti R, Masci I, Vannozzi G, Forte R, et al. Cognitively challenging physical activity benefits executive function in overweight children. Journal of Sports Sciences 2014;32:201-11.

\section{Cunningham-Sabo 2016 \{published data only\}}

Cunningham-Sabo L, Lohse B, Smith S, Browning R, Strutz E, Nigg C, et al. Fuel for Fun: a cluster-randomized controlled study of cooking skills, eating behaviors, and physical activity of 4th graders and their families. BMC Public Health 2016;16:444.

\section{Dahiya 2012 \{published data only\}}

Dahiya R, Cowin GJ, Yang Z, Almestehi M, Leong GM. Effect of a 12-month lifestyle behavioral modification intervention on fat compartmentation in overweight and obese prepubertal children. Endocrine Reviews 2012;33(3 Meeting Abstracts):OR18-4.

\section{Dai 2006 \{published data only\}}

Dai J, Jiang Z, Zhang B. Exercise and nutrition therapy for simple obesity in children. Chinese Journal of Clinical Rehabilitation 2006;10:20-2.

\section{Dalton 2013 \{published data only\}}

Dalton WT 3rd, Schetzina KE, McBee MT, Maphis L, FultonRobinson H, Ho AL, et al. Parent report of child's health-related quality of life after a primary-care-based weight management program. Childhood Obesity 2013;9:501-8.

Daniels 2009a \{published data only\}

Daniels LA, Magarey A, Battistutta D, Nicholson JM, Farrell A, Davidson $G$, et al. The NOURISH randomised control trial: positive feeding practices and food preferences in early childhood - a primary prevention program for childhood obesity. BMC Public Health 2009;9:387.

Danielsen 2013 \{published data only\}

Danielsen YS, Nordhus IH, Juliusson PB, Maehle M, Pallesen S. Effect of a family-based cognitive behavioural intervention on body mass index, self-esteem and symptoms of depression in children with obesity (aged 7-13): a randomised waiting list controlled trial. Obesity Research \& Clinical Practice 2013;7:e116-28.

Danielzik 2007 \{published data only\}

Danielzik S, Pust S, Muller MJ. School-based interventions to prevent overweight and obesity in prepubertal children: process and 4-years outcome evaluation of the Kiel Obesity Prevention Study (KOPS). Acta Paediatrica, International Journal of Paediatrics 2007;96:19-25.

\section{da Silva 2015 \{published data only\}}

da Silva DF, Bianchini JA, Lopera CA, Capelato DA, Hintze LJ, Nardo CC, et al. Impact of readiness to change behavior on the effects of a multidisciplinary intervention in obese Brazilian children and adolescents. Appetite 2015;87:229-35.

\section{Davis 1999 \{published data only\}}

Davis CE, Hunsberger S, Murray DM, Fabsitz RR, Himes JH, Stephenson LK, et al. Design and statistical analysis for the Pathways study. American Journal of Clinical Nutrition 1999;69:760S-763S.

\section{Davis 2011a \{published data only\}}

Davis AM, Blackburn KS, Dreyer M, Hampl S, Sampilo M. Treatment outcomes across three family-based behavioral pediatric obesity group treatment programs: Healthy Hawks, Zoom to Health and PHIT kids. Obesity 2011;19:S106.

\section{Davis 2011b \{published data only\}}

Davis CL, Tomporowski PD, McDowell JE, Austin BP, Miller PH, Yanasak NE, et al. Exercise improves executive function and achievement and alters brain activation in overweight children: a randomized, controlled trial. Health Psychology 2011;30:91-8.

Davis 2014 \{published data only\}

Davis CL, Pollock NK, Bassali R, Boyle CA, Waller JL, Allison JD, et al. Exercise dose and diabetes risk in overweight and obese children: a randomized control trial. Diabetes Technology and Therapeutics 2014;16:S93.

Davis 2016a \{published data only\} Davis AM, Sampilo M, Gallagher KS, Dean K, Saroja MB, Yu Q, et al. Treating rural paediatric obesity through telemedicine vs. telephone: outcomes from a cluster randomized controlled trial. Journal of Telemedicine \& Telecare 2016;22(2):86-95.

Diet, physical activity and behavioural interventions for the treatment of overweight or obese children from the age of 6 to 11 years 


\section{Davis 2016b \{published data only\}}

Davis AM, Stough CO, Black WR, Dean K, Sampilo M, Simpson S, et al. Outcomes of a weight management program conjointly addressing parent and child health. Children's Health Care 2016;45:227-40.

\section{de Mello 2004 \{published data only\}}

de Mello ED, Luft VC, Meyer F. Individual outpatient care versus group education programs. Which leads to greater change in dietary and physical activity habits for obese children?. Jornal de Pediatria 2004;80:468-74.

Dennis 2013 \{published data only\}

Dennis BA, Ergul A, Gower BA, Allison JD, Davis CL. Oxidative stress and cardiovascular risk in overweight children in an exercise intervention program. Childhood Obesity 2013;9:15-21.

\section{De Ruyter 2013 \{published data only\}}

De Ruyter JC, Katan MB, Kuijper LDJ, Liem DG, Olthof MR. The effect of sugar-free versus sugar-sweetened beverages on satiety, liking and wanting: an 18 month randomized doubleblind trial in children. PLOS ONE 2013;8:e78039.

\section{DeVault 2009 \{published data only\}}

De Vault N, Kennedy T, Hermann J, Mwavita M, Rask P, Jaworsky A. It's all about kids: preventing overweight in elementary school children in Tulsa, OK. Journal of the American Dietetic Association 2009;109:680-7.

\section{Dias 2016 \{published data only\}}

Dias KA, Coombes JS, Green DJ, Gomersall SR, Keating SE, Tjonna AE, et al. Effects of exercise intensity and nutrition advice on myocardial function in obese children and adolescents: a multicentre randomised controlled trial study protocol. BMJ Open 2016;6:e010929.

\section{Dodds 2014 \{published data only\}}

Dodds P, Wolfenden L, Chapman K, Wellard L, Hughes C, Wiggers $\mathrm{J}$. The effect of energy and traffic light labelling on parent and child fast food selection: a randomised controlled trial. Appetite 2014;73:23-30.

\section{Donnelly 2009 \{published data only\}}

Donnelly JE, Greene JL, Gibson CA, Smith BK, Washburn RA, Sullivan DK, et al. Physical Activity Across the Curriculum (PAAC): a randomized controlled trial to promote physical activity and diminish overweight and obesity in elementary school children. Preventive Medicine 2009;49:336-41.

\section{Doyle-Baker 2011 \{published data only\}}

Doyle-Baker PK, Venner AA, Lyon ME, Fung T. Impact of a combined diet and progressive exercise intervention for overweight and obese children: the B.E. H.I.P. study. Applied Physiology, Nutrition, \& Metabolism 2011;36:515-25.

\section{Dreyer 2014 \{published data only\}}

Dreyer Gillette ML, Odar Stough C, Best CM, Beck AR, Hampl SE. Comparison of a condensed 12-week version and a 24-week version of a family-based pediatric weight management program. Childhood obesity 2014;10:375-82.
DuBose 2008 \{published data only\}

DuBose KD, Mayo MS, Gibson CA, Green JL, Hill JO, Jacobsen DJ, et al. Physical activity across the curriculum (PAAC): rationale and design. Contemporary Clinical Trials 2008;29:83-93.

\section{Duckworth 2009 \{published data only\}}

Duckworth LC, Gately PJ, Radley D, Cooke CB, King RF, Hill AJ. RCT of a high-protein diet on hunger motivation and weightloss in obese children: an extension and replication. Obesity 2009;17:1808-10.

\section{Duncan 2009 \{published data only\}}

Duncan MJ, Al-Nakeeb Y, Nevill AM. Effects of a 6-week circuit training intervention on body esteem and body mass index in British primary school children. Body Image 2009;6:216-20.

Dura 2006 \{published data only\}

Dura Trave T. Treatment of childhood obesity: nutrition education, self-management and intensive follow-up. Acta Pediatrica Espanola 2006;64:328-34.

\section{Economos 2007 \{published data only\}}

Economos CD, Hyatt RR, Goldberg JP, Must A, Naumova EN, Collins JJ, et al. A community intervention reduces $\mathrm{BMI}$ z-score in children: Shape Up Somerville first year results. Obesity 2007;15:1325-36.

\section{El Hage 2012 \{published data only\}}

El Hage R, Rocher E, Chappard C, Portier H, Rochefort GY, Benhamou CL. Effect of a 6-month physical training program on hip structure analysis in obese children. Osteoporosis International 2012;23:S686.

\section{Endevelt 2014 \{published data only\}}

Endevelt R, Elkayam O, Cohen R, Peled R, Tal-Pony L, Grunwald RM, et al. An intensive family intervention clinic for reducing childhood obesity. Journal of the American Board of Family Medicine: JABFM 2014;27:321-8.

\section{Epstein 1981 \{published data only\}}

Epstein LH, Wing RR, Koeske R, Andrasik F, Ossip DJ. Child and parent weight loss in family-based behavior modification programs. Journal of Consulting \& Clinical Psychology 1981;49:674-85.

\section{Epstein 1984b \{published data only\}}

Epstein LH, Woodall K, Goreczny AJ, Wing RR, Robertson RJ. The modification of activity patterns and energy expenditure in obese young girls. Behavior Therapy 1984;15:101-8.

Epstein 1986 \{published data only\}

Epstein LH, Wing RR, Koeske R, Valoski A. Effect of parent weight on weight loss in obese children. Journal of Consulting and Clinical Psychology 1986;54:400-1.

\section{Epstein 1987a \{published data only\}}

Epstein LH, Nudelman S, Wing RR. Long-term effects of familybased treatment for obesity on nontreated family members. Behavior Therapy 1987;18:147-52.

Diet, physical activity and behavioural interventions for the treatment of overweight or obese children from the age of 6 to 11 years 
Epstein 1987b \{published data only\}

Epstein LH, Wing RR, Koeske R, Valoski A. Long-term effects of family-based treatment of childhood obesity. Journal of Consulting and Clinical Psychology 1987;55:91-5.

\section{Epstein 1987c \{published data only\}}

Epstein LH, Wing RR, Valoski A, Gooding W. Long-term effects of parent weight on child weight loss. Behavior Therapy 1987;18:219-26.

\section{Epstein 1987d \{published data only\}}

Epstein LH, Wing RR, Valoski A, Penner BC. Stability of food preferences during weight control: a study with 8- to 12 year-old children and their parents. Behavior Modification 1987;11:87-101.

\section{Epstein 1990 \{published data only\}}

Epstein LH, Valoski A, Wing RR, McCurley J. Ten-year follow-up of behavioral, family-based treatment for obese children. JAMA 1990;264:2519-23.

\section{Epstein 1993 \{published data only\}}

Epstein LH, Valoski A, McCurley J. Effect of weight loss by obese children on long-term growth. American Journal of Diseases of Children 1993;147:1076-80.

\section{Epstein 1994a \{published data only\}}

Epstein LH, McKenzie SJ, Valoski A, Klein KR, Wing RR. Effects of mastery criteria and contingent reinforcement for family-based child weight control. Addictive Behaviors 1994;19:135-45.

\section{Epstein 1994b \{published data only\}}

Epstein LH, Valoski A, Wing RR, McCurley J. Ten-year outcomes of behavioral family-based treatment for childhood obesity. Health Psychology 1994;13(5):373-83.

\section{Epstein 1995 \{published data only\}}

Epstein LH, Valoski AM, Vara LS, McCurley J, Wisniewski L, Kalarchian MA, et al. Effects of decreasing sedentary behavior and increasing activity on weight change in obese children. Health Psychology 1995;14:109-15.

Epstein 2000b \{published data only\}

Epstein LH, Paluch RA, Gordy CC, Saelens BE, Ernst MM. Problem solving in the treatment of childhood obesity. Journal of Consulting \& Clinical Psychology 2000;68:717-21.

\section{Epstein 2004 \{published data only\}}

Epstein LH, Paluch RA, Kilanowski CK, Raynor HA. The effect of reinforcement or stimulus control to reduce sedentary behavior in the treatment of pediatric obesity. Health Psychology 2004;23:371-80.

\section{Epstein 2007 \{published data only\}}

Epstein LH, Beecher MD, Graf JL, Roemmich JN. Choice of interactive dance and bicycle games in overweight and nonoverweight youth. Annals of Behavioral Medicine 2007;33:124-31.

\section{Epstein 2008a \{published data only\}}

Epstein LH, Paluch RA, Beecher MD, Roemmich JN. Increasing healthy eating vs. reducing high energy-dense foods to treat pediatric obesity. Obesity 2008;16:318-26.

Epstein 2008b \{published data only\}

Epstein LH, Roemmich JN, Robinson JL, Paluch RA, Winiewicz DD, Fuerch JH, et al. A randomized trial of the effects of reducing television viewing and computer use on body mass index in young children. Archives of Pediatrics \& Adolescent Medicine 2008;162:239-45.

\section{Epstein 2012 \{published data only\}}

Epstein LH, Raja S, Daniel TO, Paluch RA, Wilfley DE, Saelens BE, et al. The built environment moderates effects of family-based childhood obesity treatment over 2 years. Annals of Behavioral Medicine 2012;44:248-58.

\section{Erceg 2012 \{published data only\}}

Erceg DNJ. The effects of whole body vibration exercise on metabolic systems in overweight Latino boys. Dissertation Abstracts International: Section B: The Sciences and Engineering. 2012; Vol. 73:274.

\section{Escobedo 2014 \{published data only\}}

Escobedo S, Garca O, Ronquillo D, Caamao M, Rosado J. Supplementation with multiple micronutrients and polyunsaturated fatty acids does not improve school performance of obese children in a rural area of Mexico. FASEB Journal 2014;1:811.23.

\section{Escoto 2008 \{published data only\}}

Escoto Ponce de Leon MC, Mancilla Diaz JM, Camacho Ruiz EJ. A pilot study of the clinical and statistical significance of a program to reduce eating disorder risk factors in children. Eating and Weight Disorders 2008;13:111-8.

\section{Esfarjani 2013 \{published data only\}}

Esfarjani F, Khalafi M, Mohammadi F, Mansour A, Roustaee R, Zamani-Nour N, et al. Family-based intervention for controlling childhood obesity: an experience among Iranian children. International Journal of Preventive Medicine 2013;4:358-65.

\section{Estabrooks 2009 \{published data only\}}

Estabrooks PA, Shoup JA, Gattshall M, Dandamudi P, Shetterly S, $\mathrm{Xu}$ S. Automated telephone counseling for parents of overweight children: a randomized controlled trial. American Journal of Preventive Medicine 2009;36:35-42.

\section{Falbe 2015 \{published data only\}}

Falbe J, Cadiz AA, Tantoco NK, Thompson HR, Madsen KA. Active and healthy families: a randomized controlled trial of a culturally tailored obesity intervention for Latino children. Academic Pediatrics 2015;15:386-95.

\section{Farpour-Lambert 2009 \{published data only\}}

Farpour-Lambert NJ, Aggoun Y, Marchand LM, Martin XE, Herrmann FR, Beghetti M. Physical activity reduces systemic blood pressure and improves early markers of atherosclerosis in pre-pubertal obese children. Journal of the American College of Cardiology 2009;54:2396-406.

Diet, physical activity and behavioural interventions for the treatment of overweight or obese children from the age of 6 to 11 years 


\section{Ferguson 1999a \{published data only\}}

Ferguson MA, Gutin B, Le NA, Karp W, Litaker M, Humphries M, et al. Effects of exercise training and its cessation on components of the insulin resistance syndrome in obese children. International Journal of Obesity \& Related Metabolic Disorders: Journal of the International Association for the Study of Obesity 1999;23:889-95.

\section{Ferguson 1999b \{published data only\}}

Ferguson MA, Gutin B, Owens S, Barbeau P, Tracy RP, Litaker M. Effects of physical training and its cessation on the hemostatic system of obese children. American Journal of Clinical Nutrition 1999;69:1130-4.

\section{Ferrara 2013 \{published data only\}}

Ferrara P, Del Bufalo F, Ianniello F, Franceschini A, Paolini Paoletti F, Massart F, et al. Diet and physical activity "defeated" Tuberil in treatment of childhood obesity. Minerva Endocrinologica 2013;38:181-5.

\section{Ferrer 1998 \{published data only\}}

Ferrer Gonzalez P, Belda Galiana I, Segarra Aznar FM, Fenollosa Entrena B, Dalmau Serra J. Lipid and anthropometric parameter evolution during the treatment of prepubertal obese patients. Anales Espanoles de Pediatria 1998;48:267-73.

\section{Figueroa-Colon 1993 \{published data only\}}

Figueroa-Colon R, von Almen TK, Franklin FA, Schuftan C, Suskind RM. Comparison of two hypocaloric diets in obese children. American Journal of Diseases of Children 1993;147:160-6.

\section{Figueroa-Colon 1996 \{published data only\}}

Figueroa-Colon R, Franklin FA, Lee JY, von Almen TK, Suskind RM. Feasibility of a clinic-based hypocaloric dietary intervention implemented in a school setting for obese children. Obesity Research 1996;4:419-29.

\section{Firoozi 2013 \{published data only\}}

Firoozi M, Gheed Rahmat A. Virtual social network for management of obesity in children and adolescents. Iranian Journal of Pediatrics 2013;23:S9-S10.

\section{Fischer 2014 \{published data only\}}

Fischer M, Svensson V, Danielsson P, Marcus C. Effect of the omega-3 fatty acids EPA and DHA on physical activity among 8 -year old children, a randomized double blind pilot study. Obesity Reviews 2014;15:245

\section{Foger 1993 \{published data only\}}

Foger M, Bart G, Rathner G, Jager B, Fischer H, Zollner-Neussl D. Physical activity, nutritional counseling and psychological guidance in treatment of obese children. A controlled follow-up study over six months. Monatsschrift Kinderheilkunde. Organ der Deutschen Gesellschaft fur Kinderheilkunde 1993;141:491-7.

\section{Follansbee-Junger 2010 \{published data only\}}

Follansbee-Junger K, Janicke DM, Sallinen BJ. The influence of a behavioral weight management program on disordered eating attitudes and behaviors in children with overweight. Journal of the American Dietetic Association 2010:110:1653-9.

\section{Frohna 2008 \{published data only\}}

Frohna JG. Maintenance programs help sustain the initial weight loss in overweight children. Journal of Pediatrics 2008;152:443-4.

\section{Fullerton 2007a \{published data only\}}

Fullerton G, Tyler C, Johnston CA, Vincent JP, Harris GE, Foreyt JP. Quality of life in Mexican-American children following a weight management program. Obesity 2007;15:2553-6.

Fullerton 2007b \{published data only\}

Fullerton L. Quality of life in Mexican American children following a weight management program. Dissertation Abstracts International: Section B: The Sciences and Engineering. 2007; Vol. 67:4103.

\section{Furze 2008 \{published data only\}}

Furze JA, Black LL. Reducing obesity in youth: a new approach for at-risk, underserved children. Pediatric Physical Therapy 2008;20:105-6.

\section{Gajewska 2011 \{published data only\}}

Gajewska J, Weker H, Ambroszkiewicz J, Chelchowska M, Wiech M, Laskowska-Klita T. Changes in concentration of serum adiponectin multimeric forms following weight reduction programme in prepubertal obese children. Medycyna Wieku Rozwojowego 2011;15:298-305.

Galhardo 2012 \{published data only\}

Galhardo J, Hunt LP, Lightman SL, Sabin MA, Bergh C, Sodersten $\mathrm{P}$, et al. Normalizing eating behavior reduces body weight and improves gastrointestinal hormonal secretion in obese adolescents. Journal of Clinical Endocrinology \& Metabolism 2012;97:E193-201.

Garipagaoglu 2009 \{published data only\}

Garipagaoglu M, Sahip Y, Darendeliler F, Akdikmen O, Kopuz S, Sut N. Family-based group treatment versus individual treatment in the management of childhood obesity: randomized, prospective clinical trial. European Journal of Pediatrics 2009;168:1091-9.

\section{Gerards 2012 \{published data only\}}

Gerards SM, Dagnelie PC, Jansen MW, Van der Goot LO, De Vries NK, Sanders MR, et al. Lifestyle Triple P: a parenting intervention for childhood obesity. BMC Public Health 2012;12:267.

\section{Ghatrehsamani 2010 \{published data only\}}

Ghatrehsamani S, Khavarian N, Beizaei M, Ramedan R, Poursafa P, Kelishadi R. Effect of different physical activity training methods on overweight adolescents. Arya Atherosclerosis 2010;6:45-9.

\section{Goldfield 2000 \{published data only\}}

Goldfield GS, Kalakanis LE, Ernst MM, Epstein LH. Open-loop feedback to increase physical activity in obese children. International Journal of Obesity \& Related Metabolic Disorders: Journal of the International Association for the Study of Obesity 2000;24:888-92.

Diet, physical activity and behavioural interventions for the treatment of overweight or obese children from the age of 6 to 11 years 


\section{Goldfield 2001 \{published data only\}}

Goldfield GS, Epstein LH, Kilanowski CK, Paluch RA, KogutBossler B. Cost-effectiveness of group and mixed familybased treatment for childhood obesity. International Journal of Obesity \& Related Metabolic Disorders: Journal of the International Association for the Study of Obesity 2001;25:1843-9.

\section{Goldfield 2006 \{published data only\}}

Goldfield GS, Mallory R, Parker T, Cunningham T, Legg C, Lumb A, et al. Effects of open-loop feedback on physical activity and television viewing in overweight and obese children: a randomized, controlled trial. Pediatrics 2006;118:e157-66.

\section{Goldfield 2007 \{published data only\}}

Goldfield GS, Mallory R, Parker T, Cunningham T, Legg C, Lumb A, et al. Effects of modifying physical activity and sedentary behavior on psychosocial adjustment in overweight/ obese children. Journal of Pediatric Psychology 2007;32:783-93.

\section{Goldfield 2008 \{published data only\}}

Goldfield GS, Mallory R, Prud'homme D, Adamo KB. Gender differences in response to a physical activity intervention in overweight and obese children. Journal of Physical Activity \& Health 2008;5:592-606.

\section{Goldfield 2009 \{published data only\}}

Goldfield GS. Predictors of response to an intervention modifying physical activity and sedentary behavior in overweight/obese children: attitudes vs. behavior. Journal of Physical Activity \& Health 2009;6:463-6.

\section{Golley 2007 \{published data only\}}

Golley RK, Magarey AM, Baur LA, Steinbeck KS, Daniels LA. Twelve-month effectiveness of a parent-led, family-focused weight-management program for prepubertal children: a randomized, controlled trial. Pediatrics 2007;119:517-25.

\section{Golley 2011 \{published data only\}}

Golley RK, Magarey AM, Daniels LA. Children's food and activity patterns following a six-month child weight management program. International Journal of Pediatric Obesity 2011;6:409-14.

\section{Gong 2014 \{published data only\}}

Gong L, Yuan F, Teng J, Li X, Zheng S, Lin L, et al. Weight loss, inflammatory markers, and improvements of iron status in overweight and obese children. Journal of Pediatrics 2014;164:795-800.e2.

\section{Graf 2006 \{published data only\}}

Graf C, Koch B, Bjarnason-Wehrens B, Sreeram N, Brockmeier K, Tokarski W, et al. Who benefits from intervention in, as opposed to screening of, overweight and obese children?. Cardiology in the Young 2006;16:474-80.

\section{Graf 2008 \{published data only\}}

Graf C, Koch B, Falkowski G, Jouck S, Christ H, Staudenmaier K, et al. School-based prevention: effects on obesity and physical performance after 4 years. Journal of Sports Sciences 2008;26:987-94.

\section{Graham 2008 \{published data only\}}

Graham D, Appleton S, Rush E, McLennan S, Reed P, Simmons D. Increasing activity and improving nutrition through a schoolsbased programme: Project Energize. 1. Design, programme, randomisation and evaluation methodology. Public Health Nutrition 2008;11:1076-84.

\section{Graves 1988 \{published data only\}}

Graves T, Meyers AW, Clark L. An evaluation of parental problem-solving training in the behavioral treatment of childhood obesity. Journal of Consulting and Clinical Psychology 1988;56:246-50.

\section{Gregori 2014 \{published data only\}}

Gregori D, Vecchio MG, Nikolakis A, Galasso F. Even a very intense advertising promoting fruit consumption is not enough to have children eating more fruit: results from an experimental study in Italy. Obesity Facts 2014;7:176.

\section{Griffin 2013 \{published data only\}}

Griffin K, Meaney K, Hart M. The impact of a mastery motivational climate on obese and overweight children's commitment to and enjoyment of physical activity: a pilot study. American Journal of Health Education 2013;44:1-8.

\section{Grow 2014 \{published data only\}}

Grow HMG, Hencz P, Verbovski MJ, Gregerson L, Liu LL, Dossett $L$, et al. Partnering for success and sustainability in community-based child obesity intervention: seeking to help families ACT!. Family \& Community Health 2014;37:45-59.

\section{Guixeres 2009 \{published data only\}}

Guixeres J, Zaragoza I, Alcaniz M, Gomistena J, Cebolla A. A new protocol test for physical activity research in obese children (Etiobe Project). Annual Review of CyberTherapy and Telemedicine 2009;7:281-3.

\section{Gunnarsdottir 2011b \{published data only\}}

Gunnarsdottir T, Njardvik U, Olafsdottir AS, Craighead LW, Bjarnason R. The role of parental motivation in family-based treatment for childhood obesity. Obesity 2011;19:1654-62.

\section{Gunnarsdottir 2014 \{published data only\}}

Gunnarsdottir T, Einarsson SM, Njardvik U, Olafsdottir AS, Gunnarsdottir AB, Helgason T, et al. Family-based behavioral treatment for obese children - results and two year follow up. Laeknabladid 2014;100:139-45.

\section{Gunther 2007 \{published data only\}}

Gunther ALB, Buyken AE, Kroke A. Protein intake during the period of complementary feeding and early childhood and the association with body mass index and percentage body fat at 7 y of age. American Journal of Clinical Nutrition 2007;85:1626-33.

\section{Gussinyer 2008 \{published data only\}}

Gussinyer S, Garcia-Reyna NI, Carrascosa A, Gussinyer M, Yeste D, Clemente M, et al. Anthropometric, dietetic and psychological changes after application of the "Niños en movimiento" program in childhood obesity. Medicina Clinica 2008;131:245-9.

Diet, physical activity and behavioural interventions for the treatment of overweight or obese children from the age of 6 to 11 years 
Gutin 1996 \{published data only\}

Gutin B, Cucuzzo N, Islam S, Smith C, Stachura ME. Physical training, lifestyle education, and coronary risk factors in obese girls. Medicine \& Science in Sports \& Exercise 1996;28:19-23.

\section{Gutin 1999a \{published data only\}}

Gutin B, Owens S, Okuyama T, Riggs S, Ferguson M, Litaker M. Effect of physical training and its cessation on percent fat and bone density of children with obesity. Obesity Research 1999;7:208-14.

\section{Gutin 1999b \{published data only\}}

Gutin B, Ramsey L, Barbeau P, Cannady W, Ferguson M, Litaker M, et al. Plasma leptin concentrations in obese children: changes during 4-mo periods with and without physical training. American Journal of Clinical Nutrition 1999;69:388-94.

Gutin 2008 \{published data only\}

Gutin B, Yin Z, Johnson M, Barbeau P. Preliminary findings of the effect of a 3-year after-school physical activity intervention on fitness and body fat: the Medical College of Georgia Fitkid Project. International Journal of Pediatric Obesity 2008;3(Suppl 1):3-9.

\section{Habib-Mourad 2014a \{published data only\}}

Habib-Mourad C, Ghandour L, Moore H, Hwalla N, Summerbell C. An intervention to promote Healthy Eating and Physical Activity in Lebanese School children: Health-EPALS, a pilot cluster randomised controlled trial. Obesity Facts 2014;7:158-9.

\section{Habib-Mourad 2014b \{published data only\}}

Habib-Mourad C, Ghandour LA, Moore HJ, Nabhani-Zeidan M, Adetayo $\mathrm{K}$, Hwalla $\mathrm{N}$, et al. Promoting healthy eating and physical activity among school children: findings from HealthE-PALS, the first pilot intervention from Lebanon. BMC Public Health 2014;14:940.

\section{Habib-Mourad 2014c \{published data only\}}

Habib-Mourad C, Moore HJ, Nabhani-Zeidan M, Hwalla N, Summerbell C. Health-E-PALS: promoting Healthy Eating and Physical Activity in Lebanese school children - intervention development. Education \& Health 2014;32:3-8.

\section{Haemer 2013 \{published data only\}}

Haemer MA, Ranade D, Baron AE, Krebs NF. A clinical model of obesity treatment is more effective in preschoolers and Spanish speaking families. Obesity 2013;21:1004-12.

\section{Hager 2016 \{published data only\}}

Hager ER, Turner L. Successes of the Healthy Hunger-Free Kids Act. JAMA Pediatrics 2016;170:1-2.

\section{Hajihashemi 2014 \{published data only\}}

Hajihashemi P, Azadbakht L, Hashemipor M, Kelishadi R, Esmaillzadeh A. Whole-grain intake favorably affects markers of systemic inflammation in obese children: a randomized controlled crossover clinical trial. Molecular Nutrition \& Food Research 2014;58:1301-8.

\section{Hammarlund 1993 \{published data only\}}

Hammarlund VA. Development and evaluation of a weight control program for obese preadolescent children. Dissertation Abstracts International. 1993; Vol. 53:4596.

\section{Hansen 2013 \{published data only\}}

Hansen PR, Andersen LJ, Rebelo AN, Brito J, Hornstrup T, Schmidt JF, et al. Cardiovascular effects of 3 months of footbal training in overweight children examined by comprehensive echocardiography: a pilot study. Journal of Sports Sciences 2013;31:1432-40.

\section{Harder-Lauridsen 2014 \{published data only\}}

Harder-Lauridsen NM, Birk NM, Ried-Larsen M, Juul A, Andersen LB, Pedersen BK, et al. A randomized controlled trial on a multicomponent intervention for overweight school-aged children - Copenhagen, Denmark. BMC Pediatrics 2014;14:273.

\section{Hardman 2009 \{published data only\}}

Hardman CA, Horne PJ, Lowe CF. A home-based intervention to increase physical activity in girls: the Fit ' $n$ ' Fun Dudes program. Journal of Exercise Science \& Fitness 2009;7:1-8.

\section{Harrell 1998 \{published data only\}}

Harrell JS, Gansky SA, McMurray RG, Bangdiwala SI, Frauman AC, Bradley CB. School-based interventions improve heart health in children with multiple cardiovascular disease risk factors. Pediatrics 1998;102:371-80.

\section{Harrison 2006 \{published data only\}}

Harrison M, Burns CF, McGuinness M, Heslin J, Murphy NM. Influence of a health education intervention on physical activity and screen time in primary school children: 'Switch Off--Get Active'. Journal of Science \& Medicine in Sport 2006;9:388-94.

\section{Hartlieb 2015 \{published data only\}}

Hartlieb KB, Jacques-Tiura AJ, Naar-King S, Ellis DA, Jen KC, Marshall S. Recruitment strategies and the retention of obese urban racial/ethnic minority adolescents in clinical trials: the FIT Families Project, Michigan, 2010-2014. Preventing Chronic Disease: Public Health Research, Practice, and Policy 2015;12:1-10.

\section{Hashemipour 2009 \{published data only\}}

Hashemipour M, Kelishadi R, Shapouri J. Effect of zinc supplementation on insulin resistance and components of the metabolic syndrome in prepubescent obese children. Pediatric Diabetes 2009;10:94.

\section{Haszard 2015 \{published data only\}}

Haszard JJ, Skidmore PM, Williams SM, Taylor RW. Associations between parental feeding practices, problem food behaviours and dietary intake in New Zealand overweight children aged 4-8 years. Public Health Nutrition 2015;18:1036-43.

\section{Heuser 2008 \{published data only\}}

Heuser L, Herbig S. The childhood obesity challenge--Tap into Fitness: program overview and results analysis. The Journal of the Kentucky Medical Association 2008;106:118-22. 
Hollinghurst 2014 \{published data only\}

Hollinghurst S, Hunt LP, Banks J, Sharp DJ, Shield JP. Cost and effectiveness of treatment options for childhood obesity. Pediatric Obesity 2014;9:e26-e34.

\section{Holmes 2008 \{published data only\}}

Holmes T. Using reality therapy to influence health behaviors: childhood obesity. International Journal of Reality Therapy 2008;28:78-80.

\section{Horsak 2015 \{published data only\}}

Horsak B, Artner D, Baca A, Pobatschnig B, Greber-Platzer S, Nehrer $S$, et al. The effects of a strength and neuromuscular exercise programme for the lower extremity on knee load, pain and function in obese children and adolescents: study protocol for a randomised controlled trial. Trials 2015;16:586.

\section{Horton 2013 \{published data only\}}

Horton LA, Parada H, Slymen DJ, Arredondo E, Ibarra L, Ayala GX. Targeting children's dietary behaviors in a family intervention: 'Entre familia: reflejos de salud'. Salud Publica de Mexico 2013;55:397-405.

\section{Huang 2007 \{published data only\}}

Huang SH, Weng KP, Hsieh KS, Ou SF, Lin CC, Chien KJ, et al. Effects of a classroom-based weight-control intervention on cardiovascular disease in elementary-school obese children. Acta Paediatrica Taiwanica 2007;48:201-6.

\section{Huang 2015a \{published data only\}}

Huang T, Larsen KT, Jepsen JR, Moller NC, Thorsen AK, Mortensen EL, et al. Effects of an obesity intervention program on cognitive function in children: a randomized controlled trial. Obesity 2015;23:2101-8.

\section{Huang 2015b \{published data only\}}

Huang T, Larsen KT, Moller NC, Ried-Larsen M, Frandsen U, Andersen LB. Effects of a multi-component camp-based intervention on inflammatory markers and adipokines in children: a randomized controlled trial. Preventive Medicine: An International Journal Devoted to Practice and Theory 2015;81:367-72.

\section{Hystad 2013 \{published data only\}}

Hystad HT, Steinsbekk S, Odegard R, Wichstrom L, Gudbrandsen $\mathrm{OA}$. A randomised study on the effectiveness of therapist-led v. self-help parental intervention for treating childhood obesity. British Journal of Nutrition 2013;110:1143-50.

\section{Iannuzzi 2009 \{published data only\}}

Iannuzzi A, Licenziati MR, Vacca M, De Marco D, Cinquegrana G, Laccetti M, et al. Comparison of two diets of varying glycemic index on carotid subclinical atherosclerosis in obese children. Heart \& Vessels 2009;24:419-24.

\section{Ibarra-Reynoso 2015 \{published data only\}}

Ibarra-Reynoso Ldel R, Pisarchyk L, Perez-Luque EL, GaraySevilla ME, Malacara JM. Dietary restriction in obese children and its relation with eating behavior, fibroblast growth factor 21 and leptin: a prospective clinical intervention study. Nutrition \& Metabolism 2015;12:31.

\section{Ildiko 2007 \{published data only\}}

Ildiko V, Zsofia M, Janos M, Andreas P, Dora NE, Andras P, et al. Activity-related changes of body fat and motor performance in obese seven-year-old boys. Journal of Physiological Anthropology 2007;26:333-7.

\section{Innes-Hughes 2016 \{published data only\}}

Innes-Hughes C, Khanal S, Lukeis S, Rissel C. Go4Fun: an effective Australian community based obesity treatment program for children. Obesity Reviews 2016;17:124.

\section{Israel 1984 \{published data only\}}

Israel AC, Stolmaker L, Sharp JP, Silverman WK, Simon LG. An evaluation of two methods of parental involvement in treating obese children. Behavior Therapy 1984;15:266-72.

Israel 1985 \{published data only\}

Israel AC, Stolmaker L, Andrian CAG. The effects of training parents in general child management skills on a behavioral weight loss program for children. Behavior Therapy 1985; Vol. 16:169-80.

\section{Israel 1994 \{published data only\}}

Israel AC, Guile CA, Baker JE, Silverman WK. An evaluation of enhanced self-regulation training in the treatment of childhood obesity. Journal of Pediatric Psychology 1994;19:737-49.

\section{Jacobson 2009 \{published data only\}}

Jacobson D. A primary care school age Healthy Choices Intervention program [Dissertation]. Arizona: Arizona State University, 2009

\section{Jago 2013 \{published data only\}}

Jago R, Drews KL, McMurray RG, Baranowski T, Galassetti P, Foster GD, et al. BMI change, fitness change and cardiometabolic risk factors among 8th grade youth. Pediatric Exercise Science 2013;25:52-68.

James 2000 \{published data only\} James KS. A school based intervention to reduce television use decreased adiposity in children in grades 3 and 4. Evidence Based Nursing 2000;3:43.

\section{Janicke 2008a \{published data only\}} Janicke DM, Sallinen BJ, Perri MG, Lutes LD, Huerta M, Silverstein $\mathrm{JH}$, et al. Comparison of parent-only vs family-based interventions for overweight children in underserved rural settings: outcomes from project STORY. Archives of Pediatrics \& Adolescent Medicine 2008;162:1119-25.

\section{Janicke 2008b \{published data only\}}

Janicke DM, Sallinen BJ, Perri MG, Lutes LD, Silverstein JH, Huerta MG, et al. Sensible treatment of obesity in rural youth (STORY): design and methods. Contemporary Clinical Trials 2008;29:270-80.

\section{Janicke 2009 \{published data only\}}

Janicke DM, Sallinen BJ, Perri MG, Lutes LD, Silverstein JH, Brumback B. Comparison of program costs for parent-only and family-based interventions for pediatric obesity in

Diet, physical activity and behavioural interventions for the treatment of overweight or obese children from the age of 6 to 11 years 
medically underserved rural settings. Journal of Rural Health 2009;25:326-30.

\section{Janicke 2011 \{published data only\}}

Janicke DM, Gray WN, Mathews AE, Simon SL, Lim CS, DumontDriscoll $\mathrm{M}$, et al. A pilot study examining a group-based behavioral family intervention for obese children enrolled in Medicaid: differential outcomes by race. Children's Health Care 2011;40:212-31.

\section{Janicke 2013 \{published data only\}}

Janicke DM, Lim CS, Mathews AE, Shelnutt KP, Boggs SR, Silverstein JH, et al. The community-based healthy-lifestyle intervention for rural preschools (CHIRP) study: design and methods. Contemporary Clinical Trials 2013;34:187-95.

Jansen 2011 \{published data only\}

Jansen E, Mulkens S, Jansen A. Tackling childhood overweight: treating parents exclusively is effective. International Journal of Obesity 2011;35:501-9.

\section{Jensen 2012 \{published data only\}}

Jensen CD, Aylward BS, Steele RG. Predictors of attendance in a practical clinical trial of two pediatric weight management interventions. Obesity 2012;20:2250-6.

\section{Jensen 2013 \{published data only\}}

Jensen ME, Gibson PG, Collins CE, Hilton JM, Wood LG. Diet-induced weight loss in obese children with asthma: a randomized controlled trial. Clinical \& Experimental Allergy 2013;43:775-84.

\section{Jensen 2015 \{published data only\}}

Jensen BW, von Kappelgaard LM, Nielsen BM, Husby I, Bugge A, El-Naaman B, et al. Intervention effects on dietary intake among children by maternal education level: results of the Copenhagen School Child Intervention Study (CoSCIS). British Journal of Nutrition 2015;113:963-74.

\section{Jernigan 2015 \{published data only\}}

Jernigan MM, Rosenthal L, Carroll-Scott A, Peters SM, McCaslin C, Ickovics JR. Emotional health predicts changes in body mass index (BMI-z) among black and Latino youth. Clinical Pediatrics 2015;54:693-96.

\section{John 2009 \{published data only\}}

John R. Effects of parent-focused media interventions on body mass index, waist size, self-perception, family eating habits, and family activity habits in overweight Hispanic children [Dissertation]. Columbia: Teachers College, Columbia University, 2009.

\section{Johnston 2013 \{published data only\}}

Johnston CA, Moreno J, El-Mubasher A, Papaioannou MA, Woehler D. Inclusion of peers in a school-based obesity intervention. FASEB Journal 2013;27:1063.22.

Jones 2015a \{published data only\}

Jones RA, Kelly J, Cliff DP, Batterham M, Okely AD. Acceptability and potential efficacy of single-sex after-school activity programs for overweight and at-risk children: the Wollongong SPORT RCT. Pediatric Exercise Science 2015;27:535-45.

Jurg 2006 \{published data only\}

Jurg ME, Kremers SP, Candel MJ, Van der Wal MF, De Meij JS. A controlled trial of a school-based environmental intervention to improve physical activity in Dutch children: JUMP-in, kids in motion. Health Promotion International 2006;21:320-30.

Kain 2009 \{published data only\}

Kain J, Leyton B, Cerda R, Vio F, Uauy R. Two-year controlled effectiveness trial of a school-based intervention to prevent obesity in Chilean children. Public Health Nutrition 2009;12:1451-61.

Kalarchian 2013 \{published data only\}

Kalarchian MA, Levine MD, Marcus MD. Structured dietary interventions in the treatment of severe pediatric obesity: a pilot study. Bariatric Surgical Practice and Patient Care 2013;8:58-60.

\section{Kang 2008 \{published data only\}}

Kang HS, Ryu MH, Park S. The effects of a weight loss program focusing on maternal education on childhood obesity. Asian Nursing Research 2008;2:150-8.

\section{Karacabey 2009 \{published data only\}}

Karacabey K. The effect of exercise on leptin, insulin, cortisol and lipid profiles in obese children. Journal of International Medical Research 2009;37:1472-8.

\section{Kelishadi 2008 \{published data only\}}

Kelishadi R, Hashemipour M, Mohammadifard N, Alikhassy H, Adeli K. Short- and long-term relationships of serum ghrelin with changes in body composition and the metabolic syndrome in prepubescent obese children following two different weight loss programmes. Clinical Endocrinology 2008;69:721-29.

\section{Kelishadi 2009 \{published data only\}}

Kelishadi R, Zemel MB, Hashemipour M, Hosseini M, Mohammadifard N, Poursafa P. Can a dairy-rich diet be effective in long-term weight control of young children?. Journal of the American College of Nutrition 2009;28:601-10.

Kelishadi 2010 \{published data only\}

Kelishadi R, Zemel M. Three-year study on the effects of a trial of a dairy-rich diet on body composition, insulin resistance, and components of the metabolic syndrome among obese prepubescent children. Circulation 2010;122(2):e188-9.

Kerr 2000 \{published data only\}

Kerr CM. A school based, interdisciplinary curriculum in grades 6 and 7 reduced obesity in girls. Evidence Based Nursing 2000;3:13.

\section{Khadilkar 2012 \{published data only\}}

Khadilkar VV, Pandit DS, Khadilkar AV, Chiplonkar SA, Kinare AS. Diet and exercise intervention, with special reference to micronutrients, reduces cardiometabolic risk in overweight children. Indian Journal of Endocrinology and Metabolism 2012;16:124-33.

Diet, physical activity and behavioural interventions for the treatment of overweight or obese children from the age of 6 to 11 years 
Kim 2016 \{published data only\}

Kim HS, Park J, Park KY, Lee MN, Ham OK. Parent involvement intervention in developing weight management skills for both parents and overweight/obese children. Asian Nursing Research 2016;10:11-7.

\section{Kipping 2008 \{published data only\}}

Kipping RR, Payne C, Lawlor DA. Randomised controlled trial adapting US school obesity prevention to England. Archives of Disease in Childhood 2008;93:469-73.

\section{Kirschenbaum 1984 \{published data only\}}

Kirschenbaum DS, Harris ES, Tomarken AJ. Effects of parental involvement in behavioral weight loss therapy for preadolescents. Behavior Therapy 1984;15:485-500.

\section{Klesges 2008 \{published data only\}}

Klesges RC, Obarzanek E, Klesges LM, Stockton MB, Beech BM, Murray DM, et al. Memphis Girls health Enrichment Multi-site Studies (GEMS): Phase 2: design and baseline. Contemporary Clinical Trials 2008;29:42-55.

\section{Klitzman 2015 \{published data only\}}

Klitzman P, Armstrong B, Janicke DM. Distance as a predictor of treatment attendance in a family based pediatric weight management program in rural areas. Journal of Rural Health 2015;31:19-26.

\section{Kohno 1994 \{published data only\}}

Kohno H, Tanaka H, Honda S. Therapeutic assessment of childhood obesity with body composition measured by bioelectrical impedance analysis. Fukuoka igaku zasshi = Hukuoka acta medica 1994;85:267-70.

\section{Kokkvoll 2014 \{published data only\}}

Kokkvoll A, Grimsgaard S, Odegaard R, Flaegstad T, Njolstad I. Single versus multiple-family intervention in childhood overweight--Finnmark Activity School: a randomised trial. Archives of Disease in Childhood 2014;99:225-31.

\section{Kolko 2010 \{published data only\}}

Kolko RP, Teim KR, Stein RI, Saelens BE, Perri MG, Schechtman KB, et al. Self-and parent-reported psychosocial symptoms in overweight children. Obesity 2010;18:S116.

\section{Krafft 2014a \{published data only\}}

Krafft CE, Pierce JE, Schwarz NF, Chi L, Weinberger AL, Schaeffer DJ, et al. An eight month randomized controlled exercise intervention alters resting state synchrony in overweight children. Neuroscience 2014;256:445-55.

\section{Krafft 2014b \{published data only\}}

Krafft CE, Schaeffer DJ, Schwarz NF, Chi L, Weinberger AL, Pierce JE, et al. Improved frontoparietal white matter integrity in overweight children is associated with attendance at an after-school exercise program. Developmental Neuroscience 2014;36:1-9.

\section{Krafft 2014c \{published data only\}}

Krafft CE, Schwarz NF, Chi L, Weinberger AL, Schaeffer DJ, Pierce JE, et al. An 8-month randomized controlled exercise trial alters brain activation during cognitive tasks in overweight children. Obesity 2014;22:232-42.

Kriemler 2010 \{published data only\}

Kriemler S, Zahner L, Schindler C, Meyer U, Hartmann T, Hebestreit $\mathrm{H}$, et al. Effect of school based physical activity programme (KISS) on fitness and adiposity in primary schoolchildren: cluster randomised controlled trial. BMJ 2010;340:c785.

\section{Kuni 2015 \{published data only\}}

Kuni B, Ruhling NE, Hegar U, Roth C, Schmitt H. Ball games and nutrition counseling improve postural control in overweight children. BMC Pediatrics 2015;15:205.

\section{Larsen 2010 \{published data only\}}

Larsen TM, Dalskov S, Van Baak M, Jebb S, Kafatos A, Pfeiffer A, et al. The Diet, Obesity and Genes (Diogenes) Dietary Study in eight European countries - a comprehensive design for longterm intervention. Obesity Reviews 2010;11:76-91.

\section{Larsen 2016 \{published data only\}}

Larsen KT, Huang T, Ried-Larsen M, Andersen LB, Heidemann M, Moller NC. A multi-component day-camp weight-loss program is effective in reducing BMI in children after one year: a randomized controlled trial. PLOS ONE 2016;11:e0157182.

\section{Lau 2015 \{published data only\}}

Lau PW, Wong DP, Ngo JK, Liang Y, Kim CG, Kim HS. Effects of high-intensity intermittent running exercise in overweight children. European Journal of Sport Science EJSS: Official Journal of the European College of Sport Science 2015;15:182-90.

\section{Leach 2008 \{published data only\}}

Leach RA, Yates JM. Nutrition and youth soccer for childhood overweight: a pilot novel chiropractic health education intervention. Journal of Manipulative \& Physiological Therapeutics 2008;31:434-41.

\section{Li 2010 \{published data only\}}

Li Y, Hu X, Zhang Q, Liu A, Fang H, Hao L, et al. The nutritionbased comprehensive intervention study on childhood obesity in China (NISCOC): a randomised cluster controlled trial. BMC Public Health 2010;10:229.

\section{Looney 2012 \{published data only\}}

Looney SM, Raynor HA. Are changes in consumption of "healthy" foods related to changes in consumption of "unhealthy" foods during pediatric obesity treatment? International Journal of Environmental Research \& Public Health 2012;9:1368-78.

\section{Lopes 2009 \{published data only\}}

Lopes L, Lopes V, Pereira B. Physical activity levels in normal weight and overweight Portuguese children: an intervention study during an elementary school recess. International Electronic Journal of Health Education 2009;12:175-84.

\section{Loughrey 2009 \{published data only\}}

Loughrey A. Tackling childhood obesity: the whole-school approach. British Journal of School Nursing 2009;4:147-8.

Diet, physical activity and behavioural interventions for the treatment of overweight or obese children from the age of 6 to 11 years 
Luley 2010 \{published data only\}

Luley C, Blaik A, Aronica S, Dierkes J, Kropf S, Westphal S. Evaluation of three new strategies to fight obesity in families. Journal of Nutrition and Metabolism 2010;2010:1-10.

\section{Madsen 2013 \{published data only\}}

Madsen K, Thompson H, Adkins A, Crawford Y. Schoolcommunity partnerships: a cluster-randomized trial of an afterschool soccer program. JAMA Pediatrics 2013;167:321-6.

\section{Makkes 2011 \{published data only\}}

Makkes S, Halberstadt J, Renders CM, Bosmans JE, Van der Baan-Slootweg OH, Seidell JC. Cost-effectiveness of intensive inpatient treatments for severely obese children and adolescents in the Netherlands; a randomized controlled trial (HELIOS). BMC Public Health 2011;11:518.

\section{Maloney 2012 \{published data only\}}

Maloney AE, Threlkeld KA, Cook WL. Comparative effectiveness of a 12-week physical activity intervention for overweight and obese youth: exergaming with "Dance Dance Revolution". Games for Health Journal 2012;1:96-103.

\section{Manchester 1978 \{published data only\}}

Manchester CF. A study of the effects of three weight counseling techniques and one nutrition education technique on the weight, skinfold measures, and self concepts of black, urban, obese elementary school children. Dissertation Abstracts International. 1978; Vol. 38:6539-40.

\section{Marcus 2009 \{published data only\}}

Marcus C, Nyberg G, Nordenfelt A, Karpmyr M, Kowalski J, Ekelund U. A 4-year, cluster-randomized, controlled childhood obesity prevention study: STOPP. International Journal of Obesity 2009;33:408-17.

\section{Marild 2013 \{published data only\}}

Marild S, Gronowitz E, Forsell C, Dahlgren J, Friberg P. A controlled study of lifestyle treatment in primary care for children with obesity. Pediatric Obesity 2013;8:207-17.

\section{Maron 2014 \{published data only\}}

Maron L, Maeder M, Kirchhoff E, Ardelt-Gattinger E, L'Allemand D, Laimbacher J. Individual therapy equals group therapy in significantly improving mental and physical health in obese children. Swiss Medical Weekly 2014;144:20S.

\section{Martinez 2008 \{published data only\}}

Martinez Vizcaino V, Salcedo Aguilar F, Franquelo Gutierrez R, Solera Martinez M, Sanchez Lopez M, Serrano Martinez S, et al. Assessment of an after-school physical activity program to prevent obesity among 9- to 10-year-old children: a cluster randomized trial. International Journal of Obesity 2008;32:12-22.

\section{Matheson 2015 \{published data only\}}

Matheson BE, Camacho C, Peterson CB, Rhee KE, Rydell SA, Zucker NL, et al. The relationship between parent feeding styles and general parenting with loss of control eating in treatmentseeking overweight and obese children. International Journal of Eating Disorders 2015;7:1047-55.

\section{Mayurachat 2013 \{published data only\}}

Mayurachat K, Warunee F, Jutamas C, Patcharaporn A, Kennedy C. An Intervention Study of Changing Eating Behaviors and Reducing Weight in Thai Children Aged 10-12. Pacific Rim International Journal of Nursing Research 2013;17:317-28.

\section{Mazzeo 2008 \{published data only\}}

Mazzeo SE, Gow RW, Stern M, Gerke CK. Developing an intervention for parents of overweight children. International Journal of Child and Adolescent Health 2008;1:355-63.

\section{Mazzeo 2011 \{published data only\}}

Mazzeo SE, Kelly NR, Thornton L, Stern M, Gow RW, Evans RK, et al. Parent skills training to enhance weight loss in overweight children: evaluation of NOURISH. Obesity 2011;19:S106.

\section{Mazzeo 2014 \{published data only\}}

Mazzeo SE, Kelly NR, Stern M, Gow RW, Cotter EW, Thornton LM, et al. Parent skills training to enhance weight loss in overweight children: evaluation of NOURISH. Eating Behaviors 2014;15(2):225-9.

\section{McFarland 2014 \{published data only\}}

McFarland MA. Teens in Smyth County, Virginia get cooking with "Teen Cuisine". Journal of the Academy of Nutrition \& Dietetics 2014;114:A99.

\section{McGuigan 2009 \{published data only\}}

McGuigan MR, Tatasciore M, Newton RU, Pettigrew S. Eight weeks of resistance training can significantly alter body composition in children who are overweight or obese. Journal of Strength \& Conditioning Research 2009;23:80-5.

\section{Medrano 2015 \{published data only\}}

Medrano M, Maiz E, Maldonado-Martin S, Arenaza L, RodriguezVigil B, Ortega FB, et al. The effect of a multidisciplinary intervention program on hepatic adiposity in overweight-obese children: protocol of the EFIGRO study. Contemporary Clinical Trials 2015;45:346-55.

\section{Minossi 2014 \{published data only\}}

Minossi V, Cecchetto FH, Pellanda LC. Healthy habits education for overweight children impacts both children and caregivers: a randomized clinical trial. Global Heart 2014;1:e226-7.

\section{Minossi 2015 \{published data only\}}

Minossi V, Pellanda LC. The "Happy Heart" educational program for changes in health habits in children and their families: protocol for a randomized clinical trial. BMC Pediatrics 2015;15:19.

\section{Moens 2012 \{published data only\}}

Moens $\mathrm{E}$, Braet C. Training parents of overweight children in parenting skills: a 12-month evaluation. Behavioural \& Cognitive Psychotherapy 2012;40:1-18.

\section{Moreno 2015 \{published data only\}}

Moreno JP, Johnston CA, Hernandez DC, LeNoble J, Papaioannou MA, Foreyt JP. Impact of parental weight status on a school-based weight management programme designed for Mexican-American children. Pediatric Obesity 2015;5:5.

Diet, physical activity and behavioural interventions for the treatment of overweight or obese children from the age of 6 to 11 years 


\section{Morgan 2014 \{published data only\}}

Morgan AZ, Ulrich P, Simmons KP, Gropper SS, Connell LJ, Daniels MK, et al. Effectiveness of a multi-faceted, school-based health intervention program with 4th graders in Alabama. Children and Youth Services Review 2014;37:46-54.

\section{Mo-suwan 1998 \{published data only\}}

Mo-suwan L, Pongprapai S, Junjana C, Puetpaiboon A. Effects of a controlled trial of a school-based exercise program on the obesity indexes of preschool children. American Journal of Clinical Nutrition 1998;68:1006-11.

\section{Muckelbauer 2009a \{published data only\}}

Muckelbauer R, Libuda L, Clausen K, Reinehr T, Kersting M. A simple dietary intervention in the school setting decreased incidence of overweight in children. Obesity Facts 2009;2:282-5.

\section{Muckelbauer 2009b \{published data only\}}

Muckelbauer R, Libuda L, Clausen K, Toschke AM, Reinehr T, Kersting M. Promotion and provision of drinking water in schools for overweight prevention: randomized, controlled cluster trial. Pediatrics 2009;123:e661-7.

\section{Munsch 2008 \{published data only\}}

Munsch S, Roth B, Michael T, Meyer AH, Biedert E, Roth S, et al. Randomized controlled comparison of two cognitive behavioral therapies for obese children: mother versus mother-child cognitive behavioral therapy. Psychotherapy \& Psychosomatics 2008;77:235-46.

\section{Murphy 2009 \{published data only\}}

Murphy EC, Carson L, Neal W, Baylis C, Donley D, Yeater R. Effects of an exercise intervention using Dance Dance Revolution on endothelial function and other risk factors in overweight children. International Journal of Pediatric Obesity 2009;4:205-14.

\section{Mustila 2012 \{published data only\}}

Mustila T, Raitanen J, Keskinen P, Saari A, Luoto R. Lifestyle counseling during pregnancy and offspring weight development until four years of age: follow-up study of a controlled trial. Journal of Negative Results in Biomedicine 2012;11:11.

\section{Muth 2008 \{published data only\}}

Muth ND, Chatterjee A, Williams D, Cross A, Flower K. Making an IMPACT: effect of a school-based pilot intervention. North Carolina Medical Journal 2008;69:432-40.

\section{NCT00284557 \{published data only\}}

NCT00284557. A primary care behavioral approach for addressing childhood overweight. clinicaltrials.gov/ct2/show/ NCT00284557 (last accessed 10th October 2016).

\section{Nemet 2006 \{published data only\}}

Nemet D, Berger-Shemesh E, Wolach B, Eliakim A. A combined dietary-physical activity intervention affects bone strength in obese children and adolescents. International Journal of Sports Medicine 2006;27:666-71.

\section{Nemet 2013a \{published data only\}}

Nemet D, Geva D, Pantanowitz M, Igbaria N, Meckel Y, Eliakim A. Long term effects of a health promotion intervention in low socioeconomic Arab- Israeli kindergartens. BMC Pediatrics 2013;13:45

\section{Nemet 2013b \{published data only\}}

Nemet D, Oren S, Pantanowitz M, Eliakim A. Effects of a multidisciplinary childhood obesity treatment intervention on adipocytokines, inflammatory and growth mediators. Hormone Research in Pediatrics 2013;79:325-32.

\section{Nogueira 2014 \{published data only\}}

Nogueira RC, Weeks BK, Beck BR. An in-school exercise intervention to enhance bone and reduce fat in girls: the CAPO Kids trial. Bone 2014;68:92-9.

Nogueira 2015 \{published data only\} Nogueira RC, Weeks BK, Beck BR. Targeting bone and fat with novel exercise for peripubertal boys: The CAPO Kids trial. Pediatric Exercise Science 2015;27:128-39.

\section{Nowicka 2010 \{published data only\}}

Nowicka P, Price G, Suri V, Shaw M, Mercado J, Tamborlane WV, et al. Providing a cost-effective comprehensive weight management program for highly obese children - not a mission impossible. Obesity 2010;18:S148-9.

Nuutinen 1992 \{published data only\}

* Nuutinen 0 , Knip M. Predictors of weight reduction in obese children. European Journal of Clinical Nutrition 1992;46:785-94.

O'Malley 2011 \{published data only\}

O'Malley G. Aerobic exercise enhances executive function and academic achievement in sedentary, overweight children aged 7-11 years. Journal of Physiotherapy 2011;57:255.

\section{Okely 2010 \{published data only\}}

Okely AD, Collins CE, Morgan PJ, Jones RA, Warren JM, Cliff DP, et al. Multi-site randomized controlled trial of a childcentered physical activity program, a parent-centered dietarymodification program, or both in overweight children: the HIKCUPS study. Journal of Pediatrics 2010;157:388-94.e1.

\section{Oliveras 2013 \{published data only\}}

Oliveras Lopez MJ, Muros Molina J, Martin F, Lopez Garcia De La Serrana H. Influence of moderate-vigorous physical activity and extra virgin olive oil in children catalase activity. Annals of Nutrition and Metabolism 2013;63:616.

\section{Parente 2006 \{published data only\}}

Parente EB, Guazzelli I, Ribeiro MM, Silva AG, Halpern A, Villares SM. Obese children lipid profile: effects of hypocaloric diet and aerobic physical exercise. Arquivos Brasileiros de Endocrinologia e Metabologia 2006;50:499-504.

\section{Parillo 2012 \{published data only\}}

Parillo M, Licenziati MR, Vacca M, De Marco D, lannuzzi A. Metabolic changes after a hypocaloric, low-glycemic-index diet in obese children. Journal Of Endocrinological Investigation 2012;35:629-33.

Diet, physical activity and behavioural interventions for the treatment of overweight or obese children from the age of 6 to 11 years 


\section{Parra-Medina 2011 \{published data only\}}

Parra-Medina D, Mojica CM, Parma DL, Rubalcava L, Ramos Al. Preliminary results from an obesity management intervention in a rural pediatric practice: The NEST study. Obesity 2011;19:S110.

\section{Pedrosa 2011a \{published data only\}}

Pedrosa C, Oliveira BM, Albuquerque I, Simoes-Pereira C, Vazde-Almeida MD, Correia F. Markers of metabolic syndrome in obese children before and after 1-year lifestyle intervention program. European Journal of Nutrition 2011;50:391-400.

\section{Pedrosa 2011b \{published data only\}}

Pedrosa C, Oliveira BM, Albuquerque I, Simoes-Pereira C, Vazde-Almeida MD, Correia F. Metabolic syndrome, adipokines and ghrelin in overweight and obese schoolchildren: results of a 1 -year lifestyle intervention programme. European Journal of Pediatrics 2011;170:483-92.

\section{Perman 2008 \{published data only\}}

Perman JA, Young TL, Stines E, Hamon J, Turner LM, Rowe MG. A community-driven obesity prevention and intervention in an elementary school. The Journal of the Kentucky Medical Association 2008;106:104-8.

\section{Perry 1979 \{published data only\}}

Perry RP, LeBow MD, Buser MM. An exploration of obese observational learning in modifying selected eating responses of obese children. International Journal of Obesity 1979;3:193-9.

\section{Petty 2009 \{published data only\}}

Petty KH, Davis CL, Tkacz J, Young-Hyman D, Waller JL. Exercise effects on depressive symptoms and self-worth in overweight children: a randomized controlled trial. Journal of Pediatric Psychology 2009;34:929-39.

\section{Plachta-Danielzik 2007 \{published data only\}}

Plachta-Danielzik S, Pust S, Asbeck I, Czerwinski-Mast M, Langnase K, Fischer C, et al. Four-year follow-up of schoolbased intervention on overweight children: the KOPS study. Obesity 2007;15:3159-69.

\section{Plummer 2014 \{published data only\}}

Plummer MLC, Brown S. From cook to chef: a new perspective on child care. Journal of the Academy of Nutrition \& Dietetics 2014;114:A59.

\section{Polacsek 2009 \{published data only\}}

Polacsek M, Orr J, Letourneau L, Rogers V, Holmberg R, O'Rourke K, et al. Impact of a primary care intervention on physician practice and patient and family behavior: Keep ME Healthy-The Maine Youth Overweight Collaborative. Pediatrics 2009;123:258-66.

\section{Pontin 2004 \{published data only\}}

Pontin D. A school based programme to reduce carbonated drink consumption reduced obesity in children. Evidence Based Nursing 2004;7:105.

\section{Poulsen 2011 \{published data only\}}

Poulsen AA, Desha L, Ziviani J, Griffiths L, Heaslop A, Khan A, et al. Fundamental movement skills and self-concept of children who are overweight. International Journal of Pediatric Obesity 2011;6:e464-71.

\section{Prado 2009 \{published data only\}}

Prado DM, Silva AG, Trombetta IC, Ribeiro MM, Nicolau CM, Guazzelli IC, et al. Weight loss associated with exercise training restores ventilatory efficiency in obese children. International Journal of Sports Medicine 2009;30:821-6.

\section{Puder 2009 \{published data only\}}

Puder JJ, Zahner L, Schindler C, Meyer U, Hebestreit H, Rocca HBL, et al. A school-based physical activity program increases fitness and decreases adiposity and cardiovascular risk factors in primary school children: a cluster-randomized trial. Diabetologia 2009;52(S1):S330-1.

\section{Qu 2014 \{published data only\}}

Qu S. Evaluation on the effectiveness of intervention comprehensive program on child obesity, using Generalized Estimating Equation. Zhonghua Liu Xing Bing Xue Za Zhi = Zhonghua Liuxingbingxue Zazhi 2014;35:773-8.

\section{Racine 2010 \{published data only\}}

Racine NM, Watras AC, Carrel AL, Allen DB, Mcvean JJ, Clark RR, et al. Effect of conjugated linoleic acid on body fat accretion in overweight or obese children. The American Journal of Clinical Nutrition 2010;91:1157-64.

\section{Ramon-Krauel 2013 \{published data only\}}

Ramon-Krauel M, Salsberg SL, Ebbeling CB, Voss SD, Mulkern RV, Apura MM, et al. A low-glycemic-load versus low-fat diet in the treatment of fatty liver in obese children. Childhood Obesity 2013;9:252-60.

\section{Rank 2012 \{published data only\}}

Rank M, Siegrist M, Wilks DC, Haller B, Wolfarth B, Langhof H, et al. Long-term effects of an inpatient weight-loss program in obese children and the role of genetic predisposition-rationale and design of the LOGIC-trial. BMC Pediatrics 2012;12:30.

\section{Rausch 2013 \{published data only\}}

Rausch Herscovici C, Kovalskys I, De Gregorio MJ. Gender differences and a school-based obesity prevention program in Argentina: a randomized trial. Pan American Journal of Public Health 2013;34:75-82.

\section{Raynor 2002 \{published data only\}}

Raynor HA, Kilanowski CK, Esterlis I, Epstein LH. A costanalysis of adopting a healthful diet in a family-based obesity treatment program. Journal of the American Dietetic Association 2002;102:645-56.

\section{Raynor 2012 \{published data only\}}

Raynor HA, Osterholt KM, Hart CN, Jelalian E, Vivier P, Wing RR. Efficacy of U.S. paediatric obesity primary care guidelines: two randomized trials. Pediatric Obesity 2012;7:28-38.

Diet, physical activity and behavioural interventions for the treatment of overweight or obese children from the age of 6 to 11 years 
Reinehr 2006 \{published data only\}

Reinehr T, de Sousa G, Toschke AM, Andler W. Long-term followup of cardiovascular disease risk factors in children after an obesity intervention. American Journal of Clinical Nutrition 2006;84:490-6.

\section{Reinehr 2009 \{published data only\}}

Reinehr T, Kleber M, Toschke AM. Lifestyle intervention in obese children is associated with a decrease of the metabolic syndrome prevalence. Atherosclerosis 2009;207:174-80.

\section{Reinehr 2011 \{published data only\}}

Reinehr T. Child-centred physical activity programme and parent-centred dietary programme alone or combined lead to sustainable reductions in BMI in 5-10-year-olds: diet alone or diet plus activity programmes seemed most effective. Evidence Based Nursing 2011;14:12-13.

\section{Resaland 2014 \{published data only\}}

Resaland G. Key results and implications from the Sogndal school intervention study-a prospective controlled intervention study of two-year school-based 60 minutes daily physical activity on cardiovascular disease risk factors. Obesity Facts 2014;7:29.

\section{Resnick 2009 \{published data only\}}

Resnick EA, Bishop M, O'Connell A, Hugo B, Isern G, Timm A, et al. The CHEER study to reduce BMI in elementary school students: a school-based, parent-directed study in Framingham, Massachusetts. Journal of School Nursing 2009;25:361-72.

\section{Resnicow 2012 \{published data only\}}

Resnicow K, McMaster F, Woolford S, Slora E, Bocian A, Harris D, et al. Study design and baseline description of the BMI2 trial: reducing paediatric obesity in primary care practices. Pediatric Obesity 2012;7:3-15.

\section{Riddiford-Harland 2012 \{published data only\}}

Riddiford-Harland D, Steele J, Baur L, Cliff D, Okely A, Morgan P, et al. Effects of a physical activity program on plantar pressures in overweight and obese children: RCT findings at 12 months follow-up. Obesity Research and Clinical Practice 2012;6:76.

\section{Riddiford-Harland 2016 \{published data only\}}

Riddiford-Harland DL, Steele JR, Cliff DP, Okely AD, Morgan PJ, Baur LA. Does participation in a physical activity program impact upon the feet of overweight and obese children?. Journal of Science \& Medicine in Sport 2016;19:51-5.

\section{Riggs 2007 \{published data only\}}

Riggs NR, Kobayakawa Sakuma KL, Pentz MA. Preventing risk for obesity by promoting self-regulation and decision-making skills: pilot results from the PATHWAYS to health program (PATHWAYS). Evaluation Review 2007;31:287-310.

\section{Robertson 2012 \{published data only\}}

Robertson W, Thorogood M, Inglis N, Grainger C, StewartBrown S. Two-year follow-up of the 'Families for Health' programme for the treatment of childhood obesity. Child: Care, Health \& Development 2012;38:229-36.

\section{Robinson 1999 \{published data only\}}

Robinson TN. Reducing children's television viewing to prevent obesity: a randomized controlled trial. JAMA 1999;282:1561-7.

Rodearmel 2006 \{published data only\}

Rodearmel SJ, Wyatt HR, Barry MJ, Dong F, Pan D, Israel RG, et al. A family-based approach to preventing excessive weight gain. Obesity 2006;14:1392-401.

\section{Rohrer 2008 \{published data only\}}

Rohrer TR, Rizzo VF, Casar JJ, Muelbredt O, Sprengart S, Gortner L, et al. Changes in hepatic risk factors, metabolic variables, body composition, and physical fitness in obese children after a one-year weight loss program. Journal of Pediatric Endocrinology and Metabolism 2008;21:837-45.

Rooney 2005 \{published data only\}

Rooney BL, Gritt LR, Havens SJ, Mathiason MA, Clough EA. Growing healthy families: family use of pedometers to increase physical activity and slow the rate of obesity. WMJ 2005; 104:54-60.

\section{Rosado 2008 \{published data only\}}

Rosado JL, del R Arellano M, Montemayor K, Garcia OP, del C Caamano M. An increase of cereal intake as an approach to weight reduction in children is effective only when accompanied by nutrition education: a randomized controlled trial. Nutrition Journal 2008;7:28.

\section{Safavi 2013 \{published data only\}}

Safavi M, Farajian S, Kelishadi R, Mirlohi M, Hashemipour M. The effects of synbiotic supplementation on some cardio-metabolic risk factors in overweight and obese children: a randomized triple-masked controlled trial. International Journal of Food Sciences \& Nutrition 2013;64:687-93.

\section{Salcedo 2010 \{published data only\}}

Salcedo Aguilar F, Martinez-Vizcaino V, Sanchez Lopez M, Solera Martinez M, Franquelo Gutierrez R, Serrano Martinez S, et al. Impact of an after-school physical activity program on obesity in children. Journal of Pediatrics 2010;157:36-42.e3.

\section{Salehi-Abargouei 2014 \{published data only\}}

Salehi-Abargouei A, Asemi M, Mehrabani S, Feizi A, Safavi SM. Effect of low-fat milk consumption compared to apple juice and water on the energy intake among 10-12-year-old obese boys: a three-way cross-over clinical trial. International Journal of Preventive Medicine 2014;5:1405-11.

\section{Sallis 1993 \{published data only\}}

Sallis JF, McKenzie TL, Alcaraz JE, Kolody B, Hovell MF, Nader PR. Project SPARK. Effects of physical education on adiposity in children. Annals of the New York Academy of Sciences 1993;699:127-36

\section{Salmon 2008 \{published data only\}}

Salmon J, Ball K, Hume C, Booth M, Crawford D. Outcomes of a group-randomized trial to prevent excess weight gain, reduce screen behaviours and promote physical activity in 10year-old children: switch-play. International Journal of Obesity 2008:32:601-12.

Diet, physical activity and behavioural interventions for the treatment of overweight or obese children from the age of 6 to 11 years 
Sanchez-Gomez 2012 \{published data only\}

Sanchez-Gomez LM, Fernandez-Luque MJ, Ruiz-Diaz L, SanchezAlcalde R, Sierra-Garcia B, Mayayo-Vicente S, et al. A clusterrandomised clinical trial comparing two cardiovascular health education strategies in a child population: the Savinghearts project. BMC Public Health 2012;12:1024.

\section{Schaeffer 2014 \{published data only\}}

Schaeffer DJ, Krafft CE, Schwarz NF, Chi L, Rodrigue AL, Pierce JE, et al. An 8-month exercise intervention alters frontotemporal white matter integrity in overweight children. Psychophysiology 2014;51:728-33.

\section{Seabra 2014 \{published data only\}}

Seabra AC, Seabra AF, Brito J, Krustrup P, Hansen PR, Mota J, et al. Effects of a 5-month football program on perceived psychological status and body composition of overweight boys. Scandinavian Journal of Medicine \& Science in Sports 2014;24(Suppl 1):10-6.

\section{Senediak 1985 \{published data only\}}

Senediak C, Spence SH. Rapid versus gradual scheduling of therapeutic contact in a family based behavioural weight control programme for children. Behavioural Psychotherapy 1985;13:265-87.

\section{Sgro 2009 \{published data only\}}

Sgro M, McGuigan MR, Pettigrew S, Newton RU. The effect of duration of resistance training interventions in children who are overweight or obese. Journal of Strength and Conditioning Research 2009;23:1263-70.

\section{Shalitin 2009 \{published data only\}}

Shalitin S, Ashkenazi-Hoffnung L, Yackobovitch-Gavan M, Nagelberg N, Karni Y, Hershkovitz E, et al. Effects of a twelveweek randomized intervention of exercise and/or diet on weight loss and weight maintenance, and other metabolic parameters in obese preadolescent children. Hormone Research 2009;72:287-301.

\section{Sherman 1992 \{published data only\}}

Sherman JB, Alexander MA, Gomez D, Kim M, Marole P. Intervention program for obese school children. Journal of Community Health Nursing 1992;9:183-90.

\section{Slusser 2013 \{published data only\}}

Slusser WM, Sharif MZ, Toller Erausquin J, Kinsler JJ, Collin D, Prelip ML. Improving pverweight among at-risk minority youth: results of a pilot intervention in after-school programs. Journal of Health Care for the Poor \& Underserved 2013;24:12-24.

\section{Small 2014 \{published data only\}}

Small L, Bonds-McClain D, Melnyk B, Vaughan L, Gannon AM. The preliminary effects of a primary care-based randomized treatment trial with overweight and obese young children and their parents. Journal of Pediatric Health Care 2014;28:198-207.

\section{Sothern 2000a \{published data only\}}

Sothern MS, Loftin JM, Udall JN, Suskind RM, Ewing TL, Tang SC, et al. Safety, feasibility, and efficacy of a resistance training program in preadolescent obese children. American Journal of the Medical Sciences 2000;319:370-5.

Sothern 2000b \{published data only\}

Sothern MS, Udall JN Jr, Suskind RM, Vargas A, Blecker U. Weight loss and growth velocity in obese children after very low calorie diet, exercise, and behavior modification. Acta Paediatrica, International Journal of Paediatrics 2000;89:1036-43.

\section{Soto-Sanchez 2014 \{published data only\}}

Soto-Sanchez JP, Pavez Saldiviar NF, Bravo-Gatica JI, White Ortiz AR, Jaque Fernandez Fl, Vargas-Gyllen $\mathrm{Cl}$, et al. Pilot study about the effectivity of an intervention based on games in nutritional status and muscle strength on children. Nutricion Hospitalaria 2014;30:147-52.

\section{Speroni 2007 \{published data only\}}

Speroni KG, Earley C, Atherton M. Evaluating the effectiveness of the Kids Living Fit program: a comparative study. Journal of School Nursing 2007;23:329-36.

\section{Spriet 2014 \{published data only\}}

Spriet SW, Davis KL. Diet-induced weight loss in obese children with asthma: a randomized controlled trial. Pediatrics 2014;134(Suppl 3):S170.

\section{Steele 2012 \{published data only\}}

Steele RG, Aylward BS, Jensen CD, Cushing CC, Davis AM, Bovaird JA. Comparison of a family-based group intervention for youths with obesity to a brief individual family intervention: a practical clinical trial of positively fit. Journal of Pediatric Psychology 2012;37:53-63.

\section{Steele 2014 \{published data only\}}

Steele RG, Jensen CD, Gayes LA, Leibold HC. Medium is the message: moderate parental control of feeding correlates with improved weight outcome in a pediatric obesity intervention. Journal of Pediatric Psychology 2014;39:708-17.

\section{Stettler 2015 \{published data only\}}

Stettler N, Wrotniak BH, Hill DL, Kumanyika SK, Xanthopoulos MS, Nihtianova S, et al. Prevention of excess weight gain in paediatric primary care: beverages only or multiple lifestyle factors. The Smart Step Study, a clusterrandomized clinical trial. Pediatric Obesity 2015;10:267-74.

\section{Stevens 2003 \{published data only\}}

Stevens J, Story M, Ring K, Murray DM, Cornell CE, Gittelsohn J. The impact of the Pathways intervention on psychosocial variables related to diet and physical activity in American Indian schoolchildren. Preventive Medicine 2003;37:S70-9.

\section{Stewart 2009 \{published data only\}}

Stewart L, Reilly JJ, Hughes AR. Evidence-based behavioral treatment of obesity in children and adolescents. Child and Adolescent Psychiatric Clinics of North America 2009;18:189-98.

\section{Stone 2003 \{published data only\}}

Stone EJ, Norman JE, Davis SM, Stewart D, Clay TE, Caballero B, et al. Design, implementation, and quality control in the

Diet, physical activity and behavioural interventions for the treatment of overweight or obese children from the age of 6 to 11 years 
Pathways American-Indian multicenter trial. Preventive Medicine 2003;37:S13-23.

\section{St-Onge 2009 \{published data only\}}

St-Onge MP, Goree LL, Gower B. High-milk supplementation with healthy diet counseling does not affect weight loss but ameliorates insulin action compared with low-milk supplementation in overweight children. Journal of Nutrition 2009;139:933-8.

\section{Stovitz 2014 \{published data only\}}

Stovitz SD, Berge JM, Wetzsteon RJ, Sherwood NE, Hannan PJ, Himes JH. Stage 1 treatment of pediatric overweight and obesity: a pilot and feasibility randomized controlled trial. Childhood Obesity 2014;10:50-7.

\section{Sweeney 2010 \{published data only\}}

Sweeney K. A tool kit for battling childhood obesity: implementing the $A B C D$ program for childhood obesity in pediatric primary care. Journal of Pediatric Healthcare 2010;24:e7-8.

\section{Sze 2015 \{published data only\}}

Sze YY, Daniel TO, Kilanowski CK, Collins RL, Epstein LH. Webbased and mobile delivery of an episodic future thinking intervention for overweight and obese families: a feasibility study. JMIR Mhealth and Uhealth 2015;3(4):e97.

\section{Tak 2007 \{published data only\}}

Tak YR, An JY, Kim YA, Woo HY. The effects of a physical activity-behavior modification combined intervention (PABMintervention) on metabolic risk factors in overweight and obese elementary school children. Daehan Ganho Haghoeji 2007;37:902-13.

\section{Tanas 2011 \{published data only\}}

Tanas R, Pedretti S, Gilli G, Gagnayre R, Marcolongo R. Clinical evaluation of a family-based therapeutic education programme for obese and overweight children and adolescents. Education Therapeutique du Patient / Therapeutic Patient Education 2011;3:S111-20.

\section{Taveras 2014 \{published data only\}}

Taveras EM, Marshall R, Horan CM, Gillman MW, Hacker K, Kleinman KP, et al. Improving children's obesity-related health care quality: process outcomes of a cluster-randomized controlled trial. Obesity 2014;22:27-31.

Taylor 2006 \{published data only\}

Taylor RW, McAuley KA, Williams SM, Barbezat W, Nielsen G, Mann JI. Reducing weight gain in children through enhancing physical activity and nutrition: the APPLE project. International Journal of Pediatric Obesity 2006;1:146-52.

Taylor 2007 \{published data only\}

Taylor RW, McAuley KA, Barbezat W, Strong A, Williams SM, Mann JI. APPLE Project: 2-y findings of a community-based obesity prevention program in primary school-age children. American Journal of Clinical Nutrition 2007;86:735-42.

\section{Teevale 2015 \{published data only\}}

Teevale T, Taufa S, Percival T. Acceptability and non-compliance in a family-led weight-management programme for obese Pacific children. Public Health Nutrition 2015;18:2625-33.

\section{Ten 2016 \{published data only\}}

Ten Hoor GA, Plasqui G, Ruiter RAC, Kremers SPJ, Rutten GM, Schols AMWJ, et al. A new direction in psychology and health: resistance exercise training for obese children and adolescents. Psychology \& Health 2016;31:1-8.

\section{Theim 2012 \{published data only\}}

Theim KR, Sinton MM, Stein RI, Saelens BE, Thekkedam SC, Welch RR, et al. Preadolescents' and parents' dietary coping efficacy during behavioral family-based weight control treatment. Journal of Youth \& Adolescence 2012;41:86-97.

Thompson 2013 \{published data only\}

Thompson D, Mahabir R, Bhatt R, Boutte C, Cantu D, Vazquez I, et al. Butterfly Girls; promoting healthy diet and physical activity to young African American girls online: rationale and design. BMC Public Health 2013;13:709.

Tirlea 2016 \{published data only\}

Tirlea L, Truby H, Haines TP. Pragmatic, randomized controlled trials of the Girls on the Go! program to improve self-esteem in girls. American Journal of Health Promotion 2016;30:231-41.

\section{Todd 2008 \{published data only\}}

Todd MK, Reis-Bergan MJ, Sidman CL, Flohr J A, JamesonWalker K, Spicer-Bartolau T, et al. Effect of a family-based intervention on electronic media use and body composition among boys aged 8-11 years: a pilot study. Journal of Child Health Care 2008;12:344-58.

\section{Trinh 2013 \{published data only\}}

Trinh A, Campbell M, Ukoumunne OC, Gerner B, Wake M. Physical activity and 3-year BMI change in overweight and obese children. Pediatrics 2013;131:e470-7.

\section{Trost 2014 \{published data only\}}

Trost SG, Sundal D, Foster GD, Lent MR, Vojta D. Effects of a pediatric weight management program with and without active video games a randomized trial. JAMA Pediatrics 2014;168:407-13.

\section{Tucker 2014 \{published data only\}}

Tucker JM, Eisenmann JC, Howard K, Guseman EH, Yee KE, DeLaFuente K, et al. FitKids360: design, conduct, and outcomes of a stage 2 pediatric obesity program. Journal of Obesity 2014;2014:1-9.

\section{Uysal 2014 \{published data only\}}

Uysal Y, Wolters B, Knop C, Reinehr T. Components of the metabolic syndrome are negative predictors of weight loss in obese children with lifestyle intervention. Clinical Nutrition 2014;33:620-5.

\section{Vandongen 1995 \{published data only\}}

Vandongen R, Jenner DA, Thompson C, Taggart AC, Spickett EE, Burke $\mathrm{V}$, et al. A controlled evaluation of a fitness and nutrition

Diet, physical activity and behavioural interventions for the treatment of overweight or obese children from the age of 6 to 11 years 
intervention program on cardiovascular health in 10- to 12-yearold children. Preventive Medicine 1995;24:9-22.

\section{Van Grieken 2013 \{published data only\}}

Van Grieken A, Veldhuis L, Renders CM, Borsboom GJ, Van der Wouden JC, Hirasing RA, et al. Population-based childhood overweight prevention: outcomes of the 'Be Active, Eat Right' study. PLOS ONE 2013;8:e65376.

\section{Van Grieken 2014 \{published data only\}}

Van Grieken A, Renders CM, Veldhuis L, Looman CW, Hirasing RA, Raat $\mathrm{H}$. Promotion of a healthy lifestyle among 5year-old overweight children: health behavior outcomes of the 'Be active, eat right' study. BMC Public Health 2014;14:59.

\section{Vargo 2012 \{published data only\}}

Vargo K, Kennedy E, Fisher J, Sprogis E, Worley S. 41. Sustaining change: long term maintenance of weight loss following completion of a weight management program for children and adolescents. Journal of Adolescent Health 2012;50:S34-5.

\section{Vasickova 2011 \{published data only\}}

Vasickova L, Stavek P, Suchanek P. Possible effect of DHA intake on body weight reduction and lipid metabolism in obese children. Neuroendocrinology Letters 2011;32(Suppl 2):64-7.

\section{Verbeken 2013a $\{$ published data only\}}

Verbeken S, Braet C, Goossens L, Van Der Oord S. Executive function training with game elements for obese children: a novel treatment to enhance self-regulatory abilities for weightcontrol. Behaviour Research And Therapy 2013;51:290-9.

\section{Verbeken 2013b \{published data only\}}

Verbeken S, Braet C, Goossens L, Van Der Oord S. Executive function training with game elements for obese children: a novel treatment to enhance self-regulatory abilities for weightcontrol. Obesity Facts 2013;6:40

\section{Verduci 2011 \{published data only\}}

Verduci E, Radaelli G, Salvioni M, Riva E, Giovannini M. Plasma long-chain fatty acids profile and metabolic outcomes in normolipidaemic obese children after one-year nutritional intervention. Acta Paediatrica, International Journal of Paediatrics 2011;100:585-9.

\section{Verduci 2013 \{published data only\}}

Verduci E, Salvioni M, Arrizza C, Banderali G, Riva E, Giovannini M. Docosahexaenoic acid supplementation and insulin resistance in obese children in a double-blind randomised controlled clinical trial: a pilot study. Annals of Nutrition and Metabolism 2013;63:1327.

\section{Vetter 2014 \{published data only\}}

Vetter M, O'Connor H, O'Dwyer N, Orr R. Learning 'on the move': a combined numeracy and physical activity program for primary school children. Journal of Science and Medicine in Sport 2014;18:e63.

\section{Vido 1993 \{published data only\}}

Vido L, Facchin P, Antonello I, Gobber D, Rigon F. Childhood obesity treatment: double blinded trial on dietary fibres (glucomannan) versus placebo. Padiatrie und Padologie 1993;28:133-6.

\section{Virgen 2007 \{published data only\}}

Virgen Ortiz A, Muñiz Murguía JJ, de la Mota AJ, Ruiz Orozco S, Sánchez ÓAN. Effects of intervention program on overweight and obesity of school children in Colima, Mexico [Efectos de programa de intervención en sobrepeso y obesidad de niños escolares en Colima, México]. Salud Publica de México 2007;49:389-91.

\section{Vos 2011 \{published data only\}}

Vos RC, Wit JM, Pijl H, Kruyff CC, Houdijk EC. The effect of family-based multidisciplinary cognitive behavioral treatment in children with obesity: study protocol for a randomized controlled trial. Trials 2011;12:110.

\section{Vrablik 2014 \{published data only\}}

Vrablik M, Dobiasova M, Zlatohlavek L, Urbanova Z, Ceska R. Biomarkers of cardiometabolic risk in obese/overweight children: effect of lifestyle intervention. Physiological Research/ Academia Scientiarum Bohemoslovaca 2014;63:743-52.

Wake 2011 \{published data only\}

Wake M, Price A, Clifford S, Ukoumunne OC, Hiscock H. Does an intervention that improves infant sleep also improve overweight at age 6? Follow-up of a randomised trial. Archives of Disease in Childhood 2011;96:526-32.

Walker 2008 \{published data only\}

Walker K. Mechanisms of self-esteem change in overweight children participating in a family-based weight management program. Dissertation Abstracts International: Section B: The Sciences and Engineering. 2008; Vol. 68:6341.

Walsh 2014 \{published data only\}

Walsh SM, Palmer W, Welsh JA, Vos MB. Challenges and successes of a multidisciplinary pediatric obesity treatment program. Nutrition in Clinical Practice 2014;29:780-5.

\section{Wang 2013 \{published data only\}}

Wang L, Dalton WT 3rd, Schetzina KE, Fulton-Robinson H, Holt N, Ho AL, et al. Home food environment, dietary intake, and weight among overweight and obese children in Southern Appalachia. Southern Medical Journal 2013;106:550-7.

\section{Ward 2011 \{published data only\}}

Ward DS, Vaughn AE, Bangdiwala KI, Campbell M, Jones DJ, Panter AT, et al. Integrating a family-focused approach into child obesity prevention: rationale and design for the My Parenting SOS study randomized control trial. BMC Public Health 2011;11:431.

\section{Watowicz 2014 \{published data only\}}

Watowicz RP, Tindall A, Eneli IU. Implementation of an inpatient protein-sparing modified fast for severely obese adolescents. Journal of the Academy of Nutrition \& Dietetics 2014;114:A35.

Diet, physical activity and behavioural interventions for the treatment of overweight or obese children from the age of 6 to 11 years 
Wheeler 1976 \{published data only\}

Wheeler ME, Hess KW. Treatment of juvenile obesity by successive approximation control of eating. Journal of Behavior Therapy and Experimental Psychiatry 1976;7:235-41.

\section{Wijesuriya 2011 \{published data only\}}

Wijesuriya M, Gulliford M, Vasantharajah L, Viberti G, Gnudi L, Karalliedde J. DIABRISK-SL prevention of cardio-metabolic disease with life style modification in young urban Sri Lankan's - study protocol for a randomized controlled trial. Trials 2011;12:209.

\section{Wile 1992 \{published data only\}}

Wile H, McIntyre L. Weight control counselling in children: is it effective?. Journal of the Canadian Dietetic Association 1992;53:168-71.

\section{Williamson 2008 \{published data only\}}

Williamson DA, Champagne CM, Harsha D, Han H, Martin CK, Newton R Jr, et al. Louisiana (LA) Health: design and methods for a childhood obesity prevention program in rural schools. Contemporary Clinical Trials 2008;29:783-95.

\section{Williamson 2010 \{published data only\}}

Williamson DA, Champagne CM, Harsha D, Han H, Martin CK, Newton R, et al. Efficacy of two obesity prevention programs in rural schools: primary outcomes for the Louisiana (LA) health study. Obesity Reviews 2010;11:59.

\section{Williamson 2012 \{published data only\}}

Williamson DA, Champagne CM, Harsha DW, Han H, Martin CK, Newton RL Jr, et al. Effect of an environmental school-based obesity prevention program on changes in body fat and body weight: a randomized trial. Obesity 2012;20:1653-61.

\section{Wislo 2013 \{published data only\}}

Wislo VM, McGaffey A, Scopaz KA, D'Amico FJ, Jewell IK, Bridges MW, et al. Fitwits: preparing residency-based physicians to discuss childhood obesity with preteens. Clinical Pediatrics 2013;52:1107-17.

\section{Wohlfarth 2013 \{published data only\}}

Wohlfarth R, Mutschler B, Beetz A, Kreuser F, Korsten-Reck U. Dogs motivate obese children for physical activity: key elements of a motivational theory of animal-assisted interventions. Frontiers in Psychology 2013;4:796.

Wong 2013 \{published data only\} Wong EM, Cheng MM. Effects of motivational interviewing to promote weight loss in obese children. Journal of Clinical Nursing 2013;22:2519-30.

\section{Wright 2013 \{published data only\}}

Wright JA, Phillips BD, Watson BL, Newby PK, Norman GJ, Adams WG. Randomized trial of a family-based, automated, conversational obesity treatment program for underserved populations. Obesity 2013;21:E369-78.

\section{Wyatt 2011 \{published data only\}}

Wyatt KM, Lloyd JJ, Creanor S, Logan S. The development, feasibility and acceptability of a school-based obesity prevention programme: results from three phases of piloting BMJ Open 2011;1:e000026.

Xu 2012 \{published data only\}

Xu F, Ware RS, Tse LA, Wang Z, Hong X, Song A, et al. A schoolbased comprehensive lifestyle intervention among Chinese kids against obesity (CLICK-Obesity): rationale, design and methodology of a randomized controlled trial in Nanjing City, China. BMC Public Health 2012;12:316.

Yackobovitch-Gavan 2009 \{published data only\} Yackobovitch-Gavan M, Nagelberg N, Phillip M, AshkenaziHoffnung L, Hershkovitz E, Shalitin S. The influence of diet and/ or exercise and parental compliance on health-related quality of life in obese children. Nutrition Research 2009;29:397-404.

\section{Yam 2012 \{published data only\}}

Yam PS, Morrison R, Penpraze V, Westgarth C, Ward DS, Mutrie N, et al. Children, parents, and pets exercising together (CPET) randomised controlled trial: study rationale, design, and methods. BMC Public Health 2012;12:208.

\section{Yu 2008 \{published data only\}}

Yu CC, Sung RY, Hau KT, Lam PK, Nelson EA, So RC. The effect of diet and strength training on obese children's physical self-concept. Journal of Sports Medicine \& Physical Fitness 2008;48:76-82.

\section{Zahner 2006 \{published data only\}}

Zahner L, Puder JJ, Roth R, Schmid M, Guldimann R, Puhse U, et al. A school-based physical activity program to improve health and fitness in children aged 6-13 years ("Kinder-Sportstudie KISS"): study design of a randomized controlled trial. BMC Public Health 2006;6:147. [ISRCTN15360785]

\section{Zask 2012 \{published data only\}}

Zask A, Adams JK, Brooks LO, Hughes DF. Tooty Fruity Vegie: an obesity prevention intervention evaluation in Australian preschools. Health Promotion Journal of Australia 2012;23:10-5.

\section{Zhang 2011a \{published data only\}}

Zhang H, Peng Y, Liu Z, Li S, Lv Z, Tian L, et al. Effects of acupuncture therapy on abdominal fat and hepatic fat content in obese children: a magnetic resonance imaging and proton magnetic resonance spectroscopy study. Journal of Alternative \& Complementary Medicine 2011;17:413-20.

\section{Zhang 2011b \{published data only\}}

Zhang L, McIntosh WA. Children's weight status and maternal and paternal feeding practices. Journal of Child Health Care 2011;15:389-400.

\section{Zheng 2015 \{published data only\}}

Zheng M, Allman-Farinelli M, Heitmann BL, Toelle B, Marks G, Cowell $C$, et al. Liquid versus solid energy intake in relation to body composition among Australian children. Journal of Human Nutrition \& Dietetics 2015;28:70-9.

\section{Zorba 2011 \{published data only\}}

Zorba E, Cengiz T, Karacabey K. Exercise training improves body composition, blood lipid profile and serum insulin levels in

Diet, physical activity and behavioural interventions for the treatment of overweight or obese children from the age of 6 to 11 years 
obese children. Journal of Sports Medicine \& Physical Fitness 2011;51:664-9.

\section{References to studies awaiting assessment}

ACTRN12611000862943 \{published data only\}

* ACTRN12611000862943. Whanau Pakari: a multidisciplinary intervention for child and adolescent obesity. www.anzctr.org.au/Trial/Registration/TrialReview.aspx? id=343300 (accessed 10th October 2016).

Anderson YC, Wynter LE, Moller KR, Cave TL, Dolan GM, Grant CC, et al. The effect of a multi-disciplinary obesity intervention compared to usual practice in those ready to make lifestyle changes: design and rationale of Whanau Pakari. BMC obesity 2015;2(41):1

\section{ISRCTN45032201 \{published data only\}}

Fleming J, Kamal A, Harrison E, Hamborg T, Stewart-Brown S, Thorogood M, et al. Evaluation of recruitment methods for a trial targeting childhood obesity: Families for Health randomised controlled trial. Trials 2015;16(1):1.

* ISRCTN45032201. Trial of 'Families for Health': helping families with children who are overweight to manage their weight. www.isrctn.com/ISRCTN45032201 (last accessed 10th October 2016).

Robertson W, Stewart-Brown S, Stallard N, Petrou S, Griffiths F, Thorogood M, et al. Evaluation of the effectiveness and costeffectiveness of Families for Health V2 for the treatment of childhood obesity: study protocol for a randomized controlled trial. Trials 2013;14:81.

\section{ISRCTN97887613 \{published data only\}}

ISRCTN97887613. Exercise looks after you: piloting the programme to prevent obesity in children. www.isrctn.com/ ISRCTN97887613 (last accessed 10th October 2016).

\section{JPRN-UMIN000014896 \{published data only\}}

JPRN-UMIN000014896. Study on the medical checkup system for prevention of lifestyle diseases including diabetes in underage groups, especially infants, elementary and junior high school children \{Study on the Prevention and Treatment of Obesity by Behavioral Approach (Lifestyle modification approach) for Elementary School Children\}. upload.umin.ac.jp/ cgi-open-bin/ctr_e/ctr_view.cgi?recptno=R000017197 (last accessed 10th October 2016).

\section{Jung 1978 \{published data only\}}

Jung K, Maaser R, Ingenillem M, Post C, Woltermann N, Landwehr $\mathrm{L}$, et al. A comprehensive sports program geared to children - illustrated on untrained obese 9-13 year old children. Medizinische Welt 1978;29:301-09.

\section{NCT00528164 \{published data only\}}

Hare ME, Coday M, Williams NA, Richey PA, Tylavsky FA, Bush AJ. Methods and baseline characteristics of a randomized trial treating early childhood obesity: the Positive Lifestyles for Active Youngsters (Team PLAY) trial. Contemporary Clinical Trials 2012;33(3):534-49.
* NCT00528164. Team PLAY (Positive Lifestyles for Active Youngsters) (Team PLAY). www.clinicaltrials.gov/ct2/show/ NCT00528164 (last accessed 10th October 2016).

Williams NA, Coday M, Somes G, Tylavsky FA, Richey PA, Hare M. Risk factors for poor attendance in a family-based pediatric obesity intervention program for young children. Journal of Developmental \& Behavioral Pediatrics 2010;31(9):705-12.

Williams NA, Fournier J, Coday M, Richey PA, Tylavsky FA, Hare ME. Body esteem, peer difficulties and perceptions of physical health in overweight and obese urban children aged 5 to 7 years. Child: Care, Health and Development 2013;39(6):825-34.

NCT00723853 \{published data only\}

NCT00723853. REACH-OUT: Chicago Children's Diabetes Prevention Program (REACH-OUT). clinicaltrials.gov/ct2/show/ NCT00723853 (last accessed 10th October 2016).

\section{NCT00759746 \{published data only\}}

Best JR, Theim KR, Gredysa DM, Stein RI, Welch RR, Saelens BE, et al. Behavioral economic predictors of overweight children's weight loss. Journal of Consulting and Clinical Psychology 2012;80(6):1086-96.

* NCT00759746. Childhood obesity treatment: a maintenance approach (COMPASS). clinicaltrials.gov/ct2/show/NCT00759746 (last accessed 10th October 2016).

\section{NCT00851201 \{published data only\}}

Khan UI, McGinn AP, Isasi CR, Groisman-Perelstein A, Diamantis PM, Ginsberg M, et al. Differences in cardiometabolic risk between insulin-sensitive and insulin-resistant overweight and obese children. Childhood Obesity 2015;11(3):289-96.

* NCT00851201. Family Weight Management Study. clinicaltrials.gov/ct2/show/NCT00851201 (last accessed 10th October 2016)

\section{NCT01110096 \{published data only\}}

NCT01110096. Family Based Intervention in Childhood obesitY clinicaltrials.gov/ct2/show/NCT01110096 (last accessed 10th October 2016).

\section{NCT01290016 \{published data only\}}

Cohen TR, Hazell TJ, Vanstone CA, Plourde H, Rodd CJ, Weiler HA. A family-centered lifestyle intervention to improve body composition and bone mass in overweight and obese children 6 through 8 years: a randomized controlled trial study protocol. BMC Public Health 2013;13:383.

* NCT01290016. Youth lifestyle intervention with food and exercise. clinicaltrials.gov/ct2/show/NCT01290016 (last accessed 10th October 2016)

\section{NCT01506245 \{published data only\}}

Farpour-Lambert N, Maggio A, Martin X, Bucher Della Torre S, Beghetti, M. Effects of family-based behavioral therapy in group or in individual setting in pre-pubertal obese children: a randomized controlled trial. Obesity Facts 2012;5:221.

Diet, physical activity and behavioural interventions for the treatment of overweight or obese children from the age of 6 to 11 years 
* NCT01506245. Exercise and behavioral therapy in obese children. clinicaltrials.gov/ct2/show/NCT01506245 (last accessed 10th October 2016).

\section{NCT01610219 \{published data only\}}

NCT01610219. Lifestyle modification for type 2 diabetes prevention in overweight youth. clinicaltrials.gov/ct2/show/ NCT01610219 (last accessed 10th October 2016).

\section{NCT01662570 \{published data only\}}

NCT01662570. Beverage choice and lifestyle modification in overweight youth. clinicaltrials.gov/ct2/show/NCT01662570 (last accessed 10th October 2016).

\section{NCT02724943 \{published data only\}}

Hoelscher DM, Butte NF, Barlow S, Vandewater EA, Sharma SV, Huang $T$, et al. Incorporating primary and secondary prevention approaches to address childhood obesity prevention and treatment in a low-income, ethnically diverse population: study design and demographic data from the Texas Childhood Obesity Research Demonstration (TX CORD) study. Childhood Obesity 2015;11(1):71-91.

* NCT02724943. Texas Childhood Obesity Research Demonstration (TX CORD) project. clinicaltrials.gov/ct2/show/ NCT02724943 (last accessed 10th October 2016).

Oluyomi AO, Byars A, Byrd-Williams C, Sharma SV, Durand C, Hoelscher DM, et al. The utility of Geographical Information Systems (GIS) in systems-oriented obesity intervention projects: the selection of comparable study sites for a quasiexperimental intervention design-TX CORD. Childhood Obesity 2015;11(1):58-70.

\section{NCT02771951 \{published data only\}}

NCT02771951. Clinical/behavioral approach to overweight in Latino youth. clinicaltrials.gov/ct2/show/NCT02771951 (last accessed 10th October 2016).

\section{NCT02779647 \{published data only\}}

NCT02779647. Play as a method to reduce overweight and obesity in children. clinicaltrials.gov/ct2/show/NCT02779647 (last accessed 10th October 2016).

\section{NCT02794090 \{published data only\}}

NCT02794090. Childhood obesity treatment - telephone coaching vc usual care. clinicaltrials.gov/ct2/show/ NCT02794090 (last accessed 10th October 2016).

\section{Shapiro 1976 \{published data only\}}

Shapiro Joan R. A comparison of various reward and monitoring procedures in the behavioral treatment of overweight children. Dissertation Abstracts International. 1976; Vol. 36:5816-7.

\section{Terwilliger 2008 \{published data only\}}

Terwilliger SH. A study of children enrolled in a school-based physical activity program with attention to overweight and depression [Dissertation]. New York: State University of New York at Binghamton, 2008.

\section{References to ongoing studies \\ ACTRN12613001037796 \{published data only\}}

* ACTRN12613001037796. Effect of exercise intensity on cardiac and vascular function, and intra-abdominal fat in obese children and adolescents. www.anzctr.org.au/Trial/ Registration/TrialReview.aspx?id=364948 (last accessed 10th October 2016).

Dias KA, Coombes JS, Green DJ, Gomersall SR, Keating SE, Tjonna AE, et al. Effects of exercise intensity and nutrition advice on myocardial function in obese children and adolescents: a multicentre randomised controlled trial study protocol. BMJ Open 2016;6(4):e010929.

ChicTR-IOB-15005874 \{published data only\}

* ChiCTR-IOB-15005874. Effects of weight management program on postural stability and neuromuscular function among obese children. www.chictr.org.cn/showprojen.aspx? proj=10313 (last accessed 10th October 2016).

Sun F, Wang LJ, Wang, L. Effects of weight management program on postural stability and neuromuscular function among obese children: study protocol for a randomized controlled trial. Trials 2015;16:143.

DRKS00007879 \{published data only\}

DRKS00007879. Development and evaluation of a computer-based self-regulation training for obese children and adolescents. drks-neu.uniklinik-freiburg.de/drks_ web/navigate.do? navigationld=trial.HTML\&TRIAL_ ID=DRKS00007879 (last accessed 10th October 2016).

\section{ISRCTN81798055 \{published data only\}}

ISRCTN81798055. Child weigHt mANaGement for Ethnically diverse communities study (CHANGE). www.isrctn.com/ ISRCTN81798055 (last accessed 10th October 2016).

\section{Moore 2013 \{published data only\}}

Moore SM, Borawski EA, Cuttler L, levers-Landis CE, Love TE. IMPACT: a multi-level family and school intervention targeting obesity in urban youth. Contemporary Clinical Trials 2013;36(2):574-86

\section{NCT01221220 \{published data only\}}

NCT01221220. Environmental strategies \& behavior change to reduce overeating in obese children. clinicaltrials.gov/ct2/ show/NCT01221220 (last accessed 10th October 2016).

\section{NCT01574352 \{published data only\}}

Larsen KT, Huang T, Moller NC, Andersen LB, Ried-Larsen M. Effectiveness of a one-year multi-component day-camp intervention for overweight children: study protocol of the Odense overweight intervention study (OOIS). BMC Public Health 2014;14:313.

* NCT01574352. The Odense Overweight Intervention Study (OOIS). clinicaltrials.gov/ct2/show/NCT01574352 (last accessed 10th October 2016). 


\section{NCT01642836 \{published data only\}}

* NCT01642836. Clinic, family \& community collaboration to treat overweight and obese children (Stanford GOALS). clinicaltrials.gov/ct2/show/NCT01642836 (last accessed 10th October 2016).

Robinson TN, Matheson D, Desai M, Wilson DM, Weintraub DL, Haskell WL, et al. Family, community and clinic collaboration to treat overweight and obese children: Stanford GOALS - a randomized controlled trial of a three-year, multi-component, multi-level, multi-setting intervention. Contemporary Clinical Trials 2013;36(2):421-35.

\section{NCT01736748 \{published data only\}}

* NCT01736748. Dynamo: a tailored lifestyle promotion intervention among pediatric patients with cardiometabolic risk factors. clinicaltrials.gov/ct2/show/NCT01736748 (last accessed 10th October 2016).

\section{NCT02082080 \{published data only\}}

NCT02082080. Prevention and control of obesity in primary school children in Tehran. clinicaltrials.gov/ct2/show/ NCT02082080 (last accessed 10th October 2016).

\section{NCT02124460 \{published data only\}}

* NCT02124460. Connect 4 Health: an intervention to improve childhood obesity outcomes. clinicaltrials.gov/ct2/show/ NCT02124460 (last accessed 10th October 2016).

Taveras EM, Marshall R, Sharifi M, Avalon E, Fiechtner L, Horan C, et al. Connect for Health: design of a clinicalcommunity childhood obesity intervention testing best practices of positive outliers. Contemporary Clinical Trials 2015;45(Pt B):287-95.

\section{NCT02258126 \{published data only\}}

NCT02258126. Effect of exercise on hepatic fat in overweight children. clinicaltrials.gov/ct2/show/NCT02258126 (last accessed 10th October 2016).

\section{NCT02343367 \{published data only\}}

NCT02343367. Health4Kids intervention trial for Hispanic families. clinicaltrials.gov/ct2/show/NCT02343367 (last accessed 10th October 2016).

\section{NCT02560493 \{published data only\}}

NCT02560493. Gaming technology to encourage healthy weight and activity in youth. clinicaltrials.gov/ct2/show/NCT02560493 (last accessed 10th October 2016).

\section{NCT02573142 \{published data only\}}

NCT02573142. Integrated child obesity treatment study: Bull City healthy and fit. clinicaltrials.gov/ct2/show/NCT02573142 (last accessed 10th October 2016).

\section{NCT02684214 \{published data only\}}

NCT02684214. Implementing prevention plus for childhood overweight and obesity in food secure and insecure families. clinicaltrials.gov/ct2/show/NCT02684214 (last accessed 10th October 2016).

\section{NCT02711488 \{published data only\}}

NCT02711488. Managing adolescent obesity at local level by combining primary and secondary intervention. clinicaltrials.gov/ct2/show/NCT02711488 (last accessed 10th October 2016).

\section{NCT02720302 \{published data only\}}

NCT02720302. Treatment of overweight in children on distance. clinicaltrials.gov/ct2/show/NCT02720302 (last accessed 10th October 2016).

\section{NCT02773823 \{published data only\}}

NCT02773823. A behavior intervention study on cardiovascular health among Chinese obese schoolchildren. clinicaltrials.gov/ ct2/show/NCT02773823 (last accessed 10th October 2016).

RBR-8ttw64 \{published data only\}

RBR-8ttw64. Effects of dietary guidance in children attending outpatient preventive cardiology: randomized clinical trial. www.ensaiosclinicos.gov.br/rg/RBR-8ttw64/ (last accessed 10th October 2016).

\section{Additional references}

\section{Altman 2003}

Altman DG, Bland JM. Interaction revisited: the difference between two estimates. BMJ 2003;326(7382):219. [PUBMED: 12543843]

\section{Bocca 2013}

Bocca G, Ongering EC, Stolk RP, Sauer PJ. Insulin resistance and cardiovascular risk factors in 3- to 5-yearold overweight or obese children. Hormone Research in Paediatrics 2013;80(3):201-6.

\section{Boutron 2014}

Boutron I, Altman DG, Hopewell S, Vera-Badillo F, Tannock I, Ravaud P. Impact of spin in the abstracts of articles reporting results of randomized controlled trials in the field of cancer: the SPIIN randomized controlled trial. Journal of Clinical Oncology 2014;32:4120-6.

\section{Brown 2016 [pers comm]}

Brown T (Teesside University). [Personal communication]. Conversation with: L Ells (Teesside University) 1 December 2016.

\section{Butland 2007}

Butland BJS, Kopelman P, Mcpherson K, Thomas S, Mardell J, Parry V. FORESIGHT: Tackling Obesities: Future Choices Project Report. In: SCIENCE, G. O. F. (ed.) 2nd ed. FORESIGHT, 2007.

\section{Cataldo 2016}

Cataldo R, Huang J, Calixte R, Wong AT, Bianchi-Hayes J, Pati S. Effects of overweight and obesity on motor and mental development in infants and toddlers. Pediatric Obesity 2016;11(5):389-96. [DOI: 10.1111/ijpo.12077]

Diet, physical activity and behavioural interventions for the treatment of overweight or obese children from the age of 6 to 11 years 


\section{CMO 2015}

Chief Medical Officer. Annual report of the Chief Medical Officer: surveillance volume, 2012: on the state of the public's health. www.gov.uk/government/publications/chief-medical-officerannual-report-surveillance-volume-2012 (accessed 30 March 2015).

\section{Cole 2000}

Cole TJ, Bellizzi MC, Flegal KM, Dietz WH. Establishing a standard definition for child overweight and obesity worldwide: international survey. BMJ 2000;320(7244):1240-3.

\section{Cole 2007}

Cole TJ, Flegal KM, Nicholls D, Jackson AA. Body mass index cut offs to define thinness in children and adolescents: international survey. BMJ 2007;335(7612):194.

\section{Cole 2012}

Cole TJ, Lobstein T. Extended international (IOTF) body mass index cut-offs for thinness, overweight and obesity. Pediatr Obes 2012;7(4):284-94.

\section{Colquitt 2016}

Colquitt JL, Loveman E, O'Malley C, Azevedo LB, Mead E, Al-Khudairy L, et al. Diet, physical activity, and behavioural interventions for the treatment of overweight or obesity in preschool children up to the age of 6 years. Cochrane Database of Systematic Reviews 2016, Issue 3. [DOI: 10.1002/14651858.CD012105]

\section{Cook 2003}

Cook S, Weitzman M, Auinger P, Nguyen M, Dietz WH. Prevalence of a metabolic syndrome phenotype in adolescents: findings from the thirdNational Health and Nutrition Examination Survey, 1988-1994. Arch Pediatr Adolesc Med 2003;157:821-7.

\section{Craig 2008}

Craig P, Dieppe P, Maclntyre S, Miche S, Nazareth I, Pettigrew M. Developing and evaluating complex interventions: the new Medical Research Council guidance. BMJ 2008;337:a1655.

\section{Daniels 2009b}

Daniels SR. Complications of obesity in children and adolescents. International Journal of Obesity 2009;33(Suppl 1):S60-5.

\section{Deeks 2011}

Deeks JJ, Higgins JPT, Altman DG (editors). Chapter 9: Analysing data and undertaking meta-analyses. In: Higgins JPT, Green $\mathrm{S}$ (editors). Cochrane Handbook for Systematic Reviews of Interventions Version 5.1.0 (updated March 2011). The Cochrane Collaboration, 2011. Available from handbook.cochrane.org.

\section{Douketis 2005}

Douketis JD, Paradis G, Keller H, Martineau C. Canadian guidelines for body weight classification in adults: applicationin clinical practice to screen for overweight andobesity and to assess disease risk. CMAJ 2005;172:995-8.

\section{Eady 2008}

Eady AM, Wilczynski NL, Haynes RB. PsycINFO search strategies identified methodologically sound therapy studies and review articles for use by clinicians and researchers. Journal of Clinical Epidemiology 2008;61(1):34-40.

\section{Egan 2013}

Egan K, Ettinger A, Bracken M. Childhood body mass index and subsequent physician-diagnosed asthma: a systematic review and meta-analysis of prospective cohort studies. BMC Pediatrics 2013;13(1):121.

\section{Ells 2015a}

Ells LJ, Hancock C, Copley VR, Mead E, Dinsdale H, Kinra S, et al. Prevalence of severe childhood obesity in England: 2006-2013. Archives of Disease in Childhood 2015;100:631-6.

\section{Ells 2015b}

Ells LJ, Mead E, Atkinson G, Corpeleijn E, Roberts K, Viner R, et al. Surgery for the treatment of obesity in children and adolescents. Cochrane Database of Systematic Reviews 2015, Issue 6. [DOI: 10.1002/14651858.CD011740]

\section{Ells 2016 [pers comm]}

Ells L (Teesside University). [Personal communication]. Conversation with: T Brown (Teesside University) 1 December 2016.

\section{Follmann 1992}

Follmann D, Elliot P, Suh I, Cutler J. Variance imputation for overviews of clinical trials with continuous response. Journal of Clinical Epidemiology 1992;45(7):769-73.

\section{Ford 2010}

Ford AL, Hunt LP, Cooper A, Shield JPH. What reduction in BMI SDS is required in obese adolescents to improve body composition and cardiometabolic health?. Archives of Disease in Childhood 2010;95:256-61.

\section{Freedman 2006}

Freedman DS, Khan LK, Serdula MK, Ogden CL, Dietz WH. Racial and ethnic differences in secular trends for childhood BMI, weight, and height. Obesity 2006;14(2):301-8.

\section{Golan 2004}

Golan M, Crow S. Targetting parents exclusively in the treatment of childhood obesity: long-term results. Obesity Research 2004;12:357-61.

\section{GRADEproGDT 2015 [Computer program]}

GRADE Working Group, McMaster University. GRADEproGDT. Version accessed 4th July 2016. Hamilton (ON): GRADE Working Group, McMaster University, 2014.

\section{Griffiths 2010}

Griffiths LJ, Parsons TJ, Hill AJ. Self-esteem and quality of life in obese children and adolescents: a systematic review. International Journal of Pediatric Obesity 2010;5(4):282-304. 


\section{Higgins 2002}

Higgins JPT, Thompson SG. Quantifying heterogeneity in a meta-analysis. Statistics in Medicine 2002;21(11):1539-58.

\section{Higgins 2003}

Higgins JPT, Thompson SG, Deeks JJ, Altman DG. Measuring inconsistency in meta-analyses. BMJ 2003;327(7414):557-60.

\section{Higgins 2011a}

Higgins JPT, Altman DG, Gøtzsche PC, Jüni P, Moher D, Oxman AD, et al. The Cochrane Collaboration's tool for assessing risk of bias in randomised trials. BMJ 2011;343:d5928.

\section{Higgins 2011b}

Higgins JPT, Altman DG, Sterne JAC (editors). Chapter 8: Assessing risk of bias in included studies. In: Higgins JPT, Green $S$ (editors). Cochrane Handbook for Systematic Reviews of Interventions Version 5.1.0 (updated March 2011). The Cochrane Collaboration, 2011. Available from handbook.cochrane.org.

\section{Higgins 2011c}

Higgins JPT, Deeks JJ, Altman DG (editors). Chapter 16: Special topics in statistics. In: Higgins JPT, Green S (editors), Cochrane Handbook for Systematic Reviews of Interventions Version 5.1.0 (updated March 2011). The Cochrane Collaboration, 2011. Available from handbook.cochrane.org.

\section{Ho 2012}

Ho M, Garnett SP, Baur L, Burrows T, Stewart L, Neve M, et al. Effectiveness of lifestyle interventions in child obesity: systematic review with meta-analysis. Pediatrics 2012;130:e1647-71.

\section{Hróbjartsson 2013}

Hróbjartsson A, Thomsen AS, Emanuelsson F, Tendal B, Hilden J, Boutron I, et al. Observer bias in randomized clinical trials with measurement scale outcomes: a systematic review of trials with both blinded and nonblinded assessors. Canadian Medical Association Journal 2013;185(4):E201-11.

\section{HSCIC 2013}

Health and Social Care Information Centre. National Child Measurement Programme - England, 2012-13 school year. content.digital.nhs.uk/catalogue/PUB13115.

\section{Jelliffe 1966}

Jelliffe DB. The assessment of the nutritional status of the community. Monograph No. 3. Geneva, 1966.

\section{Jones 2015b}

Jones CW, Keil LG, Holland WC, Caughey MC, Platts-Mills TF. Comparison of registered and published outcomes in randomized controlled trials: a systematic review. BMC Medicine 2015;13:282. [DOI: 10.1186/s12916-015-0520-3]

\section{Kelly 2013}

Kelly AS, Barlow SE, Rao G, Inge TH, Hayman LL, Steinberger J, et al. Severe obesity in children and adolescents: identification, associated health risks, and treatment approaches: a scientific statement from the American Heart Association. Circulation 2013;128(15):1689-712.

\section{Kirkham 2010}

Kirkham JJ, Dwan KM, Altman DG, Gamble C, Dodd S, Smyth R, et al. The impact of outcome reporting bias in randomised controlled trials on a cohort of systematic reviews. BMJ 2010;340:c365. [DOI: 10.1136/bmj.c365]

\section{Knai 2012}

Knai C, Lobstein T, Darmon N, Rutter H, McKee M.

Socioeconomic patterning of childhood overweight status in Europe. International Journal of Environmental Research and Public Health 2012;9(4):1472-89.

\section{Kremers 2006}

Kremers SP, De Bruijn GJ, Visscher TL, Van Mechelen W, De Vries NK, Brug J. Environmental influences on energy balance-related behaviors: a dual-process view. International Journal of Behaviour, Nutrition and Physical Activity 2006;15(3):9.

\section{Kromeyer-Hauschild 2001}

Kromeyer-Hauschild K, Wabitsch M, Kunze D, Geller F, Geiß HC, Hesse V, et al. Percentiles of body mass index in children and adolescents evaluated from different regional German studies. Monatsschrift fur Kinderheilkunde 2001;149(8):807-18.

\section{Kuczmarski 2002}

Kuczmarski RJ, Ogden CL, Guo SS, Grummer-Strawn LM, Flegal KM, Mei Z, et al. CDC growth charts for the United States: Methods and development.. Vital Health Statistics. 2002;246(11):1-90.

\section{Landgraf 1999}

Landgraf JM, Abetz L, Ware JE. Child health questionnaire (CHQ) : a user's manual. 2nd Edition. Boston, MA: Landgraf \& Ware, 1999

\section{Leclercq 2013}

Leclercq E, Leeflang MM, Van Dalen EC, Kremer LC. Validation of search filters for identifying pediatric studies in PubMed. The Journal of Pediatrics 2013;162(3):629-34.

\section{Liberati 2009}

Liberati A, Altman DG, Tetzlaff J, Mulrow C, Gøtzsche PC, loannidis JPA, et al. The PRISMA statement for reporting systematic and meta-analyses of studies that evaluate interventions: explanation and elaboration. PLoS Medicine 2009;6(7):1-28. [DOI: 10.1371/journal.pmed.1000100]

\section{Lobstein 2004}

Lobstein T, Baur L, Uauy R. Obesity in children and young people: a crisis in public health. Obesity Reviews 2004;5(Suppl 1):4-104.

\section{Loveman 2015}

Loveman E, Al-Khudairy L, Johnson RE, Robertson W, Colquitt JL, Mead EL, et al. Parent-only interventions for childhood overweight or obesity in children aged 5 to 11 years. Cochrane Database of Systematic Reviews 2015, Issue 12. [DOI: 10.1002/14651858.CD012008]

Diet, physical activity and behavioural interventions for the treatment of overweight or obese children from the age of 6 to 11 years 


\section{Mathieu 2009}

Mathieu S, Boutron I, Moher D, Altman DG, Ravaud P. Comparison of registered and published primary outcomes in randomized controlled trials. JAMA 2009;302:977-84.

\section{Mead 2016a}

Mead E, Atkinson G, Richter B, Metzendorf MI, Baur L, Finer N, et al. Drug interventions for the treatment of obesity in children and adolescents. Cochrane Database of Systematic Reviews 2016, Issue 11. [DOI: 10.1002/14651858.CD012436]

\section{Mead 2016b}

Mead E, Batterham AM, Atkinson G, Ells LJ. Predicting future weight status from measurements made in early childhood: a novel longitudinal approach applied to Millennium Cohort Study data. Nutrition \& Diabetes 2016;6:e200.

\section{Meader 2014}

Meader N, King K, Llewellyn A, Norman G, Brown J, Rodgers M, et al. A checklist designed to aid consistency and reproducibility of GRADE assessments: development and pilot validation. Systematic Reviews 2014;3:82.

\section{Metropolitian Life Insurance Company 1959}

Metropolitan Life Insurance Company. New weight standards for men and women. Statistical Bulletin 1959;40:1-4.

\section{Must 1991}

Must A, Dallal GE, Dietz WH. Reference data for obesity: 85th and 95th percentilesof body mass index (wt/ht2) and triceps skinfold thickness. Am J Clin Nutr 1991;53:839-46.

\section{Narang 2012}

Narang I, Mathew JL. Childhood obesity and obstructive sleep apnea. Journal of Nutrition and Metabolism 2012;2012:134202 (Article ID). [DOI: 10.1155/2012/134202; PUBMED: 22957216]

\section{NCB 2015}

National Children's Bureau. Poor beginnings. Health inequalities among young children across England. www.ncb.org.uk/sites/default/files/uploads/documents/Policy_ docs/ncb_poor_beginnings_report_final_for_web.pdf 2015.

\section{Ng 2014}

Ng M, Fleming T, Robinson M, Thomson B, Graetz N, Margono C, et al. Global, regional, and national prevalence of overweight and obesity in children and adults during 1980-2013: a systematic analysis of the Global Burden of Disease Study 2013. Lancet 2014;384(9945):766-81.

\section{NOO NHS 2011}

National Obesity Observatory on behalf of the Public Health Observatories in England. A simple guide to classifying body mass index in children, 2011. www.noo.org.uk/uploads/ doc/vid_11601_A_simple_guide_to_classifying_BMI_in_ children.pdf (accessed 3 November 2015).

\section{Olds 2011}

Olds T, Maher C, Zumin S, Péneau S, Lioret S, Castetbon K, et al. Evidence that the prevalence of childhood overweight is plateauing: data from nine countries. International Journal of Pediatric Obesity 2011;6(5-6):342-60.

\section{Parsons 1999}

Parsons TJ, Power C, Logan S, Summerbell CD. Childhood predictors of adult obesity: a systematic review. International Journal of Obesity 1999;23(Suppl 8):S1-S107.

\section{Paulis 2014}

Paulis WD, Silva S, Koes BW, Van Middelkoop M. Overweight and obesity are associated with musculoskeletal complaints as early as childhood: a systematic review. Obesity Reviews 2014;15(1):52-67.

\section{Puhl 2007}

Puhl RM, Latner JD. Stigma, obesity, and the health of the nation's children. Psychology Bulletin 2007;133(4):557-80.

\section{Raat 2002}

Raat H, Bonsel GJ, Essink-Bot ML, Landgraf JM, Gemke RJ. Reliability and validity of comprehensive health status measures in children: the child health questionnaire in relation to the health utilities index. Journal of Clinical Epidemiology 2002;55:67-76.

\section{Rajput 2014}

Rajput N, Tuohy P, Mishra S, Smith A, Taylor B. Overweight and obesity in 4-5-year-old children in New Zealand: Results from the first 4 years (2009-2012) of the B4School Check programme. Journal of Paediatrics and Child Health 2014;51(3):334-43.

\section{Ravens-Sieberer 2000}

Ravens-Sieberer U, Bullinger M. KINDL-R. Questionnaire for the assessment of health-related quality of life in children and adolescents [KINDL-R. Fragebogen zur Erfassung der gesundheitsbezogenen Lebensqualität bei Kindern und Jugendlichen. Revidierte Form]. Manual 2000.

\section{Reilly 2003}

Reilly JJ, Methven E, McDowell ZC, Hacking B, Alexander D, Stewart $\mathrm{L}$, et al. Health consequences of obesity. Archives of Diseases in Childhood 2003;88(9):748-52.

\section{Reilly 2011}

Reilly JJ, Kelly J. Long-term impact of overweight and obesity in childhood and adolescence on morbidity and premature mortality in adulthood: systematic review. International Journal of Obesity 2011;35(7):891-8.

\section{RevMan 2014 [Computer program]}

Nordic Cochrane Centre, The Cochrane Collaboration. Review Manager 5 (RevMan 5). Version 5.3. Copenhagen: Nordic Cochrane Centre, The Cochrane Collaboration, 2014.

\section{Robinson 1968}

Robinson, $\mathrm{CH}$. Fundamentals of Normal Nutrition. New York: MacMillan, 1968. 


\section{Rokholm 2010}

Rokholm B, Baker JL, Sørenson TI. The levelling off of the obesity epidemic since the year 1999 - a review of evidence and perspectives. Obesity Reviews 2010;11(12):835-46.

\section{Rosner 1998}

Rosner B, Prineas R, Loggie J, Daniels SR. Percentiles for body mass index in U.S. children 5 to 17 years of age. Journal of Pediatrics 1998;132:211-22.

\section{Sbruzzi 2013}

Sbruzzi G, Eibel B, Barbiero SM, Petkowicz RO, Ribeiro RA, Cesa CC, et al. Educational interventions in childhood obesity: a systematic review with meta-analysis of randomized clinical trials. Preventive Medicine 2013;56(5):254-64.

\section{Schünemann 2011}

Schünemann HJ, Oxman AD, Higgins JPT, Vist GE, Glasziou P, Guyatt GH. Chapter 11: Presenting results and 'Summary of findings' tables. In: Higgins JPT, Green S (editors), Cochrane Handbook for Systematic Reviews of Interventions Version 5.1.0 (updated March 2011). The Cochrane Collaboration, 2011. Available from handbook.cochrane.org.

\section{Shrewsbury 2008}

Shrewsbury V, Wardle J. Socioeconomic status and adiposity in childhood: a systematic review of cross-sectional studies 1990-2005. Obesity 2008;16(2):275-84.

\section{Singh 2008}

Singh AS, Mulder C, Twisk JW, Van Mechelen W, Chinapaw MJ. Tracking of childhood overweight into adulthood: a systematic review of the literature. Obesity Reviews 2008;9(5):474-88.

\section{Skinner 2014}

Skinner AC, Skelton JA. Prevalence and trends in obesity and severe obesity among children in the United States 1999-2012. JAMA Pediatrics 2014;168(6):561-6.

\section{Sterne 2011}

Sterne JA, Sutton AJ, loannidis JP, Terrin N, Jones DR, Lau J, et al. Recommendations for examining and interpreting funnel plot asymmetry in meta-analyses of randomised controlled trials. BMJ 2011;343:d4002.

\section{Stevens 2010}

Stevens KJ. The Child Health Utility 9D (CHU9D) - a new paediatric preference based measure ofhealth related quality of life. Pro Newsletter 2010;43:11-2.

\section{Tang-Peronard 2008}

Tang-Peronard JL, Heitmann BL. Stigmatization of obese children and adolescents, the importance of gender. Obesity Reviews 2008;9(6):522-34.

\section{Tilator Oy Ltd 2004 [Computer program]}

Tilator Oy Ltd. Pediator software for growth data [computer program]. Version Version Release 6.7.4. Säkylä, Finland, 2004.

\section{Varni 2003}

Varni JW, Burwinkle TM, Seid M, Skarr D. The PedsQL 4.0 as a pediatric population health measure: feasibility, reliability, and validity. Ambulatory Pediatrics 2003;3(6):329-41.

\section{Varni 2007}

Varni JW, Limbers C, Burwinkle TM. Literature review: healthrelated quality of life measurement in pediatric oncology: hearing the voices of the children. Journal of Pediatric Psychology 2007;32(9):1151-63.

\section{Wang 2012}

Wang Y, Lim H. The global childhood obesity epidemic and the association between socio-economic status and childhood obesity. International Review of Psychiatry 2012;24(3):176-88.

\section{Waters 2011}

Waters E, de Silva Sanigorski A, Burford BJ, Brown T, Campbell KJ, Gao Y, et al. Interventions for preventing obesity in children. Cochrane Database of Systematic Reviews 2011, Issue 12. [DOI: 10.1002/14651858.CD001871.pub3]

\section{Whitaker 1997}

Whitaker RC, Wright JA, Pepe MS, Seidel KD, Dietz WH. Predicting obesity in young adulthood from childhood and parental obesity. The New England Journal of Medicine 1997;337(13):869-73.

\section{WHO 2015}

World Health Organization. Fact sheet on overweight and obesity. www.who.int/mediacentre/factsheets/fs311/en (accessed 30 March 2015).

\section{Wille 2010}

Wille N, Badia X, Bonsel G, Burstrom K, Cavrini G, Devlin N, et al. Development of the EQ-5D-Y:a child-friendly version of the EQ-5D. Quality of Life Research 2010;19:875-86.

\section{Wong 2006a}

Wong SS, Wilczynski NL, Haynes RB. Developing optimal search strategies for detecting clinically sound treatment studies in Embase. Journal of the Medical Library Association 2006;94(1):41-7.

\section{Wong 2006b}

Wong SS, Wilczynski NL, Haynes RB. Optimal CINAHL search strategies for identifying therapy studies and review articles. Journal of Nursing Scholarship 2006;38(2):194-9.

\section{References to other published versions of this review Oude Luttikhuis 2009}

Oude Luttikhuis H, Baur L, Jansen H, Shrewbury VA, O'Malley C, Stolk RP, et al. Interventions for treating obesity in children. Cochrane Database of Systematic Reviews 2009, Issue 1. [DOI: 10.1002/14651858.CD001872.pub2]

\section{Summerbell 2003}

Summerbell CD, Ashton V, Campbell KJ, Edmunds L, Kelly S, Waters $\mathrm{E}$. Interventions for treating obesity in children.

Diet, physical activity and behavioural interventions for the treatment of overweight or obese children from the age of 6 to 11 years 
Cochrane Database of Systematic Reviews 2003, Issue 3. [DOI:

10.1002/14651858.CD001872]

CHARACTERISTICS OF STUDIES

Characteristics of included studies [ordered by study ID]

Alves 2008

\begin{tabular}{|c|c|}
\hline Methods & $\begin{array}{l}\text { Parallel RCT } \\
\text { Randomisation ratio: 1:1 } \\
\text { Superiority design }\end{array}$ \\
\hline Participants & $\begin{array}{l}\text { Inclusion criteria: } \mathrm{BMI} \geq 85 \text { th percentile (CDC growth charts), absence of clinical evidence of heart dis- } \\
\text { ease (congenital or acquired), respiratory failure or type } 1 \text { diabetes, do not use drugs which interfere } \\
\text { with cardiac response during exercise (e.g. beta blockers) } \\
\text { Exclusion criteria: - } \\
\text { Diagnostic criteria: see above }\end{array}$ \\
\hline
\end{tabular}

$\begin{array}{ll}\text { Interventions } & \text { Number of study centres: } 1 \\ \text { Run-in period: no } & \\ \text { Extension period: none } & \text { Intervention: exercise group } \\ \text { Comparator: no-care control }\end{array}$

Outcomes Outcome measures reported in abstract: weight, BMI

Study details

Trial terminated early: no

Trial ID: -

\begin{tabular}{ll}
\hline Publication details & Language of publication: Portuguese \\
Funding: non-commercial funding (Cnpq, Brazilian Government) \\
Publication status: peer-reviewed journal
\end{tabular}

Stated aim for study

Quote from publication: "To verify the effectiveness of an exercise intervention to control excess of body weight without the incorporation of diet guidelines in children who lives in a deprived area in a developing country"

Notes -

\section{Risk of bias}

\begin{tabular}{lll}
\hline Bias & Authors' judgement & Support for judgement \\
\hline $\begin{array}{l}\text { Random sequence genera- } \\
\text { tion (selection bias) }\end{array}$ & Low risk & $\begin{array}{l}\text { Quote from publication: "Patients were listed consecutively and after ran- } \\
\text { domly selected, without spare, to compose the group intervention" }\end{array}$ \\
& Comment: adequate randomisation method \\
\hline
\end{tabular}

Allocation concealment Low risk Comment: study author confirmed allocation was concealed
(selection bias)

Diet, physical activity and behavioural interventions for the treatment of overweight or obese children from the age of 6 to 11 years (Review)

Copyright (c) 2017 The Cochrane Collaboration. Published by John Wiley \& Sons, Ltd. 
Alves 2008 (Continued)

Blinding of participants High risk Comment: study author confirmed study was not blinded and personnel (performance bias)

Objective outcomes

Blinding of outcome as-
sessment (detection bias) $\quad$ High risk Comment: study author confirmed study was not blinded

Objective outcomes

Incomplete outcome data Low risk Comment: dropout rates fairly low
(attrition bias)

Objective outcomes

\begin{tabular}{lll}
\hline $\begin{array}{l}\text { Selective reporting (re- } \\
\text { porting bias) }\end{array}$ & Unclear risk & Comment: no protocol available \\
\hline Other bias & Unclear risk & Comment: unclear if the study was at risk of any other bias \\
\hline
\end{tabular}

Arauz Boudreau 2013

$\begin{array}{ll}\text { Methods } & \text { Parallel RCT } \\ & \text { Randomisation ratio: 3:2 during the first half of the study, and then 2:2 during the second half of the } \\ \text { study to adequately fill the group classes }\end{array}$

\section{Superiority design}

Participants Inclusion criteria: Latino children aged 9-12 years, overweight or obese ( $\geq 85$ th percentile or $\geq 95$ th percentile, CDC growth charts. Had received primary care at a single community health centre

Exclusion criteria: children who had chronic diseases (other than asthma)

Diagnostic criteria: see above

Number of study centres: 1
Run-in period: no
Extension period: no
Intervention: lifestyle intervention and coaching on lifestyle behaviours
Comparator: waiting-list control

Outcomes Outcome measures reported in abstract: attendance, barriers to changing lifestyles to control obesity, HRQoL, obesity markers, BMI, physical activity

Study details Trial terminated early: no

Trial ID: -

\section{Publication details Language of publication: English}

Funding: Robert Wood Johnson Foundation; Massachusetts General Hospital Multicultural Affairs Career Development Award; Massachusetts General Hospital Disparities Solution Center; Harvard Catalyst Clinical Research Center (Grant no. UL1 RR025758-01); NIH; National Center for Research Resources; and General Clinical Research Centers Program (non-commercial)

Publication status: peer-reviewed journal 
Arauz Boudreau 2013 (Continued)

Stated aim for study Quote from publication: "To assess the feasibility and effectiveness of a family-centred, primary carebased approach to control childhood obesity through lifestyle choices"

Notes

\section{Risk of bias}

Bias Authors' judgement Support for judgement

Random sequence genera- Unclear risk Comment: no description of the randomisation method tion (selection bias)

Allocation concealment $\quad$ Unclear risk
(selection bias)

Blinding of participants Unclear risk Comment: no subjective outcomes measured
and personnel (performance bias)

Subjective outcomes

Blinding of participants High risk
and personnel (perfor-
mance bias)
Objective outcomes

Quote from publication: "Although participants were randomized, because of the waitlist study design, neither participants nor study team members were blinded to group allocation"

Comment: participants and study personnel were not blinded to study group

Blinding of outcome as- High risk sessment (detection bias)

Subjective outcomes

Quote from publication: "Although participants were randomized, because of the waitlist study design, neither participants nor study team members were blinded to group allocation"

Comment: participants and study personnel were not blinded to study group

\begin{tabular}{|c|c|c|}
\hline $\begin{array}{l}\text { Blinding of outcome as- } \\
\text { sessment (detection bias) } \\
\text { Objective outcomes }\end{array}$ & High risk & $\begin{array}{l}\text { Quote from publication: "Although participants were randomized, because o } \\
\text { the waitlist study design, neither participants nor study team members were } \\
\text { blinded to group allocation" }\end{array}$ \\
\hline
\end{tabular}

Objective outcomes

Comment: outcome assessors were not blinded to study group

\begin{tabular}{|c|c|c|}
\hline $\begin{array}{l}\text { Incomplete outcome data } \\
\text { (attrition bias) }\end{array}$ & High risk & $\begin{array}{l}\text { Quote from publication: "A total of } 67 \%(12 / 18) \text { control and } 61 \%(14 / 23) \text { inter- } \\
\text { vention participants took part in first and second visits" }\end{array}$ \\
\hline
\end{tabular}

Subjective outcomes

Comment: attrition rates were high

\begin{tabular}{lll}
\hline Incomplete outcome data & High risk & Quote from publication: "A total of 67\% (12/18) control and 61\% (14/23) inter- \\
(attrition bias) & & vention participants took part in first and second visits"
\end{tabular}

Objective outcomes

Comment: attrition rates were high

Selective reporting (re- Unclear risk Comment: unable to find clinical trial record/protocol
porting bias)

Other bias Unclear risk Comment: unclear if the study was at risk of any other bias

Barkin 2011

Methods Parallel RCT

Diet, physical activity and behavioural interventions for the treatment of overweight or obese children from the age of 6 to 11 years 
Barkin 2011 (Continued)

Randomisation ratio: 1:1

Superiority design

Participants Inclusion criteria: Latino children who were 8-11 years, BMI $\geq 85 \%$ adjusted for age and gender (CDC growth charts), parent $>18$ years and committed to participating in the intervention

Exclusion criteria: -

Diagnostic criteria: see above

Number of study centres: 2
Run-in period: no
Extension period: no
Intervention: group physical activity and goal setting
Comparator: standard care counselling and health education session

Outcomes Outcome measures reported in abstract: BMI (parents and children)

Study details

Trial terminated early: no

Trial ID: -

Publication details

Language of publication: English

Funding: National Institutes of Health (NICHD Grant No. R21 HD050990-02) and 'The Collaborative to Strengthen Families and Neighborhoods' - part funded by The Duke Endowment (non-commercial)

Publication status: peer-reviewed journal

\begin{tabular}{ll}
\hline Stated aim for study & $\begin{array}{l}\text { Quote from publication: "To assess whether body mass index (BMI) change in preadolescents reflected } \\
\text { that of their participating parent." }\end{array}$
\end{tabular}

Notes

\section{Risk of bias}

\begin{tabular}{lll}
\hline Bias & Authors' judgement & Support for judgement \\
\hline $\begin{array}{l}\text { Random sequence genera- } \\
\text { tion (selection bias) }\end{array}$ & Unclear risk & Comment: no description of randomisation method \\
\hline
\end{tabular}

\begin{tabular}{|c|c|c|}
\hline $\begin{array}{l}\text { Allocation concealment } \\
\text { (selection bias) }\end{array}$ & Unclear risk & Comment: unclear if allocation was concealed. No mention in text \\
\hline $\begin{array}{l}\text { Blinding of participants } \\
\text { and personnel (perfor- } \\
\text { mance bias) } \\
\text { Objective outcomes }\end{array}$ & Unclear risk & $\begin{array}{l}\text { Comment: unclear if participant and study personnel were blinded. No men- } \\
\text { tion in text }\end{array}$ \\
\hline
\end{tabular}

\begin{tabular}{ll}
$\begin{array}{l}\text { Blinding of outcome as- } \\
\text { sessment (detection bias) } \\
\text { Objective outcomes }\end{array}$ & Comment: unclear if outcome assessors were blinded. No mention in text \\
\hline $\begin{array}{l}\text { Incomplete outcome data } \\
\begin{array}{l}\text { (attrition bias) } \\
\text { Objective outcomes }\end{array}\end{array}$ & $\begin{array}{l}\text { Quote from publication: "For this community-based randomized controlled } \\
\text { trial, we had a } 68 \% \text { retention rate, consistent with other studies of this kind." }\end{array}$
\end{tabular}

Diet, physical activity and behavioural interventions for the treatment of overweight or obese children from the age of 6 to 11 years 
Barkin 2011 (Continued)

"The completers (those who completed both baseline and 6-month data) did not differ significantly on the variables of interest compared with those who did not complete the study (refer to Table 3)."

Comment: attrition rates were high and bias assessed as high even with multiple imputation method used. Only $45 \%$ of participants were followed up

\begin{tabular}{ll}
\hline $\begin{array}{l}\text { Selective reporting (re- } \\
\text { porting bias) }\end{array}$ & High risk \\
& $\begin{array}{l}\text { Comment: they only report baseline and change from baseline BMI measure- } \\
\text { ments for both groups combined, don't report them individually for interven- } \\
\text { tion and control groups. No clinical trial register or protocol to assess report- } \\
\text { ing of outcomes }\end{array}$
\end{tabular}

Other bias Unclear risk Comment: unclear if the study was at risk of any other bias

Bathrellou 2010

\begin{tabular}{ll}
\hline Methods & Parallel RCT \\
& Randomisation ratio: $1: 1$ \\
& Superiority design
\end{tabular}

Participants

Inclusion criteria: overweight or obese children (IOTF growth references), aged 7-12 years

Exclusion criteria: chronic physical or mental illness

Diagnostic criteria: see above

\begin{tabular}{|c|c|}
\hline \multirow[t]{5}{*}{ Interventions } & Number of study centres: 1 \\
\hline & Run-in period: no \\
\hline & Extension period: no \\
\hline & Intervention: behavioural intervention with parental involvement \\
\hline & Comparator: behavioural intervention without parental involvement \\
\hline Outcomes & Outcome measures reported in abstract: percent overweight \\
\hline \multirow[t]{2}{*}{ Study details } & Trial terminated early: no \\
\hline & Trial ID: - \\
\hline \multirow[t]{3}{*}{ Publication details } & Language of publication: English \\
\hline & $\begin{array}{l}\text { Funding: part funded by the Department of Nutrition and Dietetics Graduate programme (non-com- } \\
\text { mercial) }\end{array}$ \\
\hline & Publication status: peer-reviewed journal \\
\hline Stated aim for study & $\begin{array}{l}\text { Quote from publication: "In this context, the aim of the present study was to evaluate the effectiveness } \\
\text { of involving parents in an intense childhood obesity programme involving lifestyle intervention based } \\
\text { on cognitive behavioral therapy (CBT) principles and assigning high self-management to the children" }\end{array}$ \\
\hline Notes & - \\
\hline
\end{tabular}

\section{Risk of bias}

Diet, physical activity and behavioural interventions for the treatment of overweight or obese children from the age of 6 to 11 years 
Bathrellou 2010 (Continued)

\begin{tabular}{lll} 
Bias & Authors' judgement & Support for judgement \\
\hline $\begin{array}{l}\text { Random sequence genera- } \\
\text { tion (selection bias) }\end{array}$ & Unclear risk & Comment: no description of randomisation method
\end{tabular}

Allocation concealment Unclear risk Comment: unclear if allocation was concealed

(selection bias)

Blinding of participants Unclear risk Comment: unclear if participant and study personnel were blinded
and personnel (perfor-
mance bias)
Objective outcomes

\begin{tabular}{ll}
\hline Blinding of outcome as- \\
sessment (detection bias)
\end{tabular}$\quad$ Unclear risk Comment: unclear if outcome assessors were blinded

Objective outcomes

\begin{tabular}{|c|c|c|}
\hline $\begin{array}{l}\text { Incomplete outcome data } \\
\text { (attrition bias) } \\
\text { Objective outcomes }\end{array}$ & High risk & $\begin{array}{l}\text { Quote from publication: "Although most children attended the intensive } \\
\text { phase of the intervention ( } 88 \%) \text {, only three quarters of the children completed } \\
\text { all stages of the } 18 \text {-month follow-up assessment." }\end{array}$ \\
\hline
\end{tabular}

Comment: relatively high dropout rates at the end of the follow-up

\begin{tabular}{ll}
\hline $\begin{array}{l}\text { Selective reporting (re- } \\
\text { porting bias) }\end{array}$ & $\begin{array}{l}\text { Comment: methods paper lists a number of outcomes they plan to measure } \\
\text { including diet, physical activity, biochemical \& metabolic and psychological } \\
\text { measures. However, in the results of the publication only BMI and percent } \\
\text { overweight are mentioned - and only percent overweight results are given (in } \\
\text { graph), not BMI - potential reporting bias }\end{array}$ \\
\hline
\end{tabular}

Other bias Unclear risk Comment: unclear if the study was at risk of any other bias

Berry 2007

\begin{tabular}{|c|c|}
\hline Methods & $\begin{array}{l}\text { Cluster RCT } \\
\text { Randomisation ratio: 1:1 } \\
\text { Superiority design }\end{array}$ \\
\hline Participants & $\begin{array}{l}\text { Inclusion criteria: children aged } 7-17 \text { years who assented, children whose BMI > 85th percentile (CDC } \\
\text { growth charts), parents who consented and had a BMI > 25, English or Spanish speaking parents and } \\
\text { children, any ethnic group (white, black or Hispanic), no major diagnosis that would affect participa- } \\
\text { tion } \\
\text { Exclusion criteria: - } \\
\text { Diagnostic criteria: see above }\end{array}$ \\
\hline Interventions & $\begin{array}{l}\text { Number of study centres: } 1 \\
\text { Run-in period: no } \\
\text { Extension period: no } \\
\text { Intervention: nutrition and exercise education programme (NEEP) plus coping skills training (CPT) } \\
\text { Comparator: nutrition and exercise education programme (NEEP) only }\end{array}$ \\
\hline
\end{tabular}

Diet, physical activity and behavioural interventions for the treatment of overweight or obese children from the age of 6 to 11 years 
Berry 2007 (Continued)

Outcomes

Outcome measures reported in abstract: BMI, body fat percentage, pedometer steps, parental behaviour outcomes

Study details

\section{Trial terminated early: no}

Trial ID: -

\begin{tabular}{ll}
\hline Publication details & Language of publication: English \\
& Funding: research grants (non-commercial) \\
& Publication status: peer-reviewed journal \\
\hline Stated aim for study & $\begin{array}{l}\text { Quote from publication: "The purpose of this pilot study was to determine the effects of the addition of } \\
\text { coping skills training for obese multiethnic parents whose overweight children were attending a weight } \\
\text { management program." }\end{array}$
\end{tabular}

Notes -

\section{Risk of bias}

Bias Authors' judgement Support for judgement

Random sequence genera- Low risk $\begin{array}{ll}\text { tion (selection bias) } & \text { ed to join the study, they were randomized by class, using the "sealed enve- } \\ \text { lope technique" in blocks of 8-10 parent-child dyads to either the experimental }\end{array}$ group or the control group"

Comment: adequate randomisation method

\begin{tabular}{|c|c|c|}
\hline $\begin{array}{l}\text { Allocation concealment } \\
\text { (selection bias) }\end{array}$ & Low risk & $\begin{array}{l}\text { Quote from publication: "sealed envelope technique" } \\
\text { Comments: it's likely allocation was concealed }\end{array}$ \\
\hline $\begin{array}{l}\text { Blinding of participants } \\
\text { and personnel (perfor- } \\
\text { mance bias) } \\
\text { Subjective outcomes }\end{array}$ & Low risk & $\begin{array}{l}\text { Comment: study author confirmed via email that participants and personnel } \\
\text { were blinded }\end{array}$ \\
\hline $\begin{array}{l}\text { Blinding of participants } \\
\text { and personnel (perfor- } \\
\text { mance bias) } \\
\text { Objective outcomes }\end{array}$ & Low risk & $\begin{array}{l}\text { Comment: study author confirmed via email that participants and personnel } \\
\text { were blinded }\end{array}$ \\
\hline $\begin{array}{l}\text { Blinding of outcome as- } \\
\text { sessment (detection bias) } \\
\text { Subjective outcomes }\end{array}$ & Low risk & $\begin{array}{l}\text { Quote from publication: "Trained research assistants blinded to the study } \\
\text { group collected clinical and psychosocial data" } \\
\text { Comment: outcome assessors were blinded }\end{array}$ \\
\hline $\begin{array}{l}\text { Blinding of outcome as- } \\
\text { sessment (detection bias) } \\
\text { Objective outcomes }\end{array}$ & Low risk & $\begin{array}{l}\text { Quote from publication: "Trained research assistants blinded to the study } \\
\text { group collected clinical and psychosocial data" } \\
\text { Comments: outcome assessors were blinded }\end{array}$ \\
\hline $\begin{array}{l}\text { Incomplete outcome data } \\
\text { (attrition bias) } \\
\text { Subjective outcomes }\end{array}$ & Unclear risk & $\begin{array}{l}\text { Quote from publication: "dropout rates and loss to follow up were moderate" } \\
\text { Comments: potential attrition bias }\end{array}$ \\
\hline $\begin{array}{l}\text { Incomplete outcome data } \\
\text { (attrition bias) }\end{array}$ & Unclear risk & Quote from publication: "dropout rates and loss to follow up were moderate" \\
\hline
\end{tabular}

Diet, physical activity and behavioural interventions for the treatment of overweight or obese children from the age of 6 to 11 years 
Berry 2007 (Continued)

Objective outcomes Comments: potential attrition bias

Selective reporting (re- Unclear risk Comment: no protocol or clinical trial register entry available
porting bias)

Other bias High risk Comment: was a cluster-RCT and did not adjust for clustering in their analyses

Berry 2014

Methods

\section{Cluster-RCT}

Randomisation ratio: $1: 1$

Superiority design

Participants Inclusion criteria: children and parents able to speak, read and write in English, children in the 2nd-4th grade (age $7-11$ years), children with a BMI $\geq 85$ th percentile (CDC growth charts), at least 1 biological parent with a BMI $\geq 25 \mathrm{~kg} / \mathrm{m} 2$ and parent must live with the child, child self-consent and parental consent to participate

Exclusion criteria: if parent or child had congenital heart disease, a heart murmur, family history of sudden death or claustrophobia, if parent or child were participating in other weight management programme, Asian descent (due to lower BMI cut-offs for overweight and obesity)

Diagnostic criteria: see above

Interventions

\section{Number of study centres: 8}

Run-in period: no

Extension period: no

Intervention: nutrition and exercise education and coping skills intervention

Comparator: waiting list control, usual care

Outcomes Outcome measures reported in abstract: BMI percentile children, triceps growth rate, subscapular skinfolds growth rate, dietary knowledge, glasses of soda/d, eating, exercise self-efficacy parental BMI, parental triceps growth, parental subscapular skinfolds growth, parental nutrition knowledge, parental exercise knowledge, parental water and unsweetened drinks consumption, parental eating self-efficacy, parental emotional eating self-efficacy, parental exercise self-efficacy

Study details Trial terminated early: no

Trial ID: NCT01378806

\section{Publication details Language of publication: English}

Funding: National Institute of Health and the National Institute of Nursing Research (1R01NR010254-05) (non-commercial)

Publication status: peer-reviewed journal

Stated aim for study Quote from publication: "The purpose of this study was to test a 2-phased nutrition and exercise education, coping skills training, and exercise intervention programme for overweight or obese low-income ethnic minority 2nd to 4 th grade children and their parents in rural North Carolina, USA" 
Berry 2014 (Continued)

Risk of bias

\begin{tabular}{lll}
\hline Bias & Authors' judgement & Support for judgement \\
\hline $\begin{array}{l}\text { Random sequence genera- } \\
\text { tion (selection bias) }\end{array}$ & Unclear risk & $\begin{array}{l}\text { Quote from publication: "Schools were randomized to either the experimen- } \\
\text { tal or the control group for the first enrollment and exchanged conditions for } \\
\text { the second enrolment. The sequence of each school was randomized before } \\
\text { the start of the study and was stratified by county. A total of } 18 \text { months had } \\
\text { passed and the first group had completed their time in the study prior to the } \\
\text { second enrollment in each school. This design preserved a balance of treat- } \\
\text { ment groups within each site to avoid confounding site effects with interven- } \\
\text { tion effects" }\end{array}$
\end{tabular}

Comment: randomisation process described but there were baseline differences likely due to the cluster randomisation - potential bias

\begin{tabular}{|c|c|c|}
\hline $\begin{array}{l}\text { Allocation concealment } \\
\text { (selection bias) }\end{array}$ & Low risk & $\begin{array}{l}\text { Quote from publication: "Participants and staff were blinded to group assign- } \\
\text { ment from enrolment until implementation." }\end{array}$ \\
\hline
\end{tabular}

Comment: allocation was concealed

\begin{tabular}{|c|c|c|}
\hline $\begin{array}{l}\text { Blinding of participants } \\
\text { and personnel (perfor- } \\
\text { mance bias) } \\
\text { Subjective outcomes }\end{array}$ & Low risk & $\begin{array}{l}\text { Comment: study author confirmed via email that participants and personnel } \\
\text { were blinded }\end{array}$ \\
\hline
\end{tabular}

\begin{tabular}{ll}
\hline $\begin{array}{l}\text { Blinding of participants } \\
\text { and personnel (perfor- }\end{array}$ & Low risk
\end{tabular}
and personnel (perforwere blinded

Objective outcomes

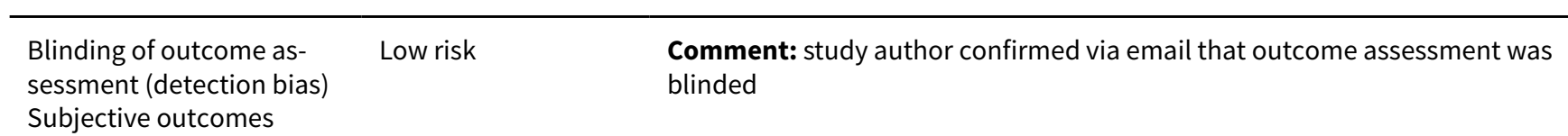

\begin{tabular}{ll}
\hline $\begin{array}{l}\text { Blinding of outcome as- } \\
\text { sessment (detection bias) }\end{array} \quad$ Low risk & $\begin{array}{l}\text { Comment: study author confirmed via email that outcome assessment was } \\
\text { blinded }\end{array}$
\end{tabular}

Sessment (detection

Incomplete outcome data Low risk

(attrition bias)

Subjective outcomes
Quote from publication: "To assess the extent of selection bias owing to attri- tion, the mean values for parent BMI and for child BMI percentiles were com- pared between those participants who did not contribute data beyond the Phase I intervention and those who did. There were no significant differences between these groups, either overall or by experimental group $(P=0.35)$."

Comment: sensitivity analysis performed between completers and dropouts low dropout overall

$\begin{array}{ll}\begin{array}{l}\text { Incomplete outcome data } \\ \text { (attrition bias) }\end{array} & \text { Low risk } \\ \text { Objective outcomes } & \begin{array}{l}\text { Quote from publication: "To assess the extent of selection bias owing to attri- } \\ \text { tion, the mean values for parent BMI and for child BMI percentiles were com- } \\ \text { pared between those participants who did not contribute data beyond the } \\ \text { Phase I intervention and those who did. There were no significant differences } \\ \text { between these groups, either overall or by experimental group }(\mathrm{P}=0.35) . "\end{array}\end{array}$

Comment: sensitivity analysis performed between completers and dropouts low dropout overall

\begin{tabular}{|c|c|c|}
\hline $\begin{array}{l}\text { Selective reporting (re- } \\
\text { porting bias) }\end{array}$ & Low risk & $\begin{array}{l}\text { Comment: no differences found between publication and protocol/clinical tri- } \\
\text { al register entry }\end{array}$ \\
\hline
\end{tabular}

Diet, physical activity and behavioural interventions for the treatment of overweight or obese children from the age of 6 to 11 years 
Berry 2014 (Continued)

Other bias Unclear risk Comment: was a cluster-RCT and adjusted for clustering in their analyses

Boutelle 2014

\begin{tabular}{ll}
\hline Methods & Parallel RCT \\
& Randomisation ratio:1:1 \\
& Superiority design \\
\hline Participants & $\begin{array}{l}\text { Inclusion criteria: overweight children ( } \geq 85 \text { th percentile, CDC growth charts), age } 8 \text { - } 12 \text { years, the chil- } \\
\text { dren ate }>10 \% \text { of their daily caloric intake in the free access paradigm, children must also like cheese } \\
\text { pizza (the dinner provided) }\end{array}$ \\
& $\begin{array}{l}\text { Exclusion criteria: non-English speakers/readers, already participating in a formal weight loss pro- } \\
\text { gramme, have a medical condition or taking medication which could influence growth or weight, and } \\
\text { eating, food allergies or dietary restrictions, having a disability which would prevent them from partici- } \\
\text { pating }\end{array}$
\end{tabular}

Diagnostic criteria: see above

\begin{tabular}{ll}
\hline Interventions & Number of study centres: 1 \\
Run-in period: no & \\
Extension period: no \\
Intervention: regulation of cues (ROC) programme \\
Comparator: usual care control group
\end{tabular}

Outcomes Outcome measures reported in abstract: acceptability ratings, child food responsiveness, eating in the absence of hunger, body weight measures

Study details Trial terminated early: no

Trial ID: NCT01442142

Publication details Language of publication: English

Funding: University of Minnesota, Faculty Development Grant (R01DK094475 and K02HL112042) (noncommercial)

Publication status: peer-reviewed journal

\begin{tabular}{lll}
\hline Stated aim for study & $\begin{array}{l}\text { Quote from publication: "This study evaluated the feasibility, acceptability, and initial efficacy of an in- } \\
\text { tervention based on Schachter's externality theory; the Regulation of Cues (ROC) program." }\end{array}$ \\
\hline Notes & - \\
\hline Risk of bias & Authors' judgement & Support for judgement \\
\hline Bias & Low risk & $\begin{array}{l}\text { Quote from publication: "the project coordinator used a computer-generated } \\
\text { randomization table to assign participants to } 1 \text { of 2 possible treatment condi- } \\
\text { tion (ROC or control) by sex" }\end{array}$
\end{tabular}

Diet, physical activity and behavioural interventions for the treatment of overweight or obese children from the age of 6 to 11 years 78 (Review)

Copyright (c) 2017 The Cochrane Collaboration. Published by John Wiley \& Sons, Ltd. 
Boutelle 2014 (Continued)

Comment: randomisation method well described

Allocation concealment Low risk Comment: study author confirmed via email that allocation was concealed
(selection bias)

\begin{tabular}{|c|c|c|}
\hline $\begin{array}{l}\text { Blinding of participants } \\
\text { and personnel (perfor- } \\
\text { mance bias) } \\
\text { Subjective outcomes }\end{array}$ & High risk & $\begin{array}{l}\text { Comment: study author confirmed via email that participants were not blind- } \\
\text { ed }\end{array}$ \\
\hline
\end{tabular}

Blinding of participants High risk and personnel (performance bias)

Objective outcomes
Comment: study author confirmed via email that participants were not blinded

\begin{tabular}{|c|c|c|}
\hline $\begin{array}{l}\text { Blinding of outcome as- } \\
\text { sessment (detection bias) }\end{array}$ & Low risk & $\begin{array}{l}\text { Comment: study author confirmed via email that outcome assessment was } \\
\text { blinded }\end{array}$ \\
\hline
\end{tabular}

Subjective outcomes

\begin{tabular}{lll}
\hline $\begin{array}{l}\text { Blinding of outcome as- } \\
\text { sessment (detection bias) } \\
\text { Objective outcomes }\end{array}$ & Low risk & $\begin{array}{l}\text { Comment: study author confirmed via email that outcome assessment was } \\
\text { blinded }\end{array}$
\end{tabular}

Incomplete outcome data Low risk

(attrition bias)

Quote from publication: "As can be seen in Figure 1, treatment completion

Subjective outcomes rate was high for the ROC intervention"

Comment: $95 \%$ and $82 \%$ of intervention and control group completed the follow-up - relatively low dropout rates

\begin{tabular}{|c|c|c|}
\hline $\begin{array}{l}\text { Incomplete outcome data } \\
\text { (attrition bias) } \\
\text { Objective outcomes }\end{array}$ & Low risk & $\begin{array}{l}\text { Quote from publication: "As can be seen in Figure 1, treatment completion } \\
\text { rate was high for the ROC intervention" } \\
\text { Comment: } 95 \% \text { and } 82 \% \text { of intervention and control group completed the fol- } \\
\text { low-up - relatively low dropout rates }\end{array}$ \\
\hline
\end{tabular}

\begin{tabular}{|c|c|c|}
\hline $\begin{array}{l}\text { Selective reporting (re- } \\
\text { porting bias) }\end{array}$ & Unclear risk & $\begin{array}{l}\text { Comment: clinical trial entry reports that there were three intervention } \\
\text { groups and } 1 \text { control group; however, there is only } 1 \text { intervention group in the } \\
\text { publication }\end{array}$ \\
\hline
\end{tabular}

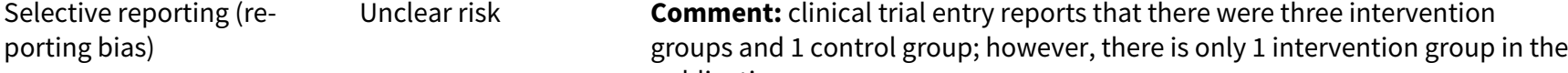

Other bias Unclear risk Comment: unclear if the study was at risk of any other bias

\section{Bryant 2011}

\begin{tabular}{|c|c|}
\hline Methods & $\begin{array}{l}\text { Parallel RCT } \\
\text { Randomisation ratio: 1:1 } \\
\text { Superiority design }\end{array}$ \\
\hline Participants & $\begin{array}{l}\text { Inclusion criteria: aged 8-16 years, BMI > 98th percentile (UK } 1990 \text { growth references), parent or carer } \\
\text { who spoke fluent English } \\
\text { Exclusion criteria: a medical cause for obesity, severe learning difficulties, significant medical or psy- } \\
\text { chiatric problems, siblings already enrolled in the study } \\
\text { Diagnostic criteria: see above }\end{array}$ \\
\hline Interventions & Number of study centres: 1 \\
\hline
\end{tabular}

Diet, physical activity and behavioural interventions for the treatment of overweight or obese children from the age of 6 to 11 years 
Bryant 2011 (Continued)

\section{Run-in period: no}

Extension period: no

Intervention: WATCH IT intervention

Comparator: waiting-list control

\begin{tabular}{ll}
\hline Outcomes & Outcome measures reported in abstract: recruitment, blinding succe \\
\hline Study details & Trial terminated early: no \\
& Trial ID: ISRCTN95431788 \\
\hline Publication details & Language of publication: English \\
& Funding: the Wellcome Trust Ltd. (078174/Z05/Z) (non-commercial) \\
& Publication status: peer-reviewed journal
\end{tabular}

$\begin{array}{ll}\text { Stated aim for study } & \begin{array}{l}\text { Quote from publication: "Our aim was to conduct a feasibility trial of the evaluation of WATCH IT, a } \\ \text { community obesity intervention for children and adolescents" }\end{array}\end{array}$

Notes -

\section{Risk of bias}

Bias Authors' judgement Support for judgement

Random sequence genera- Low risk tion (selection bias)

\begin{abstract}
Quote from publication: "After informed consent (parental consent and child assent) and baseline assessment, participants were randomised to either WATCH IT or a waiting list control for 12 months using a remote automated telephone randomisation system. Randomisation was stratified by BMI standard deviation score (SDS; $\leq 3.0$ vs. $>3.0$ ), age ( $\leq 12$ years vs. $>12$ years), gender, and maternal level of education (less than General Certificate of Secondary Education (GCSE) or equivalent (attainment reached at the age of 16 years) vs. higher)."
\end{abstract}

Comment: randomisation method well described

\begin{tabular}{|c|c|c|}
\hline $\begin{array}{l}\text { Allocation concealment } \\
\text { (selection bias) }\end{array}$ & Low risk & Comment: allocation was concealed (as confirmed by study author) \\
\hline $\begin{array}{l}\text { Blinding of participants } \\
\text { and personnel (perfor- } \\
\text { mance bias) } \\
\text { Subjective outcomes }\end{array}$ & High risk & $\begin{array}{l}\text { Quote from publication: "An assessor-blinded randomised controlled feasi- } \\
\text { bility trial" } \\
\text { Comment: participants and study personnel were not blinded }\end{array}$ \\
\hline $\begin{array}{l}\text { Blinding of participants } \\
\text { and personnel (perfor- } \\
\text { mance bias) } \\
\text { Objective outcomes }\end{array}$ & High risk & $\begin{array}{l}\text { Quote from publication: "An assessor-blinded randomised controlled feasi- } \\
\text { bility trial" } \\
\text { Comment: participants and study personnel were not blinded }\end{array}$ \\
\hline $\begin{array}{l}\text { Blinding of outcome as- } \\
\text { sessment (detection bias) } \\
\text { Subjective outcomes }\end{array}$ & Low risk & $\begin{array}{l}\text { Quote from publication: "Follow-up assessments performed after randomi- } \\
\text { sation were conducted by assessors who were blinded to the treatment alloca- } \\
\text { tion for each family." } \\
\text { Comment: outcome assessment was blinded }\end{array}$ \\
\hline
\end{tabular}


Bryant 2011 (Continued)

Blinding of outcome as- Low risk Quote from publication: "Follow-up assessments performed after randomisessment (detection bias) sation were conducted by assessors who were blinded to the treatment allocaObjective outcomes tion for each family."

Comment: outcome assessment was blinded

Incomplete outcome data Unclear risk (attrition bias)

Subjective outcomes
Quote from publication: "Retention strategies were not formalised within the protocol, but we had an acceptable level of dropout ( $24 \%$ withdrawal overall)."

Comment: $75.7 \%$ follow-up - some losses to follow-up

Incomplete outcome data Unclear risk

Objective outcomes
Quote from publication: "Retention strategies were not formalised within the protocol, but we had an acceptable level of dropout ( $24 \%$ withdrawal overall)."

Comment: $75.7 \%$ follow-up - some losses to follow-up

\begin{tabular}{lll}
\hline $\begin{array}{l}\text { Selective reporting (re- } \\
\text { porting bias) }\end{array}$ & Unclear risk & $\begin{array}{l}\text { Comment: clinical trial entry retrospectively entered. Also, publication speci- } \\
\text { fies this study was a feasibility study - hence, it doesn't report results of some } \\
\text { of the outcome measures, e.g. HRQoL }\end{array}$ \\
\hline Other bias & Unclear risk & Comment: unclear if the study was at risk of any other bias \\
\hline
\end{tabular}

Coppins 2011

Cross-over RCT (however, analysed as a parallel RCT)
Randomisation ratio: 1:1
Superiority design
Non-inferiority design: (specify 1- or 2-sided confidence interval)
Equivalence design: (specify 1- or 2-sided confidence interval)
Controlled clinical trial (CCT)

Inclusion criteria: BMI > 91st centile (SIGN 2010 guidelines), children with intellectual disability were
included if they were judged to be able to participate in the intervention, age 6-14 years

Exclusion criteria: medical conditions which might impede physical activity - GPs were asked to notify the dietitian of such conditions (none were disclosed)

Diagnostic criteria: see above

Number of study centres: 1
Run-in period: no
Extension period: no
Intervention: multi-component family-focused education package
Comparator: waiting-list control

Outcomes Composite outcome measures reported: BMI z scores, weight, attendance


Coppins 2011 (Continued)

Trial ID: ISRCTN55734850

\section{Publication details Language of publication: English}

Funding: Wessex Medical Research and The Public Health Department in States of Jersey funded the project. Department of Education, Sports and Culture, States of Jersey funded all the activities. The Channel Islands Co-op funded the food for all the healthy eating workshops; and Jersey Bowl sponsored the Family Project Xmas party (non-commercial)

Publication status: peer-reviewed journal

\begin{tabular}{ll} 
Stated aim for study & $\begin{array}{l}\text { Quote from publication: "To determine if a multi-component family focused education package is more } \\
\text { effective than a waiting list control group in treating overweight and obese children" }\end{array}$ \\
\hline Notes & $\begin{array}{l}\text { Participants in the intervention and control groups crossed over into the other condition after } 12 \\
\text { months - however, in the publication results are presented as if the trial was a parallel RCT. Hence, re- } \\
\text { sults are presented up to } 12 \text { months before the crossover }\end{array}$
\end{tabular}

\section{Risk of bias}

\begin{tabular}{|c|c|c|}
\hline Bias & Authors' judgement & Support for judgement \\
\hline \multirow[t]{2}{*}{$\begin{array}{l}\text { Random sequence genera- } \\
\text { tion (selection bias) }\end{array}$} & Unclear risk & $\begin{array}{l}\text { Quote from publication: from author (via email): "Simple random test/con- } \\
\text { trol each time a patient came forward." }\end{array}$ \\
\hline & & Comment: unclear if this method would have introduced bias \\
\hline $\begin{array}{l}\text { Allocation concealment } \\
\text { (selection bias) }\end{array}$ & Low risk & Comment: allocation was concealed (as confirmed by study author) \\
\hline $\begin{array}{l}\text { Blinding of participants } \\
\text { and personnel (perfor- } \\
\text { mance bias) }\end{array}$ & High risk & $\begin{array}{l}\text { Quote from publication: "A waiting list control group may also not have been } \\
\text { the best comparison, as enrolment into the study may have had a placebo ef- } \\
\text { fect." "The lead investigator was also not blind to treatment allocation" }\end{array}$ \\
\hline Subjective outcor & & Comment: participants and study personnel were not blinded \\
\hline
\end{tabular}

\begin{tabular}{|c|c|c|}
\hline $\begin{array}{l}\text { Blinding of participants } \\
\text { and personnel (perfor- } \\
\text { mance bias) }\end{array}$ & High risk & $\begin{array}{l}\text { Quote from publication: "A waiting list control group may also not have been } \\
\text { the best comparison, as enrolment into the study may have had a placebo ef- } \\
\text { fect." "The lead investigator was also not blind to treatment allocation" }\end{array}$ \\
\hline
\end{tabular}

Comment: participants and study personnel were not blinded

\begin{tabular}{lll}
\hline Blinding of outcome as- & High risk & $\begin{array}{l}\text { Quote from publication: A waiting list control group may also not have been } \\
\text { the best comparison, as enrolment into the study may have had a placebo ef- } \\
\text { sessment (detection bias) }\end{array}$ \\
$\begin{array}{l}\text { Subjective outcomes } \\
\text { fect." "The lead investigator was also not blind to treatment allocation" }\end{array}$
\end{tabular}

Comment: assume assessors were not blinded either

Blinding of outcome as- High risk sessment (detection bias) Objective outcomes
Quote from publication: "A waiting list control group may also not have been the best comparison, as enrolment into the study may have had a placebo effect." "The lead investigator was also not blind to treatment allocation"

Comment: assume assessors were not blinded either
Incomplete outcome data Low risk (attrition bias)

Subjective outcomes
Quote from publication: "After the study was completed, we calculated the actual power of the study for an effect size of 0.3 for BMI SDS and it was about $60 \%$."

Comment: dropout rates were low 
Coppins 2011 (Continued)

Incomplete outcome data Low risk Quote from publication: "After the study was completed, we calculated the (attrition bias)

Objective outcomes actual power of the study for an effect size of 0.3 for BMI SDS and it was about 60\%."

Comment: dropout rates were low
Selective reporting (re- Unclear risk Comment: potential selective reporting as lifestyle outcomes only briefly reporting bias)

Other bias Unclear risk ported with significant or not significant $P$ values

Croker 2012

Methods

\section{Parallel RCT}

Randomisation ratio: 1:1

Superiority design

Participants

Inclusion criteria: 8-12 years, overweight or obese (IOTF definition), at least 1 parent/guardian willing to participate in the intervention, parent and child could speak English well enough to take part in the groups and understand the materials

Exclusion criteria: had an identified medical cause for obesity (e.g. hypothyroidism, Prada Willi syndrome), had type 2 diabetes, taking obesity medication, undergoing obesity treatment, had significant learning difficulties, the parent or child had significant mental health problems, were currently receiving psychological or psychiatric treatment including psychotropic medication

Diagnostic criteria: see above

Interventions

Number of study centres: 1

Run-in period: no

Extension period: no

Intervention: family-based behavioural treatment (FBBT)

Comparator: waiting-list control

Outcomes Outcome measures reported in abstract: BMI SDS, BMI, systolic blood pressure, QoL, eating attitudes, body composition, psychosocial outcomes, adverse events

\section{Study details Trial terminated early: no}

Trial ID: ISRCTN51382628

\section{Publication details Language of publication: English}

Funding: Cancer Research UK, Great Ormond Street Hospital and Weight Concern (non-commercial)

Publication status: peer-reviewed journal

Stated aim for study

Quote from publication: "To examine the acceptability and effectiveness of 'family-based behavioural treatment' (FBBT) for childhood obesity in an ethnically and socially diverse sample of families in a UK National Health Service (NHS) setting"

Diet, physical activity and behavioural interventions for the treatment of overweight or obese children from the age of 6 to 11 years 
Croker 2012 (Continued)

Notes

\section{Risk of bias}

Bias Authors' judgement Support for judgement

Random sequence genera- Low risk tion (selection bias)
Quote from publication: "Randomisation was carried out by a statistician; each child was given an ID code, and computer-generated random numbers were used to allocate them to a treatment condition."

Comment: low risk of selection bias from randomisation method described
Quote from publication: (from author via email): "allocation was not known until they were randomised. This was a group programme and we randomised in waves, so waited until we had recruited enough families to run a treatment group. Families were informed of their group allocation as soon as they had been randomised."

Comment: allocation was concealed (as confirmed by author)

$\begin{array}{lll}\begin{array}{l}\text { Blinding of participants } \\ \text { and personnel (perfor- }\end{array} & \text { High risk } & \begin{array}{l}\text { Quote from publication: "It was not possible to blind families or clinicians to } \\ \text { treatment allocation because of the nature of the intervention" }\end{array}\end{array}$

mance bias)

Subjective outcomes

Comment: participants and study personnel were not blinded

$\begin{array}{lll}\begin{array}{l}\text { Blinding of participants } \\ \text { and personnel (perfor- }\end{array} & \text { High risk } & \begin{array}{l}\text { Quote from publication: "It was not possible to blind families or clinicians to } \\ \text { treatment allocation because of the nature of the intervention" }\end{array}\end{array}$

mance bias)

Objective outcomes

Comment: participants and study personnel were not blinded

Blinding of outcome as- Unclear risk sessment (detection bias)

Subjective outcomes

Quote from publication: "the researcher collecting anthropometric data was blinded to group allocation unless families disclosed this information"

Comment: unclear if subjective outcomes were measured by a researcher who was blinded to the study group (only mentions anthropometric data which was an objective outcome)

Blinding of outcome as- $\quad$ Low risk
sessment (detection bias)

Objective outcomes

Quote from publication: "the researcher collecting anthropometric data was blinded to group allocation unless families disclosed this information"

Comment: outcome assessors measuring objective measures (anthropometric data) were blinded to study group

Incomplete outcome data High risk

(attrition bias)

Quote from publication: "22 of the children randomised to the treatment group completed the 6 month intervention (59\% of those randomised and $73 \%$ of those starting treatment)"

Comment: high dropout in the intervention group. Missing data replaced by baseline carried forward which is a highly criticised method

\section{Incomplete outcome data High risk}

(attrition bias)

Objective outcomes

Quote from publication: "22 of the children randomised to the treatment group completed the 6 month intervention (59\% of those randomised and $73 \%$ of those starting treatment)"

Comment: high dropout in the intervention group. Missing data replaced by baseline carried forward which is a highly criticised method

\begin{tabular}{|c|c|c|}
\hline $\begin{array}{l}\text { Selective reporting (re- } \\
\text { porting bias) }\end{array}$ & High risk & $\begin{array}{l}\text { Comment: potential reporting bias as study trial register states they aimed to } \\
\text { measure additional outcomes not reported in this publication }\end{array}$ \\
\hline
\end{tabular}

Diet, physical activity and behavioural interventions for the treatment of overweight or obese children from the age of 6 to 11 years 
Croker 2012 (Continued)

Other bias Unclear risk Comment: unable to assess if any other biases present

Davis 2013

$\begin{array}{ll}\text { Methods } & \text { Parallel RCT } \\ & \text { Randomisation ratio: } 1: 1 \\ & \text { Superiority design }\end{array}$

Participants

Inclusion criteria: criteria for school participation included having rural designation (in a town or county with a population $<20,000$ ) and telemedicine capabilities (common in rural districts for distance learning), child living in rural Kansas and attending elementary school, child being overweight/obese for age/gender ( $\geq 85$ th percentile, CDC growth charts), parent able to speak English

Exclusion criteria: developmental disability preventing child from participating, being immobile and preventing the child from increasing exercise

Diagnostic criteria: see above

\begin{tabular}{ll}
\hline Interventions & Number of study centres: 1 for each study \\
Run-in period: no \\
Extension period: no \\
Intervention: telemedicine intervention \\
Comparator: physician-visit intervention
\end{tabular}

Outcomes

Composite outcome measures reported: BMI z, dietary behaviours, physical activity behaviours

Study details Trial terminated early: no

Trial ID: -

\section{Publication details Language of publication: English}

Funding: National Institutes of Health (DK068221) (non-commercial)

Publication status: peer-reviewed journal

Stated aim for study

Quote from publication: "The objective of the current study was to examine the effectiveness of a multidisciplinary weekly family-based behavioral group delivered via telemedicine to rural areas, compared with a standard physician visit intervention"

\begin{tabular}{lll}
\hline Notes & - & \\
\hline Risk of bias & Authors' judgement & Support for judgement \\
\hline Bias & Low risk & $\begin{array}{l}\text { Quote from publication: "Children within each school were ranked based } \\
\text { on an obesity factor (child BMI percentile plus primary parent BMI and strati- } \\
\text { fied based on a household factor (single or dual parent household), and gen- } \\
\text { dion (selection bias) } \\
\text { der, according to previous research, which indicates these factors are closely } \\
\text { linked to obesity and to treatment outcome. One child from each stratification } \\
\text { was then randomly assigned (via a random numbers table) to the telemedicine }\end{array}$
\end{tabular}


Davis 2013 (Continued)

intervention (TM) with the other half of the pair being assigned to the physician visits (PV) intervention."

Comment: low risk of selection bias from randomisation method described

\begin{tabular}{lll}
\hline $\begin{array}{l}\text { Allocation concealment } \\
\text { (selection bias) }\end{array}$ & Low risk & Comment: author confirmed allocation was concealed via email contact \\
\hline $\begin{array}{l}\text { Blinding of participants } \\
\text { and personnel (perfor- } \\
\text { mance bias) }\end{array}$ & Unclear risk & $\begin{array}{l}\text { Quote from publication: (from study author via email) "participants were } \\
\text { blinded, and assessment personnel were blinded. Intervention personnel were } \\
\text { not blinded." }\end{array}$
\end{tabular}

Subjective outcomes

Comment: participants were blinded but study personnel were not

\begin{tabular}{|c|c|c|}
\hline $\begin{array}{l}\text { Blinding of participants } \\
\text { and personnel (perfor- } \\
\text { mance bias) }\end{array}$ & Unclear risk & $\begin{array}{l}\text { Quote from publication: (from study author via email) "participants were } \\
\text { blinded, and assessment personnel were blinded. Intervention personnel were } \\
\text { not blinded." }\end{array}$ \\
\hline
\end{tabular}

Objective outcomes

Comment: participants were blinded but study personnel were not

\begin{tabular}{|c|c|c|}
\hline $\begin{array}{l}\text { Blinding of outcome as- } \\
\text { sessment (detection bias) }\end{array}$ & Low risk & $\begin{array}{l}\text { Quote from publication: (from author via email): "Yes, the assessment staff } \\
\text { were blinded." }\end{array}$ \\
\hline
\end{tabular}

Comment: assessment staff were blinded to study group

\begin{tabular}{ll}
\hline $\begin{array}{l}\text { Blinding of outcome as- } \\
\text { sessment (detection bias) }\end{array} \quad$ Low risk & $\begin{array}{l}\text { Quote from publication: (from study author via email): "Yes, the assessment } \\
\text { staff were blinded." }\end{array}$
\end{tabular}

Objective outcomes

Comment: participants were blinded but study personnel were not

\begin{tabular}{lll}
\hline Incomplete outcome data & Unclear risk & Quote from publication: · "In terms of other outcome measures, attrition was \\
(attrition bias) & not significantly different by group, but there was a trend for slightly higher at- \\
Subjective outcomes & trition in the TM group compared with the PV group."
\end{tabular}

(attrition bias)
Subjective outcomes

Comment: potential attrition bias due to moderate dropout rates in intervention group

\begin{tabular}{|c|c|c|}
\hline $\begin{array}{l}\text { Incomplete outcome data } \\
\text { (attrition bias) } \\
\text { Objective outcomes }\end{array}$ & Unclear risk & $\begin{array}{l}\text { Quote from publication: "In terms of other outcome measures, attrition was } \\
\text { not significantly different by group, but there was a trend for slightly higher at- } \\
\text { trition in the TM group compared with the PV group." }\end{array}$ \\
\hline
\end{tabular}

Comment: potential attrition bias due to moderate dropout rates in intervention group

Selective reporting (re- Low risk Comment: no differences between protocol and publication found
porting bias)

Other bias Unclear risk Comment: unable to assess if any other biases present

\begin{tabular}{ll} 
Davoli 2013 & \\
\hline Methods & Parallel RCT \\
& Randomisation ratio: $1: 1$ \\
& Superiority design \\
\hline Participants & $\begin{array}{l}\text { Inclusion criteria: overweight children ( } \geq 85 \text { th BMI percentile but } \leq 95 \text { th }- \text { CDC growth charts), age 4-7 } \\
\text { years, live in the Reggio Emilia Province and assisted by that paediatrician for at least } 12 \text { months }\end{array}$ \\
\hline
\end{tabular}

Diet, physical activity and behavioural interventions for the treatment of overweight or obese children from the age of 6 to 11 years 
Exclusion criteria: metabolic pathologic conditions and all pathologic conditions related to overweight and obesity, families who did not consider childhood overweight/obesity being a problem and were not interested in advice to lose weight

Diagnostic criteria: see above

\begin{tabular}{ll}
\hline Interventions & Number of study centres: 69 (paediatricians working from their own centres in Reggio Emilia) \\
Run-in period: no & Extension period: no \\
Intervention: family paediatrician-led motivational interviewing & Comparator: usual care plus a booklet on obesity prevention \\
\hline Outcomes & Outcome measures reported in abstract: attendance, BMI, parent-reported lifestyle behaviours \\
\hline Study details & Trial terminated early: no \\
& Trial ID: NCT01822626 \\
\hline Publication details & $\begin{array}{l}\text { Language of publication: English } \\
\text { Punding: no external funding (non-commercial) }\end{array}$ \\
\hline Stated aim for study & $\begin{array}{l}\text { Quote from publication: "The aim of this study was to evaluate the effect of family pediatrician-led mo- } \\
\text { tivational interviews (MIs) on BMI of overweight (85th } \geq B M I \text { percentile } \leq 95 \text { th) children aged } 4 \text { to } 7 \text { years" } \\
\text { "The objective of the current study was to examine the effectiveness of a multidisciplinary weekly fam- } \\
\text { ily-based behavioral group delivered via telemedicine to rural areas, compared with a standard physi- } \\
\text { cian visit intervention" }\end{array}$ \\
\hline
\end{tabular}

\section{Notes}

\section{Risk of bias}

Bias Authors' judgement Support for judgement

Random sequence genera- Low risk tion (selection bias)
Quote from publication: "Eligible children whose parents signed the informed consent form were centrally allocated to intervention or control groups according to a randomization list created by the Epidemiology Unit by using the package RALLOC (Stata version 11.0; Stata Corp, College Station, TX)" "Due to the practical constraints of a maximum of 3 treated children per pediatrician, different allocation rules were used according to the number of eligible children. To balance allocation within strata, observations were opportunely weighted"

Comment: low risk of selection bias from randomisation method described

Allocation concealment Low risk
(selection bias)

Quote from publication: "Each paediatrician was informed of the group allocation by means of a corporate Intranet Web form customized for the trial (Supplemental Tutorial)."

Comment: allocation likely concealed

Blinding of participants High risk
and personnel (perfor-
mance bias)

Quote from publication: "The primary outcome was the individual variation of BMI, assessed by paediatricians unblinded to treatment groups."

Comment: unlikely that participants and study personnel were blinded

Diet, physical activity and behavioural interventions for the treatment of overweight or obese children from the age of 6 to 11 years 
Davoli 2013 (Continued)

Subjective outcomes

$\begin{array}{lll}\begin{array}{l}\text { Blinding of participants } \\ \text { and personnel (perfor- }\end{array} & \text { High risk } & \begin{array}{l}\text { Quote from publication: "The primary outcome was the individual variation } \\ \text { of BMI, assessed by paediatricians unblinded to treatment groups." }\end{array}\end{array}$

mance bias)

Objective outcomes

Comment: unlikely that participants and study personnel were blinded

Blinding of outcome as- High risk sessment (detection bias)

Subjective outcomes
Quote from publication: "Both primary and secondary outcomes were assessed by the pediatricians without any blinding."

Comment: assessment staff were not blinded to study group
Blinding of outcome as- High risk

sessment (detection bias)

Objective outcomes
Quote from publication: "Both primary and secondary outcomes were assessed by the pediatricians without any blinding."

Comment: assessment staff were not blinded to study group
Incomplete outcome data Low risk

(attrition bias)

Subjective outcomes
Quote from publication: "Compliance to the 1-year intervention was high, even for a population-based study involving almost all the pediatricians in the RE Province and a relevant sample of their overweight patients"

Comment: $95 \%$ of participants completed the 1-year intervention - dropout low

\begin{tabular}{|c|c|c|}
\hline $\begin{array}{l}\text { Incomplete outcome data } \\
\text { (attrition bias) } \\
\text { Objective outcomes }\end{array}$ & Low risk & $\begin{array}{l}\text { Quote from publication: "Compliance to the } 1 \text {-year intervention was high, } \\
\text { even for a population-based study involving almost all the pediatricians in the } \\
\text { RE Province and a relevant sample of their overweight patients" } \\
\text { Comment: } 95 \% \text { of participants completed the } 1 \text { year intervention - dropout } \\
\text { low }\end{array}$ \\
\hline $\begin{array}{l}\text { Selective reporting (re- } \\
\text { porting bias) }\end{array}$ & Low risk & Comment: no differences between protocol and publication found \\
\hline Other bias & Unclear risk & Comment: unable to assess if any other biases present \\
\hline
\end{tabular}

de Niet 2012

\begin{tabular}{ll}
\hline Methods & Parallel RCT \\
& Randomisation ratio: $1: 1$ \\
& Superiority design
\end{tabular}

Participants

Inclusion criteria: overweight or obese (defined by Cole 2000 international survey), parent participation in the BFC (behavioural lifestyle treatment), sufficient knowledge of the Dutch language, parent and child fluent in Dutch language and show motivation to the programme (assessed by motivational interviewing)

Exclusion criteria: behavioural programmes (score $>70$ on Child Behaviour Checklist (CBCL), any disease causing overweight that can be treated with drugs, mental retardation

Diagnostic criteria: see above

Interventions

Number of study centres: 8

Run-in period: no

Extension period: no

Diet, physical activity and behavioural interventions for the treatment of overweight or obese children from the age of 6 to 11 years 
de Niet 2012 (Continued)

Treatment before study : all participants took part in 3 months of behavioural lifestyle treatment Intervention: short message service maintenance treatment and behavioural lifestyle treatment

Comparator: behavioural lifestyle treatment only

Outcomes Composite outcome measures reported: physical health scores, number of SMS sent, weight loss,
$\mathrm{BMI}$, dropout rates

Study details $\quad$ Trial terminated early: no

Trial ID: ISRCTN33476574

\section{Publication details Language of publication: English}

Funding: Vodafone (the Netherlands), and grants were received from the Erasmus University Medical Centre Rotterdam - MRACE (Medical Research Advice Committee) grant no. 2006-26 and Innovation Fund Insurances (Innovatiefonds Verzekeringen)

grant no. 06-334 (commercial and non-commercial)

Publication status: peer-reviewed journal

Stated aim for study Quote from publication: "The effect of a short message service maintenance treatment on body mass index and psychological well-being in overweight and obese children: a randomized controlled trial"

Notes -

\section{Risk of bias}

Bias Authors' judgement Support for judgement

Random sequence genera- Low risk tion (selection bias)
Quote from publication: "Randomization allocation in a 1:1 ratio was applied in a randomized block design. The blocks were formed by the treatment groups"

Comment: randomisation method described

Allocation concealment Low risk
(selection bias)
Quote from publication: "The randomization allocation was printed on paper in a sealed envelope. An equal number of SMSMT and control notes were put in the envelopes. The researcher randomized the children to the SMSMT or control group by picking an envelope from a basket"

Comment: allocation likely concealed

\begin{tabular}{|c|c|c|}
\hline $\begin{array}{l}\text { Blinding of participants } \\
\text { and personnel (perfor- } \\
\text { mance bias) } \\
\text { Subjective outcomes }\end{array}$ & High risk & $\begin{array}{l}\text { Comment: study author confirmed nobody was blinded to the study group in } \\
\text { the trial }\end{array}$ \\
\hline $\begin{array}{l}\text { Blinding of participants } \\
\text { and personnel (perfor- } \\
\text { mance bias) } \\
\text { Objective outcomes }\end{array}$ & High risk & $\begin{array}{l}\text { Comment: study author confirmed nobody was blinded to the study group in } \\
\text { the trial }\end{array}$ \\
\hline $\begin{array}{l}\text { Blinding of outcome as- } \\
\text { sessment (detection bias) } \\
\text { Subjective outcomes }\end{array}$ & High risk & $\begin{array}{l}\text { Comment: study author confirmed nobody was blinded to the study group in } \\
\text { the trial }\end{array}$ \\
\hline $\begin{array}{l}\text { Blinding of outcome as- } \\
\text { sessment (detection bias) }\end{array}$ & High risk & $\begin{array}{l}\text { Comment: study author confirmed nobody was blinded to the study group in } \\
\text { the trial }\end{array}$ \\
\hline
\end{tabular}

Diet, physical activity and behavioural interventions for the treatment of overweight or obese children from the age of 6 to 11 years (Review)

Copyright @ 2017 The Cochrane Collaboration. Published by John Wiley \& Sons, Ltd. 
de Niet 2012 (Continued)

Objective outcomes

Incomplete outcome data Unclear risk (attrition bias)

Subjective outcomes
Quote from publication: "Only 10 children in the intervention group dropped out of the BFC treatment (14\%) in the period between 3 and 12 months compared to 21 children in the control group (31\%)."

Comment: potential attrition bias as more dropped out in control group
Incomplete outcome data Unclear risk (attrition bias)

Objective outcomes
Quote from publication: "Only 10 children in the intervention group dropped out of the BFC treatment (14\%) in the period between 3 and 12 months compared to 21 children in the control group (31\%)."

Comment: potential attrition bias as more dropped out in control group

\begin{tabular}{|c|c|c|}
\hline $\begin{array}{l}\text { Selective reporting (re- } \\
\text { porting bias) }\end{array}$ & Unclear risk & $\begin{array}{l}\text { Comment: raw data for many outcomes not reported in tables or text but giv- } \\
\text { en in graphs or reported as either significant or non-significant }\end{array}$ \\
\hline
\end{tabular}

Other bias Unclear risk Comment: unable to assess if any other biases were present

Diaz 2010

\begin{tabular}{ll} 
Diaz 2010 & Parallel RCT \\
\hline Methods & Randomisation ratio: 1:1 \\
& Superiority design \\
\hline
\end{tabular}

Participants Inclusion criteria: age 9-17 years, BMI > 95th percentile (CDC growth charts) or BMI > 90th percentile + WC > 90th percentile, willingness to attend the group sessions, caregivers showing an interest in weight control

Exclusion criteria: glucose intolerance of type 2 diabetes, psychiatric disorders, medical condition that would preclude participating in the study, medication that affects weight or involvement in another weight loss programme, participants who had lost weight during the 4 months before the study

Diagnostic criteria: see above

\begin{tabular}{ll}
\hline Interventions & Number of study centres: 1 \\
& Run-in period: no \\
& Extension period: no \\
& Treatment before study : $5 \%$ of volunteers took part in a previous cross-sectional study \\
& Intervention: behavioural curriculum plus registered dieticians and physician consultations \\
& Comparator: physician consultations only \\
\hline Outcomes & Outcome measures reported in abstract: completion rates, body weight, BMI, insulin sensitivity \\
\hline Study details & Trial terminated early: no \\
& Trial ID: - \\
\hline Publication details & $\begin{array}{l}\text { Language of publication: English } \\
\text { Funding: grant from the International Atomic Energy Agency (ARCAL 6/059) and CONACyT (R/182996) } \\
\text { (non-commercial) }\end{array}$
\end{tabular}

Diet, physical activity and behavioural interventions for the treatment of overweight or obese children from the age of 6 to 11 years 90 (Review)

Copyright $\odot 2017$ The Cochrane Collaboration. Published by John Wiley \& Sons, Ltd. 
Diaz 2010 (Continued)

Publication status: peer-reviewed journal

Stated aim for study

Quote from publication: "The main objective of this study was to compare a lifestyle intervention-primary care physician supported by a registered dietitian (RD) and a behavioral curriculum - to a brief primary care physician intervention for treating pediatric obesity in the primary care setting"

\section{Notes}

\section{Risk of bias}

\begin{tabular}{lll}
\hline Bias & Authors' judgement & Support for judgement \\
\hline $\begin{array}{ll}\text { Random sequence genera- } \\
\text { tion (selection bias) }\end{array}$ & Low risk & $\begin{array}{l}\text { Quote from publication: "Once measurements were completed, the study } \\
\text { statistician randomly assigned participants } 1: 1 \text { to the lifestyle intervention or } \\
\text { the control group by simple randomization, stratified according to sex. The } \\
\text { randomization sequence was generated by a computer" }\end{array}$
\end{tabular}

Comment: randomisation method described

\begin{tabular}{lll}
\hline $\begin{array}{l}\text { Allocation concealment } \\
\text { (selection bias) }\end{array}$ & Low risk & Comment: study author confirmed via email that allocation was concealed \\
\hline $\begin{array}{l}\text { Blinding of participants } \\
\text { and personnel (perfor- }\end{array}$ & High risk & $\begin{array}{l}\text { Quote from publication: (from author via email) "Only study personnel who } \\
\text { mance bias) }\end{array}$ \\
$\begin{array}{l}\text { Objective outcomes } \\
\text { personnel who measured body composition by dual-energy x-ray absorptiom- } \\
\text { etry and performed blood work." }\end{array}$
\end{tabular}

Comment: study author confirmed participants were not blinded

$\begin{array}{lll}\text { Blinding of outcome as- } & \text { Low risk } & \text { Quote from publication: (from author via email): "Study personnel who mea- } \\ \text { sessment (detection bias) } & \text { sured the primary outcomes were blinded to group assignments, as were per- } \\ \text { Objective outcomes } & \text { sonnel who measured body composition by dual-energy x-ray absorptiometry } \\ & \text { and performed blood work." }\end{array}$

Comment: those who measured objective outcome were blinded to study group

\begin{tabular}{|c|c|c|}
\hline $\begin{array}{l}\text { Incomplete outcome data } \\
\text { (attrition bias) } \\
\text { Objective outcomes }\end{array}$ & Unclear risk & $\begin{array}{l}\text { Quote from publication: "A limitation of this study was the high attrition } \\
\text { rates" "We also applied an intention-to-treat analysis at } 12 \text { months in the pri- } \\
\text { mary outcomes of the study. Considering the risk of bias of procedures for an- } \\
\text { alyzing incomplete data, we made an effort to obtain the primary outcomes } \\
\text { (weight and BMI) of all participants who dropped out of the study }(n=33) \text { mea- } \\
\text { suring children at their homes. However, we were able to measure the prima- } \\
\text { ry outcomes only in } 23 \text { drop outs. Thus, intention-to-treat analysis included } 66 \\
(87 \%) \text { of the original } 76 \text { randomized participants (lifestyle group, } n=33 \text {; control } \\
\text { group, } n=33) . "\end{array}$ \\
\hline
\end{tabular}

Comment: high risk of bias due to high attrition rates; however, the study authors measured 23/33 dropouts in their own homes and presented this presented this for weight and raw BMI; therefore, rated as unclear due to disparity

Selective reporting (re- Unclear risk Comment: no clinical trial register entry or protocol available
porting bias)

Other bias Unclear risk Comment: unclear if the study was at risk of any other bias


Duffy 1993

\begin{tabular}{ll}
\hline Methods & Parallel RCT \\
& Randomisation ratio: $1: 1$ \\
& Superiority design
\end{tabular}

Participants Inclusion criteria: age $7-13$ years, exceeding $15 \%$ of ideal weight for age, height and sex (reference
used unclear), 1 parent willing to attend sessions

Exclusion criteria: none

Diagnostic criteria: unclear

Number of study centres: 1
Run-in period: no
Extension period: no
Intervention: cognitive self-management training plus behaviour therapy
Comparator: behaviour therapy plus attention placebo control methods

Outcomes Outcome measures reported in abstract: percentage overweight, number of red foods/d

\begin{tabular}{|c|c|c|}
\hline Study details & \multicolumn{2}{|c|}{$\begin{array}{l}\text { Trial terminated early: no } \\
\text { Trial ID: - }\end{array}$} \\
\hline \multirow[t]{3}{*}{ Publication details } & \multirow{3}{*}{\multicolumn{2}{|c|}{$\begin{array}{l}\text { Language of publication: English } \\
\text { Funding: unclear } \\
\text { Publication status: peer-reviewed journal }\end{array}$}} \\
\hline & & \\
\hline & & \\
\hline Stated aim for study & \multicolumn{2}{|c|}{$\begin{array}{l}\text { Quote from publication: "The present study was therefore designed to evaluate the benefits of cog- } \\
\text { nitive self-management techniques in enhancing the effectiveness of a traditional behavioural ap- } \\
\text { proach." }\end{array}$} \\
\hline Notes & \multicolumn{2}{|l|}{-} \\
\hline \multicolumn{3}{|l|}{ Risk of bias } \\
\hline Bias & Authors' judgement & Support for judgement \\
\hline $\begin{array}{l}\text { Random sequence genera- } \\
\text { tion (selection bias) }\end{array}$ & Unclear risk & Comment: no description of the randomisation process \\
\hline $\begin{array}{l}\text { Allocation concealment } \\
\text { (selection bias) }\end{array}$ & Unclear risk & Comment: not clear whether allocation was concealed \\
\hline $\begin{array}{l}\text { Blinding of participants } \\
\text { and personnel (perfor- } \\
\text { mance bias) } \\
\text { Objective outcomes }\end{array}$ & Unclear risk & Comment: unclear if participants/study personnel were blinded \\
\hline $\begin{array}{l}\text { Blinding of outcome as- } \\
\text { sessment (detection bias) } \\
\text { Objective outcomes }\end{array}$ & Unclear risk & Comment: unclear if outcome assessors were blinded to study group \\
\hline
\end{tabular}

Diet, physical activity and behavioural interventions for the treatment of overweight or obese children from the age of 6 to 11 years 
Duffy 1993 (Continued)

Incomplete outcome data High risk Quote from publication: "Of the 27 children who commenced treatment, 21

(attrition bias) completed therapy and were available for post-treatment and 3-month fol-

Objective outcomes low-up". "At the 6-month follow-up, four children who had completed the programme were not available, leaving eight in the BT + APC condition and nine in the CBT group"

Comment: dropout rate high at 6 months' follow up (37\%) and no ITT analysis

\begin{tabular}{|c|c|c|}
\hline $\begin{array}{l}\text { Selective reporting (re- } \\
\text { porting bias) }\end{array}$ & Unclear risk & Comment: no clinical trial register entry or protocol available \\
\hline
\end{tabular}

Other bias Unclear risk Comment: unclear if the study was at risk of any other bias

Duggins 2010

\begin{tabular}{ll}
\hline Methods & Parallel RCT \\
& Randomisation ratio: 1:1 \\
& Superiority design
\end{tabular}

Participants

Inclusion criteria: age 5-17 years, BMI above the 85th percentile for age and sex (CDC growth charts)

Exclusion criteria: no criteria for exclusion

Diagnostic criteria: see above

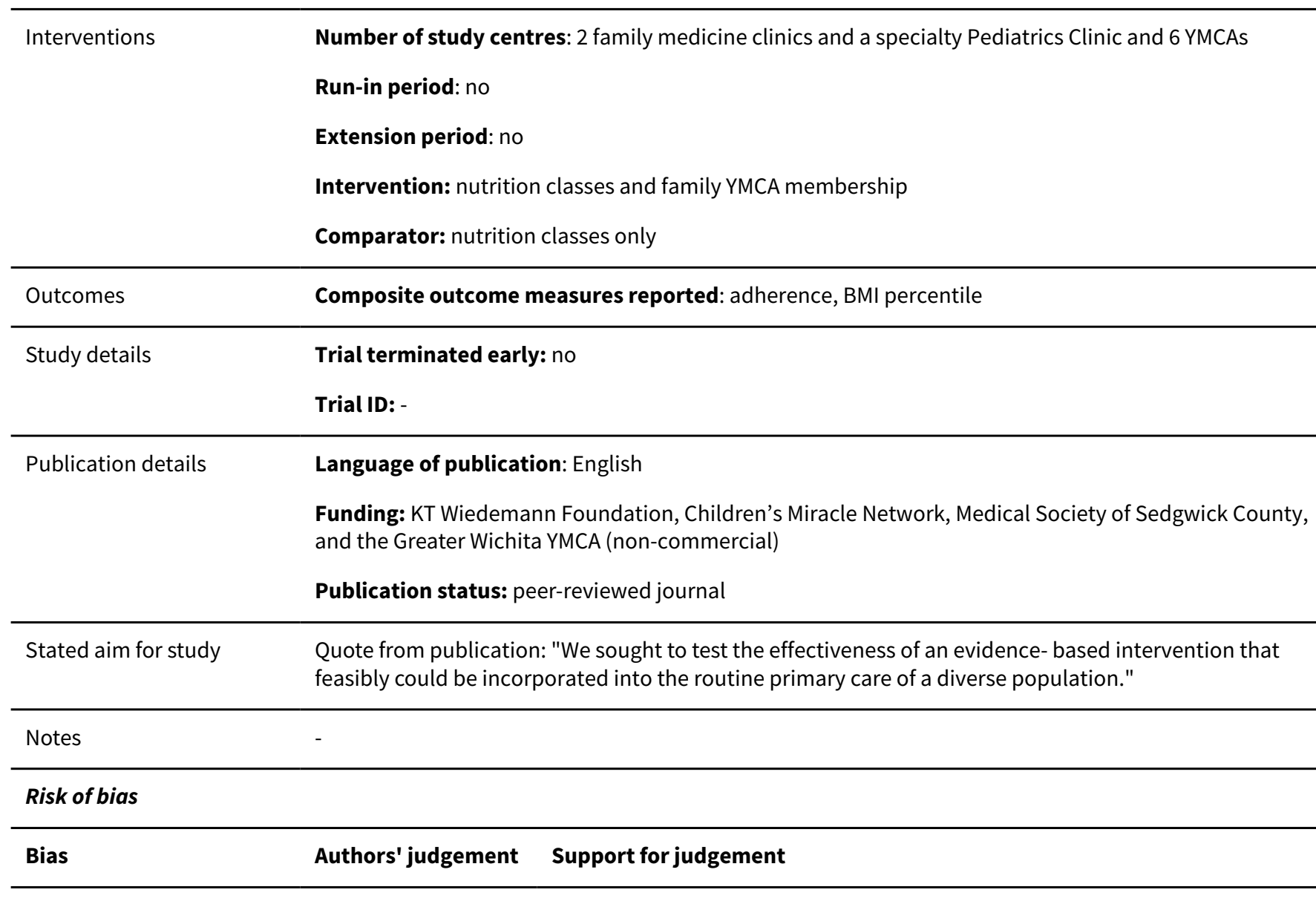

Diet, physical activity and behavioural interventions for the treatment of overweight or obese children from the age of 6 to 11 years 
Duggins 2010 (Continued)

Random sequence genera- Low risk tion (selection bias)
Quote from publication: "The study physician enrolled participants using a computer-generated randomization list"

Comment: randomisation process adequately described

Allocation concealment Low risk
(selection bias)

Quote from publication: "The allocation sequence was concealed before randomization by using sequentially numbered envelopes containing the groupappropriate materials"

Comment: allocation was concealed
Blinding of participants High risk and personnel (performance bias)

Subjective outcomes
Quote from publication: "Given the nature of the intervention neither clinicians nor participants were blind to the treatment allocation once randomization occurred."

Comment: participants and study personnel were not blinded to study group

Blinding of participants High risk
and personnel (perfor-
mance bias)
Objective outcomes

Blinding of outcome as- High risk sessment (detection bias)

Subjective outcomes
Quote from publication: "Given the nature of the intervention neither clinicians nor participants were blind to the treatment allocation once randomization occurred."

Comment: participants and study personnel were not blinded to study group
Quote from publication: "Participants' height and weight were collected and entered into the medical record at baseline and at 2 months, 4 months, 6 months, 9 months, and 12 months after enrollment by the nonblinded nursing staff."

Comment: assessment staff were not blinded

\begin{tabular}{l}
\hline Blinding of outcome as- \\
sessment (detection bias) \\
Objective outcomes
\end{tabular}

Blinding of outcome as- High risk

Objective outcomes
Quote from publication: "Participants' height and weight were collected and entered into the medical record at baseline and at 2 months, 4 months, 6 months, 9 months, and 12 months after enrollment by the nonblinded nursing staff."

Comment: assessment staff were not blinded
Quote from publication: "17 children were excluded from analysis, leaving evaluable data from 30 children in the control group and 36 in the treatment group." "Overall attendance at scheduled study-related visits was poor"

Comment: $80 \%$ of participants were included in the ITT analysis however the publication does not specify how many completed the study. Furthermore attendance at sessions very low

Incomplete outcome data Unclear risk
(attrition bias)
Objective outcomes

Incomplete outcome data Unclear risk (attrition bias)

Objective outcomes

Incomplete outcome data High risk

(attrition bias)

Subjective outcomes
Quote from publication: "17 children were excluded from analysis, leaving evaluable data from 30 children in the control group and 36 in the treatment group." "Overall attendance at scheduled study-related visits was poor"

Comment: $80 \%$ of participants were included in the ITT analysis however the publication does not specify how many completed the study. Furthermore attendance at sessions very low

\begin{tabular}{|c|c|c|}
\hline $\begin{array}{l}\text { Selective reporting (re- } \\
\text { porting bias) }\end{array}$ & Unclear risk & $\begin{array}{l}\text { Comment: no protocol or clinical trials register available. However, publica- } \\
\text { tion only reports dietary outcomes for whole group, does not split them by } \\
\text { group or comment on statistical significance. Also do not report standard de- } \\
\text { viations for change in BMI or BMI percentile. Risk of selective reporting bias } \\
\text { therefore unclear }\end{array}$ \\
\hline
\end{tabular}

Other bias Unclear risk Comment: unclear if the study was at risk of any other bias

Diet, physical activity and behavioural interventions for the treatment of overweight or obese children from the age of 6 to 11 years 
Eddy Ives 2012

\begin{tabular}{|c|c|}
\hline Methods & $\begin{array}{l}\text { Parallel RCT } \\
\text { Randomisation ratio: 1:1 } \\
\text { Superiority design }\end{array}$ \\
\hline Participants & $\begin{array}{l}\text { Inclusion criteria: age } 10-14 \text { years of both sexes, overweight or obese (BMI 85th-95th or }>95 \text { th per- } \\
\text { centiles, depending on age and sex, WHO classification) } \\
\text { Exclusion criteria: morbid obesity, secondary obesity, bulimia nervosa, mental retardation, difficultie } \\
\text { understanding the recommendations, current or recent participation in another clinical trial } \\
\text { Diagnostic criteria: see above }\end{array}$ \\
\hline Interventions & $\begin{array}{l}\text { Number of study centres: } 48 \\
\text { Run-in period: no } \\
\text { Extension period: no } \\
\text { Intervention: dietary and physical exercise recommendations during } 6 \text { sessions } \\
\text { Comparator: dietary and physical exercise recommendations in } 2 \text { sessions only (waiting list control) }\end{array}$ \\
\hline
\end{tabular}

Outcomes Outcome measures reported in abstract: completion rates, BMI z scores, WC z score, food habits, physical activities

$\begin{array}{ll}\text { Study details } & \text { Trial terminated early: no } \\ & \text { Trial ID: ISRCTN35399598 (retrospectively entered) }\end{array}$

\section{Publication details Language of publication: English}

Funding: IX Research Award Nutribén 2007 (non-commercial)

Publication status: peer-reviewed journal

Stated aim for study Quote from publication: "to assess the efficiency of an educational intervention on lifestyle habits to reduce the body mass index in adolescents."

Notes -

\section{Risk of bias}

Bias Authors' judgement Support for judgement

Random sequence genera- Low risk tion (selection bias)
Quote from publication: "We obtained the informed consent of those who chose to participate, and randomly allocated each adolescent to one of the study groups based on a sequence of random numbers generated in a centralised manner from the Research Unit that participated in the study."

Comment: randomisation process adequately described

\begin{tabular}{lll}
\hline $\begin{array}{l}\text { Allocation concealment } \\
\text { (selection bias) }\end{array}$ & Low risk & $\begin{array}{l}\text { Comment: allocation likely concealed due to randomisation method (as de- } \\
\text { scribed above) }\end{array}$ \\
\hline $\begin{array}{l}\text { Blinding of participants } \\
\begin{array}{l}\text { and personnel (perfor- } \\
\text { mance bias) }\end{array}\end{array}$ & High risk & $\begin{array}{l}\text { Comment: study author confirmed via email that participants and study per- } \\
\text { sonnel were not blinded to study group }\end{array}$ \\
\hline
\end{tabular}

Diet, physical activity and behavioural interventions for the treatment of overweight or obese children from the age of 6 to 11 years (Review)

Copyright $\odot 2017$ The Cochrane Collaboration. Published by John Wiley \& Sons, Ltd. 
Eddy Ives 2012 (Continued)

Subjective outcomes

$\begin{array}{lll}\begin{array}{l}\text { Blinding of participants } \\ \text { and personnel (perfor- }\end{array} & \text { High risk } & \begin{array}{l}\text { Comment: study author confirmed via email that participants and study per- } \\ \text { sonnel were not blinded to study group }\end{array}\end{array}$

mance bias)

Objective outcomes

\begin{tabular}{lll}
\hline $\begin{array}{l}\text { Blinding of outcome as- } \\
\text { sessment (detection bias) }\end{array}$ & High risk & $\begin{array}{l}\text { Comment: study author confirmed via email that assessment staff were not } \\
\text { blinded }\end{array}$
\end{tabular}

sessment (detection bias) blinded

\begin{tabular}{lll}
\hline $\begin{array}{l}\text { Blinding of outcome as- } \\
\text { sessment (detection bias) } \\
\text { Objective outcomes }\end{array}$ & High risk & $\begin{array}{l}\text { Comment: study author confirmed via email that assessment staff were not } \\
\text { blinded }\end{array}$ \\
\hline $\begin{array}{l}\text { Incomplete outcome data } \\
\text { (attrition bias) } \\
\text { Subjective outcomes }\end{array}$ & Unclear risk & $\begin{array}{l}\text { Quote from publication: "Thus, 174 participants were randomised, and } 125 \\
(71.8 \%) \text { completed the follow up" }\end{array}$ \\
\hline $\begin{array}{l}\text { Incomplete outcome data } \\
\text { (attrition bias) } \\
\text { Objective outcomes }\end{array}$ & Unclear risk & $\begin{array}{l}\text { Quote from publication: "Thus, } 174 \text { participants were randomised, and } 125 \\
(71.8 \%) \text { completed the follow up" }\end{array}$ \\
\hline $\begin{array}{l}\text { Selective reporting (re- } \\
\text { porting bias) }\end{array}$ & Unclear risk & Comment: relatively moderate dropout rates - may have introduced bias \\
\hline $\begin{array}{l}\text { Other bias } \\
\text { Comment: clinical trial entry registered retrospectively }\end{array}$ \\
\hline
\end{tabular}

Epstein 1984a

\begin{tabular}{ll}
\hline Methods & Parallel RCT \\
& Randomisation ratio: $1: 1: 1$ \\
& Superiority design \\
\hline Participants & $\begin{array}{l}\text { Inclusion criteria: children age } 8-12 \text { years, child and parent between } 20 \%-80 \% \text { of their ideal weight } \\
\text { for height, age and sex (Jelliffe } 1966), \text { parent and child had triceps skinfold thickness }>85 \text { th percentile, } \\
\text { parent willing to participate in all treatment meetings }\end{array}$
\end{tabular}

Exclusion criteria: child had a current psychiatric contact or a learning disability, medical problem that contraindicated exercise (parent or child)

Diagnostic criteria: see above

\begin{tabular}{l} 
Number of study centres: unclear \\
Run-in period: no \\
Extension period: no \\
Intervention 1: diet-plus-exercise group \\
Intervention 2: diet only \\
Comparator: waiting list control \\
\hline
\end{tabular}

Diet, physical activity and behavioural interventions for the treatment of overweight or obese children from the age of 6 to 11 years 
Epstein 1984a (Continued)

Outcomes

Outcome measures reported in abstract: weight, parental weight, lipids, triglycerides, cholesterol, HDL, fitness

Study details

\section{Trial terminated early: no}

Trial ID: -

\section{Language of publication: English}

Funding: part by Grant HD12520 from the National Institute of Child Health and Human Behavior (noncommercial)

Publication status: peer-reviewed journal
Quote from publication: "The present study reports the comparison of diet with diet-plus-life-style exercise in a sample of overweight children and parents enrolled in the family-based obesity treatment program previously developed in this laboratory"

\section{Notes}

\section{Risk of bias}

\begin{tabular}{|c|c|c|}
\hline Bias & Authors' judgement & Support for judgement \\
\hline $\begin{array}{l}\text { Random sequence genera- } \\
\text { tion (selection bias) }\end{array}$ & Unclear risk & Comment: no description of randomisation process \\
\hline $\begin{array}{l}\text { Allocation concealment } \\
\text { (selection bias) }\end{array}$ & Low risk & Comment: study author confirmed via email that allocation was concealed \\
\hline $\begin{array}{l}\text { Blinding of participants } \\
\text { and personnel (perfor- } \\
\text { mance bias) } \\
\text { Subjective outcomes }\end{array}$ & High risk & $\begin{array}{l}\text { Comment: study author confirmed via email that participants and study per- } \\
\text { sonnel were not blinded to study group }\end{array}$ \\
\hline $\begin{array}{l}\text { Blinding of participants } \\
\text { and personnel (perfor- } \\
\text { mance bias) } \\
\text { Objective outcomes }\end{array}$ & High risk & $\begin{array}{l}\text { Comment: study author confirmed via email that participants and study per- } \\
\text { sonnel were not blinded to study group }\end{array}$ \\
\hline $\begin{array}{l}\text { Blinding of outcome as- } \\
\text { sessment (detection bias) } \\
\text { Subjective outcomes }\end{array}$ & High risk & $\begin{array}{l}\text { Comment: study author confirmed via email that assessment staff were not } \\
\text { blinded }\end{array}$ \\
\hline $\begin{array}{l}\text { Blinding of outcome as- } \\
\text { sessment (detection bias) } \\
\text { Objective outcomes }\end{array}$ & High risk & $\begin{array}{l}\text { Comment: study author confirmed via email that assessment staff were not } \\
\text { blinded }\end{array}$ \\
\hline $\begin{array}{l}\text { Incomplete outcome data } \\
\text { (attrition bias) } \\
\text { Subjective outcomes }\end{array}$ & Low risk & $\begin{array}{l}\text { Quote from publication: "At } 6 \text { months, results were available for } 47 \text { (89\%) of } \\
\text { the original } 53 \text { families, with } 15,18 \text {, and } 14 \text { families measured per group." } \\
\text { Comment: attrition rates were low }\end{array}$ \\
\hline $\begin{array}{l}\text { Incomplete outcome data } \\
\text { (attrition bias) } \\
\text { Objective outcomes }\end{array}$ & Low risk & $\begin{array}{l}\text { Quote from publication: "At } 6 \text { months, results were available for } 47 \text { ( } 89 \%) \text { of } \\
\text { the original } 53 \text { families, with } 15,18 \text {, and } 14 \text { families measured per group." } \\
\text { Comment: attrition rates were low }\end{array}$ \\
\hline
\end{tabular}


Epstein 1984a (Continued)

Selective reporting (re- Unclear risk Comment: no protocol or clinical trials register entry porting bias)

Other bias Unclear risk Comment: unclear if the study was at risk of any other bias

Epstein 1985a

\begin{tabular}{ll}
\hline Methods & Parallel RCT \\
& Randomisation ratio: $1: 1: 1$ \\
& Superiority design
\end{tabular}

Participants

Inclusion criteria: children aged 8-12 years, child and parent $>20 \%$ over their ideal weight for height

(Metropolitian Life Insurance Company 1959; Robinson 1968)

Exclusion criteria: parent and child with a problem that would interfere with exercise

Diagnostic criteria: see above

Number of study centres: unclear
Run-in period: no
Extension period: no
Intervention 1: diet plus programmed aerobic exercise programme
Intervention 2: diet plus lifestyle exercise programme
Comparator: diet plus low intensity callisthenic exercise programme

\begin{tabular}{ll}
\hline Outcomes & Outcome measures reported in abstract: weight, parental weight \\
\hline Study details & Trial terminated early: no \\
& Trial ID: - \\
\hline
\end{tabular}

\section{Publication details Language of publication: English}

Funding: GRANT HD12520 from National Institute of Child Health and Human Development (non-commercial)

Publication status: peer-reviewed journal

\begin{tabular}{|c|c|}
\hline Stated aim for study & $\begin{array}{l}\text { Quote from publication: "The present study is designed to assess the reliability of the effects of diet } \\
\text { plus lifestyle versus diet plus programmed aerobic exercise over an extended two year observation in- } \\
\text { terval." }\end{array}$ \\
\hline Notes & - \\
\hline \multicolumn{2}{|l|}{ Risk of bias } \\
\hline Bias & Authors' judgement Support for judgement \\
\hline $\begin{array}{l}\text { Random sequence genera- } \\
\text { tion (selection bias) }\end{array}$ & Comment: no description of randomisation process \\
\hline
\end{tabular}

Diet, physical activity and behavioural interventions for the treatment of overweight or obese children from the age of 6 to 11 years 
Epstein 1985a (Continued)

Allocation concealment Low risk Comment: study author confirmed via email that allocation was concealed (selection bias)

Blinding of participants High risk and personnel (performance bias)

Subjective outcomes
Comment: study author confirmed via email that participants and study personnel were not blinded to study group

\begin{tabular}{lll}
\hline Blinding of participants & High risk & $\begin{array}{l}\text { Comment: study author confirmed via email that participants and study per- } \\
\text { and personnel (perfor- }\end{array}$
\end{tabular}

mance bias)

Objective outcomes

$\begin{array}{lll}\begin{array}{l}\text { Blinding of outcome as- } \\ \text { sessment (detection bias) }\end{array} & \text { High risk } & \begin{array}{l}\text { Comment: study author confirmed via email that assessment staff were not } \\ \text { blinded }\end{array}\end{array}$

Subjective outcomes

$\begin{array}{lll}\begin{array}{l}\text { Blinding of outcome as- } \\ \text { sessment (detection bias) } \\ \text { Objective outcomes }\end{array} & \text { High risk } & \begin{array}{l}\text { Comment: study author confirmed via email that assessment staff were not } \\ \text { blinded }\end{array}\end{array}$

Objective outcomes

Incomplete outcome data Low risk
(attrition bias)
Subjective outcomes

Subjective outcomes
Quote from publication: "Complete data were available for 35 families, which represent $85 \%$ of the families beginning the study"

Comment: attrition rates fairly were low

\begin{tabular}{ll}
\hline $\begin{array}{l}\text { Incomplete outcome data } \\
\text { (attrition bias) }\end{array}$ & Low risk \\
Quote from publication: "Complete data were available for 35 families, which \\
represent $85 \%$ of the families beginning the study"
\end{tabular}

Incomplete outcome data Low risk

Objective outcomes

Comment: attrition rates fairly were low

Selective reporting (re- Unclear risk Comment: no protocol or clinical trials register entry
porting bias)

Other bias Unclear risk Comment: unclear if the study was at risk of any other bias

Epstein 1985b

\begin{tabular}{ll}
\hline Methods & Parallel RCT \\
& Randomisation ratio: $1: 1$ \\
& Superiority design \\
\hline Participants & $\begin{array}{l}\text { Inclusion criteria: girls between 8-12 years, at least } 20 \% \text { over her ideal weight for height and age } \\
\text { (Jeliffe 1966), at least } 1 \text { parent willing to participate } \\
\text { Exclusion criteria: medical problems that would contraindicate weight loss, exercise or fitness testing } \\
\text { Diagnostic criteria: see above }\end{array}$
\end{tabular}

Interventions Number of study centres: 1

Run-in period: no

Extension period: no

Intervention: diet and exercise education

Diet, physical activity and behavioural interventions for the treatment of overweight or obese children from the age of 6 to 11 years 
Epstein 1985b (Continued)

Comparator: diet education only

\begin{tabular}{ll}
\hline Outcomes & Outcome measures reported in abstract: weight, percent overweight, fitness \\
\hline Study details & Trial terminated early: no \\
& Trial ID: -
\end{tabular}

Publication details Language of publication: English

Funding: Grant HD 16411 from National Institute of child health and human development (non-commercial)

Publication status: peer-reviewed journal

Stated aim for study

Quote from publication: "The present study was designed to re-evaluate the role of exercise plus diet in weight control by having children participate in a structured exercise program during the first 6 weeks of exercise, which may facilitate the development of appropriate exercise behavior."

\section{Notes}

\section{Risk of bias}

\begin{tabular}{lll}
\hline Bias & Authors' judgement & Support for judgement \\
\hline $\begin{array}{l}\text { Random sequence genera- } \\
\text { tion (selection bias) }\end{array}$ & Low risk & $\begin{array}{l}\text { Quote from publication: "After acceptance into the program, families were } \\
\text { assigned to one of two treatment groups by a stratified random assignment } \\
\text { procedure. Children were stratified on the basis of age, percent overweight, } \\
\text { and physical work capacity, and were then randomly assigned to either the di- } \\
\text { et plus exercise group (group 1) or the diet without exercise group (group 2)." } \\
\text { Comment: randomisation process described }\end{array}$ \\
\hline
\end{tabular}

Allocation concealment Low risk Comment: study author confirmed via email that allocation was concealed
(selection bias)

\begin{tabular}{|c|c|c|}
\hline $\begin{array}{l}\text { Blinding of participants } \\
\text { and personnel (perfor- } \\
\text { mance bias) } \\
\text { Subjective outcomes }\end{array}$ & High risk & $\begin{array}{l}\text { Comment: study author confirmed via email that participants and study per- } \\
\text { sonnel were not blinded to study group }\end{array}$ \\
\hline
\end{tabular}

\begin{tabular}{|c|c|c|}
\hline $\begin{array}{l}\text { Blinding of participants } \\
\text { and personnel (perfor- } \\
\text { mance bias) } \\
\text { Objective outcomes }\end{array}$ & High risk & $\begin{array}{l}\text { Comment: study author confirmed via email that participants and study per- } \\
\text { sonnel were not blinded to study group }\end{array}$ \\
\hline
\end{tabular}

\begin{tabular}{|c|c|c|}
\hline $\begin{array}{l}\text { Blinding of outcome as- } \\
\text { sessment (detection bias) } \\
\text { Subjective outcomes }\end{array}$ & High risk & $\begin{array}{l}\text { Comment: study author confirmed via email that assessment staff were not } \\
\text { blinded }\end{array}$ \\
\hline $\begin{array}{l}\text { Blinding of outcome as- } \\
\text { sessment (detection bias) } \\
\text { Objective outcomes }\end{array}$ & High risk & $\begin{array}{l}\text { Comment: study author confirmed via email that assessment staff were not } \\
\text { blinded }\end{array}$ \\
\hline $\begin{array}{l}\text { Incomplete outcome data } \\
\text { (attrition bias) } \\
\text { Subjective outcomes }\end{array}$ & Unclear risk & $\begin{array}{l}\text { Quote from publication: "Twenty of the } 23 \text { children completing treatment } \\
(86.96 \%) \text { attended the } 6 \text {-month assessment, and } 19 \text { children }(82.61 \%) \text { attend- } \\
\text { ed the } 1 \text {-year assessment. There was no difference in the dropout rate be- } \\
\text { tween groups" }\end{array}$ \\
\hline
\end{tabular}


Comment: even though dropout rates were relatively low, there was no sensitivity analysis or missing data imputation, and furthermore original sample size was small. Hence attrition rate may have led to bias

Incomplete outcome data Unclear risk (attrition bias)

Objective outcomes
Quote from publication:"Twenty of the 23 children completing treatment $(86.96 \%)$ attended the 6 -month assessment, and 19 children $(82.61 \%)$ attended the 1-year assessment. There was no difference in the dropout rate between groups"

Comment: even though dropout rates were relatively low, there was no sensitivity analysis or missing data imputation, and furthermore original sample size was small. Hence attrition rate may have led to bias

Selective reporting (re- Unclear risk $\quad$ Comment: no protocol or clinical trials register entry
porting bias)

\section{Epstein 1985c}

\begin{tabular}{ll}
\hline Methods & Parallel RCT \\
& Randomisation ratio: $1: 1$ \\
& Superiority design \\
\hline Participants & Inclusion criteria: obese female children (obesity defined by Robinson 1968), 5-8 years of age \\
& Exclusion criteria: none \\
& Diagnostic criteria: see above
\end{tabular}

Interventions

\section{Number of study centres: 1}

Run-in period: no

\section{Extension period: no}

Intervention: behaviourally-oriented programme that emphasised parent management

Comparator: provided equal education and attention but not behavioural principles

\begin{tabular}{ll}
\hline Outcomes & Composite outcome measures reported: weight \\
\hline Study details & Trial terminated early: no \\
& Trial ID: - \\
\hline Publication details & Language of publication: English \\
& $\begin{array}{l}\text { Funding: part by Grant HD16411 from the national Institute of child health and human development } \\
\text { (non-commercial) }\end{array}$ \\
& Publication status: peer-reviewed journal \\
\hline Stated aim for study & $\begin{array}{l}\text { Quote from publication: "The purpose of this study was to evaluate the effectiveness of family-based } \\
\text { treatment for childhood obesity for 5-to-8 year old children" }\end{array}$ \\
\hline Notes & - \\
\hline \hline
\end{tabular}

Diet, physical activity and behavioural interventions for the treatment of overweight or obese children from the age of 6 to 11 years 
Epstein 1985c (Continued)

\section{Risk of bias}

\begin{tabular}{lll}
\hline Bias & Authors' judgement & Support for judgement \\
\hline $\begin{array}{l}\text { Random sequence genera- } \\
\text { tion (selection bias) }\end{array}$ & Unclear risk & Comment: no description of randomisation method \\
\hline $\begin{array}{l}\text { Allocation concealment } \\
\text { (selection bias) }\end{array}$ & Low risk & Comment: study author confirmed via email that allocation was concealed \\
\hline $\begin{array}{l}\text { Blinding of participants } \\
\text { and personnel (perfor- } \\
\text { mance bias) } \\
\begin{array}{l}\text { Subjective outcomes } \\
\text { nigh risk }\end{array}\end{array}$ & Comment: study author confirmed via email that participants and study per- \\
\hline
\end{tabular}

\begin{tabular}{|c|c|c|}
\hline $\begin{array}{l}\text { Blinding of participants } \\
\text { and personnel (perfor- } \\
\text { mance bias) } \\
\text { Objective outcomes }\end{array}$ & High risk & $\begin{array}{l}\text { Comment: study author confirmed via email that participants and study per- } \\
\text { sonnel were not blinded to study group }\end{array}$ \\
\hline
\end{tabular}

Blinding of outcome as- High risk sessment (detection bias)

Subjective outcomes
Comment: study author confirmed via email that assessment staff were not blinded
Blinding of outcome as- High risk sessment (detection bias)

Objective outcomes
Comment: study author confirmed via email that assessment staff were not blinded

\begin{tabular}{|c|c|c|}
\hline $\begin{array}{l}\text { Incomplete outcome data } \\
\text { (attrition bias) }\end{array}$ & Unclear risk & $\begin{array}{l}\text { Quote from publication: "Five families dropped out after the preliminary } \\
\text { meeting because of conflicting obligations" }\end{array}$ \\
\hline
\end{tabular}

Subjective outcomes

Comment: moderate dropout rates, unclear if attrition bias occurred

$\begin{array}{lll}\text { Incomplete outcome data } & \text { Unclear risk } & \begin{array}{l}\text { Quote from publication: "Five families dropped out after the preliminary } \\ \text { (attrition bias) }\end{array}\end{array}$

Objective outcomes

Comment: moderate dropout rates, unclear if attrition bias occurred

Selective reporting (re- Unclear risk Comment: no protocol or clinical trials register entry
porting bias)

\begin{tabular}{lll}
\hline Other bias $\quad$ Unclear risk $\quad$ Comment: unclear if the study was at risk of any other bias \\
\hline
\end{tabular}

Epstein 2000a

\begin{tabular}{ll}
\hline Methods & Parallel RCT \\
& Randomisation ratio: $1: 1: 1$ \\
& Superiority design \\
\hline Participants & $\begin{array}{l}\text { Inclusion criteria: child }>20 \% \text { overweight (Must 1991), } 1 \text { parent willing to attend meetings, child read- } \\
\text { ing third-grade level or higher } \\
\text { Exclusion criteria: if a either parent was }>100 \% \text { overweight, a family member on an alternative weight } \\
\text { management programme, parent or child having psychiatric problems, parent or child having activity } \\
\text { restrictions }\end{array}$
\end{tabular}

Diet, physical activity and behavioural interventions for the treatment of overweight or obese children from the age of 6 to 11 years 
Epstein 2000a (Continued)

Diagnostic criteria: see above

Number of study centres: 1
Run-in period: no
Extension period: no
Intervention 1: behavioural weight-control programme plus parent and child problem solving
Intervention 2: behavioural weight-control programme plus child problem solving only
Comparator: standard treatment with no additional problem solving

Outcomes Composite outcome measures reported: BMI, child behaviour problems, parental distress, parent problem solving, child problem solving, parental weight, eating disorder symptoms

Study details Trial terminated early: no

Trial ID: -

\begin{tabular}{ll}
\hline Publication details & Language of publication: English \\
Funding: in part by Grant HD20829 (non-commercial) \\
Publication status: peer-reviewed journal
\end{tabular}

Stated aim for study Quote from publication: "This study was designed to determine the effects of adding problem-solving training for parents and children or children alone to a comprehensive family-based behavioral childhood obesity treatment"

Notes

\section{Risk of bias}

\begin{tabular}{|c|c|c|}
\hline Bias & Authors' judgement & Support for judgement \\
\hline $\begin{array}{l}\text { Random sequence genera- } \\
\text { tion (selection bias) }\end{array}$ & Low risk & $\begin{array}{l}\text { Quote from publication: (from study author via email) "After participants are } \\
\text { screened to ensure they met eligibility criteria for the specific study, families } \\
\text { are randomized to treatment groups using a random number algorithm which } \\
\text { assigned a random number that was limited to the number of groups, for ex- } \\
\text { ample in a two group study group } 1 \text { or } 2 \text {. Groups are then checked to make } \\
\text { sure they are not different in child and parent relative body weight (BMI, per- } \\
\text { cent overweight, z-BMI), usually SES, and sometimes other study specific base- } \\
\text { line values of other measures. If groups are not equal randomization is repeat- } \\
\text { ed" }\end{array}$ \\
\hline
\end{tabular}

Comment: unlikely this randomisation method introduced selection bias

Allocation concealment $\quad$ Low risk Comment: study author confirmed via email that allocation was concealed
(selection bias)

\begin{tabular}{|c|c|c|}
\hline $\begin{array}{l}\text { Blinding of participants } \\
\text { and personnel (perfor- }\end{array}$ & High risk & $\begin{array}{l}\text { Comment: study author confirmed via email that participants and study per- } \\
\text { sonnel were not blinded to study group }\end{array}$ \\
\hline
\end{tabular}

mance bias)

Subjective outcomes

Blinding of participants High risk and personnel (performance bias)
Comment: study author confirmed via email that participants and study personnel were not blinded to study group 
Epstein 2000a (Continued)

Objective outcomes

$\begin{array}{lll}\text { Blinding of outcome as- } & \text { High risk } & \begin{array}{l}\text { Comment: study author confirmed via email that assessment staff were not } \\ \text { blinded }\end{array}\end{array}$

Subjective outcomes

\begin{tabular}{lll}
\hline $\begin{array}{l}\text { Blinding of outcome as- } \\
\text { sessment (detection bias) } \\
\text { Objective outcomes }\end{array}$ & High risk & $\begin{array}{l}\text { Comment: study author confirmed via email that assessment staff were not } \\
\text { blinded }\end{array}$
\end{tabular}

Objective outcomes

Incomplete outcome data High risk
(attrition bias)

Subjective outcomes
Quote from publication: "Attrition was 3\%, 11\%, and 15\% at 6, 12, and 24 months, respectively"

Comment: low attrition rates

Incomplete outcome data Low risk
(attrition bias)
Objective outcomes

Objective outcomes

\begin{tabular}{lll}
\hline $\begin{array}{l}\text { Selective reporting (re- } \\
\text { porting bias) }\end{array}$ & High risk & $\begin{array}{l}\text { Comment: no protocol or clinical trials register entry available. Also, in the ad- } \\
\text { ditional publication all three groups were grouped together for analysis - po- } \\
\text { tential reporting bias due to non-significant results }\end{array}$ \\
\hline Other bias & Unclear risk & Comment: unclear if the study was at risk of any other bias \\
\hline
\end{tabular}

Quote from publication: "Attrition was 3\%, 11\%, and 15\% at 6, 12, and 24 months, respectively"

Comment: low attrition rates

Epstein 2001

\begin{tabular}{ll}
\hline Methods & Parallel RCT \\
& Randomisation ratio: $1: 1$ \\
& Superiority design
\end{tabular}

\begin{tabular}{ll}
\hline Participants & Inclusion criteria: children aged 8-12-years, child $\geq 85$ th BMI percentile but $<100 \%$ over average BMI \\
for age and sex (using standards derived from the National Health and Nutrition Examination Survey III) \\
child at or $>85$ th, 1 parent willing to attend the weekly treatment meetings
\end{tabular}

Exclusion criteria: either parent over 100\% overweight, a parent or child on another weight-control programme, medical restrictions to the parent or child that would prevent exercise, current psychiatric disorders in parents or child, a history of eating disorders in the parents

Diagnostic criteria: see above

Number of study centres: 1
Run-in period: no
Extension period: no
Intervention: a combination of reducing sedentary behaviour and increasing physical activity
Comparator: targeting increasing physical activity only

\begin{tabular}{ll}
\hline Outcomes & Outcome measures reported in abstract: percent overweight, adherence \\
\hline Study details & Trial terminated early: no \\
& Trial ID: - \\
\hline \hline
\end{tabular}

Diet, physical activity and behavioural interventions for the treatment of overweight or obese children from the age of 6 to 11 years 
Epstein 2001 (Continued)
Publication details
Language of publication: English

Funding: in part by Grant HD34284 (non-commercial)

Publication status: peer-reviewed journal

Stated aim for study

Quote from publication: "The primary goal was to evaluate sex differences in child weight control programs that targeted increasing physical activity (increase) or the combination of reducing sedentary behavior and increasing physical activity (combined). A second goal was to evaluate the benefits of family-based interventions on non-targeted siblings."

Notes -

\title{
Risk of bias
}

Bias Authors' judgement Support for judgement

Random sequence genera- Low risk tion (selection bias)

Quote from publication: (from author via email) "After participants are screened to ensure they met eligibility criteria for the specific study, families are randomized to treatment groups using a random number algorithm which assigned a random number that was limited to the number of groups, for example in a two group study group 1 or 2 . Groups are then checked to make sure they are not different in child and parent relative body weight (BMI, percent overweight, z-BMI), usually SES, and sometimes other study specific baseline values of other measures. If groups are not equal randomization is repeated"

Comment: unlikely this randomisation method introduced selection bias

Allocation concealment Low risk Comment: study author confirmed via email that allocation was concealed
(selection bias)

Blinding of participants

High risk

and personnel (perfor-

Comment: study author confirmed via email that participants and study per-

mance bias)

Subjective outcomes

Blinding of participants High risk
and personnel (perfor-
mance bias)
Objective outcomes

Comment: study author confirmed via email that participants and study personnel were not blinded to study group sonnel were not blinded to study group

$\begin{array}{lll}\text { Blinding of outcome as- } & \text { High risk } & \begin{array}{l}\text { Comment: study author confirmed via email that assessment staff were not } \\ \text { blinded }\end{array} \\ \text { Subjective outcomes } & \end{array}$

Subjective outcomes

\begin{tabular}{|c|c|c|}
\hline $\begin{array}{l}\text { Blinding of outcome as- } \\
\text { sessment (detection bias) }\end{array}$ & High risk & $\begin{array}{l}\text { Comment: study author confirmed via email that assessment staff were not } \\
\text { blinded }\end{array}$ \\
\hline
\end{tabular}

Objective outcomes

Incomplete outcome data Low risk
(attrition bias)

Subjective outcomes

\begin{abstract}
Quote from publication: "The final sample with complete data for targeted children at baseline, 6-month, and 12-month measurements was based on 56 of the 67 families that were randomized (84\%), which included 245 family members."
\end{abstract}

Comment: low attrition rates

\begin{tabular}{|c|c|c|}
\hline $\begin{array}{l}\text { Incomplete outcome data } \\
\text { (attrition bias) }\end{array}$ & Low risk & $\begin{array}{l}\text { Quote from publication: "The final sample with complete data for targeted } \\
\text { children at baseline, 6-month, and 12-month measurements was based on }\end{array}$ \\
\hline
\end{tabular}

Diet, physical activity and behavioural interventions for the treatment of overweight or obese children from the age of 6 to 11 years 
Epstein 2001 (Continued) Objective outcomes
56 of the 67 families that were randomized (84\%), which included 245 family members."

Comment: low attrition rates

\begin{tabular}{lll}
\hline $\begin{array}{l}\text { Selective reporting (re- } \\
\text { porting bias) }\end{array}$ & Unclear risk & Comment: no protocol or clinical trials register entry \\
\hline Other bias & Unclear risk & Comment: unclear if the study was at risk of any other bias \\
\hline
\end{tabular}

\section{Epstein 2005}

\begin{tabular}{ll}
\hline Methods & Parallel RCT \\
& Randomisation ratio: 1:1 \\
& Superiority design \\
\hline Participants & $\begin{array}{l}\text { Inclusion criteria: children aged 8-12-years, overweight or obese }(\geq 85 \text { th BMI percentile, CDC growth } \\
\text { charts), a parent willing to attend treatment meetings, child reading level at a minimum of third-grade } \\
\text { level }\end{array}$
\end{tabular}

Exclusion criteria: if any family members are participating in another weight-control programme, parent or child with medical restrictions on diet or physical activity, which could interfere with participation in the study, current psychiatric, addictive or eating disorders in parents or child

Diagnostic criteria: see above

Number of study centres: 1
Run-in period: no
Extension period: no
Intervention: standardised family-based behavioural weight control programme plus reinforcement
for increasing alternatives to eating
Comparator: standardised family-based behavioural weight control programme only

Outcomes

Outcome measures reported in abstract: BMI z score, alternatives to eating, physical activity, energy intake

\section{Study details Trial terminated early: no}

Trial ID: -

\begin{tabular}{ll}
\hline Publication details & Language of publication: English \\
Funding: supported in part by grant HD 39792 awarded to the lead study author (non-commercial) \\
Publication status: peer-reviewed journal
\end{tabular}

Stated aim for study Quote from publication: "Two experiments that attempt to increase alternatives to eating in obese youth are presented"

\section{Notes}

\section{Risk of bias}

Diet, physical activity and behavioural interventions for the treatment of overweight or obese children from the age of 6 to 11 years 
Epstein 2005 (Continued)
Bias
Authors' judgement Support for judgement

Random sequence genera- Low risk

tion (selection bias)

Quote from publication: "After participants are screened to ensure they met eligibility criteria for the specific study, families are randomized to treatment groups using a random number algorithm which assigned a random number that was limited to the number of groups, for example in a two group study group 1 or 2. Groups are then checked to make sure they are not different in child and parent relative body weight (BMI, percent overweight, z-BMI), usually SES, and sometimes other study specific baseline values of other measures. If groups are not equal randomization is repeated"

Comment: unlikely this randomisation method introduced selection bias

Allocation concealment Low risk Comment: study author confirmed via email that allocation was concealed
(selection bias)

\begin{tabular}{lll}
\hline $\begin{array}{l}\text { Blinding of participants } \\
\text { and personnel (perfor- }\end{array}$ & High risk & $\begin{array}{l}\text { Comment: study author confirmed via email that participants and study per- } \\
\text { sonnel were not blinded to study group }\end{array}$
\end{tabular}

mance bias)

Subjective outcomes

\begin{tabular}{|c|c|c|}
\hline $\begin{array}{l}\text { Blinding of participants } \\
\text { and personnel (perfor- } \\
\text { mance bias) } \\
\text { Objective outcomes }\end{array}$ & High risk & $\begin{array}{l}\text { Comment: study author confirmed via email that participants and study per- } \\
\text { sonnel were not blinded to study group }\end{array}$ \\
\hline
\end{tabular}

Blinding of outcome as-
sessment (detection bias) Unclear risk Comment: unclear if assessment staff were blinded to study group

Blinding of outcome as- $\quad$ Unclear risk Comment: unclear if assessment staff were blinded to study group
sessment (detection bias)
Objective outcomes

\begin{tabular}{|c|c|c|}
\hline $\begin{array}{l}\text { Incomplete outcome data } \\
\text { (attrition bias) } \\
\text { Subjective outcomes }\end{array}$ & Unclear risk & $\begin{array}{l}\text { Quote from publication: "Complete height and weight data at } 24 \text { months } \\
\text { was available for } 35 \text { of the } 41 \text { families "The intention to treat analysis replaced } \\
\text { missing data with return to baseline values." }\end{array}$ \\
\hline
\end{tabular}

Comment: dropout rates were moderate and ITT analysis was used - however they replaced missing data with baseline values which is not a robust imputation method. Bias may still have occurred

\begin{tabular}{|c|c|c|}
\hline $\begin{array}{l}\text { Incomplete outcome data } \\
\text { (attrition bias) } \\
\text { Objective outcomes }\end{array}$ & Unclear risk & $\begin{array}{l}\text { Quote from publication: "Complete height and weight data at } 24 \text { months } \\
\text { was available for } 35 \text { of the } 41 \text { families "The intention to treat analysis replaced } \\
\text { missing data with return to baseline values." }\end{array}$ \\
\hline
\end{tabular}

Comment: dropout rates were moderate and ITT analysis was used - however they replaced missing data with baseline values which is not a robust imputation method. Bias may still have occurred

\begin{tabular}{|c|c|c|}
\hline $\begin{array}{l}\text { Selective reporting (re- } \\
\text { porting bias) }\end{array}$ & Unclear risk & $\begin{array}{l}\text { Comment: unclear as no protocol or clinical trials register. Study found no sig- } \\
\text { nificant differences between groups - raw data reported either in the text or } \\
\text { in graphical format (not presented in a table) hence will be difficult to extract. } \\
\text { Potential selective reporting due to non-significant results }\end{array}$ \\
\hline
\end{tabular}

\begin{tabular}{ll}
\hline Other bias $\quad$ Unclear risk $\quad$ Comment: unclear if the study was at risk of any other bias \\
\hline
\end{tabular}

Diet, physical activity and behavioural interventions for the treatment of overweight or obese children from the age of 6 to 11 years 
Epstein 2015

\begin{tabular}{ll}
\hline Methods & Parallel RCT \\
& Randomisation ratio: 1:1 \\
& Superiority design \\
\hline Participants & $\begin{array}{l}\text { Inclusion criteria: children aged 8-12-years, > 85th BMI percentile (CDC growth charts), } 1 \text { over- } \\
\text { weight/obese (BMI } \geq 25) \text { parent willing to attend treatment meetings, child reading level at a minimum } \\
\text { of third-grade level }\end{array}$
\end{tabular}

Exclusion criteria: taking weight-altering drugs, if any family members are participating in another weight-control programme, parent or child with diet or physical activity restrictions, which could interfere with participation in the study, psychiatric problems in child or parent

Diagnostic criteria: see above

Interventions Number of study centres: 1

Run-in period: prior to initiating the pilot, 21 families were seen to develop treatment methods, and provide therapists experience with the intervention

Extension period: no

Intervention: family-based treatment + variety of high energy-dense foods

Comparator: family-based treatment only

\begin{tabular}{ll}
\hline Outcomes & Composite outcome measures reported: percent overweight, BMI z score, RED foods, parent BMI \\
\hline Study details & Trial terminated early: no \\
& Trial ID: NCT01208870 \\
\hline
\end{tabular}

\section{Publication details Language of publication: English}

Funding: funded in part by a grant from the National Institute of Diabetes and Digestive and Kidney Diseases UO1 DK088380 awarded to lead author (non-commercial)

Publication status: peer-reviewed journal

Stated aim for study $\quad \begin{aligned} & \text { Quote from publication: "The aims of the pilot study were to assess effects of variety of both child and } \\ & \text { parent weight loss, and to assess whether reduced variety of high energy dense foods was associated } \\ & \text { with weight loss." }\end{aligned}$

$$
\text { Notes }
$$

\section{Risk of bias}

Bias Authors' judgement Support for judgement

Random sequence genera- Low risk tion (selection bias)
Quote from publication: (from author via email) "After participants are screened to ensure they met eligibility criteria for the specific study, families are randomized to treatment groups using a random number algorithm which assigned a random number that was limited to the number of groups, for example in a two group study group 1 or 2 . Groups are then checked to make sure they are not different in child and parent relative body weight (BMI, percent overweight, z-BMI), usually SES, and sometimes other study specific baseline values of other measures. If groups are not equal randomization is repeated"

Comment: randomisation method described 
Epstein 2015 (Continued)

Allocation concealment Low risk Comment: study author confirmed via email that allocation was concealed
(selection bias)

Blinding of participants High risk and personnel (performance bias)

Subjective outcomes

\section{Blinding of participants High risk} and personnel (performance bias)

Objective outcomes
Comment: study author confirmed via email that participants and study personnel were not blinded to study group

$\begin{array}{lll}\text { Blinding of outcome as- } & \text { Unclear risk } \quad \text { Comment: unclear if subjective outcomes were measured by blinded staff } \\ \text { sessment (detection bias) }\end{array}$
sessment (detection bias)

Subjective outcomes

\begin{tabular}{|c|c|c|}
\hline $\begin{array}{l}\text { Blinding of outcome as- } \\
\text { sessment (detection bias) } \\
\text { Objective outcomes }\end{array}$ & Low risk & $\begin{array}{l}\text { Quote from publication: "Height and weight measurements were taken at } \\
0 \text { and } 6 \text { months by staff blind to treatment assignment using a digital weight } \\
\text { scale and stadiometer calibrated daily." }\end{array}$ \\
\hline
\end{tabular}
Objective outcomes $\quad$ scale and stadiometer calibrated daily."

Comment: study author confirmed via email that participants and study personnel were not blinded to study group

Comment: objective anthropometric outcomes were measured by blinded staff

Incomplete outcome data Unclear risk $\quad$ Comment: attrition rates unknown
(attrition bias)

(attrition bias)

Subjective outcomes

Incomplete outcome data Unclear risk Comment: attrition rates unknown
(attrition bias)
Objective outcomes

\begin{tabular}{ll}
\hline $\begin{array}{l}\text { Selective reporting (re- } \quad \text { Low risk } \\
\text { porting bias) }\end{array}$ & $\begin{array}{l}\text { Comment: no differences found between clinical trial register entry and the } \\
\text { publication }\end{array}$
\end{tabular}

\begin{tabular}{ll}
\hline Other bias $\quad$ Unclear risk Comment: unclear if the study was at risk of any other bias \\
\hline
\end{tabular}

Faude 2010

\begin{tabular}{ll}
\hline Methods & Parallel RCT \\
& Randomisation ratio: 1:1 \\
& Superiority design
\end{tabular}

Participants Inclusion criteria: children aged 8-12 years, overweight (large German reference sample - Kromeyer-Hauschild 2001)

Exclusion criteria: children not actively involved in regular sports activities, children not exposed to any nutritional or pharmacological intervention, adverse cardiovascular conditions, and chronic metabolic or orthopaedic disorders

Diagnostic criteria: see above

Interventions Number of study centres: 1

Run-in period: no

Diet, physical activity and behavioural interventions for the treatment of overweight or obese children from the age of 6 to 11 years 
Faude 2010 (Continued)

\section{Extension period: no}

Intervention: football training programme (FB)

Comparator: established standard sports programme (STD)

Outcomes Composite outcome measures reported: maximal performance capacity, submaximal heart rate, mo-
tor skills, self-esteem, body composition, psychometric variables

Study details Trial terminated early: no

Trial ID: -

\section{Publication details Language of publication: English}

Funding: FIFA/FMARC (Fédération International de Football Associations, FIFA - Medical Assessment and Research Center) (commercial)

Publication status: peer-reviewed journal

\begin{tabular}{ll}
\hline Stated aim for study & $\begin{array}{l}\text { Quote from publication: "The present study aimed at analyzing the efficacy of a 6-month football train- } \\
\text { ing program compared with a standard exercise program on health and fitness parameters in over- } \\
\text { weight children" }\end{array}$ \\
\hline
\end{tabular}

\section{Notes}

\section{Risk of bias}

\begin{tabular}{lll}
\hline Bias & Authors' judgement & Support for judgement \\
\hline $\begin{array}{ll}\text { Random sequence genera- } \\
\text { tion (selection bias) }\end{array}$ & Unclear risk & $\begin{array}{l}\text { Quote from publication: "Children underwent a stratified randomization in- } \\
\text { to two groups (according to age, gender, body mass index (BMI) percentile and } \\
\text { maximal performance in cycling ergometry)." }\end{array}$
\end{tabular}

Comment: randomisation method not described in enough detail

Allocation concealment $\quad$ Unclear risk Comment: unclear if allocation was concealed
(selection bias)

Blinding of participants Unclear risk and personnel (performance bias)

Subjective outcomes

\begin{abstract}
Quote from publication: "The training programs were carried out in two different locations at the same time of the day on the same days of the week (Monday, Tuesday, Thursday, 16:00-17:00 hour). This was decided to blind the groups to the training program of the other group"
\end{abstract}

Comment: participants were likely blinded to study group - unclear if personnel were blinded however

Quote from publication: "The training programs were carried out in two different locations at the same time of the day on the same days of the week (Monday, Tuesday, Thursday, 16:00-17:00 hour). This was decided to blind the groups to the training program of the other group"

Comment: participants were likely blinded to study group - unclear if personnel were blinded however

Quote from publication: "Cycling ergometry was conducted by a trained institutional investigator who was blinded for group randomization to avoid inves-

Comment: unclear if subjective outcomes were measured by blinded staff
Blinding of outcome assessment (detection bias)

Subjective outcomes tigator bias"

Unclear risk

tutional investic
tigator bias"

$\begin{array}{ll}\begin{array}{l}\text { Blinding of participants } \\ \text { and personnel (perfor- }\end{array} & \begin{array}{l}\text { Quote from publication: "The training programs were carried out in two dif- } \\ \text { ferent locations at the same time of the day on the same days of the week } \\ \text { mance bias) }\end{array} \\ \text { Objective outcomes } & \text { (Monday, Tuesday, Thursday, 16:00-17:00 hour). This was decided to blind the } \\ \text { groups to the training program of the other group" }\end{array}$


Faude 2010 (Continued)

Blinding of outcome as- Unclear risk sessment (detection bias)

Objective outcomes
Quote from publication: "Cycling ergometry was conducted by a trained institutional investigator who was blinded for group randomization to avoid investigator bias"

Comment: unclear whether other objective outcomes were measured by blinded staff

Quote from publication: "17 children (44\%) dropped out during the study period due to insufficient compliance $(\mathrm{N}=12)$, private or school problems $(\mathrm{N}=4)$ or change of residence $(\mathrm{N}=1)$. No significant differences were observed between drop-outs and children who completed the training $(P>0.10)$."

Comment: even though no differences were observed between dropouts and completers, attrition rate was high and would likely have introduced bias. Plus ITT analysis was not used

Incomplete outcome data High risk

(attrition bias)

Quote from publication: "17 children (44\%) dropped out during the study period due to insufficient compliance $(\mathrm{N}=12)$, private or school problems $(\mathrm{N}=4)$ or change of residence $(\mathrm{N}=1)$. No significant differences were observed between drop-outs and children who completed the training $(P>0.10)$."

Comment: even though no differences were observed between dropouts and completers, attrition rate was high and would likely have introduced bias. Plus ITT analysis was not used

Selective reporting (re- Unclear risk Comment: no protocol or clinical trial register entry available
porting bias)
Flodmark 1993

\begin{tabular}{ll} 
Methods & Parallel RCT \\
& Randomisation ratio: $1: 1$ \\
& Superiority design \\
\hline Participants & Inclusion criteria: children aged $10-11$ years, obese $(\mathrm{BMI}>23 \mathrm{~kg} / \mathrm{m} 2)$ \\
& Exclusion criteria: none \\
& Diagnostic criteria: see above
\end{tabular}

Interventions

Number of study centres: unclear

Run-in period: no

Extension period: no

Intervention: family therapy

Comparator: conventional treatment
Outcome measures reported in abstract: BMI, triceps thickness, subscapular thickness, suprailiac skinfold thickness, physical fitness 
Flodmark 1993 (Continued)

Trial ID: -

\section{Publication details Language of publication: English}

Funding: the Golje Foundation, the Swedish Medical Associations, the Albert Pahlsson Foundation, the Swedish Society of Medicine, the Johanna Andersson Foundation, "Forenade Liv" mutual group life insurance company Stockholm, the medical faculty of the University of Lund (commercial and non-commercial)

Publication status: peer-reviewed journal

\begin{tabular}{ll}
\hline Stated aim for study & Quote from publication: "To evaluate the effect of family therapy on childhood obesity" \\
\hline Notes & -
\end{tabular}

\section{Risk of bias}

\begin{tabular}{lll}
\hline Bias & Authors' judgement & Support for judgement \\
\hline $\begin{array}{l}\text { Random sequence genera- } \\
\text { tion (selection bias) }\end{array}$ & Unclear risk & $\begin{array}{l}\text { Quote from publication: "44 obese children were divided into two treatment } \\
\text { groups" }\end{array}$ \\
& $\begin{array}{l}\text { Comment: in the abstract the study authors do not say that the children were } \\
\text { randomised. They do in the main text of the publication but do not describe } \\
\text { the process and there are also concerns over imbalance of sexes in the two } \\
\text { groups. The study also includes a non-randomised control group - unclear } \\
\text { why they were not randomised as well }\end{array}$ \\
\hline
\end{tabular}

\begin{tabular}{lll}
\hline $\begin{array}{l}\text { Allocation concealment } \\
\text { (selection bias) }\end{array}$ & Unclear risk & Comment: unclear if allocation was concealed \\
\hline $\begin{array}{l}\text { Blinding of participants } \\
\text { and personnel (perfor- } \\
\text { mance bias) }\end{array}$ & Unclear risk & $\begin{array}{l}\text { Comment: unclear if participants and study personnel were blinded to study } \\
\text { group }\end{array}$ \\
Objective outcomes & & \\
\hline
\end{tabular}

Blinding of outcome as- Unclear risk Comment: unclear if assessment staff were blinded to study group sessment (detection bias)

Objective outcomes

\begin{tabular}{lll}
\hline $\begin{array}{l}\text { Incomplete outcome data } \\
\text { (attrition bias) } \\
\text { Objective outcomes }\end{array}$ & Low risk & $\begin{array}{l}\text { Quote from publication: "intention to treat analysis were made of the weight } \\
\text { and height data for } 39 \text { of } 44 \text { children in the two treatment groups" } \\
\text { Comment: dropout rates relatively low }\end{array}$ \\
\hline $\begin{array}{l}\text { Selective reporting (re- } \\
\text { porting bias) }\end{array}$ & Unclear risk & Comment: no protocol or clinical trials register entry available \\
\hline Other bias & Unclear risk & Comment: unclear if the study was at risk of any other bias \\
\hline
\end{tabular}

Gillis 2007

\begin{tabular}{ll}
\hline Methods & Parallel RCT \\
& Randomisation ratio: $1: 1$ \\
& Superiority design \\
\hline
\end{tabular}

Diet, physical activity and behavioural interventions for the treatment of overweight or obese children from the age of 6 to 11 years 
Gillis 2007 (Continued)

Participants

Inclusion criteria: aged 7-16 years, BMI > 90th percentile (CDC growth charts)

Exclusion criteria: none

Diagnostic criteria: see above

Number of study centres: 2
Run-in period: no
Extension period: no
Intervention: exercise and diet education with weekly diaries and telephone calls
Comparator: exercise and diet education only

\begin{tabular}{ll}
\hline Outcomes & Outcome measures reported in abstract: attitude, BMI SDS, LDL \\
\hline Study details & Trial terminated early: no \\
& Trial ID: - \\
\hline Publication details & Language of publication: English \\
& Funding: personal funds (non-commercial) \\
& Publication status: peer-reviewed journal \\
\hline Stated aim for study & $\begin{array}{l}\text { Quote from publication: "We aimed to determine whether in a small pilot group, treated over a 6- } \\
\text { month period, this intervention strategy could show at least a trend toward improving obesity-related } \\
\text { attitudes, reducing weight and decreasing adverse metabolic consequences of obesity" }\end{array}$
\end{tabular}

Notes -

\section{Risk of bias}

\begin{tabular}{lll}
\hline Bias & Authors' judgement & Support for judgement \\
\hline $\begin{array}{ll}\text { Random sequence genera- } \\
\text { tion (selection bias) }\end{array}$ & High risk & $\begin{array}{l}\text { Quote from publication: "Patients were allocated alternately to one of the } \\
\text { groups as they enrolled." } \\
\end{array}$ \\
& Comment: potential selection bias introduced through this method
\end{tabular}

\begin{tabular}{lll}
\hline $\begin{array}{l}\text { Allocation concealment } \\
\text { (selection bias) }\end{array}$ & High risk & $\begin{array}{l}\text { Comment: study author confirmed via email that allocation was not con- } \\
\text { cealed before randomisation }\end{array}$ \\
\hline $\begin{array}{l}\text { Blinding of participants } \\
\text { and personnel (perfor- } \\
\text { mance bias) }\end{array}$ & High risk & Comment: study author confirmed via email that the study was not blinded \\
$\begin{array}{l}\text { Subjective outcomes } \\
\text { Com }\end{array}$ & \\
\hline
\end{tabular}

Blinding of participants High risk Comment: study author confirmed via email that the study was not blinded
and personnel (perfor-
mance bias)
Objective outcomes
Blinding of outcome as- sessment (detection bias)


Gillis 2007 (Continued)

Blinding of outcome as- High risk Comment: study author confirmed via email that the study was not blinded sessment (detection bias)

Objective outcomes

$\begin{array}{ll}\begin{array}{l}\text { Incomplete outcome data } \\ \text { (attrition bias) }\end{array} & \text { High risk } \\ \begin{array}{l}\text { Subjective outcomes } \\ \end{array} & \begin{array}{l}\text { Quote from publication: "After } 6 \text { months } 11 / 14(78.6 \%) \text { intervention and } 7 / 13 \\ (53.8 \%) \text { control participants remained in the trial" }\end{array} \\ & \text { Comment: high dropout rates in the control group }\end{array}$

Incomplete outcome data High risk

(attrition bias)

Quote from publication: "After 6 months 11/14 (78.6\%) intervention and 7/13

Objective outcomes

(53.8\%) control participants remained in the trial"

Comment: high dropout rates in the control group

\begin{tabular}{|c|c|c|}
\hline $\begin{array}{l}\text { Selective reporting (re- } \\
\text { porting bias) }\end{array}$ & Unclear risk & $\begin{array}{l}\text { Comment: no protocol or clinical trials register entry available to assess re- } \\
\text { porting bias }\end{array}$ \\
\hline
\end{tabular}

Other bias Unclear risk Comment: unclear if the study was at risk of any other bias

Gunnarsdottir 2011a

\begin{tabular}{ll}
\hline Methods & Parallel RCT \\
& Randomisation ratio: $1: 1$ \\
& Superiority design
\end{tabular}

Participants

Inclusion criteria: children 8-12 years, BMI SDS > 2.4 (BMI reference values for Swedish children Karlberg, Luo \& Albertsson-Wikland, 2001), simple obesity (obesity not due to an identifiable medical cause), 1 parent willing to participate fully in the treatment with the child, neither parent nor child receiving other obesity treatment, children with comorbid emotional, behavioural and/or learning-related disorders were not excluded as long as they could comprehend intervention material and self-monitor

\section{Exclusion criteria: none}

Diagnostic criteria: see above

\begin{tabular}{ll}
\hline Interventions & Number of study centres: 1 \\
& Run-in period: no \\
& Extension period: no \\
& Intervention: Epstein's family-based behavioural treatment (FBBT) \\
& Comparator: standard care (waiting-list control) \\
\hline Outcomes & Composite outcome measures reported: BMI-SDS \\
\hline Study details & Trial terminated early: no \\
& Trial ID: - \\
\hline Publication details & Language of publication: English \\
& $\begin{array}{l}\text { Funding: Landspitali University Hospital Research Fund, The Icelandic Research Fund for Graduate } \\
\text { Students, University of Iceland Research Fund, and a grant from Thorvaldssen Society (non-commer- } \\
\text { cial) }\end{array}$
\end{tabular}

Diet, physical activity and behavioural interventions for the treatment of overweight or obese children from the age of 6 to 11 years 
Gunnarsdottir 2011a (Continued)

Publication status: peer-reviewed journal

Stated aim for study Quote from publication: "To assess the acceptability and effectiveness of Epstein's family-based behavioural treatment (FBBT) for childhood obesity in a medical setting in Iceland"

Notes -

\section{Risk of bias}

Bias Authors' judgement Support for judgement

Random sequence genera- Unclear risk Comment: randomisation method not described
tion (selection bias)

Allocation concealment
(selection bias) Unclear risk Comment: unclear if allocation was concealed

\begin{tabular}{|c|c|c|}
\hline $\begin{array}{l}\text { Blinding of participants } \\
\text { and personnel (perfor- } \\
\text { mance bias) } \\
\text { Subjective outcomes }\end{array}$ & Unclear risk & $\begin{array}{l}\text { Comment: unclear whether participants or study personnel were blinded to } \\
\text { study group }\end{array}$ \\
\hline
\end{tabular}

\begin{tabular}{|c|c|c|}
\hline $\begin{array}{l}\text { Blinding of participants } \\
\text { and personnel (perfor- } \\
\text { mance bias) } \\
\text { Objective outcomes }\end{array}$ & Unclear risk & $\begin{array}{l}\text { Comment: unclear whether participants or study personnel were blinded to } \\
\text { study group }\end{array}$ \\
\hline
\end{tabular}

Blinding of outcome as- $\quad$ Unclear risk Comment: unclear whether outcome assessors were blinded to study group
sessment (detection bias)
Subjective outcomes

Blinding of outcome as- Unclear risk

Comment: unclear whether outcome assessors were blinded to study group sessment (detection bias)

objective outcomes

$\begin{array}{ll}\begin{array}{l}\text { Incomplete outcome data } \\ \text { (attrition bias) }\end{array} & \begin{array}{l}\text { Quote from publication: "Three families dropped out prematurely (Figure } 1 \\ \text { shows flow of participants during study process). The children dropping out all } \\ \text { Subjective outcomes }\end{array} \\ \begin{array}{l}\text { had emotional, behavioural, and/or learning-related comorbidities." "Of the } \\ \text { three families who dropped out before the study ended, two families dropped } \\ \text { out for reasons unrelated to the intervention but one family was unable to } \\ \text { cope with the high at-home demands of the program" }\end{array}\end{array}$

Comment: $3 / 16$ (19\%) families dropped out of the study at 4 months. It is unclear whether these 13 participants were followed up until the end of the study. Furthermore all dropouts had comorbidities even though the study stated 2 of the families dropped out for reasons unrelated to the intervention - risk of bias is unclear

Incomplete outcome data Unclear risk

(attrition bias)

Objective outcomes
Quote from publication: "Three families dropped out prematurely (Figure 1 shows flow of participants during study process). The children dropping out all had emotional, behavioural, and/or learning-related comorbidities." "Of the three families who dropped out before the study ended, two families dropped out for reasons unrelated to the intervention but one family was unable to cope with the high at-home demands of the program"

Comment: $3 / 16$ (19\%) families dropped out of the study at 4 months. It is unclear whether these 13 participants were followed up until the end of the study. Furthermore all dropouts had comorbidities even though the study stat- 
Gunnarsdottir 2011a (Continued)

ed 2 of the families dropped out for reasons unrelated to the intervention - risk of bias is unclear

\begin{tabular}{|c|c|c|}
\hline $\begin{array}{l}\text { Selective reporting (re- } \\
\text { porting bias) }\end{array}$ & High risk & $\begin{array}{l}\text { Comment: no clinical trials register entry or protocol. They did not do a com- } \\
\text { parison of the intervention and control outcomes - did not present raw data } \\
\text { for physical activity or fruit and vegetable consumption for each group sepa- } \\
\text { rately }\end{array}$ \\
\hline
\end{tabular}

Other bias Unclear risk Comment: unable to assess if any other biases are present

Hamilton-Shield 2014

\begin{tabular}{|c|c|}
\hline Methods & $\begin{array}{l}\text { Parallel RCT } \\
\text { Randomisation ratio: 1:1 } \\
\text { Superiority design }\end{array}$ \\
\hline Participants & $\begin{array}{l}\text { Inclusion criteria: obese ( } \geq 95 \text { th percentile, UK } 1990 \text { references) children aged 5-11 years old } \\
\text { Exclusion criteria: parents unable to read English; secondary care evaluation was required if: possible } \\
\text { genetic cause of obesity, possible endocrine disorder, possible comorbidity, features of an overt eating } \\
\text { disorder, iatrogenic causes of obesity } \\
\text { Diagnostic criteria: see above }\end{array}$ \\
\hline Interventions & $\begin{array}{l}\text { Number of study centres: } 9 \\
\text { Run-in period: no } \\
\text { Extension period: no } \\
\text { Intervention: standard care plus Mandolean training } \\
\text { Comparator: standard care only }\end{array}$ \\
\hline Outcomes & $\begin{array}{l}\text { Outcome measures reported in abstract: progression to the main trial, recruitment numbers, atten- } \\
\text { dance }\end{array}$ \\
\hline
\end{tabular}

Study details Trial terminated before regular end (for benefit/because of adverse events): yes

Trial ID: ISRCTN90561114

Publication details Language of publication: English

Funding: NIHR Health Technology Assessment programme (non-commercial)

Publication status: peer-reviewed journal

\begin{tabular}{ll} 
Stated aim for study & $\begin{array}{l}\text { Quote from publication: "This pilot study aimed to test recruitment strategies, treatment adherence, } \\
\text { clinic attendance and participants' experiences of using a device [Mandolean }{ }^{\circledR} \text { (previously Mandome- } \\
\text { ter } \\
\text { advice in treating obesity in primary school-aged children" }\end{array}$ \\
\hline Notes & $\begin{array}{l}\text { This trial was terminated due to recruitment issues and technical issues relating to the Mandolean } \\
\text { equipment }\end{array}$
\end{tabular}

\section{Risk of bias}

Diet, physical activity and behavioural interventions for the treatment of overweight or obese children from the age of 6 to 11 years 
Hamilton-Shield 2014 (Continued)
Bias
Authors' judgement Support for judgement

Random sequence genera- Low risk tion (selection bias)

Quote from publication: "Participants were randomised into one of two groups: (1) standard care plus Mandolean therapy or (2) standard care alone. Participants were randomised using the Bristol Randomised Trials Collaboration randomisation service"

Comment: randomisation method was well described

\begin{tabular}{ll}
\hline $\begin{array}{l}\text { Allocation concealment } \\
\text { (selection bias) }\end{array}$ & Quote from publication: "Concealment of allocation was ensured by use \\
& of an automated web-based randomisation service hosted by the Bristol \\
& Randomised Trials Collaboration, a UKCRC (UK Clinical Research Collabora- \\
tion)-registered clinical trials unit."
\end{tabular}

Comment: allocation was concealed

\begin{tabular}{lll}
\hline $\begin{array}{l}\text { Blinding of participants } \\
\text { and personnel (perfor- } \\
\text { mance bias) }\end{array}$ & Unclear risk & $\begin{array}{l}\text { Comment: unclear whether participants or study personnel were blinded to } \\
\text { study group }\end{array}$ \\
$\begin{array}{l}\text { Subjective outcomes } \\
\text { Blinding of participants }\end{array}$ & Unclear risk & $\begin{array}{l}\text { Comment: unclear whether participants or study personnel were blinded to } \\
\text { study group }\end{array}$ \\
$\begin{array}{l}\text { and personnel (perfor- } \\
\text { mance bias) }\end{array}$ & \\
Objective outcomes &
\end{tabular}
sessment (detection bias)

Subjective outcomes

Blinding of outcome as- Unclear risk Comment: unclear whether outcome assessors were blinded to study group
sessment (detection bias)
Objective outcomes

Incomplete outcome data High risk Quote from publication: "None of the criteria for progression to the main trial (attrition bias) were reached. Despite numerous pathways being available for referral, only 21

Subjective outcomes (13 to standard care, eight to intervention arm; 58\%) of the target 36 families were recruited in the pilot phase. Less than $20 \%$ of those randomised to Mandolean used the device at least five times a week. The $>60 \%$ target for slowing down of eating speed by 3 months was unmet. Attendance at the weight management clinic in general practice hubs for both arms of the study at 3 months was $44 \%$ against a target of $80 \% . "$

Comment: attendance at the sessions was very low and the trial was not completed - high attrition bias

Incomplete outcome data High risk

(attrition bias)

Objective outcomes
Quote from publication: "None of the criteria for progression to the main trial were reached. Despite numerous pathways being available for referral, only 21 (13 to standard care, eight to intervention arm; 58\%) of the target 36 families were recruited in the pilot phase. Less than $20 \%$ of those randomised to Mandolean used the device at least five times a week. The $>60 \%$ target for slowing down of eating speed by 3 months was unmet. Attendance at the weight management clinic in general practice hubs for both arms of the study at 3 months was $44 \%$ against a target of $80 \%$."

Comment: attendance at the sessions was very low and the trial was not completed - high attrition bias 
Hamilton-Shield 2014 (Continued)

$\begin{aligned} & \text { Selective reporting (re- } \quad \text { High risk } \\ & \text { porting bias) }\end{aligned}$
any outcome data of those who participated

Other bias

High risk

Comment: this study was terminated before its endpoint

Ho 2016

\begin{tabular}{ll}
\hline Methods & Parallel RCT \\
& Randomisation ratio:1:1 \\
& Superiority design \\
\hline Participants & Inclusion criteria: 8-16 years, BMI $\geq 85^{\text {th }}$ centile for age and gender \\
& Exclusion criteria: currently taking a weight loss medication, enrolled in any organised weight loss \\
programmes or exercise programmes, consumed more than 30\% of all meals at restaurants, had a his- \\
tory of gastrointestinal disorder, psychiatric illness under the care of a physician, Cushing's syndrome, \\
hypothalamic or genetic aetiology of obesity, uncontrolled or untreated thyroid disease, a current di- \\
agnosis of cancer, history of an eating disorder such as bulimia or anorexia nervosa, any surgery in the \\
past 3 months, any surgery planned in the ensuing 6 months or any other chronic illness that could af- \\
fect weight change
\end{tabular}

Diagnostic criteria: BMI percentile (population reference not stated)

Number of study centres: 1
Run-in period: no
Extension period: no
Intervention: standard nutrition counselling plus portion control equipment
Control: standard nutrition counselling only

Outcomes Outcome measures reported in abstract: BMI z score

Study details

\section{Trial terminated early: no}

Trial ID: NCT00881478

\section{Publication details Language of publication: English}

Funding: non-commercial funding and commercial donation, research grant from the Alberta Children's Hospital Foundation (Calgary, Alberta, Canada). Some of the portion control tools were donated for use in the study by The Diet Plate

Publication status: peer-reviewed journal

\begin{tabular}{ll}
\hline Stated aim for study & $\begin{array}{l}\text { Quote from publication: "to assess the effect of a family intervention using a portion control tool on } \\
\text { BMI z score in children." }\end{array}$ \\
\hline Notes & - \\
\hline Risk of bias & Authors' judgement Support for judgement \\
\hline Bias &
\end{tabular}

Diet, physical activity and behavioural interventions for the treatment of overweight or obese children from the age of 6 to 11 years 
Ho 2016 (Continued)

Random sequence genera- Low risk tion (selection bias)

Quote from publication: "A computer-based random number sequence generator was used to create the random allocation..."

Comment: low risk of selection bias from the randomisation method used

Allocation concealment Low risk

(selection bias)

Quote from publication: "Sequentially numbered sealed envelopes were used to conceal the sequence until participants were assigned. The random allocation sequence was generated by a research assistant, while enrolment and assignment of participants to groups was done by the research coordinator.."

Comment: allocation was likely concealed, hence low risk of selection bias

\section{Blinding of participants High risk} and personnel (perfor-

mance bias)

Subjective outcomes

Quote from publication: "Participants and care givers were not blinded to the intervention since they were instructed on use of the portion control tools."

Comment: investigator-assessed. Participants weren't blinded due to the nature of the intervention in addition it is currently not stated whether trial personnel were blinded - this presents potentially high risk of bias

$\begin{array}{ll}\begin{array}{l}\text { Blinding of participants } \\ \text { and personnel (perfor- } \\ \text { mance bias) }\end{array} & \text { High risk } \\ \begin{array}{l}\text { Objective outcomes } \\ \text { intervention since they were instructed on use of the portion control tools." }\end{array} \\ \begin{array}{l}\text { Comment: investigator-assessed. Participants weren't blinded due to the na- } \\ \text { ture of the intervention in addition it is currently not stated whether trial per- } \\ \text { sonnel were blinded - this presents potentially high risk of bias }\end{array}\end{array}$

Blinding of outcome as- Unclear risk sessment (detection bias)

Subjective outcomes

Quote from publication: "Participants and care givers were not blinded to the intervention since they were instructed on use of the portion control tools.."

Comment: unclear if outcome assessment was blinded

Blinding of outcome as- Unclear risk sessment (detection bias)

Objective outcomes

Quote from publication: "Participants and care givers were not blinded to the intervention since they were instructed on use of the portion control tools."

Comment: unclear if outcome assessment was blinded

Incomplete outcome data Low risk
(attrition bias)
Subjective outcomes

\section{Comment: reported and ITT analysis conducted}

\begin{tabular}{lll}
\hline $\begin{array}{l}\text { Incomplete outcome data } \\
\text { (attrition bias) } \\
\text { Objective outcomes }\end{array}$ & Low risk & Comment: reported and ITT analysis conducted \\
\hline $\begin{array}{l}\text { Selective reporting (re- } \\
\text { porting bias) }\end{array}$ & Low risk & Comment: study was conducted as described in the trials register \\
\hline Other bias & Low risk & $\begin{array}{l}\text { Comment: no other bias identified - this was a generally well conducted and } \\
\text { reported study }\end{array}$
\end{tabular}

Hughes 2008

\begin{tabular}{ll}
\hline Methods & Parallel RCT \\
& Randomisation ratio: $1: 1$ \\
Superiority design
\end{tabular}

Diet, physical activity and behavioural interventions for the treatment of overweight or obese children from the age of 6 to 11 years 
Hughes 2008 (Continued)

Participants
Inclusion criteria: obese children (BMI $\geq 98$ th centile, UK 1990 references) aged 5-11 years, attending a standard elementary school, at least 1 parent who perceived their child's weight as a problem and willing to make changes to their lifestyle

Exclusion criteria: child with an underlying medical cause for their obesity, serious co-morbidity requiring urgent treatment, had received treatment for obesity in the past year

Diagnostic criteria: see above

\section{Number of study centres: 1}

Run-in period: no

Extension period: no

Intervention: behavioural programme

Comparator: standard care

Outcome measures reported in abstract: BMI z scores, weight, total physical activity, percentage time spent in sedentary behaviour and light intensity physical activity, parental views of the treatment

Trial terminated early: no

Trial ID: ISRCTN41383109

\section{Publication details Language of publication: English}

Funding: grant from the Scottish Executive Health Department. The funder's role was limited to peer review of the original grant application (non-commercial)

Publication status: peer-reviewed journal
Quote from publication: "The objective of this study was to determine whether a generalizable best practice individualized behavioral intervention reduced BMI z score relative to standard dietetic care among overweight children"

\section{Notes}

\section{Risk of bias}

Bias Authors' judgement Support for judgement

Random sequence genera- Low risk tion (selection bias)

Quote from publication: "For ensuring concealment, the study code was sent to a statistician, who produced a computer-generated randomization list and allocated participants to the intervention or control group. Randomization was in blocks of 10 (ratio 1:1) and was stratified by gender and study center (Edinburgh or Glasgow). The statistician informed the research dietitians, who were delivering the intervention of the group allocation and who then informed participants of their groups."

Comment: randomisation method was well described

Allocation concealment Low risk
(selection bias)

Low risk
Quote from publication: "For ensuring concealment, the study code was sent to a statistician, who produced a computer-generated randomization list and allocated participants to the intervention or control group" 
Hughes 2008 (Continued)

Blinding of participants High risk Quote from publication: "The primary aim of this assessor-blinded RCT" and personnel (perfor-

Comment: participants or study personnel were not blinded to study group

mance bias)

Subjective outcomes

\section{Blinding of participants High risk} and personnel (perfor-

mance bias)

Objective outcomes
Quote from publication: "The primary aim of this assessor-blinded RCT"

Comment: participants or study personnel were not blinded to study group

Blinding of outcome as-
sessment (detection bias) $\quad$ Quote from publication: "The primary aim of this assessor-blinded RCT"

Subjective outcomes

Comment: outcomes investigators were blinded to study group

Blinding of outcome as- Low risk sessment (detection bias)

Objective outcomes
Quote from publication: "The primary aim of this assessor-blinded RCT"

Comment: outcomes investigators were blinded to study group

Quote from publication: "Of the 134 children who were randomly assigned, $97(72.4 \%)$ attended the 6 month follow up and 86 (64.2\%) attended at 12 months"

Comment: dropout rates were quite high

Quote from publication: "Of the 134 children who were randomly assigned, $97(72.4 \%)$ attended the 6 month follow up and 86 (64.2\%) attended at 12 months"

Comment: dropout rates were quite high

High risk

(attrition bias)

Objective outcomes

Comment: no differences between protocol and publication porting bias)

$\begin{array}{ll}\text { Methods } & \text { Parallel RCT } \\ & \text { Randomisation ratio: } 1: 1 \\ & \text { Superiority design }\end{array}$

Participants

Inclusion criteria: child 8-12 years, $\mathrm{BMI} \geq 97$ th percentile (CDC growth charts) - severely obese, adult willing to participate in the programme

Exclusion criteria: mental retardation, pervasive development disorder or psychosis, psychiatric symptoms that require alternative treatment, genetic obesity syndrome, currently undertaking obesity treatment, inability to take part in prescribed daily activity, medical conditions which contraindicate usual care, medication which affects body weight (stable doses of stimulant or antidepressant medication allowed)

Diagnostic criteria: see above 
Kalarchian 2009 (Continued)

\section{Extension period: no}

Intervention: family-based, behavioural weight control group

Comparator: usual care

Outcomes

Composite outcome measures reported: percent overweight, medical outcomes, parental BMI, binge eating, eating disorder symptoms, self-esteem

\section{Study details $\quad$ Trial terminated early: no}

Trial ID: NCT00177229

\section{Publication details Language of publication: English}

Funding: National Institutes of Health grants to Dr Marcus at the University of Pittsburgh (grant R01 HD38425 and minority supplement grant HD38425-02S1), University of Pittsburgh Obesity and Nutrition Research Center (grant P30 DK46204), Children's Hospital of Pittsburgh General Clinical Research Center (grant M01-RR00084), and University of Pittsburgh Clinical and Translational Science Institute (Clinical and Translational Science Award UL1-RR024153) (non-commercial)

Publication status: peer-reviewed journal

Stated aim for study

Quote from publication: "We evaluated the efficacy of family-based, behavioural weight control in the management of severe pediatric obesity"

Notes -

\section{Risk of bias}

Bias Authors' judgement Support for judgement

Random sequence genera- Low risk tion (selection bias)

Quote from publication: "After baseline assessments, participants were assigned randomly to study conditions (1:1) through permuted block randomization with stratification according to race, with a block size of 2,4 , or 6 ."

Comment: randomisation method described

\begin{tabular}{lll}
\hline $\begin{array}{l}\text { Allocation concealment } \\
\text { (selection bias) }\end{array}$ & Low risk & Comment: study author confirmed via email that allocation was concealed \\
\hline $\begin{array}{l}\text { Blinding of participants } \\
\text { and personnel (perfor- } \\
\text { mance bias) }\end{array}$ & High risk & $\begin{array}{l}\text { Comment: study author confirmed participants and study personnel were not } \\
\text { blinded to study group }\end{array}$
\end{tabular}

mance bias)

Subjective outcomes

$\begin{array}{lll}\begin{array}{l}\text { Blinding of participants } \\ \text { and personnel (perfor- }\end{array} & \text { High risk } & \begin{array}{l}\text { Comment: study author confirmed participants and study personnel were not } \\ \text { blinded to study group }\end{array}\end{array}$
mance bias)

Objective outcomes

Blinding of outcome as- $\quad$ Unclear risk
sessment (detection bias)

Subjective outcomes

Quote from publication: "Assessors did not provide the intervention but were not blinded to the treatment condition."

Comment: unclear because even though assessors were not involved in the intervention, they were not blinded to study group

Blinding of outcome as- $\quad$ Unclear risk
sessment (detection bias)
Objective outcomes

Quote from publication: "Assessors did not provide the intervention but were not blinded to the treatment condition." 
Comment: unclear because even though assessors were not involved in the intervention, they were not blinded to study group

Incomplete outcome data Low risk

(attrition bias)

Subjective outcomes
Quote from publication: "However, 18- month assessment completers differed from noncompleters with respect to baseline child BMI ( 31.7 vs 34.0 $\mathrm{kg} / \mathrm{m} 2 ; t=-2.14 ; P=.037$ ), percent overweight (87.4\% vs $101.8 \% ; t=2.36 ; P$ $=.023$ ), and number of people in the household (4.11 vs 3.67 persons; $t=2.13$; $P=.035)$." "Finally, there was a significant proportion of missing data in the ITT analyses for medical risk factors, which suggests that replication is needed before firm conclusions about medical outcomes can be drawn."

Comment: relatively low amount of missing data

Incomplete outcome data Low risk
(attrition bias)

Objective outcomes
Quote from publication: "However, 18- month assessment completers differed from noncompleters with respect to baseline child BMI (31.7 vs 34.0 $\mathrm{kg} / \mathrm{m} 2 ; t=-2.14 ; P=.037$ ), percent overweight ( $87.4 \%$ vs $101.8 \% ; t=2.36 ; P$ $=.023$ ), and number of people in the household (4.11 vs 3.67 persons; $t=2.13$; $P=.035)$." "Finally, there was a significant proportion of missing data in the ITT analyses for medical risk factors, which suggests that replication is needed before firm conclusions about medical outcomes can be drawn."

Comment: relatively low amount of missing data
Selective reporting (re- High risk porting bias)
Comment: primary outcome on clinical trials register was BMI and cardiovascular risk factors, while in publication it was percentage overweight

Other bias Unclear risk Comment: unable to assess if any other bias were present

Kalavainen 2007

\begin{tabular}{ll}
\hline Methods & Parallel RCT \\
& Randomisation ratio: $1: 1$ \\
& Superiority design \\
\hline Participants & $\begin{array}{l}\text { Inclusion criteria: child age 7-9 years, attending primary school, presence of weight for height from } \\
120 \%-200 \% \text { (Finnish national growth charts - Tilator Oy Ltd 2004) } \\
\text { Exclusion criteria: disease or medication causing obesity, obvious movement disturbance, major } \\
\text { mental problems in child or parents, family members participating in another weight-management } \\
\text { programme }\end{array}$
\end{tabular}

Diagnostic criteria: see above

\begin{tabular}{ll}
\hline Interventions & Number of study centres: 1 \\
Run-in period:no & Extension period: no \\
Intervention: family-centred group programme \\
Comparator: routine treatment \\
\hline Outcomes & $\begin{array}{l}\text { Outcome measures reported in abstract: weight for height, BMI, BMI SDS, participation rate, attrition } \\
\text { rates, cost effectiveness, waist/height, metabolic risk factors, triglycerides, fasting insulin }\end{array}$
\end{tabular}


Kalavainen 2007 (Continued)

Trial ID: -

\section{Publication details Language of publication: English}

Funding: in part by grants from Kuopio University Hospital, the Scientific Foundation of Finnish Association of Academic Agronomists, Finnish Cultural Foundation of Northern Savo, Juho Vainio Foundation, Ministry of Social Affairs and Health, Social Insurance Institution, and the Finnish Cultural Foundation (non-commercial)

Publication status: peer-reviewed journal

Stated aim for study $\quad \begin{aligned} & \text { Quote from publication: "The aim of the study was to compare the efficacy of group treatment stress- } \\ & \text { ing a health-promoting lifestyle with routine counselling in the treatment of childhood obesity" }\end{aligned}$
ing a health-promoting lifestyle with routine counselling in the treatment of childhood obesity"

Notes -

\section{Risk of bias}

Bias Authors' judgement Support for judgement

Random sequence genera- Low risk tion (selection bias)

Quote from publication: "Three children with weight for height $\geq 120 \%$ at the individual interview, but $115-117 \%$ at the baseline measurement, were included (two allocated into the routine treatment and one to group treatment). The children were then stratified on the basis of their weight for height in four blocks, that is weight for height $<120 \%, 120-139 \%, 140-160 \%$ and $>160 \%$, and thereafter they were randomly allocated within each block, using closed envelopes, to either routine or group program. The siblings (three pairs in this study) were randomized together, and the stratification was based on the higher weight for height of the siblings."

Comment: randomisation method well described

\begin{tabular}{|c|c|c|}
\hline $\begin{array}{l}\text { Allocation concealment } \\
\text { (selection bias) }\end{array}$ & Low risk & $\begin{array}{l}\text { Quote from publication: "they were randomly allocated within each block, } \\
\text { using closed envelopes, to either routine or group program. }\end{array}$ \\
\hline
\end{tabular}

Comment: used closed envelopes so assume allocation was concealed

\begin{tabular}{|c|c|c|}
\hline $\begin{array}{l}\text { Blinding of participants } \\
\text { and personnel (perfor- }\end{array}$ & High risk & $\begin{array}{l}\text { Comment: Heale } 2008 \text { (see Kalavainen } 2007 \text { for reference) states study was } \\
\text { unblinded }\end{array}$ \\
\hline
\end{tabular}
mance bias)

Objective outcomes

\begin{tabular}{|c|c|c|}
\hline $\begin{array}{l}\text { Blinding of outcome as- } \\
\text { sessment (detection bias) }\end{array}$ & High risk & $\begin{array}{l}\text { Comment: Heale } 2008 \text { (see Kalavainen } 2007 \text { for reference) states study was } \\
\text { unblinded }\end{array}$ \\
\hline
\end{tabular}

Objective outcomes

Incomplete outcome data Low risk

(attrition bias)

Objective outcomes
Quote from publication: "The number of children participating in the 2-year follow-up was 69 ( 35 in routine counselling and 34 in the group program) and in the 3-year follow-up was 68 (34 in both treatment arms)."

Comment: 70 children were randomised and 68 were followed up at 3 years very low dropout rates, unlikely to have attrition bias

$\begin{array}{ll}\text { Selective reporting (re- } \quad \text { Unclear risk } & \text { Comment: } 1 \text { publication reports that they only measured height and weight - } \\ \text { porting bias) } & \text { however, in a later paper results of additional outcomes were reported (meta- } \\ & \text { bolic and body composition) but were compared with a healthy-weight chil- } \\ & \text { dren's group. Results of these outcomes were not significant - potential report- } \\ \text { ing bias }\end{array}$

Other bias Unclear risk Comment: unclear if the study was at risk of any other bias

Diet, physical activity and behavioural interventions for the treatment of overweight or obese children from the age of 6 to 11 years 
Kirk 2012

\begin{tabular}{ll}
\hline Methods & Parallel RCT \\
& Randomisation ratio: 1:1:1 \\
& Superiority design \\
\hline Participants & $\begin{array}{l}\text { Inclusion criteria: age 7-12 years, fasting blood glucose level } \leq 100 \mathrm{mg} / \mathrm{dL}, \mathrm{BMI} z \text { score of } 1.60-2.65 \\
\text { (CDC growth charts), absence of development or physical disabilities, ability to function independently } \\
\text { in group exercise sessions, parent/guardian commitment to the study sessions }\end{array}$
\end{tabular}

Exclusion criteria: medical conditions such as: cardiac, pulmonary or liver disease; hyperlipidaemia, diabetes or significant mental illness, taking medications which may alter bone density, lipid or glucose metabolism or appetite (e.g. stimulants)

Diagnostic criteria: see above

Number of study centres: 1
Run-in period: no
Extension period: no
Intervention 1: low carbohydrate diet + group exercise/education sessions
Intervention 2: reduced glycaemic load diet + group exercise/education sessions
Comparator: standard portion-controlled diet + group exercise/education sessions

Outcomes Outcome measures reported in abstract: completion rates, daily caloric intake, adherence, BMI $\mathrm{z}$ score, WC, percent body fat

\section{Study details}

Trial terminated early: no

Trial ID: NCT00215111

Publication details Language of publication: English

Funding: Thrasher Research Fund and an Institutional Clinical and Translational Science Award (National Institutes of Health (NIH)/National Center for Research Resources grant, 5UL1RR026314-02) (noncommercial)

Publication status: peer-reviewed journal

\begin{tabular}{ll}
\hline Stated aim for study & Quote from publication: "To compare the effectiveness and safety of carbohydrate (CHO)-modified di- \\
ets with a standard portion-controlled (PC) diet in obese children"
\end{tabular}

\section{Notes}

\section{Risk of bias}

\begin{tabular}{|c|c|c|}
\hline Bias & Authors' judgement & Support for judgement \\
\hline $\begin{array}{l}\text { Random sequence genera- } \\
\text { tion (selection bias) }\end{array}$ & Low risk & $\begin{array}{l}\text { Quote from publication: "The subjects were stratified by pubertal develop- } \\
\text { ment ( } 4 \text { categories) and BMI } z \text { score ( } 2 \text { categories: } \leq 2.1 \text { SD or }>2.1 \text { SD). Within } \\
\text { these } 8 \text { strata, randomly permuted block sizes were used to generate the ran- } \\
\text { domized allocation sequence. Subjects were randomly assigned to one of } 3 \text { di- } \\
\text { et groups-LC ( } n=35), R G L(n=36) \text {, or PC }(n=31) \text {-and informed of their diet } \\
\text { assignment at the initial intervention visit." }\end{array}$ \\
\hline
\end{tabular}

Diet, physical activity and behavioural interventions for the treatment of overweight or obese children from the age of 6 to 11 years 
Kirk 2012 (Continued)

Comment: randomisation method well described

\begin{tabular}{|c|c|c|}
\hline $\begin{array}{l}\text { Allocation concealment } \\
\text { (selection bias) }\end{array}$ & Low risk & $\begin{array}{l}\text { Quote from publication: "Within these } 8 \text { strata, randomly permuted block } \\
\text { sizes were used to generate the randomized allocation sequence" }\end{array}$ \\
\hline & & $\begin{array}{l}\text { Comment: assume allocation was concealed via the randomisation method } \\
\text { used }\end{array}$ \\
\hline
\end{tabular}

\begin{tabular}{|c|c|c|}
\hline $\begin{array}{l}\text { Blinding of participants } \\
\text { and personnel (perfor- } \\
\text { mance bias) }\end{array}$ & High risk & $\begin{array}{l}\text { Quote from publication: "Neither subjects nor study staff members were } \\
\text { blinded to diet assignment." }\end{array}$ \\
\hline Subjective outcomes & & Comment: participants and study personnel were not blinded to study group \\
\hline
\end{tabular}

\begin{tabular}{|c|c|c|}
\hline $\begin{array}{l}\text { Blinding of participants } \\
\text { and personnel (perfor- } \\
\text { mance bias) }\end{array}$ & High risk & $\begin{array}{l}\text { Quote from publication: "Neither subjects nor study staff members were } \\
\text { blinded to diet assignment." }\end{array}$ \\
\hline Objective outcomes & & Comment: participants and study personnel were not blinded to study group \\
\hline
\end{tabular}

Blinding of outcome as- High risk sessment (detection bias)

Subjective outcomes
Quote from publication: "Neither subjects nor study staff members were blinded to diet assignment."

Comment: assessment staff were not blinded to study group

Blinding of outcome as-
sessment (detection bias)
Objective outcomes risk

sessment (detection bias)

Objective outcomes
Quote from publication: "Neither subjects nor study staff members were blinded to diet assignment."

Comment: assessment staff were not blinded to study group
Incomplete outcome data Unclear risk
(attrition bias)

Subjective outcomes
Quote from publication: "Retention of subjects for follow-up assessments was $82 \%$ at the 12 -month follow-up and did not differ significantly among the 3 diet groups at any time point (RGL: 3 months, $92 \%$; 6 months, $89 \%$; 12 months, $89 \%$; PC: 3 months, $94 \%$; 6 months, $87 \%$; 12 months, $90 \%$; LC: 3 months, 69\%; 6 months, 69\%; 12 months, 69\%)"

Comment: dropout rates moderate and they used ITT analysis. But was unclear how they replaced missing data

Quote from publication: "Retention of subjects for follow-up assessments was $82 \%$ at the 12 -month follow-up and did not differ significantly among the 3 diet groups at any time point (RGL: 3 months, $92 \%$; 6 months, $89 \%$; 12 months, $89 \%$; PC: 3 months, $94 \%$; 6 months, $87 \%$; 12 months, $90 \%$; LC: 3 months, 69\%; 6 months, 69\%; 12 months, 69\%)"

Comment: dropout rates moderate and they used ITT analysis. But was unclear how they replaced missing data

Comment: the majority of outcomes given in the clinical trials register were measured and reported in the publication

$\begin{array}{ll}\begin{array}{l}\text { Selective reporting (re- } \\ \text { porting bias) }\end{array} & \text { Low risk } \\ \end{array}$

Other bias Unclear risk Comment: unclear if the study was at risk of any other bias

Larsen 2015

\begin{tabular}{ll} 
Methods & Parallel RCT \\
& Randomisation ratio: $1: 1$ \\
& Superiority design \\
\hline
\end{tabular}

Diet, physical activity and behavioural interventions for the treatment of overweight or obese children from the age of 6 to 11 years 
Larsen 2015 (Continued)

Participants

Inclusion criteria: overweight (IOTF criteria), children aged 5-9 years, registered with a GP on the island of Fumen

Exclusion criteria: families unable to speak Danish, previous or current participation in other overweight/obesity project, mental or physical disabilities, endocrine causes of obesity, signs of precocious puberty

Diagnostic criteria: see above

\begin{tabular}{ll}
\hline Interventions & Number of study centres: 60 \\
Run-in period: no \\
Extension period: no \\
Intervention: an education programme in addition to health consultations \\
Comparator: health consultations only
\end{tabular}

Outcomes Outcome measures reported in abstract: BMI z scores, attendance

Study details

Trial terminated early: no

Trial ID: -

Publication details Language of publication: English

Funding: Health Insurance Foundation, Rhode's Foundation, the Egmont Foundation, the Tryg Foundation, Institute of Clinical Research, Faculty of Health Sciences, University of Southern Denmark, and Odense University Hospital. (commercial and non-commercial)

Publication status: peer-reviewed journal

Stated aim for study Quote from publication: "To evaluate the effect of two intervention modalities concerning overweight and obesity among children in general practice."

Notes

\section{Risk of bias}

Bias Authors' judgement Support for judgement

Random sequence genera- Low risk Quote from publication: "Participants were randomized using a random tion (selection bias) n number table prepared before recruitment of participants for the study."

Comment: low risk of bias from the method described

Allocation concealment Low risk (selection bias)
Quote from publication: "In order to ensure concealment of the allocated intervention at the time of enrolment of participants, the participants were randomized in blocks of two for patients enrolled in a single-handed practice, and in blocks of four or six for patients enrolled in a group practice. The size of the blocks and the allocation sequence were unknown to the general practitioners (GPs). Besides information to the patients regarding the study and obtainment of oral and written consent, the GPs did not take part in either the allocation process, or information to the families on results of the randomization. The GPs informed the study investigator about the patient's acceptance of participation in the study. The study investigator allocated the patient according to the random number table and informed the family by telephone or letter."

Comment: allocation was concealed 
Larsen 2015 (Continued)

Blinding of participants Unclear risk Comment: unclear whether participants and study personnel were blinded to and personnel (perforstudy group

mance bias)

Objective outcomes

Blinding of outcome as-
sessment (detection bias) $\quad$ Unclear risk Comment: unclear whether assessment staff were blinded to study group

Objective outcomes

Incomplete outcome data High risk

(attrition bias)

Objective outcomes
Quote from publication: "A total of 10 children in Model 1 and 16 children in Model 2 succeeded in a full two-year follow-up."

Comment: only $29 \%$ of the control group and $36 \%$ of the intervention group completed the 2-year follow-up

Selective reporting (re- Unclear risk Comment: no protocol or clinical trial register entry porting bias)

Other bias Unclear risk Comment: unclear if the study was at risk of any other bias

Lison 2012

$\begin{array}{ll}\text { Methods } & \text { Parallel RCT } \\ & \text { Randomisation ratio: approximately 2:2:1 } \\ & \text { Superiority design }\end{array}$

Participants

Inclusion criteria: white children and adolescents aged 6-16 years, both sexes, overweight or obese ( $\geq$ 85th percentile, Cole's LMS method - Cole 2000), recruited at the obesity and cardiovascular risk unit, Consorcio Hospital General Unversitario, Valencia ,Spain

Exclusion criteria: secondary obesity syndromes or acute illnesses, severe obesity (z score $>2.5$ )

Diagnostic criteria: see above

\begin{tabular}{ll}
\hline Interventions & Number of study centres: 1 \\
& Run-in period: no \\
& Extension period: no \\
& Intervention 1: hospital clinic group exercise-diet programme \\
& Intervention 2: home-based combined exercise-diet programme \\
& Comparator: usual care control group \\
\hline Outcomes & Outcome measures reported in abstract: BMI z score, WC, percentage body fat, attendance \\
\hline Study details & Trial terminated early: no \\
& Trial ID: NCT01503281 \\
\hline Publication details & Language of publication: English \\
& Funding: grants from the Comunidad Valenciana Government (GV06/227) (non-commercial) \\
\hline \hline
\end{tabular}

Diet, physical activity and behavioural interventions for the treatment of overweight or obese children from the age of 6 to 11 years 
Lison 2012 (Continued)

Stated aim for study Quote from publication: "The aim of this study was to compare the effect of a hospital clinic group- versus home-based combined exercise- diet program for the treatment of childhood obesity"

Notes

\section{Risk of bias}

Bias Authors' judgement Support for judgement

Random sequence genera- High risk tion (selection bias)

Quote from publication: "Patients were assigned to experimental groups on the basis of the day of the week in which they attended the outpatient clinic. Patients who attended on Mondays and Wednesdays were assigned to the GRX and those on Tuesdays and Thursdays to the HOX. Those who attended on Fridays were assigned to the control group."

Comment: potential bias as participants would have been able to predict which group they would be allocated to

\begin{tabular}{lll}
\hline $\begin{array}{l}\text { Allocation concealment } \\
\text { (selection bias) }\end{array}$ & High risk & $\begin{array}{l}\text { Comment: unlikely that allocation was concealed due to randomisation } \\
\text { method used }\end{array}$
\end{tabular}

Blinding of participants High risk
and personnel (performance bias)

Objective outcomes

Quote from publication: "An open study design was used" "The paediatrician who attended these visits was blinded to group allocation criteria."

Comment: only the paediatrician was blinded to study group

$\begin{array}{ll}\begin{array}{l}\text { Blinding of outcome as- } \\ \text { sessment (detection bias) } \\ \text { Objective outcomes }\end{array} & \text { Low risk }\end{array} \quad \begin{aligned} & \text { Quote from publication: "All outcome measures were recorded at baseline } \\ & \text { and at the end of the program by a trained nurse who was blinded to group al- } \\ & \text { location." }\end{aligned}$

Comment: the nurse taking the measurements was blinded

\begin{tabular}{lll}
\hline Incomplete outcome data & Unclear risk & $\begin{array}{l}\text { Quote from publication: "The number of treatment completers was similar } \\
\text { (attrition bias) }\end{array}$ \\
Objective outcomes & spectively)."
\end{tabular}

Comment: the number followed up was moderate; however, the number who actually completed the treatment was relatively low

$\begin{array}{ll}\begin{array}{l}\text { Selective reporting (re- Low risk } \\ \text { porting bias) }\end{array} & \begin{array}{l}\text { Comment: outcomes given in the clinical trials register the same as reported } \\ \text { in the publication. No other differences found }\end{array}\end{array}$

Other bias Unclear risk Comment: unable to assess whether any other biases are present

Lochrie 2013

\begin{tabular}{ll}
\hline Methods & Parallel RCT \\
& Randomisation ratio: 1:1 \\
& Superiority design \\
\hline Participants & Inclusion criteria: aged 8-11 years, age-and sex-adjusted BMI $\geq 85$ th percentile (CDC growth charts) \\
& $\begin{array}{l}\text { Exclusion criteria: impaired glucose tolerance, diabetes mellitus type 2, metabolic syndrome, hyper- } \\
\text { tension or significant learning problems }\end{array}$
\end{tabular}

Diet, physical activity and behavioural interventions for the treatment of overweight or obese children from the age of 6 to 11 years 
Lochrie 2013 (Continued)

Diagnostic criteria: see above

$\begin{array}{ll}\text { Interventions } & \text { Number of study centres: } 1 \\ \text { Run-in period: no } & \text { Extension period: no } \\ \text { Intervention: family-based intervention } \\ \text { Comparator: education session }\end{array}$

Outcomes Outcome measures reported in abstract: BMI z scores, triglycerides, psychosocial data

Study details Trial terminated early: no

Trial ID: NCT01146314

\begin{tabular}{ll}
\hline Publication details & Language of publication: English \\
& Funding: American Diabetes Association (non-commercial) \\
& Publication status: peer-reviewed journal
\end{tabular}

Stated aim for study Quote from publication: "This article examined immediate post-treatment and follow-up results of a randomized controlled trial of a 6-month lifestyle intervention involving diet, education, physical exercise, behavior change, and psychosocial methods for overweight or obese school-age children ages 8 to 11 to decrease risk factors associated with medical complications of obesity."

Notes -

\section{Risk of bias}

\begin{tabular}{lll}
\hline Bias & Authors' judgement & Support for judgement \\
\hline $\begin{array}{ll}\text { Random sequence genera- } \\
\text { tion (selection bias) }\end{array}$ & Low risk & $\begin{array}{l}\text { Quote from publication: "Randomization was stratified based on BMI (85th } \\
\text { to 95th or }>95 \text { th percentiles). For both of the lists, participants were random- } \\
\text { ized using a random sequence of } 1 \mathrm{~s} \text { and } 2 \mathrm{~s}, \text { such that } 75 \text { were assigned to the } \\
\text { IG and } 75 \text { were assigned to the EG" }\end{array}$ \\
& Comment: randomisation process described
\end{tabular}

\begin{tabular}{|c|c|c|}
\hline $\begin{array}{l}\text { Allocation concealment } \\
\text { (selection bias) }\end{array}$ & Low risk & Comment: study author confirmed via email that allocation was concealed \\
\hline $\begin{array}{l}\text { Blinding of participants } \\
\text { and personnel (perfor- } \\
\text { mance bias) } \\
\text { Subjective outcomes }\end{array}$ & High risk & $\begin{array}{l}\text { Comment: study author confirmed participants and personnel were not blind- } \\
\text { ed }\end{array}$ \\
\hline $\begin{array}{l}\text { Blinding of participants } \\
\text { and personnel (perfor- } \\
\text { mance bias) } \\
\text { Objective outcomes }\end{array}$ & High risk & $\begin{array}{l}\text { Comment: study author confirmed participants and personnel were not blind- } \\
\text { ed }\end{array}$ \\
\hline
\end{tabular}

$\begin{array}{lll}\text { Blinding of outcome as- } & \text { High risk } \quad \text { Comment: study author confirmed outcome assessors were not blinded } \\ \text { sessment (detection bias) }\end{array}$

Subjective outcomes

Blinding of outcome as-

High risk

Comment: study author confirmed outcome assessors were not blinded sessment (detection bias)

Diet, physical activity and behavioural interventions for the treatment of overweight or obese children from the age of 6 to 11 years 
Lochrie 2013 (Continued)

Objective outcomes

Incomplete outcome data High risk (attrition bias)

Subjective outcomes
Quote from publication: "We recognize that this study did not achieve adequate retention of participants. Only $68 \%$ of participants completed the baseline and post-treatment evaluation and 55\% completed the follow-up evaluation."

Comment: high dropout

Quote from publication: "We recognize that this study did not achieve adequate retention of participants. Only $68 \%$ of participants completed the baseline and post-treatment evaluation and $55 \%$ completed the follow-up evaluation."

Comment: high dropout
Incomplete outcome data High risk

(attrition bias)

Objective outcomes
Comment: clinical trial entry similar to publication but publication does not given raw data for any outcomes (only shows BMI z score in a graph but no SDs) - for other outcomes they just say the difference was not statistically significant

Other bias Unclear risk Comment: unclear if the study was at risk of any other bias

Looney 2014

\begin{tabular}{ll}
\hline Methods & Parallel RCT \\
& Randomisation ratio: 1:1:1 \\
& Superiority design \\
\hline Participants & Inclusion criteria: aged 4-10 years, overweight or obese, BMI $\geq 85$ th percentile (CDC growth charts) \\
& Exclusion criteria: medication condition that affected growth, physical activity or dietary intake, child \\
& was participating in another weight loss programme and/or taking weight loss medication, primary \\
caretaker did not want to take part, or did not speak or read English, child did not speak English, fami- \\
ly did not have a working telephone number, child spent < $50 \%$ at the primary caretaker's home, family \\
was planning to move out of the East Tennessee area during the study
\end{tabular}

Diagnostic criteria: see above

Number of study centres: 1
Run-in period: no
Extension period: no
Intervention 1: newsletter and growth monitoring plus behavioural counselling
Intervention 2: newsletter and growth monitoring
Comparator: newsletter only

Outcomes Outcome measures reported in abstract: BMI z score, servings per/d of sugar-sweetened beverages

Study details

\section{Trial terminated early: no}

Trial ID: NCT01358448

Publication details Language of publication: English

Diet, physical activity and behavioural interventions for the treatment of overweight or obese children from the age of 6 to 11 years 
Funding: Amy Joye Memorial Research Award from the Academy of Nutrition and Dietetics Foundation (non-commercial)

Publication status: peer-reviewed journal

Stated aim for study

Quote from publication: "This pilot randomized controlled trial investigated the effect of 3 low-intensi-

ty ( $\leq 25$ contact hours over 6 months) pediatric obesity treatments on $z$-BMI"

Notes

\section{Risk of bias}

\begin{tabular}{|c|c|c|}
\hline Bias & Authors' judgement & Support for judgement \\
\hline $\begin{array}{l}\text { Random sequence genera- } \\
\text { tion (selection bias) }\end{array}$ & Low risk & $\begin{array}{l}\text { Quote from publication: "Sealed blank envelopes with condition assign- } \\
\text { ments enclosed were used to randomize families in blocks of 3." } \\
\text { Comment: randomisation process described }\end{array}$ \\
\hline $\begin{array}{l}\text { Allocation concealment } \\
\text { (selection bias) }\end{array}$ & Low risk & Comment: study author confirmed via email that allocation was concealed \\
\hline $\begin{array}{l}\text { Blinding of participants } \\
\text { and personnel (perfor- } \\
\text { mance bias) } \\
\text { Subjective outcomes }\end{array}$ & High risk & $\begin{array}{l}\text { Quote from publication: (from study author via email) "Study personnel were } \\
\text { not blinded. Participants cannot be blinded as in this type of intervention we } \\
\text { are asking them to complete specific tasks depending upon what condition } \\
\text { there were randomized too" }\end{array}$ \\
\hline & & $\begin{array}{l}\text { Comment: study author confirmed participants and personnel were not blind- } \\
\text { ed }\end{array}$ \\
\hline
\end{tabular}

Blinding of participants High risk and personnel (perfor-

Quote from publication: (from study author via email) "Study personnel were mance bias)

Objective outcomes not blinded. Participants cannot be blinded as in this type of intervention we are asking them to complete specific tasks depending upon what condition there were randomized too"

Comment: study author confirmed participants and personnel were not blinded

Blinding of outcome as-
sessment (detection bias) $\quad$ High risk Comment: study author confirmed outcome assessors were not blinded

Subjective outcomes

Blinding of outcome as-
$\begin{aligned} & \text { sessment (detection bias) } \\ & \text { Objective outcomes }\end{aligned}$

Incomplete outcome data Low risk

Quote from publication: "There were no significant differences between con-

Subjective outcomes ditions for retention at the 6 -month assessments ( $N, 7 / 8$ completed an assessment vs $\mathrm{N}+\mathrm{GM}, 7 / 7$ completed an assessment vs $\mathrm{N}+\mathrm{GM}+\mathrm{BC}, 7 / 7$ completed an assessment)."

Comment: only 1 lost to follow-up and 1 with missing data at 6 months (anthropometrics only)

\begin{tabular}{|c|c|c|}
\hline $\begin{array}{l}\text { Incomplete outcome data } \\
\text { (attrition bias) } \\
\text { Objective outcomes }\end{array}$ & Low risk & $\begin{array}{l}\text { Quote from publication: "There were no significant differences between con- } \\
\text { ditions for retention at the } 6 \text {-month assessments ( } N, 7 / 8 \text { completed an assess- } \\
\text { ment vs } N+G M, 7 / 7 \text { completed an assessment vs } N+G M+B C, 7 / 7 \text { completed } \\
\text { an assessment)." }\end{array}$ \\
\hline
\end{tabular}

Diet, physical activity and behavioural interventions for the treatment of overweight or obese children from the age of 6 to 11 years (Review)

Copyright $\odot 2017$ The Cochrane Collaboration. Published by John Wiley \& Sons, Ltd. 
Comment: only 1 lost to follow-up and 1 with missing data at 6 months (anthropometrics only)

\begin{tabular}{lll}
\hline $\begin{array}{l}\text { Selective reporting (re- } \\
\text { porting bias) }\end{array}$ & Unclear risk & $\begin{array}{l}\text { Comment: study still ongoing in clinical trials register. Cost-effectiveness giv- } \\
\text { en as a secondary outcome in trials register but not reported in publication - } \\
\text { perhaps will be included in an additional publication }\end{array}$ \\
\hline Other bias & Unclear risk & Comment: unclear if the study was at risk of any other bias \\
\hline
\end{tabular}

Maddison 2011

\begin{tabular}{ll}
\hline Methods & Parallel RCT \\
& Randomisation ratio: $1: 1$ \\
& Superiority design
\end{tabular}

Participants

Inclusion criteria: aged 10-14 years, overweight or obese (IOTF cut offs), owned a Playstation 2 or 3 gaming console (Sony Computer Entertainment Inc, Tokyo, Japan), but no active video games (including EyeToy (Sony) or NintendoWii, played $\geq 2$ h of video games per week, only 1 child per household was eligible to take part in the study

Exclusion criteria: contraindications to performing physical activity (e.g. medical conditions)

Diagnostic criteria: see above

\begin{tabular}{ll}
\hline Interventions & Number of study centres: 1 \\
Run-in period: no & \\
Extension period: no \\
Intervention: active video game package \\
Comparator: no-care control group \\
\hline Outcomes & $\begin{array}{l}\text { Outcome measures reported in abstract: BMl, percentage body fat, daily time spent playing active } \\
\text { video games and nonactive video games }\end{array}$
\end{tabular}

Trial ID: ACTRN12607000632493

\section{Publication details Language of publication: English}

Funding: Health Research Council of New Zealand (grant 07/077B), a Heart Foundation of New Zealand Fellowship (RM), a Heart Foundation of New Zealand Senior Fellowship (CNM), and a Tertiary Education Commission Bright Futures Doctoral Scholarship (LF). Sony Computer Entertainment Europe provided the gaming software for the study (commercial and non-commercial)

Publication status: peer-reviewed journal

\begin{tabular}{ll}
\hline Stated aim for study & Quote from publication: "The aim of this study was to evaluate the effect of active video games over a \\
6-mo period on weight, body composition, physical activity, and physical fitness"
\end{tabular}

$$
\text { Notes }
$$

\section{Risk of bias}

Diet, physical activity and behavioural interventions for the treatment of overweight or obese children from the age of 6 to 11 years 
Maddison 2011 (Continued)

Bias Authors' judgement Support for judgement

Random sequence genera- Low risk tion (selection bias)
Quote from publication: "The randomization is via a computerized central randomization service and stratified by sex and ethnicity."

Comment: randomisation process well described

\begin{tabular}{|c|c|c|}
\hline $\begin{array}{l}\text { Allocation concealment } \\
\text { (selection bias) }\end{array}$ & Low risk & $\begin{array}{l}\text { Quote from publication: "However allocation concealment (up to the point of } \\
\text { randomization) was maintained" }\end{array}$ \\
\hline
\end{tabular}

Comment: allocation was concealed

\begin{tabular}{|c|c|c|}
\hline $\begin{array}{l}\text { Blinding of participants } \\
\text { and personnel (perfor- } \\
\text { mance bias) } \\
\text { Subjective outcomes }\end{array}$ & High risk & $\begin{array}{l}\text { Quote from publication: "It was not possible to blind participants to their ex- } \\
\text { perimental group allocation." "It was also not possible to blind study staff ad- } \\
\text { ministering interventions and assessing outcomes to experimental group allo- } \\
\text { cation for pragmatic reasons." }\end{array}$ \\
\hline
\end{tabular}

Comment: participants and study personnel were not blinded to study group

Blinding of participants High risk and personnel (performance bias)

Objective outcomes
Quote from publication: "It was not possible to blind participants to their experimental group allocation." "It was also not possible to blind study staff administering interventions and assessing outcomes to experimental group allocation for pragmatic reasons."

Comment: participants and study personnel were not blinded to study group

\section{Blinding of outcome as- High risk} sessment (detection bias) Subjective outcomes
Quote from publication: "It was also not possible to blind study staff administering interventions and assessing outcomes to experimental group allocation for pragmatic reasons."

Comment: staff who assessed outcomes were not blinded to study group
Blinding of outcome as- High risk sessment (detection bias) Objective outcomes
Quote from publication: "It was also not possible to blind study staff administering interventions and assessing outcomes to experimental group allocation for pragmatic reasons."

Comment: staff who assessed outcomes were not blinded to study group
Incomplete outcome data Unclear risk (attrition bias)

Subjective outcomes
Quote from publication: "Treatment evaluations were performed on the principle of intention to treat for the primary outcome and by using the approach of the last observation carried forward when data were missing."

Comment: even though they used ITT analysis and included all participants in the analysis, dropout rates were moderate (around 20\%) at follow up
Incomplete outcome data Unclear risk

(attrition bias)

Objective outcomes
Quote from publication: "Treatment evaluations were performed on the principle of intention to treat for the primary outcome and by using the approach of the last observation carried forward when data were missing."

Comment: even though they used ITT analysis and included all participants in the analysis, dropout rates were moderate (around 20\%) at follow-up

\begin{tabular}{|c|c|c|}
\hline $\begin{array}{l}\text { Selective reporting (re- } \\
\text { porting bias) }\end{array}$ & Low risk & $\begin{array}{l}\text { Comment: no differences found in publication versus clinical trials regis- } \\
\text { ter/protocol }\end{array}$ \\
\hline
\end{tabular}

Other bias Unclear risk Comment: unclear if the study was at risk of any other bias


Maddison 2014

\begin{tabular}{ll}
\hline Methods & Parallel RCT \\
& Randomisation ratio: 1:1 \\
& Superiority design \\
\hline Participants & $\begin{array}{l}\text { Inclusion criteria: aged 9-12 years, lived in the greater Auckland metropolitan area, overweight or } \\
\text { obese (Cole 2007), used electronic media (e.g. television, video games) for at least } 15 \mathrm{~h} / \text { week, speak } \\
\text { and understand English, a primary caregiver participating in the study (aged } 18 \text { or above) and could } \\
\text { speak and understand English }\end{array}$
\end{tabular}

Exclusion criteria: medical condition precluding them from performing regular physical activity, if they lived in more than 1 household and spent equal time at both households

Diagnostic criteria: see above

\begin{tabular}{ll}
\hline Interventions & Number of study centres: 1 \\
Run-in period: no \\
Extension period: no \\
Intervention: SWITCH intervention group \\
Comparator: usual care control group
\end{tabular}

Outcomes Outcome measures reported in abstract: BMI z score, moderate-intensity physical activity, percentage body fat

Study details Trial terminated early: no

Trial ID: ACTRN12611000164998

\section{Publication details Language of publication: English}

Funding: Health Research Council of New Zealand (10/077). Dr Ralph Maddison supported by a Heart Foundation Research Fellowship (Grant 1211). Professor Cliona Ni Mhurchu supported by the National Heart Foundation Senior Fellowship (Grant 1380). Dr Louise Foley supported by a Heart Foundation of New Zealand Postdoctoral Fellowship (non-commercial)

Publication status: peer-reviewed journal

$\begin{array}{ll}\text { Stated aim for study } & \text { Quote from publication: "The SWITCH (Screen-Time Weight-loss Intervention Targeting Children at } \\ \text { Home) study aimed to determine the effect of a home-based, family-delivered intervention to reduce } \\ \text { screen-based sedentary behaviour on body composition, sedentary behaviour, physical activity, and } \\ \text { diet over } 24 \text { weeks in overweight and obese children." }\end{array}$

Notes

\section{Risk of bias}

\begin{tabular}{lll}
\hline Bias & Authors' judgement & Support for judgement \\
\hline $\begin{array}{ll}\text { Random sequence genera- } \\
\text { tion (selection bias) }\end{array}$ & Low risk & Quote from publication: "Eligible participants were randomised at a 1:1 ra- \\
& & tio to the intervention or control groups via centralised computer randomi- \\
& sation, using stratified blocked randomisation (with variable block sizes) to \\
& maintain balance across important prognostic factors. Two stratification fac- \\
& tors were considered: sex (male and female) and ethnicity (Māori, Pacific, and \\
& non-Māori/non-Pacific)."
\end{tabular}

Comment: randomisation process well described 
Maddison 2014 (Continued)

Allocation concealment Low risk Quote from publication: "Allocation concealment was maintained up to the (selection bias)

point of randomisation"

Comment: allocation was concealed

Blinding of participants High risk and personnel (performance bias)

Subjective outcomes
Quote from publication: "Blinding of participants and research assistants was not possible due to the nature of the intervention."

Comment: participants and study personnel were not blinded to study group

\begin{tabular}{|c|c|c|}
\hline $\begin{array}{l}\text { Blinding of participants } \\
\text { and personnel (perfor- } \\
\text { mance bias) } \\
\text { Obiective outcomes }\end{array}$ & High risk & $\begin{array}{l}\text { Quote from publication: "Blinding of participants and research assistants } \\
\text { was not possible due to the nature of the intervention." } \\
\text { Comment: participants and study personnel were not blinded to study group }\end{array}$ \\
\hline
\end{tabular}

Blinding of outcome as- High risk
sessment (detection bias)

Subjective outcomes

Quote from publication: "Blinding of participants and research assistants was not possible due to the nature of the intervention."

Comment: staff who assessed outcomes were not blinded to study group

$\begin{array}{lll}\begin{array}{l}\text { Blinding of outcome as- } \\ \text { sessment (detection bias) }\end{array} & \text { High risk } & \begin{array}{l}\text { Quote from publication: "Blinding of participants and research assistants } \\ \text { was not possible due to the nature of the intervention." }\end{array}\end{array}$

Objective outcomes

Comment: staff who assessed outcomes were not blinded to study group

Incomplete outcome data Low risk

(attrition bias)

Subjective outcomes
Quote from publication: "Children were randomly assigned to the intervention $(n=127)$ and control $(n=124)$ groups, with $121(95 \%)$ and $117(94 \%)$ completing 24 weeks' follow up."

Comment: low dropout rates

\begin{tabular}{|c|c|c|}
\hline $\begin{array}{l}\text { Incomplete outcome data } \\
\text { (attrition bias) } \\
\text { Objective outcomes }\end{array}$ & Low risk & $\begin{array}{l}\text { Quote from publication: "Children were randomly assigned to the interven- } \\
\text { tion }(n=127) \text { and control }(n=124) \text { groups, with } 121(95 \%) \text { and } 117(94 \%) \text { com- } \\
\text { pleting } 24 \text { weeks' follow up." }\end{array}$ \\
\hline
\end{tabular}

Comment: low dropout rates

\begin{tabular}{lll}
\hline $\begin{array}{l}\text { Selective reporting (re- } \\
\text { porting bias) }\end{array}$ & Low risk & $\begin{array}{l}\text { Comment: } \text { no differences found between publication and clinical trials regis- } \\
\text { ter }\end{array}$ \\
\hline Other bias & Unclear risk & Comment: unclear if the study was at risk of any other bias \\
\hline
\end{tabular}

\section{Markert 2014}

\begin{tabular}{|c|c|}
\hline Methods & $\begin{array}{l}\text { Parallel RCT } \\
\text { Randomisation ratio: 1:1 } \\
\text { Superiority design }\end{array}$ \\
\hline Participants & $\begin{array}{l}\text { Inclusion criteria: BMI SDS over the 90th centile (German reference values, Kromeyer-Hauschild 2001), } \\
\text { age 4-17 years } \\
\text { Exclusion criteria: none } \\
\text { Diagnostic criteria: see above }\end{array}$ \\
\hline
\end{tabular}

Interventions Number of study centres: unclear

Diet, physical activity and behavioural interventions for the treatment of overweight or obese children from the age of 6 to 11 years 
Markert 2014 (Continued)

\section{Run-in period: no}

Extension period: no

Intervention: telephone-based adiposity prevention for families (TAFF)

Comparator: no-care control

Outcomes
consumption, participation rates

Study details

Trial terminated early: no

Trial ID: DRKS00000803

\section{Publication details Language of publication: English}

Funding: Federal Ministry of Education and Research, Germany (Integrated Research and Treatment Center IFB "AdiposityDiseases," FKZ: 01E01001), the Roland-Ernst-Stiftung für Gesundheitsforschung, Dresden, Germany, and the Saxonian Ministry for Social Affairs, Germany (non-commercial)

Publication status: peer-reviewed journal

$\begin{array}{ll}\text { Stated aim for study } & \text { Quote from publication: "The aim of this paper is to present one-year results of the T.A.F.F. program, a } \\ \text { randomized controlled obesity prevention program based on telephone counseling for families with } \\ \text { overweight children or adolescents" }\end{array}$
overweight children or adolescents"

Notes -

\section{Risk of bias}

\begin{tabular}{lll}
\hline Bias & Authors' judgement & Support for judgement \\
\hline $\begin{array}{ll}\text { Random sequence genera- } \\
\text { tion (selection bias) }\end{array}$ & Low risk & $\begin{array}{l}\text { Quote from publication: "Randomization to the intervention or control group } \\
\text { was performed with a 1:1 allocation ratio and stratified according to sex and } \\
\text { age group (4-9 years, } 10-13 \text { years, 14-17 years) using electronically generated } \\
\text { four-bloc-random-lists." }\end{array}$
\end{tabular}

Comment: randomisation process well described

\begin{tabular}{ll}
\hline $\begin{array}{l}\text { Allocation concealment } \\
\text { (selection bias) }\end{array}$ & Quote from publication: "The lists were generated before the start of the trial \\
and assignment to trial arm was performed consecutively by a member of the & team who did not have contact with participants and was not involved in data \\
analysis. Enrolment of participants was carried out by the respective preven- \\
tion manager."
\end{tabular}

Comment: it was likely that allocation was concealed

\begin{tabular}{|c|c|c|}
\hline $\begin{array}{l}\text { Blinding of participants } \\
\text { and personnel (perfor- } \\
\text { mance bias) } \\
\text { Subjective outcomes }\end{array}$ & Unclear risk & $\begin{array}{l}\text { Comment: unclear whether participants or study personnel were blinded to } \\
\text { study group }\end{array}$ \\
\hline $\begin{array}{l}\text { Blinding of participants } \\
\text { and personnel (perfor- } \\
\text { mance bias) } \\
\text { Objective outcomes }\end{array}$ & Unclear risk & $\begin{array}{l}\text { Comment: unclear whether participants or study personnel were blinded to } \\
\text { study group }\end{array}$ \\
\hline
\end{tabular}

Blinding of outcome as- Unclear risk Comment: unclear whether assessment staff were blinded to study group sessment (detection bias) 
Markert 2014 (Continued)

Subjective outcomes

Blinding of outcome as- Unclear risk Comment: unclear whether assessment staff were blinded to study group
sessment (detection bias)

Objective outcomes

Incomplete outcome data High risk (attrition bias)

Subjective outcomes

Quote from publication: "Since the participants were not seen face to face, it was not always easy to encourage them to be weighed and measured and to return study material at the appropriate time. However, the effect of lag-times was analyzed and not found to have a significant impact on the results."

Comment: dropout rate in intervention group was high (62.8\%)

Incomplete outcome data High risk (attrition bias)

Objective outcomes

Quote from publication: "Since the participants were not seen face to face, it was not always easy to encourage them to be weighed and measured and to return study material at the appropriate time. However, the effect of lag-times was analyzed and not found to have a significant impact on the results."

Comment: dropout rate in intervention group was high (62.8\%)

\begin{tabular}{lll}
\hline $\begin{array}{l}\text { Selective reporting (re- } \\
\text { porting bias) }\end{array}$ & Unclear risk & $\begin{array}{l}\text { Comment: there is a clinical trials register entry but it was retrospectively en- } \\
\text { tered. The protocol was also published after recruitment and baseline mea- } \\
\text { sures were taken. }\end{array}$ \\
\hline Other bias & Unclear risk & Comment: unclear if the study was at risk of any other bias \\
\hline
\end{tabular}

McCallum 2007

\begin{tabular}{ll}
\hline Methods & Parallel RCT \\
& Randomisation ratio: $1: 1$ \\
& Superiority design \\
\hline Participants & $\begin{array}{l}\text { Inclusion criteria: overweight or mildly obese (IOTF cut points), not receiving ongoing weight manage- } \\
\text { ment in a secondary or tertiary care programme and their parents provided contact details } \\
\text { Exclusion criteria: any chromosomal, endocrine or medical condition/disability/medications which } \\
\text { may impact on their weight or growth }\end{array}$
\end{tabular}

Diagnostic criteria: see above

\begin{tabular}{ll}
\hline Interventions & Number of study centres: 29 \\
& Run-in period: no \\
& Extension period: no \\
& Intervention: LEAP Intervention \\
Comparator: no-care control group
\end{tabular}

Outcomes Outcome measures reported in abstract: attrition, BMI, nutrition scores, daily physical activity, health status, body image, cost-effectiveness

Study details

Trial terminated early: no

Trial ID: ISRCTN45068927

Diet, physical activity and behavioural interventions for the treatment of overweight or obese children from the age of 6 to 11 years 
McCallum 2007 (Continued)

\section{Publication details Language of publication: English}

Funding: lead author (McCallum) was funded via Public Health Postgraduate National Health and Medical Research Council Scholarship (ID 216745). The LEAP trial was funded by a grant from the Australian Health Ministers' Advisory Council for Priority Driven Research (AHMAC PDR 2001/15) (non-commercial)

Publication status: peer-reviewed journal

$\begin{array}{ll}\text { Stated aim for study } & \begin{array}{l}\text { Quote from publication: "The study aims to reduce incremental gain in body mass index (BMI) of over- } \\ \text { weight/obese children aged 5-9 years" }\end{array}\end{array}$

Notes -

\section{Risk of bias}

Bias Authors' judgement Support for judgement

Random sequence genera- Low risk tion (selection bias)
Quote from publication: "Randomization was performed by a third-party biostatistician using a pre-generated computerized sequence."

Comment: randomisation process well described

\begin{tabular}{|c|c|c|}
\hline Allocation concealment & Low risk & Quote from publication: "Blinding was maintained throughout allocation and \\
\hline
\end{tabular}
(selection bias) data collection."

Comment: allocation was concealed

Blinding of participants High risk and personnel (performance bias)

Subjective outcomes
Quote from publication: "Blinding was maintained throughout allocation and data collection. Following randomization, intervention families were contacted by a non-blinded member of the research team and the first GP appointment made. Control families were notified of their status via letter and were not identified to the GPs at any time."

Comment: participants were not blinded to study group

Blinding of participants High risk
and personnel (perfor-
mance bias)
Objective outcomes

Blinding of participants High risk mance bias)

Objective outcomes
Quote from publication: "Blinding was maintained throughout allocation and data collection. Following randomization, intervention families were contacted by a non-blinded member of the research team and the first GP appointment made. Control families were notified of their status via letter and were not identified to the GPs at any time."

Comment: participants were not blinded to study group

\begin{tabular}{|c|c|c|}
\hline $\begin{array}{l}\text { Blinding of outcome as- } \\
\text { sessment (detection bias) }\end{array}$ & Low risk & $\begin{array}{l}\text { Quote from publication: "Assessors of the 6- and 12-month follow-ups were } \\
\text { blinded to randomization status." }\end{array}$ \\
\hline
\end{tabular}
Subjective outcomes

Comment: assessment staff were blinded to study group

\begin{tabular}{lll}
\hline $\begin{array}{l}\text { Blinding of outcome as- } \\
\begin{array}{l}\text { sessment (detection bias) } \\
\text { Objective outcomes }\end{array}\end{array}$ & Low risk & $\begin{array}{l}\text { Quote from publication: "Assessors of the 6- and 12-month follow-ups were } \\
\text { blinded to randomization status." } \\
\text { Comment: assessment staff were blinded to study group }\end{array}$ \\
\hline $\begin{array}{l}\text { Incomplete outcome data } \\
\begin{array}{l}\text { (attrition bias) } \\
\text { Subjective outcomes }\end{array}\end{array}$ & Low risk & $\begin{array}{l}\text { Quote from publication: "A total of } 12(15 \%) \text { subjects in the intervention } \\
\text { group and five (6\%) subjects in the control group were not visited at 15 } \\
\text { months." }\end{array}$ \\
& Comment: dropout rates were low \\
\hline
\end{tabular}


McCallum 2007 (Continued)

Incomplete outcome data Low risk Quote from publication: "A total of 12 (15\%) subjects in the intervention

(attrition bias) group and five (6\%) subjects in the control group were not visited at 15

Objective outcomes months."

Comment: dropout rates were low

Selective reporting (re- Low risk Comment: no differences found between publication and protocol
porting bias)

Other bias Low risk Comment: no other bias identified - low risk of bias in majority of other do-
mains

\section{Mirza 2013}

\begin{tabular}{ll}
\hline Methods & Parallel RCT \\
& Randomisation ratio: 1:1 \\
& Superiority design \\
\hline Participants & $\begin{array}{l}\text { Inclusion criteria: Hispanic children aged 7-15, BMI } \geq 95 \text { th percentile for age and sex (CDC growth } \\
\text { charts) and otherwise healthy }\end{array}$ \\
& Exclusion criteria: any known medical conditions which would interfere with the study's objec- \\
tives/procedures (e.g. type 2 diabetes, Cushing's syndrome, severe asthma, use of medications known \\
to promote weight gain or loss)
\end{tabular}

Diagnostic criteria: see above

\begin{tabular}{ll}
\hline Interventions & Number of study centres: 1 \\
Run-in period: no \\
Extension period: no \\
Intervention: low-glycaemic load dietary group \\
Comparator: conventional low-fat dietary group
\end{tabular}

Outcomes

Outcome measures reported in abstract: completion rates, glycaemic load, BMI z score, WC, systolic blood pressure, BMI, insulin resistance, components of metabolic syndrome

\section{Study details}

\section{Trial terminated early: no}

Trial ID: NCT01068197

\section{Publication details Language of publication: English}

Funding: NIH grants K23-RR022227 (NMM), MO1-RR-020359, and UL1RR031988, which were awarded by the National Center for Research Resources to support the General Clinical Research Center and the Children's Research Institute at Children's National Medical Center, and ZIA-HD-00641 and the following foundations and organizations: Consumer Health Foundation, The Jessie Ball DuPont Foundation, and United Way of the National Capital Area. J Yanovski is supported by the Intramural Research Program of the Eunice Kennedy Shriver National Institute of Child Health and Human Development and the National Institute on Minority Health and Health Disparities of the NIH. D Ludwig is supported in part by career award K24DK082730 from the National Institute of Diabetes and Digestive and Kidney Diseases (non-commercial)

Publication status: peer-reviewed journal 
Mirza 2013 (Continued)

Stated aim for study

Quote from publication: "We compared the effects of an LGD and a low-fat diet (LFD) on body composition and components of metabolic syndrome in obese Hispanic youth"

Notes

\section{Risk of bias}

Bias Authors' judgement Support for judgement

Random sequence genera- Low risk tion (selection bias)

Quote from publication: "The order in which groups occurred was determined by random assignment in blocks of 2 within strata determined by the BMI percentile, sex, and pubertal stage"

Comment: randomisation process well described

Allocation concealment $\quad$ Unclear risk
(selection bias)
(selection bias)

\section{Blinding of participants High risk} and personnel (perfor-

mance bias)

Subjective outcomes
Quote from publication: "Because of the nature of the dietary intervention, the study was not a double-blind randomized study. Participants were not informed of their dietary group assignment but could ascertain their group on the basis of the diets offered."

Comment: participants were not blinded to study group

$\begin{array}{ll}\text { Blinding of participants } \quad \text { High risk } & \begin{array}{l}\text { Quote from publication: "Because of the nature of the dietary intervention, } \\ \text { the study was not a double-blind randomized study. Participants were not in- } \\ \text { mance bias) }\end{array} \\ \begin{array}{l}\text { formed of their dietary group assignment but could ascertain their group on } \\ \text { thective outcomes }\end{array} & \begin{array}{l}\text { the basis of the diets offered." }\end{array}\end{array}$

Comment: participants were not blinded to study group

Blinding of outcome as- Low risk sessment (detection bias) Subjective outcomes
Quote from publication: "The staff who obtained primary and secondary outcome measurements did not take part in the interventions and were blinded to subject group assignments.

Comment: assessment staff were blinded to study group

$\begin{array}{lll}\begin{array}{l}\text { Blinding of outcome as- } \\ \text { sessment (detection bias) } \\ \text { Objective outcomes }\end{array} & \text { Low risk } & \begin{array}{l}\text { Quote from publication: "The staff who obtained primary and secondary out- } \\ \text { come measurements did not take part in the interventions and were blinded to } \\ \text { subject group assignments. }\end{array}\end{array}$

Comment: assessment staff were blinded to study group

\begin{tabular}{|c|c|c|}
\hline $\begin{array}{l}\text { Incomplete outcome data } \\
\text { (attrition bias) } \\
\text { Subjective outcomes }\end{array}$ & High risk & $\begin{array}{l}\text { Quote from publication: "Seventy-nine percent of LGD and LFD enrolees } \\
\text { completed the 3-mo study, } 61 \% \text { of enrolees completed } 1 \text { y of follow-up, and } \\
54.9 \% \text { enrolees completed } 2 \text { y of follow-up (Figure } 1 \text { )." }\end{array}$ \\
\hline
\end{tabular}

Comment: dropout rates were quite high

Incomplete outcome data High risk
(attrition bias)

Objective outcomes

Quote from publication: "Seventy-nine percent of LGD and LFD enrolees completed the 3 -mo study, $61 \%$ of enrolees completed 1 y of follow-up, and $54.9 \%$ enrolees completed 2 y of follow-up (Figure 1 )."

Comment: dropout rates were quite high

Selective reporting (re- High risk
porting bias)

Comment: some outcomes not reported - cholesterol, BP, WC, glucose, total body fat mass, fat-free mass - some reported in supplementary data but only as combined groups, not separately. Did report WC and SBP in abstract but

Diet, physical activity and behavioural interventions for the treatment of overweight or obese children from the age of 6 to 11 years 


Other bias Unclear risk Comment: unable to assess if any biases were present

\section{NCT02436330}

\begin{tabular}{ll}
\hline Methods & Parallel RCT \\
& Randomisation ratio: $2: 1$ \\
& Superiority design
\end{tabular}

Participants Inclusion criteria: child aged 8-16 years, BMI $\geq 85$ th percentile, English speaking, approval by Primary

Care Doctor

Exclusion criteria: participants with medical, developmental or psychiatric diagnoses which precluded participation in both the physical activity and classroom portions of the curriculum, participants who were taking medications that positively or negatively affected weight

Diagnostic criteria: BMI percentile reference unclear

\begin{tabular}{|c|c|c|}
\hline \multirow[t]{5}{*}{ Interventions } & \multicolumn{2}{|c|}{ Number of study centres: unclear } \\
\hline & \multicolumn{2}{|l|}{ Run-in period: no } \\
\hline & \multicolumn{2}{|l|}{ Extension period: no } \\
\hline & \multicolumn{2}{|c|}{ Intervention: exergaming and didactic healthy teaching } \\
\hline & \multicolumn{2}{|c|}{ Control: didactic healthy teaching only } \\
\hline Outcomes & \multicolumn{2}{|c|}{ Outcome measures reported in abstract: no publication } \\
\hline \multirow[t]{2}{*}{ Study details } & \multicolumn{2}{|c|}{ Trial terminated early: no } \\
\hline & \multicolumn{2}{|l|}{ Trial ID: NCT02436330 } \\
\hline \multirow[t]{3}{*}{ Publication details } & \multicolumn{2}{|c|}{ Language of publication: English } \\
\hline & \multicolumn{2}{|l|}{ Funding: unclear } \\
\hline & \multicolumn{2}{|c|}{ Publication status: other (results from ClinicalTrials.gov) } \\
\hline Stated aim for study & \multicolumn{2}{|c|}{$\begin{array}{l}\text { Quote from publication: "Primary objective: to assess impact of the program on BMI z-scores. Se- } \\
\text { condary objectives: to measure impact on cardiovascular fitness, self-worth, sedentary screen time, } \\
\text { and the influence of exergaming component on attendance and participation." }\end{array}$} \\
\hline Notes & \multicolumn{2}{|c|}{ Clinical trials register entry only - no published results } \\
\hline \multicolumn{3}{|l|}{ Risk of bias } \\
\hline Bias & Authors' judgement & Support for judgement \\
\hline $\begin{array}{l}\text { Random sequence genera- } \\
\text { tion (selection bias) }\end{array}$ & Unclear risk & $\begin{array}{l}\text { Quote from publication: "enrolled sequentially and randomized 2:1 in experi- } \\
\text { mental and control groups." }\end{array}$ \\
\hline & & Comment: no further information about randomisation provided \\
\hline
\end{tabular}




\section{NCT02436330 (Continued)}

Allocation concealment (selection bias)
Unclear risk

Quote from publication: "enrolled sequentially and randomized 2:1 in experimental and control groups..."

Comment: unclear if allocation was concealed

$\begin{array}{lll}\text { Blinding of participants } & \text { High risk } & \text { Quote from publication: "Masking: open label..." } \\ \text { and personnel (perfor- } & & \text { Comment: investigator-assessed } \\ \text { mance bias) } & & \end{array}$

Subjective outcomes

\begin{tabular}{|c|c|c|}
\hline $\begin{array}{l}\text { Blinding of participants } \\
\text { and personnel (perfor- } \\
\text { mance bias) } \\
\text { Objective outcomes }\end{array}$ & High risk & $\begin{array}{l}\text { Quote from publication: "Masking: open label..." } \\
\text { Comment: investigator-assessed }\end{array}$ \\
\hline
\end{tabular}

\begin{tabular}{lll}
\hline Blinding of outcome as- & High risk & Quote from publication: "Masking: open label..." \\
sessment (detection bias) & & Comment: investigator-assessed \\
Subjective outcomes & & Com
\end{tabular}

\begin{tabular}{lll}
\hline $\begin{array}{l}\text { Blinding of outcome as- } \\
\text { sessment (detection bias) } \\
\text { Objective outcomes }\end{array}$ & High risk & Quote from publication: "Masking: open label..." \\
Comment: investigator-assessed
\end{tabular}

\begin{tabular}{lll}
\hline $\begin{array}{l}\text { Incomplete outcome data } \\
\text { (attrition bias) } \\
\text { Objective outcomes }\end{array}$ & High risk & $\begin{array}{l}\text { Comment: in intervention arm 14/60 lost to follow-up and 11/60 withdrew; in } \\
\text { the control arm 4/24 lost to follow-up and } 7 / 24 \text { withdrew. Thus over } 40 \% \text { of all } \\
\text { intervention participants did not complete }\end{array}$ \\
\hline $\begin{array}{l}\text { Selective reporting (re- } \\
\text { porting bias) }\end{array}$ & High risk & $\begin{array}{l}\text { Comment: although all outcomes were reported as stated on the registry, on- } \\
\text { ly completers' analyses were presented (and numbers varied for different out- } \\
\text { comes). Also the tests used have not undergone peer review as part of formal } \\
\text { publication }\end{array}$ \\
\hline Other bias & Unclear risk & $\begin{array}{l}\text { Comment: results have only been extracted from trials register therefore can } \\
\text { only be treated as provisional }\end{array}$ \\
\hline
\end{tabular}

Nemet 2005

\begin{tabular}{ll}
\hline Methods & Parallel RCT \\
& Randomisation ratio: $5: 4$ \\
& Superiority design \\
\hline Participants & Inclusion criteria: age 6-16, obese children and adolescents (CDC growth charts) \\
& $\begin{array}{l}\text { Exclusion criteria: organic cause for obesity, receiving medication which may interfere with growth or } \\
\text { weight control (e.g. corticosteroids, thyroid hormones) } \\
\text { Diagnostic criteria: see above }\end{array}$ \\
\hline Interventions & Number of study centres: 1 \\
& Run-in period: no
\end{tabular}

Diet, physical activity and behavioural interventions for the treatment of overweight or obese children from the age of 6 to 11 years 
Nemet 2005 (Continued)

\section{Extension period: no}

Intervention: combined dietary and exercise programme

Comparator: usual care control group

Outcomes Outcome measures reported in abstract: body weight, BMI, body fat percentage, total cholesterol,
LDL, fitness, leisure-time physical activity

Study details Trial terminated early: no

Trial ID: -

$\begin{array}{ll}\text { Publication details } & \text { Language of publication: English } \\ \text { Funding: grant from the Israeli Heart Fund (non-commercial) } \\ \text { Publication status: peer-reviewed journal }\end{array}$

Stated aim for study

Quote from publication: "To examine prospectively the short- and long-term effects of a 3-month, combined dietary-behavioral-physical activity intervention on anthropometric measures, body composition, dietary and leisure-time habits, fitness, and lipid profiles among obese children"

\section{Notes}

\section{Risk of bias}

Bias Authors' judgement Support for judgement

Random sequence genera- Low risk tion (selection bias)

Quote from publication: "Thirty children and adolescents were assigned randomly, with a computerized, random number generator, to participate in our 3-month, combined dietary and exercise program for the treatment of childhood obesity, at the Child Health and Sports Center, Meir General Hospital, Tel Aviv University"

Comment: randomisation process well described

\begin{tabular}{lll}
\hline $\begin{array}{l}\text { Allocation concealment } \\
\text { (selection bias) }\end{array}$ & Unclear risk & Comment: unclear if allocation was concealed \\
\hline $\begin{array}{l}\text { Blinding of participants } \\
\text { and personnel (perfor- } \\
\text { mance bias) }\end{array}$ & Unclear risk & $\begin{array}{l}\text { Comment: } \text { not clear if participants and study personnel were blinded to study } \\
\text { group }\end{array}$ \\
Subjective outcomes & &
\end{tabular}

\begin{tabular}{|c|c|c|}
\hline $\begin{array}{l}\text { Blinding of participants } \\
\text { and personnel (perfor- } \\
\text { mance bias) }\end{array}$ & Unclear risk & $\begin{array}{l}\text { Comment: not clear if participants and study personnel were blinded to study } \\
\text { group }\end{array}$ \\
\hline
\end{tabular}

mance bias)

Objective outcomes

\begin{tabular}{lll}
\hline $\begin{array}{l}\text { Blinding of outcome as- } \\
\text { sessment (detection bias) } \\
\text { Subjective outcomes }\end{array}$ & Unclear risk & Comment: not clear if outcome assessors were blinded to study group \\
\hline $\begin{array}{l}\text { Blinding of outcome as- } \\
\text { sessment (detection bias) } \\
\text { Objective outcomes }\end{array}$ & Unclear risk & Comment: not clear if outcome assessors were blinded to study group \\
\hline $\begin{array}{l}\text { Incomplete outcome data } \\
\text { (attrition bias) }\end{array}$ & Unclear risk & $\begin{array}{l}\text { Quote from publication: "Twenty-four subjects completed the 3-month pro- } \\
\text { gram, and } 20 \text { of them returned for evaluation } 1 \text { year later. (intervention)" }\end{array}$
\end{tabular}

Diet, physical activity and behavioural interventions for the treatment of overweight or obese children from the age of 6 to 11 years 
Nemet 2005 (Continued) Subjective outcomes
"Twenty-two control subjects completed the 3-month evaluation, and 20 of them returned for evaluation after 1 year."

Comment: moderate missing data, potential attrition bias

Incomplete outcome data Unclear risk

(attrition bias)

Quote from publication: "Twenty-four subjects completed the 3-month program, and 20 of them returned for evaluation 1 year later. (intervention)"

"Twenty-two control subjects completed the 3-month evaluation, and 20 of them returned for evaluation after 1 year."

Comment: moderate missing data, potential attrition bias

Selective reporting (re- Unclear risk Comment: no clinical trials register entry or protocol available
porting bias)

Other bias Unclear risk Comment: unable to assess if any biases were present

Nova 2001

Methods

Parallel RCT

Randomisation ratio: $2: 5$

Superiority design

Participants Inclusion criteria: child aged 3-12 years, excess weight, $\geq 20$ of ideal body weight, attended a family paediatrician's office 15 November 1997-31 March 1998

Exclusion criteria: none

Diagnostic criteria: see above

Number of study centres: unclear
Run-in period: no
Extension period: no
Intervention: enhanced approach
Comparator: routine approach

Outcomes

Outcome measures reported in abstract: percentage overweight, physical activity, computer or television use, dietary behaviour, attendance

Study details

\section{Trial terminated early: no}

Trial ID: -

\begin{tabular}{ll}
\hline Publication details & Language of publication: English \\
& Funding: NIH (the national institute of nursing research) (non-commercial) \\
& Publication status: peer-reviewed journal
\end{tabular}

Stated aim for study

Quote from publication: "To compare two types of intervention intended to reduce weight in obese children that can be carried out in the family paediatricians (FPs) office"

Notes

Diet, physical activity and behavioural interventions for the treatment of overweight or obese children from the age of 6 to 11 years 
Nova 2001 (Continued)

Risk of bias

\begin{tabular}{|c|c|c|}
\hline Bias & Authors' judgement & Support for judgement \\
\hline $\begin{array}{l}\text { Random sequence genera- } \\
\text { tion (selection bias) }\end{array}$ & Unclear risk & Comment: randomisation process not described \\
\hline $\begin{array}{l}\text { Allocation concealment } \\
\text { (selection bias) }\end{array}$ & Unclear risk & Comment: unclear if allocation was concealed \\
\hline $\begin{array}{l}\text { Blinding of participants } \\
\text { and personnel (perfor- } \\
\text { mance bias) } \\
\text { Subjective outcomes }\end{array}$ & Unclear risk & $\begin{array}{l}\text { Comment: not clear if participants and study personnel were blinded to study } \\
\text { group }\end{array}$ \\
\hline $\begin{array}{l}\text { Blinding of participants } \\
\text { and personnel (perfor- } \\
\text { mance bias) } \\
\text { Objective outcomes }\end{array}$ & Unclear risk & $\begin{array}{l}\text { Comment: not clear if participants and study personnel were blinded to study } \\
\text { group }\end{array}$ \\
\hline
\end{tabular}

Blinding of outcome as-

Unclear risk

Comment: not clear if outcome assessors were blinded to study group

sessment (detection bias)

Subjective outcomes

\begin{tabular}{|c|c|c|}
\hline $\begin{array}{l}\text { Blinding of outcome as- } \\
\text { sessment (detection bias) }\end{array}$ & Unclear risk & Comment: not clear if outcome assessors were blinded to study group \\
\hline
\end{tabular}

sessment (detection bias)

Objective outcomes

Incomplete outcome data High risk

(attrition bias)

Subjective outcomes
Quote from publication: "On average $70 \%$ of all children attended their 12 month follow up visit. However, if we consider the population of any single FP, we observe a huge dispersion around this mean value: two fps in $A$ and four in group $B$ maintained all their enrolled children, whereas three FPs in A and two in group B lost $>75 \%$ of participants"

Comment: in some areas attrition rates were high

Incomplete outcome data High risk
(attrition bias)

Objective outcomes

Quote from publication: "On average $70 \%$ of all children attended their 12 month follow up visit. However, if we consider the population of any single FP, we observe a huge dispersion around this mean value: two fps in $A$ and four in group $B$ maintained all their enrolled children, whereas three FPs in A and two in group B lost $>75 \%$ of participants"

Comment: in some areas attrition rates were high

\begin{tabular}{lll}
\hline $\begin{array}{l}\text { Selective reporting (re- } \\
\text { porting bias) }\end{array}$ & High risk & $\begin{array}{l}\text { Comment: no protocol or clinical trials register available. Potential reporting } \\
\text { bias by not reporting BMI at follow-up. Raw results not given for behavioural } \\
\text { measures. No results given for 24-month follow-up }\end{array}$ \\
\hline Other bias & Unclear risk & Comment: unclear if the study was at risk of any other bias \\
\hline
\end{tabular}

\begin{tabular}{ll}
\hline Methods & Parallel RCT \\
& Randomisation ratio: $1: 1$
\end{tabular}

Diet, physical activity and behavioural interventions for the treatment of overweight or obese children from the age of 6 to 11 years 
Nowicka 2009 (Continued)

\section{Superiority design}

Participants Inclusion criteria: obesity defined by the IOTF cut-points

Exclusion criteria: receiving any other obesity treatment, identifiable medical cause for obesity (with the exception of those with elevated blood lipids and asthma)

Diagnostic criteria: see above

\begin{tabular}{ll}
\hline Interventions & Number of study centres: 1 \\
Run-in period: no & Extension period: no \\
Intervention: summer camp \\
Comparator: no-care control
\end{tabular}

Outcomes Outcome measures reported in abstract: BMI z score

Study details

Trial terminated early: no

Trial ID: -

\begin{tabular}{ll}
\hline Publication details & Language of publication: English \\
& $\begin{array}{l}\text { Funding: study was funded by Swedish Savings Bank Foundation, the Swedish Sports Confederation } \\
\text { and Östra Göinge municipality. Research related to this paper was supported by the Sven Jerring Foun- } \\
\text { dation, Regional Research Support, and the Faculty of Medicine at Lund University, Sweden (non-com- } \\
\text { mercial) }\end{array}$ \\
& Publication status: peer-reviewed journal \\
\hline Stated aim for study & $\begin{array}{l}\text { Quote from publication: "The general aim of this study was to evaluate the effect of management of } \\
\text { childhood obesity by promoting increased physical activity, in comparison with an untreated waiting } \\
\text { list control group." }\end{array}$
\end{tabular}

\section{$-$}


Nowicka 2009 (Continued)

Blinding of outcome as- High risk Comment: study author confirmed via email that outcome assessors were not sessment (detection bias)

blinded to study group

Subjective outcomes

Blinding of outcome as- High risk sessment (detection bias)

Objective outcomes
Comment: study author confirmed via email that outcome assessors were not blinded to study group

\begin{tabular}{|c|c|c|}
\hline $\begin{array}{l}\text { Incomplete outcome data } \\
\text { (attrition bias) } \\
\text { Subjective outcomes }\end{array}$ & Unclear risk & $\begin{array}{l}\text { Quote from publication: " } 13 \text { did not want to be in the control group" } \\
\text { Comment: } 13 \text { of the control group dropped out - potential attrition bias }\end{array}$ \\
\hline
\end{tabular}

ive outcomes

Quote from publication: "13 did not want to be in the control group"

Incomplete outcome data Unclear risk

(attrition bias)

Objective outcomes

Comment: 13 of the control group dropped out - potential attrition bias

Selective reporting (re- Unclear risk

porting bias)

Comment: no protocol or clinical trials register entry available

Other bias Unclear risk Comment: unclear if the study was at risk of any other bias

\section{O'Connor 2013}

\begin{tabular}{ll}
\hline Methods & Parallel RCT \\
& Randomisation ratio: 1:1 \\
& Superiority design \\
\hline Participants & $\begin{array}{l}\text { Inclusion criteria: healthy children aged 5-8 years, overweight (BMI } \geq 85 \%) \text { but not morbidly obese } \\
\text { (BMI }<99 \%)(C D C \text { growth charts), attended participating Texas Children's Pediatric Associate (TCPA) } \\
\text { clinics, and were Texas Children's Health Plan (TCHP) members, only } 1 \text { child per family was eligible }\end{array}$ \\
& $\begin{array}{l}\text { Exclusion criteria: medical consequences of obesity (e.g. hypertension) that required intensive treat- } \\
\text { ment, taking medications which could affect a child's weight status, medical problems which would } \\
\text { cause difficulties in participating in the programme, if the child was participating in other weight loss } \\
\text { programmes, parent was unable to read or write in English or Spanish, parents had participated in for- } \\
\text { mative studies to develop the Helping HAND intervention }\end{array}$
\end{tabular}

Diagnostic criteria: see above

$\begin{array}{ll}\text { Interventions } & \text { Number of study centres: } 4 \\ \text { Run-in period: no } \\ \text { Extension period: no } \\ \text { Intervention: 'Helping HAND' obesity intervention } \\ \text { Comparator: waiting-list control }\end{array}$

Outcomes

Outcome measures reported in abstract: attrition, BMI z score, dietary intake, physical activity, hours of TV per week

Study details

Trial terminated early: no

Trial ID: NCT01195012 
O'Connor 2013 (Continued)

\section{Publication details Language of publication: English}

Funding: US Department of Agriculture (USDA/ARS) Children's Nutrition Research Center, Department of Pediatrics, BCM funded in part by the USDA/ARS (Cooperative Agreement 6250-51000) and the Gillson Longenbaugh Foundation BCM Seed Funds (non-commercial)

Publication status: peer-reviewed journal

$\begin{array}{ll}\text { Stated aim for study } & \text { Quote from publication: "Test the feasibility of Helping HAND (Healthy Activity and Nutrition Direc- } \\ \text { tions), an obesity intervention for 5- to 8-year-old children in primary care clinics" }\end{array}$

Notes -

\section{Risk of bias}

Bias Authors' judgement Support for judgement

Random sequence genera- Low risk tion (selection bias)
Quote from publication: "Families who met all criteria were enrolled and randomized to immediately starting Helping HAND (intervention group: IG) or wait-listed for the programme (control group: CG) via a random number sequence protocol developed by the project statistician"

Comment: randomisation process described
Allocation concealment Low risk (selection bias)
Quote from publication: (from author via email) "Yes, participants were recruited and baseline data obtained prior to them being randomized to the intervention of waitlist control group"

Comment: study author confirmed via email that allocation was concealed Blinding of participants High risk
and personnel (performance bias)

Subjective outcomes
Quote from publication: "No, it was not possible to blind participants to the study group since this was a feasibility study and the control group did not receive an intervention (wait-listed). For the same reasons and due to the budget available for this feasibility study, study staff were not blinded to condition. We did have a different staff team conduct the assessment from those that delivered the program."

Comment: participants and study personnel were not blinded to study group

\begin{tabular}{|c|c|c|}
\hline $\begin{array}{l}\text { Blinding of participants } \\
\text { and personnel (perfor- } \\
\text { mance bias) } \\
\text { Objective outcomes }\end{array}$ & High risk & $\begin{array}{l}\text { Quote from publication: "No, it was not possible to blind participants to the } \\
\text { study group since this was a feasibility study and the control group did not re- } \\
\text { ceive an intervention (wait-listed). For the same reasons and due to the budget } \\
\text { available for this feasibility study, study staff were not blinded to condition. We } \\
\text { did have a different staff team conduct the assessment from those that deliv- } \\
\text { ered the program." }\end{array}$ \\
\hline
\end{tabular}

Comment: participants and study personnel were not blinded to study group

\begin{tabular}{|c|c|c|}
\hline $\begin{array}{l}\text { Blinding of outcome as- } \\
\text { sessment (detection bias) } \\
\text { Subjective outcomes }\end{array}$ & High risk & $\begin{array}{l}\text { Quote from publication: "Because of limited staffing those who collected da- } \\
\text { ta could not be blinded to participant group assignment at post assessment } \\
\text { for this pilot study." }\end{array}$ \\
\hline & & $\begin{array}{l}\text { Comment: study author confirmed via email that outcome assessors were not } \\
\text { blinded to study group }\end{array}$ \\
\hline
\end{tabular}

\begin{tabular}{lll}
\hline Blinding of outcome as- & High risk & $\begin{array}{l}\text { Quote from publication: "Because of limited staffing those who collected da- } \\
\text { ta could not be blinded to participant group assignment at post assessment } \\
\text { sessment (detection bias) }\end{array}$ \\
Objective outcomes & for this pilot study."
\end{tabular}


Comment: study author confirmed via email that outcome assessors were not blinded to study group

\begin{tabular}{|c|c|c|}
\hline $\begin{array}{l}\text { Incomplete outcome data } \\
\text { (attrition bias) } \\
\text { Subjective outcomes }\end{array}$ & Low risk & $\begin{array}{l}\text { Quote from publication: "There was } 20 \% \text { attrition from Helping HAND (at- } \\
\text { tended } 4 / 6 \text { sessions)." } \\
\text { Comment: relatively low attrition rates }\end{array}$ \\
\hline $\begin{array}{l}\text { Incomplete outcome data } \\
\text { (attrition bias) } \\
\text { Objective outcomes }\end{array}$ & Low risk & $\begin{array}{l}\text { Quote from publication: "There was } 20 \% \text { attrition from Helping HAND (at- } \\
\text { tended } 4 / 6 \text { sessions)." } \\
\text { Comment: relatively low attrition rates }\end{array}$ \\
\hline $\begin{array}{l}\text { Selective reporting (re- } \\
\text { porting bias) }\end{array}$ & Unclear risk & $\begin{array}{l}\text { Comment: clinical trials register only states that family attendance was the } \\
\text { primary outcome - does not provide any secondary or other outcomes }\end{array}$ \\
\hline Other bias & Unclear risk & Comment: unclear if the study was at risk of any other bias \\
\hline
\end{tabular}

Reinehr 2010

\begin{tabular}{|c|c|}
\hline \multirow[t]{3}{*}{ Methods } & Parallel RCT \\
\hline & Randomisation ratio: $1: 1$ \\
\hline & Superiority design \\
\hline \multirow[t]{3}{*}{ Participants } & $\begin{array}{l}\text { Inclusion criteria: aged 8-16 years, overweight, BMI > 90th percentile and }<97 \text { th percentile using Ger- } \\
\text { man percentiles (Kromeyer-Hauschild 2001), apparently healthy and not on any medication, attending } \\
\text { a regular school }\end{array}$ \\
\hline & Exclusion criteria: obese children \\
\hline & Diagnostic criteria: see above \\
\hline \multirow[t]{5}{*}{ Interventions } & Number of study centres: 2 \\
\hline & Run-in period: no \\
\hline & Extension period: no \\
\hline & Intervention: 'Obeldicks Light' lifestyle intervention \\
\hline & Comparator: waiting list control \\
\hline Outcomes & $\begin{array}{l}\text { Outcome measures reported in abstract: dropout rates, BMI SDS, WC, blood pressure, skinfold thick- } \\
\text { ness, fat mass (BIA and skinfold thickness), dietary intake (energy, fat, sugar), HRQoL, self-esteem }\end{array}$ \\
\hline \multirow[t]{2}{*}{ Study details } & Trial terminated early: no \\
\hline & Trial ID: NCT00422916 \\
\hline \multirow[t]{3}{*}{ Publication details } & Language of publication: English \\
\hline & $\begin{array}{l}\text { Funding: German Federal Ministry of Research (grant numbers 01EL619 and 01EL0603) (non-commer- } \\
\text { cial) }\end{array}$ \\
\hline & Publication status: peer-reviewed journal \\
\hline
\end{tabular}


Reinehr 2010 (Continued)

Stated aim for study Quote from publication: "Our primary hypothesis was that this lifestyle intervention is effective in reducing the degree of overweight based on standard deviation scores of body mass index"

Notes

\section{Risk of bias}

Bias Authors' judgement Support for judgement

Random sequence genera- Low risk tion (selection bias)

Quote from publication: "The children were randomized in the control group (CG) (waiting period of 6 months) or in the intervention group (IG) (6 months intervention) using a computer"

Comment: randomisation process described

\begin{tabular}{lll}
\hline $\begin{array}{l}\text { Allocation concealment } \\
\text { (selection bias) }\end{array}$ & Low risk & Comment: study author confirmed via email that allocation was concealed \\
\hline $\begin{array}{l}\text { Blinding of participants } \\
\text { and personnel (perfor- } \\
\text { mance bias) }\end{array}$ & High risk & $\begin{array}{l}\text { Quote from publication: "The study was an open randomized controlled trial } \\
\text { since blinding was not possible due to the nature of the intervention." }\end{array}$ \\
$\begin{array}{l}\text { Subjective outcomes } \\
\text { Comment: participants and study personnel were not blinded to study group }\end{array}$
\end{tabular}

Blinding of participants High risk Quote from publication: "The study was an open randomized controlled trial and personnel (perfor- $\quad$ since blinding was not possible due to the nature of the intervention."

mance bias)

Objective outcomes

Comment: participants and study personnel were not blinded to study group

Blinding of outcome as-
sessment (detection bias)

Subjective outcomes

Quote from publication: "The study was an open randomized controlled trial since blinding was not possible due to the nature of the intervention."

Comment: outcome assessors were not blinded to study group

\section{Blinding of outcome as- High risk} sessment (detection bias)

Objective outcomes
Quote from publication: "The study was an open randomized controlled trial since blinding was not possible due to the nature of the intervention."

\section{Incomplete outcome data Low risk (attrition bias)}

Subjective outcomes
Comment: outcome assessors were not blinded to study group

Quote from publication: "Only one child (3\%) dropped out of the intervention group, and 5 children (16\%) dropped out of the control group"

Comment: in addition there were 5 families who withdrew consent prior to baseline measurements. Dropout rates quite low
Incomplete outcome data Low risk

(attrition bias)

Objective outcomes
Quote from publication: "Only one child (3\%) dropped out of the intervention group, and 5 children (16\%) dropped out of the control group"

Comment: in addition there were 5 families who withdrew consent prior to baseline measurements. Dropout rates quite low

$\begin{array}{ll}\begin{array}{l}\text { Selective reporting (re- } \\ \text { porting bias) }\end{array} & \text { High risk } \\ & \begin{array}{l}\text { measuring QoL. The clinical trials register entry specifies QoL as a secondary } \\ \text { measure and an additional publication (Finne 2013, see Reinehr 2010) but } \\ \text { does not present results for intervention and control separately even though } \\ \text { they were measured at these time points }\end{array}\end{array}$

Other bias Unclear risk Comment: unable to assess whether any other biases are present


Rodearmel 2007

\begin{tabular}{ll}
\hline Methods & Parallel RCT \\
& Randomisation ratio: 1:1 \\
& Superiority design \\
\hline Participants & $\begin{array}{l}\text { Inclusion criteria: aged 7-14 years, overweight or risk for overweight (BMI } \geq 85 \text { th percentile for age and } \\
\text { gender based on CDC growth charts), at least } 1 \text { parent/guardian to participate in the study }\end{array}$ \\
& $\begin{array}{l}\text { Exclusion criteria: children or parents with medical or physical conditions that prevented them for } \\
\text { participating in physical activity (assessed by health history questionnaire), pregnancy or lactation } \\
\text { (child or parent) } \\
\text { Diagnostic criteria: see above }\end{array}$
\end{tabular}

Number of study centres: 1
Run-in period: no
Extension period: no
Intervention: 'America on the Move' intervention group
Comparator: self-monitoring group

\begin{tabular}{ll}
\hline Outcomes & Outcome measures reported in abstract: BMI for age, parental weight, steps/d \\
\hline Study details & Trial terminated early: no \\
& Trial ID: - \\
\hline
\end{tabular}

Publication details Language of publication: English

Funding: McNeil Nutritionals, LLC, and National Institutes of Health grant DK42549 (commercial and non-commercial)

Publication status: peer-reviewed journal

Stated aim for study Quote from publication: "The intent of this study was to evaluate whether small changes in diet and
physical activity, as promoted by the America on the Move initiative, could prevent excessive weight gain in overweight children"

\begin{tabular}{|c|c|c|}
\hline Notes & - & \\
\hline \multicolumn{3}{|l|}{ Risk of bias } \\
\hline Bias & Authors' judgement & Support for judgement \\
\hline \multirow[t]{2}{*}{$\begin{array}{l}\text { Random sequence genera- } \\
\text { tion (selection bias) }\end{array}$} & Unclear risk & $\begin{array}{l}\text { Quote from study author (via email): "During initial telephone contact, fam- } \\
\text { ilies with a child meeting eligibility criteria were randomized to the control or } \\
\text { experimental groups using the next assignment provided by a simple random- } \\
\text { ization schedule" }\end{array}$ \\
\hline & & Comment: unclear if this method would have resulted in selection bias \\
\hline $\begin{array}{l}\text { Allocation concealment } \\
\text { (selection bias) }\end{array}$ & Low risk & Comment: study author confirmed via email that allocation was concealed \\
\hline
\end{tabular}


Rodearmel 2007 (Continued)

Blinding of participants

Unclear risk mance bias)

Subjective outcomes and personnel (perfor-

Quote from study author (via email): "Participants were not aware that there were two study groups. At the point of randomization they were only told about their assigned group. Personnel were not blinded to study group."

Comment: participants potentially were blinded to study group but personnel were not - unclear level of bias

Quote from study author (via email): "Participants were not aware that there were two study groups. At the point of randomization they were only told about their assigned group. Personnel were not blinded to study group."

Comment: participants potentially were blinded to study group but personnel were not - unclear level of bias

$\begin{array}{lll}\text { Blinding of outcome as- } & \text { High risk } & \begin{array}{l}\text { Comment: study author confirmed via email that assessors were not blinded } \\ \text { sessment (detection bias) }\end{array}\end{array}$
Subjective outcomes

Blinding of participants Unclear risk and personnel (perfor-

mance bias)

Objective outcomes

\begin{tabular}{|c|c|c|}
\hline $\begin{array}{l}\text { Blinding of outcome as- } \\
\text { sessment (detection bias) } \\
\text { Objective outcomes }\end{array}$ & High risk & $\begin{array}{l}\text { Comment: study author confirmed via email that assessors were not blinded } \\
\text { to study group }\end{array}$ \\
\hline
\end{tabular}

Quote from publication: "Overall, the dropout rate for target children was $16 \%$, with the rate slightly but not statistically significantly higher in AOM than in SM families."

Comment: low dropout rates
Low risk

Subjective outcomes
Quote from publication: "Overall, the dropout rate for target children was $16 \%$, with the rate slightly but not statistically significantly higher in AOM than in SM families."

Comment: low dropout rates
Incomplete outcome data Low risk

(attrition bias)

Objective outcomes
Comment: no protocol or clinical trials register entry available

Selective reporting (re- Unclear risk porting bias)
Unclear risk
Comment: unclear if the study was at risk of any other bias

Sacher 2010

\begin{tabular}{|c|c|}
\hline Methods & $\begin{array}{l}\text { Parallel RCT } \\
\text { Randomisation ratio: 1:1 } \\
\text { Superiority design }\end{array}$ \\
\hline Participants & $\begin{array}{l}\text { Inclusion criteria: aged } 8-12 \text { years, obese (BMI } \geq 98 \text { th percentile, UK 1990), no apparent clinical prob- } \\
\text { lems, comorbidities, physical disabilities or learning difficulties which would interfere with taking part, } \\
\text { at least } 1 \text { parent/carer who could attend the programme sessions } \\
\text { Exclusion criteria: none } \\
\text { Diagnostic criteria: see above }\end{array}$ \\
\hline
\end{tabular}

Interventions

Number of study centres: 5

Run-in period: no

Diet, physical activity and behavioural interventions for the treatment of overweight or obese children from the age of 6 to 11 years 
Sacher 2010 (Continued)

\section{Extension period: no}

Intervention: MEND program

Comparator: waiting list control

Outcomes Outcome measures reported in abstract: BMI z score, WC z score, cardiovascular fitness, physical ac-
tivity, sedentary activity, self-esteem, attendance

Study details Trial terminated early: no

Trial ID: ISRCTN30238779

\section{Publication details Language of publication: English}

Funding: National Institute for Health Research, Sainsbury's Supermarkets Ltd., Bromley Mytime, Bromley Primary Care Trust (PCT), Great Ormond Street Hospital for Children NHS Trust, London Borough of Lewisham, MEND Central Ltd., New Cross Gate New Deal for Communities, Parkwood Leisure, Southwark PCT, The Lewisham Hospital NHS Trust, UCL Institute of Child Health, and Waveney PCT (commercial and non-commercial)

Publication status: peer-reviewed journal

Stated aim for study

Quote from publication: "The aim of this study was to evaluate the effectiveness of the Mind, Exercise, Nutrition, Do it (MEND) Program, a multicomponent community-based childhood obesity intervention (www.mendcentral.org)."

Notes -

\section{Risk of bias}

Bias Authors' judgement Support for judgement

Random sequence genera- Low risk tion (selection bias)

Quote from publication: "randomization was conducted by an independent researcher using a random permuted block design with blocks of size 6 . The randomization schedule was computer generated"

Comment: randomisation process well described

Allocation concealment Low risk Comment: study author confirmed via email that allocation was concealed
(selection bias)

Blinding of participants High risk and personnel (performance bias)

Subjective outcomes

Quote from study author (via email): "As we used a delayed intervention control group, it was not possible to blind participants to the study group. Study personnel were not blinded"

Comment: participants and study personnel were not blinded to study group

\author{
Blinding of participants \\ and personnel (perfor- \\ mance bias) \\ Objective outcomes
}

High risk

Quote from study author (via email): "As we used a delayed intervention control group, it was not possible to blind participants to the study group. Study personnel were not blinded"

Comment: participants and study personnel were not blinded to study group

Blinding of outcome as- $\quad$ Low risk
sessment (detection bias)
Subjective outcomes

Blinding of outcome as- Low risk Subjective outcomes
Quote from study author (via email): "Study personnel were not blinded but all measurements were repeated and double checked by blinded additional research staff."

Comment: even though study personnel were not blinded, measurements were checked by blinded staff 
Sacher 2010 (Continued)

Blinding of outcome as- Low risk sessment (detection bias)

Objective outcomes
Quote from study author (via email): "Study personnel were not blinded but all measurements were repeated and double checked by blinded additional research staff."

Comment: even though study personnel were not blinded, measurements were checked by blinded staff

\section{Incomplete outcome data High risk}

(attrition bias)

Subjective outcomes

Quote from publication: "Of the 60 intervention children, 54 started and all 54 completed the intensive phase of the intervention (9-week MEND Program), while $62 \%$ of the 60 were seen at 6 months and $83 \%$ either at 6 or 12 months"

Comment: dropout rates relatively low in control group but moderate in intervention - potential attrition bias

Incomplete outcome data High risk
(attrition bias)

Objective outcomes

Quote from publication: "Of the 60 intervention children, 54 started and all 54 completed the intensive phase of the intervention (9-week MEND Program), while $62 \%$ of the 60 were seen at 6 months and $83 \%$ either at 6 or 12 months"

Comment: dropout rates relatively low in control group but moderate in intervention - potential attrition bias

\begin{tabular}{lll}
\hline $\begin{array}{l}\text { Selective reporting (re- } \\
\text { porting bias) }\end{array}$ & High risk & $\begin{array}{l}\text { Comment: outcomes reported in clinical trials register entry report outcomes } \\
\text { not reported in the publication (family functioning, child mental health, di- } \\
\text { etary intake) - potential reporting bias }\end{array}$ \\
\hline Other bias & Unclear risk & Comment: unable to assess if any other biases are present
\end{tabular}

Saelens 2013

$\begin{array}{ll}\text { Methods } & \text { Parallel RCT } \\ & \text { Randomisation ratio: 1:1 } \\ & \text { Superiority design }\end{array}$

Participants

Inclusion criteria: children aged 7-11 years, above the 85th percentile for age- and gender-specific BMI but not $>175 \%$ above median BMI for age and gender (CDC growth charts), at least 1 overweight parent (BMI $\geq 25$ ), no existing thought disorder, suicidality, substance abuse disorder, no disability or illness stopping them from engaging in at least moderate intensity activity, English speaking and at least second grade reading level, no current or prior diagnosed eating disturbance, live $<50$ miles from the treatment site, parent/caregiver willing to attend treatment sessions and engage in the behaviour change around eating and physical activity, parents were allowed to participate in other weight programmes if the behavioural changes recommended were consistent with the study's targets

Exclusion criteria: conditions known to promote obesity (e.g. Prader-Willi), participating in another weight control programme, recently started taking medications which affect weight (e.g. stimulants)

Diagnostic criteria: see above

Interventions Number of study centres: 1

Run-in period: no

Extension period: no

Intervention: self-directed approach

Comparator: prescribed approach

Diet, physical activity and behavioural interventions for the treatment of overweight or obese children from the age of 6 to 11 years 
Saelens 2013 (Continued)

Outcomes Outcome measures reported in abstract: BMI z score, parental BMI

\begin{tabular}{ll}
\hline Study details & Trial terminated early: no \\
& Trial ID: NCT00746629 \\
\hline Publication details & Language of publication: English \\
& $\begin{array}{l}\text { Funding: Eunice Kennedy Shriver National Institute of Child Health and Human Development of the } \\
\text { National Institutes of Health under award number R21HD054871 and the Seattle Children's Hospital } \\
\text { Research Institute (non-commercial) }\end{array}$
\end{tabular}

Publication status: peer-reviewed journal

Stated aim for study

Quote from publication: "To examine the efficacy of an adjunct motivational and autonomy-enhancing intervention (self-directed) for behavioral family-based pediatric obesity relative to the standard prescription of uniform behavioural skills use and interventionist goal assignment (prescribed)"

\section{Notes}

\section{Risk of bias}

\begin{tabular}{lll}
\hline Bias & Authors' judgement & Support for judgement \\
\hline $\begin{array}{ll}\text { Random sequence genera- } \\
\text { tion (selection bias) }\end{array}$ & Low risk & $\begin{array}{l}\text { Quote from publication: "Families were randomly assigned to receive either } \\
\text { the prescribed or self-directed approach, with child gender and child level of } \\
\text { overweight }[<\text { or }>60 \% \text { above median body mass index (BMI) for age and gen- } \\
\text { der] as stratification variables. Randomization blocks were randomly selected } \\
\text { to be either four or six participating families" }\end{array}$ \\
& Comment: randomisation process well described \\
\hline
\end{tabular}

$\begin{aligned} & \text { Allocation concealment } \\ & \text { (selection bias) }\end{aligned}$ Low risk Comment: study author confirmed via email that allocation was concealed

Blinding of participants Unclear risk
and personnel (performance bias)

Subjective outcomes

Quote from publication: "During consenting, families were provided a brief description of each approach, but were otherwise blind to approach differences during treatment."

Comment: participants blinded to which was the intervention and which was the control group. Unclear if study personnel were

Blinding of participants Unclear risk
and personnel (perfor-
mance bias)
Objective outcomes
Quote from publication: "During consenting, families were provided a brief description of each approach, but were otherwise blind to approach differ- ences during treatment."

Comment: participants blinded to which was the intervention and which was the control group. Unclear if study personnel were

\begin{tabular}{lll}
\hline $\begin{array}{l}\text { Blinding of outcome as- } \\
\begin{array}{l}\text { sessment (detection bias) } \\
\text { Subjective outcomes }\end{array}\end{array}$ & Low risk & $\begin{array}{l}\text { Quote from publication: "Assessors were not interventionists and were blind } \\
\text { to approach differences" }\end{array}$ \\
\hline $\begin{array}{l}\text { Blinding of outcome as- } \\
\text { Sessment (detection bias) } \\
\text { Objective outcomes }\end{array}$ & Low risk & $\begin{array}{l}\text { Quote from publication: "Assessors were not interventionists and were blind } \\
\text { to approach differences" }\end{array}$ \\
& Comment: outcome assessors were blinded to study group \\
\hline
\end{tabular}


Saelens 2013 (Continued)

Incomplete outcome data High risk Quote from publication: "There were 57 assessment completers at post(attrition bias) treatment, 58 at 3-month follow-up, 54 at 6-month follow-up, 52 at 1-year fol-

Subjective outcomes low-up, and 46 at 2-year follow-up."

Comment: dropout rates fairly high (48\%). Did use an imputation method to replace some data but attrition bias likely to still exist

Incomplete outcome data High risk (attrition bias)

Objective outcomes
Quote from publication: "There were 57 assessment completers at posttreatment, 58 at 3-month follow-up, 54 at 6-month follow-up, 52 at 1-year follow-up, and 46 at 2-year follow-up."

Comment: dropout rates fairly high (48\%). Did use an imputation method to replace some data but attrition bias likely to still exist
Selective reporting (re-
Unclear risk
Comment: no clinical trial entry or protocol available porting bias)
Satoh 2007

\begin{tabular}{|c|c|}
\hline Methods & $\begin{array}{l}\text { Parallel RCT } \\
\text { Randomisation ratio: } 2: 1 \\
\text { Superiority design }\end{array}$ \\
\hline Participants & $\begin{array}{l}\text { Inclusion criteria: aged } 8-14 \text { years, obesity (definition adopted from The Ministry of Health, Labor and } \\
\text { Welfare in Japan, body weight exceeded } 120 \% \text { of standard body weight corresponding to height for age } \\
\text { and sex obtained from national statistics for Japanese school children 1990) } \\
\text { Exclusion criteria: none } \\
\text { Diagnostic criteria: see above }\end{array}$ \\
\hline
\end{tabular}

Number of study centres: 3

Run-in period: no

Extension period: no

Treatment before study : before starting dietary guidance both intervention and control subjects and their parents received conventional dietary guidance

Intervention: dietary guidance using an easily handled model nutritional balance chart (MNBC)

Comparator: usual care

Outcomes Outcome measures reported in abstract: percentage overweight, nutritional balance (sugar and
beans)

Study details

Trial terminated early: no

Trial ID: -

Publication details

Funding: unclear 
Satoh 2007 (Continued)

Publication status: peer-reviewed journal

Stated aim for study Quote from publication: "In the present study, an easily handled model nutritional balance chart (MNBC) for obese children and their families was investigated"

Notes -

\section{Risk of bias}

Bias Authors' judgement Support for judgement

\begin{tabular}{lll}
\hline $\begin{array}{l}\text { Random sequence genera- } \\
\text { tion (selection bias) }\end{array}$ & Unclear risk & Comment: randomisation process not described \\
\hline $\begin{array}{l}\text { Allocation concealment } \\
\text { (selection bias) }\end{array}$ & Unclear risk & Comment: not clear if allocation was concealed \\
\hline $\begin{array}{l}\text { Blinding of participants } \\
\text { and personnel (perfor- } \\
\text { mance bias) }\end{array}$ & Unclear risk & $\begin{array}{l}\text { Comment: } \text { not clear whether study personnel or participants were blinded to } \\
\text { study group }\end{array}$ \\
$\begin{array}{l}\text { Subjective outcomes } \\
\begin{array}{l}\text { Blinding of participants } \\
\text { and personnel (perfor- } \\
\text { mance bias) }\end{array}\end{array}$ & Unclear risk & $\begin{array}{l}\text { Comment: } \text { not clear whether study personnel or participants were blinded to } \\
\text { Study group }\end{array}$ \\
\hline
\end{tabular}

$\begin{array}{ll}\text { Blinding of outcome as- } & \text { Unclear risk }\end{array}$ Comment: not clear if outcome assessors were blinded to study group

Subjective outcomes

Blinding of outcome as-

sessment (detection bias)

Objective outcomes
Unclear risk

Comment: not clear if outcome assessors were blinded to study group
Incomplete outcome data High risk

(attrition bias)

Subjective outcomes
Quote from publication: "Among the 43 obese children, 29 were randomly chosen for the obesity intervention groups and the other 14 children comprised the control group. Three children in the intervention group refused to participate in the study and five children in the intervention group withdrew after 1 month of intervention, leaving 21 remaining children in the intervention group. Among the 14 children in the control group, six children refused to participate in the study, leaving eight remaining children in the control group. These two groups were stable during the entire length of the study."

Comment: dropout high in both groups at 6 months (around 47\%) - attrition bias likely

\section{Incomplete outcome data High risk} (attrition bias)

Objective outcomes
Quote from publication: "Among the 43 obese children, 29 were randomly chosen for the obesity intervention groups and the other 14 children comprised the control group. Three children in the intervention group refused to participate in the study and five children in the intervention group withdrew after 1 month of intervention, leaving 21 remaining children in the intervention group. Among the 14 children in the control group, six children refused to participate in the study, leaving eight remaining children in the control group. These two groups were stable during the entire length of the study."

Comment: dropout high in both groups at 6 months (around 47\%) - attrition bias likely 
Satoh 2007 (Continued)
Selective reporting (re-
Unclear risk
Comment: no clinical trial entry or protocol available porting bias)

Other bias

Unclear risk

Comment: unclear if the study was at risk of any other bias

Schwingshandl 1999

\begin{tabular}{ll}
\hline Methods & Parallel RCT \\
& Randomisation ratio: $1: 1$ \\
& Superiority design
\end{tabular}

Participants

Inclusion criteria: obese children and adolescents (unclear how obesity was defined)

Exclusion criteria: none

Diagnostic criteria: unclear

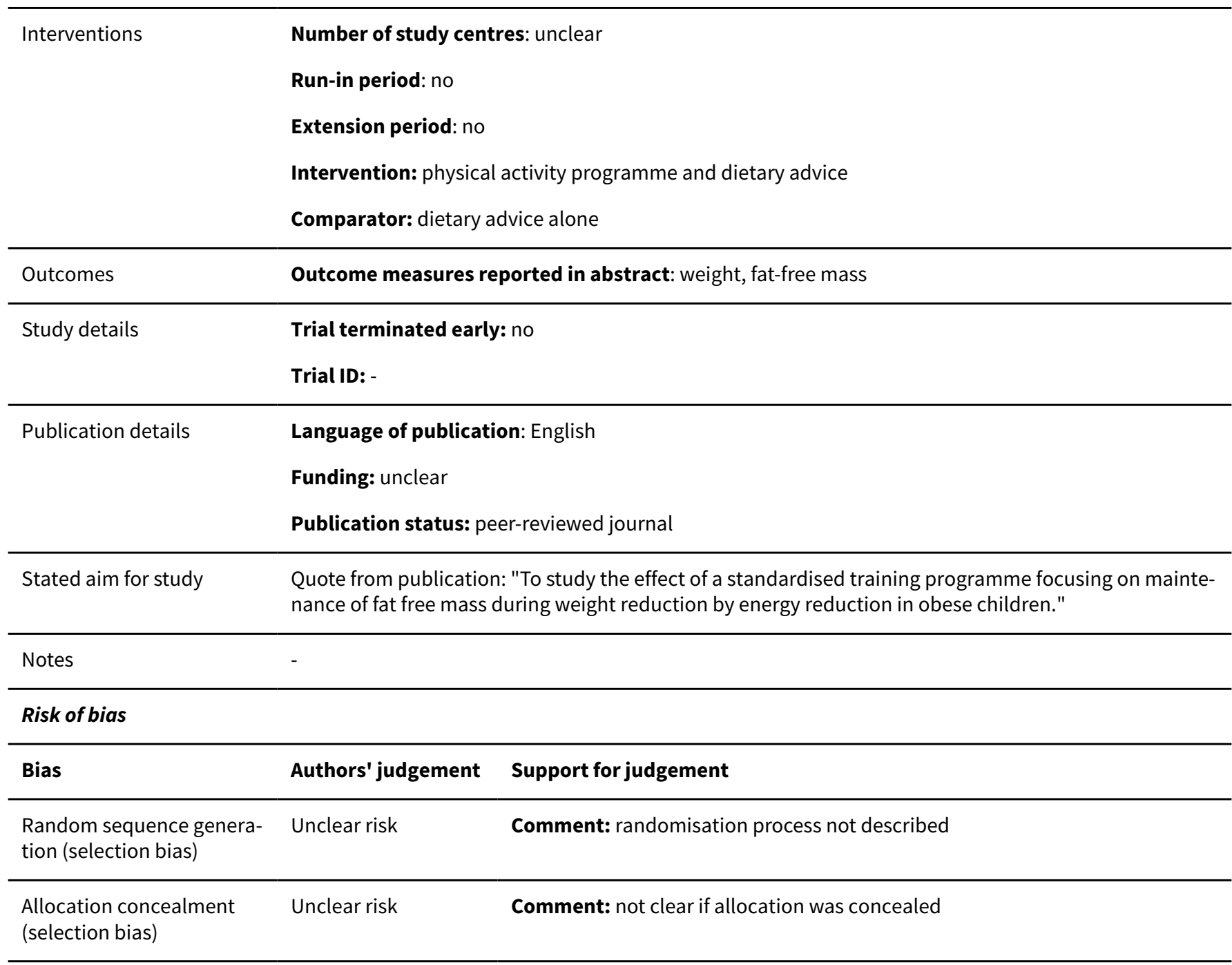

Diet, physical activity and behavioural interventions for the treatment of overweight or obese children from the age of 6 to 11 years 
Schwingshandl 1999 (Continued)

Blinding of participants Unclear risk Comment: not clear whether study personnel or participants were blinded to and personnel (perforstudy group mance bias)

Objective outcomes

Blinding of outcome as-
sessment (detection bias) $\quad$ Unclear risk Comment: not clear if outcome assessors were blinded to study group

Objective outcomes

Incomplete outcome data High risk

(attrition bias)

Objective outcomes
Quote from publication: "Thirty obese children and adolescents (14 group A, 16 group $B$ ) participated in the 12 week long programme; 20 children (10 group $A, 10$ group B) were also reassessed after one year"

Comment: dropout rates relatively high

Comment: no clinical trials register entry or protocol available. The authors do not provide data for BMI at 12 months' follow-up (only provide at 12 weeks)
Selective reporting (re- High risk porting bias)
Comment: unclear if the study was at risk of any other bias

Serra-Paya 2015

\begin{tabular}{|c|c|}
\hline Methods & $\begin{array}{l}\text { Parallel RCT } \\
\text { Randomisation ratio: 1:1 } \\
\text { Superiority design }\end{array}$ \\
\hline Participants & $\begin{array}{l}\text { Inclusion criteria: aged } 6-12 \text { years, overweight or obese (IOTF), sedentary, }<2 \mathrm{~h} / \text { week of physical ac- } \\
\text { tivity outside of school, live in or near the municipality of Lleida (Spain) and their healthcare paediatric } \\
\text { unit has been accepted to take part, at least } 1 \text { parent/guardian able to participate } \\
\text { Exclusion criteria: co-morbidities e.g. Cushing's disease, or serious chronic illness, use of medication } \\
\text { that might affect weight loss or adaptations to exertion, previous enrolment in other obesity treatment } \\
\text { interventions, regular participation in physical exercise programmes in the past } 6 \text { months } \\
\text { Diagnostic criteria: see above }\end{array}$ \\
\hline Interventions & $\begin{array}{l}\text { Number of study centres: } 16 \\
\text { Run-in period: no } \\
\text { Extension period: no } \\
\text { Intervention: Nereu programme } \\
\text { Comparator: counselling group }\end{array}$ \\
\hline Outcomes & $\begin{array}{l}\text { Outcome measures reported in abstract: BMI SDS, moderate-intense physical activity, daily fruit } \\
\text { servings, daily soft drink consumption }\end{array}$ \\
\hline Study details & $\begin{array}{l}\text { Trial terminated early: no } \\
\text { Trial ID: NCT01878994 }\end{array}$ \\
\hline Publication details & $\begin{array}{l}\text { Language of publication: English } \\
\text { Funding: partially funded by the Instituto de Salud Carlos III in Spain, from the Ministry of Economy } \\
\text { and Competitiveness (Grant PI12/02220) co-funded by FEDER and the Institute of Physical Education of }\end{array}$ \\
\hline
\end{tabular}

Diet, physical activity and behavioural interventions for the treatment of overweight or obese children from the age of 6 to 11 years 
Serra-Paya 2015 (Continued)

Catalonia (INEFC), University of Lleida, Spain, (Grants: VCP/3570/2010, 29th October, DOGC NÚM. 5753 -

11.11.2010; VCP/28/2009, 14th January, DOGC NÚM. 5302 - 22/01/2009) (non-commercial)

Publication status: peer-reviewed journal

Stated aim for study

Quote from publication: "To evaluate the effectiveness of the Nereu Program in improving anthropometric parameters, physical activity and sedentary behaviours, and dietary intake."

Notes

\section{Risk of bias}

\begin{tabular}{lll}
\hline Bias & Authors' judgement & Support for judgement \\
\hline $\begin{array}{ll}\text { Random sequence genera- } \\
\text { tion (selection bias) }\end{array}$ & Low risk & $\begin{array}{l}\text { Quote from publication: "Each cooperating healthcare paediatric unit was } \\
\text { provided a sort list (using a computer-generated random number) of their eli- } \\
\text { gible patients who met the inclusion criteria (age and BMIsd), according to the } \\
\text { data contained in clinical records. These eligible children had been randomly } \\
\text { assigned to one of the study arms, stratified by age group in each HPU" }\end{array}$ \\
& Comment: randomisation process described in detail
\end{tabular}

\begin{tabular}{|c|c|c|}
\hline $\begin{array}{l}\text { Allocation concealment } \\
\text { (selection bias) }\end{array}$ & Unclear risk & $\begin{array}{l}\text { Comment: unclear if randomisation process would introduce selection bias } \\
\text { through allocation concealment }\end{array}$ \\
\hline
\end{tabular}

\begin{tabular}{|c|c|c|}
\hline $\begin{array}{l}\text { Blinding of participants } \\
\text { and personnel (perfor- } \\
\text { mance bias) } \\
\text { Subjective outcomes }\end{array}$ & Low risk & $\begin{array}{l}\text { Comment: study author confirmed via email that they were both blinded to } \\
\text { study group }\end{array}$ \\
\hline $\begin{array}{l}\text { Blinding of participants } \\
\text { and personnel (perfor- } \\
\text { mance bias) } \\
\text { Objective outcomes }\end{array}$ & Low risk & $\begin{array}{l}\text { Comment: study author confirmed via email that they were both blinded to } \\
\text { study group }\end{array}$ \\
\hline
\end{tabular}

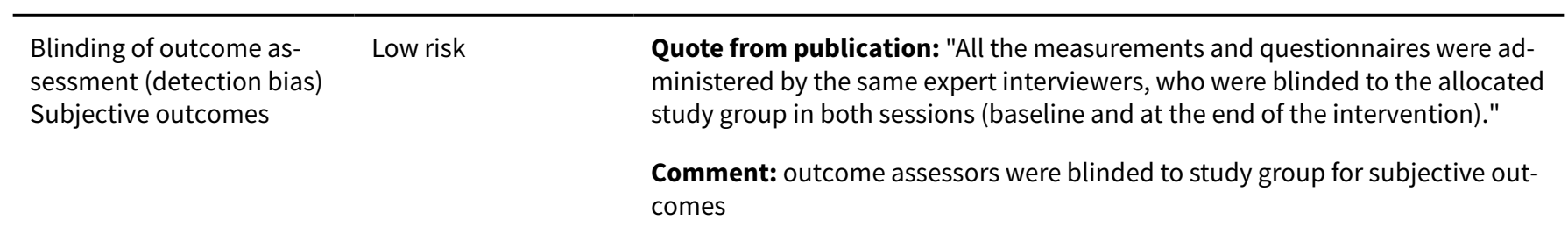

\begin{tabular}{lll}
\hline $\begin{array}{l}\text { Blinding of outcome as- } \\
\text { sessment (detection bias) } \\
\text { Objective outcomes }\end{array}$ & Unclear risk & Comment: not clear if outcome assessors were blinded to study group \\
\hline $\begin{array}{l}\text { Incomplete outcome data } \\
\text { (attrition bias) }\end{array}$ & Unclear risk & $\begin{array}{l}\text { Quote from publication: "Despite high program adherence, the rate of loss- } \\
\text { es and missing values affected the effect size, depending on the parameter, } \\
\text { which limited the statistical power to detect differences between groups in the } \\
\text { changes observed" }\end{array}$
\end{tabular}

Comment: moderate dropout rates - potential attrition bias

\begin{tabular}{ll}
\hline $\begin{array}{l}\text { Incomplete outcome data } \\
\text { (attrition bias) }\end{array}$ & Unclear risk \\
Objective outcomes & $\begin{array}{l}\text { Quote from publication: "Despite high program adherence, the rate of loss- } \\
\text { es and missing values affected the effect size, depending on the parameter, } \\
\text { which limited the statistical power to detect differences between groups in the } \\
\text { changes observed" }\end{array}$
\end{tabular}

Comment: moderate dropout rates - potential attrition bias 
Serra-Paya 2015 (Continued)

Selective reporting (re- Unclear risk Comment: publication did not report some of the outcomes given in the proporting bias) tocol - e.g. QOL - or endpoint (12 months after intervention). Perhaps will be reported in another publication

Other bias Low risk Comment: no other bias identified - study generally low risk of bias in other domains

Siwik 2013

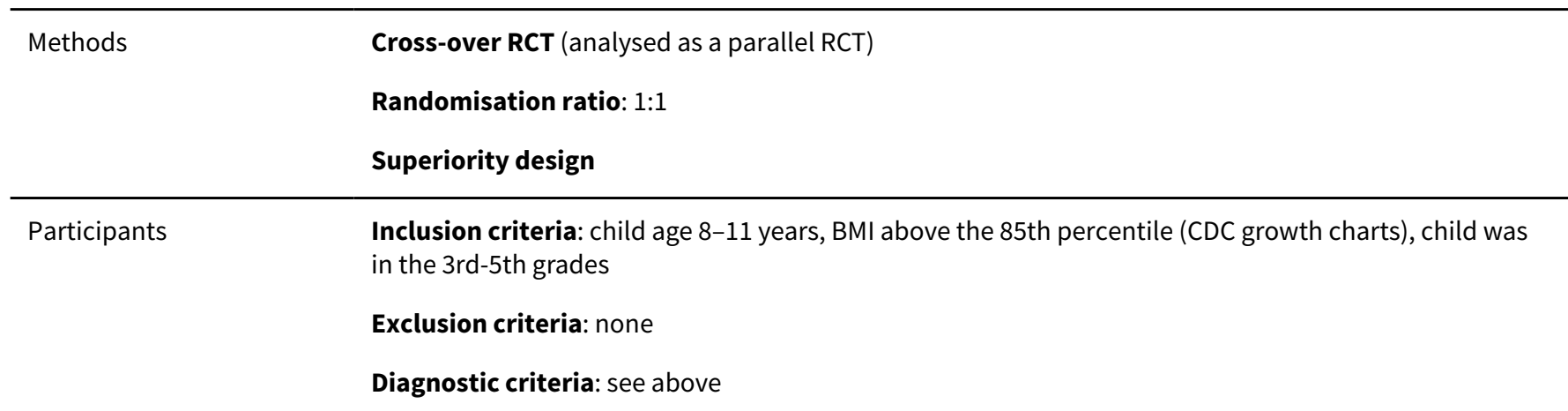

\begin{tabular}{ll}
\hline Interventions & Number of study centres: 1 \\
Run-in period: no \\
Extension period: no \\
Intervention: 'Choices' group office-visit intervention \\
Comparator: lagged control group
\end{tabular}

Outcomes Outcome measures reported in abstract: BMI z score, weight for age $z$ score, low and high METs, behaviours and attitudes

Study details Trial terminated early: no

Trial ID: NCT01674920

\section{Publication details Language of publication: English}

Funding: National Institutes of Health grant R21 HD50962 (non-commercial)

Publication status: peer-reviewed journal

Stated aim for study Quote from publication: "To improve coping skills and increase the likelihood of success in making lifestyle changes, we enhanced the concept of "choices" by providing an innovative approach to problem-solving skills designed to strengthen resiliency. We developed a group office curriculum and conducted an early phase trial to test the efficacy of the program using a lagged intervention/control design."

Notes -

\section{Risk of bias}

Bias Authors' judgement Support for judgement


Siwik 2013 (Continued)

Random sequence genera- Low risk tion (selection bias)
Quote from publication: "Allocation was done using design-adaptive allocation that minimizes the differences between groups as participants enter the study. Balancing factors were sex, age, and BMI."

Comment: randomisation process described
Allocation concealment
Unclear risk
Comment: not clear whether allocation was concealed

(selection bias)

\begin{tabular}{|c|c|c|}
\hline $\begin{array}{l}\text { Blinding of participants } \\
\text { and personnel (perfor- }\end{array}$ & Unclear risk & $\begin{array}{l}\text { Comment: unclear if participants and study personnel were blinded to study } \\
\text { group }\end{array}$ \\
\hline
\end{tabular}

mance bias)

Subjective outcomes

\begin{tabular}{|c|c|c|}
\hline $\begin{array}{l}\text { Blinding of participants } \\
\text { and personnel (perfor- } \\
\text { mance bias) } \\
\text { Objective outcomes }\end{array}$ & Unclear risk & $\begin{array}{l}\text { Comment: unclear if participants and study personnel were blinded to study } \\
\text { group }\end{array}$ \\
\hline
\end{tabular}

\begin{tabular}{|c|c|c|}
\hline $\begin{array}{l}\text { Blinding of outcome as- } \\
\text { sessment (detection bias) } \\
\text { Subjective outcomes }\end{array}$ & Unclear risk & Comment: unclear if outcome assessors were blinded to study group \\
\hline
\end{tabular}

\begin{tabular}{lll}
\hline $\begin{array}{l}\text { Blinding of outcome as- } \\
\text { sessment (detection bias) } \\
\text { Objective outcomes }\end{array}$ & Unclear risk & Comment: unclear if outcome assessors were blinded to study group \\
\hline $\begin{array}{l}\text { Incomplete outcome data } \\
\text { (attrition bias) }\end{array}$ & Low risk & $\begin{array}{l}\text { Quote from publication: "Two families were unable to attend sessions, but } \\
\text { the children received nearly all the measurements and are included in all } \\
\text { analyses" }\end{array}$ \\
& $\begin{array}{l}\text { Comment: only } 3 \text { children were not available for follow-up measurements and } \\
\text { missing data were imputed }\end{array}$
\end{tabular}

\begin{tabular}{|c|c|c|}
\hline $\begin{array}{l}\text { Incomplete outcome data } \\
\text { (attrition bias) } \\
\text { Objective outcomes }\end{array}$ & Low risk & $\begin{array}{l}\text { Quote from publication: "Two families were unable to attend sessions, but } \\
\text { the children received nearly all the measurements and are included in all } \\
\text { analyses" }\end{array}$ \\
\hline
\end{tabular}

Comment: only 3 children were not available for follow-up measurements and missing data were imputed

\begin{tabular}{lll}
\hline $\begin{array}{l}\text { Selective reporting (re- } \\
\text { porting bias) }\end{array}$ & Low risk & Comment: clinical trials register entry available - no bias \\
\hline Other bias & Unclear risk & Comment: unclear if the study was at risk of any other bias \\
\hline
\end{tabular}

Taveras 2015

\begin{tabular}{ll}
\hline Methods & Cluster RCT \\
& Randomisation ratio: $1: 1: 1$ \\
& Superiority design \\
\hline Participants & $\begin{array}{l}\text { Inclusion criteria: child aged } 6-12.9 \text { years, BMI } \geq 90 \text { th percentile for age and sex at baseline well child } \\
\text { visit (CDC growth charts), child has received well child care at Harvard Vanguard Medical Associates } \\
\text { (HVMA) within the past } 15 \text { months, at least } 1 \text { parent able to communicate in English }\end{array}$
\end{tabular}

Diet, physical activity and behavioural interventions for the treatment of overweight or obese children from the age of 6 to 11 years 
Exclusion criteria: if child has already enrolled in study, family planning to leave HVMA within the study time frame, their clinician feels the study is not appropriate for them, had chronic medical conditions which impacted on their diet/physical activity

Diagnostic criteria: see above

Number of study centres: 14
Run-in period: no
Extension period: no
Intervention 1: computerised point-of-care alerts plus direct to parent outreach and support
Intervention 2: computerised point-of-care alerts only
Comparator: usual care

Outcomes

Outcome measures reported in abstract: BMI, Healthcare Effectiveness Data and Information Set (HEDIS) performance measures for obesity

\section{Study details}

\section{Trial terminated early: no}

Trial ID: NCT01537510

Publication details Language of publication: English

Funding: this study was supported by award R18 AE000026 from the American Recovery and Reinvestment Act (Dr Taveras) (non-commercial)

Publication status: peer-reviewed journal

Stated aim for study

Quote from publication: "To examine the extent to which computerized clinical decision support (CDS) delivered to pediatric clinicians at the point of care of obese children, with or without individualized family coaching, improved body mass index (BMI; calculated as weight in kilograms divided by height in meters squared) and quality of care"

\section{Notes}

\section{Risk of bias}

Bias Authors' judgement Support for judgement

Random sequence genera- Low risk tion (selection bias)
Quote from publication: "We used a stratified block randomization scheme to assign practices to one of the 3 study arms. Strata were based on the volume of children aged 6.0 to 12.9 with a BMI 95th percentile seen for well-child visits at each site from April 2010 through March 2011. A biostatistician (KPK) blinded to the names of the practices ordered them on this characteristic, then introduced a false practice at a random spot within the order to make the number of "practices" evenly divisible by 3 . Strata consisted of consecutive groups of three practices from this ordered list. He then used a pseudo-random number generator in SAS 9.2 (SAS Institute, Cary NC) to assign one practice from each strata to each of the arms, with the exception that the false practice was deterministically assigned to the usual care arm. This resulted in 5 practices in each of the intervention arms and 4 in the usual care arm."

Comment: randomisation process described
Comment: unlikely that selection bias would have occurred from the ran-
Allocation concealment Low risk (selection bias) domisation process described above

domisation process described above


Taveras 2015 (Continued)

Blinding of participants Unclear risk Quote from publication: "Study participants and the pediatricians in each and personnel (perforpractice are blinded to specific study hypotheses but not to intervention asmance bias)

Subjective outcomes signment"

Comment: were not blinded to treatment group, but did not know study hypothesis - unclear if any bias would have occurred

\begin{tabular}{|c|c|c|}
\hline $\begin{array}{l}\text { Blinding of participants } \\
\text { and personnel (perfor- } \\
\text { mance bias) }\end{array}$ & Unclear risk & $\begin{array}{l}\text { Quote from publication: "Study participants and the pediatricians in each } \\
\text { practice are blinded to specific study hypotheses but not to intervention as- } \\
\text { signment" }\end{array}$ \\
\hline Objective outcomes & & $\begin{array}{l}\text { Comment: were not blinded to treatment group, but did not know study hy- } \\
\text { pothesis - unclear if any bias would have occurred }\end{array}$ \\
\hline
\end{tabular}

$\begin{array}{ll}\begin{array}{l}\text { Blinding of outcome as- } \\ \text { sessment (detection bias) }\end{array} & \text { Low risk }\end{array} \quad \begin{aligned} & \text { Quote from publication: "Research staff performing all assessments is blind- } \\ & \text { ed to specific study hypotheses and to intervention assignment" }\end{aligned}$

Subjective outcomes

Comment: outcomes assessors were blinded to treatment group

\begin{tabular}{|c|c|c|}
\hline $\begin{array}{l}\text { Blinding of outcome as- } \\
\text { sessment (detection bias) } \\
\text { Objective outcomes }\end{array}$ & Low risk & $\begin{array}{l}\text { Quote from publication: "Research staff performing all assessments is blind- } \\
\text { ed to specific study hypotheses and to intervention assignment" }\end{array}$ \\
\hline
\end{tabular}

Comment: outcomes assessors were blinded to treatment group

\begin{tabular}{|c|c|c|}
\hline $\begin{array}{l}\text { Incomplete outcome data } \\
\text { (attrition bias) } \\
\text { Subjective outcomes }\end{array}$ & Low risk & $\begin{array}{l}\text { Quote from publication: "we obtained BMI from } 518 \text { children }(94.4 \% \text { and } \\
\text { HEDIS measurement from } 491 \text { visits }(89.4 \%) \text {." } \\
\text { Comment: relatively low dropout rates }\end{array}$ \\
\hline $\begin{array}{l}\text { Incomplete outcome data } \\
\text { (attrition bias) } \\
\text { Objective outcomes }\end{array}$ & Low risk & $\begin{array}{l}\text { Quote from publication: "we obtained BMI from } 518 \text { children ( } 94.4 \% \text { and } \\
\text { HEDIS measurement from } 491 \text { visits }(89.4 \%) \text {." } \\
\text { Comment: relatively low dropout rates }\end{array}$ \\
\hline $\begin{array}{l}\text { Selective reporting (re- } \\
\text { porting bias) }\end{array}$ & Unclear risk & $\begin{array}{l}\text { Comment: clinical trial mentions measuring costs and health behaviours- but } \\
\text { these are not reported in the publication - may be published in an additional } \\
\text { paper }\end{array}$ \\
\hline
\end{tabular}

Other bias Low risk Comment: was a cluster RCT and adjusted for clustering in their analyses

Taylor 2015

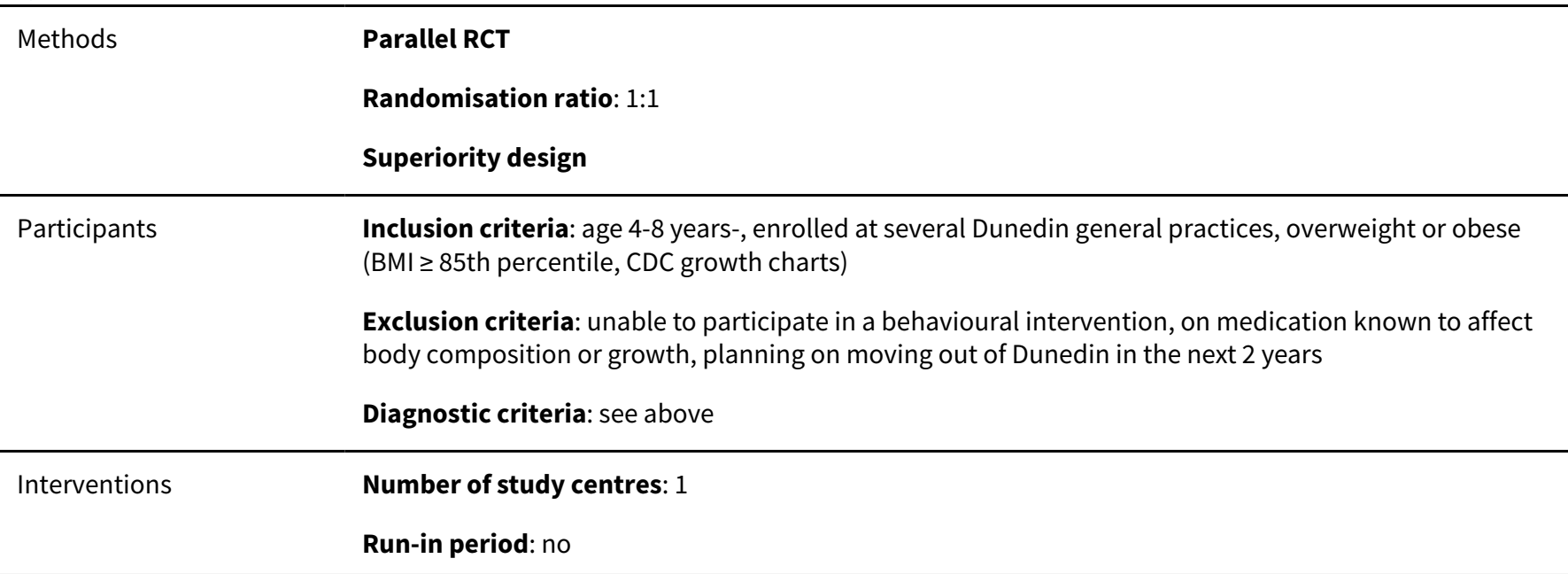

Diet, physical activity and behavioural interventions for the treatment of overweight or obese children from the age of 6 to 11 years 
Taylor 2015 (Continued)

\section{Extension period: no}

Intervention: tailored package family-based intervention

Comparator: usual care

Outcomes

Outcome measures reported in abstract: BMI, BMI z score, WC, fruit and vegetables intake, noncore food intake, noncore food availability, physical activity, parental feeding practices, parenting, QoL, child sleep, behaviours, satisfaction

Study details

\section{Trial terminated early: no}

Trial ID: ACTRN12609000749202

\section{Publication details Language of publication: English}

Funding: Health Research Council of New Zealand. Dr Dawson was in receipt of a Freemasons New Zealand Fellowship at the time the data were collected. Dr R.W. Taylor is funded by the KPS Research Fellowship (non-commercial)

Publication status: peer-reviewed journal

\section{Stated aim for study}

Quote from publication: "To determine whether a 2-year family-based intervention using frequent contact and limited expert involvement was effective in reducing excessive weight compared with usual care."

Notes -

\section{Risk of bias}

Bias Authors' judgement Support for judgement

Random sequence genera- Low risk tion (selection bias)

Quote from publication: "Randomization to intervention condition occurred using random block lengths (Stata 12.0, StataCorp) after stratifying for feedback condition."

Comment: randomisation process described

\begin{tabular}{|c|c|c|}
\hline $\begin{array}{l}\text { Allocation concealment } \\
\text { (selection bias) }\end{array}$ & Low risk & $\begin{array}{l}\text { Quote from publication: "We also met virtually all study quality criteria, in- } \\
\text { cluding blinding of outcome assessors to treatment, allocation concealment, } \\
\text { and appropriate statistical analyses" }\end{array}$ \\
\hline
\end{tabular}

Comment: allocation was concealed

\begin{tabular}{lll}
\hline $\begin{array}{l}\text { Blinding of participants } \\
\text { and personnel (perfor- }\end{array}$ & High risk & $\begin{array}{l}\text { Quote from publication: "Participants were not blinded to intervention con- } \\
\text { dition because the } 2 \text { conditions differed in the amount of contact." }\end{array}$
\end{tabular}
and personnel (perfor-

Comment: participants were not blinded to study group

Subjective outcomes

High risk

Blinding of participants High risk
and personnel (perfor-
mance bias)
Objective outcomes

Blinding of outcome as- Low risk sessment (detection bias)

Subjective outcomes
Quote from publication: "Participants were not blinded to intervention condition because the 2 conditions differed in the amount of contact."

Comment: participants were not blinded to study group

(including screening),
vention allocation."

Comment: outcomes assessors were blinded to treatment group 
Taylor 2015 (Continued)

Blinding of outcome as- Low risk Quote from publication: "Outcome assessments were undertaken at baseline sessment (detection bias)

Objective outcomes (including screening), 12 and 24 months by trained assistants blinded to intervention allocation."

Comment: outcomes assessors were blinded to treatment group

Incomplete outcome data Low risk (attrition bias)

Subjective outcomes
Quote from publication: "A major strength of our study is the high retention, with $88 \%$ of children at study end."

Comment: relatively low dropout rates
Incomplete outcome data Low risk (attrition bias)

Objective outcomes
Quote from publication: "A major strength of our study is the high retention, with $88 \%$ of children at study end."

Comment: relatively low dropout rates

\begin{tabular}{|c|c|c|}
\hline $\begin{array}{l}\text { Selective reporting (re- } \\
\text { porting bias) }\end{array}$ & Low risk & $\begin{array}{l}\text { Comment: no differences found between publication and online clinical trials } \\
\text { register }\end{array}$ \\
\hline
\end{tabular}

Other bias Unclear risk Comment: unable to assess if any other bias present

Vann 2013

\begin{tabular}{|c|c|}
\hline Methods & $\begin{array}{l}\text { Parallel RCT } \\
\text { Randomisation ratio: 1:1:1:1 } \\
\text { Superiority design }\end{array}$ \\
\hline Participants & $\begin{array}{l}\text { Inclusion criteria: age 3-18 years (sample were 4-17 years), enrolled at Healthy Lifestyle Clinic at Uni- } \\
\text { versity of South Carolina, overweight or obese (CDC growth charts), had a DVD player } \\
\text { Exclusion criteria: none } \\
\text { Diagnostic criteria: see above }\end{array}$ \\
\hline Interventions & $\begin{array}{l}\text { Number of study centres: } 1 \\
\text { Run-in period: no } \\
\text { Extension period: no } \\
\text { Intervention 1: pedometer + DVD group } \\
\text { Intervention 2: pedometer group } \\
\text { Intervention 3: fitness DVD group } \\
\text { Control: usual care }\end{array}$ \\
\hline
\end{tabular}

Outcomes Outcome measures reported in abstract: adherence, BMI

Study details Trial terminated early: no

Trial ID: -

Publication details

\section{Language of publication: English}

Funding: Richland Memorial Hospital Research and Education Foundation (non-commercial) 
Vann 2013 (Continued)

Publication status: peer-reviewed journal

Stated aim for study Quote from publication: "The primary research aims were as follows: 1) Increase physical activity of obese children and adolescents 2) Encourage at least 10,000 steps per patient daily 3) Increase awareness that physical activity can lead to improved overall health status. The ultimate goal was to determine if the use of pedometers and/or fitness DVDs will improve physical activity parameters in the Healthy Lifestyles patient population."

Notes -

\section{Risk of bias}

Bias Authors' judgement Support for judgement

Random sequence genera- Low risk tion (selection bias)
Quote from study author (via email): "Study participants were randomly assigned in equal groups to four arms: 1) control group; 2) Pedometer group; 3) Fitness DVD group; 4) Pedometer and fitness DVD"

Comment: randomisation process described

Allocation concealment Low risk
(selection bias)

Quote from study author (via email): "Yes. The persons involved in recruitment of subjects were not a part of the allocation process."

Comment: allocation was concealed

\begin{tabular}{|c|c|c|}
\hline $\begin{array}{l}\text { Blinding of participants } \\
\text { and personnel (perfor- } \\
\text { mance bias) }\end{array}$ & Unclear risk & $\begin{array}{l}\text { Quote from study author (via email): "Participants were blinded to group se- } \\
\text { lection. They only knew we were conducting a study which evaluated exercise } \\
\text { patterns in their population. So, it is technically a single blinded study." }\end{array}$ \\
\hline
\end{tabular}

Subjective outcomes

Comment: participants were blinded to group selection - not study personnel

\begin{tabular}{|c|c|c|}
\hline $\begin{array}{l}\text { Blinding of participants } \\
\text { and personnel (perfor- } \\
\text { mance bias) }\end{array}$ & Unclear risk & $\begin{array}{l}\text { Quote from study author (via email): "Participants were blinded to group se- } \\
\text { lection. They only knew we were conducting a study which evaluated exercise } \\
\text { patterns in their population. So, it is technically a single blinded study." }\end{array}$ \\
\hline
\end{tabular}

Objective outcomes

Comment: participants were blinded to group selection - not study personnel

\begin{tabular}{lll}
\hline $\begin{array}{l}\text { Blinding of outcome as- } \\
\text { sessment (detection bias) } \\
\text { Subjective outcomes }\end{array}$ & High risk & Comment: study author confirmed assessors were not blinded \\
\hline $\begin{array}{l}\text { Blinding of outcome as- } \\
\text { sessment (detection bias) } \\
\text { Objective outcomes }\end{array}$ & High risk & Comment: study author confirmed assessors were not blinded \\
\hline
\end{tabular}

Incomplete outcome data High risk (attrition bias)

Subjective outcomes

\begin{abstract}
Quote from publication: "While the participants seemed eager to participate in this study at its onset, there was a drastic drop in patient follow through as the study proceeded"
\end{abstract}

Comment: a large amount of missing data. Only 14/28 (50\%) were followed up at end of the study
Quote from publication: "While the participants seemed eager to participate in this study at its onset, there was a drastic drop in patient follow through as the study proceeded"

Comment: a large amount of missing data. Only 14/28 (50\%) were followed up at end of the study 
Vann 2013 (Continued)

Selective reporting (re- Unclear risk $\quad$ Comment: no protocol or clinical trials register entry
porting bias)

Other bias Unclear risk Comment: unclear if the study was at risk of any other bias

Wafa 2011

\begin{tabular}{ll}
\hline Methods & Parallel RCT \\
& Randomisation ratio: $1: 1$ \\
& Superiority design
\end{tabular}

Participants

Inclusion criteria: aged 7-11 years, obese (BMI > 95th percentile, CDC growth charts), at least 1 parent who perceived their child's weight status as a problem and were willing to participate in the intervention

Exclusion criteria: the child had a serious co-morbidity requiring treatment

Diagnostic criteria: see above

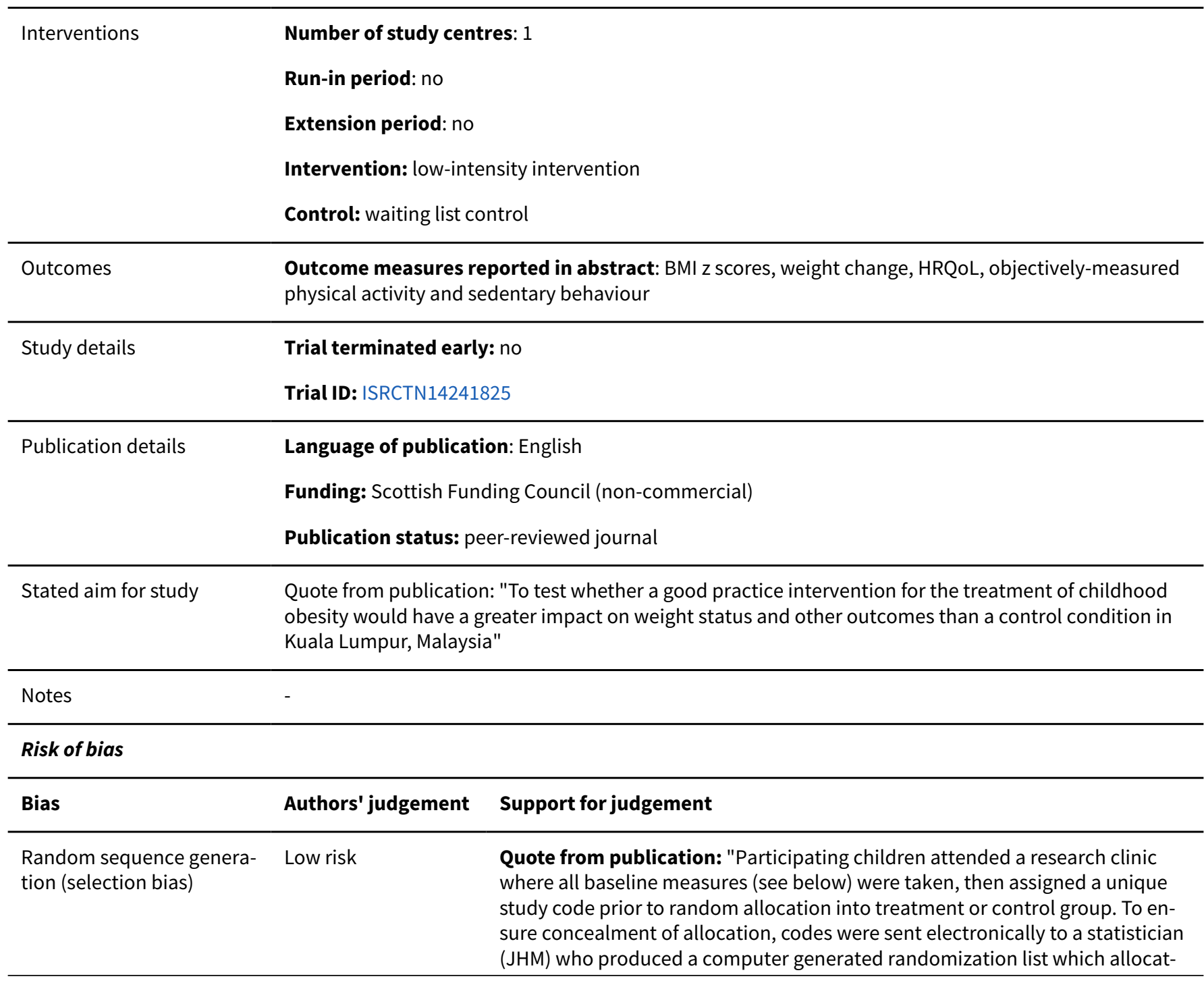

Diet, physical activity and behavioural interventions for the treatment of overweight or obese children from the age of 6 to 11 years 
Wafa 2011 (Continued)

ed participants to intervention or control group so that groups were balanced in blocks of 20. The statistician informed the researchers responsible for delivering the intervention $(\mathrm{HNH}, \mathrm{LN})$ of the allocation, and families were invited to intervention or waiting list control groups as appropriate."

Comment: randomisation process described

\begin{tabular}{|c|c|c|}
\hline $\begin{array}{l}\text { Allocation concealment } \\
\text { (selection bias) }\end{array}$ & Low risk & $\begin{array}{l}\text { Comment: allocation was concealed via the randomisation method described } \\
\text { above }\end{array}$ \\
\hline
\end{tabular}

Blinding of participants High risk and personnel (performance bias)

Subjective outcomes
Quote from study author (via email): "personnel who measured outcomes were blinded to group allocation, participating families were not (not possible/realistic we thought)"

Comment: participants and personnel were not blinded to study group

Blinding of participants High risk
and personnel (perfor-
mance bias)
Objective outcomes

Blinding of participants High risk mance bias)

Objective outcomes

Blinding of outcome as- $\quad$ Low risk
sessment (detection bias)

Subjective outcomes
Quote from study author (via email): "personnel who measured outcomes were blinded to group allocation, participating families were not (not possible/realistic we thought)"

Comment: participants and personnel were not blinded to study group
Quote from publication: "Outcome measures were made at baseline and again at six months ( $25-27$ weeks) after the start of the intervention by the same trained researcher (SWW) who was blinded to group allocation and was not involved in delivery of the treatment program

\begin{tabular}{lll}
\hline $\begin{array}{l}\text { Blinding of outcome as- } \\
\text { sessment (detection bias) }\end{array}$ & Low risk & Quote from publication: "Outcome measures were made at baseline and \\
Objective outcomes & again at six months ( $25-27$ weeks) after the start of the intervention by the \\
same trained researcher (SWW) who was blinded to group allocation and was \\
not involved in delivery of the treatment program
\end{tabular}

Comment: study author confirmed assessors were blinded

Incomplete outcome data High risk
(attrition bias)
Subjective outcomes

Subjective outcomes

Quote from publication: "Of the 107 participants entered at baseline, 80 (75\%) attended for outcome measures at the six-month follow-up."

Comment: moderate dropout rates that were higher in the intervention group

\begin{tabular}{lll}
\hline $\begin{array}{l}\text { Incomplete outcome data } \\
\text { (attrition bias) } \\
\text { Objective outcomes }\end{array}$ & High risk & $\begin{array}{l}\text { Quote from publication: "Of the 107 participants entered at baseline, 80 } \\
(75 \%) \text { attended for outcome measures at the six-month follow-up." } \\
\text { Comment: } \text { moderate dropout rates that were higher in the intervention group }\end{array}$ \\
\hline $\begin{array}{l}\text { Selective reporting (re- } \\
\text { porting bias) }\end{array}$ & Low risk & $\begin{array}{l}\text { Comment: } \text { no differences found between online clinical trial entry and publi- } \\
\text { cation }\end{array}$ \\
\hline \begin{tabular}{l} 
Other bias \\
\hline
\end{tabular} & Unclear risk & Comment: unable to assess if any other biases were present \\
\hline
\end{tabular}

Wake 2009

\begin{tabular}{ll}
\hline Methods & Parallel RCT \\
& Randomisation ratio: $1: 1$ \\
Superiority design
\end{tabular}

Diet, physical activity and behavioural interventions for the treatment of overweight or obese children from the age of 6 to 11 years 170 (Review)

Copyright @ 2017 The Cochrane Collaboration. Published by John Wiley \& Sons, Ltd. 
Wake 2009 (Continued)

Participants
Inclusion criteria: age 5 years-10th birthday, attending participating practices between May 2005-July 2006, not receiving an ongoing weight management programme, overweight or obese (IOTF cut points)

Exclusion criteria: BMI z score was $\geq 3.0$

Diagnostic criteria: see above

\section{Outcomes \\ Outcome measures reported in abstract: attrition, BMI, BMI z scores, physical activity (accelerome- try), nutrition scores (diary), harm, costs}

Control: no-care control group

\section{Number of study centres: 45}

Run-in period: no

Extension period: no

Intervention: LEAP2 behavioural intervention
Study details

\section{Trial terminated early: no}

Trial ID: ISRCTN52511065
Language of publication: English

Funding: Australian National Health and Medical Research Council (NH\&MRC) Project Grant 334309. M Wake is supported by NH\&MRC Career Development Award 284556; L Gold by NH\&MRC Capacity Building Grant 425855; and OC Ukoumunne by NH\&MRC Capacity Building Grant 436914

(non-commercial)

Publication status: peer-reviewed journal
Quote from publication: "To determine whether ascertainment of childhood obesity by surveillance followed by structured secondary prevention in primary care improved outcomes in overweight or mildly obese children."

\begin{tabular}{|c|c|c|}
\hline Notes & - & \\
\hline \multicolumn{3}{|l|}{ Risk of bias } \\
\hline Bias & Authors' judgement & Support for judgement \\
\hline \multirow[t]{2}{*}{$\begin{array}{l}\text { Random sequence genera- } \\
\text { tion (selection bias) }\end{array}$} & Low risk & $\begin{array}{l}\text { Quote from publication: "Randomisation by child was stratified by GP and by } \\
\text { overweight versus obese status; it was performed by an independent biostatis- } \\
\text { tician using computer generated random numbers." }\end{array}$ \\
\hline & & Comment: randomisation process described \\
\hline \multirow[t]{2}{*}{$\begin{array}{l}\text { Allocation concealment } \\
\text { (selection bias) }\end{array}$} & Low risk & $\begin{array}{l}\text { Quote from publication: "The randomisation sequence was concealed from } \\
\text { the study investigators, and the researchers collecting data remained blind to } \\
\text { participants' trial status until follow-up was complete." }\end{array}$ \\
\hline & & Comment: allocation was concealed \\
\hline $\begin{array}{l}\text { Blinding of participants } \\
\text { and personnel (perfor- } \\
\text { mance bias) }\end{array}$ & High risk & $\begin{array}{l}\text { Quote from study author (via email): "Randomisation and outcomes mea- } \\
\text { surement, but not participants, were blinded to group assignment" }\end{array}$ \\
\hline Subjective outcomes & & Comment: participants were not blinded to study group \\
\hline
\end{tabular}

Diet, physical activity and behavioural interventions for the treatment of overweight or obese children from the age of 6 to 11 years 
Wake 2009 (Continued)

Blinding of participants High risk Quote from study author (via email): "Randomisation and outcomes meaand personnel (perforsurement, but not participants, were blinded to group assignment" mance bias) Objective outcomes

Comment: participants were not blinded to study group Blinding of outcome as- $\quad$ Low risk
sessment (detection bias)

Subjective outcomes
Quote from publication: "Randomisation and outcomes measurement, but not participants, were blinded to group assignment"

Comment: assessment staff were blinded to study group

\begin{tabular}{|c|c|c|}
\hline $\begin{array}{l}\text { Blinding of outcome as- } \\
\text { sessment (detection bias) }\end{array}$ & Low risk & $\begin{array}{l}\text { Quote from publication: "Randomisation and outcomes measurement, but } \\
\text { not participants, were blinded to group assignment" }\end{array}$ \\
\hline
\end{tabular}

sessment (detection bias)

Comment: assessment staff were blinded to study group

\begin{tabular}{|c|c|c|}
\hline $\begin{array}{l}\text { Incomplete outcome data } \\
\text { (attrition bias) } \\
\text { Subjective outcomes }\end{array}$ & Low risk & $\begin{array}{l}\text { Quote from publication: "attrition was } 3.1 \% \text { at } 6 \text { months and } 6.2 \% \text { at } 12 \\
\text { months." } \\
\text { Comment: attrition rates were fairly low for } 12 \text { months' follow-up }\end{array}$ \\
\hline $\begin{array}{l}\text { Incomplete outcome data } \\
\text { (attrition bias) } \\
\text { Objective outcomes }\end{array}$ & Low risk & $\begin{array}{l}\text { Quote from publication: "attrition was } 3.1 \% \text { at } 6 \text { months and } 6.2 \% \text { at } 12 \\
\text { months." } \\
\text { Comment: attrition rates were fairly low for } 12 \text { months' follow-up }\end{array}$ \\
\hline $\begin{array}{l}\text { Selective reporting (re- } \\
\text { porting bias) }\end{array}$ & Unclear risk & $\begin{array}{l}\text { Comment: clinical trials register entry was retrospectively entered so difficult } \\
\text { to assess reporting bias }\end{array}$ \\
\hline Other bias & Low risk & $\begin{array}{l}\text { Comment: no other bias identified - study generally of low risk of bias in other } \\
\text { domains }\end{array}$ \\
\hline
\end{tabular}

\section{Wake 2013}

\begin{tabular}{ll}
\hline Methods & Parallel RCT \\
& Randomisation ratio: 1:1 \\
& Superiority design \\
\hline Participants & $\begin{array}{l}\text { Inclusion criteria: aged 3-11 (but not including their 11th birthday), obese (BMI } \geq 95 \text { th percentile, CDC } \\
\text { growth charts) } \\
\text { Exclusion criteria: receiving ongoing weight management in a secondary or tertiary care programme, } \\
\text { known endocrine or genetic cause for their obesity, major disability or health conditions precluding } \\
\text { participation, family did not speak English sufficiently enough to complete questionnaires and partici- } \\
\text { pate in the study } \\
\text { Diagnostic criteria: see above }\end{array}$ \\
\hline
\end{tabular}

Interventions

\section{Number of study centres: 22}

Run-in period: no

Extension period: no

Intervention: HopSCOTCH (the shared care obesity trial) intervention

Control: usual care 
Wake 2013 (Continued)

Outcomes

Outcome measures reported in abstract: attrition, attendance, BMI, BMI z scores, benefit or harm on secondary outcomes

Study details

Trial terminated early: no

Trial ID: ACTRN12608000055303

$\begin{array}{ll}\text { Publication details } & \text { Language of publication: English } \\ & \text { Funding: the Australian National Health and Medical Research Council (NHMRC Project Grant 491212) } \\ \text { (non-commercial) }\end{array}$

Publication status: peer-reviewed journal

Stated aim for study Quote from publication: "The aim of the HopSCOTCH trial is to develop, implement and trial an innova-
tive shared-care approach to manage childhood obesity."

Notes -

\section{Risk of bias}

Bias Authors' judgement Support for judgement

Random sequence genera- Low risk

Quote from publication: "Randomisation occurred via a concealed, computtion (selection bias) erised random number sequence stratified by general practitioner and pregenerated by the Clinical Epidemiology and Biostatistics Unit at the Royal Children's Hospital. Once enrolled (i.e. on receipt of written informed consent and baseline questionnaire) a research assistant, who was not otherwise involved with the trial, randomised children to either the shared-care or usual-care arm."

Comment: randomisation process described

\begin{tabular}{ll}
\hline $\begin{array}{l}\text { Allocation concealment } \\
\text { (selection bias) }\end{array}$ & Low risk \\
& Quote from publication: "All families were advised of their child's allocation
\end{tabular}

Comment: it was likely that allocation was concealed

\begin{tabular}{|c|c|c|}
\hline $\begin{array}{l}\text { Blinding of participants } \\
\text { and personnel (perfor- } \\
\text { mance bias) } \\
\text { Subjective outcomes }\end{array}$ & High risk & $\begin{array}{l}\text { Quote from publication: "Researchers collecting outcome measurements, } \\
\text { but not participants, were blinded to group assignment." } \\
\text { Comment: participants were not blinded to study group }\end{array}$ \\
\hline $\begin{array}{l}\text { Blinding of participants } \\
\text { and personnel (perfor- } \\
\text { mance bias) } \\
\text { Objective outcomes }\end{array}$ & High risk & $\begin{array}{l}\text { Quote from publication: "Researchers collecting outcome measurements, } \\
\text { but not participants, were blinded to group assignment." } \\
\text { Comment: participants were not blinded to study group }\end{array}$ \\
\hline $\begin{array}{l}\text { Blinding of outcome as- } \\
\text { sessment (detection bias) } \\
\text { Subjective outcomes }\end{array}$ & Low risk & $\begin{array}{l}\text { Quote from publication: "Researchers collecting outcome measurements, } \\
\text { but not participants, were blinded to group assignment." } \\
\text { Comment: assessment staff were blinded to study group }\end{array}$ \\
\hline $\begin{array}{l}\text { Blinding of outcome as- } \\
\text { sessment (detection bias) } \\
\text { Objective outcomes }\end{array}$ & Low risk & $\begin{array}{l}\text { Quote from publication: "Researchers collecting outcome measurements, } \\
\text { but not participants, were blinded to group assignment." } \\
\text { Comment: assessment staff were blinded to study group }\end{array}$ \\
\hline $\begin{array}{l}\text { Incomplete outcome data } \\
\text { (attrition bias) }\end{array}$ & Low risk & $\begin{array}{l}\text { Quote from publication: "Figure } 2 \text { shows that, of the } 118 \text { eligible children en- } \\
\text { rolled who provided baseline data, } 107(91 \%) \text { contributed outcome data." }\end{array}$ \\
\hline
\end{tabular}

Diet, physical activity and behavioural interventions for the treatment of overweight or obese children from the age of 6 to 11 years 
Wake 2013 (Continued)

Subjective outcomes
Comment: attrition rates were fairly low at follow-up

\begin{tabular}{lll}
\hline $\begin{array}{l}\text { Incomplete outcome data } \\
\text { (attrition bias) } \\
\text { Objective outcomes }\end{array}$ & Low risk & $\begin{array}{l}\text { Quote from publication: "Figure 2 shows that, of the 118 eligible children en- } \\
\text { rolled who provided baseline data, } 107(91 \%) \text { contributed outcome data." }\end{array}$ \\
\hline $\begin{array}{l}\text { Selective reporting (re- } \\
\text { porting bias) }\end{array}$ & Low risk & $\begin{array}{l}\text { Comment: } \text { attrition rates were fairly low at follow-up } \\
\text { als register entry }\end{array}$ \\
\hline Other bias & Low risk & $\begin{array}{l}\text { Comment: } \text { no other bias identified - study generally of low risk of bias in other } \\
\text { domains }\end{array}$ \\
\hline
\end{tabular}

Waling 2012

\begin{tabular}{ll} 
Methods & Parallel RCT \\
& Randomisation ratio: $1: 1$ \\
& Superiority design \\
\hline
\end{tabular}

Participants Inclusion criteria: age- and gender-adjusted BMI $\geq 25 \mathrm{~kg} / \mathrm{m} 2$ (Cole 2000, international survey), born between 1995 and 1998, live in or nearby the city of Umea

Exclusion criteria: chronic diseases that could influence metabolic parameters, attention deficit disorders, lack of access to internet

Diagnostic criteria: see above

\begin{tabular}{ll}
\hline Interventions & Number of study centres: 1 \\
Run-in period: no \\
Extension period: no \\
Intervention: family-based intervention \\
Control: usual care control group
\end{tabular}

Outcomes Outcome measures reported in abstract: BMI z scores, WC, waist/hip ratio, apo A/apo B ratio, physical activity level, steps/d, screen time, energy expenditure, time spent at > $3 \mathrm{MET}$, energy intake, refined sugar, dietary fibre, saturated fatty acids

Study details Trial terminated early: no

Trial ID: NCT01012206

Publication details Language of publication: English

Funding: Vardal Foundation for Healthcare Sciences and Allergy Research; the Swedish Research Council for Environment, Agricultural Sciences and Spatial Planning; the Swedish Research Council; the Medical Faculty and the Faculty of Social Sciences at Umeå University; Västerbotten County Council; Dr PersFood AB; Majblommans Riksforbund, the Magnus Bergvall Foundation; Jamtland Council Research Unit (commercial and non-commercial)

Publication status: peer-reviewed journal

\footnotetext{
Stated aim for study

Quote from publication: "To evaluate the effect of a family-based intervention on anthropometric and metabolic markers in overweight and obese children."
} 
Waling 2012 (Continued)

Notes

\section{Risk of bias}

\begin{tabular}{lll}
\hline Bias & Authors' judgement & Support for judgement \\
\hline $\begin{array}{ll}\text { Random sequence genera- } \\
\text { tion (selection bias) }\end{array}$ & Low risk & $\begin{array}{l}\text { Quote from publication: "The children were consecutively randomised (1: 1) } \\
\text { and stratified by gender into either an intervention group or a control group by } \\
\text { the researchers." }\end{array}$
\end{tabular}

Comment: randomisation process described

\begin{tabular}{lll}
\hline $\begin{array}{l}\text { Allocation concealment } \\
\text { (selection bias) }\end{array}$ & Unclear risk & Comment: author of study was unclear if allocation was concealed \\
\hline $\begin{array}{l}\text { Blinding of participants } \\
\text { and personnel (perfor- } \\
\text { mance bias) }\end{array}$ & High risk & $\begin{array}{l}\text { Quote from publication: "Neither the researchers nor the participants were } \\
\text { blinded." }\end{array}$ \\
$\begin{array}{l}\text { Subjective outcomes } \\
\text { Comment: participants and study personnel were not blinded to study group }\end{array}$ \\
$\begin{array}{l}\text { Blinding of participants } \\
\text { and personnel (perfor- } \\
\text { mance bias) }\end{array}$ & High risk & $\begin{array}{l}\text { Quote from publication: "Neither the researchers nor the participants were } \\
\text { blinded." }\end{array}$ \\
Objective outcomes & Comment: participants and study personnel were not blinded to study group
\end{tabular}

Blinding of outcome as- Unclear risk

sessment (detection bias)

Subjective outcomes

Quote from publication: "The nurse did not receive information about the allocation group of the child, but blindedness cannot be assured"

Comment: unclear if assessment staff were blinded to study group

Blinding of outcome as- Unclear risk

sessment (detection bias)

Objective outcomes
Quote from publication: "The nurse did not receive information about the allocation group of the child, but blindedness cannot be assured"

Comment: unclear if assessment staff were blinded to study group
Incomplete outcome data High risk

(attrition bias)

Subjective outcomes
Quote from publication: "From baseline to the 1-year measurement, $42 \%$ of the children in the intervention group and $33 \%$ of the children in the control group dropped out (Figure 1), which left 58 children who had completed the 1year measurement"

Comment: attrition rates were quite high
Incomplete outcome data High risk

(attrition bias)

Objective outcomes
Quote from publication: "From baseline to the 1-year measurement, $42 \%$ of the children in the intervention group and $33 \%$ of the children in the control group dropped out (Figure 1), which left 58 children who had completed the 1year measurement"

Comment: attrition rates were quite high

Comment: no differences between publications and clinical trials register entry observed

\begin{tabular}{|c|c|c|}
\hline $\begin{array}{l}\text { Selective reporting (re- } \\
\text { porting bias) }\end{array}$ & Low risk & $\begin{array}{l}\text { Comment: no differences between publications and clinical trials register en- } \\
\text { try observed }\end{array}$ \\
\hline
\end{tabular}

Other bias Unclear risk Comment: unclear if the study was at risk of any other bias

\section{Warschburger 2016}

Methods Parallel RCT


Warschburger 2016 (Continued)

\section{Randomisation ratio: $1: 1$ \\ Superiority design}

Participants

Inclusion criteria: children aged 7-12 years (extended to 13 years due to recruitment problems), obese children: $\mathrm{BMI}>$ 97th percentile (Kromeyer-Hauschild 2001 - German references), parent participation at the beginning of their child's inpatient stay,

Exclusion criteria: parents who had already done parent training, parents with inadequate language skills or severe mental disorders, children had secondary causes of obesity or suffering from severe mental health problems

Diagnostic criteria: see above

Number of study centres: 1
Run-in period: no
Extension period: no
Intervention: parental CBT training group plus child inpatient intervention
Control: parental information-only group plus child inpatient intervention

Outcomes Outcome measures reported in abstract: BMI SDS, QoL, healthy food intake, exercise

Study details

Trial terminated early: no

Trial ID: ISRCTN24655766

\begin{tabular}{ll}
\hline Publication details & Language of publication: English \\
Funding: DFG (German Research Foundation) grant (WA 1143/4-1; 4-2) (non-commercial) \\
Publication status: peer-reviewed journal
\end{tabular}

Stated aim for study

Quote from publication: "The main goal was to develop a brief behaviourally oriented parent training program that enhances 'obesity-specific' parenting skills in order to prevent relapse"

Notes -

\section{Risk of bias}

\begin{tabular}{lll}
\hline Bias & Authors' judgement & Support for judgement \\
\hline $\begin{array}{l}\text { Random sequence genera- } \\
\text { tion (selection bias) }\end{array}$ & Low risk & $\begin{array}{l}\text { Quote from publication: "A stratified (gender, age groups (7-10 or11-13 } \\
\text { years), and clinic) and blocked (block size } 8 \text { ) computerized randomization was } \\
\text { performed centrally at the Institute of Medical Epidemiology, Biometry and In- } \\
\text { formatics at the University Halle-Wittenberg, which sent the results of the ran- } \\
\text { domization by fax to the study centers within one day." }\end{array}$
\end{tabular}

Comment: randomisation process described

\begin{tabular}{ll}
\hline $\begin{array}{l}\text { Allocation concealment } \\
\text { (selection bias) }\end{array}$ & Low risk
\end{tabular}$\quad \begin{aligned} & \text { Comment: the randomisation method described was unlikely to introduce se- } \\
& \text { lection bias. Author confirmed allocation was concealed }\end{aligned}$

Blinding of participants $\quad$ High risk Comment: unlikely participants were blinded as some crossed over
and personnel (perfor-
mance bias)
Subjective outcomes

Diet, physical activity and behavioural interventions for the treatment of overweight or obese children from the age of 6 to 11 years 
Warschburger 2016 (Continued)
Blinding of participants
High risk
Comment: unlikely participants were blinded as some crossed over and personnel (perfor-

mance bias)

Objective outcomes

Blinding of outcome as- $\quad$ Low risk
sessment (detection bias)
Subjective outcomes

Subjective outcomes
Low risk

Blinding of outcome as-

sessment (detection bias)

Objective outcomes
Quote from publication: "At the follow-ups, children were asked to visit their physicians, who were blind to trial-group assignment and the study goals."

Comment: assessment staff were blinded to study group

\begin{tabular}{ll}
\hline $\begin{array}{l}\text { Incomplete outcome data } \\
\text { (attrition bias) }\end{array}$ & High risk \\
$\begin{array}{l}\text { Subjective outcomes } \\
\text { Quote from publication: "Limitations include the relatively high attrition rate, } \\
\text { which might have caused a sample bias for the follow-up analyses." }\end{array}$ \\
Comment: attrition rates were quite high
\end{tabular}

Quote from publication: "At the follow-ups, children were asked to visit their physicians, who were blind to trial-group assignment and the study goals."

Comment: assessment staff were blinded to study group

\begin{tabular}{ll}
\hline $\begin{array}{l}\text { Incomplete outcome data } \\
\text { (attrition bias) }\end{array}$ & High risk \\
$\begin{array}{l}\text { Objective outcomes } \\
\text { Quote from publication: "Limitations include the relatively high attrition rate, } \\
\text { which might have caused a sample bias for the follow-up analyses." }\end{array}$ \\
Comment: attrition rates were quite high
\end{tabular}

\begin{tabular}{lll}
\hline $\begin{array}{l}\text { Selective reporting (re- } \\
\text { porting bias) }\end{array}$ & Unclear risk & Comment: clinical trials register entry retrospectively entered \\
\hline Other bias & Unclear risk & Comment: unclear if the study was at risk of any other bias \\
\hline
\end{tabular}

\begin{tabular}{|c|c|}
\hline \multirow[t]{3}{*}{ Methods } & Parallel RCT \\
\hline & Randomisation ratio: $1: 1$ \\
\hline & Superiority design \\
\hline \multirow[t]{3}{*}{ Participants } & $\begin{array}{l}\text { Inclusion criteria: aged 7-15 years, obese (> 97th percentile, according to European Childhood Obesity } \\
\text { Group and the German Working Group on Pediatric Obesity) }\end{array}$ \\
\hline & Exclusion criteria: none \\
\hline & Diagnostic criteria: see above \\
\hline \multirow[t]{5}{*}{ Interventions } & Number of study centres: 1 \\
\hline & Run-in period: no \\
\hline & Extension period: no \\
\hline & Intervention: active intervention group \\
\hline & Control: usual care control group \\
\hline
\end{tabular}

Outcomes Outcome measures reported in abstract: BMI z score, BMI, fat mass, SBP

Study details Trial terminated early: no

Diet, physical activity and behavioural interventions for the treatment of overweight or obese children from the age of 6 to 11 years 
Weigel 2008 (Continued)

Trial ID: -

\section{Publication details Language of publication: English}

Funding: Bavarian State Ministry of Environment, Public Health, and Consumer Protection and the health insurance company SBK, Germany. The "Sea Lion Club" was financed by health insurance companies and by membership fees from the parents (commercial and non-commercial)

Publication status: peer-reviewed journal

Stated aim for study

Quote from publication: "The authors performed a group-based program for obese children and adolescents in Bavaria, Germany to enable them to establish a health-oriented lifestyle and to reduce overweight. The authors compared this program with a control approach based on the patients' own initiative."

Notes -

\section{Risk of bias}

\begin{tabular}{lll}
\hline Bias & Authors' judgement & Support for judgement \\
\hline $\begin{array}{l}\text { Random sequence genera- } \\
\text { tion (selection bias) }\end{array}$ & Unclear risk & Comment: randomisation process not described \\
\hline $\begin{array}{l}\text { Allocation concealment } \\
\text { (selection bias) }\end{array}$ & Unclear risk & Comment: unclear if allocation was concealed \\
\hline $\begin{array}{l}\text { Blinding of participants } \\
\text { and personnel (perfor- } \\
\text { mance bias) } \\
\text { Objective outcomes }\end{array}$ & Unclear risk & $\begin{array}{l}\text { Comment: unclear if participants and study personnel were blinded to study } \\
\text { group }\end{array}$ \\
\hline
\end{tabular}

Blinding of outcome as-
sessment (detection bias) $\quad$ Unclear risk $\quad$ Comment: unclear if assessment staff were blinded to study group
sessment (detection bias)

Objective outcomes

\section{Incomplete outcome data Low risk} (attrition bias)

Objective outcomes

Quote from publication: "Generally, $83 \%$ to $100 \%$ of participants attended each session, and there was only 1 dropout in the "Sea Lion Club." "Conversely, in the control group, 6 children were lost to follow up despite telephone calls, and none joined the local sports club as offered 12 months after their first visit."

Comment: dropout rate very low in intervention group and low in control group

\begin{tabular}{lll}
\hline $\begin{array}{l}\text { Selective reporting (re- } \\
\text { porting bias) }\end{array}$ & Unclear risk & Comment: no protocol or clinical trials register entry available \\
\hline Other bias & Unclear risk & Comment: unclear if the study was at risk of any other bias \\
\hline
\end{tabular}

Weintraub 2008

\begin{tabular}{ll}
\hline Methods & Parallel RCT \\
& Randomisation ratio: $1: 1$ \\
& Superiority design
\end{tabular}

Diet, physical activity and behavioural interventions for the treatment of overweight or obese children from the age of 6 to 11 years (Review)

Copyright (c) 2017 The Cochrane Collaboration. Published by John Wiley \& Sons, Ltd. 
Weintraub 2008 (Continued)

Participants
Inclusion criteria: BMI percentile $\geq 85$ th percentile for age and sex (CDC growth charts), in grades 4 and 5 in a low-income community in northern California

Exclusion criteria: had a medical condition or were taking medications which affected growth, had conditions which limited their participation in the study

Diagnostic criteria: see above

\begin{tabular}{ll}
\hline Outcomes & $\begin{array}{l}\text { Outcome measures reported in abstract: completion rates, BMI z scores, total physical activity, mod- } \\
\text { erate physical activity, vigorous physical activity }\end{array}$
\end{tabular}

Number of study centres: 1

Run-in period: no

Extension period: no

Intervention: after-school team sports programme

Control: "active placebo" control erate physical activity, vigorous physical activity

\begin{tabular}{ll}
\hline Study details & Trial terminated early: no \\
& Trial ID: NCT00186173 \\
\hline Publication details & $\begin{array}{l}\text { Language of publication: English } \\
\text { Funding: co-operative agreement from the CDC through the Association of American Medical Colleges } \\
\text { (grants U36/CCU319276 and AAMCID MM-0851-05/05) (non-commercial) }\end{array}$
\end{tabular}

Publication status: peer-reviewed journal

\begin{tabular}{ll}
\hline Stated aim for study & $\begin{array}{l}\text { Quote from publication: "To evaluate the feasibility, acceptability, and efficacy of an after-school team } \\
\text { sports program for reducing weight gain in low-income overweight children." }\end{array}$ \\
\hline Notes & -
\end{tabular}

\section{Risk of bias}

\begin{tabular}{lll}
\hline Bias & Authors' judgement & Support for judgement \\
\hline $\begin{array}{ll}\text { Random sequence genera- } \\
\text { tion (selection bias) }\end{array}$ & Low risk & $\begin{array}{l}\text { Quote from publication: "After completing baseline assessments, children } \\
\text { were randomized using a computer by the Database Manager (K.F.H.) to either } \\
\text { an after-school team sports program or a traditional nutrition and health edu- } \\
\text { cation program." }\end{array}$
\end{tabular}

Comment: randomisation process described

\begin{tabular}{ll}
\hline $\begin{array}{l}\text { Allocation concealment } \\
\text { (selection bias) }\end{array}$ & Low risk
\end{tabular}

Comment: allocation was concealed

\begin{tabular}{lll}
\hline $\begin{array}{l}\text { Blinding of participants } \\
\text { and personnel (perfor- }\end{array}$ & Unclear risk & Comment: unclear if participants and personnel were blinded \\
mance bias) & \\
Subjective outcomes & \\
\hline $\begin{array}{l}\text { Blinding of participants } \\
\text { and personnel (perfor- } \\
\text { mance bias) }\end{array}$ & Unclear risk & Comment: unclear if participants and personnel were blinded
\end{tabular}

Diet, physical activity and behavioural interventions for the treatment of overweight or obese children from the age of 6 to 11 years 
Weintraub 2008 (Continued)

Objective outcomes

\begin{tabular}{lll}
\hline $\begin{array}{l}\text { Blinding of outcome as- } \\
\text { sessment (detection bias) }\end{array}$ & High risk & $\begin{array}{l}\text { Quote from publication: "Owing to limited staffing for this pilot study, data } \\
\text { collectors were not blinded at follow-up assessments" }\end{array}$
\end{tabular}

Subjective outcomes

Comment: data collectors were not blinded to study group

Blinding of outcome as- $\quad$ High risk
sessment (detection bias)

Quote from publication: "Owing to limited staffing for this pilot study, data

Objective outcomes collectors were not blinded at follow-up assessments"

Comment: data collectors were not blinded to study group

$\begin{array}{ll}\begin{array}{l}\text { Incomplete outcome data } \\ \text { (attrition bias) }\end{array} & \text { Qow risk } \\ \text { Subjective outcomes } & \text { Comment: no missing data }\end{array}$

\begin{tabular}{lll}
\hline $\begin{array}{l}\text { Incomplete outcome data } \\
\text { (attrition bias) } \\
\text { Objective outcomes }\end{array}$ & Low risk & Quote from publication: "No participants were lost to follow-up" \\
Comment: no missing data
\end{tabular}

Other bias Unclear risk Comment: unclear if the study was at risk of any other bias

Wilfley 2007

\begin{tabular}{ll}
\hline Methods & Parallel RCT \\
& Randomisation ratio: 1:1:1 \\
& Superiority design
\end{tabular}

Participants

Inclusion criteria: children aged 7-12 years who were 20\%-100\% overweight (CDC growth charts) and had at least 1 parent with $\mathrm{BMI}>25$

Exclusion criteria: families were excluded if either the child or parent was currently involved in psychological or weight loss treatment, was using appetite- or weight-affecting medications, or had a psychiatric condition (e.g. eating disorder, psychosis) that would interfere with participation

Diagnostic criteria: see above

Interventions Number of study centres: 1

Run-in period: no

Extension period: no

Treatment before study : all participants received a weight-loss treatment focusing on dietary modification, physical activity increases and behaviour change skills ( 5 months' weight-loss treatment prior to randomisation)

Intervention 1: behavioural skills maintenance group

Intervention 2: social facilitation maintenance group

Control: no-care control group 
Wilfley 2007 (Continued)

Outcomes

\section{Study details}

Publication details

Outcome measures reported in abstract: BMI z scores, percentage overweight, weight, attendance, parental weight change, parent BMI, behaviour problems, adherence

\section{Trial terminated early: no}

Trial ID: NCT00301197

Funding: Grant 5R01HD36904-5 from the National Institute of Child Health and Human Development (NICHD; grant 1K24MH070446-01 from the National Institute of Mental Health (Dr Wilfley); and grant 1K23DK060476-01 from the National Institute of Diabetes and Digestive and Kidney Diseases (Dr Saelens) (non-commercial)

Publication status: peer-reviewed journal

Stated aim for study

Quote from publication: "To determine the short-term and long-term efficacy of 2 distinct weight maintenance approaches vs no continued treatment control following standard family based behavioral weight loss treatment for childhood overweight, and to examine children's social functioning as a moderator of outcome."

\section{Notes}

\section{Risk of bias}

Bias Authors' judgement Support for judgement

Random sequence genera- Low risk tion (selection bias)
Quote from publication: "Random assignment was conducted by using computer-generated random numbers."

Comment: randomisation method described

Allocation concealment Unclear risk Comment: unclear if allocation was concealed
(selection bias)

Blinding of participants Unclear risk Comment: unclear if participants and personnel were blinded
and personnel (performance bias)

Subjective outcomes

Blinding of participants Unclear risk Comment: unclear if participants and personnel were blinded
and personnel (perfor-
mance bias)
Objective outcomes

\begin{tabular}{lll}
\hline $\begin{array}{l}\text { Blinding of outcome as- } \\
\begin{array}{l}\text { sessment (detection bias) } \\
\text { Subjective outcomes }\end{array}\end{array}$ & High risk & $\begin{array}{l}\text { Quote from publication: "It was not possible to keep assessors blind to treat- } \\
\text { ment condition" }\end{array}$ \\
\hline $\begin{array}{l}\text { Blinding of outcome as- } \\
\text { Sessment (detection bias) } \\
\text { Objective outcomes }\end{array}$ & High risk & $\begin{array}{l}\text { Quote from publication: "It was not possible to keep assessors blind to treat- } \\
\text { ment condition" }\end{array}$ \\
\hline $\begin{array}{l}\text { Incomplete outcome data } \\
\text { (attrition bias) } \\
\text { Subjective outcomes }\end{array}$ & Low risk & Comment: assessment staff were not blinded to study group \\
\hline
\end{tabular}


Wilfley 2007 (Continued)

Incomplete outcome data Low risk Comment: 42/51 completed behavioural intervention, 43/50 completed social (attrition bias) group and $37 / 49$ completed control group (total $81.3 \%$ retention)

Objective outcomes

\begin{tabular}{lll}
\hline $\begin{array}{l}\text { Selective reporting (re- } \\
\text { porting bias) }\end{array}$ & Low risk & $\begin{array}{l}\text { Comment: no differences found between publication and clinical trial register } \\
\text { entry }\end{array}$ \\
\hline Other bias & Unclear risk & Comment: unable to assess whether any other biases were present
\end{tabular}

Woo 2004

$\begin{array}{ll}\text { Methods } & \text { Parallel RCT } \\ \text { Randomisation ratio: } 1: 1 \text { (but } 2 \text { initial arms become } 3 \text { arms after } 6 \text { weeks) } \\ \text { Superiority design }\end{array}$

Participants

Inclusion criteria: children aged 9-12 years, BMI $\geq 21 \mathrm{~kg} / \mathrm{m} 2$ (CDC growth charts), no known medical illness and no alternative cause of obesity, no family history of premature cardiovascular disease, not taking regular medications or vitamin supplementation, resting brachial artery diameter $>2.55 \mathrm{~mm}$

Exclusion criteria: history of diabetes, renal disease or cardiovascular disease, sexual maturity status was more advanced than Tanner stage 2

Diagnostic criteria: see above

\begin{tabular}{|c|c|}
\hline Interventions & $\begin{array}{l}\text { Number of study centres: } 1 \\
\text { Run-in period: no } \\
\text { Extension period: none } \\
\text { Intervention 1: diet plus supervised structured exercise programme with continuing training } \\
\text { Intervention 2: diet plus supervised structured exercise programme with detraining } \\
\text { Control: diet modification only }\end{array}$ \\
\hline Outcomes & $\begin{array}{l}\text { Outcome measures reported in abstract: waist-to-hip-ratio, cholesterol, arterial endothelial function, } \\
\text { carotid wall thickening, body fat, lipid profiles, vascular function }\end{array}$ \\
\hline Study details & $\begin{array}{l}\text { Trial terminated early: no } \\
\text { Trial ID: - }\end{array}$ \\
\hline Publication details & $\begin{array}{l}\text { Language of publication: English } \\
\text { Funding: Hong Kong Institute of Heart Health Promotion, the Shaw Foundation, and the Research } \\
\text { Grant Council of Hong Kong (CUHK4060/2000M) (non-commercial) } \\
\text { Publication status: peer-reviewed journal }\end{array}$ \\
\hline Stated aim for study & $\begin{array}{l}\text { Quote from publication: "To assess the reversibility of such early arterial damage in children, we stud- } \\
\text { ied obese children before and after random assignment to an intervention program of diet alone or di- } \\
\text { et with exercise training to define potentially effective strategies to improve obesity-related vascular } \\
\text { abnormalities." }\end{array}$ \\
\hline Notes & - \\
\hline
\end{tabular}

Diet, physical activity and behavioural interventions for the treatment of overweight or obese children from the age of 6 to 11 years 
Woo 2004 (Continued)

\section{Risk of bias}

\begin{tabular}{lll}
\hline Bias & Authors' judgement & Support for judgement \\
\hline $\begin{array}{l}\text { Random sequence genera- } \\
\text { tion (selection bias) }\end{array}$ & Low risk & $\begin{array}{l}\text { Comment: study author confirmed via email that randomisation was done via } \\
\text { a computer - likely no selection bias }\end{array}$
\end{tabular}

Allocation concealment Low risk

Comment: study author confirmed via email that allocation was concealed (selection bias)

Blinding of participants High risk and personnel (performance bias)

Subjective outcomes
Quote from publication: "Both groups (children and parents) participated in the same diet education program and were interviewed by the same dietitian, who was blinded to the exercise program allocation"

Comment: dietitian blinded but author confirmed participants and study personnel were not

\section{Blinding of participants High risk} and personnel (perfor-

mance bias)

Objective outcomes
Quote from publication: "Both groups (children and parents) participated in the same diet education program and were interviewed by the same dietitian, who was blinded to the exercise program allocation"

Comment: dietitian blinded but author confirmed participants and study personnel were not
Blinding of outcome as- Low risk sessment (detection bias)

Subjective outcomes
Quote from publication: "All ultrasound-derived vascular functions were measured by a blinded investigator, and the high reproducibility between serial observations and in control subjects over time have been documented by us previously."

Comment: study author confirmed all outcome investigators were blinded to study group

Blinding of outcome as- Low risk sessment (detection bias)

Objective outcomes
Quote from publication: "All ultrasound-derived vascular functions were measured by a blinded investigator, and the high reproducibility between serial observations and in control subjects over time have been documented by us previously."

Comment: study author confirmed all outcome investigators were blinded to study group

Incomplete outcome data Unclear risk

Comment: unclear how many dropouts there were and how they were treated

(attrition bias)

Subjective outcomes

\begin{tabular}{lll}
$\begin{array}{l}\text { Incomplete outcome data } \\
\text { (attrition bias) } \\
\text { Objective outcomes }\end{array}$ & Unclear risk & Comment: unclear how many dropouts there were and how they were treated \\
\hline $\begin{array}{l}\text { Selective reporting (re- } \\
\text { porting bias) }\end{array}$ & Unclear risk & Comment: no protocol or clinical trials register entry available \\
\hline Other bias & High risk & $\begin{array}{l}\text { Comment: outcomes reported in this refer to } 3 \text { arms that were randomised in- } \\
\text { to } 2 \text { arms originally, then } 1 \text { arm is split (non-randomly) }\end{array}$
\end{tabular}

\section{Wright 2012}


Wright 2012 (Continued)

Randomisation ratio: $2: 3$

Superiority design

Participants

Inclusion criteria: 8-12 years, English or Spanish speaking, BMI $\geq 85$ th percentile (CDC growth charts), had no physical limitations that prevented regular exercise

Exclusion criteria: none

Diagnostic criteria: see above

\begin{tabular}{ll}
\hline Interventions & Number of study centres: 5 \\
& Run-in period: no \\
& Extension period: no \\
& Intervention: Kids N Fitness (KNF) intervention \\
& Control: general education (GE) \\
\hline Outcomes & $\begin{array}{l}\text { Outcome measures reported in abstract: BMI, BMI z scores, dietary intake (vegetables, fruit, fruit } \\
\text { juice), self-efficacy of healthy food choices, parent and community involvement, TV viewing, daily phys- } \\
\text { ical activity, physical education class attendance }\end{array}$
\end{tabular}

Study details Trial terminated early: no

Trial ID: -

Publication details Language of publication: English

Funding: partly supported by a grant from the National Institutes of Health/National Institute on Minority Health and Health disparities (NIH/NIMHD) Loan repayment programme and a grant from the Robert Wood Johnson Foundation (grant no. 64195). (non-commercial)

Publication status: peer-reviewed journal

Stated aim for study
$\begin{aligned} & \text { Q CSHP with parental, school and home based components to promote optimal nutrition will reduce } \\ & \text { BMI percentiles and } z \text { scores and improve dietary behaviours in a sample of low-income, school aged } \\ & \text { children" }\end{aligned}$

Notes -

\section{Risk of bias}

\begin{tabular}{lll}
\hline Bias & Authors' judgement & Support for judgement \\
\hline $\begin{array}{l}\text { Random sequence genera- } \\
\text { tion (selection bias) }\end{array}$ & Unclear risk & Comment: randomisation process not described \\
\hline $\begin{array}{l}\text { Allocation concealment } \\
\text { (selection bias) }\end{array}$ & Unclear risk & Comment: unclear if allocation was concealed \\
\hline $\begin{array}{l}\text { Blinding of participants } \\
\text { and personnel (perfor- } \\
\text { mance bias) }\end{array}$ & Unclear risk & $\begin{array}{l}\text { Comment: unclear if participants and study personnel were blinded to study } \\
\text { groupjective outcomes }\end{array}$ \\
\hline
\end{tabular}


Wright 2012 (Continued)

Blinding of participants and personnel (perfor-

Objective outcomes

$\begin{array}{ll}\text { Blinding of outcome as- } & \text { Unclear risk } \quad \text { Comment: unclear if assessment staff were blinded to study group } \\ \text { sessment (detection bias) } & \end{array}$

Subjective outcomes

Blinding of outcome as-
sessment (detection bias)
Objective outcomes

\begin{tabular}{|c|c|c|}
\hline $\begin{array}{l}\text { Incomplete outcome data } \\
\text { (attrition bias) } \\
\text { Subjective outcomes }\end{array}$ & High risk & $\begin{array}{l}\text { Quote from publication: "Thirty children }(25 \%) \text { in the KNF@ group were lost } \\
\text { to follow-up at } 12 \text { months, compared to } 31 \text { children }(23 \%) \text { in the GE group ( } P= \\
0.75) . "\end{array}$ \\
\hline
\end{tabular}

Comment: high dropout rates, potential attrition bias

\begin{tabular}{|c|c|c|}
\hline $\begin{array}{l}\text { Incomplete outcome data } \\
\text { (attrition bias) } \\
\text { Objective outcomes }\end{array}$ & High risk & $\begin{array}{l}\text { Quote from publication: "Thirty children }(25 \%) \text { in the KNF@ group were lost } \\
\text { to follow-up at } 12 \text { months, compared to } 31 \text { children }(23 \%) \text { in the GE group ( } P= \\
0.75) . "\end{array}$ \\
\hline
\end{tabular}

Comment: high dropout rates, potential attrition bias

\begin{tabular}{lll}
\hline $\begin{array}{l}\text { Selective reporting (re- } \\
\text { porting bias) }\end{array}$ & Unclear risk & $\begin{array}{l}\text { Comment: no protocol or clinical trials register available so unable to assess } \\
\text { reporting bias }\end{array}$ \\
\hline Other bias & High risk & Comment: was cluster RCT but did not adjust for clustering in their analyses \\
\hline
\end{tabular}

AMA: American Medical Association; apo A: apolipoprotein A; apo B: apolipoprotein B;

BFC: Big Friends Club; BIA: bioimpedence analysis; BMI: body mass index; BMI SDS: standardised body mass index;

CDC: Centre for Disease Control and Prevention; CSHP: coordinated school health program;

DVD: digital versatile disc;

GP: general practitioners;

HAND: Healthy Activity and Nutrition Directions; HDL: high density lipoprotein; HRQoL: health-related quality of life;

IOTF: International Obesity Taskforce; ITT: intention-to-treat;

LDL: low-density lipoprotein; LEAP: Live, Eat and Play; LEAP2: Live, Eat and Play 2; LFD: low fat diet; LGD: low-glycaemic diet; LMS: Lambda-

Mu-Sigma;

MEND: Mind, Exercise, Nutrition; MET(s): metabolic equivalents;

$\mathrm{N}$ : number

PCT: primary care trust;

QoL: quality of life;

RCT: randomised controlled trial; RE: Reggio Emilia; ROC: Regulation of Cues; RED: high energy dense;

$\mathrm{NIH}$ : National Institutes of Health; NIHR: National Institute for Health Research;

SBP: systolic blood pressure;

SIGN: Scottish Intercollegiate Guidelines Network; SMSMT: short message service maintenance treatment; SWITCH: Screen-Time Weightloss Intervention Targeting Children at Home;

TAFF: telephone based adiposity prevention for families;

WC: waist circumference; WHO: World Health Organization;

YMCA: Young Men's Christian Association; z-BMI: standardised BMI

Characteristics of excluded studies [ordered by study ID]

\begin{tabular}{ll}
\hline Study & Reason for exclusion \\
\hline Adam 2009 & Not an RCT \\
\hline
\end{tabular}

Diet, physical activity and behavioural interventions for the treatment of overweight or obese children from the age of 6 to 11 years 


\begin{tabular}{|c|c|}
\hline Study & Reason for exclusion \\
\hline Albala 2008 & Duration $<6$ months \\
\hline Alberga 2013 & Duration of follow-up $<6$ months from baseline \\
\hline Alexander 2014 & Prevention \\
\hline Amini 2016 & Duration: 18 weeks only \\
\hline Andre 2015 & Participants: adolescents \\
\hline Astrup 2013 & Prevention: not all children overweight/obese at baseline \\
\hline Bachman 2010 & Secondary data analysis \\
\hline Baker 2012 & Not an RCT \\
\hline Ball 2012 & Parent-only intervention \\
\hline Banks 2011 & 2 alternative interventions, head-to-head trial (no control group) \\
\hline Banks 2012a & 2 alternative interventions, head-to-head trial (no control group) \\
\hline Banks 2012b & 2 alternative interventions, head-to-head trial (no control group) \\
\hline Banks 2014 & 2 alternative interventions, head-to-head trial (no control group) \\
\hline Banos 2009 & Not an RCT \\
\hline Baranowski 2011 & Aim - not to treat childhood obesity \\
\hline Barbeau 2007 & Prevention - not all overweight \\
\hline Bau 2016 & Participants: mean age at recruitment $=13$ years old (adolescents) \\
\hline Bauer 2010 & Not an RCT \\
\hline Bayat 2014 & Duration of follow-up $<6$ months from baseline, non-randomised \\
\hline Bean 2012 & Parent-only intervention \\
\hline Bean 2014 & Parent-only intervention \\
\hline Benestad 2014 & Not an RCT: 2 different treatments, no clear control group \\
\hline Benson 2008 & Aim of study - not to treat childhood obesity \\
\hline Bernstein 2015 & Thesis - not an RCT \\
\hline Best 2016 & Aim of study - not to treat childhood obesity \\
\hline Bloom 2013 & Duration (6-month follow-up only given for intervention group) \\
\hline Bocca 2014 & Participants: preschool children \\
\hline Bohnert 2013 & Aim - not all children overweight/obese at baseline (prevention) \\
\hline
\end{tabular}

Diet, physical activity and behavioural interventions for the treatment of overweight or obese children from the age of 6 to 11 years 


\begin{tabular}{|c|c|}
\hline Study & Reason for exclusion \\
\hline Boutelle 2011 & 2 alternative interventions, head-to-head trial (no control group) \\
\hline Boutelle 2013 & Duration: follow-up $<6$ months for 1 group \\
\hline Braden 2014 & Not an RCT \\
\hline Braet 1997a & 2 alternative interventions, head-to-head trial (no control group) \\
\hline Braet 1997b & 2 alternative interventions, head-to-head trial (no control group) \\
\hline Braet 2000 & 2 alternative interventions, head-to-head trial (no control group) \\
\hline Buhari 2014 & Prevention \\
\hline Burrows 2008 & Parent-only intervention \\
\hline Burrows 2010a & Parent-only intervention \\
\hline Burrows 2010b & Parent-only intervention \\
\hline Burrows 2011 & Parent-only intervention \\
\hline Bush 2007 & Prevention \\
\hline Bustos 1997 & Not an RCT \\
\hline Caballero 2003a & Prevention \\
\hline Caballero 2003b & Prevention \\
\hline Canas 2012a & Not a lifestyle intervention \\
\hline Canas 2012b & Not a lifestyle intervention \\
\hline Canas 2014 & Not a lifestyle intervention: main part of trial was carotenoid supplementation \\
\hline Carrel 2005 & Participants: adolescents \\
\hline Carrel 2007 & Participants: adolescents \\
\hline Cash 2009 & Prevention study \\
\hline Catenacci 2014 & Prevention: included healthy weight children \\
\hline Cespedes 2014 & Participants: preschool children \\
\hline Chen 2013 & Not an RCT \\
\hline Chen 2015 & Not an RCT \\
\hline Chen 2016 & Not an RCT \\
\hline Chirita-Emandi 2014 & Not a lifestyle intervention \\
\hline Chongviriyaphan 2010 & Duration of follow-up $<6$ months from baseline \\
\hline
\end{tabular}




\begin{tabular}{|c|c|}
\hline Study & Reason for exclusion \\
\hline Cohen 2012 & Duration 12 weeks \\
\hline Collins 2010 & Parent-only intervention \\
\hline Cooperberg 2014 & Participants: preschool children \\
\hline Coppinger 2016 & $\begin{array}{l}\text { Protocol for obesity prevention intervention (will include both healthy and overweight chil- } \\
\text { dren, therefore not treatment) }\end{array}$ \\
\hline Cradock 2016 & Prevention study - included normal weight children \\
\hline Crova 2014 & Prevention \\
\hline Cunningham-Sabo 2016 & Prevention \\
\hline da Silva 2015 & Participants: adolescents \\
\hline Dahiya 2012 & Secondary data analysis: comparison with normal weight children \\
\hline Dai 2006 & Duration of follow-up $<6$ months from baseline \\
\hline Dalton 2013 & Parent-only intervention \\
\hline Daniels 2009a & Aim of study - not to treat overweight/obese children \\
\hline Danielsen 2013 & Duration: control group only followed up for 12 weeks then given intervention \\
\hline Danielzik 2007 & Prevention \\
\hline Davis 1999 & Prevention \\
\hline Davis 2011a & Not an RCT \\
\hline Davis 2011b & Aim - not to treat overweight/obese children \\
\hline Davis 2014 & Duration - only 13 weeks' follow-up \\
\hline Davis 2016a & 2 alternative interventions, head-to-head trial (no control group) \\
\hline Davis 2016b & Duration of follow up $<6$ months from baseline \\
\hline de Mello 2004 & 2 alternative interventions, head-to-head trial (no control group) \\
\hline De Ruyter 2013 & Aim of study - not to treat childhood obesity \\
\hline Dennis 2013 & Duration of follow-up $<6$ months from baseline \\
\hline DeVault 2009 & Not an RCT \\
\hline Dias 2016 & Duration - 12 weeks \\
\hline Dodds 2014 & Prevention \\
\hline Donnelly 2009 & Aim - not to treat childhood obesity \\
\hline
\end{tabular}

Diet, physical activity and behavioural interventions for the treatment of overweight or obese children from the age of 6 to 11 years 


\begin{tabular}{|c|c|}
\hline Study & Reason for exclusion \\
\hline Doyle-Baker 2011 & Duration of follow-up $<6$ months from baseline \\
\hline Dreyer 2014 & Participants: adolescents \\
\hline DuBose 2008 & Not an RCT \\
\hline Duckworth 2009 & Duration of follow-up $<6$ months from baseline \\
\hline Duncan 2009 & Aim of study - not to treat childhood obesity \\
\hline Dura 2006 & Not an RCT, clinical record reviews \\
\hline Economos 2007 & Not an RCT \\
\hline El Hage 2012 & Aim to investigate hip strength in obese children \\
\hline Endevelt 2014 & Not an RCT \\
\hline Epstein 1981 & 2 alternative interventions, head-to-head trial (no control group) \\
\hline Epstein 1984b & Duration of follow-up $<6$ months from baseline \\
\hline Epstein 1986 & 2 alternative interventions, head-to-head trial (no control group) \\
\hline Epstein 1987a & Prevention - not all overweight \\
\hline Epstein 1987b & 2 alternative interventions, head-to-head trial (no control group) \\
\hline Epstein 1987c & 2 alternative interventions, head-to-head trial (no control group) \\
\hline Epstein 1987d & Not an RCT \\
\hline Epstein 1990 & 2 alternative interventions, head-to-head trial (no control group) \\
\hline Epstein 1993 & Secondary data analysis: aim to assess height growth of children \\
\hline Epstein 1994a & 2 alternative interventions, head-to-head trial (no control group) \\
\hline Epstein 1994b & 10-year follow-up (study 2 = Epstein 1984a) - however, does not follow up the control group \\
\hline Epstein 1995 & Not an RCT - unclear which is the control group \\
\hline Epstein 2000b & 2 alternative interventions, head-to-head trial (no control group) \\
\hline Epstein 2004 & 2 alternative interventions, head-to-head trial (no control group) \\
\hline Epstein 2007 & Not an RCT \\
\hline Epstein 2008a & 2 alternative interventions, head-to-head trial (no control group) \\
\hline Epstein 2008b & Duration of follow-up $<6$ months from baseline \\
\hline Epstein 2012 & Not an RCT \\
\hline Erceg 2012 & Not an RCT \\
\hline
\end{tabular}

Diet, physical activity and behavioural interventions for the treatment of overweight or obese children from the age of 6 to 11 years 


\begin{tabular}{|c|c|}
\hline Study & Reason for exclusion \\
\hline Escobedo 2014 & Not a lifestyle intervention - diet supplements \\
\hline Escoto 2008 & Aim - not to treat obesity \\
\hline Esfarjani 2013 & Parent-only intervention \\
\hline Estabrooks 2009 & Parent-only intervention \\
\hline Falbe 2015 & Duration - 10 weeks \\
\hline Farpour-Lambert 2009 & Follow-up from baseline $<6$ months \\
\hline Ferguson 1999a & Duration: crossover, 4 months only \\
\hline Ferguson 1999b & Duration: crossover, 4 months only \\
\hline Ferrara 2013 & Duration only $60 \mathrm{~d}$ ( 2 months) \\
\hline Ferrer 1998 & Not an RCT \\
\hline Figueroa-Colon 1993 & 2 alternative interventions, head-to-head trial (no control group) \\
\hline Figueroa-Colon 1996 & 2 alternative interventions, head-to-head trial (no control group) \\
\hline Firoozi 2013 & Duration of study - 6 weeks \\
\hline Fischer 2014 & Not a lifestyle intervention \\
\hline Foger 1993 & Not an RCT \\
\hline Follansbee-Junger 2010 & Not an RCT \\
\hline Frohna 2008 & Commentary on Wilfley 2007 \\
\hline Fullerton 2007a & Participants: adolescents \\
\hline Fullerton 2007b & Participants: adolescents \\
\hline Furze 2008 & Not an RCT \\
\hline Gajewska 2011 & Duration of follow-up $<6$ months from baseline \\
\hline Galhardo 2012 & Participants: adolescents \\
\hline Garipagaoglu 2009 & 2 alternative interventions, head-to-head trial (no control group) \\
\hline Gerards 2012 & Parent-only intervention \\
\hline Ghatrehsamani 2010 & Duration 3 months \\
\hline Goldfield 2000 & Duration of follow-up $<6$ months from baseline \\
\hline Goldfield 2001 & 2 alternative interventions, head-to-head trial (no control group) \\
\hline Goldfield 2006 & Duration of follow-up $<6$ months from baseline \\
\hline
\end{tabular}




\begin{tabular}{|c|c|}
\hline Study & Reason for exclusion \\
\hline Goldfield 2007 & Duration of follow-up $<6$ months from baseline \\
\hline Goldfield 2008 & Not an RCT \\
\hline Goldfield 2009 & Not an RCT \\
\hline Golley 2007 & Parent-only intervention \\
\hline Golley 2011 & Not an RCT \\
\hline Gong 2014 & Prevention study \\
\hline Graf 2006 & Not an RCT \\
\hline Graf 2008 & Not an RCT \\
\hline Graham 2008 & Aim - not to treat overweight/obese children \\
\hline Graves 1988 & 2 alternative interventions, head-to-head trial (no control group) \\
\hline Gregori 2014 & Duration of follow-up $<6$ months from baseline \\
\hline Griffin 2013 & Not an RCT \\
\hline Grow 2014 & Not an RCT \\
\hline Guixeres 2009 & Not an RCT \\
\hline Gunnarsdottir 2011b & Not an RCT \\
\hline Gunnarsdottir 2014 & Not an RCT (single group) \\
\hline Gunther 2007 & Not an RCT \\
\hline Gussinyer 2008 & Not an RCT \\
\hline Gutin 1996 & Not an RCT \\
\hline Gutin 1999a & Duration of follow-up $<6$ months from baseline \\
\hline Gutin 1999b & Duration of follow-up $<6$ months from baseline \\
\hline Gutin 2008 & Prevention \\
\hline Habib-Mourad 2014a & Prevention \\
\hline Habib-Mourad 2014b & Prevention \\
\hline Habib-Mourad 2014c & Prevention \\
\hline Haemer 2013 & Not an RCT \\
\hline Hager 2016 & Not an intervention study \\
\hline Hajihashemi 2014 & Duration of follow-up $<6$ months from baseline \\
\hline
\end{tabular}




\begin{tabular}{|c|c|}
\hline Study & Reason for exclusion \\
\hline Hammarlund 1993 & Duration of follow-up $<6$ months from baseline \\
\hline Hansen 2013 & Not an RCT \\
\hline Harder-Lauridsen 2014 & Duration of follow-up $<6$ months from baseline \\
\hline Hardman 2009 & Duration of follow-up $<6$ months from baseline \\
\hline Harrell 1998 & Duration of follow-up $<6$ months from baseline (between 8 and 10 weeks) \\
\hline Harrison 2006 & Prevention \\
\hline Hartlieb 2015 & Participants: adolescents \\
\hline Hashemipour 2009 & Not a lifestyle intervention \\
\hline Haszard 2015 & Secondary analysis of RCT data \\
\hline Heuser 2008 & Prevention - not all overweight \\
\hline Hollinghurst 2014 & 2 alternative interventions, head-to-head trial (no control group) \\
\hline Holmes 2008 & Not an RCT (discussion paper) \\
\hline Horsak 2015 & Protocol only with primary aim not to treat overweight \\
\hline Horton 2013 & Duration - 14 weeks \\
\hline Huang 2007 & Duration of follow-up $<6$ months from baseline \\
\hline Huang 2015a & Participants: adolescents \\
\hline Huang 2015b & Participants: adolescents \\
\hline Hystad 2013 & 2 alternative interventions, head-to-head trial (no control group) \\
\hline Iannuzzi 2009 & Not an RCT - no control group \\
\hline Ibarra-Reynoso 2015 & Duration - 2 months \\
\hline Ildiko 2007 & Not an RCT \\
\hline Innes-Hughes 2016 & Not an RCT \\
\hline Israel 1984 & Not an RCT \\
\hline Israel 1985 & Duration - control group only 9 weeks long \\
\hline Israel 1994 & 2 alternative interventions, head-to-head trial (no control group) \\
\hline Jacobson 2009 & Thesis - duration of follow up $<6$ months from baseline \\
\hline Jago 2013 & Prevention \\
\hline James 2000 & Commentary on a prevention intervention \\
\hline
\end{tabular}




\begin{tabular}{|c|c|}
\hline Study & Reason for exclusion \\
\hline Janicke $2008 a$ & Parent-only intervention \\
\hline Janicke 2008b & Parent-only intervention \\
\hline Janicke 2009 & Parent-only intervention \\
\hline Janicke 2011 & Duration of follow-up $<6$ months from baseline \\
\hline Janicke 2013 & Participants: preschool children \\
\hline Jansen 2011 & Parent-only intervention \\
\hline Jensen 2012 & Duration of follow-up $<6$ months from baseline \\
\hline Jensen 2013 & Duration only 10 weeks \\
\hline Jensen 2015 & Not an RCT \\
\hline Jernigan 2015 & Not an RCT \\
\hline John 2009 & Participants: preschool children \\
\hline Johnston 2013 & Prevention \\
\hline Jones 2015a & Includes children that were not actually overweight or obese (but determined as 'at risk') \\
\hline Jurg 2006 & Prevention \\
\hline Kain 2009 & Prevention \\
\hline Kalarchian 2013 & Not an RCT \\
\hline Kang 2008 & Duration of follow-up $<6$ months from baseline \\
\hline Karacabey 2009 & Duration of follow-up $<6$ months from baseline \\
\hline Kelishadi 2008 & 2 alternative interventions, head-to-head trial (no control group) \\
\hline Kelishadi 2009 & Participants: preschool children \\
\hline Kelishadi 2010 & Participants: preschool children \\
\hline Kerr 2000 & Prevention \\
\hline Khadilkar 2012 & Duration of follow-up $<6$ months from baseline \\
\hline Kim 2016 & Duration - 5 weeks \\
\hline Kipping 2008 & Prevention \\
\hline Kirschenbaum 1984 & Alternative interventions, head-to-head trial (no control group) \\
\hline Klesges 2008 & Prevention \\
\hline Klitzman 2015 & Parent-only intervention \\
\hline
\end{tabular}

Diet, physical activity and behavioural interventions for the treatment of overweight or obese children from the age of 6 to 11 years 


\begin{tabular}{|c|c|}
\hline Study & Reason for exclusion \\
\hline Kohno 1994 & Not an RCT \\
\hline Kokkvoll 2014 & 2 alternative interventions, head-to-head trial (no control group) \\
\hline Kolko 2010 & Participants: preschool children \\
\hline Krafft 2014a & Aim not to treat obesity, aim to assess brain function \\
\hline Krafft 2014b & Aim not to treat obesity, aim to assess brain function \\
\hline Krafft 2014c & Aim not to treat obesity, aim to assess brain function \\
\hline Kriemler 2010 & Prevention \\
\hline Kuni 2015 & Aim of study - not to treat childhood obesity \\
\hline Larsen 2010 & Prevention \\
\hline Larsen 2016 & Participants: adolescents \\
\hline Lau 2015 & Duration of follow-up $<6$ months from baseline \\
\hline Leach 2008 & Aim of study - not to treat childhood obesity \\
\hline Li 2010 & 2 alternative interventions, head-to-head trial (no control group) \\
\hline Looney 2012 & Secondary data analysis \\
\hline Lopes 2009 & Prevention \\
\hline Loughrey 2009 & Not an RCT (discussion paper) \\
\hline Luley 2010 & Alternative interventions (no control group) \\
\hline Madsen 2013 & Aim of study - not to treat childhood obesity \\
\hline Makkes 2011 & Participants: adolescents \\
\hline Maloney 2012 & Participants: adolescents \\
\hline Manchester 1978 & Not an RCT \\
\hline Marcus 2009 & Prevention \\
\hline Marild 2013 & 2 alternative interventions, head-to-head trial (no control group) \\
\hline Maron 2014 & Not an RCT \\
\hline Martinez 2008 & Prevention \\
\hline Matheson 2015 & Not a lifestyle intervention \\
\hline Mayurachat 2013 & Duration only 18 weeks \\
\hline Mazzeo 2008 & Parent-only intervention \\
\hline
\end{tabular}

Diet, physical activity and behavioural interventions for the treatment of overweight or obese children from the age of 6 to 11 years 


\begin{tabular}{|c|c|}
\hline Study & Reason for exclusion \\
\hline Mazzeo 2011 & Parent-only intervention \\
\hline Mazzeo 2014 & Parent-only intervention \\
\hline McFarland 2014 & Not an RCT \\
\hline McGuigan 2009 & Not an RCT \\
\hline Medrano 2015 & Duration - 22 weeks \\
\hline Minossi 2014 & Duration of follow-up $<6$ months from baseline \\
\hline Minossi 2015 & $\begin{array}{l}\text { Protocol - inclusion criteria will allow the inclusion of non-overweight children with co-morbid- } \\
\text { ity such as hypertension, dyslipidaemia or diabetes (prevention study) }\end{array}$ \\
\hline Mo-suwan 1998 & Prevention \\
\hline Moens 2012 & Parent-only intervention \\
\hline Moreno 2015 & Secondary data analysis of 2 RCTs \\
\hline Morgan 2014 & Prevention \\
\hline Muckelbauer 2009a & Aim - not to treat overweight/obese children \\
\hline Muckelbauer 2009b & Aim - not to treat overweight/obese children \\
\hline Munsch 2008 & Parent-only intervention \\
\hline Murphy 2009 & Not an RCT \\
\hline Mustila 2012 & Not an RCT \\
\hline Muth 2008 & Prevention \\
\hline NCT00284557 & Not all children were overweight or obese (inclusion criteria stated "at risk of overweight") \\
\hline Nemet 2006 & Duration - 3 months' follow-up, not an RCT \\
\hline Nemet 2013a & Duration - 3 months' follow-up, not an RCT \\
\hline Nemet 2013b & Prevention - not all overweight \\
\hline Nogueira 2014 & Trial was not exclusively in overweight children - therefore not a treatment trial \\
\hline Nogueira 2015 & Trial was not exclusively in overweight children - therefore not a treatment trial \\
\hline Nowicka 2010 & Not an RCT \\
\hline Nuutinen 1992 & Not an RCT \\
\hline O'Malley 2011 & Aim of study - not to treat childhood obesity \\
\hline Okely 2010 & Parent-only intervention \\
\hline
\end{tabular}

Diet, physical activity and behavioural interventions for the treatment of overweight or obese children from the age of 6 to 11 years 


\begin{tabular}{|c|c|}
\hline Study & Reason for exclusion \\
\hline Oliveras 2013 & Not a lifestyle intervention \\
\hline Parente 2006 & Duration - only 5 months' follow-up \\
\hline Parillo 2012 & Not an RCT - 2 alternative interventions \\
\hline Parra-Medina 2011 & Duration - only 18 weeks' follow-up \\
\hline Pedrosa 2011a & 2 alternative interventions, head-to-head trial (no control group) \\
\hline Pedrosa 2011b & 2 alternative interventions, head-to-head trial (no control group) \\
\hline Perman 2008 & Not an RCT \\
\hline Perry 1979 & Aim: to assess eating behaviours, not treat obesity \\
\hline Petty 2009 & Aim - not to treat overweight/obese children \\
\hline Plachta-Danielzik 2007 & Prevention \\
\hline Plummer 2014 & Not an RCT \\
\hline Polacsek 2009 & Not an RCT \\
\hline Pontin 2004 & Commentary, prevention \\
\hline Poulsen 2011 & Not an RCT \\
\hline Prado 2009 & Duration of follow-up $<6$ months from baseline \\
\hline Puder 2009 & Prevention \\
\hline Qu 2014 & Prevention - not all overweight \\
\hline Racine 2010 & Not a lifestyle intervention \\
\hline Ramon-Krauel 2013 & Aim - to treat fatty liver \\
\hline Rank 2012 & Not an RCT \\
\hline Rausch 2013 & Prevention \\
\hline Raynor 2002 & 2 alternative interventions, head-to-head trial (no control group) \\
\hline Raynor 2012 & Parent-only intervention \\
\hline Reinehr 2006 & Not an RCT \\
\hline Reinehr 2009 & Not an RCT \\
\hline Reinehr 2011 & Commentary on a parent-only intervention \\
\hline Resaland 2014 & Prevention \\
\hline Resnick 2009 & Parent-only intervention \\
\hline
\end{tabular}




\begin{tabular}{|c|c|}
\hline Study & Reason for exclusion \\
\hline Resnicow 2012 & Parent-only intervention \\
\hline Riddiford-Harland 2012 & Parent-only intervention: analysis from the HIKCUPS study \\
\hline Riddiford-Harland 2016 & Secondary analysis of RCT data examining foot-related outcomes \\
\hline Riggs 2007 & Prevention \\
\hline Robertson 2012 & Not an RCT \\
\hline Robinson 1999 & Prevention \\
\hline Rodearmel 2006 & Duration of follow-up $<6$ months from baseline \\
\hline Rohrer 2008 & Not an RCT \\
\hline Rooney 2005 & Prevention - not all children overweight at baseline \\
\hline Rosado 2008 & Duration of follow-up $<6$ months from baseline \\
\hline Safavi 2013 & Duration of follow up 8 weeks from baseline ( $<6$ months) \\
\hline Salcedo 2010 & Prevention (not all overweight) \\
\hline Salehi-Abargouei 2014 & Duration of follow-up $<6$ months from baseline \\
\hline Sallis 1993 & Prevention \\
\hline Salmon 2008 & Prevention \\
\hline Sanchez-Gomez 2012 & Prevention \\
\hline Schaeffer 2014 & Aim of study - not to treat childhood obesity \\
\hline Seabra 2014 & Duration of follow-up $<6$ months from baseline \\
\hline Senediak 1985 & 2 alternative interventions, head-to-head trial (no control group) \\
\hline Sgro 2009 & Not an RCT \\
\hline Shalitin 2009 & Not an RCT- no control group identified \\
\hline Sherman 1992 & Not an RCT \\
\hline Slusser 2013 & Prevention - includes healthy weight children \\
\hline Small 2014 & Participants: preschool children \\
\hline Sothern $2000 a$ & Not an RCT \\
\hline Sothern $2000 b$ & Not an RCT \\
\hline Soto-Sanchez 2014 & Not an RCT \\
\hline Speroni 2007 & Prevention \\
\hline
\end{tabular}




\begin{tabular}{|c|c|}
\hline Study & Reason for exclusion \\
\hline Spriet 2014 & Commentary paper \\
\hline St-Onge 2009 & Duration of follow-up $<6$ months from baseline \\
\hline Steele 2012 & 2 alternative interventions, head-to-head trial (no control group) \\
\hline Steele 2014 & Secondary data analysis \\
\hline Stettler 2015 & Prevention study \\
\hline Stevens 2003 & Aim - not to treat overweight/obese children \\
\hline Stewart 2009 & Not an RCT \\
\hline Stone 2003 & Aim - not to treat overweight/obese children \\
\hline Stovitz 2014 & Duration of follow-up $<6$ months from baseline \\
\hline Sweeney 2010 & Not an RCT \\
\hline Sze 2015 & Duration - 4 weeks \\
\hline Tak 2007 & Duration of follow-up $<6$ months from baseline \\
\hline Tanas 2011 & Not an RCT \\
\hline Taveras 2014 & Not a lifestyle intervention \\
\hline Taylor 2006 & Not an RCT \\
\hline Taylor 2007 & Aim of study - not to treat childhood obesity \\
\hline Teevale 2015 & Qualitative study \\
\hline Ten 2016 & Not an intervention study \\
\hline Theim 2012 & Not an RCT \\
\hline Thompson 2013 & Not all overweight or obese \\
\hline Tirlea 2016 & Participants: adolescents - mean age $>12$ \\
\hline Todd 2008 & Duration of follow-up $<6$ months from baseline \\
\hline Trinh 2013 & Not an RCT \\
\hline Trost 2014 & Duration of follow-up $<6$ months from baseline \\
\hline Tucker 2014 & Not an RCT \\
\hline Uysal 2014 & Not an RCT \\
\hline Van Grieken 2013 & Participants: preschool children \\
\hline Van Grieken 2014 & Participants: preschool children \\
\hline
\end{tabular}




\begin{tabular}{|c|c|}
\hline Study & Reason for exclusion \\
\hline Vandongen 1995 & Prevention \\
\hline Vargo 2012 & Not an RCT \\
\hline Vasickova 2011 & Duration of follow-up $<6$ months from baseline \\
\hline Verbeken 2013a & Duration of follow-up $<6$ months from baseline \\
\hline Verbeken 2013b & Duration - 12 weeks follow up (<6 months from baseline) \\
\hline Verduci 2011 & Not an RCT \\
\hline Verduci 2013 & Not a lifestyle intervention \\
\hline Vetter 2014 & Prevention \\
\hline Vido 1993 & Not a lifestyle intervention \\
\hline Virgen 2007 & Not an RCT \\
\hline Vos 2011 & Participants: adolescents \\
\hline Vrablik 2014 & Participants: adolescents, mean age $>12$ \\
\hline Wake 2011 & Aim of study - not to treat childhood obesity \\
\hline Walker 2008 & Parent-only intervention \\
\hline Walsh 2014 & Not an RCT \\
\hline Wang 2013 & Not an RCT - uses baseline data from another study \\
\hline Ward 2011 & Aim of study - not to treat childhood obesity \\
\hline Watowicz 2014 & Not an RCT \\
\hline Wheeler 1976 & Duration of follow-up from baseline not clear \\
\hline Wijesuriya 2011 & Participants: adults \\
\hline Wile 1992 & Not an RCT \\
\hline Williamson 2008 & Prevention \\
\hline Williamson 2010 & Prevention \\
\hline Williamson 2012 & Prevention \\
\hline Wislo 2013 & Not an RCT \\
\hline Wohlfarth 2013 & Duration of follow-up $<6$ months from baseline \\
\hline Wong 2013 & Duration of follow-up $<6$ months from baseline \\
\hline Wright 2013 & Duration of follow-up $<6$ months from baseline \\
\hline
\end{tabular}




\begin{tabular}{ll}
\hline Study & Reason for exclusion \\
\hline Wyatt 2011 & Prevention \\
\hline Xu 2012 & Prevention \\
\hline Yackobovitch-Gavan 2009 & Not an RCT \\
\hline Yam 2012 & Prevention \\
\hline Yu 2008 & Duration of follow-up <6 months from baseline \\
\hline Zahner 2006 & Prevention \\
\hline Zask 2012 & Aim of study - not to treat childhood obesity \\
\hline Zhang 2011a & Not a lifestyle intervention \\
\hline Zhang 2011b & Not an RCT \\
\hline Zheng 2015 & Not an RCT \\
\hline Zorba 2011 & Duration of follow-up <6 months from baseline \\
\hline
\end{tabular}

$\mathrm{RCT}$ : randomised controlled trial

Characteristics of studies awaiting assessment [ordered by study ID]

\section{ACTRN12611000862943}

\begin{tabular}{|c|c|}
\hline Methods & $\begin{array}{l}\text { Type of study: interventional } \\
\text { Allocation: randomised } \\
\text { Intervention model: parallel } \\
\text { Masking: open label } \\
\text { Primary purpose: treatment }\end{array}$ \\
\hline Participants & $\begin{array}{l}\text { Condition: obesity } \\
\text { Enrollment: target } 107 \\
\text { Inclusion criteria: } \\
\text { - } 5-16 \text { year old boys and girls } \\
\text { - BMI > } 98 \text { th WHO centile } \\
\text { - significant weight-related co-morbidities and ready to change } \\
\text { Exclusion criteria: } \\
\text { - significant co-morbidities that would make programme participation impossible }\end{array}$ \\
\hline Interventions & $\begin{array}{l}\text { Intervention: } 1 \text {-h home visits with diet and activity assessment and education, then weekly } 1.5-\mathrm{h} \\
\text { activity session for } 40 \text { weeks and psychology group }(2 \times 1 \text {-h sessions }) \\
\text { Control: brief dietary education and diet, activity and well-being assessments }\end{array}$ \\
\hline Outcomes & $\begin{array}{l}\text { Primary outcome: reduction of } 0.5 \text { SDS at } 0,6,12,18 \text { and } 24 \text { months, quality-of-life improvements } \\
\text { and physical activity improvements as the same time points }\end{array}$ \\
\hline
\end{tabular}

Diet, physical activity and behavioural interventions for the treatment of overweight or obese children from the age of 6 to 11 years 
ACTRN12611000862943 (Continued)

Secondary outcomes: improvements in dietary and sedentary behaviours and improved glycaemic control

\begin{tabular}{|c|c|}
\hline Study identifier & ACTRN12611000862943 \\
\hline Official title & Whanau Pakari: a multidisciplinary intervention for child and adolescent obesity \\
\hline Stated purpose of study & $\begin{array}{l}\text { "Our objectives are firstly to undertake a multi-disciplinary intervention which is accessible and ap- } \\
\text { propriate for those most affected by child obesity. Secondary, we aim to assess whether a quantita- } \\
\text { tive RFC questionnaire is useful in predicting response to the intervention." }\end{array}$ \\
\hline Notes & $\begin{array}{l}\text { Study author reply: } 14 / 10 / 16 \text {. "I have just submitted the } 12 \text {-month outcome paper today. I am not } \\
\text { sure of your timeframes, but if you like, I can put you on our communications update list, so you } \\
\text { hear as soon as it is published." }\end{array}$ \\
\hline
\end{tabular}

\section{ISRCTN45032201}

Type of study: interventional
Methods
Intervention model: parallel
Masking: investigator-blind
Primary purpose: treatment
Condition: childhood overweight and obesity
Enrolment: target 120 families
Inclusion criteria:
Participants child overweight (> 91st centile) or obese (>98th centile) child
- age $7-11$ years
Exclusion criteria:
- insufficiently able to speak English (child or parent)
- metabolic or other medical cause of obesity
- severe learning difficulties or behavioural problems in the child

Interventions

Intervention: 10-week family-based intervention

(group-based with children and parents, focusing on parenting, social and emotional development, and changing behaviour)

Control: receive usual care given in their area

Outcomes
BMI/BMI z score (primary), waist circumference, percentage body fat, habitual activity via ac-
celerometer, quality of life, fruit and vegetable consumption, parental BMI, parental well-being,
family eating and activity, quality of parent-child relationships, Parenting style Health state valua-
tion, economic evaluation, process evaluation
Endpoint $=12$ months

Study identifier ISRCTN45032201

Diet, physical activity and behavioural interventions for the treatment of overweight or obese children from the age of 6 to 11 years 
ISRCTN45032201 (Continued)

Official title

"A randomised controlled trial evaluating the effectiveness and cost-effectiveness of "Families for Health", a family-based childhood obesity treatment intervention delivered in a community setting for ages 7 to $11 "$
"Our objectives are to:

1. Assess the effectiveness of the 'Families for Health' programme in reducing BMI z-score in children aged 7 to 11 who are overweight and obese

2. Evaluate the cost-effectiveness and cost-utility of the 'Families for Health' programme

3. Investigate parents' and children's views of the programme and their observations on approaches to maximising impact

4. To investigate facilitators' views of the programme and their observations on approaches to maximising impact"

$\begin{array}{ll}\text { Type of study: interventional } \\ \text { Methods } & \text { Allocation: randomised } \\ \text { Intervention model: parallel } \\ \text { Masking: unclear } \\ \text { Primary purpose: treatment } \\ \text { Condition: obesity } \\ \text { Enrolment: target } 200 \\ \text { Inclusion criteria: } \\ \text { Participants children aged } 8 \text { - } 12 \text { years } \\ \text { - obesity type I and II (BMI } \geq \text { age- and gender-adjusted } 95 \text { percentile) } \\ \text { - capacity to walk for } 10 \text { min }\end{array}$

\section{Exclusion criteria:}

- diabetes type I

- hyperactivity

- morbid obesity

- contraindications to do exercise (biological or mental)

Interventions

Intervention: the 6-month programme includes two 1-h sessions of an exercise programme per week. Each session includes 20-min physical exercise to improve fitness, a 30-min activity to improve sport skills, and 10-min of healthy behaviour-changing advice (nutrition, possibilities of doing physical exercise during leisure time)

Control: usual care only

Lutcomes of physical activity, fitness tests, healthcare costs, health-related quality of life, nutritional in-
take, blood samples


ISRCTN97887613 (Continued)

\begin{tabular}{ll} 
Stated purpose of study & Not given \\
\hline Notes & Trial record retrospectively registered. Trial completed. Emailed study author (April 2016) - no reply
\end{tabular}

\begin{tabular}{|c|c|}
\hline Methods & $\begin{array}{l}\text { Type of study: interventional } \\
\text { Allocation: randomised } \\
\text { Intervention model: parallel } \\
\text { Masking: open label } \\
\text { Primary purpose: treatment }\end{array}$ \\
\hline Participants & $\begin{array}{l}\text { Condition: obesity } \\
\text { Enrolment: target } 300 \\
\text { Inclusion criteria: } \\
\text { - elementary school children ( } 6-12 \text { years) } \\
\text { - a percent relative body weight of } \geq 20 \% \\
\text { Exclusion criteria: } \\
\text { - participants who were treated or educated for obesity in medical setting(s) }\end{array}$ \\
\hline Interventions & $\begin{array}{l}\text { Intervention: educational, counselling, training (pedometer, limit screen time) } \\
\text { Control: record pedometer count and screen time without intervention }\end{array}$ \\
\hline Outcomes & Percent relative body weight, cardiovascular risk factors \\
\hline Study identifier & JPRN-UMIN000014896 \\
\hline Official title & $\begin{array}{l}\text { Study on the medical check-up system for prevention of behaviour changing diseases including di- } \\
\text { abetes in underage groups, especially infants, elementary and junior high school children [Study } \\
\text { on the Prevention and Treatment of Obesity by Behavioral Approach (Lifestyle modification ap- } \\
\text { proach) for Elementary School Children] }\end{array}$ \\
\hline Stated purpose of study & "Study on the treatment of childhood obesity by behavioral approach" \\
\hline Notes & Study completed. Study author reply: 11 October 2016. Results have not been published yet \\
\hline
\end{tabular}

Jung 1978

\section{Methods}

Participants

Interventions

\section{Outcomes}

Diet, physical activity and behavioural interventions for the treatment of overweight or obese children from the age of 6 to 11 years 
Jung 1978 (Continued)

Study identifier

Official title

Stated purpose of study

Notes

Cannot obtain full publication from the

\begin{tabular}{|c|c|}
\hline Methods & $\begin{array}{l}\text { Type of study: interventional, efficacy } \\
\text { Allocation: randomised } \\
\text { Intervention model: parallel } \\
\text { Masking: single-blind (assessors) } \\
\text { Primary purpose: treatment }\end{array}$ \\
\hline Participants & $\begin{array}{l}\text { Conditions: obesity } \\
\text { Enrolment: } 270 \\
\text { Inclusion criteria: } \\
\text { - Age 4-7 years } \\
\text { - Boys or girls, and of any race } \\
\text { - BMI > 85thpercentile for age } \\
\text { - Children and parents must speak and understand English } \\
\text { Exclusion criteria: } \\
\text { - Currently participating in a clinical trial, but they may be involved in an observational study } \\
\text { - Physical disabilities which inhibit physical activity } \\
\text { - Taking drugs known to alter glucose homeostasis } \\
\text { - History of diabetes mellitus } \\
\text { - History of significant hepatic, renal, gastrointestinal or cardiovascular disease } \\
\text { - Diagnosis of hypertension requiring limited physical activity } \\
\text { - Psychological disabilities limiting participation } \\
\text { - Other medical or behavioural factors which might interfere with the study (judged by principal } \\
\text { investigator) } \\
\text { - Unable to speak and understand English } \\
\text { - No telephone or transportation }\end{array}$ \\
\hline
\end{tabular}

Interventions

Intervention: Team PLAY Group (6-month family-centered intervention to increase physical activity and healthy eating patterns, primarily directed at parents)

Control: standard care group (primary care physician)

\begin{tabular}{ll}
\hline Outcomes & $\begin{array}{l}\text { BMI (primary), body composition (DEXA), physical activity via accelerometry, dietary changes, Body } \\
\text { Esteem Scale, Flexibility and Cohesion Evaluation Scales, MacArthur Behavior and Health Ques- } \\
\text { tionnaire }\end{array}$ \\
\hline Study identifier & NCT00528164 \\
\hline
\end{tabular}

Diet, physical activity and behavioural interventions for the treatment of overweight or obese children from the age of 6 to 11 years 
NCT00528164 (Continued)

Official title Treating childhood obesity with family lifestyle change

Stated purpose of study

"The purpose of this study is to determine whether an intense family-centered program to help children, 4 to 7 years old, control their weight is more effective than the advice and referrals their health provider gives in the primary care office."

Notes

There are three publications attached to the trial register. 1 is a protocol, the second provides baseline results and measures of attendance, and the third is a secondary data analysis examining the relationship between BMI and self-esteem. Therefore, emailed study author to ask when the full set of outcome results shall be published.

Study author reply: 12 October 2016. "I am sorry to report to you that our results have not been published. The study has been completed. We are working on the outcome manuscript - hope to have it published soon."

\section{NCT00723853}

\begin{tabular}{|c|c|}
\hline Methods & $\begin{array}{l}\text { Type of study: interventional } \\
\text { Allocation: randomised } \\
\text { Intervention model: parallel } \\
\text { Masking: open label } \\
\text { Primary purpose: prevention }\end{array}$ \\
\hline Participants & $\begin{array}{l}\text { Condition: type } 2 \text { diabetes and obesity } \\
\text { Enrolment: } 131 \\
\text { Inclusion criteria: } \\
\text { - age } 9-12 \text { years } \\
\text { - overweight (> } 85 \text { th percentile BMI for age and gender) } \\
\text { - African American } \\
\text { - family history of type } 2 \text { diabetes in a first or second degree relative } \\
\text { - parents are secondary participants } \\
\text { Exclusion criteria: } \\
\text { - Inability to give informed consent or unwillingness to be randomised } \\
\text { - Prior diagnosis of diabetes in the child planning to participate } \\
\text { - Pregnancy (women who become pregnant during the study will be omitted from the analysis. } \\
\text { Pregnant women will not participate in the exercise sessions) } \\
\text { - Uncontrolled hypertension (SBP > } 160 \text { or DBP > } 100 \text { ) } \\
\text { - Uncontrolled dyslipidaemia by NCEP III criteria } \\
\text { - Evidence of significant cardiovascular, pulmonary disease, or other serious illness } \\
\text { - Musculoskeletal disease serious enough to prevent participation in exercise sessions } \\
\text { - Known or suspected major psychiatric disorder } \\
\text { - Inability to participate in aerobic exercise activities } \\
\text { - Inability to comply with a calorie- or fat-restricted diet } \\
\text { - Age over } 65 \text { years }\end{array}$ \\
\hline
\end{tabular}


NCT00723853 (Continued)

Control: Reach-In Program, Standard of Care
Height, weight, waist and hip circumference, body fat by BIA, biochemical markers (glucose tolerance, lipid panel, insulin, hemoglobin A-1-C)

\begin{tabular}{ll}
\hline Study identifier & NCT00723853 \\
\hline Official title & REACH-OUT: Chicago Children's Diabetes Prevention Program \\
\hline Stated purpose of study & $\begin{array}{l}\text { "The purpose of this research study is to evaluate two nutrition and exercise programs in children } \\
\text { ages } 9-12 \text { who are at risk for developing type } 2 \text { diabetes. This study also includes the involvement } \\
\text { of parents or guardians who are willing to participate in these programs with the child." }\end{array}$ \\
\hline Notes & Study completed. Emailed study author (April 2016) - no reply \\
\hline
\end{tabular}

\section{NCT00759746}

\section{Methods}

Type of study: interventional

Allocation: randomised

Intervention model: parallel

Masking: open label

Primary purpose: treatment

\section{Participants}

\section{Condition: childhood overweight and obesity}

\section{Enrolment: 482}

\section{Inclusion criteria:}

- age 7-11 years

- $\geq 85$ th percentile for weight

- at least 1 parent of the participating child must be overweight (BMI $\geq 25)$

- 1 parent must agree to attend all parent/child treatment meetings as the participating parent

- participants must be able to speak and comprehend English

\section{Exclusion criteria:}

- participating parent or child having a thought disorder, suicidality, bipolar disorder, or drug or alcohol dependence

- participating parent or child having a physical disability or illness that prevents performance of physical activity at level equivalent to a brisk walk or that places severe restriction on diet

- participating parent or child being on a medication regimen that affects weight

- participating parent or child being involved in active psychiatric treatment for an ongoing problem that causes either social or occupational impairment

- parents (participating and nonparticipating) and children having an eating disorder (i.e. anorexia nervosa, bulimia nervosa, binge eating disorder) or having subclinical levels of eating disturbance (i.e. reporting key eating disorder behaviours of purging, fasting, or binge eating more than 2 times per month)

Interventions

Intervention 1: behavioural: SFM + low dose (intervention focuses on helping families create a social environment that supports weight maintenance)

Intervention 2: behavioural: SFM + high dose 
NCT00759746 (Continued)

Control: behavioural: weight maintenance education

\begin{tabular}{ll}
\hline Outcomes & Child percent overweight \\
\hline Study identifier & NCT00759746 \\
\hline Official title & Childhood obesity treatment: a maintenance approach \\
\hline Stated purpose of study & $\begin{array}{l}\text { "The purpose of this study is to determine the effect of dose and content of an enhanced weight } \\
\text { maintenance treatment on children's ability to maintain weight loss following a standard weight } \\
\text { loss treatment." }\end{array}$ \\
\hline Notes & $\begin{array}{l}\text { Study completed. Study author did not reply October 2016. Only 16-week data are currently pub- } \\
\text { lished }\end{array}$ \\
\hline
\end{tabular}

\section{NCT00851201}

\section{Methods}

Type of study: interventional

Allocation: randomised

Intervention model: parallel

Masking: open label

Primary purpose: prevention

\begin{tabular}{|c|c|}
\hline \multirow[t]{5}{*}{ Participants } & Condition: obesity, diabetes \\
\hline & Enrolment: 506 \\
\hline & Inclusion: \\
\hline & - age $7-12$ years \\
\hline & - $\mathrm{BMI}>$ 85th percentile for sex \\
\hline
\end{tabular}

\section{Exclusion:}

- health or condition that would interfere with study participation,

- unwilling or inability to provide parent/guardian consent or child assent

- intention to move from area

Intervention: intensive behaviour changing: same as control but add on: 1) 12 core group modules for parents (to address roles and skills) and for children (to enhance motivation and skills and to provide physical activity), 3) Tailored support using a 'toolbox' approach from community health workers as extensions of the Family Weight Management professional education staff, and 4) monthly after-core follow-up groups

Control: 1-Standard Intervention: 1) an initial consult, which includes an overview of behaviour-changing goals, 2) quarterly follow-up, 3) and a monthly newsletter

Outcomes

BMI percentile for age and sex, biomarkers (e.g. glucose, insulin, lipids), dietary intake, and physical activity measures

\begin{tabular}{ll}
\hline Study identifier & NCT00851201 \\
\hline Official title & Comprehensive approach to family weight management \\
\hline
\end{tabular}

Diet, physical activity and behavioural interventions for the treatment of overweight or obese children from the age of 6 to 11 years 
NCT00851201 (Continued)

Stated purpose of study "The purpose of this study is to address the Healthy People 2010 obesity prevention objective"

Notes Study completed. Baseline data available but no follow-up data identified

\section{NCT01110096}

\section{Methods}

Type of study: interventional

Allocation: randomised

Intervention model: parallel

Masking: open label

Primary purpose: treatment

Condition: obesity
Enrolment: 100
Inclusion:
- age $7-12$ years
- BMI > iso-BMI 30 (Coles index)
Exclusion:
- syndromatic obesity
- obesity related to diseases
- local community not involved
- child has present follow-up because of obesity in secondary health care
- parent has current/planned follow-up because of obesity in secondary health care

Interventions Intervention: 2-week family camp: Parent Management Training - Oregon (PMTO), motivational interviewing, dynamic group therapy

Control: 4- $d$ family behaviour-changing school

\begin{tabular}{ll}
\hline Outcomes & BMI SDS, quality of life, physical fitness, behaviour, blood samples \\
\hline Study identifier & NCT01110096 \\
\hline Official title & $\begin{array}{l}\text { Randomised controlled clinical trial comparing two family interventions to treat obesity in children } \\
\text { between } 7 \text { and } 12 \text { years }\end{array}$ \\
\hline Stated purpose of study & $\begin{array}{l}\text { "The study compares the effect on BMI of two different treatment options for obesity in childhood. } \\
\text { Families with at least } 1 \text { obese child and parent are invited to join the project. The hypothesis is } \\
\text { that family camp gives an additional reduction in BMI compared to a less intensive family lifestyle } \\
\text { school." }\end{array}$ \\
\hline Notes & $\begin{array}{l}\text { Author reply: } 27 \text { November } 2016 . \\
\text { before publishing, we just sent the final proof to the journal. I have not yet received the exact date } \\
\text { for publishing (I assume within a week or two), but I will send you the article as soon as it is pub- } \\
\text { lished." }\end{array}$ \\
\hline
\end{tabular}


NCT01290016

\begin{tabular}{ll}
\hline Type of study: interventional \\
Allocation: randomised \\
Intervention model: parallel \\
Masking: open label \\
Primary purpose: treatment \\
Condition: obesity \\
Enrollment: 132 \\
Inclusion criteria: \\
- Aged 6-12 years \\
- boys and girls, who consume less than 2 servings of milk/milk products \\
- receptive to recommendations \\
- BMI > 97 WHO centile \\
Exclusion criteria: \\
- serious or chronic illnesses of childhood \\
- medication use in last 3 months known to affect bone or mineral metabolism \\
- diabetes \\
- non dietary hyperlipidaemia
\end{tabular}

Interventions

Intervention: arm 1: family counselling to maintain 2 servings of dairy/d and physical activity improvement instructions; arm 2 as arm 1 but advised to eat 4 dairy servings (for ages 6-8 years); arm 3: as arm 2 but for $9-12$ years

Control: diet and exercise information only

Outcomes Primary outcome: body composition at 0, 3, 6, 9 and 12 months

Secondary outcomes: blood biochemistry, satiety and bone mass

\begin{tabular}{ll}
\hline Study identifier & NCT01290016 \\
\hline Official title & MY LIFE Study - McGill Youth Lifestyle Intervention With Food and Exercise Study \\
\hline Stated purpose of study & $\begin{array}{l}\text { "The aim of this study is to determine the effects of a 1 y family centered lifestyle intervention, fo- } \\
\text { cused on both nutrient dense food including increased intakes of milk and alternatives, plus total } \\
\text { and weight bearing PA, on body composition and bone mass in overweight or obese children." }\end{array}$ \\
\hline Notes & $\begin{array}{l}\text { Study author reply: } 11 \text { October 2016."Thank you for asking, our work is in press with Can J Public } \\
\text { Health, we do not yet have page proofs." }\end{array}$ \\
\hline
\end{tabular}


NCT01506245 (Continued)

Primary purpose: treatment

Condition: childhood obesity
Enrollment: 75
Inclusion criteria:
- childhood obesity (>97 percentile WHO references)
Exclusion criteria:
-
- geing involved in any weight control, physical activity, behaviour therapy, or gastric surgery pro-
- medications or hormones, which may influence cardiovascular function, body composition, lipid
- or glucose metabolism in the preceding 6 months
- genetic disorder or another chronic disease

Interventions

Intervention: family-based behavioural therapy (6 months) either in group or in individual setting. Parents can choose between the 2 types of therapy

Control: no intervention

\begin{tabular}{ll}
\hline Outcomes & BMI, total body and abdominal fat, waist circumference, blood pressure, arterial intima-media \\
thickness, arterial flow-mediated dilation, arterial stiffness, cardiorespiratory fitness, physical ac- \\
tivity, biological markers, quality of life, child's behaviour, parental psychological health
\end{tabular}

\begin{tabular}{ll}
\hline Study identifier & NCT01506245 \\
\hline Official title & $\begin{array}{l}\text { Exercise training and family-based behavioural treatment in pre-pubertal obese children and their } \\
\text { mother }\end{array}$ \\
\hline Stated purpose of study & $\begin{array}{l}\text { "The aim of this study is to compare the effects of exercise training and family-based behavioural } \\
\text { treatment, either in individual or in group setting, in pre-pubertal children and their mother" }\end{array}$ \\
\hline Notes & $\begin{array}{l}\text { Estimated completion date June } 2012 \text { - trial record not updated since Janaury 2012. Emailed study } \\
\text { author (April 2016) - no reply. Conference abstract identified }\end{array}$ \\
\hline
\end{tabular}

\begin{tabular}{ll}
\hline Methods & Type of study: interventional \\
Allocation: randomised \\
Intervention model: parallel \\
Masking: open label \\
Primary purpose: treatment \\
\hline Condition: diabetes mellitus (type 2), obesity \\
Enrollment: 52 \\
Inclusion criteria: \\
- age 4-8 year
\end{tabular}

Diet, physical activity and behavioural interventions for the treatment of overweight or obese children from the age of 6 to 11 years 
NCT01610219 (Continued)

- age- and sex-specific $B M I \geq 95$ th percentile

\section{Exclusion criteria:}

- children with serious medical conditions

- children who show signs of elevated psychopathology, as assessed by the Child Behavior Checklist $(\mathrm{CBCL})$

- children of parents with significantly elevated psychiatric disorders

Interventions

Intervention: Lifestyle modification for diabetes prevention (traffic light diet, self-monitoring, parental behavioural training, promoting physical activity)

Control: Nutrition and physical activity family-based intervention (no behavioural skills training, goal setting, self-monitoring or physical activity tool kit)

Outcomes

BMI/BMI z score (primary), \% overweight (primary), waist circumference (primary), blood pressure (primary), pulse (primary), physical activity via accelerometer (primary), glucose (primary), insulin (primary), lipid profile measures (primary), dietary intake (primary), parent BMI

\begin{tabular}{ll}
\hline Study identifier & NCT01610219 \\
\hline Official title & Lifestyle modification for type 2 diabetes prevention in overweight youth \\
\hline Stated purpose of study & $\begin{array}{l}\text { "The objective of proposed study was to test a family-based intervention designed to reduce excess } \\
\text { body weight, improve metabolic and cardiovascular profile, and improve diet and physical activity } \\
\text { levels in 4 - 8 year old youth who are "at risk" for T2D" }\end{array}$
\end{tabular}

Type of study: interventional
Allocation: randomised
Intervention model: parallel
Masking: open label
Primary purpose: treatment
Condition: obesity
Enrollment: 65
Inclusion criteria:
- ages 4-8 years old
- BMI $\geq 85$ th percentile (based on age and sex)
- consumes large ( $\geq 16$ oz/d) sugar sweetened beverages
Exclusion criteria:
- child has a serious medical condition
- signs of elevated psychopathology are present, as assessed by the Child Behavior Checklist (CBCL)
- parent demonstrates elevated psychiatric problems or eating disorders
- failure of parent or child to meet BMI criteria


NCT01662570 (Continued)

Interventions

Intervention: Beverage choice lifestyle modification

Control: Nutrition education (NE)

BMI, BMI z score, BMI percentile, child percent overweight, waist circumference, energy intake, sugar-sweetened beverage intake, treatment acceptance/satisfaction, child preferences and motivation for sugar-sweetened beverages

\begin{tabular}{ll}
\hline Study identifier & NCT01662570 \\
\hline Official title & Beverage choice and lifestyle modification in overweight youth \\
\hline Stated purpose of study & $\begin{array}{l}\text { "This research study developed and tested a "Beverage Choice and Lifestyle Modification" (BCLM) } \\
\text { intervention for } 4 \text { to } 8 \text { year old children who are at-risk for being overweight or are overweight and } \\
\text { who consume large amounts of sugar sweetened beverages and juice." }\end{array}$ \\
\hline Notes & Study completed. Emailed author (April 2016) - no reply \\
\hline
\end{tabular}

Type of study: interventional
Allocation: randomised
Intervention model: parallel
Masking: open label
Primary purpose: treatment
Condition: obesity
Enrollment: 549
Inclusion criteria:
- Age 2-12 years
- BMI $\geq 85$ th centile
Exclusion criteria:
- obesity-related complications that would interfere with participant
- underlying causes of obesity
- severe psychological problems
- participation in an obesity treatment programme in the year prior to enrolment

Interventions

Intervention: the intervention consists of: (1) BMI screening, (2) Next Steps brief counselling materials for the healthcare provider, (3) a 3-month intensive Mind Exercise Nutrition Do It! and Coordinated Approach To Child Health (MEND/CATCH) phase, which included the Mind Exercise Nutrition Do it! with adapted CATCH activities, and (5) a 9-month transition MEND/CATCH Transition phase of monthly reinforcement sessions for parents and children, and twice-weekly Young Men's Christian Association (YMCA) sports for children. Community Health Workers (CHWs) serve as programme liaisons and assist in delivering all intervention group sessions as well as tracking families

Control: (active comparator - additional component to a behaviour-changing intervention and usual care) Next Steps brief clinical intervention which is a 12-month clinic-based programme conducted at 12 partner healthcare clinics and entailed (1) EHR changes to support childhood obesity clinical visits; (2) BMI screening, (3) Next Steps brief counselling materials for the healthcare 
NCT02724943 (Continued)

provider, and (4) Next Steps self-paced booklet for parents and children to work on nutrition and physical activity targets in a self-directed manner. Families were encouraged to seek repeated clinical visits to address child obesity.

inary outcome: change in obesity prevalence at baseline, 3 and 12 months

Secondary outcomes: waist-to-height ratio, fat-free mass, blood pressure, fitness, quality of life at the same time points

\begin{tabular}{|c|c|}
\hline Study identifier & NCT02724943 \\
\hline Official title & Texas Childhood Obesity Research Demonstration (TX CORD) Project \\
\hline \multirow[t]{2}{*}{ Stated purpose of study } & $\begin{array}{l}\text { "Aim 1: To implement and evaluate the efficacy of a systems approach to child obesity on reducing } \\
\text { BMI (expressed as \%95th percentile) by embedding a 12-month family-based secondary preven- } \\
\text { tion program within a community primary prevention program. The secondary prevention weight } \\
\text { management program will target overweight/obese children and their families in the primary pre- } \\
\text { vention catchment areas in Austin and Houston. Overweight/obese children (total } \mathrm{N}=576 \text { ), aged } \\
2-12 \text { years, will be randomly assigned to either the } 12 \text {-month secondary prevention program (ex- } \\
\text { perimental) or the community primary prevention program alone (control), in equal age subgroups } \\
\text { (2-5, 6-8, and 9-12 years). Analyses will be conducted by age group, and outcomes will include BMI } \\
\text { as expressed as \%95th percentile), obesity-related behaviors, quality of life, and program use indi- } \\
\text { cators. }\end{array}$ \\
\hline & $\begin{array}{l}\text { Aim 2: To quantify the incremental cost-effectiveness of the } 12 \text {-month family-based secondary } \\
\text { prevention program relative to primary prevention alone for child obesity. Activity Based Costing } \\
\text { methods will be used to quantify the incremental cost of delivering the secondary prevention pro- } \\
\text { gram relative to optimized healthcare. These costs will then be combined with the effectiveness } \\
\text { data to quantify the incremental cost-effectiveness of the community-based intervention." }\end{array}$ \\
\hline Notes & $\begin{array}{l}\text { Study author did not reply, October 2016. Study completed. Protocol and baseline data available } \\
\text { but no follow-up data identified }\end{array}$ \\
\hline
\end{tabular}

\section{NCT02771951}

Methods

Type of study: interventional

Allocation: randomised

Intervention model: factorial assignment

Masking: single-blind (outcome assessor)

Primary purpose: treatment

\section{Participants}

\section{Condition: obesity}

\section{Enrollment: 297}

\section{Inclusion criteria:}

- aged 6-11 years

- Latino boys and girls

- clinic visit within past 24 months prior to enrolment in study

- BMI \% for age/gender between 75th-99.9th centiles

- plan on living in target area for following 18 months

- have transportation to participating clinic 
NCT02771951 (Continued)

Exclusion criteria: not provided

Interventions

Intervention: participants receive 7 group classes taught by trained clinic health educators; in addition to a series of phone calls; clinical visits with a mid-level provider; and 6 booster group classes over 1 year

Control: usual care provision of up to 2 visits with a usual care health educator over 1 year

\begin{tabular}{ll}
\hline Outcomes & $\begin{array}{l}\text { Primary outcome: BMI over } 1 \text { year } \\
\text { Secondary outcomes: not stated }\end{array}$ \\
\hline Study identifier & NCT02771951 \\
\hline Official title & Clinical/behavioral approach to overweight in Latino youth: luces de cambio \\
\hline Stated purpose of study & No official aim stated \\
\hline Notes & Study completed. Study author reply: 11 October 2016. "Still ongoing...give us a few months." \\
& $\begin{array}{l}\text { Unclear whether healthy weight children are included as it doesn't state which BMI growth refer- } \\
\text { ence is being used - however authors state that the overweight participants recruited from paedi- } \\
\text { atric clinics }\end{array}$
\end{tabular}

\section{NCT02779647}

\begin{tabular}{ll}
\hline Methods & Allocation: randomised \\
Intervention model: parallel \\
Masking: open label \\
Primary purpose: treatment \\
Condition: obesity \\
Enrollment: 54 \\
Inclusion criteria: \\
- age 8-12 years \\
• obese boys and girls \\
Exclusion criteria: \\
• hormonal, orthopedic, respiratory or other complications
\end{tabular}

Interventions

Intervention: physical activity programme (4 x 90 min sessions/wk for 9 months and nutrition advice for children and parents)

Control: nutrition advice only

Outcomes Primary outcome: body composition over 12 months

Secondary outcomes: physical activity (1 month), sleep apneas ( 6 months)

Study identifier

NCT02779647

Diet, physical activity and behavioural interventions for the treatment of overweight or obese children from the age of 6 to 11 years 
NCT02779647 (Continued)

Official title Play as a method to reduce overweight and obesity in children. Kids-Play Study

Stated purpose of study $\quad$ "The aim of this study is to analyse an intervention based on play as a means of improving the body composition of children with overweight or obesity."

Notes Study author did not reply, October 2016. Study completed

\section{NCT02794090}

\begin{tabular}{ll}
\hline Type of study: interventional \\
Allocation: randomised \\
Intervention model: parallel \\
Masking: open label \\
Primary purpose: treatment \\
Condition: obesity \\
Enrollment: 37 \\
Inclusion criteria: \\
Participants \\
- boge 5-14 years \\
- parents had to attend at least 4 or 7 meetings in the parental education group \\
Exclusion criteria: \\
- not speaking Swedish \\
- obesity-related syndromes
\end{tabular}

Interventions

Intervention: telephone consultation every month (except summer holidays) for 18 months. The treating nurse communicating with 1 of the parents

Control: usual care according to regular treatment routines at the clinic

Outcomes Primary outcome: BMI z score - baseline, during intervention and follow-up in total 3.7 years

Secondary outcomes: health care personnel time, families' experience of the programme

\begin{tabular}{ll}
\hline Study identifier & NCT02794090 \\
\hline Official title & $\begin{array}{l}\text { Exclusive telephone coaching in maintaining weight loss - an randomized controlled trial of child- } \\
\text { hood obesity treatment }\end{array}$ \\
\hline Stated purpose of study & No official aim stated \\
\hline Notes & $\begin{array}{l}\text { Author reply: } 12 \text { October 2016. "The paper is submitted and we are waiting for response from our } \\
\text { first revision." }\end{array}$ \\
\hline
\end{tabular}

Diet, physical activity and behavioural interventions for the treatment of overweight or obese children from the age of 6 to 11 years 
Shapiro 1976

Methods

\section{Participants}

Interventions

\section{Outcomes}

Study identifier

\section{Official title}

Stated purpose of study

\begin{tabular}{ll}
\hline Notes & $\begin{array}{l}\text { Cannot obtain full publication from the } \\
\text { British Library }\end{array}$
\end{tabular}

Terwilliger 2008

Methods

Participants

Interventions

\section{Outcomes}

Study identifier

Official title

Stated purpose of study

Notes

Thesis - unable to obtain

BIA: Bioelectrical impedance analysis; BMI: body mass index; CBCL: Child Behavior Checklist; DBP: diastolic blood pressure; DEXA: dual energy X-ray absorptiometry; EHR: electronic health records; N: number; NCEP: National Cholesterol Education Program; PA: physical activity; SBP: systolic blood pressure; SDS: standard deviation score; SFM: Social Facilitation Maintenance; T2D: Type II diabetes; WHO: World Health Organization

\section{Characteristics of ongoing studies [ordered by study ID]}

\section{ACTRN12613001037796}

\section{Trial name or title}

Effect of exercise intensity on cardiac and vascular function, and intra-abdominal fat in obese children and adolescents

Methods

Type of study: interventional, efficacy/safety

Allocation: randomised

Intervention model: parallel

Masking: blinded (masking used). The people assessing the outcomes. The people analysing the results/data 
Primary purpose: treatment

\begin{tabular}{|c|c|}
\hline Participants & $\begin{array}{l}\text { Condition: obesity } \\
\text { Enrolment: target } 60 \\
\text { Inclusion criteria: } \\
\text { - } 7-16 \text { years } \\
\text { - boys and girls } \\
\text { - obese (CDC growth charts) - those above 99th centile will b } \\
\text { - all ethnic groups } \\
\text { - blood pressure < } 95 \text { th percentile } \\
\text { - fasting total cholesterol }<5.5 \text { mmol/L and low-density lipop } \\
\text { - participants willing to be randomised to high or moderate in } \\
\text { - } \text { able to follow protocol } \\
\text { Exclusion criteria: } \\
\text { - participating in another study } \\
\text { - smoking } \\
\text { - diabetes } \\
\text { - taking medications such as steroids } \\
\text { - coronary heart disease or congenital cardiac abnormalities } \\
\text { - family history of hypertrophic obstructive cardiomyopathy } \\
\text { - abnormalities during the resting or exercise stress echo } \\
\text { - orthopaedic and/or neurological limitations to exercise } \\
\text { - considerable pulmonary disease } \\
\text { - self-reported kidney failure } \\
\text { - major organ transplant } \\
\text { - epilepsy or history of seizures } \\
\text { attention deficit hypersensitivity disorder diagnosis }\end{array}$ \\
\hline
\end{tabular}

Intervention 1: a high-intensity interval training group (dietary sessions over 3 months, physical activity training sessions plus home training)

Intervention 2: a moderate-intensity exercise group (same as above but moderate intensity training instead of high)

Outcomes

Other outcome(s): peak systolic (S') tissue velocity (primary), intra-abdominal fat via MRI (primary), arterial endothelial-dependent dilatation (primary), arterial stiffness, VO2 max, body composition via DEXA, oxidised LDL, adiponectin, total nitrate, HOMA, blood pressure, diet, accelerometry data, height, weight, waist circumference

Starting date

Start date: estimated first participant enrolled: 1 October 2013

Completion date: unclear. Estimated last participant enrolled: 1 January 2015

Contact information

Study identifier
Responsible party/principal investigator: Miss Katrin Dias, The University of Queensland, Australia, katrin.dias@uqconnect.edu.au 
ACTRN12613001037796 (Continued)

Stated purpose of study

Quote: "The objective of the study is to investigate the effects of high intensity exercise intensity on myocardial and arterial function, intra-abdominal fat and cardiovascular disease risk factors in obese children and adolescents over one year."

Notes

Study author reply 12 October 2016: "While the study is still ongoing (12-month follow up), we are currently collating results from the three-month supervised phase of the study. We aim to submit two papers with these results to journals by the end of the year. Given the time taken from submission to publication, I would expect them to be published between mid to end 2017."

\section{ChiCTR-IOB-15005874}

Trial name or title

Effects of weight management program on postural stability and neuromuscular function among obese children

Type of study: interventional
Allocation: randomised
Intervention model: parallel
Masking: single-blind
Primary purpose: treatment
Condition: obesity
Enrollment: target 120
Inclusion criteria:
Participants children aged 8 -10 years
- obese (IOTF definition
- tanner stage 1
- can participate in 3 exercise classes/week for 6 months,
- 1 parent willing to attend treatment meetings and no family member involved in another weight
control programme

\section{Exclusion criteria:}

- cannot communicate in Chinese

- diabetic

- suffer from psychiatric disorder

- angina in past 3 months or severe dyspnoea at rest

- syndromic of medicinal cause of obesity

- other illness that prevents participation

Interventions

Intervention: weight management: combined diet and exercise programme: dietary intervention only ( 6 dietetic visits) and weekly nurse telephone support. Exercise: 50 min session at sports centre $1 \mathrm{x} /$ week to be repeated twice that week at home

Control: 60 min weekly education session

Outcomes Primary outcome: body height and weight

Secondary outcomes: waist and hip circumference, $\%$ body fat, movement biomechanistics and postural stability tests 
ChiCTR-IOB-15005874 (Continued)

Completion date: unclear until completed

\begin{tabular}{ll}
\hline Contact information & Responsible party/principal investigator: wanglin@sus.edu.cn \\
\hline Study identifier & ChiCTR-IOB-15005874 \\
\hline Official title & Not provided \\
\hline Stated purpose of study & $\begin{array}{l}\text { "The present study attempts to investigate the effect of a six-month weight management program } \\
\text { on postural stability and neuromuscular control among obese children" }\end{array}$ \\
\hline Notes & $\begin{array}{l}\text { Study author reply: 11 October 2016. "The study is ongoing now. In fact, we meet a few problems in } \\
\text { participant's recruitment and funding support, therefore, there are not any data currently." }\end{array}$ \\
\hline
\end{tabular}

DRKS00007879

Trial name or title $\quad \begin{aligned} & \text { Development and evaluation of a computer-based self-regulation training for obese children and } \\ & \text { adolescents }\end{aligned}$

adolescents

\begin{tabular}{l} 
Type of study: interventional \\
Allocation: randomised \\
Intervention model: parallel \\
Masking: blinded patient/subject, investigator/therapist, caregiver, assessor \\
Primary purpose: treatment \\
Condition: obesity \\
Enrollment: target 226 \\
Inclusion criteria: \\
Participants 8 -16 years \\
- boys and girls \\
- BMI > 97 centile \\
Exclusion criteria: \\
- secondary obesity \\
- hyperkinetic disorder with medication \\
\hline
\end{tabular}

Interventions

Intervention: self-regulation training with the developed computer program (Approach-Avoidance-Training), in addition to treatment as usual (inpatient rehab treatment), conducted over 6 sessions (10-15 min each), over 2 consecutive weeks.

Control: placebo training (similar to the intervention computer program but with no learning effect) in addition to treatment as usual (inpatient rehab treatment), conducted over 6 sessions (10-15 min each), over 2 consecutive weeks 
DRKS00007879 (Continued)

Starting date

Start date: 6 March 2015

Completion date: unclear until study has completed

\section{Contact information Responsible party/principal investigator:}

Prof. Petra Warschburger, Karl-Liebknecht-Str. 24/25, 14476 Potsdam, Germany. E-mail: warschb at uni-potsdam.de

\begin{tabular}{ll}
\hline Study identifier & DRKS00007879 \\
\hline Official title & $\begin{array}{l}\text { Development and evaluation of a computer-based self-regulation training for obese children and } \\
\text { adolescents }\end{array}$ \\
\hline Stated purpose of study & No formal aims provided \\
\hline Notes & $\begin{array}{l}\text { Author reply: } 12 \text { October 2016. "Yes, our study is still ongoing and we expect the results in April/May } \\
\text { next year. A paper of our pilot study is in preparation." }\end{array}$ \\
\hline
\end{tabular}

\section{ISRCTN81798055}

Trial name or title

Child weigHt mANaGement for Ethnically diverse communities study (CHANGE)

Methods

Type of study: interventional

Allocation: randomised

Intervention model: parallel

Masking: -

Primary purpose: prevention

Condition: childhood obesity
Enrolment: estimate 160-180
Inclusion criteria:
- phase 1
$*$ aged 4-11 years
$*$ overweight/obese
$*$ Bangladeshi and Pakistani parents and carers
$*$ offered the existing children's weight management service.
phase 2
$*$ aged 4-11 years
$*$ overweight/obese
Exclusion criteria:
- phase 2
$*$ families who self-refer to the service but do not have an overweight or obese child (defined as
$\geq 91$ st centile) aged 4-11 year will be excluded from the study


ISRCTN81798055 (Continued)

Control: existing children's weight management programme, 7 weeks

Completion rates (primary), height, weight, BMI, waist circumference, percentage body fat (BIA), dietary intake, physical activity (accelerometry), parent-reported sedentary behaviours, health-related quality of life, a health utility measure, body image, self-concept

Starting date

Start date: 1 September 2014 (recruitment)

Completion date: unclear. 28 February 2017 (recruitment)

\begin{tabular}{ll}
\hline Contact information & Responsible party/principal investigator: Dr Miranda Pallan, University of Birmingham, UK \\
\hline Study identifier & ISRCTN81798055 \\
\hline Official title & $\begin{array}{l}\text { Development of a culturally adapted weight management programme for children of Pakistani and } \\
\text { Bangladeshi origin }\end{array}$ \\
\hline Stated purpose of study & $\begin{array}{l}\text { "Therefore the main aim of this study is to develop and assess the feasibility and acceptability of a } \\
\text { weight management programme for children aged 4-11 years and their families, tailored to be cul- } \\
\text { turally relevant to Bangladeshi and Pakistani communities, but also suitable for delivery to an eth- } \\
\text { nically diverse population." }\end{array}$ \\
\hline Notes & Study not yet completed \\
\hline
\end{tabular}

Moore 2013

\begin{tabular}{ll}
\hline Trial name or title & Acronym: IMPACT \\
\hline Methods & Type of study: interventional \\
& Allocation: randomised \\
& Intervention model: parallel \\
& Masking: assessor blinded \\
& Primary purpose: treatment \\
\hline
\end{tabular}

Participants

Condition: childhood obesity

Enrolment: unknown

Inclusion criteria:

- entering 6th grade

- $\mathrm{BMI} \geq 85$ th percentile determined from height and weight measurements (CDC growth charts)

- provision of consent by parents and assent by children

\section{Exclusion criteria:}

- medications that alter appetite or weight

- stage 2 hypertension or stage 1 hypertension with end organ damage (e.g. left ventricular hypertrophy, microalbuminuria)

- sickle cell disease

- severe behavioural problems that preclude group participation as reported by parent/guardian

- involvement in another weight management programme

- family expectation to move from the region within 1 year

Diet, physical activity and behavioural interventions for the treatment of overweight or obese children from the age of 6 to 11 years 
Control: SystemCHANGE intervention (based on system process improvement theory and focuses on redesign of the activities in a family's daily routines related to home, school, and work to support positive behaviour changes)

$\begin{array}{ll}\text { Outcomes } & \text { Weight, height, waist circumference, triceps skinfold, BMI, dietary intake, physical activity (ac- } \\ & \text { celerometry), blood pressure, haemogloblin A1c (HbA1c), glucose, total cholesterol, LDL-choles- } \\ \text { terol, HDL-cholesterol, triglycerides, high-sensitivity C-reactive protein (hs-CRP), insulin and ala- } & \text { nine aminotransferase (ALT), child's self-efficacy, social support, motivation, and family prob- } \\ \text { lem-solving, systems thinking, self-regulation child's self-efficacy, social support, motivation, and } \\ \text { family problem-solving, self-regulation, sleep, stress levels, cardiovascular risk, socioeconomic and } \\ \text { demographic factors, environmental (home, school, neighbourhood) factors, peer norms }\end{array}$

Starting date

Start date: unclear

Completion date: unclear

Responsible party/principal investigator: Shirley Moore, Frances Payne Bolton School of Nursing, Case Western Reserve University, Cleveland, USA.smm8@case.edu

\begin{tabular}{ll}
\hline Study identifier & - \\
\hline Official title & A multi-level family and school intervention targeting obesity in urban youth \\
\hline Stated purpose of study & $\begin{array}{l}\text { "The primary aim of this study is to compare the effects of three distinct behavioral obesity man- } \\
\text { agement interventions on BMl in overweight/obese middle school, urban youth." }\end{array}$ \\
\hline Notes & $\begin{array}{l}\text { Author reply: } 16 \text { October 2016. "This study is still ongoing. We will not be unblended until spring } \\
2017 \text { and cannot share results prior to publication of the results (hopefully fall 2017)." }\end{array}$ \\
\hline
\end{tabular}

\section{NCT01221220}

\section{Trial name or title}

Environmental strategies \& behavior change to reduce overeating in obese children

Type of study: interventional

Allocation: randomised

Intervention model: parallel

Masking: single-blind (assessor)

Primary purpose: treatment

\section{Participants}

\section{Condition: obesity}

Enrolment: estimated 160

\section{Inclusion criteria:}

- 8-15 years

- obese based on 95th percentile (CDC growth references)

- parent and child must both want to join the study

- parent and child must agree to attend sessions and not miss more than 2 consecutive sessions 
NCT01221220 (Continued)

\section{Exclusion criteria:}

- diagnosed with a medical condition affecting growth (e.g. type 1 or 2 diabetes, chronic renal diseases, pregnancy)

- taking medication known to affect growth

- have a condition which would limit their participation in the study or the assessments

- not able to read or understand English or Spanish, or unable to compete consent forms

- within the next 18 months the family plans to move from the San Francisco Bay area

Interventions

Intervention: behavioural: Standard Packard Pediatric Weight Control Program plus home-based advising on environmental changes (6 months program)

Control: Standard Packard Pediatric Weight Control Program only

\begin{tabular}{ll}
\hline Outcomes & BMI (primary), waist circumference, triceps skinfold, resting heart rate, dietary intake, weight con- \\
cerns, depressive symptoms, daily energy intake, physical activity, blood pressure, fasting blood \\
lipids
\end{tabular}

Starting date

Start date: September 2010

Completion date: February 2015 (final assessment)

\begin{tabular}{ll}
\hline Contact information & Responsible party/principal investigator: Thomas Robinson, Stanford University, USA \\
\hline Study identifier & NCT01221220 \\
\hline Official title & Environmental strategies \& behavior change to reduce overeating in obese children \\
\hline Stated purpose of study & $\begin{array}{l}\text { "There is a need for effective weight control methods for obese children. Environmental strategies } \\
\text { such as reducing the size of dishware and serving utensils, storing food out of view and reducing } \\
\text { food consumption while watching television may reduce food intake without requiring conscious, } \\
\text { cognitive self-control. The investigators propose to test these methods when added to a current } \\
\text { state-of-the-art behavioral program." }\end{array}$ \\
\hline Notes & Author reply: 11 October 16. Ongoing. Not yet published \\
\hline
\end{tabular}

NCT01574352

\begin{tabular}{ll}
\hline Trial name or title & Acronym: OOIS \\
\hline Methods & Type of study: interventional \\
& Allocation: randomised \\
& Intervention model: parallel \\
& Masking: single blind (investigator) \\
& Primary purpose: treatment
\end{tabular}

Participants

Condition: children, overweight, obesity, metabolic syndrome

Enrolment: 100

Inclusion criteria:

- live in municipality of Odense, Denmar

- overweight or obese (BMI) - IOTF

Diet, physical activity and behavioural interventions for the treatment of overweight or obese children from the age of 6 to 11 years 
NCT01574352 (Continued)

\section{Exclusion criteria:}

- participating in other similar research studies

- following a special school programme

- the use of weight-altering medicine 3 months before the baseline

- motor-skill determined handicap which hinders participation

Interventions

Intervention: behavioural: intervention camp (6-week day-camp: physical activity, health education, healthy foods, social activities)

Control: behavioural: small intervention

(weekly 1-h session over 6 weeks plus 2 parental diet and exercise information sessions)

$\begin{array}{ll}\text { Outcomes } & \text { BMI (primary), cognitive function, motor skills, body composition by DEXA, brain-derived neu- } \\ \text { rotrophic factor, blood pressure, subclinical atherosclerosis, cardio-respiratory fitness, insulin, glu- } \\ \text { cose, blood lipids, C-reactive protein, waist/hip circumference, clustered CVD risk factor, physical } \\ \text { strength measured by hand grip and Sargent vertical jump } \\ \text { Endpoint: } 12 \text { months }\end{array}$

Starting date

Start date: April 2012

Completion date: July 2017

Contact information

Study identifier

Official title

Stated purpose of study

Responsible party/principal investigator: Lars Bo Andersen, Professor, Center of Research in Childhood Health (RICH), University of Southern Denmark

NCT01574352

The Odense Overweight Intervention Study (OOIS): a randomized controlled trial on overweight prevention in children
"This study is carried through as a randomized controlled trial which investigates the effect of participating in a 6 week health promoting resident for overweight fifth grade children camp followed by 42 weeks of family support"

Notes Data collection is complete.

Protocol: Larsen et al. Effectiveness of a 1-year multi-component day-camp intervention for overweight children: study protocol of the Odense overweight intervention study (OOIS). BMC Public Health 2014, 14:313

\begin{tabular}{ll}
\hline Trial name or title & Acronym: Stanford GOALS \\
\hline Methods & Type of study: interventional \\
& Allocation: randomised \\
& Intervention model: parallel \\
& Masking: single blind (outcome assessment) \\
& Primary purpose: treatment \\
\hline
\end{tabular}


NCT01642836 (Continued)

\author{
Enrolment: 240 \\ Inclusion criteria: \\ - 7-11 years \\ - $\mathrm{BMI} \geq 85$ th percentile for age and sex on the $2000 \mathrm{CDC}$ BMI reference
}

\title{
Exclusion criteria:
}

- have been diagnosed with a medical condition affecting growth (a genetic or metabolic disease/syndrome associated with obesity, type 1 diabetes, type 2 diabetes taking medication, chronic gastrointestinal diseases, chronic renal diseases, uncorrected structural heart disease, heart failure, heart transplant, anorexia nervosa or bulimia nervosa or binge eating disorder (present or past), AIDS or HIV infection, pregnancy);

- take medications affecting growth (systemic corticosteroids $>2$ weeks in the past year, insulin, oral hypoglycaemic, thyroid hormone, growth hormone);

- have a condition limiting their participation in the interventions (e.g. unable to participate in routine physical education classes at school, requiring oxygen supplementation for exertion, developmental or physical disability preventing participation in interventions, children or parents/guardians who cannot medically participate in mild dietary restrictions and/or increased physical activity for any reason);

- have a condition limiting participation in the assessments (child or primary caregiver not able to read surveys in English or Spanish, child 2 or more grade levels delayed in school for reading and writing in native language);

- are unable to read, understand or complete informed consent in English or Spanish;

- plan to move from the San Francisco Bay Area within the next 36 months;

- are deemed to have another characteristic that makes them unsuitable for participation in the study in the judgment of the Principal Investigator

Interventions

Intervention: multi-component, multi-level, multi-setting (MMM) - sports programme, homebased family intervention, behavioural counselling

Control: enhanced standard care/health and nutrition education intervention

BMI (primary), physical activity (accelerometry), waist circumference, triceps skinfold thickness,
resting blood pressure, resting heart rate, cholesterol, triglycerides, insulin, glucose, haemoglobin
Alc, HsCRP, ALT, screen time and other sedentary behaviours, energy intake, waist-to-height ratio,
weight concerns, depressive symptoms, school performance, sleep habits

Endpoint: 3 years

Starting date

Start date: July 2012

Completion date: April 2017

\begin{tabular}{ll}
\hline Contact information & Responsible party/principal investigator: Thomas N Robinson, Stanford University, USA \\
\hline Study identifier & NCT01642836 \\
\hline Official title & Clinic, family \& community collaboration to treat overweight and obese children \\
\hline Stated purpose of study & $\begin{array}{l}\text { "Primary Research Question: Will a 3-year, innovative, interdisciplinary, multi-component, mul- } \\
\text { ti-level, multi-setting (MMM) community-based intervention to treat overweight and obese children } \\
\text { significantly reduce BMI compared to an enhanced standard care/health and nutrition education } \\
\text { active comparison intervention?" }\end{array}$ \\
\hline
\end{tabular}

\section{Notes}

Ongoing, finished recruiting

Protocol: Robinson TN, Matheson D, Desai M, Wilson DM, Weintraub DL, Haskell WL, McClain A, McClure S, Banda JA, Sanders LM, Haydel KF, Killen JD. Family, community and clinic collabora-

Diet, physical activity and behavioural interventions for the treatment of overweight or obese children from the age of 6 to 11 years 


\begin{tabular}{|c|c|}
\hline Trial name or title & Acronym: CIRCUIT \\
\hline \multirow[t]{5}{*}{ Methods } & Type of study: interventional \\
\hline & Allocation: randomised \\
\hline & Intervention model: parallel \\
\hline & Masking: double-blind (subject, caregiver, investigator, outcomes assessor) \\
\hline & Primary purpose: prevention \\
\hline \multirow[t]{7}{*}{ Participants } & Condition: obesity \\
\hline & Enrolment: 100 \\
\hline & Inclusion: \\
\hline & - children aged 6-18 years \\
\hline & - $\mathrm{BMI}>$ 95th percentile for age and sex \\
\hline & Exclusion: \\
\hline & $\begin{array}{l}\text { - children with a physical or psychological condition that would impair their ability to participate } \\
\text { in physical activity }\end{array}$ \\
\hline \multirow[t]{2}{*}{ Interventions } & Intervention: sensor-based PA intervention \\
\hline & Control: traditional PA counselling \\
\hline Outcomes & Change in physical activity levels (primary), blood pressure, glucose homeostasis, lipid status, BMI \\
\hline \multirow[t]{2}{*}{ Starting date } & Start date: January 2015 \\
\hline & Completion date: January 2019 \\
\hline Contact information & Responsible party/principal investigator: Melanie Henderson, St. Justine's Hospital, Canada \\
\hline Study identifier & NCT01736748 \\
\hline Official title & $\begin{array}{l}\text { Implementing Dynamo: a tailored lifestyle promotion intervention among pediatric patients with } \\
\text { cardiometabolic risk factors }\end{array}$ \\
\hline Stated purpose of study & $\begin{array}{l}\text { "Its primary goal is to promote physical activity and reduce sedentary time to improve children's } \\
\text { cardiometabolic profile" }\end{array}$ \\
\hline Notes & This study was not yet open for participant recruitment (as of March 2016) \\
\hline
\end{tabular}


NCT02082080

\begin{tabular}{|c|c|}
\hline Trial name or title & Prevention and control of obesity in primary school children in Tehran \\
\hline Methods & $\begin{array}{l}\text { Type of study: interventional } \\
\text { Allocation: randomised } \\
\text { Intervention model: parallel } \\
\text { Masking: open label } \\
\text { Primary purpose: prevention }\end{array}$ \\
\hline Participants & $\begin{array}{l}\text { Condition: childhood obesity prevention } \\
\text { Enrolment: estimated } 360 \\
\text { Inclusion criteria: } \\
\text { - BMI z score } \geq 1 \text { (WHO) } \\
\text { - students in the fifth or sixth grades (age 9-14) } \\
\text { Exclusion criteria: } \\
\text { - metabolic disorders (hypo or hyperthyroidism) } \\
\text { - any disease which interferes with adherence to the intervention } \\
\text { - intake of any appetite-reducing drug } \\
\text { - doing professional sports } \\
\text { - being on a weight-reduction diet }\end{array}$ \\
\hline
\end{tabular}

Interventions Intervention: education and social support intervention

Control: no care

\begin{tabular}{ll}
\hline Outcomes & Primary outcome measure(s): Pain on the 11-point Short Pain Scale (SPS-11), BMI \\
\hline Starting date & Start date: December 2012 \\
& Completion date: June 2013 \\
\hline Contact information & Responsible party/principal investigator: Tehran University of Medical Sciences, Iran \\
\hline Study identifier & NCT02082080 \\
\hline Official title & Prevention and control of obesity in primary school children in Tehran \\
\hline Stated purpose of study & $\begin{array}{l}\text { "This study evaluates the effect of an interventional model for preventing and controlling over- } \\
\text { weight and obesity in male and female fifth-graders" }\end{array}$ \\
\hline Notes & Unable to find contact details \\
\hline
\end{tabular}

\section{NCT02124460}

\section{Trial name or title}

Methods
Connect 4 Health: an intervention to improve childhood obesity outcomes

Type of study: interventional

Allocation: randomised 
NCT02124460 (Continued)

Intervention model: parallel

Masking: single-blind (outcomes assessor)

Primary purpose: treatment

Condition: overweight, obesity
Enrolment: 721
Inclusion criteria:
- aged $2.0-12.9$ years at baseline primary care visit,
- BMI $\geq 85$ th percentile for age and sex at baseline primary care visit
- at least 1 parent has an active email address
- at least 1 parent is comfortable reading and speaking in English

\section{Exclusion criteria:}

- children who do not have at least 1 parent/legal guardian who is able to follow study procedures for 1 year

- families who plan to leave Harvard Vanguard Medical Associates within the study time frame

- families for whom the primary care clinician thinks the intervention is inappropriate, e.g. emotional or cognitive difficulties

- children who have a sibling already enrolled in the study

- children with chronic conditions that substantially interfere with growth or physical activity participation

Interventions

Intervention: Connect 4 Health: using health coaches for behavioural counselling and community connections

Control: enhanced primary care

\begin{tabular}{ll}
\hline Outcomes & BMI (primary), quality of life (primary), Quality and Family-Centeredness of Pediatric Obesity Care, \\
specified behavioural outcomes, process measures, socioeconomic variables, geographic variables
\end{tabular}

Starting date

Start date: June 2014

Completion date: November 2016

\begin{tabular}{ll}
\hline Contact information & Responsible party/principal investigator: Elsie M Taveras, Massachusetts General Hospital, USA \\
\hline Study identifier & NCT02124460 \\
\hline Official title & Improving childhood obesity outcomes: testing best practices of positive outliers
\end{tabular}

Stated purpose of study "The primary specific aims are to examine the extent to which the intervention, compared to the control condition, results in: A smaller age-associated increase in BMI over a 12-month period.

Improved parental and child ratings of pediatric health-related quality of life."

Notes This study is ongoing, but not recruiting participants (as of March 2016)

\section{NCT02258126}

\begin{tabular}{ll}
\hline Trial name or title & Acronym: EFIGRO \\
\hline Methods & Type of study: interventional \\
\hline
\end{tabular}

Diet, physical activity and behavioural interventions for the treatment of overweight or obese children from the age of 6 to 11 years 
NCT02258126 (Continued)

Allocation: randomised

Intervention model: parallel

Masking: open label

Primary purpose: treatment

\begin{tabular}{|c|c|}
\hline Participants & $\begin{array}{l}\text { Condition: } \\
\text { - non-alcoholic fatty liver disease } \\
\text { - obesity } \\
\text { - metabolic syndrome } \\
\text { Enrolment: } 160 \\
\text { Inclusion criteria: } \\
\text { - } 9-11 \text { years } \\
\text { - overweight or obesity status } \\
\text { Exclusion criteria: } \\
\text { - medical conditions that hamper their participation in the exercise programme } \\
\text { - secondary obesity }\end{array}$ \\
\hline Interventions & $\begin{array}{l}\text { Intervention: multidisciplinary intervention programme - education programme, behavioural ad- } \\
\text { vice, supervised exercise } \\
\text { Control: healthy behaviour-changing education including supportive therapy and behavioural ad- } \\
\text { vice for both children and parents to improve nutrition and physical activity }\end{array}$ \\
\hline Outcomes & $\begin{array}{l}\text { Hepatic fat (primary), insulin sensitivity, serum lipid profile, liver enzymes, dietary habits, physical } \\
\text { activity, body composition, blood pressure, leptin, adiponectin, C-reactive protein (CRP) }\end{array}$ \\
\hline Starting date & $\begin{array}{l}\text { Start date: November } 2014 \\
\text { Completion date: June } 2018\end{array}$ \\
\hline Contact information & $\begin{array}{l}\text { Responsible party/principal investigator: Idoia Labayen, Department of Nutrition and Food } \\
\text { Sciences, Faculty of Pharmacy, University of the Basque Country, Spain }\end{array}$ \\
\hline Study identifier & NCT02258126 \\
\hline Official title & The effect of exercise on hepatic fat in overweight children; the EFIGRO Study \\
\hline Stated purpose of study & $\begin{array}{l}\text { "The objective of the present study is to evaluate the effect of } 6 \text { months exercise intervention pro- } \\
\text { gram on hepatic fat fraction in overweight children" }\end{array}$ \\
\hline Notes & Recruiting participants (as of March 2016) \\
\hline
\end{tabular}

NCT02343367

Trial name or title

Methods
Acronym: $\mathrm{H} 4 \mathrm{~K}$

Type of study: interventional

Allocation: randomised 
NCT02343367 (Continued)

\section{Intervention model: parallel}

Masking: double-blind (caregiver, investigator, outcomes assessor)

Primary purpose: treatment

Condition: paediatric obesity
Enrolment: estimated 460
Inclusion criteria:
- aged $6-11$ years
- identified as Hispanic (by parent/guardian)
- overweight or obese (85th to < 99th percentile for age and gender)
- parent/guardian to participate in intervention
Exclusion criteria:
- child who has a mental, emotional, or physical handicap which may interfere with participation
- cardiovascular, pulmonary, or digestive disease diagnosis
- parent without a cell phone, or parent unable/unwilling to receive text messages
child or parent planning to move from the local area during study

Interventions

Intervention: experimental: paediatric obesity management (standard care plus counselling session face-to face and over the telephone, newsletters, text messages, information on community events)

Control: active comparator: standard care - brief behavioural counselling and education materials

Outcomes

Weight (primary), waist circumference (primary), BMI z score (primary), fasting insulin, fasting glucose, cholesterol, MVPA assessed by accelerometry, consumption of sugar-sweetened beverages, consumption of fruit and vegetables

Starting date

Start date: January 2015

Completion date: October 2018 (final assessment)

\begin{tabular}{ll}
\hline Contact information & $\begin{array}{l}\text { Responsible party/principal investigator: Deborah Parra-Medina, The University of Texas Health } \\
\text { Science Center at San Antonio, USA }\end{array}$ \\
\hline Study identifier & NCT02343367 \\
\hline Official title & Pediatric obesity management intervention trial for Hispanic families \\
\hline Stated purpose of study & $\begin{array}{l}\text { "Our proposed randomized controlled trial, the Health4Kids (H4K) Trial for Hispanic Families, aims } \\
\text { to improve Hispanic children's body composition by testing a comprehensive, culturally and lin- } \\
\text { guistically relevant, family-oriented intervention for overweight and obese (body mass index (BMI) } \\
\text { between the 85th and 99.9thth (<99th) percentile for age and gender) Hispanic children ages 6-11 } \\
\text { in pediatric clinics in San Antonio, Texas, a largely Hispanic city." }\end{array}$ \\
\hline Notes & Currently recruiting participants (March 2016) \\
\hline
\end{tabular}

\section{NCT02560493}

\begin{tabular}{ll}
\hline Trial name or title & Acronym: GameSquad \\
\hline Methods & Type of study: interventional \\
\hline
\end{tabular}

Diet, physical activity and behavioural interventions for the treatment of overweight or obese children from the age of 6 to 11 years 
NCT02560493 (Continued)
Allocation: randomised
Intervention model: parallel
Masking: single-blind (outcome assessor)
Primary purpose: treatment

Condition: obesity
Enrollment: target 46
Inclusion criteria:
- aged $10-12$ years
- overweight or obese (according to CDC charts)
- boys and girls
- at least 1 family member willing to undertake 3 h/week exergaming
Exclusion criteria:
- pregnancy
- impairments of normal ambulation
- previous cardiovascular disease, muscular-skeletal injury or epilepsy

Interventions

Intervention: $3 \mathrm{~h} /$ week of exergame play and encouraged to achieve recommended 60 active min/ d

Control: no intervention

\begin{tabular}{ll}
\hline Outcomes & Primary outcome: BMI z score over 6 months \\
& $\begin{array}{l}\text { Secondary outcomes: body fat, blood pressure, physical activity, diet and health behaviours over } \\
6 \text { months }\end{array}$ \\
\hline Starting date & $\begin{array}{l}\text { Start date: October } 2015 \\
\text { Completion date: March } 2017\end{array}$ \\
\hline Contact information & $\begin{array}{l}\text { Responsible party/principal investigator: Amanda Staiano, Principal Investigator, Pennington } \\
\text { Biomedical Research Center }\end{array}$ \\
\hline Study identifier & NCT02560493 \\
\hline Official title & $\begin{array}{l}\text { Gaming technology to encourage healthy weight and activity in youth } \\
\text { and 2) demonstrating the potential of exergaming to reduce body fat and improve children's car- } \\
\text { diovascular health." }\end{array}$ \\
\hline Noted purpose of study & \begin{tabular}{l} 
Study has recruited but is ongoing \\
\hline
\end{tabular}
\end{tabular}

\section{NCT02573142}

\begin{tabular}{ll}
\hline Trial name or title & Acronym: $\mathrm{BCHF}$ \\
\hline Methods & Type of study: interventional \\
& Allocation: randomised
\end{tabular}

Diet, physical activity and behavioural interventions for the treatment of overweight or obese children from the age of 6 to 11 years 
NCT02573142 (Continued)

Intervention model: parallel

Masking: open label

Primary purpose: treatment

Condition: obesity
Enrollment: estimated: 100
Inclusion criteria:
- aged 5-11 years
- boys and girls
BMI $\geq 95$ th centile
Exclusion criteria:
"Inability to read and write in English; Family currently has paid membership to a gym or fitness
center; Parent with severe medical or mental health condition limiting ability to attend appoint-
ments; Child with severe medical or mental health condition limiting ability to attend appoint-
ments or participate in behavioral therapies; Parent and child live greater than 30 miles (48.2km)
from the Duke Healthy Lifestyles clinic; Plan to move out of state in next 6 months; Child with
medical condition as cause of obesity (e.g., hypothyroidism, Cushing's Syndrome, Prader-Willi
syndrome, drug-induced obesity)"

Interventions

Intervention: Bull City Fit Intervention, where participants will receive standard of care clinical treatment in the Duke Healthy Lifestyles clinic and unlimited access to a community-based wellness programme that includes physical fitness activities and cooking classes

Control: This active control is comprised of education only, where participants will receive standard of care clinical treatment in the Duke Healthy Lifestyles clinic and educational materials describing community-based resources for physical activity and how to access them

$\begin{array}{ll}\text { Outcomes } & \text { Primary outcome: BMI } 3 \text { and } 6 \text { months post enrolment } \\ & \text { Secondary outcomes: adherence, health habits and cardiovascular fitness at } 3 \text { and } 6 \text { months post } \\ \text { enrolment }\end{array}$

Starting date Start date: October 2015

Completion date: anticipated: October 2017

\begin{tabular}{ll}
\hline Contact information & Principal investigator: Sarah C Armstrong, sarah.c.armstrong@duke.edu \\
\hline Study identifier & NCT02573142 \\
\hline Official title & Integrated child obesity treatment study: Bull City Healthy and Fit (BCHF) \\
\hline Stated purpose of study & $\begin{array}{l}\text { "The primary aim of this study is to reduce body mass index (BMI) among children ages 5-11 who } \\
\text { are obese by integrating behavioral treatment strategies in both clinic (Healthy Lifestyles) and } \\
\text { community (Bull City Fit) settings." }\end{array}$ \\
\hline Notes & Currently still recruiting \\
\hline
\end{tabular}




\section{Methods}

\section{Type of study: interventional}

Allocation: randomised

Intervention model: parallel

Masking: single-blind (outcome assessor)

Primary purpose: treatment

Participants

\section{Condition: obesity}

Enrollment: target 120

\section{Inclusion criteria:}

- 4-10 years

- boys and girls

- patients at designated clinics

- caregiver willing to participate

\section{Exclusion criteria:}

- caregiver not able to speak English
Intervention: "PP+: Following baseline assessment, children will continue to receive standard care at CHS and the monthly newsletter. Additionally, each family will be provided with a scale; wall growth chart to measure height; a BMI wheel to calculate BMI; a BMI-for-age growth chart; a binder for intervention materials; a self-monitoring diary to record child's monthly height, weight, BMI and BMI percentile; and picture-based diaries to monitor daily energy balance behaviours. Family materials provided at each session will outline a process to measure growth and include information about how children grow, as well as cover behavioral parenting strategies to assist with changing child behavior for energy balance behaviors.

Families will meet in person with a $\mathrm{BHC}$ at the $\mathrm{CHS}$ clinic in which they receive care for 30 minutes during months 1,3 , and 5 . In these sessions, child height and weight will be taken, and BMI will be plotted on the BMI-for-age growth chart. Families will receive feedback about growth and the weight status of their child. Additionally, the session materials will be reviewed and behavioral parenting strategies will be encouraged to aid with changing two dietary and two leisure-time activity (energy balance) behaviors of the child. As is traditional in a family-based approach, the caretaker will also change the same energy balance behaviors as the child, as adult caretakers can then model healthy behaviors for the child, assisting the child in learning the new weight-related behaviors.13 Thus, both the caretaker and child will be encouraged to change and self-monitor energy balance behaviors with the use of the picture-based diaries.

During months 2, 4, and 6, BHCs will complete a 20-minute phone call with the caretaker. Caretakers will be asked to measure the height and weight of their child, calculate BMI and plot it on the BMI-for-age growth chart prior to the call. During the call, the BHC will provide feedback on the changes in child growth since the previous contact. Additionally, the BHC will discuss the family's progress on achieving child and caretaker energy balance behavior goals and implementation of behavioral parenting strategies.

The child's energy balance behavioral goals will be to consume $<3$ sugar-sweetened beverage (e.g., regular carbonated soft drinks, sports drinks, lemonades, ice teas, flavoured milk, juice drinks $<100 \%$ juice, and punches) servings/wk, $\geq 1 \frac{1}{2}$ cups/day of whole vegetables and $\geq 1$ cup/day of whole fruit, engage in $\geq 60$ minutes/day of moderate- to vigorous-intensity physical activity, and reduce TV viewing to $<2$ hours/day. The caretaker's energy balance behavioral goals will be to consume $<3$ sugar-sweetened beverage servings/wk, $\geq 21 / 2$ cups/day of whole vegetables and $\geq 1 \frac{1}{2}$ cups/day of whole fruit, engage in $\geq 150$ minutes of moderate- to vigorous-intensity physical activ-

Diet, physical activity and behavioural interventions for the treatment of overweight or obese children from the age of 6 to 11 years 
ity per week, and reduce TV viewing to $<10$ hours/wk. To increase self-efficacy, the goals will be incrementally increased, with families implementing the full programme goals at month four. Additionally, children and caretakers will be asked to achieve at least three of the five goals each day (child) or week (adult caretaker)."

Control: "PP: This condition will be identical to PP+ except that caretakers will not receive any energy balance behavior goals. Additionally, the caretaker will not self-monitor energy balance behaviors. The focus will be on all other behavioral parenting strategies to assist the child with mak ing changes in the targeted behaviors (i.e., stimulus control, positive reinforcement, and assisting child in self-monitoring energy-balance behaviors)."

NB both conditions will be given to high and low household food security

Outcomes

Primary outcomes: baseline to 6 month: demographics, weight history, weight, child and care-giver dietary intake, activity levels, quality control, parent weight history, height, BMI, BMI z score

Secondary outcomes: participant rate and characteristics of non participators, programme adherence, implementation costs, programme sustainability

\begin{tabular}{ll}
\hline Starting date & $\begin{array}{l}\text { Start date: April } 2016 \\
\text { Completion date: April } 2018\end{array}$ \\
\hline Contact information & Responsible party/principal investigator: Hollie Raynor, University of Tennessee, USA \\
\hline Study identifier & NCT02684214 \\
\hline Official title & Not stated \\
\hline Stated purpose of study & Not stated \\
\hline Notes & Trial only just started \\
\hline
\end{tabular}

\begin{tabular}{ll}
\hline Trial name or title & Acronym: PAAPAS-DC \\
\hline Methods & Type of study: interventional \\
& Allocation: randomised \\
& Intervention model: parallel \\
& Masking: single-blind (outcome ass \\
& Primary purpose: treatment \\
& Condition: obesity \\
Participants & Enrollment: estimated: 3000 \\
& Inclusion criteria: \\
& - 9 -15 years \\
& boys and girls \\
\hline
\end{tabular}

Exclusion criteria: 
NCT02711488 (Continued)

$$
\text { - pregnancy }
$$

Interventions

Intervention: participants will be subject to primary prevention activities at school level combined with secondary prevention at home

Control: no intervention

Outcomes Primary outcome: BMI over 1 year

Secondary outcomes: body composition, physical activity, diet and adherence over 1 year

Starting date

Start date: March 2016

Completion date: anticipated December 2016

Contact information

Responsible party/principal investigator: Rosely Sichieri, MD, PhD. Full Professor of Epidemiology, Rio de Janeiro State University, Brasil

\begin{tabular}{ll}
\hline Study identifier & NCT02711488 \\
\hline Official title & $\begin{array}{l}\text { Managing adolescent obesity at local level by combining primary and secondary intervention (PAA- } \\
\text { PAS-DC) }\end{array}$ \\
\hline Stated purpose of study & No formal aims provided \\
\hline Notes & $\begin{array}{l}\text { Not yet completed, mean age of participants at baseline will determine whether this trial is includ- } \\
\text { ed in the review or the adolescent review }\end{array}$ \\
\hline
\end{tabular}

\section{NCT02720302}

\begin{tabular}{|c|c|}
\hline Trial name or title & Acronym: TeleSOFT \\
\hline Methods & $\begin{array}{l}\text { Type of study: interventional } \\
\text { Allocation: randomised } \\
\text { Intervention model: parallel } \\
\text { Masking: open label } \\
\text { Primary purpose: treatment }\end{array}$ \\
\hline Participants & $\begin{array}{l}\text { Condition: obesity } \\
\text { Enrollment: target } 120 \\
\text { Inclusion criteria: } \\
\text { - } \text { aged } 9-12 \text { years } \\
\text { - boys and girls } \\
\text { - } \text { overweight but not obese according to the IOTF classification } \\
\text { Exclusion criteria: } \\
\text { - non Swedish speaking } \\
\text { - monogenic obesity } \\
\text { - present at school less than } 80 \% \text { in the previous year } \\
\text { - no foster care for the child or siblings }\end{array}$ \\
\hline
\end{tabular}

Diet, physical activity and behavioural interventions for the treatment of overweight or obese children from the age of 6 to 11 years 
NCT02720302 (Continued)

Interventions
Intervention: SOFT - a programme based on 'systemic and solution-focused theories to change lifestyle', shown to facilitate positive effects on children in terms of obesity, physical fitness, self-esteem and family functioning

Control: TeleSOFT - where therapists communicate with the overweight child and family by the SOFT method at distance via video

\begin{tabular}{ll}
\hline Outcomes & $\begin{array}{l}\text { Primary outcome: BMI z score at baseline and } 12 \text { months } \\
\text { Secondary outcomes: change in body fat, activity levels, metabolic health, session rating and di- } \\
\text { etary habits }\end{array}$ \\
\hline Starting date & Start date: March 2016 \\
& Completion date: anticipated June 2021 \\
\hline Contact information & Responsible party/principal investigator: Inge Lissau, inlis18@gmail.com \\
\hline Study identifier & NCT02720302 \\
\hline Official title & $\begin{array}{l}\text { Treatment of overweight in children on distance. A comparison between consultations on the hos- } \\
\text { pital with video-consultations on distance }\end{array}$ \\
\hline Stated purpose of study & "aim to treat overweight in children 9-11 years of age" \\
\hline Notes & Currently recruiting \\
\hline
\end{tabular}

\section{NCT02773823}

\begin{tabular}{ll}
\hline Trial name or title & A behavior intervention study on cardiovascular health among chinese obese schoolchildren \\
\hline Methods & Type of study: interventional \\
Allocation: randomised & Intervention model: parallel \\
Masking: open label \\
Primary purpose: treatment \\
Condition: obesity \\
Enrollment: target 200 \\
Inclusion criteria: \\
Participants \\
- aged 8-12 years \\
- boys and girls \\
Exclusion criteria: \\
- history of cardiovascular disease \\
- disability
\end{tabular}


NCT02773823 (Continued)

Control: no intervention

Outcomes Primary outcome: body weight, BMI, blood pressure, blood lipids, glucose and cardiorespiratory fitness at 8 months

Secondary outcomes: well-being and depression at 8 months

\begin{tabular}{ll}
\hline Starting date & $\begin{array}{l}\text { Start date: November } 2015 \\
\text { Completion date: June 2017 (anticipated) }\end{array}$ \\
\hline Contact information & Responsible party/principal investigator: Qiqiang He, 4657473@qq.com \\
\hline Study identifier & NCT02773823 \\
\hline Official title & A comprehensive intervention study on Klotho gene methylation and cardiovascular risk factors \\
\hline Stated purpose of study & No formal aim stated \\
\hline Notes & Study not completed \\
\hline
\end{tabular}

\section{RBR-8ttw64}

$\begin{array}{ll}\text { Trial name or title } & \begin{array}{l}\text { Effects of dietary guidance in children attending outpatient preventive cardiology: randomized } \\ \text { clinical trial }\end{array}\end{array}$

\begin{tabular}{|c|c|}
\hline Methods & $\begin{array}{l}\text { Type of study: interventional } \\
\text { Allocation: randomised } \\
\text { Intervention model: parallel } \\
\text { Masking: open label } \\
\text { Primary purpose: treatment }\end{array}$ \\
\hline Participants & $\begin{array}{l}\text { Condition: overweight, obesity, heart disease } \\
\text { Enrolment: } 74 \\
\text { Inclusion criteria: } \\
\text { - aged } 7-11 \text { years } \\
\text { - overweight or obese according to the criteria of the World Health Organization } \\
\text { - boys and girls } \\
\text { - parents or caregivers signed an informed consent form } \\
\text { - reside in the state of Rio Grande do Sul } \\
\text { Exclusion criteria: } \\
\text { - children with neurological disorders that interfere with learning } \\
\text { - cognitive deficits e.g. Disorder Attention Deficit Hyperactivity Disorder } \\
\text { - contraindications for physical activity group } \\
\text { - using drugs that interfere with the body weight or lipid profile, such as statins, ritonavir, } \\
\text { furosemide, hydrochlorothiazide, propranolol, nadolol, prednisolone among others }\end{array}$ \\
\hline
\end{tabular}

Interventions

Intervention: nutritional education group

Diet, physical activity and behavioural interventions for the treatment of overweight or obese children from the age of 6 to 11 years 
RBR-8ttw64 (Continued)

Control: conventional treatment with a nutritionist

\begin{tabular}{ll}
\hline Outcomes & Total cholesterol (primary), BMI \\
\hline Starting date & First enrolment: October 2013 \\
& Last enrolment: April 2014 \\
\hline Contact information & $\begin{array}{l}\text { Responsible party/principal investigator: Vanessa Minossi, Instituto de Cardiologia Fundação } \\
\text { Universitária de Cardiologia- IC/FUC, Brazil, pellanda.pesquisa@gmail.com }\end{array}$ \\
\hline Study identifier & RBR-8ttw64 \\
\hline Official title & $\begin{array}{l}\text { Effects of dietary guidance in children attending outpatient preventive cardiology: randomized } \\
\text { clinical trial }\end{array}$ \\
\hline Stated purpose of study & $\begin{array}{l}\text { "The objective of this study is to evaluate the effectiveness of an innovative, simple and cost ef- } \\
\text { fective educational program to improve eating habits, physical activity and the knowledge about } \\
\text { healthy habits in children, as well as in their families, as compared to routine outpatient care." }\end{array}$ \\
\hline Notes & \begin{tabular}{l} 
Recruiting (as of March 2016) \\
\hline
\end{tabular}
\end{tabular}

A1c (HbA1c): haemoglobin; ALT: alanine aminotransferase; BIA: bioelectrical impedance analysis; BMI: body mass index; CDC: United States Centers for Disease Control and Prevention; CHS: Community Health Systems; CVD: cardiovascular disease; DEXA: dual energy X-ray absorptiometry; HDL-cholesterol: high density lipoprotein cholesterol; HOMA; homeostasis assessment model; hs-CRP: high-sensitivity Creactive protein; IOTF: International Obesity Task Force; LDL-cholesterol: low density lipoprotein cholesterol; MVPA: moderate-to-vigorous physical activity; PA: physical activity; PP: Prevention Plus; VO2 max: maximum volume of oxygen; WHO: World Health Organization

\section{DATA AND ANALYSES}

\section{Comparison 1. Behaviour-changing interventions versus no treatment/usual care}

\begin{tabular}{|c|c|c|c|c|}
\hline Outcome or subgroup title & No. of studies & $\begin{array}{l}\text { No. of partici- } \\
\text { pants }\end{array}$ & Statistical method & Effect size \\
\hline 1 Change in BMI (all trials) & 24 & 2785 & $\begin{array}{l}\text { Mean Difference (IV, Random, 95\% } \\
\mathrm{Cl} \text { ) }\end{array}$ & $-0.53[-0.82,-0.24]$ \\
\hline $\begin{array}{l}2 \text { Change in BMI z score (all } \\
\text { trials) }\end{array}$ & 37 & 4019 & $\begin{array}{l}\text { Mean Difference (IV, Random, 95\% } \\
\mathrm{Cl} \text { ) }\end{array}$ & $-0.06[-0.10,-0.02]$ \\
\hline 3 Change in weight (all trials) & 17 & 1774 & $\begin{array}{l}\text { Mean Difference (IV, Random, 95\% } \\
\mathrm{Cl} \text { ) }\end{array}$ & $-1.45[-1.88,-1.02]$ \\
\hline 4 Serious adverse events & 31 & 4096 & Risk Ratio (M-H, Random, 95\% Cl) & $0.57[0.17,1.93]$ \\
\hline $\begin{array}{l}5 \text { Health-related quality of } \\
\text { life (parent-report measures) }\end{array}$ & 5 & 718 & $\begin{array}{l}\text { Std. Mean Difference (IV, Random, } \\
95 \% \mathrm{CI})\end{array}$ & $0.13[-0.06,0.32]$ \\
\hline 5.1 PedsQL caregiver-report & 4 & 526 & $\begin{array}{l}\text { Std. Mean Difference (IV, Random, } \\
95 \% \mathrm{CI} \text { ) }\end{array}$ & $0.13[-0.13,0.40]$ \\
\hline $\begin{array}{l}5.2 \text { CHQ-PF50 - global score, } \\
\text { parental report }\end{array}$ & 1 & 192 & $\begin{array}{l}\text { Std. Mean Difference (IV, Random, } \\
95 \% \mathrm{CI} \text { ) }\end{array}$ & $0.14[-0.14,0.42]$ \\
\hline
\end{tabular}

Diet, physical activity and behavioural interventions for the treatment of overweight or obese children from the age of 6 to 11 years 


\begin{tabular}{|c|c|c|c|c|}
\hline Outcome or subgroup title & No. of studies & $\begin{array}{l}\text { No. of partici- } \\
\text { pants }\end{array}$ & Statistical method & Effect size \\
\hline $\begin{array}{l}6 \text { Health-related quality of } \\
\text { life (child-report measures) }\end{array}$ & 3 & 164 & $\begin{array}{l}\text { Std. Mean Difference (IV, Random, } \\
95 \% \mathrm{Cl} \text { ) }\end{array}$ & $0.15[-0.34,0.64]$ \\
\hline 6.1 PedsQL child-report & 2 & 142 & $\begin{array}{l}\text { Std. Mean Difference (IV, Random, } \\
95 \% \mathrm{Cl} \text { ) }\end{array}$ & $0.10[-0.60,0.79]$ \\
\hline 6.2 KINDL-R child-report & 1 & 22 & $\begin{array}{l}\text { Std. Mean Difference (IV, Random, } \\
95 \% \mathrm{CI} \text { ) }\end{array}$ & $0.33[-0.51,1.18]$ \\
\hline $\begin{array}{l}7 \text { Self-esteem (Harter global } \\
\text { score) }\end{array}$ & 2 & 144 & $\begin{array}{l}\text { Mean Difference (IV, Random, 95\% } \\
\mathrm{Cl} \text { ) }\end{array}$ & $0.19[-0.04,0.42]$ \\
\hline 8 Waist circumference & 11 & 1325 & $\begin{array}{l}\text { Mean Difference (IV, Random, 95\% } \\
\mathrm{CI})\end{array}$ & $-2.41[-3.59,-1.23]$ \\
\hline 9 Overweight & 3 & 347 & $\begin{array}{l}\text { Mean Difference (IV, Random, 95\% } \\
\mathrm{CI})\end{array}$ & $-3.27[-7.47,0.92]$ \\
\hline 10 Body fat & 10 & & $\begin{array}{l}\text { Mean Difference (IV, Random, 95\% } \\
\mathrm{Cl} \text { ) }\end{array}$ & Subtotals only \\
\hline 10.1 Bioelectrical impedance & 5 & 1004 & $\begin{array}{l}\text { Mean Difference (IV, Random, 95\% } \\
\mathrm{Cl} \text { ) }\end{array}$ & $-1.25[-2.62,0.12]$ \\
\hline $\begin{array}{l}\text { 10.2 Dual energy X-ray ab- } \\
\text { sorptiometry }\end{array}$ & 5 & 443 & $\begin{array}{l}\text { Mean Difference (IV, Random, 95\% } \\
\mathrm{Cl} \text { ) }\end{array}$ & $-1.04[-2.88,0.80]$ \\
\hline 11 Diet & 2 & 168 & $\begin{array}{l}\text { Mean Difference (IV, Random, 95\% } \\
\mathrm{Cl} \text { ) }\end{array}$ & $\begin{array}{l}-161.53[-583.79 \\
260.73]\end{array}$ \\
\hline 12 Television viewing & 2 & 55 & $\begin{array}{l}\text { Mean Difference (IV, Random, 95\% } \\
\mathrm{Cl} \text { ) }\end{array}$ & $-6.60[-12.88,-0.31]$ \\
\hline $\begin{array}{l}13 \text { Physical activity (ac- } \\
\text { celerometer MVPA) }\end{array}$ & 6 & 744 & $\begin{array}{l}\text { Mean Difference (IV, Random, 95\% } \\
\mathrm{Cl} \text { ) }\end{array}$ & $-0.76[-5.30,3.78]$ \\
\hline $\begin{array}{l}14 \text { Change in BMI - type of } \\
\text { control }\end{array}$ & 24 & 2785 & $\begin{array}{l}\text { Mean Difference (IV, Random, 95\% } \\
\mathrm{CI})\end{array}$ & $-0.53[-0.82,-0.24]$ \\
\hline $\begin{array}{l}14.1 \text { Intervention versus no } \\
\text { treatment }\end{array}$ & 11 & 1452 & $\begin{array}{l}\text { Mean Difference (IV, Random, 95\% } \\
\mathrm{Cl} \text { ) }\end{array}$ & $-0.43[-0.87,-0.00]$ \\
\hline $\begin{array}{l}14.2 \text { Intervention versus usu- } \\
\text { al care }\end{array}$ & 13 & 1333 & $\begin{array}{l}\text { Mean Difference (IV, Random, 95\% } \\
\mathrm{Cl} \text { ) }\end{array}$ & $-0.67[-1.12,-0.21]$ \\
\hline $\begin{array}{l}15 \text { Change in BMI z score - } \\
\text { type of control }\end{array}$ & 37 & 4019 & $\begin{array}{l}\text { Mean Difference (IV, Random, 95\% } \\
\mathrm{Cl} \text { ) }\end{array}$ & $-0.06[-0.10,-0.02]$ \\
\hline 15.1 No treatment & 15 & 1709 & $\begin{array}{l}\text { Mean Difference (IV, Random, 95\% } \\
\mathrm{Cl} \text { ) }\end{array}$ & $-0.06[-0.12,0.01]$ \\
\hline 15.2 Usual care & 22 & 2310 & $\begin{array}{l}\text { Mean Difference (IV, Random, 95\% } \\
\mathrm{Cl} \text { ) }\end{array}$ & $-0.06[-0.11,-0.02]$ \\
\hline
\end{tabular}




\begin{tabular}{|c|c|c|c|c|}
\hline Outcome or subgroup title & No. of studies & $\begin{array}{l}\text { No. of partici- } \\
\text { pants }\end{array}$ & Statistical method & Effect size \\
\hline $\begin{array}{l}16 \text { Change in weight - type of } \\
\text { control }\end{array}$ & 17 & 1774 & $\begin{array}{l}\text { Mean Difference (IV, Random, 95\% } \\
\mathrm{Cl} \text { ) }\end{array}$ & $-1.45[-1.88,-1.02]$ \\
\hline 16.1 No treatment & 7 & 906 & $\begin{array}{l}\text { Mean Difference (IV, Random, 95\% } \\
\mathrm{Cl} \text { ) }\end{array}$ & $-1.73[-2.47,-0.98]$ \\
\hline 16.2 Usual care & 10 & 868 & $\begin{array}{l}\text { Mean Difference (IV, Random, 95\% } \\
\mathrm{Cl} \text { ) }\end{array}$ & $-1.31[-1.84,-0.78]$ \\
\hline $\begin{array}{l}17 \text { Change in BMI - type of in- } \\
\text { tervention }\end{array}$ & 24 & 2785 & $\begin{array}{l}\text { Mean Difference (IV, Random, 95\% } \\
\mathrm{CI})\end{array}$ & $-0.53[-0.82,-0.24]$ \\
\hline 17.1 Diet only & 1 & 73 & $\begin{array}{l}\text { Mean Difference (IV, Random, 95\% } \\
\mathrm{Cl} \text { ) }\end{array}$ & $-0.12[-0.85,0.61]$ \\
\hline 17.2 Physical activity only & 4 & 443 & $\begin{array}{l}\text { Mean Difference (IV, Random, 95\% } \\
\mathrm{Cl} \text { ) }\end{array}$ & $-0.29[-0.50,-0.09]$ \\
\hline $\begin{array}{l}17.3 \text { Diet and physical activi- } \\
\text { ty }\end{array}$ & 2 & 209 & $\begin{array}{l}\text { Mean Difference (IV, Random, 95\% } \\
\mathrm{Cl} \text { ) }\end{array}$ & $-1.03[-3.43,1.38]$ \\
\hline $\begin{array}{l}\text { 17.4 Diet and behavioural } \\
\text { therapy }\end{array}$ & 1 & 39 & $\begin{array}{l}\text { Mean Difference (IV, Random, 95\% } \\
\mathrm{Cl} \text { ) }\end{array}$ & $-0.7[-3.65,2.25]$ \\
\hline $\begin{array}{l}17.5 \text { Physical activity and be- } \\
\text { havioural therapy }\end{array}$ & 1 & 230 & $\begin{array}{l}\text { Mean Difference (IV, Random, 95\% } \\
\mathrm{Cl} \text { ) }\end{array}$ & $-0.01[-1.29,1.27]$ \\
\hline $\begin{array}{l}\text { 17.6 Diet, physical activity } \\
\text { and behavioural therapy }\end{array}$ & 15 & 1791 & $\begin{array}{l}\text { Mean Difference (IV, Random, 95\% } \\
\mathrm{CI})\end{array}$ & $-0.67[-1.12,-0.23]$ \\
\hline $\begin{array}{l}18 \text { Change in BMI z score - } \\
\text { type of intervention }\end{array}$ & 37 & 4019 & $\begin{array}{l}\text { Mean Difference (IV, Random, 95\% } \\
\mathrm{CI} \text { ) }\end{array}$ & $-0.06[-0.10,-0.02]$ \\
\hline 18.1 Diet only & 1 & 73 & $\begin{array}{l}\text { Mean Difference (IV, Random, 95\% } \\
\mathrm{CI})\end{array}$ & $-0.05[-0.17,0.07]$ \\
\hline 18.2 Physical activity only & 3 & 365 & $\begin{array}{l}\text { Mean Difference (IV, Random, 95\% } \\
\mathrm{Cl} \text { ) }\end{array}$ & $-0.05[-0.23,0.14]$ \\
\hline $\begin{array}{l}\text { 18.3 Diet and physical activi- } \\
\text { ty }\end{array}$ & 7 & 577 & $\begin{array}{l}\text { Mean Difference (IV, Random, 95\% } \\
\mathrm{CI})\end{array}$ & $-0.03[-0.10,0.04]$ \\
\hline $\begin{array}{l}\text { 18.4 Diet and behavioural } \\
\text { therapy }\end{array}$ & 2 & 152 & $\begin{array}{l}\text { Mean Difference (IV, Random, 95\% } \\
\mathrm{CI})\end{array}$ & $-0.07[-0.16,0.03]$ \\
\hline $\begin{array}{l}18.5 \text { Physical activity and be- } \\
\text { havioural therapy }\end{array}$ & 1 & 230 & $\begin{array}{l}\text { Mean Difference (IV, Random, 95\% } \\
\mathrm{Cl} \text { ) }\end{array}$ & $-0.03[-0.26,0.20]$ \\
\hline $\begin{array}{l}\text { 18.6 Diet, physical activity } \\
\text { and behavioural therapy }\end{array}$ & 24 & 2622 & $\begin{array}{l}\text { Mean Difference (IV, Random, 95\% } \\
\mathrm{Cl} \text { ) }\end{array}$ & $-0.08[-0.13,-0.02]$ \\
\hline $\begin{array}{l}19 \text { Change in weight - type of } \\
\text { intervention }\end{array}$ & 17 & 1774 & $\begin{array}{l}\text { Mean Difference (IV, Random, 95\% } \\
\mathrm{Cl} \text { ) }\end{array}$ & $-1.45[-1.88,-1.02]$ \\
\hline
\end{tabular}




\begin{tabular}{|c|c|c|c|c|}
\hline Outcome or subgroup title & No. of studies & $\begin{array}{l}\text { No. of partici- } \\
\text { pants }\end{array}$ & Statistical method & Effect size \\
\hline 19.1 Diet only & 1 & 73 & $\begin{array}{l}\text { Mean Difference (IV, Random, 95\% } \\
\mathrm{Cl} \text { ) }\end{array}$ & $-0.12[-2.45,2.21]$ \\
\hline 19.2 Physical activity only & 3 & 422 & $\begin{array}{l}\text { Mean Difference (IV, Random, 95\% } \\
\mathrm{CI})\end{array}$ & $-1.34[-1.94,-0.73]$ \\
\hline $\begin{array}{l}\text { 19.3 Diet and physical activi- } \\
\text { ty }\end{array}$ & 1 & 125 & $\begin{array}{l}\text { Mean Difference (IV, Random, 95\% } \\
\mathrm{Cl} \text { ) }\end{array}$ & $-0.15[-3.81,3.51]$ \\
\hline $\begin{array}{l}19.4 \text { Physical activity and be- } \\
\text { havioural therapy }\end{array}$ & 1 & 230 & $\begin{array}{l}\text { Mean Difference (IV, Random, 95\% } \\
\mathrm{CI})\end{array}$ & $0.21[-4.29,4.71]$ \\
\hline $\begin{array}{l}\text { 19.5 Diet, physical activity } \\
\text { and behavioural therapy }\end{array}$ & 11 & 924 & $\begin{array}{l}\text { Mean Difference (IV, Random, 95\% } \\
\mathrm{Cl} \text { ) }\end{array}$ & $-1.76[-2.41,-1.11]$ \\
\hline $\begin{array}{l}20 \text { Change in BMI - attrition } \\
\text { bias }\end{array}$ & 24 & 2785 & $\begin{array}{l}\text { Mean Difference (IV, Random, 95\% } \\
\mathrm{Cl})\end{array}$ & $-0.53[-0.82,-0.24]$ \\
\hline $20.1 \mathrm{High}$ & 4 & 238 & $\begin{array}{l}\text { Mean Difference (IV, Random, 95\% } \\
\mathrm{Cl} \text { ) }\end{array}$ & $-0.47[-1.04,0.10]$ \\
\hline 20.2 Low & 15 & 1910 & $\begin{array}{l}\text { Mean Difference (IV, Random, 95\% } \\
\mathrm{Cl} \text { ) }\end{array}$ & $-0.50[-0.93,-0.07]$ \\
\hline 20.3 Unclear & 5 & 637 & $\begin{array}{l}\text { Mean Difference (IV, Random, 95\% } \\
\mathrm{Cl} \text { ) }\end{array}$ & $-0.72[-1.45,0.01]$ \\
\hline $\begin{array}{l}21 \text { Change in BMI z score - at- } \\
\text { trition bias }\end{array}$ & 37 & 4019 & $\begin{array}{l}\text { Mean Difference (IV, Random, 95\% } \\
\mathrm{Cl} \text { ) }\end{array}$ & $-0.06[-0.10,-0.02]$ \\
\hline 21.1 Low & 17 & 1745 & $\begin{array}{l}\text { Mean Difference (IV, Random, 95\% } \\
\mathrm{Cl} \text { ) }\end{array}$ & $-0.08[-0.16,-0.01]$ \\
\hline 21.2 Unclear & 9 & 897 & $\begin{array}{l}\text { Mean Difference (IV, Random, 95\% } \\
\mathrm{Cl} \text { ) }\end{array}$ & $-0.05[-0.13,0.03]$ \\
\hline 21.3 High & 11 & 1377 & $\begin{array}{l}\text { Mean Difference (IV, Random, 95\% } \\
\mathrm{Cl} \text { ) }\end{array}$ & $-0.03[-0.06,0.01]$ \\
\hline $\begin{array}{l}22 \text { Change in weight - attri- } \\
\text { tion bias }\end{array}$ & 17 & 1774 & $\begin{array}{l}\text { Mean Difference (IV, Random, 95\% } \\
\mathrm{Cl} \text { ) }\end{array}$ & $-1.45[-1.88,-1.02]$ \\
\hline 22.1 Low & 9 & 986 & $\begin{array}{l}\text { Mean Difference (IV, Random, 95\% } \\
\mathrm{Cl} \text { ) }\end{array}$ & $-1.20[-1.73,-0.67]$ \\
\hline 22.2 Unclear & 4 & 553 & $\begin{array}{l}\text { Mean Difference (IV, Random, 95\% } \\
\mathrm{CI})\end{array}$ & $-1.73[-3.54,0.07]$ \\
\hline $22.3 \mathrm{High}$ & 4 & 235 & $\begin{array}{l}\text { Mean Difference (IV, Random, 95\% } \\
\mathrm{CI})\end{array}$ & $-1.99[-2.80,-1.17]$ \\
\hline 23 Change in weight - setting & 17 & 1774 & $\begin{array}{l}\text { Mean Difference (IV, Random, 95\% } \\
\mathrm{Cl} \text { ) }\end{array}$ & $-1.45[-1.88,-1.02]$ \\
\hline
\end{tabular}




\begin{tabular}{|c|c|c|c|c|}
\hline Outcome or subgroup title & No. of studies & $\begin{array}{l}\text { No. of partici- } \\
\text { pants }\end{array}$ & Statistical method & Effect size \\
\hline 23.1 Schools & 1 & 55 & $\begin{array}{l}\text { Mean Difference (IV, Random, 95\% } \\
\mathrm{Cl} \text { ) }\end{array}$ & $-1.20[-4.20,1.80]$ \\
\hline 23.2 Community & 1 & 78 & $\begin{array}{l}\text { Mean Difference (IV, Random, 95\% } \\
\mathrm{Cl} \text { ) }\end{array}$ & $-1.37[-1.99,-0.75]$ \\
\hline 23.3 Child's home & 3 & 625 & $\begin{array}{l}\text { Mean Difference (IV, Random, 95\% } \\
\mathrm{Cl} \text { ) }\end{array}$ & $-0.26[-1.97,1.45]$ \\
\hline 23.4 Primary care & 2 & 191 & $\begin{array}{l}\text { Mean Difference (IV, Random, 95\% } \\
\mathrm{CI})\end{array}$ & $-2.02[-5.28,1.24]$ \\
\hline $\begin{array}{l}\text { 23.5 Secondary care (outpa- } \\
\text { tient) }\end{array}$ & 4 & 248 & $\begin{array}{l}\text { Mean Difference (IV, Random, 95\% } \\
\mathrm{Cl} \text { ) }\end{array}$ & $-1.52[-2.77,-0.27]$ \\
\hline 23.6 Research clinic & 4 & 374 & $\begin{array}{l}\text { Mean Difference (IV, Random, 95\% } \\
\mathrm{Cl} \text { ) }\end{array}$ & $-1.88[-2.75,-1.02]$ \\
\hline 23.7 Mixed & 2 & 203 & $\begin{array}{l}\text { Mean Difference (IV, Random, 95\% } \\
\mathrm{CI} \text { ) }\end{array}$ & $-0.54[-3.17,2.08]$ \\
\hline $\begin{array}{l}24 \text { Change in BMI z score - } \\
\text { setting }\end{array}$ & 37 & 4019 & $\begin{array}{l}\text { Mean Difference (IV, Random, 95\% } \\
\mathrm{Cl} \text { ) }\end{array}$ & $-0.06[-0.10,-0.03]$ \\
\hline 24.1 Schools & 2 & 76 & $\begin{array}{l}\text { Mean Difference (IV, Random, 95\% } \\
\mathrm{Cl} \text { ) }\end{array}$ & $-0.01[-0.17,0.15]$ \\
\hline 24.2 Community & 2 & 76 & $\begin{array}{l}\text { Mean Difference (IV, Random, 95\% } \\
\mathrm{CI})\end{array}$ & $0.04[-0.04,0.11]$ \\
\hline 24.3 Child's home & 6 & 998 & $\begin{array}{l}\text { Mean Difference (IV, Random, 95\% } \\
\mathrm{CI} \text { ) }\end{array}$ & $-0.06[-0.12,-0.00]$ \\
\hline 24.4 Primary care & 8 & 864 & $\begin{array}{l}\text { Mean Difference (IV, Random, 95\% } \\
\mathrm{Cl} \text { ) }\end{array}$ & $-0.06[-0.12,-0.01]$ \\
\hline $\begin{array}{l}24.5 \text { Secondary care (outpa- } \\
\text { tient) }\end{array}$ & 10 & 583 & $\begin{array}{l}\text { Mean Difference (IV, Random, 95\% } \\
\mathrm{Cl} \text { ) }\end{array}$ & $-0.12[-0.25,0.01]$ \\
\hline 24.6 Hospital inpatient & 1 & 523 & $\begin{array}{l}\text { Mean Difference (IV, Random, 95\% } \\
\mathrm{Cl} \text { ) }\end{array}$ & $0.02[-0.06,0.10]$ \\
\hline 24.7 Research clinic & 4 & 388 & $\begin{array}{l}\text { Mean Difference (IV, Random, 95\% } \\
\mathrm{Cl} \text { ) }\end{array}$ & $-0.03[-0.07,0.02]$ \\
\hline 24.8 Mixed & 5 & 511 & $\begin{array}{l}\text { Mean Difference (IV, Random, 95\% } \\
\mathrm{CI} \text { ) }\end{array}$ & $-0.09[-0.16,-0.01]$ \\
\hline 25 Change in BMI - setting & 24 & 2785 & $\begin{array}{l}\text { Mean Difference (IV, Random, 95\% } \\
\mathrm{Cl} \text { ) }\end{array}$ & $-0.55[-0.85,-0.26]$ \\
\hline 25.1 Schools & 1 & 21 & $\begin{array}{l}\text { Mean Difference (IV, Random, 95\% } \\
\mathrm{Cl} \text { ) }\end{array}$ & $-0.57[-4.94,3.80]$ \\
\hline
\end{tabular}




\begin{tabular}{|c|c|c|c|c|}
\hline Outcome or subgroup title & No. of studies & $\begin{array}{l}\text { No. of partici- } \\
\text { pants }\end{array}$ & Statistical method & Effect size \\
\hline 25.2 Community & 1 & 78 & $\begin{array}{l}\text { Mean Difference (IV, Random, 95\% } \\
\mathrm{Cl} \text { ) }\end{array}$ & $-0.53[-1.05,-0.01]$ \\
\hline 25.3 Child's home & 4 & 667 & $\begin{array}{l}\text { Mean Difference (IV, Random, 95\% } \\
\mathrm{Cl} \text { ) }\end{array}$ & $-0.32[-0.86,0.22]$ \\
\hline 25.4 Primary care & 6 & 1055 & $\begin{array}{l}\text { Mean Difference (IV, Random, 95\% } \\
\mathrm{Cl} \text { ) }\end{array}$ & $-0.10[-0.35,0.14]$ \\
\hline $\begin{array}{l}25.5 \text { Secondary care (outpa- } \\
\text { tient) }\end{array}$ & 7 & 384 & $\begin{array}{l}\text { Mean Difference (IV, Random, 95\% } \\
\mathrm{CI})\end{array}$ & $-1.46[-2.42,-0.50]$ \\
\hline 25.6 Research clinic & 3 & 295 & $\begin{array}{l}\text { Mean Difference (IV, Random, 95\% } \\
\mathrm{Cl} \text { ) }\end{array}$ & $-0.24[-0.86,0.37]$ \\
\hline 25.7 Mixed & 3 & 285 & $\begin{array}{l}\text { Mean Difference (IV, Random, 95\% } \\
\mathrm{Cl} \text { ) }\end{array}$ & $-0.79[-1.87,0.30]$ \\
\hline $\begin{array}{l}26 \text { Change in BMI - post-inter- } \\
\text { vention follow-up }\end{array}$ & 24 & 2785 & $\begin{array}{l}\text { Mean Difference (IV, Random, 95\% } \\
\mathrm{Cl} \text { ) }\end{array}$ & $-0.53[-0.82,-0.24]$ \\
\hline $\begin{array}{l}26.1 \text { No post-intervention fol- } \\
\text { low-up }\end{array}$ & 15 & 1573 & $\begin{array}{l}\text { Mean Difference (IV, Random, 95\% } \\
\mathrm{Cl} \text { ) }\end{array}$ & $-0.68[-1.10,-0.27]$ \\
\hline $\begin{array}{l}26.2 \text { Post-intervention fol- } \\
\text { low-up }<6 \text { months }\end{array}$ & 3 & 153 & $\begin{array}{l}\text { Mean Difference (IV, Random, 95\% } \\
\mathrm{Cl} \text { ) }\end{array}$ & $-1.49[-2.93,-0.05]$ \\
\hline $\begin{array}{l}26.3 \text { Post-intervention fol- } \\
\text { low-up } 6 \text { months to }<12 \\
\text { months }\end{array}$ & 2 & 282 & $\begin{array}{l}\text { Mean Difference (IV, Random, 95\% } \\
\mathrm{Cl} \text { ) }\end{array}$ & $-0.59[-2.34,1.15]$ \\
\hline $\begin{array}{l}\text { 26.4 Post-intervention fol- } \\
\text { low-up } 12 \text { months or more }\end{array}$ & 4 & 777 & $\begin{array}{l}\text { Mean Difference (IV, Random, 95\% } \\
\mathrm{Cl} \text { ) }\end{array}$ & $-0.07[-0.34,0.20]$ \\
\hline $\begin{array}{l}27 \text { Change in BMI z score - } \\
\text { post-intervention follow-up }\end{array}$ & 37 & 4019 & $\begin{array}{l}\text { Mean Difference (IV, Random, 95\% } \\
\mathrm{Cl} \text { ) }\end{array}$ & $-0.06[-0.10,-0.02]$ \\
\hline $\begin{array}{l}\text { 27.1 No post-intervention fol- } \\
\text { low-up }\end{array}$ & 21 & 2278 & $\begin{array}{l}\text { Mean Difference (IV, Random, 95\% } \\
\mathrm{CI} \text { ) }\end{array}$ & $-0.09[-0.15,-0.04]$ \\
\hline $\begin{array}{l}27.2 \text { Post-intervention fol- } \\
\text { low-up }<6 \text { months }\end{array}$ & 6 & 228 & $\begin{array}{l}\text { Mean Difference (IV, Random, 95\% } \\
\mathrm{CI})\end{array}$ & $-0.06[-0.15,0.04]$ \\
\hline $\begin{array}{l}27.3 \text { Post-intervention fol- } \\
\text { low-up } 6 \text { months to }<12 \\
\text { months }\end{array}$ & 3 & 168 & $\begin{array}{l}\text { Mean Difference (IV, Random, 95\% } \\
\mathrm{Cl} \text { ) }\end{array}$ & $0.04[-0.09,0.16]$ \\
\hline $\begin{array}{l}27.4 \text { Post-intervention fol- } \\
\text { low-up } 12 \text { months or more }\end{array}$ & 7 & 1345 & $\begin{array}{l}\text { Mean Difference (IV, Random, 95\% } \\
\mathrm{Cl} \text { ) }\end{array}$ & $-0.01[-0.06,0.03]$ \\
\hline $\begin{array}{l}28 \text { Change in weight - post-in- } \\
\text { tervention follow-up }\end{array}$ & 17 & 1774 & $\begin{array}{l}\text { Mean Difference (IV, Random, 95\% } \\
\mathrm{Cl} \text { ) }\end{array}$ & $-1.45[-1.88,-1.02]$ \\
\hline $\begin{array}{l}28.1 \text { No post-intervention fol- } \\
\text { low-up }\end{array}$ & 12 & 1365 & $\begin{array}{l}\text { Mean Difference (IV, Random, 95\% } \\
\mathrm{Cl} \text { ) }\end{array}$ & $-1.49[-1.94,-1.04]$ \\
\hline
\end{tabular}

Diet, physical activity and behavioural interventions for the treatment of overweight or obese children from the age of 6 to 11 years 


\begin{tabular}{|c|c|c|c|c|}
\hline Outcome or subgroup title & No. of studies & $\begin{array}{l}\text { No. of partici- } \\
\text { pants }\end{array}$ & Statistical method & Effect size \\
\hline $\begin{array}{l}\text { 28.2 Post-intervention fol- } \\
\text { low-up }<6 \text { months }\end{array}$ & 1 & 32 & $\begin{array}{l}\text { Mean Difference (IV, Random, 95\% } \\
\mathrm{CI})\end{array}$ & $-0.50[-9.28,8.29]$ \\
\hline $\begin{array}{l}28.3 \text { Post-intervention fol- } \\
\text { low-up } 6 \text { months to }<12 \\
\text { months }\end{array}$ & 1 & 40 & $\begin{array}{l}\text { Mean Difference (IV, Random, 95\% } \\
\mathrm{CI} \text { ) }\end{array}$ & $-4.60[-17.49,8.29]$ \\
\hline $\begin{array}{l}28.4 \text { Post-intervention fol- } \\
\text { low-up } 12 \text { months or more }\end{array}$ & 3 & 337 & $\begin{array}{l}\text { Mean Difference (IV, Random, 95\% } \\
\mathrm{CI})\end{array}$ & $-1.01[-2.49,0.47]$ \\
\hline $\begin{array}{l}29 \text { Change in BMI - type of } \\
\text { parental involvement }\end{array}$ & 24 & 2785 & $\begin{array}{l}\text { Mean Difference (IV, Random, 95\% } \\
\mathrm{CI} \text { ) }\end{array}$ & $-0.53[-0.82,-0.24]$ \\
\hline 29.1 Parent involvement & 20 & 2217 & $\begin{array}{l}\text { Mean Difference (IV, Random, 95\% } \\
\mathrm{CI})\end{array}$ & $-0.65[-1.04,-0.25]$ \\
\hline 29.2 No parental involvement & 3 & 422 & $\begin{array}{l}\text { Mean Difference (IV, Random, 95\% } \\
\mathrm{CI} \text { ) }\end{array}$ & $-0.29[-0.50,-0.09]$ \\
\hline 29.3 Parent targeted & 1 & 146 & $\begin{array}{l}\text { Mean Difference (IV, Random, 95\% } \\
\mathrm{CI})\end{array}$ & $0.0[-0.81,0.81]$ \\
\hline $\begin{array}{l}30 \text { Change in BMI z score - } \\
\text { type of parental involvement }\end{array}$ & 37 & 4019 & $\begin{array}{l}\text { Mean Difference (IV, Random, 95\% } \\
\mathrm{CI})\end{array}$ & $-0.06[-0.10,-0.02]$ \\
\hline 30.1 Parent involvement & 32 & 2927 & $\begin{array}{l}\text { Mean Difference (IV, Random, 95\% } \\
\mathrm{CI})\end{array}$ & $-0.07[-0.11,-0.03]$ \\
\hline 30.2 No parental involvement & 2 & 344 & $\begin{array}{l}\text { Mean Difference (IV, Random, 95\% } \\
\mathrm{CI})\end{array}$ & $-0.03[-0.24,0.19]$ \\
\hline 30.3 Parent targeted & 3 & 748 & $\begin{array}{l}\text { Mean Difference (IV, Random, 95\% } \\
\mathrm{CI})\end{array}$ & $0.01[-0.06,0.08]$ \\
\hline $\begin{array}{l}31 \text { Change in weight - type of } \\
\text { parental involvement }\end{array}$ & 17 & 1774 & $\begin{array}{l}\text { Mean Difference (IV, Random, 95\% } \\
\mathrm{CI} \text { ) }\end{array}$ & $-1.45[-1.88,-1.02]$ \\
\hline 31.1 Parent involvement & 13 & 1273 & $\begin{array}{l}\text { Mean Difference (IV, Random, 95\% } \\
\mathrm{CI} \text { ) }\end{array}$ & $-1.32[-2.09,-0.55]$ \\
\hline 31.2 No parental involvement & 3 & 422 & $\begin{array}{l}\text { Mean Difference (IV, Random, 95\% } \\
\mathrm{CI})\end{array}$ & $-1.34[-1.94,-0.73]$ \\
\hline 31.3 Parent targeted & 1 & 79 & $\begin{array}{l}\text { Mean Difference (IV, Random, 95\% } \\
\mathrm{CI})\end{array}$ & $-2.0[-3.02,-0.98]$ \\
\hline $\begin{array}{l}32 \text { Change in BMI z score - } \\
\text { baseline BMI z score }\end{array}$ & 37 & 4019 & $\begin{array}{l}\text { Mean Difference (IV, Random, 95\% } \\
\mathrm{CI})\end{array}$ & $-0.06[-0.10,-0.02]$ \\
\hline $\begin{array}{l}32.1 \text { Baseline BMI z score < } \\
2.67 \text { units }\end{array}$ & 29 & 3549 & $\begin{array}{l}\text { Mean Difference (IV, Random, 95\% } \\
\mathrm{CI})\end{array}$ & $-0.07[-0.11,-0.03]$ \\
\hline $\begin{array}{l}32.2 \text { Baseline BMI } z \text { score } \geq \\
2.67 \text { units }\end{array}$ & 8 & 470 & $\begin{array}{l}\text { Mean Difference (IV, Random, 95\% } \\
\mathrm{CI} \text { ) }\end{array}$ & $-0.03[-0.11,0.05]$ \\
\hline
\end{tabular}


Analysis 1.1. Comparison 1 Behaviour-changing interventions versus no treatment/usual care, Outcome 1 Change in BMI (all trials).

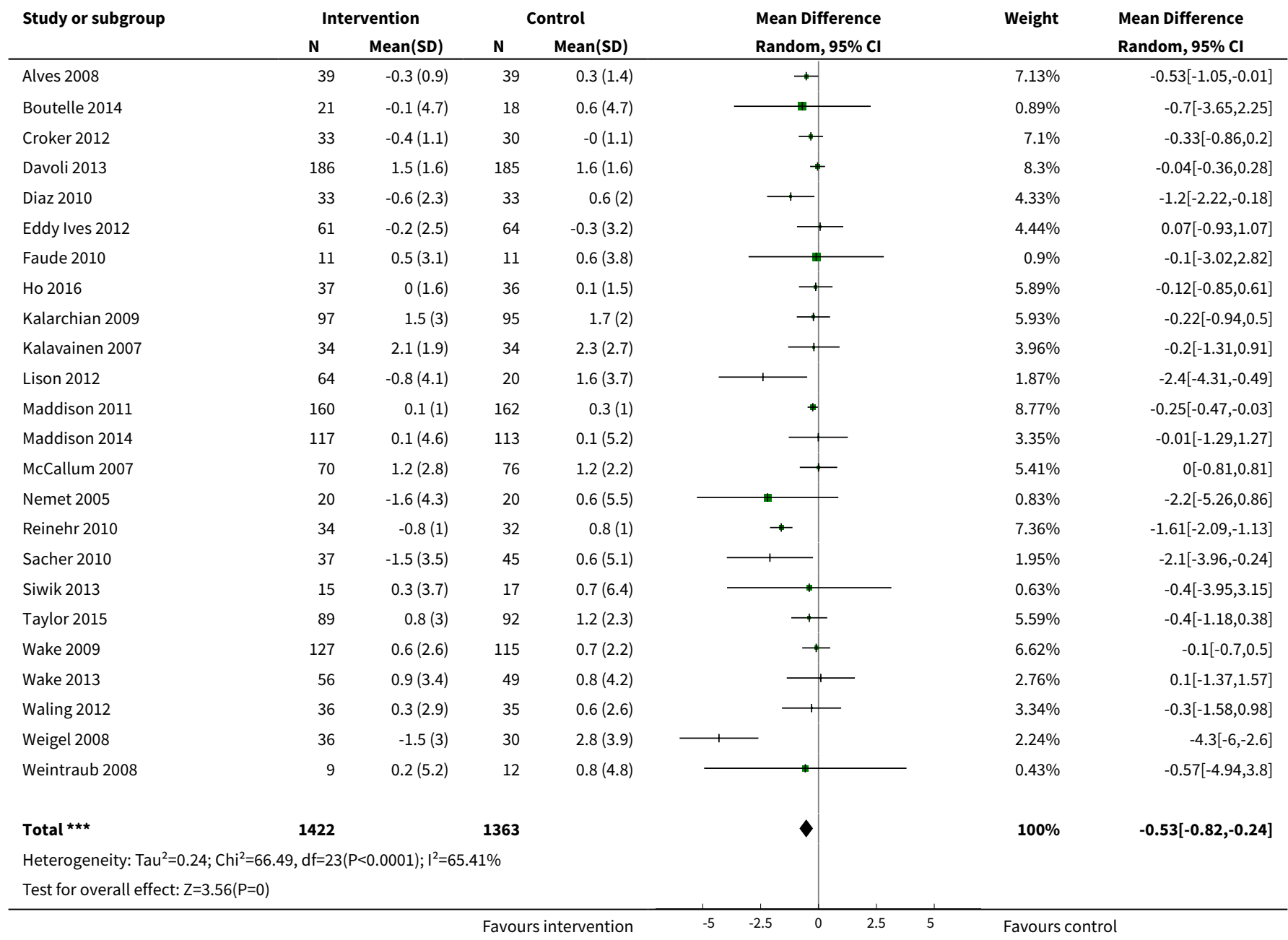

Analysis 1.2. Comparison 1 Behaviour-changing interventions versus no treatment/usual care, Outcome 2 Change in BMI $z$ score (all trials).

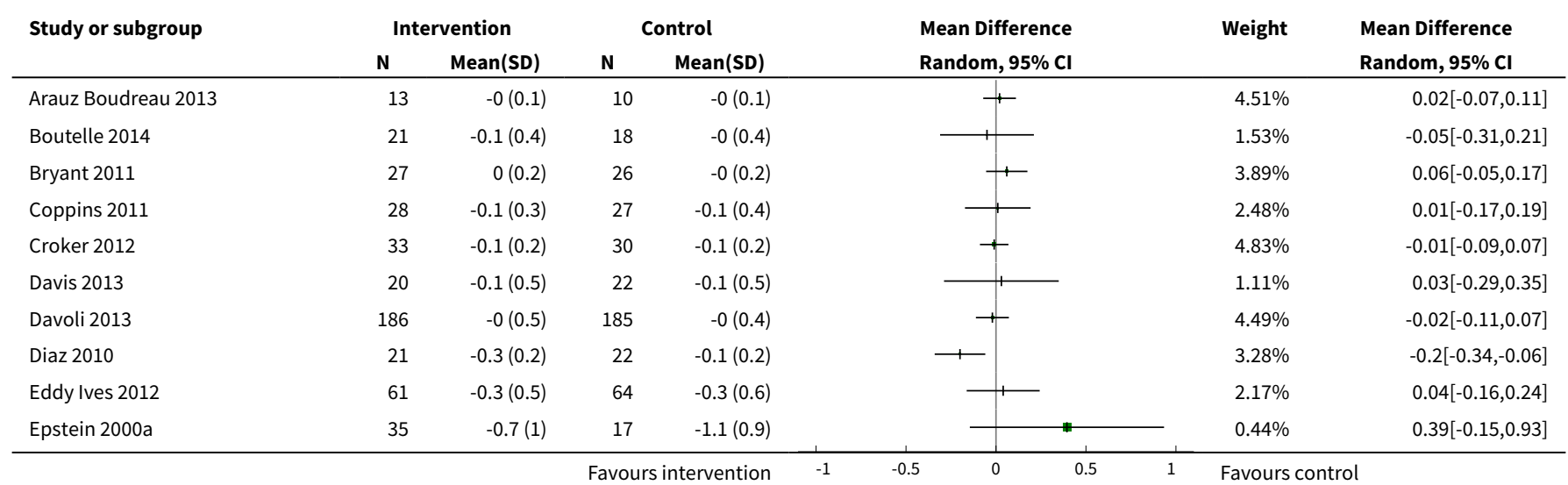

Diet, physical activity and behavioural interventions for the treatment of overweight or obese children from the age of 6 to 11 years 245 (Review)

Copyright (c) 2017 The Cochrane Collaboration. Published by John Wiley \& Sons, Ltd. 


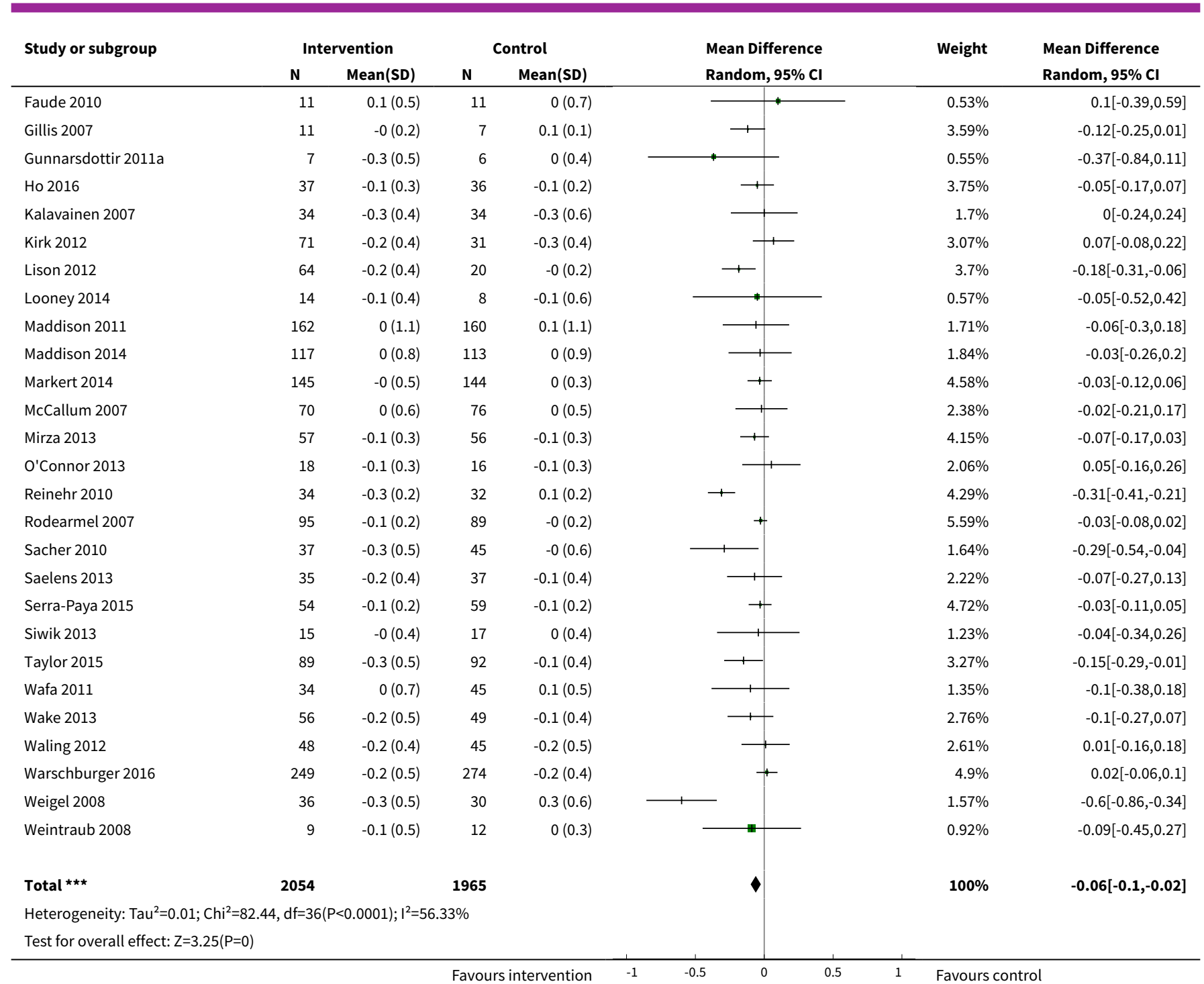

Analysis 1.3. Comparison 1 Behaviour-changing interventions versus no treatment/usual care, Outcome 3 Change in weight (all trials).

\begin{tabular}{|c|c|c|c|c|c|c|c|}
\hline \multirow[t]{2}{*}{ Study or subgroup } & \multicolumn{2}{|c|}{ Intervention } & \multicolumn{2}{|c|}{ Control } & \multirow{2}{*}{$\begin{array}{l}\text { Mean Difference } \\
\text { Random, } 95 \% \mathrm{Cl}\end{array}$} & \multirow[t]{2}{*}{ Weight } & \multirow{2}{*}{$\begin{array}{l}\text { Mean Difference } \\
\text { Random, } 95 \% \mathrm{Cl}\end{array}$} \\
\hline & $\mathbf{N}$ & $\operatorname{Mean}(S D)$ & $\mathbf{N}$ & Mean(SD) & & & \\
\hline Alves 2008 & 39 & $0.6(1.3)$ & 39 & $2(1.5)$ & + & $48.51 \%$ & $-1.37[-1.99,-0.75]$ \\
\hline Coppins 2011 & 28 & $3.9(5.5)$ & 27 & $5.1(5.8)$ & 1 & $2.07 \%$ & $-1.2[-4.2,1.8]$ \\
\hline Croker 2012 & 33 & $0.8(2.8)$ & 30 & $2.8(2.9)$ & + & $9.41 \%$ & $-1.99[-3.4,-0.58]$ \\
\hline Diaz 2010 & 33 & $2.1(6.1)$ & 33 & $5.6(5.9)$ & 1 & $2.22 \%$ & $-3.5[-6.4,-0.6]$ \\
\hline Eddy Ives 2012 & 61 & $3.2(9.4)$ & 64 & $3.4(11.4)$ & - & $1.39 \%$ & $-0.15[-3.81,3.51]$ \\
\hline Epstein 2000a & 45 & $9(13)$ & 32 & $7.2(17)$ & 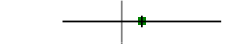 & $0.38 \%$ & $1.78[-5.23,8.78]$ \\
\hline Faude 2010 & 11 & $4.1(13.5)$ & 11 & $3.8(11.7)$ & & $0.17 \%$ & $0.3[-10.26,10.86]$ \\
\hline Ho 2016 & 37 & $2.6(5.2)$ & 36 & $2.7(5)$ & $\longrightarrow$ & $3.43 \%$ & $-0.12[-2.45,2.21]$ \\
\hline Kalarchian 2009 & 97 & $11.8(6.9)$ & 95 & $13.4(5.4)$ & $\rightarrow$ & $6.12 \%$ & $-1.58[-3.32,0.16]$ \\
\hline Kalavainen 2007 & 34 & $17.3(5.2)$ & 34 & $17.1(7.4)$ & 1 & $2.02 \%$ & $0.2[-2.84,3.24]$ \\
\hline Maddison 2011 & 162 & $2(13.1)$ & 160 & $2.8(14.7)$ & -1 & $2.01 \%$ & $-0.72[-3.76,2.32]$ \\
\hline
\end{tabular}

Diet, physical activity and behavioural interventions for the treatment of overweight or obese children from the age of 6 to 11 years 246 (Review)

Copyright (c) 2017 The Cochrane Collaboration. Published by John Wiley \& Sons, Ltd. 


\begin{tabular}{|c|c|c|c|c|c|c|c|}
\hline \multirow[t]{2}{*}{ Study or subgroup } & \multicolumn{2}{|c|}{ Intervention } & \multicolumn{2}{|c|}{ Control } & \multirow{2}{*}{$\begin{array}{l}\text { Mean Difference } \\
\text { Random, } 95 \% \mathrm{Cl}\end{array}$} & \multirow[t]{2}{*}{ Weight } & \multirow{2}{*}{$\begin{array}{l}\text { Mean Difference } \\
\text { Random, } 95 \% \mathrm{Cl}\end{array}$} \\
\hline & $\mathbf{N}$ & Mean(SD) & $\mathbf{N}$ & Mean(SD) & & & \\
\hline Maddison 2014 & 117 & $2.8(16.3)$ & 113 & $2.6(18.4)$ & 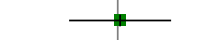 & $0.92 \%$ & $0.21[-4.29,4.71]$ \\
\hline Nemet 2005 & 20 & $0.6(16.7)$ & 20 & $5.2(24.2)$ & & $0.11 \%$ & $-4.6[-17.49,8.29]$ \\
\hline Siwik 2013 & 15 & $3.4(8.8)$ & 17 & $3.9(15.9)$ & . & $0.24 \%$ & $-0.5[-9.28,8.29]$ \\
\hline Taylor 2015 & 89 & $7.5(10.4)$ & 92 & $8.1(8)$ & 1 & $2.53 \%$ & $-0.6[-3.31,2.11]$ \\
\hline Waling 2012 & 36 & $6.7(11.4)$ & 35 & $8.8(10.9)$ & $\longrightarrow+$ & $0.69 \%$ & $-2.1[-7.29,3.09]$ \\
\hline Total $\star \star \star ~$ & 891 & & 883 & & $\checkmark$ & $100 \%$ & $-1.45[-1.88,-1.02]$ \\
\hline \multicolumn{8}{|c|}{ Heterogeneity: $\mathrm{Tau}^{2}=0 ; \mathrm{Chi}^{2}=8.95, \mathrm{df}=16(\mathrm{P}=0.92) ; \mathrm{I}^{2}=0 \%$} \\
\hline \multicolumn{3}{|c|}{ Test for overall effect: $Z=6.59(P<0.0001)$} & & & & & \\
\hline
\end{tabular}

Analysis 1.4. Comparison 1 Behaviour-changing interventions versus no treatment/usual care, Outcome 4 Serious adverse events.

\begin{tabular}{|c|c|c|c|c|c|}
\hline Study or subgroup & $\begin{array}{c}\text { Intervention } \\
\mathbf{n} / \mathbf{N} \\
\end{array}$ & $\begin{array}{c}\text { Control } \\
\mathrm{n} / \mathrm{N}\end{array}$ & $\begin{array}{c}\text { Risk Ratio } \\
\text { M-H, Random, 95\% Cl }\end{array}$ & Weight & $\begin{array}{c}\text { Risk Ratio } \\
\text { M-H, Random, } 95 \% \text { CI } \\
\end{array}$ \\
\hline Alves 2008 & $0 / 39$ & $0 / 39$ & & & Not estimable \\
\hline Boutelle 2014 & $0 / 18$ & $0 / 18$ & & & Not estimable \\
\hline Bryant 2011 & $0 / 35$ & $0 / 35$ & & & Not estimable \\
\hline Coppins 2011 & $0 / 28$ & $0 / 28$ & & & Not estimable \\
\hline Croker 2012 & $0 / 37$ & $0 / 35$ & & & Not estimable \\
\hline Davoli 2013 & $0 / 187$ & $0 / 185$ & & & Not estimable \\
\hline Diaz 2010 & $0 / 38$ & $0 / 38$ & & & Not estimable \\
\hline Eddy Ives 2012 & $0 / 87$ & $0 / 87$ & & & Not estimable \\
\hline Gillis 2007 & $0 / 14$ & $0 / 13$ & & & Not estimable \\
\hline Hamilton-Shield 2014 & $0 / 26$ & $0 / 35$ & & & Not estimable \\
\hline Ho 2016 & $0 / 48$ & $0 / 51$ & & & Not estimable \\
\hline Kalavainen 2007 & $0 / 35$ & $0 / 35$ & & & Not estimable \\
\hline Lison 2012 & $0 / 86$ & $0 / 24$ & & & Not estimable \\
\hline Looney 2014 & $0 / 14$ & $0 / 8$ & & & Not estimable \\
\hline Maddison 2011 & $2 / 160$ & $4 / 162$ & & $52.57 \%$ & $0.51[0.09,2.73]$ \\
\hline Maddison 2014 & $2 / 127$ & $3 / 124$ & 4 & $47.43 \%$ & $0.65[0.11,3.83]$ \\
\hline Mirza 2013 & $0 / 57$ & $0 / 56$ & & & Not estimable \\
\hline Nemet 2005 & $0 / 30$ & $0 / 24$ & & & Not estimable \\
\hline Nowicka 2009 & $0 / 20$ & $0 / 28$ & & & Not estimable \\
\hline O'Connor 2013 & $0 / 20$ & $0 / 20$ & & & Not estimable \\
\hline Reinehr 2010 & $0 / 39$ & $0 / 32$ & & & Not estimable \\
\hline Sacher 2010 & $0 / 60$ & $0 / 56$ & & & Not estimable \\
\hline Taylor 2015 & $0 / 104$ & $0 / 102$ & & & Not estimable \\
\hline Vann 2013 & $0 / 21$ & $0 / 7$ & & & Not estimable \\
\hline Wake 2009 & $0 / 139$ & $0 / 119$ & & & Not estimable \\
\hline Wake 2013 & $0 / 62$ & $0 / 56$ & & & Not estimable \\
\hline Waling 2012 & $0 / 58$ & $0 / 47$ & & & Not estimable \\
\hline Warschburger 2016 & $0 / 336$ & $0 / 350$ & & & Not estimable \\
\hline
\end{tabular}

Diet, physical activity and behavioural interventions for the treatment of overweight or obese children from the age of 6 to 11 years 


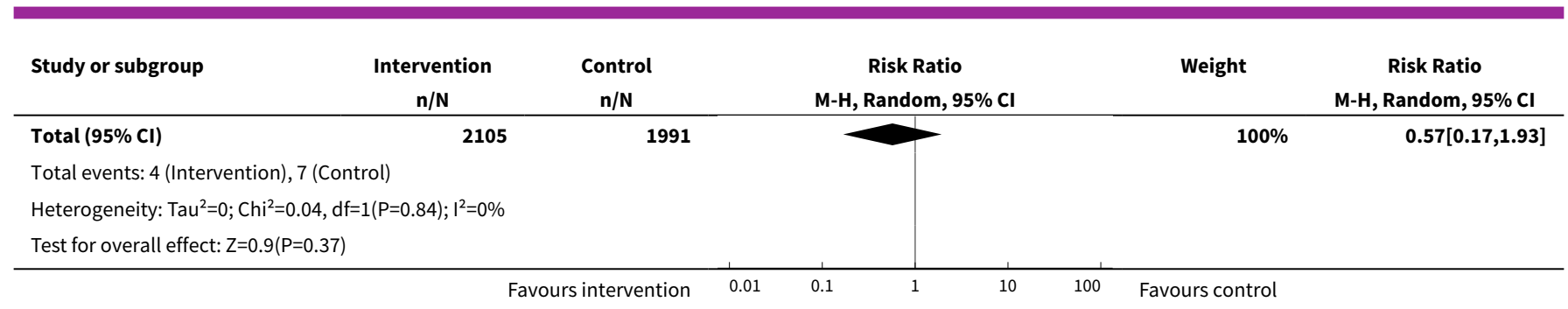

\section{Analysis 1.5. Comparison 1 Behaviour-changing interventions versus no treatment/ usual care, Outcome 5 Health-related quality of life (parent-report measures).}

\begin{tabular}{|c|c|c|c|c|c|c|c|}
\hline \multirow[t]{2}{*}{ Study or subgroup } & \multicolumn{2}{|c|}{ Intervention } & \multicolumn{2}{|c|}{ Control } & \multirow{2}{*}{$\begin{array}{c}\text { Std. Mean Difference } \\
\text { Random, } 95 \% \mathrm{Cl}\end{array}$} & \multirow[t]{2}{*}{ Weight } & \multirow{2}{*}{$\begin{array}{c}\text { Std. Mean Difference } \\
\text { Random, } 95 \% \mathrm{Cl}\end{array}$} \\
\hline & $\mathbf{N}$ & Mean(SD) & $\mathbf{N}$ & $\operatorname{Mean}(S D)$ & & & \\
\hline \multicolumn{8}{|c|}{ 1.5.1 PedsQL caregiver-report } \\
\hline Croker 2012 & 33 & $3.8(9.1)$ & 30 & $3(9.1)$ & & $11.69 \%$ & $0.09[-0.41,0.58]$ \\
\hline McCallum 2007 & 70 & $1.2(13.3)$ & 76 & $3.6(13.3)$ & —- & $21.36 \%$ & $-0.18[-0.51,0.15]$ \\
\hline Wafa 2011 & 34 & $3.9(19.3)$ & 46 & $-4.2(15.5)$ & $\rightarrow$ & $13.58 \%$ & $0.47[0.02,0.92]$ \\
\hline Wake 2009 & 124 & $4.3(13.3)$ & 113 & $1.3(13.5)$ & - & $28.15 \%$ & $0.22[-0.03,0.48]$ \\
\hline Subtotal $\star \star \star$ & 261 & & 265 & & & $74.79 \%$ & $0.13[-0.13,0.4]$ \\
\hline \multicolumn{8}{|c|}{ Heterogeneity: $\mathrm{Tau}^{2}=0.04 ; \mathrm{Chi}^{2}=6.17, \mathrm{df}=3(\mathrm{P}=0.1) ; \mathrm{I}^{2}=51.35 \%$} \\
\hline \multicolumn{8}{|c|}{ Test for overall effect: $Z=1(P=0.32)$} \\
\hline \multicolumn{8}{|c|}{ 1.5.2 CHQ-PF50 - global score, parental report } \\
\hline Kalarchian 2009 & 97 & $4.1(24.5)$ & 95 & $0.5(27.7)$ & & $25.21 \%$ & $0.14[-0.14,0.42]$ \\
\hline Subtotal $\star \star \star$ & 97 & & 95 & & & $25.21 \%$ & $0.14[-0.14,0.42]$ \\
\hline \multicolumn{8}{|c|}{ Test for overall effect: $Z=0.96(P=0.34)$} \\
\hline Total ${ }^{\star \star \star}$ & 358 & & 360 & & & $100 \%$ & $0.13[-0.06,0.32]$ \\
\hline \multicolumn{8}{|c|}{ Heterogeneity: $\mathrm{Tau}^{2}=0.02 ; \mathrm{Chi}^{2}=6.17, \mathrm{df}=4(\mathrm{P}=0.19) ; \mathrm{I}^{2}=35.17 \%$} \\
\hline \multicolumn{8}{|c|}{ Test for overall effect: $Z=1.37(P=0.17)$} \\
\hline \multicolumn{8}{|c|}{ Test for subgroup differences: $\mathrm{Chi}^{2}=0, \mathrm{df}=1(\mathrm{P}=0.98), \mathrm{I}^{2}=0 \%$} \\
\hline
\end{tabular}

\section{Analysis 1.6. Comparison 1 Behaviour-changing interventions versus no treatment/ usual care, Outcome 6 Health-related quality of life (child-report measures).}

\begin{tabular}{|c|c|c|c|c|c|c|c|}
\hline \multirow[t]{2}{*}{ Study or subgroup } & \multicolumn{2}{|c|}{ Intervention } & \multicolumn{2}{|c|}{ Control } & \multirow{2}{*}{$\begin{array}{c}\text { Std. Mean Difference } \\
\text { Random, } 95 \% \mathrm{Cl} \\
\end{array}$} & \multirow[t]{2}{*}{ Weight } & \multirow{2}{*}{$\begin{array}{c}\text { Std. Mean Difference } \\
\text { Random, } 95 \% \mathrm{Cl}\end{array}$} \\
\hline & $\mathbf{N}$ & Mean(SD) & $\mathbf{N}$ & Mean(SD) & & & \\
\hline \multicolumn{8}{|c|}{ 1.6.1 PedsQL child-report } \\
\hline Croker 2012 & 33 & $0.8(11.8)$ & 30 & $4(11.8)$ & $\longrightarrow$ & $37.69 \%$ & $-0.27[-0.76,0.23]$ \\
\hline Wafa 2011 & 34 & $5(11.6)$ & 45 & $-1.4(16.1)$ & & $40.42 \%$ & $0.44[-0.01,0.89]$ \\
\hline Subtotal ${ }^{\star \star \star}$ & 67 & & 75 & & & $78.11 \%$ & $0.1[-0.6,0.79]$ \\
\hline \multicolumn{8}{|c|}{ Heterogeneity: $\mathrm{Tau}^{2}=0.19 ; \mathrm{Chi}^{2}=4.27, \mathrm{df}=1(\mathrm{P}=0.04) ; \mathrm{I}^{2}=76.57 \%$} \\
\hline \multicolumn{8}{|c|}{ 1.6.2 KINDL-R child-report } \\
\hline Faude 2010 & 11 & $5(8.9)$ & 11 & $0.4(16.5)$ & 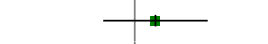 & $21.89 \%$ & $0.33[-0.51,1.18]$ \\
\hline \multirow[t]{2}{*}{ 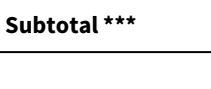 } & 11 & & 11 & & & $21.89 \%$ & $0.33[-0.51,1.18]$ \\
\hline & & & Favou & ervention & -2 & Favour & \\
\hline
\end{tabular}

Diet, physical activity and behavioural interventions for the treatment of overweight or obese children from the age of 6 to 11 years 


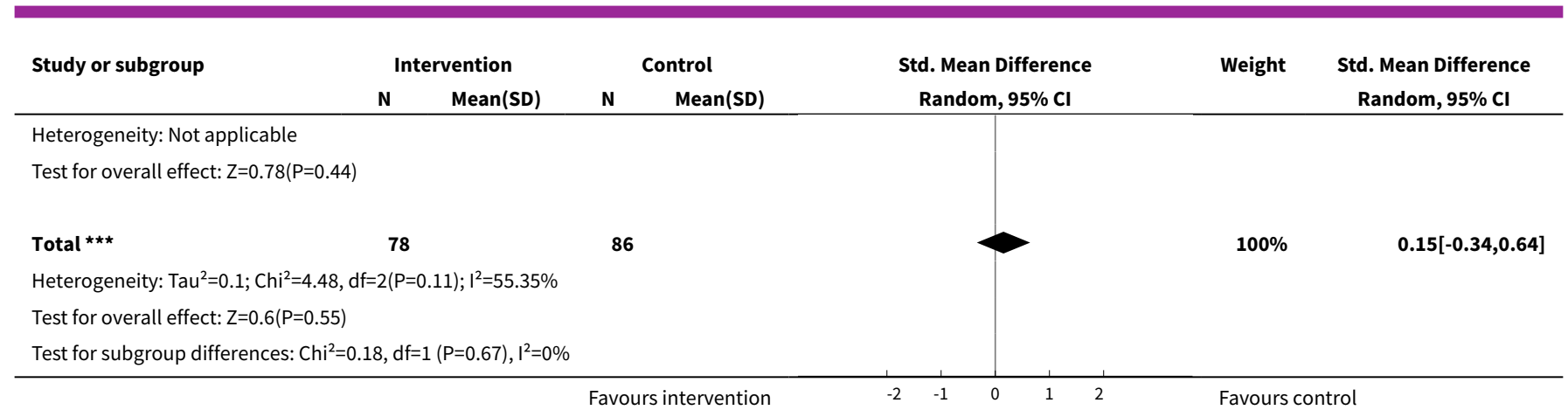

Analysis 1.7. Comparison 1 Behaviour-changing interventions versus no treatment/usual care, Outcome 7 Self-esteem (Harter global score).

\begin{tabular}{|c|c|c|c|c|c|c|c|}
\hline \multirow[t]{2}{*}{ Study or subgroup } & \multicolumn{2}{|c|}{ Intervention } & \multicolumn{2}{|c|}{ Control } & \multirow{2}{*}{$\begin{array}{l}\text { Mean Difference } \\
\text { Random, } 95 \% \mathrm{Cl}\end{array}$} & \multirow[t]{2}{*}{ Weight } & \multirow{2}{*}{$\begin{array}{l}\text { Mean Difference } \\
\text { Random, } 95 \% \mathrm{Cl}\end{array}$} \\
\hline & $\mathbf{N}$ & Mean(SD) & $\mathbf{N}$ & Mean(SD) & & & \\
\hline Croker 2012 & 33 & $0.2(0.6)$ & 30 & $0.1(0.6)$ & $\frac{1}{1}$ & $46.02 \%$ & $0.06[-0.26,0.38]$ \\
\hline Sacher 2010 & 37 & $0.4(0.7)$ & 44 & $0.1(0.7)$ & -1 & $53.98 \%$ & $0.3[0.01,0.59]$ \\
\hline Total $\star \star \star ~$ & 70 & & 74 & & & $100 \%$ & $0.19[-0.04,0.42]$ \\
\hline \multicolumn{8}{|c|}{ Heterogeneity: $\mathrm{Tau}^{2}=0.01 ; \mathrm{Chi}^{2}=1.21, \mathrm{df}=1(\mathrm{P}=0.27) ; \mathrm{I}^{2}=17.46 \%$} \\
\hline \multicolumn{8}{|c|}{ Test for overall effect: $Z=1.58(P=0.11)$} \\
\hline
\end{tabular}

Analysis 1.8. Comparison 1 Behaviour-changing interventions versus no treatment/usual care, Outcome 8 Waist circumference.

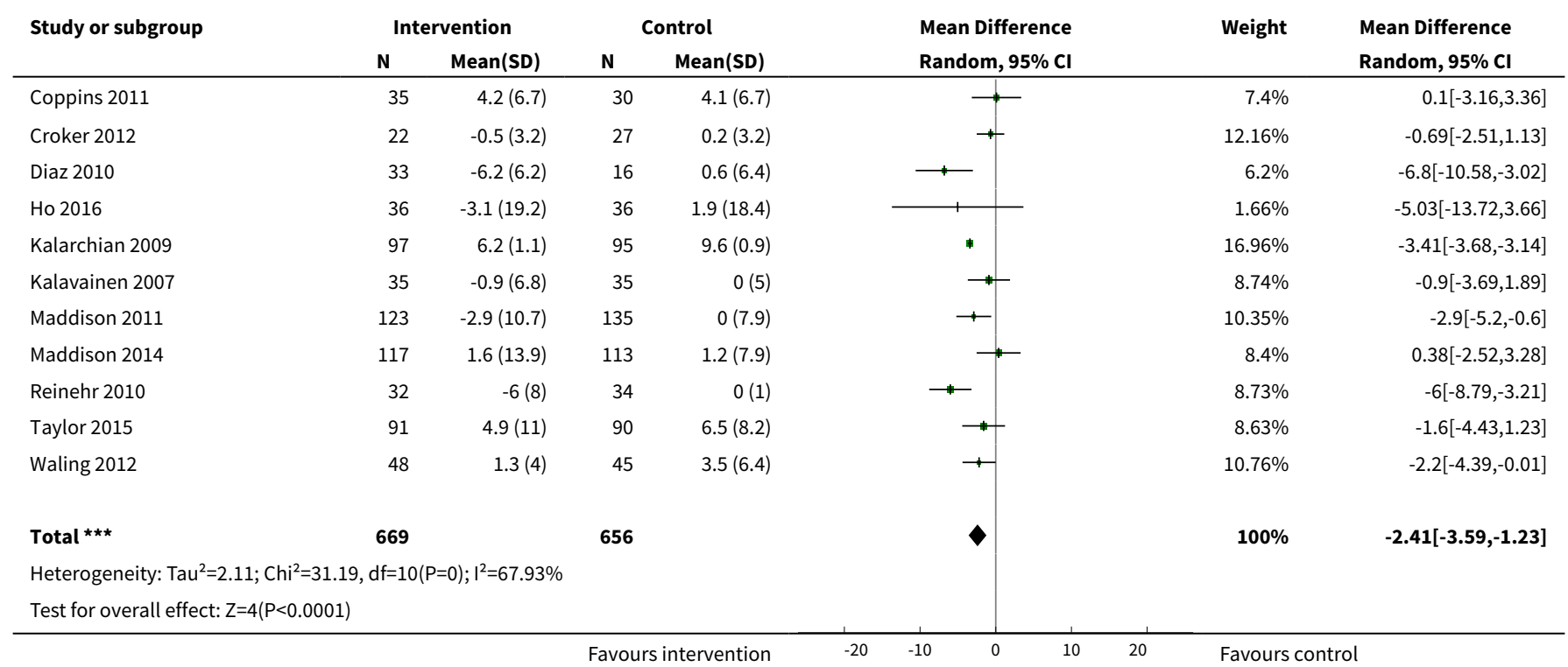

Diet, physical activity and behavioural interventions for the treatment of overweight or obese children from the age of 6 to 11 years 


\section{Analysis 1.9. Comparison 1 Behaviour-changing interventions} versus no treatment/usual care, Outcome 9 Overweight.

\begin{tabular}{|c|c|c|c|c|c|c|c|}
\hline \multirow[t]{2}{*}{ Study or subgroup } & \multicolumn{2}{|c|}{ Intervention } & \multicolumn{2}{|c|}{ Control } & \multirow{2}{*}{$\begin{array}{l}\text { Mean Difference } \\
\text { Random, 95\% Cl }\end{array}$} & \multirow[t]{2}{*}{ Weight } & \multirow{2}{*}{$\begin{array}{l}\text { Mean Difference } \\
\text { Random, } 95 \% \mathrm{CI}\end{array}$} \\
\hline & $\mathbf{N}$ & $\operatorname{Mean}(S D)$ & $\mathbf{N}$ & $\operatorname{Mean}(S D)$ & & & \\
\hline Kalarchian 2009 & 97 & $-1.2(1.7)$ & 97 & $-0.2(0.1)$ & 1 & $54.75 \%$ & $-0.99[-1.32,-0.66]$ \\
\hline Nova 2001 & 50 & $-8.5(9.7)$ & 80 & $-2.9(10.8)$ & $\Psi$ & $39.2 \%$ & $-5.58[-9.17,-1.99]$ \\
\hline Satoh 2007 & 15 & $-8(21.5)$ & 8 & $1(17.1)$ & + & $6.05 \%$ & $-9[-25.09,7.09]$ \\
\hline 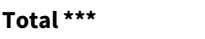 & 162 & & 185 & & 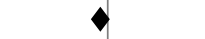 & $100 \%$ & $-3.27[-7.47,0.92]$ \\
\hline \multicolumn{3}{|c|}{ Test for overall effect: $Z=1.53(P=0.13)$} & & & & & \\
\hline
\end{tabular}

\section{Analysis 1.10. Comparison 1 Behaviour-changing interventions} versus no treatment/usual care, Outcome 10 Body fat.

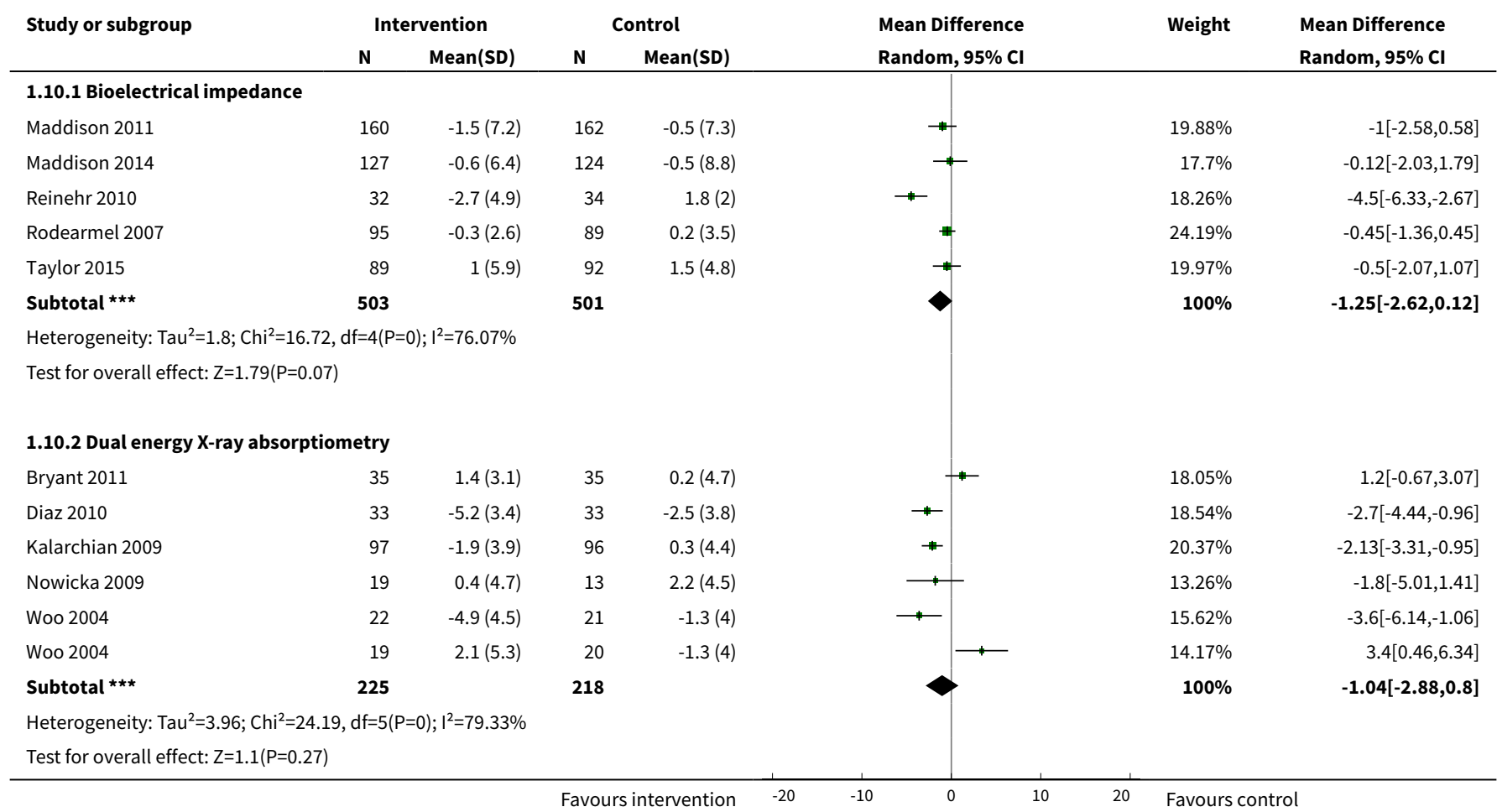

Analysis 1.11. Comparison 1 Behaviour-changing interventions versus no treatment/usual care, Outcome 11 Diet.

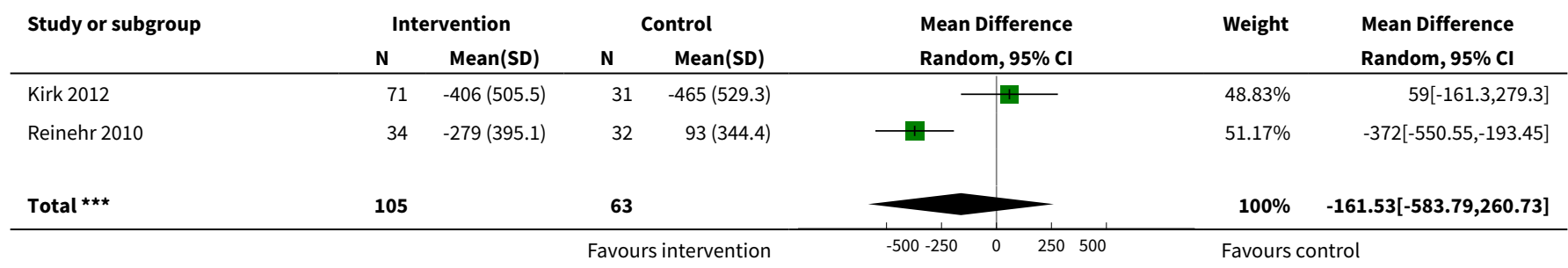

Diet, physical activity and behavioural interventions for the treatment of overweight or obese children from the age of 6 to 11 years 


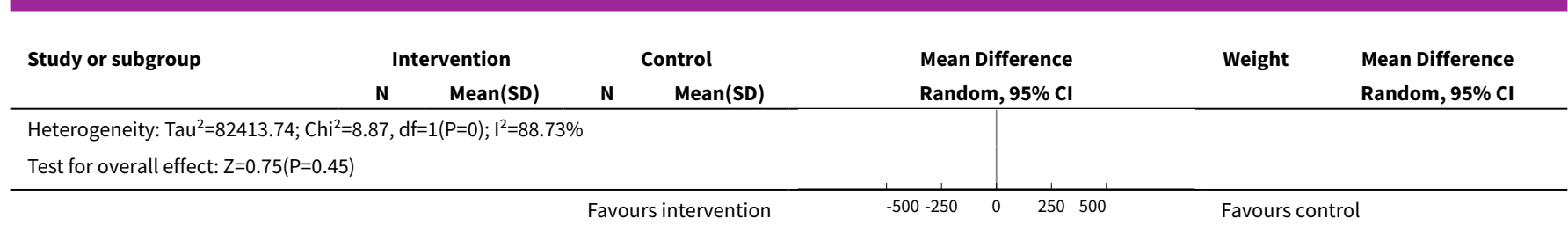

Analysis 1.12. Comparison 1 Behaviour-changing interventions versus no treatment/usual care, Outcome 12 Television viewing.

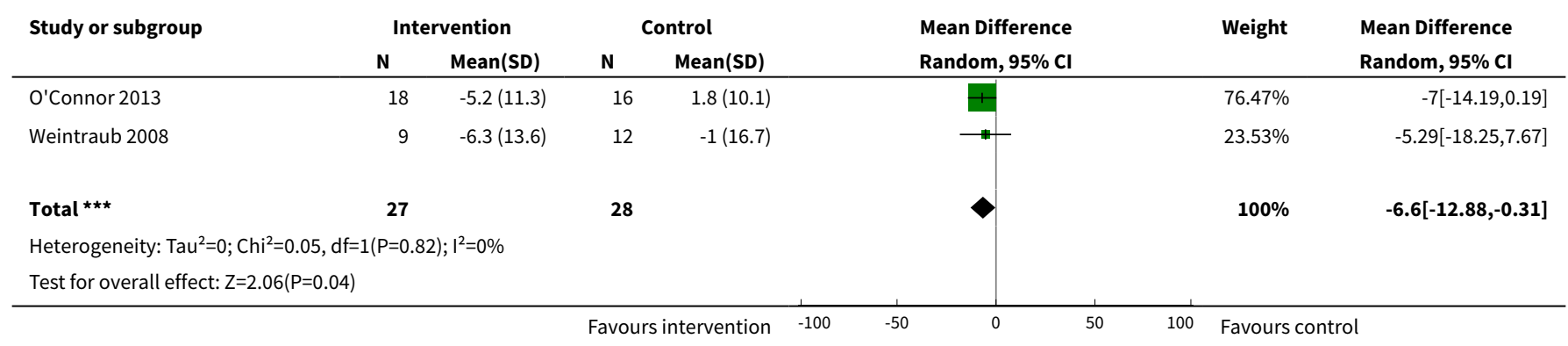

Analysis 1.13. Comparison 1 Behaviour-changing interventions versus no treatment/usual care, Outcome 13 Physical activity (accelerometer MVPA).

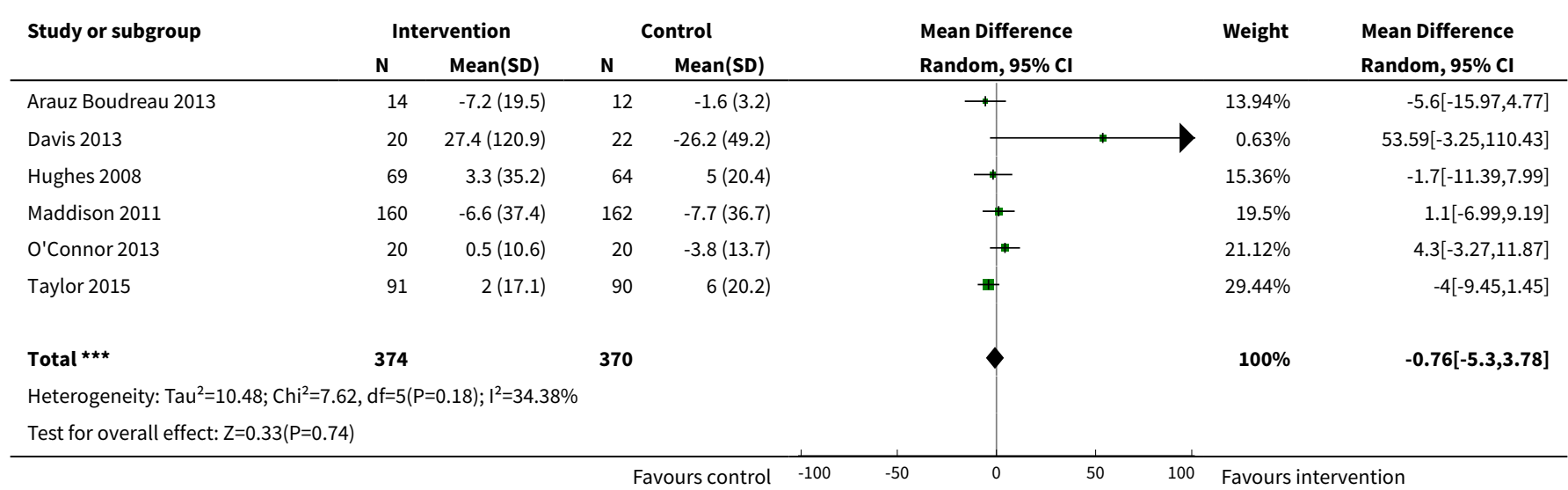

Analysis 1.14. Comparison 1 Behaviour-changing interventions versus no treatment/usual care, Outcome 14 Change in BMI - type of control.

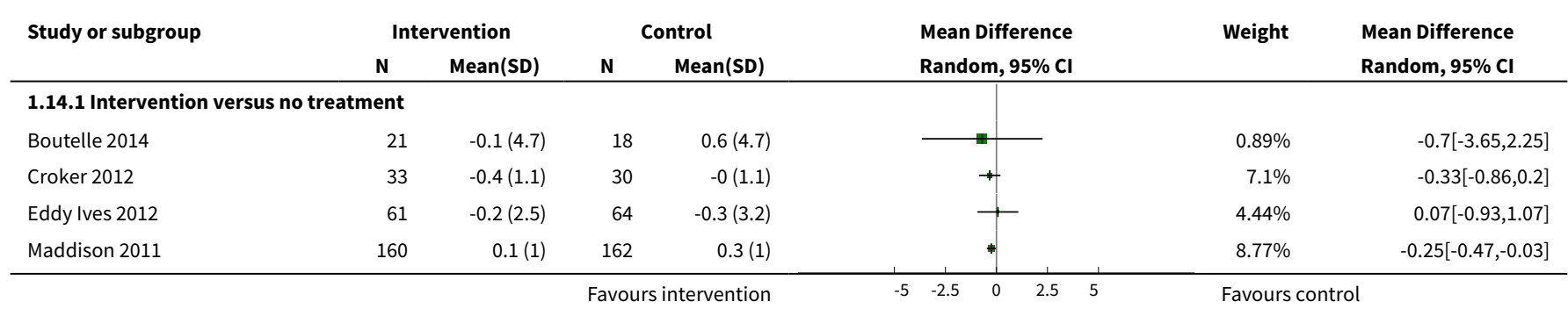




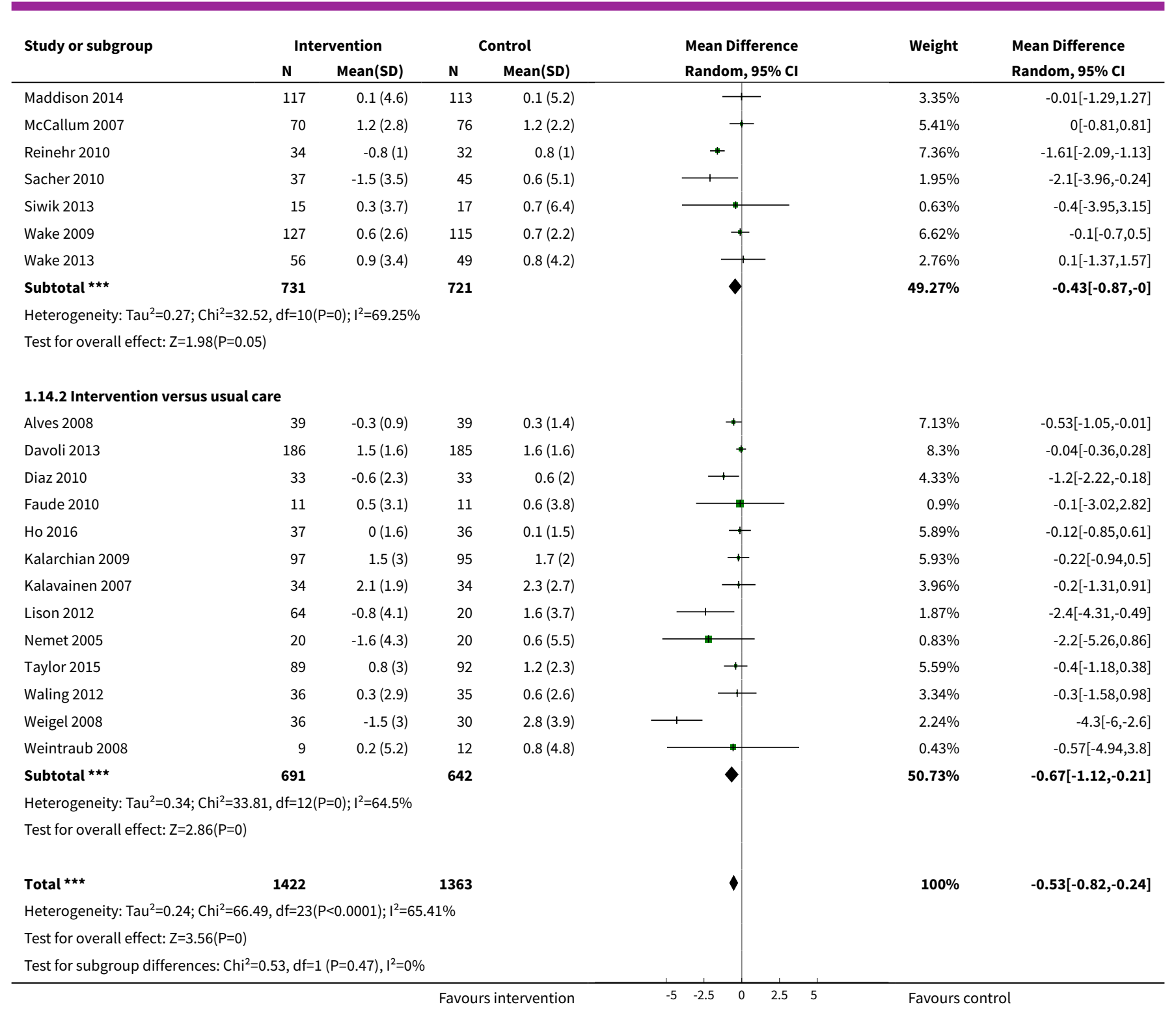

Analysis 1.15. Comparison 1 Behaviour-changing interventions versus no treatment/usual care, Outcome 15 Change in BMI z score - type of control.

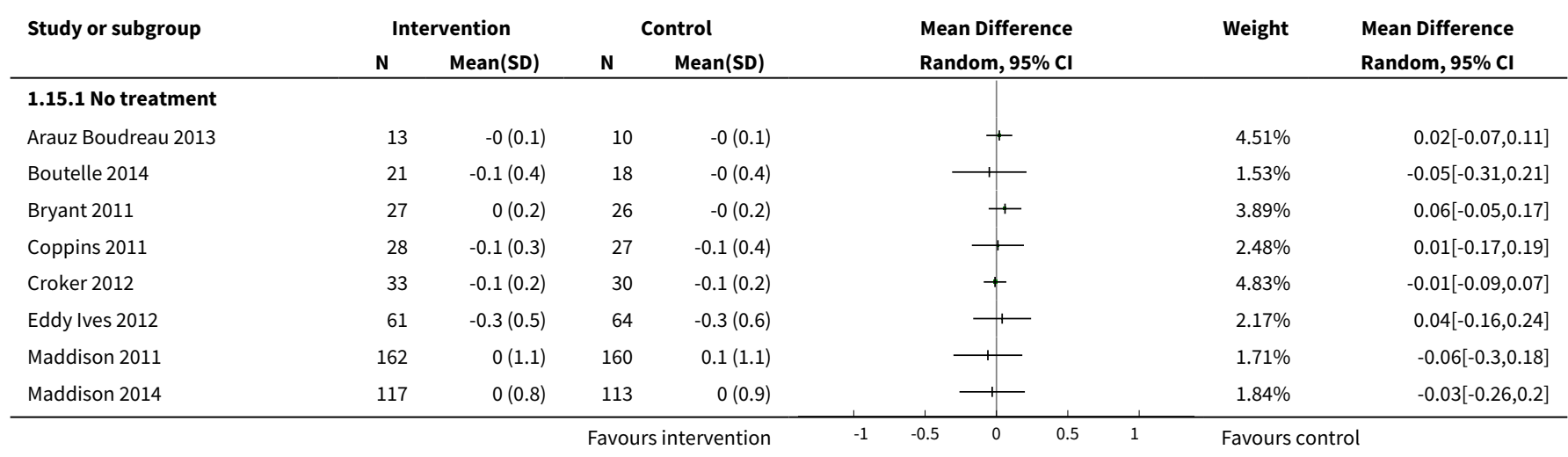

Diet, physical activity and behavioural interventions for the treatment of overweight or obese children from the age of 6 to 11 years 252 (Review)

Copyright (c) 2017 The Cochrane Collaboration. Published by John Wiley \& Sons, Ltd. 


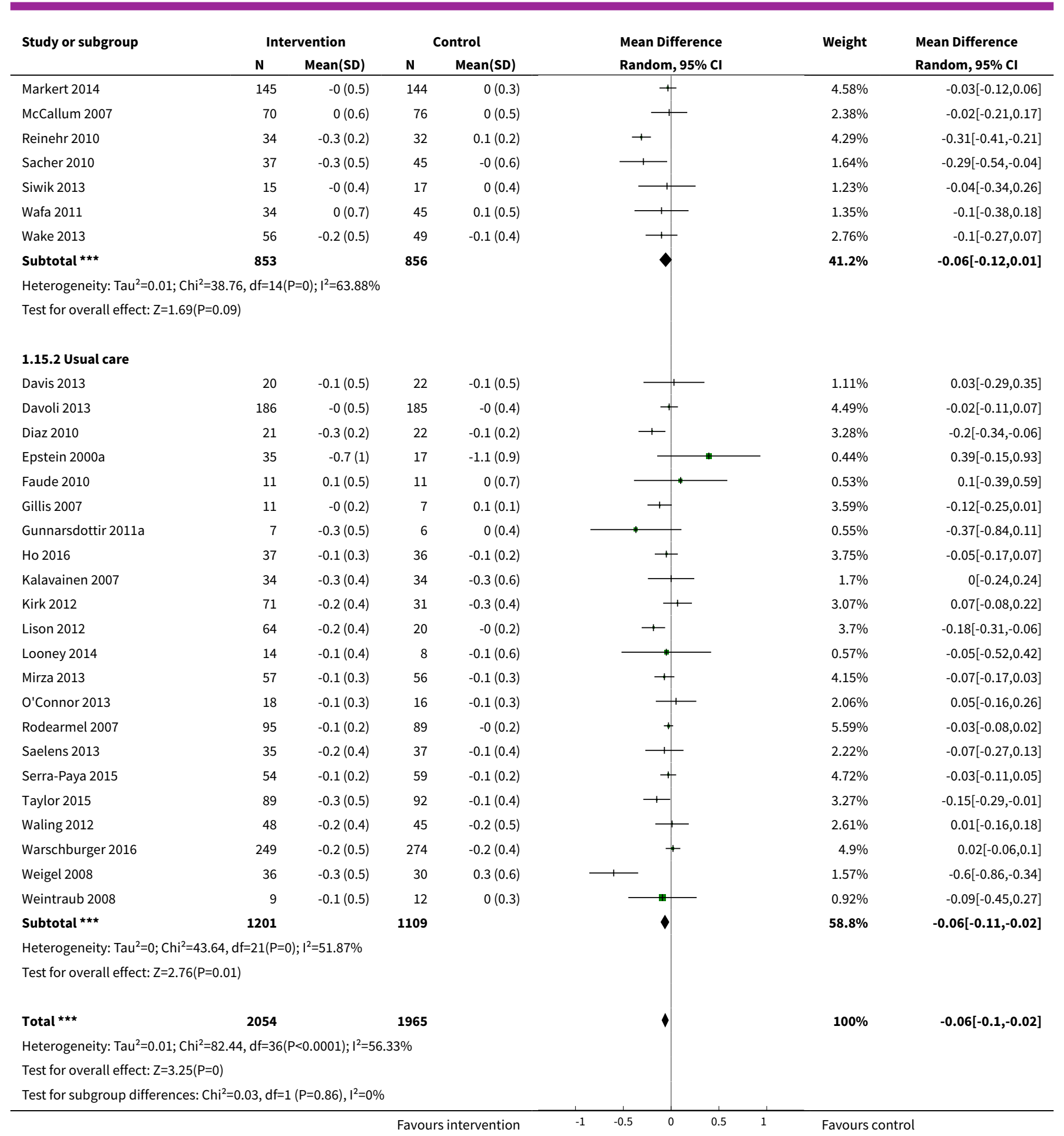

Diet, physical activity and behavioural interventions for the treatment of overweight or obese children from the age of 6 to 11 years 
Analysis 1.16. Comparison 1 Behaviour-changing interventions versus no treatment/usual care, Outcome 16 Change in weight - type of control.

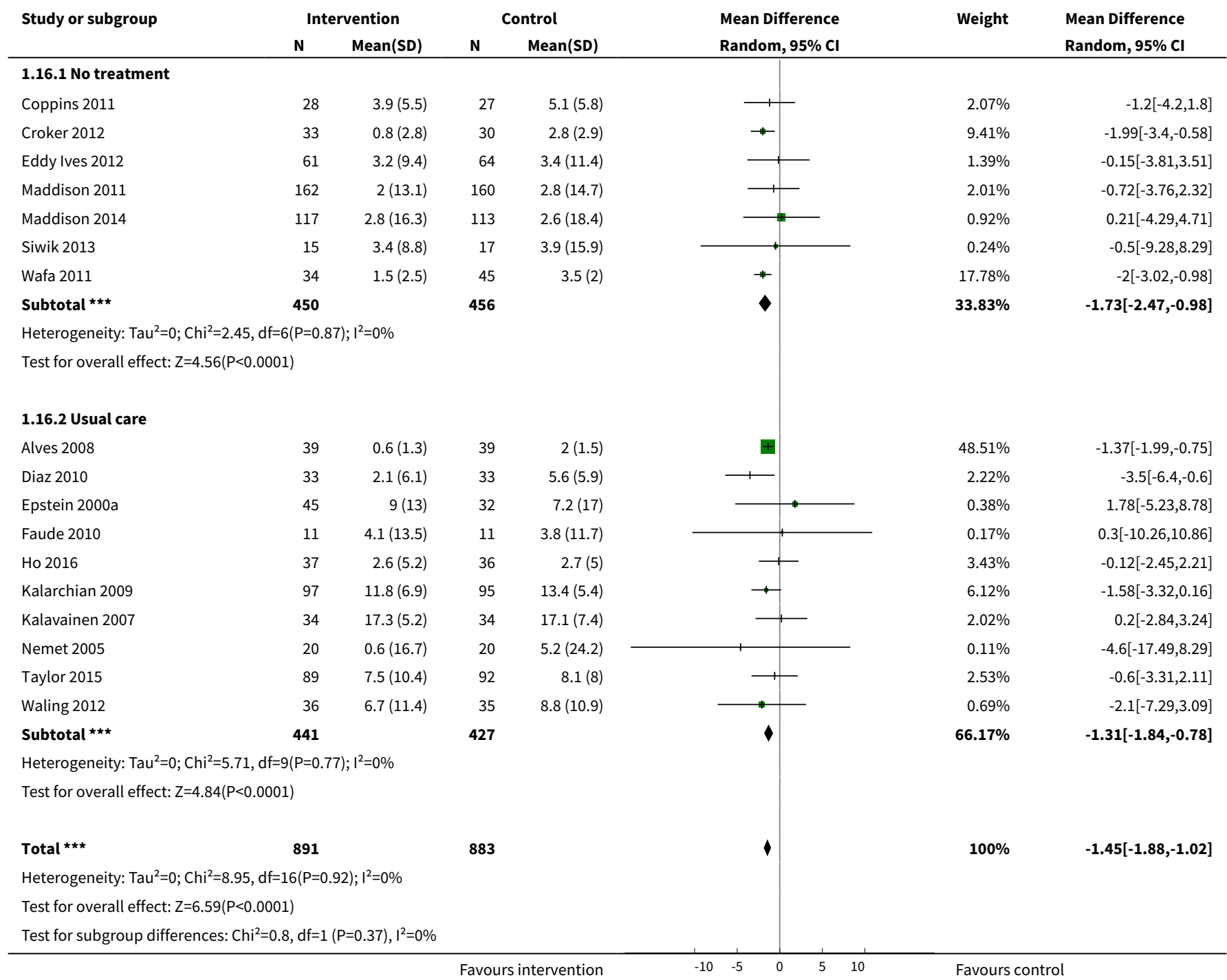

Analysis 1.17. Comparison 1 Behaviour-changing interventions versus no treatment/usual care, Outcome 17 Change in BMI - type of intervention.

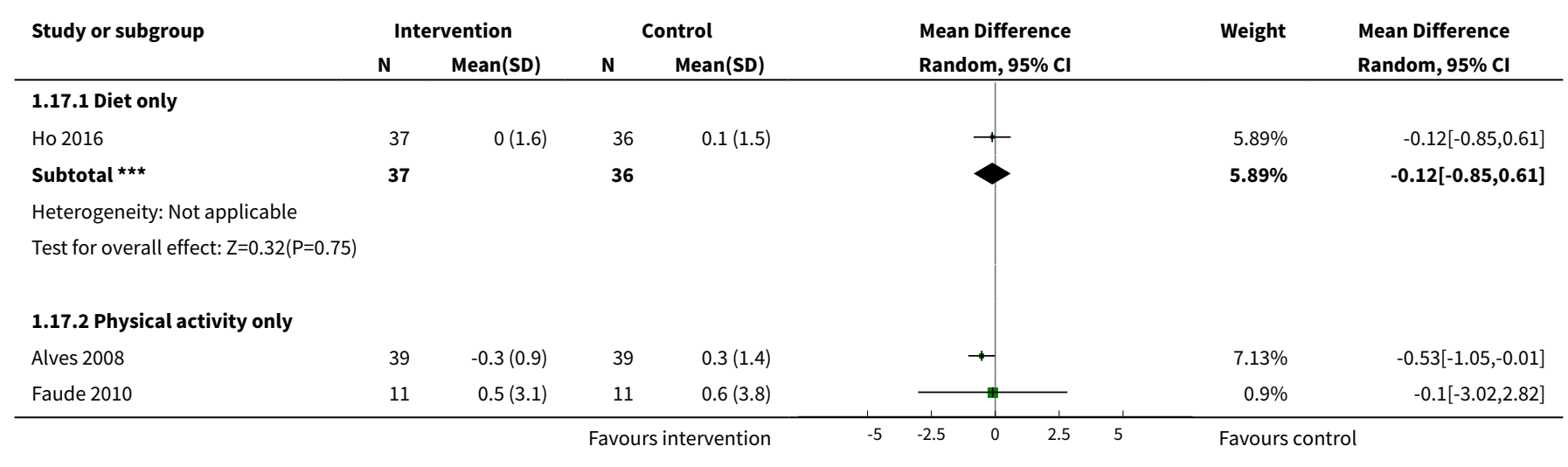

Diet, physical activity and behavioural interventions for the treatment of overweight or obese children from the age of 6 to 11 years 


\begin{tabular}{|c|c|c|c|c|c|c|c|}
\hline \multirow[t]{2}{*}{ Study or subgroup } & \multicolumn{2}{|c|}{ Intervention } & \multicolumn{2}{|c|}{ Control } & \multirow{2}{*}{$\begin{array}{l}\text { Mean Difference } \\
\text { Random, } 95 \% \mathrm{Cl}\end{array}$} & \multirow[t]{2}{*}{ Weight } & \multirow{2}{*}{$\begin{array}{l}\text { Mean Difference } \\
\text { Random, } 95 \% \mathrm{Cl}\end{array}$} \\
\hline & $\mathbf{N}$ & $\operatorname{Mean}(S D)$ & $\mathbf{N}$ & Mean(SD) & & & \\
\hline Maddison 2011 & 160 & $0.1(1)$ & 162 & $0.3(1)$ & $*$ & $8.77 \%$ & $-0.25[-0.47,-0.03]$ \\
\hline Weintraub 2008 & 9 & $0.2(5.2)$ & 12 & $0.8(4.8)$ & $\because$ & $0.43 \%$ & $-0.57[-4.94,3.8]$ \\
\hline Subtotal $\star \star \star ~$ & 219 & & 224 & & $\checkmark$ & $17.23 \%$ & $-0.29[-0.5,-0.09]$ \\
\hline \multicolumn{8}{|c|}{ Heterogeneity: Tau $^{2}=0 ; \mathrm{Chi}^{2}=0.97, \mathrm{df}=3(\mathrm{P}=0.81) ; \mathrm{I}^{2}=0 \%$} \\
\hline \multicolumn{8}{|c|}{ Test for overall effect: $Z=2.82(P=0)$} \\
\hline \multicolumn{8}{|c|}{ 1.17.3 Diet and physical activity } \\
\hline Eddy Ives 2012 & 61 & $-0.2(2.5)$ & 64 & $-0.3(3.2)$ & 1 & $4.44 \%$ & $0.07[-0.93,1.07]$ \\
\hline Lison 2012 & 64 & $-0.8(4.1)$ & 20 & $1.6(3.7)$ & 1 & $1.87 \%$ & $-2.4[-4.31,-0.49]$ \\
\hline Subtotal ${ }^{\star \star \star}$ & 125 & & 84 & & & $6.31 \%$ & $-1.03[-3.43,1.38]$ \\
\hline \multicolumn{8}{|c|}{ Heterogeneity: $\mathrm{Tau}^{2}=2.45 ; \mathrm{Chi}^{2}=5.04, \mathrm{df}=1(\mathrm{P}=0.02) ; \mathrm{I}^{2}=80.17 \%$} \\
\hline \multicolumn{8}{|c|}{ Test for overall effect: $Z=0.84(P=0.4)$} \\
\hline \multicolumn{8}{|c|}{ 1.17.4 Diet and behavioural therapy } \\
\hline Boutelle 2014 & 21 & $-0.1(4.7)$ & 18 & $0.6(4.7)$ & - & $0.89 \%$ & $-0.7[-3.65,2.25]$ \\
\hline Subtotal $\star \star \star$ & 21 & & 18 & & & $0.89 \%$ & $-0.7[-3.65,2.25]$ \\
\hline \multicolumn{8}{|c|}{ Heterogeneity: Not applicable } \\
\hline \multicolumn{8}{|c|}{ Test for overall effect: $Z=0.46(P=0.64)$} \\
\hline \multicolumn{8}{|c|}{ 1.17.5 Physical activity and behavioural therapy } \\
\hline Maddison 2014 & 117 & $0.1(4.6)$ & 113 & $0.1(5.2)$ & - & $3.35 \%$ & $-0.01[-1.29,1.27]$ \\
\hline Subtotal ${ }^{\star \star \star}$ & 117 & & 113 & & & $3.35 \%$ & $-0.01[-1.29,1.27]$ \\
\hline \multicolumn{8}{|c|}{ Heterogeneity: Not applicable } \\
\hline \multicolumn{8}{|c|}{ Test for overall effect: $Z=0.02(P=0.99)$} \\
\hline \multicolumn{8}{|c|}{ 1.17.6 Diet, physical activity and behavioural therapy } \\
\hline Croker 2012 & 33 & $-0.4(1.1)$ & 30 & $-0(1.1)$ & + & $7.1 \%$ & $-0.33[-0.86,0.2]$ \\
\hline Davoli 2013 & 186 & $1.5(1.6)$ & 185 & $1.6(1.6)$ & + & $8.3 \%$ & $-0.04[-0.36,0.28]$ \\
\hline Diaz 2010 & 33 & $-0.6(2.3)$ & 33 & $0.6(2)$ & + & $4.33 \%$ & $-1.2[-2.22,-0.18]$ \\
\hline Kalarchian 2009 & 97 & $1.5(3)$ & 95 & $1.7(2)$ & + & $5.93 \%$ & $-0.22[-0.94,0.5]$ \\
\hline Kalavainen 2007 & 34 & $2.1(1.9)$ & 34 & $2.3(2.7)$ & - & $3.96 \%$ & $-0.2[-1.31,0.91]$ \\
\hline McCallum 2007 & 70 & $1.2(2.8)$ & 76 & $1.2(2.2)$ & $\longrightarrow$ & $5.41 \%$ & $0[-0.81,0.81]$ \\
\hline Nemet 2005 & 20 & $-1.6(4.3)$ & 20 & $0.6(5.5)$ & - & $0.83 \%$ & $-2.2[-5.26,0.86]$ \\
\hline Reinehr 2010 & 34 & $-0.8(1)$ & 32 & $0.8(1)$ & $\rightarrow$ & $7.36 \%$ & $-1.61[-2.09,-1.13]$ \\
\hline Sacher 2010 & 37 & $-1.5(3.5)$ & 45 & $0.6(5.1)$ & + & $1.95 \%$ & $-2.1[-3.96,-0.24]$ \\
\hline Siwik 2013 & 15 & $0.3(3.7)$ & 17 & $0.7(6.4)$ & $\rightarrow$ & $0.63 \%$ & $-0.4[-3.95,3.15]$ \\
\hline Taylor 2015 & 89 & $0.8(3)$ & 92 & $1.2(2.3)$ & 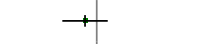 & $5.59 \%$ & $-0.4[-1.18,0.38]$ \\
\hline Wake 2009 & 127 & $0.6(2.6)$ & 115 & $0.7(2.2)$ & + & $6.62 \%$ & $-0.1[-0.7,0.5]$ \\
\hline Wake 2013 & 56 & $0.9(3.4)$ & 49 & $0.8(4.2)$ & - & $2.76 \%$ & $0.1[-1.37,1.57]$ \\
\hline Waling 2012 & 36 & $0.3(2.9)$ & 35 & $0.6(2.6)$ & 1 & $3.34 \%$ & $-0.3[-1.58,0.98]$ \\
\hline Weigel 2008 & 36 & $-1.5(3)$ & 30 & $2.8(3.9)$ & - & $2.24 \%$ & $-4.3[-6,-2.6]$ \\
\hline Subtotal $* \star \star$ & 903 & & 888 & & $>$ & $66.35 \%$ & $-0.67[-1.12,-0.23]$ \\
\hline \multicolumn{8}{|c|}{ Heterogeneity: $\mathrm{Tau}^{2}=0.48 ; \mathrm{Chi}^{2}=57.99, \mathrm{df}=14(\mathrm{P}<0.0001) ; \mathrm{I}^{2}=75.86 \%$} \\
\hline \multicolumn{8}{|c|}{ Test for overall effect: $Z=2.94(P=0)$} \\
\hline Total $\star \star \star ~$ & 1422 & & 1363 & & $\boldsymbol{\vartheta}$ & $100 \%$ & $-0.53[-0.82,-0.24]$ \\
\hline \multicolumn{8}{|c|}{ Heterogeneity: $\mathrm{Tau}^{2}=0.24 ; \mathrm{Chi}^{2}=66.49, \mathrm{df}=23(\mathrm{P}<0.0001) ; \mathrm{I}^{2}=65.41 \%$} \\
\hline \multicolumn{8}{|c|}{ Test for overall effect: $Z=3.56(P=0)$} \\
\hline Test for subgroup dif & $32, \mathrm{df}=$ & $(P=0.65), I^{2}=0$ & & & & & \\
\hline
\end{tabular}

Diet, physical activity and behavioural interventions for the treatment of overweight or obese children from the age of 6 to 11 years 
Analysis 1.18. Comparison 1 Behaviour-changing interventions versus no treatment/usual care, Outcome 18 Change in BMI z score - type of intervention.

\begin{tabular}{|c|c|c|c|c|c|c|c|}
\hline \multirow{3}{*}{$\begin{array}{l}\text { Study or subgroup } \\
1.18 .1 \text { Diet only }\end{array}$} & \multicolumn{2}{|c|}{ Intervention } & \multicolumn{2}{|c|}{ Control } & \multirow{2}{*}{$\begin{array}{l}\text { Mean Difference } \\
\text { Random, } 95 \% \mathrm{Cl}\end{array}$} & \multirow[t]{2}{*}{ Weight } & \multirow{2}{*}{$\begin{array}{l}\text { Mean Difference } \\
\text { Random, } 95 \% \mathrm{Cl}\end{array}$} \\
\hline & \multirow[t]{2}{*}{$\mathbf{N}$} & \multirow[t]{2}{*}{ Mean(SD) } & \multirow[t]{2}{*}{$\mathbf{N}$} & \multirow[t]{2}{*}{ Mean(SD) } & & & \\
\hline & & & & & & & \\
\hline Ho 2016 & 37 & $-0.1(0.3)$ & 36 & $-0.1(0.2)$ & & $3.73 \%$ & $-0.05[-0.17,0.07]$ \\
\hline 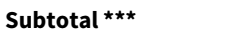 & 37 & & 36 & & & $3.73 \%$ & $-0.05[-0.17,0.07]$ \\
\hline \multicolumn{8}{|c|}{ Heterogeneity: Not applicable } \\
\hline \multicolumn{8}{|c|}{ Test for overall effect: $Z=0.81(P=0.42)$} \\
\hline \multicolumn{8}{|c|}{ 1.18.2 Physical activity only } \\
\hline Faude 2010 & 11 & $0.1(0.5)$ & 11 & $0(0.7)$ & + & $0.52 \%$ & $0.1[-0.39,0.59]$ \\
\hline Maddison 2011 & 162 & $0(1.1)$ & 160 & $0.1(1.1)$ & - & $1.7 \%$ & $-0.06[-0.3,0.18]$ \\
\hline Weintraub 2008 & 9 & $-0.1(0.5)$ & 12 & $0(0.3)$ & I & $0.91 \%$ & $-0.09[-0.45,0.27]$ \\
\hline Subtotal ${ }^{\star \star \star}$ & 182 & & 183 & & & $3.13 \%$ & $-0.05[-0.23,0.14]$ \\
\hline \multicolumn{8}{|c|}{ Heterogeneity: $\operatorname{Tau}^{2}=0 ; \mathrm{Chi}^{2}=0.41, \mathrm{df}=2(\mathrm{P}=0.81) ; \mathrm{I}^{2}=0 \%$} \\
\hline \multicolumn{8}{|c|}{ 1.18.3 Diet and physical activity } \\
\hline Bryant 2011 & 27 & $0(0.2)$ & 26 & $-0(0.2)$ & + & $3.88 \%$ & $0.06[-0.05,0.17]$ \\
\hline Eddy Ives 2012 & 61 & $-0.3(0.5)$ & 64 & $-0.3(0.6)$ & + & $2.16 \%$ & $0.04[-0.16,0.24]$ \\
\hline Gillis 2007 & 11 & $-0(0.2)$ & 7 & $0.1(0.1)$ & 十 & $3.58 \%$ & $-0.12[-0.25,0.01]$ \\
\hline Kirk 2012 & 71 & $-0.2(0.4)$ & 31 & $-0.3(0.4)$ & 1 & $3.05 \%$ & $0.07[-0.08,0.22]$ \\
\hline Lison 2012 & 64 & $-0.2(0.4)$ & 20 & $-0(0.2)$ & 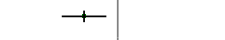 & $3.68 \%$ & $-0.18[-0.31,-0.06]$ \\
\hline Looney 2014 & 7 & $-0.1(0.3)$ & 4 & $-0.1(0.4)$ & & $0.54 \%$ & $-0.01[-0.49,0.47]$ \\
\hline Rodearmel 2007 & 95 & $-0.1(0.2)$ & 89 & $-0(0.2)$ & & $5.61 \%$ & $-0.03[-0.08,0.02]$ \\
\hline Subtotal $\star \star \star$ & 336 & & 241 & & & $22.5 \%$ & $-0.03[-0.1,0.04]$ \\
\hline \multicolumn{8}{|c|}{ Heterogeneity: $\mathrm{Tau}^{2}=0 ; \mathrm{Chi}^{2}=12.52, \mathrm{df}=6(\mathrm{P}=0.05) ; \mathrm{I}^{2}=52.1 \%$} \\
\hline \multicolumn{8}{|c|}{ Test for overall effect: $Z=0.94(P=0.35)$} \\
\hline Mirza 2013 & 57 & $-0.1(0.3)$ & 56 & $-0.1(0.3)$ & + & $4.15 \%$ & $-0.07[-0.17,0.03]$ \\
\hline 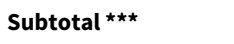 & 78 & & 74 & & & $5.66 \%$ & $-0.07[-0.16,0.03]$ \\
\hline \multicolumn{8}{|c|}{ Heterogeneity: $\operatorname{Tau}^{2}=0 ; \mathrm{Chi}^{2}=0.02, \mathrm{df}=1(\mathrm{P}=0.89) ;\left.\right|^{2}=0 \%$} \\
\hline \multicolumn{8}{|c|}{ Test for overall effect: $Z=1.36(P=0.17)$} \\
\hline \multicolumn{8}{|c|}{ 1.18.5 Physical activity and behavioural therapy } \\
\hline Maddison 2014 & 117 & $0(0.8)$ & 113 & $0(0.9)$ & - & $1.82 \%$ & $-0.03[-0.26,0.2]$ \\
\hline Subtotal $\star \star \star$ & 117 & & 113 & & & $1.82 \%$ & $-0.03[-0.26,0.2]$ \\
\hline Heterogeneity: Not ap & & & & & & & \\
\hline Test for overall effect: & & & & & & & \\
\hline 1.18.6 Diet, physical & havio & l therapy & & & & & \\
\hline Arauz Boudreau 2013 & 13 & $-0(0.1)$ & 10 & $-0(0.1)$ & - & $4.51 \%$ & $0.02[-0.07,0.11]$ \\
\hline Coppins 2011 & 28 & $-0.1(0.3)$ & 27 & $-0.1(0.4)$ & - & $2.46 \%$ & $0.01[-0.17,0.19]$ \\
\hline Croker 2012 & 33 & $-0.1(0.2)$ & 30 & $-0.1(0.2)$ & - & $4.83 \%$ & $-0.01[-0.09,0.07]$ \\
\hline Davis 2013 & 20 & $-0.1(0.5)$ & 22 & $-0.1(0.5)$ & & $1.1 \%$ & $0.03[-0.29,0.35]$ \\
\hline Davoli 2013 & 186 & $-0(0.5)$ & 185 & $-0(0.4)$ & - & $4.49 \%$ & $-0.02[-0.11,0.07]$ \\
\hline Diaz 2010 & 21 & $-0.3(0.2)$ & 22 & $-0.1(0.2)$ & $一$ & $3.26 \%$ & $-0.2[-0.34,-0.06]$ \\
\hline Epstein 2000a & 35 & $-0.7(1)$ & 17 & $-1.1(0.9)$ & & $0.43 \%$ & $0.39[-0.15,0.93]$ \\
\hline Gunnarsdottir 2011a & 7 & $-0.3(0.5)$ & 6 & $0(0.4)$ & - & $0.55 \%$ & $-0.37[-0.84,0.11]$ \\
\hline Kalavainen 2007 & 34 & $-0.3(0.4)$ & 34 & $-0.3(0.6)$ & - & $1.68 \%$ & $0[-0.24,0.24]$ \\
\hline & & & rav & rvention & -0.5 & rave & \\
\hline
\end{tabular}

Diet, physical activity and behavioural interventions for the treatment of overweight or obese children from the age of 6 to 11 years 


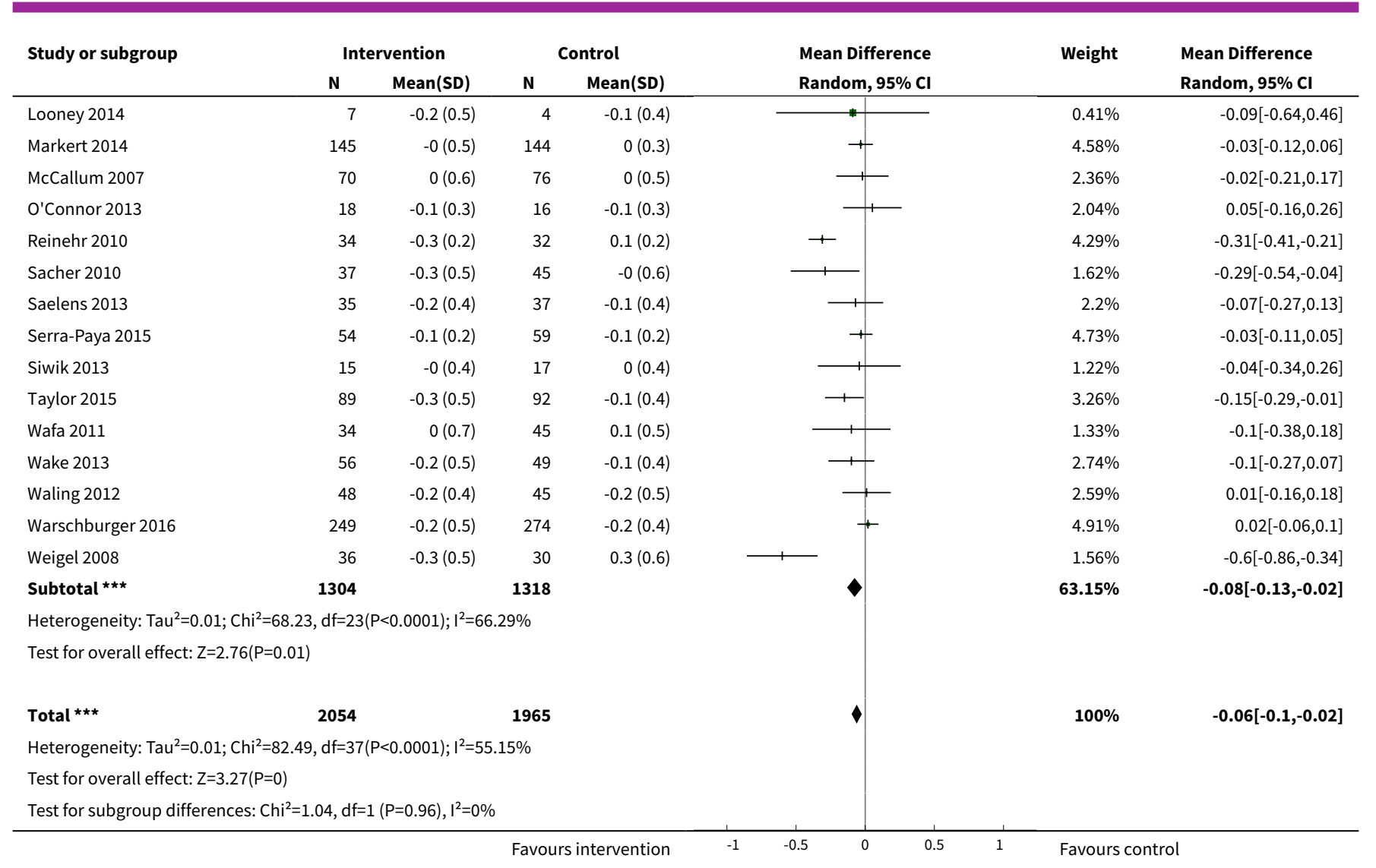

Analysis 1.19. Comparison 1 Behaviour-changing interventions versus no treatment/usual care, Outcome 19 Change in weight - type of intervention.

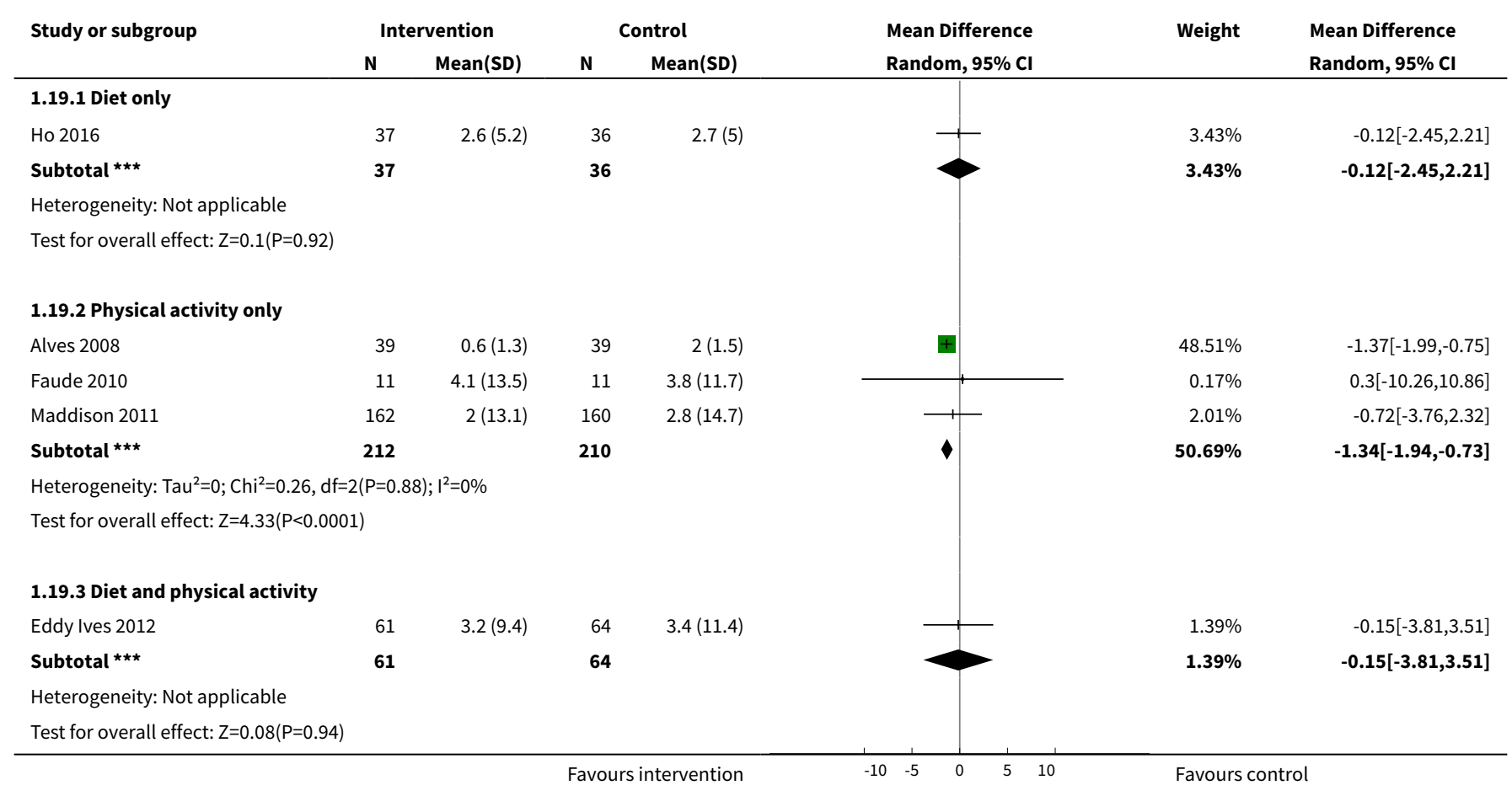

Diet, physical activity and behavioural interventions for the treatment of overweight or obese children from the age of 6 to 11 years 


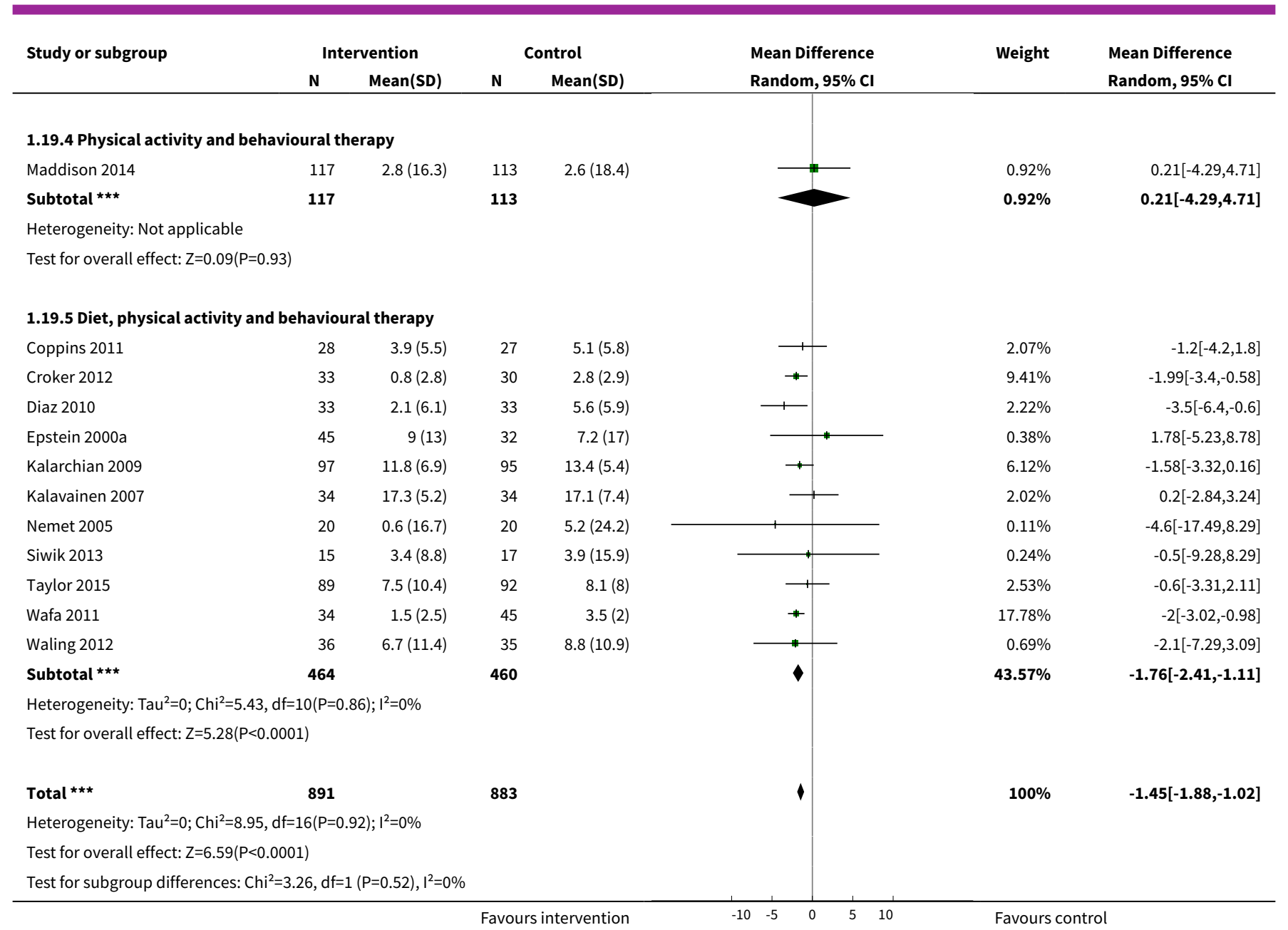

\section{Analysis 1.20. Comparison 1 Behaviour-changing interventions versus no treatment/usual care, Outcome 20 Change in BMI - attrition bias.}

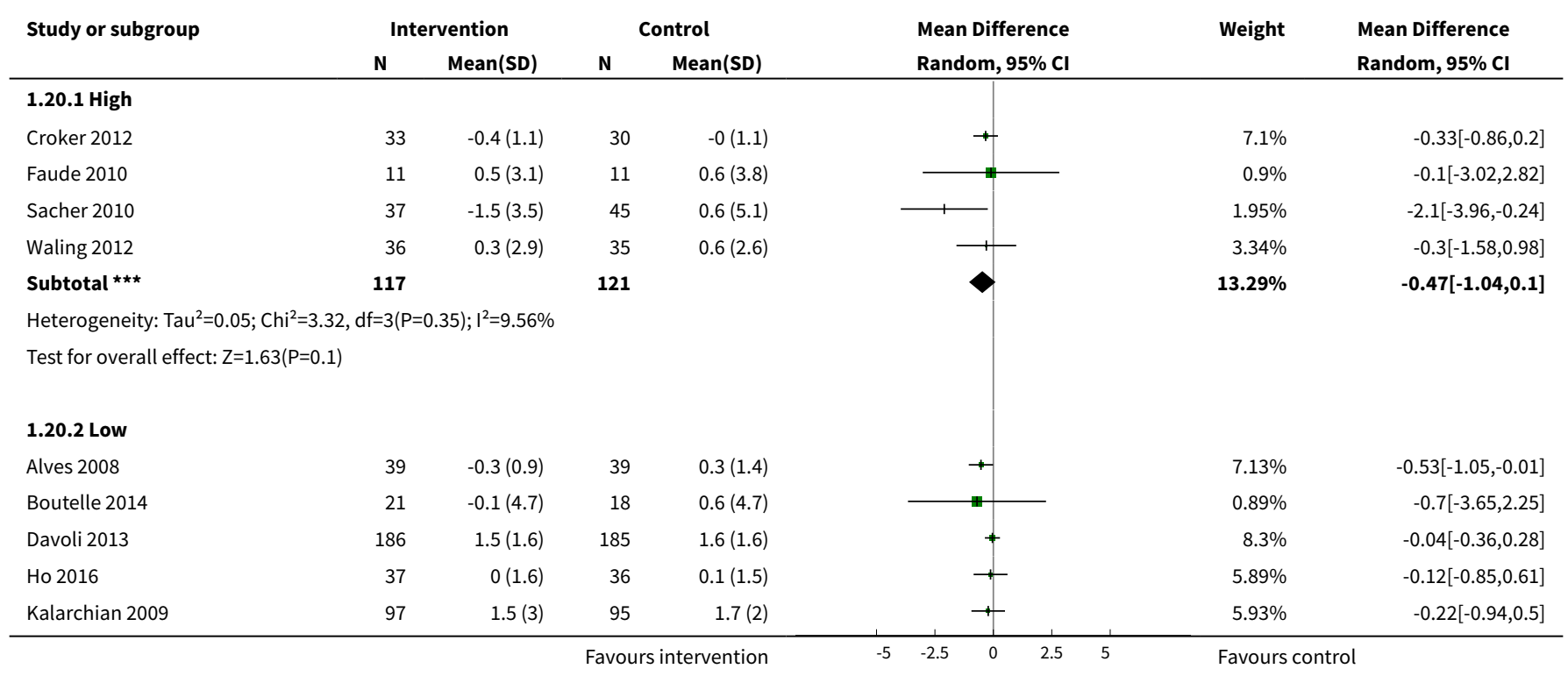

Diet, physical activity and behavioural interventions for the treatment of overweight or obese children from the age of 6 to 11 years 258 (Review)

Copyright (c) 2017 The Cochrane Collaboration. Published by John Wiley \& Sons, Ltd. 


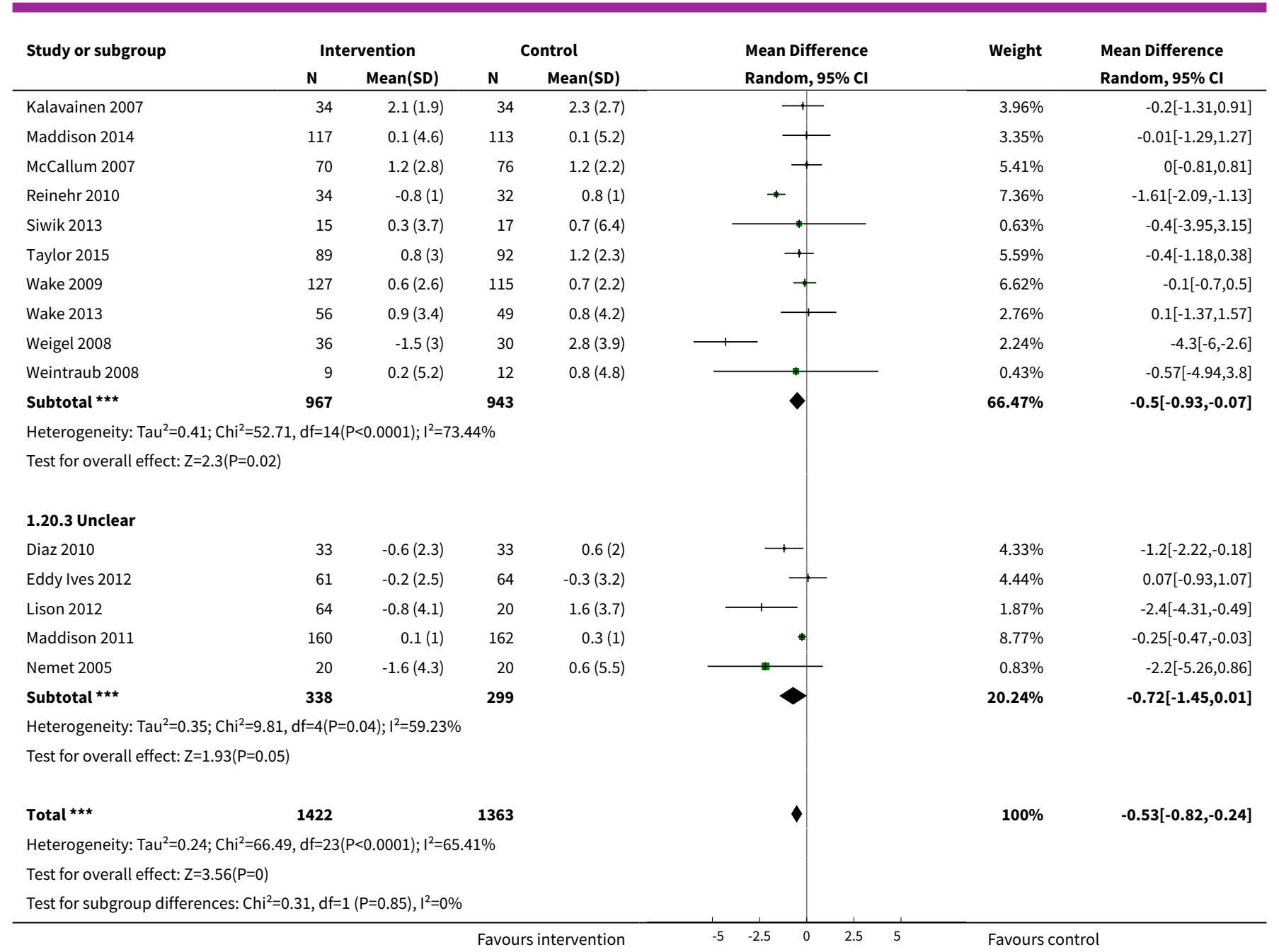

\section{Analysis 1.21. Comparison 1 Behaviour-changing interventions versus no treatment/usual care, Outcome 21 Change in BMI $z$ score - attrition bias.}

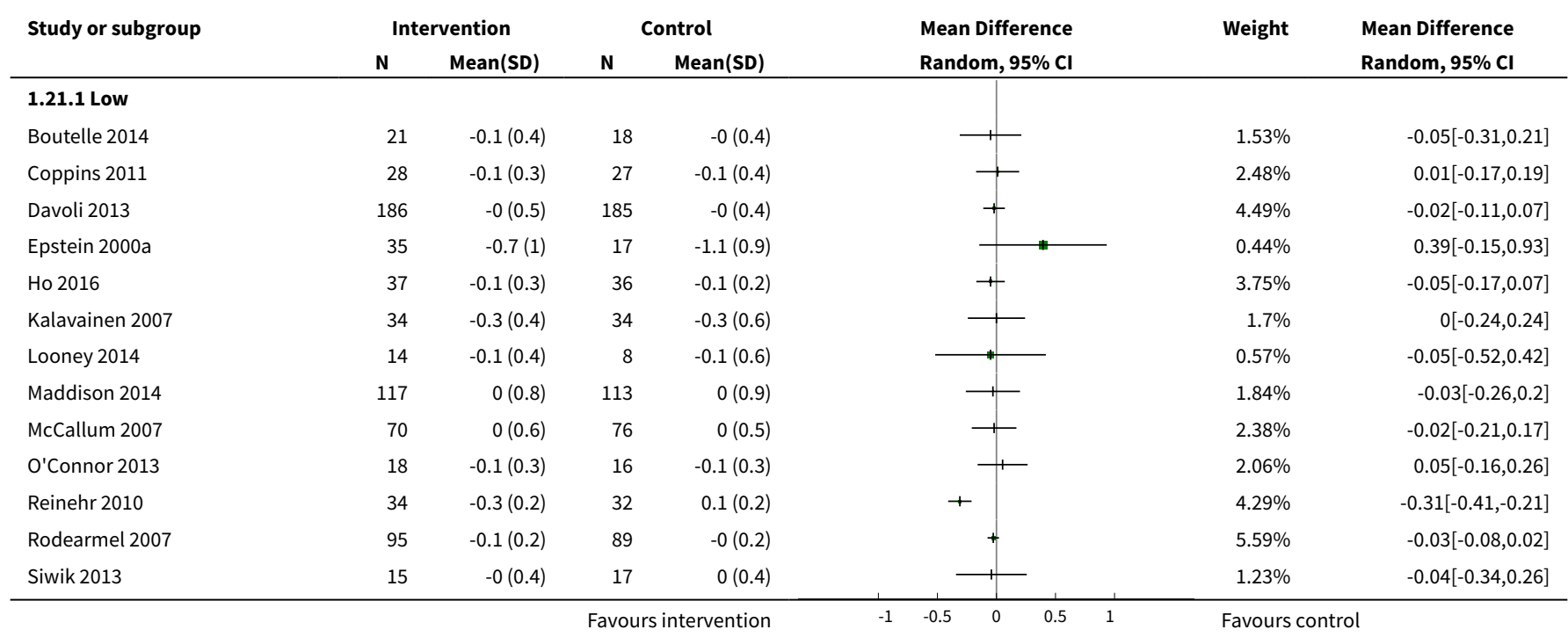

Diet, physical activity and behavioural interventions for the treatment of overweight or obese children from the age of 6 to 11 years 259 (Review)

Copyright (c) 2017 The Cochrane Collaboration. Published by John Wiley \& Sons, Ltd. 


\begin{tabular}{|c|c|c|c|c|c|c|c|}
\hline \multirow{3}{*}{$\begin{array}{l}\text { Study or subgroup } \\
\text { Taylor } 2015\end{array}$} & \multicolumn{2}{|c|}{ Intervention } & \multicolumn{2}{|c|}{ Control } & \multirow{2}{*}{$\begin{array}{l}\text { Mean Difference } \\
\text { Random, 95\% CI }\end{array}$} & \multirow{3}{*}{$\begin{array}{l}\text { Weight } \\
3.27 \%\end{array}$} & \multirow{3}{*}{$\begin{array}{l}\begin{array}{l}\text { Mean Difference } \\
\text { Random, 95\% Cl }\end{array} \\
-0.15[-0.29,-0.01]\end{array}$} \\
\hline & \multirow{2}{*}{$\frac{\mathbf{N}}{89}$} & \multirow{2}{*}{$\frac{\operatorname{Mean}(\mathbf{S D})}{-0.3(0.5)}$} & \multirow{2}{*}{$\frac{\mathbf{N}}{92}$} & \multirow{2}{*}{$\frac{\operatorname{Mean}(\text { SD) }}{-0.1(0.4)}$} & & & \\
\hline & & & & & + & & \\
\hline Wake 2013 & 56 & $-0.2(0.5)$ & 49 & $-0.1(0.4)$ & + & $2.76 \%$ & $-0.1[-0.27,0.07]$ \\
\hline Weigel 2008 & 36 & $-0.3(0.5)$ & 30 & $0.3(0.6)$ & 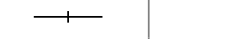 & $1.57 \%$ & $-0.6[-0.86,-0.34]$ \\
\hline Weintraub 2008 & 9 & $-0.1(0.5)$ & 12 & $0(0.3)$ & $\longrightarrow$ & $0.92 \%$ & $-0.09[-0.45,0.27]$ \\
\hline Subtotal $* \star \star$ & 894 & & 851 & & $\nabla$ & $40.88 \%$ & $-0.08[-0.16,-0.01]$ \\
\hline \multicolumn{8}{|c|}{ Heterogeneity: $\mathrm{Tau}^{2}=0.01 ; \mathrm{Chi}^{2}=50.36, \mathrm{df}=16(\mathrm{P}<0.0001) ; \mathrm{I}^{2}=68.23 \%$} \\
\hline \multicolumn{8}{|c|}{ Test for overall effect: $Z=2.32(P=0.02)$} \\
\hline \multicolumn{8}{|l|}{ 1.21.2 Unclear } \\
\hline Bryant 2011 & 27 & $0(0.2)$ & 26 & $-0(0.2)$ & + & $3.89 \%$ & $0.06[-0.05,0.17]$ \\
\hline Davis 2013 & 20 & $-0.1(0.5)$ & 22 & $-0.1(0.5)$ & 1 & $1.11 \%$ & $0.03[-0.29,0.35]$ \\
\hline Diaz 2010 & 21 & $-0.3(0.2)$ & 22 & $-0.1(0.2)$ & + & $3.28 \%$ & $-0.2[-0.34,-0.06]$ \\
\hline Eddy Ives 2012 & 61 & $-0.3(0.5)$ & 64 & $-0.3(0.6)$ & 1 & $2.17 \%$ & $0.04[-0.16,0.24]$ \\
\hline Gunnarsdottir 2011a & 7 & $-0.3(0.5)$ & 6 & $0(0.4)$ & +- & $0.55 \%$ & $-0.37[-0.84,0.11]$ \\
\hline Kirk 2012 & 71 & $-0.2(0.4)$ & 31 & $-0.3(0.4)$ & 十 & $3.07 \%$ & $0.07[-0.08,0.22]$ \\
\hline Lison 2012 & 64 & $-0.2(0.4)$ & 20 & $-0(0.2)$ & + & $3.7 \%$ & $-0.18[-0.31,-0.06]$ \\
\hline Maddison 2011 & 162 & $0(1.1)$ & 160 & $0.1(1.1)$ & 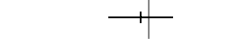 & $1.71 \%$ & $-0.06[-0.3,0.18]$ \\
\hline Serra-Paya 2015 & 54 & $-0.1(0.2)$ & 59 & $-0.1(0.2)$ & + & $4.72 \%$ & $-0.03[-0.11,0.05]$ \\
\hline 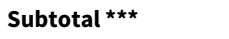 & 487 & & 410 & & 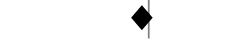 & $24.21 \%$ & $-0.05[-0.13,0.03]$ \\
\hline \multicolumn{8}{|c|}{ Heterogeneity: $\mathrm{Tau}^{2}=0.01 ; \mathrm{Chi}^{2}=17.84, \mathrm{df}=8(\mathrm{P}=0.02) ; \mathrm{I}^{2}=55.17 \%$} \\
\hline \multicolumn{8}{|c|}{ Test for overall effect: $Z=1.25(P=0.21)$} \\
\hline \multicolumn{8}{|l|}{ 1.21.3 High } \\
\hline Arauz Boudreau 2013 & 13 & $-0(0.1)$ & 10 & $-0(0.1)$ & + & $4.51 \%$ & $0.02[-0.07,0.11]$ \\
\hline Croker 2012 & 33 & $-0.1(0.2)$ & 30 & $-0.1(0.2)$ & + & $4.83 \%$ & $-0.01[-0.09,0.07]$ \\
\hline Faude 2010 & 11 & $0.1(0.5)$ & 11 & $0(0.7)$ & - & $0.53 \%$ & $0.1[-0.39,0.59]$ \\
\hline Gillis 2007 & 11 & $-0(0.2)$ & 7 & $0.1(0.1)$ & + & $3.59 \%$ & $-0.12[-0.25,0.01]$ \\
\hline Markert 2014 & 145 & $-0(0.5)$ & 144 & $0(0.3)$ & + & $4.58 \%$ & $-0.03[-0.12,0.06]$ \\
\hline Mirza 2013 & 57 & $-0.1(0.3)$ & 56 & $-0.1(0.3)$ & + & $4.15 \%$ & $-0.07[-0.17,0.03]$ \\
\hline Sacher 2010 & 37 & $-0.3(0.5)$ & 45 & $-0(0.6)$ & 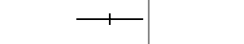 & $1.64 \%$ & $-0.29[-0.54,-0.04]$ \\
\hline Saelens 2013 & 35 & $-0.2(0.4)$ & 37 & $-0.1(0.4)$ & 1 & $2.22 \%$ & $-0.07[-0.27,0.13]$ \\
\hline Wafa 2011 & 34 & $0(0.7)$ & 45 & $0.1(0.5)$ & -1 & $1.35 \%$ & $-0.1[-0.38,0.18]$ \\
\hline Waling 2012 & 48 & $-0.2(0.4)$ & 45 & $-0.2(0.5)$ & + & $2.61 \%$ & $0.01[-0.16,0.18]$ \\
\hline Warschburger 2016 & 249 & $-0.2(0.5)$ & 274 & $-0.2(0.4)$ & + & $4.9 \%$ & $0.02[-0.06,0.1]$ \\
\hline Subtotal ${ }^{\star \star \star}$ & 673 & & 704 & & 1 & $34.91 \%$ & $-0.03[-0.06,0.01]$ \\
\hline \multicolumn{8}{|c|}{ Heterogeneity: $\operatorname{Tau}^{2}=0 ; \mathrm{Chi}^{2}=10.54, \mathrm{df}=10(\mathrm{P}=0.39) ; \mathrm{I}^{2}=5.15 \%$} \\
\hline \multicolumn{8}{|c|}{ Test for overall effect: $Z=1.43(P=0.15)$} \\
\hline Total $\star \star \star ~$ & 2054 & & 1965 & & $\checkmark$ & $100 \%$ & $-0.06[-0.1,-0.02]$ \\
\hline \multicolumn{8}{|c|}{ Heterogeneity: $\mathrm{Tau}^{2}=0.01 ; \mathrm{Chi}^{2}=82.44, \mathrm{df}=36(\mathrm{P}<0.0001) ; \mathrm{I}^{2}=56.33 \%$} \\
\hline \multicolumn{8}{|c|}{ Test for overall effect: $Z=3.25(P=0)$} \\
\hline Test for subgroup diffe & $09, \mathrm{df}=$ & $(P=0.35), I^{2}=4$. & & & & & \\
\hline
\end{tabular}

Diet, physical activity and behavioural interventions for the treatment of overweight or obese children from the age of 6 to 11 years 
Analysis 1.22. Comparison 1 Behaviour-changing interventions versus no treatment/usual care, Outcome 22 Change in weight - attrition bias.

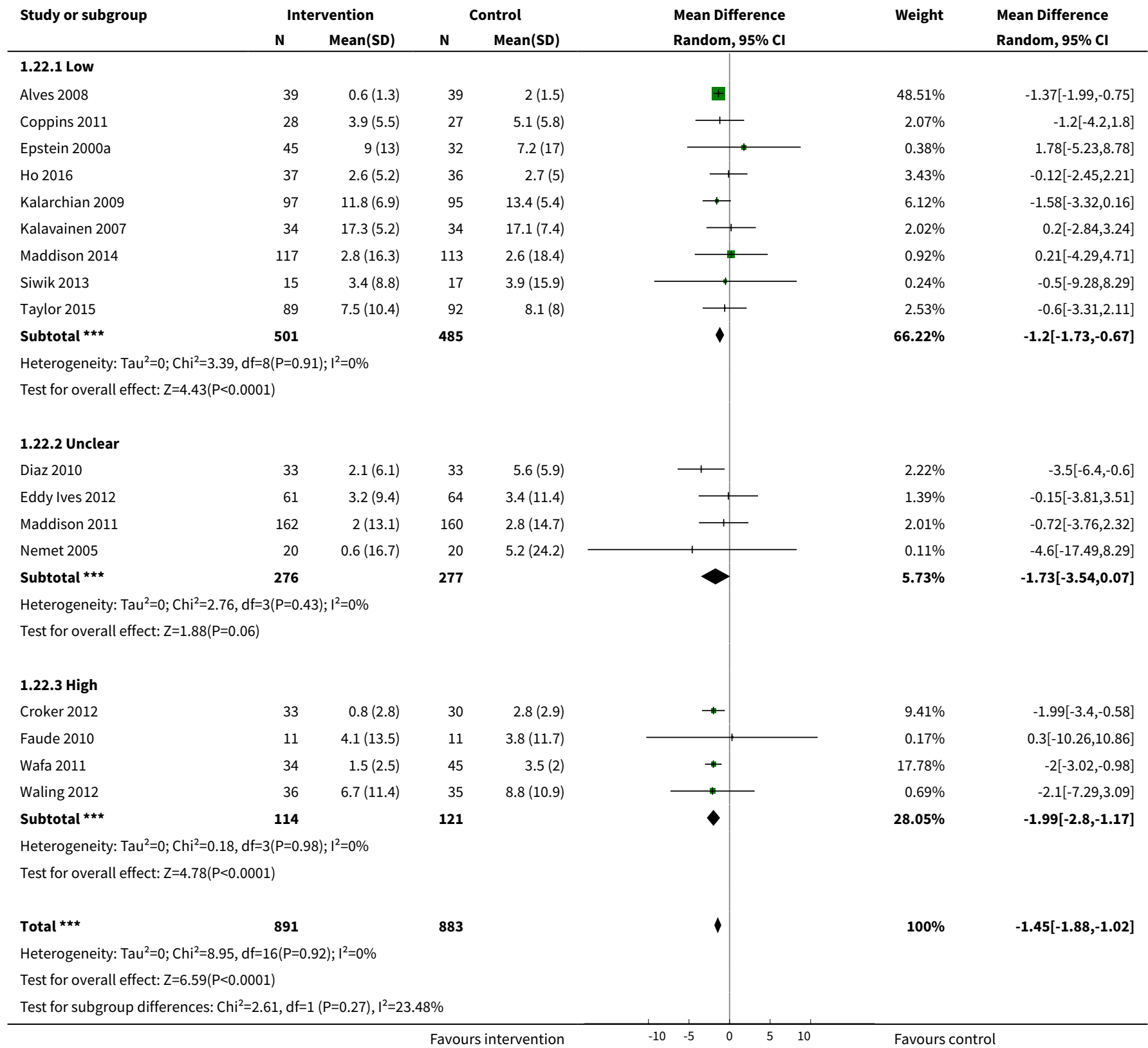

\section{Analysis 1.23. Comparison 1 Behaviour-changing interventions versus no treatment/usual care, Outcome 23 Change in weight - setting.}

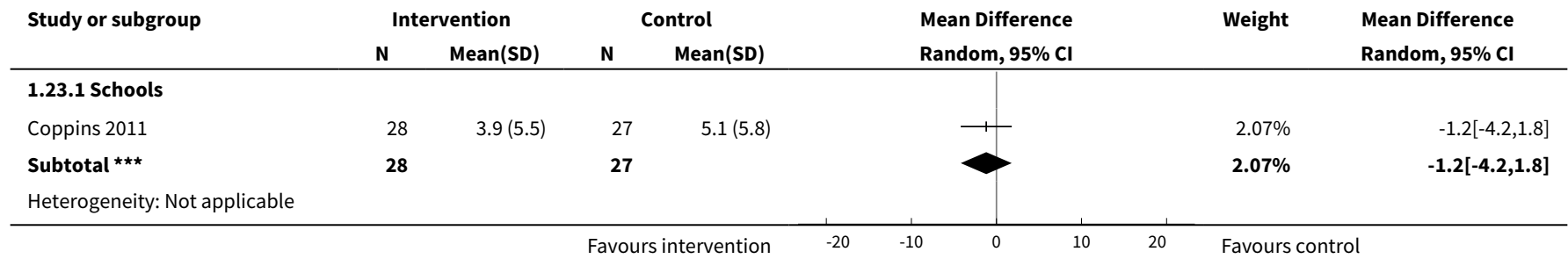

Diet, physical activity and behavioural interventions for the treatment of overweight or obese children from the age of 6 to 11 years 


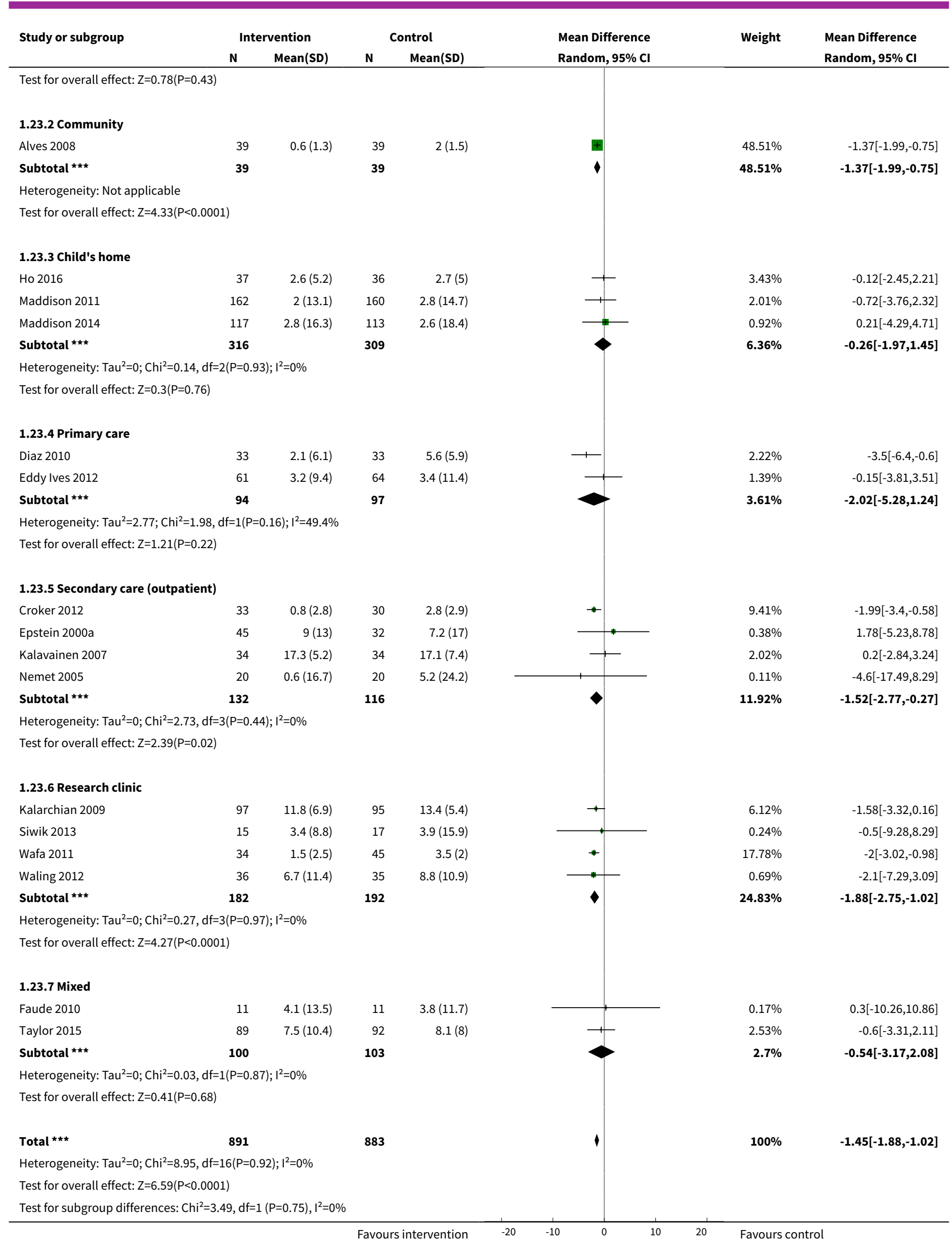

Diet, physical activity and behavioural interventions for the treatment of overweight or obese children from the age of 6 to 11 years 
Analysis 1.24. Comparison 1 Behaviour-changing interventions versus no treatment/usual care, Outcome 24 Change in BMI z score - setting.

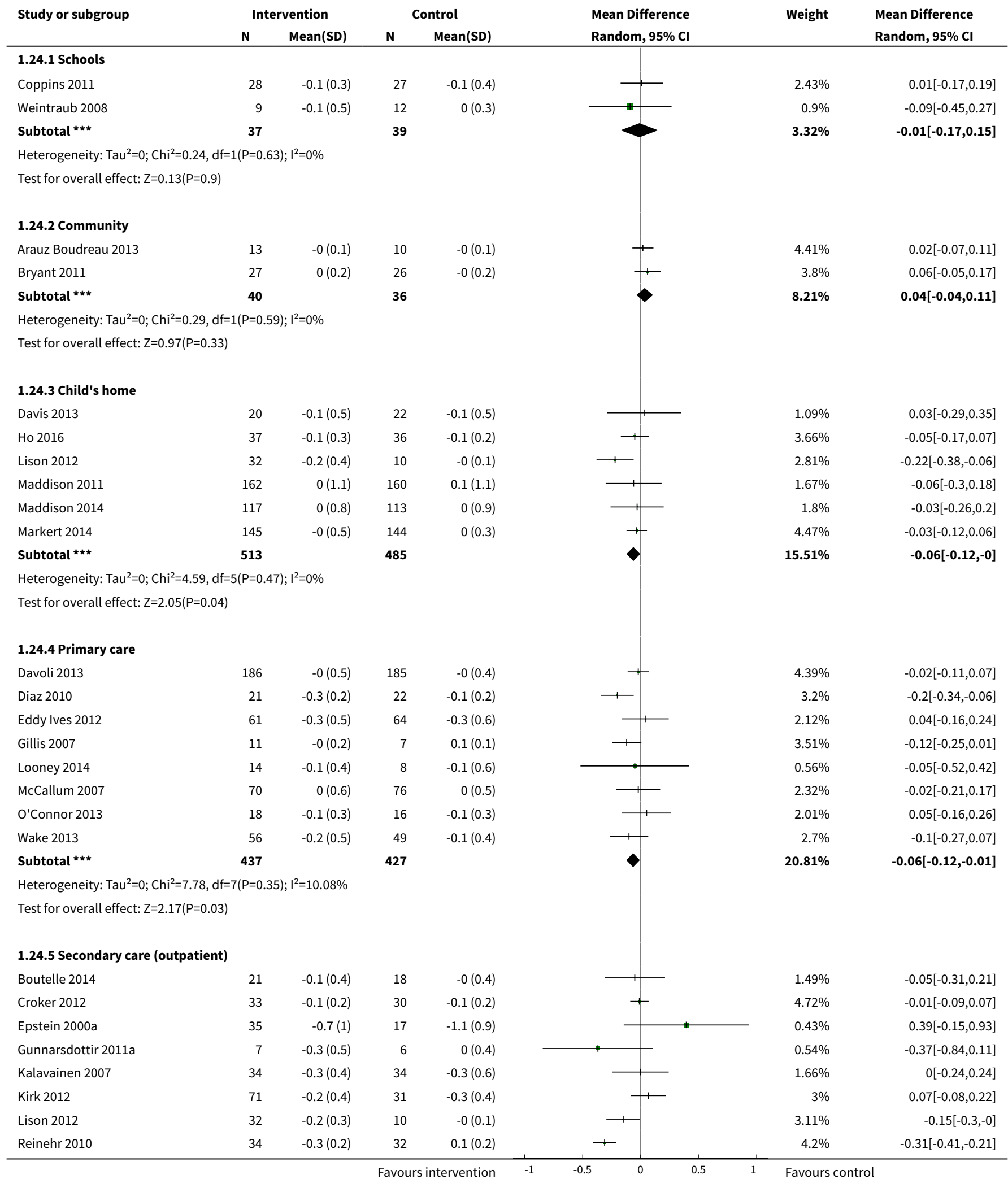

Diet, physical activity and behavioural interventions for the treatment of overweight or obese children from the age of 6 to 11 years 


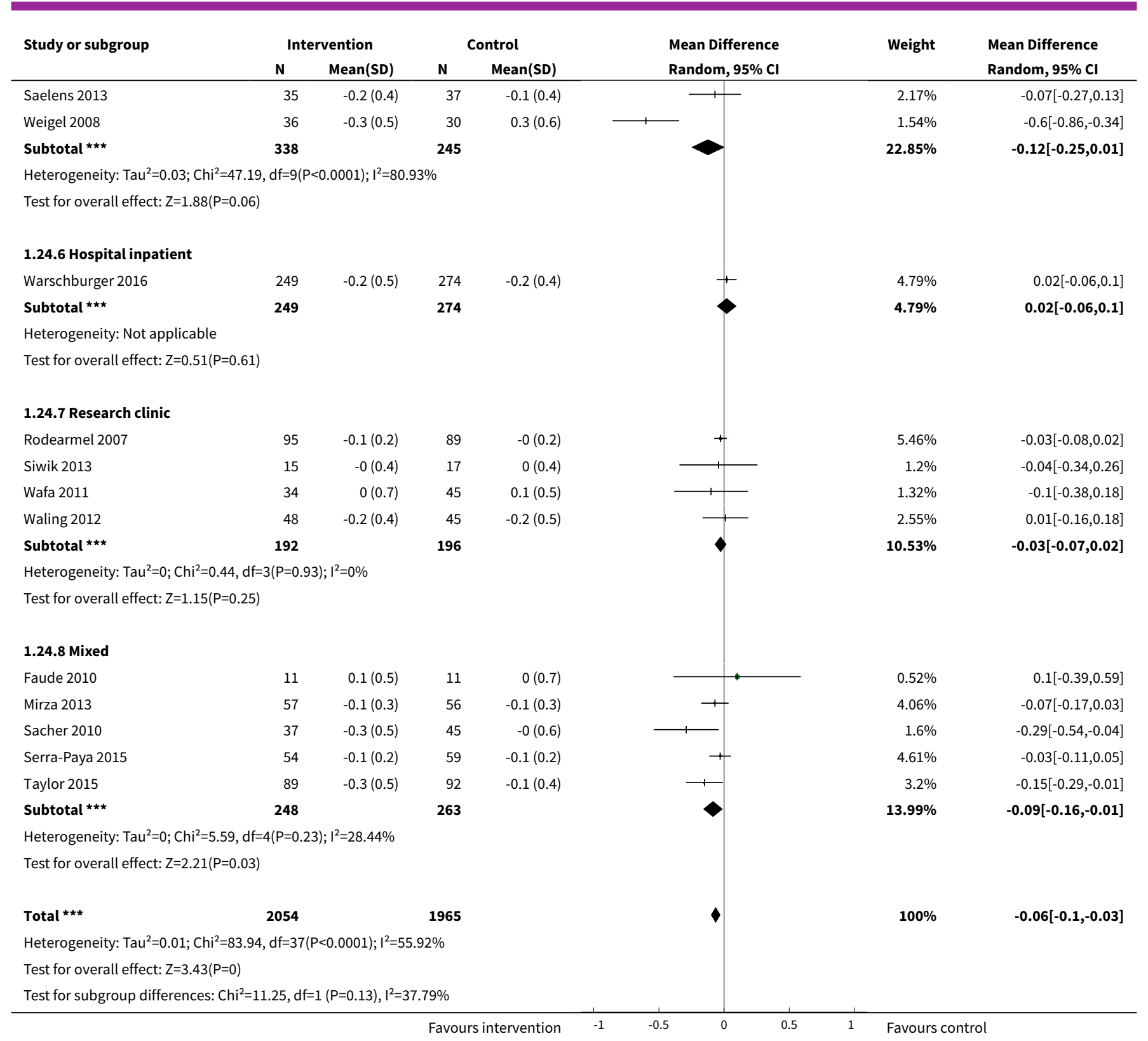

Analysis 1.25. Comparison 1 Behaviour-changing interventions versus no treatment/usual care, Outcome 25 Change in BMI - setting.

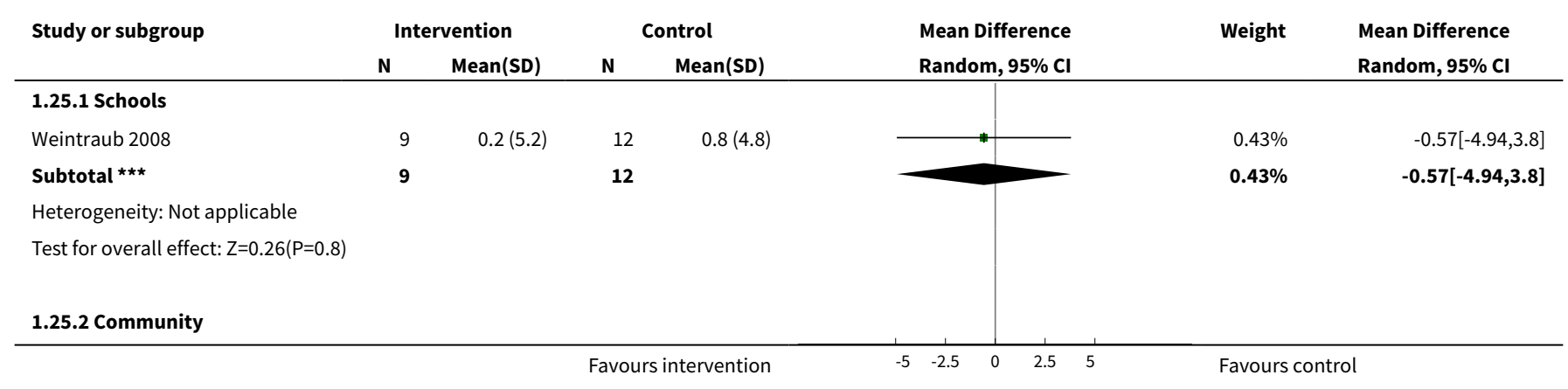

Diet, physical activity and behavioural interventions for the treatment of overweight or obese children from the age of 6 to 11 years 264 (Review)

Copyright ( 2017 The Cochrane Collaboration. Published by John Wiley \& Sons, Ltd. 


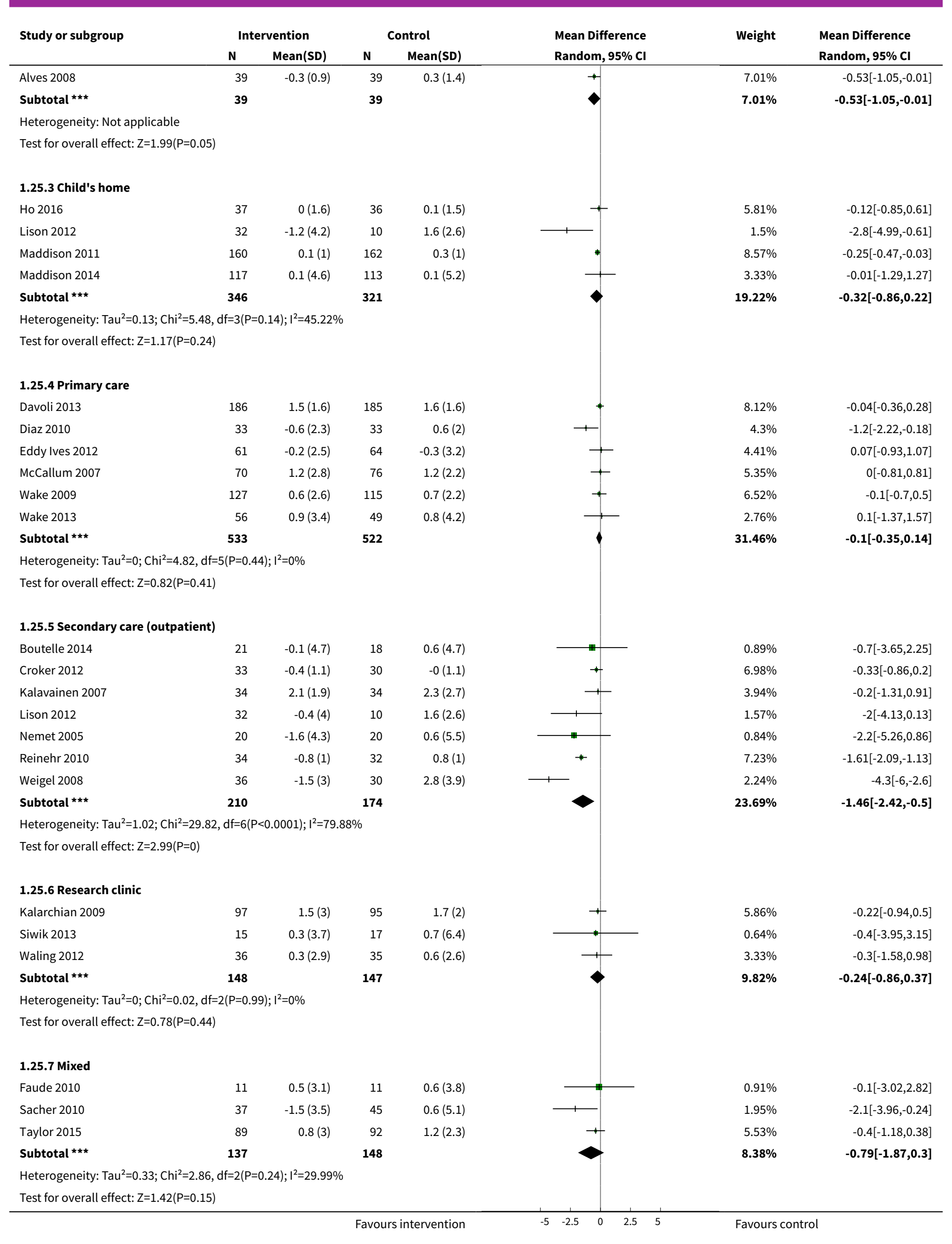

Diet, physical activity and behavioural interventions for the treatment of overweight or obese children from the age of 6 to 11 years 265 (Review)

Copyright (c) 2017 The Cochrane Collaboration. Published by John Wiley \& Sons, Ltd. 


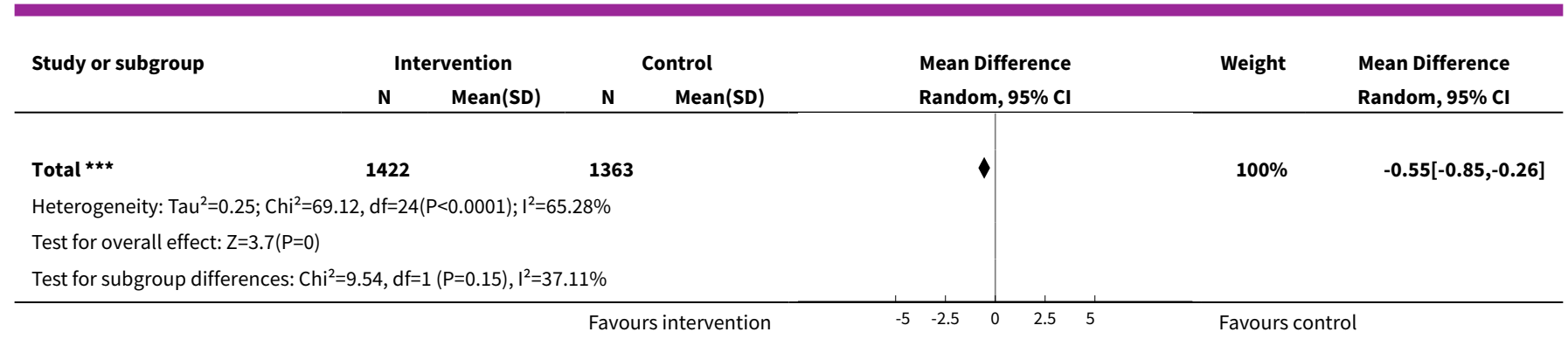

Analysis 1.26. Comparison 1 Behaviour-changing interventions versus no treatment/usual care, Outcome 26 Change in BMI - post-intervention follow-up.

\begin{tabular}{|c|c|c|c|c|c|c|c|}
\hline \multirow[t]{2}{*}{ Study or subgroup } & \multicolumn{2}{|c|}{ Intervention } & \multicolumn{2}{|c|}{ Control } & \multirow{2}{*}{$\begin{array}{l}\text { Mean Difference } \\
\text { Random, } 95 \% \mathrm{CI}\end{array}$} & \multirow[t]{2}{*}{ Weight } & \multirow{2}{*}{$\begin{array}{l}\text { Mean Difference } \\
\text { Random, } 95 \% \mathrm{Cl}\end{array}$} \\
\hline & $\mathbf{N}$ & Mean(SD) & $\mathbf{N}$ & Mean(SD) & & & \\
\hline \multicolumn{8}{|c|}{ 1.26.1 No post-intervention follow-up } \\
\hline Alves 2008 & 39 & $-0.3(0.9)$ & 39 & $0.3(1.4)$ & $\rightarrow$ & $7.13 \%$ & $-0.53[-1.05,-0.01]$ \\
\hline Croker 2012 & 33 & $-0.4(1.1)$ & 30 & $-0(1.1)$ & $\rightarrow$ & $7.1 \%$ & $-0.33[-0.86,0.2]$ \\
\hline Diaz 2010 & 33 & $-0.6(2.3)$ & 33 & $0.6(2)$ & 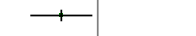 & $4.33 \%$ & $-1.2[-2.22,-0.18]$ \\
\hline Eddy Ives 2012 & 61 & $-0.2(2.5)$ & 64 & $-0.3(3.2)$ & 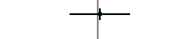 & $4.44 \%$ & $0.07[-0.93,1.07]$ \\
\hline Faude 2010 & 11 & $0.5(3.1)$ & 11 & $0.6(3.8)$ & & $0.9 \%$ & $-0.1[-3.02,2.82]$ \\
\hline Ho 2016 & 37 & $0(1.6)$ & 36 & $0.1(1.5)$ & + & $5.89 \%$ & $-0.12[-0.85,0.61]$ \\
\hline Lison 2012 & 64 & $-0.8(4.1)$ & 20 & $1.6(3.7)$ & + & $1.87 \%$ & $-2.4[-4.31,-0.49]$ \\
\hline Maddison 2011 & 160 & $0.1(1)$ & 162 & $0.3(1)$ & $*$ & $8.77 \%$ & $-0.25[-0.47,-0.03]$ \\
\hline Maddison 2014 & 117 & $0.1(4.6)$ & 113 & $0.1(5.2)$ & & $3.35 \%$ & $-0.01[-1.29,1.27]$ \\
\hline Reinehr 2010 & 34 & $-0.8(1)$ & 32 & $0.8(1)$ & + & $7.36 \%$ & $-1.61[-2.09,-1.13]$ \\
\hline Taylor 2015 & 89 & $0.8(3)$ & 92 & $1.2(2.3)$ & & $5.59 \%$ & $-0.4[-1.18,0.38]$ \\
\hline Waling 2012 & 36 & $0.3(2.9)$ & 35 & $0.6(2.6)$ & - & $3.34 \%$ & $-0.3[-1.58,0.98]$ \\
\hline Weigel 2008 & 36 & $-1.5(3)$ & 30 & $2.8(3.9)$ & & $2.24 \%$ & $-4.3[-6,-2.6]$ \\
\hline Weintraub 2008 & 9 & $0.2(5.2)$ & 12 & $0.8(4.8)$ & $\rightarrow$ & $0.43 \%$ & $-0.57[-4.94,3.8]$ \\
\hline 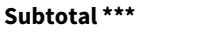 & 815 & & 758 & & & $65.49 \%$ & $-0.68[-1.1,-0.27]$ \\
\hline \multicolumn{8}{|c|}{ Heterogeneity: $\mathrm{Tau}^{2}=0.37 ; \mathrm{Chi}^{2}=53.88, \mathrm{df}=14(\mathrm{P}<0.0001) ; \mathrm{I}^{2}=74.01 \%$} \\
\hline \multicolumn{8}{|c|}{ Test for overall effect: $Z=3.21(P=0)$} \\
\hline \multicolumn{8}{|c|}{ 1.26.2 Post-intervention follow-up $<6$ months } \\
\hline Boutelle 2014 & 21 & $-0.1(4.7)$ & 18 & $0.6(4.7)$ & & $0.89 \%$ & $-0.7[-3.65,2.25]$ \\
\hline Sacher 2010 & 37 & $-1.5(3.5)$ & 45 & $0.6(5.1)$ & & $1.95 \%$ & $-2.1[-3.96,-0.24]$ \\
\hline Siwik 2013 & 15 & $0.3(3.7)$ & 17 & $0.7(6.4)$ & $*$ & $0.63 \%$ & $-0.4[-3.95,3.15]$ \\
\hline Subtotal $\star \star \star$ & 73 & & 80 & & & $3.46 \%$ & $-1.49[-2.93,-0.05]$ \\
\hline \multicolumn{8}{|c|}{ Heterogeneity: $\operatorname{Tau}^{2}=0 ; \mathrm{Chi}^{2}=1.05, \mathrm{df}=2(\mathrm{P}=0.59) ; \mathrm{I}^{2}=0 \%$} \\
\hline \multicolumn{8}{|c|}{ 1.26.3 Post-intervention follow-up 6 months to $<12$ months } \\
\hline Nemet 2005 & 20 & $-1.6(4.3)$ & 20 & $0.6(5.5)$ & & $0.83 \%$ & $-2.2[-5.26,0.86]$ \\
\hline Wake 2009 & 127 & $0.6(2.6)$ & 115 & $0.7(2.2)$ & & $6.62 \%$ & $-0.1[-0.7,0.5]$ \\
\hline Subtotal $\star \star \star$ & 147 & & 135 & & & $7.45 \%$ & $-0.59[-2.34,1.15]$ \\
\hline \multicolumn{8}{|c|}{ Heterogeneity: $\operatorname{Tau}^{2}=0.94 ; \mathrm{Chi}^{2}=1.75, \mathrm{df}=1(\mathrm{P}=0.19) ; \mathrm{I}^{2}=42.71 \%$} \\
\hline \multicolumn{8}{|c|}{ Test for overall effect: $Z=0.67(P=0.5)$} \\
\hline \multicolumn{8}{|c|}{ 1.26.4 Post-intervention follow-up 12 months or more } \\
\hline Davoli 2013 & 186 & $1.5(1.6)$ & 185 & $1.6(1.6)$ & + & $8.3 \%$ & $-0.04[-0.36,0.28]$ \\
\hline
\end{tabular}

Diet, physical activity and behavioural interventions for the treatment of overweight or obese children from the age of 6 to 11 years 


\begin{tabular}{|c|c|c|c|c|c|c|c|}
\hline \multirow[t]{2}{*}{ Study or subgroup } & \multicolumn{2}{|c|}{ Intervention } & \multicolumn{2}{|c|}{ Control } & \multirow{2}{*}{$\begin{array}{l}\text { Mean Difference } \\
\text { Random, } 95 \% \mathrm{CI}\end{array}$} & \multirow[t]{2}{*}{ Weight } & \multirow{2}{*}{$\begin{array}{l}\text { Mean Difference } \\
\text { Random, } 95 \% \mathrm{Cl}\end{array}$} \\
\hline & $\mathbf{N}$ & $\operatorname{Mean}(S D)$ & $\mathbf{N}$ & Mean(SD) & & & \\
\hline Kalarchian 2009 & 97 & $1.5(3)$ & 95 & $1.7(2)$ & + & $5.93 \%$ & $-0.22[-0.94,0.5]$ \\
\hline Kalavainen 2007 & 34 & $2.1(1.9)$ & 34 & $2.3(2.7)$ & 1 & $3.96 \%$ & $-0.2[-1.31,0.91]$ \\
\hline McCallum 2007 & 70 & $1.2(2.8)$ & 76 & $1.2(2.2)$ & $\longrightarrow$ & $5.41 \%$ & $0[-0.81,0.81]$ \\
\hline Subtotal *** & 387 & & 390 & & $>$ & $23.59 \%$ & $-0.07[-0.34,0.2]$ \\
\hline \multicolumn{8}{|c|}{ Heterogeneity: $\mathrm{Tau}^{2}=0 ; \mathrm{Chi}^{2}=0.28, \mathrm{df}=3(\mathrm{P}=0.96) ; \mathrm{I}^{2}=0 \%$} \\
\hline \multicolumn{8}{|c|}{ Test for overall effect: $Z=0.51(P=0.61)$} \\
\hline Total *** & 1422 & & 1363 & & $\bullet$ & $100 \%$ & $-0.53[-0.82,-0.24]$ \\
\hline \multicolumn{8}{|c|}{ Heterogeneity: $\operatorname{Tau}^{2}=0.24 ; \mathrm{Chi}^{2}=66.49, \mathrm{df}=23(\mathrm{P}<0.0001) ; \mathrm{I}^{2}=65.41 \%$} \\
\hline \multicolumn{8}{|c|}{ Test for overall effect: $Z=3.56(P=0)$} \\
\hline Test for subgroup dif & $74, \mathrm{df}=$ & $P=0.03), I^{2}=6$ & & & & & \\
\hline
\end{tabular}

Analysis 1.27. Comparison 1 Behaviour-changing interventions versus no treatment/ usual care, Outcome 27 Change in BMI z score - post-intervention follow-up.

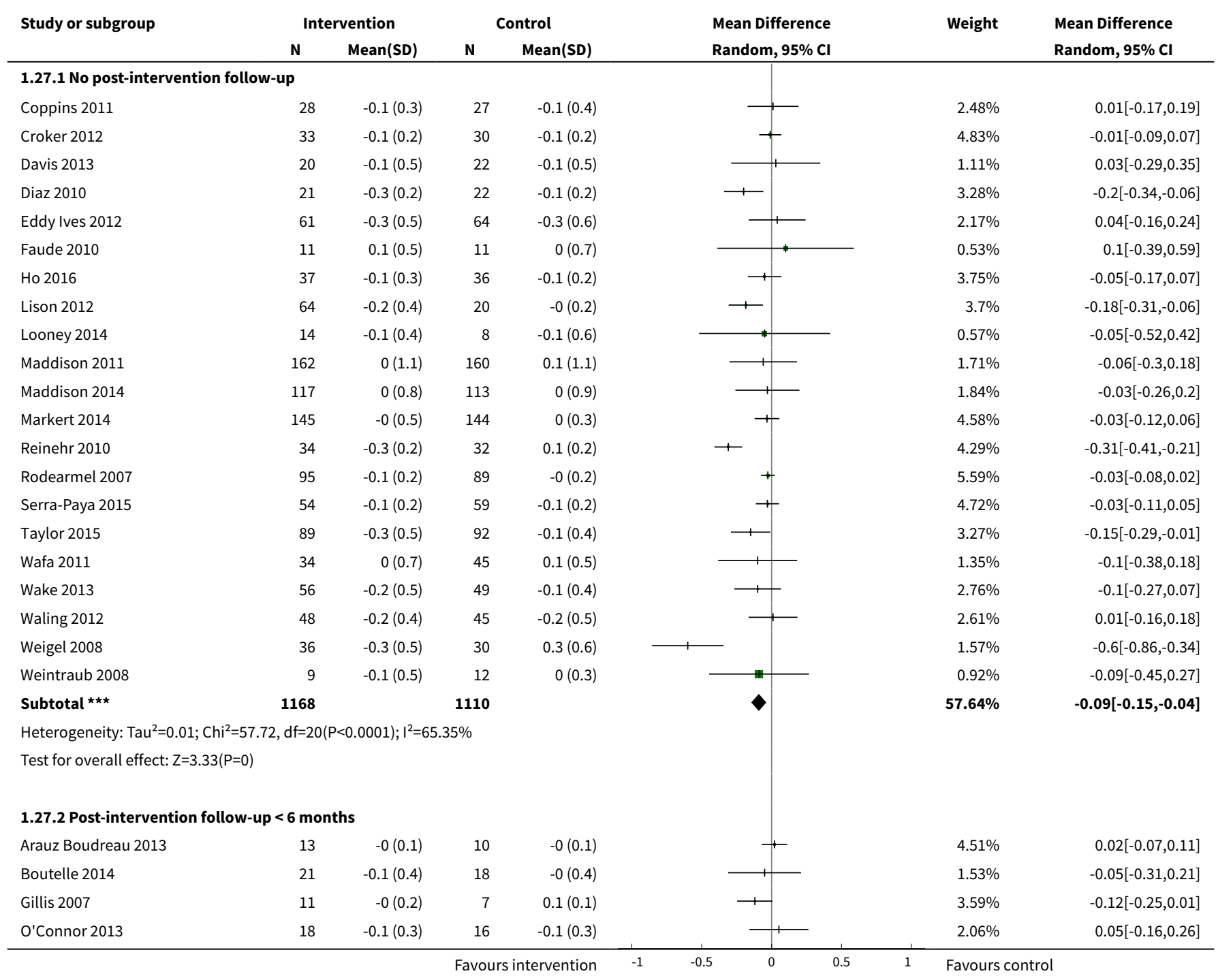




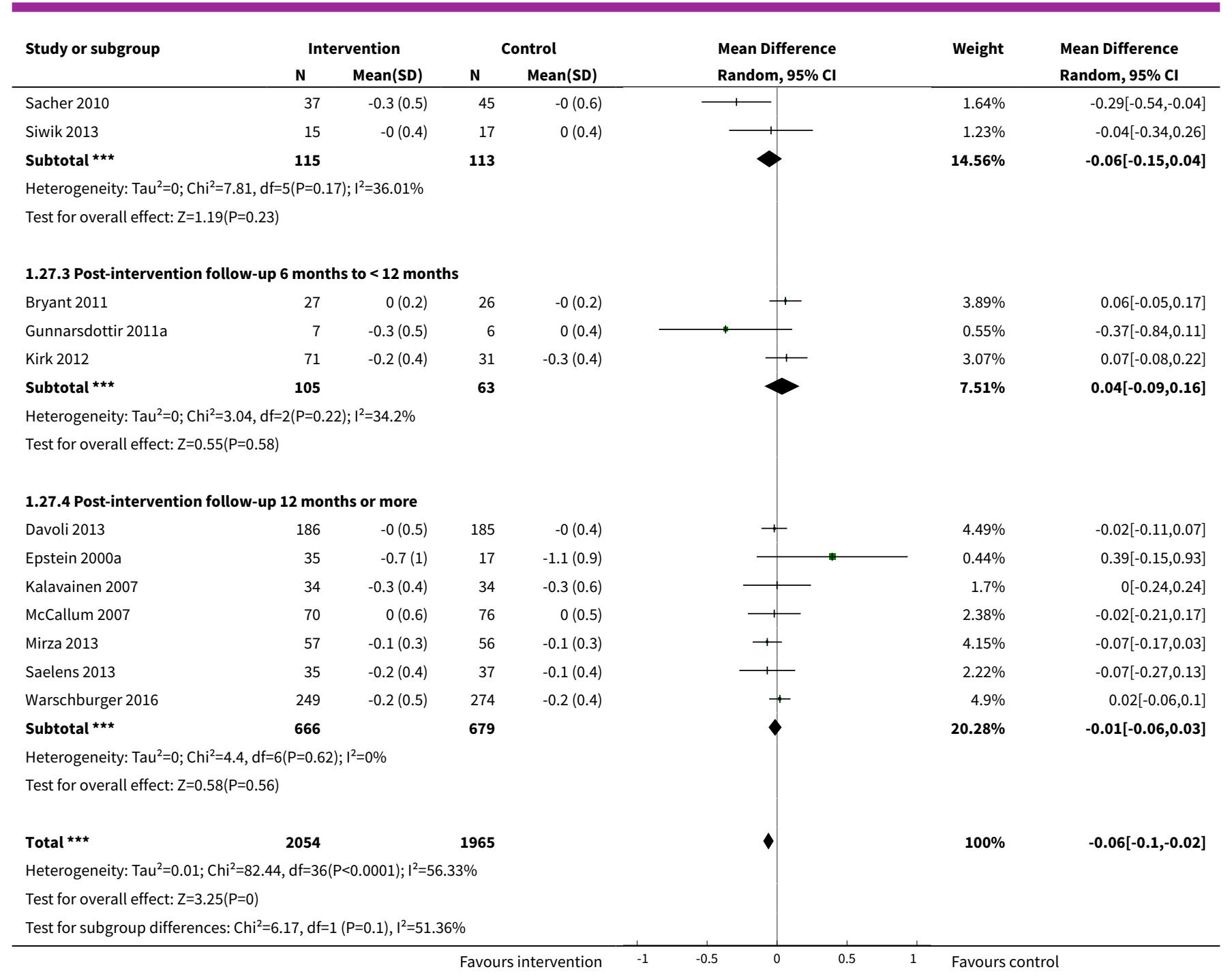

Analysis 1.28. Comparison 1 Behaviour-changing interventions versus no treatment/usual care, Outcome 28 Change in weight - post-intervention follow-up.

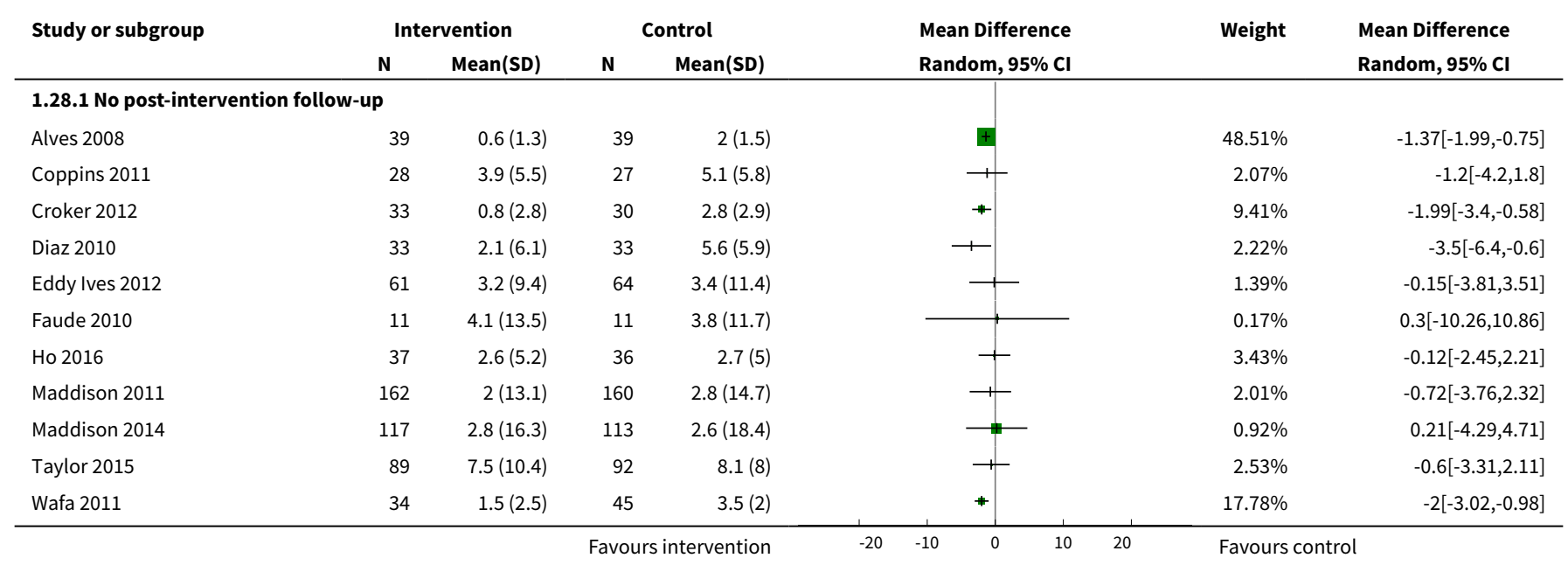

Diet, physical activity and behavioural interventions for the treatment of overweight or obese children from the age of 6 to 11 years 268 (Review)

Copyright (c) 2017 The Cochrane Collaboration. Published by John Wiley \& Sons, Ltd. 


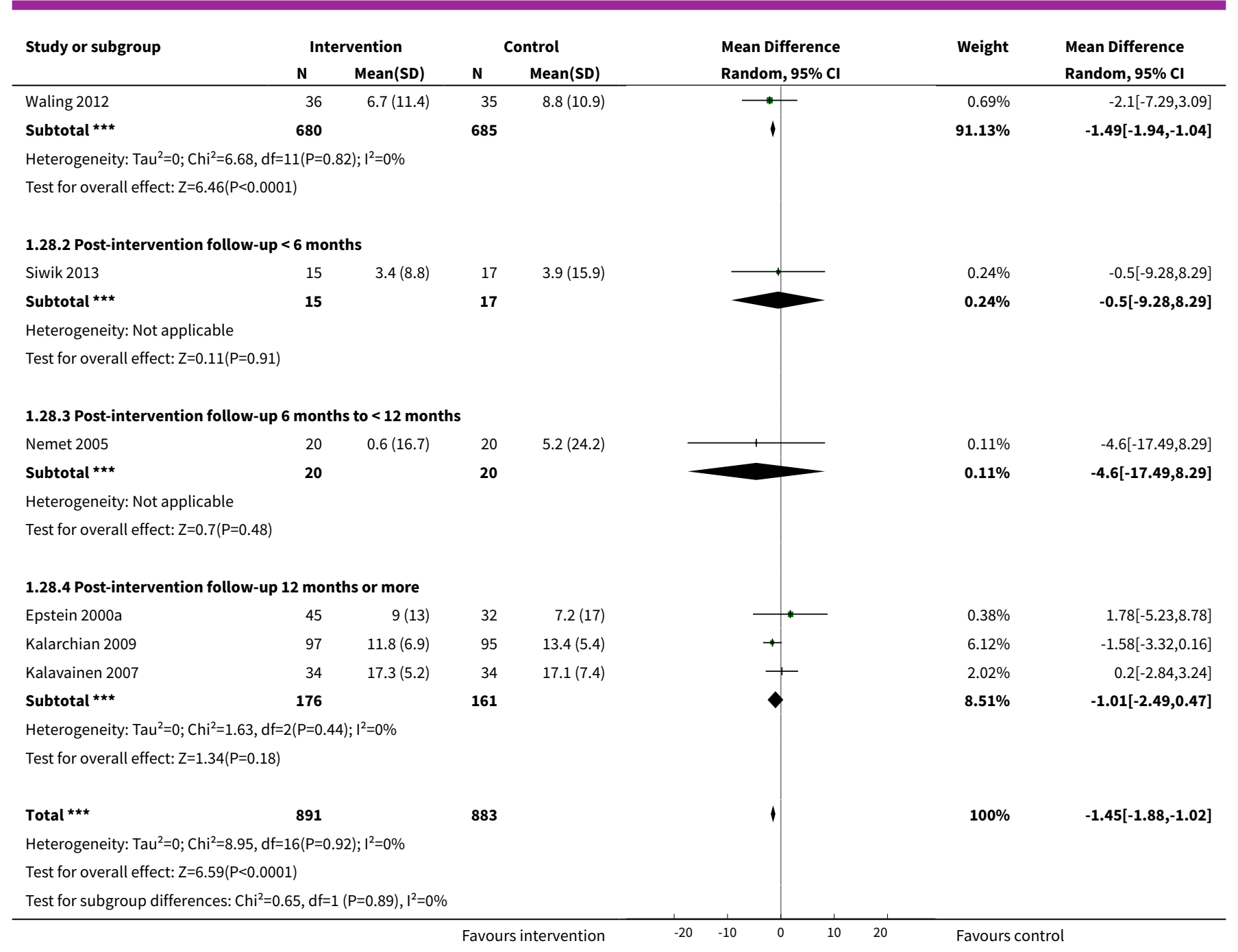

Analysis 1.29. Comparison 1 Behaviour-changing interventions versus no treatment/usual care, Outcome 29 Change in BMI - type of parental involvement.

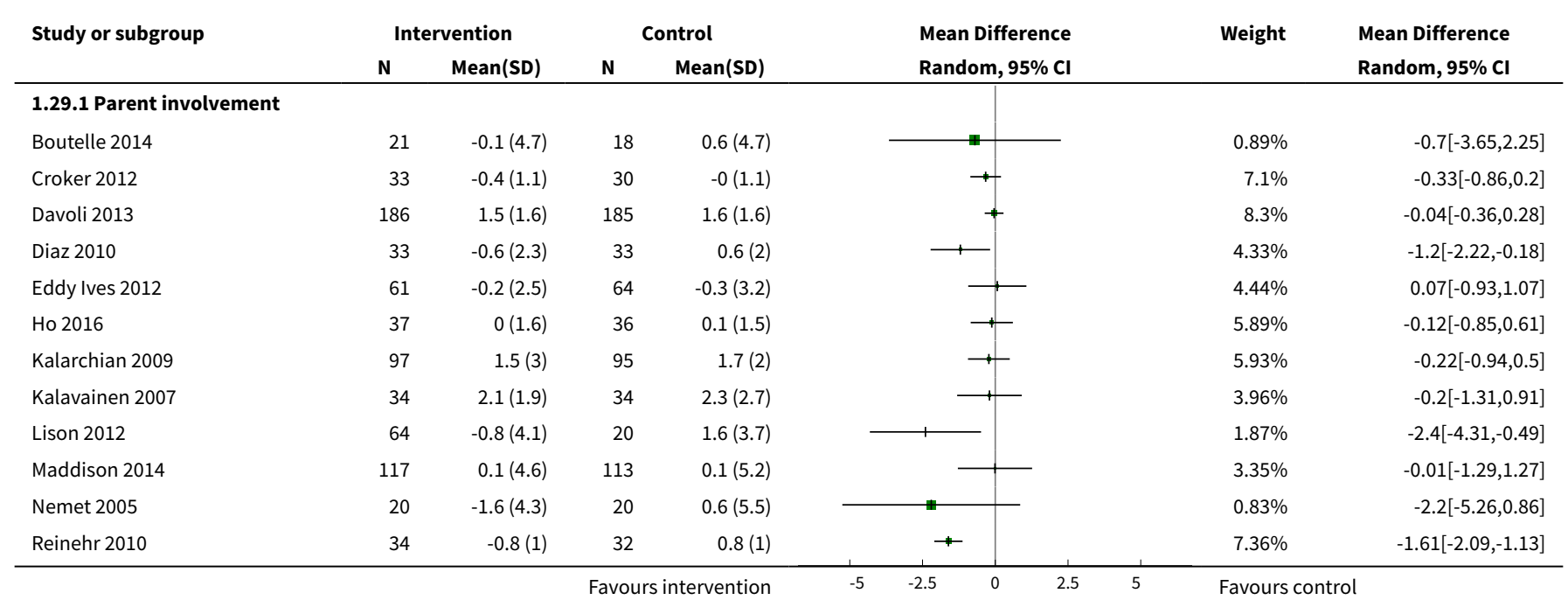

Diet, physical activity and behavioural interventions for the treatment of overweight or obese children from the age of 6 to 11 years 269 (Review)

Copyright @ 2017 The Cochrane Collaboration. Published by John Wiley \& Sons, Ltd. 


\begin{tabular}{|c|c|c|c|c|c|c|c|}
\hline \multirow{3}{*}{$\begin{array}{l}\text { Study or subgroup } \\
\text { Sacher } 2010\end{array}$} & \multicolumn{2}{|c|}{ Intervention } & \multicolumn{2}{|c|}{ Control } & \multirow{2}{*}{$\begin{array}{l}\text { Mean Difference } \\
\text { Random, } 95 \% \mathrm{Cl}\end{array}$} & \multirow{3}{*}{$\begin{array}{r}\text { Weight } \\
1.95 \%\end{array}$} & \multirow{3}{*}{$\begin{array}{l}\begin{array}{l}\text { Mean Difference } \\
\text { Random, } 95 \% \text { Cl }\end{array} \\
-2.1[-3.96,-0.24]\end{array}$} \\
\hline & \multirow{2}{*}{$\frac{\mathbf{N}}{37}$} & \multirow{2}{*}{$\frac{\operatorname{Mean}(\text { SD) }}{-1.5(3.5)}$} & \multirow{2}{*}{$\frac{\mathbf{N}}{45}$} & \multirow{2}{*}{$\frac{\operatorname{Mean}(\mathrm{SD})}{0.6(5.1)}$} & & & \\
\hline & & & & & 1 & & \\
\hline Siwik 2013 & 15 & $0.3(3.7)$ & 17 & $0.7(6.4)$ & * & $0.63 \%$ & $-0.4[-3.95,3.15]$ \\
\hline Taylor 2015 & 89 & $0.8(3)$ & 92 & $1.2(2.3)$ & $\rightarrow$ & $5.59 \%$ & $-0.4[-1.18,0.38]$ \\
\hline Wake 2009 & 127 & $0.6(2.6)$ & 115 & $0.7(2.2)$ & $\rightarrow$ & $6.62 \%$ & $-0.1[-0.7,0.5]$ \\
\hline Wake 2013 & 56 & $0.9(3.4)$ & 49 & $0.8(4.2)$ & & $2.76 \%$ & $0.1[-1.37,1.57]$ \\
\hline Waling 2012 & 36 & $0.3(2.9)$ & 35 & $0.6(2.6)$ & 1 & $3.34 \%$ & $-0.3[-1.58,0.98]$ \\
\hline Weigel 2008 & 36 & $-1.5(3)$ & 30 & $2.8(3.9)$ & & $2.24 \%$ & $-4.3[-6,-2.6]$ \\
\hline Weintraub 2008 & 9 & $0.2(5.2)$ & 12 & $0.8(4.8)$ & 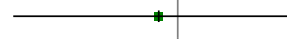 & $0.43 \%$ & $-0.57[-4.94,3.8]$ \\
\hline 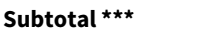 & 1142 & & 1075 & & 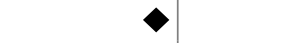 & $77.79 \%$ & $-0.65[-1.04,-0.25]$ \\
\hline \multicolumn{8}{|c|}{ Heterogeneity: $\operatorname{Tau}^{2}=0.43 ; \mathrm{Chi}^{2}=63.15, \mathrm{df}=19(\mathrm{P}<0.0001) ; \mathrm{I}^{2}=69.91 \%$} \\
\hline \multicolumn{8}{|c|}{ Test for overall effect: $Z=3.2(P=0)$} \\
\hline \multicolumn{8}{|c|}{ 1.29.2 No parental involvement } \\
\hline Alves 2008 & 39 & $-0.3(0.9)$ & 39 & $0.3(1.4)$ & $\rightarrow$ & $7.13 \%$ & $-0.53[-1.05,-0.01]$ \\
\hline Faude 2010 & 11 & $0.5(3.1)$ & 11 & $0.6(3.8)$ & & $0.9 \%$ & $-0.1[-3.02,2.82]$ \\
\hline Maddison 2011 & 160 & $0.1(1)$ & 162 & $0.3(1)$ & * & $8.77 \%$ & $-0.25[-0.47,-0.03]$ \\
\hline 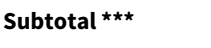 & 210 & & 212 & & 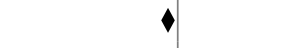 & $16.8 \%$ & $-0.29[-0.5,-0.09]$ \\
\hline \multicolumn{8}{|c|}{ Heterogeneity: $\mathrm{Tau}^{2}=0 ; \mathrm{Chi}^{2}=0.95, \mathrm{df}=2(\mathrm{P}=0.62) ; \mathrm{I}^{2}=0 \%$} \\
\hline \multicolumn{8}{|c|}{ Test for overall effect: $Z=2.81(P=0)$} \\
\hline \multicolumn{8}{|c|}{ 1.29.3 Parent targeted } \\
\hline McCallum 2007 & 70 & $1.2(2.8)$ & 76 & $1.2(2.2)$ & $\rightarrow$ & $5.41 \%$ & $0[-0.81,0.81]$ \\
\hline Subtotal $\star \star \star ~$ & 70 & & 76 & & & $5.41 \%$ & $0[-0.81,0.81]$ \\
\hline \multicolumn{8}{|c|}{ Heterogeneity: Not applicable } \\
\hline \multicolumn{8}{|c|}{ Test for overall effect: Not applicable } \\
\hline Total $\star \star \star ~$ & 1422 & & 1363 & & $\boldsymbol{\gamma}$ & $100 \%$ & $-0.53[-0.82,-0.24]$ \\
\hline \multicolumn{8}{|c|}{ Heterogeneity: $\mathrm{Tau}^{2}=0.24 ; \mathrm{Chi}^{2}=66.49, \mathrm{df}=23(\mathrm{P}<0.0001) ; \mathrm{I}^{2}=65.41 \%$} \\
\hline \multicolumn{8}{|c|}{ Test for overall effect: $Z=3.56(P=0)$} \\
\hline \multicolumn{8}{|c|}{ Test for subgroup differences: $\mathrm{Chi}^{2}=3.2, \mathrm{df}=1(\mathrm{P}=0.2), \mathrm{I}^{2}=37.56 \%$} \\
\hline & & & Favou & ervention & -2.5 & Favours c & \\
\hline
\end{tabular}

Analysis 1.30. Comparison 1 Behaviour-changing interventions versus no treatment/ usual care, Outcome 30 Change in BMI $z$ score - type of parental involvement.

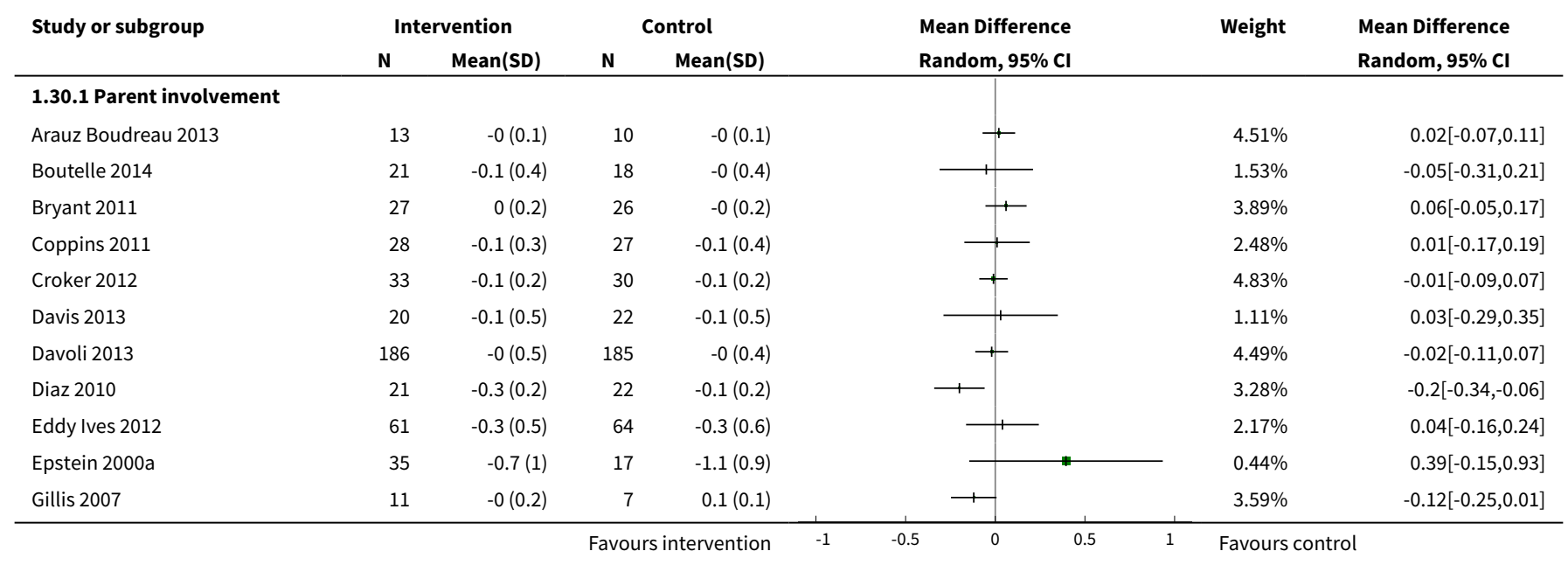

Diet, physical activity and behavioural interventions for the treatment of overweight or obese children from the age of 6 to 11 years 270 (Review)

Copyright (c) 2017 The Cochrane Collaboration. Published by John Wiley \& Sons, Ltd. 


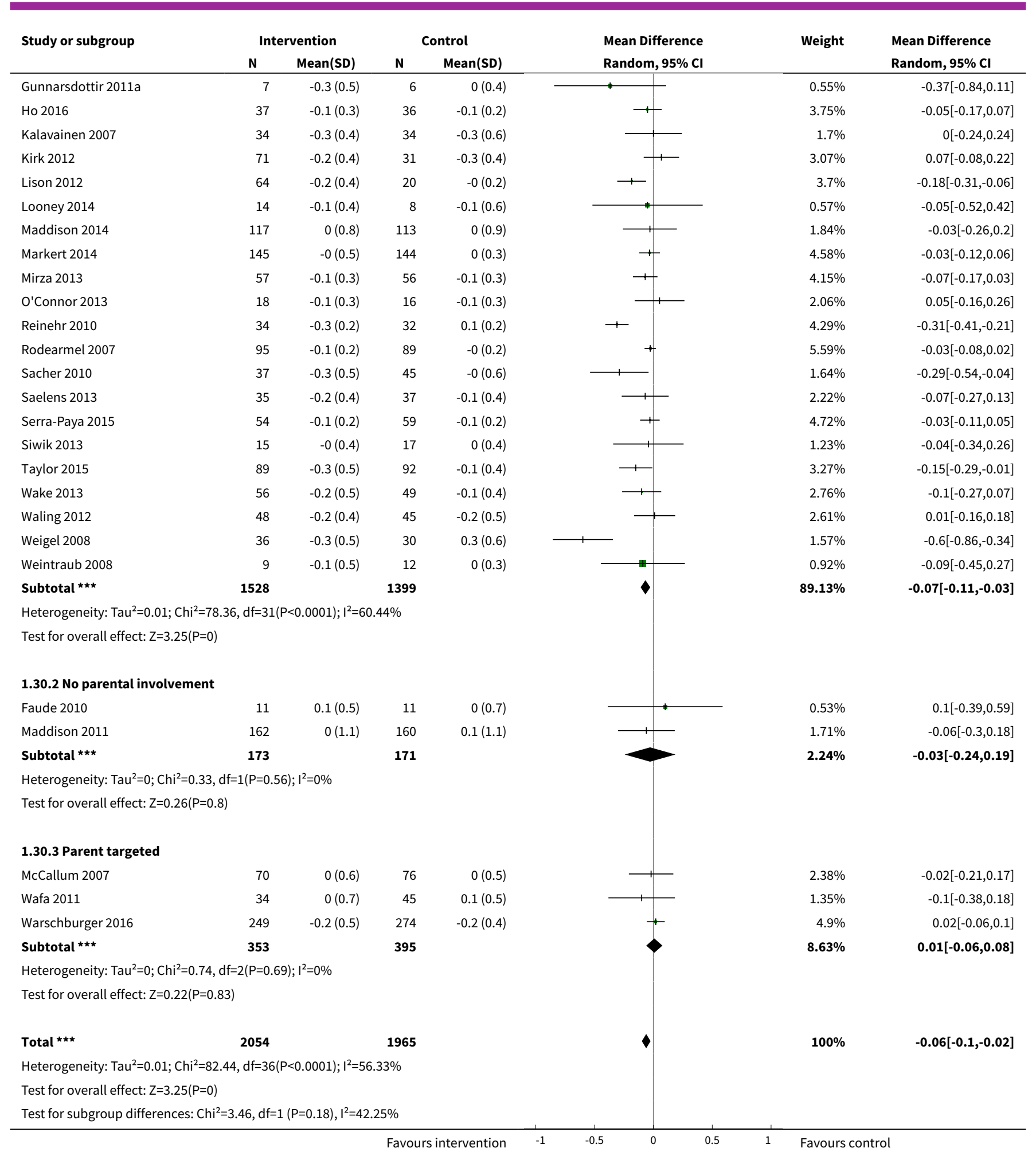

Diet, physical activity and behavioural interventions for the treatment of overweight or obese children from the age of 6 to 11 years 
Analysis 1.31. Comparison 1 Behaviour-changing interventions versus no treatment/usual care, Outcome 31 Change in weight - type of parental involvement.

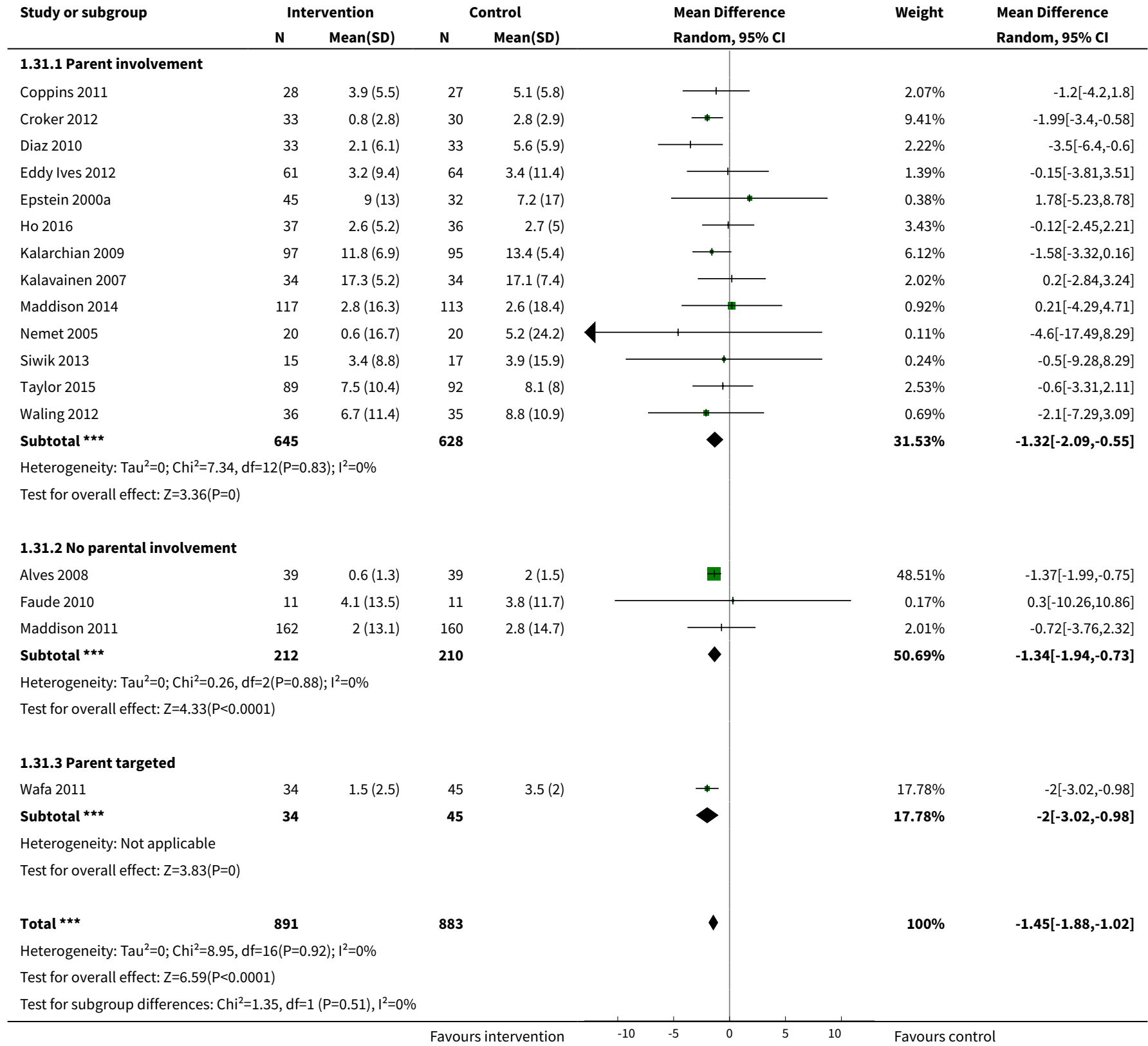

Analysis 1.32. Comparison 1 Behaviour-changing interventions versus no treatment/usual care, Outcome 32 Change in BMI z score - baseline BMI z score.

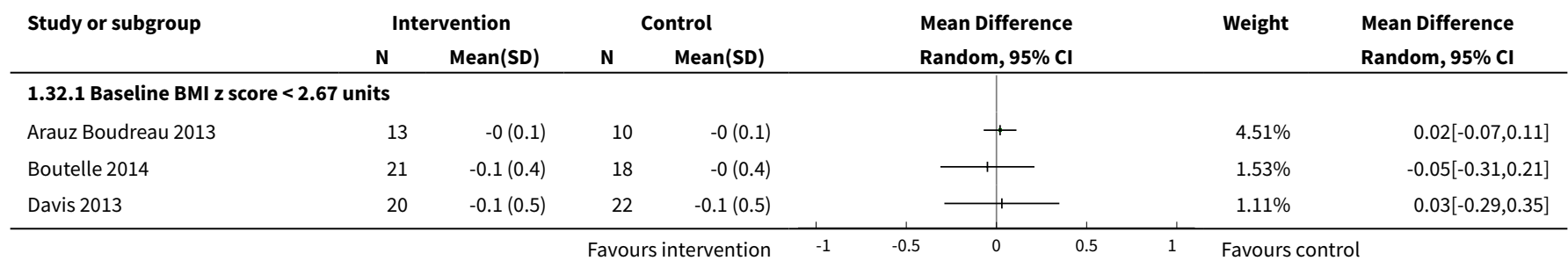

Diet, physical activity and behavioural interventions for the treatment of overweight or obese children from the age of 6 to 11 years 


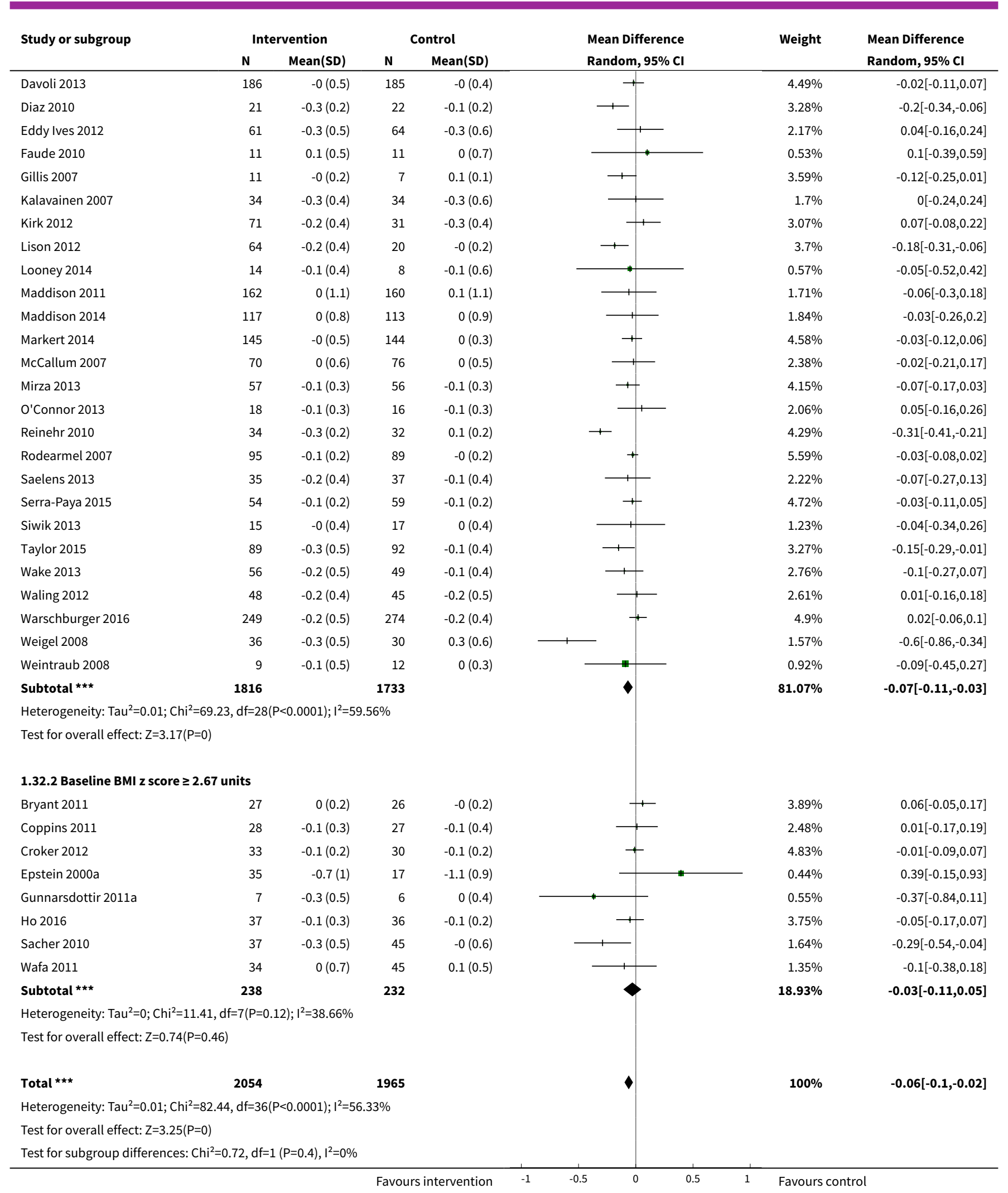

Diet, physical activity and behavioural interventions for the treatment of overweight or obese children from the age of 6 to 11 years 
Comparison 2. Behaviour-changing interventions plus component versus behaviour-changing intervention without component

\begin{tabular}{llllll}
\hline $\begin{array}{l}\text { Outcome or subgroup } \\
\text { title }\end{array}$ & No. of studies & $\begin{array}{l}\text { No. of partici- } \\
\text { pants }\end{array}$ & Statistical method & Effect size \\
\hline 1 Change in BMI & 4 & 195 & Mean Difference (IV, Random, 95\% Cl) & $-0.75[-1.42,-0.09]$ \\
\hline 2 Change in BMI z score & 5 & 212 & Mean Difference (IV, Random, 95\% Cl) & $-0.03[-0.10,0.04]$ \\
\hline 3 Change in weight & 4 & 106 & Mean Difference (IV, Random, 95\% Cl) & $1.59[-4.58,7.77]$ \\
\hline
\end{tabular}

Analysis 2.1. Comparison 2 Behaviour-changing interventions plus component versus behaviour-changing intervention without component, Outcome 1 Change in BMI.

\begin{tabular}{|c|c|c|c|c|c|c|c|}
\hline \multirow[t]{2}{*}{ Study or subgroup } & \multicolumn{2}{|c|}{ Intervention } & \multicolumn{2}{|c|}{ Control } & \multirow{2}{*}{$\begin{array}{l}\text { Mean Difference } \\
\text { Random, 95\% Cl }\end{array}$} & \multirow[t]{2}{*}{ Weight } & \multirow{2}{*}{$\begin{array}{l}\text { Mean Difference } \\
\text { Random, } 95 \% \mathrm{CI}\end{array}$} \\
\hline & $\mathbf{N}$ & Mean(SD) & $\mathbf{N}$ & Mean(SD) & & & \\
\hline Epstein 1985c & 7 & $-3.7(2.7)$ & 11 & $-1.3(3.2)$ & 1 & $5.72 \%$ & $-2.4[-5.14,0.34]$ \\
\hline Epstein 2001 & 13 & $-0.3(1.4)$ & 14 & $1(1.7)$ & $\rightarrow$ & $27.28 \%$ & $-1.27[-2.44,-0.1]$ \\
\hline Epstein 2001 & 14 & $-1.8(1.9)$ & 15 & $-0.6(1.4)$ & $\rightarrow-$ & $26.4 \%$ & $-1.11[-2.31,0.09]$ \\
\hline Flodmark 1993 & 20 & $1.1(2.8)$ & 19 & $1.6(3.3)$ & $\longrightarrow$ & $10.96 \%$ & $-0.5[-2.45,1.45]$ \\
\hline Woo 2004 & 22 & $0.1(2.4)$ & 21 & $-0.2(2.3)$ & + & $20.01 \%$ & $0.3[-1.1,1.7]$ \\
\hline Total *** & 95 & & 100 & & & $100 \%$ & $-0.75[-1.42,-0.09]$ \\
\hline \multicolumn{8}{|c|}{ Heterogeneity: $\mathrm{Tau}^{2}=0.07 ; \mathrm{Chi}^{2}=5.51, \mathrm{df}=5(\mathrm{P}=0.36) ; \mathrm{I}^{2}=9.17 \%$} \\
\hline \multicolumn{3}{|c|}{ Test for overall effect: $Z=2.21(P=0.03)$} & & & & & \\
\hline
\end{tabular}

Analysis 2.2. Comparison 2 Behaviour-changing interventions plus component versus behaviour-changing intervention without component, Outcome 2 Change in BMI z score.

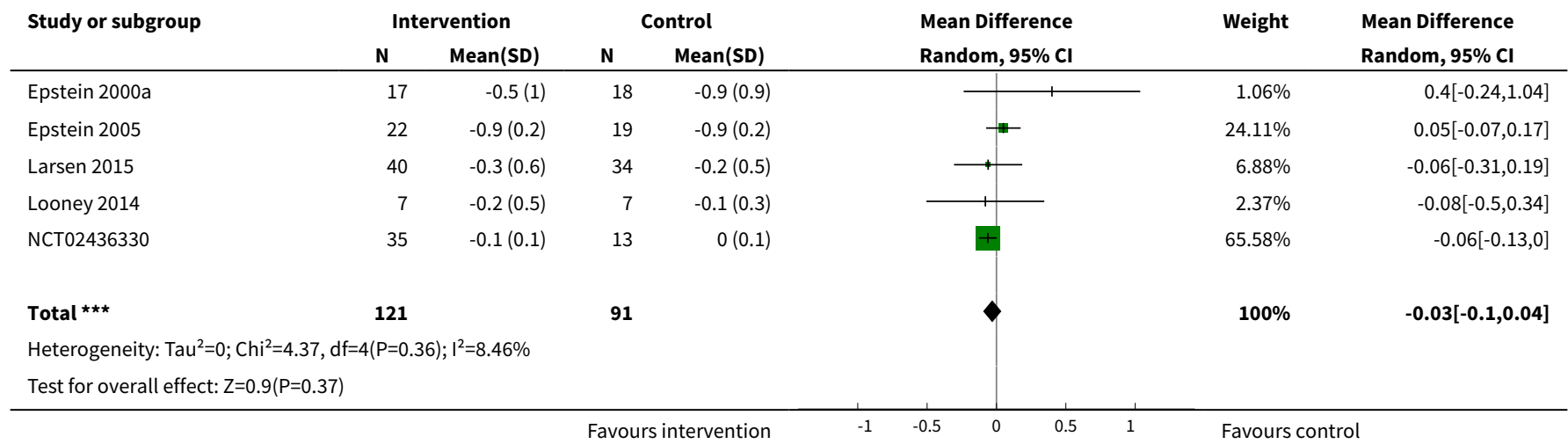


Analysis 2.3. Comparison 2 Behaviour-changing interventions plus component versus behaviour-changing intervention without component, Outcome 3 Change in weight.

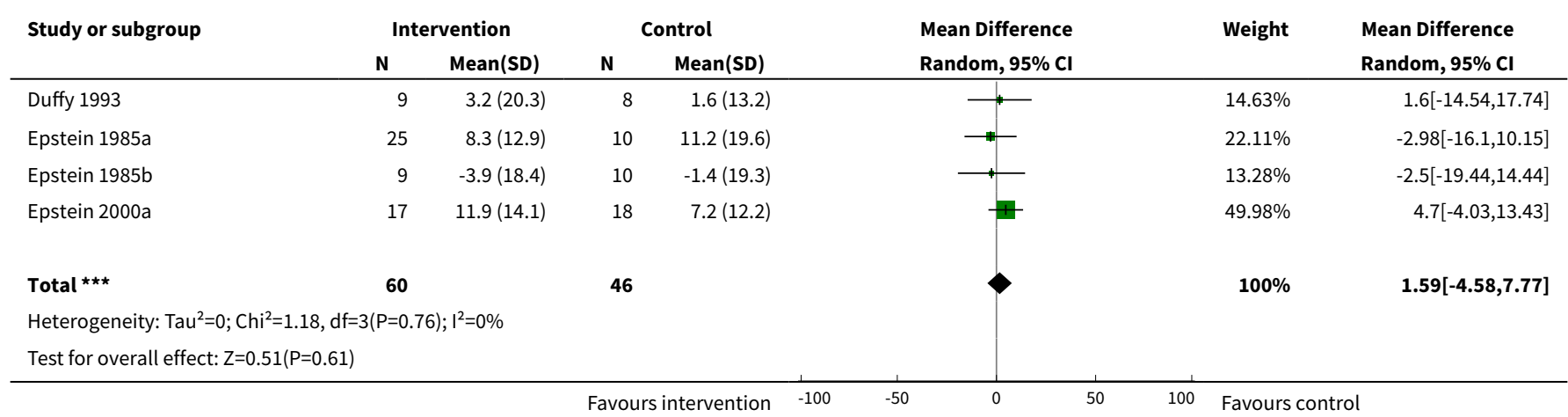

\section{Comparison 3. Cluster RCTs versus comparator}

\begin{tabular}{lllll}
\hline $\begin{array}{l}\text { Outcome or subgroup ti- } \\
\text { tle }\end{array}$ & No. of studies & $\begin{array}{l}\text { No. of partici- } \\
\text { pants }\end{array}$ & Statistical method & Effect size \\
\hline 1 Change in BMI & 2 & 629 & Mean Difference (IV, Random, 95\% Cl) & $-0.49[-1.24,0.27]$ \\
\hline
\end{tabular}

Analysis 3.1. Comparison 3 Cluster RCTs versus comparator, Outcome 1 Change in BMI.

\begin{tabular}{|c|c|c|c|c|c|c|c|}
\hline \multirow[t]{2}{*}{ Study or subgroup } & \multicolumn{2}{|c|}{ Intervention } & \multicolumn{2}{|c|}{ Control } & \multirow{2}{*}{$\begin{array}{l}\text { Mean Difference } \\
\text { Random, } 95 \% \mathrm{Cl}\end{array}$} & \multirow[t]{2}{*}{ Weight } & \multirow{2}{*}{$\begin{array}{l}\text { Mean Difference } \\
\text { Random, } 95 \% \mathrm{Cl}\end{array}$} \\
\hline & $\mathbf{N}$ & $\operatorname{Mean}(S D)$ & $\mathbf{N}$ & $\operatorname{Mean}(S D)$ & & & \\
\hline Berry 2007 & 40 & $-1.2(6.4)$ & 40 & $-0.3(5.9)$ & $\longrightarrow$ & $7.89 \%$ & $-0.9[-3.59,1.79]$ \\
\hline Taveras 2015 & 365 & $0.7(4.5)$ & 184 & $1.2(4.4)$ & & $92.11 \%$ & $-0.45[-1.24,0.33]$ \\
\hline 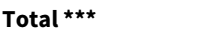 & 405 & & 224 & & & $100 \%$ & $-0.49[-1.24,0.27]$ \\
\hline \multicolumn{8}{|c|}{ Heterogeneity: $\mathrm{Tau}^{2}=0 ; \mathrm{Chi}^{2}=0.1, \mathrm{df}=1(\mathrm{P}=0.75) ; \mathrm{I}^{2}=0 \%$} \\
\hline \multicolumn{8}{|c|}{ Test for overall effect: $Z=1.27(P=0.2)$} \\
\hline
\end{tabular}

\section{Comparison 4. Maintenance intervention versus no treatment/usual care}

\begin{tabular}{lllll}
\hline Outcome or subgroup title & No. of studies & $\begin{array}{l}\text { No. of partici- } \\
\text { pants }\end{array}$ & Statistical method & Effect size \\
\hline 1 Change in BMI z score & 2 & 263 & $\begin{array}{l}\text { Mean Difference (IV, Random, 95\% } \\
\text { Cl) }\end{array}$ & $-0.07[-0.19,0.04]$ \\
\hline
\end{tabular}


Analysis 4.1. Comparison 4 Maintenance intervention versus no treatment/usual care, Outcome 1 Change in BMI z score.

\begin{tabular}{|c|c|c|c|c|c|c|c|}
\hline \multirow[t]{2}{*}{ Study or subgroup } & \multicolumn{2}{|c|}{ Intervention } & \multicolumn{2}{|c|}{ Control } & \multirow{2}{*}{$\begin{array}{l}\text { Mean Difference } \\
\text { Random, 95\% Cl }\end{array}$} & \multirow[t]{2}{*}{ Weight } & \multirow{2}{*}{$\begin{array}{l}\text { Mean Difference } \\
\text { Random, } 95 \% \mathrm{Cl}\end{array}$} \\
\hline & $\mathbf{N}$ & Mean(SD) & $\mathbf{N}$ & Mean(SD) & & & \\
\hline de Niet 2012 & 73 & $-0.1(0.6)$ & 68 & $-0(0.5)$ & \# & $41.48 \%$ & $-0.03[-0.21,0.15]$ \\
\hline Wilfley 2007 & 85 & $0(0.4)$ & 37 & $0.1(0.4)$ & + & $58.52 \%$ & $-0.11[-0.26,0.05]$ \\
\hline 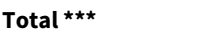 & 158 & & 105 & & $\gamma$ & $100 \%$ & $-0.07[-0.19,0.04]$ \\
\hline \multicolumn{8}{|c|}{ Heterogeneity: $\mathrm{Tau}^{2}=0 ; \mathrm{Chi}^{2}=0.38, \mathrm{df}=1(\mathrm{P}=0.54) ; \mathrm{I}^{2}=0 \%$} \\
\hline
\end{tabular}




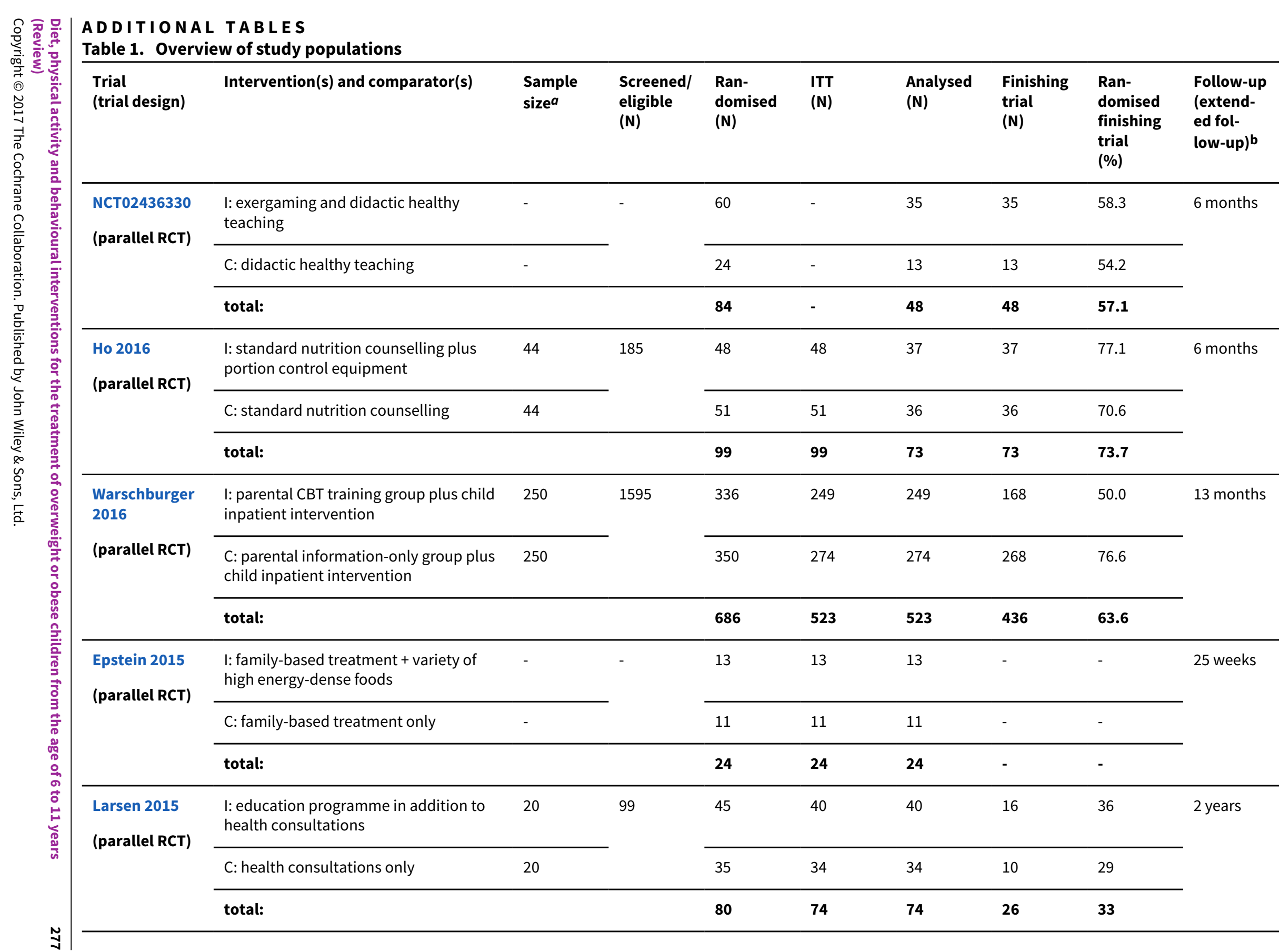




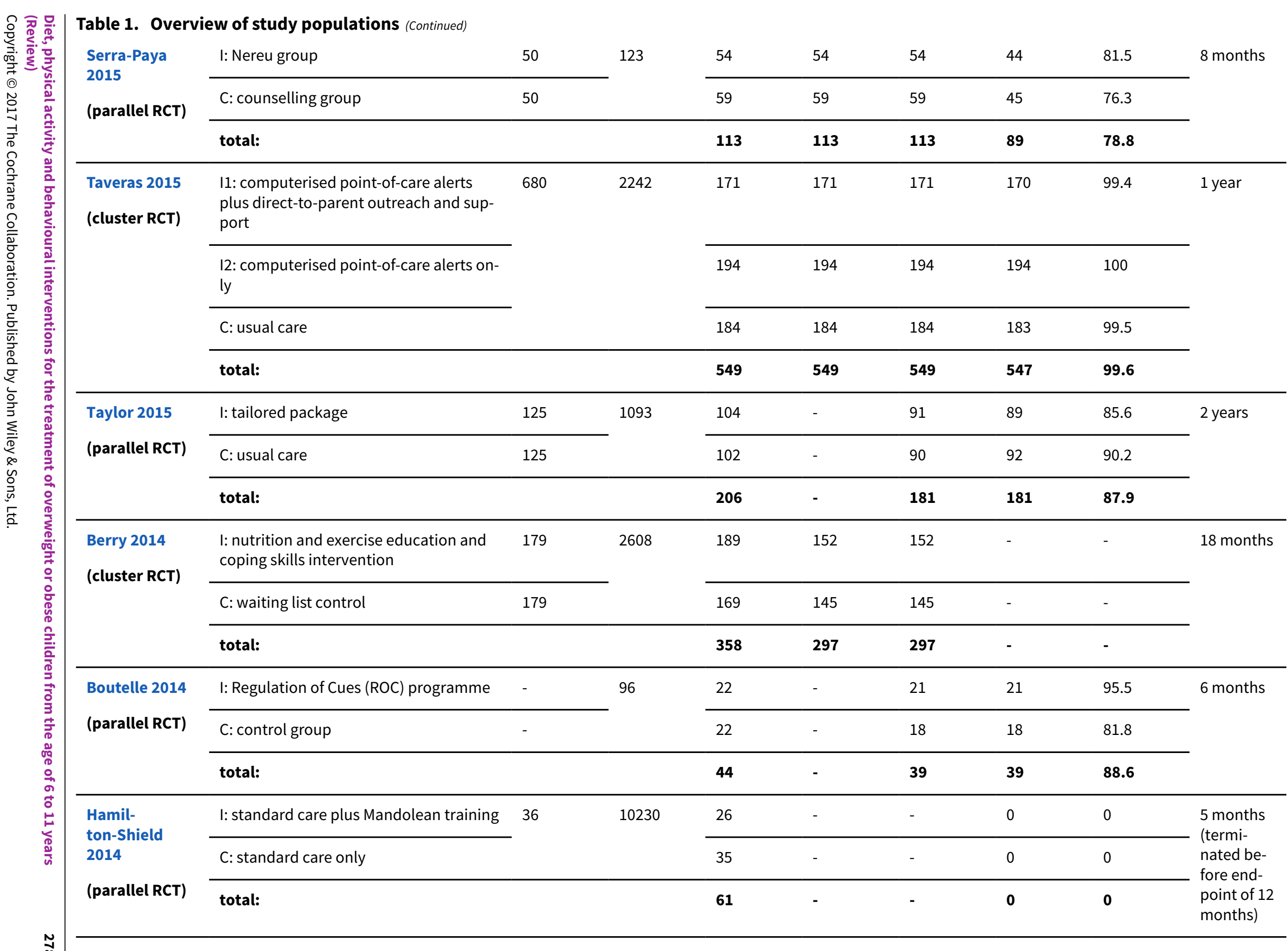


Table 1. Overview of study populations (Continued)

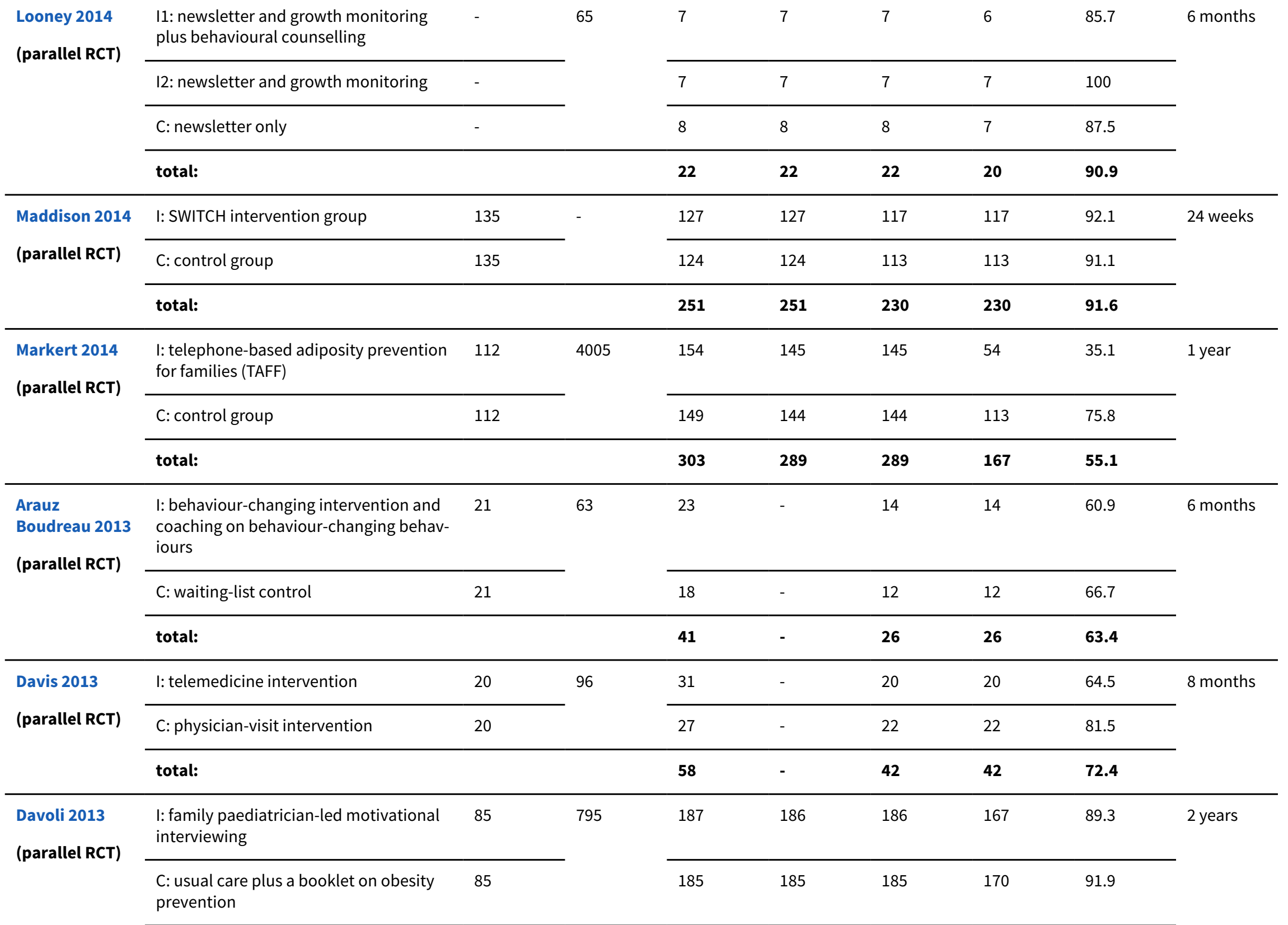




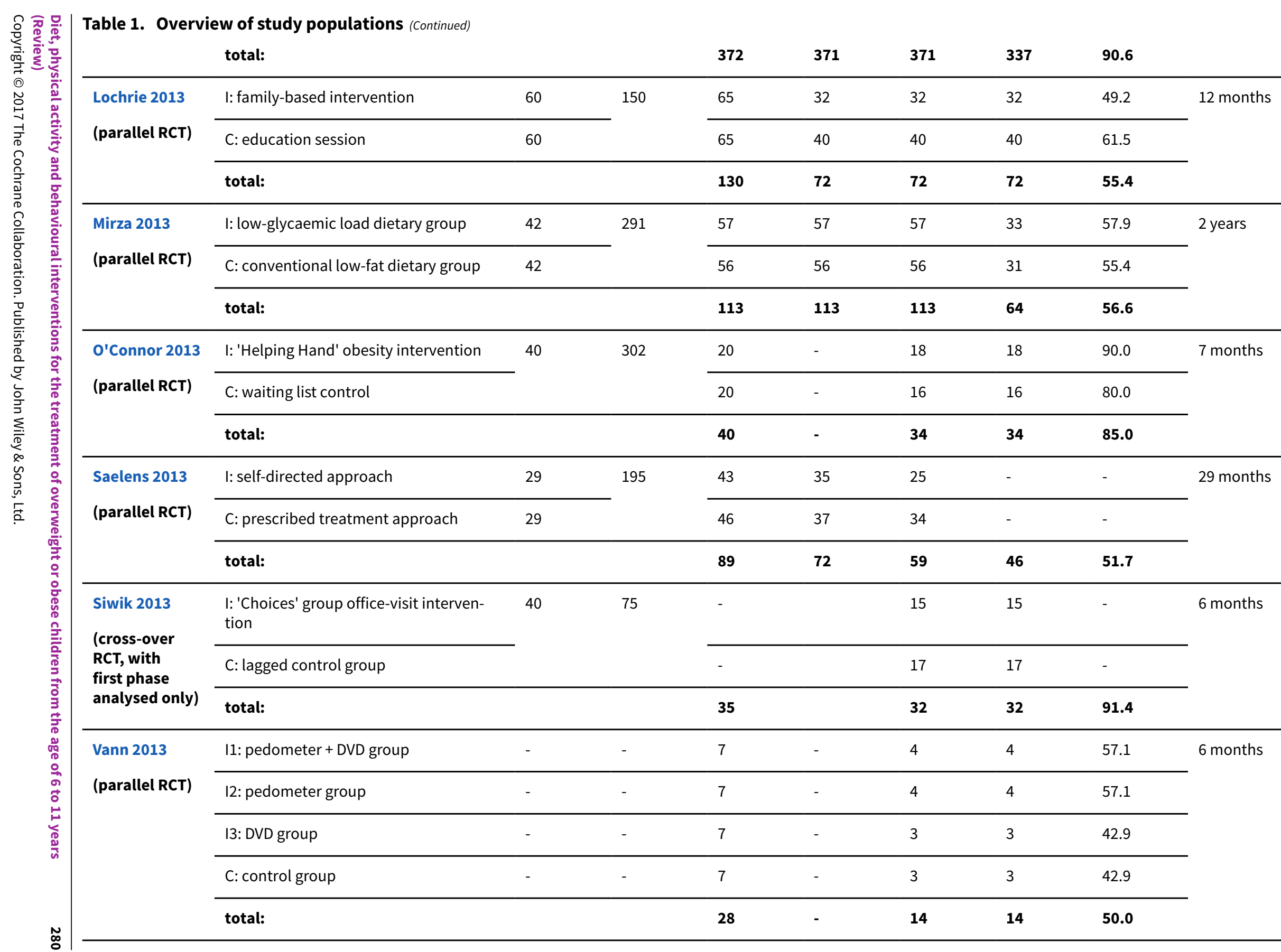


Table 1. Overview of study populations (Continued)

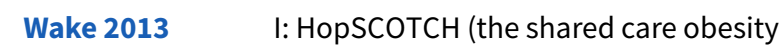

c: usual care

\begin{tabular}{lllll}
\hline 56 & 56 & 51 & 51 & 91.1 \\
\hline $\mathbf{1 1 8}$ & $\mathbf{1 1 8}$ & $\mathbf{1 0 7}$ & $\mathbf{1 0 7}$ & $\mathbf{9 0 . 7}$
\end{tabular}

total:

118

$118 \quad 118$

\begin{tabular}{lll}
\hline Croker 2012 & I: family-based behavioural treatment & 48 \\
\cline { 2 - 3 } (parallel RCT) & C: waiting list control & 99 \\
\cline { 2 - 3 } & &
\end{tabular}

\begin{tabular}{|c|c|c|c|c|c|c|c|c|c|}
\hline & total: & & & 72 & 72 & 60 & 49 & 68.0 & \\
\hline de Niet 2012 & $\begin{array}{l}\text { I: short message service maintenance } \\
\text { treatment and behaviour-changing }\end{array}$ & 64 & 144 & 73 & 73 & 73 & 63 & 86.3 & 9 months \\
\hline
\end{tabular}

treatment and behaviour-changing

(parallel RCT) treatment

\begin{tabular}{ll} 
C: behaviour-changing treatment only 64 \\
\hline
\end{tabular}

total:

$68 \quad 68$

\begin{tabular}{lllll}
\hline 68 & 68 & 67 & 47 & 70.1 \\
\hline $\mathbf{1 4 1}$ & $\mathbf{1 4 1}$ & $\mathbf{1 4 0}$ & $\mathbf{1 1 0}$ & $\mathbf{7 8 . 6}$ \\
\hline
\end{tabular}

\begin{tabular}{lllll}
68 & 68 & 67 & 47 & 70.1 \\
\hline $\mathbf{1 4 1}$ & $\mathbf{1 4 1}$ & $\mathbf{1 4 0}$ & $\mathbf{1 1 0}$ & $\mathbf{7 8 . 6}$ \\
\hline
\end{tabular}

\begin{tabular}{lllll}
68 & 68 & 67 & 47 & 70.1 \\
\hline $\mathbf{1 4 1}$ & $\mathbf{1 4 1}$ & $\mathbf{1 4 0}$ & $\mathbf{1 1 0}$ & $\mathbf{7 8 . 6}$ \\
\hline
\end{tabular}

70.1

\begin{tabular}{llll}
\hline Eddy Ives 2012 & $\begin{array}{l}\text { I: dietary and physical exercise recom- } \\
\text { mendations during 6 sessions }\end{array}$ & 110 & 211 \\
\cline { 2 - 3 } (parallel RCT) & $\begin{array}{l}\text { C: dietary and physical exercise recom- } \\
\text { mendations at 2 sessions only }\end{array}$ & 110 & 87
\end{tabular}

mendations at 2 sessions only

$87 \quad 6$

$61 \quad 61$

61

70.1

\begin{tabular}{|c|c|c|c|c|c|c|c|c|c|}
\hline & total: & & & 174 & 125 & 125 & 125 & 71.8 & \\
\hline \multirow{4}{*}{$\begin{array}{l}\text { Kirk } 2012 \\
\text { (parallel RCT) }\end{array}$} & $\begin{array}{l}\text { I1: low carbohydrate diet plus group ex- } \\
\text { ercise/education sessions }\end{array}$ & - & - & 35 & 35 & 35 & 25 & 71.4 & \multirow[t]{4}{*}{12 months } \\
\hline & $\begin{array}{l}\text { 12: reduced glycaemic load diet plus } \\
\text { group exercise/education sessions }\end{array}$ & - & 440 & 36 & 36 & 36 & 32 & 88.9 & \\
\hline & $\begin{array}{l}\text { C: standard portion-controlled diet plus } \\
\text { group exercise/education sessions }\end{array}$ & - & & 31 & 31 & 31 & 28 & 90.3 & \\
\hline & total: & & & 102 & 102 & 102 & 85 & 83.3 & \\
\hline Lison 2012 & $\begin{array}{l}\text { I1: hospital clinic group exercise-diet } \\
\text { programme }\end{array}$ & 20 & 120 & 45 & 32 & 32 & 32 & 71.1 & 6 months \\
\hline
\end{tabular}




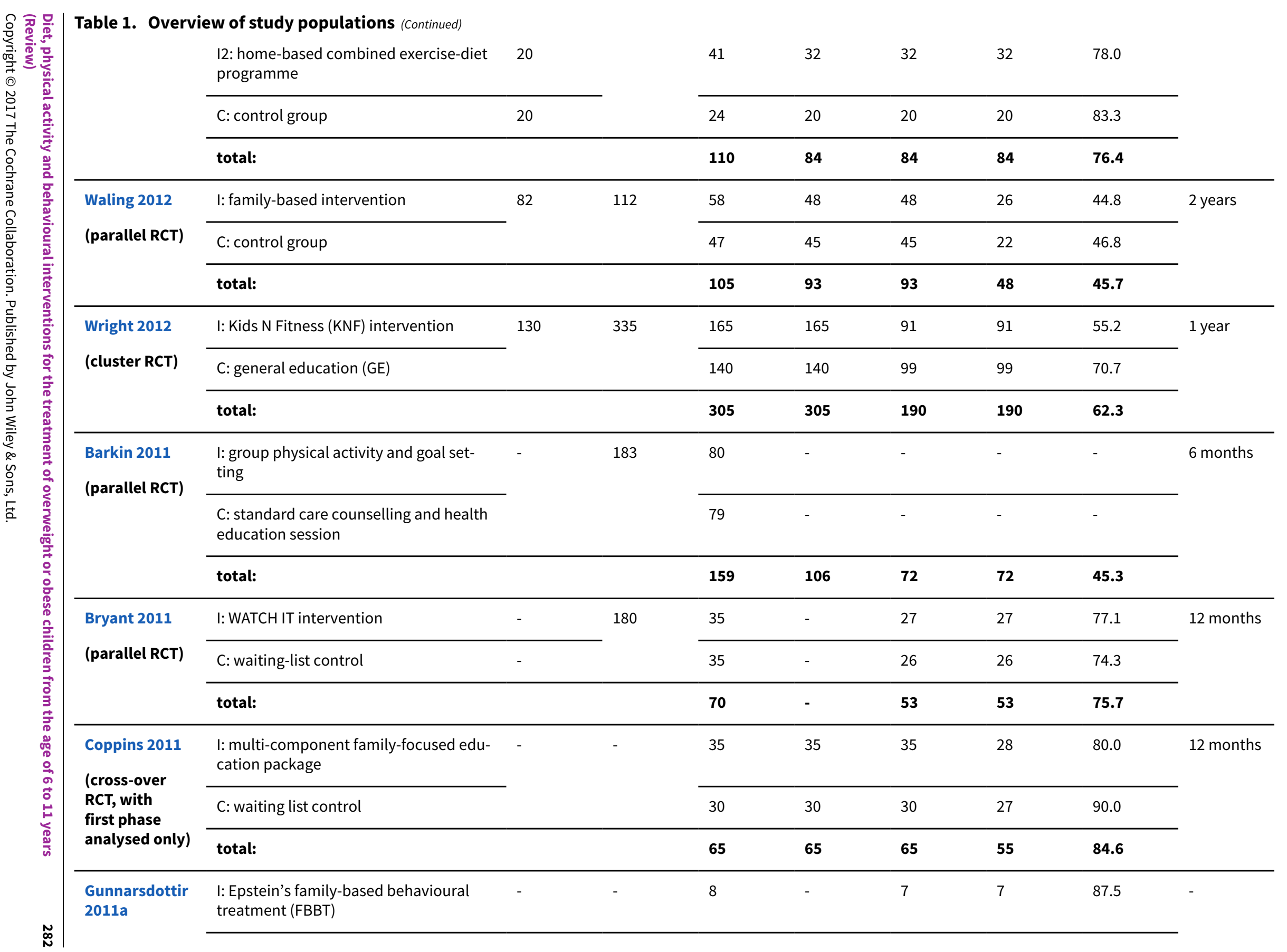




\begin{tabular}{|c|c|c|c|c|c|c|c|c|c|c|c|}
\hline 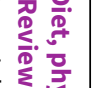 & (parallel RCT) & C: standard care (waiting-list control) & - & - & 8 & - & 6 & 6 & 75.0 & & 0,1 \\
\hline$\frac{\varrho}{2}$ & & total: & & & 16 & - & 13 & 13 & 81.3 & 12 months & \\
\hline$\stackrel{\vec{\Sigma}}{\gtrless}$ & Maddison 2011 & I: active video game package & 165 & 1932 & 160 & 160 & 160 & 123 & 77.0 & 24 weeks & \\
\hline$\frac{z}{\bar{z}}$ & (parallel RCT) & C: control group & 165 & & 162 & 162 & 162 & 135 & 83.3 & & \\
\hline$\stackrel{2}{\underline{2}}$ & & total: & & & 322 & 322 & 322 & 258 & 80.1 & & 罾哥 \\
\hline 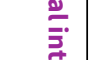 & Wafa 2011 & I: low intensity intervention & 30 & 365 & 52 & 34 & 34 & 34 & 65.4 & 6 months & \\
\hline స్ & (parallel RCT) & C: waiting-list control & 30 & & 55 & 45 & 45 & 45 & 81.8 & & \\
\hline $\begin{array}{l}5 \\
\overrightarrow{3} \\
0\end{array}$ & & total: & & & 107 & 79 & 79 & 79 & 73.8 & & \\
\hline 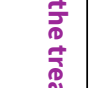 & $\begin{array}{l}\text { Bathrellou } \\
2010\end{array}$ & $\begin{array}{l}\text { I: behavioural intervention with parental } \\
\text { involvement }\end{array}$ & - & - & 24 & - & 23 & 16 & 66.7 & 18 months & \\
\hline 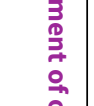 & (parallel RCT) & $\begin{array}{l}\text { C: behavioural intervention without } \\
\text { parental involvement }\end{array}$ & & & 23 & - & 19 & 16 & 69.6 & & \\
\hline$\sum_{\Sigma}^{\frac{0}{2}}$ & & total: & & & 47 & - & 42 & 32 & 68.1 & & \\
\hline 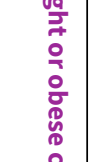 & $\begin{array}{l}\text { Diaz } 2010 \\
\text { (parallel RCT) }\end{array}$ & $\begin{array}{l}\text { I: behavioural curriculum plus registered } \\
\text { dieticians and physician consultations }\end{array}$ & 26 & 134 & 38 & 33 & $\begin{array}{l}33 \text { (pri- } \\
\text { mary out- } \\
\text { comes } \\
\text { ITT) }\end{array}$ & 21 & 55.3 & 12 months & \\
\hline 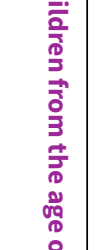 & & & & & & & $\begin{array}{l}21 \text { (sec- } \\
\text { ondary } \\
\text { outcomes, } \\
\text { com- } \\
\text { pleters' } \\
\text { analysis) }\end{array}$ & & & & 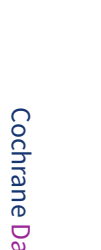 \\
\hline 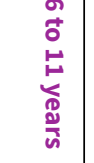 & & C: physician consultations only & 26 & & 38 & 33 & $\begin{array}{l}33 \text { (pri- } \\
\text { mary out- } \\
\text { comes, } \\
\text { ITT) }\end{array}$ & 22 & 57.9 & & 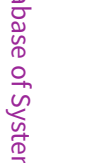 \\
\hline$\underset{\omega}{\mathbb{\infty}}$ & & & & & & & $\begin{array}{l}22 \text { (sec- } \\
\text { ondary } \\
\text { outcomes, }\end{array}$ & & & & 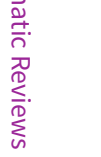 \\
\hline
\end{tabular}




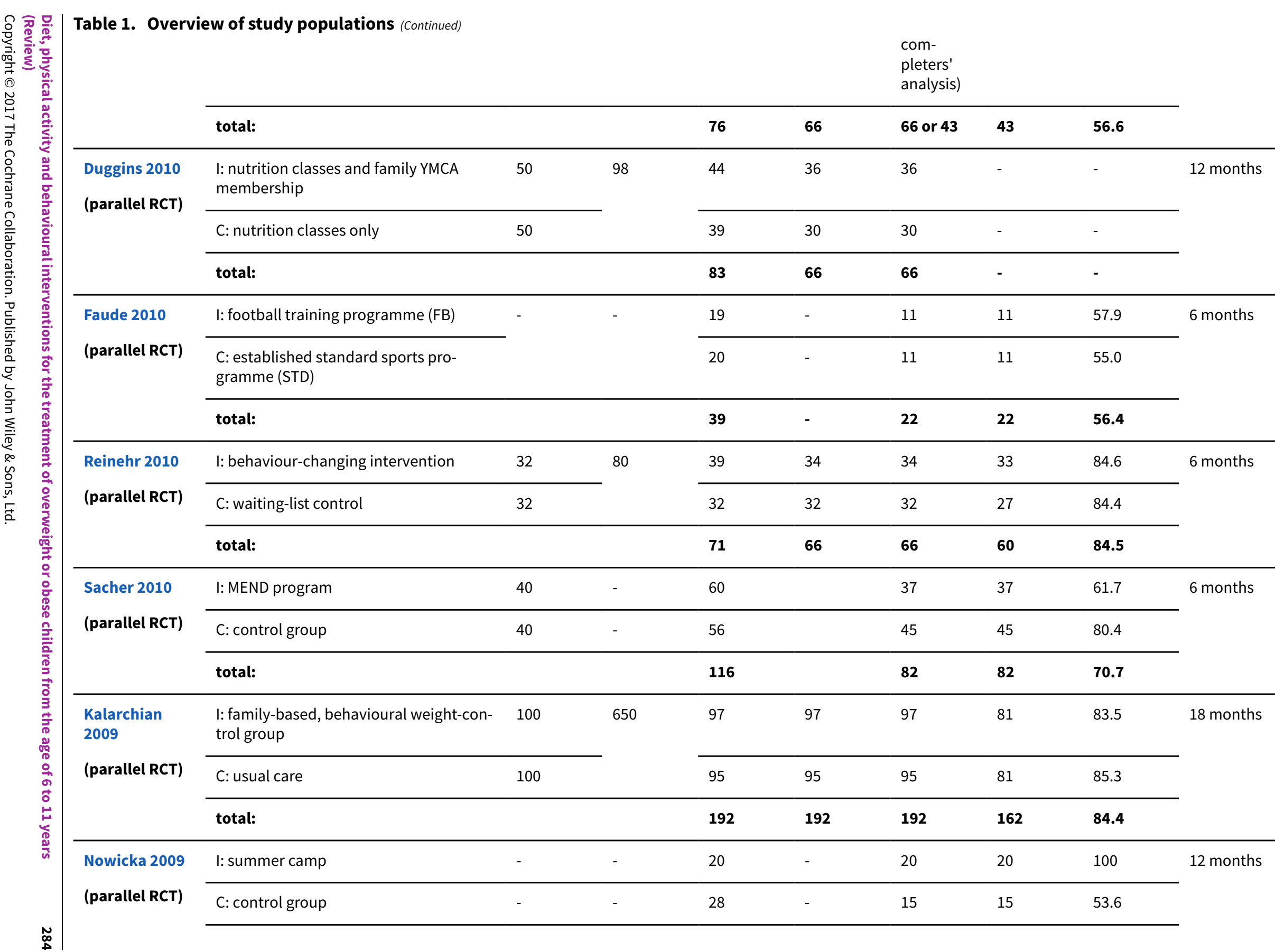




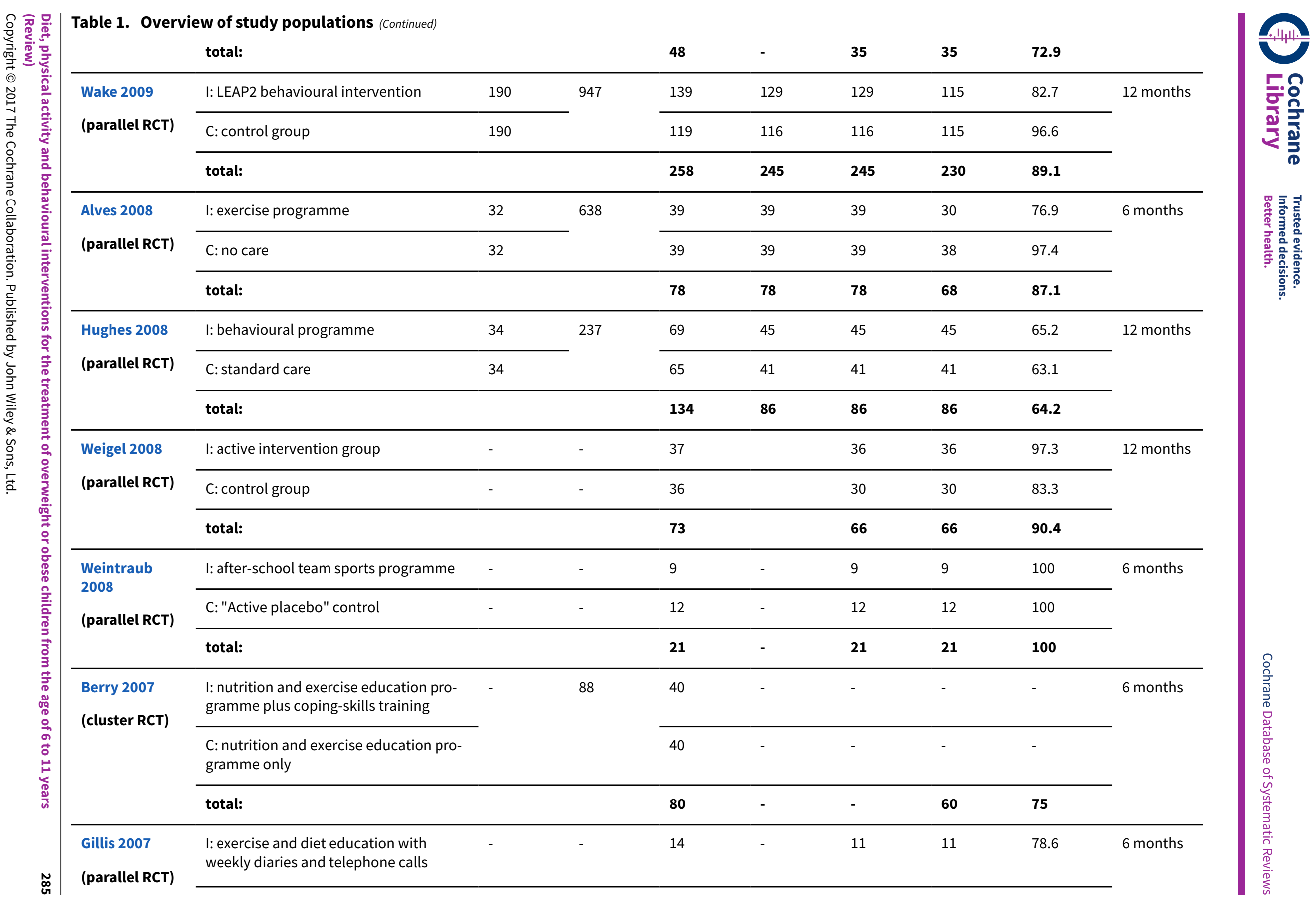




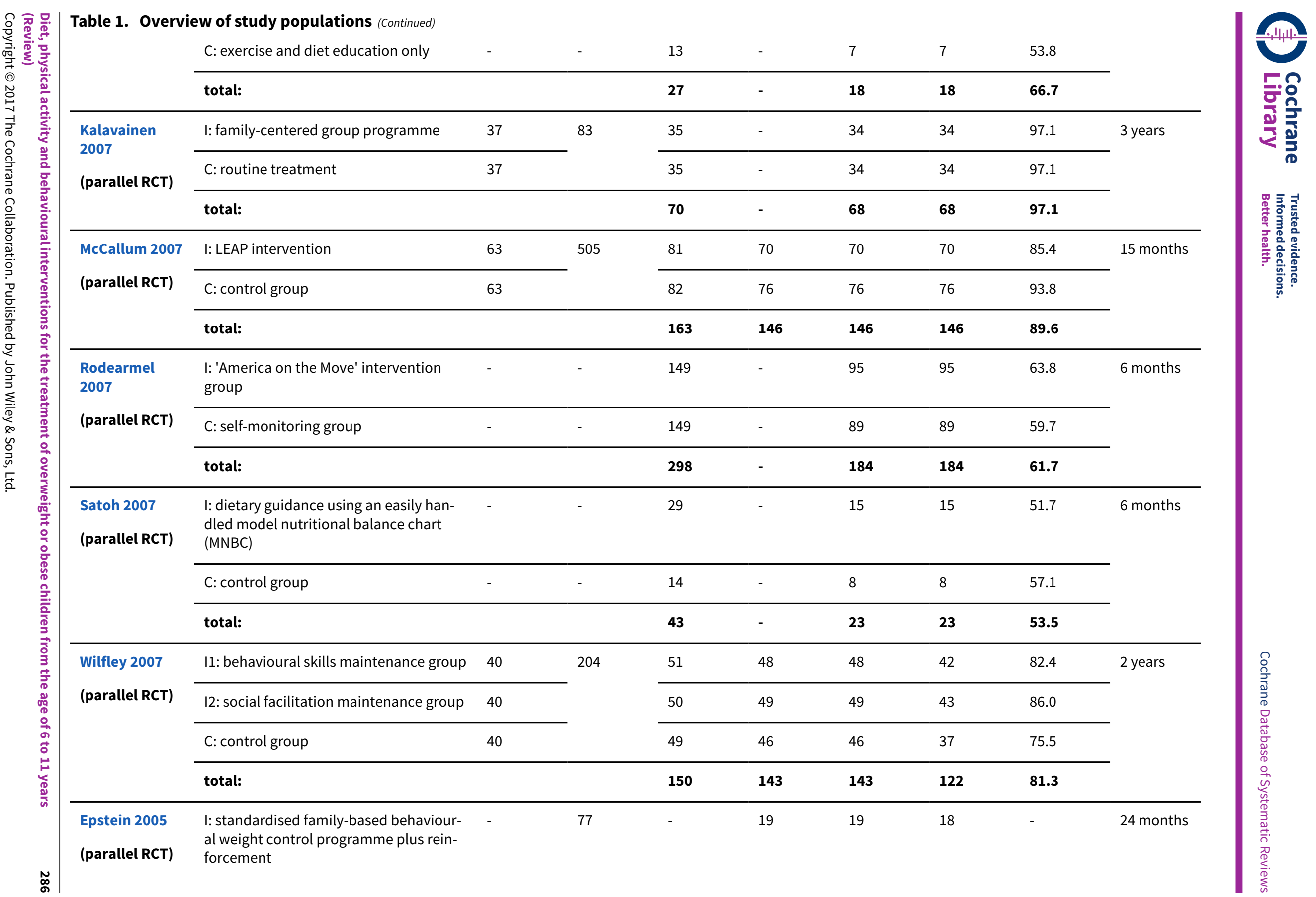




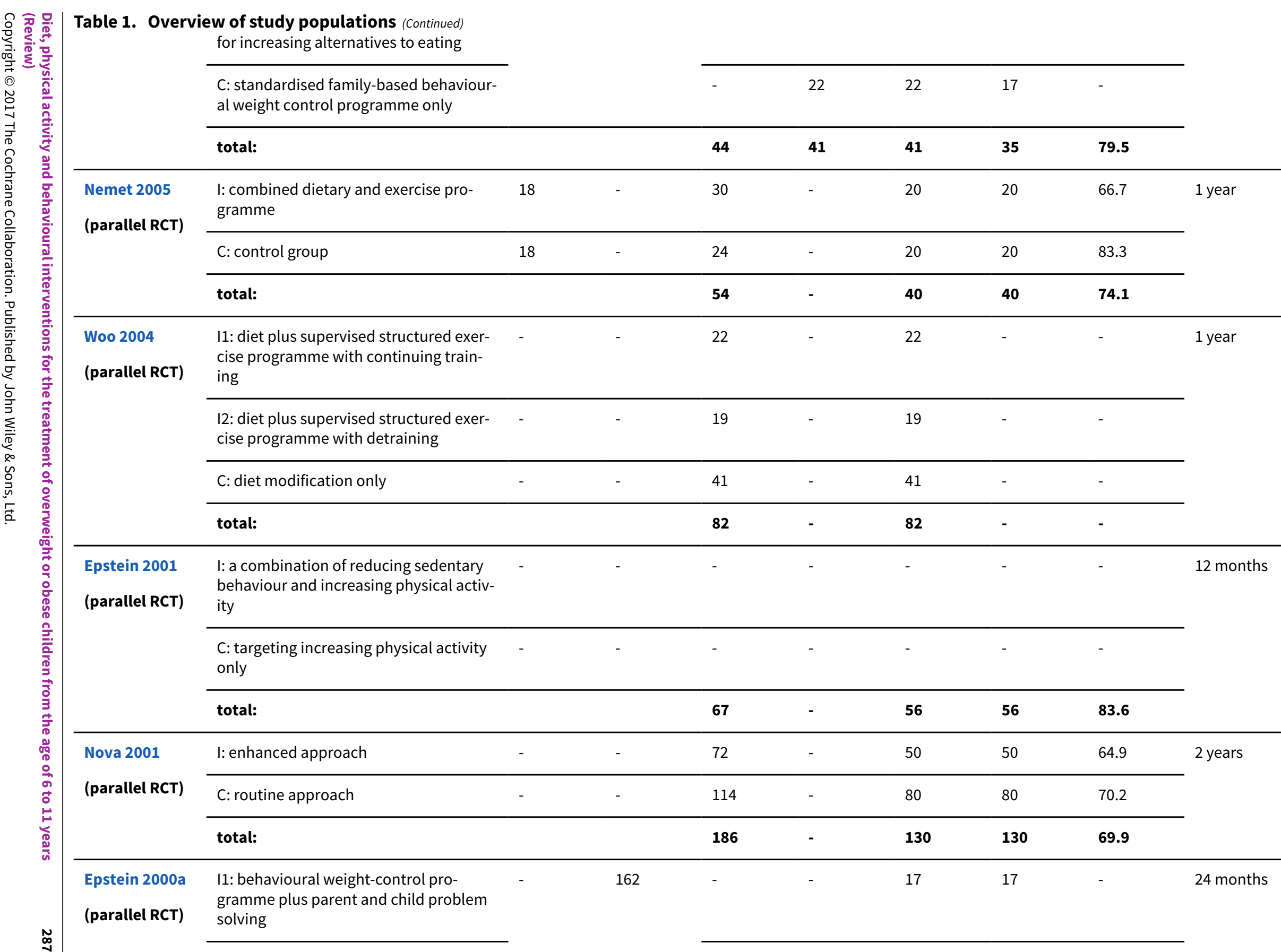




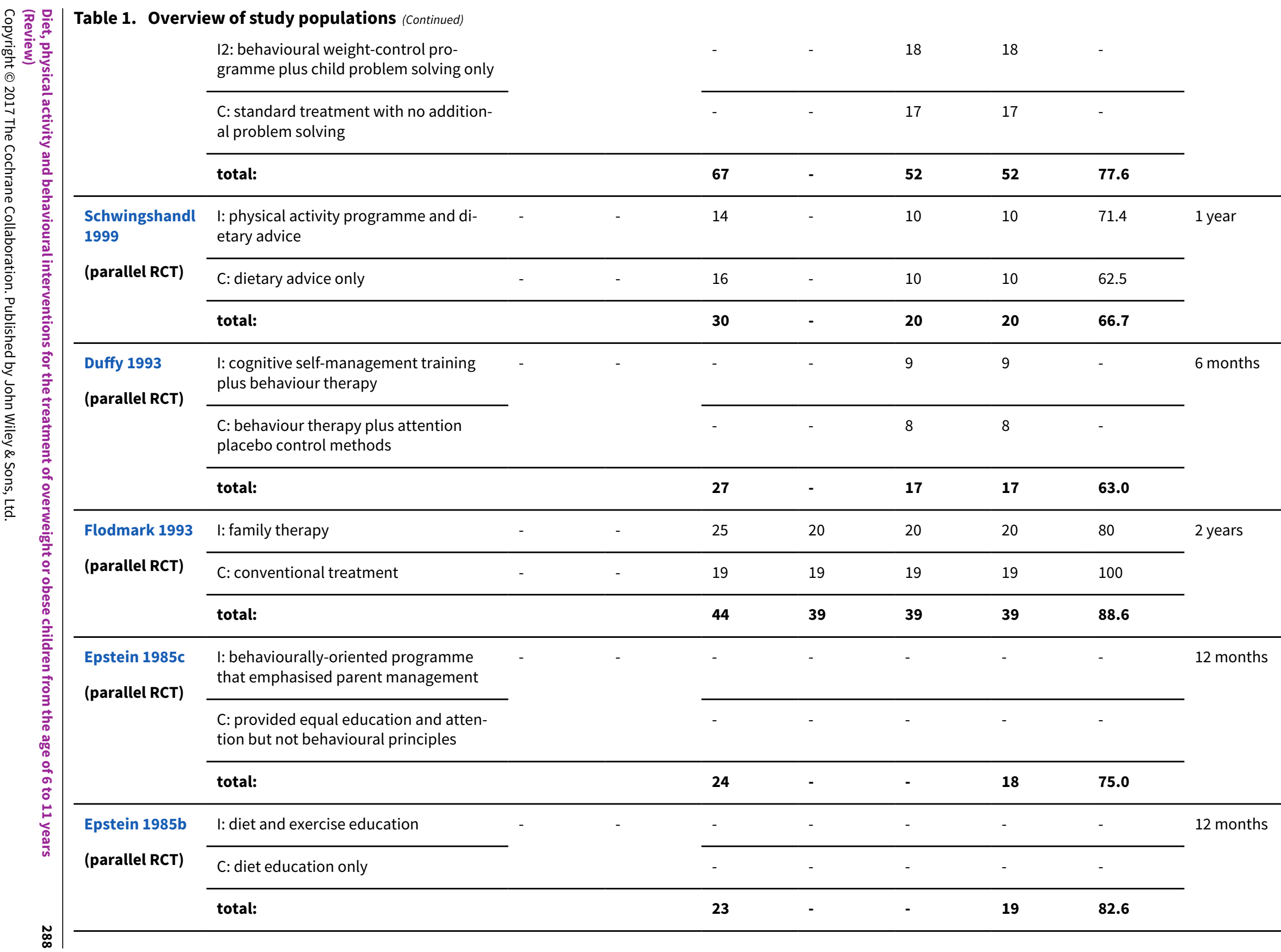




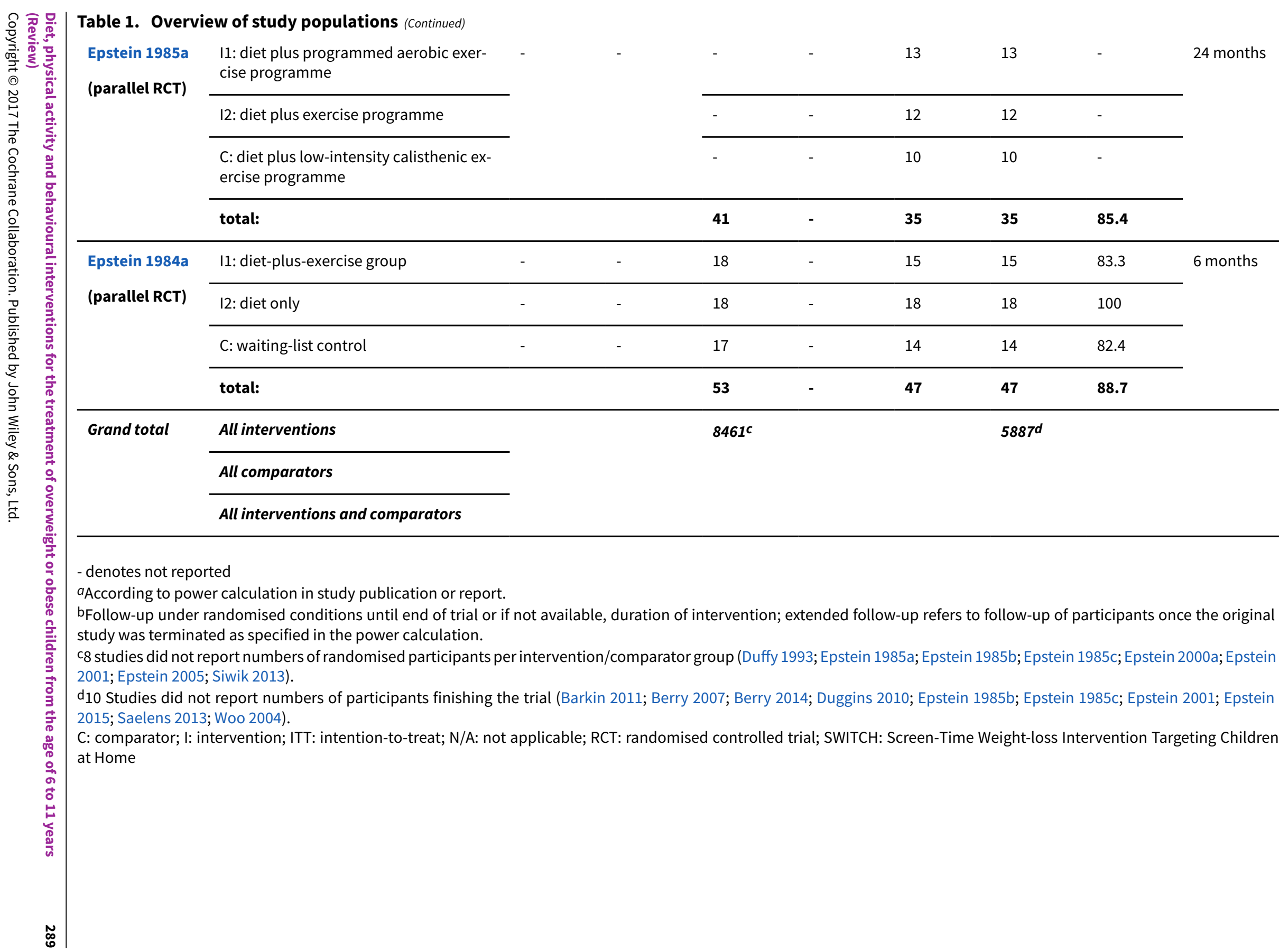

${ }^{a}$ According to power calculation in study publication or report.

-up under randomised conditions until end of trator if not available, duration of intervention; extended follow-up refers to follow-up of participants once the original

c8 studies did not report numbers of randomised participants per intervention/comparator group (Duffy 1993; Epstein 1985a; Epstein 1985b; Epstein 1985c; Epstein 2000a; Epstein d 10 Studies did not report numbers of participants finishing the trial (Barkin 2011; Berry 2007; Berry 2014; Duggins 2010; Epstein 1985b; Epstein 1985c; Epstein 2001; Epstein 2015; Saelens 2013; Woo 2004).

C: comparator; I: intervention; ITT: intention-to-treat; N/A: not applicable; RCT: randomised controlled trial; SWITCH: Screen-Time Weight-loss Intervention Targeting Children 
Table 2. Sensitivity analyses

\begin{tabular}{|c|c|c|c|c|c|}
\hline Analysis & $\begin{array}{l}\text { Number of } \\
\text { studies }\end{array}$ & $\begin{array}{l}\text { Number of partici- } \\
\text { pants }\end{array}$ & $\begin{array}{l}\text { Mean difference } \\
(95 \% \mathrm{Cl})\end{array}$ & Chi $^{2}$ (P value) & I2 statistic \\
\hline $\begin{array}{l}\text { Change in BMI (all trials) } \\
\text { Analysis } 1.1\end{array}$ & 24 & $\begin{array}{l}\text { Intervention: } 1422 \\
\text { Comparator: } 1363\end{array}$ & $\begin{array}{l}-0.53(-0.82 \text { to } \\
-0.24)\end{array}$ & $\begin{array}{l}66.49(< \\
0.00001)\end{array}$ & $65 \%$ \\
\hline $\begin{array}{l}\text { Change in BMI (removing stud- } \\
\text { ies with imputed data) }\end{array}$ & 9 & $\begin{array}{l}\text { Intervention: } 653 \\
\text { Comparator: } 646\end{array}$ & $\begin{array}{l}-0.48(-0.83 \text { to } \\
-0.13)\end{array}$ & $\begin{array}{l}33.87(< \\
0.0001)\end{array}$ & $76 \%$ \\
\hline $\begin{array}{l}\text { Change in BMI z score (all tri- } \\
\text { als) Analysis } 1.2\end{array}$ & 37 & $\begin{array}{l}\text { Intervention: } 2054 \\
\text { Comparator: } 1965\end{array}$ & $\begin{array}{l}-0.06(-0.10 \text { to } \\
-0.02)\end{array}$ & $\begin{array}{l}82.44(< \\
0.0001)\end{array}$ & $56 \%$ \\
\hline $\begin{array}{l}\text { Change in BMI z score (remov- } \\
\text { ing studies with imputed data) }\end{array}$ & 15 & $\begin{array}{l}\text { Intervention: } 800 \\
\text { Comparator: } 791\end{array}$ & $\begin{array}{l}-0.05(-0.10 \text { to } \\
0.00)\end{array}$ & $\begin{array}{l}41.49 \\
(0.0001)\end{array}$ & $66 \%$ \\
\hline $\begin{array}{l}\text { Change in weight (all trials) } \\
\text { Analysis } 1.3\end{array}$ & 17 & $\begin{array}{l}\text { Intervention: } 891 \\
\text { Comparator: } 883\end{array}$ & $\begin{array}{l}-1.45(-1.88 \text { to } \\
-1.02)\end{array}$ & $8.95(0.92)$ & $0 \%$ \\
\hline $\begin{array}{l}\text { Change in weight (removing } \\
\text { studies with imputed data) }\end{array}$ & 8 & $\begin{array}{l}\text { Intervention: } 335 \\
\text { Comparator: } 339\end{array}$ & $\begin{array}{l}-1.54(-1.99 \text { to } \\
-1.09)\end{array}$ & $5.95(0.55)$ & $0 \%$ \\
\hline
\end{tabular}

BMI: body mass index

BMI z score: "A BMI z score or standard deviation score indicates how many units (of the standard deviation) a child's BMI is above or below the average BMI value for their age group and sex. For instance, a $\mathrm{z}$ score of 1.5 indicates that a child is 1.5 standard deviations above the average value, and a z score of -1.5 indicates a child is 1.5 standard deviations below the average value" (NOO NHS 2011)

\section{A P PENDICES}

\section{Appendix 1. Search strategies}

\section{Cochrane Central Register of Controlled Trials (CENTRAL; Cochrane Library)}

\section{Part I: Obesity}

1. [mh^ Obesity]

2. [mh^"Obesity, Morbid"]

3. [mh ^"Obesity, Abdominal"]

4. [mh ^"Pediatric Obesity"]

5. [mh^ ^overweight]

6. [mh^"Weight Loss"]

7. (adipos ${ }^{\star}$ or obes $\left.{ }^{\star}\right): t i, a b$

8. (overweight* or ("over" next weight $\left.\left.{ }^{\star}\right)\right): t i, a b$ 
(Continued)

9. ("weight" near/1 (reduc* or los ${ }^{\star}$ or control ${ }^{\star}$ or manage $\left.\left.{ }^{\star}\right)\right): t i, a b$

10. $\{$ or \#1-\#9\}

\section{Part II: Intervention}

11. [mh "Behavior Therapy"]

12. [mh "Counseling"]

13. [mh^"Family Therapy"]

14. [mh^"Social Support"]

15. [mh^^"Program Evaluation"]

16. [mh "Exercise"]

17. [mh "Exercise Therapy"]

18. [mh "Physical Education and Training"]

19. [mh "Exercise Movement Techniques"]

20. [mh ^"Motor Activity"]

21. [mh Diet]

22. [mh "Diet Therapy"]

23. [mh ^"Patient Education as Topic"]

24. [mh^"Health Education"]

25. [mh "Health Behavior"]

26. [mh "Health Promotion"]

27. [mh^"School Health Services"]

28. [mh^"School Nursing"]

29. [mh^"Life style"]

30. (("obesity" near/4 "intervention") or "program" or "programme" or "camp" or "camps"):ti,ab

31. ("lifestyle" or "life style"):ti,ab

32. exercis*:ti,ab

33. (physic ${ }^{\star}$ next $\left(\right.$ activ $^{\star}$ or fit $\left.\left.{ }^{\star}\right)\right):$ ti,ab

34. (walk* or jog* or swim or ("weight" next lift*) or danc* or "aerobics"):ti,ab

35. ((physic ${ }^{\star}$ or strength ${ }^{\star}$ or resist* or "circuit" or "weight" or aerob* or "cross" or "endurance" or structur $\left.{ }^{\star}\right)$ near/4 train $\left.{ }^{\star}\right): t i, a b$

36. ("behavioral" or "behavioural" or (("behavior" or "behaviour") next "modification") or psychoth* or "psychosocial"):ti,ab

37. (("group" or "family" or cognit* or behav*) next therap*):ti,ab

38. (counseling or counselling):ti,ab

39. educat*:ti,ab

40. (("parent" or "parents" or "family") next ("based" or "focused" or "directed" or "centered" or "only" or "led")):ti,ab

41. (diet* or "healthy nutrition" or (nutrition ${ }^{\star}$ next ("knowledge" or educat ${ }^{\star}$ or therap* or program or intervention $\left.{ }^{\star}\right)$ )):ti,ab

Diet, physical activity and behavioural interventions for the treatment of overweight or obese children from the age of 6 to 11 years 
(Continued)

42. $\{$ or \#11-\#41\}

Part III: Part I + Part II and additional MeSH/subheading combination

43. \#10 and \#42

44. [mh^^obesity] or [mh^"Obesity, Morbid"] or [mh^^Overweight]

45. [mh /DH,PC,RH,TH,PX] [diet therapy or prevention \& control or rehabilitation or therapy or psychology]

46. \#44 and \#45

47. \#43 or \#46

Part IV: Population [adapted from Leclercq 2013 ]

48. [mh ^Adolescent]

49. [mh Child]

50. $\left[\mathrm{mh}^{\wedge} \operatorname{Infant}\right]$

51. [mh ^Pediatrics]

52. "minors":ti,ab

53. ("boy" or "boys" or "boyhood"):ti,ab

54. girl $^{\star}: \mathrm{ti}, \mathrm{ab}$

55. ("kid" or "kids"):ti,ab

56. infant ${ }^{\star}: \mathrm{ti}, \mathrm{ab}$

57. ("baby" or "babies"):ti,ab

58. ("toddler" or "toddlers"):ti,ab

59. ("child" or "childs" or children* or childhood* or childcare* or schoolchild*):ti,ab

60. adolescen*:ti,ab

61. juvenil ${ }^{\star}: \mathrm{ti}, \mathrm{ab}$

62. youth*:ti,ab

63. (teen ${ }^{\star}$ or preteen $\left.{ }^{\star}\right): t i, a b$

64. (underage* or ("under" next age*)):ti,ab

65. pubescen $^{\star}: \mathrm{ti}, \mathrm{ab}$

66. (paediatric ${ }^{\star}$ or pediatric $\left.{ }^{\star}\right): \mathrm{ti}, \mathrm{ab}$

67. $\{$ or \#48-\#66\}

Part V: Part III AND IV and additional MeSH/subheading combination

68. \#47 and \#67

69. [mh^^"Pediatric Obesity"]

70. $[\mathrm{mh} / \mathrm{DH}, \mathrm{PC}, \mathrm{RH}, \mathrm{TH}, \mathrm{PX}]$

71. \#69 and \#70

72. \#68 or \#71

Diet, physical activity and behavioural interventions for the treatment of overweight or obese children from the age of 6 to 11 years 


\section{MEDLINE (Ovid SP)}

\section{Part I: Obesity}

\section{Obesity/}

2. Obesity, Morbid/

3. Obesity, Abdominal/

4. Pediatric Obesity/

5. Overweight/

6. Weight Loss/

7. $\left(\right.$ adipos $^{\star}$ or obes $\left.{ }^{\star}\right)$.tw.

8. (overweight ${ }^{\star}$ or over weight $\left.{ }^{\star}\right)$.tw.

9. (weight adj1 (reduc ${ }^{\star}$ or los ${ }^{\star}$ or control ${ }^{\star}$ or manage $\left.{ }^{\star}\right)$ ).tw.

10. or/1-9

\section{Part II: Intervention}

11. exp Behavior Therapy/

12. exp Counseling/

13. Family Therapy/

14. Social Support/

15. Program Evaluation/

16. exp Exercise/

17. exp Exercise Therapy/

18. exp "Physical Education and Training"/

19. exp Exercise Movement Techniques/

20. Motor Activity/

21. exp Diet/

22. exp Diet Therapy/

23. Patient Education as Topic/

24. Health Education/

25. exp Health Behavior/

26. exp Health Promotion/

27. School Health Services/

28. School Nursing/

29. Life style/

30. ((obesity adj3 intervention) or program or programme or camp?).tw.

31. (lifestyle or life style).tw.

Diet, physical activity and behavioural interventions for the treatment of overweight or obese children from the age of 6 to 11 years 
(Continued)

32. exercis ${ }^{\star}$. tw.

33. (physic ${ }^{\star}$ adj $\left(\right.$ activ $^{\star}$ or fit*) ).tw.

34. (walk* or jog* or swim* or weight lift ${ }^{\star}$ or danc ${ }^{\star}$ or aerobics).tw.

35. ((physic ${ }^{\star}$ or strength* or resist* or circuit or weight or aerob* or cross or endurance or structur $\left.{ }^{\star}\right)$ adj3 train $\left.{ }^{\star}\right)$.tw.

36. (behavio?ral or behavio?r modification or psychoth* or psychosocial).tw.

37. ((group or family or cognit* or behav*) adj therap $\left.{ }^{\star}\right)$.tw.

38. counsel?ing.tw.

39. educat*.tw

40. ((parent? or family) adj (based or focused or directed or centered or only or led)).tw.

41. (diet* or healthy nutrition or (nutrition* adj (knowledge or educat* or therap* or program* or intervention $\left.\left.{ }^{\star}\right)\right)$ ).tw.

42. or $/ 11-41$

Part III: Part I + Part II and additional MeSH/subheading combination

43. 10 and 42

44. Obesity/ or Obesity, Morbid/ or Overweight/ or Weight Loss/

45. diet therapy.fs. or prevention \& control.fs. or rehabilitation.fs. or therapy.fs. or psychology.fs.

46. 44 and 45

47.43 or 46

Part IV: Population [adapted from Leclercq 2013 ]

48. Adolescent/

49. $\exp$ Child/

50. Infant/

51. Pediatrics/

52. minors.tw.

53. (boy or boys or boyhood).tw.

54. girl*.tw.

55. infant*.tw

56. (baby or babies).tw.

57. toddler?.tw.

58. (kid or kids).tw.

59. (child or childs or children ${ }^{\star}$ or childhood ${ }^{\star}$ or childcare ${ }^{\star}$ or schoolchild ${ }^{\star}$ ).tw.

60. adolescen*.tw.

61. juvenil*.tw.

62. youth*.tw.

63. $\left(\right.$ teen $^{\star}$ or preteen $\left.{ }^{\star}\right)$.tw.

Diet, physical activity and behavioural interventions for the treatment of overweight or obese children from the age of 6 to 11 years 

(Continued)
64. (underage ${ }^{\star}$ or under age $e^{\star}$.tw.
65. pubescen*.tw.
66. p?ediatric ${ }^{\star}$. tw.
67. or/48-66

Part V: Part III AND IV and additional MeSH/subheading combination

68.47 and 67

69. Pediatric Obesity/

70. diet therapy.fs. or prevention \& control.fs. or rehabilitation.fs. or therapy.fs. or psychology.fs.

71.69 and 70

72.68 or 71

\section{Part VI: Study filter [Cochrane Handbook 2008 RCT filter - sensitivity and precision maximizing version]}

73. randomized controlled trial.pt.

74. controlled clinical trial.pt.

75. randomi?ed.ab.

76. placebo.ab.

77. clinical trials as topic/

78. randomly.ab.

79. trial.ti.

80. or/73-79

81. exp animals/ not humans/

82. 80 not 81

Part VII: Part V+Part VI

83. 72 and 82

\section{Embase (Ovid SP)}

Part l: Obesity

1. obesity/

2. morbid obesity/

3. abdominal obesity/

4. childhood obesity/

5. weight reduction/

6. weight control/

7. (adipos* or obes $\left.{ }^{\star}\right) . t w$.

8. (overweight ${ }^{\star}$ or over weight*).tw.

9. (weight adj1 (reduc ${ }^{\star}$ or los ${ }^{\star}$ or control* or manage $\left.{ }^{\star}\right)$ ).tw.

Diet, physical activity and behavioural interventions for the treatment of overweight or obese children from the age of 6 to 11 years 
(Continued)

10. or $/ 1-9$

\section{Part II: Intervention}

11. behavior therapy/

12. cognitive therapy/

13. exp counseling/

14. family therapy/

15. social support/

16. exp program evaluation/

17. exp exercise/

18. exp physical education/

19. exp physical activity/

20. exp motor activity/

21. training/

22. exp diet/

23. exp diet therapy/

24. nutritional health/

25. child nutrition/

26. feeding behavior/

27. patient education/

28. health promotion/

29. health literacy/

30. nutrition education/

31. health education/

32. school health education/

33. school health service/

34. lifestyle/

35. lifestyle modification/

36. ((obesity adj3 intervention) or program or programme or camp?).tw.

37. (lifestyle or life style).tw.

38. exercis ${ }^{\star} . t w$.

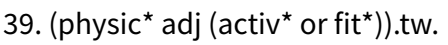

40. (walk* or jog* or swim* or weight lift* or danc ${ }^{\star}$ or aerobics).tw.

41. ((physic ${ }^{\star}$ or strength* or resist* or circuit or weight or aerob* or cross or endurance or structur $\left.{ }^{\star}\right)$ adj3 train $\left.{ }^{\star}\right)$.tw.

42. (behavio?ral or behavio?r modification or psychoth* or psychosocial).tw.

Diet, physical activity and behavioural interventions for the treatment of overweight or obese children from the age of 6 to 11 years 
(Continued)

43. ((group or family or cognit* or behav $\left.{ }^{\star}\right)$ adj therap $\left.{ }^{\star}\right)$.tw.

44. counsel?ing.tw.

45. educat ${ }^{\star}$. tw.

46. ((parent? or family) adj (based or focused or directed or centered or only or led)).tw.

47. (diet* or healthy nutrition or (nutrition* adj (knowledge or educat* or therap* or program* or intervention*))).tw.

48. or/11-47

Part III: Part I + Part II and additional MeSH/subheading combination

49. 10 and 48

50. obesity/ or morbid obesity/

51. pc.fs or rh.fs or th.fs. [prevention.fs. or rehabilitation.fs. or therapy.fs.]

52. 50 and 51

53.49 or 52

Part IV: Population [adapted from Leclercq 2013 ]

54. juvenile/

55. adolescent/

56. child/

57. infant/

58. baby/

59. toddler/

60. preschool child/

61. school child/

62. pediatrics/

63. minors.tw

64. (boy or boys or boyhood).tw.

65. girl ${ }^{\star} . t w$.

66. infant*.tw.

67. (baby or babies).tw.

68. toddler?.tw.

69. (kid or kids).tw.

70. (child or childs or children ${ }^{\star}$ or childhood ${ }^{\star}$ or childcare ${ }^{\star}$ or schoolchild ${ }^{\star}$ ).tw.

71. adolescen ${ }^{\star}$.tw.

72. juvenil*.tw

73. youth ${ }^{\star}$.tw.

74. (teen ${ }^{\star}$ or preteen $\left.{ }^{\star}\right)$.tw.

Diet, physical activity and behavioural interventions for the treatment of overweight or obese children from the age of 6 to 11 years 
(Continued)

75. (underage $e^{\star}$ or under age $e^{\star}$ ).tw.

76. pubescen*.tw.

77. p?ediatric ${ }^{\star}$.tw.

78. or/54-77

Part V: Part III AND IV and additional MeSH/subheading combination

79.53 and 78

80. childhood obesity/

81. pc.fs or rh.fs or th.fs. [prevention.fs. or rehabilitation.fs. or therapy.fs.]

82. 80 and 81

83.79 or 82

Part VI: Study filter [Wong 2006a filter-SDSSGS version]

84. random*.tw. or clinical trial ${ }^{\star}$.mp. or exp treatment outcome/

Part VII: Part V + Part VI

85.83 and 84

\section{PsycINFO (Ovid SP)}

\section{Part I: Obesity}

1. exp Overweight

2. $\left(\right.$ adipos $^{\star}$ or obes $\left.{ }^{\star}\right)$. tw.

3. (overweight ${ }^{\star}$ or over weight*).tw.

4. or/1-3

Part II: Intervention

5. Weight Control/

6. Weight Loss/

7. Aerobic Exercise/

8. Diets/

9. exp Exercise/

10. Movement Therapy/

11. Dance Therapy/

12. exp Physical Activity/

13. Physical Fitness/

14. Health Behavior/

15. Health Promotion/

16. Health Knowledge/

17. Health Literacy/

Diet, physical activity and behavioural interventions for the treatment of overweight or obese children from the age of 6 to 11 years 
(Continued)
18. Health Education/
19. Client Education/

20. Lifestyle/

21. Physical Education/

22. exp Program Evaluation/

23. Educational Programs/

24. Educational Therapy/

25. exp Program Development/

26. School Based Intervention/

27. School Counseling/

28. Counseling/

29. Group Counseling/

30. Family Therapy/

31. Support Groups/

32. Social Support/

33. School Counselors/

34. exp Behavior Modification/

35. Cognitive Behavior Therapy/

36. Cognitive Therapy/

37. ((obesity adj3 intervention) or program or programme or camp?).tw.

38. (lifestyle or life style).tw.

39. exercis*.tw.

40. (physic ${ }^{\star}$ adj (activ ${ }^{\star}$ or fit $\left.\left.{ }^{\star}\right)\right) . t w$.

41. (walk* or jog* or swim* or weight lift* or danc ${ }^{\star}$ or aerobics).tw.

42. ((physic ${ }^{\star}$ or strength* or resist* $^{\star}$ or circuit or weight or aerob* or cross or endurance or structur $\left.{ }^{\star}\right)$ adj3 train $\left.{ }^{\star}\right)$.tw.

43. (behavio?ral or behavio?r modification or psychoth* or psychosocial).tw.

44. ((group or family or cognit ${ }^{\star}$ or behav $\left.{ }^{\star}\right)$ adj therap*).tw.

45. counsel?ing.tw.

46. educat*.tw.

47. ((parent? or family) adj (based or focused or directed or centered or only or led)).tw.

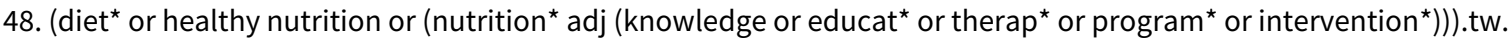

49. or/5-48

Part III: Part I+ Part II

50.4 and 49

Diet, physical activity and behavioural interventions for the treatment of overweight or obese children from the age of 6 to 11 years 
(Continued)

Part IV: Population [adapted from Leclercq 2013 ]

51. minors.tw.

52. (boy or boys or boyhood).tw.

53. girl .tw.

54. infant ${ }^{\star}$. tw

55. (baby or babies).tw.

56. toddler?.tw.

57. (kid or kids).tw.

58. (child or childs or children* or childhood ${ }^{\star}$ or childcare* or schoolchild ${ }^{\star}$ ).tw.

59. adolescen ${ }^{\star}$. tw.

60. juvenil ${ }^{\star} . t w$.

61. youth*.tw.

62. $\left(\right.$ teen $^{\star}$ or preteen $\left.{ }^{\star}\right)$.tw.

63. (underage ${ }^{\star}$ or under age ${ }^{\star}$ ).tw.

64. pubescen $^{\star}$. tw.

65. p?ediatric*.tw.

66. or/51-65

Part V: Part III AND IV and additional MeSH/subheading combination

67.50 and 66

Part VI: Study filter [ Eady 2008 filter - BS version]

68. control ${ }^{\star}$.tw. OR random*.tw. OR exp Treatment/

Part VII: Part V+Part VI

69.67 and 68

\section{CINAHL (EBSCOhost)}

Part l: Obesity

S1. MH "Obesity+"

S2. TX (adipos* or obes $\left.^{\star}\right)$

S3. TX (overweight* or "over weight*")

\section{S4. S1 OR S2 OR S3}

Part II: Intervention

S5. MH "Weight Loss"

S6. MH "Behavior Modification+"

S7. MH "Counseling"

S8. MH "Family Therapy"

Diet, physical activity and behavioural interventions for the treatment of overweight or obese children from the age of 6 to 11 years 
(Continued)

S9. MH "Support, Psychosocial"

S10.MH "Support Groups"

S11.MH "Program Evaluation"

S12.MH "Program Implementation"

S13.MH "Exercise+"

S14.MH "Sports+"

S15.MH "Therapeutic Exercise+"

S16.MH "Physical Fitness"

S17.MH "Physical Education and Training+"

S18.MH "Health Education+"

S19.MH "Diet+"

S20.MH "Diet Therapy+"

S21.MH "Health Behavior"

S22.MH "Eating Behavior"

S23.MH "Health Promotion"

S24.MH "School Health Services+"

S25.MH "Life style changes"

S26.MH "Life style"

S27.TX (weight N1 (reduc* or los ${ }^{\star}$ or control* or manage*))

S28.TX ((obesity N3 intervention) OR program OR programme OR camp\#)

S29.TX (lifestyle or "life style")

S30.TX exercis*

S31.TX (physic* N1 (activ* or fit*))

S32.TX (walk* or jog* or swim* or weight lift* or danc* or aerobics)

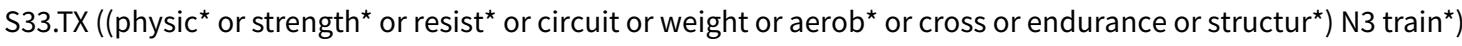

S34.TX (behavio\#ral or behavio\#r modification or psychoth* or psychosocial)

S35.TX ((group or family or cognit* or behav*) N1 therap*)

S36.TX counsel\#ing

S37.TX educat*

S38.TX ((parent\# or family) N1 (based or focused or directed or centered or only or led))

S39.TX (diet* or "healthy nutrition" or (nutrition* N1 (knowledge or educat* or therap* or program * or intervention*))

S40.S5 OR S6 OR S7 OR S8 OR S9 OR S10 OR S11 OR S12 OR S13 OR S14 OR S15 OR S16 OR S17 OR S18 OR S19 OR S20 OR S21 OR S22 OR S23 OR S24 OR S25 OR S26 OR S27 OR S28 OR S29 OR S30 OR S31 OR S32 OR S33 OR S34 OR S35 OR S36 OR S37 OR S38 OR S39

Part III: Part I + Part II and additional MeSH/subheading combination

\section{S41.S4 AND S40}

Diet, physical activity and behavioural interventions for the treatment of overweight or obese children from the age of 6 to 11 years 
(Continued)

S42.(MH "Obesity+/DH/ED/PC/PF/RH/TH") [diet therapy or education or prevention \& control or psychosocial factors or rehabilitation or therapy]

\section{S43.S41 OR S42}

Part IV: Population [based on Leclercq 2013 ]

S44.MH "Adolescence"

S45.MH "Child+"

S46.MH "Infant"

S47.MH "Pediatrics"

S48.TX minors

S49.TX (boy OR boys OR boyhood)

S50.TX girl*

S51.TX infant*

S52.TX (baby OR babies)

S53.TX toddler\#

S54.TX (kid OR kids)

S55.TX (child OR childs OR children* OR childhood* OR childcare* OR schoolchild*)

S56.TX adolescen*

S57.TX juvenil*

S58.TX youth*

S59.TX (teen* or preteen ${ }^{\star}$ )

S60.TX (underage* or under age*)

S61.TX pubescen*

S62.TX (paediatric ${ }^{\star}$ OR pediatric*)

S63.S44 OR S45 OR S46 OR S47 OR S48 OR S49 OR S50 OR S51 OR S52 OR S53 OR S54 OR S55 OR S56 OR S57 OR S58 OR S59 OR S60 OR S62

Part V: Part III AND IV and additional MeSH/subheading combination

\section{S64.S43 AND S63}

S65.(MH "Pediatric Obesity/DH/ED/PC/PF/RH/TH") [diet therapy or education or prevention \& control or psychosocial factors or rehabilitation or therapy]

\section{S66.S64 OR S65}

Part VI: Study filter [Wong 2006b filter - SDSSGS version]

S67.MH "treatment outcomes+" OR MH "experimental studies+" or random*

Part VII: Part V+ Part VI

S68.S66 AND S67

\section{LILACS (IAHx)}

Diet, physical activity and behavioural interventions for the treatment of overweight or obese children from the age of 6 to 11 years 302 (Review)

Copyright @ 2017 The Cochrane Collaboration. Published by John Wiley \& Sons, Ltd. 
(Continued)

((((MH:"Obesity" OR MH:"Obesity, Morbid" OR MH:"Obesity, Abdominal" OR MH:"Pediatric Obesity" OR MH:"Overweight" OR adipos\$ OR obes\$ OR overweight\$ OR "over weight" OR sobrepes\$ OR "exceso de peso" OR "excesso de peso") AND (MH:"Weight Loss" OR MH:"Exercise" OR MH:"Exercise Therapy" OR MH:"Physical Education and Training" OR MH:"Exercise Movement Techniques" OR MH:"Weight Reduction Programs" OR MH:"Motor Activity" OR MH:"Behavior Therapy" OR MH:"Counseling" OR MH:"Family Therapy" OR MH:"Social Support" OR MH:"Program Evaluation" OR MH:"Diet" OR MH:"Diet Therapy" OR MH:"Patient Education as Topic" OR MH:"Health Education" OR MH:"Health Behavior" OR MH:"Health Promotion" OR MH:"Weight Reduction Programs" OR MH:"School Health Services" OR MH:"Life style" OR exerci\$ OR ejerci\$ OR ((physic\$ OR fisic\$) AND (activ\$ OR ativid\$ OR fit\$ OR educac\$ OR entrenam\$ OR treinam\$)) OR ((physic\$ OR fisic\$ OR strength\$ OR forca OR fuerza OR resist\$ OR circuit\$ OR weight OR aerob\$ OR endurance OR structur OR estructur\$) AND train\$ OR treina\$ OR entrena\$) OR program\$ OR "estilo de vida" OR padres OR pais OR familia OR familias OR familiar OR terapia OR orienta\$ OR educa\$ OR diet\$ OR nutric\$ OR "weight reduction" OR "weight loss" OR "weight control" OR "control de peso")) OR (MH:"Obesity/diet therapy" OR MH:"Obesity, Morbid/diet therapy" OR MH:"Overweight/diet therapy" OR MH:"Obesity/prevention \& control" OR MH:"Obesity, Morbid/prevention \& control " OR MH:"Overweight/prevention \& control" OR MH:"Obesity/rehabilitation" OR MH:"Obesity, Morbid/rehabilitation" OR MH:"Overweight/rehabilitation" OR MH:"Obesity/therapy" OR MH:"Obesity, Morbid/therapy" OR MH:"Overweight/therapy" OR MH:"Obesity/psychology" OR MH:"Obesity, Morbid/psychology" OR MH:"Overweight/psychology")) AND (MH:"Adolescent" OR MH:"Child" OR MH:"Pediatrics" OR MH:"Infant" OR minors OR boy OR boys OR girl\$ OR kid OR kids OR child OR childs OR children\$ OR childhood\$ OR childcare\$ OR schoolchild\$ OR escolar\$ OR adolescen\$ OR preadolescen\$ OR juvenil\$ OR juventud\$ OR youth\$ OR teen\$ OR preteen\$ OR underage\$ OR pubescen\$ OR paediatri\$ OR pediatri\$ OR joven $\$$ OR jovem\$ OR niños OR niñas OR crianca\$ OR menin\$ OR "menor de edad" OR "menores de edad" OR "menor de idade" OR "menores de idade")) OR MH:"Pediatric Obesity/diet therapy" OR MH:"Pediatric Obesity/prevention \& control" OR MH:"Pediatric Obesity/rehabilitation" OR MH:"Pediatric Obesity/therapy" OR MH:"Pediatric Obesity/psychology"

[activated filter "Controlled Clinical Trial"]

\section{ICTRP Search Portal (advanced search)}

[activated "Search for clinical trials in children"]:

in Title: obes* OR overweight*

$\mathrm{OR}$

in Condition: obes* OR overweight*

Recruitment Status: ALL

\section{ClinicalTrials.gov (advanced search)}

Conditions: obese OR overweight OR obesity

Study type: Interventional Studies

Age Group: Child (birth-17)

\section{Appendix 2. Description of interventions}

\section{Intervention(s) (route, frequency, total dose/day)}

NCT02436330

\author{
Exergaming and didactic healthy teaching
} ergaming and $1 \mathrm{~h}$ of didactic classes teaching behavioural and dietary curricula). Followed by monthly 1-h maintenance didactic teaching for 6-month period)
(6 months of 10 weekly 2 - $\mathrm{h}$ sessions ( $1 \mathrm{~h}$ of ex-

\begin{tabular}{lll}
$\begin{array}{l}\text { Adequatea in- } \\
\text { tervention (Yes/ } \\
\text { No) }\end{array}$ & $\begin{array}{l}\text { Comparator(s) } \\
\text { (route, frequency, } \\
\text { total dose/day) }\end{array}$ & $\begin{array}{l}\text { Adequatea com- } \\
\text { parator (Yes/No) }\end{array}$ \\
\hline N/A & $\begin{array}{l}\text { Didactic healthy } \\
\text { teaching }\end{array}$ & Yes \\
& $\begin{array}{l}\text { (6 months of 10 } \\
\text { weekly 1-h sessions } \\
\text { of didactic classes } \\
\text { teaching behavioural } \\
\text { and dietary curricula. }\end{array}$ \\
\end{tabular}

Diet, physical activity and behavioural interventions for the treatment of overweight or obese children from the age of 6 to 11 years (Review)

Copyright $\odot 2017$ The Cochrane Collaboration. Published by John Wiley \& Sons, Ltd. 
Followed by monthly 1 -h didactic health for 6-month period)

Ho 2016 Standard nutrition counselling plus portion
control equipment $(1 \times 1 \mathrm{~h}$ nutrition counselling with dietician plus $10-15$ counselling on using a calibrated dinner plate and breakfast cereal bowl to assist with portion control. 6 monthly phone calls to assess compliance and dietician recommendations)

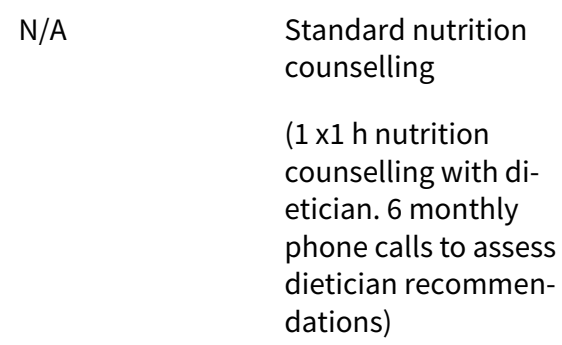

Parental CBT training group plus child in-pa$\mathrm{N} / \mathrm{A}$ tient intervention
Parental informa- tion-only group plus child in-patient inter- vention
(Inpatient treatment for children
Parents only re- ceived brief writ- ten guide after child completed inpatient stay

Yes (Inpatient treatment for children only for 3-6 weeks Involved behaviour-changing intervention: nutrition education, diet modification, CBT

Parental group - $2 \mathrm{~d}$ CBT training e.g. self-monitoring, stimulus control ter child completed intervention)

Follow-up telephone interview 3 months later)

\begin{tabular}{|c|c|c|c|c|}
\hline Epstein 2015 & $\begin{array}{l}\text { Family-based treatment + variety of high ener- } \\
\text { gy-dense foods } \\
\text { ( } 12 \text { weekly meetings, } 2 \text { biweekly then } 1 \text { month- } \\
\text { ly ( } 25 \text { weeks in total). Separate large group } \\
\text { meetings for parents and children ( } 50-60 \text { min) } \\
\text { then small group counselling ( } 20-30 \text { min). Traf- } \\
\text { fic light diet, } \leq 2 \text { red foods/ } d \text { (chose } 2 \text { red foods } \\
\text { to target monthly). Given activity advice ( } 60 \\
\text { min/d MVPA). Behavioural treatment (self- } \\
\text { monitoring, positive reinforcement)) }\end{array}$ & $\mathrm{N} / \mathrm{A}$ & $\begin{array}{l}\text { Family-based treat- } \\
\text { ment only } \\
\text { (Same intervention } \\
\text { but not required to } \\
\text { participate in the } \\
\text { variety meal plan } \\
\text { choosing } 2 \text { red foods } \\
\text { monthly) }\end{array}$ & Yes \\
\hline Larsen 2015 & $\begin{array}{l}\text { An education programme in addition to health } \\
\text { consultations } \\
\text { (Monthly consultations in general practice } \\
\text { (year } 1 \text { ), every two months in year } 2 \text {. Also re- } \\
\text { ceived } 3 \text { educational programmes ( } 3 \text { h each) in } \\
\text { groups. Led by dietitian, physical exercise in- } \\
\text { structor and psychologist } \\
\text { Promote healthy lifestyles and inspire enjoy- } \\
\text { able activities + healthy diet } \\
\text { Aimed at families - no behavioural compo- } \\
\text { nent) }\end{array}$ & $\mathrm{N} / \mathrm{A}$ & $\begin{array}{l}\text { Health consultations } \\
\text { only } \\
\text { (Families received } \\
\text { health consultations } \\
\text { in general practices } \\
\text { but did not receive } \\
\text { the } 3 \text { educational } \\
\text { sessions) }\end{array}$ & Yes \\
\hline Serra-Paya 2015 & Nereu programme & $\mathrm{N} / \mathrm{A}$ & Counselling group & Yes \\
\hline
\end{tabular}


(Supervised PA sessions for children (3 per week, 8 months). Family theoretical and practical sessions for parents (once per week, $60 \mathrm{~min}$ each) - families could share experiences and commitments. Behaviour strategy sessions for family: increase PA, improve eating habits. Weekend activities - e.g. ski/water park party, 3 weekends)
(8 monthly, 10 min sessions with family. Delivered by child's paediatrician. Aim to increase PA and learn healthy behaviours)

Taveras 2015 I1: computerised point-of-care alerts plus di-
rect-to-parent outreach and support.
(Visits with paediatrician focusing on individual
and family-level behaviours. Include decrease
sugar, increase MVPA, improve sleep, reduce
screen time. Computerised CDS system alerts
included growth charts, guidelines, document-
ing BMI percentile + behaviours, referrals. Al-
so families assigned to health coach who used
motivational interviewing by telephone at 1, 3,
6 and 9 months - also took part in text message
service)

12: computerised point-of-care alerts only

N/A

(Same as above but did not receive the motivational interviewing or texts)

N/A
Usual care

Yes

(received the current standard of care offered by their paediatric office. This included well child visits and follow-up appointments for weight checks with their primary care provider, subspecialist, or a nutritionist. They also received generic health-related materials in the mail from the study team. Clinicians in the usual care arm did not have access to the computerised point-of-care alerts for the duration of the intervention.)

Taylor $2015 \quad$ Tailored package family-based intervention
(families attended for a single multidisciplinary
session with a consultant for 1-2 h to develop
specific goals suitable for each family. This ini-
tial session was followed by regular sessions
with a mentor to discuss progress and pro-
vide support. Mentor meetings were month-
ly in year 1 and every 3 months in year 2 (al-
ternating between face-to-face at Universi-
ty/or at home (30-40 min) or phone calls (5-10
min)). Individual goals were negotiated and re-
sources provided to families. Behavioural tar-
gets included parenting, dietary intake and PA.
Length = 2 years)

Usual care

(met with trained researcher at baseline and 6 months - first appointment (30-45 $\mathrm{min}$ ) the parent received individualised feedback about their child's diet and activity habits. Generalised guidance was then provided. At the second appointment the progress was reviewed and additional support provided (15-30 min))

\begin{tabular}{|c|c|c|c|c|}
\hline Berry 2014 & $\begin{array}{l}\text { Nutrition and exercise education and coping } \\
\text { skills intervention } \\
\text { (Phase } 1 \text { - } 60 \text { min education + } 45 \text { min exercise } \\
\text { once per week, } 12 \text { weeks. Phase } 2 \text { - sessions } \\
\text { once per month for } 9 \text { months. Behavioural } \\
\text { component - coping skills, cognitive restruc- } \\
\text { turing, problem solving. }\end{array}$ & $\mathrm{N} / \mathrm{A}$ & $\begin{array}{l}\text { Waiting-list control, } \\
\text { usual care } \\
\text { (Usual care for } 18 \\
\text { months then given } \\
\text { intervention) }\end{array}$ & Yes \\
\hline
\end{tabular}

Diet, physical activity and behavioural interventions for the treatment of overweight or obese children from the age of 6 to 11 years 
Diet + exercise advice - moderate intensity exercise, portion control, calories. Aimed at child and parent, led by dietitian/nurse practitioner + exercise trainer)

$\begin{array}{ll}\text { Boutelle } 2014 & \text { Regulation of Cues (ROC) programme } \\ \text { (12 sessions over } 12 \text { weeks then } 2 \text { biweekly v } & \text { its (45 min each) } \\ & \text { Child + parent sessions by psychologists, co- } \\ \text { therapists and undergraduates. Psychoed- } \\ \text { ucation, parenting skills, self-monitoring of } \\ \text { hunger, overeating. No PA component) }\end{array}$

\section{Hamilton-Shield Standard care plus Mandolean training} 2014

(5 standard care sessions (every 3 months for 12 months), 3 supportive telephone calls were provided to help participants to engage in behaviours discussed in the face-to-face sessions. Eatwell plate, nutrition goals, activity $60 \mathrm{~min} / \mathrm{d}$, motivational interviewing techniques. Families were encouraged to set their own dietary goals and targets, with practical advice and guidance from the practice nurse. Mandolean therapy - 4 sessions with the nurse therapist over the first 2 months, in addition to standard care appointments. Mandolean portable weighing scales measure portion size + eating speed)
I1: newsletter and growth-monitoring plus behavioural counselling

(Newsletter monthly (6 months) - PA and nutrition advice. Received growth monitoring materials (e.g. diary, BMI wheel, scale)

Behavioural counselling $3 \times 30 \mathrm{~min}+3 \times 20 \mathrm{~min}$ $\left(2 \frac{1}{2} h\right)$

Self-monitoring, modelling, stimulus control, positive reinforcement MVPA $>60 \mathrm{~min}$, reduce $\mathrm{TV}<2 \mathrm{~h} / \mathrm{d}$, reduce sugar, increase fruit + vegetables)

12: newsletter and growth monitoring

(Monthly contact ( $3 \times 15 \mathrm{~min}$ in person and $3 \mathrm{x}$ 10 phone calls $-1 \mathrm{~h} 15$ total). No behavioural counselling)

N/A

$\begin{array}{ll}\text { N/A } & \begin{array}{l}\text { Usual care control } \\ \text { group }\end{array}\end{array}$

(No care for 4 months then received a binder with treatment materials included)

$\begin{array}{ll}\text { N/A } & \text { Standard care only } \quad \text { Yes } \\ & \text { (No Mandolean train- } \\ & \text { ing given) }\end{array}$

Newsletter only

(No contact - only received monthly newsletter)

Yes

SWITCH intervention group

N/A

Usual care control

Yes

(Face-to-face meetings over 20 weeks and monthly newsletter + website. Based on social cognitive theory: praise, positive reinforcement etc. Decrease sedentary behaviours - TV monitoring device (30 token for $30 \mathrm{~min}$ ). Children group

given activity pack for non-screen activities e.g.

(Given access to the website but no other contact) 
(Continued)

tennis ball, cards. Child and parent involved -

no nutritional component)

\begin{tabular}{ll}
\hline Markert 2014 & $\begin{array}{l}\text { Telephone based adiposity prevention for fam- } \\
\text { ilies (TAFF) }\end{array}$ \\
& (14 calls every 3-4 weeks (plus 2 optional \\
& coaching sessions), 20-30 min each, Newsletter \\
& (14 issues) over 1 year. Based on family thera- \\
py approaches + solution focused systematic & therapy. Newsletter - psychological support, \\
& stress, diet behaviour, PA)
\end{tabular}

Arauz Boudreau Behaviour-changing intervention and coaching N/A

2013 on behaviour changing

( 5 power-up sessions over 5 weeks ( $1.5 \mathrm{~h}$ each) - 1 session 3 months later. Children and parents involved in sessions. Interactive games and activities (e.g. indoor jump rope). Topics included portion control, healthy snacking, TV viewing. Led by health educator, physical therapist, nutritionist, paediatrician. Health coaching at least once, then follow-ups during 6 months. Focus on social barriers and goal setting

Behavioural, diet and PA components)

\begin{tabular}{|c|c|c|c|c|}
\hline Davis 2013 & $\begin{array}{l}\text { Telemedicine intervention } \\
\text { ( } 8 \text { weekly groups over Telemedicine ( } 1 \text { h each) } \\
\text { then monthly for } 6 \text { months. Parents and chil- } \\
\text { dren taught separately and met at end for goal } \\
\text { setting. Topics included behaviour modifica- } \\
\text { tion (e.g. goal setting), activity monitoring, } \\
\text { Stop Light Diet, nutritional recommendations } \\
\text { Led by psychologists or graduate stu- } \\
\text { dents/postdoctoral fellows) }\end{array}$ & $\mathrm{N} / \mathrm{A}$ & $\begin{array}{l}\text { Physician-visit inter- } \\
\text { vention } \\
\text { (One visit with a pri- } \\
\text { mary care physician } \\
\text { to talk about a list of } \\
\text { topics e.g. exercise) }\end{array}$ & Yes \\
\hline Davoli 2013 & $\begin{array}{l}\text { Family paediatrician-led motivational inter- } \\
\text { viewing } \\
\text { ( } 5 \text { individual meetings over } 12 \text { months for child } \\
\text { and parent. Family paediatrician-led motiva- } \\
\text { tional interviewing based on the transtheoreti- } \\
\text { cal model of addiction and behaviour change. } \\
\text { The child and parents agreed on } 2 \text { objectives at } \\
\text { each meeting ( } 1 \text { concerning dietary improve- } \\
\text { ments and } 1 \text { concerning PA improvements) } \\
\text { that were clearly defined and achievable. Dur- } \\
\text { ing each subsequent meeting, the degree of } \\
\text { achievement of the objectives set at the previ- } \\
\text { ous meeting was assessed; the objectives were } \\
\text { then reinforced or redefined and recorded ac- } \\
\text { cordingly.) }\end{array}$ & $\mathrm{N} / \mathrm{A}$ & $\begin{array}{l}\text { Usual care plus a } \\
\text { booklet on obesity } \\
\text { prevention } \\
\text { (Received a booklet } \\
\text { about obesity pre- } \\
\text { vention and usual } \\
\text { care from a paedia- } \\
\text { trician) }\end{array}$ & Yes \\
\hline Lochrie 2013 & $\begin{array}{l}\text { Family-based intervention } \\
\text { ( } 8 \text { weekly sessions, } 4 \text { bimonthly then } 2 \text { monthly } \\
\text { (60-90 min) - } 6 \text { months }\end{array}$ & $\mathrm{N} / \mathrm{A}$ & $\begin{array}{l}\text { Education session } \\
\text { (One 1-h group ses- } \\
\text { sion led by a dietit- }\end{array}$ & Yes \\
\hline
\end{tabular}

Diet, physical activity and behavioural interventions for the treatment of overweight or obese children from the age of 6 to 11 years 
Outpatient group sessions by a psychologist and dietitian (child + parent). Nutrition, behaviour modification, psychosocial intervention, exercise. Applied maintenance sessions integrated participants in the community. Goal setting and nutrition topics reinforced at each session) ian. General recommendations on PA and nutrition

No behaviour change or psychosocial strategies or techniques)

\begin{tabular}{|c|c|c|c|}
\hline Mirza 2013 & $\begin{array}{l}\text { Low-glycemic load dietary group } \\
\text { (Nutrition sessions: } 12 \text { weekly group sessions, } \\
\text { separate for parents + children. Plus weekly } \\
\text { family session - met with child + parent individ- } \\
\text { ually. Behaviour changes - self-monitoring, so- } \\
\text { cial reinforcement, contingency. Parents giv- } \\
\text { en parenting classes to target diet + activity be- } \\
\text { haviours. Increase PA, reduce sedentary behav- } \\
\text { iour. }\end{array}$ & $\mathrm{N} / \mathrm{A}$ & $\begin{array}{l}\text { Conventional low-fat } \\
\text { dietary group } \\
\text { (Diet advice based } \\
\text { on low fat diet in- } \\
\text { stead. Limit fat and } \\
\text { increase grains. } \\
\text { Other components } \\
\text { were the same) }\end{array}$ \\
\hline
\end{tabular}

Lower glycaemic load, replace carbohydrates with protein + fat - given recipes)

\begin{tabular}{|c|c|}
\hline \multirow[t]{3}{*}{ O'Connor 2013} & $\begin{array}{l}\text { "Helping Hand" obesity intervention } \\
\text { (6 sessions, once per month (follow-up } 2 \text { weeks } \\
\text { after each session) }\end{array}$ \\
\hline & $\begin{array}{l}\text { Behaviour selected each month (e.g. be more } \\
\text { active, eat more fruit) }\end{array}$ \\
\hline & $\begin{array}{l}\text { Goals set and behaviour monitored - parents } \\
\text { completed worksheets) }\end{array}$ \\
\hline
\end{tabular}
doctor (usual paediatric care). Offered intervention at 7 months)

(20 sessions over $21 / 22$ weeks (20-30 min individual family, 40-50 min separate child + parents groups). Skills: Food monitoring, contingency management, environmental control etc. Given more autonomy in making choices about skills to use, self-efficacy. Develop tailored realistic and meaningful goals. Increase exercise, decrease sedentary activities, Stoplight Eating Plan

\begin{tabular}{|c|c|c|c|c|}
\hline Siwik 2013 & $\begin{array}{l}\text { "Choices" group office-visit intervention } \\
\text { (12 weekly individual and group check-ins } \\
\text { (child and parent). Reunions at 3-4 and 6-8 } \\
\text { months after. Motivational interviewing, re- } \\
\text { ceived certificate of accomplishment if goals } \\
\text { met. Set goals to increase PA - promoted ac- } \\
\text { tivities (e.g. dance, soccer). Increase water, de- } \\
\text { crease sugar, portion control, decrease fast } \\
\text { foods) }\end{array}$ & $\mathrm{N} / \mathrm{A}$ & $\begin{array}{l}\text { C: lagged control } \\
\text { group } \\
\text { (Given intervention } \\
\text { at } 6 \text { months) }\end{array}$ & Yes \\
\hline Vann 2013 & $\begin{array}{l}\text { I1: pedometer + DVD group } \\
\text { (Given a pedometer (goal of } 10,000 \text { steps dai- } \\
\text { ly) + age-appropriate fitness DVD. Involved in a }\end{array}$ & $\mathrm{N} / \mathrm{A}$ & $\begin{array}{l}\text { Usual care } \\
\text { (Not given a fitness } \\
\text { DVD or pedometer }\end{array}$ & Yes \\
\hline
\end{tabular}

Diet, physical activity and behavioural interventions for the treatment of overweight or obese children from the age of 6 to 11 years 
(Continued)

weight-management programme - no behavioural component. Nutrition advice given and also encouraged to use a Xbox-Kinect in the clinic) but still involved in weight-management programme)
12: pedometer group

(As above but no fitness DVD)

13: fitness DVD group

(As above but no pedometer)

\begin{tabular}{ll}
\hline Wake 2013 & $\begin{array}{l}\text { HopSCOTCH (the shared care obesity trial) in- } \\
\text { tervention }\end{array}$ \\
& (One initial appointment with obesity spe- \\
& cialist consultant. Then 11 GP consultations \\
& over 15 months (15-30 min each). Weight man- \\
& agement counselling, goal setting, tracking \\
& progress. Advice on healthy eating by a dietit- \\
& ian, followed up by GP. PA and sedentary be- \\
& haviour advice followed up by GP)
\end{tabular}

Croker $2012 \quad$ Family-based behavioural treatment (FBBT)

(12 sessions ( $1.5 \mathrm{~h}$ each) plus 3 maintenance sessions - over 6 months. Child sessions run by dietitian and parent sessions by a clinician. Based on learning theory and behaviour-modification techniques. Encouraged to reduce sedentary behaviours, increase activity. Used the traffic light system and Eatwell Plate)

\begin{tabular}{|c|c|c|c|c|}
\hline de Niet 2012 & $\begin{array}{l}\text { Short message service maintenance treatment } \\
\text { and behaviour-changing treatment } \\
\text { ( } 3 \text { months of behaviour-changing treatment ( } 8 \\
\text { sessions) before randomisation. Then sessions } \\
\text { at } 6,9 \text { and } 12 \text { months - behavioural-modifica- } \\
\text { tion techniques. SMSMT - self-monitoring and } \\
\text { feedback weekly } \\
\text { Nutrition and PA self-monitoring and advice } \\
\text { given) }\end{array}$ & $\mathrm{N} / \mathrm{A}$ & $\begin{array}{l}\text { Behaviour-changing } \\
\text { treatment only } \\
\text { (Received behaviour } \\
\text { changing treatment } \\
\text { but no SMSMT) }\end{array}$ & Yes \\
\hline Eddy Ives 2012 & $\begin{array}{l}\text { Dietary and physical exercise recommenda- } \\
\text { tions during } 6 \text { sessions } \\
\text { (At baseline the child and parents/tutor re- } \\
\text { ceived dietary and physical exercise recom- } \\
\text { mendations which were then also provided at } \\
1,3,6,9 \text { and } 12 \text { months. Included increasing } \\
\text { exercise to } 45 \text { min daily, reducing TV and com- } \\
\text { puter use, eating three meals/d, eating slowly } \\
\text { and using small plates, eating fruit and vegeta- } \\
\text { bles and monitoring sugar consumption. Ses- } \\
\text { sions led by paediatricians and were 30-45 min } \\
\text { long) }\end{array}$ & $N / A$ & $\begin{array}{l}\text { Dietary and physical } \\
\text { exercise recommen- } \\
\text { dations at } 2 \text { sessions } \\
\text { only } \\
\text { (Received the same } \\
\text { recommendations } \\
\text { as the intervention } \\
\text { group but only at } \\
\text { baseline and } 12 \\
\text { months. Offered the } \\
\text { intervention after } 12 \\
\text { months) }\end{array}$ & Yes \\
\hline
\end{tabular}

Usual care Yes

(No support given but told to visit GP for usual care)

$\begin{array}{ll}\text { N/A } & \text { Waiting-list control Yes } \\ & \text { (No care, then given } \\ & \text { intervention after 6 } \\ & \text { months) }\end{array}$


(Continued)

$\begin{array}{lll}\text { Kirk 2012 } & \begin{array}{l}\text { I1: low carbohydrate diet + group exercise/edu- } \\ \text { cation sessions }\end{array} & \text { N/A } \\ \begin{array}{l}\text { (Biweekly 1-h exercise sessions, 12 weekly par- } \\ \text { ent-child sessions (30-min individual coun- } \\ \text { selling or 90-min group sessions) - over 3 } \\ \text { months. Exercise led by exercise specialist, en- } \\ \begin{array}{l}\text { courage to be active for } \geq 30 \text { min/d. Limit car- } \\ \text { bohydrate intake and increase high protein } \\ \text { foods (measure ketones). No behavioural com- } \\ \text { ponent) }\end{array}\end{array} & \begin{array}{l}\text { tion-controlled diet + } \\ \text { group exercise/edu- } \\ \text { cation sessions }\end{array} \\ & \text { (Same PA sessions. } \\ \begin{array}{l}\text { I2: reduced glycaemic load diet + group exer- } \\ \text { cise/education sessions }\end{array} & \text { N/A } & \begin{array}{l}\text { Diet - consume age- } \\ \text { appropriate amount } \\ \text { of grains, vegetables, } \\ \text { fruit etc. }\end{array} \\ & \begin{array}{l}\text { Calorie target re- } \\ \text { evaluated frequency) }\end{array}\end{array}$

(Same PA sessions but told to limit high-gly-

caemic index foods)

\begin{tabular}{|c|c|c|c|c|}
\hline \multirow[t]{2}{*}{ Lison 2012} & $\begin{array}{l}\text { I1: hospital clinic group exercise-diet pro- } \\
\text { gramme } \\
\text { ( } 5 \times 60 \text { min exercise sessions per week ( } 120 \text { ses- } \\
\text { sions, } 6 \text { months). Moderate aerobic activity + } \\
\text { resistance training, increase intensity each ses- } \\
\text { sion. Two 1-h educational sessions conduct- } \\
\text { ed by paediatricians at the hospital. Promote } \\
\text { Mediterranean diet, additional support (e.g. } \\
\text { food labels). No behavioural component) }\end{array}$ & $\mathrm{N} / \mathrm{A}$ & $\begin{array}{l}\text { Usual care control } \\
\text { group } \\
\text { (At the two hospital } \\
\text { visits they were in- } \\
\text { structed about di- } \\
\text { et and behaviour } \\
\text { changes but never } \\
\text { received any exercise } \\
\text { sessions) }\end{array}$ & Yes \\
\hline & $\begin{array}{l}\text { 12: home-based combined exercise-diet pro- } \\
\text { gramme } \\
\text { (performed exercise at home and completed } \\
\text { log book. Same nutrition information as above) }\end{array}$ & $N / A$ & & \\
\hline Waling 2012 & $\begin{array}{l}\text { Family-based intervention } \\
\text { (14 sessions } 1-2 \text { times per month ( } 90-120 \text { min) } \\
\text { over } 12 \text { months + assignments. } 2^{\text {nd }} \text { year: Inter- } \\
\text { net-based email system for counselling, chat } \\
\text { rooms, assignments. Formulate goals - crav- } \\
\text { ings, hunger control, stress, self-image, self- } \\
\text { perception. Pedometer task, indoor + outdoor } \\
\text { games (e.g. line dancing). Healthy foods, fruit + } \\
\text { vegetables, cooking, recipes, reduce sugar) }\end{array}$ & $\mathrm{N} / \mathrm{A}$ & $\begin{array}{l}\text { No-care control } \\
\text { group } \\
\text { (One information } \\
\text { session over } 2 \text { years - } \\
\text { no care) }\end{array}$ & Yes \\
\hline Wright 2012 & $\begin{array}{l}\text { Kids N Fitness (KNF) intervention } \\
\text { ( } 6 \text { weekly } 90 \text {-min sessions (after-school) plus } \\
\text { school + community activities. Involved PA ses- } \\
\text { sions and activities } \\
\text { Nutrition education - healthy lifestyle behav- } \\
\text { iours, food pyramid } \\
\text { Also a parental support group - bimonthly edu- } \\
\text { cational newsletter) }\end{array}$ & $N / A$ & $\begin{array}{l}\text { General education } \\
\text { (GE) } \\
\text { (Standard PA pro- } \\
\text { gramme in school. } \\
\text { No education or oth- } \\
\text { er activities offered) }\end{array}$ & Yes \\
\hline Barkin 2011 & $\begin{array}{l}\text { Group PA and goal setting } \\
\text { ( } 6 \text { sessions over } 6 \text { months for child and par- } \\
\text { ent. } 1 \text { clinic visit, received behaviour-modifi- }\end{array}$ & $N / A$ & $\begin{array}{l}\text { Standard care coun- } \\
\text { selling and health } \\
\text { education session }\end{array}$ & Yes \\
\hline
\end{tabular}

Diet, physical activity and behavioural interventions for the treatment of overweight or obese children from the age of 6 to 11 years 
(Continued)

cation counselling by a physician (trained in brief principles of motivational interviewing) and also a 45-min group health education session. Five monthly PA sessions ( $1 \mathrm{~h}$ long) at a recreational center. Each session included 20 min skills-building didactic based on American Heart Association educational materials, 30 min of group PA. Parent and child completed a goal setting contract. Behavioural and PA components, no dietary component.)
( 2 sessions of standard care counselling by a physician and a 45-min health education session. Nutrition advice addressed both nutrition and PA. Programme manager responded to group questions

\begin{tabular}{|c|c|c|c|c|}
\hline Bryant 2011 & $\begin{array}{l}\text { WATCH IT intervention } \\
\text { ( } 30 \text { min of motivational counselling weekly for } \\
4 \text { months (child + parent). Plus weekly 1-h ses- } \\
\text { sions of PA given by sports coaches. Nutrition } \\
\text { advice given through a Healthy Eating Lifestyle } \\
\text { Programme. Given by health trainers instead of } \\
\text { medical professionals) }\end{array}$ & $\mathrm{N} / \mathrm{A}$ & $\begin{array}{l}\text { Waiting-list control } \\
\text { (No care for } 12 \\
\text { months then offered } \\
\text { the intervention) }\end{array}$ & Yes \\
\hline Coppins 2011 & $\begin{array}{l}\text { Multi-component family-focused education } \\
\text { package } \\
\text { ( } 2 \text { workshops ( } 8 \mathrm{~h} \text { in total), held } 1-2 \text { weeks } \\
\text { apart (child + parent). Plus } 2 \text { PA sessions ( } 1 \mathrm{~h} / \\
\text { week). Workshops - behaviour change, psycho- } \\
\text { logical well-being, healthy eating) }\end{array}$ & $\mathrm{N} / \mathrm{A}$ & $\begin{array}{l}\text { Waiting-list control } \\
\text { (No care, given in- } \\
\text { tervention after } 12 \\
\text { months) }\end{array}$ & Yes \\
\hline $\begin{array}{l}\text { Gunnarsdottir } \\
\text { 2011a }\end{array}$ & $\begin{array}{l}\text { Epstein's family-based behavioural treatment } \\
\text { (FBBT) } \\
\text { (11 weeks of treatments ( } 4 \text { months) - } 11 \text { group } \\
\text { education sessions ( } 60 \text { min each) and } 11 \text { in- } \\
\text { dividual consulting sessions ( } 30 \text { min each). } \\
\text { Trained parents in behaviour modifications } \\
\text { such as stimulus control. Group sessions fo- } \\
\text { cused on behaviour changes (exercise, Traf- } \\
\text { fic Light Diet). Child and parent attended indi- } \\
\text { vidual sessions together - participants were } \\
\text { weighed, and daily food and activity records } \\
\text { were analysed and graphed for weekly changes } \\
\text { in body weight, fruit and vegetable consump- } \\
\text { tion and PA; goal setting and problem solving } \\
\text { were among the factors discussed.) }\end{array}$ & $\mathrm{N} / \mathrm{A}$ & $\begin{array}{l}\text { Standard care (waiti- } \\
\text { ing-list control) } \\
\text { (One or two 30-min } \\
\text { consultations with a } \\
\text { paediatric endocri- } \\
\text { nologist. One or two } \\
60 \text {-min nutritional } \\
\text { counselling sessions. } \\
\text { Participants offered } \\
\text { the intervention after } \\
12 \text { months) }\end{array}$ & Yes \\
\hline Maddison 2011 & $\begin{array}{l}\text { Active video game package } \\
\text { (Given a Song PlayStation EyeToy upgrade - } \\
\text { received } 5 \text { games during } 6 \text { months. Encourage } \\
\text { to increase activity and substitute non-active } \\
\text { video game play. No behavioural or nutrition } \\
\text { component) }\end{array}$ & $\mathrm{N} / \mathrm{A}$ & $\begin{array}{l}\text { No-care control } \\
\text { group } \\
\text { (Continued with nor- } \\
\text { mal video play } \\
\text { Given PlayStation } \\
\text { update at } 6 \text { months) }\end{array}$ & Yes \\
\hline Wafa 2011 & $\begin{array}{l}\text { Low-intensity intervention } \\
\text { ( } 8 \times 1 \text {-h group session with a dietician over } 26 \\
\text { weeks (parents only). } 8 \text { PA sessions for children } \\
\text { - led by exercise instructor. Behaviour-change } \\
\text { techniques (parenting skills, relapse). A clinical }\end{array}$ & $\mathrm{N} / \mathrm{A}$ & $\begin{array}{l}\text { Waiting-list control } \\
\text { (Offered the inter- } \\
\text { vention at } 6 \text { months) }\end{array}$ & Yes \\
\hline
\end{tabular}

Diet, physical activity and behavioural interventions for the treatment of overweight or obese children from the age of 6 to 11 years (Review)

Copyright $\odot 2017$ The Cochrane Collaboration. Published by John Wiley \& Sons, Ltd. 
psychologist provided support in 1 session. In-

crease PA, decreasing sedentary behaviours.

Changes in diet, food labels, cooking, traffic

light plan, family meals)

Bathrellou $2010 \quad \begin{aligned} & \text { Behavioural intervention with parental involve- } \\ & \text { ment }\end{aligned}$
(Multidisciplinary programme (CBT principles),
12 weekly sessions (2 h each). The interven-
tion had 3 components: delivery of a behav-
ioural curriculum (Programa Cambia), consul-
tations with registered dieticians and physician
consultations. The behavioural curriculum in-
cluded 12 weekly sessions of $2 \mathrm{~h}$ and was based
on the health belief model and a simple food
guide developed by the authors (a Health Nu-
trition Traffic Light system). Parental involve-
ment in 2 individual sessions and last 10 min of
other sessions. Monthly booster sessions from
3-9 months. Dietary and PA advice regarding
energy balance. Goal setting and self-monitor-
ing encouraged)

Diaz 2010 Behavioural curriculum plus registered dieticians and physician consultations

N/A

Behavioural inter-

Yes

vention without

parental involvement

(Same multidisciplinary programme but no parental involvement in the sessions)

(12 behavioural sessions over 12 weeks ( 2 h each). 12 dietician consultations over 12 weeks. Then monthly physician consultations (10-15 min) - total 6 months. Behavioural modification, exercise goal setting, traffic light diet (child + parent))

$\begin{array}{ll}\text { N/A Physician consulta- } & \text { Yes } \\ \text { tions only }\end{array}$

tions only

(Monitored BMI and blood pressure and encouraged PA, reduce sedentary behaviour, nutrition advice and behavioural techniques

12 monthly sessions (10-15 min each))

Duggins $2010 \quad$ Nutrition classes and family YMCA membership N/A

(4 nutrition sessions over 9 months (dietitian-led): eating habits, meal planning. Handbook on food choices, PA, sedentary behaviours. Every participant and their parents or guardians were scheduled to attend the nutrition classes (within 6 weeks of enrolment and Nutrition classes onYes ly

(Received the same 4 nutrition classes but had no YMCA membership) 1 week later, at 6 months and 9 months). Also, received a no-cost 1-year family membership to a YMCA (swimming, jogging). YMCA diaries were completed by the participant during each visit to the YMCA throughout the 12month study duration. No behavioural component)

\begin{tabular}{|c|c|c|c|c|}
\hline Faude 2010 & $\begin{array}{l}\text { Football training programme (FB) } \\
\text { ( } 6 \text { months, } 3 \mathrm{~d} / \text { week ( } 1 \text {-h sessions). } 10 \% \text { warm } \\
\text { up, } 50 \% \text { small-sided games, } 20 \% \text { techniques, } \\
20 \% \text { fitness with ball. No behavioural or nutri- } \\
\text { tional component) }\end{array}$ & $\mathrm{N} / \mathrm{A}$ & $\begin{array}{l}\text { Established stan- } \\
\text { dard sports pro- } \\
\text { gramme (STD) (10\% } \\
\text { warm up, } 40 \% \text { aero- } \\
\text { bic endurance activ- } \\
\text { ities, } 20 \% \text { co-ordina- } \\
\text { tion/flexibility, } 15 \%\end{array}$ & Yes \\
\hline
\end{tabular}

Diet, physical activity and behavioural interventions for the treatment of overweight or obese children from the age of 6 to 11 years 
sessions

\begin{tabular}{|c|c|c|c|c|}
\hline Reinehr 2010 & $\begin{array}{l}\text { "Obeldicks Light" behaviour-changing inter- } \\
\text { vention } \\
3 \text { months intensive phase ( } 6 \text { × 1.5-h child } \\
\text { groups sessions, } 6 \text { × 1.5-h parent evening, } 1 \text { nu- } \\
\text { trition counselling + } 1 \text { PA training (1 per week, } \\
1.5 \mathrm{~h} \text { ) } \\
\text { Establishing phase ( } 3 \text { months) - } 1 \text { nutritional } \\
\text { counselling, } 3 \times 30 \text { min individual counselling } \\
\text { and PA training continued } \\
\text { Behavioural counselling based on systemic + } \\
\text { solution-focused theories } \\
\text { Exercise included ball games, reduce sedentary } \\
\text { behaviours } \\
\text { "Optimized mixed diet", diet guidelines, traffic } \\
\text { light system, nutrition course }\end{array}$ & $\mathrm{N} / \mathrm{A}$ & $\begin{array}{l}\text { Waiting-list control } \\
\text { Intervention offered } \\
\text { after } 6 \text { months }\end{array}$ & Yes \\
\hline Sacher 2010 & $\begin{array}{l}\text { MEND program } \\
18 \text { sessions over } 9 \text { weeks ( } 2 \mathrm{~h} \text { each) - behav- } \\
\text { ioural, nutrition, PA } \\
\text { Given access to swimming pool for } 21 \text { weeks } \\
\text { Behavioural - stimulus control, goal setting, re- } \\
\text { inforcement (child and parent) } \\
\text { Child took part in non-competitive group play } \\
\text { Healthy eating advice, weekly targets, food } \\
\text { habits, recipes, supermarket tours }\end{array}$ & $\mathrm{N} / \mathrm{A}$ & $\begin{array}{l}\text { Waiting-list control } \\
\text { Offered intervention } \\
\text { after } 6 \text { months }\end{array}$ & Yes \\
\hline Kalarchian 2009 & $\begin{array}{l}\text { Family-based, behavioural weight control } \\
\text { group } \\
\text { ( } 20 \text { group meetings (child + parent separate, } \\
60 \text { min) for } 6 \text { months. } 6 \text { booster sessions be- } \\
\text { tween months } 6-12 \text {. Behavioural: self-monitor- } \\
\text { ing, goals, stimulus control, positive reinforce- } \\
\text { ment. Encouraged to increase PA and decrease } \\
\text { sedentary behaviours. Stoplight eating Plan } \\
\text { with daily energy range based on body weight) }\end{array}$ & $\mathrm{N} / \mathrm{A}$ & $\begin{array}{l}\text { Usual care } \\
\text { ( } 2 \text { nutrition consulta- } \\
\text { tions based on Stop- } \\
\text { light eating plan. Of- } \\
\text { fered intervention at } \\
18 \text { months) }\end{array}$ & Yes \\
\hline Nowicka 2009 & $\begin{array}{l}\text { Summer camp } \\
\text { (Week-long summer camp - children tried out } \\
\text { at least } 2 \text { sports/d. Meals served during camp } \\
\text { were nutritionally balanced + portion con- } \\
\text { trolled. A coach was assigned to support child's } \\
\text { favourite sport for a further } 6 \text { months. No be- } \\
\text { havioural component) }\end{array}$ & $\mathrm{N} / \mathrm{A}$ & No-care control & Yes \\
\hline Wake 2009 & LEAP2 behavioural intervention & $\mathrm{N} / \mathrm{A}$ & $\begin{array}{l}\text { No-care control } \\
\text { group }\end{array}$ & Yes \\
\hline
\end{tabular}

Diet, physical activity and behavioural interventions for the treatment of overweight or obese children from the age of 6 to 11 years 
( 4 consultations over 12 weeks with a GP (child and parent)

Behavioural changes - goals, family-based reinforcement techniques

Target PA and nutrition (e.g. lower fat, breakfast))

\begin{tabular}{ll}
\hline Alves 2008 & Exercise programme \\
& (Exercises programme $3 \times$ per week (6 months), \\
& 50 min sessions for child only. Moderate inten- \\
& sity exercises such as dancing. Taught by phys- \\
ical education teacher. No behavioural or diet & component)
\end{tabular}

N/A No-care control Yes

(8 appointments during 26 weeks (total $5 \mathrm{~h}$ ). Used family-centred approach, various behavioural-change techniques, modified traffic-light approach and restrict sedentary behaviour)

Standard care
(3-4 outpatient ap-
pointments (total
1.5 h) typical dietet-
ic care - direct-a-par-
ent, mainly less focus
on exercise/seden-
tary behaviour)

Usual care control Yes group

(Therapeutic care at 0 and 6 months. $\mathrm{PA}$ and diet recommendations. Coping strategies) vegetable template, food logbooks. Led by dietitians, sports coaches, psychologists Length $=12$ months)

\begin{tabular}{|c|c|c|c|c|}
\hline Weintraub 2008 & $\begin{array}{l}\text { After-school team sports programme } \\
\text { Offered } 3 \mathrm{~d} / \text { week ( } 21 / 4 \mathrm{~h} \text { ) for } 5 \text { months, then } 4 \\
\mathrm{~d} / \text { week from month } 5 \text { ( } 6 \text { months in total) } \\
\text { Supportive team building, warm up, stretching, } \\
\text { soccer skills } \\
\text { Matches held quarterly with children, parents } \\
\text { and coaches } \\
\text { No behavioural or nutrition arm }\end{array}$ & $\mathrm{N} / \mathrm{A}$ & $\begin{array}{l}\text { "active placebo" } \\
\text { control } \\
25 \text { sessions on nutri- } \\
\text { tion and health edu- } \\
\text { cation } \\
\text { After school meet- } \\
\text { ings for } 6 \text { months }\end{array}$ & Yes \\
\hline Berry 2007 & $\begin{array}{l}\text { Nutrition and exercise education programme } \\
\text { (NEEP) plus coping skills training (CST) } \\
\text { ( } 24 \text { weekly sessions aimed at child and par- } \\
\text { ents. Parent received } 6 \text { weeks of NEEP and } 6 \\
\text { weeks of CST. Children received } 6 \text { weeks of } \\
\text { NEEP, } 6 \text { weeks of behavioural-modification } \\
\text { with NEEP and } 12 \text { weeks of exercise. NEEP in- } \\
\text { volved exercise classes, diet and PA education. } \\
\text { CST - cognitive behaviour modification, barri- } \\
\text { ers, problem solving (parents only)) }\end{array}$ & $\mathrm{N} / \mathrm{A}$ & $\begin{array}{l}\text { Nutrition and exer- } \\
\text { cise education pro- } \\
\text { gramme only } \\
\text { (No CST classes given } \\
\text { to parents) }\end{array}$ & Yes \\
\hline
\end{tabular}

Diet, physical activity and behavioural interventions for the treatment of overweight or obese children from the age of 6 to 11 years 
(Continued)

\begin{tabular}{|c|c|c|c|c|}
\hline Gillis 2007 & $\begin{array}{l}\text { Exercise and diet education with weekly diaries } \\
\text { and telephone calls } \\
\text { (30 min talk about healthy diet + exercise at } \\
\text { baseline and } 3 \text { months. During } 3 \text { months, } \\
\text { weekly phone calls to review weekly diaries. } \\
\text { Modify behaviours - weekly diaries to record } \\
\text { exercise + food ingested } 1 \text { day of the week) }\end{array}$ & $\mathrm{N} / \mathrm{A}$ & $\begin{array}{l}\text { Exercise and diet ed- } \\
\text { ucation only } \\
\text { (Received initial in- } \\
\text { struction. Did not } \\
\text { record food/exercise } \\
\text { in diaries or receive } \\
\text { phone calls) }\end{array}$ & Yes \\
\hline Kalavainen 2007 & $\begin{array}{l}\text { Family-centered group programme } \\
\text { (15 sessions ( } 90 \text { min) separately for child and } \\
\text { parent ( } 1 \text { joint at end) - } 6 \text { months. Based on } \\
\text { principles of behavioural and solution orientat- } \\
\text { ed therapy. Promote healthy lifestyle and well- } \\
\text { being instead of weight loss. Increase exercise } \\
\text { and decrease sedentary behaviours } \\
\text { Promote healthy diet using Finnish recommen- } \\
\text { dations) }\end{array}$ & $\mathrm{N} / \mathrm{A}$ & $\begin{array}{l}\text { Routine treatment } \\
\text { Modified from the } \\
\text { counselling practice } \\
\text { for obese children in } \\
\text { Finland. } \\
\text { Given booklets and } \\
\text { children had } 30 \text { min } \\
\text { individual appoint- } \\
\text { ments with a school } \\
\text { nurse) }\end{array}$ & Yes \\
\hline McCallum 2007 & $\begin{array}{l}\text { LEAP Intervention } \\
\text { (Parents attended } 4 \text { consultations over } 12 \\
\text { weeks. Family folder used to assist and record } \\
\text { goals - behaviour change. Topic sheets - chose } \\
\text { goals e.g. be more active, lower fat, drink wa- } \\
\text { ter. Reinforcement techniques used to encour- } \\
\text { age parental participation) }\end{array}$ & $\mathrm{N} / \mathrm{A}$ & $\begin{array}{l}\text { No-care control } \\
\text { group } \\
\text { (Carried on seeing } \\
\text { GP if required) }\end{array}$ & Yes \\
\hline
\end{tabular}

Rodearmel 2007 'America on the Move' intervention group

N/A

Self-monitoring

Yes

(6 meetings with study staff over 24 weeks - no behavioural component. Told to wear pedometers, increase PA by 2000 steps/d. Told to eliminate $100 \mathrm{kcal} / \mathrm{d}$, replace sugar with sucralose sweeteners. Food labelling, caloric content, eat breakfast, 5 x fruit + veg/d)

group

(Told to monitor usual behaviour during study. Wear pedometers and complete sweets survey. Did not receive any information on exercise)

\begin{tabular}{|c|c|c|c|c|}
\hline Satoh 2007 & $\begin{array}{l}\text { Dietary guidance using an easily handled mod- } \\
\text { el nutritional balance chart (MNBC) } \\
\text { (Meal chart completed } 3 \text { days of the week (nu- } \\
\text { trition component only) } \\
\text { Investigators placed black dots on balance } \\
\text { chart according to content of the meal chart } \\
\text { (e.g. meat, green and yellow vegetables, sug- } \\
\text { ar). Investigator responded with advice, com- } \\
\text { ments and encouragement. No behavioural } \\
\text { component) }\end{array}$ & $\mathrm{N} / \mathrm{A}$ & $\begin{array}{l}\text { Usual care } \\
\text { (Received dietary } \\
\text { guidance before the } \\
\text { study started from } \\
\text { nutritionists at hos- } \\
\text { pitals. Then received } \\
\text { conventional dietary } \\
\text { guidance once per } \\
\text { month) }\end{array}$ & Yes \\
\hline Wilfley 2007 & $\begin{array}{l}\text { I1: behavioural-skills maintenance group } \\
\text { (16 weekly sessions ( } 20 \text { min family }+40 \text { min } \\
\text { separate child + parent) }\end{array}$ & $\mathrm{N} / \mathrm{A}$ & $\begin{array}{l}\text { No-care control } \\
\text { group }\end{array}$ & Yes \\
\hline
\end{tabular}

Diet, physical activity and behavioural interventions for the treatment of overweight or obese children from the age of 6 to 11 years 
Motivation for weight loss and promoting small changes in eating and exercise. Identify highrisk situations for overeating or missing PA. Preplanning, problem solving, cognitive restructuring, positive self-talk)

12: social-facilitation maintenance group

(Based on premise that relapse results from absence of a supportive social environment for weight control (instead of focusing on behavioural skills). Encourage child to form friendships, address body image concerns + teasing. Same nutrition and PA advice as above)

Epstein $2005 \quad \begin{aligned} & \text { Standardised family-based behavioural weight } \\ & \text { control programme plus reinforcement for in- } \\ & \text { creasing alternatives to eating }\end{aligned}$
(14 sessions (6 months), 6 booster sessions
$6-12$ months, as needed to 24 months. Rein-
forcement system to motivate children for be-
haviour change. Points received for meeting
goals and alternative behaviour to eating. Gen-
eral PA information (moderate intensity), traf-
fic light diet)

$\mathrm{N} / \mathrm{A}$

Standardised family-based behavioural weight control programme only

(Received no reinforcement through alternative behaviour to eating)

\section{Nemet 2005}

Combined dietary and exercise programme
N/A

(4 evening lectures over 3 months: therapeutic nutritional approach. 6 dietitian sessions + exercise programme twice weekly. Exercise programme - endurance activities, coordination + flexibility. Encouraged: + 30-45 min/week of exercise and decrease sedentary behaviours. Nutrition education - food pyramid, cooking, balanced hypocaloric diet)

Usual-care control Yes
group

(Referred to an ambulatory nutrition consultation at least once during study Instructed to perform exercise $3 \times$ per week)

Woo $2004 \quad$ I1: diet plus supervised structured exercise pro- N/A
gramme with continuing training

Diet modification on- Yes ly

(Diet education twice weekly for 6 weeks then every two months until 12 months. Plus 6 weeks of exercise training $(2 \times /$ week $)$ then weekly for 1 year ( 75 minutes). 18 exercise stations: aerobic exercise, resistance training, agility. Exercise intensity at $60 \%-70 \%$ predicted maximum heart rate (during aerobic). Balanced hypocaloric diet - low fat, high in complex carbohydrates, protein)

(No exercise training throughout 12 months)

12: diet plus supervised structured exercise pro- N/A gramme with detraining

(Stopped exercise training after 6 weeks but continued with the diet programme)

$\begin{array}{llll}\text { Epstein } 2001 & \begin{array}{l}\text { A combination of reducing sedentary behav- } \\ \text { iour and increasing PA }\end{array} & \text { N/A } & \begin{array}{l}\text { Targeting increasing } \\ \text { PA only }\end{array}\end{array}$

Diet, physical activity and behavioural interventions for the treatment of overweight or obese children from the age of 6 to 11 years 
(Continued)

(16 weekly meetings then 2 biweekly and 2
monthly - 6 months in total. Participants met
with the therapist individually for 30 min the
first week and on subsequent weeks they al-
ternated between separate child and parent
30-min group meetings and individual meet-
ings. Workbooks on self-monitoring, behaviour
change, positive reinforcement. Traffic light di-
et, food labels, increase PA (up to 180 min per
week), decrease sedentary (final goal of $15 \mathrm{~h} /$
week). Families were provided additional infor-
mation about food labels, shopping, and cur-
rent findings in the research on obesity and nu-
trition

(Participants were given the same intervention but targeted increased PA only and did not focus on reducing sedentary behaviours

$\begin{array}{ll}\text { Nova } 2001 & \text { Enhanced approach } \\ & \text { (Given specific diet (approximately } 1400 \text { calo- } \\ \text { ries), and guidelines on PA. Encouraged active } & \text { parental commitment and gave a alimenta- } \\ & \text { ry diary. Paediatrician reviewed the diary and } \\ & \text { evaluation accuracy (9 times over } 24 \text { mo). Par- } \\ & \text { ents rated commitment to the intervention - } \\ & \text { no behavioural component) }\end{array}$

\section{Epstein 2000a}

$$
\begin{aligned}
& \text { I1: behavioural weight-control programme plus } \\
& \text { parent and child problem solving } \\
& \text { (16 weekly meetings then } 2 \text { monthly meetings } \\
& -6 \text { months. Behaviour change techniques - } \\
& \text { stimulus control, self-monitoring. Problem } \\
& \text { solving training, group + individual content. } \\
& \text { Workbooks on increasing exercise and traffic } \\
& \text { light diet) }
\end{aligned}
$$

12: behavioural weight-control programme plus N/A child problem solving only

(Problem solving only for children - parents not involved)

$\begin{array}{ll}\text { N/A } & \text { Routine approach } \\ & \text { (Received leaflets } \\ & \text { with general infor- } \\ & \text { mation about obesi- } \\ & \text { ty and risks, advice } \\ & \text { on healthy eating } \\ & \text { and an invitation to } \\ & \text { take part in some PA) }\end{array}$

Standard treatment Yes

\begin{tabular}{|c|c|c|c|c|}
\hline $\begin{array}{l}\text { Schwingshandl } \\
1999\end{array}$ & $\begin{array}{l}\text { PA programme and dietary advice } \\
\text { (Individualised training programme - twice } \\
\text { weekly ( } 60-70 \text { min), } 12 \text { weeks. Walk up peri- } \\
\text { od, exercises such as lying leg press (sets, resis- } \\
\text { tance increased). Dietary advice about energy } \\
\text { requirements, nutrients, fibre, fluids, vitamins) }\end{array}$ & N/A & $\begin{array}{l}\text { Dietary advice alone } \\
\text { (Dietary advice only - } \\
\text { no training sessions) }\end{array}$ & Yes \\
\hline Duffy 1993 & $\begin{array}{l}\text { Cognitive self-management training plus be- } \\
\text { haviour therapy } \\
\text { ( } 8 \text { weekly sessions ( } 90 \text { min each). Stimulus con- } \\
\text { trol, nutritional education (traffic light), in- } \\
\text { creasing exercise. Parents taught goal setting } \\
\text { and positive reinforcements CBT - monitoring } \\
\text { negative thoughts, problem solving, self-rein- } \\
\text { forcement) }\end{array}$ & $\mathrm{N} / \mathrm{A}$ & $\begin{array}{l}\text { Behaviour therapy } \\
\text { plus attention place- } \\
\text { bo control methods } \\
\text { (No CBT - instead } \\
\text { they received a } \\
\text { placebo component } \\
\text { which was relaxation } \\
\text { training) }\end{array}$ & Yes \\
\hline Flodmark 1993 & Family therapy & $\mathrm{N} / \mathrm{A}$ & $\begin{array}{l}\text { Conventional treat- } \\
\text { ment }\end{array}$ & Yes \\
\hline
\end{tabular}
with no additional problem solving

(Same sessions as intervention group

But homework assignments not based on problem solving)

Diet, physical activity and behavioural interventions for the treatment of overweight or obese children from the age of 6 to 11 years 
(Continued)

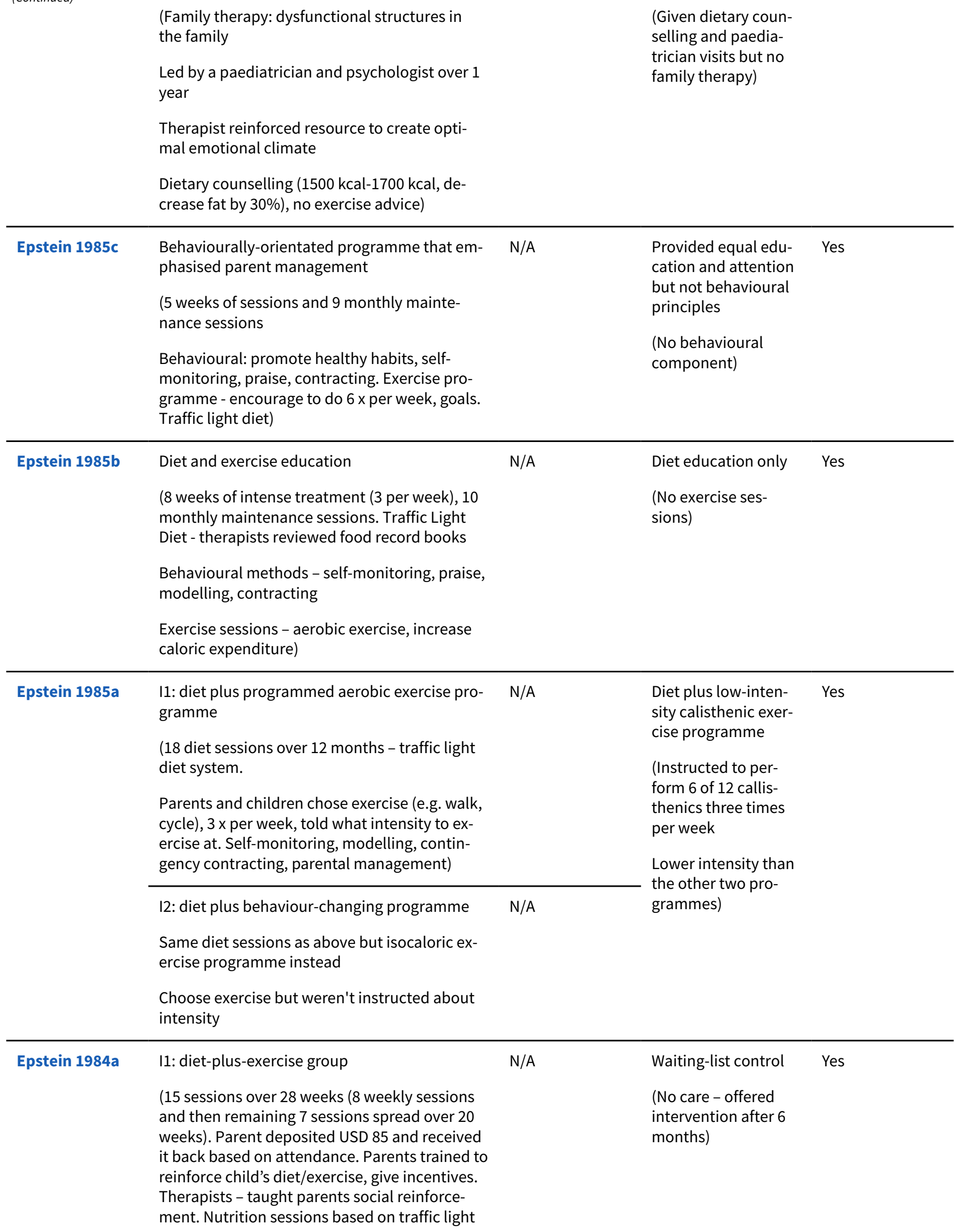

Diet, physical activity and behavioural interventions for the treatment of overweight or obese children from the age of 6 to 11 years 
(Continued)

diet; exercise programme required increasing caloric expenditure above normal through a series of gradual steps. Each of the 15 sessions involved a group discussion (parents and children separated))

12: diet only

(did not receive the life-style change exercise programme. Instead given information on lowexpenditure stretching and callisthenics, and were not provided any suggestions or supports for systematic exercise)

\section{- denotes not reported}

aThe term 'adequate' refers to sufficient use of the intervention/comparator with regard to dose, dose escalation, dosing scheme, provision for contraindications and other features necessary to establish a fair contrast between intervention and comparator

BMI: body mass index; C: comparator; CBT: cognitive behavioural therapy; CDS: clinical decision support; CST: coping skills training; GP: general practitioner; I: intervention; kcal: calories; MVPA: moderate to vigorous physical activity; N/A: not applicable; NEEP: nutrition and exercise education program; PA: physical activity; SMSMT: SMS maintenance treatment; SWITCH: Screen-Time Weight-loss Intervention Targeting Children at Home; YMCA: Young Men's Christian Association 


\begin{tabular}{|c|c|c|c|c|c|c|c|c|c|c|c|}
\hline & Appendix 3 & Baseline characteri & s (I) & & & & & & & & \\
\hline 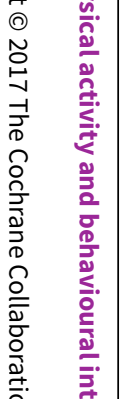 & & $\begin{array}{l}\text { Intervention(s) and } \\
\text { comparator(s) }\end{array}$ & $\begin{array}{l}\text { Dura- } \\
\text { tion of in- } \\
\text { terven- } \\
\text { tion/du- } \\
\text { ration of } \\
\text { follow-up } \\
\text { (days, } \\
\text { weeks, } \\
\text { months, } \\
\text { years) }\end{array}$ & $\begin{array}{l}\text { Descrip- } \\
\text { tion of } \\
\text { partici- } \\
\text { pants }\end{array}$ & $\begin{array}{l}\text { Trial peri- } \\
\text { od } \\
\text { (year to } \\
\text { year) }\end{array}$ & Country & Setting & $\begin{array}{l}\text { Ethnic groups } \\
(\%(\mathrm{~N}))\end{array}$ & Socioeconomic status & $\begin{array}{l}\text { Duration } \\
\text { of be- } \\
\text { ing over- } \\
\text { weight/obese } \\
\text { (mean/ } \\
\text { range } \\
\text { years } \\
\text { (SD), or as } \\
\text { reported) }\end{array}$ & 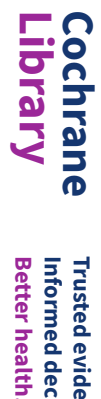 \\
\hline 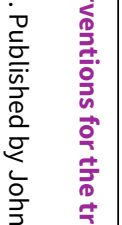 & NCT02436330 & $\begin{array}{l}\text { I: exergaming and di- } \\
\text { dactic healthy teach- } \\
\text { ing }\end{array}$ & $\begin{array}{l}6 \text { months } \\
\text { (0 months) }\end{array}$ & $\begin{array}{l}\text { Children } \\
8-16 \text { years } \\
\text { old with } \\
\text { BMI } \geq 85 \text { th } \\
\text { percentile }\end{array}$ & $\begin{array}{l}\text { April 2011- } \\
\text { Septem- } \\
\text { ber } 2013\end{array}$ & USA & Unclear & $\begin{array}{l}\text { Asian: } 8 \text { (5) } \\
\text { Black or African } \\
\text { American: } 27 \text { (16) } \\
\text { White: } 65 \text { (39) }\end{array}$ & - & - & 5 \\
\hline 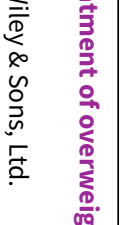 & & $\begin{array}{l}\text { C: didactic healthy } \\
\text { teaching }\end{array}$ & & & & & & $\begin{array}{l}\text { Asian: } 17 \text { (4) } \\
\text { Black or African } \\
\text { American: } 25 \text { (6) } \\
\text { White: } 58 \text { (14) }\end{array}$ & - & - & \\
\hline 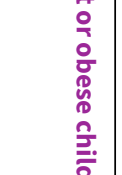 & Ho 2016 & $\begin{array}{l}\text { I: standard nutrition } \\
\text { counselling plus por- } \\
\text { tion control equip- } \\
\text { ment }\end{array}$ & $\begin{array}{l}6 \text { months } \\
\text { (0 months) }\end{array}$ & $\begin{array}{l}\text { Age 8-16 } \\
\text { over- } \\
\text { weight or } \\
\text { obese chil- }\end{array}$ & $2009-2014$ & Canada & Home & - & - & - & \\
\hline $\begin{array}{l}\frac{3}{7} \\
\overrightarrow{0} \\
3\end{array}$ & & $\begin{array}{l}\text { C: standard nutrition } \\
\text { counselling }\end{array}$ & $\begin{array}{l}6 \text { months } \\
\text { (0 months) }\end{array}$ & & & & Unclear & - & & - & $\delta$ \\
\hline $\begin{array}{l}0 \\
0 \\
0 \\
\text { og } \\
0 \\
0 \\
0 \\
0 \\
0 \\
0 \\
1\end{array}$ & $\begin{array}{l}\text { Warschburg- } \\
\text { er } 2016\end{array}$ & $\begin{array}{l}\text { I: parental CBT train- } \\
\text { ing group plus child } \\
\text { inpatient interven- } \\
\text { tion }\end{array}$ & $\begin{array}{l}3-6 \\
\text { weeks (12 } \\
\text { months) }\end{array}$ & $\begin{array}{l}\text { Obese } \\
\text { children } \\
\text { age } 7-13\end{array}$ & $\begin{array}{l}\text { First ran- } \\
\text { domisa- } \\
\text { tion on } 1 \\
\text { Novem- }\end{array}$ & Germany & $\begin{array}{l}\text { Inpatient } \\
\text { rehabili- } \\
\text { tation set- } \\
\text { ting }\end{array}$ & All white & - & - & 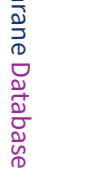 \\
\hline$\underset{\sim}{\tilde{O}}$ & & $\begin{array}{l}\text { C: parental informa- } \\
\text { tion-only group plus } \\
\text { child in-patient inter- } \\
\text { vention }\end{array}$ & & & $\begin{array}{l}\text { and last } \\
\text { on } 3 \text { March } \\
2011-\text { first } \\
\text { 1-year fol- } \\
\text { low-up on } \\
27 \text { Janu- }\end{array}$ & & & & - & - & 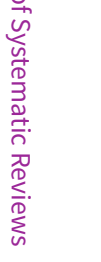 \\
\hline
\end{tabular}




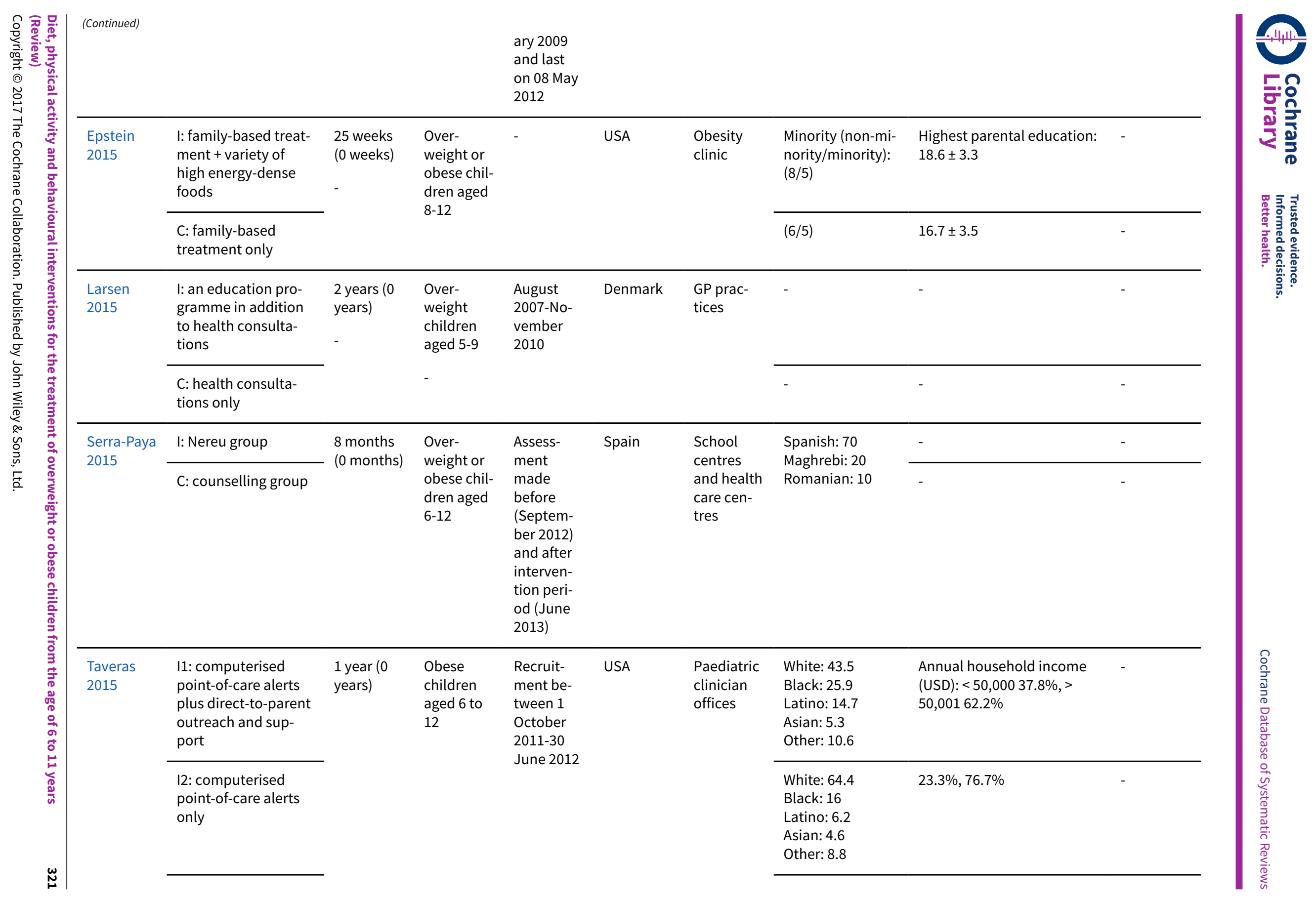




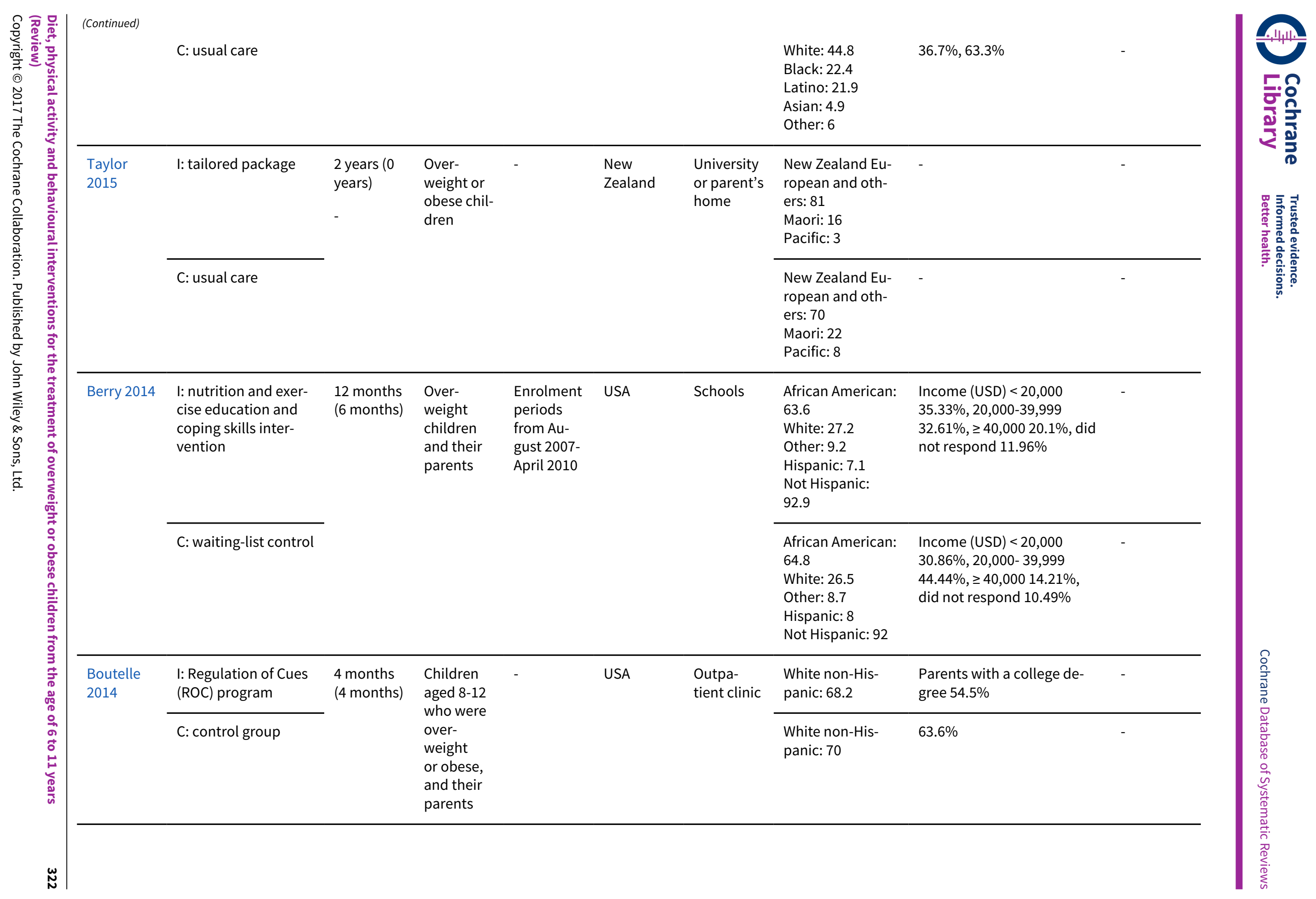




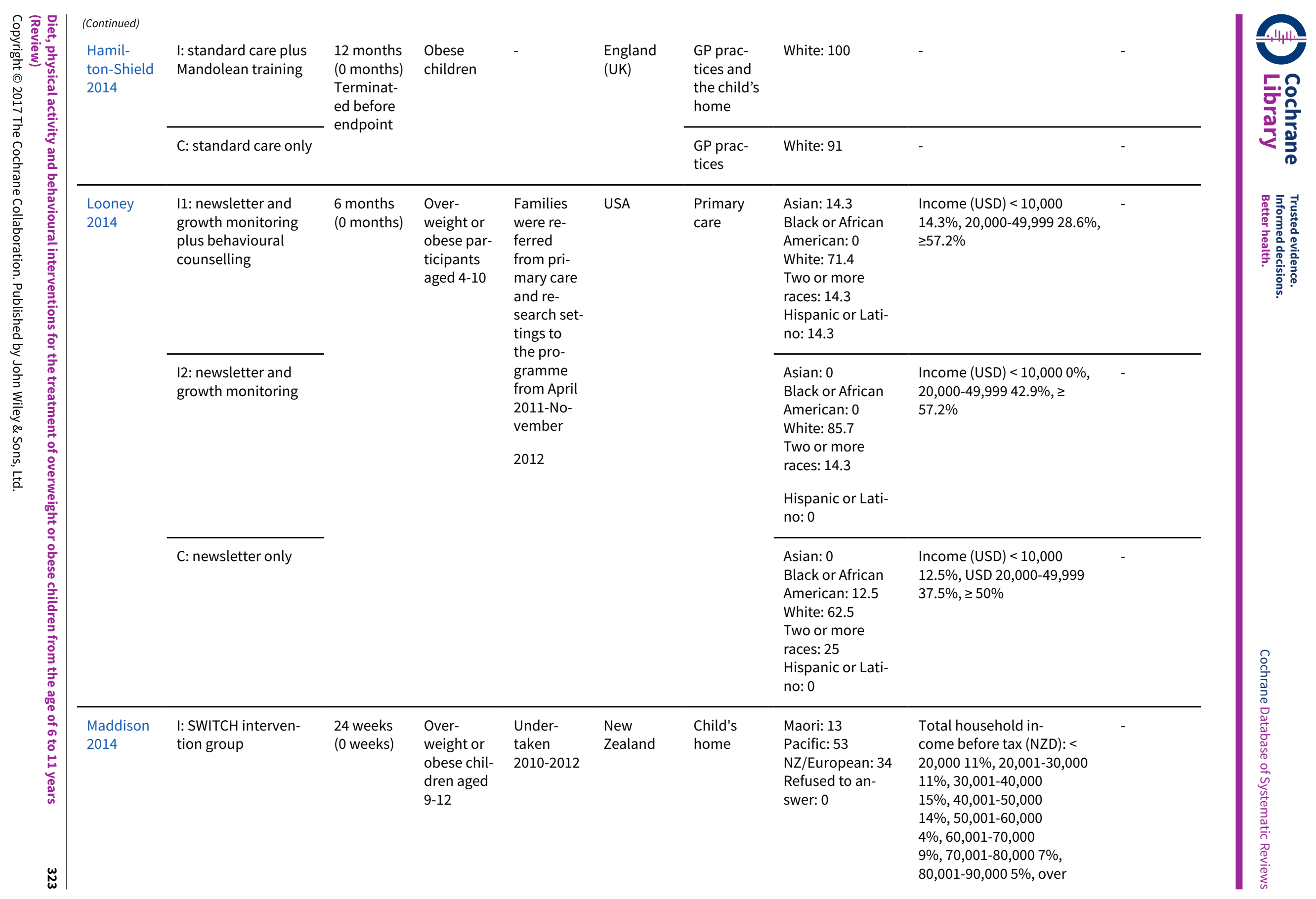




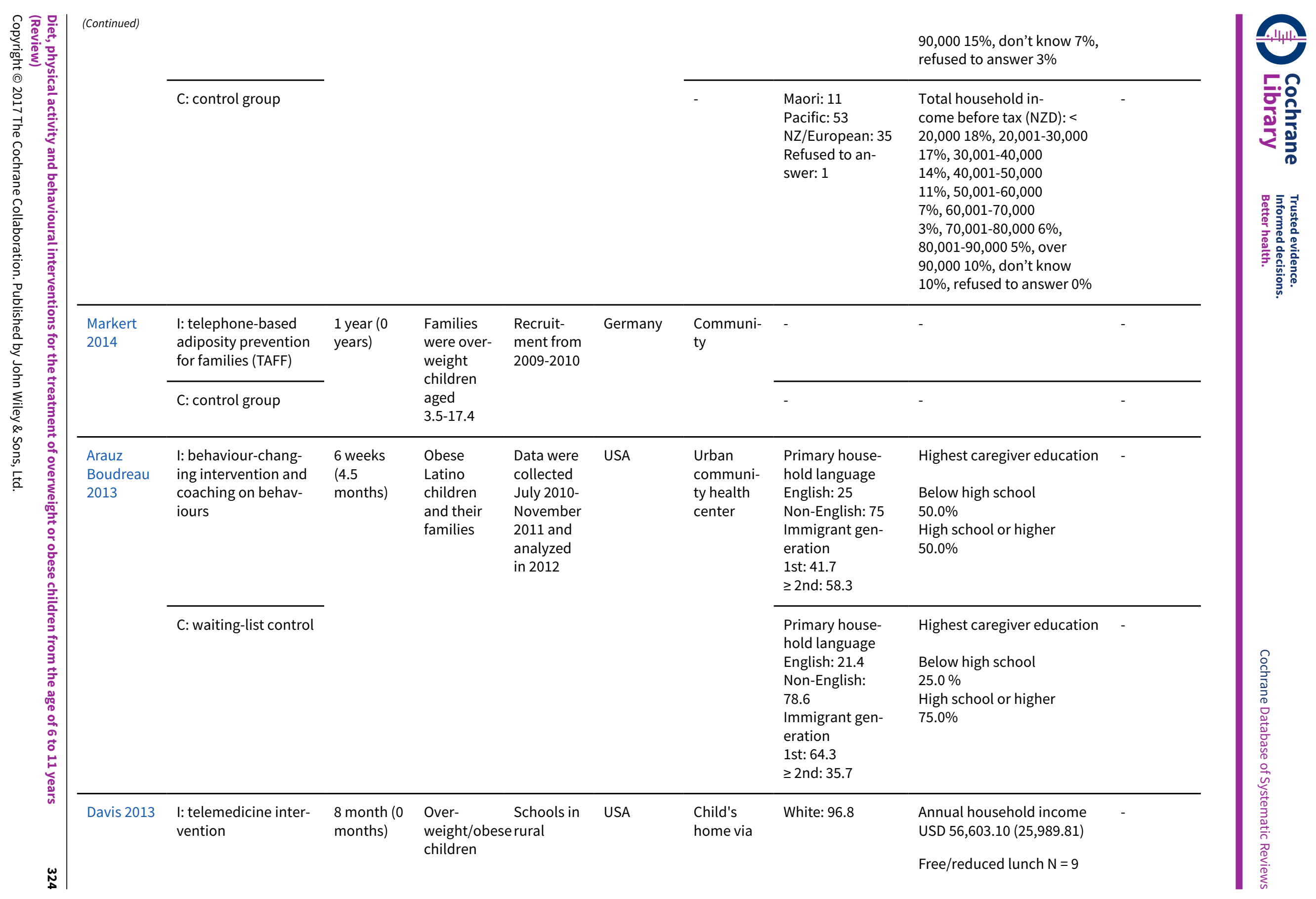




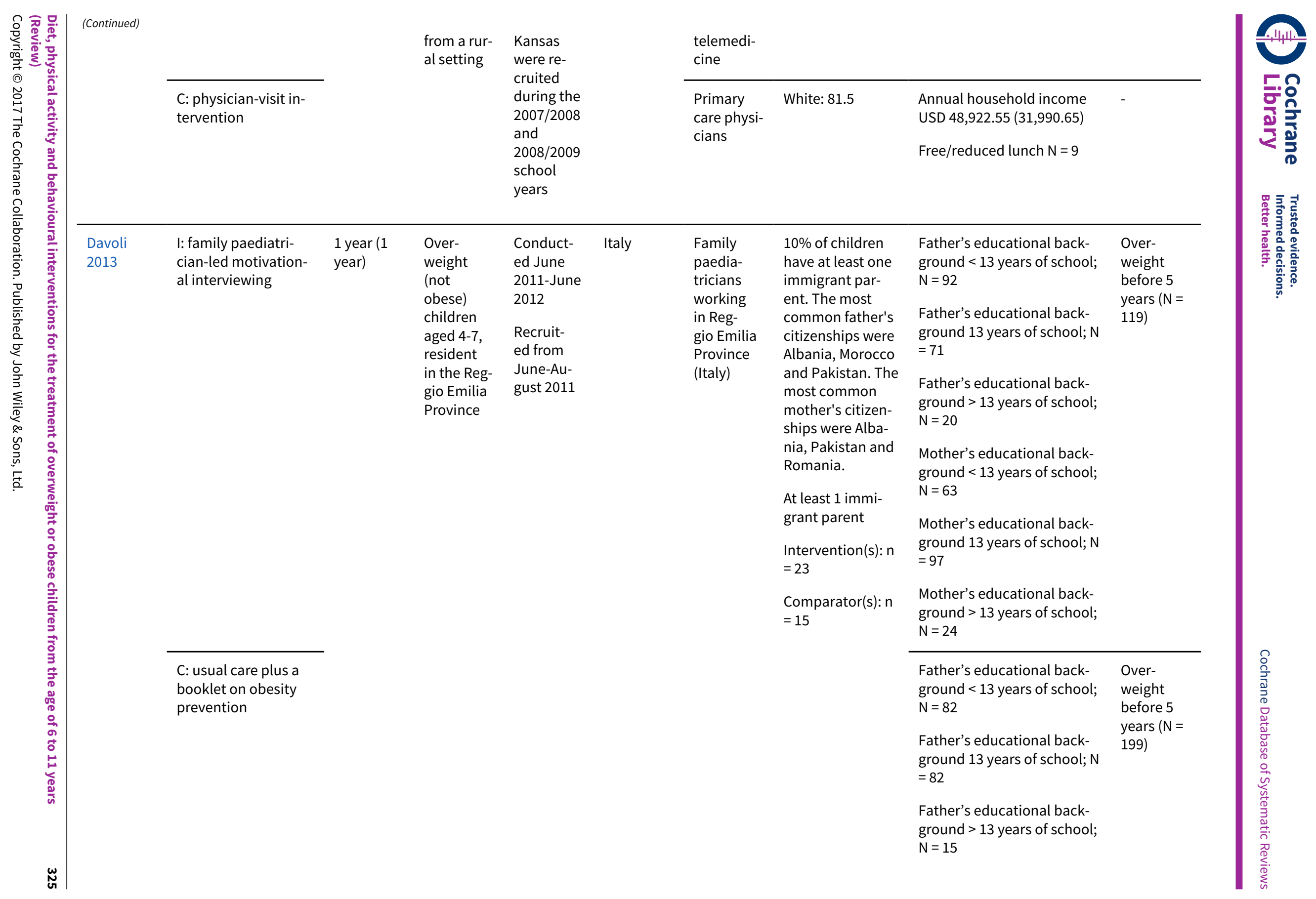




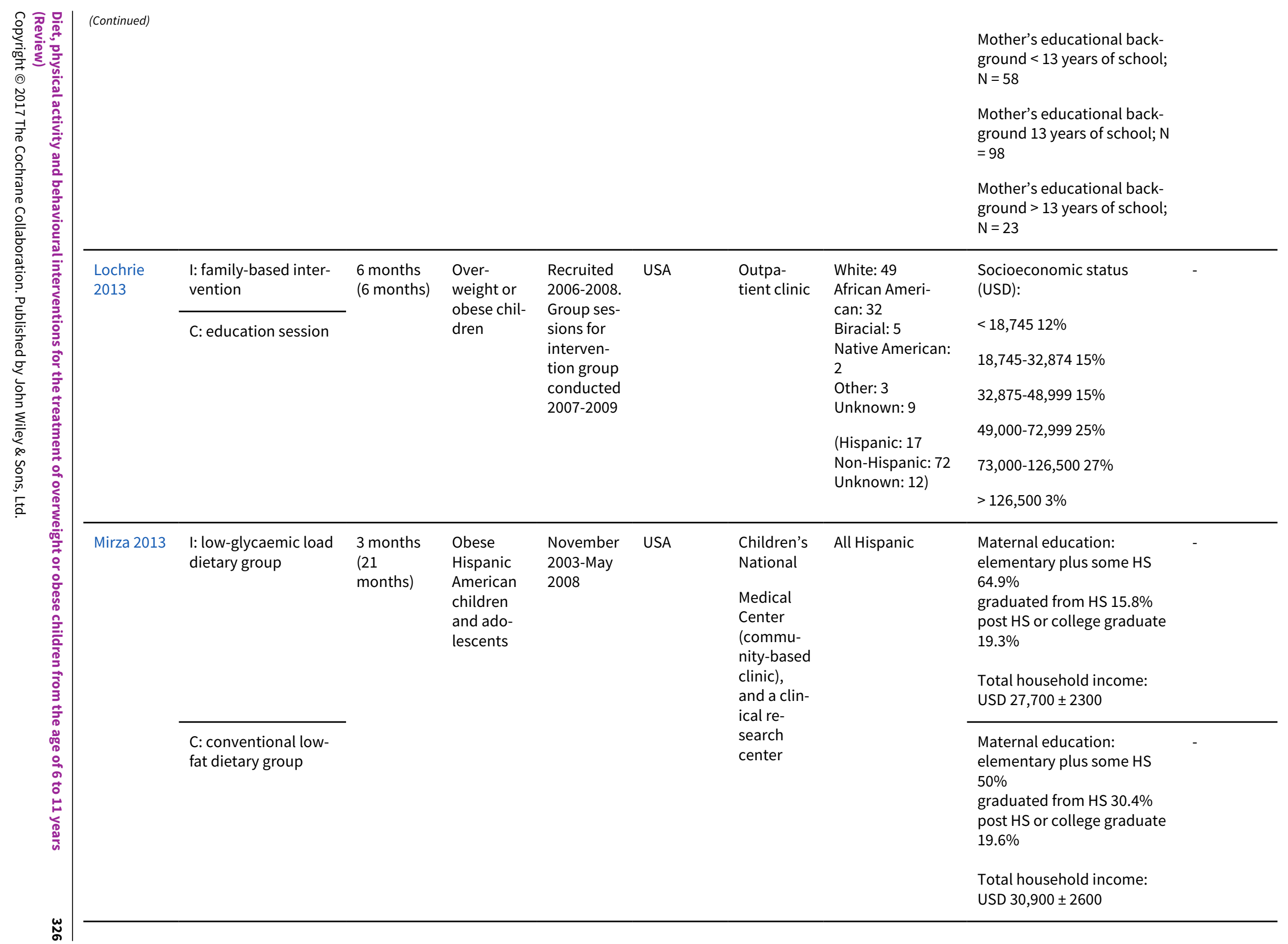




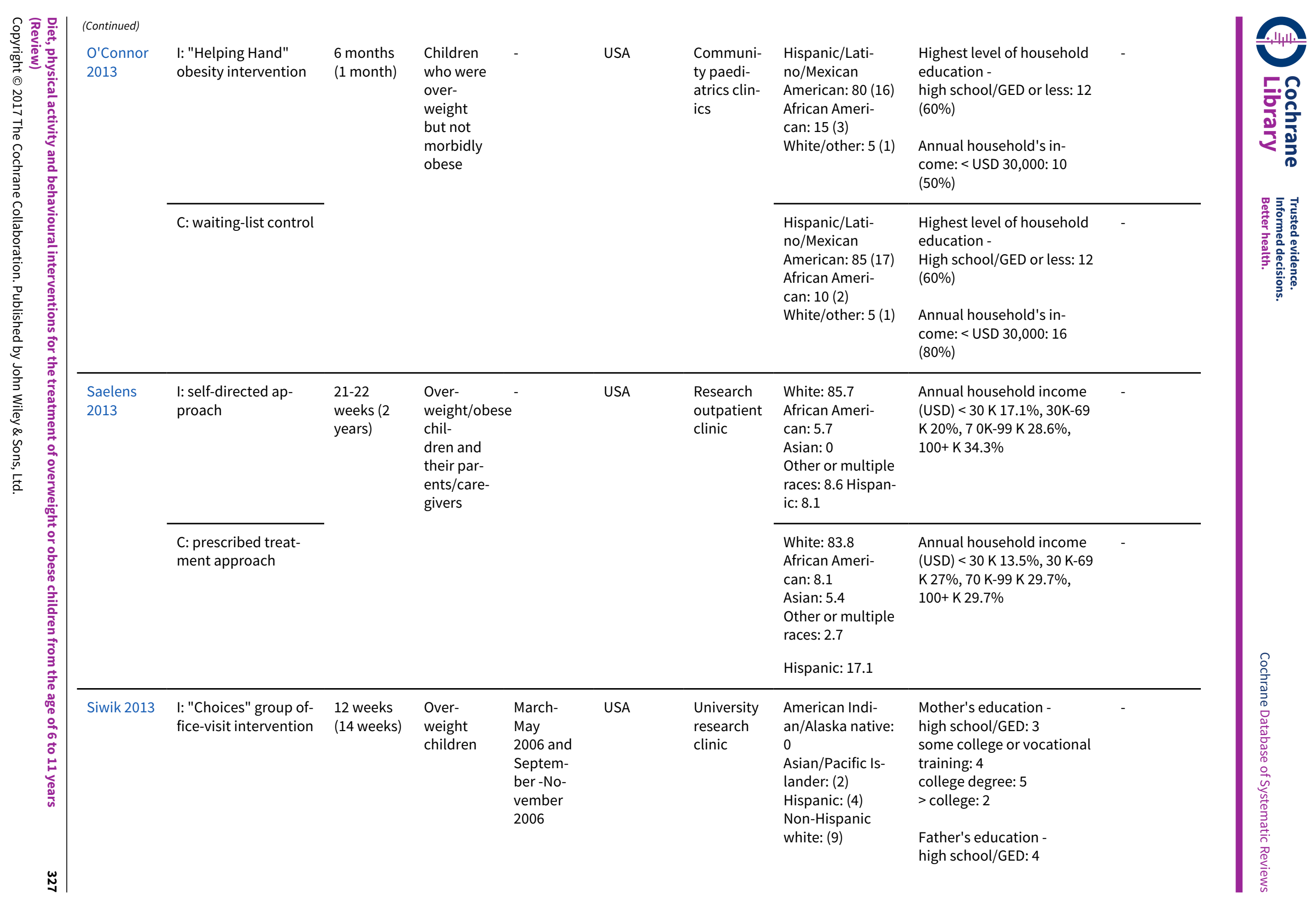


C: lagged control

group some college or vocational

training: 5

college degree: 3

> college: 2

Family income (USD) -

$<50,000: 6$

$\geq 50,000-100,000: 4$

$>100,000: 3$

\section{American Indi- \\ Mother's education -}

an/Alaska native: high school/GED: 0

(1)

Asian/Pacific Is-

lander: (1)

Hispanic: (3)

Non-Hispanic

white: (12) some college or vocational

training: 5

college degree: 2

> college: 5

Father's education -

high school/GED: 3

some college or vocational

training: 4

college degree: 2

$>$ college: 3

Family income (USD) -

$<50,000: 4$

$\geq 50,000-100,000: 4$

$>100,000: 4$

\begin{tabular}{|c|c|c|c|c|c|c|c|c|c|}
\hline \multirow[t]{4}{*}{ Vann 2013} & $\begin{array}{l}\text { I1: pedometer + DVD } \\
\text { group }\end{array}$ & \multirow[t]{4}{*}{$\begin{array}{l}6 \text { months } \\
\text { (0 months) }\end{array}$} & \multirow{4}{*}{$\begin{array}{l}\text { Over- } \\
\text { weight or } \\
\text { obese chil- } \\
\text { dren aged } \\
4-17\end{array}$} & \multirow[t]{4}{*}{$\begin{array}{l}\text { April } 2011 \\
\text { enrolled }\end{array}$} & \multirow[t]{4}{*}{ USA } & \multirow[t]{4}{*}{$\begin{array}{l}\text { University } \\
\text { clinic }\end{array}$} & \multirow{4}{*}{$\begin{array}{l}\text { Majority of par- } \\
\text { ticipants were } \\
\text { African-American: } \\
79\end{array}$} & - & - \\
\hline & 12: pedometer group & & & & & & & - & - \\
\hline & I3: DVD group & & & & & & & - & - \\
\hline & C: control group & & & & & & & - & - \\
\hline Wake 2013 & C: usual care & $\begin{array}{l}15 \text { months } \\
\text { (0 months) }\end{array}$ & $\begin{array}{l}\text { Obese,aged } \\
3-10\end{array}$ & $\begin{array}{l}\text { Novem- } \\
\text { ber } 2009- \\
\text { July } 2010\end{array}$ & Australia & $\begin{array}{l}\text { GP prac- } \\
\text { tices }\end{array}$ & $\begin{array}{l}\text { Largely white } \\
\text { (including mid- } \\
\text { dle eastern) with } \\
\text { some Asian and } \\
\text { Indian }\end{array}$ & $1030(45.3)$ & - \\
\hline
\end{tabular}




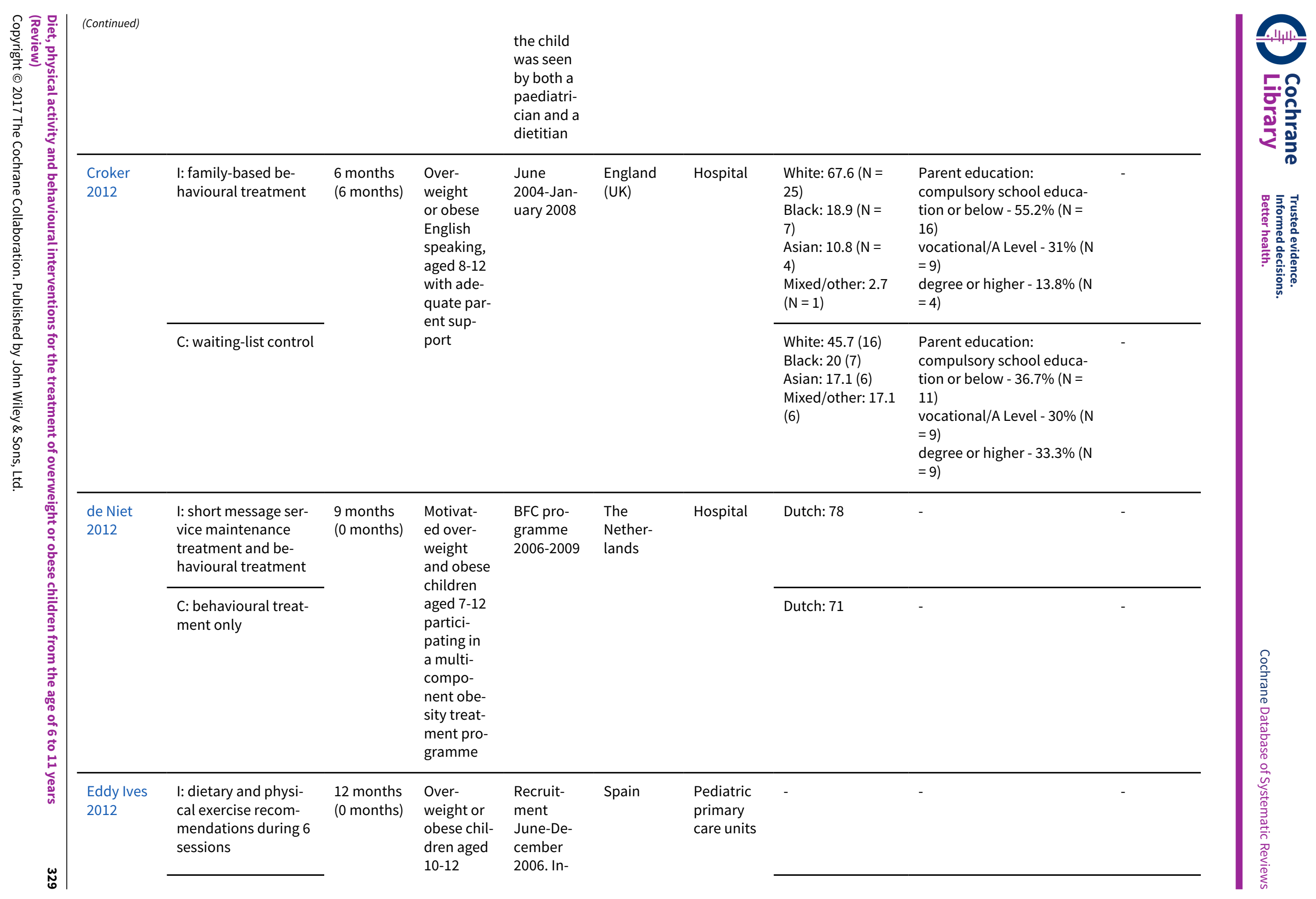




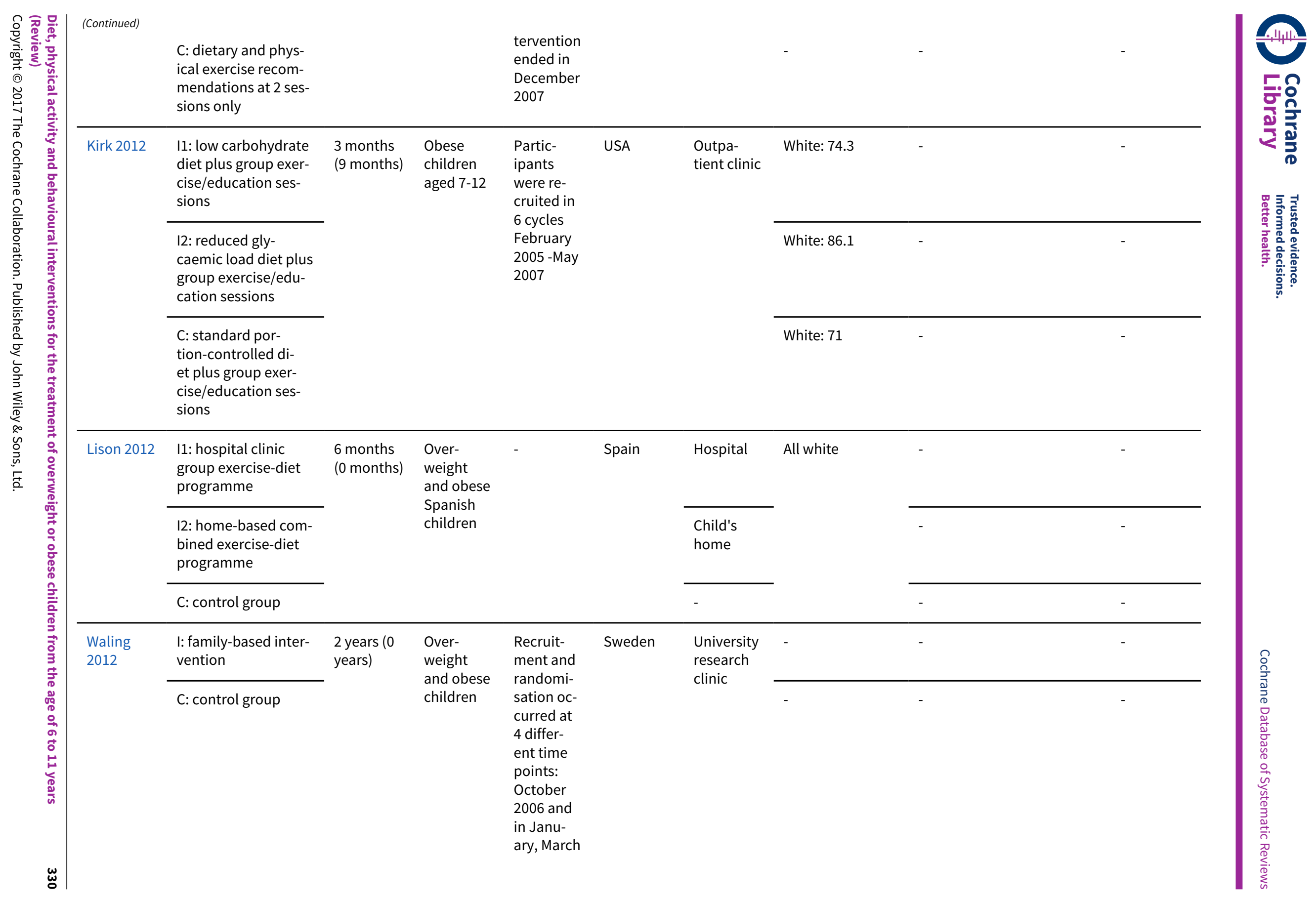




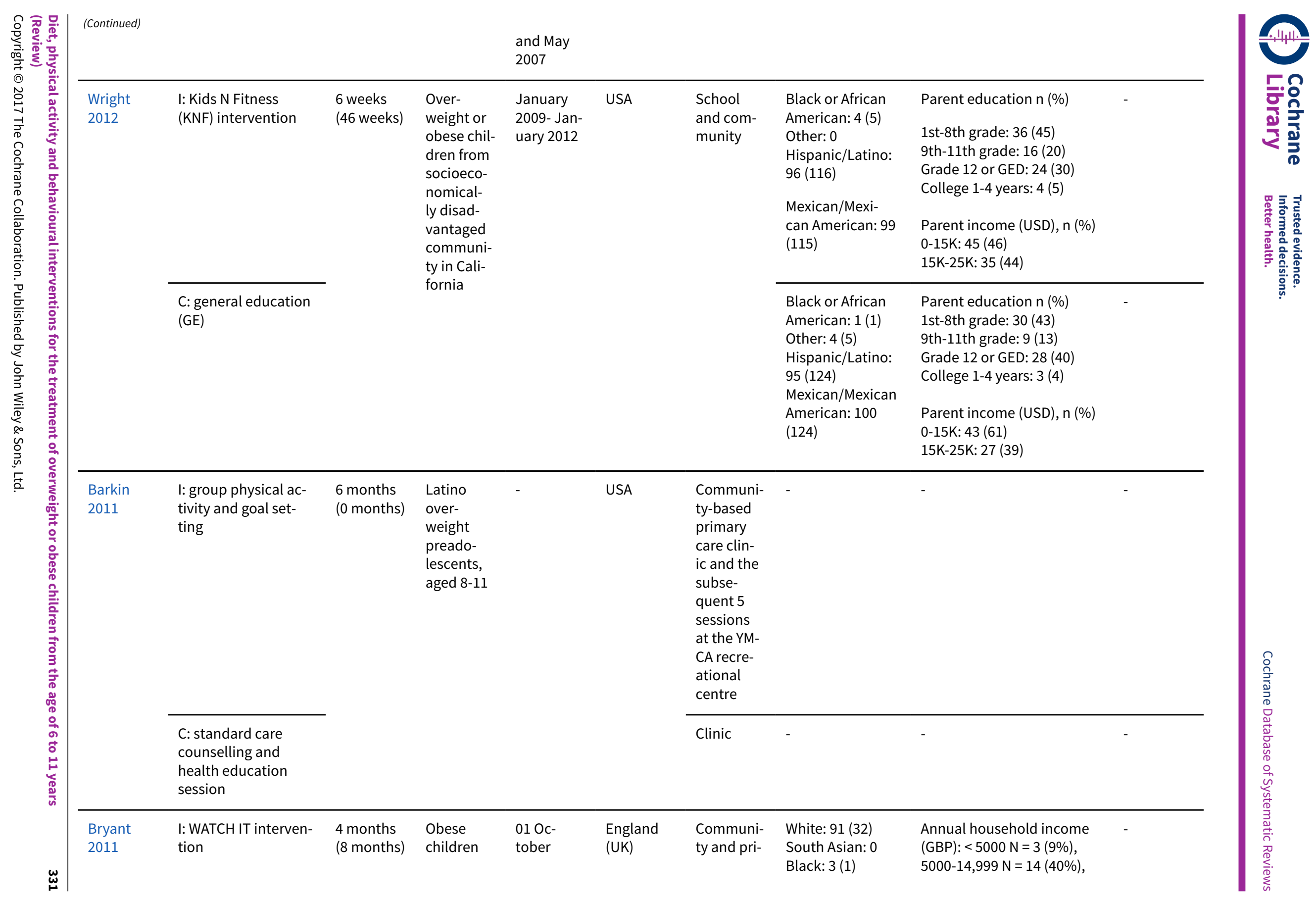




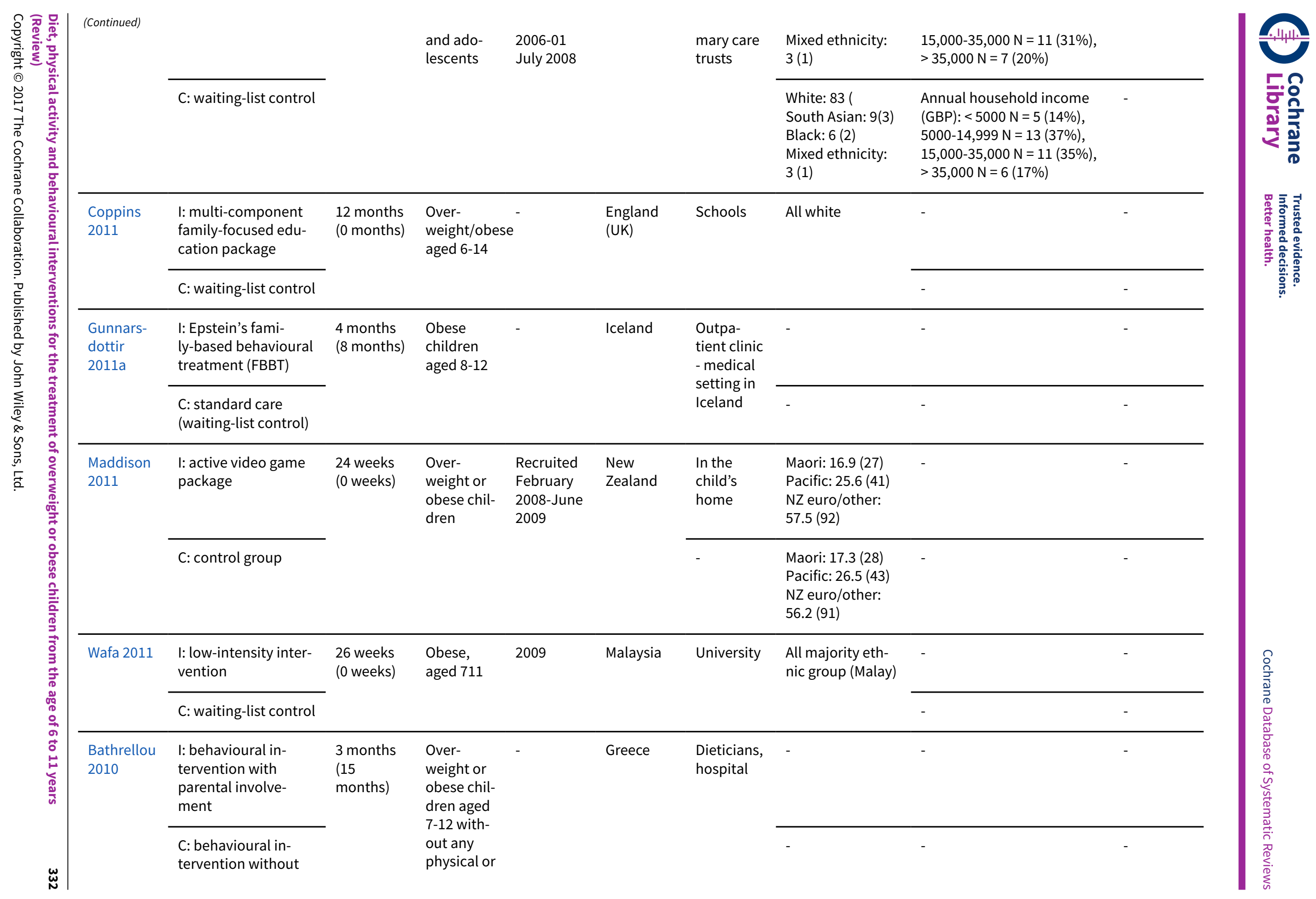




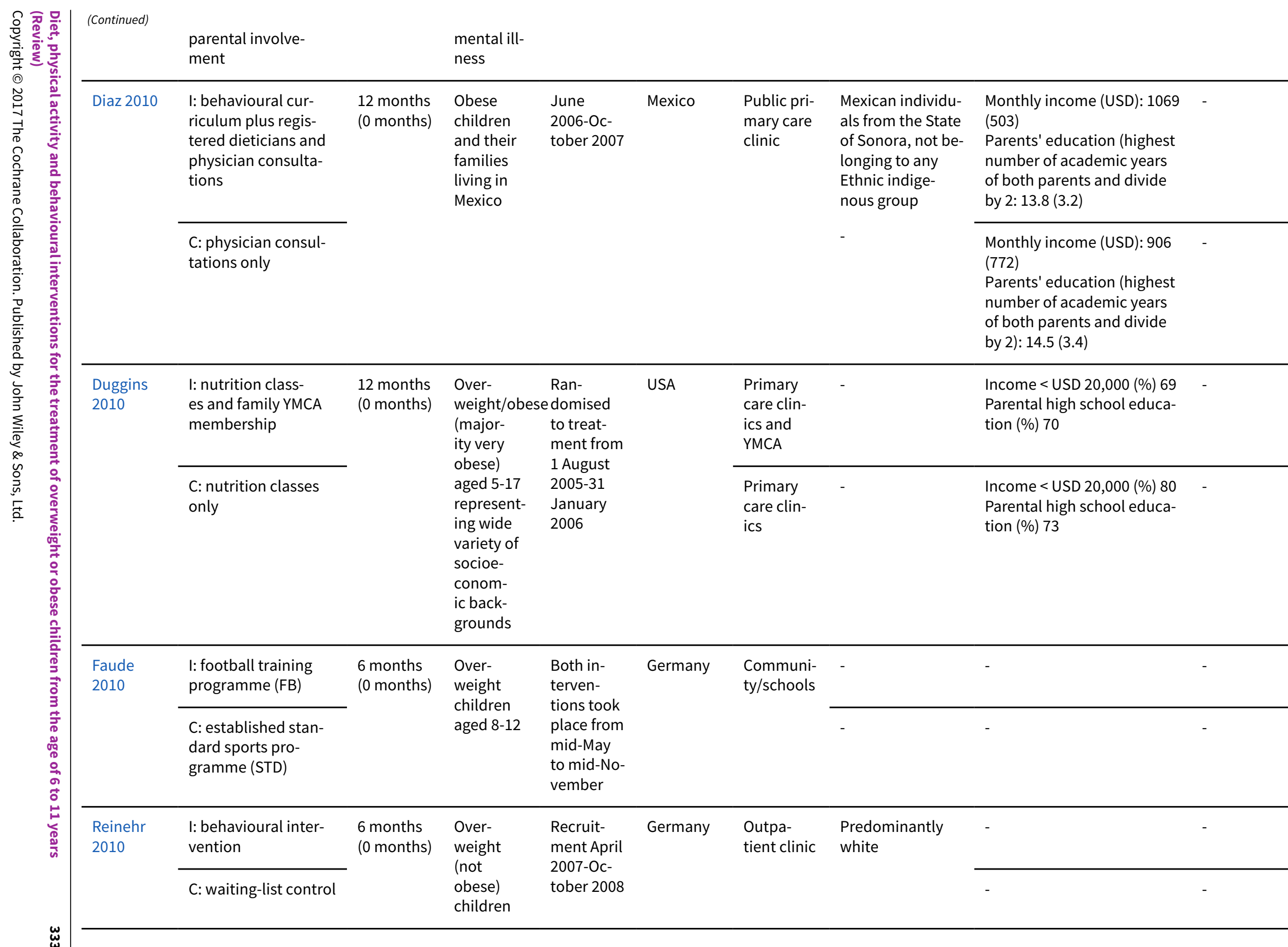




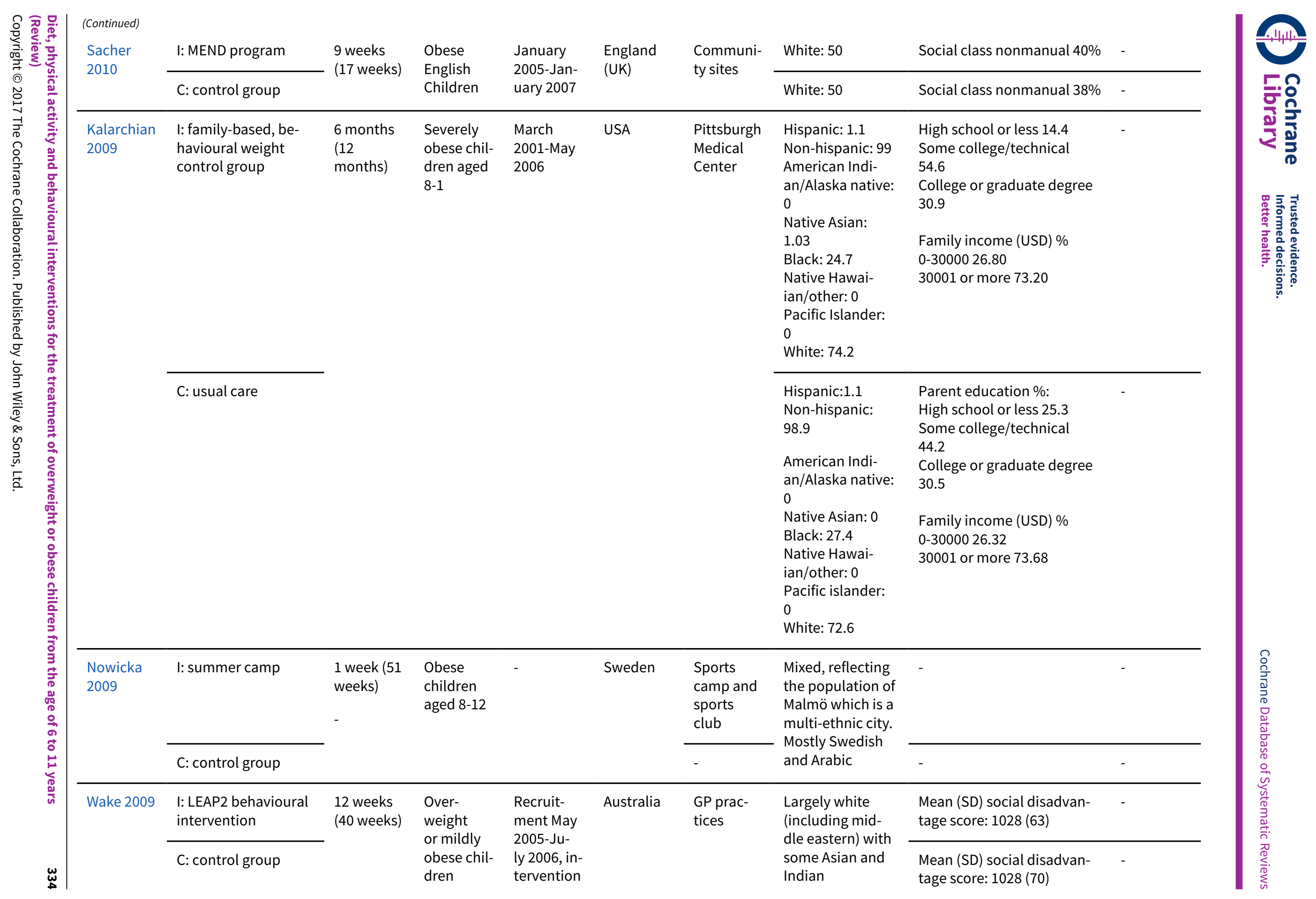




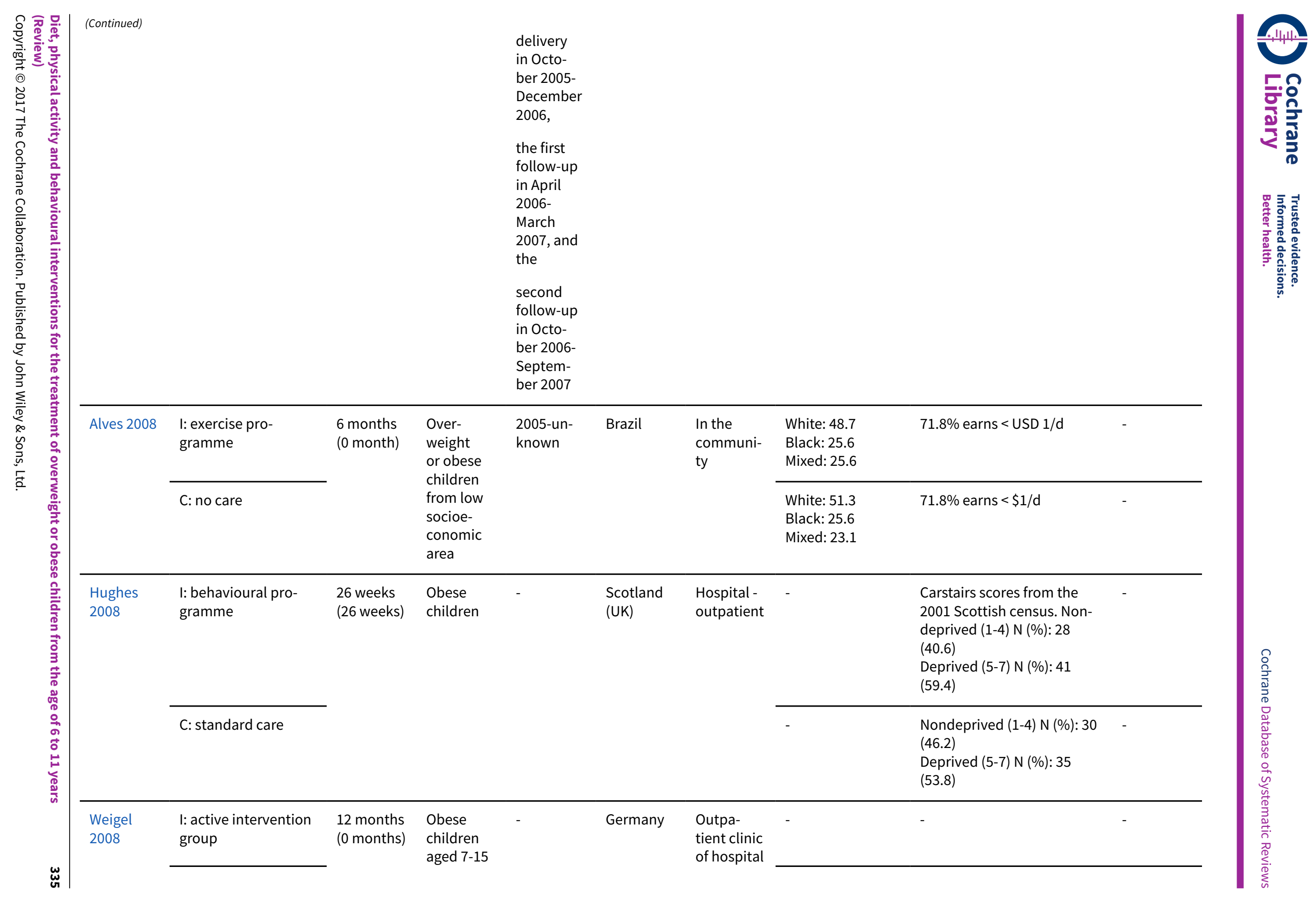




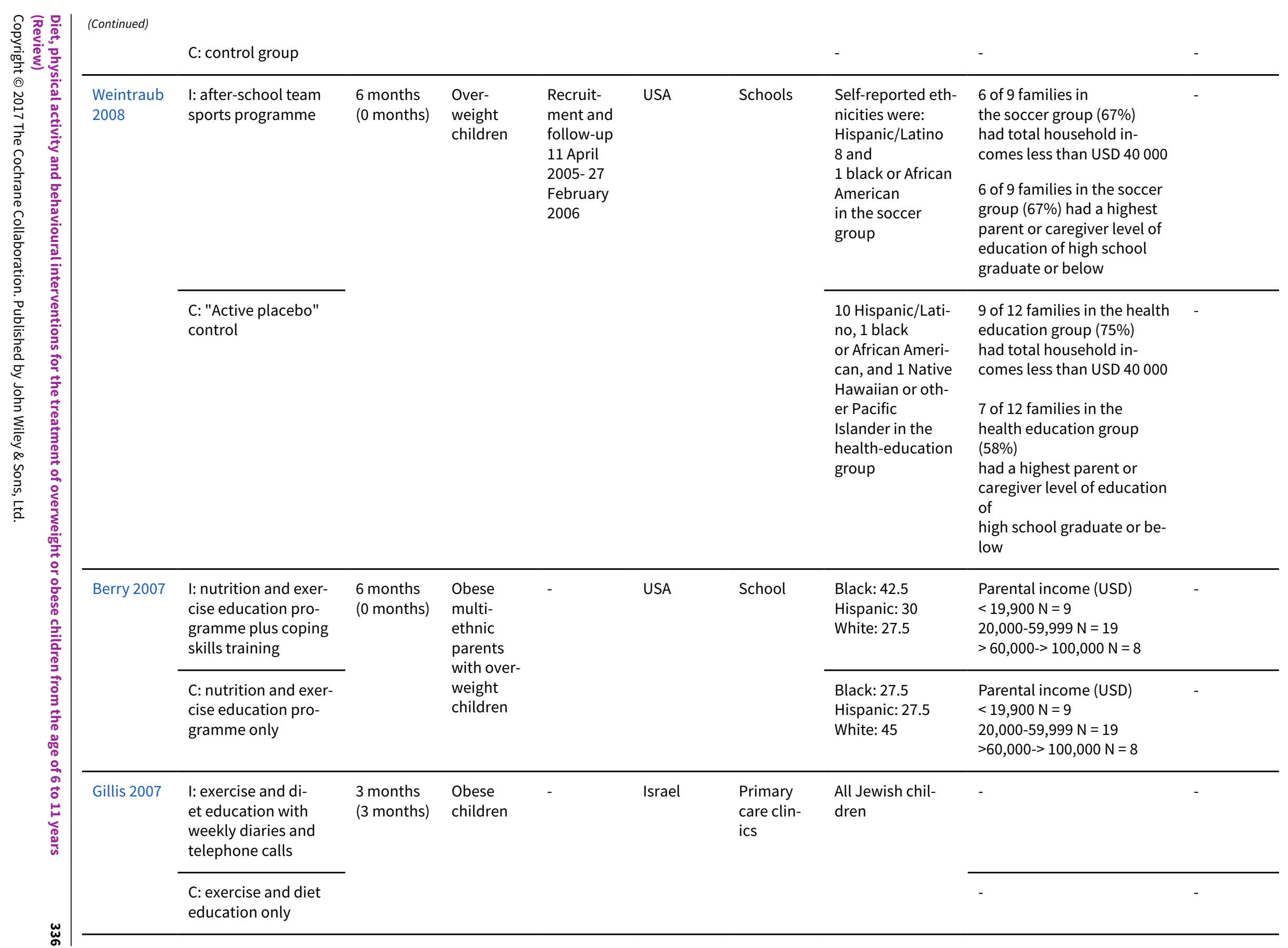




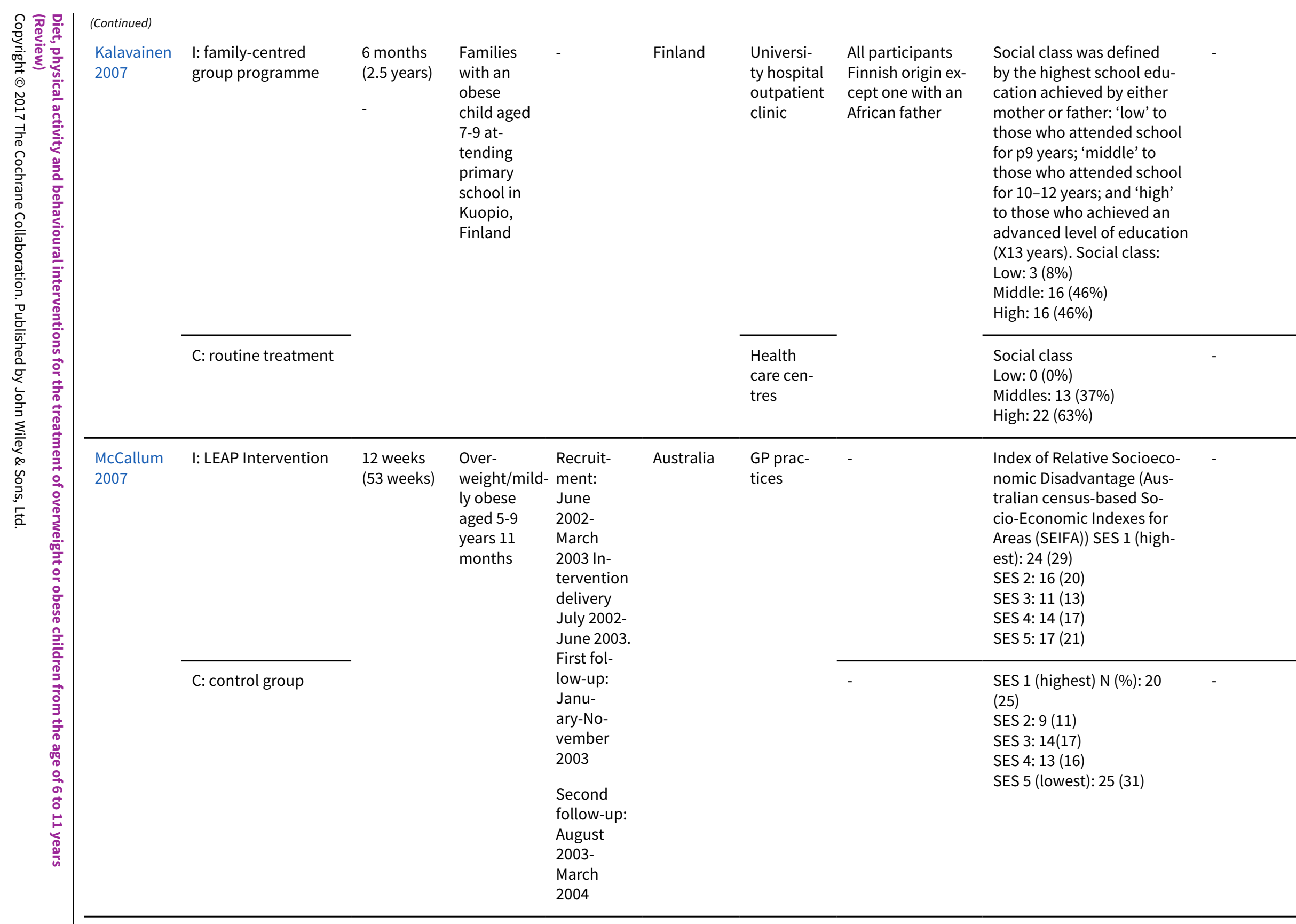




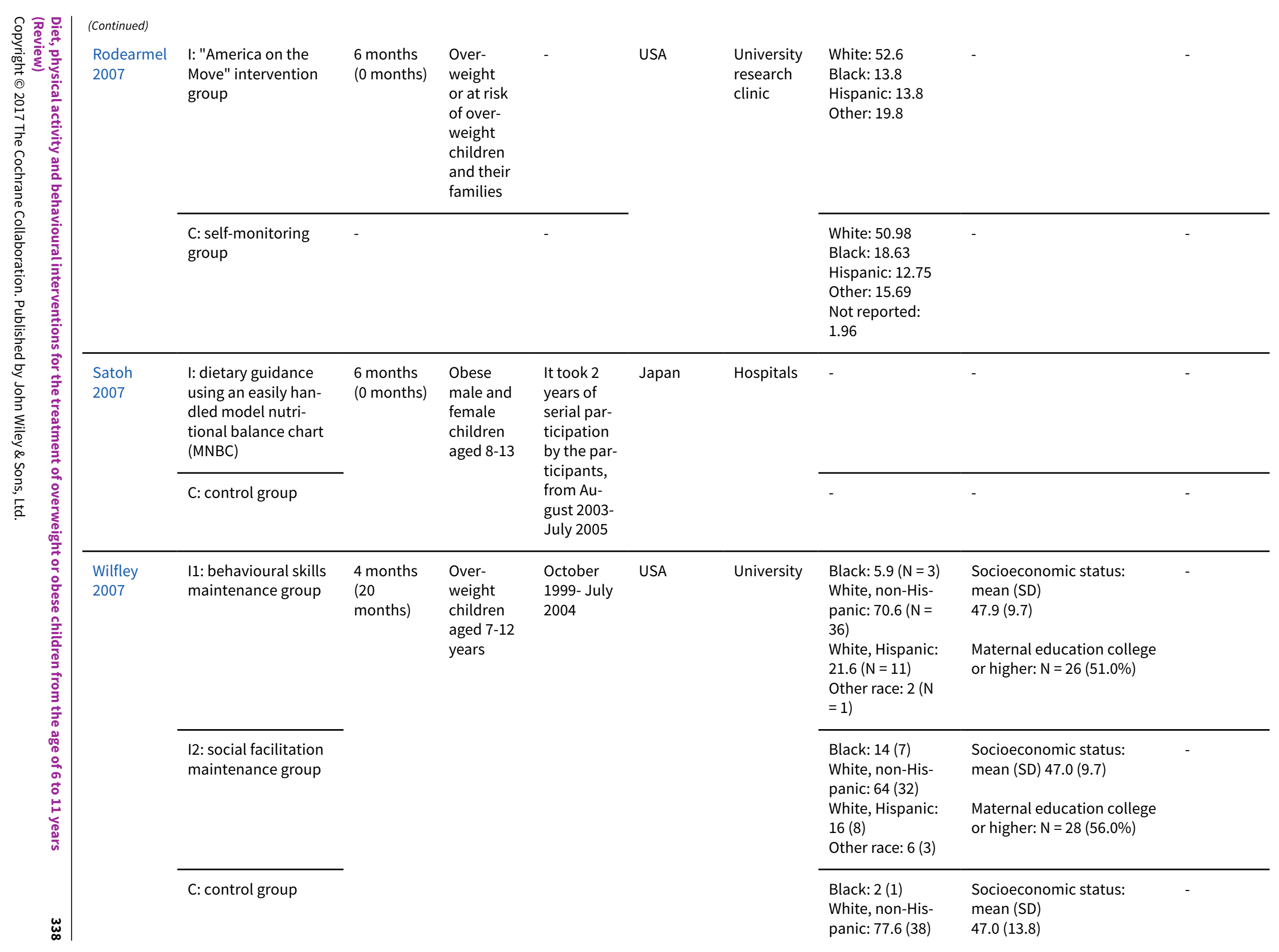




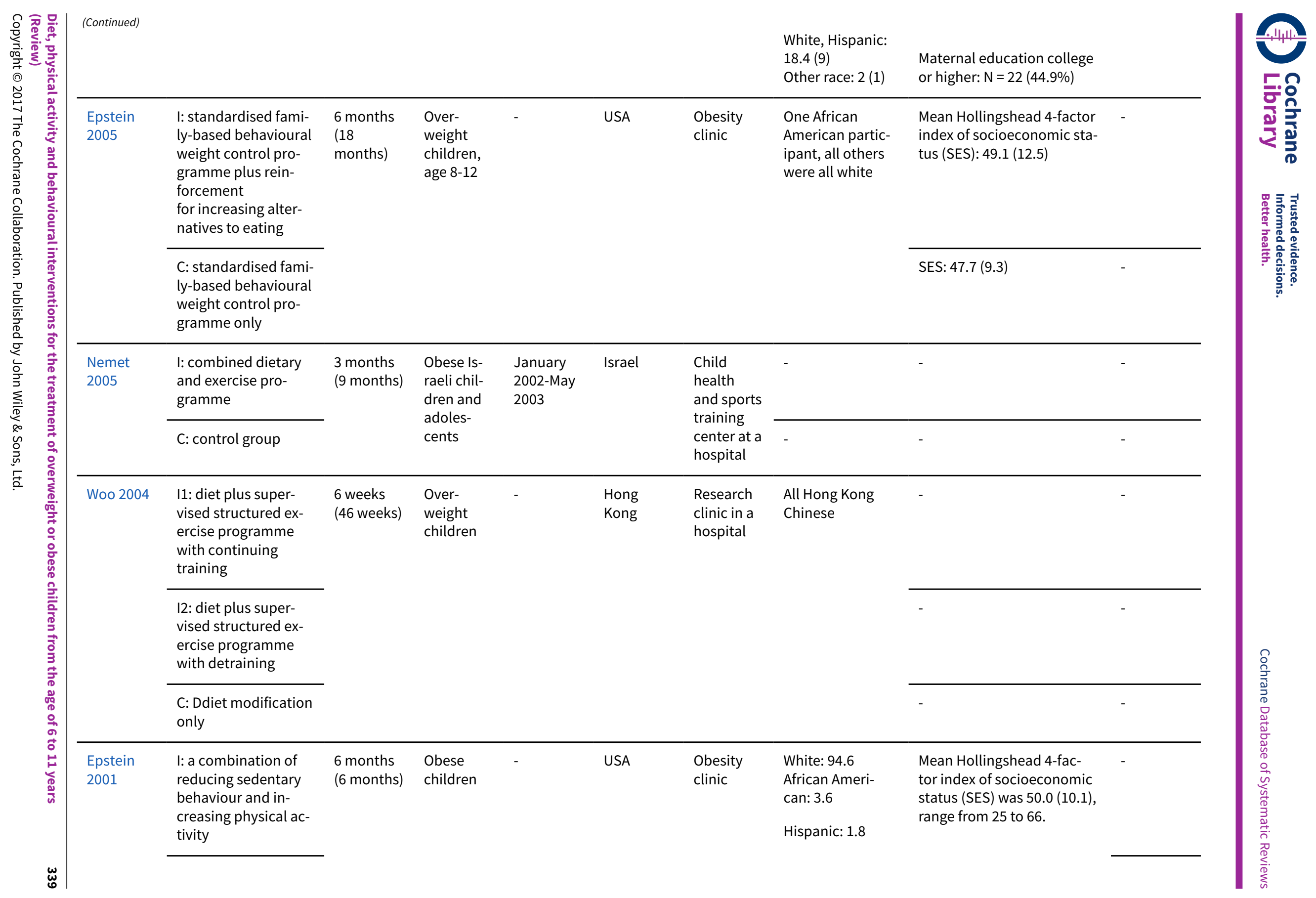




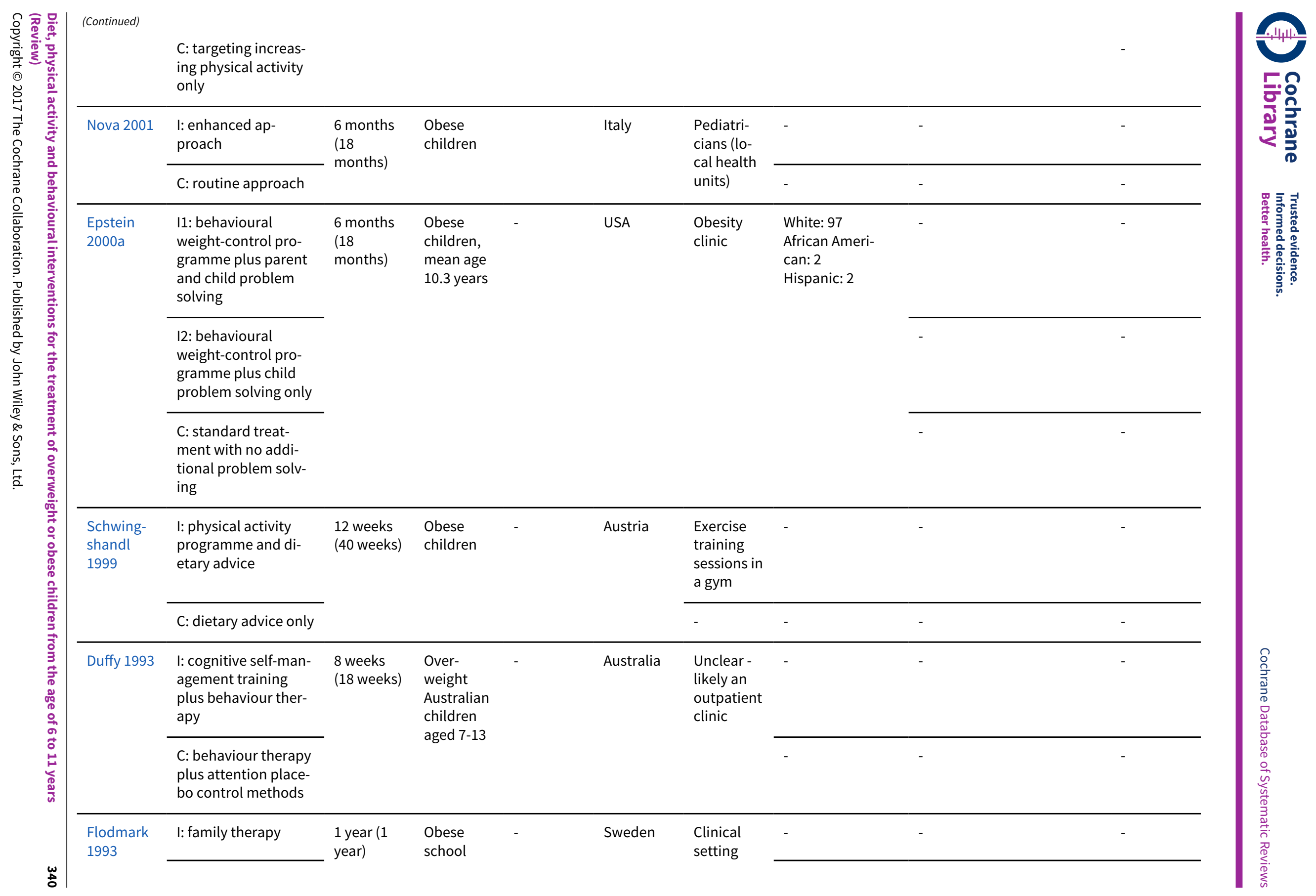




\begin{tabular}{|c|c|c|c|c|c|c|c|c|c|c|}
\hline 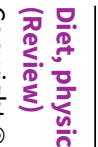 & (Continued) & $\begin{array}{l}\text { C: conventional } \\
\text { treatment }\end{array}$ & & $\begin{array}{l}\text { children } \\
\text { aged } 10-11\end{array}$ & & & & - & - & - \\
\hline 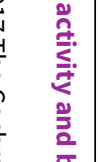 & $\begin{array}{l}\text { Epstein } \\
1985 c\end{array}$ & $\begin{array}{l}\text { I: behaviourally-ori- } \\
\text { ented programme } \\
\text { that emphasised par- } \\
\text { ent management }\end{array}$ & $\begin{array}{l}5 \text { weeks } \\
\text { (47 weeks) }\end{array}$ & $\begin{array}{l}\text { Obese } \\
\text { girls aged } \\
5-8\end{array}$ & - & USA & $\begin{array}{l}\text { Obesity } \\
\text { clinic }\end{array}$ & All white & - & - \\
\hline 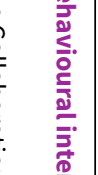 & & $\begin{array}{l}\text { C: provided equal ed- } \\
\text { ucation and atten- } \\
\text { tion but not behav- } \\
\text { ioural principles }\end{array}$ & & & & & & & - & - \\
\hline 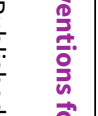 & $\begin{array}{l}\text { Epstein } \\
1985 b\end{array}$ & $\begin{array}{l}\text { I: diet and exercise } \\
\text { education }\end{array}$ & $\begin{array}{l}8 \text { weeks } \\
\text { (10 } \\
\text { months) }\end{array}$ & $\begin{array}{l}\text { Obese } \\
\text { girls aged } \\
8-12\end{array}$ & - & USA & $\begin{array}{l}\text { Obesity } \\
\text { clinic }\end{array}$ & All white & - & - \\
\hline 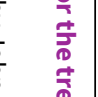 & & $\begin{array}{l}\text { C: diet education on- } \\
\text { ly }\end{array}$ & & & & & & & - & - \\
\hline 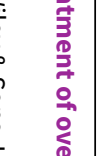 & $\begin{array}{l}\text { Epstein } \\
1985 a\end{array}$ & $\begin{array}{l}\text { I1: diet plus pro- } \\
\text { grammed aerobic ex- } \\
\text { ercise programme }\end{array}$ & $\begin{array}{l}12 \text { months } \\
\text { (12 } \\
\text { months) }\end{array}$ & $\begin{array}{l}\text { Obese } \\
\text { children } \\
\text { aged 8-12 }\end{array}$ & - & USA & $\begin{array}{l}\text { Obesity } \\
\text { clinic }\end{array}$ & All white & - & - \\
\hline 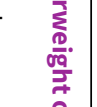 & & $\begin{array}{l}\text { I2: diet plus exercise } \\
\text { programme }\end{array}$ & & $\begin{array}{l}\text { least one } \\
\text { over- } \\
\text { wejght }\end{array}$ & & & & & - & - \\
\hline 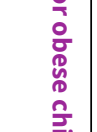 & & $\begin{array}{l}\text { C: diet plus low-in- } \\
\text { tensity callisthenic } \\
\text { exercise programme }\end{array}$ & & $\begin{array}{l}\text { parent re- } \\
\text { siding in } \\
\text { the USA }\end{array}$ & & & & & - & - \\
\hline $\begin{array}{l}\overline{0} \\
\stackrel{0}{2} \\
\overrightarrow{\vec{B}} \\
\end{array}$ & $\begin{array}{l}\text { Epstein } \\
1984 a\end{array}$ & $\begin{array}{l}\text { 11: diet-plus-exercise } \\
\text { group }\end{array}$ & $\begin{array}{l}28 \text { weeks } \\
\text { (0 weeks) }\end{array}$ & Obese & - & USA & $\begin{array}{l}\text { Obesity } \\
\text { clinic }\end{array}$ & - & - & - \\
\hline 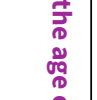 & & 12: diet only & $\begin{array}{l}28 \text { weeks } \\
\text { (0 weeks) }\end{array}$ & & & & & - & - & - \\
\hline \begin{tabular}{l|l}
0 \\
+ \\
+ \\
\\
\end{tabular} & & C: waiting-list control & $\begin{array}{l}28 \text { weeks } \\
\text { (0 weeks) }\end{array}$ & & & & & - & - & - \\
\hline & - denotes & reported & & & & & & & & \\
\hline
\end{tabular}




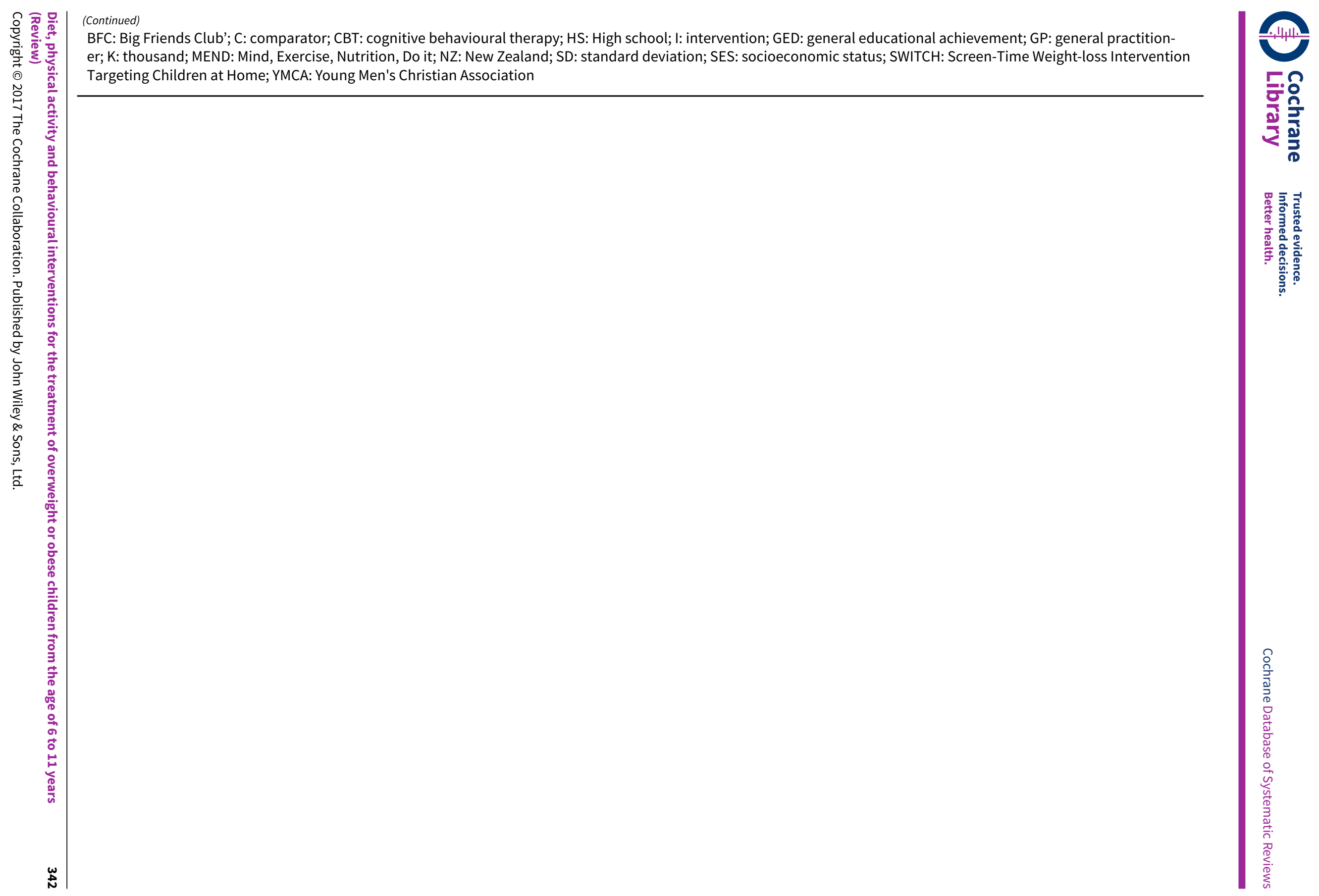


Appendix 4. Baseline characteristics (II)

\begin{tabular}{|c|c|c|c|c|c|c|c|c|}
\hline & $\begin{array}{l}\text { Intervention(s) and compara- } \\
\text { tor(s) }\end{array}$ & $\begin{array}{l}\text { Sex } \\
\text { (female \%) }\end{array}$ & $\begin{array}{l}\text { Age } \\
\text { (mean/ } \\
\text { range years } \\
\text { (SD), or as } \\
\text { reported) }\end{array}$ & $\begin{array}{l}\text { BMI / BMI z score } \\
\text { (mean } \mathrm{kg} / \mathrm{m}^{2} / \text { unit } \\
\text { (SD)) }\end{array}$ & $\begin{array}{l}\text { Body } \\
\text { weight } \\
\text { (mean kg } \\
\text { (SD)) }\end{array}$ & $\begin{array}{l}\text { Parental } \\
\text { BMI }\end{array}$ & $\begin{array}{l}\text { Comedica- } \\
\text { tions/co-in- } \\
\text { terventions } \\
\text { (\% of par- } \\
\text { ticipants) }\end{array}$ & $\begin{array}{l}\text { Comorbidi } \\
\text { ties } \\
\text { (\% of par- } \\
\text { ticipants) }\end{array}$ \\
\hline \multirow[t]{2}{*}{ NCT02436330 } & $\begin{array}{l}\text { I: exergaming and didactic } \\
\text { healthy teaching }\end{array}$ & 61.7 & $10.0(1.2)$ & BMI z score: 2.2 (2.82) & - & - & - & - \\
\hline & C: didactic healthy teaching & 50.0 & $10.1(1.1)$ & BMI z score: 2.2 (3.34) & - & - & - & - \\
\hline \multirow[t]{2}{*}{ Ho 2016} & $\begin{array}{l}\text { I: standard nutrition counselling } \\
\text { plus portion control equipment }\end{array}$ & 47.9 & $11.5(2.15)$ & $\begin{array}{l}\text { BMI } 29.80(5.63) \\
\text { BMI z score: } 2.74(0.42)\end{array}$ & - & - & - & - \\
\hline & C: standard nutrition counselling & 60.8 & $10.9(2.33)$ & $\begin{array}{l}\text { BMI } 28.53(5.67) \\
\text { BMI z score: } 2.69(0.35)\end{array}$ & - & - & - & - \\
\hline \multirow[t]{2}{*}{$\begin{array}{l}\text { Warschburg- } \\
\text { er } 2016\end{array}$} & $\begin{array}{l}\text { I: parental CBT training group } \\
\text { plus child in-patient intervention }\end{array}$ & 53.4 & $11.3(1.3)$ & BMI SDS: $2.6(0.4)$ & - & $\begin{array}{l}\text { BMI: } 29.7 \\
(7.1)\end{array}$ & $\begin{array}{l}\text { Child in-pa- } \\
\text { tient inter- }\end{array}$ & - \\
\hline & $\begin{array}{l}\text { C: parental information-only } \\
\text { group plus child in-patient inter- } \\
\text { vention }\end{array}$ & 51.5 & $11.3(1.3)$ & BMI SDS: $2.5(0.4)$ & - & $\begin{array}{l}\text { BMI: } 28.7 \\
(6.7)\end{array}$ & & - \\
\hline \multirow[t]{2}{*}{$\begin{array}{l}\text { Epstein } \\
2015\end{array}$} & $\begin{array}{l}\text { I: family-based treatment + vari- } \\
\text { ety of high energy-dense foods }\end{array}$ & 61.5 & $10.5(1.4)$ & $\begin{array}{l}\text { BMI: } 31.1(7.5) \\
\text { BMI z score: } 2.3(0.4)\end{array}$ & $73.8(22.7)$ & $\begin{array}{l}\text { BMI: } 39.5 \\
(7.5)\end{array}$ & - & - \\
\hline & C: family-based treatment only & 54.5 & $10.5(1.4)$ & $\begin{array}{l}\text { BMI: } 28.0(3.0) \\
\text { BMI z score: } 2.2(0.4)\end{array}$ & $63.0(10.4)$ & $\begin{array}{l}\text { BMI: } 37.2 \\
(6.8)\end{array}$ & - & - \\
\hline \multirow[t]{2}{*}{ Larsen 2015} & $\begin{array}{l}\text { I: an education programme in ad- } \\
\text { dition to health consultations }\end{array}$ & 66.7 & $6.1(1.1)$ & BMI z score: $2.88(0.87)$ & - & - & - & - \\
\hline & C: health consultations only & 62.9 & $6.3(1.3)$ & BMI: 2.79 (0.82) & - & - & & \\
\hline $\begin{array}{l}\text { Serra-Paya } \\
2015\end{array}$ & I: Nereu group & 50.0 & $10.1(1.98)$ & $\begin{array}{l}\text { BMI: } 25.22(3.35) \\
\text { BMI z score: } 2.47(0.51)\end{array}$ & $52.54(13.29)$ & - & - & - \\
\hline
\end{tabular}




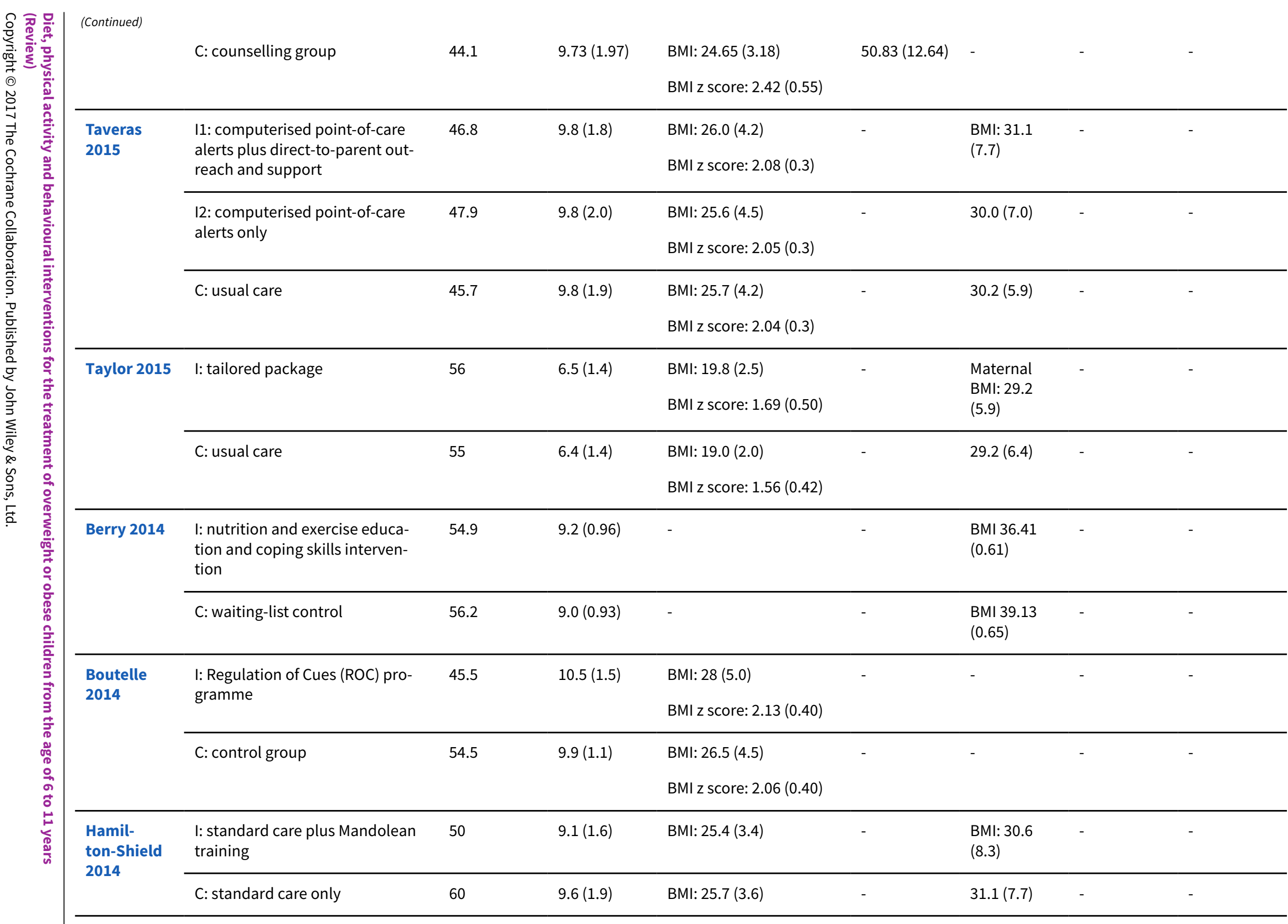




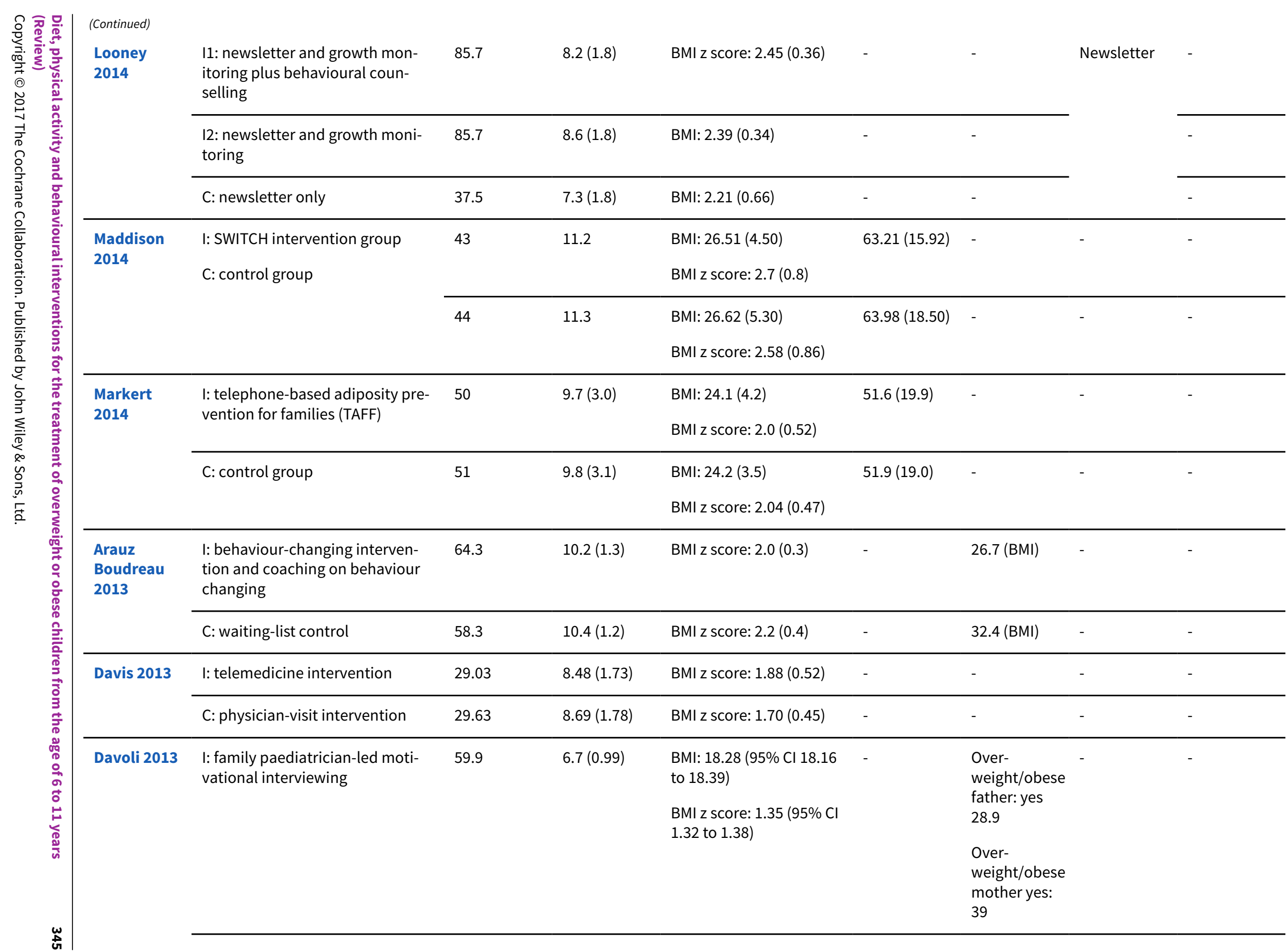




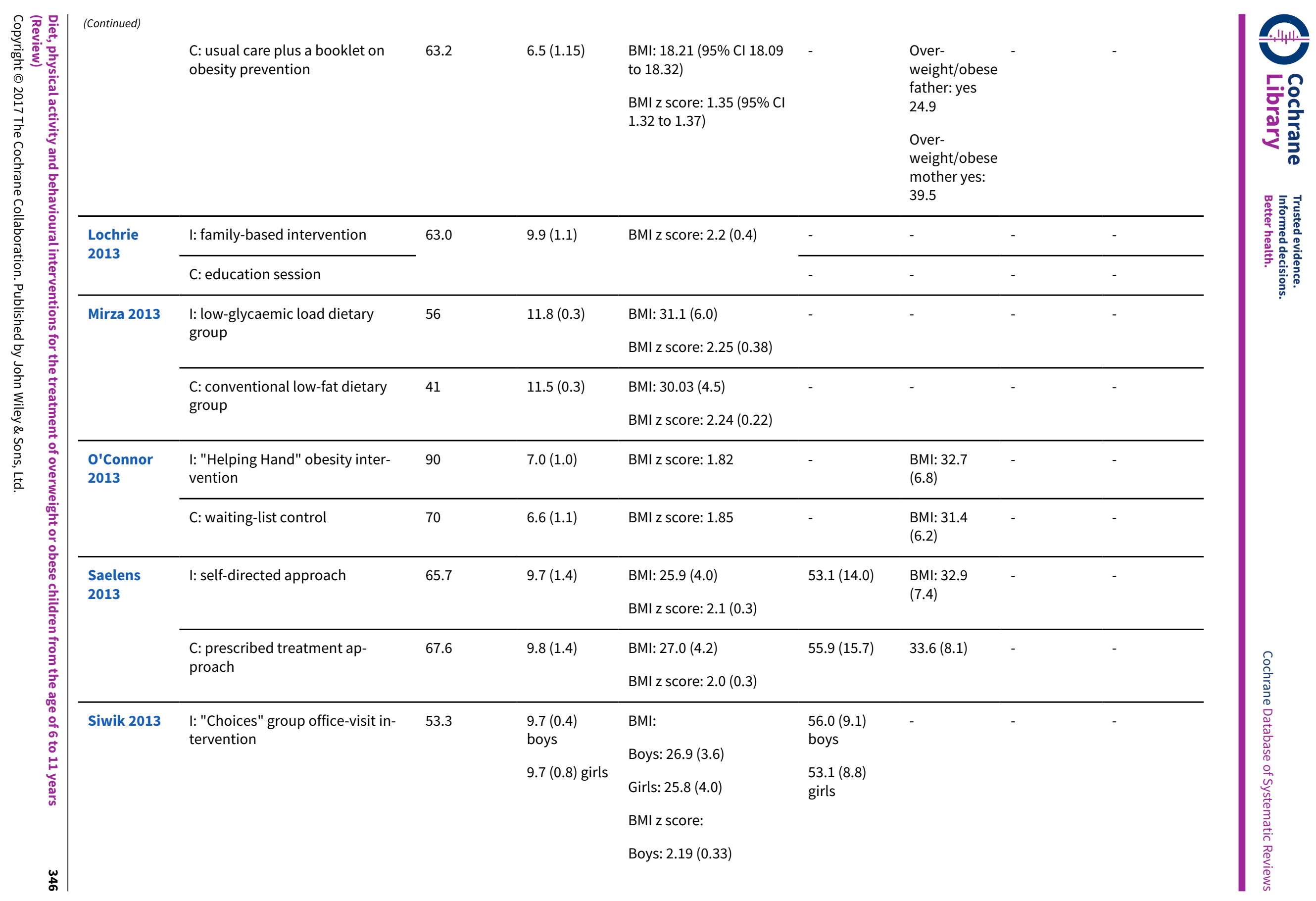




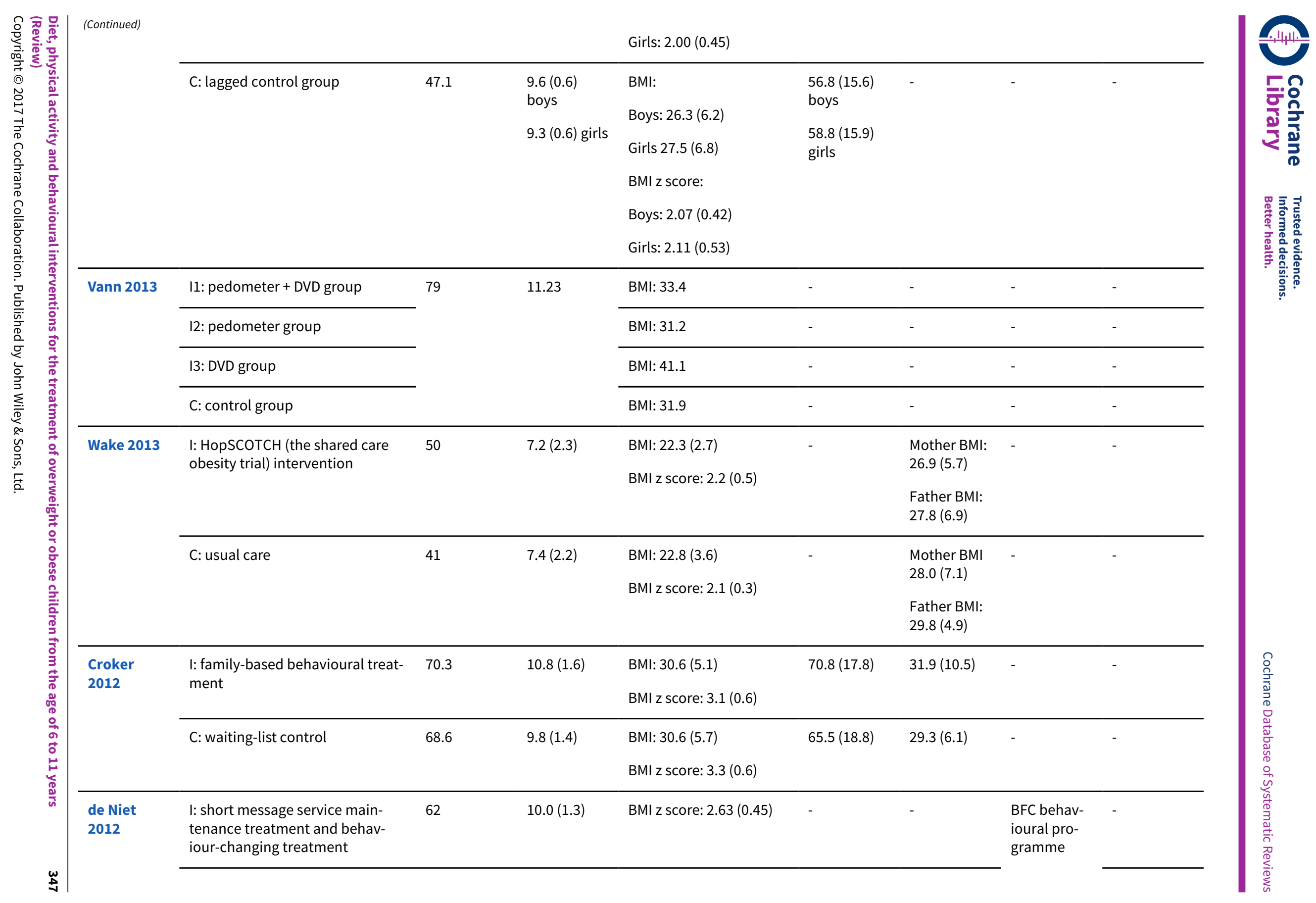




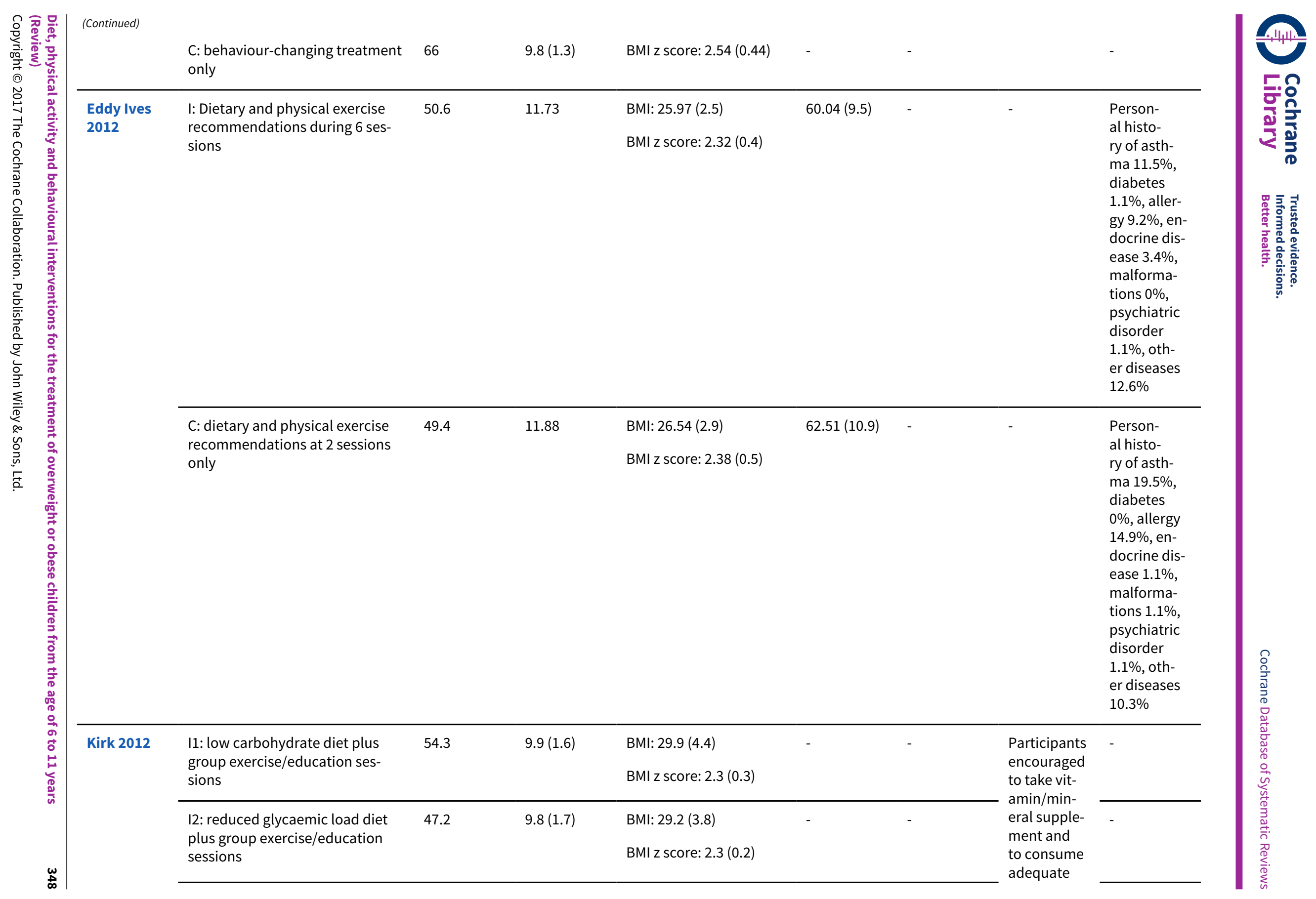




\begin{tabular}{|c|c|c|c|c|c|c|c|c|c|c|}
\hline 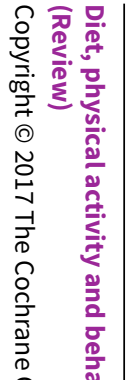 & (Continued) & $\begin{array}{l}\text { C: standard portion-controlled } \\
\text { diet plus group exercise/educa- } \\
\text { tion sessions }\end{array}$ & 74.2 & $9.7(1.3)$ & $\begin{array}{l}\text { BMI: } 29.1(3.8) \\
\text { BMI z score: } 2.3(0.3)\end{array}$ & - & - & $\begin{array}{l}\text { fluids with } \\
\text { goal of } 48 \\
\text { ounces/d, } \\
\text { preferably } \\
\text { water. Same } \\
\text { exercise ses- } \\
\text { sions given } \\
\text { to all partic- } \\
\text { ipants }\end{array}$ & - & 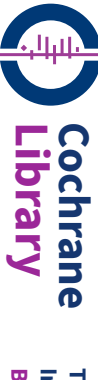 \\
\hline 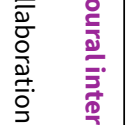 & Lison 2012 & $\begin{array}{l}\text { I1: hospital clinic group exer- } \\
\text { cise-diet programme }\end{array}$ & 51.1 & $12.3(1.9)$ & $\begin{array}{l}\text { BMI: } 28.5(3.8) \\
\text { BMI z score: } 2.11(0.33)\end{array}$ & $67.2(17.3)$ & - & - & - & 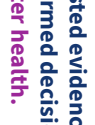 \\
\hline 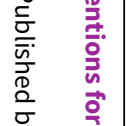 & & $\begin{array}{l}\text { 12: Home-based combined exer- } \\
\text { cise-diet programme }\end{array}$ & 48.8 & $11.9(2.2)$ & $\begin{array}{l}\text { BMI: } 29.7(3.7) \\
\text { BMI z score: } 2.10(0.26)\end{array}$ & $74.0(16.2)$ & - & - & - & \\
\hline 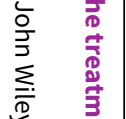 & & C: control group & 45.8 & $11.2(2.1)$ & $\begin{array}{l}\text { BMI: } 29.2(3.9) \\
\text { BMI z score: } 2.23(0.21)\end{array}$ & $69.2(18.3)$ & - & - & - & \\
\hline 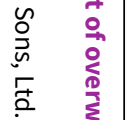 & $\begin{array}{l}\text { Waling } \\
2012\end{array}$ & I: family-based intervention & 44 & $10.5(1.15)$ & $\begin{array}{l}\text { BMI: } 23.4(2.79) \\
\text { BMI z score: } 2.03(0.88)\end{array}$ & $52.1(9.95)$ & - & - & $\begin{array}{l}\text { At baseline, } \\
3 \text { children } \\
\text { in the study }\end{array}$ & \\
\hline 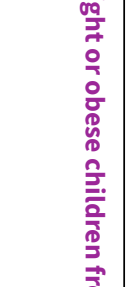 & & C: control group & 58 & $10.5(1.02)$ & $\begin{array}{l}\text { BMI: } 22.6(2.39) \\
\text { BMI z score: } 1.77(0.71)\end{array}$ & $50.4(9.99)$ & - & - & $\begin{array}{l}\text { fined as hav- } \\
\text { ing MetS, } 1 \\
\text { participant } \\
\text { in the in- } \\
\text { tervention } \\
\text { group, and } 2 \\
\text { in control }\end{array}$ & \\
\hline 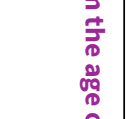 & Wright 2012 & $\begin{array}{l}\text { I: Kids N Fitness (KNF) interven- } \\
\text { tion }\end{array}$ & 58 & $9.0(1.6)$ & $\begin{array}{l}\text { BMI: } 21.89(6.26) \\
\text { BMI z score: } 2.3(0.41)\end{array}$ & - & - & - & - & 謇 \\
\hline 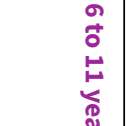 & & C: general education (GE) & 62 & $8.3(1.1)$ & $\begin{array}{l}\text { BMI: } 21.25(6.68) \\
\text { BMI z score: } 2.28(0.5)\end{array}$ & - & - & - & - & 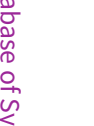 \\
\hline$\stackrel{\omega}{.}$ & Barkin 2011 & $\begin{array}{l}\text { I: group physical activity and goal } \\
\text { setting }\end{array}$ & 54.1 & $9.3(1.2)$ & BMI: $25.8(4.9)$ & - & $\begin{array}{l}\text { BMI: } 33.6 \\
(7.8) \% \\
\text { Overweight: } \\
18.9 \%\end{array}$ & - & - & . \\
\hline
\end{tabular}




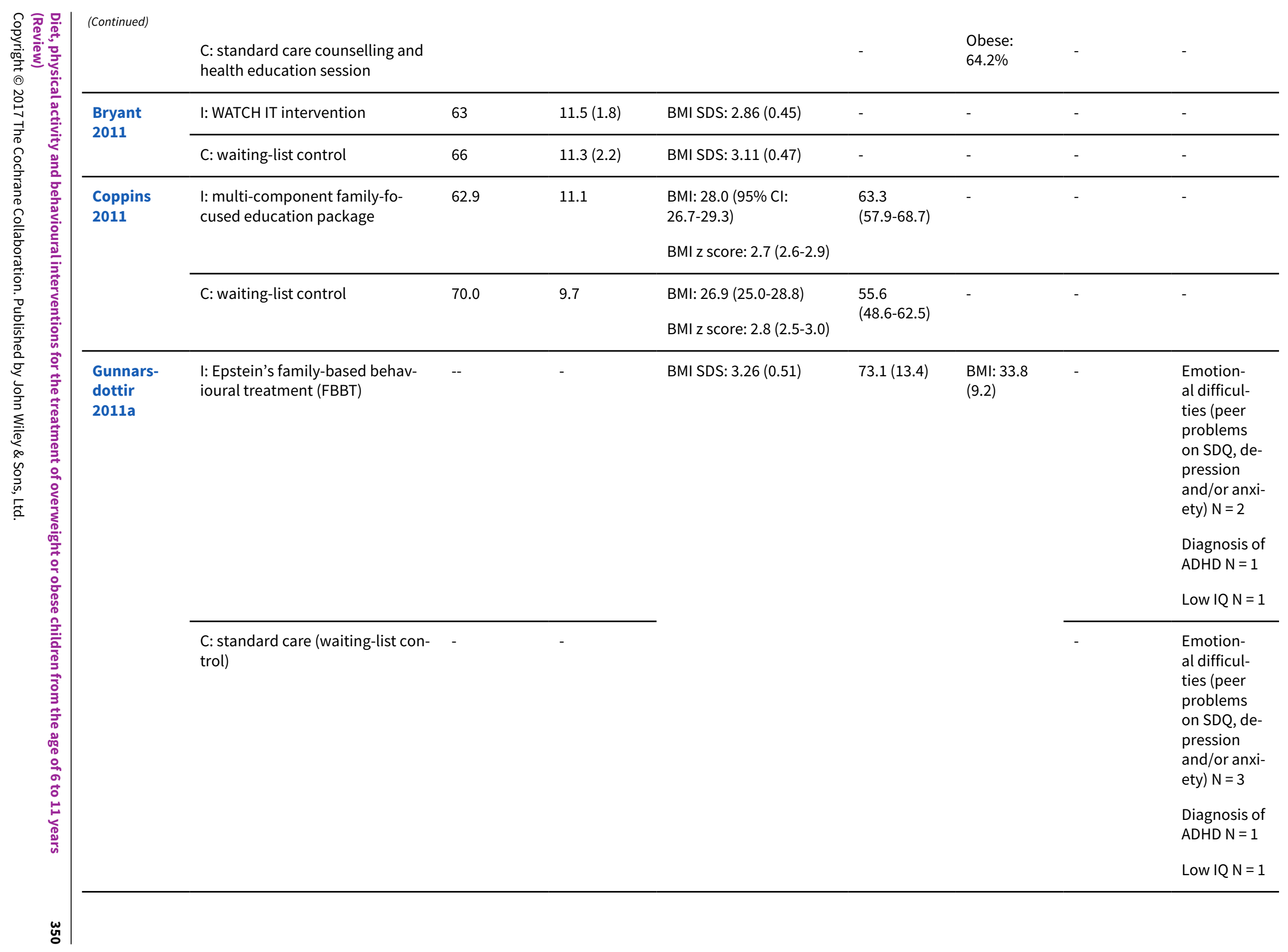




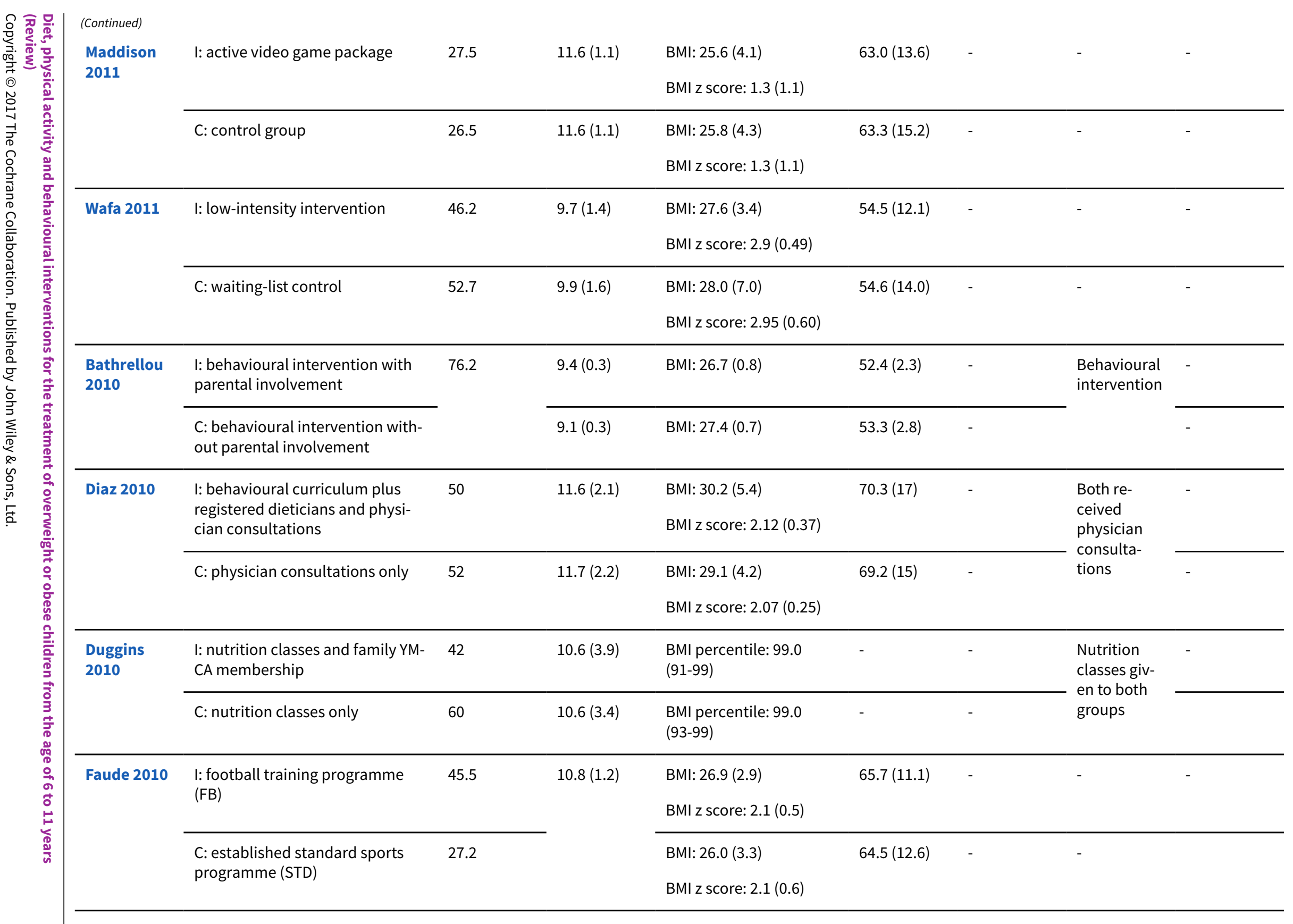




\begin{tabular}{|c|c|c|c|c|c|c|c|c|c|c|}
\hline 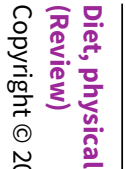 & $\begin{array}{l}\text { (Continued) } \\
\text { Reinehr } \\
2010\end{array}$ & I: behaviour-changing treatment & 62 & $11.6(1.6)$ & $\begin{array}{l}\text { BMI: } 24.2(1.5) \\
\text { BMI z score: } 1.73(0.22)\end{array}$ & - & - & - & - & ת. \\
\hline 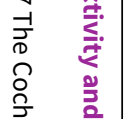 & & C: waiting-list control & 59 & $11.4(1.7)$ & $\begin{array}{l}\text { BMI: } 23.3(1.7) \\
\text { BMI z score: } 1.59(0.15)\end{array}$ & - & - & - & - & ํํㄹ \\
\hline 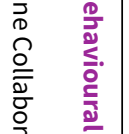 & $\begin{array}{l}\text { Sacher } \\
2010\end{array}$ & I: MEND program & 63 & $10.3(1.3)$ & $\begin{array}{l}\text { BMI: } 27.2(3.7) \\
\text { BMI z score: } 2.77(0.51)\end{array}$ & $59.2(12.5)$ & $\begin{array}{l}\text { Maternal } \\
\text { BMI: } 29.3 \\
(6.2)\end{array}$ & - & - & 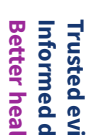 \\
\hline 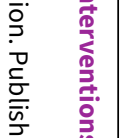 & & C: control group & 45 & $10.2(1.3)$ & $\begin{array}{l}\text { BMI: } 27.1(4.9) \\
\text { BMIz score: } 2.76(0.63)\end{array}$ & $58.3(14.8)$ & $\begin{array}{l}\text { Maternal } \\
\text { BMI: } 30.5 \\
(6.5)\end{array}$ & - & - & 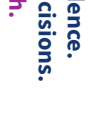 \\
\hline 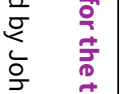 & $\begin{array}{l}\text { Kalarchian } \\
2009\end{array}$ & $\begin{array}{l}\text { I: family-based, behavioural } \\
\text { weight control group }\end{array}$ & 55.67 & $10.07(1.19)$ & BMI: $31.71(5.21)$ & $70.17(18.44)$ & $\begin{array}{l}\text { BMI: } 35.60 \\
(9.20)\end{array}$ & - & - & \\
\hline 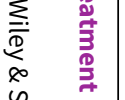 & & C: usual care & 57.89 & $10.30(1.21)$ & BMI: 32.54 (4.67) & $72.74(16.63)$ & $\begin{array}{l}\text { BMI: } 35.60 \\
(9.20)\end{array}$ & - & - & \\
\hline 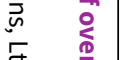 & Nowicka & I: summer camp & - & - & - & - & - & - & - & \\
\hline 墨. & & C: control group & - & - & - & - & - & - & - & \\
\hline 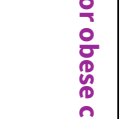 & Wake 2009 & I: LEAP2 behavioural intervention & 60 & $7.4(1.4)$ & $\begin{array}{l}\text { BMI: } 20.2(2.3) \\
\text { BMI z score: } 1.9(0.5)\end{array}$ & - & - & - & - & \\
\hline $\begin{array}{l}\frac{2}{2} \\
\frac{0}{3} \\
\frac{\overrightarrow{3}}{0} \\
\frac{0}{3}\end{array}$ & & C: control group & 61 & $7.6(1.4)$ & $\begin{array}{l}\text { BMI: } 20.3(1.9) \\
\text { BMI z score: } 1.9(0.5)\end{array}$ & - & - & - & - & $\delta$ \\
\hline $\begin{array}{ll}0 \\
0 \\
00\end{array}$ & Alves 2008 & I: exercise programme & 53.8 & $7.97(1.81)$ & BMI: 20.6 (3.33) & $35.4(12.3)$ & - & - & - & 胥 \\
\hline$\stackrel{\circ}{\stackrel{2}{\sim}}$ & & C: no care & 43.6 & $7.85(1.47)$ & BMI: $21.0(2.90)$ & $34.4(9.75)$ & - & - & - & 离 \\
\hline 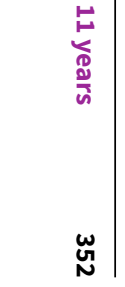 & $\begin{array}{l}\text { Hughes } \\
2008\end{array}$ & I: behavioural programme & 56.5 & $9.1(1.7)$ & $\begin{array}{l}\text { BMI z score median } \\
\text { (IQR): } 3.2 \text { ( } 2.7 \text { to } 3.6)\end{array}$ & $\begin{array}{l}\text { Median } \\
\text { (IQR): } 52.6 \\
(43.8 \text { to } \\
61.2)\end{array}$ & $\begin{array}{l}\text { Maternal } \\
\text { BMI medi- } \\
\text { an (IQR): } \\
28.0(24.2 \text { to } \\
32.8)\end{array}$ & - & - & 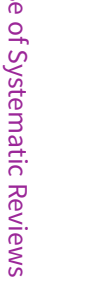 \\
\hline
\end{tabular}




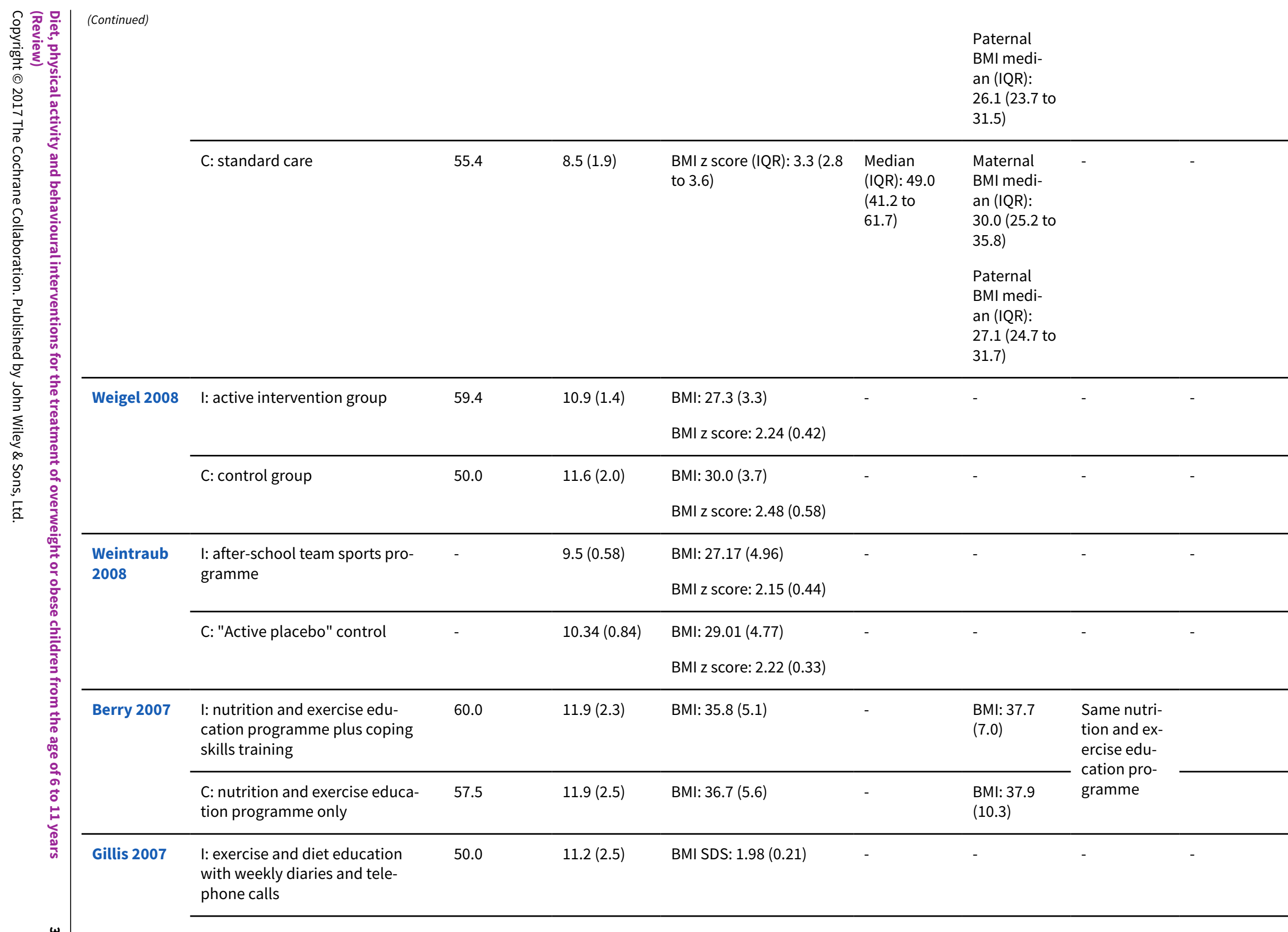




\begin{tabular}{|c|c|c|c|c|c|c|c|c|c|}
\hline 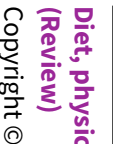 & (Continued) & $\begin{array}{l}\text { C: exercise and diet education } \\
\text { only }\end{array}$ & 53.8 & $9.0(2.2)$ & BMI SDS: $2.16(0.34)$ & - & - & - & - \\
\hline 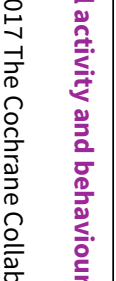 & $\begin{array}{l}\text { Kalavainen } \\
2007\end{array}$ & $\begin{array}{l}\text { I: family-centred group pro- } \\
\text { gramme }\end{array}$ & 54 & $8.1(0.9)$ & $\begin{array}{l}\text { BMI: } 23.4(2.6) \\
\text { BMI z score: } 2.6(0.6)\end{array}$ & $43.1(8.7)$ & $\begin{array}{l}\text { Mother's } \\
\text { BMI: } 26.1 \\
(5.4) \\
\text { Father's } \\
\text { BMI: } 26.9 \\
(3.8)\end{array}$ & - & $\begin{array}{l}\text { Healthy N } \\
=23(66 \%), \\
\text { asthma/al- } \\
\text { lergy N = } 10 \\
(28 \%), \text { other } \\
\text { disease } \mathrm{N}= \\
2(6 \%)\end{array}$ \\
\hline 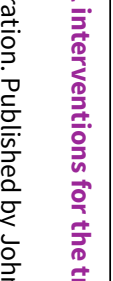 & & C: routine treatment & 66 & $8.0(0.8)$ & $\begin{array}{l}\text { BMI: } 22.9(2.5) \\
\text { BMI z score: } 2.5(0.6)\end{array}$ & $40.4(6.7)$ & $\begin{array}{l}27.0(6.3) \\
27.7(3.9)\end{array}$ & - & $\begin{array}{l}\text { Healthy N } \\
=18(52 \%), \\
\text { asthma/al- } \\
\text { lergy N = } 12 \\
(34 \%), \text { other } \\
\text { disease } \mathrm{N}= \\
5(14 \%)\end{array}$ \\
\hline 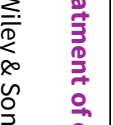 & $\begin{array}{l}\text { McCallum } \\
2007\end{array}$ & I: LEAP Intervention & 49 & $7.5(1.6)$ & $\begin{array}{l}\text { BMI: } 20.5(2.2) \\
\text { BMI z score: } 2.0(0.5)\end{array}$ & - & - & - & - \\
\hline 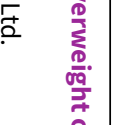 & & C: control group & 54 & $7.4(1.6)$ & $\begin{array}{l}\text { BMI: } 20.0(1.8) \\
\text { BMI z score: } 1.9(0.5)\end{array}$ & - & - & - & - \\
\hline 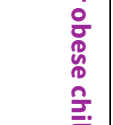 & $\begin{array}{l}\text { Rodearmel } \\
2007\end{array}$ & $\begin{array}{l}\text { I: 'America on the move' inter- } \\
\text { vention group }\end{array}$ & 50.86 & $11.11(2.08)$ & $\begin{array}{l}\text { BMI: } 25.40(4.22) \\
\text { BMIz score: } 1.76(0.45)\end{array}$ & $58.3(18.6)$ & $\begin{array}{l}\text { BMI: } 30.81 \\
(7.80)\end{array}$ & - & - \\
\hline 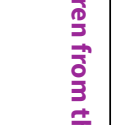 & & C: self-monitoring group & 53.92 & $11.28(2.29)$ & $\begin{array}{l}\text { BMI: } 24.75(5.04) \\
\text { BMIz score: } 1.68(0.42)\end{array}$ & $57.7(19.4)$ & $31.14(7.04)$ & - & - \\
\hline 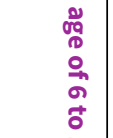 & Satoh 2007 & $\begin{array}{l}\text { I: dietary guidance using an easi- } \\
\text { ly-handled model nutritional bal- } \\
\text { ance chart (MNBC) }\end{array}$ & 52.4 & $11.0(1.5)$ & - & - & - & - & $\begin{array}{l}\text { Fatty liver } \mathrm{N} \\
=2\end{array}$ \\
\hline 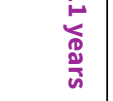 & & C: control group & 75.0 & $12.4(1.6)$ & - & - & - & - & $\begin{array}{l}\text { Fatty liver } \mathrm{N} \\
=0\end{array}$ \\
\hline & $\begin{array}{l}\text { Wilfley } \\
2007\end{array}$ & $\begin{array}{l}\text { I1: behavioural skills mainte- } \\
\text { nance group }\end{array}$ & 72.5 & $9.9(1.4)$ & BMI: 27.1 (3.3) & - & $\begin{array}{l}\text { BMI: } 35.2 \\
(5.9)\end{array}$ & - & - \\
\hline
\end{tabular}


12: social facilitation mainte-

70.0

$9.9(1.4)$

BMI: $28.2(3.3)$

$35.2(5.9)$

nance group

$9.8(1.2)(20)(B M 1: 27.3(3.7)$

$34.6(7.2)$

Epstein l: standardised family-based be- 59.1

2005 havioural weight control pro-

$10.2(1.1)$

BMI: $27.3(3.7)$

$-$

gramme plus reinforcement

BMI z score: 4.1 (1.2)

$62.4(11.2)$

BMI: 31.4

All partic-

for increasin

(5.9)

ipants re-

ceived the

same be-

haviour-

ing

C: standardised family-based

52.6

$10.1(1.3)$

BMI: 29.7 (3.4)

$64.8(10.8)$

$30.6(6.0)$

al weight

behavioural weight control pro-

BMI z score: 4.5 (1.3)

gramme

gramme only

10.9 (1.9) BMI: 28.5 (4.1)

$63.8(19.1)$

Parental

obesity, no:

programme

41.7

10 single

6 none

C: control group

45.5

$11.3(2.8)$

BMI: 27.8 (5.0)

$63.4(22.8)$

Parental

obesity, no:

7 both
11 single
4 none

\begin{tabular}{|c|c|c|c|c|c|c|c|c|}
\hline \multirow[t]{3}{*}{ Woo 2004} & $\begin{array}{l}\text { I1: diet plus supervised struc- } \\
\text { tured exercise programme with } \\
\text { continuing training }\end{array}$ & 34 & $10.0(1.0)$ & BMI: $25.3(2.4)$ & $54.6(9.5)$ & - & \multirow[t]{3}{*}{$\begin{array}{l}\text { Diet modifi- } \\
\text { cation }\end{array}$} & - \\
\hline & $\begin{array}{l}\text { 12: diet plus supervised struc- } \\
\text { tured exercise programme with } \\
\text { detraining }\end{array}$ & & & BMI: $26.1(4.0)$ & & - & & - \\
\hline & C: diet modification only & 34 & $9.9(0.9)$ & BMI: 24.7 (3.1) & $50.3(8.5)$ & - & & - \\
\hline $\begin{array}{l}\text { Epstein } \\
2001\end{array}$ & $\begin{array}{l}\text { I: combination of reducing seden- } \\
\text { tary behaviour and increasing } \\
\text { physical activity }\end{array}$ & 48.1 & $\begin{array}{l}\text { Boys } \\
10.4(1.2) \\
\text { Girls } 9.9 \\
(1.1)\end{array}$ & $\begin{array}{l}\text { Boys } 27.5(2.5) \\
\text { Girls } 27.9(4.7)\end{array}$ & $\begin{array}{l}\text { Boys } 132.8 \\
(24.8) \\
\text { Girls } 134.7 \\
(40.6)\end{array}$ & $\begin{array}{l}\text { Father's } \\
\text { BMI: } 31.1 \\
(7.3)\end{array}$ & - & - \\
\hline
\end{tabular}




\begin{tabular}{|c|c|c|c|c|c|c|c|c|c|}
\hline 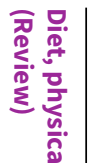 & (Continued) & & & & & & $\begin{array}{l}\text { Mother's } \\
\text { BMI: } 28.5 \\
(5.5)\end{array}$ & & \\
\hline 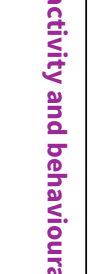 & & $\begin{array}{l}\text { C: targeting increasing physical } \\
\text { activity only }\end{array}$ & 48.3 & $\begin{array}{l}\text { Boys } 10.8 \\
(1.1) \\
\text { Girls } 10.2 \\
(1.4)\end{array}$ & $\begin{array}{l}\text { Boys } 27.3(3.8) \\
\text { Girls } 26.9(3.6)\end{array}$ & $\begin{array}{l}\text { Boys } 134.5 \\
(30.7) \\
\text { Girls } 127.8 \\
(32.4)\end{array}$ & $\begin{array}{l}\text { Father's } \\
\text { BMI: } 31.3 \\
(4.4) \\
\text { Mother's } \\
\text { BMI: } 29.8 \\
(3.4)\end{array}$ & - & \\
\hline 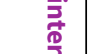 & Nova 2001 & I: enhanced approach & 47.2 & $8.6(1.9)$ & $23.75(2.65)$ & - & - & - & - \\
\hline 些. & & C: routine approach & 41.6 & $8.6(2.1)$ & $22.37(1.85)$ & - & - & - & - \\
\hline 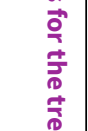 & $\begin{array}{l}\text { Epstein } \\
2000 a\end{array}$ & $\begin{array}{l}\text { I1: behavioural weight-control } \\
\text { programme plus parent and child } \\
\text { problem solving }\end{array}$ & 52.9 & $10.7(0.9)$ & $\begin{array}{l}\text { BMI z score score: } 2.8 \\
(0.9)\end{array}$ & $64.2(13.2)$ & $\begin{array}{l}\text { Weight: } 89.0 \\
(18.2)\end{array}$ & $\begin{array}{l}\text { All partic- } \\
\text { ipants re- } \\
\text { ceived a }\end{array}$ & - \\
\hline 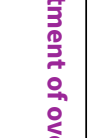 & & $\begin{array}{l}\text { I2: behavioural weight-control } \\
\text { programme plus child problem } \\
\text { solving only }\end{array}$ & 50.0 & $10.3(1.2)$ & BMI z score: $2.6(0.9)$ & $58.2(10.9)$ & $79.8(16.0)$ & $\begin{array}{l}\text { with dietary } \\
\text { + exercise } \\
\text { advice and }\end{array}$ & - \\
\hline 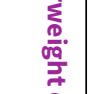 & & $\begin{array}{l}\text { C: standard treatment with no } \\
\text { additional problem solving }\end{array}$ & 52.9 & $10.0(1.2)$ & BMI z score: $2.7(0.8)$ & $57.0(11.4)$ & $87.0(23.0)$ & principles & - \\
\hline $\begin{array}{l}\circ \\
\stackrel{0}{0} \\
0 \\
0\end{array}$ & $\begin{array}{l}\text { Schwing- } \\
\text { shandl } 1999\end{array}$ & $\begin{array}{l}\text { I: Physical activity programme } \\
\text { and dietary advice }\end{array}$ & 57.1 & $11.0(2.5)$ & BMI SDS: 5.58 (2.46) & $63.3(16.5)$ & - & $\begin{array}{l}\text { Dietary ad- } \\
\text { vice }\end{array}$ & - \\
\hline$\frac{\overline{2}}{\overline{2}}$ & & C: dietary advice only & 56.3 & $12.2(2.7)$ & BMI SDS: 5.33 (1.79) & $69.2(20.6)$ & - & & - \\
\hline $\begin{array}{l}\overrightarrow{3} \\
\overline{3} \\
3 \\
\overrightarrow{0}\end{array}$ & Duffy 1993 & $\begin{array}{l}\text { I: cognitive self-management } \\
\text { training plus behaviour therapy }\end{array}$ & 78.6 & $9.9(1.7)$ & & $57.14(11.37)$ & - & $\begin{array}{l}\text { Behaviour } \\
\text { therapy }\end{array}$ & - \\
\hline $\begin{array}{l}0.9 \\
0 \\
0 \\
0 \\
0\end{array}$ & & $\begin{array}{l}\text { C: behaviour therapy plus atten- } \\
\text { tion placebo control methods }\end{array}$ & & & & $55.55(11.82)$ & - & both groups & - \\
\hline$\underset{\infty}{\stackrel{5}{*}}$ & Flodmark & I: family therapy & 56.0 & - & BMI: 24.7 (1.76) & - & - & - & - \\
\hline$v$ & & C: conventional treatment & 47.4 & - & BMI: 25.5 (2.31) & - & - & - & - \\
\hline
\end{tabular}




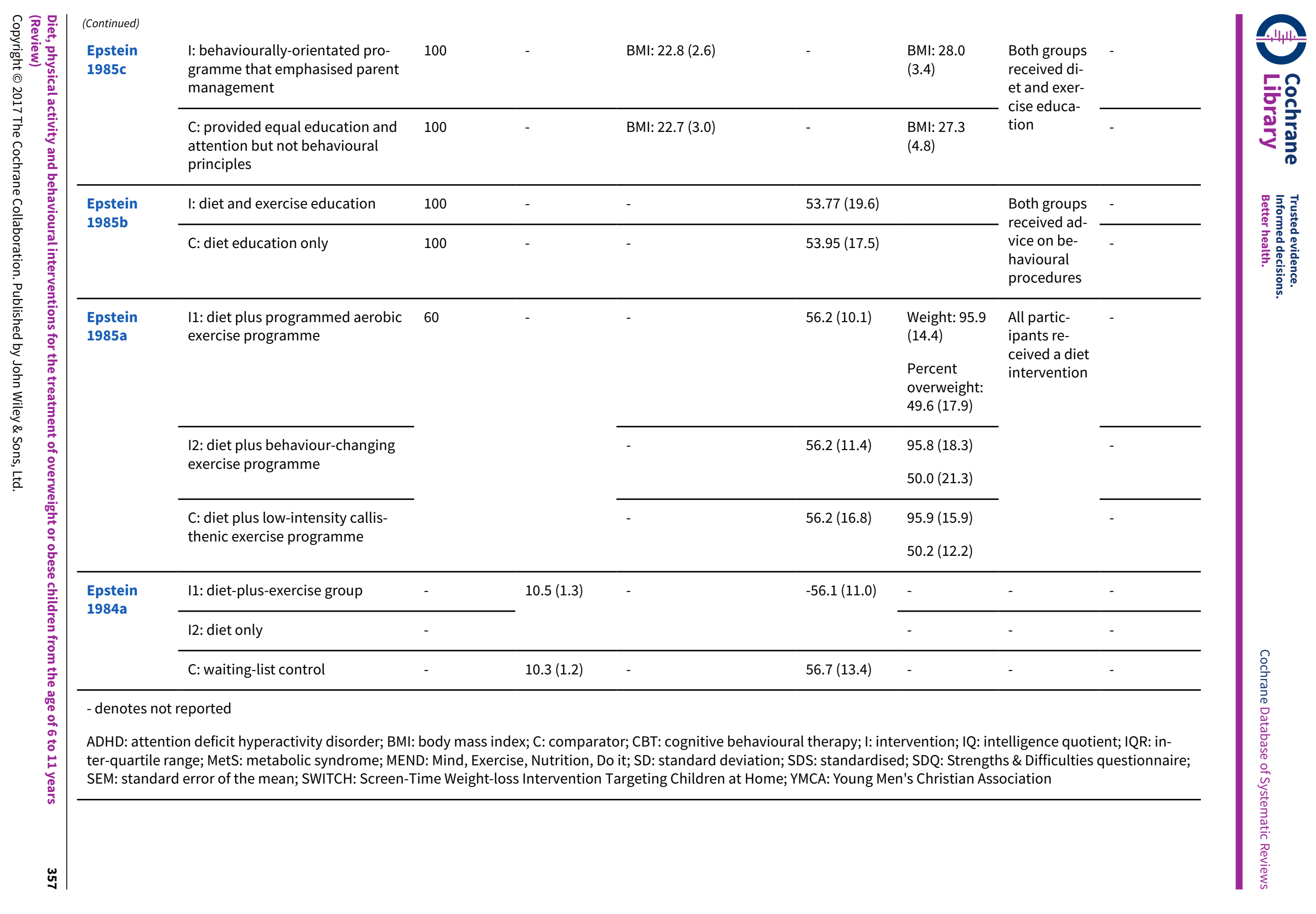




\section{Appendix 5. Matrix of study endpoints (publications and trial documents)}

\begin{tabular}{|c|c|c|}
\hline & $\begin{array}{l}\text { Endpoints quoted in trial document(s) } \\
\text { (ClinicalTrials.gov, FDA/EMA document, } \\
\text { manufacturer's website, published design } \\
\text { paper)a }\end{array}$ & Endpoints quoted in publication(s) \\
\hline \multirow[t]{4}{*}{ NCT02436330 } & Source: NCT02436330 & $\mathrm{N} / \mathrm{A}$ \\
\hline & $\begin{array}{l}\text { Primary outcome measure(s):): BMI z -score } \\
\text { change }\end{array}$ & \\
\hline & $\begin{array}{l}\text { Secondary outcome measure(s): after } \\
\text { school screen time, Saturday screen time; ac- } \\
\text { tivity levels measured by pedometers; self } \\
\text { perception as assessed using the Children } \\
\text { and Youth Physical Self-Perception Profile } \\
\text { (CY-PSPP), physical self-worth: changes in } \\
\text { physical self-worth, global self-worth score; } \\
\text { dietary change (total calorie intake, \% fat, } \\
\% \text { carbohydrates, number of vegetable serv- } \\
\text { ings, number of fruit servings, number of sug- } \\
\text { ar-sweetened beverages); attendance, WC } \\
\text { change; systolic blood pressure change; heart } \\
\text { rate change, shuttle run change }\end{array}$ & \\
\hline & Other outcome measure(s): - & \\
\hline \multirow[t]{4}{*}{ Ho 2016} & Source: NCT00881478 & Primary outcome measure(s): BMI z score \\
\hline & $\begin{array}{l}\text { Primary outcome measure(s): change in age } \\
\text { and gender-adjusted BMI z score }\end{array}$ & \\
\hline & $\begin{array}{l}\text { Secondary outcome measure(s): age and } \\
\text { gender-adjusted WC percentile, age and } \\
\text { gender-adjusted blood pressure percentile, } \\
\text { fasting lipid profile, fasting insulin and fast- } \\
\text { ing glucose, plasma visfatin level, plasma } \\
\text { adiponectin level, proportion of children } \\
\text { achieving a BMI below the 85th percentile for } \\
\text { age and gender }\end{array}$ & $\begin{array}{l}\text { Secondary outcome measure }(\mathrm{s}) \text { : BMI }(\mathrm{kg} / \mathrm{m} 2) \text {, BMI per- } \\
\text { centile, BMI z score, WC }(\mathrm{cm}) \text {, systolic BP, systolic BP per- } \\
\text { centile, systolic BP z score, diastolic BP, diastolic BP z } \\
\text { score, fasting insulin, fasting glucose, glucose at 2-h } \\
\text { OGTT, total cholesterol, triglycerides, total adiponectin, } \\
\text { high-molecular-weight adiponectin }\end{array}$ \\
\hline & Other outcome measure(s): - & $\begin{array}{l}\text { Other outcome measure(s): compliance ( } \geq 80 \% \text { of rec- } \\
\text { ommendations) }\end{array}$ \\
\hline \multirow[t]{4}{*}{ Epstein 2015} & Source: NCT01208870 & Primary outcome measure(s): - \\
\hline & $\begin{array}{l}\text { Primary outcome measure(s): responding } \\
\text { for food on the habituation task, BMI z score }\end{array}$ & \\
\hline & $\begin{array}{l}\text { Secondary outcome measure(s): dietary in- } \\
\text { take }\end{array}$ & Secondary outcome measure(s): - \\
\hline & Other outcome measure(s): - & $\begin{array}{l}\text { Other outcome measure(s): percent overweight, parent } \\
\text { BMI, adherence, fat calories, total calories, carbohydrate } \\
\text { calories, red foods, fruit and vegetables }\end{array}$ \\
\hline Larsen 2015 & $\mathrm{~N} / \mathrm{A}$ & Primary outcome measure(s): BMI z score \\
\hline
\end{tabular}

Diet, physical activity and behavioural interventions for the treatment of overweight or obese children from the age of 6 to 11 years 
Secondary outcome measure(s): WC, waist-to-height ratio

Other outcome measure(s):

Secondary outcome measure(s): PA habits (Actigraph accelerometers (GT3X+ models))

\begin{abstract}
Other outcome measure(s): (from protocol) weight, height, BMI, WC, waist-to-height ratio, triceps and subscapular skinfold thickness, blood pressure, cholesterol, triglycerides, glucose, insulin, TSH, cortisol, PA and fitness levels (ALPHA fitness test), sedentary and PA behaviour (accelerometry), dietary behaviours (dietary recall and questionnaire), PA self-efficacy, body image, PA enjoyment, HRQoL, cost-utility of the intervention, parental outcomes (anthropometric, sedentary + PA behaviours, diet, psychological aspects, economic data, pubertal stage, socioeconomic and demographic parameters, adherence, degree of satisfaction
\end{abstract}

\begin{tabular}{ll}
\hline Taveras 2015 & Source: NCT01537510 \\
& $\begin{array}{l}\text { Primary outcome measure(s): BMI, blood } \\
\text { pressure, and laboratory screening, provision } \\
\text { of nutrition and PA counselling }\end{array}$ \\
\hline
\end{tabular}

Secondary outcome measure(s): BMI, health behaviours (sugar-sweetened beverage intake, fast food, PA, TV viewing and sleep), costs

Other outcome measure(s): -

Primary outcome measure(s): BMI and quality of care (HEDIS measures)

Secondary outcome measure(s): height, BMI, weight, WC, waist-to-height ratio, PA and sedentary time (accelerometer), dietary intake (food frequency questionnaire)

\title{
Other outcome measure(s): -
}

\section{Secondary outcome measure(s): -}

Other outcome measure(s): parent's height and weight and BMI, parental acceptance and satisfaction of interventions

Taylor $2015 \quad$ Source: ACTRN12609000749202 Primary outcome measure(s): BMI z score

Primary outcome measure(s): BMI z score

Secondary outcome measure(s): PA (accelerometry), food behaviours and intake, QoL, psychological functioning
Secondary outcome measure(s): WC, height, weight, BMI, waist girth, waist-to-height ratio, percentage fat, dietary intake, home food availability, accelerometry, parental feeding practices, home environment, QoL, motivation
Other outcome measure(s): -

Source: NCT01378806

\section{Other outcome measure(s): -}

Primary outcome measure(s): BMI percentile children, decrease in BMI parents 
Secondary outcome measure(s): change in adiposity for adults and children as measured by change in WC, triceps, and subscapular skinfold measures; WC and triceps and subscapular skinfold measures; change in health behaviours as measured by nutrition and exercise in adults and children; nutrition and exercise behaviours in adults and children based on questionnaire scores; change in selfefficacy in adults and children as measured by belief that they can improve their eating and exercise behaviours; eating and exercise self-efficacy in adults and children based on questionnaire scores
Secondary outcome measure(s): decrease in adiposity and an improvement in health behaviours and self-efficacy
Other outcome measure(s): N/A

Other outcome measure(s): -

Primary outcome measure(s): -

Secondary outcome measure(s): -

Other outcome measure(s): absence of hunger, subjective bulimic episode, objective bulimic episode, objective over-eating episode, loss of control eating, overeating episodes, caloric intake, BMI, BMI z score, treatment acceptability

\section{Hamilton-Shield $2014 \quad$ Source: ISRCTN90561114 \\ Primary outcome measure(s): BMI z score}

Primary outcome measure(s): child BMI standard deviation scores (SDS)

Secondary outcome measure(s): adult eating rate, child BMI SDS, child eating rate, child ideal portion size choice, child self-determined portion size, parent BMI, parent ideal portion size choice, parent self-determined portion size, HRQoL (PedsQL, CHU9D, EQ5D, EQ5D-Y)
Secondary outcome measure(s): height and weight of parents; maintained BMI or BMI z-score value improvement at 12 months post therapy; QoL measures in child (PedsQL, CHU9D and EQ-5D-Y) and parents; resource-use questionnaire, including child's use of primary and secondary care services; change in eating speed and self-determined portion size; precise measures of changes in 'ideal portion size' and 'expected satiety levels'; changes in PA levels, measured as number of steps/ d for 1 week (pedometers)

Other outcome measure(s): -

Looney $2014 \quad$ Source: NCT01358448 Primary outcome measure(s): BMI z score

Primary outcome measure(s): weight status (BMI z score), dietary intake, leisure-time behaviours, care feeding behaviours

Secondary outcome measure(s): cost effectiveness
Secondary outcome measure(s): child dietary intake, leisure time behaviours

Other outcome measure(s): -

Other outcome measure(s): -

Maddison $2014 \quad$ Source: ACTRN12611000164998 Primary outcome measure(s): child BMI z score

Diet, physical activity and behavioural interventions for the treatment of overweight or obese children from the age of 6 to 11 years 
Primary outcome measure(s): child BMI z

score

Secondary outcome measure(s): parent BMI, parent PA, child's daily min in sedentary behaviour, child min spent in PA, child dietary intake
Secondary outcome measure(s): child BMI, weight, WC, $\%$ body fat, self-reported daily PA, total sedentary time, sleep, dietary intake, perceived enjoyment of PA and sedentary behaviour, parental BMI and self-reported PA

Other outcome measure(s): -

Other outcome measure(s): -

\begin{tabular}{ll}
\hline Markert 2014 & Source: DRKS00000803 (German Clinical Trial Primary outcome measure(s): BMI SDS \\
& Register) \\
& Primary outcome measure(s): BMI SDS
\end{tabular}

Secondary outcome measure(s): eating behaviour, nutrition, PA and leisure time habits, QoL
Secondary outcome measure(s): $\mathrm{HRQ}$ L, eating patterns, PA, leisure time habits

\section{Other outcome measure(s): - $\quad$ Other outcome measure(s): -}

\section{Arauz Boudreau 2013 Source: lists Clinicaltrials.partners.org} 2009P001721 - however, unable to find the record
Primary outcome measure(s): HRQoL, metabolic markers of obesity (lipids, glucose, insulin, HbA1c, AST/ALT, Creactive protein, IL-6, TNF- $\alpha$, cholesterol, triglycerides, HDL, VLDL, LDL), BMI, accelerometer-based PA

Secondary outcome measure(s): -

Other outcome measure(s): nutrition knowledge and intake, height, weight, BMI z scores

Primary outcome measure(s): BMI z score
Davis $2013 \quad$ Source: Gallagher et al. Treating rural pediatric obesity through telemedicine: baseline data from a randomised controlled trial. 2011. Journal of pediatric psychology. 36 (6). 687-95 (see Davis 2013)

Primary outcome measure(s): -
Secondary outcome measure(s): dietary behaviours, PA behaviours, child behaviour checklist, behavioural feeding assessment scale
Other outcome measure(s): BMI, actigraph activity monitor information, $24 \mathrm{~h}$ dietary recalls, child-behaviour checklist, behavioural paediatrics feeding assessment scale

\section{Other outcome measure(s): -}

Primary outcome measure(s): individual variation in BMI (BMI z score)

Primary outcome measure(s): BMI z score

Secondary outcome measure(s): PA behaviours variation, dietary behaviours variation
Secondary outcome measure(s): percentage of positive changes in parent-reported dietary behaviours and in PA

Other outcome measure(s): -

Other outcome measure(s): -

Lochrie 2013

Source: NCT01146314

Primary outcome measure(s): BMI

Diet, physical activity and behavioural interventions for the treatment of overweight or obese children from the age of 6 to 11 years 
Primary outcome measure(s): improvement of health status of overweight children, BMI, blood pressure, WC, and reducing the risk of the development of type 2 diabetes and metabolic syndrome

Secondary outcome measure(s): improvement of health behaviours and psychosocial adjustment;

changing health behaviours, such as eating patterns, diet, and eating behaviour; evaluate the effects of maintaining of improving adjustment to psychological stressors associated with being overweight (self-esteem, depression, behaviour)

Other outcome measure(s): -

\section{Secondary outcome measure(s): -}

Other outcome measure(s): total cholesterol, HDL, LDL, WC, abdominal girth, triceps skinfold, child depression inventory, pediatric QoL parent, pediatric QoL youth, Harter SPP global self-worth, BASC-2 parent version-externalising, BASC-2 parent version internalising, blood pressure

$\begin{array}{ll}\text { Mirza } 2013 & \text { Source: NCT01068197 } \\ & \text { Primary outcome measure(s): insulin sensi- } \\ & \text { tivity, BMI z score }\end{array}$

Secondary outcome measure(s): body fat mass, LDL, cholesterol, triglycerides, FFA, hormonal, metabolic outcomes

Other outcome measure(s): -
Primary outcome measure(s): BMI z score
Secondary outcome measure(s): changes in insulin resistance and metabolic risk markers

\begin{tabular}{|c|c|c|}
\hline & Other outcome measure(s): - & $\begin{array}{l}\text { Other outcome measure(s): dietary intake, adverse } \\
\text { events, metabolic syndrome }\end{array}$ \\
\hline \multirow[t]{4}{*}{ O'Connor 2013} & Source: NCT01195012 & Primary outcome measure(s): - \\
\hline & $\begin{array}{l}\text { Primary outcome measure(s): family atten- } \\
\text { dance }\end{array}$ & \\
\hline & Secondary outcome measure(s): - & Secondary outcome measure(s): - \\
\hline & Other outcome measure(s): - & $\begin{array}{l}\text { Other outcome measure(s): attendance, satisfaction, } \\
\text { height, weight, parent BMI, child BMI z scores and per- } \\
\text { centiles, child behaviours (diet, physical activities, TV } \\
\text { viewing), parent behaviours }\end{array}$ \\
\hline \multirow[t]{3}{*}{ Saelens 2013} & $\mathrm{~N} / \mathrm{A}$ & Primary outcome measure(s): BMI z score, parent BMI \\
\hline & & Secondary outcome measure(s): \\
\hline & & $\begin{array}{l}\text { Other outcome measure(s): parent self-efficacy and } \\
\text { confidence }\end{array}$ \\
\hline \multirow[t]{2}{*}{ Siwik 2013} & Source: NCT01674920 & Primary outcome measure(s): BMI z score \\
\hline & Primary outcome measure(s): BMI z scores & \\
\hline
\end{tabular}

Diet, physical activity and behavioural interventions for the treatment of overweight or obese children from the age of 6 to 11 years 
(Continued)

\section{Secondary outcome measure(s): weight z Secondary outcome measure(s): -}

scores

Other outcome measure(s):-

Other outcome measure(s): weight z score, height z score, BMI, weight, height, METs (low, medium, high), percent body fat, qualitative interview measures

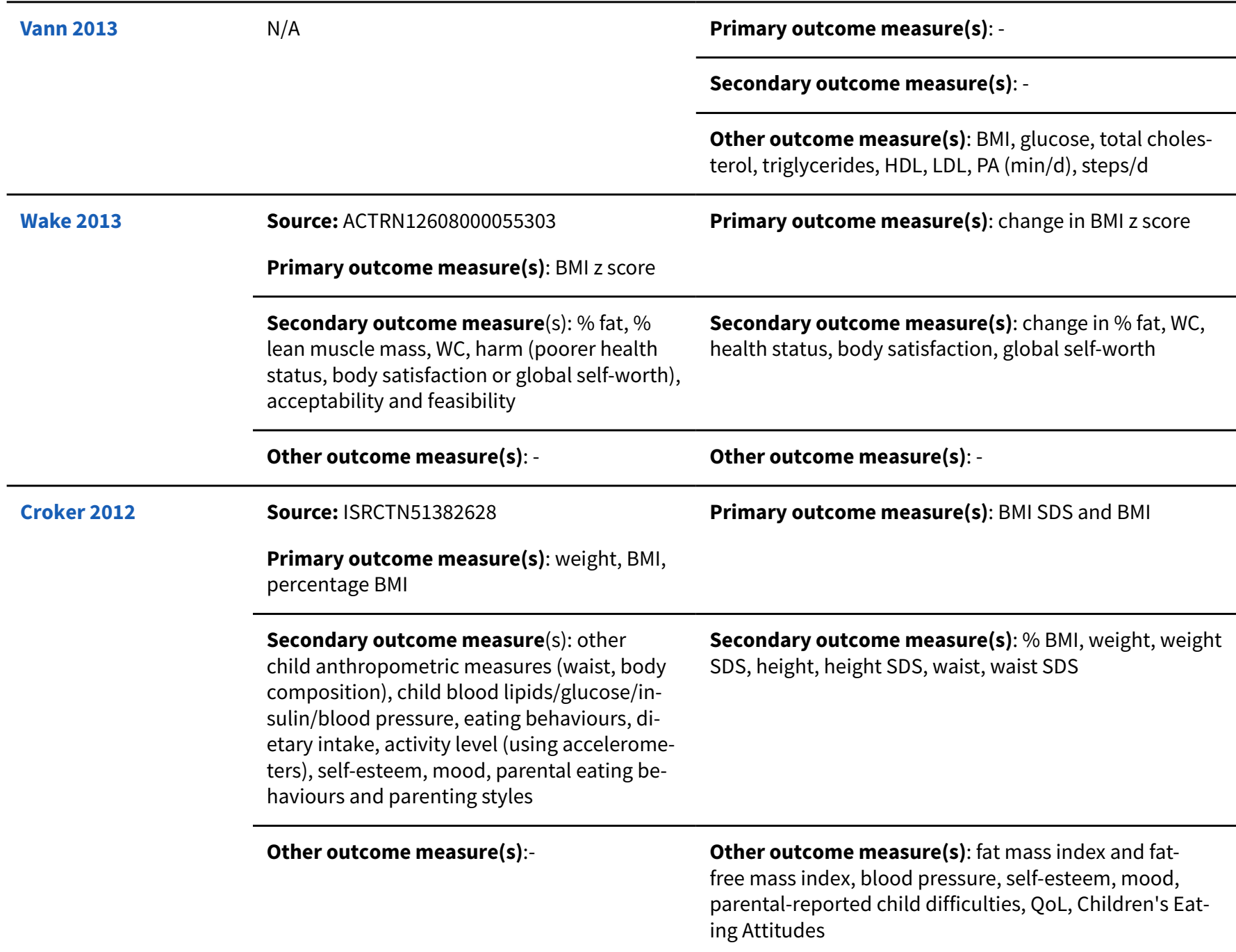

de Niet 2012

Source: ISRCTN33476574

Primary outcome measure(s): dropout rate

Secondary outcome measure(s): BMI-SDS, problem behaviour, measured with Youth Outcome Questionnaire (YOQ), family functioning, measured with the Dutch version of the Family Adaptability and Cohesion Evaluation Scale (FACES III), HRQoL measured with the Child Health Questionnaire-Parent Form (CHQ-PF-50), perceived competence, measured with Dutch version of the Self-Perception Profile for Children (SPPC), eating behaviour, measured with the Dutch Eating Behaviour Questionnaire (DEBQ)
Primary outcome measure(s): BMI SDS, eating behaviour, psychological well-being

Secondary outcome measure(s): adherence, self-reported health behaviours and mood, feasibility of the SMSMT

Diet, physical activity and behavioural interventions for the treatment of overweight or obese children from the age of 6 to 11 years 
(Continued)
Other outcome measure(s): -
Other outcome measure(s): -

\begin{tabular}{|c|c|}
\hline \multirow[t]{4}{*}{ Eddy Ives 2012} & Source: ISRCTN35399598 \\
\hline & $\begin{array}{l}\text { Primary outcome measure(s): reduction in } \\
\text { BMI }\end{array}$ \\
\hline & $\begin{array}{l}\text { Secondary outcome measure(s): social-eco- } \\
\text { nomic class, eating and PA habits, emotional } \\
\text { status (AF-5) }\end{array}$ \\
\hline & Other outcome measure(s): - \\
\hline \multirow[t]{2}{*}{ Kirk 2012} & Source: NCT00215111 \\
\hline & $\begin{array}{l}\text { Primary outcome measure(s): body weight, } \\
\text { height, BMI, WC, percent body fat, adipose } \\
\text { mass, lean body mass, bone mineral densi- } \\
\text { ty, fasting lipid profile, fasting insulin, fast- } \\
\text { ing glucose, 2-h glucose (baseline and 3- } \\
\text { month assessment), 2-h insulin (baseline and } \\
\text { 3-month assessment), interleukin-6, tumor } \\
\text { necrosis factor, C-reactive protein, serum } \\
\text { amyloid A, ketones, energy intake, macronu- } \\
\text { trient intake (carbohydrate, protein and fat), } \\
\text { micronutrient intake (vitamins and minerals), } \\
\text { dietary fibre intake, glycaemic load, psycho- } \\
\text { logical measures (Child Behavior Checklist } \\
\text { and Teach Report Form) }\end{array}$ \\
\hline
\end{tabular}

Secondary outcome measure(s): PA (3-d PA records and pedometer readings), compliance with behavioural intervention (frequency rewards were earned), attendance at group and individual sessions during initial 3month intervention, parent/guardian weight, parent/guardian body mass index, Sexual Maturity Rating, Hunger/Satiety assessment (Three-Factor Eating Questionnaire), parent/guardian perception of success for each diet assignment prior to their child being randomised to a diet group
Primary outcome measure(s): BMI and BMI z scores
Secondary outcome measure(s): abdominal perimeter, abdominal perimeter $z$ score, dietary and physical exercise habits, self-esteem indicators

\section{Other outcome measure(s):-}

Primary outcome measure(s): BMI z score, WC, \% body fat, dietary intake

Secondary outcome measure(s): clinical metabolic parameters (fasting glucose, fasting insulin, total cholesterol, triglycerides, HDL, LDL, SBP and DBP)

Other outcome measure(s): -

Other outcome measure(s): -

Source: NCT01503281

Primary outcome measure(s): -

Primary outcome measure(s): BMI, BMI-Z score

Secondary outcome measure(s): WC, percentage body fat

Secondary outcome measure(s): -

Other outcome measure(s): -
Other outcome measure(s): anthropometric values (including body weight, height, BMI, BMI-Z score, and WC), percentage body fat was also determined with a body fat analyser (TANITA TBF-410 M) 
Primary outcome measure(s): BMI

Secondary outcome measure(s): food in- Secondary outcome measure(s): take, PA

\section{Other outcome measure(s):}

Other outcome measure(s): WC, sagittal abdominal diameter, body composition analysis (DEXA), body fat, truncal fat, fat mass index, blood pressure, plasma glucose, serum lipids (cholesterol, HDL, LDL, triglycerides, apo $A$ and apo B), insulin, HbA1c, HOMA-Index, metabolic syndrome, total energy expenditure, basal metabolic index, energy intake, macronutrient intakes (e.g. protein, sucrose, fat, fibre), PA level, steps/d, metabolic equivalents, screen time

\begin{tabular}{lll}
\hline Wright 2012 & N/A & $\begin{array}{l}\text { Primary outcome measure(s): BMI z scores, dietary } \\
\text { measures }\end{array}$ \\
\cline { 2 - 2 } & N/A & $\begin{array}{l}\text { Secondary outcome measure(s): - } \\
\text { Other outcome measure(s): food preferences, knowl- } \\
\text { edge and self-efficacy }\end{array}$ \\
\hline Barkin 2011 & \begin{tabular}{l} 
Primary outcome measure(s): BMI, BMI percentile \\
\cline { 2 - 2 }
\end{tabular} & $\begin{array}{l}\text { Secondary outcome measure(s): - } \\
\text { Other outcome measure(s): - }\end{array}$ \\
\hline
\end{tabular}

Primary outcome measure(s): adiposity at 12 months (\% body fat by DEXA scan)

Secondary outcome measure(s): adiposity from bioimpedance, BMI SD score and WC at 6 and 12 months, feasibility (e.g. recruitment rate, attrition, acceptability etc.), biomedical markers of morbidity, lifestyle, psychological measures and physical fitness
Primary outcome measure(s): BMI, WC, BIA, DXA

Secondary outcome measure(s): 2 -h oral glucose tolerance, lipid level, liver function, blood pressure, fitness (step test), PA (accelerometry), parental height and weight, diet questionnaire, eating behaviour, PA questionnaire, sedentary behaviour questionnaire, QoL, strengths and difficulties questionnaire, social and cognitive competence

Other outcome measure(s): -

Primary outcome measure(s): BMI SDS

Secondary outcome measure(s): WC, body fat, lifestyle outcomes (food and activity diary, frequency of specific foods, pedometer steps, time in low, moderate and high intensity activity)

Other outcome measure(s): -

Gunnarsdottir 2011a N/A

Primary outcome measure(s): ratings of treatment acceptability (measured post treatment) and child changes in BMI-SDS 
Other outcome measure(s): daily fruit and vegetable consumption, daily exercise (min), parental BMI

Maddison 2011
Source: ACTRN12607000632493

Primary outcome measure(s): BMI z score, BMI centile

Secondary outcome measure(s): percent body fat (\%), WC (cm), physical fitness measured in VO2Max $(\mathrm{mL} / \mathrm{kg} / \mathrm{min})$ PA Questionnaire for Children score (self-report levels), average daily time spent in light-to-vigorous activities ( $\mathrm{min}$ ) as measured via accelerometry, average daily time spent in active video games ( $\mathrm{min}$ ) (self-report), average daily time spent in non-active video games (min) (selfreport)
Primary outcome measure(s): change from baseline in $\mathrm{BMl}$; in $\mathrm{kg} / \mathrm{m} 2$

Secondary outcome measure(s): changes in percentage body fat, PA, cardiorespiratory fitness, video game play, and food snacking
Other outcome measure(s): -

Source: ISRCTN14241825

Primary outcome measure(s): change in BMI SDS

Secondary outcome measure(s): HRQoL, PA and sedentary (accelerometry) and estimated fat free mass (impedance)
Secondary outcome measure(s): weight change, HRQoL, objectively measured PA and sedentary behaviour

\section{Other outcome measure(s): - $\quad$ Other outcome measure(s): -}

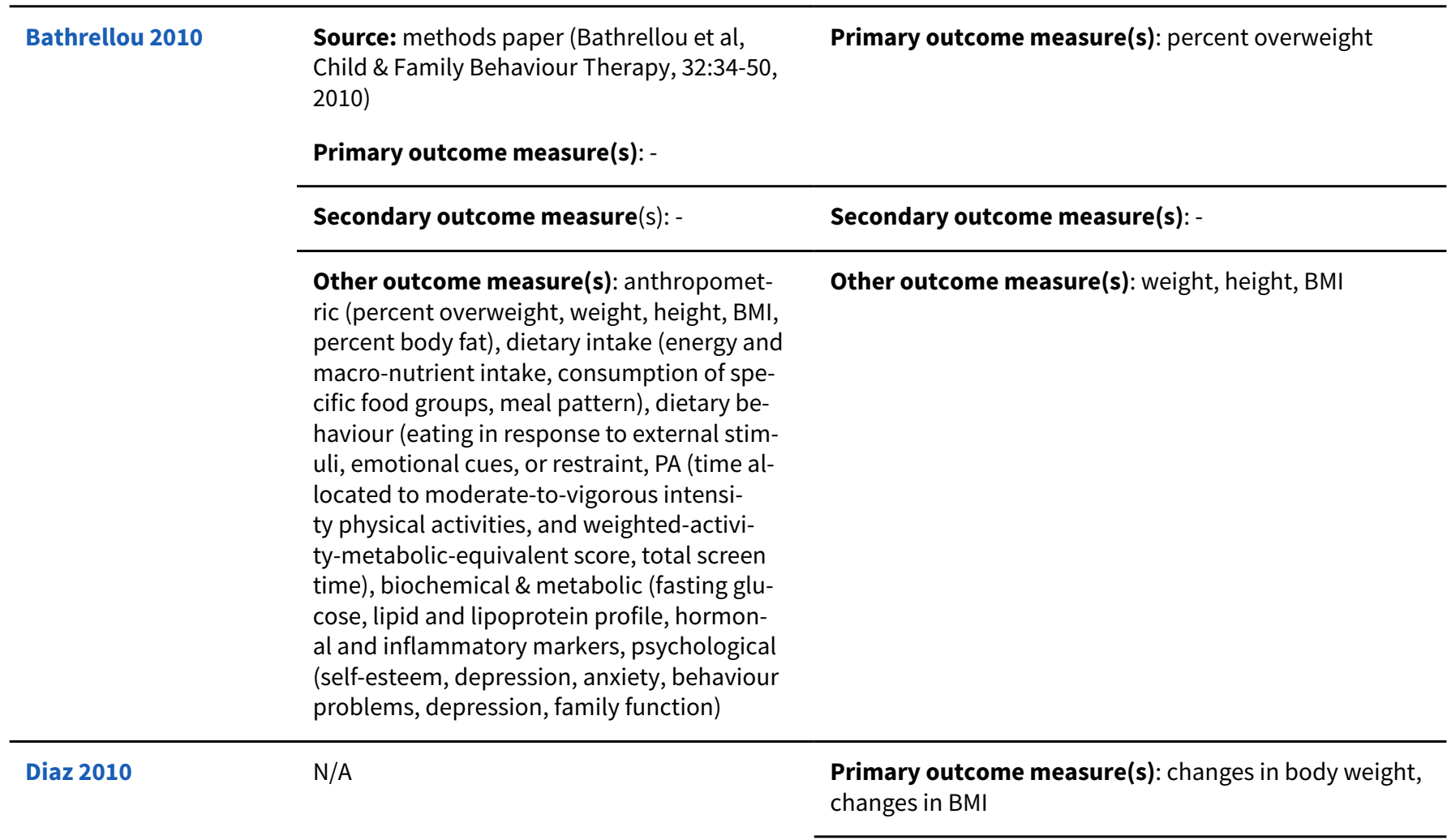

Diet, physical activity and behavioural interventions for the treatment of overweight or obese children from the age of 6 to 11 years 
Secondary outcome measure(s): changes in other obesity parameters, changes in body composition, changes in blood pressure, changes in biochemical parameters

Other outcome measure(s): -

\begin{tabular}{|c|c|c|}
\hline \multirow[t]{3}{*}{ Duggins 2010} & \multirow[t]{3}{*}{$\mathrm{N} / \mathrm{A}$} & Primary outcome measure(s): BMI percentile \\
\hline & & Secondary outcome measure(s): - \\
\hline & & $\begin{array}{l}\text { Other outcome measure(s): attendance, eating habits, } \\
\text { number of participants who met AMA weight loss tar- } \\
\text { gets, number of participants who lost weight }\end{array}$ \\
\hline Faude 2010 & $\mathrm{~N} / \mathrm{A}$ & $\begin{array}{l}\text { Primary outcome measure(s): height, weight, BMI, BMI } \\
\text { percentile, BMI z score, POmax, VO2 max, max lactate, } \\
\text { max heart rate, psychometric data (total score, physical } \\
\text { well-being, emotional well-being, self-esteem, family, } \\
\text { friends, school), training compliance and training inten- } \\
\text { sity, time one-leg standing right, time one-leg standing } \\
\text { left, sit and reach test, counter movement jump height, } \\
\text { agility test, } 20 \text { m shuttle run min, maximal heart rate dur- } \\
\text { ing shuttle run }\end{array}$ \\
\hline
\end{tabular}

Secondary outcome measure(s): -

Other outcome measure(s): -

Primary outcome measure(s): change of weight status

Secondary outcome measure(s): change of eating and exercise behaviour, and change of QoL
Secondary outcome measure(s): BMI, WC, triceps skinfold thickness, subscapularis skinfold thickness, percentage fat mass based on skinfold measurements, lean body mass, fat mass, \% body fat, SBP, DBP, dietary intake (energy, fat, protein, carbohydrate, sugar), sports activity, TV consumption, computer consumption
Other outcome measure(s): -

Source: ISRCTN30238779

Primary outcome measure(s): WC

Secondary outcome measure(s): body composition, weight, height, BMI, self- esteem, family functioning, child mental health, cardiovascular fitness and dietary intake and composition

\section{Other outcome measure(s): -}

Primary outcome measure(s): WC 
Primary outcome measure(s): BMI and car-

diovascular risk factors

Secondary outcome measure(s): eating, activity, and psychosocial functioning
Secondary outcome measure(s): -

Other outcome measure(s): -
Other outcome measure(s): changes in blood pressure, body composition, WC, and HRQoL, binge eating (in additional publication)

\begin{tabular}{ll}
\hline Nowicka 2009 N/A & \begin{tabular}{l} 
Primary outcome measure(s): - \\
\cline { 2 - 3 }
\end{tabular} \\
\cline { 2 - 3 } & Secondary outcome measure(s): - \\
& $\begin{array}{l}\text { Other outcome measure(s): BMI z score, weight, height, } \\
\text { DXA \% body fat, DXA body fat DXA lean mass, MRI subcu- } \\
\text { taneous fat caudal, fat cranial, visceral fat caudal, viscer- } \\
\text { al fat cranial, physical education, involvement in sports } \\
\text { clubs, TV viewing weekdays and weekends, computer } \\
\text { weekdays and weekends }\end{array}$ \\
\end{tabular}

\begin{tabular}{|c|c|c|}
\hline \multirow[t]{2}{*}{ Wake 2009} & $\begin{array}{l}\text { Source: ISRCTN52511065 } \\
\text { Primary outcome measure(s): BMI }\end{array}$ & Primary outcome measure(s): BMI, BMI z score \\
\hline & 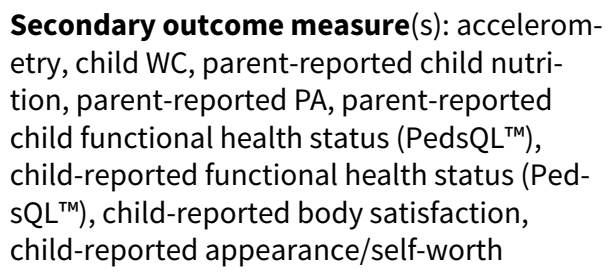 & $\begin{array}{l}\text { Secondary outcome measure(s): WC, maternal and pa- } \\
\text { ternal BMI, PA (accelerometry), PA (diary), nutrition (di- } \\
\text { ary), HRQoL, body dissatisfaction, physical appearance } \\
\text { and self-worth }\end{array}$ \\
\hline
\end{tabular}

\begin{tabular}{lll}
\cline { 2 - 3 } & Other outcome measure(s): - & Other outcome measure(s): - \\
\hline Alves 2008 & N/A & Primary outcome measure(s): weight, height, BMI \\
\cline { 2 - 2 } & Secondary outcome measure(s): - \\
\cline { 2 - 2 } & Other outcome measure(s): - \\
\hline
\end{tabular}

\begin{tabular}{|c|c|c|}
\hline \multirow[t]{4}{*}{ Hughes 2008} & Source: : protocol (Stewart 2005) & Primary outcome measure(s):BMI z score \\
\hline & $\begin{array}{l}\text { Primary outcome measure(s): change in BMI } \\
\text { z score }\end{array}$ & \\
\hline & $\begin{array}{l}\text { Secondary outcome measure(s): growth ve- } \\
\text { locity, PA and sedentary behaviour (measured } \\
\text { objectively with accelerometry), and QoL }\end{array}$ & $\begin{array}{l}\text { Secondary outcome measure(s): WC, weight, height, } \\
\text { total activity, monitored time (sedentary, light, MVPA), } \\
\text { QOL (child self -report and parent proxy report) }\end{array}$ \\
\hline & Other outcome measure(s): - & Other outcome measure(s): - \\
\hline \multirow[t]{2}{*}{ Weigel 2008} & $\mathrm{~N} / \mathrm{A}$ & Primary outcome measure(s): BMI z score \\
\hline & & Secondary outcome measure(s): - \\
\hline
\end{tabular}


Other outcome measure(s): BMI, fat mass, lean mass, SBP, DBP, triglycerides, total cholesterol, uric acid, HDL, ALT, AST, cortisol, TSH or heart rate

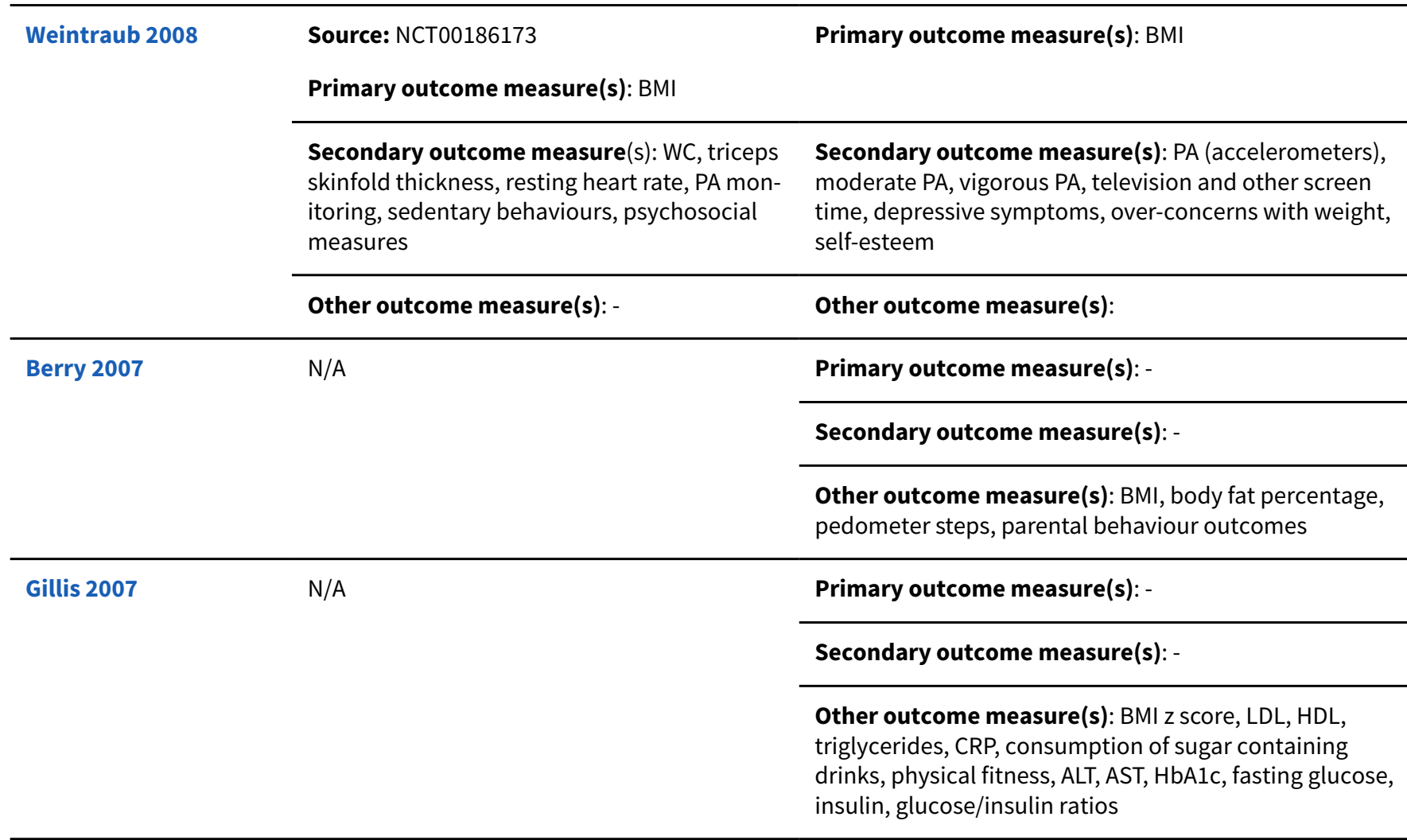

Primary outcome measure(s): the change of weight for height based on Finnish growth charts

Secondary outcome measure(s): changes in BMI and BMI standard deviation scores (BMI-SDS)

Other outcome measure(s): fat mass, lean body mass, WC, waist/height, SBP, DBP, triglycerides, LDL, HDL, total cholesterol, fasting glucose, fasting insulin, HOMA-IR (from ID4097), cost effectiveness (Kalavainen 2009)

McCallum $2007 \quad$ Source: protocol (McCallum 2005)

Primary outcome measure(s): BMI

Primary outcome measure(s): change in BMI

Secondary outcome measure(s): child WC, HRQoL, behaviour, self-esteem and family activities, parental concern regarding child's weight, readiness to change, child PA, sedentary behaviour, child and family nutrition, relationship with GP
Secondary outcome measure(s): parent reported child nutrition, PA and health status, child-reported health status, body satisfaction, appearance/self-worth
Other outcome measure(s): -

$\mathrm{N} / \mathrm{A}$
Other outcome measure(s): costs

Primary outcome measure(s): BMI for age for target children and change in BMI for parents 
Secondary outcome measure(s): change in the following anthropometric measurements: BMI (children), weight, percentage of body fat, and WC

Other outcome measure(s): steps/d, sugar intake

\begin{tabular}{|c|c|c|}
\hline \multirow[t]{3}{*}{ Satoh 2007} & \multirow[t]{3}{*}{$\mathrm{N} / \mathrm{A}$} & Primary outcome measure(s): - \\
\hline & & Secondary outcome measure(s): - \\
\hline & & $\begin{array}{l}\text { Other outcome measure(s): intake of foods (meat, fish, } \\
\text { eggs, milk, beans, green and yellow vegetables, light } \\
\text { coloured vegetables, fruit, grains, oil and sugar), per- } \\
\text { centage overweight }\end{array}$ \\
\hline \multirow[t]{4}{*}{ Wilfley 2007} & Source: NCT00301197 & $\begin{array}{l}\text { Primary outcome measure(s): BMI z score, percent } \\
\text { overweight }\end{array}$ \\
\hline & $\begin{array}{l}\text { Primary outcome measure(s): weight (child } \\
\text { and parent) }\end{array}$ & \\
\hline & $\begin{array}{l}\text { Secondary outcome measure(s): weight-re- } \\
\text { lated behaviours, psychological functioning } \\
\text { (specific and general) }\end{array}$ & $\begin{array}{l}\text { Secondary outcome measure(s): treatment specific } \\
\text { psychosocial targets }\end{array}$ \\
\hline & Other outcome measure(s): - & Other outcome measure(s): - \\
\hline \multirow[t]{3}{*}{ Epstein 2005} & $\mathrm{~N} / \mathrm{A}$ & Primary outcome measure(s): - \\
\hline & & Secondary outcome measure(s): - \\
\hline & & $\begin{array}{l}\text { Other outcome measure(s): BMI z score, percent over- } \\
\text { weight, time spent in MVPA, alternative to eating, chil- } \\
\text { dren's episodes of eating and drinking/d }\end{array}$ \\
\hline \multirow[t]{3}{*}{ Nemet 2005} & $\mathrm{~N} / \mathrm{A}$ & Primary outcome measure(s): weight change \\
\hline & & Secondary outcome measure(s): - \\
\hline & & $\begin{array}{l}\text { Other outcome measure(s): skinfold thickness, BMI, } \\
\text { body weight, screen time, habitual activity, endurance } \\
\text { time, caloric intake, carbohydrate, protein, fat, triglyc- } \\
\text { erides, cholesterol, HDL, LDL, height, BMI percentile, } \\
\text { body fat \% }\end{array}$ \\
\hline \multirow[t]{3}{*}{ Woo 2004} & N/A & $\begin{array}{l}\text { Primary outcome measure(s): arterial endothelium-de- } \\
\text { pendent dilation and intima-media thickness }\end{array}$ \\
\hline & & Secondary outcome measure(s): - \\
\hline & & $\begin{array}{l}\text { Other outcome measure(s): body weight, height, body } \\
\text { fat, fasting serum cholesterol, triglycerides, HDL, LDL, } \\
\text { waist-to-hip ratio, LDL ratio, BMI }\end{array}$ \\
\hline \multirow[t]{2}{*}{ Epstein 2001} & $\mathrm{~N} / \mathrm{A}$ & Primary outcome measure(s): - \\
\hline & & Secondary outcome measure(s): - \\
\hline
\end{tabular}


Other outcome measure(s): height, weight, percent overweight, BMI, motivation, perceived support of immediate family and friends, adherence

\begin{tabular}{|c|c|c|}
\hline Nova 2001 & $\mathrm{~N} / \mathrm{A}$ & $\begin{array}{l}\text { Primary outcome measure(s): variation in percentage } \\
\text { overweight }\end{array}$ \\
\hline
\end{tabular}

Secondary outcome measure(s): behavioural modifications (PA, PC or TV usage)

Other outcome measure(s): adherence to follow-up visits (parental commitment and compliance)

Epstein 2000a N/A

Primary outcome measure(s): -

Secondary outcome measure(s): -

Other outcome measure(s): height; weight; BMI z scores; PEPSI; CBCL: total behaviour problems, total competence, internalising behaviour problems, externalising behaviour problems, adherence, KEDS: total score, weight dissatisfaction, bingeing/purging; parental weight, height, PSI, GSI, adherence, binge eating symptoms

Secondary outcome measure(s): -

Other outcome measure(s): weight, BMI SDS, fat-free mass

Duffy 1993 N/A

Primary outcome measure(s): -

Secondary outcome measure(s): -

Other outcome measure(s): weight, height, percentage overweight, number of red foods/d

\begin{tabular}{lll}
\hline Flodmark $1993 \quad$ N/A & Primary outcome measure(s): -
\end{tabular}

Secondary outcome measure(s): -

Other outcome measure(s): BMI, triceps, subscapular, suprailiac skinfold thickness, physical fitness (w/kg for normal weight and height at pulse 170)

\begin{tabular}{lll}
\hline Epstein $1985 \mathrm{C}$ & N/A & Primary outcome measure(s): -
\end{tabular}

Secondary outcome measure(s): -

Other outcome measure(s): BMI, percent overweight, eating behaviour, parent and child self-control

Epstein 1985b N/A Primary outcome measure(s): -

Secondary outcome measure(s): -

Diet, physical activity and behavioural interventions for the treatment of overweight or obese children from the age of 6 to 11 years 
Other outcome measure(s): weight, percent overweight, physical work capacity, activity levels

\begin{tabular}{|c|c|c|}
\hline \multirow[t]{3}{*}{ Epstein 1985a } & \multirow[t]{3}{*}{$\mathrm{N} / \mathrm{A}$} & Primary outcome measure(s): - \\
\hline & & Secondary outcome measure(s): - \\
\hline & & $\begin{array}{l}\text { Other outcome measure(s): child and parent outcomes } \\
\text { - percent overweight, weight fitness, eating behaviour, } \\
\text { intervention compliance }\end{array}$ \\
\hline \multirow[t]{3}{*}{ Epstein 1984a } & \multirow[t]{3}{*}{$\mathrm{N} / \mathrm{A}$} & Primary outcome measure(s): percent overweight \\
\hline & & Secondary outcome measure(s): - \\
\hline & & $\begin{array}{l}\text { Other outcome measure(s): adherence, girth, skinfold } \\
\text { thickness, fitness and serum lipids }\end{array}$ \\
\hline
\end{tabular}

\footnotetext{
- denotes not reported

aTrial document(s) refers to all available information from published design papers and sources other than regular publications (e.g. FDA/EMA documents, manufacturer's websites, trial registers).

bPublication(s) refers to trial information published in scientific journals (primary reference, duplicate publications, companion documents or multiple reports of a primary trial).

cOther outcome measures refer to all outcomes not specified as primary or secondary outcome measures.
}

ALT: alanine transaminase; AMA: American Medical Association; Apo A: Apolipoprotein A; Apo B: Apolipoprotein B; AST: aspartate transaminase;

BASC-2: Behavior Assessment System for Children - Second Edition; BIA: Bioelectrical impedance analysis; BMI: body mass index; BMI SDS: standardised body mass index; BP: blood pressure;

CBCL: Child Behavior Checklist; CFQ: Child feeding questionnaire; CHU9D: Child Health Utility 9-Dimensions; CRP: c-reactive protein; CRPBI: Child Report of Parental Behavior Inventory;

DBP: diastolic blood pressure; DXA/DEXA: dual-energy X-ray absorptiometry;

EAH: eating in the absence of hunger; EDI: Eating Disorder Inventory; EMA: European Medicines Agency; EQ-5D-Y: European Quality of Life 5-Dimensions - youth;

FDA: Food and Drug Administration (US); FFA: free fatty acids; FFM: fat-free mass;

GP: General Practitioner; GSI: Global Severity Index; HbA1c: Glycated haemoglobin;

HDL: high-density lipoprotein; HEDIS: Healthcare Effectiveness Data and Information Set; HOMA-IR: homeostatic model assessment insulin resistance; $\mathrm{HRQ}$ oL: health-related quality of life;

IL-6: interleukin-6;

KEDS: Kids' Eating Disorders Survey;

LDL: low-density lipoprotein;

METs: metabolic equivalents; MRI: Magnetic resonance imaging; MVPA: moderate-to-vigorous physical activity;

N/A: not applicable;

OGTT: oral glucose tolerance test;

PA: physical activity; PedsQL: Pediatric Quality of Life Inventory; PEPSI: Purdue Elementary Problem-Solving Inventory; POmax: maximal power output; PSI: Problem Solving Inventory; PWC: physical work capacity;

QoL: quality of life

Diet, physical activity and behavioural interventions for the treatment of overweight or obese children from the age of 6 to 11 years 
(Continued)

QUICKI: quantitative insulin sensitivity check index;

RCT: randomised controlled trial; RED: high energy density foods; ROC: regulation of cues;

SBP: systolic blood pressure; SD: standard deviation; SMSMT: Short Message Service Maintenance Treatment; SPP: Self-Perception Profile; SWITCH: Screen-Time Weight-loss Intervention Targeting Children at Home;

TC: total cholesterol; TG: triglycerides; TNF-a: tumor necrosis factor alpha; TSH: thyroid-stimulating hormone;

VLDL: very low density lipoprotein; $\mathrm{VO}_{2 \text { max }}$ : maximal oxygen uptake;

WC: waist circumference; $\mathrm{w} / \mathrm{kg}$ : watts per kilogram

Appendix 6. High risk of outcome reporting bias according to ORBIT classification

\begin{tabular}{|c|c|c|c|c|c|}
\hline & Outcome & $\begin{array}{l}\text { High risk of } \\
\text { bias } \\
\text { (category A)a }^{\text {cate }}\end{array}$ & $\begin{array}{l}\text { High risk of } \\
\text { bias } \\
\text { (category } \\
\text { D)b }\end{array}$ & $\begin{array}{l}\text { High risk of } \\
\text { bias } \\
\text { (category E)c }\end{array}$ & $\begin{array}{l}\text { High risk of } \\
\text { bias } \\
\text { (category } \\
\text { G)d }\end{array}$ \\
\hline NCT02436330 & $\mathrm{N} / \mathrm{D}$ & & & & \\
\hline Ho 2016 & $\begin{array}{l}\text { All primary and secondary out- } \\
\text { come ITT analyses (only results for } \\
\text { completers were presented in the } \\
\text { text) }\end{array}$ & Yes & & & \\
\hline Warschburger 2016 & $\mathrm{~N} / \mathrm{D}$ & & & & \\
\hline \multirow[t]{2}{*}{ Epstein 2015} & Child BMI z score & & Yes & & \\
\hline & Dietary intake & Yes & & & \\
\hline Larsen 2015 & $\mathrm{~N} / \mathrm{D}$ & & & & \\
\hline Serra-Paya 2015 & $\mathrm{~N} / \mathrm{D}$ & & & & \\
\hline Taveras 2015 & $N / D$ & & & & \\
\hline Taylor 2015 & $\mathrm{~N} / \mathrm{D}$ & & & & \\
\hline Berry 2014 & $\mathrm{~N} / \mathrm{D}$ & & & & \\
\hline Boutelle 2014 & $\mathrm{~N} / \mathrm{D}$ & & & & \\
\hline Hamilton-Shield 2014 & $\mathrm{~N} / \mathrm{D}$ & & & & \\
\hline Looney 2014 & $\begin{array}{l}\text { Dietary intake and leisure-time be- } \\
\text { haviours }\end{array}$ & Yes & & & \\
\hline Maddison 2014 & $\mathrm{~N} / \mathrm{D}$ & & & & \\
\hline \multirow[t]{2}{*}{ Markert 2014} & QoL & Yes & & & \\
\hline & Eating patterns & Yes & & & \\
\hline
\end{tabular}

Diet, physical activity and behavioural interventions for the treatment of overweight or obese children from the age of 6 to 11 years 
(Continued)

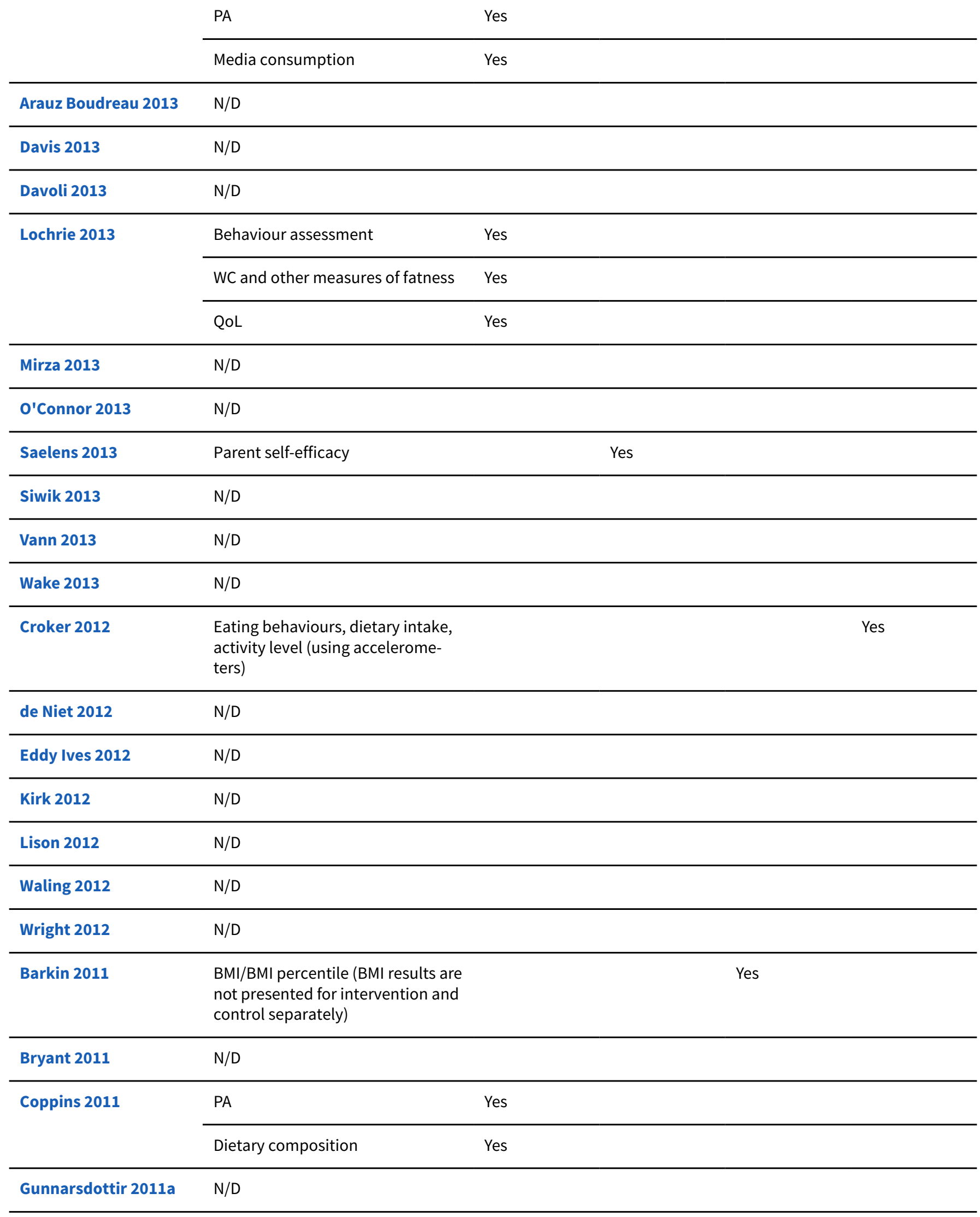


(Continued)

\begin{tabular}{|c|c|c|c|c|c|}
\hline Maddison 2011 & $\mathrm{~N} / \mathrm{D}$ & & & & \\
\hline Wafa 2011 & $\mathrm{~N} / \mathrm{D}$ & & & & \\
\hline \multirow[t]{3}{*}{ Bathrellou 2010} & Behaviour changes & & & Yes & \\
\hline & HRQOL & & & Yes & \\
\hline & Body fat distribution & & & Yes & \\
\hline Diaz 2010 & $\mathrm{~N} / \mathrm{D}$ & & & & \\
\hline Duggins 2010 & Behaviour changes - eating habits & Yes & & & \\
\hline Faude 2010 & $\mathrm{~N} / \mathrm{D}$ & & & & \\
\hline Reinehr 2010 & HRQoL & & Yes & & \\
\hline Sacher 2010 & $\begin{array}{l}\text { Dietary intake, family functioning, } \\
\text { child mental health }\end{array}$ & & & & Yes \\
\hline Kalarchian 2009 & $\begin{array}{l}\text { Self esteem, self-reported depres- } \\
\text { sive and anxiety symptoms, and } \\
\text { eating disorder symptoms (binge } \\
\text { eating) }\end{array}$ & Yes & Yes & & \\
\hline Nowicka 2009 & $\mathrm{~N} / \mathrm{D}$ & & & & \\
\hline Wake 2009 & $\mathrm{~N} / \mathrm{D}$ & & & & \\
\hline Alves 2008 & $\mathrm{~N} / \mathrm{D}$ & & & & \\
\hline Hughes 2008 & $\mathrm{~N} / \mathrm{D}$ & & & & \\
\hline Weigel 2008 & $\begin{array}{l}\text { Triglycerides, total cholesterol, } \\
\text { uric acid, HDL, ALT, AST, cortisol, } \\
\text { TSH or heart rate }\end{array}$ & Yes & & & \\
\hline
\end{tabular}

\begin{tabular}{lll}
\hline Weintraub 2008 & WC, triceps skinfold thickness & Yes \\
\hline Berry 2007 & N/D & Yes \\
\hline Gillis 2007 & Dietary habits & \\
\hline Kalavainen 2007 & N/D & Yes \\
\hline McCallum 2007 & WC, total body fat mass, fat-free & Yes \\
\hline Rodearmel 2007 & Sugar intake & \\
\hline Satoh 2007 & N/D \\
\hline Wilfley 2007 & N/D \\
\hline Epstein 2005 & Behaviour changes - diet and PA & Yes \\
\hline
\end{tabular}


(Continued)

\begin{tabular}{|c|c|c|c|}
\hline \multirow[t]{2}{*}{ Nemet 2005} & \multicolumn{3}{|l|}{$\mathrm{N} / \mathrm{D}$} \\
\hline & \multicolumn{3}{|l|}{$\mathrm{N} / \mathrm{D}$} \\
\hline Epstein 2001 & \multicolumn{3}{|l|}{$N / D$} \\
\hline Nova 2001 & PA, PC and TV viewing & Yes & \\
\hline Epstein 2000a & \multicolumn{2}{|l|}{$\begin{array}{l}\text { Behaviour changes - kids' eating } \\
\text { disorder survey } \\
\text { (not reported by group) }\end{array}$} & Yes \\
\hline Schwingshandl 1999 & BMI (at 12 months endpoint) & Yes & \\
\hline Duffy 1993 & \multicolumn{3}{|l|}{$\mathrm{N} / \mathrm{D}$} \\
\hline Flodmark 1993 & \multicolumn{3}{|l|}{$\mathrm{N} / \mathrm{D}$} \\
\hline Epstein 1985c & $\begin{array}{l}\text { Behaviour changes - eating behav- } \\
\text { iour and self-control }\end{array}$ & Yes & \\
\hline Epstein 1985b & $\mathrm{N} / \mathrm{D}$ & & \\
\hline \multirow[t]{2}{*}{ Epstein 1985a } & \multicolumn{2}{|l|}{$\mathrm{BMI}$} & Yes \\
\hline & $\begin{array}{l}\text { Behaviour changes - fitness and } \\
\text { eating behaviour }\end{array}$ & Yes & \\
\hline Epstein 1984a & $\mathrm{N} / \mathrm{D}$ & & \\
\hline
\end{tabular}

aClear that outcome was measured and analysed; trial report states that outcome was analysed but reports only that result was not significant

(Classification 'A', table 2, Kirkham 2010)

bClear that outcome was measured and analysed; trial report states that outcome was analysed but report no results

( Classification 'D', table 2, Kirkham 2010)

'Clear that outcome was measured but was not necessarily analysed; judgement says likely to have been analysed but not reported because of non-significant results

(Classification 'E', table 2, Kirkham 2010)

dUnclear whether outcome was measured; not mentioned, but clinical judgement says likely to have been measured and analysed but not reported on the basis of non-significant results

(Classification 'G', table 2, Kirkham 2010)

ALT: alanine transaminase; AST: aspartate transaminase; BMI: body mass index; HDL: High-density lipoproteins; HRQoL: health-related quality of life; ITT: intention to treat; N/A: not applicable; N/D: non detected; ORBIT: Outcome Reporting Bias In Trials; QoL: quality of life; PA: physical activity; TSH: thyroid-stimulating hormone; WC: waist circumference 


\begin{tabular}{|c|c|c|c|c|c|c|c|c|c|c|}
\hline 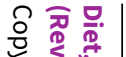 & Appendix 7. Definitior & ndpoint measurement & & & & & & & & \\
\hline 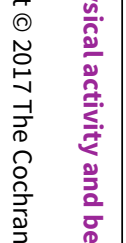 & $\begin{array}{l}\text { All-cause } \\
\text { mortality }\end{array}$ & Behaviour change & $\begin{array}{l}\text { Changes in BMI and } \\
\text { body weight }\end{array}$ & $\begin{array}{l}\text { Health- } \\
\text { related } \\
\text { quality of } \\
\text { life }\end{array}$ & Morbidity & $\begin{array}{l}\text { Other mea- } \\
\text { sures of body } \\
\text { fat distrubu- } \\
\text { tion }\end{array}$ & $\begin{array}{l}\text { Socioeco- } \\
\text { nomic ef- } \\
\text { fects }\end{array}$ & $\begin{array}{l}\text { Partic- } \\
\text { ipants } \\
\text { views } \\
\text { of the in- } \\
\text { terven- } \\
\text { tion }\end{array}$ & $\begin{array}{l}\text { Se- } \\
\text { vere/seri- } \\
\text { ous } \\
\text { adverse } \\
\text { events }\end{array}$ & 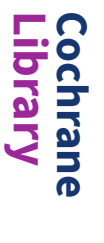 \\
\hline 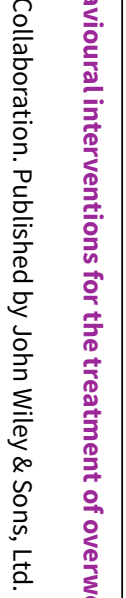 & NCT02436330NI & $\begin{array}{l}\text { PA: activity levels measured by } \\
\text { pedometer and fitness level } \\
\text { measured by shuttle run, heart } \\
\text { rate change } \\
\text { Sedentary behaviour: mea- } \\
\text { sured by: after school screen } \\
\text { time, Saturday screen time } \\
\text { Dietary intake: dietary change } \\
\text { measured by: total calorie in- } \\
\text { take, \% fat, \% carbohydrates, } \\
\text { number of vegetable servings, } \\
\text { number of fruit servings, num- } \\
\text { ber of sugar-sweetened bever- } \\
\text { ages }\end{array}$ & $\begin{array}{l}\text { BMI z score change } \\
\text { (not reported which } \\
\text { growth chart ap- } \\
\text { plied) }\end{array}$ & $\begin{array}{l}\text { Self-per- } \\
\text { ception as } \\
\text { assessed } \\
\text { using the } \\
\text { CY-PSPP: } \\
\text { physical } \\
\text { self-worth } \\
\text { changes } \\
\text { in Physi- } \\
\text { cal Self- } \\
\text { worth, } \\
\text { Global } \\
\text { Self-Worth } \\
\text { Score }\end{array}$ & $\mathrm{NI}$ & WC change & $\mathrm{NI}$ & $\mathrm{NI}$ & $\begin{array}{l}\text { Unclear } \\
\text { how they } \\
\text { were as- } \\
\text { sessed }\end{array}$ & 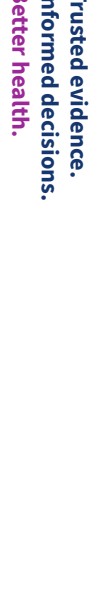 \\
\hline 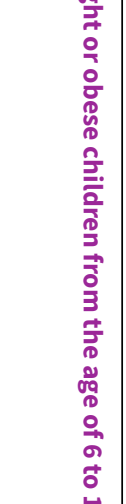 & Ho 2016 & $\mathrm{NI}$ & $\begin{array}{l}\text { Weight was mea- } \\
\text { sured in clothed par- } \\
\text { ticipants with no } \\
\text { jackets or shoes us- } \\
\text { ing a calibrated scale } \\
\text { (Seca, Germany). } \\
\text { Height was mea- } \\
\text { sured using a wall- } \\
\text { mounted, calibrated } \\
\text { stadiometer (Holtain } \\
\text { Limited, Britain). } \\
\text { Collected at } 0,3,6 \\
\text { months }\end{array}$ & $\mathrm{NI}$ & $\mathrm{NI}$ & $\begin{array}{l}\text { WC was mea- } \\
\text { sured using } \\
\text { the technique } \\
\text { - described by } \\
\text { Douketis } 2005 \\
\text { collected at } 0 \text {, } \\
3,6 \text { months }\end{array}$ & $\mathrm{NI}$ & $\mathrm{NI}$ & $\mathrm{NI}$ & 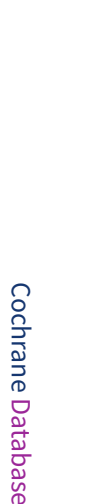 \\
\hline$\underset{\boldsymbol{J}}{\boldsymbol{w}}$ & $\begin{array}{l}\text { Warschburg- NI } \\
\text { er } 2016\end{array}$ & $\begin{array}{l}\text { Children's food intake was as- } \\
\text { sessed using a food frequency } \\
\text { list including "healthy" (fruits, } \\
\text { vegetables) and "problematic" } \\
\text { food items (e.g. sweets, salty } \\
\text { snacks). Parents rated the fre- }\end{array}$ & $\begin{array}{l}\text { Weight data of the } \\
\text { child were assessed } \\
\text { by means of a stan- } \\
\text { dard beam scale (ac- } \\
\text { curate to } 100 \mathrm{~g} \text { ) and } \\
\text { measured with a cal- }\end{array}$ & $\begin{array}{l}\text { Children's } \\
\text { HRQoL } \\
\text { was mea- } \\
\text { sured } \\
\text { using } \\
\text { the KID- }\end{array}$ & $\mathrm{NI}$ & $\mathrm{NI}$ & $\mathrm{NI}$ & $\mathrm{NI}$ & $\mathrm{NI}$ & 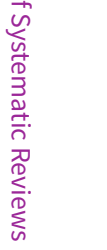 \\
\hline
\end{tabular}


ibrated stadiome-

ter (accurate to 1 tion of the items on a 5-point scale ("never" - "several times a day"). A score for the number of servings/d was calculated and converted to a 0 to 100 scale, with higher values representing more frequent consumption. Children's activity level including media consumption and exercise was evaluated. The parents were asked about the mean duration (in hours) of their child's use of television, video or computers on a 5-point Likert scale separately for weekdays and week ends. A summarised value reflecting the overall media consumption during an entire week was used. Regarding the fre quency of exercise, parents reported the mean duration (in

hours) completed during the week. $\mathrm{cm})$. A standardised

ed according to age and sex of the child (Kromeyer-Hauschild

2001). At the fol-

low-ups, children

were asked to vis

it their physicians,

who were blind to trial-group

assignment and the study goals. In or-

der to decrease at-

trition bias, families were reminded several times (by post and telephone) and reimbursed for their efforts. When unable to visit their physi-

cian, a reimburse-

ment for the use of a calibrated scale in pharmacies or a visit at home was of-

fered. Furthermore, all parents report-

ed their height and

weight, as well as the

respective data of

their partners
KINDL-R

filled in by

the par-

ents (e.g.

"Last week

my child

was proud

of him/

herself.").

On the ba-

sis of 4

subscales

(psycho-

logical

well-be-

ing, self-

esteem,

family and

peer re-

lation-

ship), a

sum score

was com -

posed.

The child's

weight-re-

lated OoL

was as-

sessed by

the GW-

QKJ, in-

cluding 11

items (e.g.

"In the last

two weeks

our child

lacked

self-con-

fidence

because

of his/her

weight")

rated on

a 5-point 


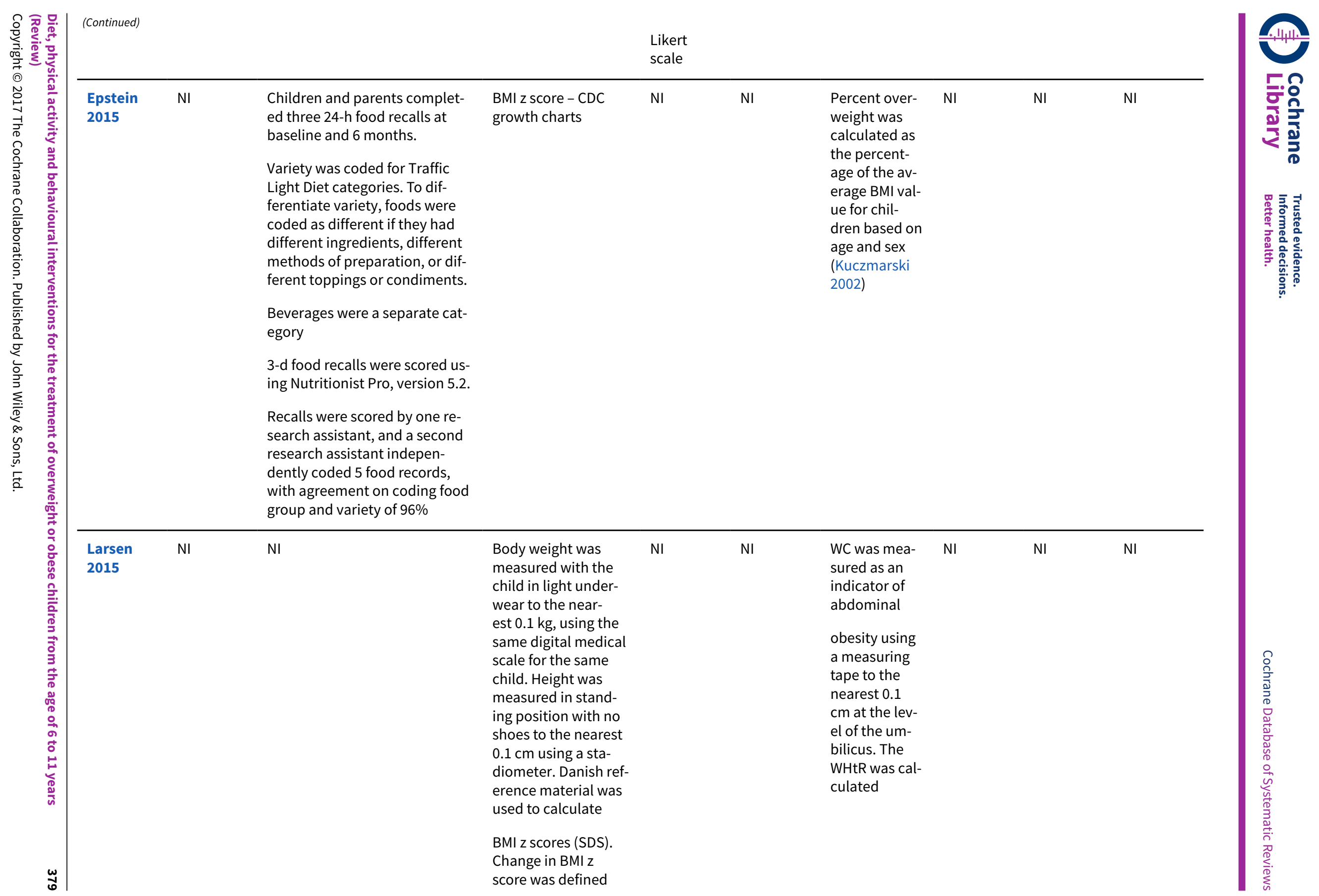




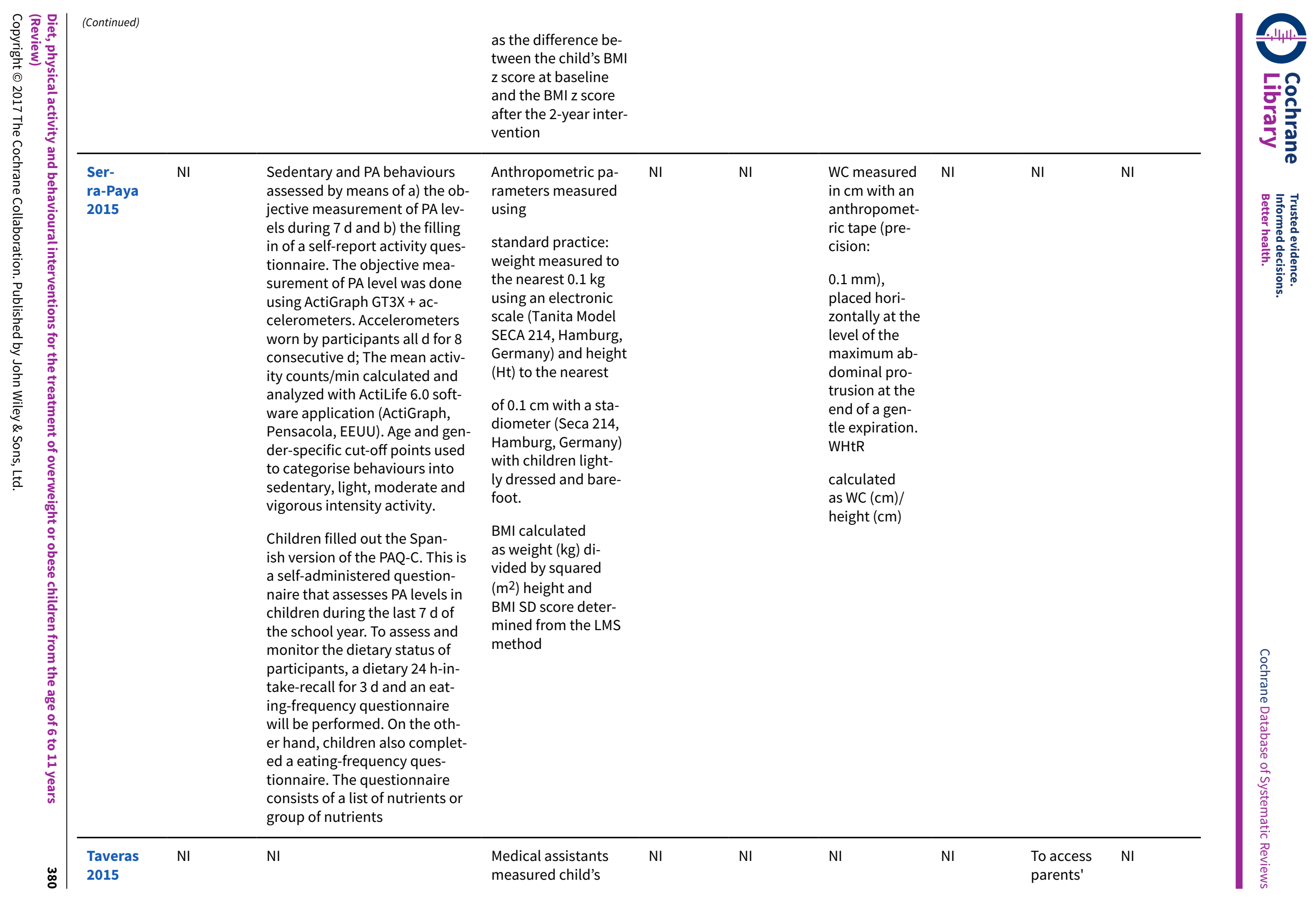


and measured the

child's height using

isfaction

a stadiometer. Cal-

with the

culated BMI and age-

interven

and sex specific BMI

tion com-

$z$ scores using $C D C$

growth charts

parents in

the inter-

vention

groups

asked

to rate

how satis-

fied they

were with

the pro-

gramme

and

whether

they

would rec-

ommend

the pro

gramme

to their

family or

friends

\begin{tabular}{|c|c|c|c|c|c|c|c|c|c|}
\hline $\begin{array}{l}\text { Taylor } \\
2015\end{array}$ & $\mathrm{NI}$ & $\begin{array}{l}\text { Children wore an accelerome- } \\
\text { ter (ActiGraph GT33, Pensaco- } \\
\text { la, FL) fitted over the right hip } \\
\text { for } 7 \mathrm{~d} \text { and } 8 \text { nights to measure } \\
\text { PA and sleep. MVPA was esti- } \\
\text { mated. Dietary intake assessed } \\
\text { using the Children's Dietary } \\
\text { Questionnaire, which assesses } \\
\text { intake patterns over the past } \\
\text { week for which intake is recom- } \\
\text { mended and foods for which } \\
\text { intake is discouraged. Portion } \\
\text { size of vegetables, meat and } \\
\text { starch-based foods assessed by } \\
3 \text { brief questions which have } \\
\text { been validated by duplicate }\end{array}$ & $\begin{array}{l}\text { Duplicate measures } \\
\text { of height (Tanita } \\
\text { portable stadiome- } \\
\text { ter), weight (Tani- } \\
\text { ta BC-418) were ob- } \\
\text { tained after stan- } \\
\text { dard techniques. BMI } \\
\text { was derived and z } \\
\text { scores calculated - } \\
\text { CDC growth charts }\end{array}$ & $\begin{array}{l}\text { QoL as- } \\
\text { sessed } \\
\text { using } \\
\text { the Ped- } \\
\text { sQL4.0, a } \\
\text { validated } \\
\text { 23- } \\
\text { item ques- } \\
\text { tionnaire } \\
\text { for chil- } \\
\text { dren aged } \\
\text { 2-18 years, } \\
\text { which as- } \\
\text { sesses } \\
\text { physical, }\end{array}$ & $\mathrm{NI}$ & $\begin{array}{l}\text { Duplicate } \\
\text { measures } \\
\text { of WC (lev- } \\
\text { el of the um- } \\
\text { bilicus) were } \\
\text { obtained af- } \\
\text { ter standard } \\
\text { techniques. } \\
\text { WHtR was cal- } \\
\text { culated. Esti- } \\
\text { mates of per- } \\
\text { centage } \\
\text { FM were ob- } \\
\text { tained by }\end{array}$ & $\mathrm{NI}$ & $\mathrm{NI}$ & $\mathrm{NI}$ \\
\hline
\end{tabular}


24-h recall measures in 7-year old children. Food availability in the home assessed using a

modified version of the Home Food Inventory. The Lifestyle Behaviour Checklist used to assess what challenges parents of overweight children face in managing their children's behaviour and how we might address this as part of the tailored package treatment. The Checklist includes 26 weight-related behaviours and asks parents to rate how much of a problem each is, and their confidence in changing each behaviour. Child behaviour assessed using the SDQ emotional,

social and

school

function-

ing.

Parent

proxy ver-

sions of

the ques-

tionnaire

used as

appropri-

ate.

As utilitie

have not

been de-

termined

for Ped-

SQL, QoL

also mea-

sured us-

ing the

Health

Utilities In-

dex. The

40-item

version

(HUI23P4E.40Q)

answered

by a par-

ent/guardian

on behalf

of each

child
BIA (Tani-

ta BC-418),

which pro-

vide a good

estimate of

change in per-

centage fat at

this age

\begin{tabular}{|c|c|c|c|c|c|c|c|c|c|}
\hline $\begin{array}{l}\text { Berry } \\
2014\end{array}$ & $\mathrm{NI}$ & $\begin{array}{l}\text { The Child Health Behavior Sur- } \\
\text { vey ( } 20 \text { items) and the Adult } \\
\text { Health Behavior Survey ( } 23 \\
\text { items) were used to collect in- } \\
\text { formation on usual food and } \\
\text { beverage intake. The Health } \\
\text { Promoting Lifestyle Profile II, } \\
\text { with four subscales, was used }\end{array}$ & $\begin{array}{l}\text { Weight was mea- } \\
\text { sured twice to the } \\
\text { nearest } 0.1 \mathrm{~kg} \text { in } \\
\text { street clothes } \\
\text { without shoes, us- } \\
\text { ing a Tanita WB110A } \\
\text { Digital Scale (Tani- }\end{array}$ & $\mathrm{NI}$ & $\mathrm{NI}$ & $\begin{array}{l}\text { WC was mea- } \\
\text { sured } 3 \text { times } \\
\text { and averaged } \\
\text { using a Fig- } \\
\text { ure Finder } \\
\text { measuring } \\
\text { tape with a } \\
\text { lock (Novel }\end{array}$ & $\mathrm{NI}$ & $\mathrm{NI}$ & $\mathrm{NI}$ \\
\hline
\end{tabular}


to measure nutrition, exercise health responsibility and stress management in parents. The 48 items have four responses (never, sometimes, often or rou

tinely) on a 4-point Likert scale. Mean subscale scores were calculated.

The $\mathrm{CATCH}$ questionnaire was used to measure health behaviours in children. The CATCH contains 130 items in seven subscales using a 3-point Likert scale.

PA of the children and parents was measured using the Actical Omni-directional accelerometer (Phillips Respironics, Bend OR, USA)

EAH: Each child participated in a standard ad libitum pizza dinner with their parents. Self-reported post-meal satiety was assessed with a cartoon representation of three levels of fullness along with two questions regarding each child's level of hunger. $10 \mathrm{~min}$ after the com pletion of the meal, each child tasted and rated palatability of small samples of snacks as "yummy," "just ok," and "yucky". Following the rating of foods the child was left alone in a room with containers of

pre-weighed portions of the snack foods as well as toys and games. After $10 \mathrm{~min}$, the co-ordinator returned to the room, and the amounts of remain-

ing food items were measured.

The total calories consumed by ta, Arlington Heights, IL, USA), and averaged. For children,

BMI percentiles were calculated with a computer using height, weight, age and gender, and for parents BMI was calculated with a computer using height and weight $(\mathrm{kg} / \mathrm{m} 2)$.

CDC charts for children

\section{Child height was}

measured using a

standard stadiome-

ter in duplicate. Chil-

dren's weight was

measured in dupli-

cate on a calibrat-

ed slide scale with-

out jackets, outer-

wear, or shoes. The

average of the two

values was used

for analysis. Chil-

dren's heights and

weights were trans-

lated to BMI-for age

percentile scores us-

the CDC growth

charts and to BMI-Z

scores
Products Inc.

Rockton, IL,

USA). Tri-

ceps and sub-

scapular skin-

folds were

measured ac-

cording to the

National

Health and

Nutrition Ex-

amination

Survey Proce-

dures on the

right side of

the body, 3

times, and av-

eraged

derived by

calculating

the child's

percent over

post-treat-

ment as-

sessment

the medi-

an BMI for

age and sex

(child's BMI-

median BMI

for age and

sex/median

BMI for age

and sex $x$ 100)

using CDC

growth charts

ticipant

in the in-

terven-

tion group

complet-

ed a treat-

ment eval-

uation

form that

asked

"How

much did

you like

the ROC

program?" 
child's estimated daily calori

respond

needs to derive the percent of

calorie needs consumed during

how true

the free access period (EAH\%).

ing state-

Dietary intake of the child was

were for

assessed with three 24-h di-

etary recalls. Average total dai-

them "Be-

cause of

caloric intake was used as a

ROC, I feel

more in

The EAH Questionnaire for Chil-

dren and Adolescents-Par-

control of

my eat-

ent Report of Child includes

three subscales: Negative Af-

fect, External Eating, and Fa-

tigue/Boredom Eating. Parents

ing.'

completed two scales from the

Addition-

ally, chil-

CEBQ regarding their child's

dren re-

eating patterns: Food Respon-

spond-

ed to the

following

question

siveness

with a yes

or no an-

swer: "Do

you think

other kids

your age

would like

the ROC

program?"

At the

post-treat-

ment as-

sessment

visit, each

parent

partici-

pant 
complet-

ed a treat-

ment eval-

uation

form that

asked

"How

much did

you like

the ROC

program?"

and "How

much do

you think

your child

liked

the ROC

program?"

Addi-

tionally,

parents

report-

ed how

much they

agreed or

disagreed

with the

following

statement

"The ROC

program

has taught

my child

to have

more con-

trol of 


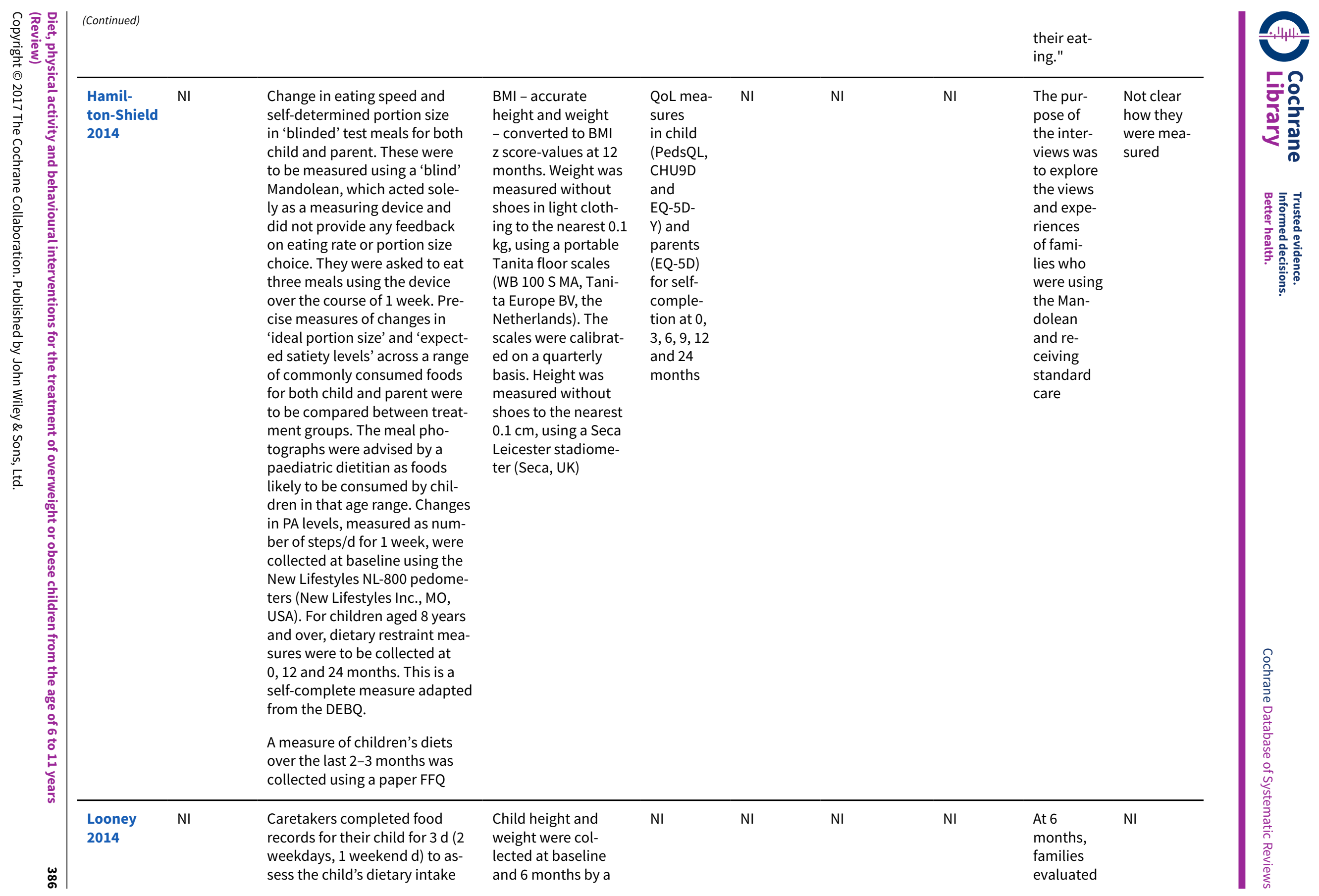




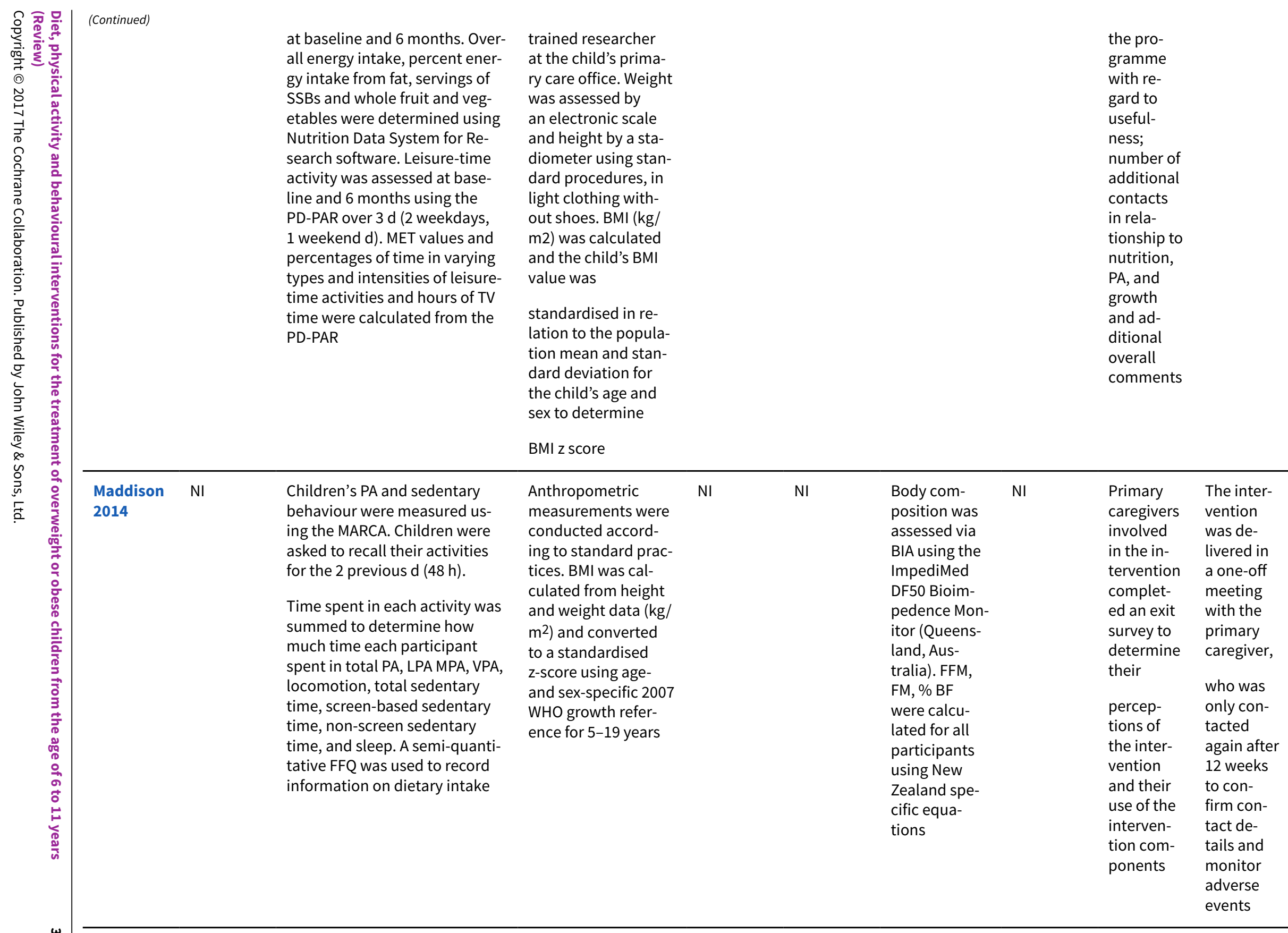




\begin{tabular}{|c|c|c|c|c|c|c|c|c|c|}
\hline 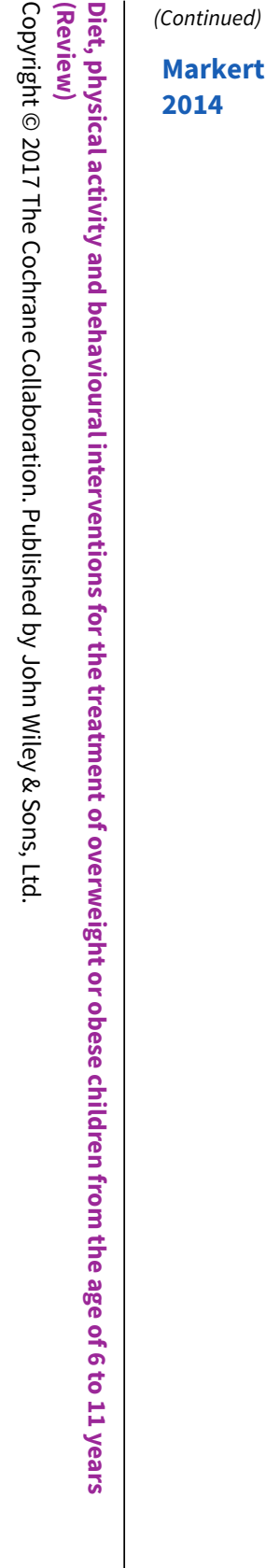 & $\mathrm{NI}$ & $\begin{array}{l}\text { The FFQ used in the KIGGS } \\
\text { study (individual eating habits) } \\
\text { and the AD-EVA questionnaires } \\
\text { (family eating habits) were } \\
\text { applied. An eating behaviour } \\
\text { score was calculated based } \\
\text { on four basic areas of eating } \\
\text { habits: number of meals/d, } \\
\text { joint meals within the family, } \\
\text { activity during meals, regular } \\
\text { mealtimes. Combining these } \\
\text { four values results in a 9-point } \\
\text { scale to run from -10 (bad) to } \\
+10 \text { (good) in steps of } 2.5 \text {. The } \\
\text { level of PA was assessed, based } \\
\text { on the questionnaires used by } \\
\text { KiGGS as well as the PA scale } \\
\text { (MoMo questionnaire). Infor- } \\
\text { mation on media consump- } \\
\text { tion (and leisure time habits) } \\
\text { was obtained applying the KIG- } \\
\text { GS-questionnaire }\end{array}$ & $\begin{array}{l}\text { Measurements of } \\
\text { body weight and } \\
\text { body height were as- } \\
\text { sessed at } 0 \text { and } 12 \\
\text { months of interven- } \\
\text { tion by } \\
\text { local paediatricians } \\
\text { with standardised } \\
\text { procedures and cen- } \\
\text { trally collected in } \\
\text { the CrescNet data- } \\
\text { base. BMI data were } \\
\text { standardised to age } \\
\text { and sex of the chil- } \\
\text { dren applying Ger- } \\
\text { man reference data } \\
\text { and were calculated } \\
\text { as BMI-SDS. A cut off } \\
\geq 1.28 \text { SDS ( } 90 \text { th cen- } \\
\text { tile) classifies over- } \\
\text { weight and a cut off } \\
\geq 1.88 \text { SDS (97th cen- } \\
\text { tile) classifies obesity } \\
\text { in German children }\end{array}$ & $\begin{array}{l}\text { HRQoL } \\
\text { was as- } \\
\text { sessed by } \\
\text { the KINDL- } \\
\text { R ques- } \\
\text { tionnaire. } \\
\text { Addition- } \\
\text { al parame- } \\
\text { ters that } \\
\text { were ob- } \\
\text { tained in- } \\
\text { clude re- } \\
\text { sources } \\
\text { and pro- } \\
\text { tection } \\
\text { factors for } \\
\text { HRQoL ex- } \\
\text { pectancy } \\
\text { for self- } \\
\text { efficacy, } \\
\text { subjective } \\
\text { life satis- } \\
\text { faction, } \\
\text { and infor- } \\
\text { mation } \\
\text { on social } \\
\text { support. } \\
\text { Items from } \\
\text { the KINDL- } \\
\text { R were } \\
\text { utilised. } \\
\text { Higher } \\
\text { score val- } \\
\text { ues cor- } \\
\text { respond } \\
\text { with a } \\
\text { higher } \\
\text { QoL, with } \\
\text { a high de- } \\
\text { gree of re- } \\
\text { liability } \\
\text { and valid- } \\
\text { ity }\end{array}$ & $\mathrm{NI}$ & $\mathrm{NI}$ & $\mathrm{NI}$ & $\mathrm{NI}$ & $\mathrm{NI}$ \\
\hline
\end{tabular}


es ranging from 0 to $\geq 3$. PA date

port and

collected using accelerometers

caregiv-

and a valid $d$ was when the ac-

er proxy

report

hours with a minimum of $10 \%$

generic

nonzero epochs per hour

core scales

The 24-h diet recall is a stan-

Height and weight

NI

dardised three-pass method,

were assessed by

developed by the US Depart-

school nurses via a

ment of Agriculture for use in

Harpenden Holtain

national dietary surveillance.

stadiometer, Mod-

Parents completed the phone

recalls regarding their child's

diet for two weekdays and one

weekend $d$ at each time point

el 603 (Holtain,

Crymych, UK) and a

portable SECA digi-

using standardised procedures.

All dietary data were analysed

tal scale (SECA, Ham-

burg, Germany).

2005. The ActiGraph (Actigraph

LLC, Pensacola, FL, USA) mea-

sured PA duration and intensi-

ty. Participants were asked to

wear the activity monitor for at

least $6 \mathrm{~h} / \mathrm{d}$ for a minimum of $3 \mathrm{~d}$

during a 1-week period. All da-

ta were run through Santech

were taken in tripli-

cate and used to cal-

culate BMI z score

and BMI percentile

for children (which

was used for edu-

cational purposes)

based on the CDC

MeterPlus soft

growth charts

CL (Achenbach, 1991) is a stan-

dardised measure that assess-

es parental report of child com-

petencies and behavioural or

emotional problems. Values for

total score, internalising behav

iour, and externalising behav-

iour were assessed.

BPFAS: The measure is com-

posed of 35 items: 25 describe

the child's feeding behaviour

and 10 describe parent's feel- 


\begin{tabular}{|c|c|c|c|c|c|c|c|c|c|c|c|}
\hline 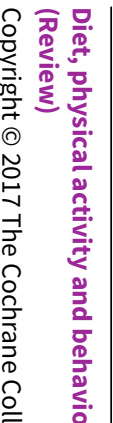 & (Continued) & & $\begin{array}{l}\text { ings about or strategies for } \\
\text { dealing with eating problems. } \\
\text { Parents are also asked to rate } \\
\text { on a scale from } 1-5 \text { how much } \\
\text { they agree or disagree with } \\
\text { each statement, as well as } \\
\text { whether each of the } 35 \text { items } \\
\text { are a problem. Higher scores } \\
\text { are suggestive of more prob- } \\
\text { lematic feeding behaviours }\end{array}$ & & & & & & & & 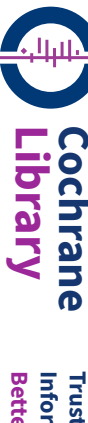 \\
\hline 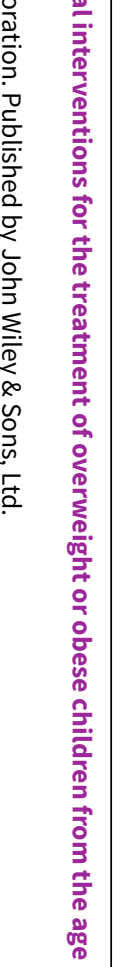 & $\begin{array}{l}\text { Davoli } \\
2013\end{array}$ & $\mathrm{NI}$ & $\begin{array}{l}\text { Secondary outcomes were the } \\
\text { percentage } \\
\text { of positive changes in par- } \\
\text { ent-reported dietary behav- } \\
\text { iours and in PA. These factors } \\
\text { were measured by using the } \\
\text { questionnaire }\end{array}$ & $\begin{array}{l}\text { The primary out- } \\
\text { come was the indi- } \\
\text { vidual } \\
\text { BMI score variation } \\
\text { as suggested by Cole } \\
\text { 2000. BMI score was } \\
\text { calculated as the } \\
\text { weight (kg) } \\
\text { divided by the } \\
\text { square of height (m). } \\
\text { The difference in BMI } \\
\text { was calculated as the } \\
\text { within-child differ- } \\
\text { ence between BMI } \\
\text { score at } 12 \text { months } \\
\text { and at baseline. BMI } \\
\text { z scores and changes } \\
\text { from overweight sta- } \\
\text { tus to normal weight } \\
\text { or obesity were al- } \\
\text { so reported to allow } \\
\text { comparability with } \\
\text { previous studies }\end{array}$ & $\mathrm{NI}$ & $\mathrm{NI}$ & $\mathrm{NI}$ & $\mathrm{NI}$ & $\mathrm{NI}$ & $\begin{array}{l}\text { Assessed } \\
\text { through } \\
\text { paedia- } \\
\text { tricians, } \\
\text { where } \\
\text { possible }\end{array}$ & 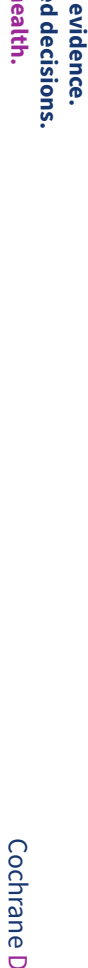 \\
\hline 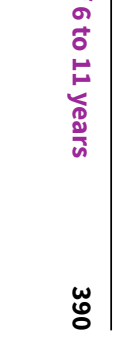 & $\begin{array}{l}\text { Lochrie } \\
2013\end{array}$ & $\mathrm{NI}$ & $\begin{array}{l}\text { The BASC-2: P (for ages 8-18) } \\
\text { is a well-validated instrument } \\
\text { for the assessment } \\
\text { of both positive and negative } \\
\text { features of }\end{array}$ & $\begin{array}{l}\text { Measurements were } \\
\text { then converted to } \\
\text { BMI, BMI percentiles, } \\
\text { and BMI z scores. BMI } \\
\text { z score reflects the } \\
\text { number of standard } \\
\text { deviations above or } \\
\text { below the average }\end{array}$ & $\begin{array}{l}\text { CDI-S is } \\
\text { a self-re- } \\
\text { port, } \\
\text { symp- } \\
\text { tom-orien- } \\
\text { tated scale }\end{array}$ & $\mathrm{NI}$ & $\begin{array}{l}\text { WC was mea- } \\
\text { sured with a } \\
\text { steel measur- } \\
\text { ing tape at the } \\
\text { high point of } \\
\text { the iliac crest } \\
\text { (to the near- } \\
\text { est } 0.1\end{array}$ & $\mathrm{NI}$ & $\mathrm{NI}$ & $\begin{array}{l}\text { BP was } \\
\text { measured } \\
\text { three } \\
\text { times with } \\
\text { the partic- } \\
\text { ipant com- } \\
\text { fortably }\end{array}$ & 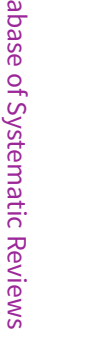 \\
\hline
\end{tabular}


behavioural adjustment in youth throughout the pediatric age range value for a child's age and gender, based on the current childhood norms measurin

depressive

symp-

The CDI-S

has 10

items,

each of

which

consists

of three

choices.

The CD

profile

contains

questions

regarding

negative

mood, in-

terperson-

al prob-

lems, in-

effective-

ness,

anhedo-

nia, and

negative

self-es-

teem.

Higher

scores

indicate

more de-

pressive

symp-

toms, as

report-

ed by the

child. Har-

ter SPP

is a child $\mathrm{cm}$ ) at min-

imal respi-

ration at the

end of nor-

mal expira-

tion. Measure

ment of WC

was trans-

formed into

percentiles

using child-

hood norms sitting re-

laxed, and

using an

appropri-

ately fit-

ted cuff

and an au-

tomated

sphygmo-

manome-

ter. Two

manual

BPs deter-

mined by

detection

Korotkoff

sounds

were done

for confir-

mation.

Only chil-

dren with

normal BP

were en-

tered into

the study 
The SPP

assesses

child and

adoles-

cent

self-per-

ception

in areas

believed

to be im-

portant

for self-

esteem:

scholas-

tic compe-

tence,

social ac-

ceptance,

athlet-

ic com-

petence,

physical

appear-

ance, be

haviour

conduct,

and global

self-worth.

\section{PedsOL}

(caregiver

and child).

Pediatric

QoL was

measured

with the

PedsQL

generic

version,

completed

separate-

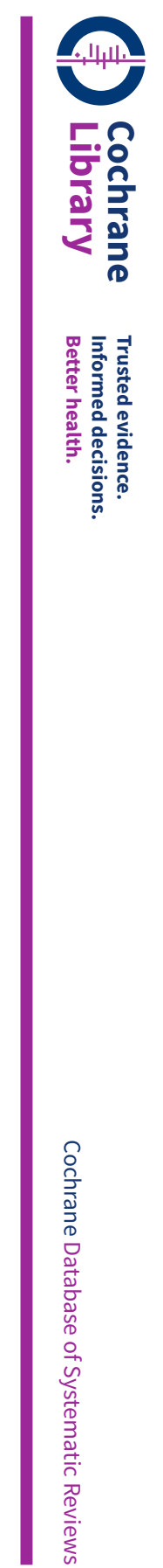


ly by care-

givers

and youth.

The

PedsQL is

a 23-item

instru-

ment with

separate

but sim-

ilar ver-

sions for

children

(ages 8-12)

and ado-

lescents

(ages

13-17) that

measures

$\mathrm{HRQOL}$

Dietary intake and composi-

BMI (in kg/m2) was

NI

tion were assessed with both

calculated. BMI z

a 24-h dietary recall and a 2-

score (BMI expressed

week dietary recall by using a

as a standard $z$ score

Block Kid FFQ. The Nutritionist

relative to CDC age-

Pro software (version 4.2; Axxya and sex-specific

norms)

an energy and macronutrient

analysis of the 24-h dietary re-

call. The daily GI was calculat-

ed by summing the weighted $G$

values for each food item. The

GL was calculated as the prod-

uct of the daily GI and total car-

bohydrate and adjusted for en-

ergy intake

$\begin{array}{llll}\begin{array}{l}\text { Changes } \\ \text { in meta- } \\ \text { bolic }\end{array} & \begin{array}{l}\text { Total BF mass } \\ \text { and FFM were } \\ \text { assessed by } \\ \text { using }\end{array} & \text { NI } & \begin{array}{l}\text { Unclear } \\ \text { how they } \\ \text { were mea- } \\ \text { sured }\end{array} \\ \begin{array}{ll}\text { syndrome } \\ \text { preva- }\end{array} & \begin{array}{l}\text { air-displace- } \\ \text { lence with } \\ \text { ment plethys- }\end{array} & \\ \text { interven- } & \begin{array}{l}\text { mography } \\ \text { tion were }\end{array} & \text { (BodPod; Life } & \\ \text { assessed } & \text { Measurement } & \\ \text { by using } & \text { Inc) } & \\ \text { metabolic } & & \\ \text { syndrome } & & \\ \text { criteria } & & \\ \text { proposed } & & \\ \text { by Cook } & & \\ \text { 2003. The } & & \\ \text { risk of } & & \\ \text { metabolic } & & \\ \text { syndrome } & & \\ \text { (present } & & \\ \text { compared } & & \\ \text { with } & & \end{array}$




\section{each time}

point and

taking in-

to account

the status

at the time

just before

(transition

modelling)

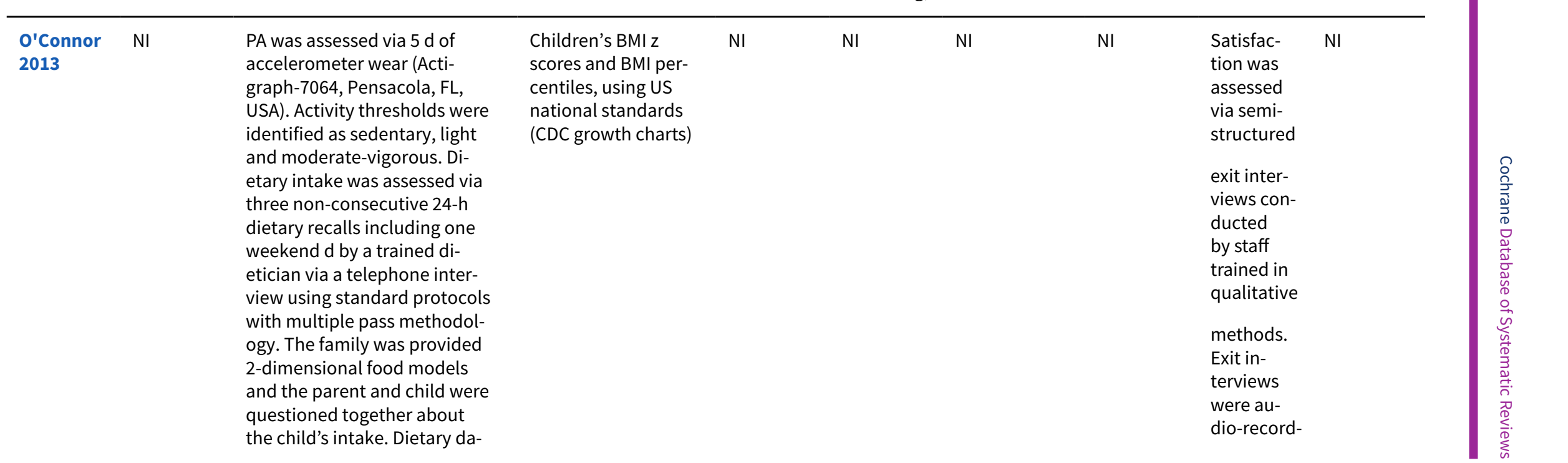


ta System, University of Min-

and trans-

nesota. TV viewing was as-

lated by

sessed by parent report of their

bilingual

staff. In-

terview re-

sponses

were

grouped

for cod-

5 main

themes:

general

feedback,

pro-

gramme

and ma-

terial,

health ad-

visor, pro-

gramme

barriers,

and pro-

gramme

improve-

ment and

sub-codes

assigned

\begin{tabular}{|c|c|c|c|c|c|c|c|c|c|}
\hline $\begin{array}{l}\text { Saelens } \\
2013\end{array}$ & $\mathrm{NI}$ & $\begin{array}{l}\text { At each assessment point, par- } \\
\text { ents rated their self-efficacy } \\
\text { or confidence to help their } \\
\text { child make and maintain eat- } \\
\text { ing and PA lifestyle changes us- } \\
\text { ing two items (response ranges } \\
\text { strongly disagree to strongly } \\
\text { agree; items were averaged). It } \\
\text { was expected that parent-pro- } \\
\text { vided self-directed interven- } \\
\text { tion would have more positive }\end{array}$ & $\begin{array}{l}\text { Children and partici- } \\
\text { pating parents were } \\
\text { weighed } 3 \\
\text { times in light cloth- } \\
\text { ing without shoes } \\
\text { using a digital } \\
\text { Scaletronix scale, } \\
\text { with more measure- } \\
\text { ments until agree- } \\
\text { ment within } 0.1 \mathrm{~kg} \text {, }\end{array}$ & $\mathrm{NI}$ & $\mathrm{NI}$ & $\mathrm{NI}$ & $\mathrm{NI}$ & $\mathrm{NI}$ & $\mathrm{NI}$ \\
\hline
\end{tabular}


changes in self-efficacy over

time than parent provided prescribed intervention and those values av-

eraged. Height

was measured with

a Heightronic sta-

diometer at least

in triplicate, until

agreement within 0.5

ues

averaged. In one in stance at 2-year fol-

low-up, child weight and height informa-

tion was obtained

only by parent-re-

port of child mea-

sures at a recent pae-

diatrician appoint-

ment.

BMI was calculated as $\mathrm{kg} / \mathrm{m} 2$. Children's percent above

median BMI and BMI

z scores were cal-

culated using $C D C$

growth charts for

age-specific median

standard deviation,

and distribution

skewness correction

and the LMS method

(Kuczmarski 2002)

Siwik NI At each data collection visit,

the children were given a vali-

dated PA recall focused on re-

calling activities before, dur-

ing, and after school of the cur-

rent and previous d. A MET (a

unit describing the energy ex-

penditure of a specific activi-
(

Reference popula-

tion data from the

CDC were used for $z$

Scores

NI

NI

NI
Weight and
$\%$ BF were
measured
on an elec-

tronic scale

with built-

in BIA (Tani- 
ty) was assigned to each activ ity. Pedometers were used as an intervention tool, but did

not contribute to the outcome measures ta, Arlington

Heights, IL) views 12 -

18 months

and again

months af-

ter inter-

vention.

matched

interview-

ers met

the chil-

dren indi-

vidually.

Initial

ques-

tions were

open-end-

ed and re-

garded

recall of

the inter-

vention;

probes on

the

easiest,

most diffi-

cult, and

continuing

lifestyle

changes

followed.

Parents

were

queried

about

project

content

(Choices

model,

thinking

patterns),

changes

in chil-

dren's be-

haviour, 


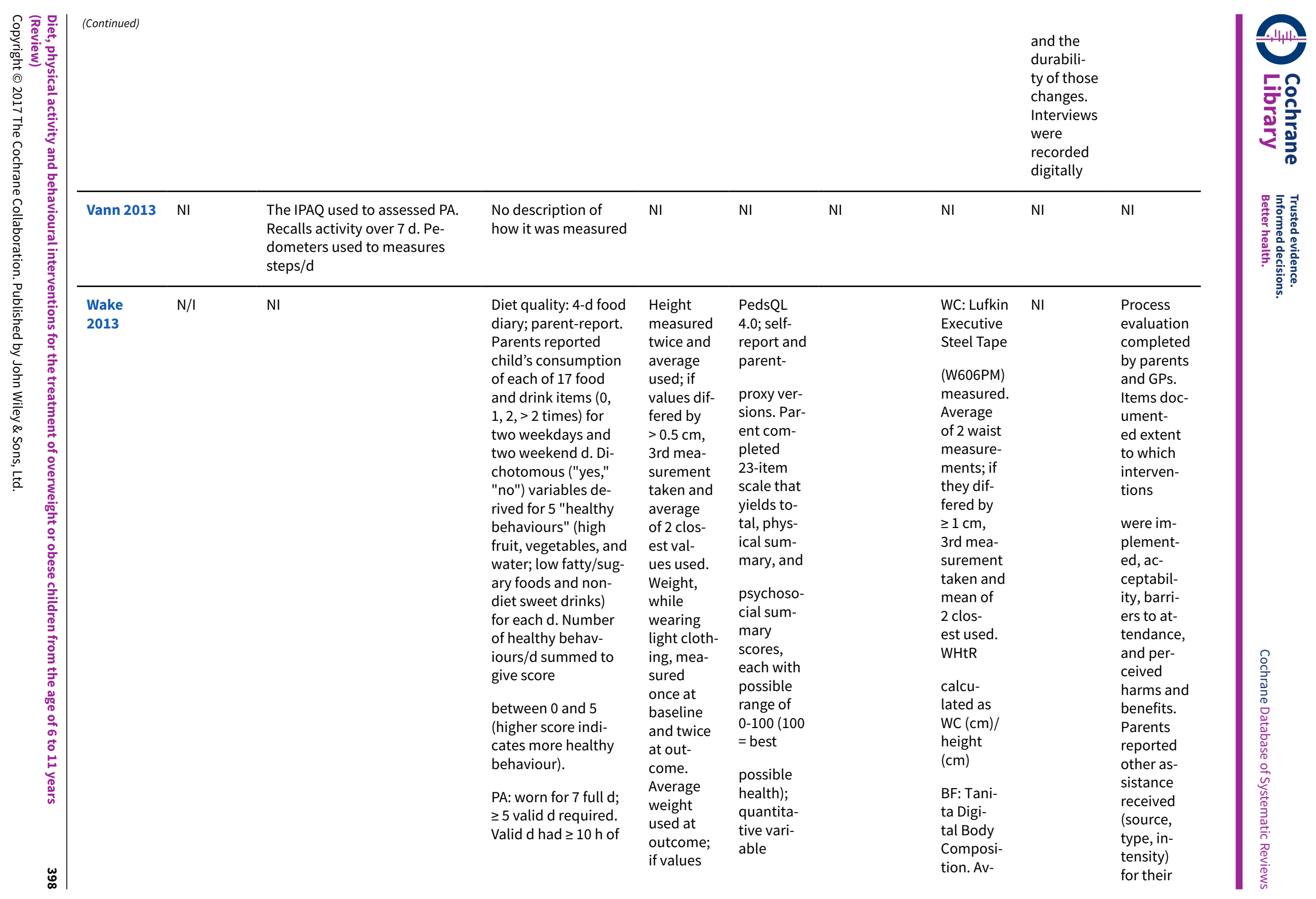


non-missing data be-

tween 06:00 $\mathrm{h}$ and

11:00 h. Missing data

were segments

with $\geq 20 \mathrm{~min}$

of consecutive " 0 "

counts, or counts $>0$

that were constant

for $\geq 10$ min. Out-

comes across all

valid d: mean activity

counts/min

and $\%$ time spent

in MVPA Actical Ac-

celerometer (Mini

Mitter) measured differed by Physical

$\geq 0.2 \mathrm{~kg}$,

3rd mea-

sure tak -

en and

average

2 clos-

est values

used. BM

calculated

as weight

(kg)/

(height

$\left.\mathrm{m}^{2}\right) ; \mathrm{z}$

score cal-

culated

according

Portable

rigid sta-

diome-

ter (mod-

el IP0955,

Invicta,

Leices-

ter, UK);

measured.

Calibrat-

ed digi-

tal scale

(model

ITHD646,T

ta, Toyko,

Japan);

BMI index

z score -

US CDC

reference

values

modi-

fied from

Harter's

PCSC; self-

report.

of state-

ments

with bi-

nary re

sponse

children

chose

statement

from each

pair clos-

est to their

compe-

tence.

Each of 6

s then

coded as

being ei-

ther "posi-

tive/better

percep-

"nega-

tive/worse

percep-

tion". 6 re-

sponses

analysed

as single

outcome

(\% pos-

itive re-

sponses

erage of

2 body

children's

weight

percent-

measure-

ments. 


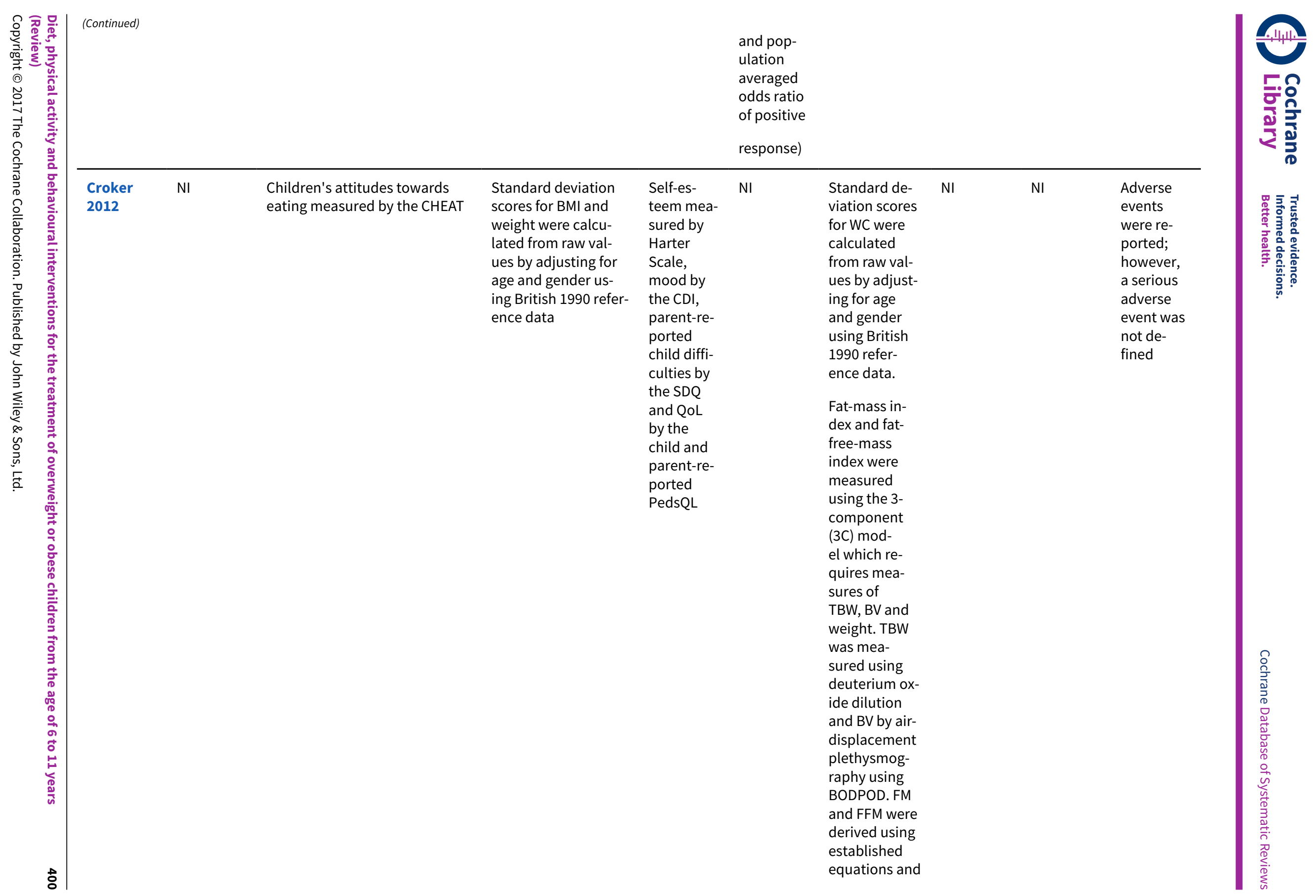




\begin{tabular}{|c|c|c|c|c|c|c|c|c|c|c|c|}
\hline 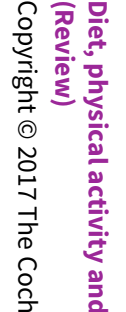 & (Continued) & & & & & & $\begin{array}{l}\text { index values } \\
\text { calculated by } \\
\text { dividing each } \\
\text { by height } \\
\text { squared to } \\
\text { take height in- } \\
\text { to account }\end{array}$ & & & & 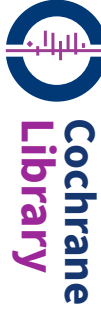 \\
\hline 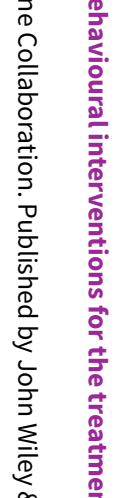 & $\begin{array}{l}\text { de Niet } \\
2012\end{array}$ & $\mathrm{NI}$ & $\begin{array}{l}\text { DEBQ measures emotional eat- } \\
\text { ing and external eating, it al- } \\
\text { so measures restrained eating } \\
\text { - high scores reflect high de-- } \\
\text { gree of eating behaviour. SP- } \\
\text { PC measures scholastic compe- } \\
\text { tence, social acceptance, ath- } \\
\text { letic competence, physical ap- } \\
\text { pearance and behavioural con- } \\
\text { duct. In addition it assess glob- } \\
\text { al self-worth. High scores re- } \\
\text { flect greater perceived compe- } \\
\text { tence or global self-worth }\end{array}$ & $\begin{array}{l}\text { BMI-SDS corrected } \\
\text { for age and gender. } \\
\text { BMI-SDS of } 1.1 \text { repre- } \\
\text { sents the threshold } \\
\text { for overweight, and } \\
\text { BMI-SDS of } 2.3 \text { indi- } \\
\text { cates obesity (Cole } \\
2000 \text { ) }\end{array}$ & $\begin{array}{l}\text { HRQoL } \\
\text { assessed } \\
\text { by the } \\
\text { CHQ-PF50 } \\
\text { and was a } \\
\text { Dutch-val- } \\
\text { idated ver- } \\
\text { sion. High- } \\
\text { er scores } \\
\text { reflect } \\
\text { best possi- } \\
\text { ble health } \\
\text { state }\end{array}$ & $\mathrm{NI}$ & $\mathrm{NI}$ & $\mathrm{NI}$ & $\mathrm{NI}$ & $\mathrm{NI}$ & 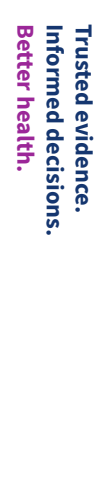 \\
\hline 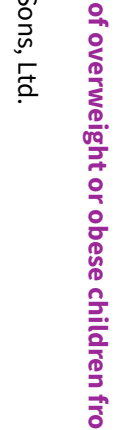 & $\begin{array}{l}\text { Eddy Ives } \\
2012\end{array}$ & $\mathrm{NI}$ & $\begin{array}{l}\text { Dietary and physical exercise } \\
\text { habits, recorded in a question- } \\
\text { naire developed specifically for } \\
\text { that study }\end{array}$ & $\begin{array}{l}\text { Evaluation of the BMI } \\
\text { and the associated } \\
\text { z score. The BMI z } \\
\text { scores were calculat- } \\
\text { ed using the growth } \\
\text { charts published by } \\
\text { the WHO }\end{array}$ & $\begin{array}{l}\text { AF-5 ques- } \\
\text { tionnaire } \\
\text { for self-es- } \\
\text { teem - di- } \\
\text { mensions } \\
\text { included } \\
\text { emotional, } \\
\text { physical, } \\
\text { academ- } \\
\text { ic, social, } \\
\text { family }\end{array}$ & $\mathrm{NI}$ & $\begin{array}{l}\text { Measured the } \\
\text { abdominal } \\
\text { perimeter and } \\
\text { the associat- } \\
\text { ed z score }\end{array}$ & $\mathrm{NI}$ & $\mathrm{NI}$ & $\mathrm{NI}$ & \\
\hline 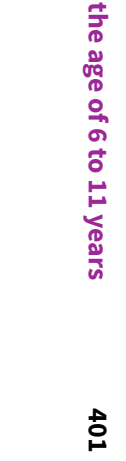 & Kirk 2012 & $\mathrm{NI}$ & $\begin{array}{l}\text { Food records were kept over } 3 \\
\text { consecutive } \\
\text { d ( } 2 \text { weekdays and } 1 \text { weekend } \\
\text { d) during the week before the } \\
\text { assessment visit. Food records } \\
\text { were analysed using the Nutri- } \\
\text { tion Data System for Windows } \\
\text { version } 4.04 \text { (Nutrition Coor- } \\
\text { dinating Center, University of } \\
\text { Minnesota, Minneapolis, Min- } \\
\text { nesota) }\end{array}$ & $\begin{array}{l}\text { Following stan- } \\
\text { dardised protocols, } \\
\text { trained research staff } \\
\text { measured height us- } \\
\text { ing a wall-mounted } \\
\text { stadiometer (Ayr- } \\
\text { ton 226; Stadiome- } \\
\text { ter.com, Sno- } \\
\text { qualmie, }\end{array}$ & $\mathrm{NI}$ & $\mathrm{NI}$ & $\begin{array}{l}\text { WC using a fi- } \\
\text { breglass tape } \\
\text { measure with } \\
\text { calibrated } \\
\text { tension de- } \\
\text { vice (Gulick } \\
\text { M-22C; Cre- } \\
\text { ative Health } \\
\text { Products, } \\
\text { Plymouth, } \\
\text { Michigan). }\end{array}$ & $\mathrm{NI}$ & $\mathrm{NI}$ & $\begin{array}{l}\text { Elevated } \\
\text { metabolic } \\
\text { outcomes } \\
\text { were as- } \\
\text { sessed }\end{array}$ & 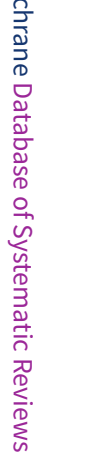 \\
\hline
\end{tabular}




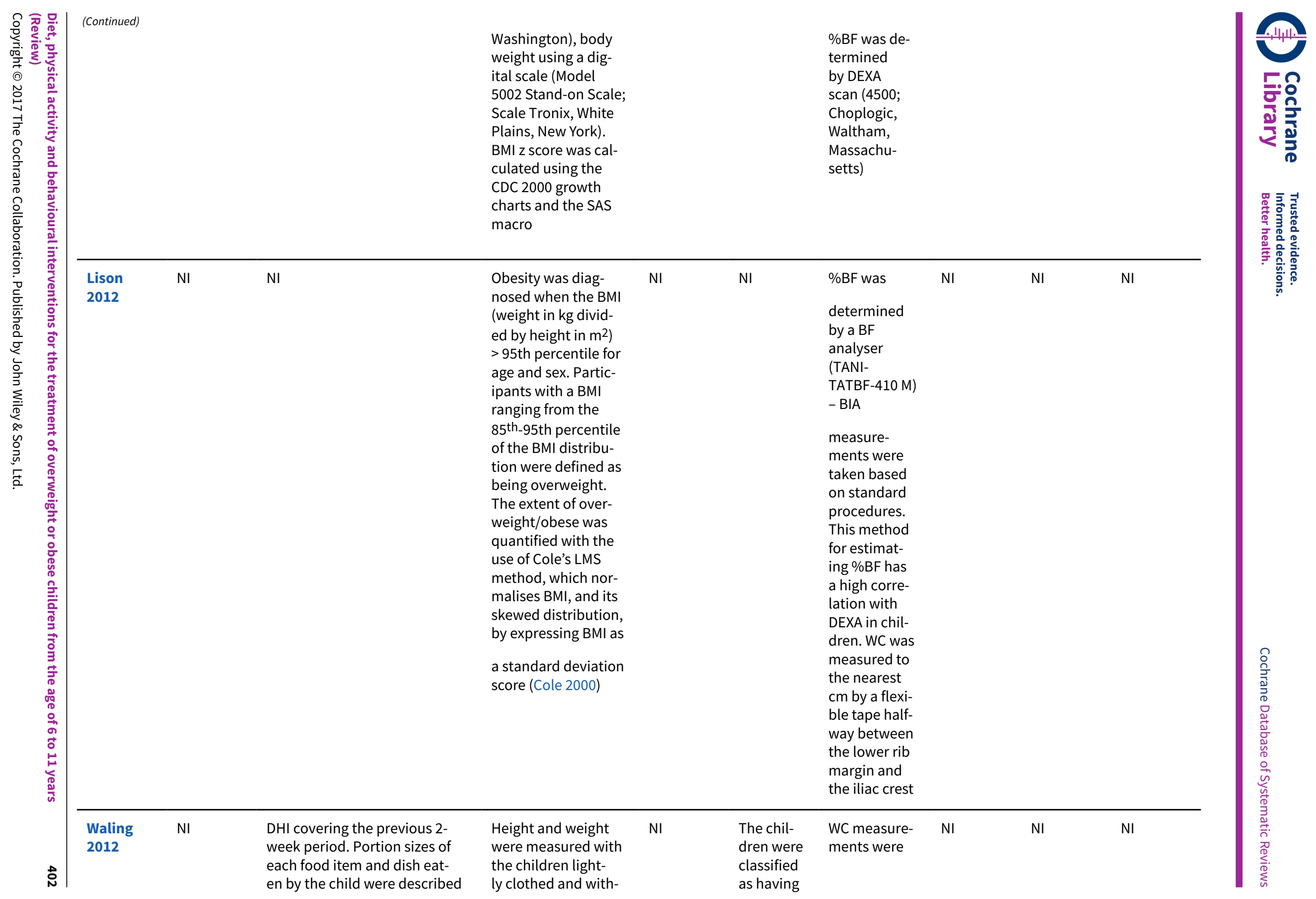


with the help of food-portion photographs, household measures, standard weights of food items, or bags of rice in different volumes. To follow the children's food habits, three 2-d food records were conducted by each child during the first year of the study. The records were scheduled to cover weekdays and weekend $\mathrm{d}$ as well as different seasons. After 1 year of participation, each child conducted a 4-day food record. Reported food intakes from the

$\mathrm{DHI}$ at baseline and the food records were entered into the dietary analysis programme Dietist XP version 3.0 to calculate the daily energy and nutrient intake. Dietist XP uses the Swedish food composition database (version March 6 2008). TEE was measured using a SenseWear armband.

At baseline, TEE was measured during $4 \mathrm{~d}$ included in the 14-d period that the $\mathrm{DHI}$ covered and for the food records, TEE was measured during the same d. PA was assessed with SenseWear Armband during 4 consecutive $d$ ( 2 weekdays and 2 weekend d) at baseline and after 2 years of participation out shoes. Height was measured to the nearest $0.1 \mathrm{~cm}$ with a wall stadiometer (HyssnaMeasuring Equipment $A B$, Sweden), and weight was measured to the nearest $0.1 \mathrm{~kg}$ with an electronic scale (AJ Medical, Sweden). The main outcome of the study, $\mathrm{BMI}$, was calculated as weight $(\mathrm{kg}) /$ height $(\mathrm{m})^{2}$ and converted to BM

z scores by using both US reference data and a Swedish reference dataset. Children were classified as normal weight, overweight, or obese using th

(1)

ing the de-

finitions of

recorded to

the Inter-

national

Diabetes

Federa-

tion; WC $\geq$

90th per-

centile

presence

of 2 or

more oth-

er clinical

features

(i.e. ele-

vated

TC, low

$\mathrm{HDL}-\mathrm{C}$,

high BP, or

increased

glucose)

$0.1 \mathrm{~cm}$ mid-

way between

the tenth rib

and the ili-

ac crest with

children in a

standing po-

sition using

a non-elastic

flexible tape.

Sagittal ab-

dominal di-

ameter was

measured to

the nearest

$0.1 \mathrm{~cm}$ using

a ruler with

the child in

a supine po-

sition from

the bed to the

top of the ab-

domen. Body

composition

analysis was

performed us

ing DEXA (Lu-

nar Prodigy

whole-body

scanner GE

Medical Sys-

tems, Madi-

son, WI, USA),

with the child

in a supine

position.

$\mathrm{BF}$ content is

expressed as

absolute val-

ues $(\mathrm{kg})$ and

as $\mathrm{FM} \%$, and

truncal fat ex-

pressed as 


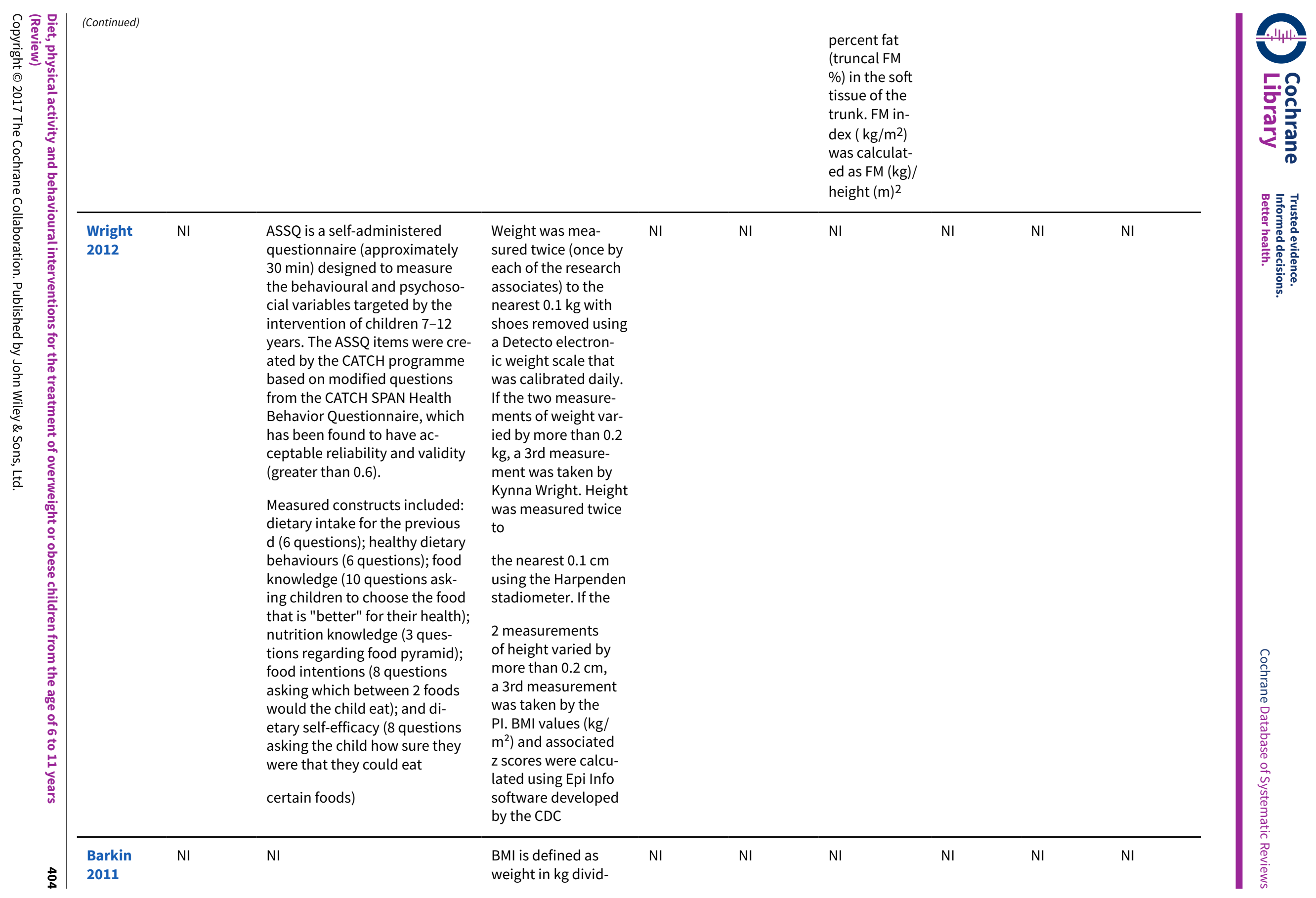


centile for age and gender was calculated by using the

CDC calculator. "Be-

cause approximate-

ly two thirds of chil-

dren were above

the 95th percentile,

we used absolute

BMI instead of BMI

percentile to avoid

a compressed per-

centile scale bound-

ed by $100 . "$

Bryant NI PA over a 7-d period measured

Height was mea-

sured to within 0.1

$\mathrm{cm}$ using a wall-

qu

PedsQL
question-
naire

$\mathrm{NI}$

Trained re-
searchers
measured
weight,

WATCH IT Diet questionnaire

designed specifically to exam-

ine foods aligned to the dietary

goals promoted as part of the

intervention), DEBQ, PAC-Q

Robinson School-Based Seden-

consistency, 2 mea-

surements were tak-

en, and an average

tary Behaviour Questionnaire, they differed by $>0.5$

SDQ, and the Harter Scale

$\mathrm{cm}$, a 3rd measure-

Perceived Social and Cognitive ment was taken, and

Competence

an average of the

closest 2 was used.

Weight was mea-

sured in light cloth-

ing with no shoes (to

within $0.1 \mathrm{~kg}$ ) using a

calibrated Seca digi-

tal weighing scale

height, WC,

and BIA (HY-

DRA ECFICF

model 4200;

Xitron tech-

nologies,

San Diego,

CA) and per-

formed a

DEXA (Lunar

Prodigy; GE

Medical Sys-

tems, Madi-

son, WI) scan

at baseline

and 6 and

12 months.

WC was mea-

sured twice

at $4 \mathrm{~cm}$ above

the umbili-

cus. Whenev-

er measure-
Feedback

interviews

or any in-

dividual

assess-

ment. Of

the $10 \%$

of families

who were

randomly

selected

to pro-

vide feed-

back at

the end of

the study,

all except

1 parent

report-

ed a posi-

tive expe-

rience
They were measured

and on

the agen-

da at each

meeting

ported 
surement was

taken, and an

average of the

closest 2 was

used

\begin{tabular}{|c|c|c|c|c|c|}
\hline $\begin{array}{l}\text { Coppins } \\
2011\end{array}$ & $\mathrm{NI}$ & $\begin{array}{l}\text { Food and activity assessed by a } \\
\text { 7-d food diary at baseline and } \\
\text { each 6-month review. Frequen- } \\
\text { cy of use of specific food was } \\
\text { extracted from food diaries - } \\
\text { this method not validated. PA } \\
\text { assessed by Yamax Digi-Walk- } \\
\text { er electronic pedometer over 7- } \\
\text { d period. Record also taken of } \\
\text { time spent in low-, moderate- }\end{array}$ & $\begin{array}{l}\text { BMI calculated using } \\
\text { weight/height } 2 \text { and } \\
\text { plotted on the Child } \\
\text { Growth Foundation } \\
\text { BMI chart (1997). BMI } \\
\text { adjusted for age and } \\
\text { gender to give BMI } \\
\text { SDS (BMI z score) } \\
\text { using British 1990 } \\
\text { Growth Reference }\end{array}$ & $\mathrm{NI}$ & $\mathrm{NI}$ \\
\hline
\end{tabular}

time spent in low-, moderateData

WC was mea-
sured in cen-
timetres to
one decimal
point using a
standard an-
thropomet-
ric tape at the
maximal ab-
dominal girth.
Results were
plotted on the
Child Growth
Foundation
WC
Chart (2005)
and convert-
ed to WC SDS
(z score) us-
ing the British
1990 Growth
Reference Da-
ta. The Tani-
ta BF Monitor
(BIA) (Chas-
mors Ltd)
was used to
analyse BF to
$\pm 0.5 \%$ preci-
sion and re-
sults were
plotted on
the Child
Growth Foun-
dation BF
Chart (2005).


measure-

ments (mm)

were taken at

the calf, sub-

scapular and

triceps sites

using the non-

dominant

side and av-

eraged. Sum

of 3 skinfolds

was calculat-

\begin{tabular}{|c|c|c|c|c|}
\hline $\begin{array}{l}\text { Gunnars- } \\
\text { dottir } \\
\text { 2011a }\end{array}$ & $\mathrm{NI}$ & $\begin{array}{l}\text { Children and parents were giv- } \\
\text { en instructions on measuring } \\
\text { and recording consumption } \\
\text { of fruits and vegetables. The } \\
\text { average number of servings/d } \\
\text { was graphed weekly to moni- } \\
\text { tor changes during treatment. } \\
\text { Children and parents were in- } \\
\text { structed to record all children's } \\
\text { PA outside of school that went } \\
\text { on for at least } 5 \text { continuous min } \\
\text { and was of at least medium } \\
\text { intensity (defined and taught } \\
\text { before the start of monitoring } \\
\text { as being equivalent to a brisk } \\
\text { walk). The average min of PA/ } \\
\text { d was graphed from week to } \\
\text { week during treatment to mon- } \\
\text { itor changes }\end{array}$ & $\begin{array}{l}\text { Weight was mea- } \\
\text { sured with a digital } \\
\text { scale (Marel type C2, } \\
\text { Marel, Reykjavík, Ice- } \\
\text { land). Height was } \\
\text { measured with a } \\
\text { wall-mounted dig- } \\
\text { ital stadiometer } \\
\text { (Ulm, Germany). BMI } \\
\text { (weight in kg m²) } \\
\text { was calculated, and } \\
\text { BMI standard devia- } \\
\text { tion scores (BMI-SDS) } \\
\text { were derived from } \\
\text { BMI reference val- } \\
\text { ues for Swedish chil- } \\
\text { dren as calculation } \\
\text { of BMI-SDS not possi- } \\
\text { ble based on the cut- } \\
\text { offs of IOTF }\end{array}$ & $\begin{array}{l}\text { Psycho- } \\
\text { logical } \\
\text { disor- } \\
\text { der/learn- } \\
\text { ing dis- } \\
\text { ability de- } \\
\text { fined by } \\
\text { the di- } \\
\text { agnoses } \\
\text { were con- } \\
\text { firmed } \\
\text { during } \\
\text { screen- } \\
\text { ing by se- } \\
\text { mi-struc- } \\
\text { tured in- } \\
\text { terviews. } \\
\text { SDQ: the } \\
\text { CDI and } \\
\text { MASC: re- } \\
\text { sults not } \\
\text { presented } \\
\text { in the pa- } \\
\text { per) }\end{array}$ \\
\hline
\end{tabular}

Psycho-

logical

$\mathrm{NI}$

disor-

ders/learn-

ing dis-

ability di-

agnosed

using

question-

naires

given in

HRQoL

section

At the end $\mathrm{NI}$
of treat-
ment, par-
ticipants
complet-
ed accept-
ability
question-
naires.
Individ-
ual treat-
ment com-
ponents
were rated
on a
5-point
Likert
scale,
where 1
represent-
ed "very
help-
ful/satis-
fied" and 5
"not help-
ful/ satis-
fied at all".
Addition-


sessed by using the $20-\mathrm{m}$ shut-

tle test that requires partici-

pants to run continuously be-

tween 2 lines, which are 20

$\mathrm{m}$ apart, in time to recorded

beeps. The output can be used

to determine VO2max in this

age group. Participants were in-

structed to wear the accelerom

eter on their right hip during

waking hours for $7 \mathrm{~d}$ after each

assessment. During the $7 \mathrm{~d}$ af-

ter each assessment, partici-

pants provided self-reports of

their daily time spent playing

all video games by using a diary

developed and tested in a pre-

vious pilot study. Participants

completed a snack food diary

to self-report the frequency and

quantity of snack foods con-

sumed for 7 consecutive $d$. The

diary consisted of pictures of

29 common categories of snack

\begin{tabular}{|c|c|c|c|c|c|c|}
\hline $\begin{array}{l}\text { The mean of } 2 \text { mea- } \\
\text { surements or the } \\
\text { median of } 3 \text { mea- } \\
\text { surements were used } \\
\text { for analysis. BMI z } \\
\text { score was derived } \\
\text { separately at each } \\
\text { time point by using } \\
\text { data from the } 2002 \\
\text { New Zealand Na- } \\
\text { tional Children's Nu- } \\
\text { trition Survey be- } \\
\text { fore calculating the } \\
\text { change in BMI z score }\end{array}$ & $\mathrm{NI}$ & $\mathrm{NI}$ & $\begin{array}{l}\text { WC was mea- } \\
\text { sured twice } \\
\text { (as for height } \\
\text { and weight) } \\
\text { to the nearest } \\
0.1 \mathrm{~cm} \text { with a } \\
\text { standard an- } \\
\text { thropomet- } \\
\text { ric tape at the } \\
\text { maximal cir- } \\
\text { cumference. } \\
\text { BF was as- } \\
\text { sessed by us- } \\
\text { ing standard- } \\
\text { ised analysis } \\
\text { procedures of } \\
\text { BIA with the } \\
\text { ImpediMed } \\
\text { DF50 Bioim- } \\
\text { pedence Mon- } \\
\text { itor (Imped- } \\
\text { iMed, } \\
\text { Queensland, } \\
\text { Australia). }\end{array}$ & $\mathrm{NI}$ & $\mathrm{NI}$ & $\begin{array}{l}\text { A serious } \\
\text { adverse } \\
\text { event was } \\
\text { defined as } \\
\text { any event } \\
\text { that re- } \\
\text { quired } \\
\text { hospital- } \\
\text { ization } \\
\text { and was } \\
\text { deter- } \\
\text { mined at } \\
12 \text { and } 24 \\
\text { weeks }\end{array}$ \\
\hline
\end{tabular}


foods and drinks. For each food or drink, 3 pictures were presented. Participants indicated the number of servings of the respective food and serving size they had consumed on each d. Each serving size was assigned a caloric value (kJ). The caloric value of all reported snacks was summed and divided by $7 \mathrm{~d}$ to give the average daily total energy consumed from snacks (kJ)
Children were

hydrated and

required to

void thei

bladder be-

fore measure

ment. A equa-

tion was used

to calculate

FM, FFM and

percentage

BF for all par-

ticipants

\section{Wafa $2011 \quad \mathrm{NI}$}

Habitual PA and sedentary

In the absence of

Malaysian reference

data for BMI for age,

behaviour were measured objectively over $5 \mathrm{~d}$ - during the waking hours - at baseline and

follow-up using a CSA/MTI GT1M accelerometer

(The Actigraph, Fort Wal-

ton Beach, Florida, USA). Ac-

celerometry data were includ

ed so long as at least $4 \mathrm{~d}$ of

monitoring with at least $10 \mathrm{~h} /$

$\mathrm{d}$ were obtained accelerome-

try counts/min (cpm) were used as a measure of total volume

of PA. Accelerometry data were

also summarised using cut-

off points as percentage of the

time spent in sedentary behav-

iour LPA and MVPA.

outcome measure was BMI z score

calculated relative to

US CDC 2000 BMI for

age reference data.

Weight was mea-

sured to $0.1 \mathrm{~kg}$ in

light indoor cloth-

ing with children

not wearing shoes,

and height was mea-

sured to $0.1 \mathrm{~cm}$ with

a portable stadiome

ter (Leicester Height

Measure, SECA, UK

and children not

wearing shoes.

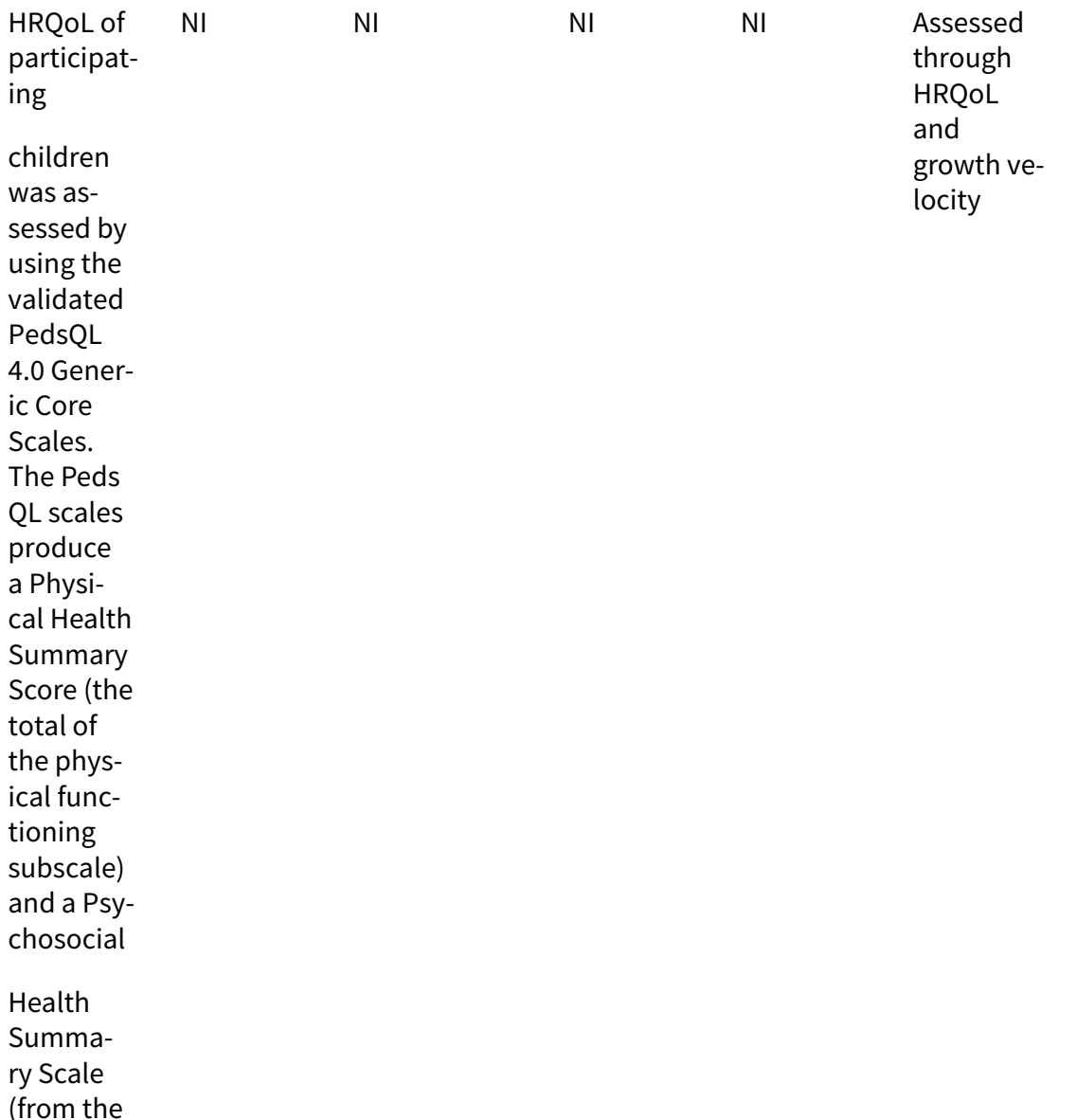




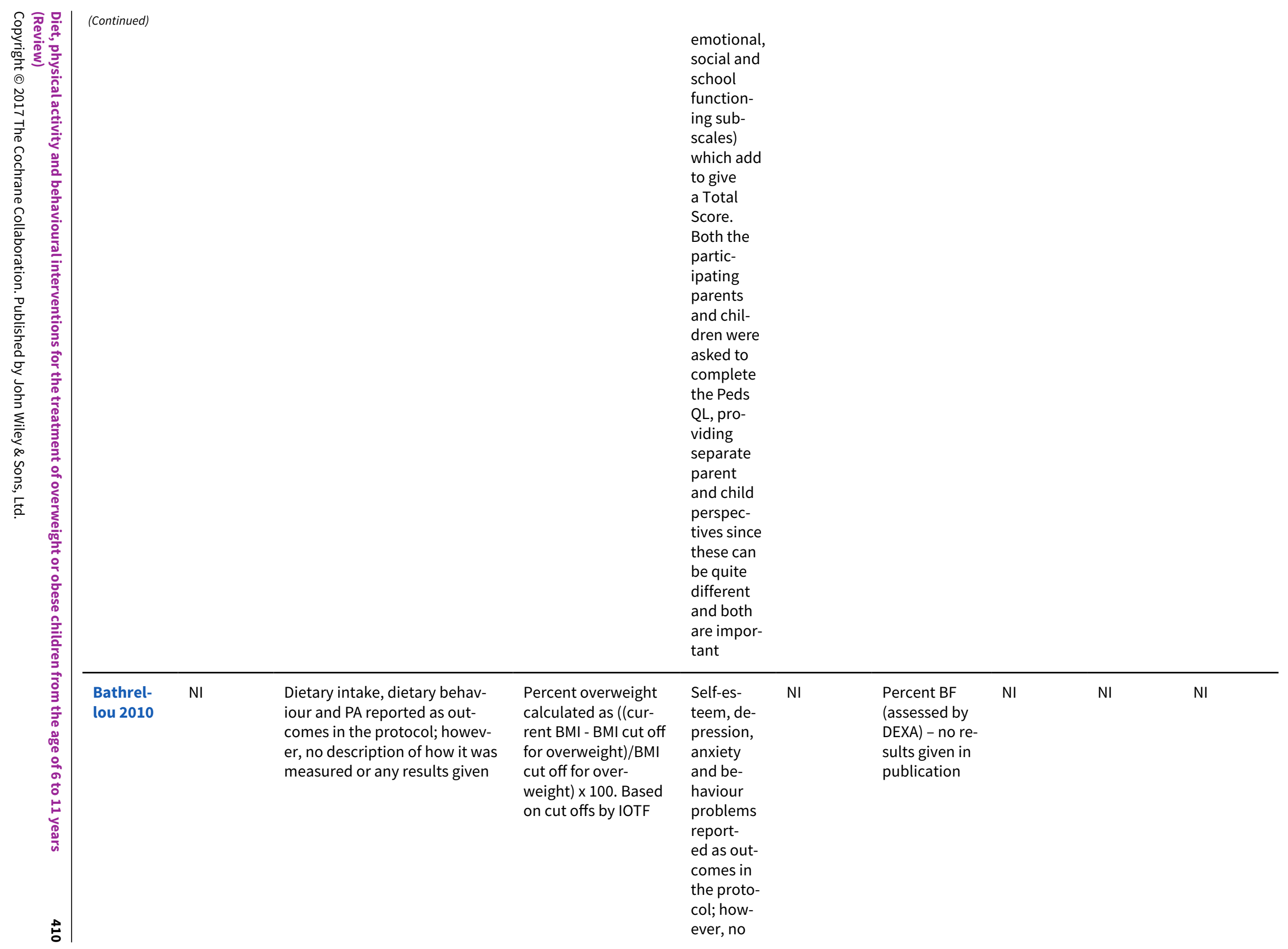




\begin{tabular}{|c|c|c|c|c|c|c|c|c|c|c|c|}
\hline 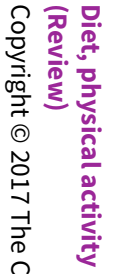 & (Continued) & & & & $\begin{array}{l}\text { descrip- } \\
\text { tion of } \\
\text { how mea- } \\
\text { sured or } \\
\text { any results } \\
\text { given }\end{array}$ & & & & & & 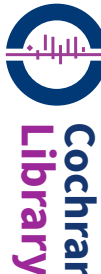 \\
\hline 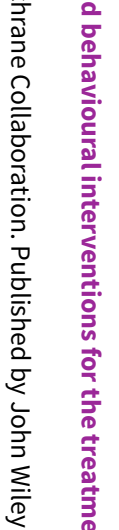 & Diaz 2010 & $\mathrm{NI}$ & $\mathrm{NI}$ & $\begin{array}{l}\text { BMI and BMI z score } \\
\text { were obtained us- } \\
\text { ing Epi Info software. } \\
\text { Based on US growth } \\
\text { charts (Kuczmarski } \\
\text { 2002) }\end{array}$ & $\mathrm{NI}$ & $\mathrm{NI}$ & $\begin{array}{l}\text { BF was deter- } \\
\text { mined by a } \\
\text { whole-body } \\
\text { DEXA } \\
\text { scan (Lunar } \\
\text { DPX-MD, GE } \\
\text { Lunar Corpo- } \\
\text { ration, Madi- } \\
\text { son, WI), and } \\
\text { WC measured } \\
\text { according to } \\
\text { established } \\
\text { guidelines }\end{array}$ & $\mathrm{NI}$ & $\mathrm{NI}$ & $\mathrm{NI}$ & 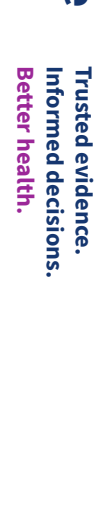 \\
\hline 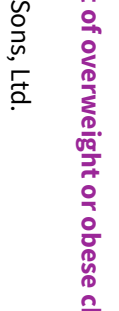 & $\begin{array}{l}\text { Duggins } \\
2010\end{array}$ & $\mathrm{NI}$ & $\begin{array}{l}\text { Eating habits assessed by ques- } \\
\text { tionnaire - based on Nation- } \\
\text { al Institutes of Health We Can! } \\
\text { Go, slow and whoa foods. Num- } \\
\text { ber of servings of different food } \\
\text { types were identified }\end{array}$ & $\begin{array}{l}\text { BMI for age per- } \\
\text { centile was deter- } \\
\text { mined using mea- } \\
\text { sured height and } \\
\text { weight and reference } \\
\text { to age- and sex-nor- } \\
\text { mative } \\
\text { data from the CDC }\end{array}$ & $\mathrm{NI}$ & $\mathrm{NI}$ & $\mathrm{NI}$ & $\mathrm{NI}$ & $\mathrm{NI}$ & $\mathrm{NI}$ & \\
\hline 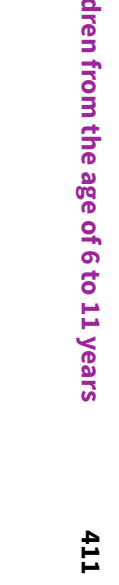 & $\begin{array}{l}\text { Faude } \\
2010\end{array}$ & $\mathrm{NI}$ & $\begin{array}{l}\text { Cycling ergometry used to mea- } \\
\text { sure aerobic capacity and heart } \\
\text { rate. Oxygen uptake was con- } \\
\text { tinuously measured until ex- } \\
\text { haustion. Motor ability tests } \\
\text { included counter-movement } \\
\text { jump, a sit and reach test, a bal- } \\
\text { ance test (by a one-leg standing } \\
\text { test), an agility test and a } 20 \text { m } \\
\text { shuttle run test }\end{array}$ & $\begin{array}{l}\text { Age and gender-stan- } \\
\text { dardised BMI z } \\
\text { scores were calcu- } \\
\text { lated using the LMS } \\
\text { method. BMI per- } \\
\text { centiles were calcu- } \\
\text { lated with respect } \\
\text { to a large German } \\
\text { reference sample } \\
\text { (Kromeyer-Hauschild } \\
\text { 2001) }\end{array}$ & $\begin{array}{l}\text { The } \\
\text { KINDL-R } \\
\text { question- } \\
\text { naire was } \\
\text { used to } \\
\text { calculate } \\
\text { HRQoL. } \\
\text { Is in Ger- } \\
\text { man lan- } \\
\text { guage and } \\
\text { comprises } \\
24 \text { items } \\
\text { on a 5- }\end{array}$ & $\mathrm{NI}$ & $\mathrm{NI}$ & $\mathrm{NI}$ & $\mathrm{NI}$ & $\mathrm{NI}$ & 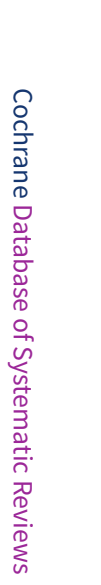 \\
\hline
\end{tabular}




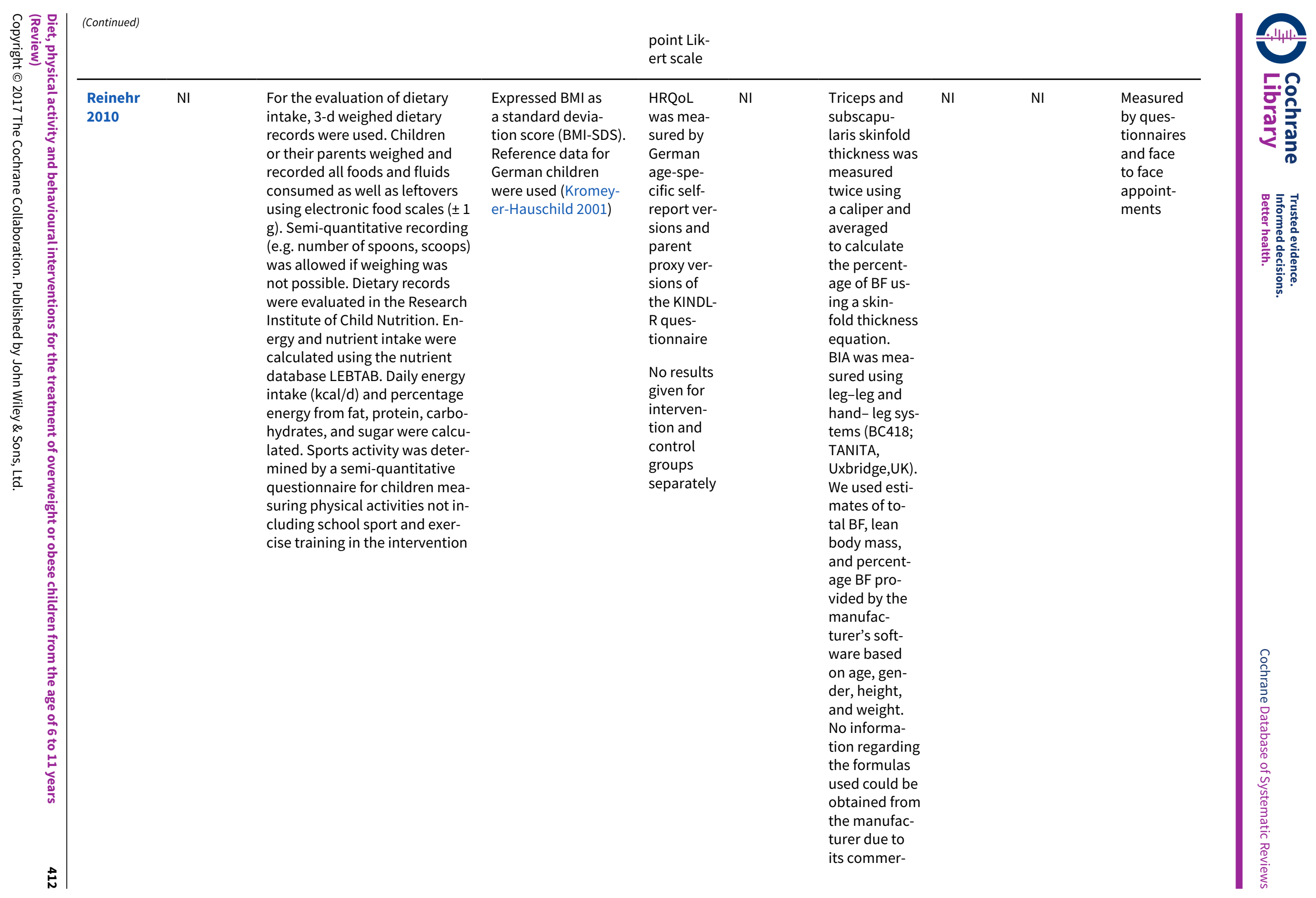




\begin{tabular}{|c|c|c|c|c|c|c|c|c|c|c|}
\hline 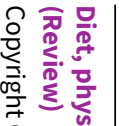 & (Continued) & & & & & & $\begin{array}{l}\text { cially sensi- } \\
\text { tive nature }\end{array}$ & & & \\
\hline 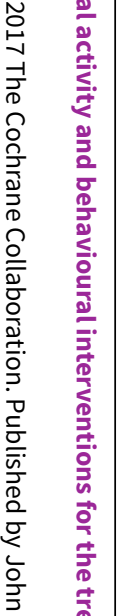 & $\begin{array}{l}\text { Sacher } \\
2010\end{array}$ & $\mathrm{NI}$ & $\begin{array}{l}\text { Levels of PA and the amount } \\
\text { of sedentary behaviours were } \\
\text { assessed using a non-validat- } \\
\text { ed questionnaire This was ad- } \\
\text { ministered by the researchers } \\
\text { to parents and children and in- } \\
\text { cluded the number and dura- } \\
\text { tion of physical } \\
\text { education lessons, time spent } \\
\text { on different types of vigorous } \\
\text { activities (e.g. sports), and time } \\
\text { spent on sedentary activities } \\
\text { (e.g. television, computer) }\end{array}$ & $\begin{array}{l}\text { Weight and height } \\
\text { were obtained for } \\
\text { both children and } \\
\text { their mothers, and } \\
\text { were subsequent- } \\
\text { ly used to calculate } \\
\text { BMI. Children were } \\
\text { classified as obese if } \\
\text { their BMI was > 98th } \\
\text { percentile for age } \\
\text { and gender using the } \\
\text { recommended cut- } \\
\text { off for treatment or } \\
\text { referral }\end{array}$ & $\begin{array}{l}\text { For self-es- } \\
\text { teem as- } \\
\text { sessment, } \\
\text { children } \\
\text { completed } \\
\text { the Har- } \\
\text { ter SPP } \\
\text { a wide- } \\
\text { ly-used as- } \\
\text { sessment } \\
\text { tool vali- } \\
\text { dated for } \\
\text { UK chil- } \\
\text { dren of } \\
\text { this age } \\
\text { group }\end{array}$ & $\mathrm{NI}$ & $\begin{array}{l}\text { Body weight, } \\
\text { height, and } \\
\text { WC were mea- } \\
\text { sured follow- } \\
\text { ing } \\
\text { standardised } \\
\text { procedures/ } \\
\text { Deuterium } \\
\text { dilution was } \\
\text { used to mea- } \\
\text { sure chil- } \\
\text { dren's TBW, } \\
\text { and hence FM } \\
\text { and FFM were } \\
\text { derived }\end{array}$ & $\mathrm{NI}$ & $\mathrm{NI}$ & $\begin{array}{l}\text { Measured } \\
\text { by log- } \\
\text { books }\end{array}$ \\
\hline 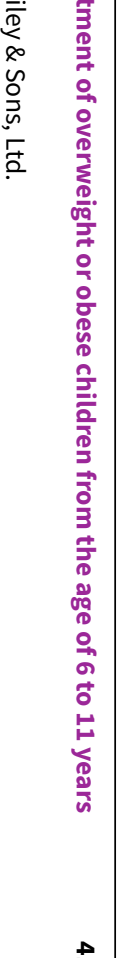 & $\begin{array}{l}\text { Kalarchi- } \\
\text { an } 2009\end{array}$ & $\mathrm{NI}$ & $\begin{array}{l}\text { Eating disorder symptoms } \\
\text { were assessed at baseline us- } \\
\text { ing the ChEAT, a } 26 \text {-item self- } \\
\text { report questionnaire designed } \\
\text { to assess attitudes and behav- } \\
\text { iours related to eating disor- } \\
\text { ders in school-aged children. } \\
\text { To assess binge eating, chil- } \\
\text { dren's responses to the ChEAT } \\
\text { item "I have gone on eating } \\
\text { binges where I feel that I might } \\
\text { not be able to stop" were cod- } \\
\text { ed as symptomatic or non- } \\
\text { symptomatic based on estab- } \\
\text { lished ChEAT scoring guide- } \\
\text { lines. Specifically, children } \\
\text { who reported eating binges } \\
\text { "always" "very often" or "of- } \\
\text { ten" were coded as sympto- } \\
\text { matic (Binge Eating Group), } \\
\text { and children who reported eat- } \\
\text { ing binges "never," "rarely" or } \\
\text { "sometimes" were coded as } \\
\text { non-symptomatic (No Binge } \\
\text { Eating Group) }\end{array}$ & $\begin{array}{l}\text { Children and adults } \\
\text { were weighed in } \\
\text { street clothes, with- } \\
\text { out shoes, by using a } \\
\text { digital scale (Scale- } \\
\text { Tronix 5002; Scale- } \\
\text { Tronix, White Plains, } \\
\text { NY). A stationary } \\
\text { stature board was } \\
\text { used for height as- } \\
\text { sessments. Child per- } \\
\text { cent overweight, cal- } \\
\text { culated as percent } \\
\text { over the median BMI } \\
\text { for age and gender }\end{array}$ & $\begin{array}{l}\text { Self-re- } \\
\text { ported de- } \\
\text { pressive } \\
\text { and anxi- } \\
\text { ety symp- } \\
\text { toms were } \\
\text { assessed } \\
\text { at base- } \\
\text { line using } \\
\text { the CDI } \\
\text { and the } \\
\text { STAIC, re- } \\
\text { spective- } \\
\text { ly. Child } \\
\text { self-es- } \\
\text { teem was } \\
\text { measured } \\
\text { using the } \\
\text { global } \\
\text { self-worth } \\
\text { score from } \\
\text { the SPPC. } \\
\text { The CDI, } \\
\text { STAIC and } \\
\text { SPPC all }\end{array}$ & $\mathrm{NI}$ & $\begin{array}{l}\text { WC was mea- } \\
\text { sured at the } \\
\text { midpoint be- } \\
\text { tween the } \\
\text { lowest rib and } \\
\text { the iliac crest. } \\
\text { Body com- } \\
\text { position was } \\
\text { determined } \\
\text { through DEXA } \\
\text { with a GE Lu- } \\
\text { nar Prodigy } \\
\text { system (GE } \\
\text { Medical Sys- } \\
\text { tems Lunar, } \\
\text { Madison, WI). } \\
\text { Percent BF, } \\
\text { total BF, and } \\
\text { FFM were de- } \\
\text { termined }\end{array}$ & $\mathrm{NI}$ & $\mathrm{NI}$ & $\mathrm{NI}$ \\
\hline
\end{tabular}


chomet-

ric prop-

erties and

are used

widely in

research.

Adults al-

so com-

pleted

the gener-

al health

percep-

tions and

global

health

subscales

of the

CHQ-PF50,

for assess-

ment of

HRQoL

\begin{tabular}{|c|c|c|c|c|c|c|c|c|c|}
\hline $\begin{array}{l}\text { Nowicka } \\
2009\end{array}$ & $\mathrm{NI}$ & $\begin{array}{l}\text { Lifestyle was measured with a } \\
\text { semi-structured questionnaire } \\
\text { with questions about daily ac- } \\
\text { tivities, transportation, and } \\
\text { sports. Although this question- } \\
\text { naire has not been validated, } \\
\text { it has been extensively used } \\
\text { in the childhood obesity unit } \\
\text { since } 2001 \text { to evaluate treat- } \\
\text { ment effects of primary obesity }\end{array}$ & $\begin{array}{l}\text { Body weight was } \\
\text { measured using } \\
\text { an electronic scale } \\
\text { (Tanita BWB-800) to } \\
\text { the nearest } 0.1 \mathrm{~kg} \\
\text { with the participant } \\
\text { wearing light cloth- } \\
\text { ing without shoes. } \\
\text { Height was mea- } \\
\text { sured using a stan- } \\
\text { dardised stadiome- } \\
\text { ter (Hyssna) to the } \\
\text { nearest } 0.5 \mathrm{~cm} \text { with- } \\
\text { out shoes. BMI was } \\
\text { calculated as weight/ } \\
\text { height } 2 \text { (kg/m²) and } \\
\text { BMI z score was } \\
\text { calculated using } \\
\text { Swedish age- and }\end{array}$ & $\mathrm{NI}$ & $\mathrm{NI}$ & $\begin{array}{l}\text { Body compo- } \\
\text { sition was al- } \\
\text { so measured } \\
\text { with DEXA } \\
\text { and MRI be- } \\
\text { fore and } 12 \\
\text { months after } \\
\text { the interven- } \\
\text { tion. DEXA, a } \\
\text { whole-body } \\
\text { fan } \\
\text { beam (Holog- } \\
\text { ic QDR 4500A; } \\
\text { equipped } \\
\text { with paedi- } \\
\text { atric software; } \\
\text { Hologic, Bed- } \\
\text { ford, MA, USA) } \\
\text { was used to } \\
\text { scan to esti- }\end{array}$ & $\mathrm{NI}$ & $\mathrm{NI}$ & $\mathrm{NI}$ \\
\hline
\end{tabular}


lean tissue.

MRI (Siemens

Sonata 1.5

Tesla, Er-

langen, Ger-

many) was

used to mea-

sure subcuta-

neous and vis-

ceral fat at the

lumbar level.

Image analy-

sis software

(Tomovision

Inc., Montre-

al, Canada)

was used to

segment the

cross-section-

al images for

adipose tis-

sue (AT) and

skeletal mus-

cle (SM). The

AT compart-

ment was fur-

ther segment-

ed into total

subcutaneous

AT, visceral

AT, and AT vol-

\begin{tabular}{|c|c|c|c|c|c|c|c|c|c|}
\hline $\begin{array}{l}\text { Wake } \\
2009\end{array}$ & $\mathrm{NI}$ & $\begin{array}{l}\text { PA: Actical Accelerometer (Mi- } \\
\text { ni Mitter). Worn for } 7 \text { full d; } \geq \\
5 \text { valid d required. Outcomes } \\
\text { across all valid d: mean activity } \\
\text { counts/min, and } \% \text { time spent } \\
\text { in MVPA. Also } 4-d \text { activity diary; } \\
\text { parent-report parent rating of } \\
\text { child's activity on } 7 \text {-point scale } \\
\text { ( } 1=\text { sedentary, } 7=\text { intense ac- } \\
\text { tivity). Nutrition: } 4 \text {-d food di- }\end{array}$ & $\begin{array}{l}\text { Weight was mea- } \\
\text { sured in light cloth- } \\
\text { ing to the nearest } \\
100 \text { g using digi- } \\
\text { tal scales (Tani- } \\
\text { ta, Japan, Mod- } \\
\text { el THD-646) and } \\
\text { height was measured } \\
\text { (twice) to the near- } \\
\text { est } 0.1 \mathrm{~cm} \text { using a }\end{array}$ & $\begin{array}{l}\text { PedsQL } \\
4.0 \text { self-re- } \\
\text { port and } \\
\text { parent } \\
\text { proxy ver- } \\
\text { sions. } 23 \\
\text { items that } \\
\text { yield total, } \\
\text { physical } \\
\text { summary, }\end{array}$ & $\mathrm{NI}$ & $\begin{array}{l}\text { WC: Lufkin Ex- } \\
\text { ecutive Steel } \\
\text { Tape (W606P- } \\
\text { M); average of } \\
\text { two WC mea- } \\
\text { surements; if } \\
\text { they differed } \\
\text { by } \geq 1 \mathrm{~cm} \text {, a } \\
\text { 3rd measure- } \\
\text { ment was }\end{array}$ & $\mathrm{NI}$ & $\mathrm{NI}$ & $\begin{array}{l}\text { Assessed } \\
\text { through } \\
\text { HRQoL, } \\
\text { body dis- } \\
\text { satisfac- } \\
\text { tion, and } \\
\text { self es- } \\
\text { teem }\end{array}$ \\
\hline
\end{tabular}


ary; parent-report. Parents reported child's consumption of each of 10 food and drink items $(0,1,2,>2$ times) for two weekdays and two weekend $d$, from which were derived dichotomous ("yes" v "no") variables

for 5 "healthy behaviours" (high fruit, vegetables, and water; low

fatty/sugary foods and non-diet sweet drinks ber of healthy behaviours/d

was summed to give a score between 0 and 5 (higher score in-

dicating more healthy behav-

iour), thus providing 4 mea-

surements at each wave portable rigid stadiometer (Invicta,

Oadby, Leicester,

Model IP0955). The average of the height measurements was used in analyses; if

the two differed by $\geq 0.5 \mathrm{~cm}$ a $3 \mathrm{rd}$ measurement was taken and the mean of the closest 2 values was used. BMI Z score was also calculated us ing the CDC 2000 sexspecific BMI-for-age growth charts and psy-

chosocia

summa-

ry scores,

each with

a possi-

ble range

$0-100$

$(100=$ best

possible

health);

quan-

titative

variable.

Physical

appear-

ance and

self worth:

modified

from Har-

ter's per-

ceived

compe-

tence

scale;

child self

report.

6 pairs

of state-

ments

with bi-

nary re-

sponse

format;

children

chose the

statement

from each

pair clos-

est to their

compe-

tence.

Each of

the 6 re-

sponses

was then taken and the

mean of the

closest 2 used 


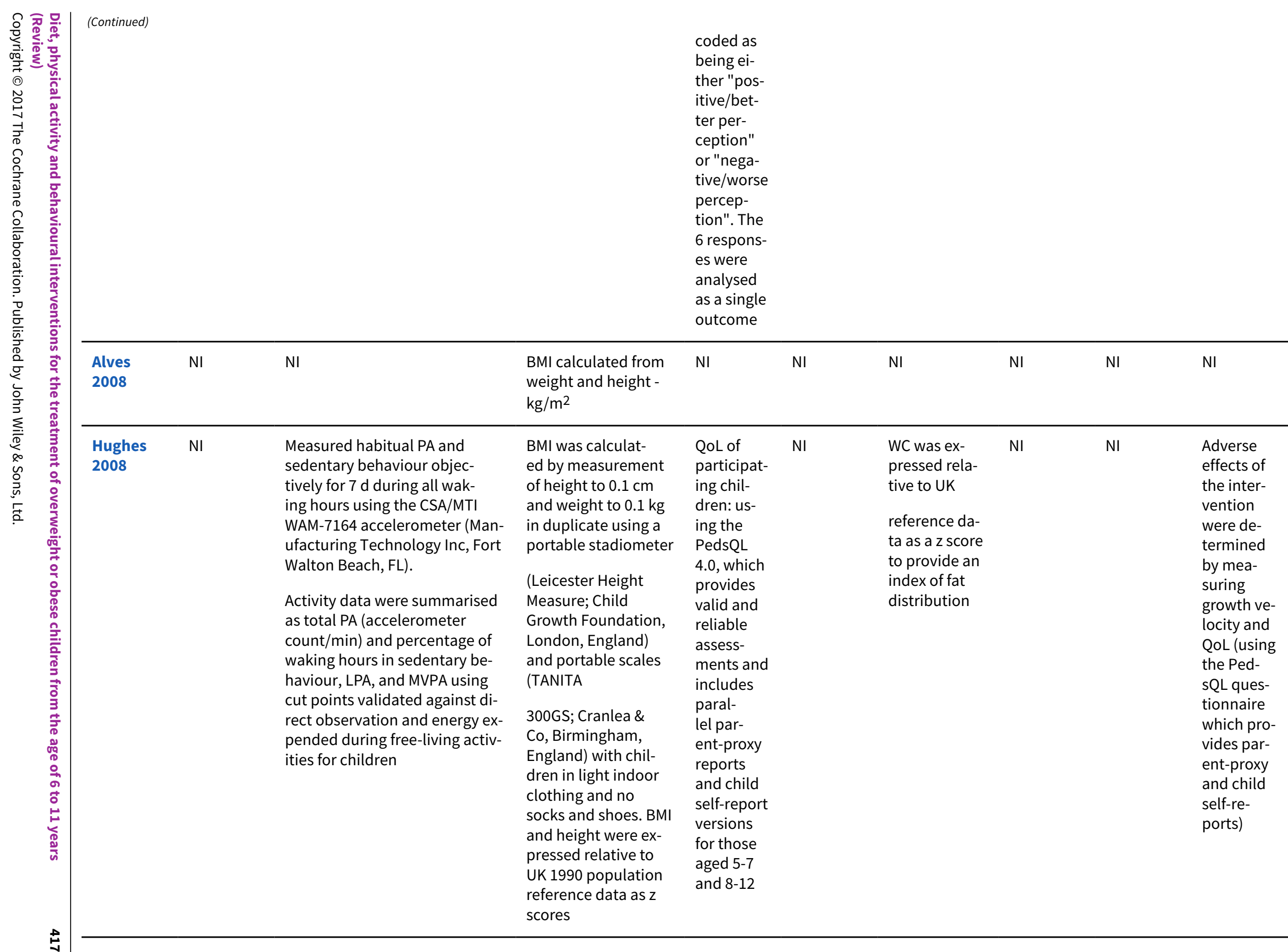


Log of PA and diet were encouraged - but was not defined as an outcome measure

Pairs of weigh

and height measurements, obtained us ing calibrated equip-

ment, were used to

calculate BMI and

were adjusted for

age and gender to

calculate BMI z score.

For control purpos-

es, recent German

reference data were

used that had been

obtained from 17,147

boys and 17,275 girls

aged $0-18$ years
$\mathrm{NI}$

FM and lean

$\mathrm{NI}$

sured by BIA

\begin{tabular}{|c|c|c|c|c|c|c|c|c|c|}
\hline $\begin{array}{l}\text { Wein- } \\
\text { traub } \\
2008\end{array}$ & $\mathrm{NI}$ & $\begin{array}{l}\text { PA was assessed on } 6 \text { consec- } \\
\text { utive d using accelerometers } \\
\text { (ActiGraph; Manufacturing } \\
\text { Technologies Inc, Fort Wal- } \\
\text { ton Beach, Florida) worn on } \\
\text { belts at the right hip. Mean dai- } \\
\text { ly counts/min, min of MPA, and } \\
\text { min of VPA 07:00 h -10:00 h } \\
\text { were used in the analysis. } \\
\text { To assess screen time, self-re- } \\
\text { port instruments were used } \\
\text { and demonstrated to be sensi- } \\
\text { tive to change in previous stud- } \\
\text { ies of reducing screen time. } \\
\text { Children reported their own } \\
\text { television viewing, videotape } \\
\text { viewing, and video game use }\end{array}$ & $\begin{array}{l}\text { The BMI was the pri- } \\
\text { mary measure of } \\
\text { BF. Height was mea- } \\
\text { sured twice with par- } \\
\text { ticipants barefoot } \\
\text { using a direct read- } \\
\text { ing } \\
\text { stadiometer (Shorr } \\
\text { Productions, Ol- } \\
\text { ney, Maryland), with } \\
\text { methods to account } \\
\text { for hair. Weight was } \\
\text { measured twice with } \\
\text { participants bare- } \\
\text { foot and wearing } \\
\text { light clothing us- } \\
\text { ing an electronic } \\
\text { scale (model 5602; } \\
\text { Scaletronix, White } \\
\text { Plains, New York). } \\
\text { The mean of the } \\
\text { replicate measures } \\
\text { was used in the } \\
\text { analysis. Age and } \\
\text { sex-standardised BMI }\end{array}$ & $\begin{array}{l}\text { The 10- } \\
\text { item } \\
\text { Rosen- } \\
\text { berg Self- } \\
\text { esteem } \\
\text { Scale was } \\
\text { used to as- } \\
\text { sess self- } \\
\text { esteem. } \\
\text { The 10- } \\
\text { item CDI } \\
\text { was used } \\
\text { to assess } \\
\text { depres- } \\
\text { sive symp- } \\
\text { toms. The } \\
\text { over-con- } \\
\text { cerns with } \\
\text { weight } \\
\text { and shape } \\
\text { subscale } \\
\text { of the } \\
\text { McKnight } \\
\text { Risk Fac- } \\
\text { tor Survey } \\
\text { wasused }\end{array}$ & $\mathrm{NI}$ & $\mathrm{NI}$ & $\mathrm{NI}$ & $\mathrm{NI}$ & $\begin{array}{l}\text { Injuries } \\
\text { and all } \\
\text { adverse } \\
\text { events } \\
\text { (any med- } \\
\text { ical ill- } \\
\text { nesses or } \\
\text { injuries } \\
\text { requiring } \\
\text { a visit to } \\
\text { a med- } \\
\text { ical pro- } \\
\text { fession- } \\
\text { al or in- } \\
\text { stitution) } \\
\text { during the } \\
\text { previous } \\
3 \text { months } \\
\text { were for- } \\
\text { mally as- } \\
\text { sessed } \\
\text { in both } \\
\text { groups at } \\
\text { baseline } \\
\text { and at all } \\
\text { the fol- }\end{array}$ \\
\hline
\end{tabular}


them

\begin{tabular}{|c|c|c|c|c|c|c|c|c|c|}
\hline $\begin{array}{l}\text { Berry } \\
2007\end{array}$ & $\mathrm{NI}$ & $\begin{array}{l}\text { Steps were counted with pe- } \\
\text { dometers (Accusplit Eagle } 170 \\
\text { Deluxe Activity Pedometer) and } \\
\text { Pedometer Walking Book which } \\
\text { were logged in a logbook for } \\
\text { the duration of the intervention }\end{array}$ & $\begin{array}{l}\text { BMI was calculated } \\
\mathrm{kg} / \mathrm{m}^{2} \text { and used BMI } \\
\text { gender- and age-spe- } \\
\text { cific growth charts } \\
\text { for children (Kucz- } \\
\text { marski 2002) }\end{array}$ & $\mathrm{NI}$ & $\mathrm{NI}$ & $\begin{array}{l}\text { BF percent- } \\
\text { age was ob- } \\
\text { tained using } \\
\text { the TBF300 } \\
\text { which uses } \\
\text { leg-to-leg } \\
\text { BIA - which } \\
\text { is a low-lev- } \\
\text { el electrical } \\
\text { signal that } \\
\text { is passed } \\
\text { through the } \\
\text { body using } \\
\text { foot elec- } \\
\text { trodes. BF } \\
\text { percentage } \\
\text { is calculated } \\
\text { based on the } \\
\text { amount of im- } \\
\text { pedance as } \\
\text { the current } \\
\text { flows from } \\
\text { one point to } \\
\text { another }\end{array}$ & $\mathrm{NI}$ & $\mathrm{NI}$ & $\mathrm{NI}$ \\
\hline
\end{tabular}

Gillis 2007 NI Physical fitness was evaluated by a modified Harvard step

BMI SDS were $\mathrm{NI}$ $\mathrm{NI}$

$\mathrm{NI}$

$\mathrm{NI}$ $\mathrm{NI}$ 


\begin{tabular}{|c|c|c|c|c|c|c|c|c|c|}
\hline 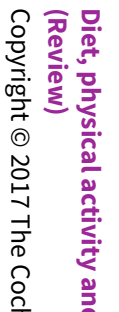 & (Continued) & $\begin{array}{l}\text { from the ground once every } 2 \\
\text { sec or as fast as the child can } \\
\text { manage for } 5 \text { min. At the } 6 \text { - } \\
\text { month visits participants from } \\
\text { both groups filled in a question- } \\
\text { naire together with their par- } \\
\text { ents regarding lifestyle changes }\end{array}$ & $\begin{array}{l}\text { upon data-from the } \\
\text { CDC }\end{array}$ & & & & & & \\
\hline 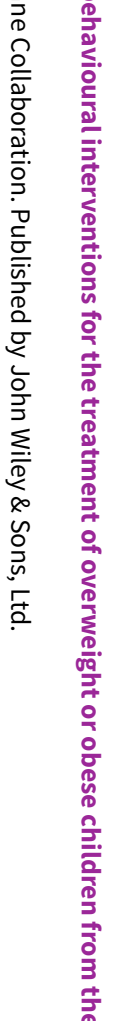 & $\begin{array}{l}\text { Kalavainen NI } \\
2007\end{array}$ & $\mathrm{NI}$ & $\begin{array}{l}\text { The primary out- } \\
\text { come measure of } \\
\text { the study was the } \\
\text { change of the weight } \\
\text { for height, which is in } \\
\text { routine use. Weight } \\
\text { for height was de- } \\
\text { fined as 'percentage } \\
\text { deviation of weight } \\
\text { from median weight } \\
\text { for height and gen- } \\
\text { der'; thus, this devi- } \\
\text { ation means the de- } \\
\text { viation in \% units, } \\
\text { the mean weight for } \\
\text { height in the popula- } \\
\text { tion being } 100 \% \text {. } \\
\text { The calculation of } \\
\text { BMI-SDS was based } \\
\text { on the British refer- } \\
\text { ence }\end{array}$ & $\mathrm{NI}$ & $\mathrm{NI}$ & $\begin{array}{l}\text { WC was mea- } \\
\text { sured at the } \\
\text { midpoint be- } \\
\text { tween the lat- } \\
\text { eral iliac crest } \\
\text { and the low- } \\
\text { est rib to the } \\
\text { nearest } 0.5 \\
\text { cm using a } \\
\text { flexible tape. } \\
\text { WHtR was cal- } \\
\text { culated by } \\
\text { dividing WC } \\
\text { (cm) by height } \\
\text { (cm) } \\
\text { FM and lean } \\
\text { body were as- } \\
\text { sessed by BIA } \\
\text { with Inbody } \\
3.0^{\circledast} \text { (Bio- } \\
\text { space, Seoul, } \\
\text { South Korea) } \\
\text { for partici- } \\
\text { pants in up- } \\
\text { right position } \\
\text { after voiding }\end{array}$ & $\mathrm{NI}$ & $\mathrm{NI}$ & $\mathrm{NI}$ \\
\hline 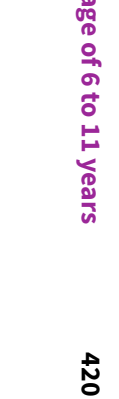 & $\begin{array}{l}\text { McCallum NI } \\
2007\end{array}$ & $\begin{array}{l}\text { Child PA, sedentary behaviour } \\
\text { and nutrition were measured } \\
\text { using 4-d food and activity di- } \\
\text { aries. Parents were given a list } \\
\text { of } 14 \text { food and drink items, } \\
\text { which were later broken down } \\
\text { into 'healthy' and 'less healthy' } \\
\text { food and drink categories by } \\
\text { the study team. They report- } \\
\text { ed their children's consump- }\end{array}$ & $\begin{array}{l}\text { The primary out- } \\
\text { come measure was } \\
\text { BMI }(\mathrm{kg} / \mathrm{m} 2) \text {, mea- } \\
\text { sured by trained re- } \\
\text { searchers using stan- } \\
\text { dard } \\
\text { protocols and equip- } \\
\text { ment. BMI z score } \\
\text { outcomes are report- }\end{array}$ & $\begin{array}{l}\text { Child } \\
\text { health } \\
\text { status } \\
\text { was mea- } \\
\text { sured us- } \\
\text { ing the To- } \\
\text { tal Scores } \\
\text { from the } \\
\text { 23-item } \\
\text { PedsQL }\end{array}$ & $\mathrm{NI}$ & $\mathrm{NI}$ & $\mathrm{NI}$ & $\mathrm{NI}$ & $\mathrm{NI}$ \\
\hline
\end{tabular}


tion of each over each of four $24 \mathrm{~h}$ periods. Higher scores in dicating better nutrition. Using the validated Bouchard after-school activity diary, parents were given a list of 7 activity categories. Average daily activity scores were calculated from parent ratings of children's activity on a scale of 1 (sedentary)-7 (intense activi-

ty) at 15-min intervals between $15.30 \mathrm{~h}$ and $18.30 \mathrm{~h}$ over $4 \mathrm{~d}$

Children's activity was also dichotomised into percentage of

time spent in low-level activity (ratings 1-3) vs higher level of activity (ratings 4-7, reported as percentage time spent MVPA ed using the US CDC 2000 gender-specific

BMI-for-age growth charts that came into wide use after commencement of the study
Parent

Proxy and

Child Self-

report,

and child

body sat-

isfaction

and phys-

ical ap-

pearance

and global

self-worth

using the

Collins

body fig-

ure per-

ception

and the

modified

Harter

scales, re-

spectively
Rodearmel NI Electronic pedometers (Ac2007 cusplit AE120, San Jose, CA)

were used. Participants were

instructed to maintain (not

change), monitor, and record

their usual lifestyle with re-

gard to PA (steps/d) during the

2-week baseline period. Each

family member, regardless of

group assignment, was asked

to record daily steps continu-

ously throughout the first 18

weeks of the study and during

the last week of the study. Both

groups were asked to complete

a sweets survey during baseline

and at the end of the 6-month

intervention.

The survey assessed partici-

pants' consumption of sugar
Study staff measured height and weight
for all participants at
each of the 6 family
meetings using a sta- diometer
(Invicta Plastics
Ltd, Leicester, Eng- land) and a calibrat- ed electronic scale
(Take-A-Weigh elec-
tronic scale, mod-
el PS-6600; Befour
Inc, Saukville, WI), re-
spectively. BMI was
calculated for all par-
ticipants, and BMI-
for-age $z$ scores and
corresponding per-
centile scores were
calculated for target

$\mathrm{NI}$

NI

NI

WC and per-

centage of BF

were assessed

by study staff

for all par-

ticipants be-

fore the in-

tervention

(family meet-

ing 1) and af-

ter interven-

tion months

3 and 6 (fami-

ly meetings 4

and 6).WC

was mea-

sured using a

Gulick II tape

measure 


\begin{tabular}{|c|c|c|c|c|c|c|c|c|c|c|c|}
\hline 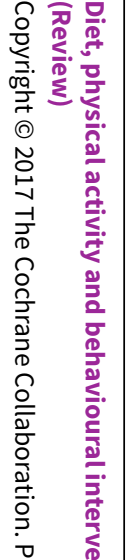 & (Continued) & & $\begin{array}{l}\text { and non-calorically sweetened } \\
\text { foods and beverages }\end{array}$ & $\begin{array}{l}\text { children. The prima- } \\
\text { ry } \\
\text { outcome was BMI } \\
\text { for age analysed on } \\
\text { the z score scale (z } \\
\text { scores are consid- } \\
\text { ered more suitable } \\
\text { for statistical analy- } \\
\text { sis than the corre- } \\
\text { sponding percentile } \\
\text { scores) }\end{array}$ & & & $\begin{array}{l}\text { (Country } \\
\text { Technology, } \\
\text { Gays Mills, } \\
\text { WI), and per- } \\
\text { centage of BF } \\
\text { was assessed } \\
\text { by BIA } \\
\text { (Biodynam- } \\
\text { ics BIA Analyz- } \\
\text { er, model 450; } \\
\text { Biodynamics } \\
\text { Corp, Seattle, } \\
\text { WA) }\end{array}$ & & & & 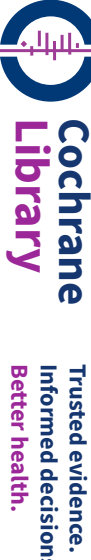 \\
\hline 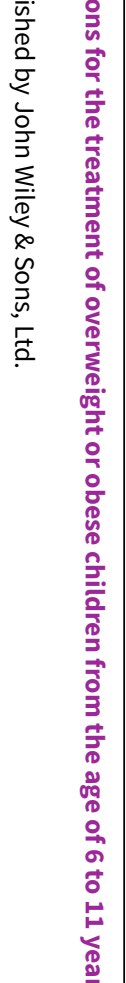 & $\begin{array}{l}\text { Satoh } \\
2007\end{array}$ & $\mathrm{NI}$ & $\begin{array}{l}\text { Nutritional balance: the actu- } \\
\text { al food intake (black dots), the } \\
\text { ideal food intake } \\
\text { following the MNBC (black } \\
\text { dots)). Thus, the nutritional bal- } \\
\text { ance based on the MNBC was } \\
\text { ideally "1". } \\
\text { Only measured in intervention } \\
\text { group }\end{array}$ & BMI not measured & $\mathrm{NI}$ & $\begin{array}{l}\text { Fatty liver } \\
\text { measured } \\
\text { at baseline } \\
\text { but not at } \\
\text { follow-up } \\
\text { (medical } \\
\text { check). } \\
\text { Blood re- } \\
\text { sults at } \\
\text { entry al- } \\
\text { so showed } \\
\text { normal re- } \\
\text { sults for } \\
\text { TC serum } \\
\text { glucose, } \\
\text { serum } \\
\text { protein, } \\
\text { serum } \\
\text { triglyc- } \\
\text { eride, and } \\
\text { haemoglo- } \\
\text { bin (but } \\
\text { was not } \\
\text { evaluat- } \\
\text { ed at fol- } \\
\text { low-up) }\end{array}$ & $\begin{array}{l}\text { Percentage } \\
\text { overweight } \\
\text { values, de- } \\
\text { fined as the } \\
\text { fractional dif- } \\
\text { ference of ac- } \\
\text { tual weight } \\
\text { to age and } \\
\text { sex-matched } \\
\text { standards de- } \\
\text { rived from na- } \\
\text { tionwide sur- } \\
\text { veys of Japan- } \\
\text { ese children }\end{array}$ & $\mathrm{NI}$ & $\begin{array}{l}\text { Partic- } \\
\text { ipants } \\
\text { asked } \\
\text { about in- } \\
\text { tervention } \\
\text { (17 partici- } \\
\text { pants) }\end{array}$ & $\mathrm{NI}$ & 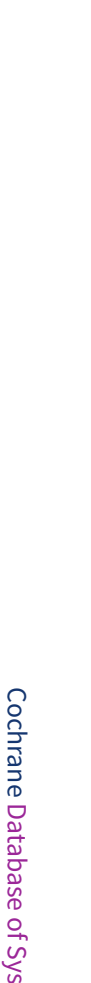 \\
\hline$\underset{\mathrm{N}}{\mathrm{N}}$ & $\begin{array}{l}\text { Wilfley } \\
2007\end{array}$ & $\mathrm{NI}$ & $\begin{array}{l}\text { The Child Dietary Self-efficacy } \\
\text { Scale evaluated children's self- } \\
\text { efficacy in choosing healthy, } \\
\text { low-fat foods. The Self-effica- }\end{array}$ & $\begin{array}{l}\text { BMI was calculated } \\
\text { from weight, which } \\
\text { was measured to } \\
\text { the nearest one- }\end{array}$ & $\mathrm{NI}$ & $\mathrm{NI}$ & $\begin{array}{l}\text { Percentage } \\
\text { overweight } \\
\text { was defined } \\
\text { as percentage }\end{array}$ & $\mathrm{NI}$ & $\mathrm{NI}$ & $\begin{array}{l}\text { Unclear } \\
\text { how they } \\
\text { were mea- } \\
\text { sured }\end{array}$ & 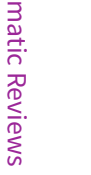 \\
\hline
\end{tabular}


cy Scale for Children's PA examined children's perceived selfefficacy in overcoming barriers to achieving weight goals and developing positive alternatives to unhealthy habits.

The Child Eating Disorder Examination assessed weight and shape concerns. The Coping with Teasing Scale measured the adequacy of children's responses to teasing.

Peer support for diet and PA was measured using the Socia Support for Eating Habits/Exercise Survey.

The levels of social problems of the children were evaluated by using the social problem subscale of the Achenbach CBCL-Parent Version fourth pound $(0.1 \mathrm{~kg})$ on a Detecto balance-beam scale

(Cardinal Scale Manufacturing, Webb

City, MI), and height, which was measured

to the nearest oneeighth inch $(0.3 \mathrm{~cm})$

\section{with a stadiometer} The BMI z scores of the children were determined using the age-specific and sexspecific median BMI above median

BMI

PA measured using $3 \mathrm{~d}$ from PD-PAR. Measures calculated included minutes sampled, average activity in METS and

mins and percentage of $\mathrm{PA}>3$ METS, with MET values based on the revised compendium of physical activities. Eating episodes calculated using $4 \mathrm{~d}$ of dietary recording in habit books - episode defined as oc casion where food was con-

sumed at a single sitting

\begin{tabular}{|c|c|c|c|c|c|c|}
\hline $\begin{array}{l}\text { Nemet } \\
2005\end{array}$ & $\mathrm{NI}$ & $\begin{array}{l}\text { Participants kept three 2-d } \\
\text { food records (at baseline, at } \\
\text { the end of the 3-month pro- } \\
\text { gramme, and } 1 \text { year later). } \\
\text { The food record data were re- } \\
\text { viewed by the project nutrition- } \\
\text { ist and checked for omissions }\end{array}$ & $\begin{array}{l}\text { Standard calibrated } \\
\text { scales and stadiome- } \\
\text { ters were used to de- } \\
\text { termine } \\
\text { height, weight, and } \\
\text { BMI. Because BMI }\end{array}$ & $\mathrm{NI}$ & $\mathrm{NI}$ & $\begin{array}{l}\text { Triceps and } \\
\text { subscapu- } \\
\text { lar skinfold } \\
\text { values were } \\
\text { measured } \\
\text { to the near- } \\
\text { est } 0.1 \mathrm{~mm} \text {, }\end{array}$ \\
\hline
\end{tabular}

\begin{tabular}{|c|c|c|c|}
\hline $\begin{array}{l}\text { Standardised BMI } \\
\text { was calculated by } \\
\text { comparing the youth } \\
\text { BMI to mean BMI of } \\
\text { population/standard } \\
\text { deviation of popu- } \\
\text { lation (Kuczmarski } \\
\text { 2002) }\end{array}$ & $\mathrm{NI}$ & $\mathrm{NI}$ & $\begin{array}{l}\text { Percent over } \\
\text { weight base } \\
\text { on compar- } \\
\text { isons of the } \\
\text { BMI to the } \\
\text { 50th per- } \\
\text { centile BMI } \mathrm{f} \\
\text { age and sex } \\
\text { using the CD } \\
\text { growth char }\end{array}$ \\
\hline $\begin{array}{l}\text { Standard calibrated } \\
\text { scales and stadiome- } \\
\text { ters were used to de- } \\
\text { termine } \\
\text { height, weight, and } \\
\text { BMI. Because BMI }\end{array}$ & $\mathrm{NI}$ & $\mathrm{NI}$ & $\begin{array}{l}\text { Triceps and } \\
\text { subscapu- } \\
\text { lar skinfold } \\
\text { values were } \\
\text { measured } \\
\text { to the near- } \\
\text { est } 0.1 \mathrm{~mm} \text {, }\end{array}$ \\
\hline
\end{tabular}

$\mathrm{NI}$

$\mathrm{NI}$

\begin{tabular}{|c|c|c|}
\hline $\mathrm{NI}$ & $\mathrm{NI}$ & $\begin{array}{l}\text { Unclear } \\
\text { how they } \\
\text { were mea- } \\
\text { sured }\end{array}$ \\
\hline
\end{tabular}


and errors. Food records were analysed with the Israeli Ministry of Health tables. Fitness was assessed with a progressive treadmill exercise test, to determine exercise endurance. Participants performed an exercise test.

All participants were familiarised with the treadmill for 5 min and performed a warmup of $1 \mathrm{~min}$ at a speed of 2.2 miles $(3.5 \mathrm{~km})$ per hour, with no incline. The exercise intensity was enhanced every 2 min. All participants were encouraged throughout the test by the staff members and exercised to the limit of their tolerance. Endurance time was measured from the end of the warm-up period to exhaustion changes with age, BMI-for-age percentiles were cal-

culated according to the CDC growth charts. The age-adjusted $z$ score corresponding to the exact percentile for a given measurement was calculated with Holtain

skinfold

calipers (CMS

Weighing

Equipment

Crymych,

United King-

dom). Mea-

surements

were made

on the right

side of the

body. All mea-

surements

(baseline, 3

months, and

1 year) were

performed

by the same

trained indi-

vidual.

Calculations

of percent-

age $B F$ were

performed

with standard

equations

\begin{tabular}{|c|c|c|c|c|c|c|c|c|c|}
\hline Woo 2004 & $\mathrm{NI}$ & $\mathrm{NI}$ & $\begin{array}{l}\text { Body weight was } \\
\text { measured with an } \\
\text { electronic body } \\
\text { weight scale (Se- } \\
\text { ca Delta Model } 707 \text { ) } \\
\text { with participants } \\
\text { dressed in a light } \\
\text { T-shirt and shorts. } \\
\text { Height was mea- } \\
\text { sured with a Harpen- } \\
\text { den statiometer }\end{array}$ & $\mathrm{NI}$ & $\mathrm{NI}$ & $\begin{array}{l}\text { BF content } \\
\text { was deter- } \\
\text { mined by } \\
\text { DEXA, with } \\
\text { the fan beam } \\
\text { model (Holog- } \\
\text { ic QDR-4500) }\end{array}$ & $\mathrm{NI}$ & $\mathrm{NI}$ & $\mathrm{NI}$ \\
\hline $\begin{array}{l}\text { Epstein } \\
2001\end{array}$ & $\mathrm{NI}$ & $\begin{array}{l}\text { Motivation to engage in PA was } \\
\text { assessed using the Children's } \\
\text { Self-Perception of Adequacy } \\
\text { and Predilection for PA Scale. } \\
\text { This self-report scale was de- }\end{array}$ & $\begin{array}{l}\text { Height was mea- } \\
\text { sured in } 0.125 \text {-inch } \\
(0.3 \mathrm{~cm}) \text { intervals ei- } \\
\text { ther using a labo- } \\
\text { ratory-construct- }\end{array}$ & $\mathrm{NI}$ & $\mathrm{NI}$ & $\begin{array}{l}\text { Percentage } \\
\text { of overweight } \\
\text { calculations } \\
\text { were based } \\
\text { on compar- }\end{array}$ & $\mathrm{NI}$ & $\mathrm{NI}$ & $\mathrm{NI}$ \\
\hline
\end{tabular}


veloped for children ages 9-16 to assess perceived adequacy, predilection, and enjoyment of PA. Reliability over a 2-week period for each factor ranged between 0.70 and 0.91 . The total scale score was strongly related to free-time PA ( $r$ between 0.59 and 0.76), teacher evaluation of PA ( $r$ between 0.50 and 0.67 ), and standardised tests of motor co-ordination ( $r$ between 0.70 and 0.82 ) on a sample of 1205 children ed height board or a stadiometer (Seca, Columbia, MD), and weight was measured in 0.25 pound $(0.1 \mathrm{~kg})$ intervals using a medical balance beam scale (Healthometer, Bridgeview, IL). Children and parents who were $\geq 85$ th $\mathrm{BMI}$ percentile were considered obese isons of the

participant

BMI to the

50th BMI per-

centile for age

and sex us-

ing standards

derived from

the Nation-

al Health and

Nutrition Ex-

amination

Survey III

\begin{tabular}{|c|c|c|c|c|c|c|c|c|c|}
\hline $\begin{array}{l}\text { Nova } \\
2001\end{array}$ & $\mathrm{NI}$ & $\begin{array}{l}\text { Change in behaviours - number } \\
\text { of hs of PA per week, number of } \\
\mathrm{h} \text { spent using the TV and PC/d. } \\
\text { This information was measured } \\
\text { by interviewing the child and } \\
\text { his/her parents }\end{array}$ & $\begin{array}{l}\text { BMI measured but } \\
\text { not reported at fol- } \\
\text { low-up }\end{array}$ & $\mathrm{NI}$ & $\mathrm{NI}$ & $\begin{array}{l}\text { Percentage } \\
\text { overweight } \\
\text { measured - } \\
\text { no explana- } \\
\text { tion }\end{array}$ & $\mathrm{NI}$ & $\mathrm{NI}$ & $\mathrm{NI}$ \\
\hline $\begin{array}{l}\text { Epstein } \\
2000 a\end{array}$ & $\mathrm{NI}$ & $\begin{array}{l}\text { Child problem solving assessed } \\
\text { by PEPSI; lower scores indicate } \\
\text { greater self-perception of prob- } \\
\text { lem solving. Child psychologi- } \\
\text { cal problems assessed by the } \\
\text { CBCL; Achenbach 1991. Total } \\
\text { competence, total behaviour } \\
\text { problems, internalising behav- } \\
\text { iour problems and externalising } \\
\text { behaviour problems reported. } \\
\text { The KEDS was used to assess } \\
\text { symptoms of disordered eating } \\
\text { and possible eating disorders }\end{array}$ & $\begin{array}{l}\mathrm{BMI}=\mathrm{kg} / \mathrm{m}^{2} \text { con- } \\
\text { verted to standard } \mathrm{z} \\
\text { scores based on sam- } \\
\text { ple BMI mean and } \\
\text { standard deviation } \\
\text { for age and gender } \\
\text { (Rosner 1998) }\end{array}$ & $\mathrm{NI}$ & $\mathrm{NI}$ & $\mathrm{NI}$ & $\mathrm{NI}$ & $\mathrm{NI}$ & $\mathrm{NI}$ \\
\hline $\begin{array}{l}\text { Schwing- } \\
\text { shandl } \\
1999\end{array}$ & $\mathrm{NI}$ & $\mathrm{NI}$ & No description & $\mathrm{NI}$ & $\mathrm{NI}$ & $\begin{array}{l}\text { Body compo- } \\
\text { sition was es- } \\
\text { timated from } \\
\text { BIA. Measure- } \\
\text { ments were } \\
\text { performed at } \\
\text { baseline and } \\
\text { after } 4,8 \text {, and } \\
12 \text { weeks. To- }\end{array}$ & $\mathrm{NI}$ & $\mathrm{NI}$ & $\mathrm{NI}$ \\
\hline
\end{tabular}




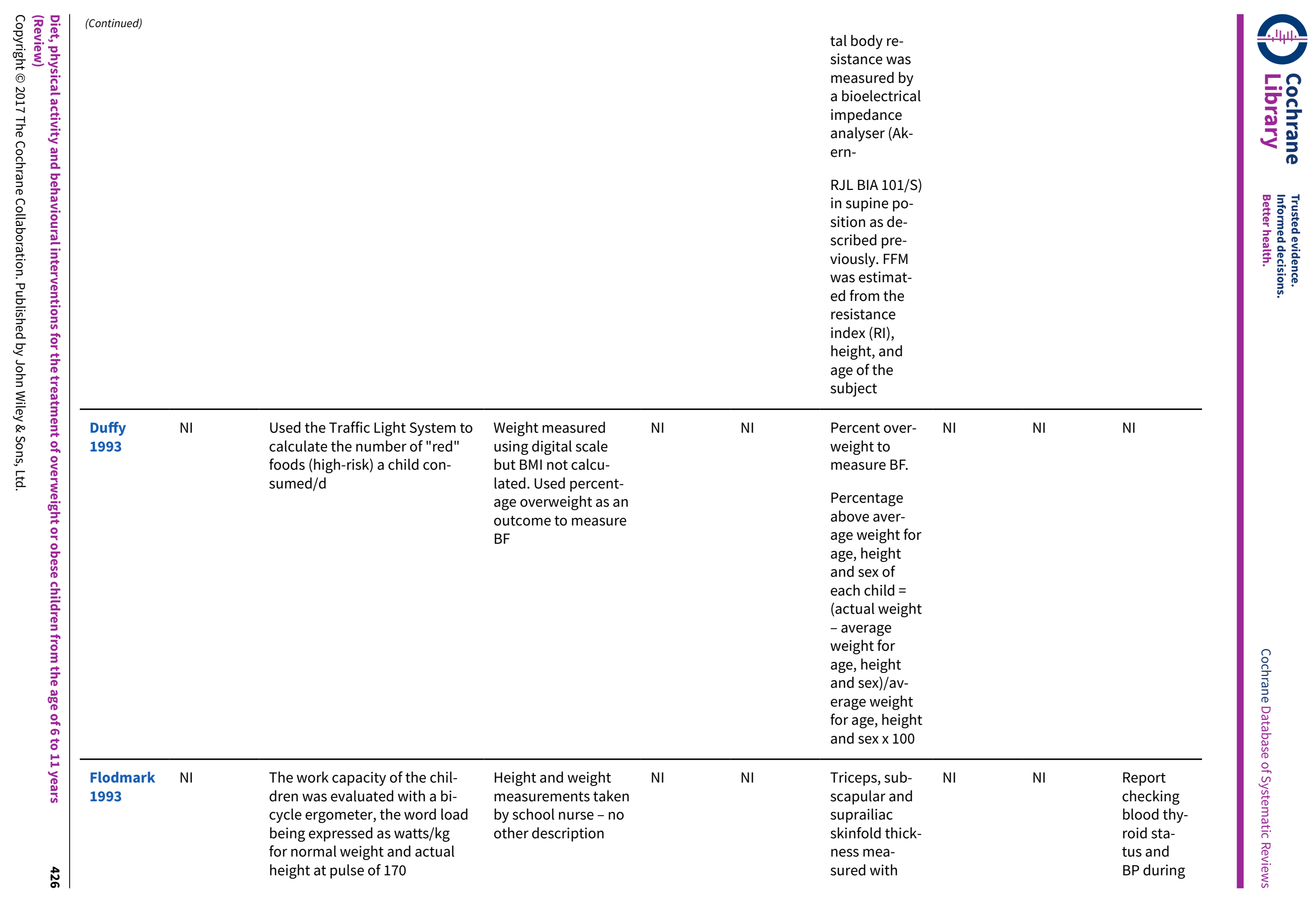




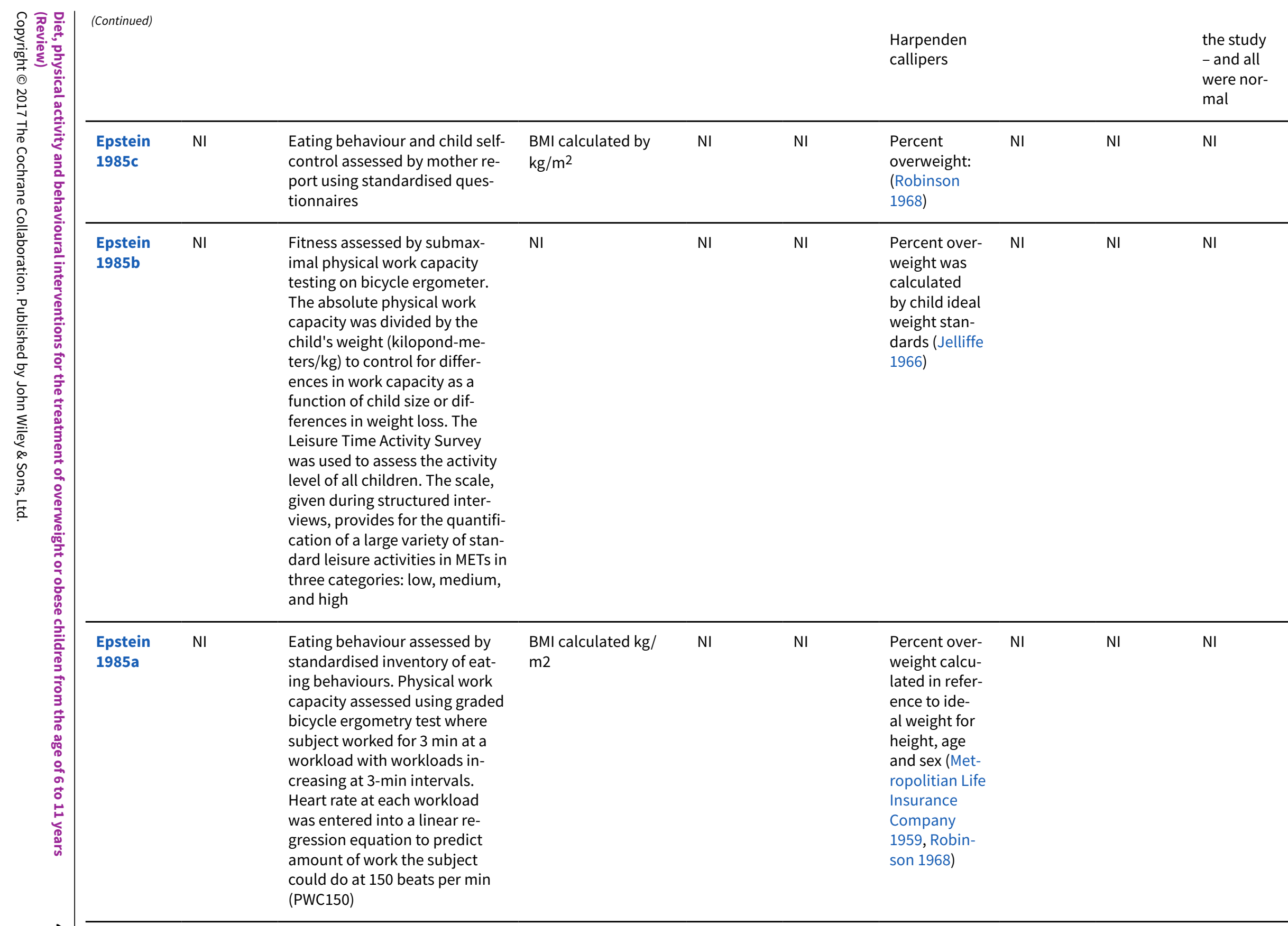


Fitness assessed using a submaximal step test and percentile rankings using recovery heart rates were calculated based on standardisation data

(3.2 mm) int

BMI was calculated

according to the fol-

lowing formula: BMI

$=$ weight $(\mathrm{kg} /$ height

(m)2

AF-5: Five-Factor Self-Concept Questionnaire; ASSQ: The Child and Adolescent Trial for Cardiovascular Health After-School Student Questionnaire

BASC-2: P Behavioral Assessment System for Children: Parent version; BF: body fat, BIA: Bioelectrical impedance analysis; BMI: body mass index; BP: blood pressure; BPFAS: Behavioral Pediatrics Feeding Assessment Scale; BV: body volume

CATCH: Child and Adolescent Health; CBCL: Child Behavior Checklist; CDC: centre for disease control and prevention; CDI:Child Depression Inventory CDI-S: Child Depression Inventory short form; CEBO: Child Eating Behaviour Questionnaire; CFCA CY-PSPP: Children and Youth Physical Self-Perception Profile; CHO-PF50: Child Health Questionnaire - PF50; CHU9D: Child Health Utility 9-Dimensions;

DEXA: dual energy X-ray absorptiometry; DEBQ: Dutch Eating Behavior Questionnaire; DHI: diet history interviews

EAH: eating in the absence of hunger; EQ-5D-Y: European Quality of Life 5-Dimensions - youth; EQ-5D: European Quality of Life 5-Dimensions

FFM: fat free mass; FM: fat mass; FFQ: food frequency questionnaire;

GI: glycaemic index; GL: glycaemic load; GP: general practitioner; GW-LQ-KJ: weight-specific quality-of life measure, children and young;

HDL: high density lipoprotein;

IOTF: International Task Force of Obesity; IPAQ: international physical activity questionnaire

KEDS: Kids Eating disorder survey; KiGGS: the German health Interview and Examination Survey for children and adolescents

LPA: low physical activity

MARCA: Multimedia Activity Recall for Children and Adolescents; MASC: Multidimensional Anxiety Scale for Children; MetS: metabolic syndrome; METs: metabolic equivalent; MPA: moderate physical activity; MRI: magnetic resonance imaging; MVPA: moderate-vigourous physical activity

N/D: not defined; N/I: not investigated; 


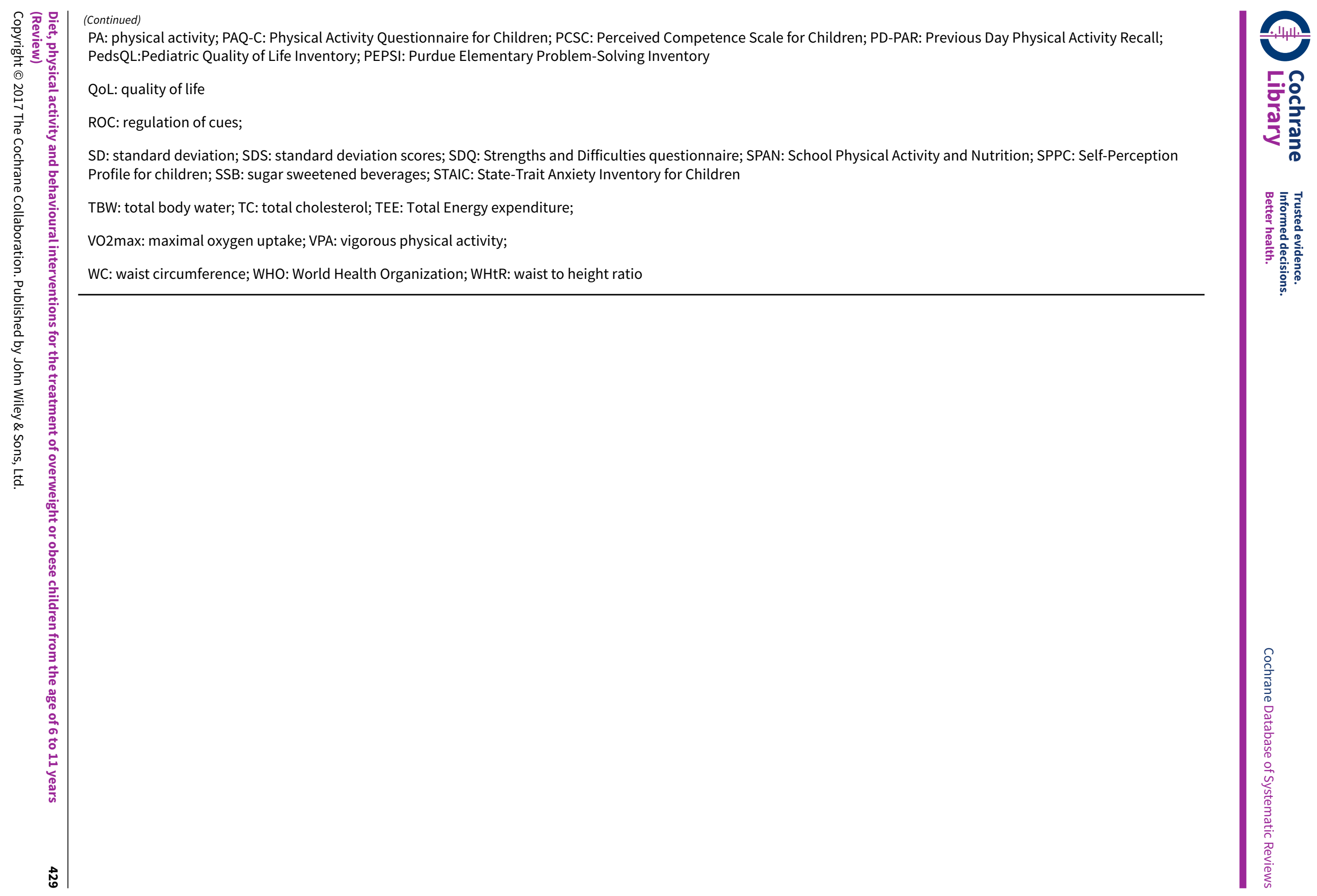




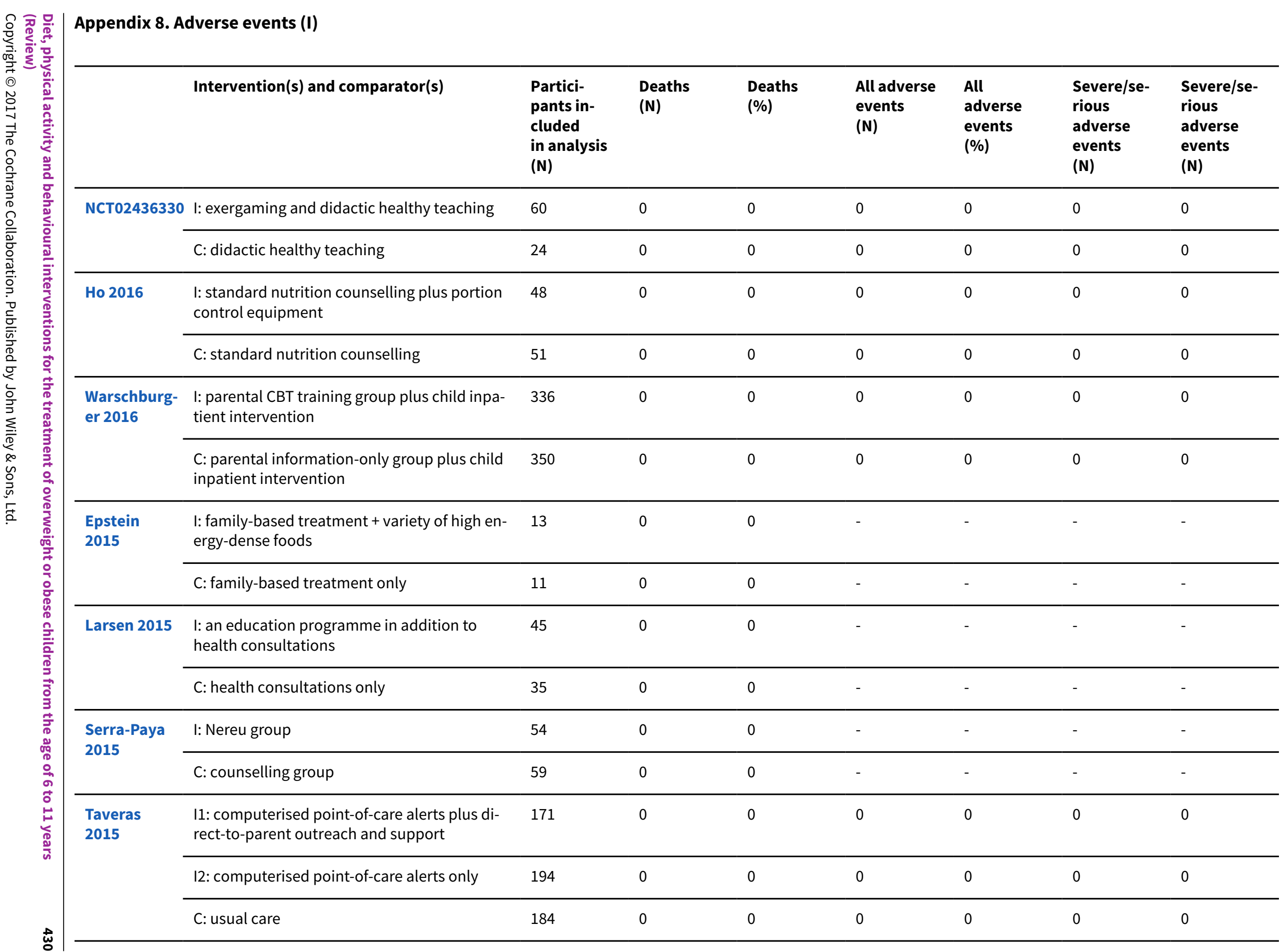




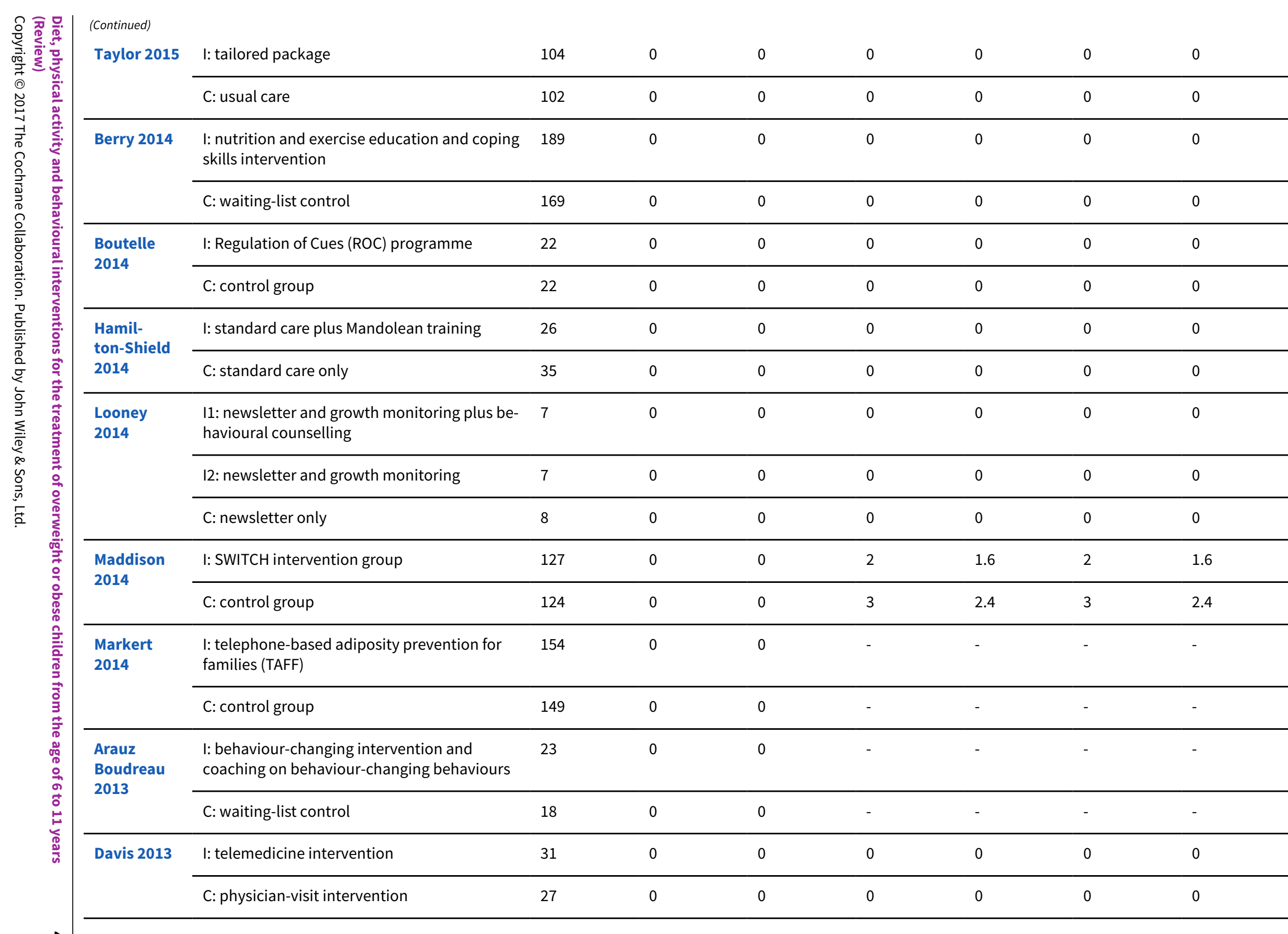




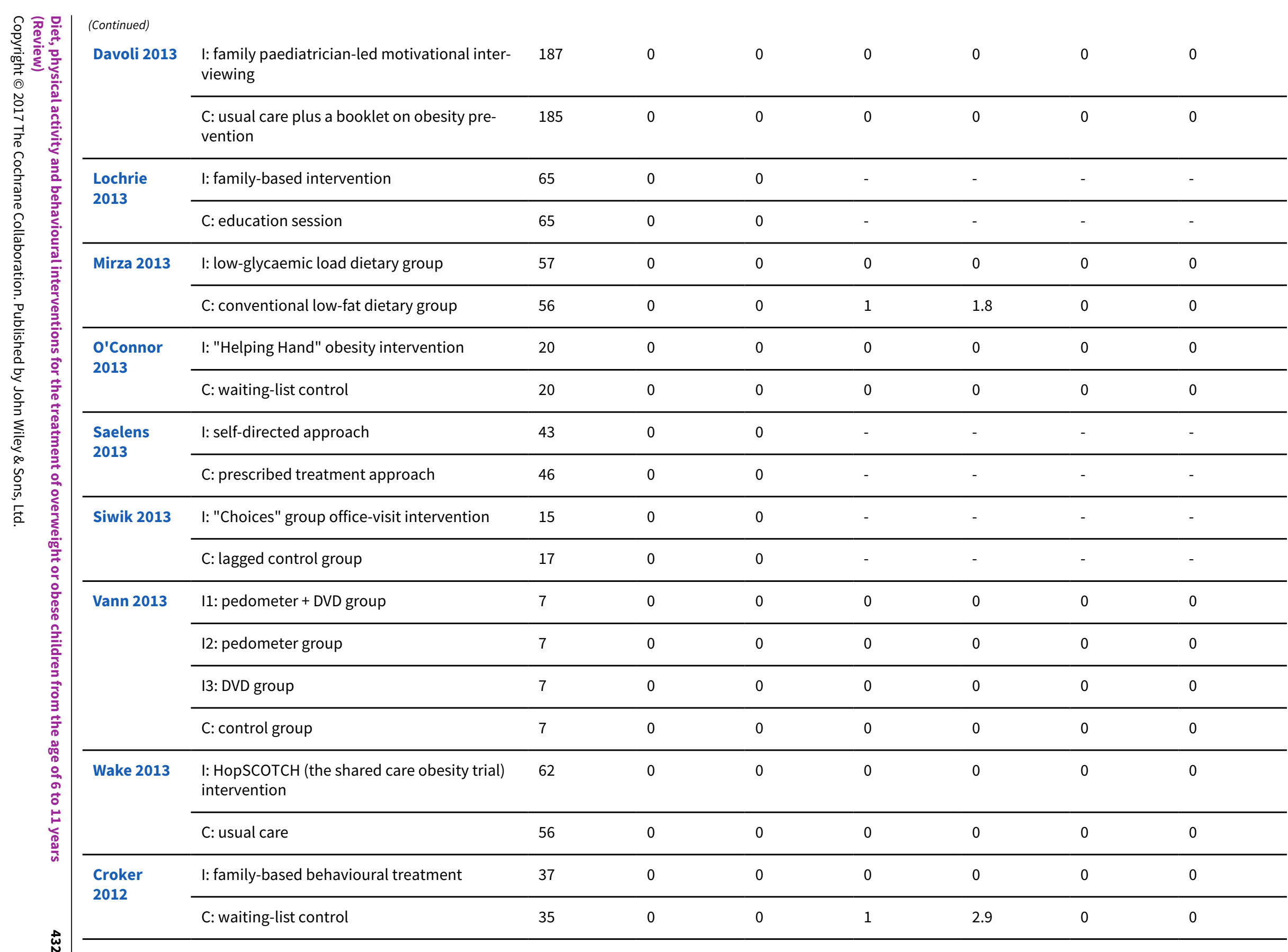




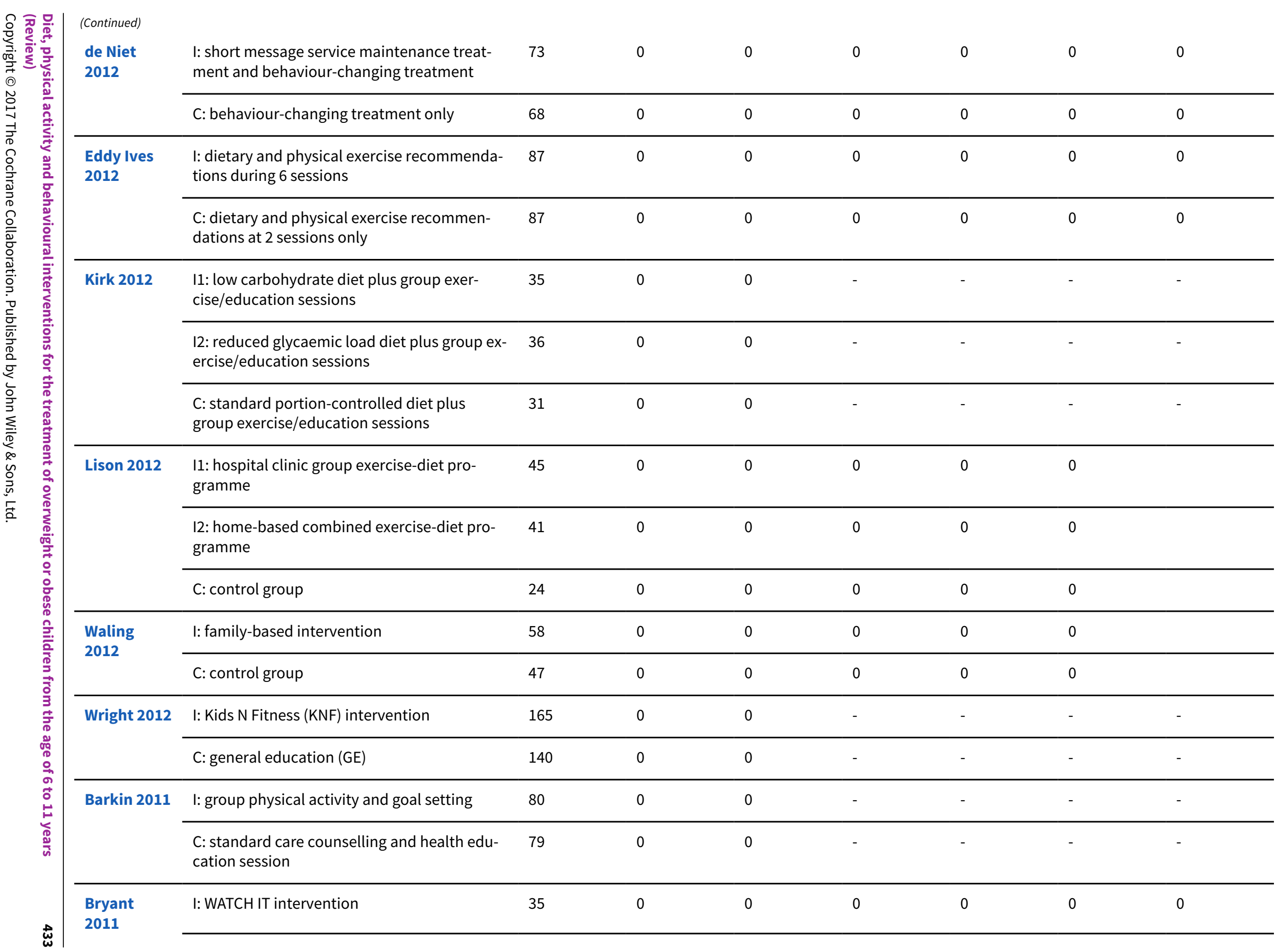




\begin{tabular}{|c|c|c|c|c|c|c|c|c|c|}
\hline 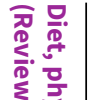 & (Continued) & C: waiting-list control & 35 & 0 & 0 & 0 & 0 & 0 & 0 \\
\hline 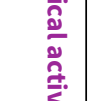 & \multirow[t]{2}{*}{$\begin{array}{l}\text { Coppins } \\
2011\end{array}$} & $\begin{array}{l}\text { I: multi-component family-focused education } \\
\text { package }\end{array}$ & 35 & 0 & 0 & 0 & 0 & 0 & 0 \\
\hline 气 & & C: waiting-list control & 30 & 0 & 0 & 0 & 0 & 0 & 0 \\
\hline 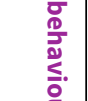 & \multirow{2}{*}{$\begin{array}{l}\text { Gunnars- } \\
\text { dottir } \\
2011 a\end{array}$} & $\begin{array}{l}\text { I: Epstein's family-based behavioural treat- } \\
\text { ment (FBBT) }\end{array}$ & 8 & 0 & 0 & - & - & - & - \\
\hline$\stackrel{\Xi}{\Xi}$ & & C: standard care (waiting-list control) & 8 & 0 & 0 & - & - & - & - \\
\hline 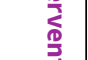 & \multirow{2}{*}{$\begin{array}{l}\text { Maddison } \\
2011\end{array}$} & I: active video game package & 160 & 0 & 0 & 2 & 1.3 & 2 & 1.3 \\
\hline$\stackrel{\circ}{3}$ & & C: control group & 162 & 0 & 0 & 6 & 2.5 & 6 & 2.5 \\
\hline 䓂 & \multirow[t]{2}{*}{ Wafa 2011} & I: low-intensity intervention & 52 & 0 & 0 & 0 & 0 & 0 & 0 \\
\hline$\stackrel{\substack{0 \\
3}}{3}$ & & C: waiting-list control & 55 & 0 & 0 & 0 & 0 & 0 & 0 \\
\hline $\begin{array}{c}\vec{l} \\
\stackrel{0}{0} \\
\dot{0} \\
0\end{array}$ & \multirow[t]{2}{*}{$\begin{array}{l}\text { Bathrellou } \\
2010\end{array}$} & $\begin{array}{l}\text { I: behavioural intervention with parental in- } \\
\text { volvement }\end{array}$ & 24 & 0 & 0 & - & - & - & - \\
\hline 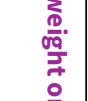 & & $\begin{array}{l}\text { C: behavioural intervention without parental } \\
\text { involvement }\end{array}$ & 23 & 0 & 0 & - & - & - & - \\
\hline $\begin{array}{l}\circ \\
\stackrel{0}{0} \\
0 \\
0 \\
0 \\
n\end{array}$ & \multirow[t]{2}{*}{ Diaz 2010} & $\begin{array}{l}\text { I: behavioural curriculum plus registered di- } \\
\text { eticians and physician consultations }\end{array}$ & 38 & 0 & 0 & 0 & 0 & 0 & 0 \\
\hline$\frac{0}{1}$ & & C: physician consultations only & 38 & 0 & 0 & 0 & 0 & 0 & 0 \\
\hline 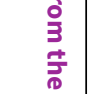 & \multirow[t]{2}{*}{$\begin{array}{l}\text { Duggins } \\
2010\end{array}$} & $\begin{array}{l}\text { I: nutrition classes and family YMCA member- } \\
\text { ship }\end{array}$ & 44 & 0 & 0 & - & - & - & - \\
\hline 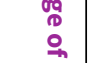 & & C: nutrition classes only & 39 & 0 & 0 & - & - & - & - \\
\hline$\stackrel{+}{\circ}$ & \multirow[t]{2}{*}{ Faude 2010} & I: football training programme (FB) & 19 & 0 & 0 & - & - & - & - \\
\hline 兽 & & $\begin{array}{l}\text { C: established standard sports programme } \\
\text { (STD) }\end{array}$ & 20 & 0 & 0 & - & - & - & - \\
\hline$\vec{A}$ & $\begin{array}{l}\text { Reinehr } \\
2010\end{array}$ & I: behaviour-changing treatment & 39 & 0 & 0 & 0 & 0 & 0 & 0 \\
\hline
\end{tabular}




\begin{tabular}{|c|c|c|c|c|c|c|c|c|c|}
\hline 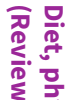 & (Continued) & C: waiting-list control & 32 & 0 & 0 & 0 & 0 & 0 & 0 \\
\hline 吾 & \multirow{2}{*}{$\begin{array}{l}\text { Sacher } \\
2010\end{array}$} & I: MEND programme & 60 & 0 & 0 & 0 & 0 & 0 & 0 \\
\hline 管. & & C: control group & 56 & 0 & 0 & 0 & 0 & 0 & 0 \\
\hline$\frac{\overline{0}}{\overline{0}}$ & \multirow[t]{2}{*}{$\begin{array}{l}\text { Kalarchian } \\
2009\end{array}$} & $\begin{array}{l}\text { I: family-based, behavioural weight control } \\
\text { group }\end{array}$ & 97 & 0 & 0 & 0 & 0 & 0 & 0 \\
\hline 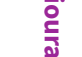 & & C: usual care & 95 & 0 & 0 & 0 & 0 & 0 & 0 \\
\hline 蛋 & \multirow{2}{*}{$\begin{array}{l}\text { Nowicka } \\
2009\end{array}$} & I: summer camp & 20 & 0 & 0 & 0 & 0 & 0 & 0 \\
\hline 普. & & C: control group & 28 & 0 & 0 & 0 & 0 & 0 & 0 \\
\hline$\stackrel{\overrightarrow{0}}{\square}$ & \multirow[t]{2}{*}{ Wake 2009} & I: LEAP2 behavioural intervention & 139 & 0 & 0 & 0 & 0 & 0 & 0 \\
\hline $\begin{array}{ll}0 \\
7 \\
0\end{array}$ & & C: control group & 119 & 0 & 0 & 0 & 0 & 0 & 0 \\
\hline 胥 & \multirow[t]{2}{*}{ Alves 2008} & I: exercise programme & 39 & 0 & 0 & 0 & 0 & 0 & 0 \\
\hline$\stackrel{0}{0}$ & & C: no care & 39 & 0 & 0 & 0 & 0 & 0 & 0 \\
\hline$\stackrel{\frac{\Phi}{0 .}}{0 .}$ & \multirow{2}{*}{$\begin{array}{l}\text { Hughes } \\
2008\end{array}$} & I: behavioural programme & 69 & 0 & 0 & 0 & 0 & 0 & 0 \\
\hline$\stackrel{0}{9}$ & & C: standard care & 65 & 0 & 0 & 0 & 0 & 0 & 0 \\
\hline 年 & \multirow[t]{2}{*}{ Weigel 2008} & I: active intervention group & 37 & 0 & 0 & - & - & - & - \\
\hline$\frac{10}{3}$ & & C: control group & 36 & 0 & 0 & - & - & - & - \\
\hline 告 & \multirow{2}{*}{$\begin{array}{l}\text { Weintraub } \\
2008\end{array}$} & I: after-school team sports programme & 9 & 0 & 0 & - & - & - & - \\
\hline$\stackrel{0}{\circ}$ & & C: "Active placebo" control & 12 & 0 & 0 & - & - & - & - \\
\hline $\begin{array}{l}0 \\
\stackrel{2}{0} \\
\stackrel{0}{\circ} \\
\end{array}$ & \multirow[t]{2}{*}{ Berry 2007} & $\begin{array}{l}\text { I: nutrition and exercise education pro- } \\
\text { gramme plus coping skills training }\end{array}$ & 40 & 0 & 0 & 0 & 0 & 0 & 0 \\
\hline$\frac{\stackrel{\nu}{u}}{3}$ & & $\begin{array}{l}\text { C: nutrition and exercise education pro- } \\
\text { gramme only }\end{array}$ & 40 & 0 & 0 & 0 & 0 & 0 & 0 \\
\hline
\end{tabular}




\begin{tabular}{|c|c|c|c|c|c|c|c|c|c|}
\hline 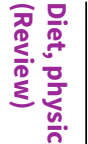 & $\begin{array}{l}\text { (Continued) } \\
\text { Gillis } 2007\end{array}$ & $\begin{array}{l}\text { I: exercise and diet education with weekly di- } \\
\text { aries and telephone calls }\end{array}$ & 14 & 0 & 0 & 0 & 0 & 0 & 0 \\
\hline בั้. & & C: exercise and diet education only & 13 & 0 & 0 & 0 & 0 & 0 & 0 \\
\hline 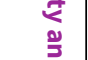 & \multirow{2}{*}{$\begin{array}{l}\text { Kalavainen } \\
2007\end{array}$} & I: family centred group programme & 35 & 0 & 0 & 0 & 0 & 0 & 0 \\
\hline $\begin{array}{l}\frac{9}{9} \\
\\
\frac{1}{2}\end{array}$ & & C: routine treatment & 35 & 0 & 0 & 0 & 0 & 0 & 0 \\
\hline 高 & \multirow{2}{*}{$\begin{array}{l}\text { McCallum } \\
2007\end{array}$} & I: LEAP Intervention & 81 & 0 & 0 & - & - & - & - \\
\hline $\begin{array}{l}\text { 蛋 } \\
\frac{1}{1}\end{array}$ & & C: control group & 82 & 0 & 0 & - & - & - & - \\
\hline 产. & \multirow{2}{*}{$\begin{array}{l}\text { Rodearmel } \\
2007\end{array}$} & I: 'America on the Move' intervention group & 116 & 0 & 0 & - & - & - & - \\
\hline$\vec{\circ}$ & & C: self-monitoring group & 102 & 0 & 0 & - & - & - & - \\
\hline 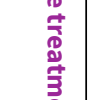 & \multirow[t]{2}{*}{ Satoh 2007} & $\begin{array}{l}\text { I: dietary guidance using an easily handled } \\
\text { model nutritional balance chart (MNBC) }\end{array}$ & 29 & 0 & 0 & - & - & - & - \\
\hline $\begin{array}{c}\overrightarrow{0} \\
0 \\
0\end{array}$ & & C: control group & 14 & 0 & 0 & - & - & - & - \\
\hline$\sum_{\substack{0 \\
\Sigma}}^{0}$ & \multirow{3}{*}{$\begin{array}{l}\text { Wilfley } \\
2007\end{array}$} & I1: behavioural skills maintenance group & 51 & 0 & 0 & 0 & 0 & 0 & 0 \\
\hline $\begin{array}{ll}\frac{1}{2} \\
0 \\
0\end{array}$ & & 12: social facilitation maintenance group & 50 & 0 & 0 & 0 & 0 & 0 & 0 \\
\hline 임 & & C: control group & 49 & 0 & 0 & 0 & 0 & 0 & 0 \\
\hline 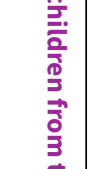 & \multirow[t]{2}{*}{$\begin{array}{l}\text { Epstein } \\
2005\end{array}$} & $\begin{array}{l}\text { I: standardised family-based behavioural } \\
\text { weight control programme plus reinforce- } \\
\text { ment } \\
\text { for increasing alternatives to eating }\end{array}$ & 19 & 0 & 0 & - & - & - & - \\
\hline $\begin{array}{c}0 \\
0 \\
0 \\
0 \\
0 \\
0 \\
0\end{array}$ & & $\begin{array}{l}\text { C: standardised family-based behavioural } \\
\text { weight control programme only }\end{array}$ & 22 & 0 & 0 & - & - & - & - \\
\hline $\begin{array}{ll}0 \\
0 \\
\end{array}$ & \multirow[t]{2}{*}{ Nemet 2005} & I: combined dietary and exercise programme & 30 & 0 & 0 & 0 & 0 & 0 & 0 \\
\hline$\frac{D}{2}$ & & C: control group & 24 & 0 & 0 & 0 & 0 & 0 & 0 \\
\hline & Woo 2004 & $\begin{array}{l}\text { I1: diet plus supervised structured exercise } \\
\text { programme with continuing training }\end{array}$ & 22 & 0 & 0 & 0 & 0 & 0 & 0 \\
\hline
\end{tabular}




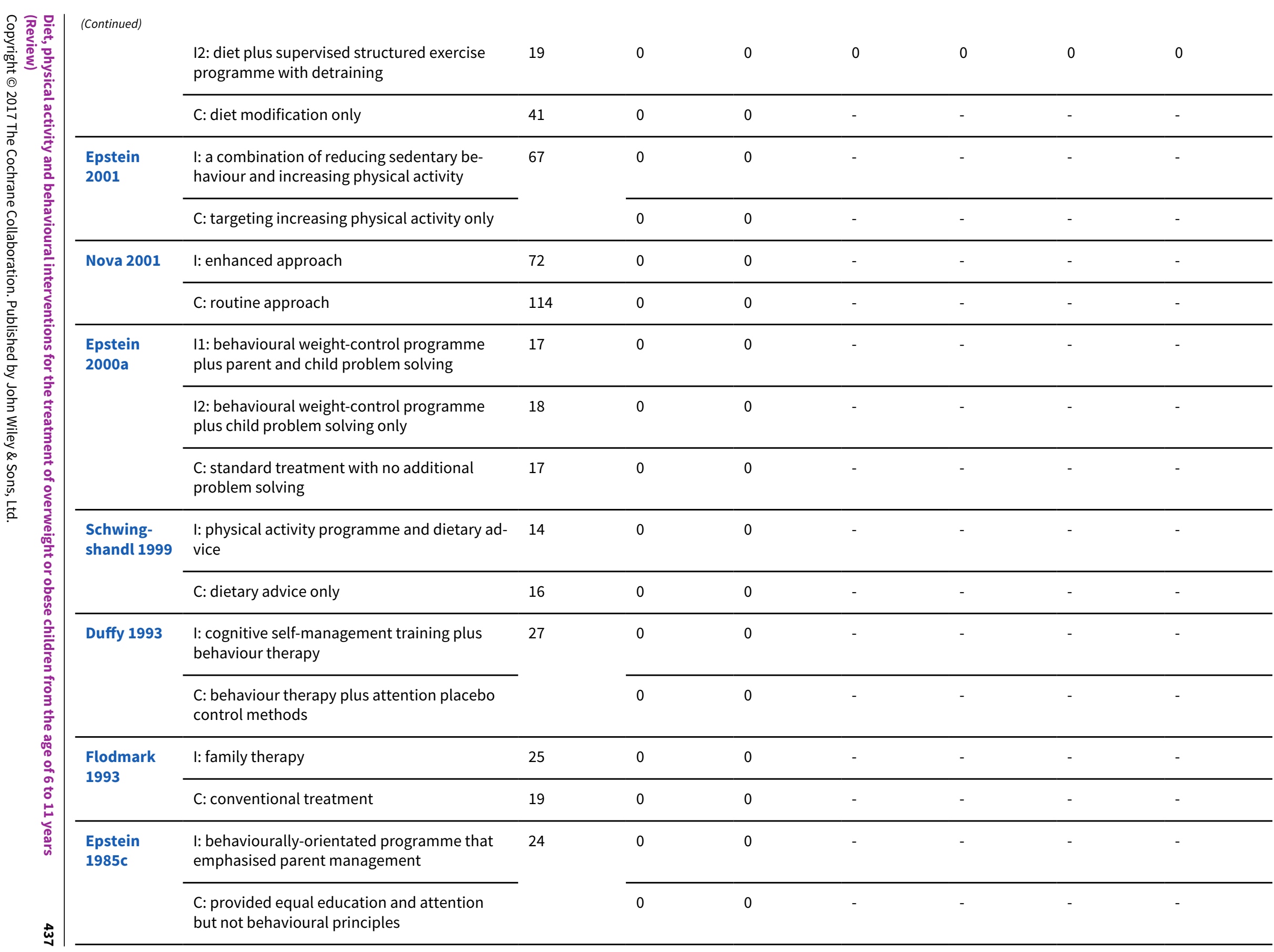




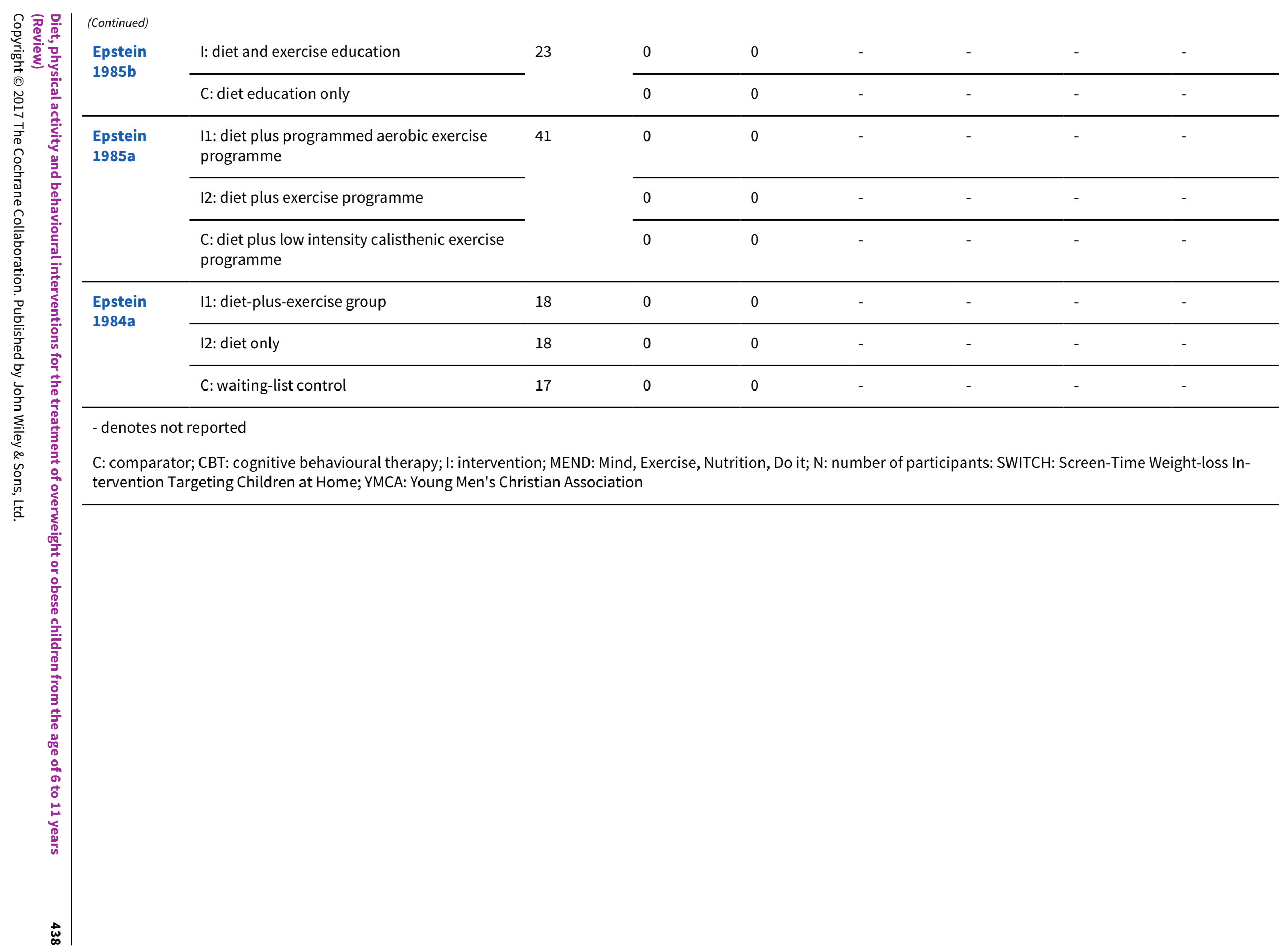




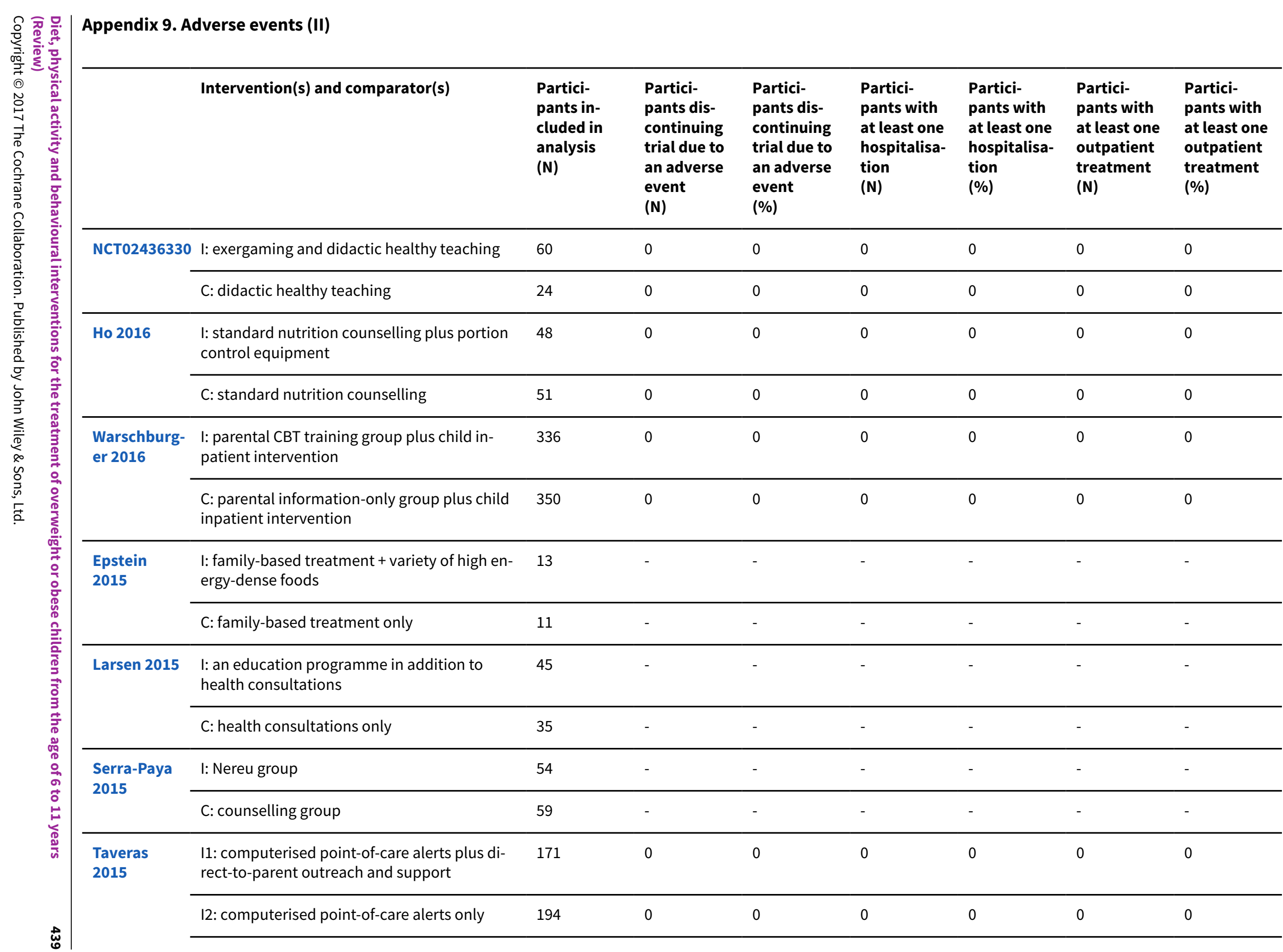




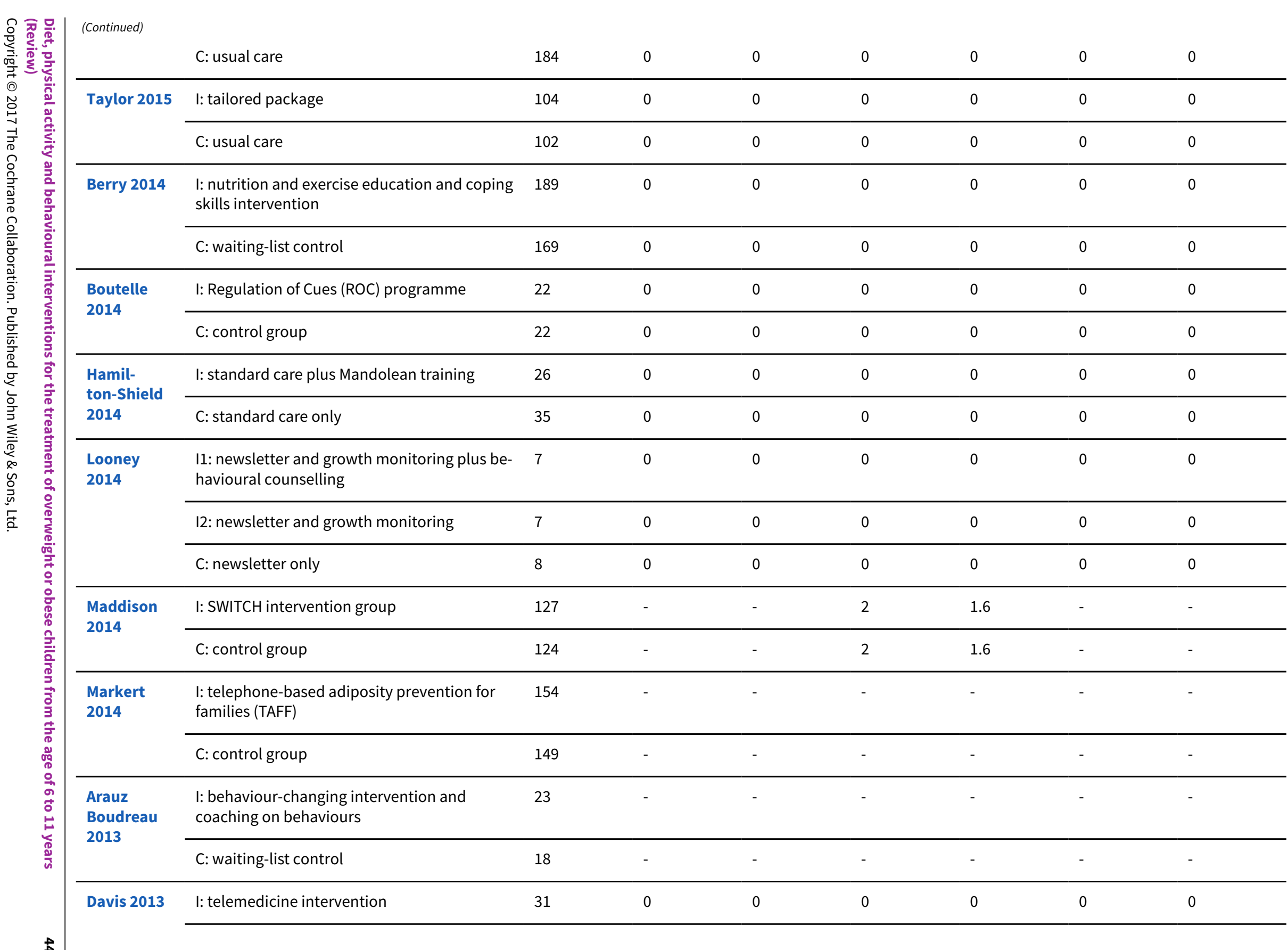




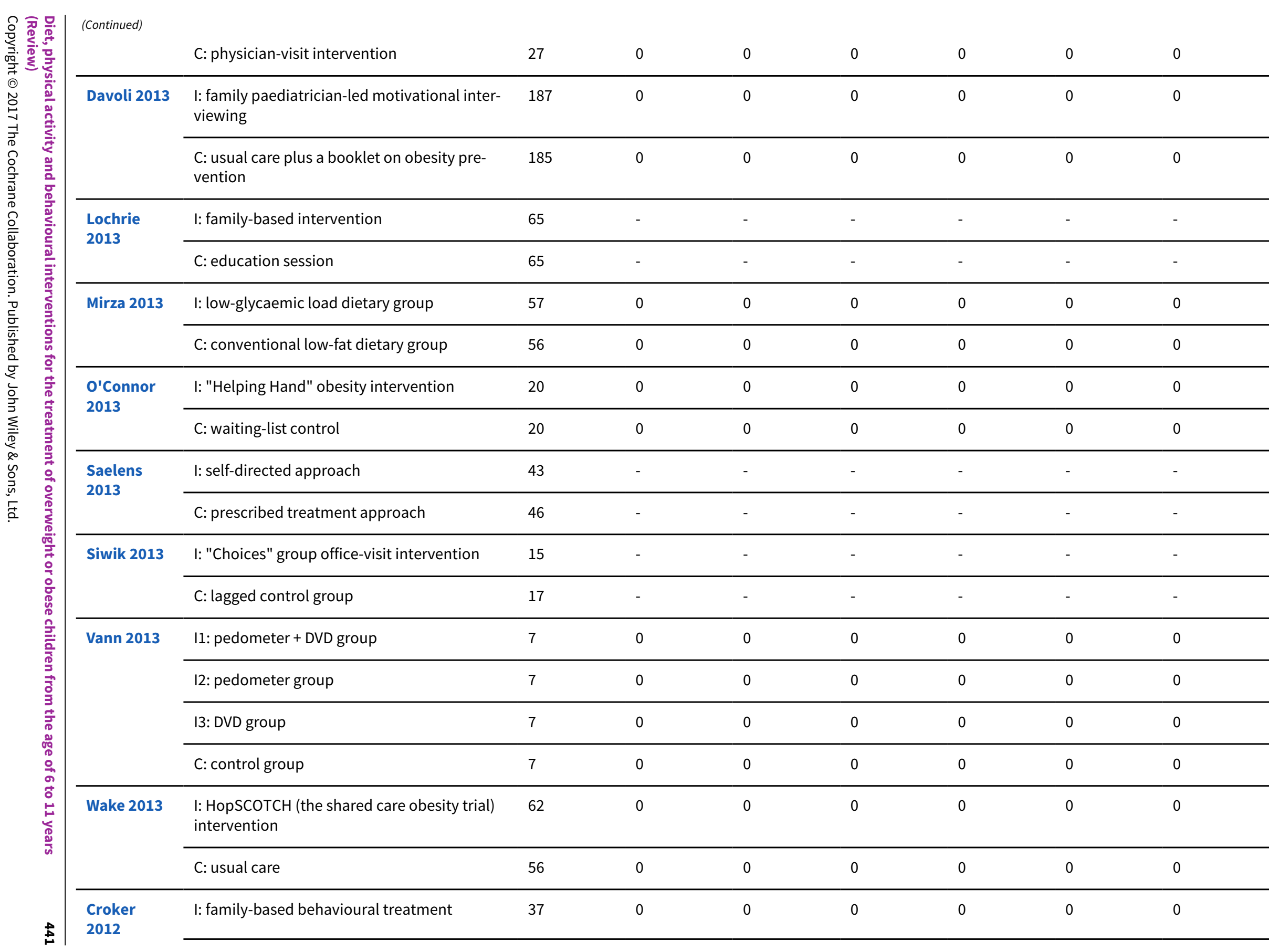




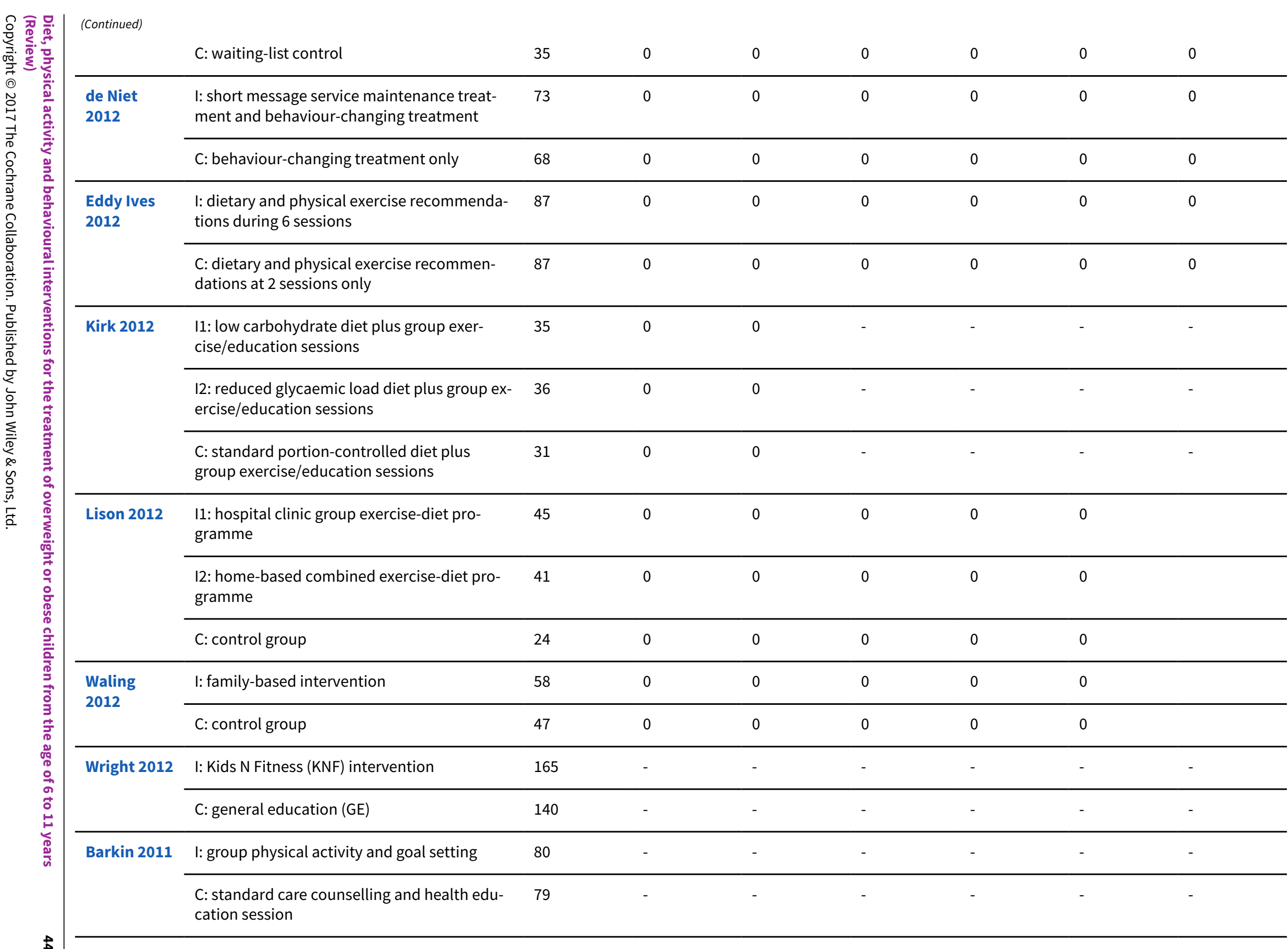




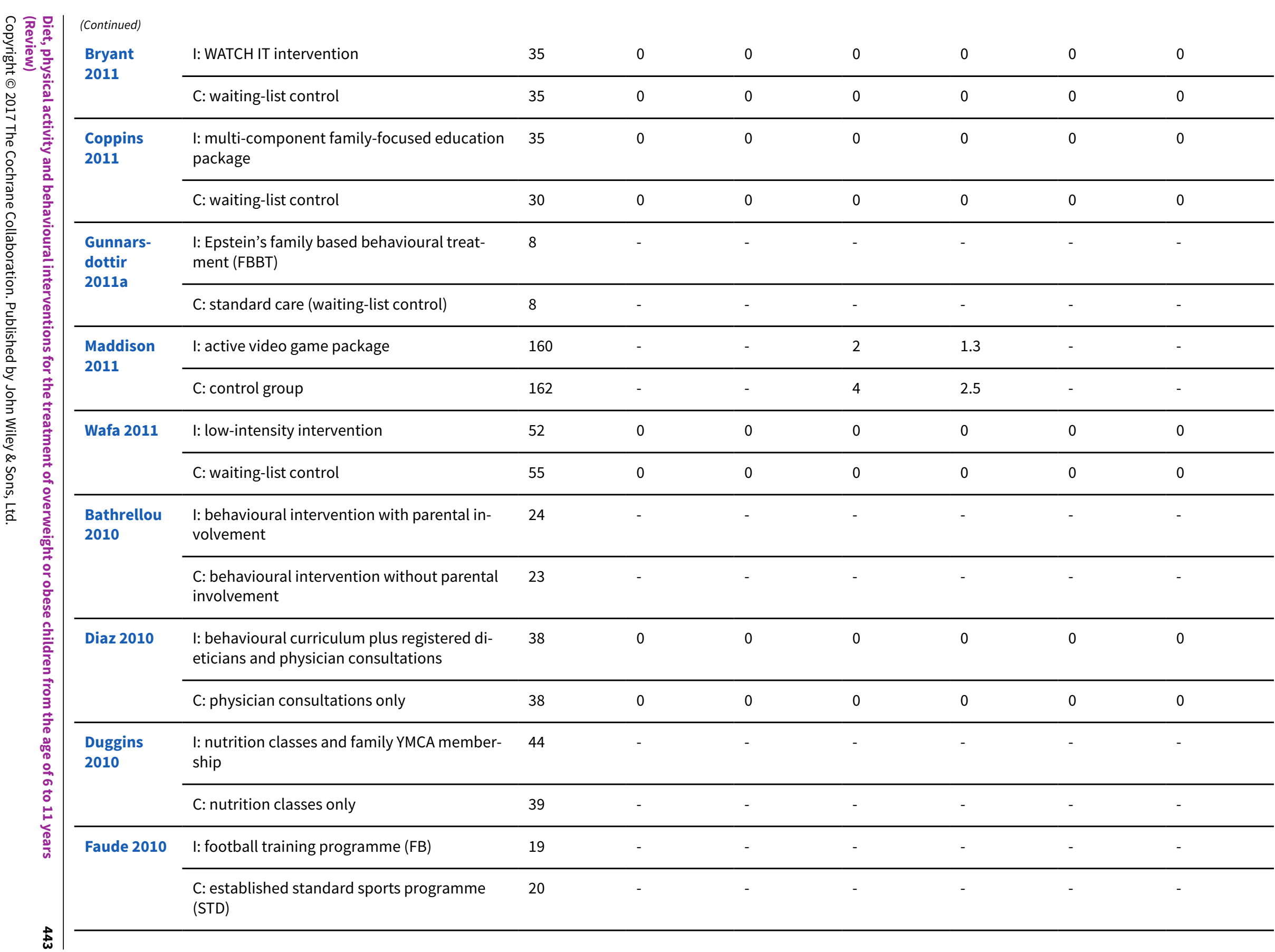




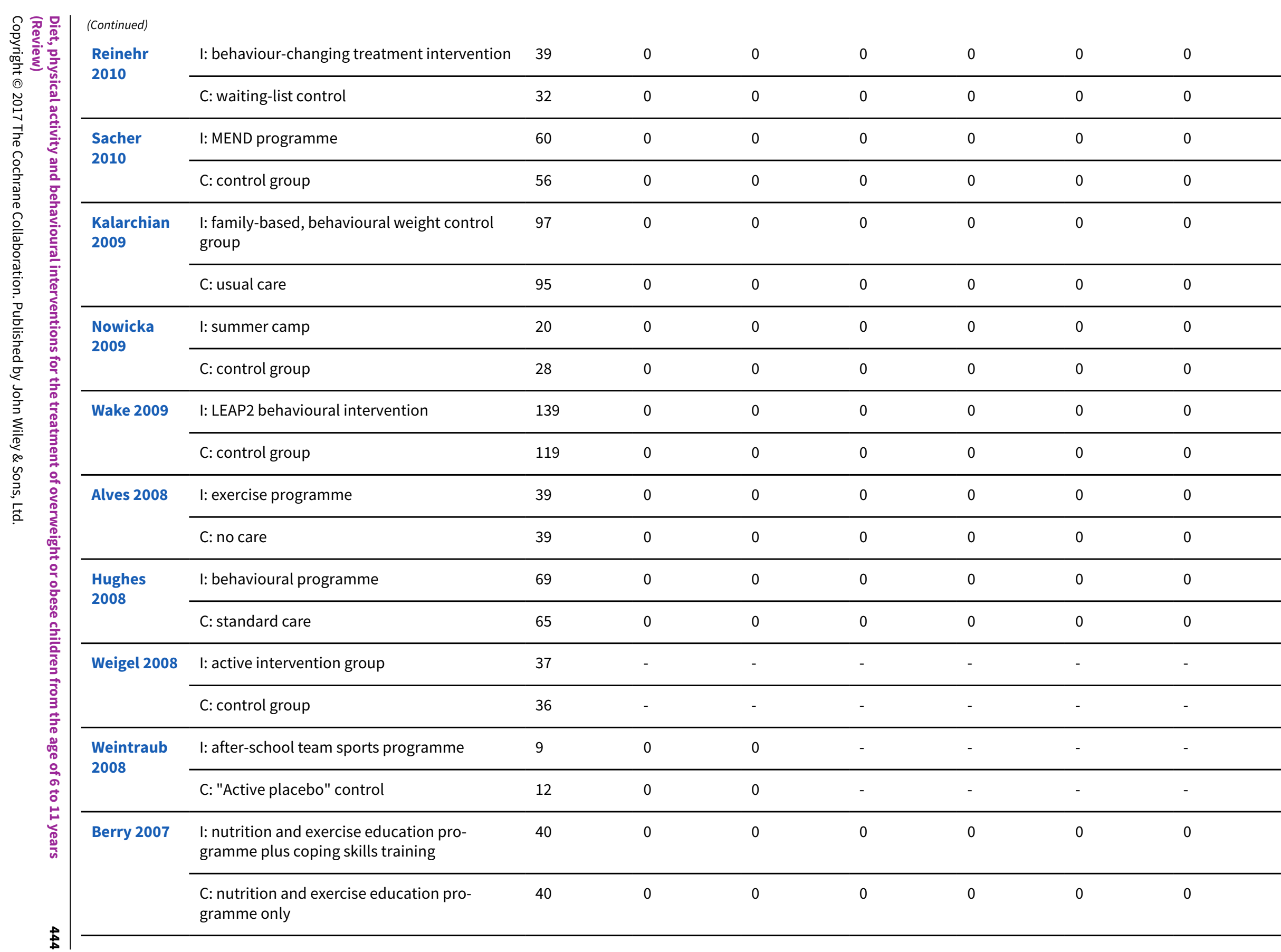




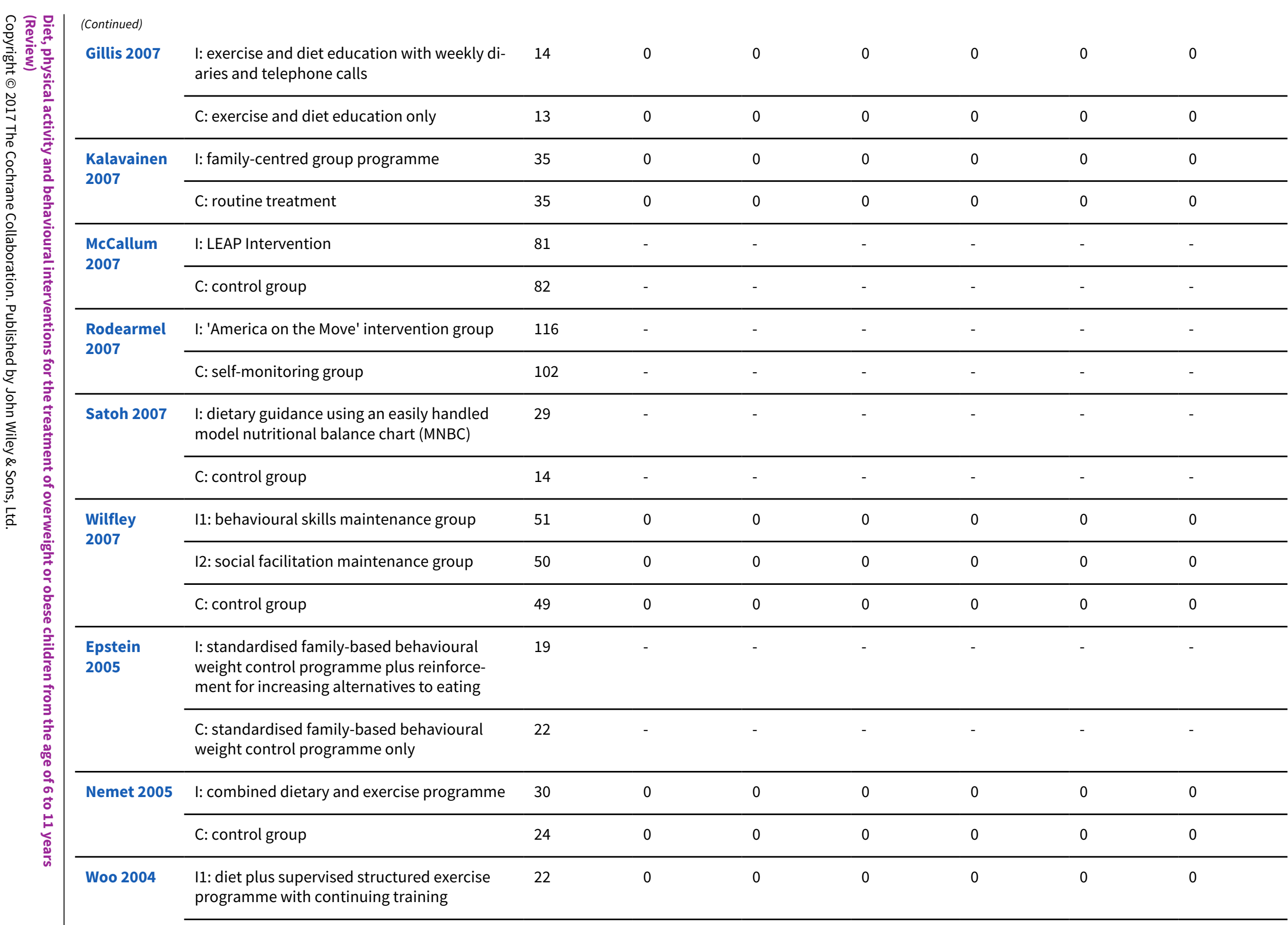




\begin{tabular}{|c|c|c|c|c|c|c|c|c|c|}
\hline 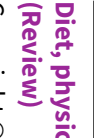 & (Continued) & $\begin{array}{l}\text { 12: diet plus supervised structured exercise } \\
\text { programme with detraining }\end{array}$ & 19 & 0 & 0 & 0 & 0 & 0 & 0 \\
\hline & & C: diet modification only & 41 & - & - & - & - & - & - \\
\hline $\begin{array}{l}\text { ह゙ } \\
\stackrel{\varrho}{\frac{\partial}{\sigma}} \\
\frac{0}{\sigma}\end{array}$ & $\begin{array}{l}\text { Epstein } \\
2001\end{array}$ & $\begin{array}{l}\text { I: a combination of reducing sedentary be- } \\
\text { haviour and increasing physical activity }\end{array}$ & 67 & - & - & - & - & - & - \\
\hline 总. & & C: targeting increasing physical activity only & & - & - & - & - & - & - \\
\hline$\stackrel{\Xi}{\Xi}$ & Nova 2001 & I: enhanced approach & 72 & - & - & - & - & - & - \\
\hline 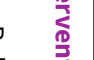 & & C: routine approach & 114 & - & - & - & - & - & - \\
\hline $\begin{array}{l}\stackrel{\circ}{\overline{3}} \\
\stackrel{\mathrm{D}^{\circ}}{2}\end{array}$ & $\begin{array}{l}\text { Epstein } \\
2000 a\end{array}$ & $\begin{array}{l}\text { I1: behavioural weight-control programme } \\
\text { plus parent and child problem solving }\end{array}$ & 17 & - & - & - & - & - & - \\
\hline 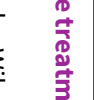 & & $\begin{array}{l}\text { I2: behavioural weight-control programme } \\
\text { plus child problem solving only }\end{array}$ & 18 & - & - & - & - & - & - \\
\hline $\begin{array}{l}\vec{z} \\
\stackrel{0}{0} \\
\stackrel{0}{0}\end{array}$ & & $\begin{array}{l}\text { C: standard treatment with no additional } \\
\text { problem solving }\end{array}$ & 17 & - & - & - & - & - & - \\
\hline 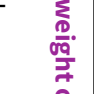 & $\begin{array}{l}\text { Schwing- } \\
\text { shandl } 1999\end{array}$ & $\begin{array}{l}\text { I: physical activity programme and dietary ad- } \\
\text { vice }\end{array}$ & 14 & - & - & - & - & - & - \\
\hline$\stackrel{\circ}{\circ}$ & & C: dietary advice only & 16 & - & - & - & - & - & - \\
\hline $\begin{array}{l}\frac{n}{2} \\
\frac{\overline{2}}{2} \\
\frac{0}{3} \\
\frac{9}{3}\end{array}$ & Duffy 1993 & $\begin{array}{l}\text { I: cognitive self-management training plus } \\
\text { behaviour therapy }\end{array}$ & 27 & - & - & - & - & - & - \\
\hline 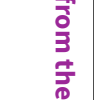 & & $\begin{array}{l}\text { C: behaviour therapy plus attention placebo } \\
\text { control methods }\end{array}$ & & - & - & - & - & - & - \\
\hline 然 & Flodmark & I: family therapy & 25 & - & - & - & - & - & - \\
\hline + & & C: conventional treatment & 19 & - & - & - & - & - & - \\
\hline 这 & $\begin{array}{l}\text { Epstein } \\
\text { 1985c }\end{array}$ & $\begin{array}{l}\text { I: behaviourally-orientated programme that } \\
\text { emphasised parent management }\end{array}$ & 24 & - & - & - & - & - & - \\
\hline$f$ & & $\begin{array}{l}\text { C: provided equal education and attention } \\
\text { but not behavioural principles }\end{array}$ & & - & - & - & - & - & - \\
\hline
\end{tabular}




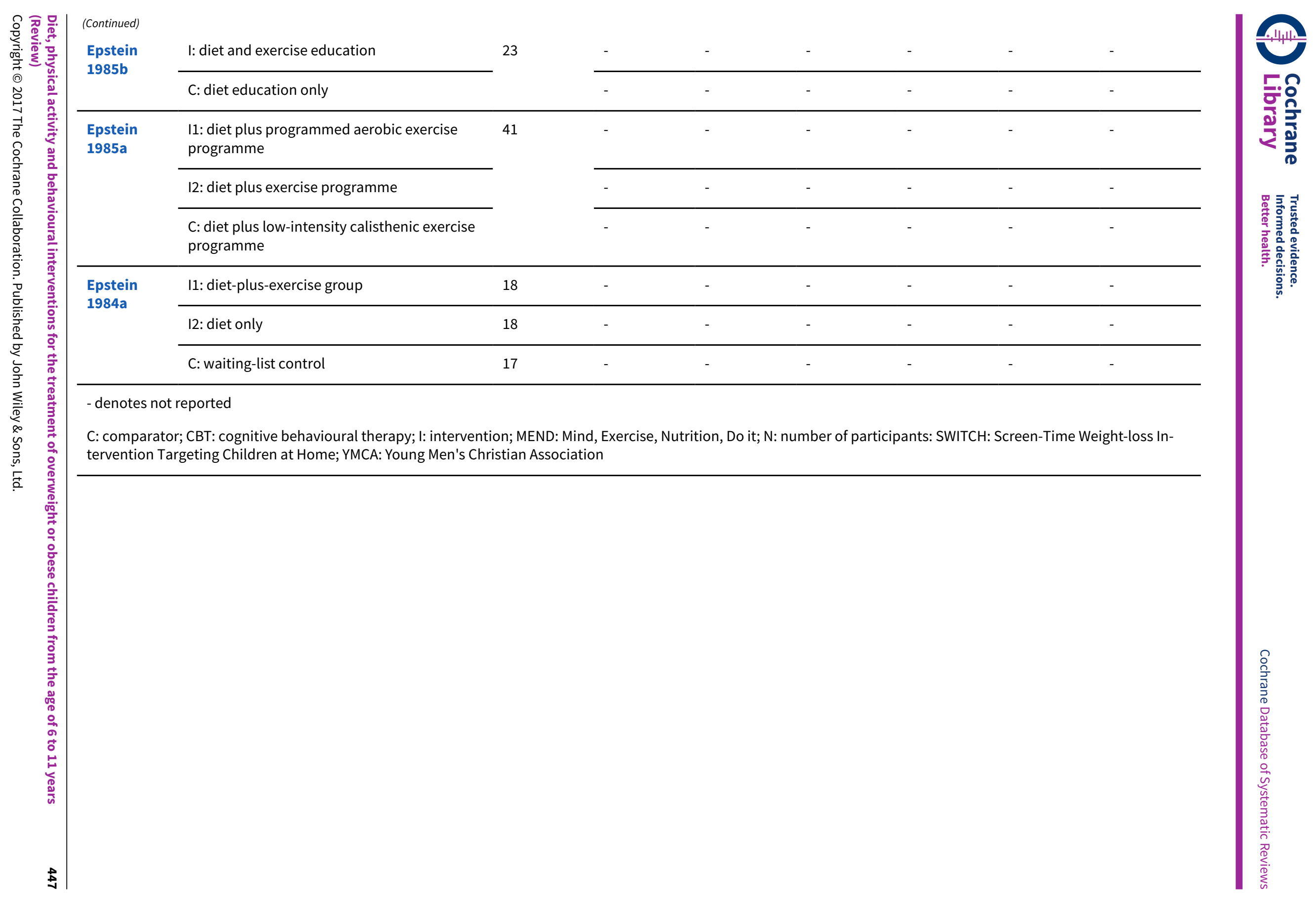


Appendix 10. Adverse events (III)

\begin{tabular}{|c|c|c|c|c|c|}
\hline & Intervention(s) and comparator(s) & $\begin{array}{l}\text { Participants } \\
\text { included in } \\
\text { analysis } \\
\text { (N) }\end{array}$ & $\begin{array}{l}\text { Participants } \\
\text { with a spe- } \\
\text { cific adverse } \\
\text { event } \\
\text { (description) }\end{array}$ & $\begin{array}{l}\text { Participants } \\
\text { with at least } \\
\text { one specif- } \\
\text { ic adverse } \\
\text { events } \\
\text { (N) }\end{array}$ & $\begin{array}{l}\text { Participants } \\
\text { with at least } \\
\text { one specif- } \\
\text { ic adverse } \\
\text { event } \\
(\%)\end{array}$ \\
\hline \multirow[t]{2}{*}{ NCT02436330 } & I: exergaming and didactic healthy teaching & 60 & 0 & 0 & 0 \\
\hline & C: didactic healthy teaching & 24 & 0 & 0 & 0 \\
\hline \multirow[t]{2}{*}{ Ho 2016} & $\begin{array}{l}\text { I: standard nutrition counselling plus por- } \\
\text { tion control equipment }\end{array}$ & 48 & 0 & 0 & 0 \\
\hline & C: standard nutrition counselling & 51 & 0 & 0 & 0 \\
\hline \multirow[t]{2}{*}{$\begin{array}{l}\text { Warschburg- } \\
\text { er } 2016\end{array}$} & $\begin{array}{l}\text { I: parental CBT training group plus child in- } \\
\text { patient intervention }\end{array}$ & 336 & 0 & 0 & 0 \\
\hline & $\begin{array}{l}\text { C: parental information-only group plus } \\
\text { child inpatient intervention }\end{array}$ & 350 & 0 & 0 & 0 \\
\hline \multirow[t]{2}{*}{ Epstein 2015} & $\begin{array}{l}\text { I: family-based treatment + variety of high } \\
\text { energy-dense foods }\end{array}$ & 13 & - & - & - \\
\hline & C: family-based treatment only & 11 & - & - & - \\
\hline \multirow[t]{2}{*}{ Larsen 2015} & $\begin{array}{l}\text { I: an education programme in addition to } \\
\text { health consultations }\end{array}$ & 45 & - & - & - \\
\hline & C: health consultations only & 35 & - & - & - \\
\hline \multirow{2}{*}{$\begin{array}{l}\text { Serra-Paya } \\
2015\end{array}$} & I: Nereu group & 54 & - & - & - \\
\hline & C: counselling group & 59 & - & - & - \\
\hline \multirow[t]{3}{*}{ Taveras 2015} & $\begin{array}{l}\text { I1: computerised point-of-care alerts plus di- } \\
\text { rect-to-parent outreach and support }\end{array}$ & 171 & 0 & 0 & 0 \\
\hline & 12: computerised point-of-care alerts only & 194 & 0 & 0 & 0 \\
\hline & C: usual care & 184 & 0 & 0 & 0 \\
\hline \multirow[t]{2}{*}{ Taylor 2015} & I: tailored package & 104 & 0 & 0 & 0 \\
\hline & C: usual care & 102 & 0 & 0 & 0 \\
\hline \multirow[t]{2}{*}{ Berry 2014} & $\begin{array}{l}\text { I: nutrition and exercise education and cop- } \\
\text { ing skills intervention }\end{array}$ & 189 & 0 & 0 & 0 \\
\hline & C: waiting-list control & 169 & 0 & 0 & 0 \\
\hline Boutelle 2014 & I: Regulation of Cues (ROC) programme & 22 & 0 & 0 & 0 \\
\hline
\end{tabular}

Diet, physical activity and behavioural interventions for the treatment of overweight or obese children from the age of 6 to 11 years 
(Continued)

\begin{tabular}{|c|c|c|c|c|c|}
\hline & C: control group & 22 & 0 & 0 & 0 \\
\hline \multirow{2}{*}{$\begin{array}{l}\text { Hamil- } \\
\text { ton-Shield } \\
2014\end{array}$} & I: standard care plus Mandolean training & 26 & 0 & 0 & 0 \\
\hline & C: standard care only & 35 & 0 & 0 & 0 \\
\hline \multirow[t]{3}{*}{ Looney 2014} & $\begin{array}{l}\text { I1: newsletter and growth monitoring plus } \\
\text { behavioural counselling }\end{array}$ & 7 & 0 & 0 & 0 \\
\hline & 12: newsletter and growth monitoring & 7 & 0 & 0 & 0 \\
\hline & C: newsletter only & 8 & 0 & 0 & 0 \\
\hline \multirow[t]{2}{*}{$\begin{array}{l}\text { Maddison } \\
2014\end{array}$} & I: SWITCH intervention group & 127 & $\begin{array}{l}\text { (1) Bowel re- } \\
\text { placement } \\
\text { surgery ("child } \\
\text { remained at } \\
\text { home, moni- } \\
\text { tored by care- } \\
\text { giver.") Coded } \\
\text { as severe } \\
\text { (2) Dislocated } \\
\text { left hip. Coded } \\
\text { as moderate } \\
\text { severity }\end{array}$ & $\begin{array}{l}\text { (1) } 1 \\
\text { (2) } 1\end{array}$ & $\begin{array}{l}\text { (1) } 0.8 \\
\text { (2) } 0.8\end{array}$ \\
\hline & C: control group & 124 & $\begin{array}{l}\text { (1) Operation } \\
\text { to remove cyst } \\
\text { ("participant } \\
\text { had operation } \\
\text { to remove cyst } \\
\text { from a testi- } \\
\text { cle.") Coded } \\
\text { as mild sever- } \\
\text { ity } \\
\text { (2) Broken an- } \\
\text { kle ("child fell } \\
\text { off a swing } \\
\text { on the play- } \\
\text { ground and } \\
\text { broke his an- } \\
\text { kle. Child is } \\
\text { now back at } \\
\text { school and is } \\
\text { doing fine"). } \\
\text { Coded as mod- } \\
\text { erate severity } \\
\text { ( } 3 \text { ) Broke } 2 \text { fin- } \\
\text { gers on left } \\
\text { hand whilst } \\
\text { playing rugby, } \\
\text { "hand now in } \\
\text { cast" coded as } \\
\text { mild severity }\end{array}$ & $\begin{array}{l}\text { (1) } 1 \\
\text { (2) } 1 \\
\text { (3) } 1\end{array}$ & $\begin{array}{l}\text { (1) } 0.8 \\
\text { (2) } 0.8 \\
\text { (3) } 0.8\end{array}$ \\
\hline
\end{tabular}

Diet, physical activity and behavioural interventions for the treatment of overweight or obese children from the age of 6 to 11 years 
(Continued)

\begin{tabular}{|c|c|c|c|c|c|}
\hline \multirow[t]{2}{*}{ Markert 2014} & $\begin{array}{l}\text { I: telephone-based adiposity prevention for } \\
\text { families (TAFF) }\end{array}$ & 154 & - & - & - \\
\hline & C: control group & 149 & - & - & - \\
\hline \multirow{2}{*}{$\begin{array}{l}\text { Arauz } \\
\text { Boudreau } \\
2013\end{array}$} & $\begin{array}{l}\text { I: behaviour-changing intervention and } \\
\text { coaching on behaviours }\end{array}$ & 23 & - & - & - \\
\hline & C: waiting-list control & 18 & - & - & - \\
\hline \multirow[t]{2}{*}{ Davis 2013} & I: telemedicine intervention & 31 & 0 & 0 & 0 \\
\hline & C: physician-visit intervention & 27 & 0 & 0 & 0 \\
\hline \multirow[t]{2}{*}{ Davoli 2013} & $\begin{array}{l}\text { I: family paediatrician-led motivational in- } \\
\text { terviewing }\end{array}$ & 187 & 0 & 0 & 0 \\
\hline & $\begin{array}{l}\text { C: usual care plus a booklet on obesity pre- } \\
\text { vention }\end{array}$ & 185 & 0 & 0 & 0 \\
\hline
\end{tabular}

\begin{tabular}{|c|c|c|c|c|c|}
\hline \multirow[t]{2}{*}{ Lochrie 2013} & I: family-based intervention & 65 & - & - & - \\
\hline & C: education session & 65 & - & - & - \\
\hline \multirow[t]{2}{*}{ Mirza 2013} & I: low-glycaemic load dietary group & 57 & 0 & 0 & 0 \\
\hline & C: conventional low-fat dietary group & 56 & $\begin{array}{l}\text { Experienced } \\
\text { a feeling of } \\
\text { faintness dur- } \\
\text { ing the blood } \\
\text { draw at the 3- } \\
\text { month post- } \\
\text { intervention } \\
\text { assessment }\end{array}$ & 1 & 1.8 \\
\hline \multirow{2}{*}{$\begin{array}{l}\text { O'Connor } \\
2013\end{array}$} & I: "Helping Hand" obesity intervention & 20 & 0 & 0 & 0 \\
\hline & C: waiting-list control & 20 & 0 & 0 & 0 \\
\hline \multirow[t]{2}{*}{ Saelens 2013} & I: self-directed approach & 43 & - & - & - \\
\hline & C: prescribed treatment approach & 46 & - & - & - \\
\hline \multirow[t]{2}{*}{ Siwik 2013} & I: "Choices" group office-visit intervention & 15 & - & - & - \\
\hline & C: lagged control group & 17 & - & - & - \\
\hline \multirow[t]{3}{*}{ Vann 2013} & I1: pedometer + DVD group & 7 & 0 & 0 & 0 \\
\hline & 13: DVD group & 7 & 0 & 0 & 0 \\
\hline & C: control group & 7 & 0 & 0 & 0 \\
\hline Wake 2013 & $\begin{array}{l}\text { I: HopSCOTCH (the shared care obesity trial) } \\
\text { intervention }\end{array}$ & 62 & 0 & 0 & 0 \\
\hline
\end{tabular}

Diet, physical activity and behavioural interventions for the treatment of overweight or obese children from the age of 6 to 11 years 
(Continued)

\begin{tabular}{|c|c|c|c|c|c|}
\hline & C: usual care & 56 & 0 & 0 & 0 \\
\hline \multirow[t]{2}{*}{ Croker 2012} & I: family-based behavioural treatment & 37 & 0 & 0 & 0 \\
\hline & C: waiting-list control & 35 & $\begin{array}{l}\text { Very high re- } \\
\text { duction in BMI } \\
(28.8) \text { and BMI } \\
\text { SDS (4.2) }\end{array}$ & 1 & 2.9 \\
\hline
\end{tabular}

\begin{tabular}{|c|c|c|c|c|c|}
\hline de Niet 2012 & $\begin{array}{l}\text { I: short message service maintenance treat- } \\
\text { ment and behaviour-changing treatment }\end{array}$ & 73 & 0 & 0 & 0 \\
\hline & C: behaviour-changing treatment only & 68 & 0 & 0 & 0 \\
\hline \multirow[t]{2}{*}{$\begin{array}{l}\text { Eddy Ives } \\
2012\end{array}$} & $\begin{array}{l}\text { I: dietary and physical exercise recommen- } \\
\text { dations during } 6 \text { sessions }\end{array}$ & 87 & 0 & 0 & 0 \\
\hline & $\begin{array}{l}\text { C: dietary and physical exercise recommen- } \\
\text { dations at } 2 \text { sessions only }\end{array}$ & 87 & 0 & 0 & 0 \\
\hline \multirow[t]{3}{*}{ Kirk 2012} & $\begin{array}{l}\text { 11: Low carbohydrate diet plus group exer- } \\
\text { cise/education sessions }\end{array}$ & \multirow{3}{*}{$\begin{array}{l}\text { All: } \\
\text { BP }=84 \\
\text { TG }=74 \\
\text { LDL }=86 \\
\text { Glucose }=86\end{array}$} & $\begin{array}{l}\text { (1) Elevated } \\
\text { BP }\end{array}$ & \multirow{3}{*}{$\begin{array}{l}\text { (1) } 3 \\
(2) 9 \\
(3) 3 \\
(4) 3\end{array}$} & \multirow{3}{*}{$\begin{array}{l}\text { (1) } 3.6 \\
\text { (2) } 12.2 \\
\text { (3) } 3.5 \\
\text { (4) } 3.5\end{array}$} \\
\hline & $\begin{array}{l}\text { 12: reduced glycaemic load diet plus group } \\
\text { exercise/education sessions }\end{array}$ & & $\begin{array}{l}\text { (2) Elevated } \\
\text { TG }\end{array}$ & & \\
\hline & $\begin{array}{l}\text { C: standard portion-controlled diet plus } \\
\text { group exercise/education sessions }\end{array}$ & & $\begin{array}{l}\text { (3) Elevated } \\
\text { LDL } \\
\text { (4) Elevated } \\
\text { Glucose }\end{array}$ & & \\
\hline \multirow[t]{3}{*}{ Lison 2012} & $\begin{array}{l}\text { I1: hospital clinic group exercise-diet pro- } \\
\text { gramme }\end{array}$ & 45 & 0 & 0 & 0 \\
\hline & $\begin{array}{l}\text { I2: home-based combined exercise-diet pro- } \\
\text { gramme }\end{array}$ & 41 & 0 & 0 & 0 \\
\hline & C: control group & 24 & 0 & 0 & 0 \\
\hline \multirow[t]{2}{*}{ Waling 2012} & I: family-based intervention & 58 & 0 & 0 & 0 \\
\hline & C: control group & 47 & 0 & 0 & 0 \\
\hline \multirow[t]{2}{*}{ Wright 2012} & I: Kids N Fitness (KNF) intervention & 165 & - & - & - \\
\hline & C: general education (GE) & 140 & - & - & - \\
\hline \multirow[t]{2}{*}{ Barkin 2011} & I: group physical activity and goal setting & 80 & - & - & - \\
\hline & $\begin{array}{l}\text { C: standard care counselling and health edu- } \\
\text { cation session }\end{array}$ & 79 & - & - & - \\
\hline \multirow[t]{2}{*}{ Bryant 2011} & I: WATCH IT intervention & 35 & 0 & 0 & 0 \\
\hline & C: waiting-list control & 35 & 0 & 0 & 0 \\
\hline
\end{tabular}


(Continued)

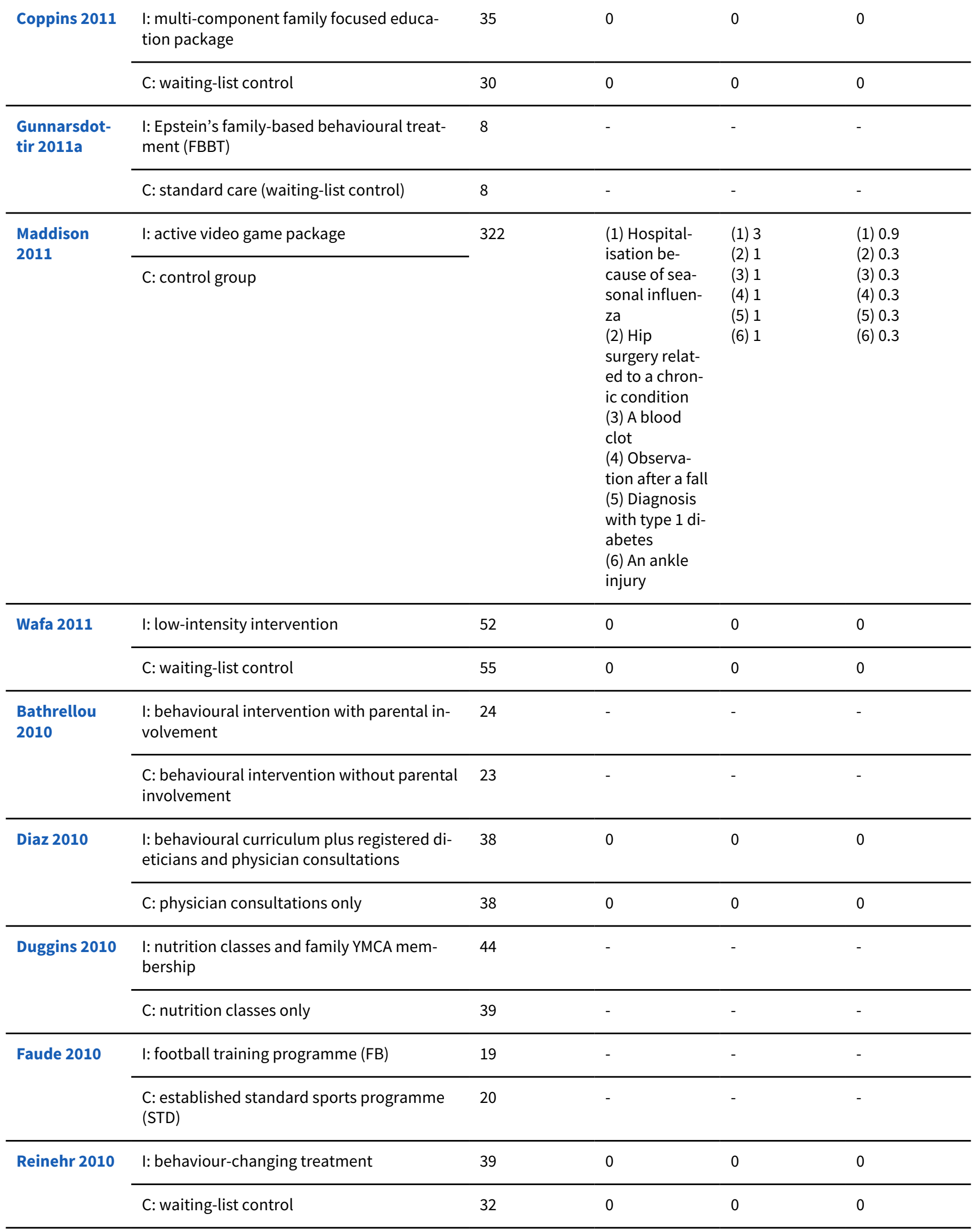

Diet, physical activity and behavioural interventions for the treatment of overweight or obese children from the age of 6 to 11 years 
(Continued)

\begin{tabular}{|c|c|c|c|c|c|}
\hline \multirow[t]{2}{*}{ Sacher 2010} & I: MEND programme & 60 & 0 & 0 & 0 \\
\hline & C: control group & 56 & 0 & 0 & 0 \\
\hline \multirow[t]{2}{*}{$\begin{array}{l}\text { Kalarchian } \\
2009\end{array}$} & $\begin{array}{l}\text { I: family-based, behavioural weight control } \\
\text { group }\end{array}$ & 97 & 0 & 0 & 0 \\
\hline & C: usual care & 95 & 0 & 0 & 0 \\
\hline \multirow{2}{*}{$\begin{array}{l}\text { Nowicka } \\
2009\end{array}$} & I: summer camp & 20 & 0 & 0 & 0 \\
\hline & C: control group & 28 & 0 & 0 & 0 \\
\hline \multirow[t]{2}{*}{ Wake 2009} & I: LEAP2 behavioural intervention & 139 & 0 & 0 & 0 \\
\hline & C: control group & 119 & 0 & 0 & 0 \\
\hline \multirow[t]{2}{*}{ Alves 2008} & I: exercise programme & 39 & 0 & 0 & 0 \\
\hline & C: no care & 39 & 0 & 0 & 0 \\
\hline \multirow[t]{2}{*}{ Hughes 2008} & I: behavioural programme & 69 & 0 & 0 & 0 \\
\hline & C: standard care & 65 & 0 & 0 & 0 \\
\hline \multirow[t]{2}{*}{ Weigel 2008} & I: active intervention group & 37 & - & - & - \\
\hline & C: control group & 36 & - & - & - \\
\hline \multirow[t]{2}{*}{$\begin{array}{l}\text { Weintraub } \\
2008\end{array}$} & I: after-school team sports programme & 9 & $\begin{array}{l}\text { (1) Skin rash } \\
\text { (2) Car colli- } \\
\text { sion } \\
\text { (3) Newly di- } \\
\text { agnosed hy- } \\
\text { pothyroidism }\end{array}$ & $\begin{array}{l}\text { (1) } 1 \\
\text { (2) } 1 \\
\text { (3) } 1\end{array}$ & $\begin{array}{l}\text { (1) } 11 \\
\text { (2) } 11 \\
\text { (3) } 11\end{array}$ \\
\hline & C: "Active placebo" control & 12 & $\begin{array}{l}\text { (1) Foot injury } \\
\text { (2) Knee pain } \\
\text { while ice skat- } \\
\text { ing } \\
\text { (3) Eye } \\
\text { pain and } \\
\text { headaches } \\
\text { (4) Ingrown } \\
\text { toenail } \\
\text { (5) Ear infec- } \\
\text { tion } \\
\text { (6) Skin rash }\end{array}$ & $\begin{array}{l}\text { (1) } 1 \\
\text { (2) } 1 \\
\text { (3) } 1 \\
\text { (4) } 1 \\
\text { (5) } 1 \\
\text { (6) } 1\end{array}$ & $\begin{array}{l}\text { (2) } 8.3 \\
\text { (3) } 8.3 \\
\text { (4) } 8.3\end{array}$ \\
\hline \multirow[t]{2}{*}{ Berry 2007} & $\begin{array}{l}\text { I: nutrition and exercise education pro- } \\
\text { gramme plus coping skills training }\end{array}$ & 40 & 0 & 0 & 0 \\
\hline & $\begin{array}{l}\text { C: nutrition and exercise education pro- } \\
\text { gramme only }\end{array}$ & 40 & 0 & 0 & 0 \\
\hline Gillis 2007 & $\begin{array}{l}\text { I: exercise and diet education with weekly } \\
\text { diaries and telephone calls }\end{array}$ & 14 & 0 & 0 & 0 \\
\hline
\end{tabular}

Diet, physical activity and behavioural interventions for the treatment of overweight or obese children from the age of 6 to 11 years 
(Continued)

\begin{tabular}{|c|c|c|c|c|c|}
\hline & C: exercise and diet education only & 13 & 0 & 0 & 0 \\
\hline \multirow{2}{*}{$\begin{array}{l}\text { Kalavainen } \\
2007\end{array}$} & I: family-centred group programme & 35 & 0 & 0 & 0 \\
\hline & C: routine treatment & 35 & 0 & 0 & 0 \\
\hline \multirow{2}{*}{$\begin{array}{l}\text { McCallum } \\
2007\end{array}$} & I: LEAP Intervention & 81 & - & - & - \\
\hline & C: control group & 82 & - & - & - \\
\hline \multirow{2}{*}{$\begin{array}{l}\text { Rodearmel } \\
2007\end{array}$} & I: 'America on the Move' intervention group & 116 & - & - & - \\
\hline & C: self-monitoring group & 102 & - & - & - \\
\hline \multirow[t]{2}{*}{ Satoh 2007} & $\begin{array}{l}\text { I: dietary guidance using an easily handled } \\
\text { model nutritional balance chart (MNBC) }\end{array}$ & 29 & - & - & - \\
\hline & C: control group & 14 & - & - & - \\
\hline \multirow[t]{3}{*}{ Wilfley 2007} & I1: behavioural skills maintenance group & 51 & 0 & 0 & 0 \\
\hline & 12: social facilitation maintenance group & 50 & 0 & 0 & 0 \\
\hline & C: control group & 49 & 0 & 0 & 0 \\
\hline Epstein 2005 & $\begin{array}{l}\text { I: standardised family-based behavioural } \\
\text { weight control programme plus reinforce- } \\
\text { ment for increasing alternatives to eating }\end{array}$ & 19 & - & - & - \\
\hline \multirow[t]{2}{*}{ Nemet 2005} & $\begin{array}{l}\text { I: combined dietary and exercise pro- } \\
\text { gramme }\end{array}$ & 30 & 0 & 0 & 0 \\
\hline & C: control group & 24 & 0 & 0 & 0 \\
\hline \multirow[t]{3}{*}{ Woo 2004} & $\begin{array}{l}\text { I1: diet plus supervised structured exercise } \\
\text { programme with continuing training }\end{array}$ & 22 & 0 & 0 & 0 \\
\hline & $\begin{array}{l}\text { 12: diet plus supervised structured exercise } \\
\text { programme with detraining }\end{array}$ & 19 & 0 & 0 & 0 \\
\hline & C: diet modification only & 41 & - & - & - \\
\hline \multirow[t]{2}{*}{ Epstein 2001} & $\begin{array}{l}\text { I: a combination of reducing sedentary be- } \\
\text { haviour and increasing physical activity }\end{array}$ & 67 & - & - & - \\
\hline & C: targeting increasing physical activity only & & - & - & - \\
\hline \multirow[t]{2}{*}{ Nova 2001} & I: enhanced approach & 72 & - & - & - \\
\hline & C: routine approach & 114 & - & - & - \\
\hline $\begin{array}{l}\text { Epstein } \\
\text { 2000a }\end{array}$ & $\begin{array}{l}\text { I1: behavioural weight-control programme } \\
\text { plus parent and child problem solving }\end{array}$ & 17 & - & - & - \\
\hline
\end{tabular}

Diet, physical activity and behavioural interventions for the treatment of overweight or obese children from the age of 6 to 11 years 
(Continued)

I2: behavioural weight-control programme
plus child problem solving only

C: standard treatment with no additional $\quad 17$

problem solving

\begin{tabular}{|c|c|c|c|c|c|}
\hline $\begin{array}{l}\text { Schwing- } \\
\text { shandl } 1999\end{array}$ & $\begin{array}{l}\text { I: physical activity programme and dietary } \\
\text { advice }\end{array}$ & 14 & - & - & - \\
\hline & C: dietary advice only & 16 & - & - & - \\
\hline \multirow[t]{2}{*}{ Duffy 1993} & $\begin{array}{l}\text { I: cognitive self-management training plus } \\
\text { behaviour therapy }\end{array}$ & 27 & - & - & - \\
\hline & $\begin{array}{l}\text { C: behaviour therapy plus attention placebo } \\
\text { control methods }\end{array}$ & & - & - & - \\
\hline \multirow{2}{*}{$\begin{array}{l}\text { Flodmark } \\
1993\end{array}$} & I: family therapy & 25 & - & - & - \\
\hline & C: conventional treatment & 19 & - & - & - \\
\hline \multirow[t]{2}{*}{ Epstein 1985c } & $\begin{array}{l}\text { I: behaviourally-orientated programme that } \\
\text { emphasised parent management }\end{array}$ & 24 & - & - & - \\
\hline & $\begin{array}{l}\text { C: provided equal education and attention } \\
\text { but not behavioural principles }\end{array}$ & & - & - & - \\
\hline \multirow{2}{*}{$\begin{array}{l}\text { Epstein } \\
\text { 1985b }\end{array}$} & I: diet and exercise education & 23 & - & - & - \\
\hline & C: diet education only & & - & - & - \\
\hline \multirow[t]{3}{*}{$\begin{array}{l}\text { Epstein } \\
1985 a\end{array}$} & $\begin{array}{l}\text { I1: diet plus programmed aerobic exercise } \\
\text { programme }\end{array}$ & 41 & - & - & - \\
\hline & 12: diet plus lifestyle programme & & - & - & - \\
\hline & $\begin{array}{l}\text { C: diet plus low-intensity calisthenic exer- } \\
\text { cise programme }\end{array}$ & & - & - & - \\
\hline \multirow{3}{*}{$\begin{array}{l}\text { Epstein } \\
1984 a\end{array}$} & I1: diet-plus-exercise group & 18 & - & - & - \\
\hline & 12: diet only & 18 & - & - & - \\
\hline & C: waiting-list control & 17 & - & - & - \\
\hline
\end{tabular}

\footnotetext{
- denotes not reported
}

BP: blood pressure; C: comparator; CBT: cognitive behavioural therapy; I: intervention; LDL: low-density lipoprotein; MEND: Mind, Exercise, Nutrition, Do it; N: number of participants: SWITCH: Screen-Time Weight-loss Intervention Targeting Children at Home; TG: triglycerides; YMCA: Young Men's Christian Association

\section{Appendix 11. Survey of study investigators providing information on included trials}




\begin{tabular}{lllll}
\hline & $\begin{array}{l}\text { Date trial author } \\
\text { contacted }\end{array}$ & Summary of information asked for & $\begin{array}{l}\text { Date trial au- } \\
\text { thor replied }\end{array}$ & $\begin{array}{l}\text { Trial author provided data } \\
\text { (short summary) }\end{array}$ \\
\hline NCT02436330 & $\begin{array}{l}16 \text { September } \\
2016\end{array}$ & To ask if results were published & $16 / 09 / 16$ & $\begin{array}{l}\text { Response from author: } \\
\text { "We are awaiting a final re- } \\
\text { sponse after two cycles of } \\
\text { edits and reviews with a } \\
\text { journal" }\end{array}$ \\
\hline Ho 2016 & & N/A & N/A \\
\hline & $\begin{array}{l}\text { Not contacted: } \\
\text { study identified } \\
\text { from latest update } \\
\text { search (July 2016) } \\
\text { and no further in- } \\
\text { formation was re- } \\
\text { quired }\end{array}$ & & \\
\hline
\end{tabular}

\begin{tabular}{lllll}
\hline $\begin{array}{l}\text { Warschburger } \\
2016\end{array}$ & 22 April 2016 & $\begin{array}{l}\text { Allocation concealment, ethnic } \\
\text { groups, adverse events, additional pa- } \\
\text { pers }\end{array}$ & 22 April 2016 & $\begin{array}{l}\text { Answered all questions - no } \\
\text { additional papers }\end{array}$ \\
\hline Epstein 2015 & 14 January 2016 & $\begin{array}{l}\text { Adverse events, blinding, allocation } \\
\text { concealment, randomisation method, } \\
\text { setting, additional papers }\end{array}$ & 03 February 2016 & $\begin{array}{l}\text { Answered general ques- } \\
\text { tions about all of their stud- } \\
\text { ies included in this review } \\
\text { but not necessarily specif- } \\
\text { ic questions related to this } \\
\text { study }\end{array}$ \\
\hline
\end{tabular}

\begin{tabular}{|c|c|c|c|c|}
\hline Larsen 2015 & 19 February 2015 & $\begin{array}{l}\text { Adverse events, blinding, additional } \\
\text { papers }\end{array}$ & No reply & $\mathrm{N} / \mathrm{A}$ \\
\hline Serra-Paya 2015 & 21 April 2016 & $\begin{array}{l}\text { Allocation, blinding, adverse events, } \\
\text { ethnic group, dropout reasons, addi- } \\
\text { tional papers }\end{array}$ & 28 April 2016 & $\begin{array}{l}\text { Answered all questions - no } \\
\text { additional papers available } \\
\text { yet }\end{array}$ \\
\hline Taveras 2015 & 21 April 2016 & $\begin{array}{l}\text { Allocation, adverse events, additional } \\
\text { papers }\end{array}$ & 25 April 2016 & $\begin{array}{l}\text { Answered all questions - no } \\
\text { additional papers currently } \\
\text { available }\end{array}$ \\
\hline Taylor 2015 & 14 January 2016 & $\begin{array}{l}\text { Adverse events, number of study cen- } \\
\text { tres, baseline differences and addition- } \\
\text { al papers }\end{array}$ & 17 January 2015 & $\begin{array}{l}\text { Answered all questions and } \\
\text { no more additional papers } \\
\text { published }\end{array}$ \\
\hline Berry 2014 & 12 January 2016 & $\begin{array}{l}\text { Blinding, adverse events, number of } \\
\text { participants measured at each time } \\
\text { point, additional papers }\end{array}$ & 14 January 2016 & $\begin{array}{l}\text { Answered questions but still } \\
\text { unsure about number fol- } \\
\text { lowed up at each time point } \\
\text { (assume it must be } 304 \text { and } \\
\text { 290). Additional papers pro- } \\
\text { vided are the ones already } \\
\text { obtained }\end{array}$ \\
\hline Boutelle 2014 & $\begin{array}{l}12 \text { January } 2016 \\
\text { Reminder- } 03 \text { Feb- } \\
\text { ruary } 2016\end{array}$ & $\begin{array}{l}\text { Allocation concealment, blinding, ad- } \\
\text { verse events, setting, ITT, additional } \\
\text { papers }\end{array}$ & $03 / 02 / 2016$ & $\begin{array}{l}\text { Answered all questions and } \\
\text { no additional papers pub- } \\
\text { lished }\end{array}$ \\
\hline $\begin{array}{l}\text { Hamilton-Shield } \\
2014\end{array}$ & $\begin{array}{l}\text { Did not contact } \\
\text { as study was ter- } \\
\text { minated and no }\end{array}$ & $\mathrm{N} / \mathrm{A}$ & $\mathrm{N} / \mathrm{A}$ & $\mathrm{N} / \mathrm{A}$ \\
\hline
\end{tabular}

Diet, physical activity and behavioural interventions for the treatment of overweight or obese children from the age of 6 to 11 years 
(Continued)

more information

was required

\begin{tabular}{llll}
\hline Looney 2014 13 January 2016 & $\begin{array}{l}\text { Allocation concealment, blinding, ad- } \\
\text { verse events, additional papers }\end{array}$ & $\begin{array}{l}13 \text { Janauary } \\
2016\end{array}$
\end{tabular}

Answered all questions and verse events, additional papers 2016 confirmed no other papers had been published

\begin{tabular}{ll}
\hline Maddison 2014 & 12 January 2016 \\
& Reminder 03 Feb- \\
& ruary 2016
\end{tabular}

Study centres, adverse events, additional papers

17 February 2016

Answered all questions above - provided extra adverse events information.

No other published papers but they do have a process paper currently under review (accepted but not published)

\begin{tabular}{|c|c|c|c|c|}
\hline Markert 2014 & $\begin{array}{l}13 \text { January } 2016 \\
\text { Reminder email } 03 \\
\text { February } 2016\end{array}$ & $\begin{array}{l}\text { Study centres, blinding, adverse } \\
\text { events, ethnic groups, additional pa- } \\
\text { pers }\end{array}$ & No reply & $\mathrm{N} / \mathrm{A}$ \\
\hline $\begin{array}{l}\text { Arauz Boudreau } \\
2013\end{array}$ & $\begin{array}{l}11 \text { December } 2015 \\
\text { Reminder email } 03 \\
\text { February } 2016\end{array}$ & $\begin{array}{l}\text { Randomisation, allocation conceal- } \\
\text { ment, adverse event, ITT, additional } \\
\text { papers }\end{array}$ & No reply & $\mathrm{N} / \mathrm{A}$ \\
\hline Davis 2013 & 13 January 2016 & $\begin{array}{l}\text { Study centres, allocation concealment, } \\
\text { blinding, setting, adverse events, addi- } \\
\text { tional papers }\end{array}$ & 19 January 2016 & $\begin{array}{l}\text { Answered all questions and } \\
\text { said there were no addition- } \\
\text { al papers }\end{array}$ \\
\hline Davoli 2013 & 13 January 2016 & $\begin{array}{l}\text { Baseline differences, ethnic groups, } \\
\text { adverse events, additional papers }\end{array}$ & 18 January 2016 & $\begin{array}{l}\text { Gave all answers and pro- } \\
\text { vided an additional paper } \\
\text { with 24-month follow-up re- } \\
\text { sults }\end{array}$ \\
\hline Lochrie 2013 & $\begin{array}{l}13 \text { January } 2016 \\
\text { Asked for SEM/SDs } \\
-26 \text { January } 2016\end{array}$ & $\begin{array}{l}\text { Definition of obesity, funding, blinding, } \\
\text { allocation concealment, ethnic groups, } \\
\text { additional papers }\end{array}$ & $\begin{array}{l}20 \text { January } 2016 \\
\text { No response to } \\
\text { the second email }\end{array}$ & $\begin{array}{l}\text { Answered questions to first } \\
\text { email - no additional pa- } \\
\text { pers }\end{array}$ \\
\hline Mirza 2013 & $\begin{array}{l}12 \text { January } 2016 \\
\text { Reminder } 03 \text { Feb- } \\
\text { ruary } 2016\end{array}$ & $\begin{array}{l}\text { Allocation concealment, additional pa- } \\
\text { pers }\end{array}$ & No reply & $\mathrm{N} / \mathrm{A}$ \\
\hline O'Connor 2013 & 12 January 2016 & $\begin{array}{l}\text { Study centres, allocation concealment, } \\
\text { blinding, adverse events, ITT, baseline } \\
\text { data, more papers }\end{array}$ & 16 January 2016 & $\begin{array}{l}\text { Answered all questions and } \\
\text { gave an additional paper }\end{array}$ \\
\hline Saelens 2013 & 13 January 2016 & $\begin{array}{l}\text { Study centres, allocation concealment, } \\
\text { setting, adverse events, additional pa- } \\
\text { pers }\end{array}$ & 13 January 2016 & $\begin{array}{l}\text { Answered questions but still } \\
\text { unclear if there were ad- } \\
\text { verse events. No additional } \\
\text { papers published }\end{array}$ \\
\hline Siwik 2013 & $\begin{array}{l}13 \text { January } 2016 \\
\text { Reminder } 03 \text { Feb- } \\
\text { ruary } 2016\end{array}$ & $\begin{array}{l}\text { Allocation, blinding, number ran- } \\
\text { domised, setting, adverse events, ITT } \\
\text { and additional papers }\end{array}$ & No reply & $\mathrm{N} / \mathrm{A}$ \\
\hline
\end{tabular}

Diet, physical activity and behavioural interventions for the treatment of overweight or obese children from the age of 6 to 11 years 
(Continued)

\begin{tabular}{|c|c|c|c|c|}
\hline Vann 2013 & $\begin{array}{l}14 \text { January } 2016 \\
\text { Asked for SDs - } 26 \\
\text { January } 2016\end{array}$ & $\begin{array}{l}\text { Adverse events, allocation conceal- } \\
\text { ment, randomisation method, blind- } \\
\text { ing, definition of obesity, ITT and addi- } \\
\text { tional papers }\end{array}$ & 20 January 2016 & $\begin{array}{l}\text { Answered questions- no ad- } \\
\text { ditional papers. Unable to } \\
\text { provide SD values }\end{array}$ \\
\hline Wake 2013 & $\begin{array}{l}13 \text { January } 2016 \\
\text { Reminder } 03 \text { Feb- } \\
\text { ruary } 2016\end{array}$ & $\begin{array}{l}\text { Ethnic groups, adverse events, addi- } \\
\text { tional papers }\end{array}$ & 01 March 2016 & $\begin{array}{l}\text { Provided answers and an } \\
\text { additional paper provided }\end{array}$ \\
\hline Croker 2012 & 13 January 2016 & $\begin{array}{l}\text { Allocation concealment, additional pa- } \\
\text { pers }\end{array}$ & 13 January 2016 & $\begin{array}{l}\text { Allocation was concealed } \\
\text { and no additional papers } \\
\text { available }\end{array}$ \\
\hline de Niet 2012 & $\begin{array}{l}12 \text { January } 2016 \\
\text { Reminder } 03 \text { Feb- } \\
\text { ruary } 2016\end{array}$ & $\begin{array}{l}\text { Allocation concealment, blinding, ad- } \\
\text { verse events, ITT, imputation method, } \\
\text { BMI SDS data error, additional papers }\end{array}$ & 18 February 2016 & Answered all questions \\
\hline Eddy Ives 2012 & 20 April 2016 & $\begin{array}{l}\text { Adverse events, additional papers, al- } \\
\text { location concealment, blinding, details } \\
\text { of intervention }\end{array}$ & 20 April 2016 & $\begin{array}{l}\text { Answered all questions and } \\
\text { confirmed no additional } \\
\text { publications were available }\end{array}$ \\
\hline Kirk 2012 & $\begin{array}{l}12 \text { January } 2016 \\
\text { Reminder } 03 \text { Feb- } \\
\text { ruary } 2016\end{array}$ & $\begin{array}{l}\text { Baseline differences, setting, adverse } \\
\text { events, missing data method, raw BMI } \\
\text { data, additional papers }\end{array}$ & No reply & $\mathrm{N} / \mathrm{A}$ \\
\hline Lison 2012 & 12 January 2016 & $\begin{array}{l}\text { Ethnic groups, adverse events, SDs, ad- } \\
\text { ditional papers }\end{array}$ & 12 January 2016 & $\begin{array}{l}\text { Author answered questions } \\
\text { and confirmed no addition- } \\
\text { al papers were published }\end{array}$ \\
\hline Waling 2012 & 13 January 2016 & $\begin{array}{l}\text { Allocation concealment, ethnic } \\
\text { groups, adverse events, additional pa- } \\
\text { pers }\end{array}$ & 22 January 2016 & $\begin{array}{l}\text { Answered questions - also } \\
\text { provided links to } 2 \text { papers }\end{array}$ \\
\hline Wright 2012 & $\begin{array}{l}\text { Email provided } \\
\text { in the publica- } \\
\text { tion did not work. } \\
\text { Emailed co-au- } \\
\text { thors, only one ad- } \\
\text { dress worked (13 } \\
\text { January } 2016 \text { ) } \\
\text { Reminder } 03 \text { Feb- } \\
\text { ruary } 2016\end{array}$ & $\begin{array}{l}\text { Study centres, randomisation method, } \\
\text { allocation concealment, blinding, ad- } \\
\text { verse events, additional papers }\end{array}$ & No reply & $\mathrm{N} / \mathrm{A}$ \\
\hline Barkin 2011 & $\begin{array}{l}11 \text { December } 2015 \\
\text { Reminder - } 03 \\
\text { February } 2016\end{array}$ & $\begin{array}{l}\text { Randomisation, allocation conceal- } \\
\text { ment, blinding, ethnic group, adverse } \\
\text { events, BMI data, additional papers }\end{array}$ & No reply & $\mathrm{N} / \mathrm{A}$ \\
\hline Bryant 2011 & $\begin{array}{l}\text { Yes (12 January } \\
2016)\end{array}$ & $\begin{array}{l}\text { Allocation concealment, adverse } \\
\text { events, ITT, additional papers }\end{array}$ & $\begin{array}{l}\text { Yes (12 January } \\
2016)\end{array}$ & $\begin{array}{l}\text { Answered all the questions } \\
\text { above and provided an ad- } \\
\text { ditional paper }\end{array}$ \\
\hline Coppins 2011 & $\begin{array}{l}16 \text { December } 2015 \\
\text { Reminder } 03 \text { Feb- } \\
\text { ruary } 2016\end{array}$ & $\begin{array}{l}\text { Randomisation, allocation conceal- } \\
\text { ment, ethnic groups, adverse events, } \\
\text { data in table 3, additional papers }\end{array}$ & 15 March 2016 & $\begin{array}{l}\text { Answered all questions - no } \\
\text { additional papers }\end{array}$ \\
\hline
\end{tabular}

Diet, physical activity and behavioural interventions for the treatment of overweight or obese children from the age of 6 to 11 years 
(Continued)

$\begin{array}{llll}\text { Gunnarsdottir } & \text { No-email ad- } & \text { N/A } & \text { N/A } \\ \text { 2011a } & \text { dress in publica- } & & \text { N/A } \\ & \text { tion did not work } & \end{array}$

\begin{tabular}{|c|c|c|c|c|}
\hline Maddison 2011 & $\begin{array}{l}12 \text { January } 2016 \\
\text { Reminder } 03 \text { Feb- } \\
\text { ruary } 2016\end{array}$ & $\begin{array}{l}\text { Sample size at follow-up, sample size } \\
\text { for mean change in BMI, additional pa- } \\
\text { pers }\end{array}$ & 17 February 2016 & $\begin{array}{l}\text { Provided sample size data } \\
\text { No additional papers }\end{array}$ \\
\hline Wafa 2011 & 13 January 2016 & $\begin{array}{l}\text { Blinding, adverse events, ethnic } \\
\text { groups, additional papers }\end{array}$ & 2 January 2016 & $\begin{array}{l}\text { Answered questions and } \\
\text { gave additional papers }\end{array}$ \\
\hline
\end{tabular}

\begin{tabular}{|c|c|c|c|c|}
\hline Bathrellou 2010 & $\begin{array}{l}14 \text { December } 2015 \\
\text { Reminder - } 03 \\
\text { February } 2016\end{array}$ & $\begin{array}{l}\text { Randomisation, allocation conceal- } \\
\text { ment, blinding, BMI data, adverse } \\
\text { events, ethnic groups, setting, ITT, ad- } \\
\text { ditional papers }\end{array}$ & No reply & $\mathrm{N} / \mathrm{A}$ \\
\hline Diaz 2010 & 12 January 2016 & $\begin{array}{l}\text { Funding source, allocation conceal- } \\
\text { ment, blinding, ethnic groups, adverse } \\
\text { events, additional papers }\end{array}$ & 21 January 2016 & $\begin{array}{l}\text { Answered all questions and } \\
\text { gave additional paper }\end{array}$ \\
\hline
\end{tabular}

\begin{tabular}{|c|c|c|c|c|}
\hline Duggins 2010 & $\begin{array}{l}12 \text { January } 2016 \\
\text { Reminder } 03 \text { Feb- } \\
\text { ruary } 16\end{array}$ & $\begin{array}{l}\text { Ethnic groups, adverse events, ITT, } \\
\text { number of participants who complet- } \\
\text { ed the study, SDs for raw BMI change, } \\
\text { additional papers }\end{array}$ & 21 March 2016 & $\begin{array}{l}\text { Was unable to provide BMI } \\
\text { data, unclear if adverse } \\
\text { events occurred. No ITT } \\
\text { analysis, did not record eth- } \\
\text { nic groups, no additional } \\
\text { papers }\end{array}$ \\
\hline
\end{tabular}

\begin{tabular}{lll}
\hline Faude 2010 & No-email ad- N/A & N/A \\
& dress provided in & \\
the publication & \\
did not work & \\
\hline
\end{tabular}

\begin{tabular}{|c|c|c|c|c|}
\hline Reinehr 2010 & 12 January 2016 & $\begin{array}{l}\text { Allocation concealed, setting, ethnic } \\
\text { groups, how were adverse events mea- } \\
\text { sured, additional papers }\end{array}$ & 12 January 2016 & $\begin{array}{l}\text { Answered questions and } \\
\text { said they were performing } \\
5-7 \text { year follow-up so paper } \\
\text { likely at the end of } 2016\end{array}$ \\
\hline Sacher 2010 & 12 January 2016 & $\begin{array}{l}\text { Allocation, blinding, adverse events, } \\
\text { ITT, additional papers }\end{array}$ & 12 January 2016 & $\begin{array}{l}\text { Answered all questions } \\
\text { above and gave references } \\
\text { to additional papers }\end{array}$ \\
\hline Kalarchian 2009 & 12 January 2016 & $\begin{array}{l}\text { Allocation concealment, blinding, ad- } \\
\text { verse events, additional papers }\end{array}$ & 13 January 2016 & $\begin{array}{l}\text { Answered all questions } \\
\text { above and said there we no } \\
\text { additional papers }\end{array}$ \\
\hline Nowicka 2009 & 12 January 2015 & $\begin{array}{l}\text { Funding, randomisation, allocation, } \\
\text { blinding, ethnic groups, baseline data, } \\
\text { adverse events, ITT, additional papers }\end{array}$ & 28 January 2015 & $\begin{array}{l}\text { Answered questions but did } \\
\text { not provide any additional } \\
\text { baseline data. No additional } \\
\text { published papers }\end{array}$ \\
\hline Wake 2009 & $\begin{array}{l}13 \text { January } 2016 \\
\text { Reminder } 03 \text { Feb- } \\
\text { ruary } 2016\end{array}$ & $\begin{array}{l}\text { Missing data, ITT, additional papers, } \\
\text { ethnic groups, type of control }\end{array}$ & 01 March 2016 & $\begin{array}{l}\text { Answered questions - no } \\
\text { relevant additional papers } \\
\text { identified }\end{array}$ \\
\hline Alves 2008 & 11 December 2015 & $\begin{array}{l}\text { Allocation concealment, blinding, } \\
\text { dropout rates, imputation method, ad- }\end{array}$ & $\begin{array}{l}11 \text { December } \\
2015\end{array}$ & $\begin{array}{l}\text { Answered all questions and } \\
\text { no additional papers pub- } \\
\text { lished relating to this study }\end{array}$ \\
\hline
\end{tabular}

Diet, physical activity and behavioural interventions for the treatment of overweight or obese children from the age of 6 to 11 years 
(Continued)

verse events, funding source, addition-

al papers

\begin{tabular}{|c|c|c|c|c|}
\hline Hughes 2008 & $12 / 01 / 2016$ & $\begin{array}{l}\text { Ethnic groups, adverse events, median } \\
\text { (IQR) reasons, additional papers }\end{array}$ & $12 / 01 / 2016$ & $\begin{array}{l}\text { Answered the questions } \\
\text { and give links to additional } \\
\text { publications }\end{array}$ \\
\hline
\end{tabular}

\begin{tabular}{|c|c|c|c|}
\hline Weigel 2008 & $\begin{array}{l}\text { Email address pro- } \\
\text { vided in the pub- } \\
\text { lication did not } \\
\text { work }\end{array}$ & $\mathrm{N} / \mathrm{A}$ & $\mathrm{N} / \mathrm{A}$ \\
\hline
\end{tabular}

\begin{tabular}{|c|c|c|c|c|}
\hline Weintraub 2008 & $\begin{array}{l}13 \text { January } 2016 \\
\text { Remainder } 03 \\
\text { February } 2016\end{array}$ & $\begin{array}{l}\text { Blinding, \% girls in each group, num- } \\
\text { ber of participants who suffered at } \\
\text { least } 1 \text { adverse event, additional pa- } \\
\text { pers }\end{array}$ & No reply & $\mathrm{N} / \mathrm{A}$ \\
\hline Berry 2007 & 12 January 2016 & $\begin{array}{l}\text { Blinding, adverse events, ITT, number } \\
\text { randomised and followed up, addition- } \\
\text { al papers }\end{array}$ & 14 January 2016 & $\begin{array}{l}\text { Answered questions above } \\
\text { but still unclear about num- } \\
\text { ber of dropouts. Did not } \\
\text { provide any additional pa- } \\
\text { pers but said the study was } \\
\text { the basis of the family part- } \\
\text { ners for health R01 study }\end{array}$ \\
\hline
\end{tabular}

Gillis 2007

12 January 2016

Funding, randomisation, allocation concealment, blinding, ethnic groups,

$\%$ girls, adverse events, ITT, additional papers

Kalavainen 200712 January $2016 \quad \begin{aligned} & \text { Adverse events, missing data method, } 12 \text { January } 2016 \\ & \text { additional papers }\end{aligned}$

\section{January 2016 Author answered ques-} tions- no additional papers reported

\begin{tabular}{|c|c|c|c|c|}
\hline McCallum 2007 & $\begin{array}{l}12 \text { January } 2016 \\
\text { Reminder } 03 \text { Feb- } \\
\text { ruary } 2016\end{array}$ & $\begin{array}{l}\text { Ethnic groups, adverse events, addi- } \\
\text { tional papers }\end{array}$ & No reply & $\mathrm{N} / \mathrm{A}$ \\
\hline Rodearmel 2007 & $\begin{array}{l}12 \text { January } 2016 \\
\text { Reminder } 03 \text { Feb- } \\
\text { ruary } 2016\end{array}$ & $\begin{array}{l}\text { Randomisation, allocation, blinding, } \\
\text { setting, adverse events, ITT, additional } \\
\text { papers }\end{array}$ & 08February 2016 & $\begin{array}{l}\text { Answered all questions but } \\
\text { wasn't sure if any adverse } \\
\text { events }\end{array}$ \\
\hline Satoh 2007 & $\begin{array}{l}\text { No - email ad- } \\
\text { dress provided did } \\
\text { not work }\end{array}$ & $\mathrm{N} / \mathrm{A}$ & $\mathrm{N} / \mathrm{A}$ & $\mathrm{N} / \mathrm{A}$ \\
\hline Wilfley 2007 & $\begin{array}{l}13 \text { January } 2016 \\
\text { Reminder } 03 \text { Feb- } \\
\text { ruary2016 }\end{array}$ & $\begin{array}{l}\text { Allocation concealment, blinding, how } \\
\text { adverse events were measured, addi- } \\
\text { tional papers }\end{array}$ & No reply & $\mathrm{N} / \mathrm{A}$ \\
\hline Epstein 2005 & $\begin{array}{l}14 \text { January } 2016 \\
\text { Reminder } 03 \text { Feb- } \\
\text { ruary } 2016\end{array}$ & $\begin{array}{l}\text { Randomisation method, allocation } \\
\text { concealment, blinding, number ran- } \\
\text { domised to each group, ethnic groups, } \\
\text { additional papers, adverse events }\end{array}$ & 03 February 2016 & $\begin{array}{l}\text { Answered general ques- } \\
\text { tions about all of their stud- } \\
\text { ies included in this review } \\
\text { but not necessarily specif- } \\
\text { ic questions related to this } \\
\text { study }\end{array}$ \\
\hline
\end{tabular}

Diet, physical activity and behavioural interventions for the treatment of overweight or obese children from the age of 6 to 11 years 
(Continued)

\begin{tabular}{|c|c|c|c|c|}
\hline Nemet 2005 & $\begin{array}{l}12 \text { January } 2016 \\
\text { Reminder } 03 \text { Feb- } \\
\text { ruary } 2016\end{array}$ & $\begin{array}{l}\text { Allocation concealed, blinding, ethnic } \\
\text { groups, ITT, follow-up time point, more } \\
\text { papers }\end{array}$ & No reply & N/A \\
\hline Woo 2004 & 13 January 2016 & $\begin{array}{l}\text { Randomisation, allocation, blind- } \\
\text { ing, baseline differences, number of } \\
\text { dropouts, setting, ethnic groups, ITT, } \\
\text { adverse events, additional papers }\end{array}$ & 14 January 2016 & $\begin{array}{l}\text { Answered all questions but } \\
\text { still unclear about dropout } \\
\text { at end of study. Additional } \\
\text { paper given }\end{array}$ \\
\hline Epstein 2001 & $\begin{array}{l}14 \text { January } 2016 \\
\text { Reminder } 03 \text { Feb- } \\
\text { ruary } 2016\end{array}$ & $\begin{array}{l}\text { Study centres, randomisation, allo- } \\
\text { cation concealment, baseline differ- } \\
\text { ences, blinding, number of partici- } \\
\text { pants randomised and completing the } \\
\text { study, setting, adverse events, ITT, raw } \\
\text { data BMI, additional papers }\end{array}$ & 03 February 2016 & $\begin{array}{l}\text { Answered general ques- } \\
\text { tions about all of their stud- } \\
\text { ies included in this review } \\
\text { but not necessarily specif- } \\
\text { ic questions related to this } \\
\text { study }\end{array}$ \\
\hline Nova 2001 & $\begin{array}{l}\text { No - email ad- } \\
\text { dress provided in } \\
\text { the publication } \\
\text { did not work }\end{array}$ & $\mathrm{N} / \mathrm{A}$ & $\mathrm{N} / \mathrm{A}$ & $\mathrm{N} / \mathrm{A}$ \\
\hline
\end{tabular}

\begin{tabular}{lll}
\hline Epstein 2000a & 14 January 2016 & $\begin{array}{l}\text { Allocation concealment, randomisa- } \\
\text { tion method, blinding, adverse events, }\end{array}$ \\
$\begin{array}{lll}\text { Reminder 03 Feb- } \\
\text { ruary 2016 Februray 2016 } \\
\text { number randomised in each group, ITT } \\
\text { and additional papers }\end{array}$
\end{tabular}

\begin{tabular}{lllll}
\hline $\begin{array}{l}\text { Schwingshandl } \\
1999\end{array}$ & $\begin{array}{l}\text { No-email ad- } \\
\text { dress provided did } \\
\text { not work }\end{array}$ & N/A & N/A \\
\hline
\end{tabular}

\begin{tabular}{lllll}
\hline Duffy 1993 & $\begin{array}{l}\text { No - unable to } \\
\text { find an email ad- } \\
\text { dress }\end{array}$ & N/A & N/A & N/A \\
\hline Flodmark 1993 & 12 January 2016 & $\begin{array}{l}\text { Study centres, allocation concealed, } \\
\text { blinding, setting, ethnic groups, ad- } \\
\text { verse events, contact during follow-up } \\
\text { period, additional papers }\end{array}$ & No reply & N/A \\
ruary 2016 & Peb- & & \\
\hline
\end{tabular}

\begin{tabular}{|c|c|c|c|c|}
\hline Epstein 1985c & $\begin{array}{l}14 \text { January } 2016 \\
\text { Reminder } 03 \text { Feb- } \\
\text { ruary } 2016\end{array}$ & $\begin{array}{l}\text { Randomisation method, allocation } \\
\text { concealment, blinding, number of par- } \\
\text { ticipants randomised in each group } \\
\text { and number which completed, setting, } \\
\text { ethnic group, mean age at baseline, } \\
\text { adverse events, ITT, additional papers }\end{array}$ & 3 February 2016 & $\begin{array}{l}\text { Answered general ques- } \\
\text { tions about all of their stud- } \\
\text { ies included in this review } \\
\text { but not necessarily specif- } \\
\text { ic questions related to this } \\
\text { study }\end{array}$ \\
\hline
\end{tabular}

\begin{tabular}{|c|c|c|c|c|}
\hline Epstein 1985b & $\begin{array}{l}14 \text { January } 2016 \\
\text { Reminder } 03 \text { Feb- } \\
\text { ruary } 2016\end{array}$ & $\begin{array}{l}\text { Allocation concealed, blinding, ethnic } \\
\text { groups, setting, number randomised } \\
\text { in each group and number completed, } \\
\text { mean age at baseline, adverse events, } \\
\text { additional papers }\end{array}$ & 03 February 2016 & $\begin{array}{l}\text { Answered general ques- } \\
\text { tions about all of their stud- } \\
\text { ies included in this review } \\
\text { but not necessarily specif- } \\
\text { ic questions related to this } \\
\text { study }\end{array}$ \\
\hline
\end{tabular}

\begin{tabular}{|c|c|c|c|c|}
\hline Epstein 1985a & $\begin{array}{l}14 \text { January } 2016 \\
\text { Reminder } 03 \text { Feb- } \\
\text { ruary } 2016\end{array}$ & $\begin{array}{l}\text { Study centres, blinding, randomisa- } \\
\text { tion method, allocation concealment, } \\
\text { baseline differences, number ran- } \\
\text { domised, mean age, setting, adverse }\end{array}$ & 03 February 2016 & $\begin{array}{l}\text { Answered general ques- } \\
\text { tions about all of their stud- } \\
\text { ies included in this review } \\
\text { but not necessarily specif- }\end{array}$ \\
\hline
\end{tabular}

Diet, physical activity and behavioural interventions for the treatment of overweight or obese children from the age of 6 to 11 years 
(Continued)

events, ethnic groups, additional papers ic questions related to this study

\begin{tabular}{|c|c|c|c|c|}
\hline Epstein 1984a & $\begin{array}{l}14 \text { January } 2016 \\
\text { Reminder } 03 \text { Feb- } \\
\text { ruary } 2016\end{array}$ & $\begin{array}{l}\text { Study centres, blinding, randomisa- } \\
\text { tion method, allocation concealment, } \\
\text { baseline differences, \% girls, setting, } \\
\text { adverse events, ITT, ethnic groups, ad- } \\
\text { ditional papers }\end{array}$ & 03 February 2016 & $\begin{array}{l}\text { Answered general ques- } \\
\text { tions about all of their stud- } \\
\text { ies included in this review } \\
\text { but not necessarily specif- } \\
\text { ic questions related to this } \\
\text { study }\end{array}$ \\
\hline
\end{tabular}

BMI: body mass index; IQR: interquartile range; ITT: intention to treat; N/A: not applicable; SD: standard deviation; SDS: standardised 


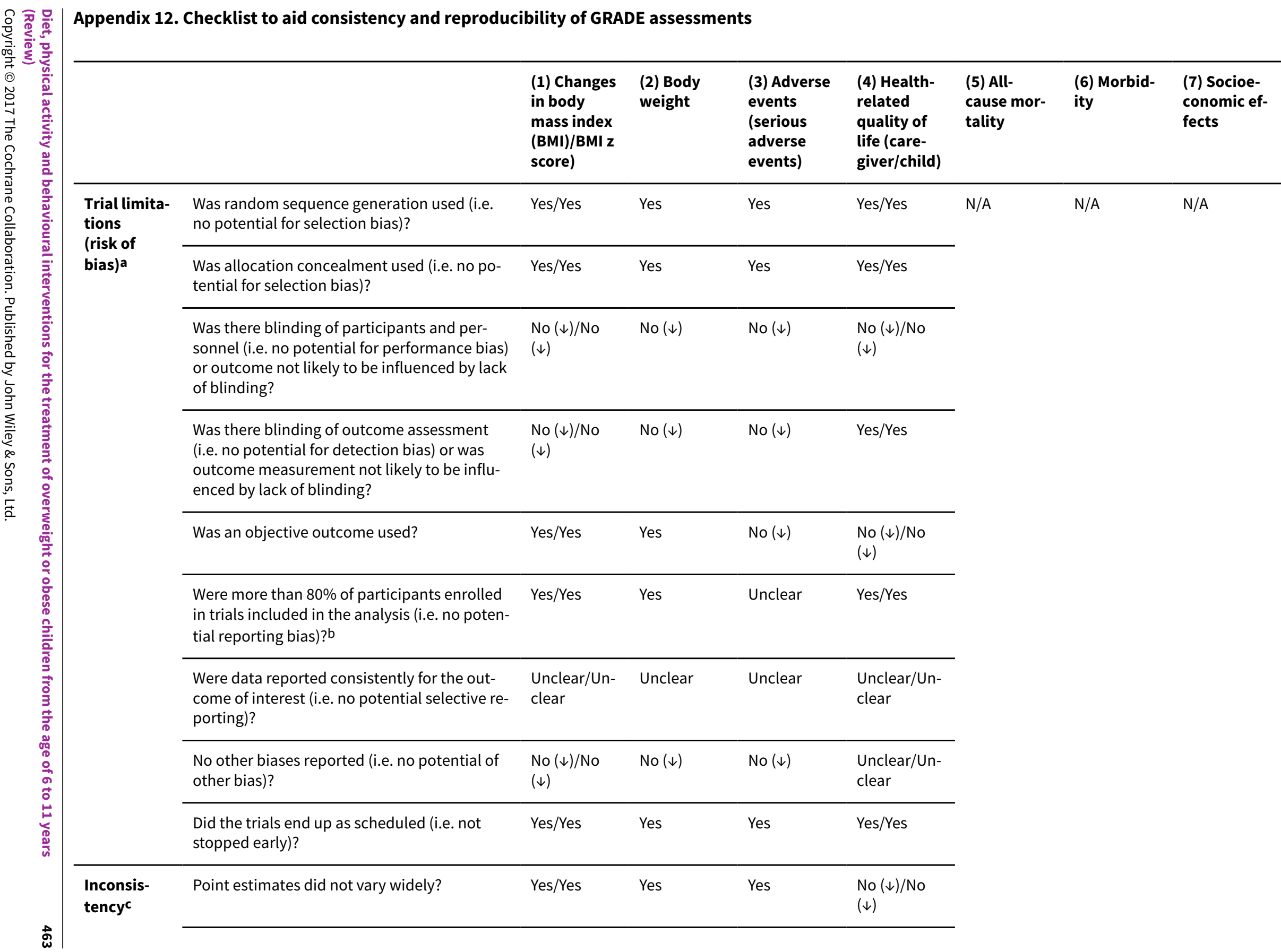




\begin{tabular}{|c|c|c|c|c|c|}
\hline 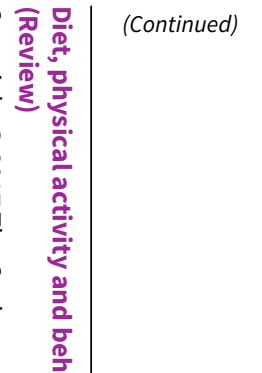 & $\begin{array}{l}\text { To what extent did confidence intervals over- } \\
\text { lap (substantial: all confidence intervals over- } \\
\text { lap at least one of the included studies point } \\
\text { estimate; some: confidence intervals over- } \\
\text { lap but not all overlap at least one point esti- } \\
\text { mate; no: at least one outlier: where the con- } \\
\text { fidence interval of some of the studies do not } \\
\text { overlap with those of most included studies)? }\end{array}$ & $\begin{array}{l}\text { Some/Sub- } \\
\text { stantial }\end{array}$ & Substantial & Substantial & $\begin{array}{l}\text { Substan- } \\
\text { tial/Some }\end{array}$ \\
\hline$\stackrel{\substack{0 \\
\vdots}}{\varrho}$ & Was the direction of effect consistent? & $\begin{array}{l}\text { No }(\downarrow) / \mathrm{No} \\
(\downarrow)\end{array}$ & No $(\downarrow)$ & Yes & $\begin{array}{l}\text { No }(\downarrow) / \mathrm{No} \\
(\downarrow)\end{array}$ \\
\hline 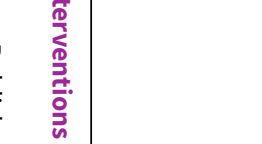 & $\begin{array}{l}\text { What was the magnitude of statistical hetero- } \\
\left.\text { geneity (as measured by } I^{2}\right)- \text { low }\left(I^{2}<40 \%\right) \text {, } \\
\left.\text { moderate }\left(I^{2} 40 \%-60 \%\right) \text {, high } I^{2}>60 \%\right) ?\end{array}$ & $\begin{array}{l}\text { High }(\downarrow) / \\
\text { Moderate }\end{array}$ & Low & Low & $\begin{array}{l}\text { Low/High } \\
(\downarrow)\end{array}$ \\
\hline 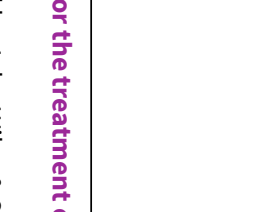 & $\begin{array}{l}\text { Was the test for heterogeneity statistically } \\
\text { significant }(P<0.1) \text { ? }\end{array}$ & $\begin{array}{l}\text { Statistically } \\
\text { significant } \\
(\downarrow) / \text { Statisti- } \\
\text { cally signifi- } \\
\text { cant }(\downarrow)\end{array}$ & $\begin{array}{l}\text { Not statisti- } \\
\text { cally signifi- } \\
\text { cant }\end{array}$ & $\begin{array}{l}\text { Not statisti- } \\
\text { cally signifi- } \\
\text { cant }\end{array}$ & $\begin{array}{l}\text { Not statisti- } \\
\text { cally signifi- } \\
\text { cant/Statis- } \\
\text { tically signif- } \\
\text { icant }(\downarrow)\end{array}$ \\
\hline $\begin{array}{l}\text { Indirect- } \\
\text { ness }\end{array}$ & $\begin{array}{l}\text { Were the populations in included studies ap- } \\
\text { plicable to the decision context? }\end{array}$ & $\begin{array}{l}\text { Applica- } \\
\text { ble/Applica- } \\
\text { ble }\end{array}$ & Applicable & Applicable & $\begin{array}{l}\text { Applica- } \\
\text { ble/Applica- } \\
\text { ble }\end{array}$ \\
\hline 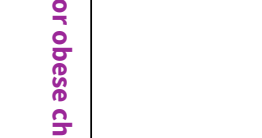 & $\begin{array}{l}\text { Were the interventions in the included studies } \\
\text { applicable to the decision context? }\end{array}$ & $\begin{array}{l}\text { Applica- } \\
\text { ble/Applica- } \\
\text { ble }\end{array}$ & Applicable & Applicable & $\begin{array}{l}\text { Applica- } \\
\text { ble/Applica- } \\
\text { ble }\end{array}$ \\
\hline $\begin{array}{l}\frac{0}{\overline{7}} \\
\stackrel{1}{3} \\
\overrightarrow{7} \\
0\end{array}$ & $\begin{array}{l}\text { Was the included outcome not a surrogate } \\
\text { outcome? }\end{array}$ & Yes/Yes & Yes & Yes & Yes/Yes \\
\hline 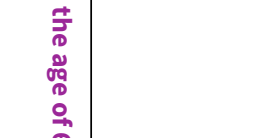 & Was the outcome timeframe sufficient? & $\begin{array}{l}\text { Suffi- } \\
\text { cient/Suffi- } \\
\text { cient }\end{array}$ & Sufficient & Sufficient & $\begin{array}{l}\text { Suffi- } \\
\text { cient } / \text { Suffi- } \\
\text { cient }\end{array}$ \\
\hline$\underset{\infty}{\stackrel{0}{5}}$ & $\begin{array}{l}\text { Were the conclusions based on direct com- } \\
\text { parisons? }\end{array}$ & Yes/Yes & Yes & Yes & Yes/Yes \\
\hline $\begin{array}{l}\text { Impreci- } \\
\text { siond }\end{array}$ & $\begin{array}{l}\text { Was the confidence interval for the pooled es- } \\
\text { timate not consistent with benefit and harm? }\end{array}$ & Yes/Yes & Yes & Yes & Yes/Yes \\
\hline
\end{tabular}


What is the magnitude of the median sample size (high: 300 participants, intermediate: 100-300 participants, low: < 100 partici-

$\operatorname{Low}(\downarrow) /$ Low $\quad$ Low $(\downarrow)$

$\quad \operatorname{Low}(\downarrow)$ $(\downarrow)$
Low $(\downarrow) /$ Low

$(\downarrow)$ pants)?b

What was the magnitude of the number of included studies (large: > 10 studies, moderate:

Large/Large Large

Large

Moder-

5-10 studies, small: $<5$ studies)?e

$(\downarrow)$

Was the outcome a common event (e.g. occurs more than $1 / 100)$ ?

Not applica- Not applica- No $(\downarrow) \quad$ Not applica-

ble/Not ap- ble ble/Notapplicable

plicable

Publication Was a comprehensive search conducted?

Yes/Yes

Yes

Yes/Yes

biase

\begin{tabular}{lllll}
\hline Was grey literature searched? & $\begin{array}{l}\text { No }(\downarrow) / \text { No } \\
(\downarrow)\end{array}$ & No $(\downarrow)$ & No $(\downarrow)$ & $\begin{array}{l}\text { No }(\downarrow) / \text { No } \\
(\downarrow)\end{array}$ \\
\hline $\begin{array}{l}\text { Were no restrictions applied to study selec- } \\
\text { tion on the basis of language? }\end{array}$ & Yes/Yes & Yes & Yes & Yes/Yes \\
\hline $\begin{array}{l}\text { There was no industry influence on studies in- } \\
\text { cluded in the review? }\end{array}$ & $\begin{array}{l}\text { No }(\downarrow) / \text { No } \\
(\downarrow)\end{array}$ & No $(\downarrow)$ & No $(\downarrow)$ & Yes/Yes \\
\hline $\begin{array}{l}\text { There was no evidence of funnel plot asym- } \\
\text { metry? }\end{array}$ & $\begin{array}{l}\text { No }(\downarrow) / \text { Un- } \\
\text { clear }\end{array}$ & Unclear & Unclear & $\begin{array}{l}\text { Unclear/Un- } \\
\text { clear }\end{array}$ \\
\hline $\begin{array}{l}\text { There was no discrepancy in findings be- } \\
\text { tween published and unpublished trials? }\end{array}$ & $\begin{array}{l}\text { Unclear/Un- } \\
\text { clear }\end{array}$ & Unclear & Unclear & $\begin{array}{l}\text { Unclear/Un- } \\
\text { clear }\end{array}$
\end{tabular}

aQuestions on risk of bias are answered in relation to the majority of the aggregated evidence in the meta-analysis rather than to individual trials.

bDepends on the context of the systematic review area.

'Questions on inconsistency are primarily based on visual assessment of forest plots and the statistical quantification of heterogeneity based on $\mathrm{I}^{2}$ (Higgins 2002 ).

dWhen judging the width of the confidence interval it is recommended to use a clinical decision threshold to assess whether the imprecision is clinically meaningful. eQuestions address comprehensiveness of the search strategy, industry influence, funnel plot asymmetry and discrepancies between published and unpublished trials.

$(\downarrow)$ : key item for potential downgrading the quality of the evidence (GRADE) as shown in the footnotes of the 'Summary of finding' table(s)

BMI: body mass index; BMI z score ("A BMI z score or standard deviation score indicates how many units (of the standard deviation) a child's BMI is above or below the average BMI value for their age group and sex. For instance, a $z$ score of 1.5 indicates that a child is 1.5 standard deviations above the average value, and a $z$ score of -1.5 indicates a child is 1.5 standard deviations below the average value" (NOO NHS 2011)) 


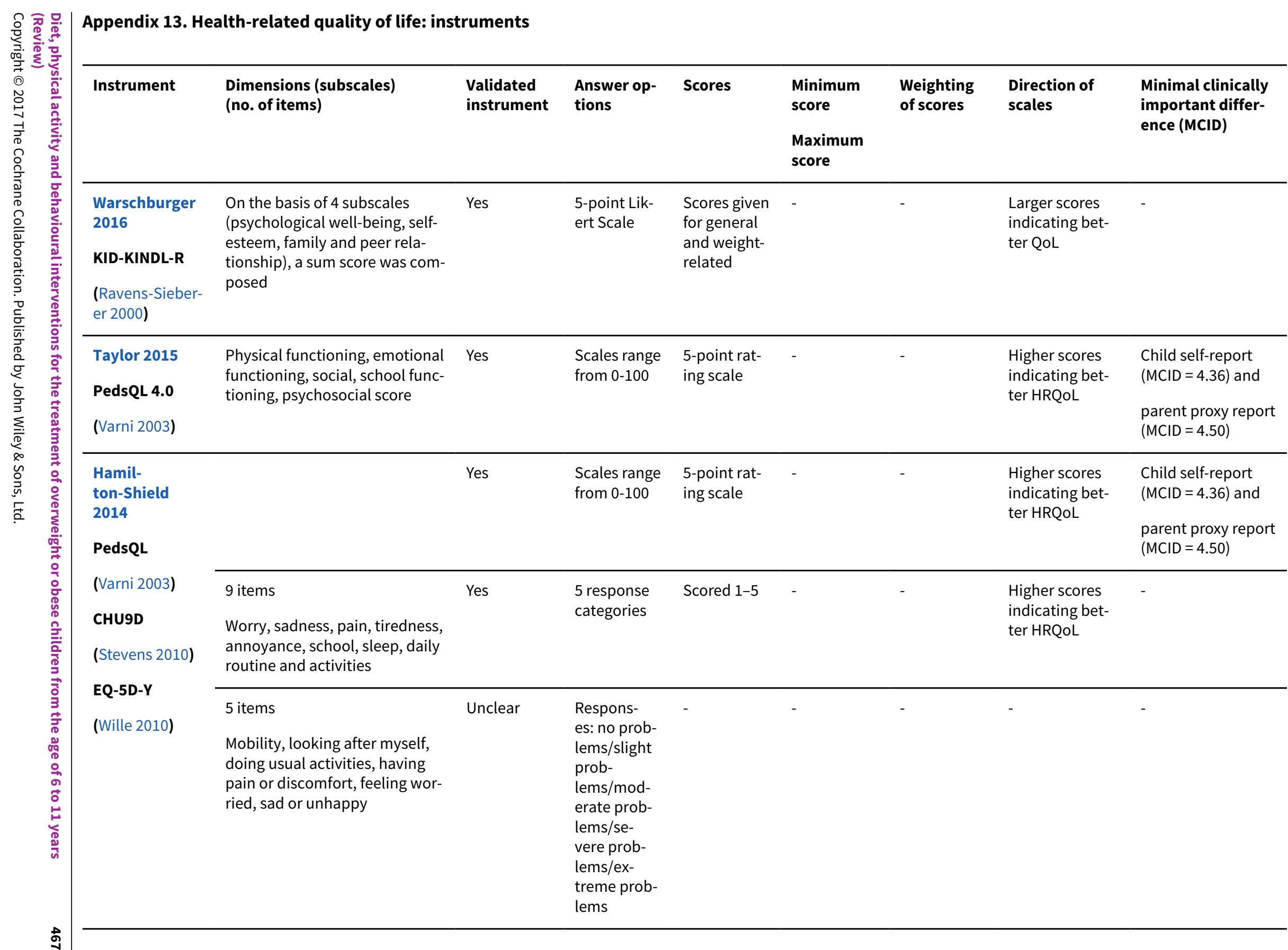




\begin{tabular}{|c|c|c|c|c|c|c|c|c|c|c|}
\hline 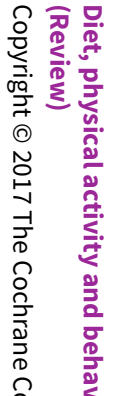 & $\begin{array}{l}\text { (Continued) } \\
\text { Markert } 2014 \\
\text { KINDL-R } \\
\text { (Ravens-Sieber- } \\
\text { er 2000) }\end{array}$ & Total score & Yes & $\begin{array}{l}\text { 5-point Lik- } \\
\text { ert Scale }\end{array}$ & $\begin{array}{l}\text { Scores given } \\
\text { for all sub- } \\
\text { scales } \\
\text { All scores } \\
\text { transformed } \\
\text { to values } \\
\text { between } \\
0-11\end{array}$ & - & - & $\begin{array}{l}\text { Larger scores } \\
\text { indicating bet- } \\
\text { ter QoL }\end{array}$ & - & 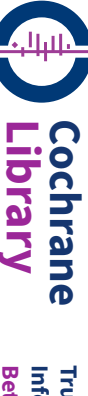 \\
\hline 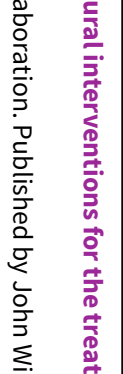 & $\begin{array}{l}\text { Arauz } \\
\text { Boudreau } 2013 \\
\text { PedsQL } \\
\text { (Varni 2003) }\end{array}$ & $\begin{array}{l}\text { 1) PedsQL child self-report } \\
\text { Physical, emotional, social, and } \\
\text { school-related aspects } \\
\text { 2) Caregiver proxy report Ped- } \\
\text { sQL generic core scales } \\
\text { Physical, emotional, social, and } \\
\text { school-related aspects }\end{array}$ & $\begin{array}{l}\text { 1) Yes } \\
\text { 2) Yes } \\
\text { (among } \\
\text { Spanish } \\
\text { and Eng- } \\
\text { lish speak- } \\
\text { ing Hispanic } \\
\text { groups) }\end{array}$ & $\begin{array}{l}\text { 1) Scale } \\
0-100 \\
\text { 2) Scale } \\
0-100\end{array}$ & $\begin{array}{l}\text { 5-point rat- } \\
\text { ing scale }\end{array}$ & - & No & $\begin{array}{l}\text { Higher values } \\
\text { means better } \\
\text { assessment }\end{array}$ & $\begin{array}{l}\text { Child self-report } \\
(M C I D=4.36) \text { and } \\
\text { parent proxy report } \\
(M C I D=4.50)\end{array}$ & 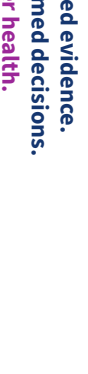 \\
\hline 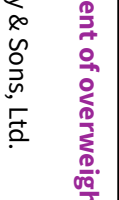 & $\begin{array}{l}\text { Lochrie } 2013 \\
\text { PedsQL } \\
\text { (Varni 2003) }\end{array}$ & Total score - parent and youth & Yes & $\begin{array}{l}\text { Scales range } \\
\text { from } 0-100\end{array}$ & $\begin{array}{l}\text { 5-point rat- } \\
\text { ing scale }\end{array}$ & - & - & $\begin{array}{l}\text { Higher scores } \\
\text { indicating bet- } \\
\text { ter HRQoL }\end{array}$ & $\begin{array}{l}\text { Child self-report } \\
(M C I D=4.36) \text { and } \\
\text { parent proxy report } \\
(M C I D=4.50)\end{array}$ & \\
\hline 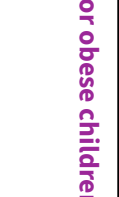 & $\begin{array}{l}\text { Wake } 2013 \\
\text { PedsQL 4.0 } \\
\text { (Varni 2003) }\end{array}$ & $\begin{array}{l}\text { Total scores: child and parent } \\
\text { reports }\end{array}$ & Yes & $\begin{array}{l}\text { Scales range } \\
\text { from } 0-100\end{array}$ & $\begin{array}{l}\text { 5-point rat- } \\
\text { ing scale }\end{array}$ & - & - & $\begin{array}{l}\text { Higher scores } \\
\text { indicating bet- } \\
\text { ter HRQoL }\end{array}$ & $\begin{array}{l}\text { Child self-report } \\
(\mathrm{MCID}=4.36) \text { and } \\
\text { parent proxy report } \\
(\mathrm{MCID}=4.50)\end{array}$ & \\
\hline 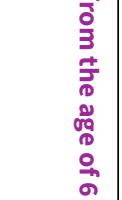 & $\begin{array}{l}\text { Croker } 2012 \\
\text { PedsQL } \\
\text { (Varni 2003) }\end{array}$ & $\begin{array}{l}\text { PedsQL total score (parent-re- } \\
\text { ported) } \\
\text { PedsQL total score (child-re- } \\
\text { ported) }\end{array}$ & Yes & Scale $0-100$ & $\begin{array}{l}\text { Scores giv- } \\
\text { en for total } \\
\text { score only }\end{array}$ & - & No & $\begin{array}{l}\text { Higher values } \\
\text { means better } \\
\text { assessment }\end{array}$ & $\begin{array}{l}\text { Child self-report } \\
(M C I D=4.36) \text { and } \\
\text { parent proxy report } \\
(M C I D=4.50)\end{array}$ & 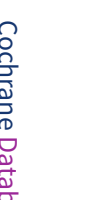 \\
\hline 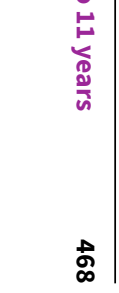 & $\begin{array}{l}\text { de Niet } 2012 \\
\text { Dutch validat- } \\
\text { ed CHQ-PF50 } \\
\text { (Raat 2002) }\end{array}$ & $\begin{array}{l}\text { CHQ physical } \\
\text { CHQ psychosocial }\end{array}$ & Yes & Scale $0-100$ & $\begin{array}{l}\text { Scores given } \\
\text { for } \\
\text { CHQ physi- } \\
\text { cal }\end{array}$ & - & No & $\begin{array}{l}\text { Higher values } \\
\text { reflect best pos- } \\
\text { sible health } \\
\text { state }\end{array}$ & - & 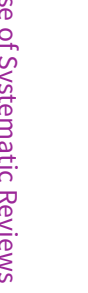 \\
\hline
\end{tabular}




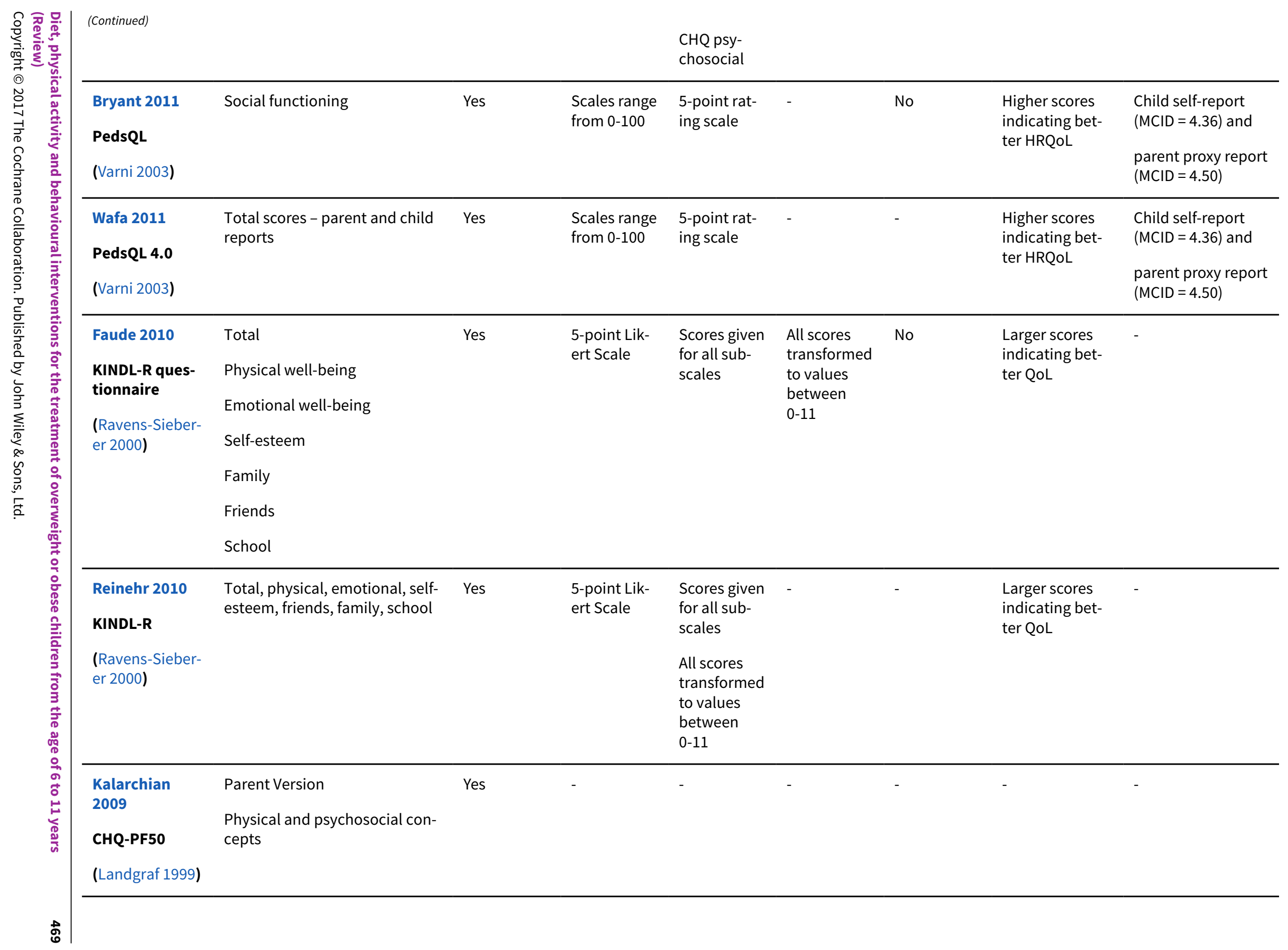




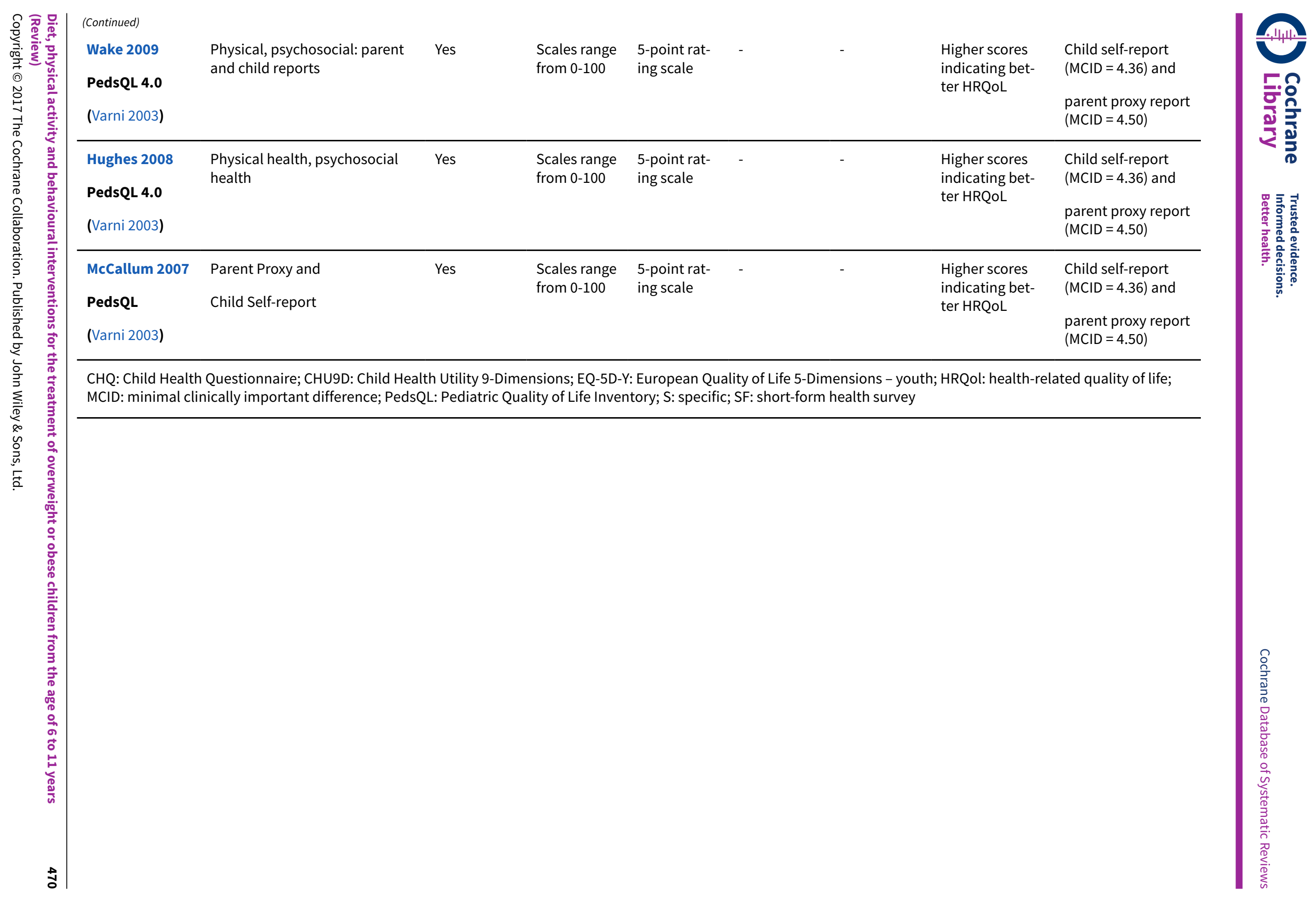


WHAT'S NEW

\begin{tabular}{|c|c|c|}
\hline Date & Event & Description \\
\hline 2 March 2017 & New search has been performed & $\begin{array}{l}\text { This is an update of the former Cochrane Review 'Interventions } \\
\text { for treating obesity in children and adolescents.' }\end{array}$ \\
\hline \multirow[t]{7}{*}{2 March 2017} & \multirow[t]{7}{*}{$\begin{array}{l}\text { New citation required and conclusions } \\
\text { have changed }\end{array}$} & $\begin{array}{l}\text { Given the rapid growth in the treatment of child and adoles- } \\
\text { cent obesity, we have split the original review ('Interventions for } \\
\text { treating obesity in children and adolescents') into six separate } \\
\text { reviews, with a specific intervention and age focus }\end{array}$ \\
\hline & & $\begin{array}{l}\text { - Diet, physical activity, and behavioural interventions for the } \\
\text { treatment of overweight or obesity in adolescents aged } 12 \text { to } \\
17 \text { years }\end{array}$ \\
\hline & & $\begin{array}{l}\text { Diet, physical activity, and behavioural interventions for the } \\
\text { treatment of overweight or obese children from the age of } 6 \text { to } \\
11 \text { years }\end{array}$ \\
\hline & & $\begin{array}{l}\text { Diet, physical activity, and behavioural interventions for the } \\
\text { treatment of overweight or obesity in preschool children up to } \\
\text { the age of } 6 \text { years }\end{array}$ \\
\hline & & $\begin{array}{l}\text { - Drug interventions for the treatment of obesity in children and } \\
\text { adolescents }\end{array}$ \\
\hline & & - Parent-only interventions for childhood overweight or obesity \\
\hline & & $\begin{array}{l}\text { - Surgery for the treatment of obesity in children and adoles- } \\
\text { cents }\end{array}$ \\
\hline
\end{tabular}

\section{H I S T O R Y}

Review first published: Issue 6, 2017

\begin{tabular}{lll}
\hline Date Event Desiption & Descion
\end{tabular}

11 October $2008 \quad$ New citation required and conclusions have changed
This review concludes that combined behavioural lifestyle interventions compared to standard care or self-help can produce a significant and clinically meaningful reduction in overweight in children and adolescents.

The search was updated to May 2008. Some amendments were made to update the search strategies. No changes have been made to other aspects of the methodology. Forty-six new studies have been included. These included information on drug interventions for treating obesity in adolescents. The added evidence suggests that lifestyle interventions appear to have positive effects in the treatment of child and adolescent obesity. Furthermore, orlistat and sibutramine were found to have beneficial effects on adiposity in obese adolescents. However, a range of adverse effects was noted.

Converted to new review format. Authorship changed with new authors and new contact person. 


\section{CONTRIBUTIONS OF AUTHORS}

All review authors read and approved the final review draft.

Emma Mead (EM): acquiring trial reports, trial selection, data extraction, data analysis, data interpretation, review draft and future review updates.

Tamara Brown (TB): acquiring trial reports, trial selection, data extraction, data analysis, data interpretation, review draft and future review updates.

Karen Rees (KR): acquiring trial reports, trial selection, data extraction, data analysis, data interpretation, review draft and future review updates.

Liane Azevedo (LA): acquiring trial reports, trial selection, data extraction, data analysis, data interpretation, review draft and future review updates.

Victoria Whittaker (VW): data analysis, data interpretation, review draft.

Dan Jones (DJ): acquiring trial reports, data extraction, data interpretation, review draft.

Joan Olajide (JO): acquiring trial reports, data extraction, data interpretation, review draft.

Giulia M Mainardi (GM): data extraction, data interpretation, review draft.

Eva Corpeleijn (EC): trial selection, data extraction, data interpretation, review draft and future review updates.

Claire O'Malley (CM): acquiring trial reports, trial selection, data extraction, data interpretation, review draft.

Elizabeth Beardsmore (EB): data extraction, data interpretation, review draft.

Lena Al-Khudairy (LA-K): acquiring trial reports, trial selection, data extraction, data interpretation, review draft and future review updates.

Louise Baur (LB): protocol draft, data interpretation, review draft and future review updates.

Maria-Inti Metzendorf (MIM): search strategy development.

Alessandro Demaio (AD): data interpretation, review draft.

Louisa J Ells (LE): protocol draft, acquiring trial reports, trial selection, data extraction, data analysis, data interpretation, review draft and update draft.

\section{DECLARATIONS OF INTEREST}

EM: none known.

TB: none known.

KR: none known

LA: none known.

VW: none known.

DJ: none known.

JO: none known.

GM: none known

EC: none known.

CM: none known.

EB: none known.

LA-K: none known.

LB: is a co-author on two of the included studies (McCallum 2007; Wake 2009).

Diet, physical activity and behavioural interventions for the treatment of overweight or obese children from the age of 6 to 11 years 
MIM: none known.

Disclaimer: Alessandro Demaio is currently a staff member of the World Health Organization. The author alone is responsible for the views expressed in this publication and they do not necessarily represent the decisions, policy or views of the World Health Organization.

LE: is seconded to Public Health England part-time as a specialist obesity advisor. The author received funding from WHO to complete this review. Louisa Ells also has a part time secondment to Public Health England, but undertook this review within her role at Teesside University.

\section{SOURCES OF SUPPORT}

\section{Internal sources}

- University Medical Center, Groningen, Netherlands.

- The Children's Hospital at Westmead, Sydney, Australia.

- Centre for Food Physical Activity and Obesity Research, University of Teesside, UK.

- The Wolfson Research Institute, University of Durham, UK.

- Evidence and Programme Guidance Unit, Department of Nutrition for Health and Development, World Health Organization, Switzerland.

Alessandro Demaio is a full time staff of the World Health Organization.

\section{External sources}

- Evidence and Programme Guidance Unit, Department of Nutrition for Health and Development, World Health Organization, Switzerland.

Dr Louisa Ells worked as a consultant for WHO during the preparation of this work.

- The Bill \& Melinda Gates Foundation, USA.

The World Health Organization gratefully acknowledges the financial contribution of The Bill \&Melinda Gates Foundation towards the development of systematic reviews of the evidence on the effects of nutrition interventions.

Donors do not fund specific guidelines and do not participate in any decision related to the guideline development process including the composition of policy questions, membership of the guideline groups, the conduct and interpretation of systematic reviews, or the formulation of recommendations.

\section{DIFFERENCES BETWEEN PROTOCOLANDREVIEW}

Given the rapid growth in the treatment of child and adolescent obesity, we have split the original review ('Interventions for treating obesity in children and adolescents') into six separate reviews, with a specific intervention and age focus:

- Diet, physical activity, and behavioural interventions for the treatment of overweight or obesity in adolescents aged 12 to 17 years.

- Diet, physical activity, and behavioural interventions for the treatment of overweight or obesity in children from the age of 6 to 11 years.

- Diet, physical activity, and behavioural interventions for the treatment of overweight or obesity in preschool children up to the age of 6 years.

- Drug interventions for the treatment of obesity in children and adolescents.

- Parent-only interventions for childhood overweight or obesity.

- Surgery for the treatment of obesity in children and adolescents.

\section{NOTES}

Portions of the background and methods sections, the appendices, additional tables and figures 1 to 3 of this review are based on a standard template established by Cochrane Metabolic and Endocrine Disorders.

\section{INDEX TERMS}

\section{Medical Subject Headings (MeSH)}

*Behavior Therapy; *Body Mass Index; *Exercise; Combined Modality Therapy; Overweight [diet therapy] [^therapy]; Pediatric Obesity [diet therapy] [*therapy]; Quality of Life; Randomized Controlled Trials as Topic

\section{MeSH check words}

Child; Humans

Diet, physical activity and behavioural interventions for the treatment of overweight or obese children from the age of 6 to 11 years 